

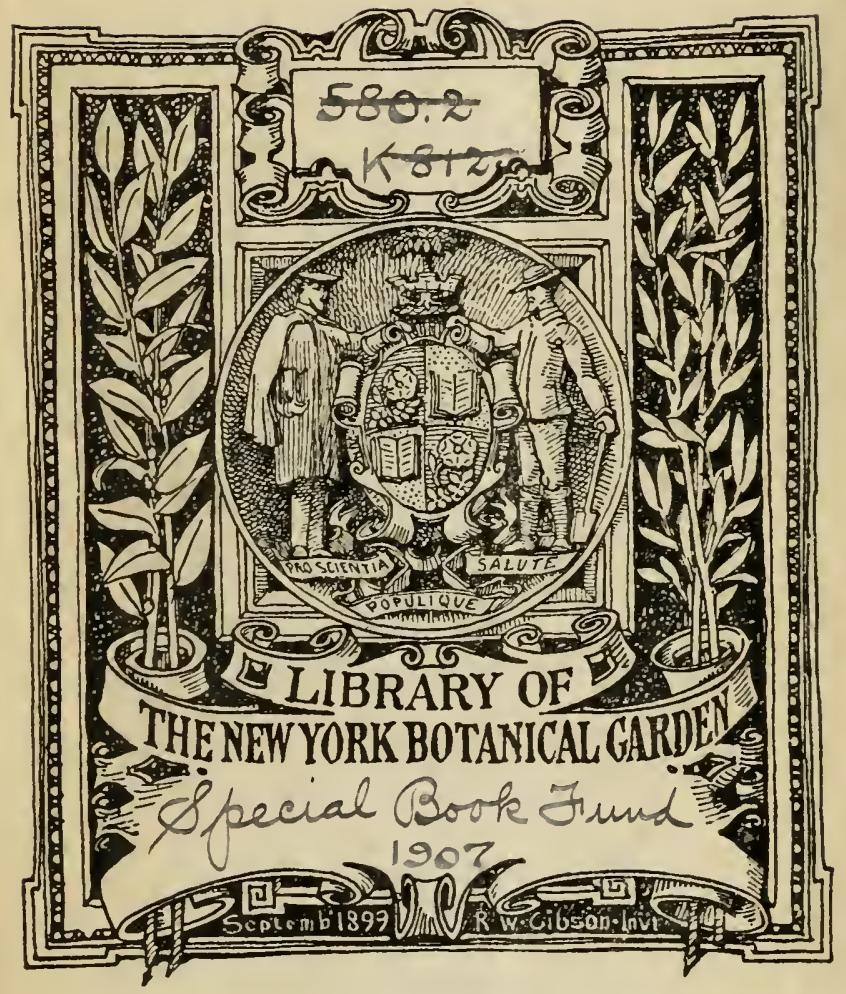




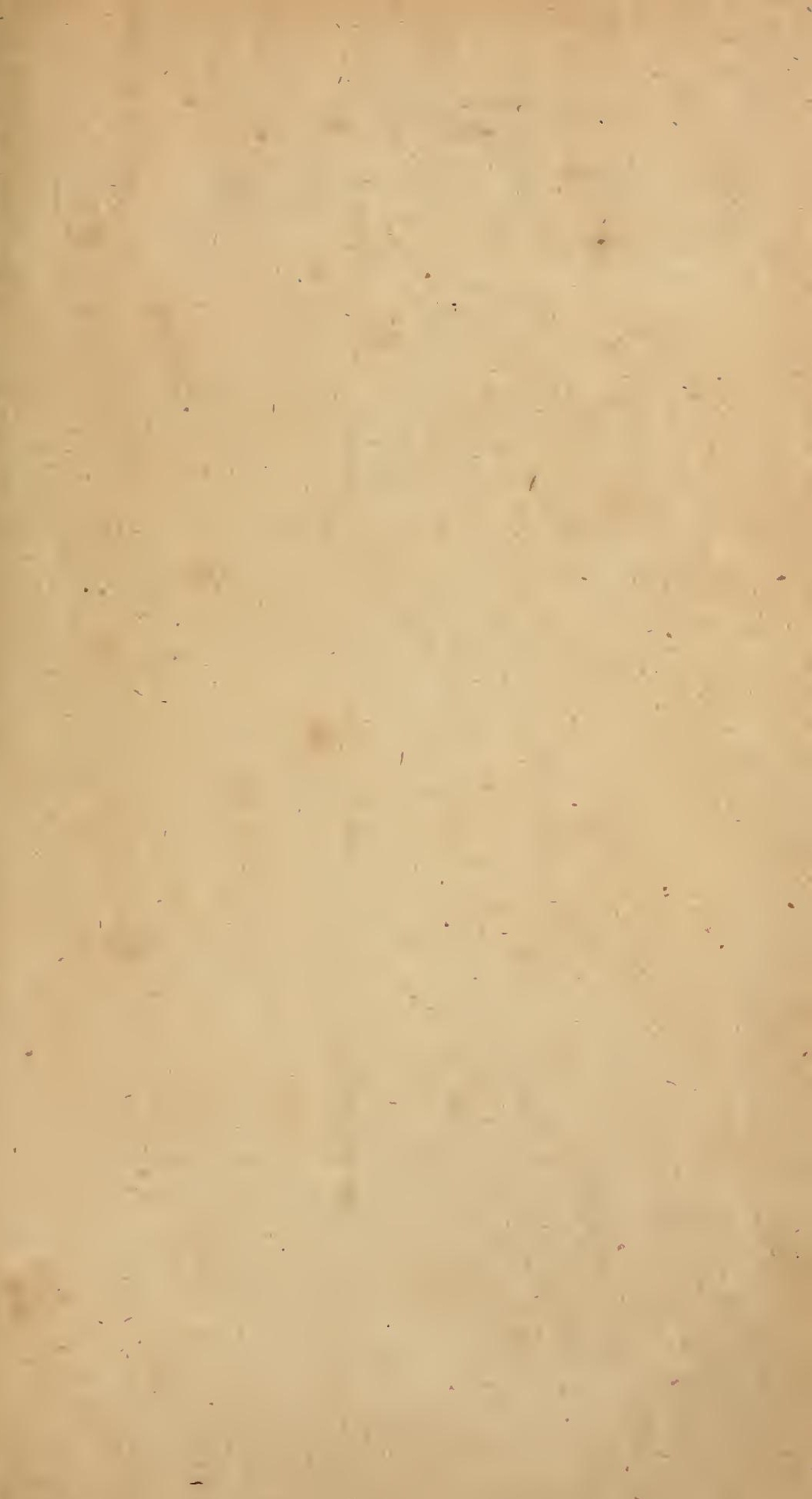




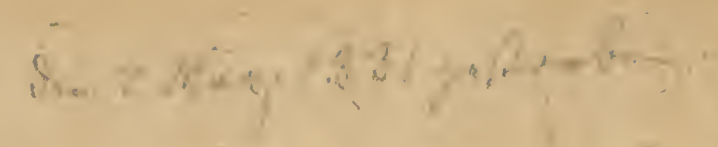




\section{$\mathfrak{B}$ o $\mathfrak{t} \mathfrak{a} \mathfrak{n} \mathfrak{i} \mathfrak{i d} \mathfrak{e} \mathfrak{B}$}

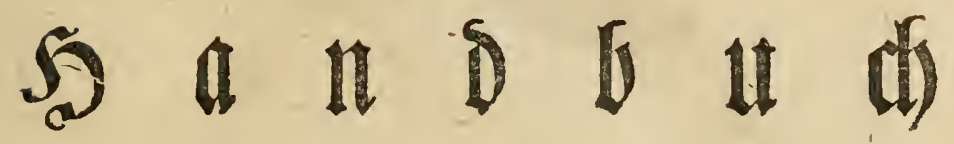

$$
\text { fum }
$$

Selbfunteridat

füs

Dentide Riebfaber ber gfimzentemose

í $\mathfrak{c} \mathfrak{x} \mathfrak{b}$ a

ano fiir

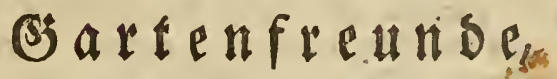

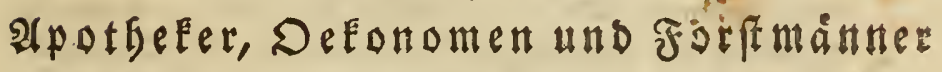
inz $\mathfrak{b} \in \mathfrak{e} \mathfrak{o n b} \mathfrak{e r}$

entholfers

oon

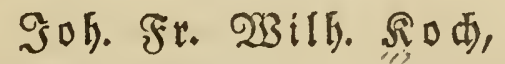

Eonfiftorial = uns Sdulraty in פagbeburg. .

\section{Errfter Theil.}

Die im Freyen ausbautrenden \$flanzen ber erften 23 Sinneiftjen

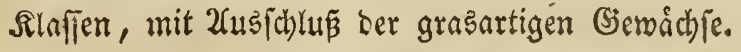

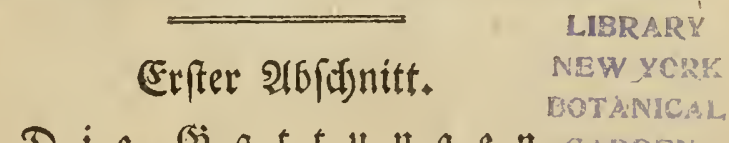

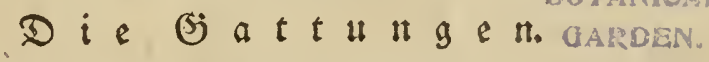

Dritte ganz umgearbeitete 2 uffage.

$\mathfrak{M} \mathfrak{a} \mathfrak{g} b \mathfrak{e} \mathfrak{b} \mathfrak{x} \mathfrak{g}$

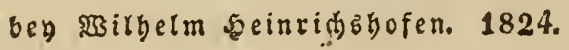




\section{Seinem godgeaditen Freunde,}

Detr

Greunde bet Ract: und ber Pflanzenfunde,

Dem eifrigen Beforberet thete Jienntnia

$$
\text { uns }
$$

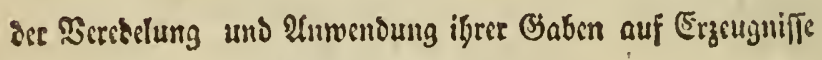

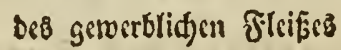

gerviomet,

bon

bet Berfaffer. 


\section{1. \\ $30.10 \circ x$ t. \\ LIBRARY \\ NEW YORK BOTANICAL QAROEN.}

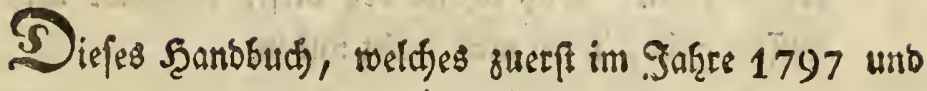

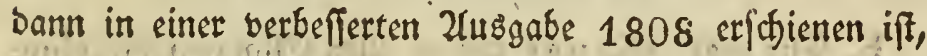

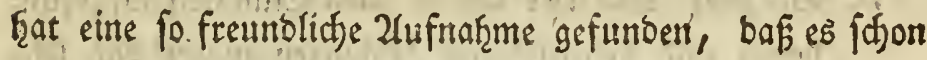

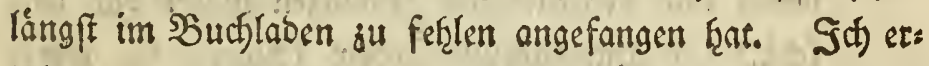

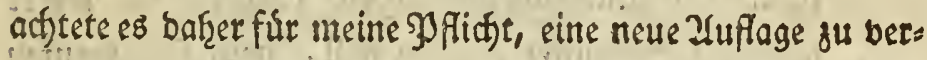
auffalten, und biefe wurtoe fdjon lantgf erfotienen fenn,

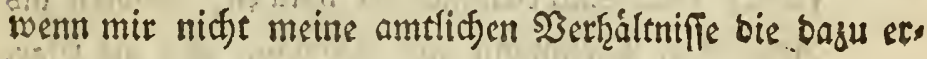
forberlide Mhife nur farajam verffattet hăatten.

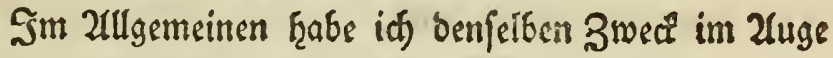
bef̧alten, welther bert borigen Zlusgaben g̈um Gronto lag:

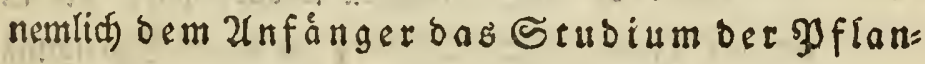
zenfunde baburd zuerleiderern, baf id folgenbe

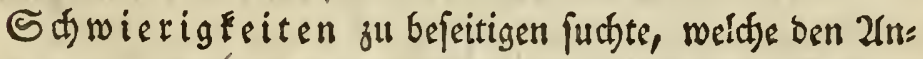

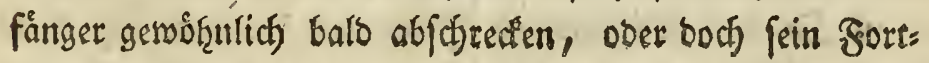
fhreiten aufhgalten.

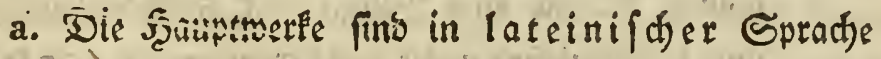
gefdricben. SWerm biefe gleid vor allen bajil geeig: net ift, fo madyt fie bod bie Yyflansertwelt Denen ju 
Tarmhauşplanjen ganz ausgefchloffen morben. Denn

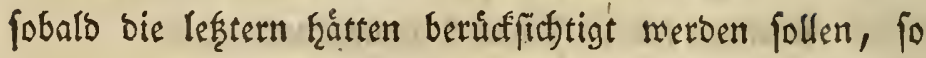

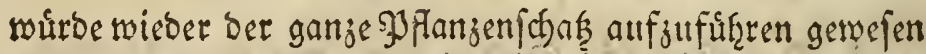
fenn, weil bie Sartenfunf fie Zlle treiben Fann, uno bie vother bemerfte jwente Schroierigfeit màre boir neliem ents ftanden. Daher finbet man f̧ier nur 811 Sattungen uno von biefen mur 5028 2rten aufgefúf̨rt. Dafs iaf fur bie

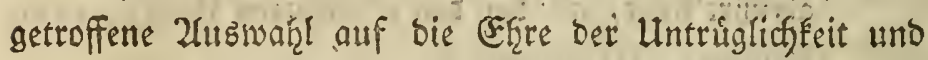

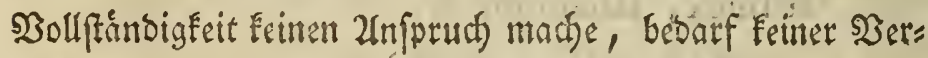
fidferung.

Die SBefđreibung ber Planjen ift burcfaub ges ånbert unb beriftigt, unb Jt biefem 3roef:, fo weit bie

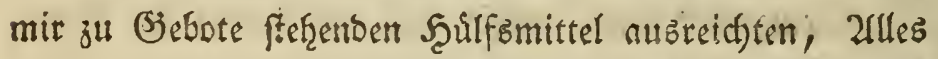
bennf̧t, momit bie verbienteften \$otanifer unfrer - Seit bie Wiffenfd)aft bereidjert hraben. Sieles verbanfe idh.in bie:

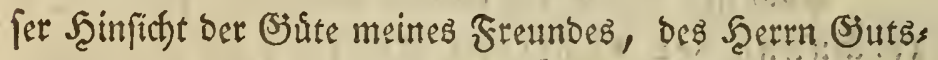
befthers Nathufius, welder mir Den freyen (Sebrauch feiner, vorguglid in allen 3reigen ber ?aturmiffenfd)aft, reichen Búcherfammlung geftattete, uno beffen Sjartenan= lagen, befonders in ofonomifarer uno forftbotanifacter şin= ficht, ungemein fefrenzmerth unb lef̧resid) fint. Son bent

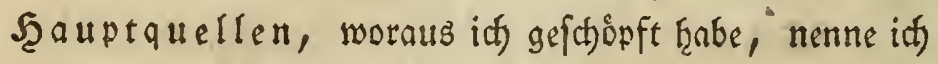
Gier nur, aufer C.L. Willdenow spec. plant. Berol. 1797-1805. - Deffen enumeratio plantarúm horti regii berolinensis. Berol. 1809. - H. F. Linck enum. pl. hort. reg. berol. altera. Berol. 18 g r. - C. I. Persoon synopsis plantarum. Paris 1805 - 7. - II. A. Schrader flora germanica. Tom. I. Gütting. 1806. - J. J. Roemer \& J. A. Schultes 
systema regetabilium. Vol. I. - VI. Stuttgardt. $1817-20 .-5$. S. Scanne benorologifthe Flora.

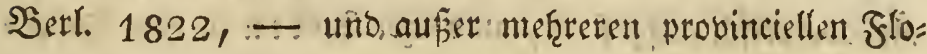
ren ben hortus Bréitherianus, - Belvederanus, Carlsruhanus : $c$.

(Enblid) in 2rbfint anf bie 2(norbrung mirb man bey ber 2 ergleicfung mit ber vorigen 2lusgabe finden, ba

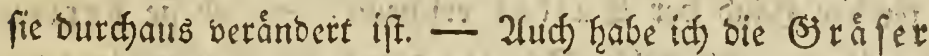
unt grabattigen Sienádj) von ben ubrigen abgetrennt unb einem befonoern Bändfen borbef̧alten. Dieje unterfecte

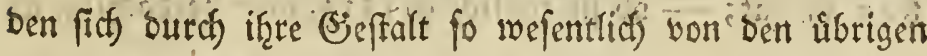

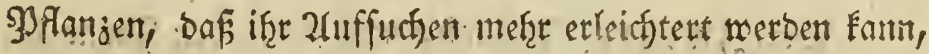
wenn fie jufammenftefien, als wenn fie mit andern ver: mifett find. 2fud) nef̧men die meiften S3artenfreunto vont

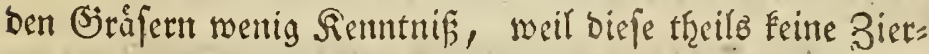
pflanjen, theils fdwerer aufjufinden fino, nogegen fie fút ben Defonomen ein ganj befonderes Sintereffe ḩaben, wel:

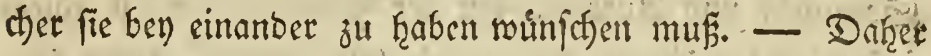

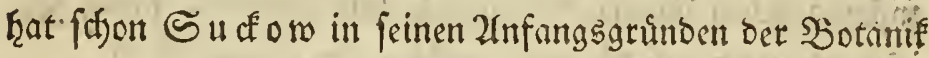
(Reipjig 1797) fie ebenfalls in einem befonbern SBante aufgeftellt, fo wie bief and in bem neueften botanipwon

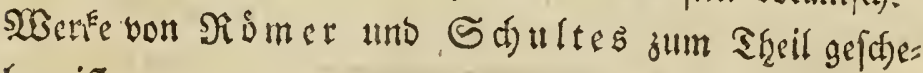
hen iff.

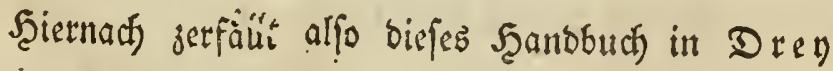
Theile.

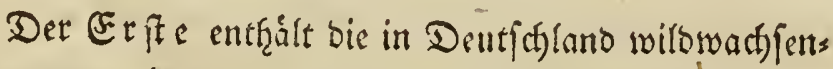
bêt, ober burd) Sultur in Freven ausdaurenden, ஒflat:

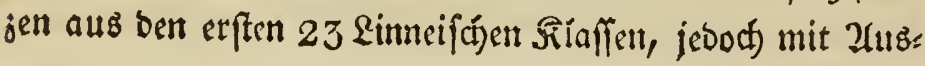




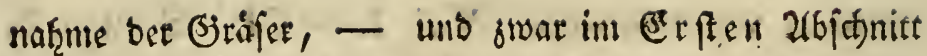
die (S) attungen. Sm 3 weyten abjunitt fint biefe alphabetarifh aufgeftellt, umb. Ifre. Arten in. eine Sabells gebradt uno ben jeoer jugleid) if̧re Dauer bemerflich ges madte, ifre $\mathfrak{J a t e r l a n d ~ u n d ~} \subseteq$ tandort, ifre Farbe, ifre.(J) erud); - ferner ob fie in Dentfhlanto, und auch

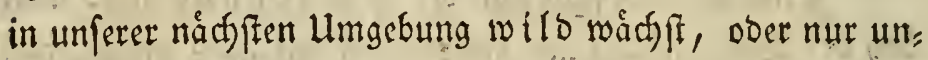
ter ber. Seand ber E nell ift, und weldje S fqeile davon als 2rannenmittel be

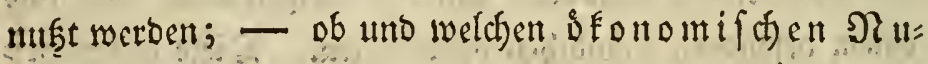
Gen fie hat; - ob fie endich giftig, ober werbadstig, ift.

Der 3 weyte. Sfeil wirs nach demfelben झlane bie Gattungen uno Zrten, fowoht Der biráfer, als ber cryptogamifden Siewådjfe, entralten.

Der $\mathfrak{D}$ ritte Sheil endich enthåalt eine Unleitung jur Senntnif bes Linneifhen Enftems, zum Unterfuchen,

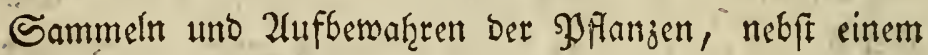

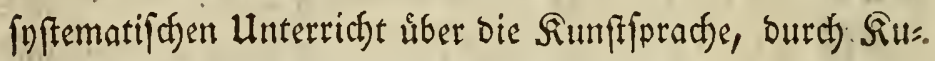
pfer erláatert unb bie erforberfict)en æegifter. 


\section{II. \\ (5) $\mathfrak{r} \mathfrak{\mathfrak { a }} \mathfrak{r} \mathfrak{u} \mathfrak{n} \mathfrak{g}$. \\ A. Der Beiden.}

1. Eit In ber Sabelle mit lateinifater Gdrift auf: gefúţrter Sy/anjenttreil beutet an, baf́p bie barauf fol= genden Unterabtheilungen bie nåfzere Seftimmung bef= felben entḩalten, 3. 3 . S. 1. unter B. bie "SBlumen Decfe" if entweber "tungetfecilt" ober "8 paltig" uno im erften Fall entweber ifretrelformig" :c. ober "/gant Fleitr."

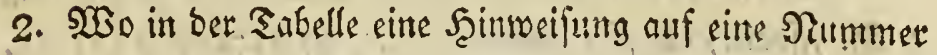
ftef̨t z. SB. S. 1. "8 fpaltig ( $\{.79) "$, da iff bief ẹin Zeidfen, baf biefe Ejattung cigentliff nicht fhierter ges

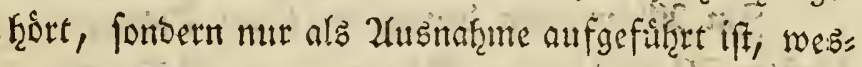

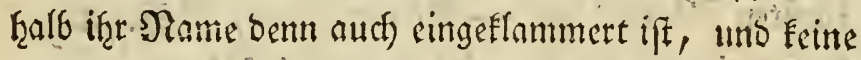
fortlaufento Nummer fૃat. Die weitere B̉eforeifung Derf̧elben f̧at man alfo unter ber allegirten ఇummer zu ju(t)en.

3. Die mit fádåger uno fleiner Estift geoututen

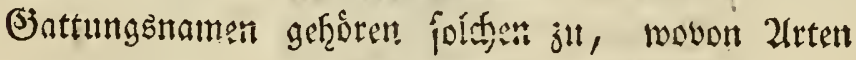

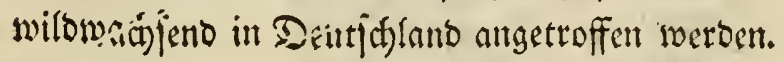

4. Die mit geraber uno gróferer Sdjrift futo laus ter Culturpflanjen.

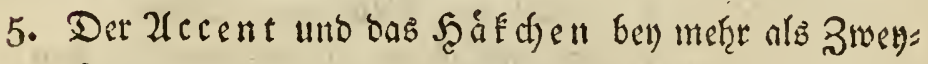
fnlbigen Namen beftimmt bie ridftige 2utsprad)e, jener, baf die Sylbe betont, - diefes, bafifie 
Furs ausgefprofien merben foll. 230 sin Doppeloocul zu betomen ift, ift ber 2lecent auf Den 3roijhenraum Şevoer geftellt.

6. Die mit einem h. Ķaben 2(rest, reldje ben uns nur als Såume, ober Stråuder, erfadsinen.

7. Die mit einem † fino $3 a$ ferplanzen, alfo nue an

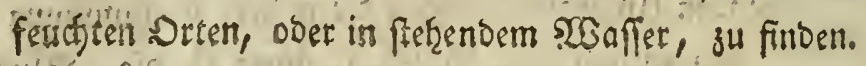

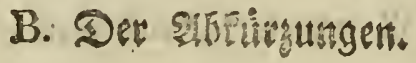

Die mehreften bedirfen Eciner Erflärung. Dent baf 3. 2B. "fipalt," theilo, mosutl" xe Praltig, theilig, unoeutria fzcife, ift von felbft flar.

Sin Zllgemeinen beutet eir $f$ am Ende cines abge:

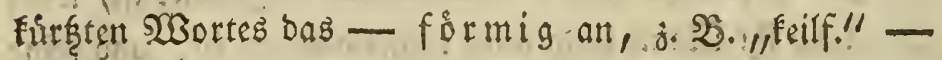
Feifformig; aufer in "einf." — sinfadf. ... "n!

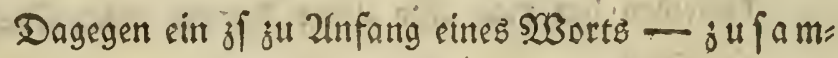
men j. SB. "s[jeneigt" - zufammengeneigt:

"2lfterfab." 2afterfaden

"Balgkapy." — ß̊ilgkapfel

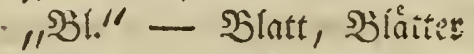

"ふ̧l6b." - SBlumenboden

"Şlbl." - Shlumenb!att, SBlumenblátter

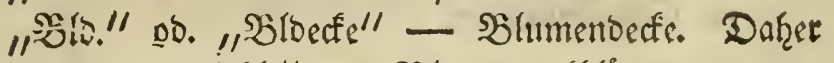
"SBlobl." - SBlumenoectblätter "Blobláttct)." - Blumendectbláttchen "B̉iblapp." - SBlumenbecflappen "Blojdupp." - Blumenbedfjuppen :C. 


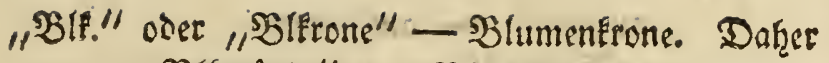
"Blfmúno." - Blumenfronmúnoung

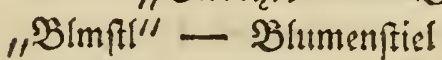

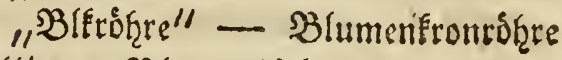

"B̉iftaub" - Blumenftaub

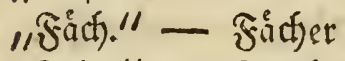

"Srtbo." — Frut)tbober

"frudt!nn." - Frudtifnoten

"(Sefd)l." - (jefdlectit

"Giriff." — Bjriffel

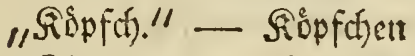

"Sifrudft" - Siernfrudt

"M." - mámliche ß̉lutţe

"Mauno." - Múnount

"Nebenbl." - Rebenblatt, blåtter

"Nect." - Nectarium

"פlectb[." - Nectariumblatt

"Dlippe" - Dberlippe

"Suerm." - Duerwano

"S." - Saame

"Scheibebl." - Scheideblatt, blatter

"Sfapf." - Saamenfapjel

"Sfrone" - Saamenfrone

"Şpreubl." - Spreubláttchen

"ভ́tbeut." - Staubbeute!

"Stfab." - Staubfaden

"Stgef." - Staubgefäfe

"Stfr." ober "Stfrud)t" - Steinfrudt

"Stw." doer "Stweg" - Staubweg

"Ulippe" - Unterlippe

"W." - meiblicbe Blutthe

"Z." - Bwitterblutţe. 
"aufleg." - aufliegeno

"auffpring." - auffpringento

"aufio." - aufwårts

"ausgeranto." - autsgerandet

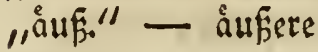

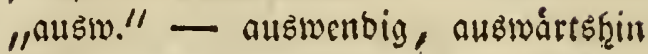

${ }_{1,6 e j . "}$ - Eejonbere

"Daur." - Daureno

"einf." - einfací

"ellipt." - elliptifch

"entgegengef." - entgegengefeşt

"eñr." - evrund

"féct)." - fádgerig

"fruotitb." - fructitbar

"gefalt." - gefoltet

"gefied." - geficoert

"geflug." - gefugelt

"gem." - gemeinfabafticf

"gettr." - getţeilt

"gemimp." - getwimpart

"glånj." - glànjento

"gleichf." - gleidfformig

"groff." - grófer

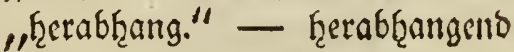

"fiurj." - Furjer

"ob." - oberer

"paarm." - warmeis

"rúctim." - rúcfmárte

"úberf̨ang." - ưberḩangeno

"unfruct)tb." - unfrutstbar

"ungettr." - ungetţeilt

"unģl." - ungleid)

"sungleidff." - ungleichformig 
"unc." - unterer

"verf." - berfehret

"bertrodn." - bertrodnens

"verwadbf." - vermadyfen

"med)f" - wed) felswocisftef̨eno

"उfgef." sufammengefét. 


\section{III. \\ Ueberfitht Der Rinffen.}

Die Slitthen fins

1. 3roitterblisthen: Staubgefáfe u. Staubivege

in Eintr 2lume vercinigt; - Die Stnubgefípe fint:

A. Inverwadfu (frey)

1. von gleidger Range. Man ziiglts

Silafie. @rite.

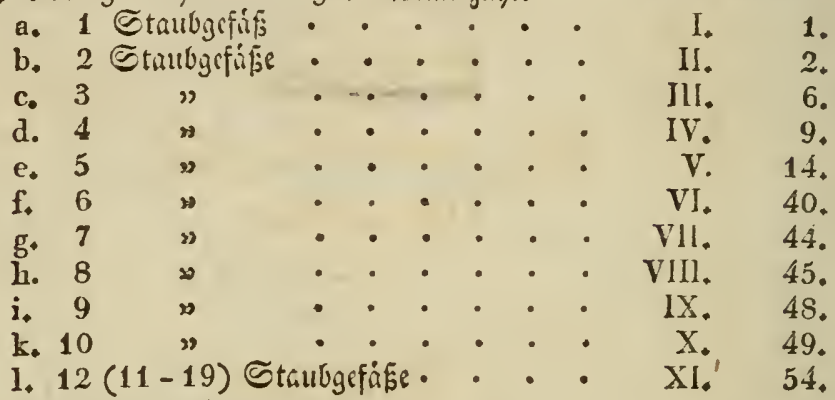

m. $201110 \mathrm{mel}) \mathrm{s}$. • •. .

a. allf ber innern Flache D. Bl ldecte . XII. 57.

b. auf Din Blumenboden - X XIII. 60.

2. you ungleider Iánge:

a. 2 länger, alb bie 2 andern i . - XIV. 64.

b. 4 " . . XV. 70 .

B. mit cinander verma

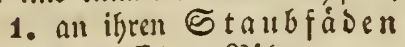

a. in 5ine giofre • • • • • • XVI, 74.

b. in §wey \artfien (1 fren, bie úbrigen verwadien) - . . . . XVII. 77.

c. in Refrere Partfien - - - XVIII, 82,

2. an ifren Etaubbeuteln. - - XIX. 83.

3. mit ben ఠtaubivegen •. •. XX. 93.

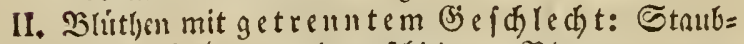

gefáfe u. Stáuburge in verichiedenen Slumen, u. zmar

1. nuf Einer \$flanze (balbgetremut). • . XXI. 94.

2. auf verfdiesenen PFlanzen (ganzgetrenut) XXII. 100.

3. getrentut uno vereinigt auf Derfelben

ญflange (yermengt) • • • • • XXIII, 106.

Ingang. Tabelle fitr blos meiblidge Blittyen - - 108. ネegifter • . . . . - 117. 


\title{
I. Rlafle.
}

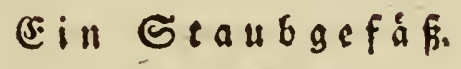
(Monandria.)

\author{
1. Oronung. \\ Ein $\subseteq t a$ u $b$ weg.
}

A. 9) (it 2 18. น. 1 blättr., 5 lapp., gleich).,

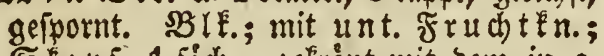
Skapf. 1 fảh., gefroont mit bem in $e^{\text {. }}$ feber. Sfrone verwandelten Blorano, Centránthus;

B. ofne $\mathfrak{B I}$ r.; Bldecke:

1. ungetheilt,

a. Ereujelform., faft.; ST $\mathrm{r}$ iff. 2 fpalt.; 2.

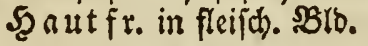

Salicornia.

b. ganz Elein; (5) riff. in ber Furche $\delta$.

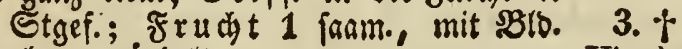
oben geránbelt

2. 8 fpalt. (F. 79.)

Hippuris:

(Alchemilla.)

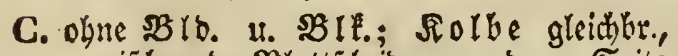
zwilchen $\delta$. sBlatticheibe, an $b$. e. Seite frudttrag.; Stbeut. Dem Frudhten. enta

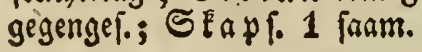

4. $t$.
Zostera.

211 


\section{Dronung.}

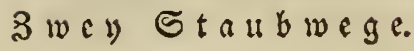

Blumenkrone:

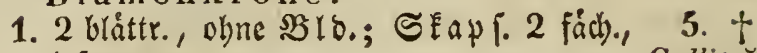
4 fanm.

Callitriche.

2. Fefilt; Bld:

a. 1 blått., 3 fpalt.; 1 S. in beetart. 6 . şlo.

b. 2 blåttr.; 1 S. oval; erhaben = flach, nadt

Blitum.

7.

Corispérmum.

\section{RIalfe.}

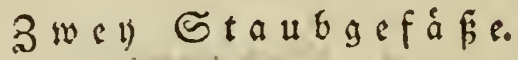

(Diandria.)

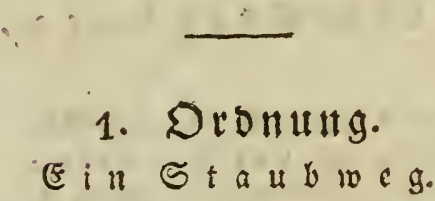

1. Mit Blumenkrone;

A. oberer Fruchtknoten;

1. Blk. I blättrig;

a. Blk. gleichförmig.

Blumenkione:

A. 4 ppaltig;

1. mit Stein fruds $t$; Bld:

a. 4 zabnig; Nufsschale:
a. Enodenbart; Stfrucht $1-2$ fád).; 8. $\hbar$ 1-2 jaam.; Slltapp. faft enr. Olěa.
b. papierart., 2 fádj., mit cingeln. S.; 9. St f $\mathrm{r}$ u d t $t$ beerart.
Phillyrĕa.

b. 4 theil.; SIltapy. gleichbr., lang; Ru $\bar{\beta}$; d) ale fnodenbart, geftreift, 1-5 faam.

10. $\hbar$

Chionánthus. 
2. mit 2 făd. Becre: Fà hater 2 faam.; 11. 万 Brb. 4 jabur.

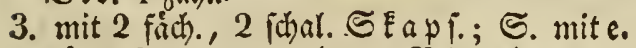
háut. Rambe umgeben; $B$ Ib. 4 jabn.; $S B$ IE. trichterf.

B. 5-8 fpalt., tellerf.; B̉eere 2 mopf. ob.

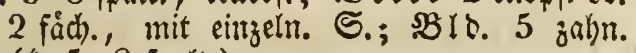
(4-5-8 patt.)

$12 . \hbar$
Syringa.

Jasmĭnum.

\section{b. Blk. ungleichförmig;}

a. mit Saamenkapsel.

Blumendecke:

\section{1 blåttrig;}

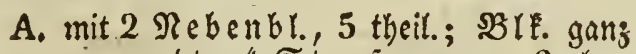
umigewandt; $4 \mathcal{S}_{\mathrm{tg}} \mathrm{f} \mathrm{f}_{\text {., }}$ wowon 2 okne

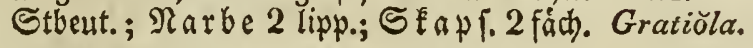

B. ohne ?lebenblatt, uno

1. gleidhf.; Blk.:

a. Saum 4 theil.: Der unt. \&app:

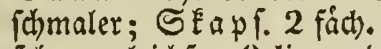

b. Fefhr ungleidhf., 2 lipp. od. rachenf., mit getheilt. Ul 1 ippe; $\mathfrak{B} 10.5$, felten 4, theil.; Et tbeut. 2 fách.; 16. 方

S. nit Erummen Epiken Justicia.

c. 2 lipp., ohne Sporn:

a. Slippe Eurz: Urippe bart.; B 10.5 theil.; St gef. unter $D$. Slippe angeorúct, jigeneigt; obere Skap i. 2 fách.

b. Ulippe aufgeblajen, fajubform.; Blbefe 4 theil.; SEapf. mit 2 ipalt. Sthaten

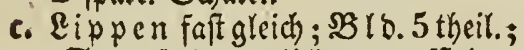
Stgef. in D. ulippe auffteig.;

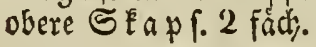

15.

Veronica.

\section{7. \\ Wrulfenia:}

18.

Calceoları̌a.

19.

Paederóta.

2. 2 lipp., 5 fpalt.; $\mathfrak{B}$ 住. rachenj., mit 20. Eporn; SEapf. 1 fåch.

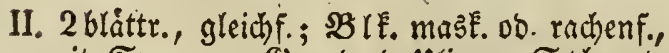
mit Sporn am Sirunde b. Ullippe; Stbeut. an b. immern Epize d. Stfáb.; Rarbe

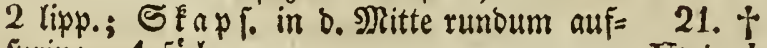
fpring., 1 fåd

Utricularĭa. 
b. mit Schote.

SBlt. 5i fialt., becherf.; SBlf. unten bauth., oben 5 fpalt., gloctenf.; Ectyote 2 fácth., mit b)åut. = seflugelt. $\subseteq$.

\section{c. mit unbedeckt. Saamen.}

(Blüthen in Quirl)

Blumendecke:

1. rơhrig; Blk: :

a. robkr., faft gleichf., 4 ipalt.: ber ob. Iapp. breiter und ausgeruno.; 3 Is. 5 ipalt.; Stgef. v. cinander abftehent; 4 S. an o. Spisce eingeorutut

23.

Lycŏpus.

b. rachenf.; S lippe gleidhbr., b. Stgef. verbúllento; SB ro. gejabnt

24.

Monàrda.

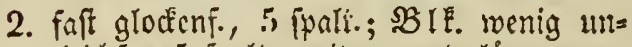
gleichf., 5 fpalt, thit c. unt. langern u. in o. S)Rtte vertieft. Iapp.; 4 hóder. $\subseteq$. Amethystèa.

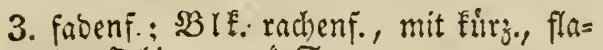
dher Solipue; 4 厄.

26.

Ziziphŏra.

4. 2 lipp.: Oberlippe d. Bld.:

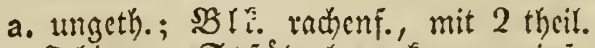
Slippe; Stfáb. lang, frumm, cinj., mit e. Babn

$27 . \hbar$
Rosmarínus:

b. 2-3 zabn., linterther ungeth.: Ulippe 2 ipalt.; bie ganze.3̧ Is. faft glodenf.; BYł. rachenfơrm.; St fád b. mite. Szuer= faben, auf belien unterm Enoe e. Drúpe น. auf b. obern 8. Stbeut.

28.

Salvŭa,

c. 3 zafn.; Urippe b. SBIf. vielipalt, haarf.; 4 S., roovon 3 yor D. SReife abfallen

Collinsonùa.

\section{Blk. 2 blättrig.}

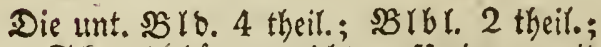

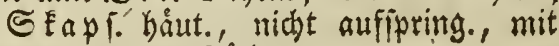
zwey 1 paam. F̌ád.

30. Fontanèsia. 
3. Blk. 4 blättrig.

Bl umendecke:

1. 1 bláttrig;

a. mit ङanmenfapjel (j. 378.) (Lythrum.)

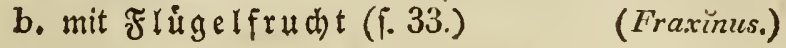

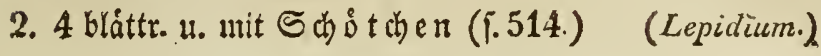

B. unterer Fruchtinoten:

Blumendecke:

1. einfact),

a. 1 blättrig (i. 36.)

(Fedia.)

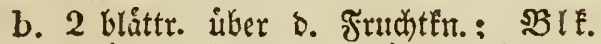
2 blåttr.; ऽfapl. 2 fích., nicht alls 31. fpring., mit 1. fuam. F́ách), Circa'ea.

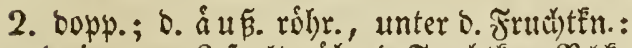

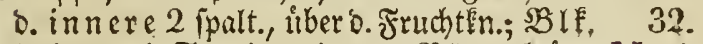
2 lipp-; 1 S. mit D. imnern sir. getrónt Morina.

II. ohne Blumenkrone.

1. Scautfrutbt in fleifh. Bib. (f. 2.) (Salicomăa.)

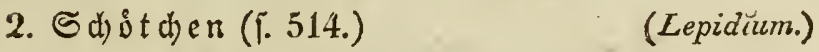

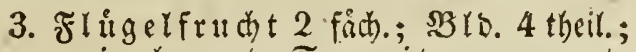
wenige hangende ङ.; mit vermengt. (3) effl. (f. 807.). Fraxunus.

4. SEap f. ůber ber 1 bråttr. SB Ibedé, 34. † 1-2 fách., 1-2 jaam.

Lemna.

III. ohne Blumendccke.

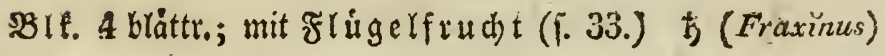


2. Dronung.

$$
\text { నwey } 5 \mathfrak{a} \mathfrak{u} \mathfrak{b} \text { we g e: }
$$

1. Mit eigentl. Bhluthe (i. 2S.) (Salvià.)

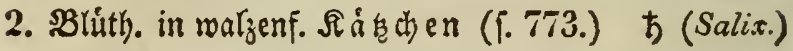

\section{Oronutig.}

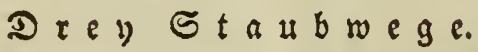

BIb. 1 bláttrig;

1. Flúgelfrudt 1 jaam.; $B$ lb. 5 theil.; SBIE. 5 blättr.; (S) riff. an b. Seite b. 35. Fructitén. (โ. S00.)

2. Skapfel (i. 380.).

Aîlànthus.

(Euphorbía.)

\section{RIafle.}

Drey Sta

(Triandria.)

1. Oronung.

E in $\mathfrak{S} \mathfrak{t} \mathfrak{a} \mathfrak{u} \mathfrak{b} \mathfrak{w} \mathfrak{e}$ a.

1. Mit Bldecke u. Blkrone;

A unterer Fruchtknoten.

Die 1 blättr. Blkrone:

1. 5 ppalt., am Şrunbe auf e. Seite hơdr.; Bld.:

a. 3-6 zahn.; $2 \mathbb{l}$ lt. ungleidh 5 palt., ohne Sporn; Sḱapf. mit baur. SBlb. 


\section{3 Staubgefáze.}

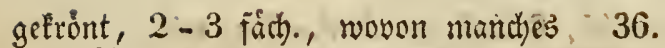
obme ङ. Fedia.

b. e. bloper nath innen gerollt. Rant auf D. Frudttfn. u. ben ber Reife in eine Efrone fid) verwantelno; Skap 1 fä́ch., mit e. feber. Skrone

37.

Valeriàna:

(Galüum).

(Asperüla.)

2. rabformig (f. 65.)
3. róbr. = triđterformig (f. 67.)

B. oberer Fruchtknoten.

Blumendecke:

1. 1 bláttrig, faft 5 theil., mit am (3rumbe 2 jahn. Lapp.; $3 B$ lé. 5 Gláttr., gantz Hlein, j̧geneigt; Skap f. 1 fächerig, 3 idjal., vielfaam.

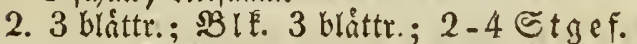
obne Stbeut., mit Freuzmeisftelyenoen Driten; Skapf. 2-3 fách.; S.an D. Ectalen geheftet

3. 4 blättrig (i. 514.)

38.

Loeflinğ̀a.

\section{II. ohne Blumenkrone.}

Mit obern. Frudtenoten;

1. mit gem. SBlboben, Freujerf. ob. Eu= gel., fleifh., Dured) zigeneigte Sdhupp. geithloft, morit die Slitthen; mit ver= mengt. (5) 5 h) r. (i. S0S.)

2. mit eigentl. Blumendecke:
a. 1 blattrig (i.170.)
b. 5 blåttr.; $1 \subseteq$. in bautfruchtart., fonfr. 'Ekap.
(Thesüum.) 41.
Polycnémium.

39.
Commelína.
(Lepidhum.)

40.

Ficus.

\section{III. ohne Bld. u. mit Scheide:}

I. Mit untern $\mathfrak{F}$ rudbt $t$ noten; bie 1 blättr. Blkrone:

1. faft gleichf., trid)terf., am Saum getbeilt,

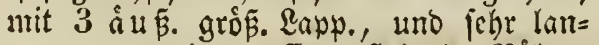
ger, unten in $b$. Eroe ftehent. SRohre; Sa)eide meift 2 fatal, wovon die ins nere viel idmaler; $\mathfrak{R a r b e}$ tief 3 ipalt., mit jigerollt. Eappen

42.

Crocus. 
2. gang gleichf., 6 theil., glodenf.=offen, mit geraber Róbre; Sheibe 1-2 ioral.; 3 Rarben etwas aufr. und abftebeno; viele runbl. S.

Ixı̌a.

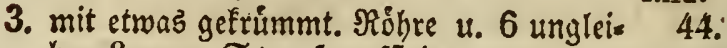
đen $\mathfrak{L a p p}$; Stger. auffteig.

4. mit 6 wedhf. zurudfeichlag. u. mechl. zigegeneigt. Lapp.; (S riff. fehlt; $3 \mathfrak{R a r b e n ~} 45$. blblattart.

5. offen, 6 theil., mit 3 innern Eleinern Iapp.

Iris.

Gladiơlus.

46.

Mora'ea.

II. mit Dbern FruׁtEnoten (โ. 540.) (Sisyrinchuัum.)

\section{Dronung.

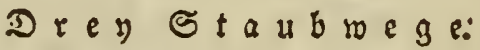

Mit obern Frudtfnot.; Blk.:

1. 1 blättr., ungleichfi.; 3 18. $2-3$ blăttr.; 47. f

Ekapr. 1 fách., 3 ídalo, 3 jaam.

2. 3 blàttrig (f. \&6.)

3. 5 blättrig ; Bld.:

Montia.

(Tilla'ea.)
a. 1 bláttrig (i. 245.).
(Alsine.)

b. 5 blättrig,

s. 5 feitig; $B \mathfrak{B} \mathfrak{b l}$. Pehr Flein, eyr.; Skap ₹. 1 fády., mit 3-4 lanjettf., 48. nadh innen georeht. Sdal. u. viel. S. Polycárpori:

b. nidbt; SE a p f. faft walzenf., 1 fách), an D. Spise aufipring., 6 phal., 49. vieljaaur.

4. Fehlt; Skapsel:

a. 1 fåcherig,

a. 1 faam.; $3 B$ Ib. 1 blättr.; 5 theil. 50: ob. 5 blättr.

b. mehriaam., 3 低alig; sB Ibede 51 . 5 bláttr.

b. 3 fádherig, 3 ichalig; $B$ ibede 52. 5 blăttr.

Mollúgo.

\section{Dronung.}

Fủnf Staubrege. (₹.35.) ち (Ailánthus.). 


\section{Rilafle.}

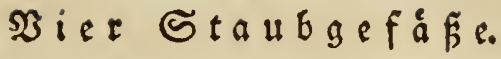

(Tetrandria.)

- 1. Orontug.

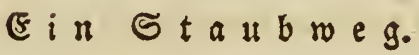

I. Mit Bldecke u. Blkrone;

A. Blk. I blättrig;

1. Frucht Isaamig;

a. Saame unbedeclit;

a. oberer Fruchtknoten.

Die gem. SBrb. Dadjziegelf.: b e f. Mro. rỏbr., 5 gुahn.; Nilippe b. SBlk. 2 theil.: Ulippe 3 theil. (auth 3-4 ipalt.); 1 S. in BIb.; $B$ Ib . fpreuart.

53.

Globularǐa.

b. unterer Fruchtknoten.

1. Mit vielblåttr.; gem. SBIb.; obere bef. Bld.:

a. cinfach; ; 1 S. mit $\mathfrak{B}$ ro. gefroont; Blbd.: ค. \{preuart., Eegelform.; $B$ l'E. rỏbr., 54: 4 fpalt.; Sk rone becherf., ungeth. Dipsăcus.

b: nađát; gem. SBlo. walzenf.=långl., 55 . 5 blủth.; $\mathfrak{B}$ İ. ungleich).

b. bopp., ffronförm., veridicdengefpalten; $\$$ IbD. Tpreuart.

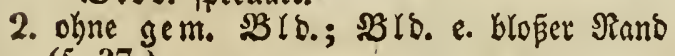
(1. 37.) Knauť̌a. 56. Scabiósa. (Valeriána.)

b. Saame bedeckt;

a. oberer Fruchtknoten

u. Saamenkapsel.

1. Mit gem. Eugel., haar. SBrbo., obne

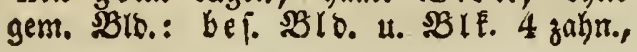


róbr. = trichterf.; Sf ap [. 2-4 fidh., nicht alfipring., mit cinjeln. S., wowon nur 1 frudbtb. ul. lántyl.

2. mit eigentl. einf. Bldecke:

a. 1 bláttrig; Blkrone:

a. rabformig (F. 175.)

b. unten rỏhr., oben welfeno (i. 177.) (Gentiána.)

c. róbr., 4 fpalt., mit abftebeno. Saum; Blo. 4 ipalt.; Stgef. Eurz; Skap 1 fách., in o. Mitte runbs um aufipring., vielfaam.

Cephalánthus.

\section{(Swertia.)}

58.

Centuncùlus.

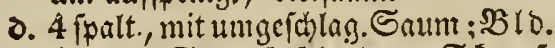
4 fpalt.; Stgef. Febr lang; Stapl. 2 fád)., in o. SMitte rumbum auf= pwring.

b. 4 bråttr.; sBre. faft glocéenf., 4 fpalt.,

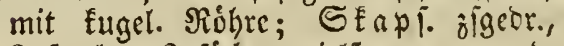
2 furch., 2 fäch., vielfaam., an $b$. Epitze auffpring.

\section{9. \\ Plantágo.}

\section{b. halboberer Fruchtknot,}

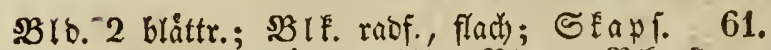

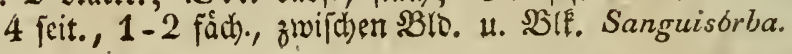

\section{c. unterer Fruchtknoten.}

1. Beere 2 fpalt., 4 faam.; 2 viertheil. SB 10. auf 1 Frud)tfn.; $\mathfrak{B}$ IE. trichterf., inm. rauch; 4 Mlarben

2. Skapiel (i. 63.)

3. 2 ob. 4 S. vor ber Reife beredt, binter: her nadt (1. 471.)

62.

Mitchélla.

(Houstonĭa.)

(Verbéna.)

\section{Frucht 2 knopfig.}

1. Mit obern Fruchten.; $\mathfrak{B l}$ lo. 5 zahn.;

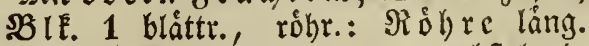
als Blo.; 4 SEIElappen abftebento,

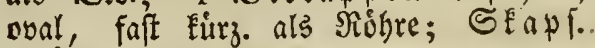
2 fách., 2 fold., 2 fram.

60.
Exácum.

mit ưt. $\mathscr{F} \mathfrak{r} u$ cht $t$ n.; die fternf. Blk.;

a. rabf. = faft glodenf.; SSlo. c. blö̧er unt= beutl. $4-5$ jnln. Rano; 2 Seeren 1 jaam. 
b. gang rabfórm.; 3BIb. wie 64; 2 Fugel. fi5. ธ.

c. glodenform., $3-4$ fpalt.; Frutht 3 born.

Galüun.

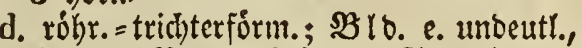

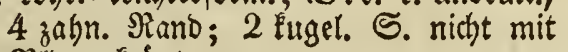
Blo. gef́rónt

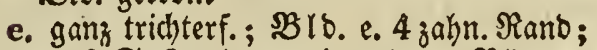
a. $2 \Subset .3$ jabn., mit o. baur. Silb. ges frónt

b. $\mathfrak{E} a p[.2$ fádherig, nidut theilbar, 2 zabn., mit 1 faam. frách.; Ranbe zigerollt

66.

Vaillantia.

\author{
67. \\ Asperŭla.
}

68.

Sherardia.

69.

Spermacóce.

\title{
B. Blk. 4 blättrig; \\ 1. oberer Fruchtknoten.
}

A. Mit Sdeibe ofme Blo. (โ. 274.)

B. mit eigentl. Bldecke:

1. 1 blattrig:

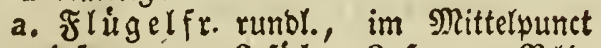
1 fram., u. 2 fách., 2 faam.; $B$ lb. 4 theil.; 31 ibl. leberart.

b. SEaplel gefárbt (i. 163.)

c. Beere 1 faam.; B Ibl. ellipt., jwithen

(Bulbocodium.) Den 4 Salojánen ftebeno; Stbeut.

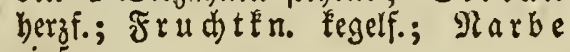
einf.

2. 4 blăttrig,

a. vor ber Eroffinung D. B̧ume abfall., ben sBlbl. entgegengef.; 4 becherf. Nect. auf b. Bibl. auflieg.; S b o te 1 fádh., 2 idjal., vielfanm.

b. bleibent;
a. mit S̄bote (i. 538.)
b. mit Beere (1. 153.)
(Cardamine).
ち (Rhamnus.)

70. 5

Ptelĕa.

ち (Evony̆mus.)

72.

Epimedrum.

\section{2. unterer Fruchtknoten.}

Die 1 blåttr. Bldecke:

1. 4 żahn. (einige mit 4 bláttr. $\mathfrak{g} \mathfrak{u} \mathfrak{l}(\mathfrak{e}) ; 73 . \hbar$ St fru cht $t$ mit 2 fåd. $\mathfrak{R} u \tilde{B}$ Cornus.

2. 4 theil.; $\Re u B 1$ fádb. , 1 faam., mit 208. 4 entgegenf. uno aus b. Blobláttch. 74. $t$ entftanbenen Dornen umgeben Trapa. 
3. faft ungeth.; B Bere 1-2 (ielten 3-4) 75. ち

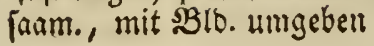

Cissus.

\section{II. ohne Blumendecke.}

1. Mit obern Frudtenoten (i. 276.)

2. mit untern $\mathfrak{F}$ rudbten.; fatt $B$ lo. e. $2-3$ blâttr., 1 blüth. Şulle; SBlE. 1 blättr., trid)terf., mit fabenf. Fỏhre;

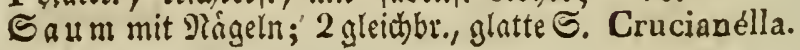

(Convallariă.)

III. ohne Blumentrone.

1. Mit obern Frudtenoten;

A. mit einjaam. Skap F.; $\$ B$ is. Exugf., mit

2 fehr Eleinen, entgegengei. u. weibj.

3ăbnen; Stgef. herwortagens

77.

Camphorósma.

B. mit unbededt. Saamen; Bld.:

1. 4 fpalt; $1 \mathfrak{S}$. langgejogen, mit SBls. beoedt; mit vermengt. (S) (1. 801.)

2. $S$ palt.; mit medj. Heinern Enppen;

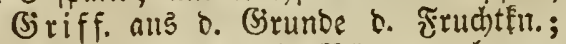
1-2 S. madt, mit sBlo. umgeber. Alchemilla.

II. mit untern Frudtenoten;

1. SEapF. 4 fáh., 4 jeit., vieljaam., mit $S 0$. t Bib. umgeb.; $B$ ID. gloffenf., 4.jpalt. Isnardia.

2. mit $\subseteq$ teinfrudt; Nufs:

a. långl., 1 faam.; Stfrubt eyr.; SBID. 4-S ppalt., gloffeñ., austoeno. ramgl., inmentig gefórbt, abfall.;

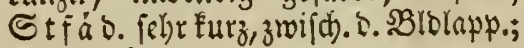
(5) riff. Fury

b. fharfgeipist, mit\$lo. bebedt (1.170) (Thesium.)

$81 . \hbar$

Elaeàgnus. 
II. obne SJifle; Bldecke:

A. 1 blattrig; Blkrone:

1. rioffertmig (i. 175.)

2. unten róbrig (ร. 177.)

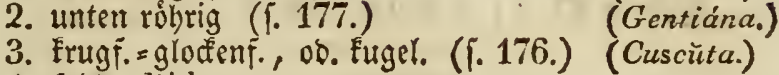

4. fehlit ; Bld. :
a. 4 zalyn. (i. 235.)
b. 4 theil. (f. 230.).
$\hbar$ (Ulmus.)
(Herniaria.)

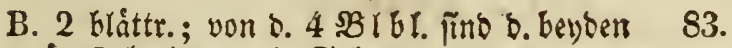

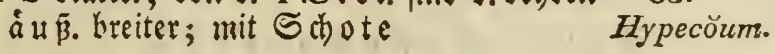

C. 4 blattrig;

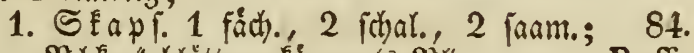

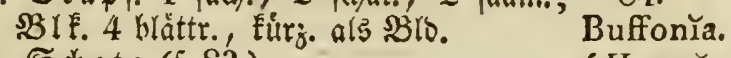

2. Sthote (f. S3.)

(Hypecŏum.)

\section{Oronung.

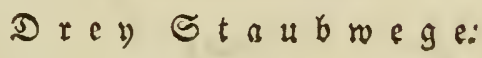

Dic 4 Blumenblätter:

1. 2 fpaltig (i. $35 \pi$.

2. ungetheilt (1.360.)

(Siléne.)

(Arenaria.)

\section{Droning. \\ $\mathfrak{B}$ i e $\mathfrak{s}$ S $\mathfrak{t} \mathfrak{a} \mathfrak{b}$ we g e.}

I. Mit SBId. u. Blkrone:

1. 1 Glättr., 4 ppalt., or. 4 thcil., rabf.; S5. 古 (3) riff. Fehlt; Beere 4 jaam.

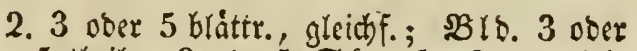
5 theil.; 3 ob. 5 Sf fapt., 2 ob. viels 86. faam., nach innen aufipring. Tilla'ea.

3. 4 blåttrig; Bldecke:

a. 1 blăttr., vielipalt.; obere SEapí 4 ob. 8 ínal., 8 fách., Eugelo, mit sins zcln. $\subseteq$.

b. 4 bråattrig; Skapsel:

a. 4 fách., 4 fachal., vieljaam. Sagina.

b. 8 fáchlorig (โ. 256.)

87.

Radiöla. 88.

(Linum.) 


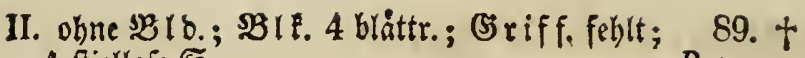
4 friellofe 5 .

Potamogéton. 90. +

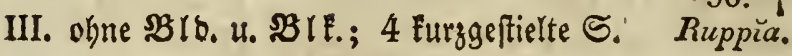

\section{Rlaffe.}

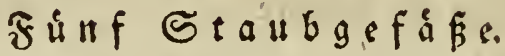

(Pentandria.)

\section{Oronung.

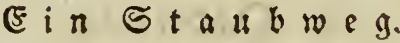

I. Mit Blumenkrone;

A, Blk. \& blättrig;

1. oberer Fruchtknot.;

a. Frucht unbedeckt;

f. Frucht I saamig.

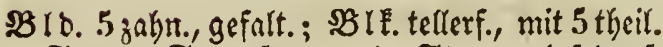
Saum; Stgef. unter $D$. Streg; 1 fabenf.

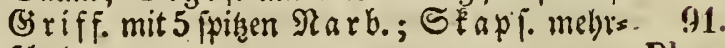
fdal.

Plumbágo.

b. Frucht 2 saamig.

(A n m. Von $9^{2}-106$. sämmtl. rauhblättrig.) SBIb. tief 5 theil., mit ungleid). Eapp. (ober 5

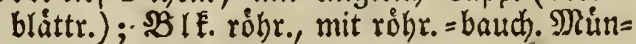
bung ur. offn. Sdhluno; Stbeut. ipie巨sf., am (Srunde surch i piralf. Lapp. unter fid zibang.; 2 Nirffe fpiegelglatt, eyr., Enochens hart, 2 fád., am Srunde b. SBlb. angehefs tet, unten burchbohrt, mit 1 [aam. Fd d). Cerinthe.

c. Frucht 4 saamig.

Die vier 1 få̀̆hy. Nüsse: 
1. abgejuntert im Sirtmbe o. SBlt. ut.

A. am (Sruntbe ourchbohrt; Blkschlund:

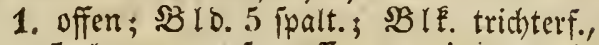
5 Iapp., auft. = offen, mit ungetl\%.

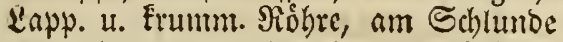
etwas hervorrag., 3igeneigt., ent. Schups pen; Ratbe ausgernoet; Ruffe am Sirunde geftrichelt

2. mit Flappen gciditorien; Blk.:

a. trichterf.: : Sif $\mathfrak{b}$ re walgenf., fo lang als 2 Bld.; ain (Strunde prismat.: Saum balb 5 phalt., aufr. = abfte= Gens, ftumpf: $S^{b}$ luno mit 5 er= bab., bersorrng., lángl., zigeneigt. Sdupp. geftlofi.; Stbeut. langl., bebedt; 许 a r b f fumpf, alsgerano.; S. lângl., ftumpf, bưftr.

b. walzcul. = glocfenf.; Si óbre ganz

94.

Anchúsa.

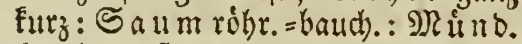
5 zalin., ftumpf, etras umgebog.: S屯luno mit 5 pfriement., in $\mathfrak{e}$. Segel zigneneigt. Strablen, D. Eúrzer als b. Saum fino; $\mathfrak{R} a r b \mathfrak{c}$ einf.; S. cyr., bod thr., langgeipict, mit b. Spizen żgeneigt, 1 fạt).

B. nicht surchbohrt; Blkschlünd:

1. offen; Bldecke:

a. 5 zahn., prismat. $=5$ leit.; $\mathfrak{B} \mathfrak{H}$. tridterf.: Sid bre walzenf., ránger als Blb.; 5 ऽau mlapp. ftumpf; Iarbe ftumpf; ausgerand.; $4 \mathbb{S}$. ftumpi, runbl.

b. 5 ipalt., mit langettf., aufr. Snpp.;

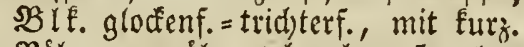
Robre u. robr., baud)., 5 jalln. Saum; Stbeut.pfeilf., am Grun= be mit 8 . Blflapp. verbumb.; Nar= be ftumpf; (S) riff. eingefdloff.; S. enr., glânz., fteinbart, 1 fách., im Srunce b. SBlo. angebeftet

c. 5 theilig; Blk:

a. glodenf.: $\mathfrak{R} \mathfrak{b}$ re febr furz: Sau m aufr., nach u. nach erweis tert, 5 fpalt.: I app. oft ungleich, ftumpf: bie 2 ob. láng.: die unt.

96.
Pulmonaria.
93.
Lycópsis. 
Fleiner, ipit, umgeibling.; Stfäb: ungl., abwártsgeneigt; $\mathfrak{R}$ a $\mathrm{r}$ be ftumpf, 2 palt.; S. rumbl., Thiefgefpist, 1 fách., glatt, fnot., in Srunde o. SBlo.

b. tridhterform., fo lang als $\$$ Bld.: : $\Re$ o h re walgenf.: $S_{\text {a }}$ um halb 5 ipalt., ftumpf, aufr.; Stbeut. lángl., im Ed)luno O. SBIE.; (S) r iff. To lang als BlEróbre; Na rbe ftumpf, 2 ipalt.; 4 S. enr.s langgeipicht, bart, glatt, im (strunde b. JiBlo.

2. gejoloffen; Blk:

a. rabf., mit 5 theil. Saum, fpic. Lapp: u. Durd 5 ausgerano., ftumpf. Servorra= gungen gefroint; 3 lo. 5 theil.; St $f \dot{\alpha} D$. pfriemenform., zjgeneigt; Stbeut. Iångl., an b. innern Sitte $b$. Stfáb. an= gebeftet, zigeneigt, abgejondert; $\Re$ a r be einf.; S. rundl., unourd)bohrt, runjl., an ber Spikce austr. rachenf, ant Sirunde fugel., ber lánge nach am auj. gebóblt. Frbo. angeheftet 100. Borágo.

b. tellerf., mit 5 theil., ftumpf. Saum; SB lo. 5 theil.; Stgef. cingeichlop.; Stbeut. fdilof; Narbe fopff; 4 abs gefond. Nuffe, am Srunde genabelt

II. an Eentralfâle geheftet u. nicht burchbohrt;

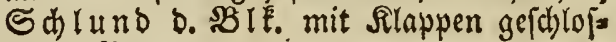
fen; Blk:

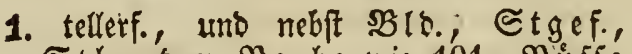
Stbeut. u. Narbe wie 101; Nitfle runoum ftachl., zigeorud

102.

Rochelía.

2. trichterf., mit Furg. Robre u. 5 ftumpf. Saumlapp.; Bld.:

a. 5 zabn., ungleitff., an b. Frudit zige= bruad, mit flact)=parall., budst., un= gleichgezahnt. Ramellen; $\mathfrak{B}$ IE $\{\mathfrak{c h} \mathfrak{u} \mathfrak{p}$. gewolbt, am Shlunde jigen.; $\Re$ a r be ftumpf; S. långl., jigebr., paarmeis nabeftebeno

b. 5 theil.; Stgef.eingeidylofl.; Narbe topff., etwas ausgerano.; Rúf fie cins georuift

103.

Asperúgo.

104:

Cynoglóssum. 


\section{5 Strubgefäje:}

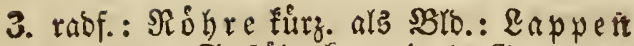
gerumbet; St $f$ åd. Eurz, in D. Sirunoe b. Sidre; Stbeut. bedeft yon 5 Furjen Sduppen; (3) riff. Kurz; Narbe bidz; S. Erugf., am Nande gezahnt

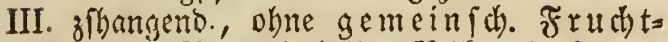

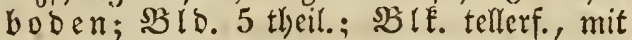
offn. Echluno, 5 fpalt. Saunt u. gefalt., cimf. od. mit e. 3abn verjebenen Buchten; Etgef. eingeichloß. ; Ratbe fchiltof., faft legelf.

105.

Picotia:

Heliotropüum.

b. Frucht bedeckt;

ค. mit Saamenkapsel;

a. Skapsel \& fächerig.

1. Mit $\mathfrak{R e c t . : ~}$ ein Strict) von 2 ber Range nach) zigeneigt. Ramellen inwend. an b. NRitte jebes sBlElapp.; $\mathfrak{B}$ lÉ. 5 fpalt., glodéenf., mit aufr., ftumpf., ausgerano. Lapp.; s及b to. 5 theil., faft fo lang als \$lle., offen, Dautr., mit pfrie menf. Lapp.; Stgef. herborrag.; Marbe 2 fpalt., fwith, abftelsento; SEapi. Eutgel., 2 ichalo, mit 1 groß口., rumbl. S.

II. obne Rectarium;

A. mit Scitlte; Bldecke:

1. 5 zahn., róhr., ect., baur.; şb te tellerf. ob. trichterf.: $\Re$ of hre walzenf., fo lamg, od. láng. als SBld.: Schlund offen, meift brujenloz: Eaum etwas abftes heno, mit 5 altsgerand. Lapp.; Stgef. ind. Blftrogre; Narbe Eugel.; Skapf. ftielrunt, fo lang als 38ld., mit 10 ppart. SRłno. u. vielen rumbl. S.

108.

Primuila.

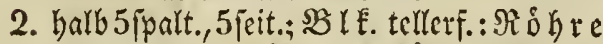
enr., mit $\triangleright$. Bro. verhfillt: $S_{a u m}$ 5 theil. wit eyr. Ilingl., ftumpf., ungeth. ob. altsgerano. Lapp.: $S_{\text {chlund }}$ mit

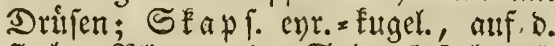
fladten 3 Bro, an ber Spitge 5 fad) aufs ipring., mit 5-10 $\mathbb{5}$.

B. ohne Jjŭlle; Bldecke:

107.

Hydrophýllum,

1. 5 zabn., bauch., zfgeneigt, mit 5 Dors nen, woyon bie obern einf., bie unt.

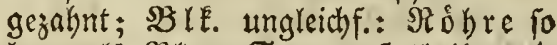
lang als 3Blb.: Saum 5 thcil., mit

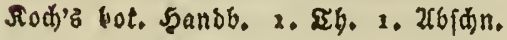


li̊ngl., ausgerano., ftumpf. Rapp., wo= wou bie 2 unt. Firr. u. ntebr abfteben; Narbe sidét.; Skap F. Eugel., im Srunde D. SBlo., 5 fhal., mit vielen faft ei)r., Fleir. $\mathbb{S}$.

110.

Coris.

2. 5 fpaltig; Blkrone:

a. tabform., mit eyr., gerumbet. Lapp.; Blolapp. fpin; Stfád. am Sirunde

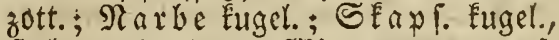
ftechendipis, in ber s)ditte runoum auf= fpring., mit vielen eyr., foft 3 feit., raub. S. im Euget. Frb

b. rabf. = faft glodenf.: Saum flach mit

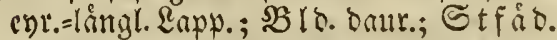
am Sirunoe breiter, juweilen jigewnehi.;

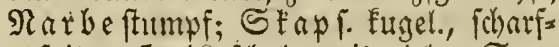
gerpint, 5-10 jhal., mit vielen $\mathbb{S}$. Lysimachia.

c. glodenf. = trichter F., gefranjt $=$ jerrif.; Slolapp. läaettf.; Stbent. pfeilf.; Narbe einf.; SEap l. lángl., mal= ànf., geftreift, an b. Spire vielzahn.,

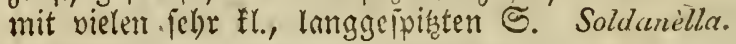

d. gainz trichterf., mit furg. Saum, wel= fent; Stbeut. nach D. SBefruchtung fpiralf.; (Siriff. aufr.; 2 runol. $\Re a r=$ ben; Sfapr. gleichbr.

e. tief 5 ipalt., offen, mit èr., ftumpf. Lnpp. ut. fort ofne Rogre; $\mathfrak{B}$ lo. glof = fenform.; mit lanzettf., cinf. DD. an D. Spike 3 jalyn. Iapp.; Stbeut, jige=

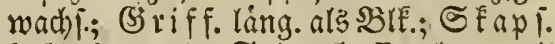
2 forl., an D. Spine 5-7 zabn., mit vielen edx. S.

115.

Cortísa.

3. balb 5 fpaltig; Blkrühre;

a. noch eimmal fo grop̃ als SBls., faft Eugel., Furz, úberlyang.: Sa a m 5 theil., mit nad) oben umgeifhlag., langettf. Iapp.: Schluno etrons bervorfteheno; SBlo. glodfenf., baur., mit eyr. Enpp.; Stfå o. febr furg, in \&. SBrerobre; Stbeut. âjgeneigt; (Siriff. láng, als ভtfî́.; Rarbe pin; Stapi. Hugel., an $\delta$. Spise 5 fad') aufipring., bebect mit Scaut, mit vielen faft evr., edf. S. Cyclämen. 


\section{5 Staubgefáp̧e.}

b. fing. als SBlo., 5 theil., mit umgeidst. Saum u. fehr langert, lanzcttf. Rapp.; Blo. Daur., mit umgeid)lag. Lapp.; Stfåd. Felur $\mathfrak{F u r}_{3}$, in b. SBlfro̊bre; Stbeut. pfeilf., in e. Stanabel jigen.; (3) riff. fabenform.; Plarbe ftumpf; Ekap F. loingl., an o. Epike aufipring., nit vielen $\mathfrak{k l}$. S.; Sputr von e. ganz tl., vielblåttr. ŞSilli e

117.

Dodecathěon.

4. 5 theilig; Blkrone:

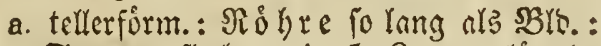
Sa a m flact), mit 5-8 eyr. =lángl., ausgeramo. Eapp.; B 1 b lapp. gleichbr., aufr. a abftebend, Denen bic Etgef. in 8. SBlfrỏhre entgegenfechen; (S) riff. kurz; Narbe Eugel.; SEap f. Fugel., langgeipizt, mit vielen Eltgel. S.; Frudiboo. gropi, fingel.

118.

Hotionia.

b. trict)terform., mit abftelyend., 5 lapp., gleidff., inment. ftachelhaar. Saum;

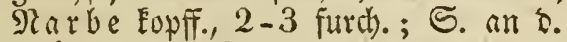
$\mathbb{M B}_{3}$ antoen o. SEapj.

119.

Menyánthes.

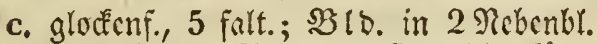
eingeidlós.; Stgef. foft glcid), Eitry. als sBlefaum; $\mathfrak{F r u d h} t$ halb 2 fách., 4 faam.; (5) riff. ungeth.; 2 $\mathfrak{N}$ a $\mathfrak{b}$ en ftumpi

d. rabform., 5 lapp., faft unglcichf., am (Srunte b. \&appenbudt. geflectit; $\mathfrak{B} 18$. glocfenf.; St gef. angenábert; S tbeut. an o. Spite mit e. Lod); 9 a a $\mathfrak{b}$ be rumbl.; Skapl. mit 2 ant Manbe cinwárts= gebog., bie Scheioemånoe tragenoen Schalen u. vielen $ธ$.

\section{1. \\ Ramondĭa.}

2) Skapsel 2 fächerig.

Blumendecke:

1. 5 zahn., róhr. = baudh., ckf., abfall., nur mit ๖. Freižrund. (Srunto baur.; sB IE. trichterf.,

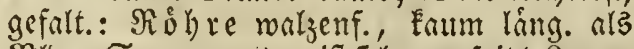
SBld.: Saum mit meift 5 langgeipišt. \&app.;

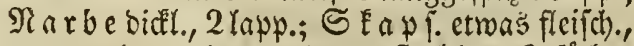
cur., glatt ob. runoum ftachl., 2 fách., 122. 4 fajal., mit vielen nierenf. $\subseteq$. Datúra. 
2. 5 ppalt., rơbr.,

a. unten bauch.; baur.; $B(1$. trid)terf., mit

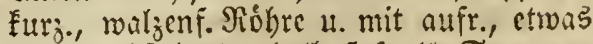
fater., abftebeno., balb 5 fpalt. Saum $n$. ftumpf. Rapy., wovon e. breiter; Stfá.

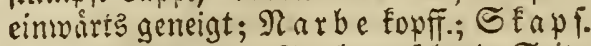
eyr., ftumpf, nit e. Furbe auf beys. Ecit., in s. Sgltte runcum anfipring., mit Dedét u. vielen $\mathbb{S}$.

b. nicht batch), SBIt. trichterf. ob. tellerf., mit 5 ipalt., gejalt., etwas offen. Saum; Stfaio. u. Narbe mie 123; Stapf. 2 fhal., an 0 . Epise 4 fach allfipring.

123.

Hyoscyămus.

124.

Nicotiána.

3. 5 theilig; Blkrone:

a. rabform., ungleichi, 5 lapp:; Stgef. abgejonocrt, ungleich, mit cinwairts ges reigt., meift am (jirunoc zott., Etfáo.; Stbcut. u. Rarbe eint.; Sfapl. 2 ichal., evr. ob. Eltgel., mit cingebog. Ed)a= Ien 1. vielen S. in ser Mitte b. Frbo. Ferbáscum.

b. trichteriơnis,

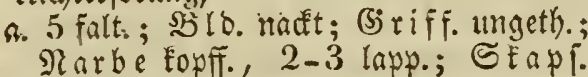 vidliant.
b. nicht faltig (f. 13S.)

c. glodenf., 5 falt.; sero. nadt, ob. mit $2 \mathrm{fl}$. פebenbl.; StgcF. Eưr (S) $r$ iff. ungeth.; 2 fadenform. গarben; Stapi. mit Stalen

126.

Ipomo'ea. (Elliša ) .

127.

Convols'ülus.

\section{3) Skapsel z fächerig.}

\section{Blkrone:}

1. telferfớrinig; Bldecke:

a. 1 bláttr., tief 5 ipait., faft margenf., baur., mit \{pik., jigencigt. Lapp.; sBlér ó hre falt walzenr. láng. als BBld., etwas ge= frummt: $S_{a} u$ m flach mit frumpf. Eapp.; b. Stbeut. pfeilf.; 3. Stfáb. lónger, mit Etbeut. biz fur Niuneung o. Rohre rei= deno; Narbc 3 palt.; Stapi. eyr.s 3 eá., 3 jhal. mit 1 faam. F́ăch.

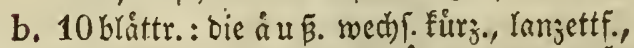
umgefdhl.: Die innern lánger, lanjettf.,

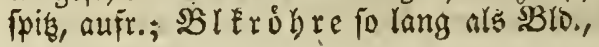


mit 5 ipaltig. Gaum u. ftumpf: Eapp.; Stbeut. jigeneigt in oen Sitechlumb; FruchtEn. jott.; (Gi riff. balb 2 ppalt.; 2 Nar ben runbl.; SEap f. evr., 2 fhar., gefärbt, elaftijch, mit 2 grozen, getwólbt., eur., fdrwiel. S., welche wie e. einjelner ausfebcn

2. glodfenf.=rabf., 5 theil.: S mit 5 Rlappen, morauf $D$. Stgef.: Saum

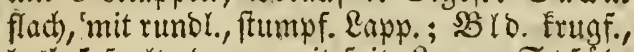
balb 5 fpalt., baur., mit fpis. $\mathfrak{L}_{\text {app.; }}$ Stfás. am Srunde breit.; Narbe 3 fpart.; ভEapi. evr. $=3$ feit., 3 idal., mit vielen sungleid)f., ctif. $\mathfrak{S}$.

129.

Galax.
130.

Polemonüum,

4) Skapsel.4 fächerig:

Brs. Febr Flein, ungleichf.; SIIf. trichs terform., 5 ppalt., ungleichf.; ङ D. Stweg cingefugt u. mebft (s) riff. abwarts= geneigt u. Kerworrageno; Stbeut. oben an 2 \&odern mit Dectel aufipring.

\section{b. mit 2 Balgkapseln.}

SIr. 5 theilig; Blkrone:

1. glodenf., 5 ipalt.; 5 Rect. Druiferi um 131. $\hbar$ Asalẹciog.

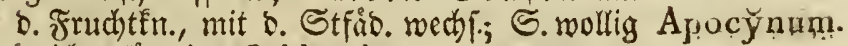

2. trichterformig; Schlund:

a. natet, zigeoreht; Stbent. zigeneigt in c. Siegel; Shalg wollig. $\subseteq$.

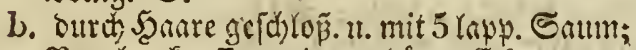
Rarbe fopfi., mit e. báut. Ede unge

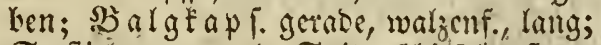
๔. ftielruno, an s. Epise fujefabgeftuat; nadt

133.

Echites.

3. tellerformig:

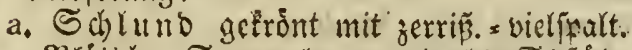
Brattch.: Saum lapp., georebt; Etfåd. in 8. Mitte ร. Shobre eingefingt; S.tbeut: pfeilf., nit ber ftumpf. Narbe in D. Mitte fibang.; (5i riff. Fabenf., an s. Spise breiter; einige $3 \mathfrak{a} \mathfrak{h} \pi$ h). im (3runbe $\delta$.

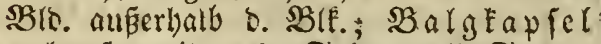
walgenf., mit an .o. Spike woll. $\sigma_{\text {. }}$

135.

Neřum. 
b. Saum 5 theil.; Stgef. eingefdhlö.; Stbeut. pfeilf.; (siriff. fadenform.; Rarbe yon bem breiteren (srunbe an Tabernaemon2 ipalt.; 5 . in weicher Naffe tána.

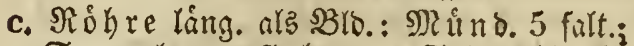
Saumlapp. flady, an $b$. Spipe focherabs geftugt; Shlolapp. gerabe, fpitz; Stfáb. an ber Spibe breiter, in kohle Schupp. úbergeheno; Stbeut. båut., zulezt zige= brebt; Narbe bopp.: bie unt. fhillof.: die ob. Fopff.; 2 Drifen am Sirunde

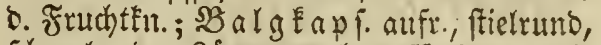
fthmal, ber aringe nach aufipring., mit längl., nadt. S.

137. $ち$

Vinca.

\section{c. mit Beere;}

21) Beere 4 samig.

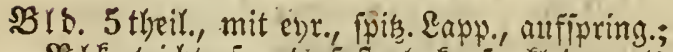
28 IE. trichterf., ob. faft glocenf., Eleiner als Bld., mit 5 ftumpf. Lapp.; Stgef. im Sirunde D. SBle.; $\mathfrak{N a r b e}$ einf. od. 2 ipalt.; Beere troden, hooenf., 2 ichal., 2 fäh., mit Eugel., fobwarz., punct.

138.

Elliš̃a.

2) Beere mehrsaamig.

Blkrone:

1. rauf., 4, 5, 6 theil.; $B$ ro. Daur., 5-10 theil.; Stbeut. lángl.; an $D$. Spize mit 2 lob bern aufipring.; Beere 2-6 faich.

139.

Solánum.

2. glodenf. = rabform., gejalt., 5 lapp.; $2 B$ IS. 5 zahn.; Stbeut. zigeneigt, o. Eange nact) aufipring.; Rarbe fopfi.; B e ere 2 fách., bebeft mit $\delta$. aufgebla ., cá., báut., wehr= lojen Bilo.

140.

Physculis.

3. ganz gloctenfơrmig,

a. noch cimmal jo lang als SB(b.; bauch.eeinr., mit 5 fpalt., elwas offer. Nitno. u. fait gleich. Lapp.; $\mathfrak{B}$ Io. 5 theilig, erft faft glodenf., bann abftehend, mit fpis., baut. Iapp.; Stfád. fabenf., am Srunde zige= neigt, oben gebogen; Narbe fopffirm.; Beere Eugel. in $\mathfrak{B l d}, 2$ fách., mit jebr vielen nierenf. S. am håut. ซ̌rboden Atröpa. 
b. nicht gang nod) eimmal fo lang als Bro, 5 fpalt.; sBIb. treujelf., 5 palt.; St fỉ.

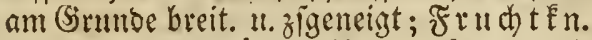
am Srunde 2 Drifjen; Şeere fugcl., mit nierenf. $\subseteq$.

142.

Mandragŏra.

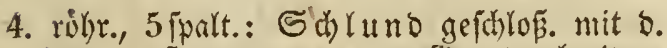
langen Soarren ber am Srrunde breitern Stfád.; SB lo. 3-5 ipalt., baur., glodenf.,

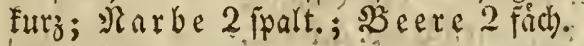

Lyciüm.

2. halboberer Fruchtknot.

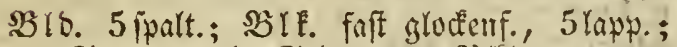

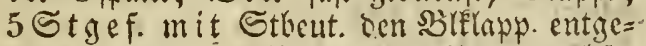
gengef.: 5 ogne Etbent. mit ifnen wechf.; 144. +

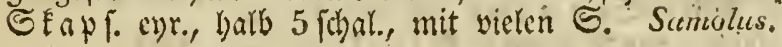

\section{3. unterer Fruchtknoten,}

a. mit Saamenkapsel.

\section{Blkrone:}

1. gleichfơrmig,

a. juterit Fegelf., mit an \$. Spise etwas wer= wad). Rapp., Kernach 5 theil., nit gleichbr., fohlafi., jullezt- ungeichkag. Eaph.; şro. harbabliteheno, mit 5 fpib. Eapp.; Stbeut. lángl.; (s) riff. 2-3 p̧palt., mit långl.,

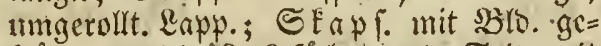
Frount, rumbl., 2-3 ficth., an $\emptyset$. Seite nit e. Eod nufipring. uno mit, vielen flẹinen, runo. S.

145.

Phytéuina.

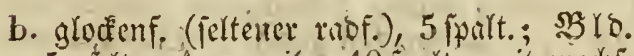
5 , felten 4, aumeilen 10 ipalt., mit wechi.

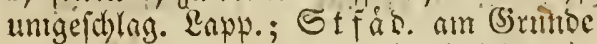
breiter; Aarbe $3-2$ lapp. ( aud 5 [palt.); Sk $a$ p. $3,2,5$ fådh., mit Seffmungen an 146 . D. Eeite u. an D. obern Epikge mit Edjaten Campanüla.

c. trid)terf., 5 jpalt., offen, nod eimmal fo. lang als bie längl., $4-5$ zaflun., unten mit Rebenbl. veriefene Bglt.; Stge f. cinge=

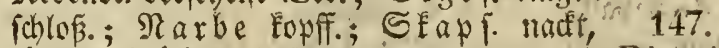
lángl., 4 fách), viclfaant.

Diervílla.

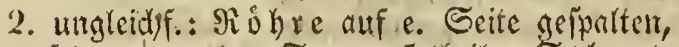
felten ungeth.: Saum 5 theil.; Etbeut. 
วfgerwachl; : Rarbe 2 lapp., jumeilen ungetb.; SEap $[.2$, felten 3 frich., an $\$$. obern Spise 2 joal.

\section{b, mit Beere.}

\section{Beere :}

1. 1 faamig (f. 64.)

2. 3 faam.; 1-3 fäb., reberart., am Strunde in Blo. geichloß̄., u. mit beren sapp: geftront, verf. enr. = 3 jeit.; $3 \mathrm{~S}$. Enochenhart, ellipt., nul b. e. Seite mit 3 ounfeln Streif.: bie åuß. Scaut dia u. fnochenthart: bie innere báut., fajt abrcibbar; 5 SB lo lapp. abfte. beno, lanjettf., Daur.; BB le. róbr., faft fo

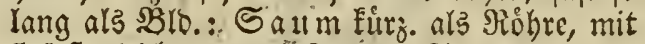
5 faft gleid)., runbl. \&app.; Stgef. einge= (d)lop.; Narbe langl., verbiaft

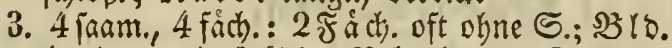
4 jabn., mit 2 Elcin. Nebenbl. am Sirunbe; $\mathfrak{B}^{\mathrm{B}}$ lE. glodfenf., 3 u. 5 fpalt., faft gleid)f.; Stgef. menig herworrag.; Nar be Eugel.

4. vielianmig,

a. einfach und abgefondert, 3 fád

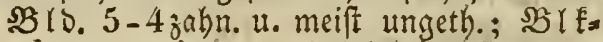
tỏb r e verlángert, mit 5 fpalt., faft gleich $F$. ob. 2 lipp. Saum; Stgef. io lang als Site; $\mathfrak{N a r b}$ e Eugel.

b. Doppelt, u. am (Sirunde melyr od. reniger

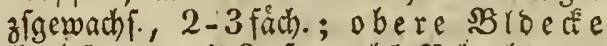
$5-4$ palt., mit 2 jigemachi. Rebenbl. am

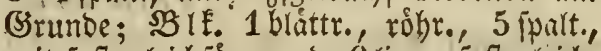
mit foft gleichform. oo. 2 lipp., faft gleich. Saum; glarbe fopff. od: verbidet

(Rubra.)

149.

Triostěum:

$150 . \hbar$
Symphoricárpos.

\section{1. $\hbar$}

Caprifolüm.

\section{B. Blk. 5 blättrig;}

\section{1. oberer Fruchtknoten;}

a. mit Beere.

\section{Bldecke:}

1. frugf., 4-5 part.; $\mathfrak{B}$ rf. 4-5blattr., zu= weilen obne Fructit zu bringen; 1 Gi riff.

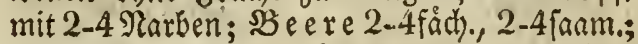
S. am Sirumbe mit e. Enorpelart., etwas her* vorftebento. Rabei

153. $\hbar$

Rhamniss. 
2. freuferf., mit 5 their., (ietten 5 ipart.), of fges

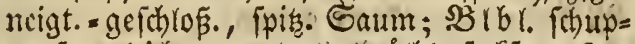
penf., gleid), rundl., gecwólbt s fact. , ziges orueft, fehr ftumpf, abftebenb, aus b. sgloein= fahnitt., mit Rågeln Den Stgef. entrgegengef.; (3) riff. halb 3 fpalt.; Starbe ftumpf, (Felí ten 3 fpalt.); Becre troden; $^{2} 3-4$ entopf. 3 fách., an $\$$. Spise eingebr., mit Snoten 154 . u. $3-4$ evr. .

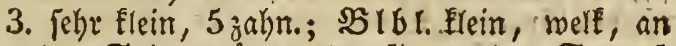
Der Spite muthenart fifluangento; Stgef.

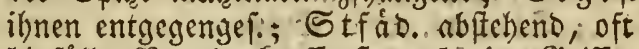
binfấl.; $\Re$ a r b e fopff., ftumpf, ofne Siriff.;

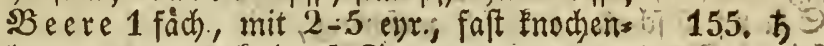

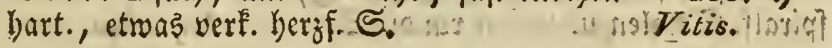

\section{b. mit Steinfruche.}

Steinfrucht:

1. balb fugel., foft fajirbf., mit e. Greit., bơut.;

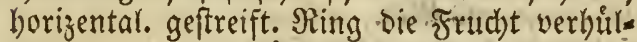
lent, trodf., $2-3$ fádb, , $2-3$ fáam., forfart. ; Bro. fladh, 5 ppalt., Daur.; mit eyr., ipir. Lapp.; 5 sibl. felye abfelento, Elein, hohy,

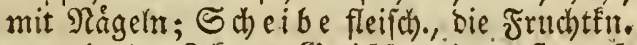
umgebent; 3 furzc Ser riff. mit e. ftumpi. Rarbe

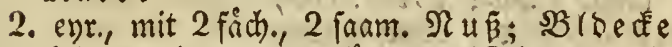
5 [part., inmens. gefåtbt, abjteheno, mit fpit. Rapp.; $\mathfrak{B}$ l b l. Febr Flein, hohl, zwifchen ben BIolapp.; Sche ibe fleifon., freistund, ben Stweg umgebeno, gefantbt; Stgef. Den \$ibr. entgengef.; 2 furze (s) riff. mit c. ftumpen $\mathfrak{R}$ a r be

\section{5 Zizy̆phus.}

\section{c. mic Saamenkapsel,}

Saamenkapsel:

I. 1 fádherig; Bldecke:

1. 1 bláttr., 5 theil., auf e. Scite unter b. oberften sBibl. gefpaften, mit am (Sirunde verläng. Lapp. (ober 5 blåttr.); šlbl. un. gleich: bns oberfte einjelne grós. u. am sirunde in e. Scurn ausgeheno; Nect.: e. brîj. Fortfak an b. Spize 2 Stgefápáp; Stbeut. an b. Spine zigemachi., baut.; 
Stfáb. nbgefonbert, wobon 2 in tem Scorn bes ab. SBibl. fteden; : at a rb e fpits

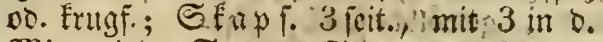
Mitte vielen S: trag. Echalenti:

2. 2 bláttr., febr flein, faft vermachj., $a b=$ fall.; B̉bl. ungl.: basp berfte rund., aufr., leicht 3 ipalt. ..e. 2rrt von Slippe bilens: 2 unterften jebrgrof, nusw. breiter, sfúminf, e. Unterlippe mad)eno; Rect. Fappenf:, ben (Sirun: D. Slume um=

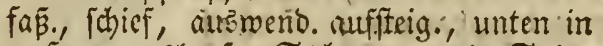
e. Scorn mublauf.; Stbeut. an o. Spike jigemachlen; Narbe einf.; ofne (Sriff.;

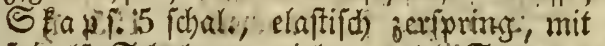
fpiralf. Sdjulen $\mathfrak{u}$. vielen runoL: $S$........

158.

Vióla.

is

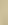

- $\operatorname{lin}(5)$

$\therefore, i x+1, y$ is stlity $^{2}=2$ तु? 159.

Impatiens... :

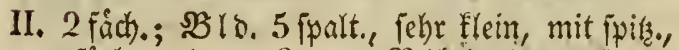
geförbt., Daur. Eapp.; SS lb t? Ianzettf. u., uebft Stger., in s. SBlo. cingefingt; Stbeut. überbang.; (S) riff. "saur.; Narbe ftumpf;

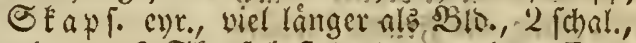
wie aus 2 Sfapj. beftehend an obern Ende ftechendipiz, alffuring : mit vielen, febr Elei nen, lơngl., glânz. S

III. 3 fåd)erig; Narb 3 palt.; Bldecke:

1. 1 bláttr., hals 5 fpalt., flad); feldr flein, mit. ftumpfo, ungl. Rapp.; SBlbl. eyr., abfteheno, grōs. als ßBto., obne Nagel, am Ranbe umgerd)(agen; Frud) tîn. febr flein auf rem grop.; flads.l 10 ftreif. BSIbD.; (3) riff. pfriemenf.; fluzer als Stgef.; Skap f. ftumipf 3 jeit., bodfr., 3 id)al., etwas fleifos., mit wenigen- 5 . balbs eingehúlt in e. Saamenlyaut mit 4 palt. ) ) iuno.

160. 万

Itěa:

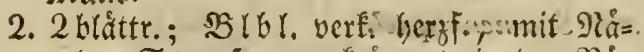
geln; Stgef. umgefrummt, in ten $\mathfrak{l}^{\circ}=$

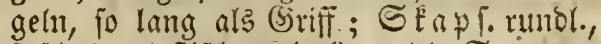
3 forl., elaftifh, mit 3 runor, $\sigma_{0} \quad$ Claytonira.

IV. 3 od. 5 faecter.; $\mathfrak{B}$ 18. 5 theil., flach, mit rumbl., Gohl. Iapp.; $\$$ (b bl. enr., flach), $a b=$ fteheno; Schcibe fleifd., 4-5 jeit., ben Srumb D. Blbecfe bebecterto; Stgef. Den Blolapp. entgergengel.; (S) riff. Furj; Narbe

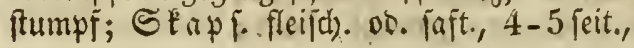

161.5

Celästrus. 


\title{
V. 5 Staubgefäfie.
}

gefárbt, 3 . 5 (d)al.: Sch alèn 2 lapp., burd

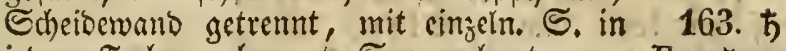
jebem Fadbe u. beerart. Saamenhaut

Evionymmus.

2. unterer Fruchtknot;

a. Frucht ibedeckt.

1. Mit Skapf; gem. Bib. baul, 10 blattr., wovon bie innern wechl. . Id)maler; - Die

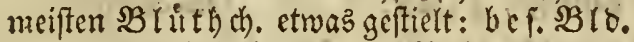
5 palt, od. 5 theil, baur.; $59 B$ rbl. tanzettf., aufr., unten verwadbf.; Stbeut to lontgh, am Srumbe zifang.; $\mathfrak{R a r b e} 2$ fpalt (an

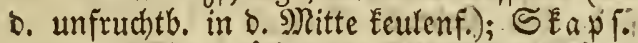
rund. =5 feit., 2 fách., mit.bel.:Bld. gefrónt, ant. D. Spike auffpring, mit vielen faft eyr. S.

164.

2. mit $\mathfrak{B c e r e ; ~ B l d e c k e : ~}$

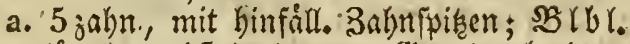
långl., abjteheno, am Csrunde breiter: Stbeut. am S3runbe 2.fpalt.: $\mathfrak{R}$ arbe

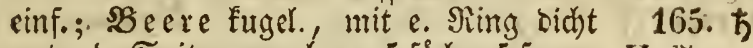

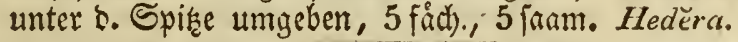

b. halb 5 [palt., baudb., mit langl., bohl., gefärbt., abjteheno. od. umgejalagenen,

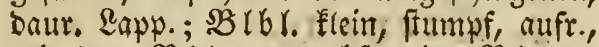
mit bent Blolapp. wechi., im Blbrano nebft ben ben Brolapp. entgegenger. Stfád.

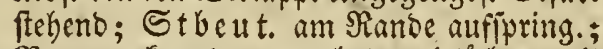
$s^{3}$ eere Eugelo, genabelt, 1 fách), mit 2 Frbd. an D. Seiten

\section{b. Saame unbedeckt.}

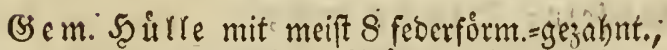
gewimp., umgefchlag. SBrattch): be F. Şitre mit 4 haarf.=feberf., nur 1 Bimftr umgebent. Blåttch.; $\$ B$ í̀. 5 blättr., , laarf. = viel ipalt.; Brbr. 2 horn.; Rarbe einf.; S. einjeln, eyr. =långl, mit BBlo. gefrơnt

\section{Jasióne.}

piy . hist

165.
166. ち
Ribes.

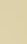 ?

Ribes

\begin{abstract}
.
\end{abstract} 政 
a. unter semielben; $\mathfrak{B} 1$ s. gefórbt; 5 lapp;

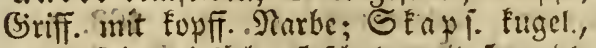
ranggeipidst; 1 fach., 5 jichal.", mit 5 rutrol. S. in fleifh. Sculle

168.

Glaux.

b. um benjerben; 'sord faft 5 their, init an b. Epitze bidern $\mathfrak{a}_{\text {app. }}$ mounit 5 froenf.

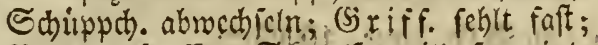
Rarbe fopff; ; Stap T. mit jigeneigt. Bib. beocat, geftreift, 1 jaam.

169.

Illecĕbrum.

2. mit untern fruchtinoten:

a. mit Rect. $\mathfrak{B}$ a a t am Srinte jedes $\mathfrak{L a p}_{\text {ap. }}$

D. 1 bláttr., inwenoig gefárbt: 'Blo., 100 burch bie auf. 0 -, $2 B 10$. ftebent. Stge

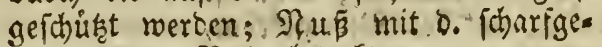

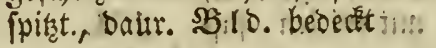

b. obre Nect.; SBlo. 5 palt, am Sorunte flact; (S riff. prisiemenform., gefvilimnit's Rarbe ppis; Stfendt. ent.; Rú lángl, eč́., 1 faam. nit tief., ungleichen Eángenfurden (mit vermengtem (d).te (t) t) (f. 809.)

171. $\frac{1}{5}$

\section{Oronung.}

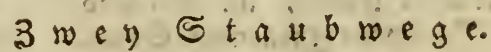

\section{Mit Blumenkrone;}

A. Blk. , blättr.; oberer

Fruchtknoten.

I. Mit 2 BalgépleIn; Same woll.;

Nectarium:

1. einfach; Blkrone:

a. raof.; 3 ro. 5 ipalt.; Nect.:

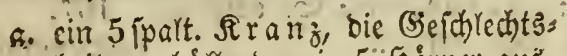
theile verbilleno $u_{0}$ in 5 Seirner aus" gebeno

172. 万 Periplöca. 173.

b. ein 5 zakn, walzenf. $\mathfrak{A} \mathfrak{r} \mathfrak{n} \mathfrak{z}$

b. glodenf., (f. 133.)
Cynánchum.

(Apocy̆num.) 
2. Dopp.: e. Siranz die (jepthled)tstbeile verbillterto $11.5 \Omega$ a pen in e. Sornthen

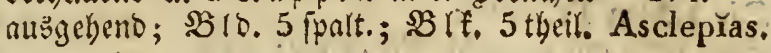

II. mit Saamentaplel;

1. mit Nectarium:

a. 2 mit $\mathfrak{B}$ inmpern umgebene $\mathfrak{D} u \mathfrak{u}$ cte nm (3)runte jebes ber lanjettf. SIfflapp.; SEI $\delta$. flach, mit 5 lamjettförm. $\mathfrak{R}_{a p p}$; Blé. rabf, mit flachem, abfehentem. Saum; Stbeut. pfeilf.; (S riff. furz mit 2 einf. Narben; SEapr. faft wal= jenf., langgepipist, 1 fád)., 2 jobl., mit vielen felyr fleinen $S$.

b. 5 od. 4 Sdupp. am (jrunbe b. Stger. 11. Denferben cutgegengel.; SB!b. 5 ob. 4 fpalt. od. theil.; $\$ B$ lE. frugf. =gludfenf. ob. Eugel., welfent, dairr., mit 5 ob. 4 fpalt. Saum; Sarben fopfiorm.; Sfa pi. 1-2 fåch., in D. Mitte runoum auffpring., mit $1-3$ jaam.:Fachern

2. ohne Nect.; SBY ipif., baur. $\mathfrak{L a p p}$; $\mathfrak{B}$ If. unten róbr., obne Deffrung, ohen 4, 5-oo. Sppalt., welfeno, v. werfichichener (sejtalt; Stfáo.

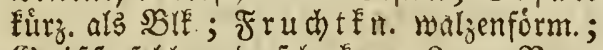
(S) riff. fehlen ob: febr furg; 2 eur. $\mathfrak{N a} x=$ ben; Stapi. lángl., ftieltunb, langge= ipist, an 0 . Spise lcict) 2 (palt., 1 fách.,

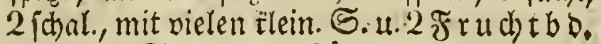
an jeber Sable der Roinge nach)

177.

Gentiàna.

\section{B. Blk. 5 blättr. u. unterer Fruchtinoten;}

1. Blüthen kopff., auf gem. Blbd.

Friichte :

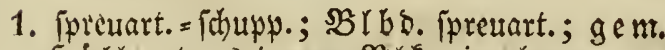
Sellle etwaz born;, Ble cingebogen

178.

Eryngüum.

2. geftreift, rippenlos, in o. aufgeidwoll. $\mathfrak{F} \times b$ o. niftelno, mit gejurchi. Nabellinie; BSlutb. foft lopff; ; ge m. Șulle wenigbláttr.; \$lte am Sianoe M., in o. Jitte ' ${ }_{\text {. }}$
Swertŭa,

\author{
St
176.
Cuscüta. \\ Stuerta
176.
Cuscüta.
}

(2) 
2. Blüth. in kopff. Dolde.

\section{Die bichten Früchte:}

1. 4 ripp., eyr., mit gewólbt. Nabellinie; $g$ em. S马 ưlte 6 bláttr., lång. als Dolbe; 2 l bl. ungeth.

2. rippenlos, längl., mit bafenf. ob. wiber= Derbafenf. SBorten umgeben; gem. Sg i̊lle wenigbláttr.; slithdy. mit vermengtem (SeId) I.; BIID. Daur.

150. Dondía.

\section{Blïth. in gebuischelter Dolde.}

(5) em. Sculle fo lang als Dolve; Frud dite lángl., mit gefurcht., runğl. Saut umgeben Astrantia.

\section{Blüth. in eigentl. Dolde;}

a. Dolde einfach.

Gemeinschaftl. Hiille:

1. breitbláttr., faft fo lang als Dolbe; Fr $\mathfrak{u}$ db te evr. od. prismat., bicht, mit 5 ftumpf. Sipp. u. ausgebóblter Rabellinie; $\mathfrak{B}$ l. glcidjbr., lanjettf., Yångl., runol.

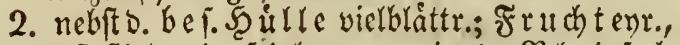

faft fticlumb, feinhanr., gerippt; $\mathfrak{B l}$ l. cinfach Odontites.

3. feblt ob. undeutl.; Frúd te mit ichmal. Rabellinie u. Siudén, an lezterm 3 riph., an

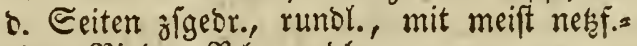
aber. Sinbe; $\mathfrak{B}$ l. runbl.

183.

Buplé urum. 184.

\section{S5. + \\ Hydrocoty̆le.}

\section{b. Dolde zusammengesetzt;}

a. Früchte eyr., diclit, gerippt; Zweige ruthenf.; Bl. zsges. od. dopp. zsges.

Früchte:

1. 3 ripp am Mưden; gem. Hülle:

a. 1blattr.; bef. S⿹ Sulte fehlt; Fridchte längl.= prism.: $\Re$ R i p pen etwas ftumpf, mit gefurcht., geftreift. Sertiefungen u. breiter, auf beyd. Seiten gerippt. $\mathfrak{N a}=$ bellinie

186.

Carum. 
b. mekrblätr., ob. feldt; $\mathfrak{F}$ ru dh te mit ge=

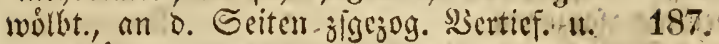
ambgebóhlt. Mabellinie " , Sison.

2. 5 rippig;

a. mit gent. Şưlle; Riplpen fharf, et= wab gefluggelt, mit gefurdst., geftreift. 188. Serticfungen

b. ohne gem. So ưlle; bes. Hiille:

A. 1 od. menigbláttr.; Ripp. D. Frudjte

(c)arf: Vertielungen:

1) gefurcht, geftreift; 3 weige ruthenf.; 189.

B̉l. Doph. zigel.: :Blàttch. fohmal Sesěli.

2) flach: Vabellinie auszebolt; 190.

Fruth te prismat. Meum.

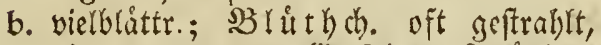
mit vermengt. (S) fid) r. Frid the wrismat., an d. Spitąe mit SBlo. uno Stweyen gefrint

191.

Oenánthe.

c. feblt; 5 gi ip p. etwas, ftumpf: Vertiefungen:

1) etwas flach, mit Saftging; ; Früchte 192. glatt

Pimpinélla.

193.

2) nebft פabertinie gang flach

Aprüm. is

b. Frïchte mit locker. Rinde.

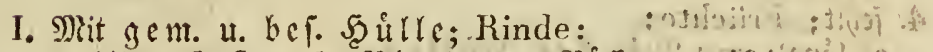

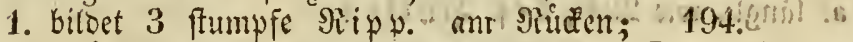

Fritch te flach, mit flact)er Nabellinie. Thysselinum.

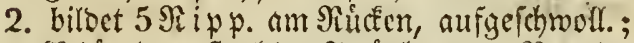
sbruth. geftrablt; Frủ chte am Rande zlgebrudet, flach, runol., in. o. Slit te Der Doloc nachenf., am $R$ a no zerrip., att $\Re$ ú def en 3 ftreif.

3. Doppelt: die å 11 B. burch 5 lapp. Fabeut mit o. 5 ect. innern zllang.; beybe Şällen vielbláttr.

Pleurospèrmum. 197.

4. forfart. $=\{$ chrontmart., biff

5. an Rande aufgeichwoll., feingeterbt;

Fril d) te freistumb, flad), zjlgebriteft;

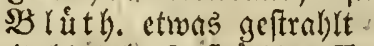

II. mit blos. bef. Sgúlte; Früchte:

195.

Hasselquistra.

Cachrys.

1. eur., bidbt: Rinde:

198.

Tordylium. 
a. \{chrwarz, 3 ripp., an b. Seite aus ber 199. Rabellinie zigezogen

Smyrnıum.

b. in ben Bertiefungen etwas hervorrageno; 200. $F r$ is th te 5 rippig Cicúta.

c. in 5 idarfe, berborrageno. Rippen auf 201. ichelleno; bế. Şullten halbumgebeno Aethúsa.

2. Fugel., Eaum gerippt, bie Rabellinie bildet burch e. Şaut ben Saftgang; $B$ litth. geftrablt

3. oval, jigeor., mit 9 ftumpf. Sipp., movon 5 mefr betworragen

202.

Coriándrum. 203.

c. Früchte stachelhaarig.

Gem. Hülle:

1. fiederf.ob. gefiedert; SBlith. meif geftrahlt;

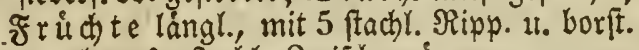
Do. eben fo ftachl. Swifchenráumen

Siler.

2. neblt ber. Scư lle vielbláttr.; bic err., sid)t., 5 ripp. Früchte:
a. hadtr. od. zott., mit etwas breitern Eaft= gángen Der Sertiefungen u. D. Nabellinie Athamánta.
206.

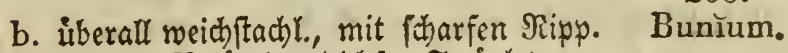
3. unbeutl.; sglit th. gleichf.; Fril ch) te evr., gerippt, uberall mit SBorften od. Stacheln bebeft

4. Feblt; Früchte:

204.
Daucus.

a. lángl., wie 204; ber. Şน์llen ungetf. Caucălis.

b. epr., bidht, mie 205. 209. Bubon.

o. Früchte pyramidenf. od. kegelf., geschnabelt.

Früchte :

1. pyramibenf., gefonnabelt, 5 ripp.,

a. Frubtfern halb jo lang, als b. Frucht; gem. Seulte fehlt, oo. an beren Etelle e. SBlatt

b. mit Stwegen gefrónt: Ripp. Tdarf; ohne gem. Soulle

2. Fegelf., mit gefurcht. Nabellinie;
a. obne Srippen u. Saftgånge, glatt, meift obne gem. Şưlle

210.

Scandix:

211.

Myrrhis. 
b: weidffachl., mit baur. Stwegef, ohne gem. Şutle

213.

Anthriscus.

\author{
c. Frïchte fast eyr., ge- \\ ript, gefurcht.
}

Frïchte :

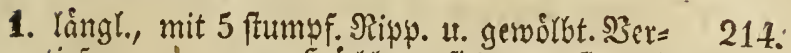
tiefungen;" gem. Se utle gefied. ob. fieberf. Anmi。

2. eyr. = prismat., etwas glatt, mit Şaut u. 7 Ripp.: Sertiefungen mit Saftgången; 215. So úl ren wenigbláttr.

3. faft prismat., mit 5 ftumpf. Ripw. u. etwas 216.

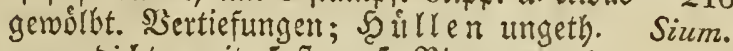

4. eyr., bicht, nit 5 ftumpf. Nipp., vor ber Sieife fein geferb̆t, mit flachen Sertiefungen 217. u. gem. u. bef. Soitrle Conйum.

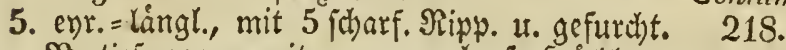
Bertiefungen; mit gem. u. bef. Şưlle Ligusticum.

\title{
f. Früchte zśgedrückt, fach, oft gefliigelt.
}

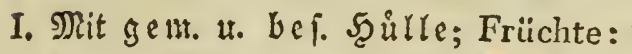

1. ganz flach, oval, am Ranoe geflig., mit 3 etwas herworrag. Ripp. am Rucfert: Ber= tiefungen und Nabellimie mit Saftgången Selinum.

2. flads, gerånbelt, nicht geflitg., mit 3 ob.

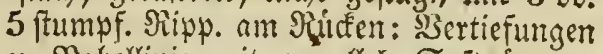
u. Nabellinie mit parallel. Eaftgángen; 220.

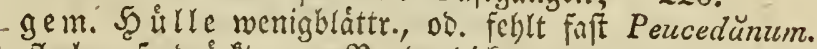

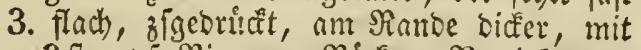

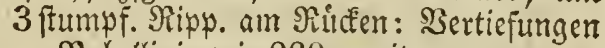
u. Rabellinie wie 220; mit vermengt. 221: (3) e fich t.

4. långl., etwas zfgeor., mit bucht. Frluggeln

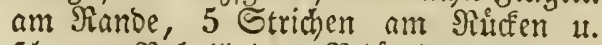
füupp. Rabellinie; $\Re$ lu th. geftrablt; 222. Şüllen fieberf.

5. oval, etwa as zigeor., mit 3 zuerfit fcharien, 223.

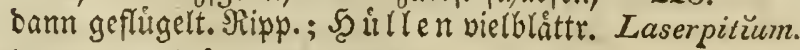

II. ofme gem. Şülle; Friichte:

Ferüla.

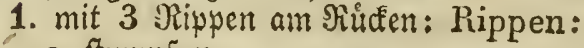

a. ftumipf 11 .

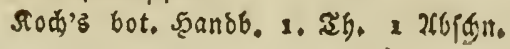


a. erbat.: Fru u ch te felb ft rumbl., zigebr., flach, am Sintre breitgeflingelt, init ge= furcht. Siertiefungen

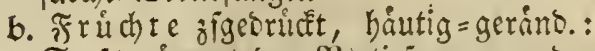
Saftgånge ter Sertiefungen u. ber Nabellin. Eeulenf.; SBl ù th. faft geftrohlt

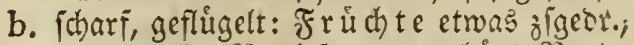

mit gefurcht. Sertiefungen u, båut. Ranoe Angelica.

2. mit unbeutl. Sipp. Do. vielmebr Etrichen am Rưfen; Fruchte owal, j̧fgetr., flnch, ge= rånto, mit ganj jarten Dertie fungen u. 2 mebr fichtbar. Saftgangen; bef. Ş itlten feblert fait

3. Faft obne Ripp., fothmal, menig zfigebr., mit

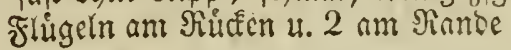

224.

Imperatoria.

225.

Heracléum. 226.

\section{II. ohne Blurnentrone;}

A. Frucht bedeckt.

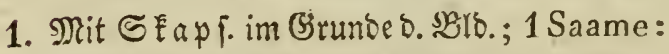

a. nierenf., zigeor., in o. Gubftang D. BLla.; Stap i. 1 fach., abfall.; $\$ B l b$. tief 5 ipalt. of. 5 bláttr., mit eyr. =långl., ftumpf., baur., bohl. Rapp.; Stgef. Det Rlolapp. cntgegengel.; hal'b unter. Frudten.; (Si riff. fehr fury, auft.

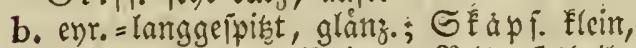
bebect, faum aufipring.; SBlo. 5 theil., fpic, abfebeno, inwent. gefiarbt, baur.; $10 \circlearrowleft$ tgef., movon 5 ohne ङtbeut. u. mit ber Blo. wechf.; (5) riff. fehlt faft; 2 gla $\mathrm{r}=$ ben. langgeipitst, fo lang als Grift.

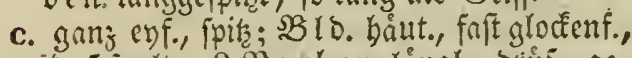
4-5 ipalt.; 2 গa a ben långl:, bruí., ge=

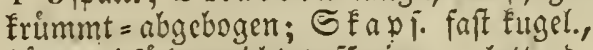
båut., 1 fåd., nibt aufipring., glatt ob. feinichup., 1 faam.; 4 od. 6 Etgef. ; M. BR(utb). Den ubrigen bengemijedt

229
Beta.

227.

Pastináca. 228.

Thapsı̆a.

230.

Herniaria.

231.

Planéra.

2. mit $\mathfrak{S} a u t f r u$ d t . vermengt. (S) Z. \$2lo. 5 th.; Stroeg jelten vollemmen; Şautfr. eingetr. - W. Blo. 2 fpalt., frucht=

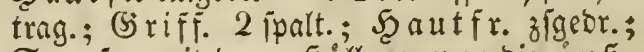
ธ. aufr., mit bopp. Şülle, wovon bie ă $\mathfrak{B}$. rimbenart. (i. S02.)

232. Atriplex. 
3. mit Steinfrucht:

a. trofien, flein, werf. =ey)r., am (5runte mit BBr. umgeten, mit 1 runoumfitachl. S.; 3 is. tief 5 theil., mit enr., fwite, Saur. \&app.; (j) riff. bimfáll.

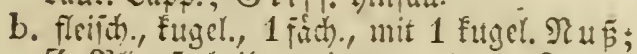

233.

Microtéa.

Z. SBls. 5 thcil., mit enr., baur. $\mathbb{\Omega}_{a p p}$; Etbcut. 4 cf.; ; 2 (j) riff. pfriemem., et= mots bid, abftebrit; Parbe cinf.;-M. $\$ 3$ lf. meît lit theil. mit 6 Stgef.

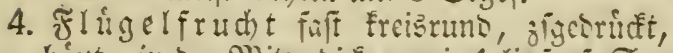
hiut., in ber Mritte bider, mit 1 linjenf. $\subseteq$.; SBIs. glectenformig, $4-5$ zubut , welfeno;

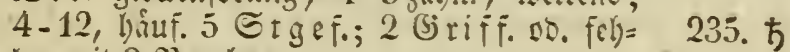
- Icn mit 2 giaben

Ulmus.

231. 万

Celtis.

\section{B. Ein einselner oberer Saame unbedectit.}

Saamenkeim :

1. Thneffenferm.; \$3Is. 5 theil. of. 5 klattr.;

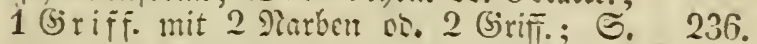
horizontal, nadt, mit SBls. cngumichló̄. Salsola.

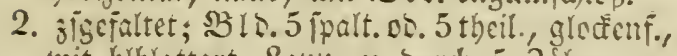
mit blblattart. appy. u. Surd) 5 asthe ge=

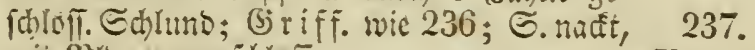
mit SBlt. engumichloll.

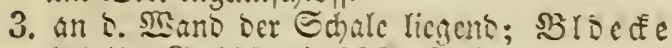
5 theil.; (Siriff. mie 236; S. Frcizrunt, mit

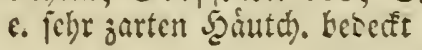

Köolǐa. 238.

Chenopodum.

\section{Orbแนng.}

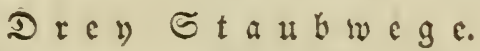

\section{Unterer Fruchtlinoten.}

Sline Briffel; Beere:

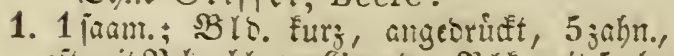

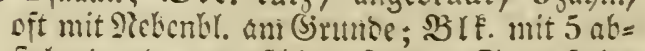
frelyent. of, umgeriflag. \&appo; $\subseteq$ tgc f. im

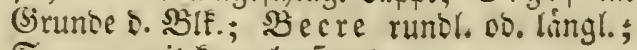
๔. eyr., mit Enorpl. S5aut 
2. 3 fanm.; BIt mit 5 gang flcincn Sáhnen;

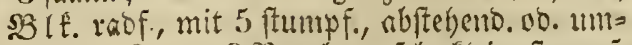
geichlag. Lapp.; 3 g a r b en febr Elein, ftumpi; B̉cere Eugel., 1 fích.; 3 S. rumgl., an b. e. Seite eck.

\section{II. oberer Fruchtknoter;}

A. mit 5 blättr. Blkronв.

I. Nit bedecter Frucht;

A. mit $\mathfrak{B e c r e ;} \mathfrak{B} l \mathrm{l}$. Hein, mit 5 baur. Laph.; 5 SBlbl. furz, enr., gerabe, etwaz

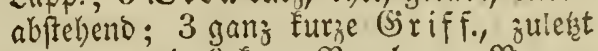

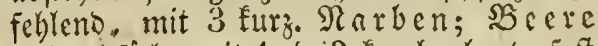
flein, 1 fich., mit 1 os. 3 enochentart., falt fugel. ভ.

B. mit Sanmentapjel; Blkrone:

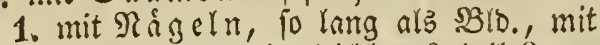
flach. Saun, mit gleichbr., 2 theil. \&app. u. burch) 2 Safhnct). an jebem sBlbl. ge= fróntent Edhluno; 310.5 palt., bis fajt jut פlitte waljenf., mit gerab, , ipils. Eapp.; (5) riff. abftebend; Sarbea cinf.; SE a P T. runol., mit BBro. bededt, 1 fåch., Hlein; 1 S. nierenf., glån. Drypis.

241. $\frac{\hbar}{5}$ Rhus.

2. oline Någel; Bldecke:

a. 1 bláttriğ,

Q. gefárbt, 5 theil., mit bohl., runol. Enpy. a. frugform. Scheitue ant Sirunde; 5 SB lbl. gerade, långl., ber SBlo. áhnl.; $2-3$ (s) riff. mit

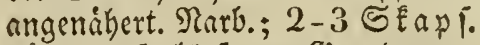
baut., aufgeblar., am (sinunde ver= wachf., nach innen auffpring., mit 2 fned)en bart., fugel., am (s) abgeftutist., narbigen $\subseteq$.

b. nicht gefárbt,

1) balb fo gró als sle., 5 thail., mit gerab., ftumpfo, orur. Rapp; Blblátt. eyr., ftumpf, bobl; 5-10 Stgef., weldie zulest ver = madjen; (Sriff. felylt; Rar ben unigerollt, feder., längl.; ङ 


\section{5 Staubgefápere}

3 ficit., Yanggeipitat; S. Elleitt, woll., an b. Mitte D. Sdjaten bang.

244.

Tamürisi.

2) faft eben io grof́, mit kobl., longl., fwic. Lapp.; sBIb l. gleich; 3-10 Etgef.; Narben ftumpf; SEapr. 1 fach., 3 funal., evr., mit SBIs. bebectet, mit febr viclen fugel. S.

245:

Alsine.

b. 5 blatttr., baur.: SB r. Yangl., hoht, nachenf.; $B$ lE. langl., ftumpf, ges rabe, am Sirutnde ichmaler, in SBlbo.; (5) riff. fehlt; 3 fwike, abfteh. Stars

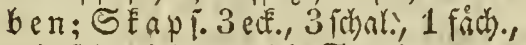
mit jehr vielen rund. $\subseteq$.

246.

Telephrัun.

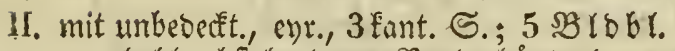
eyr., holgl, abftehend, am Rande håut., Daur. 11. an D. Frudyt zigeneigt; 5 sBlG

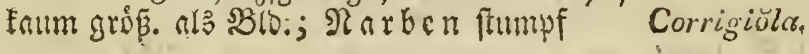

B. ohne Blumenkrone.

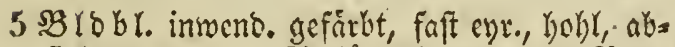
ftebeno, baur.; Stfåd. fo lang afs SBlo.; S tbeut. am Sirunde 2 palt.; ${ }^{2} a p$ f.enr., faft 3 jeit., 3 finch., 3 jhanl., nit jegr vielen

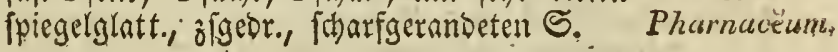

\section{Dronting. \\ $\mathfrak{B}$ i $\mathfrak{e} \mathfrak{r} \subseteq \mathfrak{S} \mathfrak{a} \mathfrak{b} \mathfrak{b} \in \mathfrak{g}$ e.}

\$B ro. 5 theil., nit långl.; abjtehend. Sapp.:

Blé. 5 blättr.; Rect. 5-10 hohl., berzf.

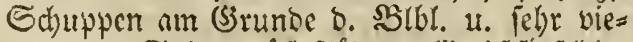
len an D. Spitze briij. Fåaen; (5) riff. fehlt; 200.4 Rarben; SEap f. falt 4 feit., 1 fák 4ichal., mit halber Edheiberwant; viele $\mathcal{S}^{\prime}$ 249. † nit baut. Sande, an b. Edjaten angebeftet Parnassia. 


\section{Dronung.}

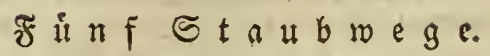

I. Nit untern Frudtenoten; Doldé

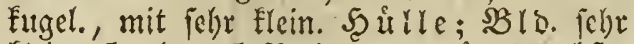
flein, 5 jahn.; $5 \mathfrak{B l b l}$. eyr. = langl., abjte $=$ heno ob. umgeid)lagen; 3-10 fajt Eugel. Rarben; şeere rumbl., gefront, mit 5 bart., langl. $\subseteq$.

II. mit obern Frudtenoten;

A. mit Şưlle u. gem. SBls.; Şülle rọhr., troff., umgeichlng., oft an o. Spike in mehrere \&app. geth.; gem. $\mathfrak{B}$ lo. nit

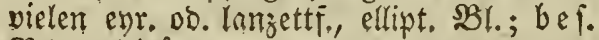
\$B! b. 1 blättr, ungeth., gefalt., gamb

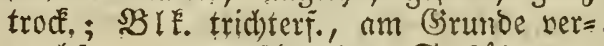
wachien, unten formaler; St $\mathfrak{f} \mathfrak{a} b$. ant

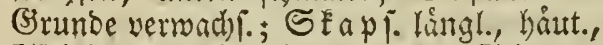
5 fpinig, mit SBlo. bebedft, ohne Edyalen; 1 S. långl., an e. langen Faben hang.; Frb b. fpreunt.

B, okne Salle u. gem. SBlbece;

1. mit Rectar.; 5 Blobl. lanjettf., rimnenf. $=$ bobl, fpics, in e. Siubre zfge $=$ neigt; SBlErone 5 blittr., mit langen, gleichkr., geraben, am Sirmmbe verbun= Denen u. D. Stgef. trag. Någeln, enr., umgeiditag., abftelyeno. Enum; Rect, 5 gan fleine welke Schupp., auswerto, an Dem (Srunbe D. Fruchtent; (5) riff. proiement.; Narbe ftump;

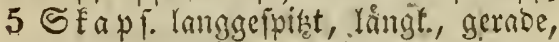
zigeor., ber Länge nad) imweno. auf fpring., mit vielen $\mathrm{El}$. S. $^{2} \quad$ Crassüla,

2. ohne Rectarium; Bldecke;

a, 1 blàttrigi

F, ungeth., gefart., glocefenform. Do: trid)terf., mit haiut., an $\delta$. Spithe gez̧alint, Saum; sBlf́rone meift 5 bláttr., Daum 1 blåttr.; St $\mathfrak{f a ̊ d}^{\circ}$. mit Siubre Doer Shlbl.; zilhng.; 5 (5) riff., felten 3 abgefondert;

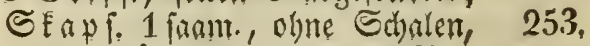
mit ber båut. SBlo. U. mit 1 S. Statice. 


\section{5 Staubgefåpe.}

b. tief5 ppalt., saur., mit gerno., [pis:

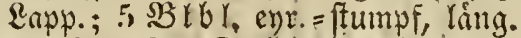
als Blbecte; Stbeut; febr llem; 5-6 (S) riff.; SEap F. eyr., etwas ctf., 1 farch., an ber Spilse 3 ober 5 fichal., mit vielen flein., fajt enr. $\mathfrak{S}$. Drosěra.

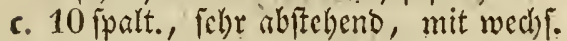
ichmalern Lapp.; 5 SB Ybl. in \$Bro.; (3) $r$ iff. aus o. Seite D. Fruthtfrt; Tar be Engel.; 5 nadte, langl. $S_{\text {. }}$ in Blo. geid)lofi.

b. 5 blåttr. Daur.; $\mathfrak{B} \mathfrak{l}$ f. 5 blåttr.: $\Re$ ågel in e. ganz furje siokre foft verwadhr., welche e. eyr. Slatte tragen; $\mathcal{S}_{t} f_{a} b$.

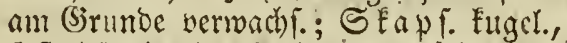
faft 5 frit.', 5-10 (b)al., 10 fích., mit 1 fanm. Fåàern; S. einjeln, eyr., lang= geipist, etwas flach, glatt

Sibbaldia.

255.
Sibbaldia.

\section{4. +}

Drosera.

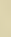

$256 ;$
Linlum.




\section{Rlaffe.}

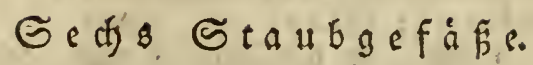

(Hexandria.)

\section{Dronung.

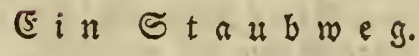

\section{Mit Blumenkrone;}
A, mit Bldecke ohne Scheide."

Mit obern Frudt
A. 1 blåttrig,
1. rabf.; $\mathfrak{B}$ rs. 6 fpalt.; $\mathfrak{B}$ eere 6 jaam.
2. rachenf. (F. 554.)
260. 5
Prinos.
(Corydülis.)

B. 3 blåttr.; sBro. 3 blåttr.; Stfåb. mit Tan. 261. gen, geglieb. Scaaren;

C. 4 bláttr.; Bldecke:

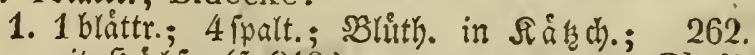
mit Soulfe (f. 810.) - Gleditschŭa.

2. 4 blăttr.; $\mathfrak{B}$ lé. f̂reuź.;
a. mit Sct) ote (i. 527.)

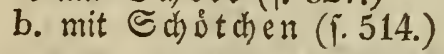
(Cleŏme,)
(Lepidŭım.)

D. 5 bråttrig; Die 1 bratttr. Bldecke:

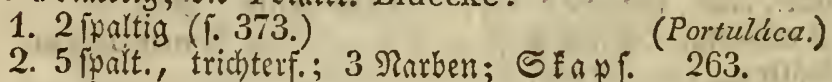
1 fách., 3 fobal., vielfaum.

Frankenı̆a.

E. 6 britttr., auf b. sBlo.; Bld.:1

1. 1 bláttrig,

a. 12 zabnig (i. 378 .) vierfanm.

2. 6 blättrig; Nect. :

- a. 2 D rufen an Den Rlageln jebes Rlbr.; (3) riff. feldt; sBeere 1 fách., 2 ober 4 faam.; Rarbe genabelt; Stfáb. anfangs an $\$$. Druje bangeno, Damn fich elaftich aufrichtens

(Lythrum.)

264.

Peplis. 


\section{6 Staubgefáfe.}

b. ๔. $6 \mathfrak{b r a ̊ t t r . ~} \mathfrak{R} \mathfrak{a} \mathfrak{n} z$ auf $\delta$. Ragefn $b$ : sBle.; $\mathfrak{B}$ rs. abfall.; Stfru cht Eugel.; 266. 1 fam.; Stbeut. am Ranbe auffipring. Caulophýllum.

B. ohne Blumen decke:

1. mit Scheide; a. unterer. Fruchtknoten.

Blumenkrone :

A. 1 bláttrig,

1. tellerf., mit. 6 theil. Saum; Scheibe 1 blo̊ttr.; Rect. Rranz 1 b!attr., glo.

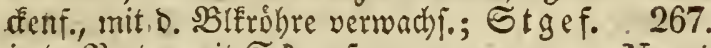
in D. Sect.; mit SEap F.

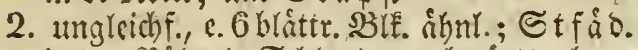

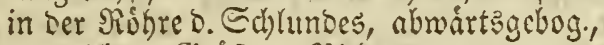

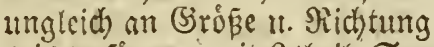

3. trid)terfórm, mit 6 theil. Saum; Nect. Narcissus. : I $\mathrm{r}$ a $\mathrm{z}$ glocfenf., 6 od. 12 zahn., mit $\delta$. Bletrofhe verwachf. 11. Die Stgef. tragento; mit SEap

B. 3 blâttr.; SBlbr. hohl; Scheide 1 bråttr.; Nect. Aranz 36lattr., mit flein., ausgerand. $\mathfrak{B l} \mathfrak{l} ; \mathfrak{N a} \mathfrak{a} \mathfrak{b} \mathfrak{e}$ cinf.

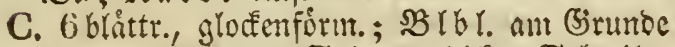
ofhangento, an D. Spitie versidt; Sdyeibe 1 bláttr.; Narbe cinf.

268.

Amarýllis.

269.

Pancratı̌um:

\section{b. oberer. Fruchtknoten.}

\section{Blumenkrone :}

1. 1 blättr., trictiterform., 6 theil.; Stgef. im 272.

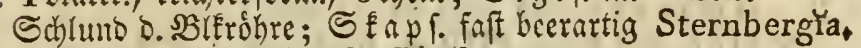

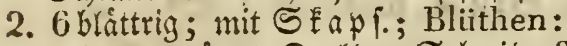
a. in e. georangt. Dolbe; Sch cibe 2bláttr.; vierbluth.; $B$ IE. offen

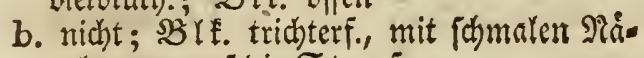 getn, worauf sie Stgef.

270.

Galânithus.

271.

Leucójum.

2. ohne Scheide;

a. unterer Fruchtkn. (j. 320.) (Polygönum.)

b. oberer Fruchtknoten;i

a. Blk. I blättrig.

1. Mit Rectar.; 3 If. 6 theil., offent Nect.: 275.

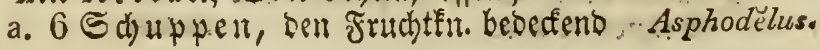


b. 3 jwenborft. Prifen (โ.325.)

(Laurus.)

2. obne Rectarium;

a, mit $B_{\text {cere: }} 3$ fáh)., 3 [aаm., vor p. Sicife = 276.

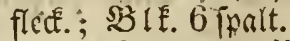

Convallaria.

b. mit S'apjel; Blkrone;

6. glocfenformig, 6 ipalt., mit 3 গect. Eod) ern am Fruchten.; SEapF. mit 3 imeift 2 jaam. Fỏhern

b. robr., mit eys. of. waljenf. siokre u. furz., 6 zahn. Saum

c. trichterf., gefrimmtroibr., gleich form.;

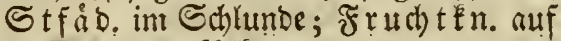
o. Sirumbe D. SBYE.

จ. trichterf. = glodenf., 6 theil.; ङ nbwärtzgeneigt; Nintbe flein, einf., etwas haar.

e. 6 tbeilig, abftebend; Stfäs. bart.; SEap T. rundl.; S: eftig!

277.

Hyacinthus, 278.

Muscíri.

279.

Polyánthes.

Ilemerocillis. 281.

Bulbine.

b. Blk, 6 blättrig.

A. mit Nectarium:

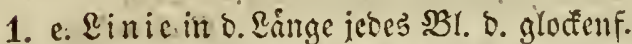
BIE., beren Rappen oft umgejolagen fino; SEapi..mit Sdualen, bie Durh e. gegit: tertes Şargervebe verbunten fino

282.

Lilium:

2. c. (5i $r \mathfrak{l} b$ d) $e \mathfrak{n}$

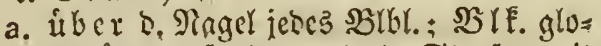
denform., fo lang als D. Stgef.; mit

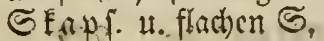

283.

Fritillaria.

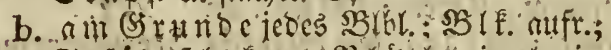

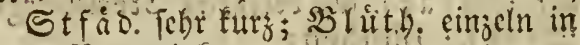
s. SBlattwint.

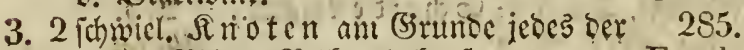

3 invern Btbl.; $2 B$ lE: glodenf:

Erythronuัum.

Burobrealect d $\mathrm{r} i \mathrm{llm}$;

1. nit (5) riffel;

a. mit 3 fách. SBe re: F́acher 2 faam.; \$Blbl. aufr., bie 3 innern an b. Epize. 286. umgeichlag.

b. mit Sa amen

s. abfteheno,

1) abfateno; Stfå fabenf. am a) Srumbe D. $\mathfrak{B l b l}$.

Asparăğrus.

287.

Scilla. 
2) daur.; Stfado. fabenf., bart.; 288.

SEapf. evr., mit eff. $\mathfrak{S}^{\prime}$. Anthericum.

b. aufr., Dautr., won ber $\$$ Nitte an $a b=$ ftehend; Stfåd. am Strunde brei=

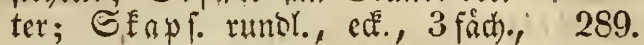
mit rundl, nad̂t. $\Subset$. Ornithogălum.

c. bie 3 innern lónger; St ge f. aufr.; Słap r. faft eyr., 3 flugel., mit 290. fuget. $\subseteq$.

2. ohme. (S) riffel; Blkrone:

a. glodenförmig

Lachenalǐa.

291.

Tillipa.

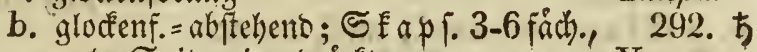

an b. Spirge eingebrudft Yucca,

II. ohne Blumenkrone. (F. 26jt.) (Peplis.)

\section{OrDnung.

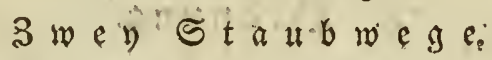

Blumendecke:

1. 2 bláttrig (1. 296.)

2. Felit (1.320.)

(Rumex.)

$\left(\mu_{0}\right.$ lygönum. $)$

\section{Droning.}

$\mathfrak{D} \mathfrak{x} \mathfrak{S} \in \mathbb{E} \mathfrak{a} \mathfrak{u} \mathfrak{b} \mathfrak{w} \mathfrak{e} \mathfrak{g}$.

I. Mit eigentl. Bldecke:

1. 1 bláttr., 3 fpalt.; 3 lk 6 blättr.; mit

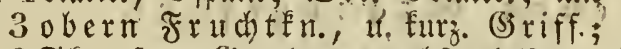

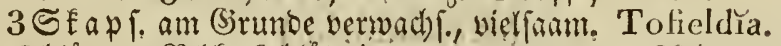

2. 3 blåttr.; Ş le. 3 blătrtig;

a. 3 ecere 3 fäch.

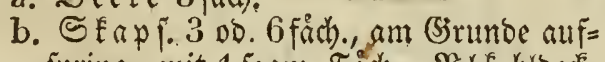
fpring., mit 1 faam. Fach; ; $\mathfrak{B l f}$. blocét= ortig; 3 গAarben obne (s) riff.; mit obern $\mathscr{F} \mathfrak{u}$ then.

c. Sanme unbebedét, eingerm, nadét, 3 fant.; $3 \mathfrak{B} \mathfrak{l b l}$. zigcneigt

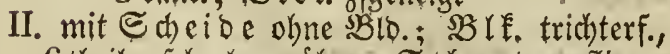
6 thcil., jehr langrógr.;. Stbent. auflieg.; 3 (s) riff.; 3 Skap f, verwachl., aufgeblaf., 297. an b. Epilze nach immen auffipring., vielfaan... Colchiccum: 
III. ohne Stheibe u. BId ; Blkrone:

A. 1 blattr., 6 theil.; mit obern Fru (b) tEn.;

1. mit 3(3riff.; Gfapi. 3 fách., 3 hom., 298. vieljanm.

2. ohne (Siriffel;

a. 1 Skap $\%$ faft fugel., an D. Spike mit 3 fach. Spalte auffuring. u. mit

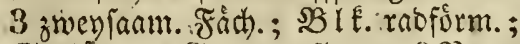

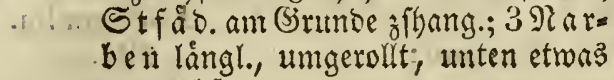

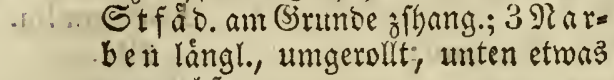
berwachi.

b. 3 SE apr. aufgethlá., meift 2 jaam.; $3 \mathfrak{F r u c b}$ tenot.; SBlE. blectartig; Stbeut. gleichbreit

B. 6blattrig; 3 bieljamige stapieln (1. 804.)

299...

Xerop̣hýllum.

300. t

Scheuchzeria.

301:

Verátrum.

\section{$\therefore$ 4. Drditug.}

פebr $\mathfrak{S t a} \mathfrak{a b}$ wege.

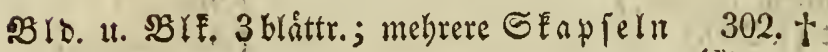
1 jaam.

Alǐsma.

\section{Rlaffe.}

Sieben Śtaubgefáfa.

(Heptandria.)

\section{$\longrightarrow$ \\ 1. Oronung. \\ Eี $\mathfrak{n} \subseteq \mathfrak{t} \mathfrak{u} \mathfrak{b} \mathfrak{w} \mathfrak{e}$.}

I. Mit eigentl. Bldecke:

A. 1 blätrrig,

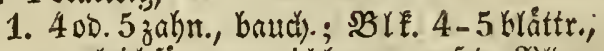
ungleichförn., reeidhaar., auf b. sBlo.; ভkapf. 3 fách.;

303. ち Aescülus. 
VII. 7 Stautgefåje.

45

2. 5 theilig ( 1.542.$)$

3. meift 7 theil.; (1. 260.)

B. T Grattr.; SBlE. 1 bláttr., 7 theil., gleichf., flach); $B^{2} \in \mathfrak{c}$ re 1 fádb., faftlob

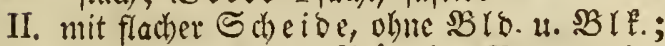

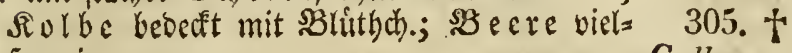
famig

Calla:
(Pelargonumm.)

(Prinos.) 万 304.

Trientalis.

2. Dronung.

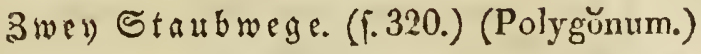

3. Orditng.

D) $\mathfrak{e y}$ Staubrege.

Dic 5 Blumenblätter:

1. 2 fpaltig (i. 357.)

2. ungetbeilt (\$. 360.)

(Siléne.)

(Arenarăa.)

4. Dronatig.

Zinf Staubráge. ( $\{.370$.) (Spergüla.)

VIII. Rlaffe.

$\mathfrak{A} \mathfrak{d} \mathfrak{t} \subseteq \mathfrak{S} \mathfrak{a} \| \mathfrak{b} \mathfrak{g} \in \mathfrak{f} \mathfrak{a} \mathfrak{b}$ ?

(Octandria.)

1. Droแtกg.

Ein S $t \mathfrak{a} \mathfrak{u} \mathfrak{b} \mathfrak{w} \mathfrak{g}$ :

Blumendecke:

I. (sinfach; Blkrone: 
A. 1 bláttrig; Bldecke:

1. 1 blattrig,

a. 4 a abnig; $\subseteq$ tfa $a$, auf sgrbo.; mit itnt. Frucht glocenf., 4-5 part., mit umgeithlag. Eapp.; SBeere 4-5 fát)., vielijam.

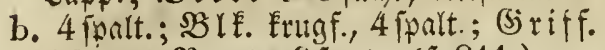
4 palt.; Beere 8 jaan. (i. 811.)

c. $S$ ipaltig (i. 260.)

2. 4 blăttr., baur.; sBle. 4 fpalt., baur.; Etfád. auf Bblbo.; Stbelt. 2 fpalt.; Skapf. hăut.; 4 fách., nnit Scheibervàndat von $D$. Máanocrn $D$. Echalen

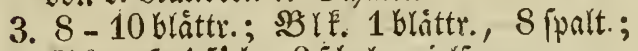

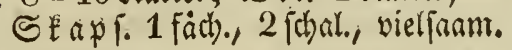

$306 . \hbar$

Vaccinǔum.

$307 . \hbar$

Diospýros。

(Prinos.) b

308. $ち$

Erica.

399.

Chlora.

B. 4 bratttrig;

1. mit Saamenkapsel:

a. 3 fácherig; Bld.:

a. 1 blättrig (1. 303.)

b. 5 blăttr.; $\stackrel{B}{B}$ IE. umgleichf.; Rect. vier 2 fpalt. Schupp.; $\mathfrak{F}$ ácher b. - aufgeblaf. SE ap 2 faam.

(Aescüluss.) ち

b. 4 fädherig,

ค. wăl BID. 4 palt.; Saame:

1) wollig

2) nadft; $\mathfrak{B}$ lolapp. abrwåttsgebog.,

311.

Epilobŭrom.

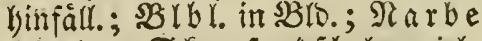
4 ipalt.; Skap i. 4 idjal., vicl= faam.; 'ভ. am 4 jeit. Frbo. in $b$. stitte

b. lappig (1. 343.)

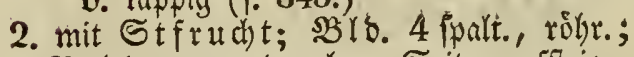
Blbl. gegen $\triangleright$. obere Seite aufferg.; श) uี 1 faam. 4 ect.

310.
Koelreuterı̆a. 
III. Felyt; Blkrone:

A. 1 blättr., 4 jpartitis,

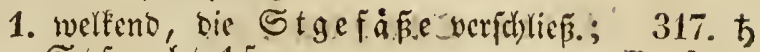
Stfrud t 1 farm.

Dapline.

2. in b. Mitte bauth); St ge f. im Schlunde cingefught, febr furtz, mit' 1 gefchnabel= $\operatorname{ten} \mathfrak{N} \amalg \tilde{\beta}$

B. Sbrăttrig (โ. 347.)

318.

Stelléra. .

(Monotrŏpa.)

\section{Oroแแ॥ g.}

$3 \mathfrak{w} \mathfrak{c} y \subseteq \mathfrak{a} u \mathfrak{b} \mathfrak{w} \mathfrak{e} g$ e.

Blumenkrone:

1. 4 blattrig; Bldecke:
a. 1 biáattr. (f. 35t.)

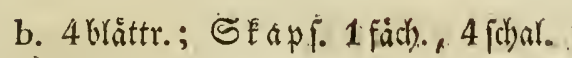
(IItydrangèa.) Moehrinğ̌a.

2. 5 bláttrig (i. 390.)

3. fefilt; $\mathfrak{B}$ ro: 4 ipartig;

(Agrimonia.)

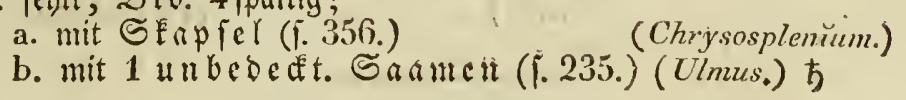

\section{Oำกung.}

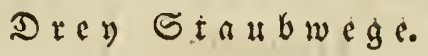

Blumenkrone:

1. 1 brättr., 4 theil., broedfart.; 1S. êt., bet 320 . becift

2. 4 blăttrig;

a. mit giect.: 4 bláttrig, ungleichf.; ; B is 321 .

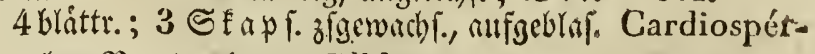

b. obne Rectarium; Blblätter:

mum.
a. 2 fpaltig ( 1.357.$)$
b. ungetheitt (โ. 360.)
(Silene.)
(Arenariur.) 


\section{Drontug.}

Siér Staubrege.

Blumenkrone:

1. 1 blättr., 4-5 palpalt.; \$Brb. 2-3 ppart.; mit

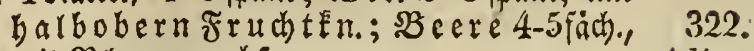
mit Sirs. vermachi.

2. 4 blàttrig; Bldecke:

a. 1 brättrig (f. 756.$)$

b. 4 blåttrig;

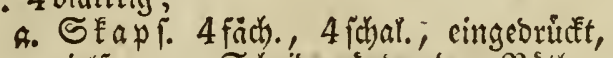
vielfaam.; Scheiberoánde ben Máthen entgegenger.

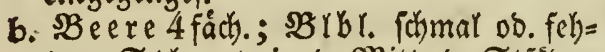
Ien; Stbeut. in b. Sitte b. Stfáden angerwach fen

3. feglt (i.324.)

Adbra.

(Myriophillum.)t

\section{3. $\%$ \\ Elatĭne.}

$324:$

Paris.

(Paris.)

\section{Dronung.}

Sieben od. 2 dit staubrege (i. 371.)

(Phytolácca.)

\section{Rlafíe.}

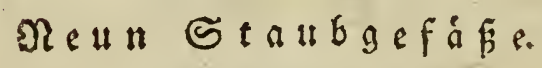

(Enneandria.)

\section{$\longrightarrow$ \\ 1. Dronung.

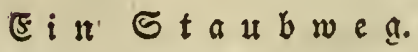

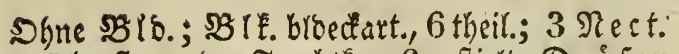
2 borft. um ben Fructetn.; 2 geffielte Drúfen am (Srunde jebes b. inn. Stfäb.; Stfrucht 325. 万 1 faam. 


\section{10 Stánbgefápéc.}

\section{Orbnung.}

Drey Staubwege:

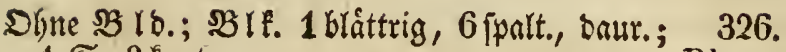
1 S. 3 Eant.

Rheum.

\section{Oronung.}

Seds Staubrege:

Shne SBIS.; SBIF. 6 blättr.: 6 SEapfeln 327. f vielfarmig

Butŏmus.

\section{Riaffe.}

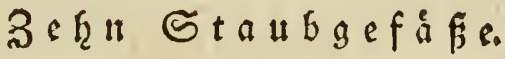

(Decandria.)

1. Ording.

E i $\mathfrak{S}$ t $\mathfrak{a} \mathfrak{u} \mathfrak{b} \mathfrak{w}$ e

\section{Blkrone I blättrig.}

Blumendecke:

I. cinfach); Blkrone:

A. cyrumb,

1. mit 5 ppalt. Mlunoung; Bld.;

a. 5 zahn. (โ. 306j.)

b. 5 theil., mit umgejchlag. Rapp.; mit obern Frudtetroten; ভ我pr. 328. 5 fách., mit Duerwano

Androméda.

2. am Srunbe D. Meindung ourchfichtig; RI I 5 theil.; mit obern FrudtEn.; Beere 5 fách).

329. $\hbar$

Arbütus.

- B. trichterf., etwas fohief; $\mathscr{B} \backslash 8.5$ theil.; Stgef. cubwártageneigt; Słapf. 5 fảch., $330 . \hbar$ vieljaam.

Rhododéndrum.

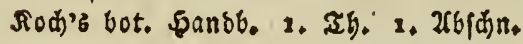


C. tellerf., mit foft 5 horn. Saum, muf beren

- Siruber bie Etbeut. anfliegen; $\$ 2$ ld ed e 5 theil.; Sf a pi. 5 fách.. vielfam. 331. $\hbar$

Kalmǐa.

II. Doppelt: die äussere:

1. 3 blattr.: bie innere 5 theil.; $\mathfrak{B l}$ le. 332.5

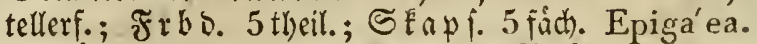

2. 2 blăttr.; bie inncre 5 ppalt.; $3 \mathfrak{B l}$. eyr., mit 10 Nect. Spizen um ben Fruchten.; die 5 fách. SEapF. mit D. innern beerart. SBlo, ungeben

333. $\hbar$

Gaultherǐa.

\section{Blk. mehrblättrig;}

A, Blk. ungleichförmig.

\section{Blumenkrone:}

I. fchmetterlingsfơrmig: Fähnchen :

1. fo lang alb bie Flugel; Gliederhiilse:

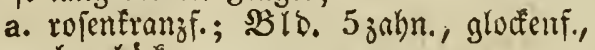 oben bojatr.
b. baudb., glatt, vieljaam.; SBlo. 5 palt., faft 2 lipp. 334.
Sophora. 335.
Podalyría.

2. nebjt ben fllig. Furger als o. Sthiffd);

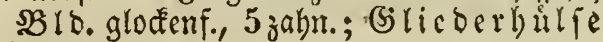
vielian.

3. furz, unter o. Flügeln veroedłt; $\mathfrak{B}$ lo. 5 zahn., unten bớfr.; (s lieberbulie vieliaam.

II. nicht fdymetterlingsf.; Bldecke:

1. 1 blåttr., 5 zahn.; $\mathfrak{B}$ lá. 3bláttr.; St gef. abroirtşgeneigt; Sfap 5.5 fóch., vicliam. Rhodóra.

2. 5 blàttrig; $\mathfrak{B l k}$. 5 blåtrig;

a. bie 3 unt. Stbeut. gerchnabelt : bie 3 obern unfrubtb., an ben lảngern Stfäben gefrummt; (B) lieberbulle båut., 2 fual., vielinam.

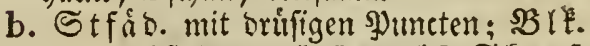

336. 方 Anagy'ris.

337.5

Cercis.

etras abftcbend; 5 zigewadb. S产ap. Dictámnus.

B. Blk. gleichförmiğ.

Blumendecke:

I. 1 bláttrig; Blkrone:

A. 1 bláttr., 5 theil., flach; BBrs. 5 ppalt.; Sfapi. 5 fåd j., vieljaam., am (Srundé aufipring.

339.

Cassia.

340. 


\section{10 Staubgefápét.}

B. 5 blåttrig;

1. ohure (s) riff.; Brblät. abftelend; 342 . 5 Skap f. bơdr., etwas dorn., vicljaam. Tribŭlus.

2. mit (3) riffel; Skapsel:

a. gelappt; $\mathfrak{B} l \mathfrak{b l}$ l. bohl; $\mathfrak{B}$ Ib b. mit 10 Pect. Sguncten umgeben; 343. B (b. 4-5 theilig Ruta.

b. nidht gelappt; Rarbe 3 ipartig; 344 . 万 SEapi. 3 fách., 3 ichal.

c. an ben Edfen auffipringend, 5 fådh.; 345. Stbeut. oben 2 born.

II. 5 blåttrig; $\mathfrak{B} \mathfrak{l}$ e. 5 blåttr.;

Pyröla.

A. mit Nectarium:

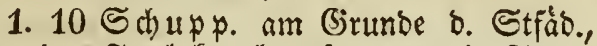
ben Fruchten. bebectend u. bie Stgef. trageno; S

2. 5 Drúfen am Srunde einiger Stfáb. (i. 544.)

B. obne Rect. (โ. 345.)

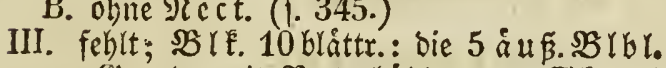
nm (Srunde mit Rectarbóblungen; $\mathcal{S}_{\mathfrak{k}} \mathfrak{a}$ F. 5 idhat.

(Geranŭum.)

(Pyrōla.)

347.

Illonotröpa.

\section{Drditug.}

\section{$3 \mathfrak{w e y}$ S $t \mathfrak{a} \mathfrak{u} \mathfrak{b} \mathfrak{e} g$ e.}

SB ro. 1 blåttr., 5 theil.; Blkrone:

I. 5 blåttrig;

A. mit obcrn frudh tenoten; Blk.:

1. mit $\mathfrak{R} \mathfrak{a}$ geln ; Bld.:

a. warlonfớrmig, lang, leberart., mit 4-8 Schupp. um (Śrumbe; SEap F. malzenf., 1 fách., viclfaam., an b. Epize aufipring.

b. róhr., ohne ভchupp.; SEap l. lingl., 1 find

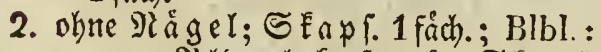

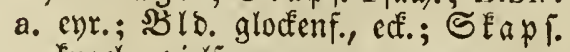
fugel., vielian.

b. fieberf., auf d. 5 fpart. BBld.; SE ap F. 2 ithal.; Sthalen gleichlang

c. ungeth., auf $\delta .5$ theil. Bld.; $\subseteq \mathfrak{E} \mathfrak{a} \mathfrak{p}$. 2 final.: Sdjoten verínicoengrop Tiarélla.

348.

Dicinthus.

349.

Saponarüa.

350.

Gypsophila. 351. Mitèlla. D 2 


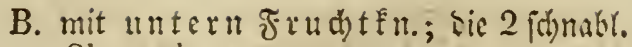
Skapsel:

1..1 fidch., vicliuant.; $\mathfrak{B}$ ls. 5 theil.

2. 2 fächerig, jwijchen scu Şömern an

II. fehlt; Rod aufipring.; SElo. 5 jalln.

A. mit oberu Frudtenoter;

1. mit 1 pam. Skapi.; 5 St Etbeut. (1. 230.)

2. mit $1-2$ unbededft. $\subseteq$. in \$ืls.;

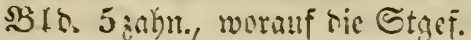

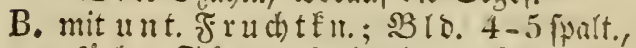

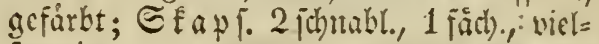
fanmig

\section{Dtsแu 119 . \\ D $\mathfrak{A y}$ Staubwagc.}

Blumerdecke:

I. 1 blàttrig; Blblätter :

A. mit $\mathfrak{l} \mathfrak{a} g e \mathfrak{l n} ; \mathfrak{B}$ le. 5 blåttr.;

1. mit Nect.: 2 Bäbne nu jesent Szlkt.,

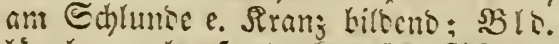
langl., waljenf. od. fègelf.; SEati. 3 Fầh.

2. chne Nect.; Bldecke:

$3.5 \%$.

Siline.,
a. aujgeblaj., ob. glodenf.; SEapi. 3 fäch.
b. längl. (i. 365.)
358.
Cucubuilns,
(Lychnis.)

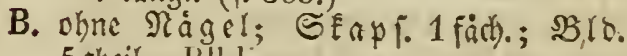
5 thcil.; Blbl.:

1. jerrifien (1.381.)

II. 5 blacttrig; Blhrone:

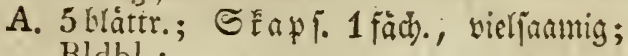
Bldbl.:

1. abjebeno; Blbl.:
2 theil.
6 jabn.
b. ungatheilt
2. midht abitcheuo, 2 jpalt. (โ. 369.)

(Reséda.)

(Tamărix.) 


\section{X. so Staubgefápát.}

B. fehlt;

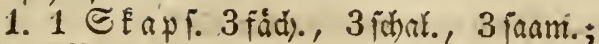
5 Rect. 2 ipalt., blblattart.; ङ tbeut. 361. mechi. unfrudutb. Cherlerüa.

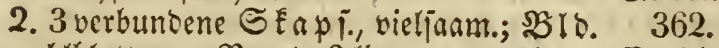
blblattart.; $\mathfrak{R}$ ect. 2 lipt.

Garidélla. III. fellt (F.320.)

(Polysürnumb.)

\section{$\therefore$ 4. Ordnnng.}

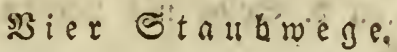

SBrs. 1 Gláttr.; Blk.:

1. mit $\mathfrak{R} a \dot{g} \mathfrak{e l n}$;

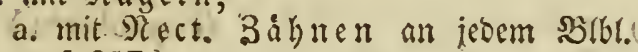
( $(.357$.

b. whre Nect. (F.3S1.)

2. vbne Rágel (î. 365.)

(Silène.)

(Resèda.)

(Lychnis.)

\section{Dronung.}

F้น

Blumendecke:

I. $\mathbf{1}$ blăttrig ;

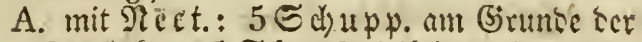

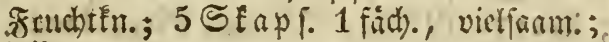

Blk.:

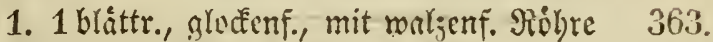

u. 5 theit. Saum; $\$ 210.5$ theil.

2. 5 blättr.; 3 rs. 5 palt.

Cotvlédon. $3 \dot{6 i t}$

Sedum.

B. obne Pest.:-Blbl.:

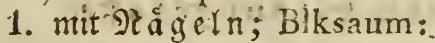

a. meift 2 finalt.; 33lo. längl, glatt; 365 .

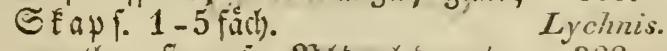

b. ungeth., ftumpf; B1t. leverart.; 366 . Stapi. 1 fäch.

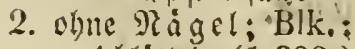
a. 1 bláttriz (f) 322.)
(Adóxa.)

b. 5 klittrig; Bld.:

a. 2 palt. (5. 373 .)

b. 5 f palt.; 5 S vielianm.

(Portulica.)

$36 \%$ :

Penthorrum. I

c. fehit (f. 367.)

(Penthorum.) 
II. 5 blättrig;

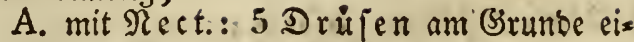
niger Stfád. (i: 544.)

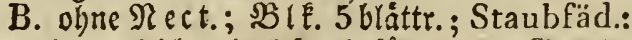
(Geranŭum.)

1. ungleich: bie 5 a $\mathfrak{u}$. verwadben; $\mathfrak{A}$ lenàgel verbunben; SEap 5 ieit., an D. Ecten elaftijt aufipring.; S. meift mit Şaut

2. gleid); Blbl.:

a. geipalten $(2-4-5$ palt:); S 1 făch., an o. Spitse mit వâhnen aufipring.

b. ungetheilt; Skapfel eyr., 1 fách., 370. 5 final.

ILI. febit (i. 371.)

Sperğ̈̆la.

(Phytolácca.)

\section{Dronting.}

Sechs Staubroge (F.369.) (Cerastüum.)

\section{Dronung.}

$3 \in \mathfrak{h} \mathfrak{n}$ Staubrege:

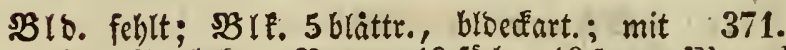
obern. Frudthtfr.; $\Re$ e e r e 10 fåd)., 10 [aam. Phytolácca.

\section{Rlaffe.}

EIf bis Neunjeh̨n Staubgefáfe.

(Dodecandria.)

1. Dronung.

Ein $\subseteq \mathfrak{t} \mathfrak{a} \mathfrak{b} \mathfrak{m} \mathfrak{e}$.

Blumenkroue:

1 blättrig,

1. 4 fpalt., glodenf.; sBis. 4 zahn.; unt.' 372. ち

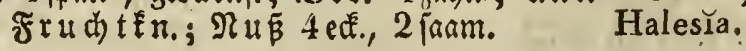




\section{I I-rg Gtaubgefápe.}

2. 8-10 their. (f. 387.)

II. 4 blättrig (î. 527.)

(Decumarǐa) $\hbar$

(Cleŏme.)

III. 5 blattrig; Bldecke :

A. 1 blàttrig,

1. 2 fpalt.; S a 1.1 fáth., in b. Mitte 373.

( rundum auffpring.; 5 fabenf. Frbo. Portuláca.

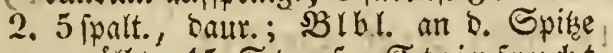
gewolbt; 15 ड̈tgef.; Steinfrutt 374. 1 faam.

B. 5 blattrig;

Nitrará. 375.

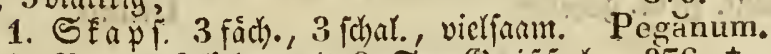

2. Seere 3 fadd., nit 2 S.; Sriffel 376. 万 3 ppalt.

C. Feblt (1. 375.)

IV. 6 bláttr., auf ठ. ภBlo,; Saamenkapsel: Aristotelía. (Pegănum.)

A, 1 fát)., nit Silo. oev Lange nach auf fpring., mit 3 fant. Frbo.; $\$ B$ lo. robr.s 377.

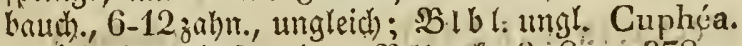
B. 2 fächerig, vielfamig; $\mathfrak{B} 1 \mathrm{~b}$ ede $6-\mathrm{S}^{-} 378$. $10-12$ zakn.

V. fehlt; $2310.3-4$ fpalt., anf D. Fruct)tent.;

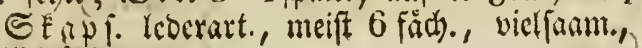
gefront

\section{DrDnแแg.}

Drey Staub vege.

SB İ. 1 blåttr.; Fruchtknoten :

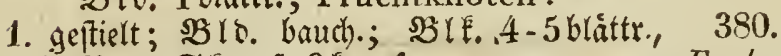
in SBro; SEapr. 3 Fnonf.

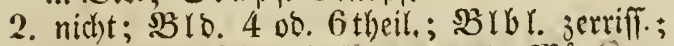

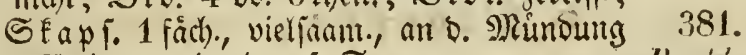
suffpring., mit nierenf. $\mathfrak{S}^{\text {. }}$

379.

Asüruin.

Euphorbia.

Reséda.

\section{Dronung.}

$\mathscr{B}$ i e $\mathfrak{x}$ St a $u b \mathfrak{e} g$ e.

1. mit Samenfapiel (โ. 381.)

2. mit unbebefft. S. (f. 402.)

(Resédà.)

(Tormentilla.) 


\section{Blumendecke:}

1. 1 blättrig (โ. 373.)

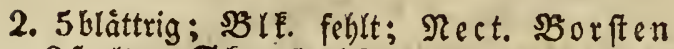
2 fpalt.; Skap 5 feit., 5 fädr., 5 fodal.; vielfanm.; $\subseteq$. mit e. borftenf. Faben an b. fáulenf. Frrbo. geheftet

\section{(Portuláca.)}

382.
Glinus.

\section{Stonung.}

ธechs $ธ$ taubwege:

1. Mit Rectarium (f. 364.)

(Sedum.)

2. obne Rect.; Bldecke:
a. 4-6theil. (i. 381.)
(Reséda.)
b. 9-12 their. (1. 383.)
(Sempervivum.)

\section{Drstung:}

Sieben od. 2 dat Staubrege

(i. 437.)

(Thalictrum.

\section{Dronung.}

3 wo if $S t a \mathfrak{u}$ wege:

BIb. 9-12 theil.; BB IE rone 8-12 blättr.; 383.

12 SEapf. vielfaam.

Sempersivum. 


\section{Rlaffe.}

\section{Siele Etrubgefáfe}

auf ber Slumenbesfe ob. Slumenlrone,

(nid)t auf Dem 3̧umentboden).

(Icosandria.)

\section{Oronung.}

Ei $\mathfrak{n}$ S $\mathfrak{t} \mathfrak{u k} \mathfrak{w} \in \mathrm{g}$.

\section{7. unierer Fruchtknoten.}

Die 1 blättr. Blumendecke:

1. 4 theil.; $\mathfrak{B}$ IE. 4 blâttr.; (S r iff. 4 ppalt.; 384. 方 Słap f. 4 fåh., vielfam.; S. mit Scaut Philadelphus.

2. 5 [palt.; $\mathfrak{B}$ If. 5 blättr.; a. mit Beere:
ค. $2-5$ fantig (1. 397.)
b. vielfaamig, 2-3 fơdy. (Mespitus:) $\frac{1}{\hbar}$ 385. $\frac{1}{10}$ Myrtus.

b. mit vielfáth., vielfaam. Seinfrucht; 386. bl S. beerart.

3. 5 theil.; $B$ If 5 brâttr.; (5) riff. 5 part.; Skap f. 5 fách. (i. 384.)

4. 8-10 theil.; B̉ le. 8-10blâttr.; SEłap. 7-10 fách. vieljaam.

Púnčca.

\section{II. oberer Fruchiknoten.}

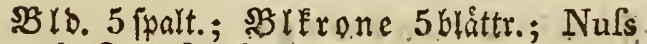

d. Steinfrucht:

1. Iỏcherig

(Philadelphus.) 5

387: $\frac{1}{\hbar}$

Decumarı̌a.

2. nicht locterig, aber mit bervorfegenten 389 . 方 Nåtben

Prunus.

\section{Oronung.}

$3 \mathfrak{w} \in \mathfrak{y} \subseteq \mathfrak{S} \mathfrak{a} \mathfrak{u} \mathfrak{b} \mathfrak{w} \in \mathfrak{g}$ e.

\section{SBlÉ. 5 blåttr.; Bldecke:}

1. 5 zaldn., tellerf., mit am Schlunde zigczog. 390 .

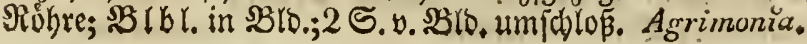


2. 5 fpalt.; 3 Beere $2-5$ farm. (1. 393.)

3. 10 ppalt., mit mechf. fleinern $2 a p p . ;$ (S riff feulenf., hinfäll.; 2 S. vert. = enr.

\section{Dronuttg.}

\section{Drey $S_{\mathfrak{a}} \mathfrak{a} \mathfrak{b} \mathfrak{w} \mathfrak{g e}$.}

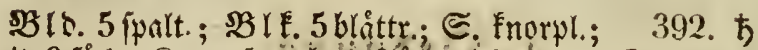

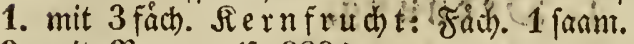

Sorbus.

2. mit Beere $(1 ., 393$.

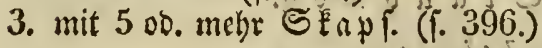

(Mespitus.) ち

(Spirciea.)

\section{Dronung.}

$\mathfrak{S}$ i e $\mathfrak{S}$ S $\mathfrak{a} \mathfrak{a b} \mathfrak{w} \times \mathfrak{g e}$.

\section{Blumenkròne:}

1. 5 blattr.; 3 Klo. 5 palt.;

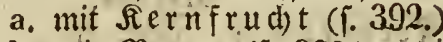
b. mit Beere (₹. 393.)

2. vielblåttrig (1. 397.)

3. fiblt (1. 39S.)

(Sorbus.) ち

(ITespilus.), 5

(Mesembryan-

thĕním.

(Tetragonia.) :

\section{Oronung.}

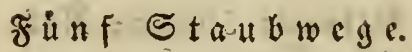

\section{Blumenkrone:}

1. 5 blätrig; $3 \mathcal{B}$ to. 5 paltig;

a. Bcere $2=5$ pamig; S. fnocbentart; 393. $\hbar$ SBlolapp. gciágt

b. Sernfrudt 5 fích.; mit unt. Frud) t= Enoten; Fächer d. Kernfr.:

a. 2 faam.; Nernfr. fleifhig

Mespilus.

394. 卢

Pyrtes.

395. 5

b. vielfaam., flebrig; $\mathbb{A}$ ernfr. fillig: Cydonia.

c. 5 od. mehr Sfapl., inwent. 2 fidal., 396. vielfan.; $\$ 21 D$. abifeheno

Spiráea. 


\section{Siele Stgef. auf $\delta$. BIb.}

2. vielbręttrig, gleidbur.; unten zíbang.; $\mathfrak{B}$ ro. 5 ppalt.; untere Skapf. freujelf., fleifit., 5 coer 10 fách., vielfam.

3. fellit;

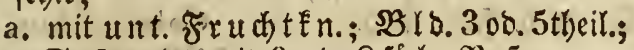
Stfrud t mit 3 od. 8 fách. Stuß

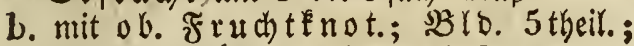
SEap 5 fåch., 5 jhal., vieljarm.

397.

Mesembryanthěmum.

398.

Tetragoňa. 'S 399.

Aizóon.

\section{Oronung.}

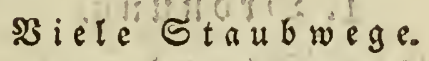

Blumendecke:

I. 1 blăttrig,

A. 5 fpaltig; Blkrone:

1. 5 blåttrig;

a. Beere zigepeft aus tleinen 1 jam: Beerchen

b. vicle borft. S. an d. innern $\mathfrak{B l} 10=$ wano angekeftet; $\mathfrak{B}$ 1D. Entugfórm., $401 . \hbar$ fleifh., am Soalfe verengt.

c. 5 od. mehr. Sfap i. (i. 396.)

2. vielblättrig (i. 397.)

B. 8 ppaltig; Blkrone:

1. 4biattr.; S. runol., nadet, auf $b$. Flei=

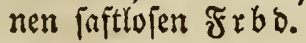

Rosa.

(Spiráea.

(Miesémbryanthĕmum.)

402.

Tormentilla. 403.

2. Sblåttr.; S. gejdumånzt, haar. Dryas.

C. 10 ipalt.; $\mathfrak{B l f}$. 5 blåttr.; Fruchtbod.: 404. 1. beerart., eyr., abfalleno

Fragaria. 405.

2. Faftlos, flein: S. rungl., runblo, nadft Potentilla.

3. fchrammig, evr., baur.; 3 lbl. Flei= 406. ner als 3 BID.

Comărum. 
4. mit bart. ob. gegliebert - begrarnten 407. S.:- SBlolap 1. ungleich

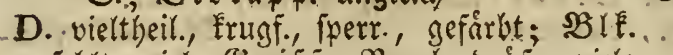

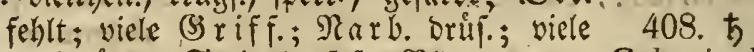

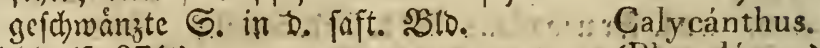

II. fellt (f. 371:).

(Phytolácca.)

\section{Rlaffe.}

Nie

auf ben Stumentoben.

(Polyandria)

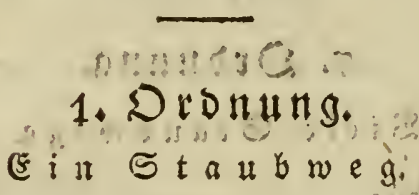

Blumenkrone:

I. 4 blåtrtig; Bldecke:

A. 2 blattrig; mit obern $\widetilde{F} \mathfrak{r}$ (b)

1. SŁav 1 fách, unter d. Daur. Narbe 409. in Eobern auffipring.

2. Sct ot e gleidbreit und

a, 1 fäch., 2 fchal., ohne 3wiphenwand; 410.

vicle fummf. S. ". Chelidonŭum.

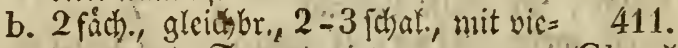
ren puntet. S.

'Glaucium...

B. 4 brittrig; bie 1 fád. Beere:

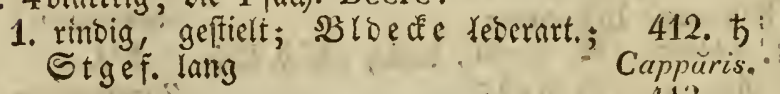
41:3.

2. mit harbtreišruno. S. Actíecto

il. 5 blåttrig; Bldecke:

A. cinfad),

1. 1 bláttrig;

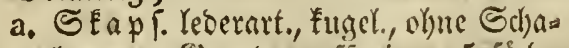

$\therefore$ len, am (Sirumbe aufipring.; 5 fíd.)., rod) 4 Fåd). vhne S., baber 1 [anm.; Bito. 5 theil.

414. ち $\cdots$

Tilia. 


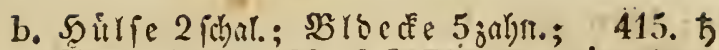
डgef. bis zu 100 (i. 807.). Acač̀.

2. 5 bláttrig, die 2 åu f́. B̉. Hleiner; Stap 1 fach., 3 idhul.; S: an ben 3ivijchenwånden befeftigt

B. Soppclt: Die áǘre 3 ut. bie obere 5 blâttr.; Giriff. mit o. fobilof. Slarbe be= becft; ভ

C. fiblt; Nectarium:

1. ein 2 ppaltig., binterwårţ gehơntes.

$$
\text { (i. 425.) }
$$

2. 2 gefticlte Sapp. unter D. oberften SBIbl. (1. 424.$)$

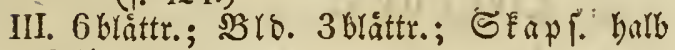
2 fibal.

IV. Sblattr.; $\mathfrak{B}$ rs. 2 blattr.; Schote cut., 1 fóch.

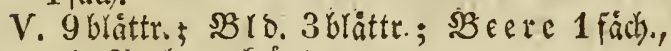
mit Marbe gefrónt

VI. vielblättr.; $\mathfrak{B}$ ID. 4 - 5 blättr.; $\mathfrak{R}$ a r be ge= ftrablt, ohne (siriff.; SBcere mit vielen vielfaam. Fådbern

\section{(Delphinŭum.)}

(Aconitum.)

Sarracenia.

416.
Helianthĕmum.

$41 \mathrm{~S}$.

Argemóne. 419.

Sanguinařa.

420.

Podophýllum.

421.
Nympha'ea.

\section{Oronung.}

$8 w \mathfrak{s}$ Staubwcge.

Blumenkrone:

1. 5 blättr.; SEฉYi. vielfaum.; Bldecke:
a. 5blattrig; Sriffel feblen; Stawie bưlīenf.
b. Fellt (i. 439.)
422.
Paeonia.
(Isopýrum.)

2. fel)lt; §io. 1 blättr., ganjranb., abgeftuigt; Stfáb. fehr lang, Eculenform.; Sir iffel 2 ppalt.; ऽfapi. 2 fách., 2 horn., mit ein= jelnen, enodbenbart. $\subseteq$.
423. $\hbar$
Fothergilla.

\section{Ordnung.}

Drey Staub wege.

Bldecke:

I. 1. blàttrig (1.381.)

II. 5 blåttrig (f. 422.)
(Reséda.)

(Paeonăa.) 
III. fehlt; Blkrone:

A. 5blăttrig; Nectarium:

1. Kappen:

a. 2 geftielte, frumme, unter bem ober $=424$.

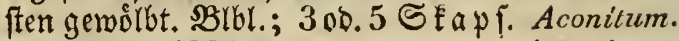

b. ftielloje (i. 439.)

2. cin 2 ipalt., bintermärts 'gebỏrntes; 425 . 3. oo. 1 Stapr.

B. 6 ob. 9 bláttrig (F. 425.)

Delphinüum.

(Delphinüum.)

4. Dronung.

$\mathfrak{B}$ i e $\mathfrak{r}$ Sta $\mathfrak{u} \mathfrak{b} w e \mathfrak{g e}$.

SIo. 4 blättr.; $\mathfrak{B}$ If. mit 4 frugf. Rect.; 426 .

4 Skap mit fdup. $\subseteq$.

\section{Dronung.}

$\mathfrak{F} \mathfrak{u} \mathfrak{n} \mathfrak{f} \quad \mathfrak{S} \mathfrak{t a} \mathfrak{u} \mathfrak{b} \mathfrak{w} \mathfrak{c} \mathfrak{g}$.

Shne SBlb.; SRle. 5blattr.; Nectarium:

1. ein 2 ipalt., hinterwairts gebơrntes (i. 425.) (Delphinŭum.)

2. 2 langgeftielte unter D. oberft.sblbl. (1. 424.) (Aconitum.)

3. ein Srang von 5 am Sirunbe gefpornten Blattchen zwi fchen o. SBlbl.; 5 abgejonoerte SEapi.

4. 5 orevfpalt. in 8 . Blerone; 5 verbumbene Stap?.

427.

Aquilegřa.

Nigélla.

\section{Oronung.}

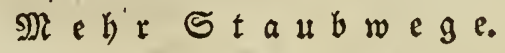

Blumendecke:

I. 3 blattrig; Blkrone:
A. 6 bláttr.; S. unbebedt, zablreid), $\mathfrak{u}=429$. geidhwaingt]
Hepatica.
B. 9 bláttrig ; 
1. Frigclfrúd te Dadyziegelf. in e. 3apf. 430. vereinigt

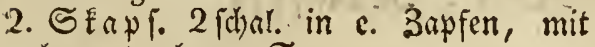
bcerart., hang. S.

II. 5 bláttrig; S. unbebecit;

Liriodéndron.

A. mit Slect.: e. Rod) innerhalb an jebem 432.

Ragel D. 5 SBlbl.

431. $\hbar$

Magnolía.

Ranuncùlus, 433.

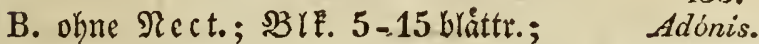
III. fel)lt ; Blkrone;

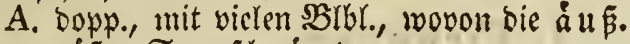

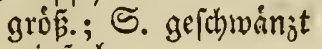

B. cinfach),

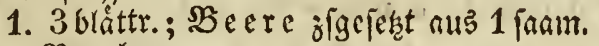
Beerthen

2. 4 bláttrig; Saame:

434. 万

Atragěne.

a. geíthwánzt mit. baur. SBro., zigeor. Clemütis.

b. nicht, fontorn geftreift, fitelrund; 437. Stfáo. Febr lang

3. 5 bláttrig;

Thalictrum.

a. mit unbedectit. Saamen:
a. geichwånzt (ई. 436.)
b. nicht (i. 437.)
(Clemătis.)
(Thalictrum.)

b. mit SaamenE aplel; Blk.:

a. Dauerno; Nectar.:

1) mehrere 2lipp. Sappen; 5 bis 6 SEapfeln vieljaam., etwaz aufr., zigebrudat

2) 5 brenipaltige innerbalb der SIÉ. (గ. 428.)

b. abfätlend; viele SEapr. vielfant.;

1) mit Rect.: ftiellope, 3 ipalt. Sappen; $\subseteq \mathfrak{E} a$ F. umgefrumnt Isopýrium.

2) obne Rectarium

438.

Helleborrus.

(Nigèlla.)

439:

$440 .+$

Caltha.

441.

4. 6 blättr.; viele S. mit feoer. Sdywang Pulsatilla.

5. 6-9bråtri; viele S. ohne ङdhwand Anemóne.

6. etwa 14 biáttr.; Pect. Seranz von

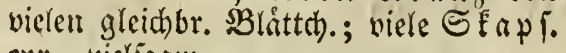
evr., vicljaam.

443.

Trollüus. 


\section{Rlaffe.}

3wey lángere t. Zwey Eurjere

Staubgefáfe.

(Didynamia.)

\section{Oronung.}

S $a \mathfrak{a} \mathfrak{e} \mathfrak{u} \mathfrak{u} \mathfrak{b} \in \mathfrak{b} \mathfrak{e} d$

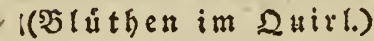

\section{Mit eigentl., I blättr. Bld.;}

A. Bld,ungetheilt, od.undeutl. gezahnt.

SIE. rachenf.; Bldecke:

1. ctwas gezabut, eyr., mit e. Nebenblättch.; \$lE. ganz .umgerwant; Stgef. in ber 444. BhlErivhre

Lavandŭla.

2. mit ungeth., nact) b. SBlithe geidtorl. u. mit e. Dedél verieken. Runo.; şlk. mit 445.

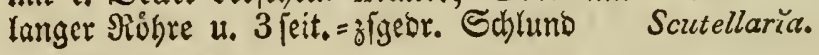

B. Bld. 5 theilig;

1. Bld. 2lipp.; Blk. rachenf.i

Oberlippe d. Blkrone:

I. ungetheilt,

1. flach, ausgerand: Schlund mit langen 446. Scanr. geithlolf.

2. oben breiter; Stfáben an ber Spize 2 gabl.; Stbeut. auf b. e. untern 2ufte; Rarbe 2 fpalt.

3. fityerf.; Stgef. peht lang

Thymus.

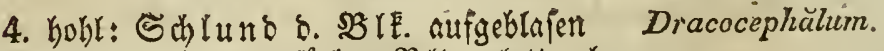

5. flach: $\mathfrak{u l i p p e ~ g e f a ́ r b t ; ~} \mathfrak{B}$ lo. glatt, glo= denfớrm., ftumpf, an o. Mưnoung fobief; Stbeut. in e. Sireuz zigelegt

447.

Prunèlla. 448.

Trichostémma. 449.

450. Melittis. 


\section{XIV, 2 li̊ng, น. 2 Eùrs. Stgef.}

II. 2 fpaltig,

1. gemólbt: Urippe nit Gerzf. Mittellapp.; 2 I 18 . troce., oben etwas flach, mit etwa aufferig. Slippe

2. Etfado. and. Spife 2 gabr.; Stbeut. auf bem e. untern 2ffte; Rarbe 4 part. Cleonia.

3. Ulippe 3 theil., mit faft gleich. $\mathfrak{L} a p p . ; 453$.

Stgef. v. einanter entfernt

III. 2 lapp.: Ut lippe 3 lapp., faft gleichfơrm.; Brs. begrannt, am Schlunde glatt, nah' D. sklithen gejabnt

Lepechinia.

451.

Melissa.

V. 4 ipalt.: bie andern ungeth.: SBlk. gang umgewand; S Sippe b. sBlo. Freizrumb: Urippe 4 fpalt.; bie $2 \mathfrak{a} u \xi$. Stfåd. am (Brunbe mit e. Fortiag

454.

Horminum.

\section{Bld. nicht 2 lippig;}

a. Blk. rachenfürmig;

a. Blk. I lippig.

\$B lo. 5 ipalt.; Oberlippe d. Blk.:

456.

1. Tehr flein, 2 zahn., furrzer als b. Stgef. Ajŭga.

2. Fehlt ganz, ftatt Derferben eine Spalte, 457 . worin d. Etgef. Yiegen

\section{Teucrŭun:}

b. Blk. 2lippig;

1) Olippe ungetheilt.

Blumendecke:

I. 5 jabnig;

1. Oberlippe d. Blkrone:

a. ausgerand., ber unterlippe faft gleid'; \$B Io. róbr., geftreift; St gef.entfernt= ftekeno unter D. Slippe

b. flach, jott.: Ut lippe 3 theil., wovon §. ) Sittellapp. ungetb.; SBIo. 5 feit.; Stbeut. mit glánz. \$Juncten

Eururus.

c. aufiteig., etwas flach, mit waljenf. 460 . Róbre; $B$ ro zåne begrannt Betončca:

d. ungeth.: Ulippe 3lapp., mit lång. Mittellapp.; $\mathfrak{B}$ ro. róhr., mit nadt. 461. Schlumbe; Stgef. entferntfehento Brachystémum.

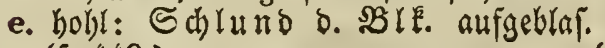
(f. 449.)

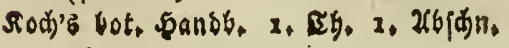

(Dracoceptrălum.) 
f. getvolbt: Ulippe 2lapp.; S d) lutto ๖. SBlE. anfigcblar., nuf beyren Seiten am Siante gráatumt

462.

Lainüum.

g. etwas geterbt, gerolbt: urippe obers balk 2 zalgn.

h. gleidhbr., Die Stföo. verbituleno (F. 23.) 463.

Galeópsis.

(Monàrda.)

2. Unterlippe d. Blk.:

a. 3 theil., wowon's. Mrittcllapp. ctwas $\mathfrak{g c}=$ ferbt; Stgef. gerabe, cntferntifebeno Hyssùpus.

b. mit geferbt. Slittcllnpp.; $\mathfrak{S}_{\text {ch) }}$ lun b. langrobst. SZlf. am Siande umge= folagent; SBIo. trode, geftreift, un= gleidgejallnt; Stgef. maibefebento

465.

Nepěta.

1I. 5 ipalt.; Slippe o. SBre. gelvorbt: Unterlippe:

1. 3 fpalt.: Rapp. fommtr. fpils; slipye gantzano; S3ts.ungl., begrannt; ऽtbcut. glutt

2. an ben Eciten umgejchlag., mit gróp., ausgerans. 2)ittellapp.; ङ tgef. nad) d. \$zefructuturg an bie Seite geosgen

2) Oberlippe zspaltig.

1. Stbent. parmeis in e. Sirely jigclegt Glechóma.

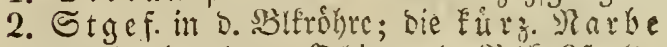
umgibt bie andere; Slippe d. SSle. 2 palt. ob. ausgerant, mit 3 theil. Uliple

469.

Siderilis.

5) Oberlippe $4 z a h u i g$.

Ulippe o. SBle lánger, ungeth., etwas fein= geferbt; $\mathfrak{B}$ Id. rókr., 5 galn.; Stgef. ent= ferntitebeno
466.
Galeobdulon.

467.
Stacliys

468 .

470.

Elsholzia.

\section{b. Blk. fast gleichf, nicht rachenförmig.}

SBlo. 5 zakit.; Blkrone:

1. trichterf., Ertmmmónr., mit ungl. Enum; e. $2 B(0 ; a i) n$ abgeftust; 2 ob. 4 S. vor $\delta$. গeife berectet, nacbber madit

471.

Verbeina.

2. 4 ppalt., mit e. breitern nusgerant. Lapp.; 472.

Stgef. aufr., entferntftelend

Mentha. 


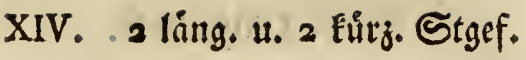

c. Bld. mehrtheilig.

Bldecke:

1. tellerf., fteif, 10 ftreif.; Dlippe b. $\Re$ Y 47 . 473. 2 fpalt., gleichbr., gerabe Marrubium.

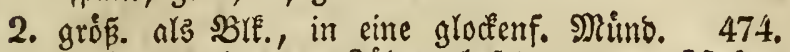
erweitert, mit born. Sáhnen beferat Moluccélla.

\section{II. mit Hülle.}

\section{Blitthen :}

1. im $\$ \Perp i r l$; Olippe d. Blk.:
a. auflieg., zigeorudt, langhaar.: ulippe breit, ungeth.; $\mathfrak{B}$ 1
Phlomis:

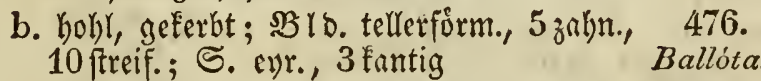
c. flach, werk =herzf., gerabe; 3 1 lo. 2 lipp.; 477. Şîlfe vierborft,, unter b. Suirl Clinopadium.

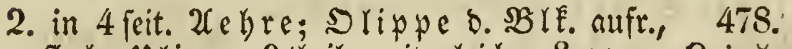
flach: Ulip pe 3 theil., mit gleidhen 2 appen Origünum.

\section{Dronultg. \\ S a me bebed t.}

\section{Blumendecke doppelt.}

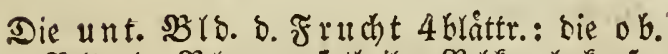
B ID. D. SBlume 5 theil.; $3 \mathbb{R}$ lE. glodenf.; 479 , 古 - Bepre 3 fádb., trodert

Linna'ex.

\section{Blumendecke einfach;}

\section{A. Bld. I blättrig;}

\section{Bld. 2 theilig.}

\$Bro. bopp.= ausgerano., fheibef., unten ber Eánge nad aufipring.; $3 g$ rE. rôbr., ungleichf., mit e. cinzigen auffteig., 4 jpaltigen Qippe;

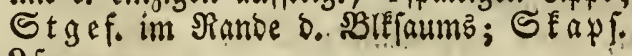
2 jaam.

480.

Hebenstreitřa. 


\section{Bld. 4theilig.}

Saamenkapsel :

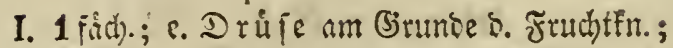
Blk.:

1. majfirt

2. rachenf., fafi gleidfr., 4 palt.; SEapi. 2 ichal., vietjanm.

II. 2 fâd)erig; tic maşfirte Blk. :

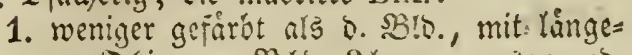
rer Dlippe; B to. 2 lapp., ausgerano. 481.

Lathriea. 482.

Orobánche.

2. 2 ripp.: Sberlippe 2 palt.: Ulippe 3 lapy., mit 2 palt. 2apy.; $B$ lo. Imal= jeniorm.; Stbeut. 2lapp.: bie 2 nie= digern enbigen am Sirunte \&. einen Sapy. in e. Dorn; Słaџ . eyt.=lángl. Euphrasia.

3. ractenf., mit jlgesrueft. Slippe; SBlo.

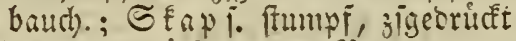

4. mit jigetridet. u. am Pianbe umgeiblag. Dlipłe; SEapT. Folei, an cincr Eeite aufipring., mit 2 bơfir. S.

Bartsia.

485.

Rhinánthus.

486.

Mrelampýriom.

3. Bld. 5 theilig.

I. Nit Saamenkapsel:

A. 1 fícherig; Bldecke:

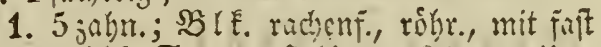
gleidf. Eaum: Slippe 2 lapp: $\mathfrak{u n}_{\mathrm{n}}$ terlippe 3 lapt.; Sfapl. Eugel., mit 1 క.

$457 \cdot \%$

2. 5 jpaltig; Blkrone:

a. glodent., 5 ppalt., gleidhf.; Stgef. paarweis = nabeftebeno; ङEaplel 2 ja) al., vieljam.

b. rachenf., 5 jpalt. (i. 4S2.)

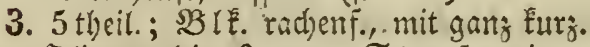
Slipye: Die 2 unt. Stgef. mit e. Enbjabr u. faft zur Seite ftehentent Etbeut.; ङ‡aџ 2 jual., vielfaam. Lindernŭa.

B. 2 fäberig; Blkrone:

1. gleidh., 5 theil.; St gef. parmeis ent=

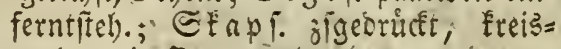
runo, mit Duervano

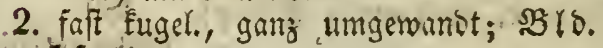
5 ipalt.

490.

Sibthorpĭa.

491.

Scrophitaria. 
3. rabf., nit kart. Stfoden

492.

4. glodenfórm., 5 ppaltig, unten baudh.; 493.

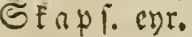

5. rachenf.

Digitális.

a. mit ait D. Esiten umgefalt. Slippe; - Blo. prismat., 5 zalnig; Marbe siff; Sfapf. vieljadm.

404.

Minuulus.

b. mit ftechendipit., an b. Spise idjief. 495. Sfapl.; S. mit saut becedit pediculíris.

6. masfirt,

a. mit Eporn n. 2 thcil. (Baumen; $S_{F}$ ap $=$ fel eyr., an b. Spise vielidjal., viels jomign

b. am sirumbe hơfr.e, mit 2 theil. (S)aus

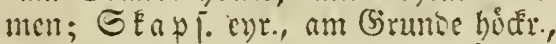
viclinum., an s. Epiz̧e mit 3 sócherm anfipring.

496.

Linariăa.

c. am Girunde gur Scite herborrag. honigtrng., amit flacher Ulipłe u. offin. Enlumo, dbue Gaumen; SEapid 498. viclfidal., vicliant.

d. mit e. 3 ipalt., 2 mal fo lang. Ul lippe;

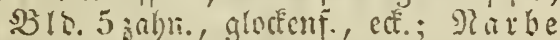

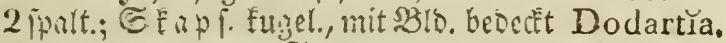

7. telferf., mit 5 thail. Enum, wovon bie

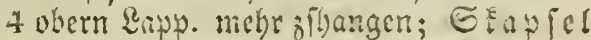
viclianm., mit bopp. Edjeibcramb, mowon

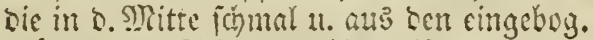
gianern ¿. Edhalen gebiltet if

500. Manulěa.

II, mit 2 finth. $S_{\text {thote }}$ 1t. hout = gefligelt.. .;

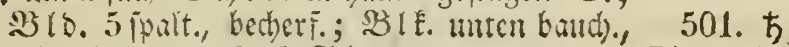
mit 5 jpalt, glodenf. Ed)

Bignona.

B. Bld. 5 blätirig.

I. פRit 2 fäh. Sfaplel; Blkrone:

1. rachenf., baudh; mit e. untehaart. 2f fter= 502.

faben jwirchen ben obern Etgef. Chelóne,

2. 2 lippig, kauch., mit $\varepsilon$, oben bartigen 503. Ufteriaber Pentsténon:

3. glcidy., mit 5 fpalt. Eaum u. aus̆geratร. 504 . Lapp.; Slippe jegr furd ungeidlagen Erínus.

II. 2Rit Sdjote (j.501.)

(Bignonǐa.) $\hbar$ 
C. Bld. mehrblättrig.

SBlo. befteht aus 3 wechf. Sparen; $B$ I febr gros, 3lappig: Róhre mit langen Şanr. gejhloflen, abroártsgebog., 3 partig; Narbe 2 fpalt.; Skapfel eyruno, 2 fách., 1-2 [aam

505.

Acánthus.

\section{Rafíe.}

Bier lángere u. Zwey fúrzere

Staubgefáa

('Tetrad y n a m i a.)

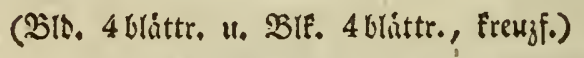

\section{Drbtumg.}

Surze, runbride હdotden.

1. Schötchen an der Spitze ausgerandet;

A. Griffel an derselben
hervorstehend.

1. Die fúr $z_{\text {. Stfioen baben an }}$. innern Seite e. 3ahn; Schitch. faft ungeth., ges rándelt, mit ftechenofpis. (Sriff. u. mit hoh)= len Stjalen, weldhe ber Échciberoand parallel ftehen

2. nicht; Schütchen:

a. eyr. vo. runo, håut., 2 fadal., vielfaam.: (S) riff. etraả lang; S. långl., meittåuf= tiggeftreift $u$. nicht gerándeft

t. aufgeidhwoll., fogarf, runzl., vieliaam., mit 2 bodfre, ftumpf. Schalen u. faft pas ralfet. Scheiberwanto

506.

Alýssum.

. verê. = herzis., vielfaam., mit gerånoelt nad)enf. Sdialen

507.

Camelína.

508.

Cochlearia.

509.

Thlaspi. 
d. 3fgetriact=flach, abgérumbet, oben u. uns

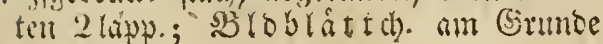
bớct.

510.

Biscutéllc.

e. ari s. Spibe cingeoritrt, an Siante ge= front mit Schalen, bie noth cinmal fo lang fins, als bie Esheisenumb n. mit 2 panm. Fich.; (5) riff. in b. Allitte ftect)entofpils, (i) icf

511.

Anastatica.

B. Griffel nicht hervorstellend.

Blumenblätter:

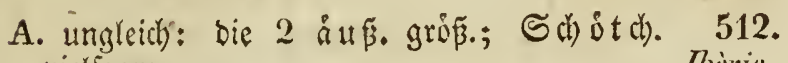
vietfanat.

Iberis.

13. gleid); Schütchen :

1. frcistuno, zfigcoriteft flack), abfoll., in 2 Stitce theitbar, 1 fåh. , obne Edalen, 1 farm.

513. Clypeǔla.

2. herjf., vierfam., mit mathenf. Schalen 11. Sltermatto

514.

Lepiclum.

3. vert $=$ herjf., in 2 Stude theilbar, 1 fanm., mit nad)enf. Edbulen u. gegittert, Ed)ei= 515. Serwanto

Iscitis.

II. Schötchen an der Spitze nicht ausgerandet;

A. Griffel an derselben hervorstehend.

Schütchen :

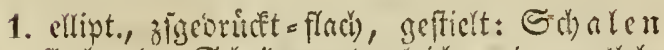
flad), ber Sdheibemano gleidh uno purallet;

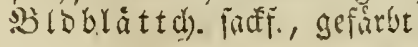

516.

Lunaria.

2. cyr., mit eyr., hohlen Ëhalen uno suer 517.

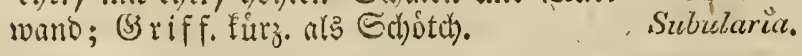

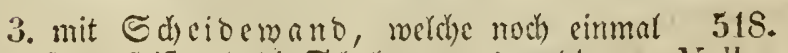
fo groṕ ift, ałs bie Schalen u. muswendigent. Vella.

4. forfart., ohne Sabalen, mit fegelf. Brifif. 519.

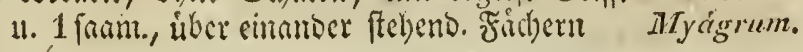




\section{B. Griffol nicht hervor- stehend.}

\section{Schötchen :}

1. oyal=långl., mit etwas flach. ut. gegen bie 520. Scheibemand parallel fekenden Schalen Draba.

2. lanzettf., faft 4 feit., in D. SNitte auf bevs. Seit. mit e. Babn, 2 gliebr., gliebermeis ab= 521 . fpring.: (S lieber 1 faam., ohne Echaren Cakîle.

3. faft 4 fláchig, olye Shalen, 2 ob. 4 fód $; 522$. rungl.

4. Eugel., 1 fam., forfartig, ohne Sdyalen; die 4 lång. Stfåd. an d. Exitye 2 gabl., auf D. eimen D. Stbeut.

Bunias.

5. faft freistund, ż fgeor.=flach, nid)t auffpring., 1 od. 3 fam.

6. nierenf., zjgeoridet, jfgerungelt, mit 1 fanm. Fád)., ohne Schalen

523.

Crambe.

524.

Peltarìa. 525.

Sennebiéra.

\section{Orontag. \\ S d) $D$ t e $n$ l $\mathfrak{n}^{\prime} \mathfrak{g} g$.}

\section{Bldblättchen zsgeneigt;}

A. Schote If ächerig.

I. Mit Nectar. Drüsen :

1. 2 zwifhen ben firm. Stgef. u. D. Stmen

u. 2 zwifhen b. långern u. D. borft. Blo.;

Scho te enot., etwas geglicoert, ftielrums, geidnabelt

2. 3 an jeber SBubt D. Blo., mit 2fusnal)me

o. unterften; $B \backslash b$ l. fammtlid) auffteig.; Schote 1 fach, 2 jal.

II, obne rect.; Sch ote längl., zfgebrucat, mit flact). Sibalen

526.

Róphanus.
Cleŏme.

528.

Ricotĩa.

I. Mit Nectarium:

$$
\text { B. Schote } 2 \text { fücherig. }
$$

\section{A. mit 4 Nect. Driisen:}

1. 2 zwifhen ben furtz. Stgef. 11. D. Stmeg u. 2 zwifhen o. ling. U. D. Blo.; BID. aufr.; ङ. fuzer.

529.

Brassica. 
2. cinzeln, ciner ungebog. Schuppe ânnl:

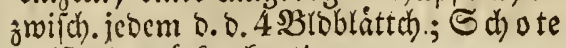
meift zigeoructet, Enotig

530.

Arŭbis.

B. 1 Dr úfe zwifhen s. Furz. Stgef.; $\mathscr{B}$ Ib. fúr. alb SBlfnágel; $\mathfrak{B}$ Ibl. gleichbr. ob. veré. eyr., fébiefgebogen; Siarbe am Srunde 2 gabl. u. an D. Spite zfgeneigt; Schote ganj gerabe

531.'

Hespěris.

C. ein bruifiger Bahn auf berben Seiten b. Fruchtên.; volt ben 4 sBloblättchen 2 am (Sirunde hodfr.; $\$ 3$ rbl. mady oben breiter= werbend; Narbe 2 lapp.; S. flad), ju= weilen gerantelt

532.

Cheiránthus.

II. obme Séctarium; Schote:

1. elaftijh jeripring., mit umgerollt. Saba. ven u. ctwas fchwammat. Scheiberoano;

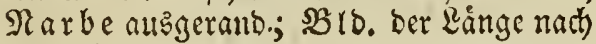
zigeneigt

2. fảułenf., gennu 4 fláchig

533.

Dentarìa.

534.

Erysimum.

3. fehr Yang, cé., geftreift; $\mathfrak{B}$ lb. u. NB/E. aufr.

\section{Bldblätıchen abstehend.}

Blumenblätter :

A. mit $\mathfrak{R} \dot{a} \mathfrak{g} \mathfrak{e} \mathfrak{l n} ;$ blkrone:

1. mit e. Driffe jwifken b. Firry. Stgef. u. D. Stweg u. jwifden ben ling. u b. SBlo; Blena áger gerabe; Sd oten geidunab., mit hervorrag., fíbelf. Sit)eidemano

2. ohne Rectar.; Schalen d. Schote:

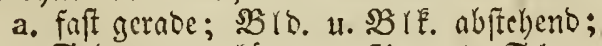
Schote an furgern, ftichumo. Echnas bil aufipring.

b. umgerollt; Narbe ungeth.; $S$ d) o te rang, elaftifd) auffpring.

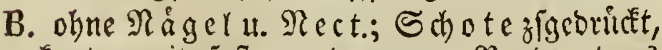
Enot., mit faft gernoen, am Ranbe etroa auggefthmeift. Schalen
536.

Sincípis.
537.

Sisymbrium.

538.

Cardamine.
539.
Heliophila: 


\section{Rlafie.}

Stankfiben in (Fine Gáule vermadjen.

(Monadelphia.)

1. Oronumg.

Drey Staubgefáb.

1 Mit 1 Stmeg; Sd cibe 2 blattr.; 631 b\%. faft gleidl); sJlo. feblt; untere SEapfel 540.

3 fáct).

Sisyrinclĭum.

\section{OrDnung.}

F⿱⺈

\section{I Staubweg.}

Bldecke:

I. 1 Glittr., 5 theil.; Blkrone:

1. 1 blăttr., rabfưrm.; bic 1 fơdh., vielfanm. Skapsel:

a. in 5. Mitte runtum aufipring. (F.111.) (Anaguillis.)

b. fingel., 5-10ichal. (i. 112) (L.ysimachür.)

2. 4 brittr. Fometterlingaf. (f. 5+3.)

3. 5 blittr., ungicid)f. (i. $5+2$.)

II 5 blittr.; $B$ Iff. 5 blittr., glcid)f; ; bic 5 am (S)runbe D. Frbo. gejchnabelt, 1 fanm, Saamendecken:

1. fpiralform. u. an ธ. imnern Ecite bart.;

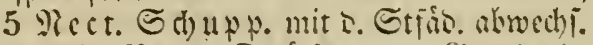
u. mit Mect. Defifen an Sinnoe b. Etzer.

2. beyses nidyt (i. 5tt.)

(Dalĕa.)

(Pelargonum.)

\section{I. 5 Staubriege (i 256.)}

$5+1$.

Erodium.

(Geruň̀um.)

(Linum.) 


\section{Didnแng. \\ Sieben Staubrege.}

BIo. 5 theil., ber oberfte Brolapy. breiter, in e. neben b. SBlattftl herabgehende haarf.

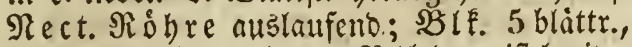
ungleidsf., bie 2 obern $\mathfrak{B}$ lbl. meift breitcr u. andersgeforbt; 10 ungleiche Stfáben, movon 3 obne. Etbeut.; 5 Sa antendecen 1 faam., begrannt, am Srunbe b. Frbo. ge= ichnabelt, mit piralf., an b. inmern Seite bart. (Sirannen

\section{2. \\ Pelargonium.}

\section{Dronung.}

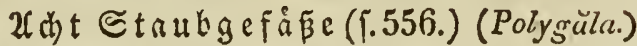

\section{Drontıng.}

3ebn Staubgefábe.

\section{I Staubweg;}

A. Blk. schmetterlingsförm.

\section{Blumenkrone:}

I. wirklich fchmetterlingsfơrmig, mit Flưgel u.

\section{Schiffit.; Bld.:}

A. 1 blåttrig,

1. 2 lippig,

a. Slippe 2 zahn.: Urippe 3 zabnig
(1. 558.)

b. Stbeut. wechjelstweis langl. und runol. (i. 559.)

c. $\mathfrak{B l}$ k. gand umgemanot (f. 557.)

d. Narbe ber lánge nach jott.; $S t f a=$ benfáule an b. Fruditen. hang.; Huilse :
ค. 1-2 janm. (Г. 560.)
b. nieljaam. (i. 561.)

(Lupinus.)

(Arăchis.

2. nid)t 2 lippig, fundern 5 theil.; 
a. Fligel u. Shiffd. mit b. Stfa= bentále verwad) $\int_{0}:$ Fåbndy. furz; Şúlfe 1 jaam., fưr. als Blbette; sहluth. in 2abren

$5+3$.
Dalěa.

b. nidht; Hiilse :
6. aufgerdowndl. fticlloz (โ. 563.)
b. runol., mit $\mathfrak{B l o}$. beder (f. 564.)

B. 2 bláttrig (i. 565.)

(Onónis.)

(Anthyllis.)

(Ulex.) $\hbar$

(Amòrpha.) $\hbar$

B. B Blk, gleichförmig.

SBIs. 5 b!dittr.; SBIf. 5 blittr., mit 5 Nect. Drúfeut an Brumbe b. láng. Etfåd.; 5 ge= ichnabelt., 1 faam. Saamendeden am Srumce o. Frbo., beren (Stammen weocr fpiz ralfurm., nod bartig fins

544.

Geranŭım.

(Oxülis.)

II. 5 Siaubwege. (1. 368) 
XVI. Stfäs. in 1 Sạule verwad) fen.

2. Sopp.: bie ȧuß. vielblåttr.; 5 Rarben; 547. Skap 5 fách., vierjam.

Hibíscus.

\section{II. viele Staubwege.}

Rit bopp: $\mathfrak{B} 1$ r.; die äussere:

A. 1 bláttrig; viele Skapseln:

1. im $\cong$ uirl fireno; äuss. Bld.:

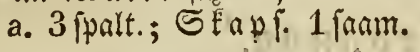

548.

Lavatéra.

549.

b. 6 od. 9 fpalt.; Stap vielfám. 'Altha'ea.

2. in e. 5 lapp. $\mathcal{R} \dot{p} p$ fd en vereinigt, 1 fanm.; 550.

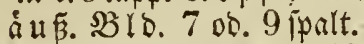

B. 3 blåttrig; vicle 1 faam. Skaps.:

1. im Souirl ftebento

Kitaibelǐa:

2. ohne Slonung gefnauelt

551.

Malva.

552.

Malŏpe.

\section{Rlafie.}

Staubfáben in 3mey \$ुartfien verwat) fen.

(Diadelphia.)

(Biff. fodmetterlingšförnig.)

\section{Ordming.}

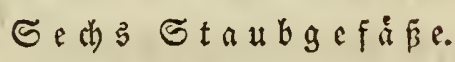

\$Bro. 2 blättr.; 3 le rone rachenf.; 2 håut. Stfáben, beren jeber 3 Stbeut. trágt;

1. Sdi of then 1 fanm., ohne Ethalen

2. bie vieljaam. Schote:
a. 2 ichalig

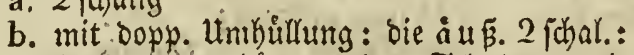
bie "innere baint., obne Edjalen, mit neesf. Fäben angebeftet

553.

Fumarı̆a.

554.

Corydülis. 555.

Cysticàpnos. 


\section{Dronung.}

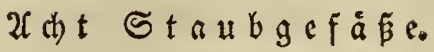

Son ben $5 \mathfrak{B l b l}$. find 2 flügelfơrm., gefárbt; 556.

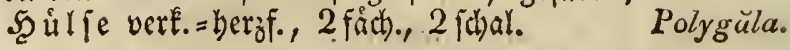

\section{Dronung.}

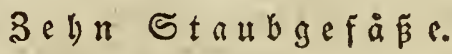

\section{Blüthen einzeln;}

A. Stfüd. undeutl.in zParthien;

(scheinbar in I Süule.)

1. Bldecke Iblättrig.

Blumendécke:

I. 2 lippig; Blkrone:

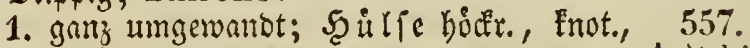
aderig, leoerart.

Arăchis.

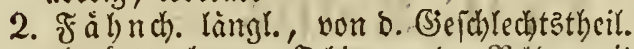
abwart3gebog.; Slippe s. BR lo. mit 2: Ulippe nit 3 jegr furzen Băhnen

3. 5 ๔ tbeut. längl. u. 5 runol.; $5 \mathfrak{u} \mathfrak{u} l \mathfrak{e}$ leocrart., aufgeithmoll., zigeoriuct

4. Stfabenfáale an b. Fructhtin. bang.; Rarbe ber \&ange nach oberbarb jottig; Hiilse:

a. 1-2 faam.; heybe Briblipp. an ber 560. 万 Spize feingezahnt

b. viclfamig, zigeorudét; Brolippen unget).

II. nicht 2 lippig; o. ichmetterlingsf. Blk,

Spartǔum.

561.

Spartiánthus.

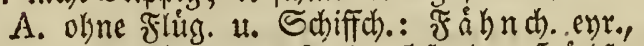

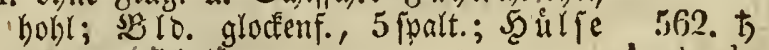
2 farm. fichelf.

B. mit Flligel u. Sdhiffthen; Huilse:

1. aufgeids moll., ftielloz; $\mathfrak{B}$ 18. 5 theil., mit gleichbr. Eapp.; F́ăhnch. gefreift; 563. Etfảb. zfgewachf., obne Eparte Onónis.

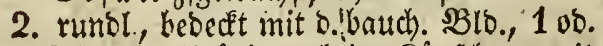
3 faam.; sblitth. auch im Sơpfd. u. mit Amòrpha.

558. Genista. 559. Lupinus. 
2. Bld. 2 blättig, mit $2 \mathrm{Nebenbl.}$

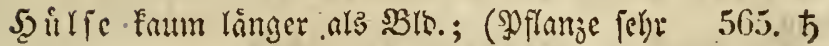
born.)

B. Sifüd. deutl. in. 2 Parthien;

(9 verwachs., I frey.)

1. mit Hiilse;

a. Bld. 2 lippig.

Narbe:

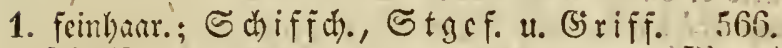
fpiralf. gimumoen

2. nicht hantig; S⿹弋 $\mathfrak{u}$ lie jigeoridft; Bldecke:

a. mit 3 pait. Slippe u. ungeth. Ulippe; 567.5

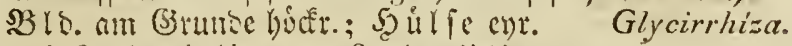

b. mit 2 zahn. Dlippe u. 3 zahtr. Urippr;

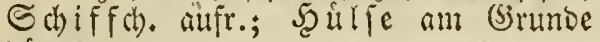
dimner

c. Ulippe mit c. lång. 3ahn; $\sigma_{d}$ iff

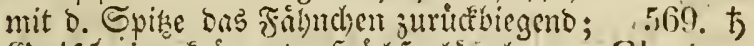

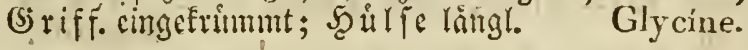

b. Bld. nicht 2 lippig;

a. Blk, undeutl. schmetterlingsfürmig.

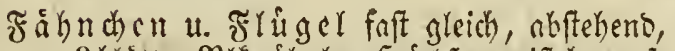

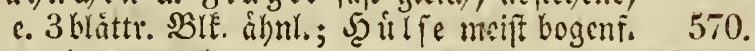

u. aber. $=$ rumjl.

568. 方

Cytřsus.

vilex. 
c. gleichbr.; SBls. am (Sirunce ftumpf: bie

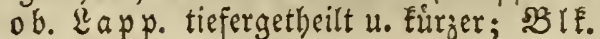
lang

d. mit e. fobiefbart. Rarbe an ser innern

Seite; Blo. oben ausgerano., 2 jahn., unten mit 3 lang., gerab. 3ábnen; $\mathfrak{g} a \mathfrak{h} n=$ d) en ausgerano.; Fruchtknoten:

a. wellenfi., gefaltet

b. nicht

575.

Ervilǐa.

576.

Vič̃a.

577.

Ervum. 5 theil.

f. abmarts o. Rånge nach bart.; Sd iff d).

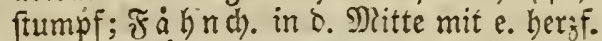
Sunct; Shille aujgeblafen, an 8.1 obern Sath mad innen aufgejobolf., faumentrag. น. aufipring.

578. 方

Colutěa.

2) Narbe unbehart.

Huilse:

1. Godererig,
a. lang; SBIS. 4 fpalt., mit 2 theil. obern Lapp.
579. ち
Robinǔa.

b. meit 2 fåd., น. ร. S. in 2 Reihen trag.:

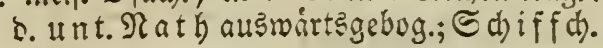
ftumpi

580.

Astragŭŭlus.

581.

Biserrŭla.

2. flach, 2 fãdy., auf bens. Esit. bucht.=gejägt, mit Duerwano; ङ chiffch. ftumpf

3. aufgeichroll., 2 jaam.; SBI Is. 5 theil., fo

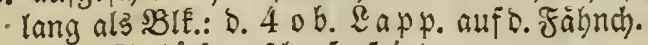
lieg.; S. boffr., icharigepint

582.1

Cicer.

4. etroas aufgeidhroll., 1 fád)., an b. obern Rath nach innen allfgetrieben $u$. jaamentrag.; ङ nicht behaart

583.

Phaca.

5. Enot., etwas aufgetrieben, mit jobief. Suer=

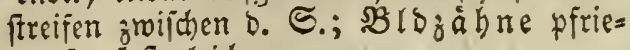
menf., faft gleich
584.
Galéga:

6. zigeoridát,

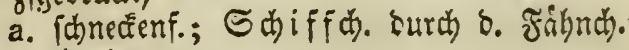 abgebogen
b. nierenf., am Rande geflugelt
585.
Medicágo.
$5 \$ 6$.
Hymenocárpos. 


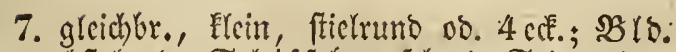
alftehend; Seth iffdy. anf bend. Scit. mit e. pfriemenf., ctwas abftebend. ভporn

587.

Indigofera,

\title{
2. mit Gliederhiilse.
}

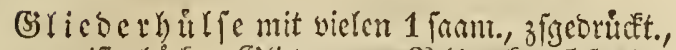

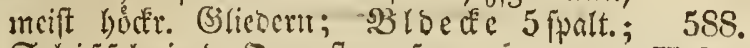
Sd)iffch. in b. Sutcr ftumpf

IIedysŭrum.

\section{Blüthen in Dolden.}

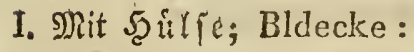

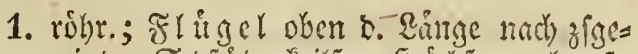
ncigt; Êtfab. feiff; Satlfe wargenf., gants gernoe

589.

Lotus.

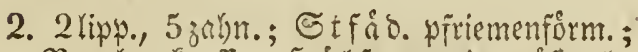
Rarbe fopffi; 5 Sulfe wenig gróp. als

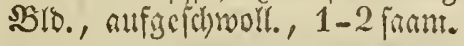

II, Gliederhïlse glieserwcis auffpringento ut:

1. gerabe, ftictrund, gealicoert; SB IS. 2 lipp.: bie 2 Sålne D. STiphe sigervadyen: urippe:3 zalmig; 33 rbr. mit Ningett:

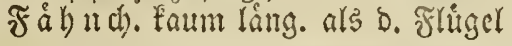

591:

Coronilla.

2. bogig, frielutus, geglicbert; $\$ 3$ rs. robry.;

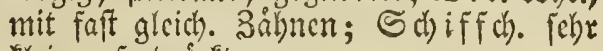
Elein, zfseoridet

590.
Dorycnŭum:

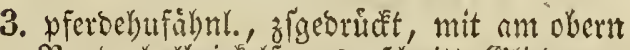

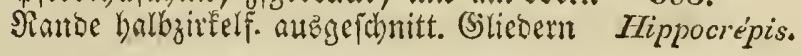

4. umgerolyt, fticlruno, gejurcht, mit sicren=

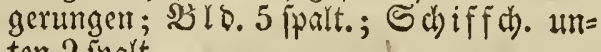
ten 2 ppalt.

\author{
59 4. \\ Scorpiúrus.
}

\section{Blïthen in Köpfchen. i:}

\section{Bldecke:}

1. bautdig (i. 564.)

2. rơbr., 5 jahtu.; 3 let. baur.: Schiffch. an=

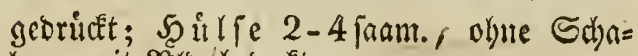
lent, thit Sild. 'bebedft

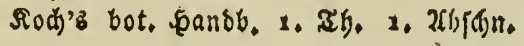

(Anthỳllis.)

Ornitrioptus.

593. 
IV. Blüthen in Trauben.

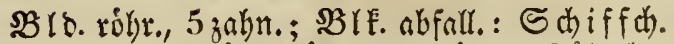

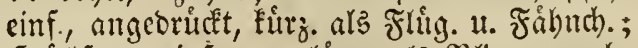

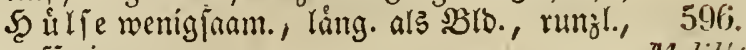
aufipring.

Melilotus.

\section{Rlafic.}

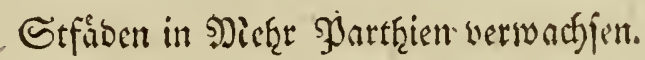

(Polyadelphia.)

1. Dronung.

Siele Strubgefåpe auf D. SBlseçe.

BIt. 5 ipalt ; 5 SIbl. lơngl,; 20 Stbent.;

Stfad. breiter werbent; Beere rinoigi 597. 万

9-1S fády., in weid)er Mlarle

Citrus.

\section{Dronung.}

Sicle Staubgefäpe auf b. BBlumenbober.

Blumendecké:

1. 1 brättr., 5 theil., ntit greich., faft eyr. $\mathbb{S}_{\text {app.; }}$ B Y 1 . 5 bláttr.; viele St fad am (Grunoé verwad) F.; Skap F. runtl., nad) 598. D. $2 \mathfrak{n}_{z}$ abl b. Brifi. $1-2-3$ od. 5 fách). Hyperǐcum.

2. 4 blăttr.: biè bey ben innern berzf., fegr

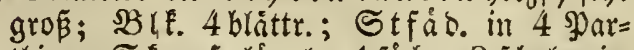
thien; Stapf. lángl., 1 fádq., 2 johal., in sBlo. 


\section{Rlaffe.}

Stabbeutel verwad fen.

(Syngenesia.)

(Brittjen zu[ammengefsect.)

\section{Oronumg.}

Sammtrich awitterblutben.

(Syngenesia aequalis.)

\section{Blüthen sämmtl. I lippig;}

A. Bld. einfach.

Blumenboden:

A. Ipreuartig; Saamenkrone:

1. fpreunrt., 56ráttr., mit begrannt. Spreubl.; 600. BB lo. bachziegelf., gam trodfen

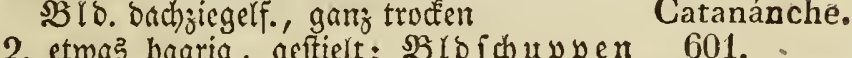
gleichbr.

3. Feber. $; \mathfrak{B l}$. bactjiegelf.

4. feblt; SBlo. oachjiegelf., born.

B. etwas borft. = fpretart.; SB Is. viclblăttr.; Strone in b. Nitte feber. 1. in Rande 5 grammig.

C. langhaarig, aber am Manbe fpreuaritig; Skrone in o. Mitte haar., friellos uno im

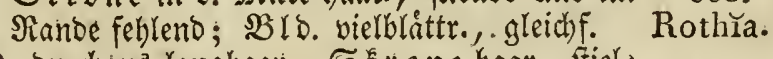

D. Durchaus langhaar. ; É trone haar., ftiel $\Rightarrow$ los; $\Re$ Ib. vieltheil, faft gleich)., runol.; $\Subset$.

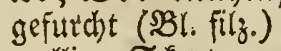

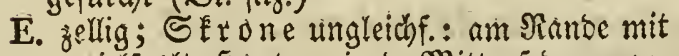
Seriơla. 602. Hypcho'eris. ' Scoly̆mus.

604. Geropógon. 605. 606. Andryăla. e. vielipalt. Scaut u. in o. Mritte feber., ge= ftielt; $\mathcal{B}$ lo. S bláttr., 8 ede.; S. am Pianoe abgeftuht, in e. ppreumt. Sirone endigento $\mathfrak{u}_{\text {. }}$

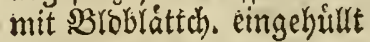

607.

Thrincia.

F. nadet; Skrone: 1. feoerig, 
a. ftiellos; $\mathfrak{B}$ Y D. bachsiegdf.

b. geftielt; Bld.:

a. 1 bliftr., Sthillig, Ereufelfurntig; $\mathfrak{S}$. quergeftreift

b. vielbrattrin

c. Dachjiegelform., mit am Sanbe ganj trocirt. Salupp.

2. băut., 5 blättr., mit 5 Sorften bajmificten abroed) felno; $\mathfrak{B}$ (d. vielblittr.

3. baarig,

a. gęticlt; 2 lo. Dachjiegelf., waljenf., am Ranbe baut.; S. glatt

b. ftiellos; die Dantjiegerf. Bld.:

a. eyrunto

b. boudbig
608.

Aparğ̌x.

609.

Árnopògon.

610.

Tragopúgon.

611.

Scorzonéra.

612.

Kriğ̌a.

B. Bld. am Grunde durctz e. Anhang vermehrt.

Blumenboden:

A. ctwas Tpreuartig; SErone viciblittrig, ipreuart.

B. ctwas borft. =ipreuart.; SEtone ungleichf:: in b. Mitte fcoer. u. im Niamie 5 grom. (1.607.)

C. zellig; Skrone unglcichf: : an Sianoe ace= zabnt u. in s. Shitte 2 oo. 4 grann.; b. unt.

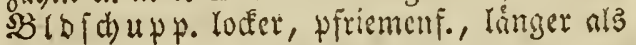
\$Bro.

D. naift; Skrone:

1. gleid)formig, -

a. meift feocr., fely furs; bie untern

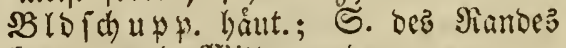
frumm u. D. SJlitte gerabe

b. feber., geftielt; S. quergeftreift

616.

Cichorĭum.

(Geropògon.)
Lactica.

614.

IIierach̆ım.

615.

Sonchus.

c. baarig,

a. etwas geftielt; unt. SBlo [d) up 620. loffer u. abjall.

Crepis.

b. geftielt; unt. Brofduppen wic 621. vorber

c. nur in o. Mitte geftielt, am Ranoe foft ober gand fiellos; unt. $\mathfrak{B} Y \mathbf{b}=$ fdu $u$ p. wie vorber

\section{Lagosěris。}

622. Borkhausia. 


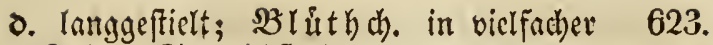
Sieilye; S. wcichftachl.

Chandrilla.

c. faft ftiellos; $\mathfrak{B l u}$ (thonen in einfacher 624 . Rieike

$\therefore$ verichiesen geftaltet:

Prenánthes,

a. Die $\mathfrak{a} \mathfrak{u} \tilde{\beta} \mathfrak{c} \mathfrak{n}$ baarf. : bie innern fprettart.=

gramenf.; $B$ ls. faft Ereišrund; S. am Sinnde oft mit SBTofdupp. beocdt und faft unseutl. Strone

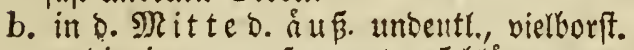

11. Die innern fpreuart., 5 blattr.: ain

Ra nde cin brut., feingezabnt. Sind

3. fellt; Saamen:

625.

Hyosěris,

626.

Hedypnöis. 627.

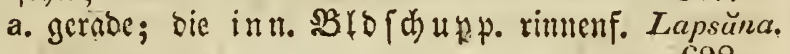

b. Erumm, abftehens 628.

Rhagadiǔlus,

C. Bld. doppelt.

SBYbo beit mact; dic gefticlte Skrone:

1. feber.: innere $\mathfrak{B l}$ lo. Sblattr., ungleidhF.: åu 5 . 5 bláttr., locier, eben jo lang als bie innere; 5 . quergeftreift

2. baarig

629.

Helmintra, 630.

Leontŏdon.

II. Blüthen in d. Mitte rölır., am Rande x lippig.

Blummenboden :

1. Pprenartig; Skrone:

a. feber.; $\mathfrak{B}$ I 5 . Dachziegelf., mit 2rnkang v. ficberf.xborn. B̧rittch.; bef. $\mathfrak{B}$ IE. 5 jabn. Atracty̆lis,

b. aแs 2 ขึ. 4 aแfr., umgeboig., fdyarfen

(Graumen beftebens; $B \mathfrak{b}$ b. flaci); ङ. 632.

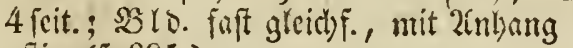

2. borftig ([. 695.)

Bidens.

(Centaluréa.)

III. Blüthen sämmtl. röhrig;

A. Blütho in. $h_{0}$ öpfchen

vereinigt;

1. BIá. ein \ach.

Blumenbolen:

A. Fprourtig; Skrone 
1, feber. ob. gezahnt; $2 B$ lo. bachjiegelform.; walzenf., webrlos

(j33.

Serratüla.

2. borlt. = fpreuart,; SBIb, fuget., mit an b. 634. Spize hačenf., eingebog. Schuppen Arctüum.

B. ipreuart.=borftig; Skrone:

1. ipreuart. = feber. (Den Fủhlbornern ber Sthmetterlinge ǻbl.); $\mathfrak{B l}$ o. geftrablt, mit lang., gefärbt. Siandichupp.

2. fpreuart. = haar., ob. fehlt; BII . oach)=

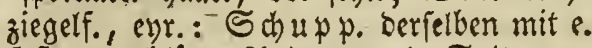
faft eyr., blattr. 2(nbang an b. Spike

C. langhaarig; Skrone:

1. feber.; $\mathfrak{B}$ lo. Dachjiegelf.: Deren Schupp.: a. bornig:- B 1 D. bauchig

b. nicht (f. 633.)

2. nur bie åu

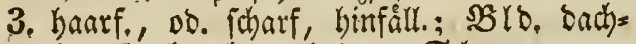
ziegelf., bauth., mit born. Schuppen

635.

Carlina.

636.

Carthümus.

4. gezabnt; $B$ Io. Dadziegelf., ohne Dornen (1. 633.)

D. borftig; Sirone feoer., ftiellos; SBro. Dachjiegelf. mit fleifh)., am Enbe breitern, ausgerano. u. an D. Spiţe Dorn. Echupen

E. zellig; SErone haarform., binfálu.; $\mathfrak{B}$ ld. sachiegelf., mit ftechendipis. Schupp.;. quergeftreift

F. nadt; $S_{B}$ ID. badjoiegelf.; Skrone:

1. einf., feder., gefürbt; $B$ lo. längl,

637.

Cnicus.

(Serratüla.)

638.

Heterotrichum.

639.

Cardiuzs.

(Serratüla.)

640.

Cynăra.

641.

Onopórdum.

642 .

Liátris.

2. Dopp.: D. áa

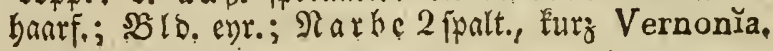

2. Bld. am Grunde durch Anhang vermehrt,

RI\$. [preuart.; SErone feber.; SID. bad)= ôiegelfơrmig

644.

Acárna,

B. Blüth. in e. Scheibenblume;

1. Bld. einfach,

Blumenboden :

A. ipreuartig; Skrone:

645.

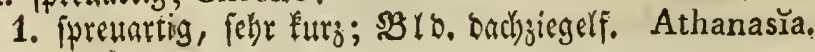




\section{Staubbeutel verwad) (en.}

2. mit 2 ob. 4 (jirannen (T. 632.)

3. fel)lt; Bib. burbfugel, bachiegelf., mit 646. nachenf, an o. Spize trodén. Șchuppen Santolina.

B. nadt; Skrone:

1. hantig; Bld. bad)jiegdforomig u.

647.

a. maljenf.; (3) riff. lang, halb 2 fpalt. Eupatorum.

b. balbeuget.; (S) riff. kaum langer als 648 . ber. SBite.

Clirysocŏma.

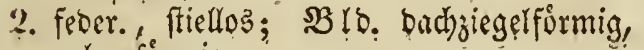
waljenfurmig.

3. itharf (i. 6t7.)

4. ans 5 faft begrannt. Schupp. beftehend; SBID. längl., init e. Doppefreilye v. BBl.; be . 3 I 1 . 4-5 jpalt.

5. feblt, od. e. Glo Dad) jiegelf., Erreistund

649.

Kuhnı̌a.

(Eupatorǔum.)

\section{Bld. am Grunde durch Anhang vermehrt,}

Der flache Blumenboden:

1. Trreuartig (i. 632.) (Bidlens.)

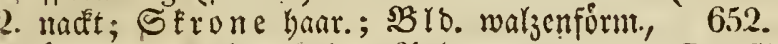
långl., nur mit e. Eleinen 2(rnhang

Cacalia.

\section{Orbaung.}

Sowohl bie switter. n! s weibl.

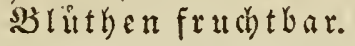

(Syngenesia superflua.)

\section{Blüthen sämmıl, s lippig.}

Blumenboden :

A. Foreunartig; Skrone:

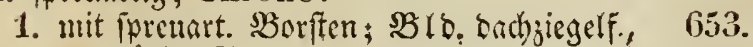

e. gefårbt. Strabl bildend

Xeranthĕmum.

2. ausgeranto.; $: B$ ro. Galbétuger., mit ange=

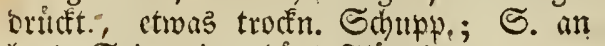
beyo. Scit. mit c. Giunt. Frtingel

B. etwas langhaar.; B̧rb. Dichsiegelfórm., mit runt., jigencigt. Sdutup.; SEtroue fehlt

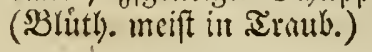

654.

Anacýclus.

655.

Artemisü. 
C. faft radt; Strone gerånbelt ober feblt; be F. SBlE. in D. Nitte 4 palt.: am Rande faft feblend

656.

Cotǐla.

D. ganz nadt; Skrone:

1. feber.; SBlD. bachziegelf., mit geråno., runo., ganz trod̋́n., gefårbt., glånz., kurz. Schupp.

2. baarig; Bldecke:

657.

Gnaphalŭum:

a. einfach, sachziegelf.,

R. malgenf., glodenf. ob. eyr., mit eyr., etwas leberart. Schupp.; W. SBluthe mit Z. BBłith. vernifot

658.

Bacchăris.

b. rumbl., mit faft gleichbr., eur. Sduup= 659. pen; SBlE. D. Si andez 3 ipalt. Conyza.

c. mit gefởbt., geråndelten ङ઼uppen (i. 657.)

b. am (3runoe mit 2 inl)anß, walzenform.,

(Gnaphalüum.) ntit an D. Spific mie verbrannt. Schupy. Senecio.

3. etwas gerånoelt; $\mathfrak{B}$ IE. D. $\Re$ andes $u n=$ beutl. 3 fpalt.; $\mathfrak{B}$ ID. Dachziegelf., halb=

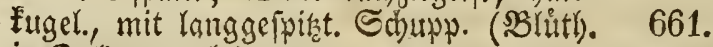
in Dolbentraub.)

4. feblt; Die Dadhsiegelf. Bld.:

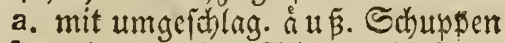

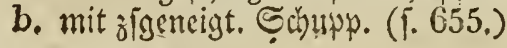

Tanacétum.

662.

Carpesŭum.

(Artemisia.)

\section{Ir. Blïthen in d. Mitte röhr., an Rainde ılippig.}

Blumenboden:

I. Ppreuartig; Skrone:

A. e. bloper Rand:

1. unbeutl., ob. 4 zabn.; S. an D. Sciten 663.

bejonders an ben siandblinth. gerintelt Buptuthálmum.

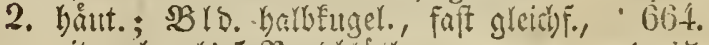
mit mehr als 5 sandblutben Anthěmis.

B. grannenfómig:

1. 2 aufr. (3ramen; SBYs. bact)ziegerf.,

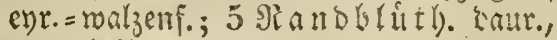
ungetbeilt

2. nur am $\Re$ a d be 3 gramn., in D. Mitte nicht; sBlo. Dach;iegelf., flact); 5 . nnt Rantie warz., it 0 . SMitte gefligelt Sauvitalì.

665. Zinnıัa.

666. 


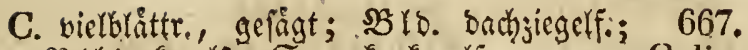
3Zlbb. fegelf.; S. verf. = Fegelf. Galinsogéa.

D. fehtt; Bldecke:

1. Soppelt: Dic äussere:

a. 5 bláttr.: bie bef. B̉b. abjtelyent; 668. Strabl balb

b. vielbláttr.: bie innere 1 blåttrig, 669. 8tbeil. Georgina.

2. cinfach,

a. halbéugcl. (i. 664.)

b. eyr., Dadyblegelf.; etra 4 breitere. Ranobluth.; (Bluthen in Dols Dentraub.)

(Anthĕmis.)

c. 4 feit., 4 theil., felbr breit

d. vielblättr., faft gleichf.; S. an b. Ranobluth. nate, ausgerand.: an b. Nittelblutb. am Siande ges fluggelt

II. zellig, balbfugel.; Skrone gezalnt =be= grannt, faft 2 horn.; $\Re$ andblut b. zahls

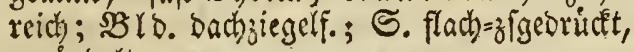
geraindelt

III. fât nacdt (โ. 656.)

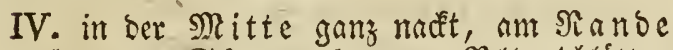
fpreuart.; Sf ro ne 5 gramt.; $B$ Id 1 blattr.,

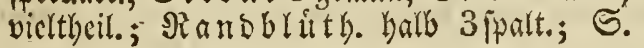
zottig

Achilléa.

671.

Tetragonothéca

V. itbcrall gan

A. 5 aufr. Grannen; $\mathfrak{B}$ ld. 1 bláttr., 5 zalyn.,

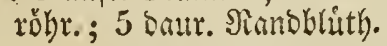

672.

Ximeneša.

\section{3. \\ Bolțonǐa,}

(Cotüla.)

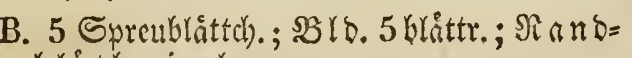
blüt cingeld

C. 8 Spreubrattchen uno begrant; $310=$ blåt th. gleich; S. fegelf.

674.

Helenüum.

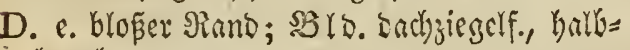
fuggel.,

1. nit etwas fpistar, am rambe trocknen Schtrpen

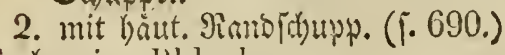

E. hantig; Bldecke:

675.

Tagètes.

676.

Schkuhrŭa,

$677 .:$

Bellum.

1. cinfucts,

a. 1blattrig; 
6. Die 5 Stfäb. ber Mittclblủh. oft ohne Stbeut.; $B$ loblattd en gleich)

679.

Arnica.

b. Die Staubbeuteliáule unten in 2 Borften auslauf.; $B$ ID. fperr. od. backziegelf.; jablreicte Ranoblutth.

680.

Inül.a.

681

c. $\Re$ anọlúth: gleichbr., fchr fchmal Erigěron.

o. etma 5 Ranoblith.; $\mathfrak{B}$ Io [dupp. 682. Dachsirgelf., geid)loll.; $2 B$ f b o. punctirt Solidágo.

e. mebr als 10 Ranoblitt).; $B$ ro. oach) jiegelf., mit abftehend. unt. Schupp.; SB \bo. grubig

683. Aster:

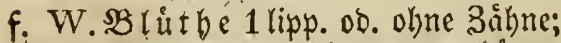
Blofchupp. gleich, etwas häut. fo lang als bie Edgeibe

b. vielbláttrig,
a. einfad), gleichformig
b. SB Io f d u p.p. in Dop'p. Sieihe, gleidhf., läng. als Scheibe; S. D. Яi and. blut h. nact u. obne Sfrone. 684. Tussiligo. 685. Cineraria:

686.

Doronǐcum.

2. am Grunde vermelnt (†. 660.)

(Senecro.)

3. boppelt: Die äussere;
a. 1 blättrig, 4 theil.: bie innere viel=

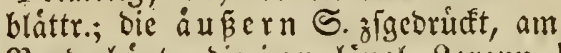 Rande bạut,: bie inn. längl., 2 grann, Helerospérmum
b. vielblåttr.: die innerẹ Sblåttr, Boebéra.

F. Fehlt; BIdecke:

1. cinfach,
a. balbfugelig,
a. mit gleichf. Schupp.; \$Brb o. fegelf.; 689. S. verf. =eyr.
Bellis.
690.

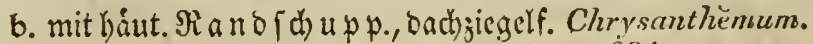

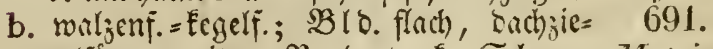
gelform., mit am Mạno trofn. Shupp. Matricaria.

2. Soppelt: bie ål ǰ. $S$ od. 10 blåttr., gleid)= 692 . fơrm.: Die innere vielbláttr., fưrjer Madra. 


\section{Oronutng.}

Die 3witterblith. frutbthar u. bie weibl. $\mathfrak{B} \mathfrak{l}$ atben unfrucbtbar.

(Syngenesia frustanea.)

Blumendecke :

I. eimfact); Blumenboden:

A. ipreuartig; Skrone:

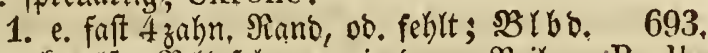
fegelf.; SB I

2. 2 blättr., himfạ́ll.; $\mathfrak{B}$ I bD. flach); $B 18.694$. bacbriegelf., etwas [perr., bláttr. Heliánthus.

3. 5 bláttr. (1. 702.) (Arctótis.)

4. feklt (5. 693.)

B. zottig (1. 702.)

(Rudbeckia.)

(Arctótis.)

C. borftig; Skrone:

1. Ganrig, verichicoengeftaltet; $\Re a n d=695$. bluth, trichterf., lảnger, ungleidhf. Centauréa,

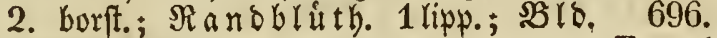
bachjiegelf.

II. boppelt; $\mathfrak{B} \backslash \mathfrak{b} \delta$. ipreuart; beyde Bld.: . Zoegéa.

1. 1 bláttr., Stheil.; S. 4 feit,; Skrone 697. 2 oo. 4 grann., baur. Cosméa.

2. vielblattr.; S. zfgebrudet, ausgerano.; 698. Strone 2 horn.

Coreòpsis,

\section{Dronngg.}

3mitterbrithen, unfrudbtbar u, weibl. Bluthen fruchtbar.

(Syngenesia necessaria.)

Blumenboden:

A. fpreuntig; Skrone: 699.

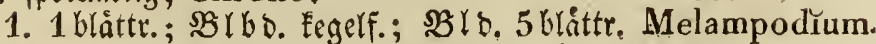

2. 4 ob. 10 jahn.; $\$$ ro. einf., 4-5bláttr.,

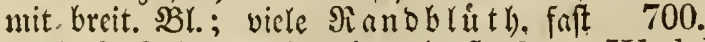
oval, $2-3$ ipalt.; $\Re$ a r ben borftenf. Wedelia.

3. geráno. $=2$ horn.; $\mathfrak{B}$ lo. Fperr., mit breit. Erbupp.; S. öfgeoridet, verf. = her fơrm., gerånolt

701.

Silphĭum. 
4. 3 blåttr.; $\$ B 1$ D. bactjiegelf., mit an ter 702. Spitze trod'n. Ectupp. Arctótis.

5. fehlt; Bldecke:

a. cinjach,

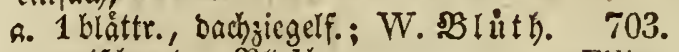
grwijhen ben Sibichupp.

b. 5 bláttr.; $\mathfrak{B l b b}$. flad); $\mathcal{S}_{\text {. verk. }}=704$. ebr., faft obne Strone. Parthen̋̆um. 705.

c. vielblattr., waljenf.; 5-^innobluth. Baltimóra.

b. mit 2(nbang am (srunoe; W. $\mathfrak{B} \backslash \dot{L} t \mathfrak{h}$. won ben \$lofdupen eingehúllt; olye Randblưth.

c. Doppelt: bie á uß̧. 4-5blåttr.: bie 707 .

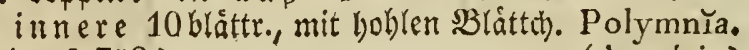

B. jottig (1. 702.)

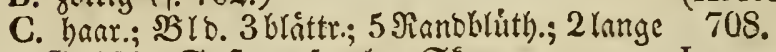

Sriff.; S. ftumpf, ohne Strone Iva.

D. nadit; Skrone:

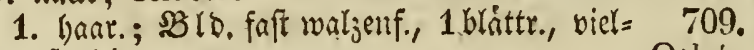
ipaltig

2. c. (Sranne von o. Daur. (Siriff.; S. jiges bruat, an ber Spire faft 2 zalnn.; W. Blut th. ohme SBlbl.; $B$ lo. vielbláttr., in cinf. Sronung (Blutb. fticlos)

E. fehlt; Bldecke:

1. 1 blàttr. (\%. T09.)

2. viclblattrig; Saame:

a. Entgel., Enochenlyart, gefärbt

b. in ber Schcibe băut.; $\mathfrak{B}$ rs. glcidf.

Othónna.

706.
icrŏpus.
707.
olymnia.
Arctótis.)

\section{Othonna.}

710.

Gymnostýles.

(Othónna.)

711. $\hbar$

Osteospérmum. 712.

Calendiula.

\section{SrDnttg.}

sBlitben ourch befonbere $3 \mathfrak{l}=$ mendeden in ber jufammenge= festen Blume abjefonbcrt.

(Syngenesia segregata.)

Mit rơkr. 3witterblutgen; beq. Blo.

1 blúth.; Blboden :

1. Gorft.; SEtoune undentl.

713.

Echinops. 


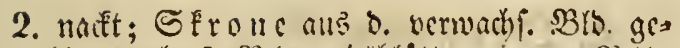

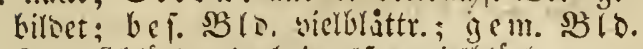

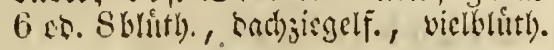
714. Brotéra.

\section{Rlaffe.}

Staubfaben แ. Staubrege

vermad fen.

(Gynandria.)

1. Oronแแg.

(5)

Die 5 Grattr. Blumenkrone:

A. mit Sporn albs. S. Sirunte ber Unterlipwe; Stbelt to am Enoe angeraachj.; Blk.:

1. etwas abftebeno

2. mit gewortht. ofern \$Bbr.

715.

Limodórum.

716.

Orchis.

B. obne Sporn it.

1. rad)enf.; Blblätter:

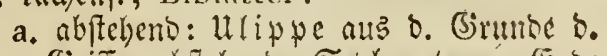
(Siriff, abjecheno; $\mathbb{S}$ tbeut. ant Enoe angewachien

b. zigeneigt; urippe flach, abwartsges bogetl; Stbeut. an b. verlingertent (Siriffel

c. bie ån. añ b. vorbern Scite uแm bern baudb. (Sruito ber allippe verbunden; Stbeut. nit b. langgeipirat. Şriffel parallel ut. Ginten eingefingt

oplirys.

718.

Serapı̆as.

719.

Neottúa.

2. aufr. =abftebent; uripue flact), bang., in ber Epise 2 palt.; Stbeutel mit 720.

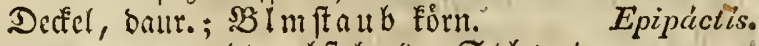

3. ganj umgewanot, abftebent; Stbent. mit Decfel; ulippe hobl=abftelent, auf= fteigeno

721.

Malàxis: 


\section{Dronuttg.}

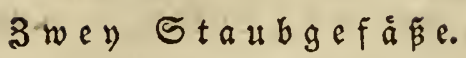

SBle. 4 brăttrig, abftebeno: Ulippe baudi)=

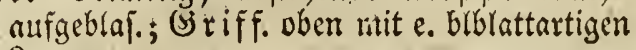
Eappen

722.

Cypripedŭum.

\section{Dronumg.}

ভ

SB lo. fchlt; BIE. 1 bláttr., 1 lipp., am (Sirunde 723.

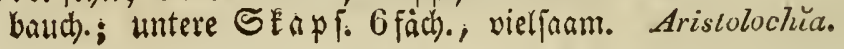

\section{RIalfe.}

Miannlid)e u. tweisl. જ̉lutţ̨en getrennt, aber auf (Einem Stamme.

(Monoecia.)

\section{Dronung. \\ $\mathbb{E} \mathfrak{i} \mathfrak{n} \mathfrak{S} \mathfrak{a} \mathfrak{a} \mathfrak{b} \mathfrak{g} \mathfrak{e} f \mathfrak{a}$}

I. Mit 1 Staubreg;

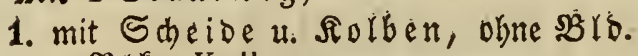

u., $\mathfrak{B} \backslash \mathfrak{k}$, ; Kolben:

a. obent nadet, in $\delta$. Mitte b. månnl. it. untten b. weibl. (Seldhledt tstheile trageno (i. 761.$)$

b. Die mit eiinanoer wed). (Bejellecht tstheile nebjt 8 . eyr. Fruchten. an Einer Seite trageno; Stbeut. ogne Stfád.; (s) rif 724 . $t$ fel 2 palt.; S Eap f. 1 faam. Zostéra.

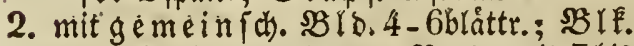
feblt; $\mathfrak{B}$ IbD. woll., am Rande mit 7 bis 
XXI. M. u. W. B̉lutţ. getrenut auf I Stamme. 95

10 M., in D. Nitte mit 3-6 W. SB(ưtl).;

M. Bro. rókr., foruppenform.: W. $\$ 318.725$.

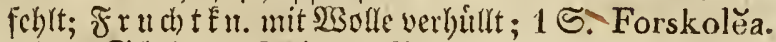

3. obne Stheibe; Julben, Blo. u. SlE.;

Stbeut. ohne Stfat.; Narbe :

a. 2 fpalt.; Sirff. fabenf.; Stapfel 726. + 1 raam.

Caulinia.

b. 5 fach; Stbedt. fugch.; Beere 1fách., 727. † vielianmig

II. mit 2 Stw $\mathfrak{g}$. ; $\mathfrak{B l E}$. felt ; M. Bld.:

Chara.

1. 1 blittr., 2 thcil.: W. 1 bláttr., 2 gorr., an D. ob. Frudten. verwachf.; 1 S. mit 728.

Bro. engumidiloflen

2. 2 blâttr. (i. 5.).

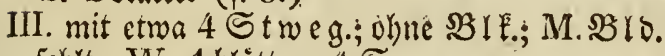

fehlt: W. 1 blättr.; $4 \mathbb{S}$.

Ceratocárpus. (Cullilriche.) + $729 .+$

Zannichellia.

\section{Dronuแg.}

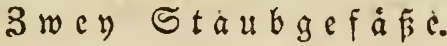

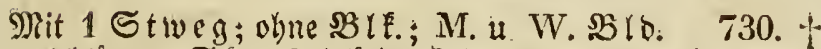

1 blättr.; Stałi. 1 fack., 2 jaam. Lemna.

\section{OrDutug.

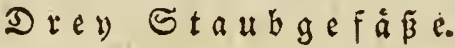

I. Nit 1 Strubreg;

A. mit eigentl. SB ro e de (†. 738.)

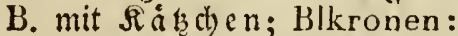

(Urtica.)

1. M: 2 bláttr: W. 6 blåtr.; SBeyde Brbeck. e. hlope Shuppe; Stfáben 2 gabl.; $\Re$ u $\bar{\beta}$ extr.

2. fellen; beyde Kätzchen:

a. Waklzenf., bie W. unter ben M.; M. BB lo. unbeutl., 3 blåttr.: W. feblt; 1 S., beffer Stiel am Sirunde mit langen Şaaren, wie mit e. Sftone, umgeben ift

b. rumbl.; $\mathfrak{B}$ eyde $\mathfrak{B l}$ r. 3 blattrig; Rarbe 2 ipalt; Stfrudt faft= loz, 1 laam.

731. $\hbar$

Comptonĭa. 


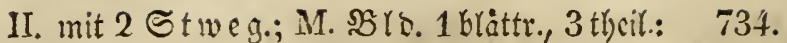
W. 5 blattr.; 3 le. fellen; $1 \subseteq$.

III. mit 3 Etweg.; $\mathfrak{B}$ ende 3 ro. 3 battr.;

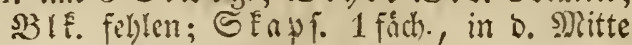
runoum aufipring., nit $1 \subseteq$.

Axýris.

735.

Amaránthus.

\section{Drontug.}

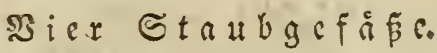

I. Mit 1 ธtaubreg; M. Bldecke:

A. 1 biătrig,

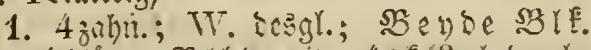
4 blattr.; 33 rbo. mit e. 4 ede. Eow burth = bobrt; mit unt. Frudtent; (j) iff.

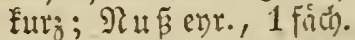

736.

Aucǔba.

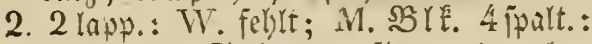

W. Fehlit; Etbeut. jibangens, ohne

Stfas.; Sriff.ppriemcll.; Parbe $2-3$ ipalt.; SEapf. 1 00. 4 fuant. Najas.

B. 4 brottrig; W. Bld.:

1. 2 bláttr.; B YE. Fehlen; e. Echerfôrm.

Rect. in s. Nitte; 1 glán. S.

2. fehlt; M. SBIE. 4 fpalt.: W. ungleid)

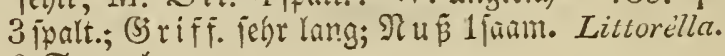

II. nit 2 ङ taub́begen;

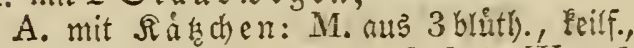
abgeftukt. Ech uppeñigepert: W. aus 2 blutt). Є(hupp.; sglê. M. 4 theil.: W. feglt; S. Jigeorindet, cyr., nadt

B. mit 'sBis.: M. 4 theil.: W. 4 blattrig; 741. 万
B lÉ. fehlen; mit $\mathfrak{B}$ eere u. 1 S.
Morus.

III. mit 3 Stroegen; M. BBro. 3 brattrig: W. 4 bláttr.; M. Szlk. 2 bráttr., mit e. Spur

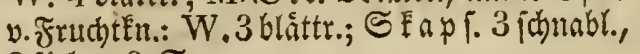
3 fád $\phi . ; 2$ S.

742. 万

Buxus.

\section{Dronung.}

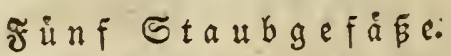

1. Mit 1 Stweg; M. gem. SBls. 1 bláttr.;

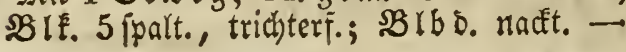


W. SBrs. 1 bróttr., ungetheilt, mit 5 jabn.

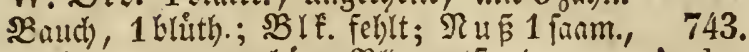

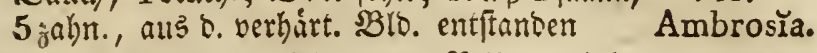

2. mit 2 Stweg.; M. gem. SBlb. Dactjic=

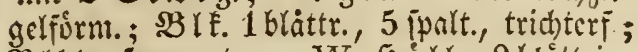
Blbo. ppreuart. - W. Soulle 2 blättrig, 1 blith.; $\mathfrak{B}$ Is. u. B IÉ. fehlen; Stfrud th 744.

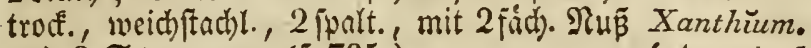

3. mit 3 Stwegen $(\lceil .735)$.

(Amaránthus.)

\section{Drd $\mathfrak{1}$ g.}

Sechs Staubgefäpe ([.296.) (Rumex.)

\section{Orb nung.

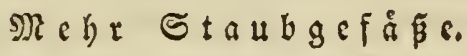

\section{Mit Kätzchen.}

M. Kätzchen:

I. nafit; M. Bldecke:

1. 5 jpalt.; Ş If. felytt; 5-10 Stgef.; -

W. B!b. 1 bláttr., gang ungeth., (d)arf;

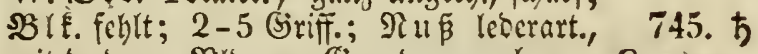
mit b. Daur. \$Blb. aln Sirutbe umgeber Quercus.

2. feglt; M. Blk.:

a. 5blättr.; 10-20 Stgef.;-W. B lb.

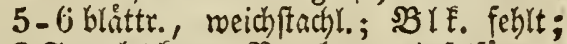
$3 \mathfrak{F r u d t} \mathfrak{f}_{\mathfrak{n}}$;. Rarben pinfelfórm.; 3 Rúfie in b. rutbum ftadl, sBbecte eingeloblofien

b. fehlt; Stbeut. Dadsiegelf.; - W. B10. 4 [palt.; Etfrudit mit 3 Eunt.

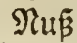

II. sachsiegeffóntmig;

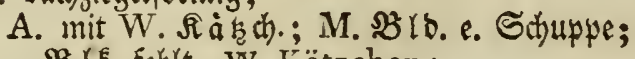

$\mathfrak{B}_{\mathfrak{Z}} \mathfrak{l}$. Fehlt; W. Kätzchen:

1. Dadbjiegerf.; W. SBloede e. 2 blüth. St)uppe; W. Blk.:

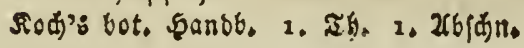

$746 . \hbar$
Castaněa. 747. 5 Salisburýa. 
a. 3 fpalt.; M. B 1 lof 10 Stgef.; - Nu $\beta$ evr., gefurdt Carpinus.

b. feblt; 10-12 Stgef.; 1 geflugelt. 749 . 万 S.

2. nact; Stgef. åt.; W. SBlode 1 . SIE. fehlen; SE apl. nufgeblar., bach)= jiegelf., ant Sirunbe 1 janm.

B. ohne W. $\Omega$ a Betüla. M. Bik. :

1. 6 theil.; 4-18 Stgef. -W. BIt. u. ß̊Frone 4 fpalt.; 2 (siriff.; unt. Fruthten.; Stfrucht leocrart., mit gefurct)t. $\mathfrak{R} u$

2. fegrt; 8 ङtgef.; - W. MB It. 2 theil., zertiffen; $\mathfrak{B}$ IE. fellt; 2 Siriff.; $\mathfrak{R}$ eyr., mit baur. SBlo. unigcben 751. 5 Iuglans.

750. 5 Ostry̆a.

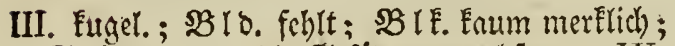
Stbeut. um bic Etfáo. verwachf.; -W.

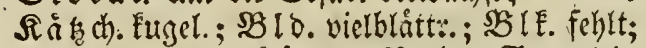
(3) riff. mit umgefrummt. Narbe; $\odot$. rutor., mit (Sriff. fted)enogefpiłt u. unten mit Sirone Platănus.

IV. Fegelfồm., mit 4 blattr. Scủlte umgeben; BIo. u. $2 \mathcal{Z}$ IE. fehlen; jebr viele Stgef.;-

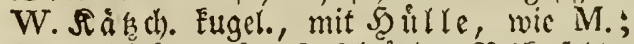
SBlb. 1 blåttr., frugf., 2 blitth.; $\mathfrak{B}$ lE. fehlt; 2 (S riff.; 2 SE apf. mit \$. \$Rlo. am Srunde umgeben, 1 fåch., vieljanm.

754. $\hbar$

Liquidámbar.

\section{II. ohne Kätzchen;}
A. mit eigentl. Blitmendecke.

M. Blumendecke:

\section{1 blättrig,}

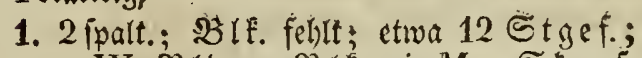

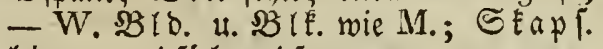
reberart., 1 făchl., 1 fanam.

2. 3 theil.; (i. 789.)

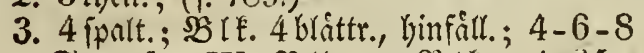
Stgef.; W. BIt. u. SBlke wie M.; 4 Streg. ohne Sriff; 4 SEnpl. mit Rinbe

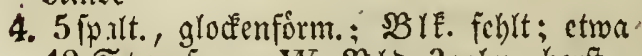

755.

Thelygŏnum. (Mercuriális.) 756.
Myriophillum. 12 Etgef.; - W. 3 Io. 3 zahn., borft.; 


\title{
XXI. M. 1. W. Blưtr. getrennt all I Stamme. 99
}

SBlf. feblt; 2 Fruchten.; 2 Rifle in o. leserart., 4 fpalt., runoum ftaci)l. Blo. eingeitblollen

5. vieltheil.; $\mathfrak{B}$ If. feldt; ctwa 16-20 Stgef. mit 3 fpir. Stbeut. - W. BB (o. 6 blàttr., Dachjiegelf.; $\mathfrak{B}\left(\mathrm{k}\right.$. Fellt ; $1 \varsigma_{\text {twe }} \mathfrak{g}$ mit fabenf. Briff.; $\mathfrak{N} u \tilde{B} 1$ făm.

II. 3 bláttr.; Sgle. 3 blớttr.; meift $2 t$ Stgef.;

- W. sBlo. u. BBlérone 3 blattr.; vicle

Stwege; vicle nafte 5 .

III. 4 blàttr ; SBlt. 4 theil.; 30-50 Stgef.;

-W. BS ID. u. SBle. wie M.; 2 Stwege; 760.

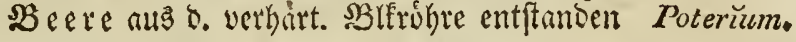

$$
\begin{aligned}
& \text { B. ohne Bld. u. Blk., mit . } \\
& \text { Scheide u. Kolben. }
\end{aligned}
$$

Sd) cide 1 blättr., fappenf.; Solben oben nadt, unten mit $W$. , in Der פ) itté mit $M$. (Seld)lechtztheilen

\author{
757. $ち$ \\ Fagus. \\ $758 .+-$
Ceratophillum,
}

759. + . .

Sagittaria.

761.
Arum.

\section{8. $\mathfrak{O} \mathfrak{D} \mathfrak{u} \mathfrak{H}$.}

Staubfaben in Eine Såule verwad) fen.

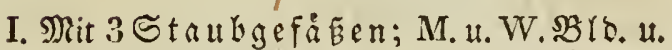

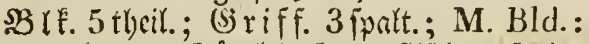

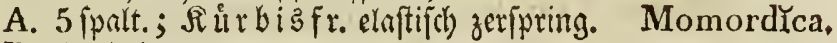

B. 5 jalynig;

1. Kiirbisfrucht:

a. 1 farmig

b. mebriannig; Saame am Rande: a. aufgeidiwollen

b. nicht

763.

Sicy̆os, 764.

Cucurbita. 765.

Cucŭmis. 766.

2. Beere faft fugel., vielfanm.

II. mit 4 Stgef.; M. Sátad). bachjiegelf.;

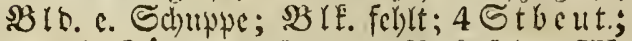

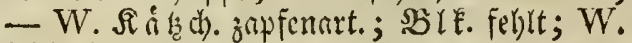
Bldschuppe:

1. 1 blith.; Sa a r be 2 goble Suncte; Stbeut. olyne Stfăb.; গরu

767. 5

Cupréssus. 
2. 2 blüth.; -W. $\mathfrak{B l E}$ feblt; $1 \Re \mathfrak{R}$ mit e. Flúgel am Siande

III. mit S-16 Stgef.; M. 28 Io. 3-4blattr.; SBlE. feblt; - W. $\mathfrak{B}$ lo. 3 blàttr.; $\mathfrak{B l f}$. feblt; 3 SSriff.; Słapf. 3 fnopf., 3 fåd. mit einjelnen $\mathfrak{S}$.

IV. mit vielen Stgef.;

768.

Thuja.

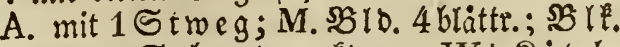

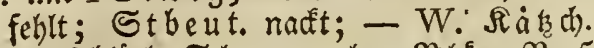

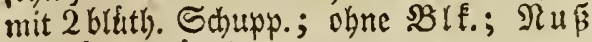
mit háut. Fłtủgel

B. mit 3 Stweg.; SEende SBlo. 5 theil. u. BlE. Fellen; - Griffel 2 jpaltig; SEap 3 fách., mit einzeln. S.

\section{9. Acalýpha,}
7\%0. $\hbar$
Pinus.

771.

Ričnus.

\section{Dronting.}

Stfäben u. Strocge vermachlett.

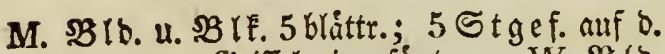
Spur v. e. (Siriffel eingefugt; - W. $\mathfrak{B}$ lo. 5 bláttr.; Sle. feblt; 3 śriff.; Stapl. 3 fách., mit 2 ..

772.

Andràcline.

\section{Rlaffe.}

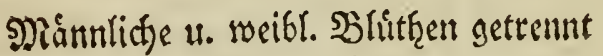
auf 3wey Stámmen.

\section{(Dioecia.)}

\section{Dronung. \\ 3wey Sta $\mathfrak{b} \mathfrak{b}$ efá}

M. $\mathfrak{A}$ a thet

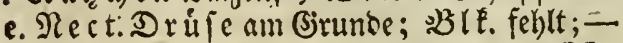

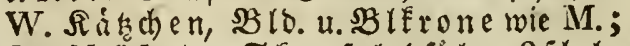

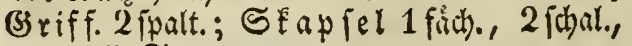
mit woll..

773. 万 Salix. 
XXII. M. น. W. Blutr̨. getr. auf 2 Stámm. ro1

\section{Dronung.}

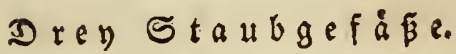

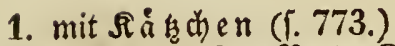

2. mit Blbeffe; Benbe Blkronen:
a. 3 blättr.; St gef. Kang; - W.9 Jriff.; SBe ere 9 farm.

\author{
Emę̌trum.
}
774. ち

(Salix.)

b. fehlen; sevbe Bldecken:
a. 3 ppalt.; - W. 1 s riff. mit runbl, Narbe; Beere 1 fádh.

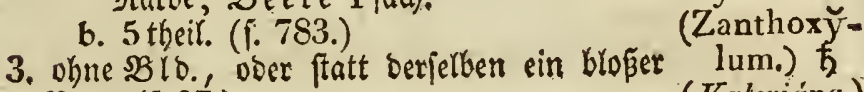

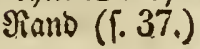
775. 万
Osýris.
(Zanthoxy̆-
lum.) $\frac{1}{2}$
(Valeriána.)

\section{DrDnIIIg.}

Şiex Staนbgefábe.

\section{I Staubrweg.}

A. Beere:

1. 1 faam.; $B$ eyde $\mathfrak{A g}$ lf. fehten; M. Bld.:

a. 2 theil.; - W. BID. 2 [palt.

b. 4 theil.; Stbeut. ohme Stfáden an b. BBld. vertwad)i.; - W. B18. 4 blättr.;

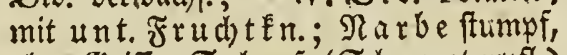
obne (Sriff.; S. herzf. (Sd)marokerpfl.)

2. 3-4 faam. (1. 153.)

B. $\tilde{F} l \mathfrak{u} g e l f r u d t(f .70$.

C. mit unbededtem $S$ aamen;

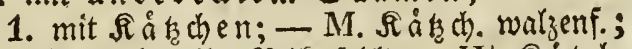

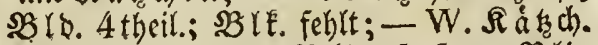
fugel., aus fentenf. Blbo. zigefert; B ID.

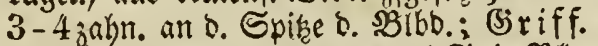
pFriemenf., zur Éeite ftebeno; 1 S. in Blb. Broussonetĭa. 2. mit $2 \mathbb{B}$ 10. (โ. 738.)

776. 万

Hippophüe.

777.

Viscum. (Rhamnuls.) क (Ptelěa.)

\section{II, 2 Staubwege.}

1. Mit långl. $\mathfrak{x}$ å

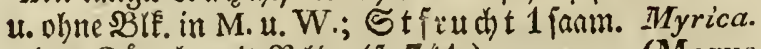

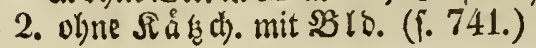
(Urtica.) 


\section{OำแIIg.

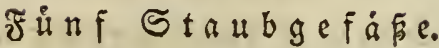

I. Mit 1 Staubweg;

1. mit $\Omega$ a tathen $(1.773$.

2. mit eigentl. B I loe e; Blk. :

(Salix.) $\hbar$
a. 5 blíttr. (i. 16i6.)
b. 5 Echuppen (1. 153.)
(Piles.)
(Rluamnus.) $\hbar$

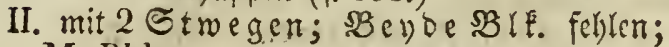

M. Bld.:

1. 1 blättr., 5 theil.; - W. $\Omega$ l o. 1 blattr., ungeth., an.o. Esite offen; $\mathfrak{R u}_{\tilde{\beta}} 2$ jhal. in SBlo.

2: 5 blàttr.;-W. W Ib. 1 blàttr., ungetheilt, ichiefabfteheno; 1 S. in D. Glattr. SBlo.

III. mit 3 Strogen (1. 241.)

IV. mit 4 Stweg.; SEeve obne BZIt.; N. BB 10.5 theil.: W. 4 ppalt.; 1 S., in verlyar. teter BIo.

V. mit 5 Stmeg.; Beyre ohne BIf,; M.

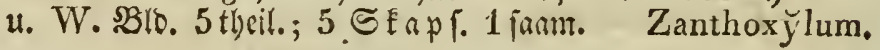

\section{0. \\ Cannütis.}

781:

Humùlus. (Rhus.) $\hbar$
782.

Spinacra. 783. 方

\section{Ordnutก.}

Sed

I. Nit 1 Streg u. unt. Frudt noten; Bevte Blkronen:

1. 6 palt., umgeront, unten verwach $\Gamma_{.}$, mit

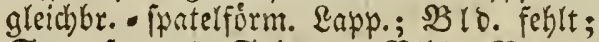
Etgef. an D. Epiren D. SBlbl.; Beere 1 jaam., genabelt oo. nit Blo. gefrónt Loránthus.

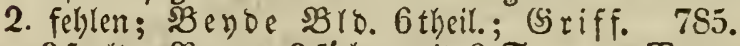
3 ipalt.; B Beere 3 fách., mit $2 \mathbb{S}$.

Tamus. II. mit 3 St $\mathfrak{a} u b$ wegen; Blk.:

1. 3 bláttr. ( 1.296 .)

2. fehlen; Sende Bld.:

a. 1 blattr., 6 tbeil.; Stap 3 fäherig, zfgeorudet; 2 háut. S.

(Riumex.)

bolattr.; 3 eere 3 fach.

b. 6blăttr.; SBeere 3 f̧ẩ

7S6. $\hbar$

Dioscoréa. 787.

Sinilax. 
XXII. M. u. W, Blưth̨. getr. auf 2 Ståmm. 103

\section{Dronung.

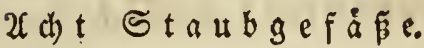

Mit 1 Staubweg;

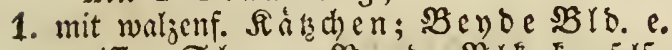
zerriflene Sdhupp.; $\mathfrak{B}$ ey o e $\mathfrak{B}$ le. Freujelf., ichicf, ungeth.; - W. $\mathfrak{N a r b c} 4$ fpaltig;

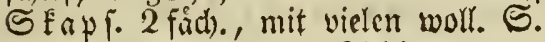

2. mit eigentl. 3 Iro. (i. S05.)

3. oline beyoe; Blk.:
a. 4 ipalt. (i. 317.)
b. 6 theil. (1. 325.)
(Daphne.) 5
(Laurus.) $\hbar$

788. 5

Popūlus.

(Acer.)

\section{Orbunng.}

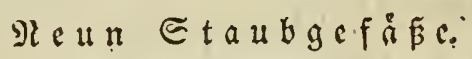

1. Mit 1 Staubweg (f. 325.)

2. nit 2 Stwegen; $\mathfrak{B}$ ey oe $B$ Io. 3 theil. u. SBlE. Fehlen; Ǵtbeut. Eugel., 2 Enopf.; 789 ,

SEapf. 2 Enopf., 2 fấb., nit 1 fanm. Fách. Mercuriális.

3. mit 6 Stwegen; M. Sd) cibe 2 blattr.; SIS. 3 palt.; 2 le. 3 blattr.; bie 3 intr.

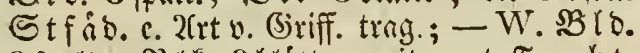
3 part.; $\mathfrak{B} \mathfrak{l f}^{2} .3$ blattr., mit unt. Frudsts fluoten; Skapl. 6 fád), vielfan.

(Laurus.) $ち$

790. : Hyckrackuris.

\section{Dronung.}

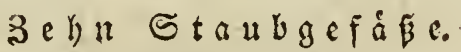

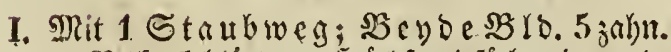

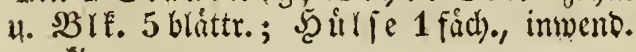
martig

II, mit 2 Staubregen (f. 358.)

III. mit 5 Staubwegen; Bldecke:

A. 1 blittrig; Blk.:

1. 5 blattrig (f. 365.)

2. fellt; \$SlD. 5 theil., mit 5 Drijen; Stbeut. 2 theil.; 5 SEapr. 1 jaam., mit $D$. erweiterten Drijen beoceft

\section{1. $\hbar$ \\ Gymnocladus. \\ (Cucubülıs.)}

(Lychnis.)

792. $ち$

Coriaria. 
- B. 5 blättrig (i. 369.)

(Cerastı̌um.)

IV. mit 10 Staubwegen (f. 371.)

(Phytolácca.)

\section{Drditutg.}

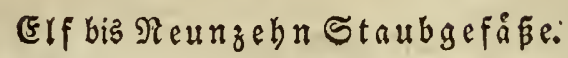

1. Mit $2 \subseteq$ taubregen;

a. mit $\mathfrak{B r E} .: 4$ ob. $6 \mathfrak{a} \mathfrak{u}$ в. U. $S$ innere

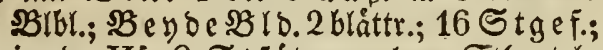
in b. W. 8 Stfáben ohne Stbeutel; Beere 1 jam.

b. ofme 2 If. (1. 789.)

793. $\hbar$

Menispérmum.

(Mercuriális.)

(Menispér-

mum.) b

2. mit 3 Staubregen;

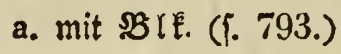

b. ohne SBIE.; M. \$Blo. 5 blåttr.; 15 långl. Stbeut. ohne Stfáb.; - W. BBlbeáe 2 zahn.; 3 unt. SÉ apf. eff., 3 horn., 1 fäh., offert, vielfanmig

794.

Datísca.

3. mit 6 Streg.; M. Sheibe 2 blåttrig; SR to. 3 theil.; $\mathfrak{B}$ IE. 3 blättr.; 20 Rect.

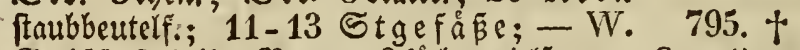
(3) riff. 2 theil.; Beere 6 fách., vieljan. Stratiòtes.

\section{Dronung. \\ Siele Staubgefáb a $\mathfrak{f}$. ber Blumendedé.}

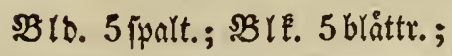

1. mit 5 Staubregen;
a. mit Skapf. (F. 396.)
(Spira'ea.)
b. mit $\Omega \mathfrak{e r n f r u d}$ t (₹. 394.)
(Pyrus.) $)$
2. mit yieten Gtaubwegen (f. 400.)
(Rubus.) क 
XXII. M. น. W. Blưt getr. auf 2 Ståmm. 105

\author{
11. Dronung. \\ Sie $\mathfrak{e}$ e Sta $\mathfrak{u} \mathfrak{b} \mathfrak{g}$ e f $\mathfrak{a} \bar{\beta}$ e. \\ a
}

1. Mit 1 ङtaubreg;
a. mit Stheibe u. Rolben (i. 761.)

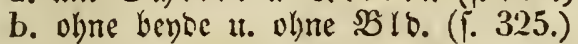
(Arum.)

2. mit vielen Staubwegen; ohne Blo.; mit 4-5 bláttr. $3 \mathcal{B}\{$ F.; Saame:

a. geichmånzt (i. 436.)

b. nid)t (1. 437 .)

\title{
12. DrDnแng.
}

\section{Staubfáben in Eine Såule}

vermadjen.

I. 3 Staubgef.u. 3 Stwege; M. $\Re a ̊$ b). enr.; $B$ lo. e. Sduppe; sBle. feglt; - W. Bribede 3 theil.; SBle. 3 blattr.; BRecre 796. 万 3 [aam., ungleid burch 3 Sinoten o. Blid. Junipěrus.

II. 5 Stgef. u. 1 Stweg; $\mathfrak{B}$ eybe $\mathfrak{B l}$. 6 brăttr., ohne $2 B l \mathfrak{l}^{2}$; mit e. ent., an $D$. Spitge burbbohrten Rectar. in d. Mitte; 797. 万 Beere mit 3 zmenjaam. Fåact.

III. 7 Stgef. u. 2 Stwege; Seyde obne

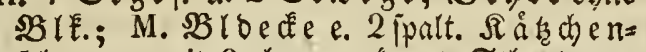
(1) uppe mit 3 obern u. 4 unt. Stbeut.; - 798. $\hbar$

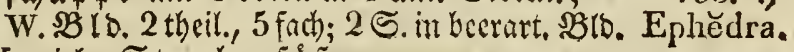
IV. viele Staubgefåze;

A. 1 Sta $\mathfrak{a b}$ weg;

1. mehrere SEapf. (i. 545.)

2. 1 ganjrand. S. in beerart. Sarbede;

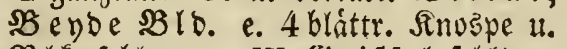
BBlf. fehlen; - W. (S riffel fehlt B. 3 Staubrege (5. 771.)

(Sida.) 799. 万

Taxus. (Rič̌nus.)

13. Ord IIItg.

Staubbeutel yerwad)[en. (โ.766.) (Bryonia.) 万 


\section{Rlarfe.}

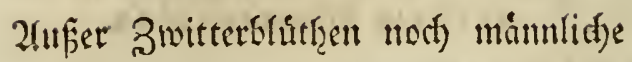

und meibl. Şlutthen.

(Polygamia.)

\section{Oronung.}

$\mathfrak{A} \mathfrak{u}$ (Finem $\subseteq$ tamme.

Zwitterbliithen:

1. mit 2 Staubgefázen;

1. mit 1 Staubweg (f. 807 .)

2. mit 3-5 ङtaubweg. (Z. F. 34.) - M. S00. 万 u. W. eben jo. Ailánthus.

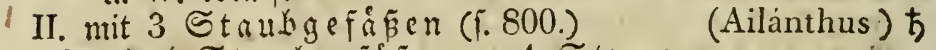
III. mit 4 ভtaubgefácen 1 Stweg; Z. Blk.:

1. 4 bláttrig (i. 70.)

2. fehlt; (Z. F. 7S.) - W. eben fo

(Ptelěa.) 万 SO1.

IV. mit 5 Staubgefáa

A. mit 1 Stweg; Z. Blk.:

1. 4-5 blåttr. (i. 303.)

2. fehlt; (Z.j.232);-W. RB. 2blattr.; Blte, (siriff. u. S. wie Z.

B. mit 2 'Etwegr; $B$ Io. 5 theil.; Brf. fchlt; Stfrud)t 1 faam.; - M. SBlo. 6 thcil.; B If. fehlt; $0 \subseteq$ Stgef.

V. mit 5 ड́tgef. u. 6 ङ troeg.; (Z. 1. 303.)

- M. $\mathfrak{B}$ lo. u. $\mathfrak{B l E}$ eben jo, mit e. Spur

$\begin{array}{ll}\text { v. Stmeg } & \text { Verátrum. } \\ \text { VI. nit } 7 \text { Stgef. 11. } 1 \text { Stroeg (f. 301.) (Aeseutus.) b }\end{array}$

VII. mit $S$ Staubgefásen;

A. mit 1 Staubweg;

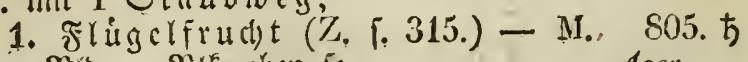
şí. u. SBlf. cben io

2. Ëanfer (i. 301.)

B. mit 2 Staubwegen (f. 805.)

VIII. nit 10 ธtgef. u. 3 ङtwegen (5.357.)

(Aescülus.) $)$

Alrĭplex:

803. 古

Celtis.

804.

(Aeseülus.) b

Acer.

(Aescütus.) †

(Acer:) $\hbar$

(Siléne.) 
IX. mit 11-19 Stgef. uno 3 Stwegen (i. 330.)

(Euphorbǐa.)

X. mit febr vielen Stgef. und 1 Stweg; 806. (Z. \{. 415.) - M. sBlb. ul. SBlk. wie Z. Acač̀a.

\section{Dronting.}

Xuf 3 wey Stám

I. Mit 2 Stgef. u. 1 Stweg; Z. Bld.: 1. fehlt od. + theil.; (Z. 1. 33.); - W. ebento Fraxĭnus. 2. ríbr., 5 zukn. (i. 153.)

(Rhamnuls.)

II. mit 3 Stgef.u. 1 Stweg; gem. B B Freujelf., fleifh., zigeneigt, bie sblitthchen verhidf.; - N. SBID. 3 theil.; $B$ If. febit;

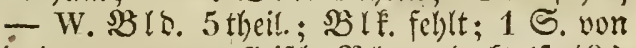
o. Daur., etwas fleifo. Bro. verbect (i. 40.) Ficus. क III. mit 5 Stgef. u. 1 Stweg; (Z. f. 170.); 809: ち - M. B B. wie Z. u. 10 ভ́tgef. Nyssa.

IV. mit 6 Stgef. ur. 1 Stweg; (Z. f. 262.);

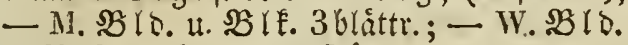

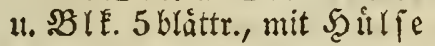

S10. $\hbar$

Gleditschụa.

V. mit 8 Stgef. u. 1 Stweg; (Z. f. 307.) - W. Bro. u. Bre eben fo, mit S faam. 811, Beere Diospýros. 


\section{$\mathfrak{A} \mathfrak{n} \mathfrak{b} \mathfrak{a} \mathfrak{n} \mathfrak{g}$.}

\section{Tabelle}

fut bie blos meibfid)en şblutţen ber $\mathfrak{Y}$ flanjen mit getrennten (Beffled)tern.

\section{Oronumg. \\ (E) $\mathfrak{i} \subseteq \mathfrak{n} \mathfrak{a} \mathfrak{u} \mathfrak{b} \mathfrak{w} \in \mathfrak{g}$.}

\section{Mit Blumenkrone.}

Blumenkrone:

\section{1 blàttrig ;}

A: mit badyziegelf. $\Re$ a

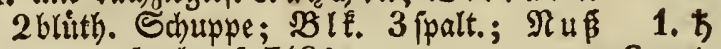
evr., gefurcht (f. 748.)

B. mit eigentl., 1 bláttr. SB roede e;

1. mit leberart. Steinfrudt $u$. ges

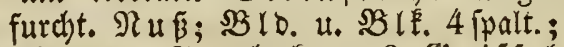
mit unt. Frud ten.; 2 Gs riffet (i. 751 .) Iuglans:

2. mit $\Omega \mathfrak{u} \mathfrak{r} \mathfrak{b} i \xi f \mathfrak{r} \mathfrak{d} d t$; Bld.:

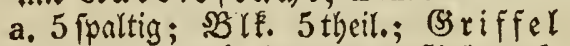
3 palt.; Rurbisfr. elaftijh auf= fpringent (1. 762.)

Carpinus.

b. 5 zalnig; Kuirbisfrucht:

q. 1 fanm.; BBrf. 5 theil.; Ģriff. 3 ipalt. (f. 763.)

b. mehriamig; Staubro. 3 part.; Saame am Rande:

1) aufgeidowollen; $B$ IF. 5 fpalt. (f. 764.$)$

3.

Momordíca.
$2 . \hbar$
Iuglans: 
C. mit unseutl. SBlo., ein bloferer Siand; B̧ॄ. 5 fpalt., am Sirunde auf c. Seite bodfr.; mit unt. Fruthtequ; SFapF. 1 fåd): : S. mit feber. Sirone (1. 37.) Valericina.

D. ohne $\mathfrak{B l}$ loefe;

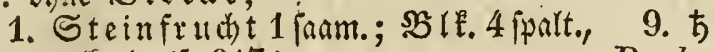
welferto (1. 317.) Daphne.

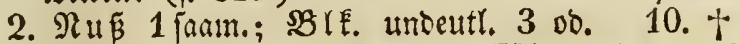
4 palt.; (5) riff. Fehr lang (F. 739.) Littorélld.

3. SBeere 1 fant.; $\mathfrak{B}$ If. 6 theil., broceft= artig, mit 3 jwanborft. Nect. Dr $\mathfrak{i}=$ fen um ben Frudtetn. (i. 315.)

11. $ち$

Lauras.

12. $\hbar$

Empěirum.

9 [aam. (i. 774.)

III. 4 blattrity;

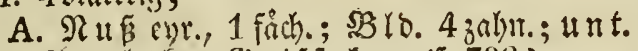
Fructeten. (5) riff. futz (F. 730.) Aucŭba.

B. Fliigelfrucht:

1. rundr., mit $1 \subseteq$. in $\delta$. Mitte; ober.

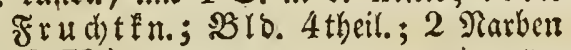
(.i. 70.$)$

2. evyr. - län.jl., mit lanzettf. Flligel, mit

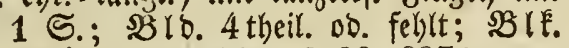
4 bleittr. ober feglt (i. 33. 807.)

IV. 5 blättrig;

A. $\widetilde{Y}$ lígeffrucht (f. vorber 15.)

B. SBere; B B bl. auf D. 5 fpalt. glodenf.

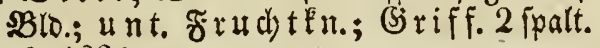
(i. 166.)

C. S2úlje; $\mathfrak{B l d}$. 5 blåttr. (i. 202. 810.) Gleditschĭa. V. 6 blättrig; Bldecke:

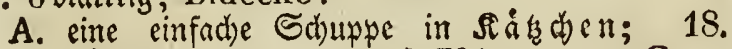

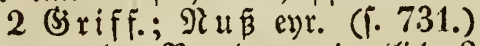

B. Doppelt: 'BCyDe unbeutlich 6 jahnig;

Blblátt. umgerollt; SBeere 1 laamig, 19. gelbl. (5. 784.)

Fraxinus.

(Fraxinus.) 方

16.

Ribes.

17. 5

Comptonia.

Loránthus,

\section{II. ohne Blimenkrone;}

A. mit Kätzchen.

Blumendecke:

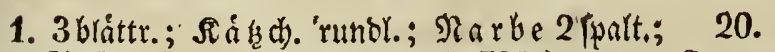
Stfruds t trock, 1 [am. (i. 733.)

Sparganium. 
2. e. Schuppe; Kätzchen:

a. walkenfürmig;

a. SEapi. 1 fach., 2 fchal., mit wollig. $21 . \hbar$ S.; (S) riff. 2 fpalt. (i. 773.) Salix.

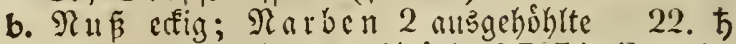
Souncte; $\Re$ ro id) up pe 1 blúth. (1.767.) Cupréssus.

b. zapfenartig; SBIS ichuppe 2 blitbig; $1 \mathfrak{R} u \tilde{\beta}$ umgeben mit e. geråno. Flúgel (i. 768.$)$

23. $\frac{\hbar}{5}$ Thuja.

3. fehlt ganz;

a. SFapieln aufgeblai., bachziegelf., am 24.

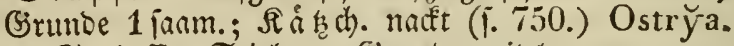

b. 1 S., dellen Etiel am Sirunde mit lan= gen Saaren, wic mit e. Sitone, umge=

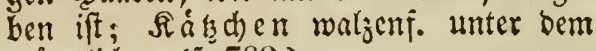
mámulichen (i. 732.)

25.

Typha.

\section{B. mit Zapfen.}

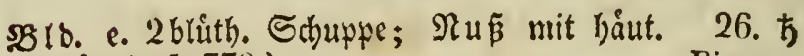
Flügel (F. 770.)
C. mit eigentl. Bldecke.

\section{Blumendecke:}

\section{1 blàttrig;}
A. mit $\mathfrak{B}$ ecre; Bldecke:
27. ち
1. 2 palt.; $\mathfrak{B}$ eere 1 faam. (f. 776.) Hippophä̈.

2. 3 ppattig; פarbe runol.; Becre 2S. 1 fách. (f. 775.)

3. 5 jabll., rơhr., mit 5 Shuppen auf $29 . \hbar$ berjelben; Beere 3-4 jam. (1. 153.) Rhamnus.

4. 6 theil.; (S) riff. 3 ipalt.; unt. Fruch $t=$
Enoten; Beere 3 fách., mit 2 S. (i. 785.)
30.
Tamus.

B. mit 1 fách. Saamenkapsel:
1. 1 jaam., leberart.; Blo eqe 2 fpaltig (1. 755.)
2. 2 [anm.; $B$ lo. 1 brittr. (i. 33.)

$$
\begin{aligned}
& 31 . \\
& \text { Thelygŏnum. } \\
& \text { 32. † } \\
& \text { Lemna. }
\end{aligned}
$$

C. mit Steinfrudt; Bldecke:

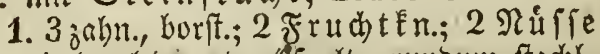
in ber leserart., 4 fpalt., runoum ftad)l. SBIb. ( $1.75 \%$. 
2. 4 fpalt ; $\Re \Perp \dot{\beta} 3$ fant. (i. 747.)

3. ungeth)., 1 bluth., am Sanche 5 zahn.; $\Re \mathfrak{B} 1$ janm., ans "der vetbărt. Sblo. entftandat (i. 743 .)

34. $\hbar$

Salisburýa.

35.

Ambroša,

36.

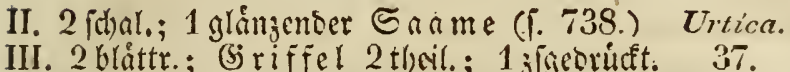
Sarme (i. 802.)

IV. 4 blattt.; แnt. Frad) tenoten; Rarbe ftumpf, obne Sriff.; SBcere nit 1 berjf. 38. S. (i. 777.) Viscum.

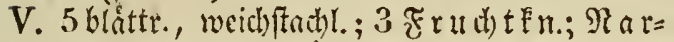
ben pimjelf.; 3 ఇúfie in o. runoum ftat)l. SBlo. (T. 746.)

39. 5

Castanĕa. VI. 6 biớttrig;

A. mit $\mathfrak{N e c t . ~ i n ~ b . ~ M i t t e ~ b e ß ~} \Re$ Bros., cyt.

it. an $\delta$. Spike burchbolirt; Beere mit

3 jwevian. F̌ăch. (i. 797.)

40. $ち$

Ruscus.

B. olne Prectarium;

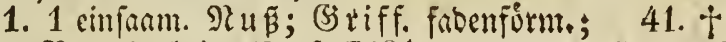
2Blo. brichjiegelf. (f. 758.)

Ceratopliýllizm.

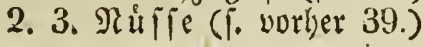

(Castanča.) ち

D. olnne Blumendecke.

1. Flugelfrucht (f. norber 15.)

2. Becre 1 fád)., vielfarm.; (Siriffel feldt; 5 Rarben (

3. Saamenkapsel:

a. 1 fanmig; Griffel:

a. priemenf.; $\mathfrak{R}$ a b e 2-3 palt. (1. 737.) Najas.

(Fraxinus.)

42.

Chara.

43. \&

44.

5. fadenf.; Narbe 2 part. (i. 726.)

Caulinia.

ig (i. vorber 43.)

(Najas.) if

defft. Sa a men;

1) iffel; Narbecinfad); frat $t=$

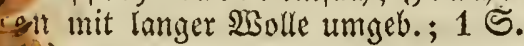

3riff.; ftatt SBlo. e. 4 Glattrige wope; 1 S. in D. ganziand., becrart. (1. (1. 799.)

45.

Forskolěa.

46. 方 


\section{Drotung.

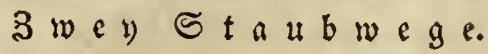

\section{Mic Blumenkrone.}

Blumenkrone:

1. 1 blattr., 4 theil.; $\mathfrak{B l}$ D. 4 blättrig; SBeere 47. aus b. verbártet. Blfto̊bre entftand. (1. 760.) Poterüum.

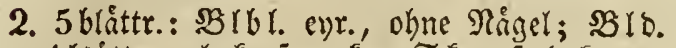
1 bláttr., glodfenf., eff.; Skaplel fugel., $4 S$. 1 fát). (1. 350.) Gypsoplizla.

3. 12 ob. 14 blittr., movon 4 ob. 6 auswärt3 u. 8 inm. ftehen; şloede 2 bléttr.; 2 bis

3 Fruchtaten; 2 Beeren 1 jamig (5. 793.)

49.

Menispérmum.

\section{II. olnne Blumienkrone;}

A. mit eigentl. Bldecke.

Blumendecke:

\section{1 blåttrig ;}

A. Skapf. 2 mopf., 2 fách., mit 1 faam. 50. Fُåd); $\mathfrak{B}$ 18. 3 theil. (1. 789.) Mercuriális.

B. $\Re$ « ; Bld. :

1. 2 theil., jerriil.; $\mathfrak{N} \mathbb{B}$ enr., mit baur. $51 . \hbar$ SBlo. umgeben (1. 752.)

Cory̆lus.

2. ungetbeilt, an ber Seite offen; $\mathfrak{R} \tilde{\beta} \quad 52$. 2 ichal. in Bro. (\%. 780.)

Cannübis.

3. ganzrand., icharf; $\mathfrak{N} \mathfrak{u} \tilde{s}$ leberart., am (srunde mit o. Daur. SBloede unigeben (1. 745.)

C. mit unbebedt. Sa amen; Bldecke:

1. cinfach,

a. ungeth., fdbiefoffen; 1 S. im (Sirunbe b. Rlo. (1. 781.)

b. 2 born.; 1 S. v. o. Brbecte bededt (i. 728.)

$53 . \hbar$
Quercus.

2. 5 fach Liber einanber geffellt, wowon jebe 1 blattrig, 2 theil.; 2 S. von ber beerart. 310. bebeçt (1. 798.).

Huınülus.

55.

Ceratocárpus. 


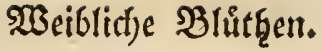

II. 2 blättrig; Staplel 2 fỏcherig, 4 jaamig (f. 5.)

III. 4 blättrig; 1 S. in berartiger SBlbede (f. 741.)

IV. 5 blåttr.; 1 S. (โ. 734.)

$57 . \hbar$

Callitriche.

58. $\hbar$

Morus.

59.

Axýris.

B. mit Kä̈tzcheu u. Hülle.

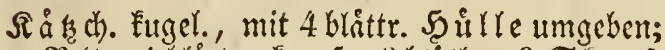
$\mathfrak{B}$ lo. 1 blättr., frugf., 2 blitth.; 2 Skapi. 1 fád)., vielfanm., am (3runbe mit $\mathfrak{B l o}$. um= geben ([. 754.)

60. $\hbar$

Liquidảmbar.

\section{C. mit. Kätzchen, ohne Hülle.}

SID. c. Shupwc;

A. Stfrucht 1 laamig; Sáthen lóngl.; 61. BIb fdu up. eyr. (1. 779.) Myrica.

B. mit unberedet. Saamen; BIb (d) uppe 2 blittly.;

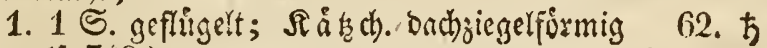
(1. 749.) Belüla.

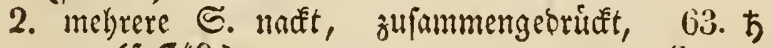
evr. (1. 740.)

Alnus.

D. mit Hülle, ohne Kätzch. und Bldecke.

Şitle 2 brattr., 1blith.; Stfrud t trocf.,

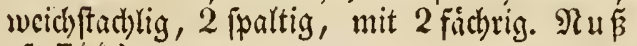
(1. 744.)

64.

Xanthŭım.

\section{DrDnung.}

D) $\mathfrak{E y} \quad \mathfrak{S} t \mathfrak{a} \mathfrak{u b w} \mathfrak{e} \mathfrak{c}$.

\section{Mit Blumenkrone.}

Blumenkrone:

1. 3 blättrig; Bldecke:

A. 1 blattr., 3 theil.; SEecre 3 fanm. mit. 65.

3 Inotch). D. ungleid)f. SBID. (โ. 796.) Tuniperus.

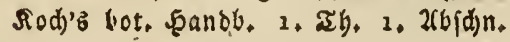


B. 3 blåttr., $2 \mathfrak{l b l . ~ z ̇ i g c t e i g t ; ~} 1$ G. 3 fant. 66. (1. 296.) Rumex.

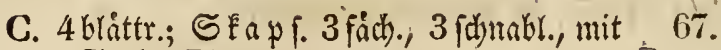
2 S. (i. $7+2$.$) Buxus.$

II. 5 blattrig;

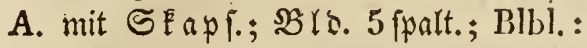

1. mit Någeln, obne Rrone an Sdhlund; SBld. aufgeblar.; Skap fel 3 fächerig (f. 358.)

68.

Cucubŭlus.

2. ohne Någel; mekrere SEap feln vicl $=69$. paamig, 2 jol)al. (f. 396.)

Spiráea.

B. mit troctin., 1 jaam. Stfrudst; $\mathfrak{B}$ Id. 70 . 5 theil. (f. 241.) Klius.

III. 12 od. 14 blattrig (F. vorber 49.) (Menispérmum.)

\section{II. oł̀ne Blumenkronē.}

Blumendecke:

A. 1 brattrig,

1. 2 zahn.: untere GEap 3 edf., 3 horn.; 71 . 1 fäh., vieljaam., offen (1. 794.) Datisca.

2. 3 theil.; Giriff. 2 fpalt.; Slap 1.3 fád).; 72. mit einzelnen S. (f. 771.) Ričnus.

3. 6 theil.; SEapl. 3 fáth., jofgedridt, mit, 73. 2 báut. S. (1. 786.) Dioscoréa.

4. gamjrandig (1. vorber 53.) (Quercus.)

B. 3 brättr.; Skapsel:

1. 1 fåch., in ter sitte runoum auffpring., 74 . mit 1 S. (i. 735.) Amaránthus.

2. 3 fåcherig, 3 fropf., mit cimjelnen $\Xi_{.} 75$. (โ. 769.) Ácalýpha.

C. 5 bláttrig; Skapsel:

1. 1 fách. ( $($. vorber $7 f$.)

2. 3 fich., mit 2 S. (1. 772.)

(Amaràn!hus.) 76.

Andráchne.

D. 6 blätrig; Seere 3 fácherig, mit $2 \mathrm{~S} .77$. (f. 787.)

Smilax. 


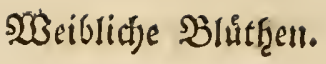

\section{Dronแng.}

Sier Staubwege.

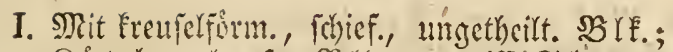

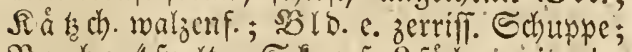

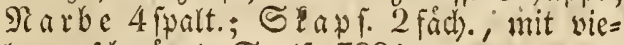
Icm gefit)wánjt. S. (†. 7S8.)

78.

Popŭlus.

II. obme $\mathfrak{B r}$; ; $\mathfrak{B}$ I

1. mit becect. S.: e. $\mathfrak{R} u \tilde{\beta}$ (F. vorber 53.) (Quercus.)

2. mit unbedecet., Saamen;

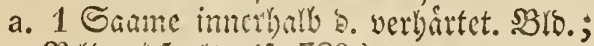
\$18. 4 palt. (i. 7S2.)
b. 4 Eamen (1. 729.)
Spinacra.
80. $\%$
Zannichellia.

\section{Drdnแกg.

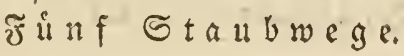

Blumenkrone:

I. 5 blåttrig; Bldecke:

1. 1 blättrig; Blblätter:

a. mit $\mathfrak{A} \mathfrak{a} g \mathfrak{l} \mathfrak{l}$ it. meift 2 fpart. Saum; Shlo. laingl., glatt; SEapf. 5 fách. S1.
(i. 365.)
Lychnis.

b. ohme Naged; 3 ro 5 f palt, ; uttere đernfrubt 5 fády: : Fádber 2 faamig S2. (i. 394.)

2. 5 bláttr.; $\$ 2$ lbl. 2 partig; Ełapier S3.

1 fåch., an D. Spiţe auffipring. (i. 369.) Cerastüum.

II. fehlt; bie 1 bráttr. Bldecke:

1. 5 thcilig,

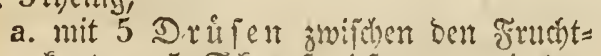
fnoten; 5 SEap 1 faam., mit sen

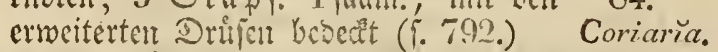

b. ohne Drifon; 5 Sfapforn 1 fam. S5. 万 (1. 783.) Zanthoxy̆lum.

2. ganzrandig ([. vorlyer 53.) (Quercus.) 万 


\section{Dionung.}

\section{Sed) Sta}

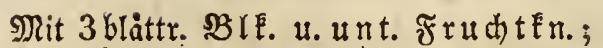

1. mit 2 blattr. $\bar{S}$ che ioe; $\mathbb{B}$ rbede 3 theil.; 20 ftaubbeutelf. Dect.; (S) riffel 2 theil.; Be ere 6 fádh., vielfanm. (i. 795.)

2. obne $S$ dhe ió; $S \backslash \delta$. 3 palt.; Skapl. 6 fád

86. + Stratiotes. IIydrochüris.

\section{Oroulng.}

xd t biz 3ehn Staubroege.

Blkrone:

1. 4 bláttr.; SBro. 4 fualt.; (S) riffel fehlt; SS. † 4 Skapl. 1 jam. (i. 756.)

2. 5 blattr., biredént.; $\mathfrak{B}$ Ib ede feblt; obere 89. Becre 10 fích., 10 janm. (f. 371.) Phytolácca.

\section{Dronung. \\ SE i}

I. Nit SRYer one; Blumendecke:

A. 1 blattr., 5 fualt.; BlE rone 3 blattr.;

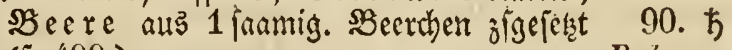
(1. 400.)

Rubus.

B. 3 bláttr.; $\mathfrak{B l E}$. 3 Glåttr.; viele nackte S. 91. † (1. 759.)

C. felylt; $\mathfrak{B}$ le. 4-5 blätr.; Saame : 1. geidhwängt (f. 436.)

2. nidht (i. 437.)

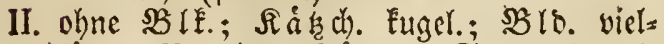
bláttr.; গ a rbe gefrimmt; S. runbl., mit (Sriff. Iharfgeipigt, am. Sirunde mit Elrone (1. 753.)

Sagritıarüa.

92.

Clemütis.

93.

Thalictrum.
94. 5

Platănus. 


\section{$\mathfrak{P} \in \mathfrak{g} i$ fiter.}

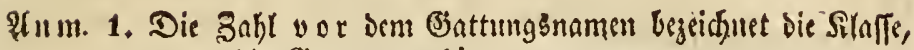
wozu bie Saattung gefórt;

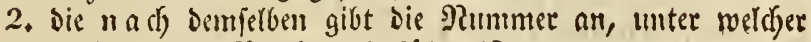
fie in Diefem 2iscrfe aufgefuifyrt iff;

3. bie biefer folgendeni umb eingeflammerten গinumern meifen auf bie Stellen bin, mo bir Battung

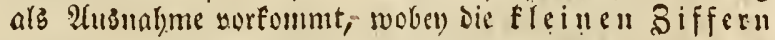
anzeigen, wie nalye ober feut fie ber angegebenen Rium= mex (teg)t;

1. Die Pinmmern endidg mit eincm W. begiegyen fid auf Dint ?tnlyang.

23. A càcìa 806. (415.)

21. Acalýpha 769. (W. 75.) 14. Acánthus 505.

19. Acárna 644.

23. Acer $805 .\left(315.788^{x}, 805^{2}\right)$

19. Achilléa 670 .

13. Aconitum 424 . $\left(417^{2}\right.$. $426^{2}$.)

13. Acta'ea 413 .

13. Adònis 433.

8. Adòxa 322. (366 2 )

7. Aescŭlus 303. (309 $8011^{2}$. $804^{x} .805^{x}$.)

5. Aethúsa 201 .

19. Agerătum 650.

12. Agrimonıัa $390,\left(319^{2}\right.$.)

10. Agrostémma 366.

23. Ailànthus 800 . (35. $52^{x}$. $800^{x}$.)

14. Ajŭga 456.
12. Aïzóon 399:

4. Alchemilla 79. (37.)

6. Alı̌sma 302.

6. Allıum 273.

21. Alnus 740. (W. 63.)

5. Alsíne 245. $\left(47^{2}\right)$

16. Altha'ea 549.

15. Alýssum 506.

21. Amaránthus 735. (744*. W. $74.75^{x}$.)

6. Amarýllis 268.

21. Ambroš̃a 743. (W. 35.)

2. Amethystéa 25.

5. Ammi 214.

17. Amórpha 562. (5434.)

5. Amsonĭa 134.

12. Amygdălus 388.

19. Anacý clus 654 .

5. Anagállis 111. $\left(540^{x}\right.$.)

10. Anagýris 336. 
14. Anarrhĭnum 498.

15. Anastatı̌ca 511.

5. Anchúsa 04.

21. Andrachne 772. (W. 76.)

10. Androměda $32 \%$.

5. Androsăce 109.

18. Andryăla 606.

13. Anemóne 442.

5. Angelǐca 226.

19. Anthĕmis 66 4. $\left(669^{x}\right.$.)

6. Anthericum 288.

5. Anthríscus 213.

17. Anthýllis 504. (5432.594.

14. Antirrhı̌num 497.

19. Aparğa 608.

5. Apı̌um 193.

5. Apocy̆num 132. (173 $3^{x}$ )

13. Aquileğ̃a 427.

15. Arăbis 530.

17. Arăchis 557. (5/12 ${ }^{3}$.)

5. Aralía 250.

10. Arbŭtus 329.

19. Arctıum 634.

19. Arctótis 702. (694. $707^{\mathrm{x}}$.)

10. Arenarı́a $360 \cdot\left(84^{3} \cdot 30^{3}\right.$ $321 \%$.)

13. Argemóné 418.

20. Aristolochǐa 723.

11. Aristotelǐa 376.

5. Armerı̃a 251.

19. Arnica 679.

19. Arnopógon 609.

5. Artedia. 222.

19. Artemisĩa. 655. $\left(662^{x}\right.$.)

21. Arum 761. $\left(723^{2} \cdot 79^{54}\right)$

11. Asărum 379.

5. Asclepras 174.

18. Asçy rum 599.

6. Asparăgus 286.

5. Asperùgo 103.

4. Asperula 67. $\left(37^{2}\right.$.)

6. Asphodelus 275.

19. Aster 683.

17. Astragălus 550 .
5. Astrantǐa 182.

5. Athamánta 205.

19. Athanaša 645 .

19. Atracty̆lis 631 .

13. Atragěne 434 .

23. Atriplex 802. (232.W.37.)

5. Atrópa 141.

21. Aucúba 736. (W. 13.)

21. Azýris 734. (W. 59.)

5. Azalěa 131.

19. Bacchruris 658.

14. Ballóta 476 .

19. Balsamita 651.

19. Baltimòra 705.

14. Bartsıa 483.

19. Bellis 689.

19. Bellium 677.

6. Berběris 265.

5. Beta 229.

14. Betonıca 460 .

21. Betüla 749. (W. 62.)

19. Bidens 632. $\left(645^{x} \cdot 65 x^{x}\right.$ )

14. Bignonı̆a 501. (50' ${ }^{x}$.)

15. Biscutélla 510.

17. Biserrǔla 581.

4. Blackburnua 71 .

1. Blitum 6.

19. Bocbéra 688.

19. Boltonı̆a 673.

5. Borágo 100 ,

19. Borckhausı̃a 622.

14. Brachystémum 461 .

15. Brassica 529.

20. Brotéra 714.

22. Broussonetra 778.

21. Bryonia 766. (799\%. W. 7.)

5. Bubon 209.

4. Buffonĩa 84.

6. Bulbíne 281 .

6. Bulbocodium 274. $\left(69^{x}\right.$.)

15. Bunı̌as $52 \%$.

5. Bunıım 206.

19 Buphthálmum 663. 
5. Buple'urum 183.

9. Butŏmus 327.

21. Buxus 742. (W. 67)

19. Cacalìa 652.

5. Cachrys 197.

15. Cakǐle 521.

2. Calceolarĭa 18.

19. Calendǔla 712.

7. Calla 305.

1. Callitrǔche 5. $\left({ }_{7} 38^{\mathbf{x}}: \mathrm{W}\right.$ : 57.)

8. Callùna 316.

13. Caltha 440.

12. Calycánthus 408.

5. Calystegìa 120.

15. Camelina 507.

5. Campanǔla 146.

3. Camphorósma 77.

22. Cannăbis 780. (W. 52.)

13. Cappăris 412.

5. Caprifolium 151.

15. Cardamine 538. $\left(72^{\mathrm{x}}\right.$.)

8. Cardiospérmum 321.

19. Cardǔus 639.

19. Carlína 635.

19. Carpesium 662.

21. Carpínus 74S. (W. 1.)

19. Cartlıămus 636.

5. Carum 186.

10. Cassia 339.

21. Casțanĕa 746. (W. 39 . $41^{\pi}$.)

2. Catálpa 22.

19. Catanánche 600 .

5. Caucălis 208.

21. Cauliñ̌a 726. (W. 4'i.)

6. Caulophýllum 266.

5. Ceanóthus 154.

5. Celástrus 161.

14. Gelša 492.

23. Celtis 803 . (234.)

19. Centauréa 695. $\left(632^{x}\right.$.)

1. Centránthus 1.

4. Centuncùlus 58.
4. Cephalánthus 57.

10. Cerastrum 369. (36ox. $370^{2} \cdot 79^{x}$. W. 83.)

21. Ceratacárpus 728. (W. 55.)

5. Ceratoceplıălus 259.

21. Ceratophýllum 75S. (W. 41.)

10. Cercis 337.

5. Cerinthe 92.

5. Chaeropliýllum 212.

21. Chara 727 . (W. 42.)

15. Cheirántlius 532.

13. Chelidonum 410.

14. Chelóne 502.

5. Chenopod̄̌n 238.

10. Cherlerı̌a 361.

2. Chionánthus 10:

8. Chilora 309.

19. Chondrílla 623.

19. Chrysanthĕnum 690. $\left(678^{x}\right.$.)

19. Chrysocóma 648 ;

10. Chrysosplenĭum 356 . (319.)

17. Cicer 582.

19. Cichorrum 616.

5. Cicúta 200.

13. Cimicifŭga 426.

19. Cineraria 685.4

2. Circá ea 31.

4. Cissus 75.

18. Citrus" 597.

5. Claytonǐa 162.

13. Clemătis $436 .\left(437^{x}: 79^{5}\right.$.

$$
\text { W. } 9^{2 .)}
$$

15. Cleŏme 527. $\left(262^{8} \cdot 37_{2^{23}}\right.$ )

14. Cleonı̌a 452.

10. Cletlira 344.

14. Clinopodium 477.

15. Clypeǒla 513.

19. Cuicus 637.

5. Cnidium 188.

15. Cochlearùa 508 .

6. Colchĭcum 297. 
2. Collinsonı̌a 29.

17. Colutĕa 578.

12. Comărum 406.

3. Commelina 39.

21. Comptonra 731. (W. 18.)

5. Conium 217.

6. Convallaria 276. $\left(7^{5}\right.$.)

5. Convolvǔlus 127.

19. Conýza 659.

19. Coreópsis 698.

5. Coriándrum 202.

22. Coriarı̌a 792. (W. 84.)

5. Coris $\mathbf{1 1 0 .}$

1. Corispérmum 7.

4. Cornus 73.

17. Coronílla 591.

5. Corrigiola 247.

5. Cortùsa 115.

17. Corydălis 554. (260x.)

21. Cory̆lus 752. (W. 51.)

19. Cosméa 697.

19. Cotǔla 656. $\left(673^{x}\right.$.)

10. Cotylédon 363.

15. Crámbe 523.

5. Crassǔla 252.

- 19. Crepis 620.

3. Crocus 42.

4. Crucianélla 76 .

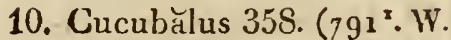
68.)

21. Cucŭmis 765. (W. 6.)

21. Cucurbita 764. W. 5.)

5. Cumínum 215.

11. Guphéa 377.

21. Cupréssus 767. (IV. 22.)

5. Cuscŭta 176. $\left(82^{3}\right.$.)

5. Cyclămen 116.

12. Cydonı̌a 395.

5. Cynánchum 173.

19. Cynăra 640.

5. Cynoglóssum 104.

20. Cypripedium 722.

17. Cysticápnos 555.

17. Cytǐsus 568.
16. Dalěa $543 .\left(540^{3}\right.$.

8. Daphne 317. (788². W.9.)

22. Datísca 794. (W. 71.).

5. Datúra 122.

5. Daucus 204.

12. Decumarĭa $387 .(372$.)

13. Delphinium 425 . ( $417^{x}$. $425^{x} .426^{x}$ )

15. Dentaria 533 .

10. Diánthus 348 .

10. Dictámnus 340 .

5. Diervílla 147.

14. Digitális 493.

22. Dioscoréa 786. (W. 73.)

23. Diuspýros 811. (307.)

4. Dipsăcus 54 .

14. Dodartǐ̀a 499.

5. Dodecathĕon 117.

17. Dolǐchos 571.

5. Dondia 180.

19. Doronǐcum 686.

17. DorycnǏum 590.

15. Draba 520.

14. Dracocephălum 449.

$$
\text { (46:) }
$$

5. Drosĕra 254.

12. Dryas 403.

5. Drypis 242.

5. $\mathrm{E}_{\text {chinophŭra } 179 .}$

19. Echínops 713.

5. Echítes 133.

5. Echrum 98.

4. Elaeágnus 81 .

8. Elatǐne 323.

5. Elliša 13S. $\left(126^{8}\right.$.)

14. Elsholž̆a 470.

22. Empĕtrum 774. (W. 12.)

22. Ephĕdra 798. (W. 56.).

10. Eviga' ea 332.

8. Epilobium 311.

4. Epimedrum 72 .

20. Epipàctis 720.

S. Eríca 308. 


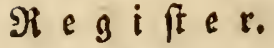

19. Erigĕron 681.

14. Erinus 504.

16. Erodium 541.

17. Ervilia 575.

17. Ervum 577.

5. Eryngĭum 178.

15. Erysimum 534:

5. Erythra' ea 114.

6. Erythronium 285.

19. Eupatorĭum $647 .\left(649^{7}.\right)$

11. Eupharbĭa 380. (33. $805^{4}$.)

14. Euphrası̆a 484.

5. Evony̆mus 163. (70x.)

4. Exàcum 60.

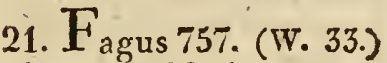

3. Fedĩa $36 .\left(3 \mathrm{o}^{4}\right.$.)

5. Ferŭla 221.

23. Ficus 8U8. (40.)

19. Filágo 703.

2. Fontanesı̌a 30.

21. Forskolěa 725. (W. 45.)

13. Fother gilla 423.

12. Fragaria 404.

6. Frankenı̌a 263.

23. Fraxǐnus S07. $\left(30^{2}, 33\right.$. $34^{x} \cdot 799^{3}$. W. $15,15^{x}, 41^{3}$.)

6. Fritilları̌a 283.

17. Fumarı̆a 553.

6. Galántlıus 270.

5. Galax 129.

17. Galéga 584 .

14. Galeobuculon 466.

14. Galeópsis 463.

19. Galinsogéa 1567.

3. Galium $65 .\left(37^{8} \cdot\right)$

10. Garidélla 362.

10. Gaultherřa 333.

8. Gaura 313.

17. Genísta 558 . $\left(542^{2}\right.$.)

5. Gentiána 177: $\left(57^{2} \cdot 82^{2}\right.$.)

19. Georgina 669.
16. Geranĭum 544. (346x . $367^{2}, 541^{x}$.)

19. Geropògon 604. $\left(616^{x}\right)$

13. Geum 407.

3. Gladiǒlus 44.

13. Glauč̆um 411.

5. Glaux 168 .

14. Glechóma 468.

23. Gleditschŭa 810 . (262 W. 17.)

11. Glinus 382.

4. Globularǐa 53.

17. Glycíne 569.

17. Glycyrrhiza 567.

19. Gnaphalıum 657, $\left(659^{\mathrm{x}}\right.$.)

16. GordonIa 546.

2. Gratiǒla 14.

22. Gymnocládus 791 .

19. Gymnostýles 710.

10. Gypsophìla 350. (W.48.)

11. $\mathrm{H}_{\text {alesı̆a } 372 .\left(544^{2} \text {.) }\right.}$

4. Hamamélis $\mathrm{S} 2$.

5. Hasselquistřa 195.

14. Hebenstreiť̌a $4 \mathrm{SO}$.

5. Hedĕra 165.

19. Hedypnơis 626.

17. Hedysărum 588.

19. Helenium 674.

14. Helianthĕmum 416.

19. Heliánthus 694.

15. Heliophǔla 539.

5. Heliotropı̌um 106.

13. Hëllebŏrus 438.

19. Helmintřa 629.

6. Helonǐas 298.

6. Hemerocállis 280.

13. Hepaťca 429.

5. Heracléum 225.

5. Herniarǐa $230 \cdot\left(82^{5} \cdot 354^{\mathrm{x}}\right.$.)

15. Hespěris 531.

19. Heterospérmum 687.

19. Heterotríchum 638.

16. Hibíscus 547.

19. Hieračum 614. 
17. Hippocrépis 593.

22. Hippophăe 776. (W. 27.)

1. Hippúris 3.

3. Holostěum 49.

14. Hormínum 454 .

5. Hottonia 118.

4. HoustonIa 63. $\left(62^{x}.\right)$

22. Humŭlus 781. (W. 54.)

6. Hyacinthus 277.

10. Hydrangěa 354. $\left(318^{2}\right.$.)

13. Hydrástis 435 .

22. Hydrochăris 790. (W. 87:)

5. Hydrocoty̆le 185.

5. Hydrophýllum 107 .

17. Hymenocárpus 586.

5. Hyoscyămus 123.

19. Hyosĕris 625.

4. Hypecŏun 83. $\left(84^{x}\right.$.)

18. Hypeřcum 598.

18. Hypocho'eris 602.

14. Hyssópus 464.

5. Jasióne 164.

2. Jasmínum 13.

15. Ibèris 512.

4. Ilex 85 .

5. Illecěbrum 169.

5. Impatǐens 159.

5. Imperatorĭa 224.

17. Indigofĕra $5 S 7$.

19. Inǔla $6 \mathrm{SO}$.

5. Ipòmó ea 126.

3. Iris 45 .

15. Isătis 515 .

4. Isnardîa 80 .

13. Isopýrum 439: ( $422^{x}$ : $424^{x}$.)

5. Itěa 160

21. Iuglans 751. (W. 2.)

22. Junipĕrus 796. (W, 65.)

2. Iusticǐa 16.

19. Iva $70 \%$.

3. Ixìa 43.

10. Kalmù 331 .
16. Kitaibelǐa 550 .

4. Knautǐa 55 .

5. Kochǐa.237.

8. Koelreuterı̆a 310 .

19. Kriǧa 612.

19. Kuhnǐa 649.

6. Iuachenalǐa 290.

19. Lactúca 613.

5. Lagoecĭa 167.

19. Lagosĕris 621.

14. Lamıum 462.

19. Lapsăna 627.

5. Laserpitrum 223.

14. Latlira' ea 481 .

17. Lathy̆rus 572 ,

9. Laurus 325. $\left(275^{x}: 788 .^{3 . \uparrow}\right.$ $79^{5}$. (WV: 11.)

14. Lavandǔla 444 .

16. Lavatúra 548.

10. Ledum $3+1$.

3. Lemña 34., (73o. W. 32.)

19. Leontǒdon 630.

14. Leonúrus 459.

14. Lepechínı̌a 453.

15. Lepidĭnฺm 514. (3o $32^{2}$ : $39^{3} \cdot 262^{2}$ )

6. Leucójum 271.

19. Liátris 642 .

5. Ligustı̌cım 218.

2. Ligústrum 11.

b. Lilium 282.

20. Limódórum 715.

14. Limosélla $4 \mathrm{SS}$.

14. Linarĭa 496.

14. Lindernǐa 489 .

14. Linná ea 479 .

6. Linum 256. $\left(88^{x}, 541^{2}\right)$

21. Liquidámbar 754.(W.6o.)

16. Liriodéndrum 430.

5. Lithospérmum 99.

21. Littorélla 739. (W. 1o.)

5. Lobelia 148.

3. Loeflingľa 38.

5. Lonicěra 152. 
22. Loránthus 784. (W. 19.)

17. Lotus 589.

15. Lunarı̆a 516 ,

17. Lupínus 559. (5423.)

10. Lychnis 365 . (358 $.362^{4}$ : $791^{2}$. W. 81.)

5. Lycium 143.

5. Lycópsis 93.

2. Lycupus 23.

5. Lysimachía 112. $\left(54 \mathrm{o}^{2}\right.$.)

11. Lythrum 378. $\left(30^{x} \cdot 263^{x}\right.$.)

19. $\mathrm{M}_{\text {a đ̆a }} 602$.

13. Magnolìa 431.

20. Maláxis 721 .

16. Malǒpe 552.

16. Malva 551.

5. Mandragora 142.

14. Manuléa 500.

14. Marrubium 473.

19. Matricarı́a 691.

17. Medicágo 585.

19. Melampodium 699.

14. Melampýrum 486.

17. Melilótus 596.

14. Melíssa 451.

14. Melíttis 450.

22. Menispérmum 793. $\left(79^{3}\right.$. W. $49 \cdot 70^{x}$.)

14. Mentha 472 .

5. Menyánthes 119 .

22. Mercuriális 789 . ( $755 \mathrm{x}$ : $79^{3 x}$. W. 5o.)

12. Mesembryanthěrnum 397. (3925. $399^{x}$. 401.)

12. Mespilus 393. $\left(384^{x} \cdot 390^{x}\right.$. 5. Meum 190.

19. Micrŏpus 706.

5. Microtéa 233.

14. Mimŭlus 494 .

3. Minuartǐa 51.

4. Mitchélla 62.

10. Mitélla 351.

8. Moehrinğّa 319.
3. Mollúgo 52.

14. Moluccélla 474.

21. Momordica 762. (W. 3.)

2. Monárdá 24. $\left(463^{x}\right.$.)

10. Monotrŏpa 347. (318x.)

3. Montı̌a 47.

3. Moráea 46.!

2. Morína 32,

21. Morus741. (779. W.58.)

6. Muscári 278.

15. Myágrum 519.

5. Myosótis 101.

5. Myosúrus 258.

22. Myríca 779. (W. 61.)

21. Myriophy'llum 756. (322: W. 88.)

5. Myrrhis 211.

12. Myrtus 385.

21. Najas 737. (W. $43.44^{x}$.)

6. Narcíssus 267.

20. Neottı̌ 719.

14. Nepěta 465.

5. Neruum 135.

5. Nicutiána 124.

12. Nigélla 42S. ( $438^{x}$.)

11. Nitrarı̆a 374.

13. Nympháea 421 .

23. Nyssa 809. (171.)

14. Ocy̆mum 455.

5. Odontités 184.

5. Oenánthe 191.

8. Oenothéra 312.

2. Olěa 5 .

17. Onónis 563. $\left(543^{x}\right.$.)

19. Onopórdum $6 \pm 1$.

5. Onósma 97.

20. Ophrys 717,

20. Orchis 716 .

14. Origănum 478.

6. Ornithogălum 289.

17. Ornithopus 592.

12. Orobánche 482 . (488 $\mathrm{x}$.)

17. Orŏbus 574. 
19. Osteospérmum 711.

21. Ostry̆a 750. (W. 24.)

22. Osýris 775. (W. 28.)

19. Othónna 709. $\left(710^{x}.\right)$

11. Oxălis 368 . (544 $4^{x}$ )

2. Paederóta 19.

13. Paeonǐa 422. ( $423^{2}$.)

5. Paliúrus: 156.

6. Pancratı̌um 269.

13. Papáver 409.

23. Parietarı̌a 801. (78.)

8. Paris 324. (324.)

5. Parnasša 249.

19. ParthenIum 704.

5. Pastináca 227.

14. Pediculáris 495.

10. Pegănum $375 .\left(376^{x}\right.$.)

16. Pelargonum 542. (3o3 . $540^{4}$.)

15. Peltarịa 524.

10. Penthŏrum 367. $\left(367^{\mathrm{x}}\right.$.)

14. Pentstémon 503.

6. Peplis 264. $\left(292^{\mathrm{x}}\right.$.)

5. Periplóca 172.

5. Peucedănum 220.

17. Phaca 583.

5. Pharnacěum 248.

17. Phaseǒlus 566.

12. Philadélphus 384. (386.)

2. Phillyrěa 9 .

14. Phlonis 475.

5. Phlox 128.

5. Physălis 140.

5. Phyte'uma 145.

10. Phytolàcca 371 . ( $324^{2}$. $370^{x} \cdot 408^{x} \cdot 79^{2}$. W. 89.)

5. Picotı̌a 105 ,

19. Picris 619 .

5. Pimpinélla 192.

2. Pinguicùla 20 .

21. Pinus 770. (W. 26.)

17. Pisum 573.

5. Planéra 231.

4. Plantágo 59.
21. Platănus 753. (W. 94.)

5. Pleurospérmum 196.

5. Plumbàgo 91.

10. Podalyrĩa 335.

13. Podophýllum 420.

5. Polemonium 130.

6. Polyánthes 279 .

3. Polycárpon 48.

3. Polycnémum 41 ,

17. Polygăla 556. $\left(542^{x}\right.$.)

8. Polygŏnum 320. $\left(27^{4^{x}}\right.$, $22^{3} \cdot 305^{x} \cdot 362^{x}$.)

19. Polymnı 707.

22. Popǔlus 788. (W. 78.)

11. Portuláca 373 . $\left(262^{3}\right.$. $366^{2} \cdot 381^{3}$.)

4. Potamogéton 89.

12. Potentilla 405.

21. Poterıum 760. (W. 47.)

19. Prenánthes 624.

5. Primŭla 108.

6. Prinos 260. $\left(3 \circ 3^{x}, 3 \circ 7^{x}\right.$.)

14. Prunélla 447.

12. Prunus 389.

4. Ptelěa 70. $\left(777^{2} \cdot 800^{2}\right.$. W. 14.)

5. Pulmónařa 96.

13. Pulsatílla 441,

12. Punı̌ca 386.

19. Pỳrĕthrum 678."

10. Pyrŏla 345. $\left(346^{2}\right)$

12. Pyrus 394. ( $79^{5^{2}}$ W. 82 .)

21. Quercus 745. (W. $53.7^{7 x}$. $7^{x} \cdot 85^{x}$.)

3. Querra 50 .

4. Radioula 57:

5. Ramondra 121:

13. Ranuncǔlus 432 .

15. Raphănus 526.

11. Reséda 381. $\left(358^{2}, 362^{3}\right.$. $381^{x} \cdot 382^{2} \cdot 423^{x}$.)

19. Rhagadiǒlus 628 . 
5. Rhamnus 153. $\left(72^{2} \cdot 7770^{x}\right.$

9. Rheum 326 .

14. Rhinánthus 485.

9. Rhododéndrum 330.

10. Rhodóra 338.

5. Rhus 241. (781. ${ }^{x}$ W. 70.)

5. Ribes 166. $\left(779^{3}, W_{0}\right.$ 16.)

21. Ričnus 771. (799 ${ }^{\circ} \cdot \mathrm{W} \cdot 7^{2}$ )

15. Ricotřa 52S。

17. Robiň̆a 579.

5. Rochelía 102.

12. Rosa 401.

2. Rosmarínus 27.

19. Rothǐa 605.

4. Rubĩa 64. (148 ${ }^{ \pm}$)

12. Rubus 400 . ( $795 \%$. W. go.)

19. Rudbecǩa 693. (694 $\left.4^{2}\right)$

6. Pumex 296. $\left(29^{2} \cdot 744^{2}\right.$. $785^{x}$. W. 66.)

4. Ruppǐa 90.

22. Ruscus 797. (W. 40.)

10. Ruta 343. (312 $\left.{ }^{x}\right)$

4. Sagína SS.

21. Sagittarı̌a 759. (W. '91.)

1. Salicornǐa 2. $\left(32^{x}\right.$.)

21. Salisburýa 747. (W. 34.)

22. Salix 773. (34'.773'.

5. Salsóla 236.

$$
779^{2} \text {. W. 21.) }
$$

2. Salvǐa 2S. $\left(3 k^{2}\right.$.)

5. Sambúcus 240.

5. Samǒlus 144.

13. Sanguinarı̆a 419.

4. Sanguisórba 61 .

5. Sanicǔla 181.

19. Santolina 646 .

19. Sanvitalı̌a 666.

10. Saponařa 349.

13. Sarracenia 417 .

14. Saturéja 458.

10. Saxifrăga 333.

4. Scabiósa 56.

5. Scandix 210 .
6. Scheuchzeria 300 .

19. Schkuhrǐa 670.

6. Scilla 287.

10. Scleránthus 355 .

19. Scoly̆mus 603.

17. Scorpiúrus 594.

19. Scorzonéra 611.

14. Scrophularĭa 491.

14. Scutellarǐa 445.

10. Sedum 364. ( $382^{x}$.)

5. Selínum 219.

11. Sempervívum 383. $\left(382^{3}\right)$

19. Senecĭo 660 . $\left(686^{x}\right.$.)

15. Sennebiéra 525 .

20. Serapǔas 718.

19. Serióla 601 .

19. Serratŭla 633. $\left(637^{x}\right.$.

$$
\left.639^{\prime} .\right)
$$

5. Sesěli 189 .

4. Sherardĩa 68.

5. Sibbaldía 255.

14. Sibthorpla 490.

21. Sic y̆os 763. (W. 4.) ?

16. Sida 545. ( $79^{8^{x}}$.)

14. Siderítis 469 .

19. Siegesbeckı̌a 668 .

10. Siléne 357. $\left(84^{2}, 3052\right.$. $\left.321^{x}, 362^{2}, 805^{3}.\right)$

5. Siler 203.

19. Silphium 701.

15. Sinápis 536.

5. Sison 187.

15. Sisymbrūum 537.

16. Sisyrinchǐum 540 . ( 46 .)

5. Sium 216.

22. Smilax 787. (W. 77.)

5. Smyrnĭum 199.

5. Solánum 139.

5. Soldanélla 113.

19. Solidágo 682 .

19. Sonchus. 615.

10. Sophǒra 334.

12. Sorbus 392. $\left(3 \mathrm{q}^{3}{ }^{3}\right.$.)

21. Sparganı̆um 733. (545. 
17. Spartiánthus 561 . $\left(542^{5}\right.$.)

17. Spartǐum 560 . $\left(542^{4}.\right)$

10. Spergǔla $370 .\left(3 \circ 5^{4}\right.$.)

4. Spermacóce 69.

22. Spinacĭa 782 . (W. 79.)

12. Spira' ea 396. $\left(3 \mathrm{g2}^{2} .401^{t}\right.$.

14. Stachys 467 :

$$
79^{5} \text {. W. } 69 \text {.) }
$$

5. Staphyléa 243.

5. Statice 253.

10. Stellarřa 359.

8. Stellèra 318.

6. Sternbergìa 272.

22. Stratiótes 795. (W. 86.)

15. Subuları̆a 517.

5. Swertı̌a 175. $\left(57^{\mathrm{x}} \cdot 82^{\mp}\right.$ )

5. Symphoricárpos 150 .

5. Symphy̆tum 95.

2. Syrínga 12.

5. Tabernaemontána 136.

19. Tagétes 675 .

5. Tamărix $244 .\left(358^{3}\right.$.)

22. Tamus 785. (W. 3o.)

19. Tanacétum 661.

22. Taxus 799. (W. 46.)

5. Telephrum 246.

12. Tetragonǐa 398. $\left(392^{\circ}\right.$.)

19. Tetragonothéca 671.

14.' Teucrum 457.

13. Thalictrum 437 . $\left(382^{4}\right.$.

5. Thapš̆a 228. $437^{2} \cdot 79^{57}$. W. $9^{3}$.)

21. Thelygŏnum 755. (W.31.)

5. Thesium 170. $\left(40^{x} \cdot 81^{x}\right.$.)

15. Thlaspi 509.

19. Thrinča 607.

21. Thuja 768. (W. 23.)

14. Thymus 446 .

5. Thysselinum 194.

10. Tiarélla 352.

3. Tiľa 41 .

4. Tilla' ea $86 \cdot\left(47^{\mathrm{T}}\right.$.)

6. Tolieldǐa 293.

19. Tolpis 617.
5. Tordylumm 198.

5. Torîlis 207.

12. Tormentílla 402 . $\left(381^{2}\right.$.)

14. Tozzı̃a 487.

6. Tradescantřa 261.

19. Tragopógon 610.

4. Trapa 74.

10. Tribǔlus 342.

14. Trichostémma $44 \mathrm{~S}$.

7. Trientális 304.

17. Trifolium 595.

6. Triglóchin 295.

17. Trigonélla 570.

6. Trillium 294.

5. Triostěum 149.

13. Trollius 443.

S. Tropaeŏlum 314.

6. Tulipa 291.

15. Turritis 535.

19. Tussílágo 684.

21. T-ypha 732. (W. 25.)

17. Ulex 565. (543.3.)

5. Ulmus 235. $\left(82^{4} .319^{3}\right.$ )

21. Urtíca 738 . $\left(730^{x} \cdot 77^{1}\right.$.) w. 36.)

2. Utricularìa 21.

6. Uvuları̌a 284.

8. $V$ accinıum 306. (327\%.)

4. Vaillantra 66.

3. Valeriána 37. $\left(566^{1} \cdot 775^{2}\right.$. w. 8.)

15. Vella 518.

23. Verátrum 804. (301.)

5. Verbáscum 125.

14. Verbéna 471. $\left(62^{2}\right.$.)

19. Vernonıa 643.

2. Veronica 15.

5. Vibúrnum 239.

17. Vicı́a 576.

5. Vinca 137.

5. Viüla 158.

22. Viscum 777. (W, 38.)

5. Vitis 155. 


\begin{tabular}{|c|c|}
\hline W $V_{\text {aldsteinřa }} 391$ & 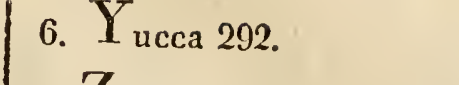 \\
\hline $\begin{array}{l}\text { 19. Wedelia } 700 . \\
\text { 2. Wulfenía } 17 \text {. }\end{array}$ & $\begin{array}{l}\text { 19. Zacintha } 618 . \\
\text { 21. Zannichellǐ 729. (W. } 80 \text { ) } \\
\text { 22. Zanthoxylum } 783 \text {. }\end{array}$ \\
\hline 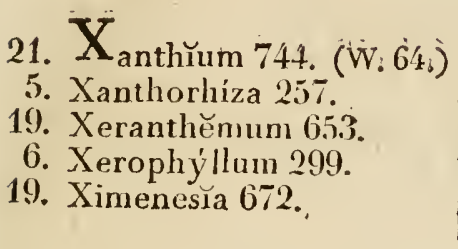 & 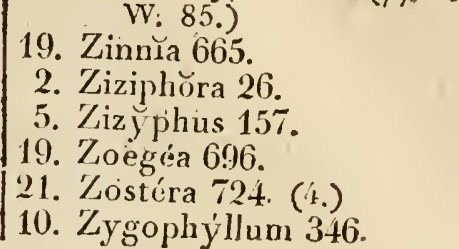 \\
\hline
\end{tabular}





\section{$\mathfrak{B}$ ot $\mathfrak{a} \mathfrak{n} \mathfrak{i}$ 无 $\mathfrak{e} \mathfrak{B}$}

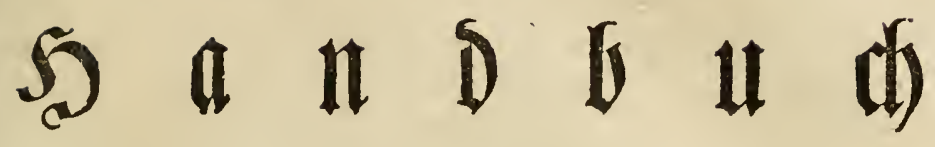
zut $\mathfrak{m}$

Selbfuntertidt

fix

Deutfibe Riebraber der $\mathfrak{P}$ flansenetunde

$$
\begin{gathered}
\dot{a} b \in c b a u p t, \\
\text { und fur }
\end{gathered}
$$

(S) $\mathfrak{a} \mathfrak{i} \mathfrak{e} \mathfrak{n} \mathfrak{f} \mathfrak{r} \mathfrak{u} \mathfrak{n} \mathfrak{D} \mathfrak{e}$

Apothefer, Defonomen und Forftainner ins $\mathfrak{b} e$ ionbere,

$$
\text { entworfen }
$$

oun

Soh. Fr. 2Bilh. SOळ,

confiftorial: und Soldrath in Magocburg.

\section{Errfiter Theil.}

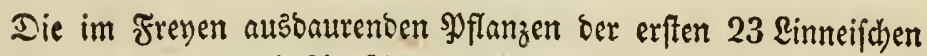

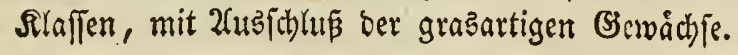

\section{Bwevter 2 bfdith}

Die $\mathfrak{A} \mathfrak{r} \mathfrak{e}$.

Dritte ganz ungearbeitete 2fuflage.

$\mathfrak{M a} \mathfrak{g} \mathfrak{b} \mathfrak{b} \mathfrak{u} \mathfrak{r} g$ ven Birgerm beinridb̧ofen. 1824. 


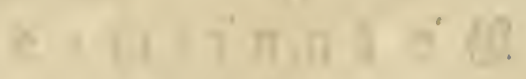

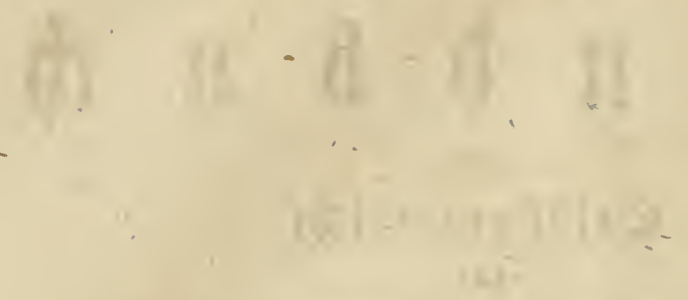

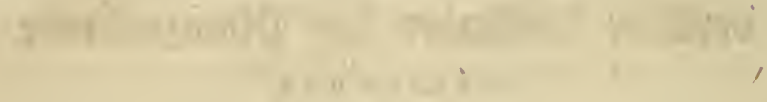

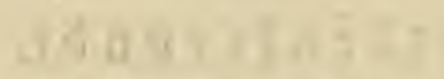

.

$$
\text { , }
$$

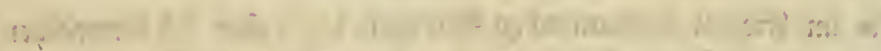

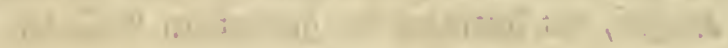

$$
\rightarrow+x+2+10
$$

$-$

$$
+1
$$

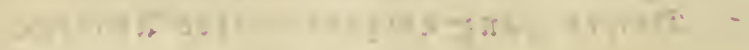
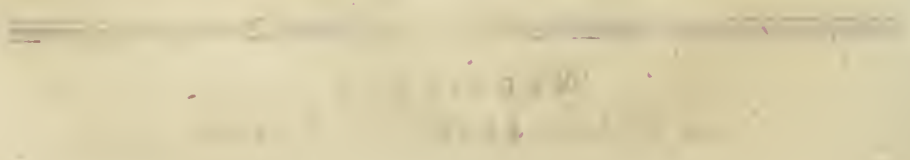


\section{$23 \circ \mathfrak{r}$ : $\mathfrak{x}$.}

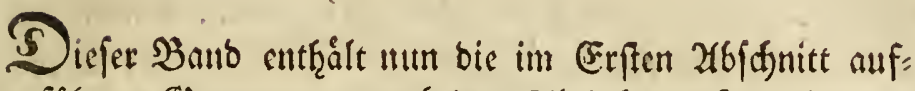

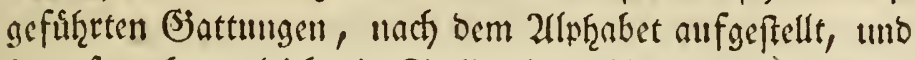
funn fontach) Jugleith bie Stelle cimes biegifferz vertreter. Die neben den (Jattungstnamen befinblichen 3aḩlen ḩaben

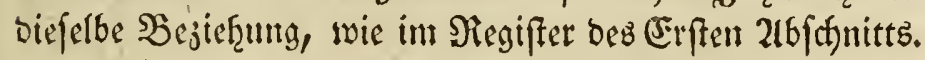

Die 2urten jeder Gjattung, fo weit fie entweder in

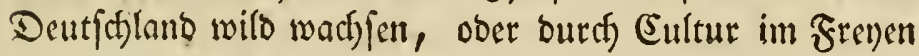
getricben angetroffen werben, fint in tabellarif der Form aufgeftellt und ganj nach bemiflben Yुlane, ber bey ben Gattungent befolgt ift.

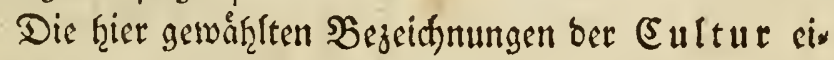
ner jeben 'art, ifrer $\mathfrak{D}$ a uer, ifres $\mathfrak{B}$ aterlandes unv

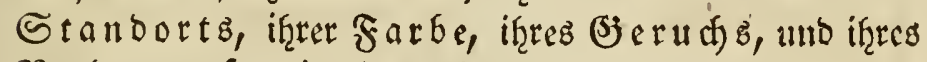

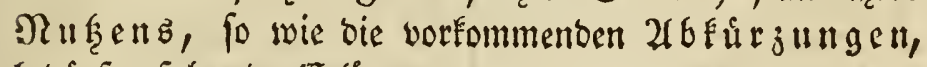
bedurfen folgente Erlánterung.

SWas zuerft ihre Eult ur betrifft, fo finbet babel) cine brenfact) $\mathfrak{B}$ ezeidfunth Statt:

1. Die mit geraber uno gróferer Sctrift georudé ten Namen beuten bicjenigen 2 rten an, weld)e it

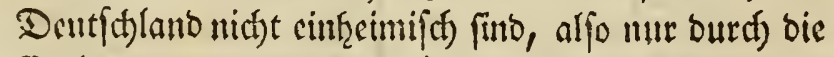
Eultur erjengt werben foumen; bagegen

2. Die mit $\{\mathfrak{d}) \mathfrak{r a ̊ g} \mathfrak{g} \mathfrak{r}$. Fleillerer Sdfrift foldfe, bie in Deutfalano wilowadfen, uno went bieje

3. jugleith eingeflammmert fint, foldhe, bie in ber

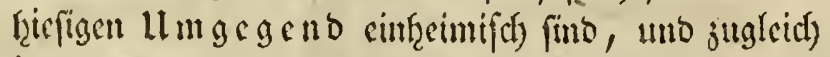

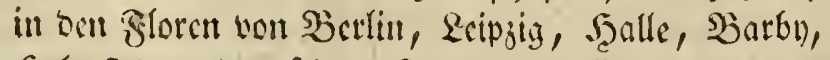
Scelmfted unt auf ben Sear angetroffen werben. 


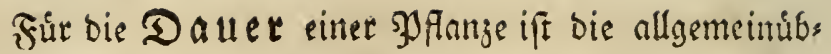

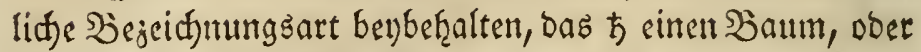

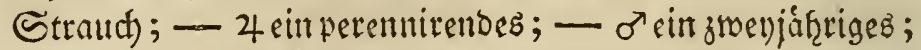

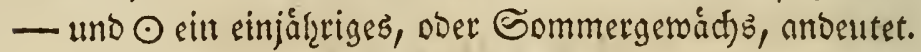

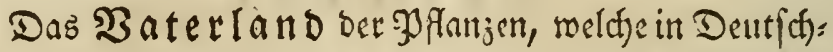
lanb nicht wild madyfen, ift anf eine leicht verftánolicje Weife angegeben, Dã E., As., Af., Am., Austr. Die 5 Erotfecile uno sin vorgefef̧tes $S, N, W, O$ die 2 elts gegend anzeigen; alfo j. 2j. SE. Sứb = Europa :c.

Der Standort D. h. berjenige B̧oben, morauf eine Yy/anze millowadjfent angetroffen wird, ber alfo nuts

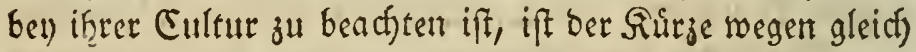
nact Dem Beicjen ber Dauter burdh Biffern bemerkt, beren Sbedeutung aus folgender Tafel erfictitich ift:
1. idattige Derter
2. Connige Derter
3. SBiejen
4. Misald
5. Buich
6. Şigel
7. ร̌u⿰ ber Berge
S. SBerge
9. 2llpen
10. bóchjte 2erpen
11. trodure Derter
12. futchtbare Eroe
13. unfructitbare Sseiden
14. Ianbreege
15. 2féperrånder
16. Salzwerfe
17. Falzige Derter
18. Nieșiano
19. Feinerer Sand
20. Fllugiano
21. fteinigter $\Re$ oben 22. Schutthaufen uns unge=
baucte Erbe
23. Felien
26. 3̊ăme
27. Strapen
28. Srachäd
29. gebaute Frbe
30. Saaten
31. 2 scinberge
32. Sartenland
33. (3razboden
34. 23 eiden, Triften
35. Meerwajier
36. Meerftrand
37. Flưfle

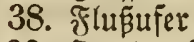
39. Suellwafier
40. Duelfen
41. SBäche
42. Sumpfwatier
43. Sumpfufer
44. ftehendes গুanafier
45. $23 a$ fifergráben
46. Torfboden
47. jumpfige SBrúd)e

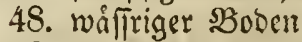
49. etwas fenchter Boben
50. úberid) wemmte Serter
24. NRuren
25. Deicher
51. Sd)marof̧erpflanze. 


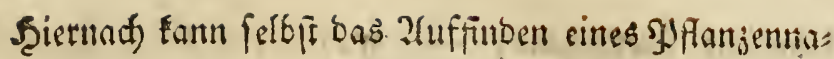
ments erleidfert werben, z. S5. wenn man fie alf fenthten Soben, oder im $\mathfrak{B a f f e r}$, gefunden hat, fo mun fie eine Der Nummern won 35 bis 50 tragen.

Die Farbe einer Sblume ift jwar weder fo beffin:

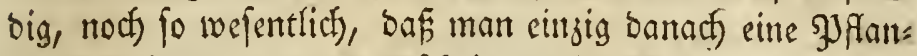
jenart erfenten uno unterf fjeiben burffte, indem Eultur,

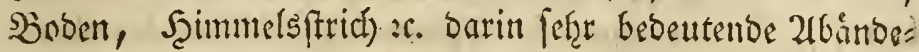
rungen berwirfen; aber wenigftens als benlánfiges, oder bes ftátigendes, Miermal verbiente fie bemerft ztl werben. Die Sejeidfnung ber Farbe gilt immer mur fur bie Blume felbft; too bie Farbe ber Sjlumendecte, Der.jbläter :c. ange= geben werben follte, find biefe ausoructiclif genannt morden.

Die Beichen fur die Farbe fint:

$$
\begin{array}{l|l}
\text { bl. - blau } & \text { r. - roth } \\
\text { brn- braun } & \text { sch. - icharlactroth } \\
\text { H. - fleirchfarbig } & \text { schw. ichward } \\
\text { gb. - gelb } & \text { v. - violett } \\
\text { grn - grün } & \text { w. - weis. } \\
\text { p. - purpurroth } &
\end{array}
$$

Das biejen Zeichen angef̨ång te ch bebeutet immer

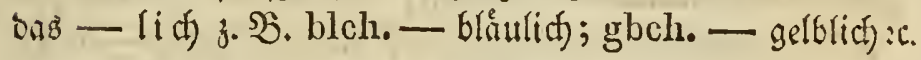

Das bemelfen vorgeferte

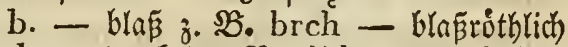

d. - bunEel z. SB. dbl. - DunEerbtau

h. - belt z. 32. hbl. - bethlat

u. - unreiil z. B. uw. - unreinmeip.

Nod) ift in biefer Seinficht zu bemerken, ba ấ alle Sarbenbeftimmungen, welche burch ein Semicolon getrenut fitto, Barietaten in 2lofitft ber Farbe bescidjnen; - finto Tie buted eimen fleinen Sonerfitrify verbunden, fo fpielt bie eine Farbe in bie andere; - fint fie endich bur(t) ein 11. (unt) verbunten, fo tragen fie bie genannten $\mathfrak{F a r b e n}$ an verffhiebenen Sheilen ber Şlutţe. 3. B. w.; r.; bl. b.

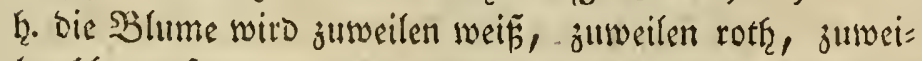
len blau gefunben; - hr. •blch. o. Ģ. Gelltroth, aber ins 


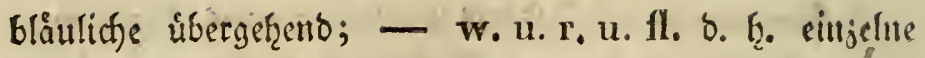

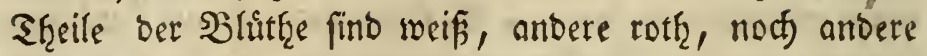
Fleifd)farbig.

Eeben fo iff hie uno ba ber Ģer SBlåtter :c. auf eine 2 Int angebentet, weldbe feiner Ertáu: terung bedarf.

23as ben $\Re$ u betrifft, fo fint in biefer Seinfid)t viererlen Beichen jubeathten:

1. Die in Den 2 potheFen gebraudtent, doer offici= nellen, כJ fanjen ḩaben vor ihrem Namen ein*, wel des in ber Tabelle wiederţolt ift unto baneben fint biejes

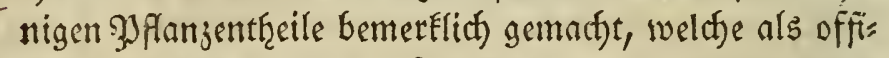

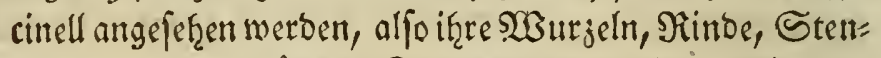
gel, Zweige, Şlâtter, Fnospen, Sproffer, B̉lumen, Saamen uno Saamenfapfeln, Fruthte, Safte, Scarj :c.

2. Die ben Sefonomen intereffirenden fino mit cinem

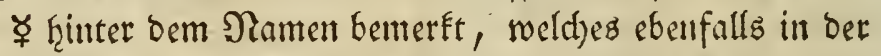

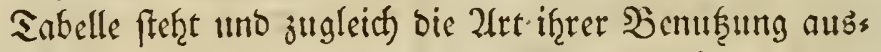

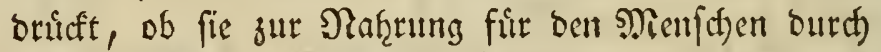
if̧ren mef̧lgebenden Enamen,' SWurzeln :c., Doer als

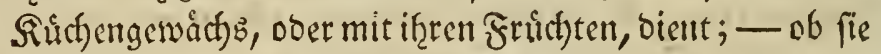

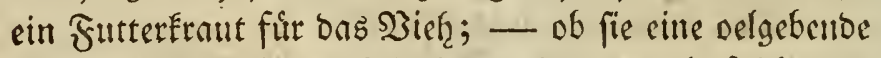
PHanje, oder cime Fabrifpllange ift unt als foldse ben Weberenen - ben Fårberenen, (mit Bemerfung ber Farbe, weldse fie giebt), - ooer ben (Sierberenen, bient; - ob fie endict) als Unfraut dem \&anb= unt Sjartent= bau frimberlict) ift.

3. Die bem Forftmann náf̧er angȩ́entoen 2leten fitto mef̧r ober mentiger alle biejenigen, Deren Damer burd) ein $ち$ bejeicfunet ift.

4. (Ein + vor bem Samen bentet sine giftige, docr bod) verbid tige, 3Planje an.

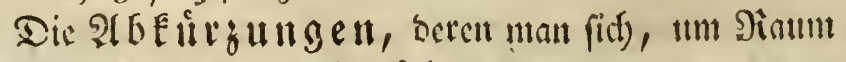
3u faren, besicut bat, fint folgende: 


\begin{tabular}{|c|c|}
\hline 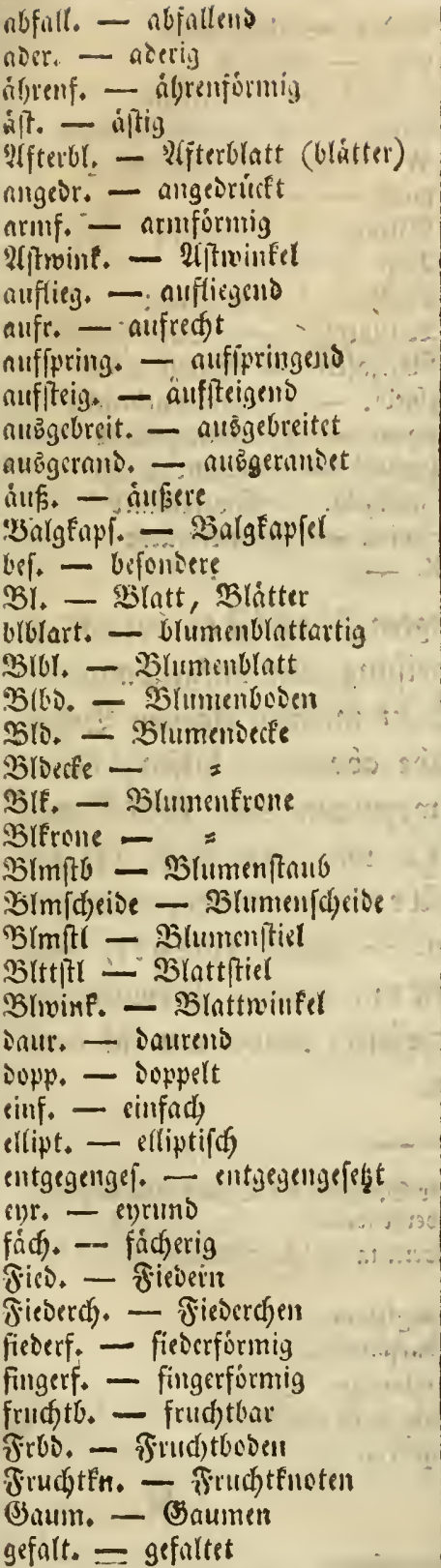 & 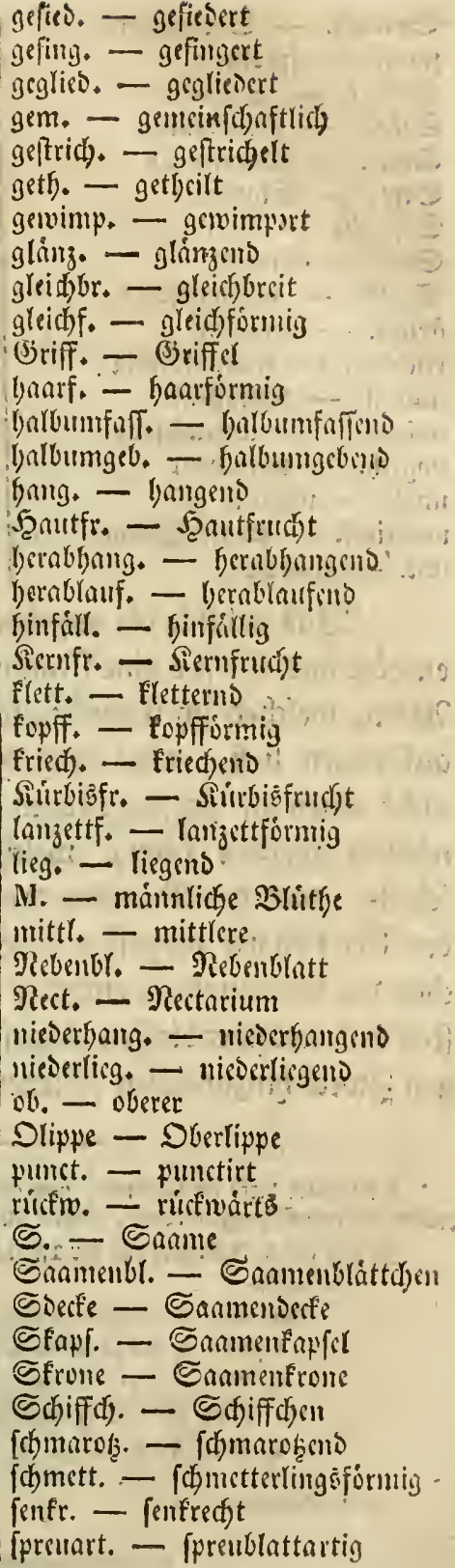 \\
\hline
\end{tabular}




\section{VIII}

Eprobr. - Eprcublitter fproff. - fprofferio Stbeut. - Stanbboutul Stfao. - Stanbfasir Stger. - Etauligefáf Stm. - Strubwer ङtfrudbt - Etrinfuctit Stgl - Etcitgel Stgrbr. - Stengerbratt ftricgl. - ftrieglidet trichterf. - trichterformig amfant. - Imfanterto unfruchto. - mifrudgtbar ungeth). - umgetheilt ungl. - tungleich ungleichf. - ungleidformig unt. - unterer

\begin{tabular}{|c|}
\hline 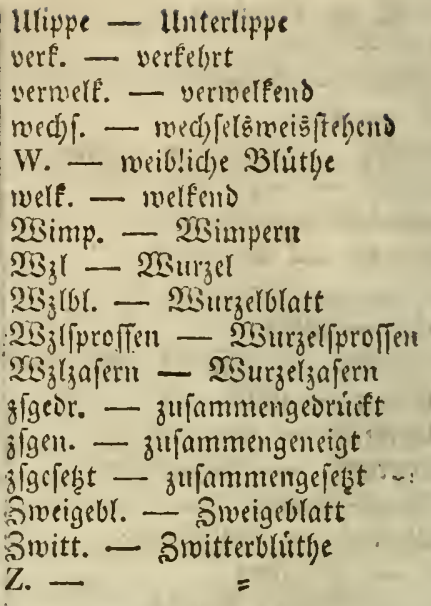 \\
\hline
\end{tabular}

lllippe - Lnterlippe

bertegrt vermelte. - vermelfend

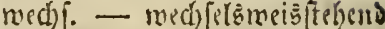
W. - meib!id)e 3rútbe wele. - welfento WBimp. - WBinpern $\mathfrak{N B}_{3} 1-\mathfrak{E}_{4}$

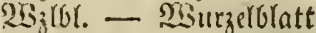
23 jrproften - NGutzerproflen

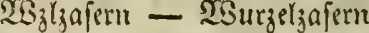
sigeor. - zu[ammengeonifft j/gen. - zujammengeneigt jigcest - zujammengejest in: Ŝneigebr. - Ŝweigeblatt Switt. - వ̂mitterblittbe

MBns enblich bie angehángten K egiffer betrifft, fo

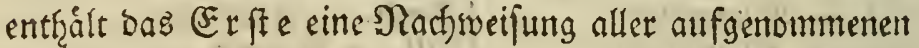

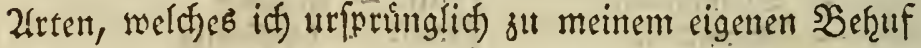
anfertigen lief, aber bod) habe aboruden laffen, ba es nicht einen' zu groffen Siaum wegnimmt uno bod Denen núf̨lich werben tann,.. Weldfe, wie das woht oft ge=

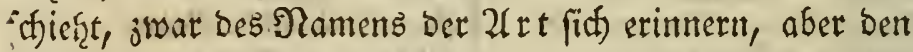
Der Siattung vergeffen Faben. - Das 3 weyte, wel= d)es bie Synonymen entfielt, if nur auf bie allerbornefrm= ften und notfrwertbigften eingefdránft, meld)e mir fúr meis nen Broef fïinzureidgen fdietten.

Salieplid) bitte id foigende, bein aller Sorgfalt bes Sesers und ber (5or: rectur bennod) fteלengebliebene, Frebler zu berbeflern:

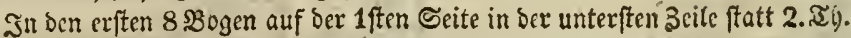

- I. 1. Th.

Sir. 829. fratt agittata lies sagittata.

» 893 ft. carphatica l. carpathica.

2) 1268 ft. tărtaricus $l$. tataricus.

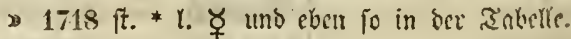

() 2595 ft. spurium l. spuria.

凡. 


\section{ACACIA. 806. (415.)}

bne Dornen; SB !. Dopp. gefico.: bef. Fie = Dern 9-11 paar.: SBlàttu). vielpaar., am (5iunbe ungl., am Enoe fpis; $B$ lit th. in $\mathrm{gc}$ báuft., faft fugel. Enoábren: Şitlien flach, båut., glatt ( $ち$ As. rch. $\Delta$ )

1.

Julibrǔssin.

\section{ACALÝPHA. 769. (W. 75.)}

W. SBluth. am Sirunde o. M. 2lebre; die gejahnt. Hiillen :

1. evr., langgeipitzt; $\mathfrak{B l}$ l. långl. = lanjettfơrm., 2.

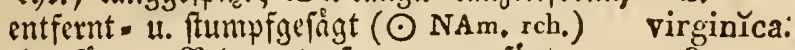

2. herzfơrm.; $\mathfrak{B}$ l. rautenf. = eyr., gelägt, am Sirunoe ganzrano. ( $\odot$ NAm.)

caroliniána.

\section{ACÁNTHUS. 505.}

Blätter:

1. Dorn., gefies.: Dorn. 6 Bolllang (4SE. 49.) spinósus.

2. ohne Dorn., budht. (4 SE. 49. bl. ; w. - * 5 . * $\mathfrak{S j l}_{\mathrm{j}}$, Siraut) mollis.

\section{ACÁRNA. 644.}

Stgl. åt.; \$El. Yanjettf., gewimp. = gezahnt,

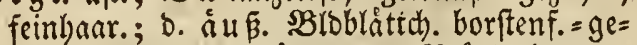

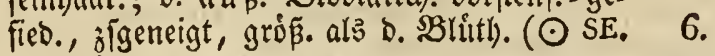
bl.; p.) cancelláta.

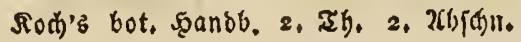




\section{ACER. 805. $\left(315.788^{x} .805^{x}.\right)$}

\section{Blätter :}

\section{I. immergrim,}

a. eyr., urigeth., einige 3 lapp.; unbeutlich 7 . gejägt, glatt ( 5 As.)

b. 'keilf., 3 lapp., ganzrand.; mit kirr. Sci= tenlapp.; Dorbentraub. wenigbluth., nufr. ( $\hbar$ As.) heterophýllum.

8. cretřcum.

II. abfallent,

A. einfact),

1. ganj cinfach, herzf., etwas zeriodnitt., ungleichgelägt; Doldentraub. aufr. (ち NAs. w.)

2. 2 lappig,

a. gefágt; sklmfte o. M. sBlutb.

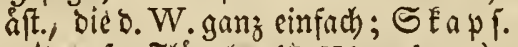
mit aufr. Flügeln (ち NAm. hgrn.) barbátum.

b. bopp. =géágt, langgeipist, glatt;

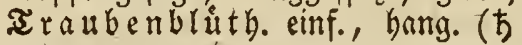
NAm. gb. grn.)

c. garizrano., gleidhlapp.: bié jủnger edf. =gezahnt; Doldentraub. wes 3. 5lappig, nigblutth., aufr. (ち grnch)

\section{9. tatarǐcum.}

a. gezilint,

a. untert graugrin u. unbehaart, un= gleitbgejabnt; $\mathfrak{x}$ aub. bang. ( 4. grn.-gb.)

b. unten feinharar., langgeipist, et= wate gejahnt; Doldentraub.

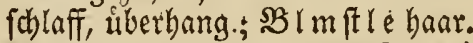

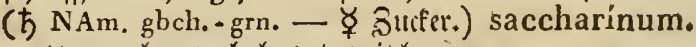

c. unten u. oben unbebaart, mit lang=

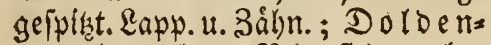
traub. aufr.; $B \mathfrak{I}$ mfte unbe= kaart ( 5 s. gra.-gb.)

b. geifigt,

a. ipis,

1) unten graugrinl.; Do o rós. aufr.;

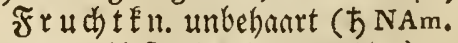
dr. - ఫ Farb. fdwar; ; bla) rubrum.

2) unten fcinbaar., faft 5 lapp.;

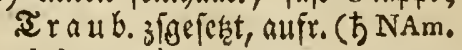
gbch.-grn.)

11.

pensylvanicum.

12.

monspessulánum.
13.

(Pséudo-Platŭnus.)

14. ఫ

15.

(platunoides.) 
b. ftumpf, runol., felyr fatwach 5 lapp., ftumpfgefägt; Doldentrauben idlaff;, aufr. (ち SE, wch.)

c. ausgejchmeift, ftuntpf, fafwad) 5 lapp., unten feinlaar.; Dolbentraub, aufr. (ち)

d. gangrand., ftumpflapp.,

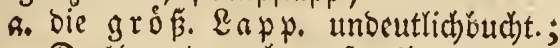
Dolbentraub. aufr. († 4. gblch.grn. - ఫ satr. rotr.)

b. UClle lánglich uno meift ungetbeilt; Seltffle etwas filz.; Dolben= tra ub. wie vorber (b) 5.gbch,-grn.)

4. hantof. =5 lapp., Yanggefpiat, gelägt, un= 18. Opălus.

19. obtusátum.

20. ఫุ (campéstre.)

21.

austriŭcum. ten feinbarig, weisgrau; $\mathfrak{B}$ lúthen in fopff. Dolb.; Fruchtłn. filz. (ち NAm.

M. gbch.: W. rch, gb.) dasycárpum. 22.
dasyca
23. ganjgetrennt. (5) éfd) l. (ち NAm.) Négúndo.

\section{ACHILLÉA. 670.670.}

\section{Blätter ungetheilt.}

\section{Bliithen :}

I. weiß oo. roth; Blätter:

A. unbehaart, gleichbreit,

1. am (S)runoe fómalet, gejägt; Stgef. 24. unbehaart (4 SE. 9. w.) Herba rota.

2. am (5nde fpis, gleich $=$ u. (d)arfgejågt; Doldentraub. jigejegt; Spreubl.:

a. and. Spite 2 fpalt.; Stgl. ripenf.; 25. Doldentraub. menigbluth. (4As.) grandiflóra.

b. ungetheilt; Stgl.:
a. rifpenf. (4 3.49. - $\mathfrak{W g}^{2}$ l., * 20. ఫ Siraut, $\mathfrak{B l m} .-\Varangle$ foutt.)

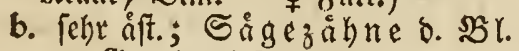 am Girunde tiefer (4) (Ptarmica.) 27. decolórans.

B. feinbatat., gleid $=u$. johalfgefogt: Stgl ripenf.; Doldentraub. wenigbluth.; 28. Ságe $\mathfrak{a} \mathfrak{a} \mathfrak{h}$ ne u. Spreubl. mievorb. (4) speciósa. C. febr faharf, flach, gezahnt: $3 \mathfrak{a} \mathfrak{b}$ ue aub= gerano., querftelone, gerwimpert = gejägt; Stgl. åft., tweitjchweif., oben feinkanr.' 29. (4 SE.) 
II. gelb; $\mathfrak{B l}$ l. långl, ftumpf, gefĩgt, gegen D. Slattfiel binner, gebilforlt; Doloentr. 30. offgef., verengt (4 SE. 33.49. - * Sirnut.) Agerătum.

\section{Blätter fiederförmig;}

A. einfachfiederförmig.

\section{Bliithen :}

I. meiß̄ oo. roth; Doldentrauben:

A. einfsct); Blätter:

1. filigig,

a. fomallanzettf., mit am (sinunbe tie. 31. fern Lapp. (4 SE.)

b. mit gleichbr., ftumpf. Lapp.: bie oberiten an b. Spibe etmas gezahnt (4 9.).

2. unbebaart: Lappen:

a. längl. = feilf., ungleichgezahnt, jenf $=$ rechtgebogen (4)

b. gleidbbr., fpits: bie unt. 2 theil.; $93 l_{\text {. }}$ jelbit fammf. = fiederf. (4 NE.) impaťens.

B. ¿ujammengejegrt; Blätter:

1. unbebaart, gleichbr., fammi. = fieberf., mit ctras gejagt. \&app. (4 SE. 9)

2. feinbaar., mit lanjettf., gejägt. Lapp. (4 As.)

3. unten etwas brar., mir gezahnt. $2 a p p . ;$ Etgl. mit gleichlyoh., unbelyant. 2(ejten; Dolbentraub. verengt ( 4 NAm.)

4. weis = filz. fammf. = fiederf., mit gang= rano., gleichbr. = pfriemenfórm. Lapp.; Dolbentraub. verengt; haar. (4 SE. 19)

II. gelb; Stglblàtt. mit ganzrano. Eappen: פ⿺廴了 Etrabl faum gróß. als SBlo. (4 NAs.)

serráta.

32.

Clavénnae.

33. squarrósa. 34.

35. alpina. 36. coronopifolıa.

37. asplenifolía.

38. pectináta.

39.

Gerbéri,

B. doppelt fiederförmig;

1. Bliithen gelb.

Blätter:!

1. Feinhaar.; Dolbentraub. ôgef.; Lappen d. Bl.: 
a. CannallanzettF., gejagt; Dorbentraub. 40. fugel.; Strahl fehr furz (4 As, 22) Eupatorium.

b. lanzettf., ganzrano. (4 As.)

41. micrántha.

2. zott.: Fiebern fehr gedràngt, mit fithmals lanzettf., ganzrano. Eapp.; Dolbentraub. รigei. (4 SE, I3)

42. tomentósa;

3. borftenart. = zott., mit lanzettf., ganzranb. Rapp.; Dorbentraub. zigejert, verengt; $\$ 3$ I ú th. róbr. (4 SE. 38)

43. compácta:

2. Blithen weils ad. roth:

\section{Blätter:}

1. unbehaart; o. öfigeferte Doldentraube:

a. etwas abftekeno; Lappen D. $2 B$ l. lam zettfơrm. gejågt (4 8)

b. gleidhod); Lappen d. Bl.:

a. zerichnitt.egejagt: Spino el geflugelt, zerichnitt. = gejägt (4 SE. 4)

b. gleichbr., fharfgefägt: Syindel gez 46 . flugelt, ganjrant. (4 SE, 6)

ligustǐca.

2. feintharig:

a. Fiebern ain Sirunbe ungeth., mit fothmals lanjettf. Eapw.; Doldentraub. zigei.; BIo. walzenf. (4)

b. Stglbl. Dopp. fieserform., mit gleithbr., ftumpf. Lapp.: $\mathfrak{W J}_{\text {a }} \mathrm{lbl}$ l. Sopp. gefied., mit 3 theil. Fieberch); Doldentraub. zigef. (4 SE. 8)

47.

ochroléuca.

tanacetifolia.

45.

istans.

48.

crithmifolia,

3. jott =Fcinbaar., mit lanzettf., ftumpf. Rapp.; 49 . Doloentraub. jiger., gleichbod) (4) lanáta.

4. ganz jott.: $\subseteq \operatorname{tglbl}$. bopp. fieberform., mit gleichbr., etwas gejalynt. Iapp. u. geflugelt., gezahnt. Spindel: $2 \mathbb{S}$; lbl. 3 fach fieberf.; Doloentraub. ofigereat, gleidhod) (4 - 50 . * Sitaut.)

(nobulis.)

C. 3 fachfiederförmig.

Mit langettf., ipif. Rapp.; Dolbentrauk. 51.

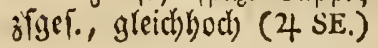
magna. 


\section{Blätter gefiedert;}

A. einfachgefiedert;

1. Bl iithen weifs od, roth.

\section{Fiedern :}

A. flact); Blätter:

1. unbehaart, Eammf, = fieberf.; bie gleidhbr. Fiedern:

a. Vanggerpist, faft 3 theil.; 2 fter bolbe

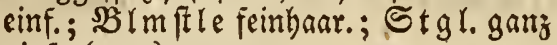
einf. (4 9)

b. ein. (4 9) atrita

b. etwas ffumpf, ganzrambig, punctirt. 53.
(4 10$)$

2. zottig: Fiebern gleid)hreit, geznlynt: 23zlbl. bopp. gefied.; Stgl. ganj einf. 54. (4 SE, Io)

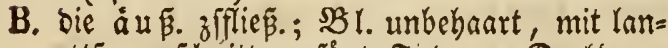
zettf., jerichnitt. = gefågt. Fiebern; Doldena

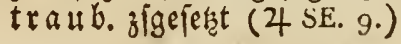

\section{nana.}

\section{5 , macrophýlla.}

\section{Bliithen gelb.}

\section{Fiedern:}

1. querftebent, bidytoadyziegelf., 3 theil., ge=

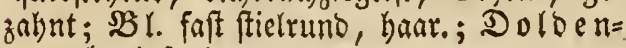
$\operatorname{tr} \mathfrak{a} u b$. einf. (4 As.)

2. flach; Blätter:

a. feinthar.: Fie bern gleidbr., ganzranb., ftumpf: Die unt. lánger; Xfteroold. cinf. (4)

b. fir

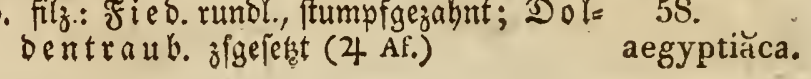

$$
\text { B. doppeltgefiedert. }
$$

\section{Blätter:}

1. unbebaart: $\mathfrak{L} a p p$. b. Fiebern gleidbreit, gezalynt (4 34. w.; r. - * Siraut, $\mathcal{1} 1 \mathrm{~m}$. - * 59. - ఫ्र Futt., (3ert.)

2. feinhaar.: Fie bern fieberf., mit gleidbr.: pfriemenf. Eapp.; Dolbentraub. jigé., gleidbuct); Bluth. rỏhr. (4)

(Millefolüum.)

60. myriophýlla. 


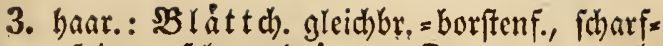
gefpitzt, febr georangt; Doldentraub. 61. wie vorber ( 4 II.)

4. unten baar.: Ficb. gleichbreit, ganzrando.; 62. Dolbentraub. einf. $\left(\begin{array}{ll}4 & 10\end{array}\right)$ (odoràta.)

\section{ACONITUM, $424,\left(417^{2} \cdot 426^{2}\right)$}

\section{Blumenkrone gelb,}

Der fappenf. Nectariumsporn;

1. Tpiralf.: Lippe eur., ausgerano.; $\mathfrak{B l}$ te belm fegelf, verlångert; $3 B l$. bandfớrm., feinl)aar., mit 3 fpalt., gejalint. $\mathfrak{a}$ app. (4 9.) † (Lycoctönum.)

2. bogenf., ftumpf: Lippe lanzettf., ausge= rano.; ShlEbelm wie vorber; SBl, unten feingaar., handf. $=5$ lapp., mit 3 theil., zer= fchnitt. = gejahnt. Lapp. (4 As.)

64.

ochroléucum.

3. gerabe, ftumpf; Lippe fpatelf., ausgerand.;

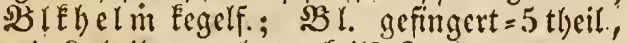

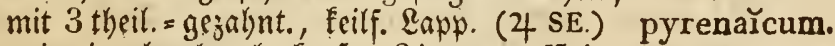

4. wie eingebroch., hadfenf.: Lippe auffeig.,

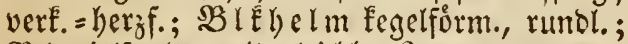

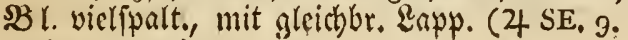
* $\mathfrak{2} \mathfrak{3} \mathfrak{l}, \mathfrak{D} \mathrm{lm}$.

* 66.
+. Anthüra.

\section{II, Blkrone weifs.}

Rect. Sporn bafenf., ftumpf: Rippe fehr furz; umgeid)lag., 2 palt.; $\mathfrak{B}$ IEhel $\mathrm{m} f e=$ gelf., mit breit. Nagel; $\mathfrak{B} l .3$ theil., mit cyr.: Feilf., 3 fpalt., gezaljnt. Lapp. (4 As.) album.

\section{III, Bllkrone blau.}

I. Mit 3 Stmegen; ber Eappenf., ftumpfe Nect. Sporn:

A. gerabe: Lippe langettformt, aufíteig., 2 fpalt.; Blf 5 theil., mit 3 theil., zerjonitt., gleichbr. Eapp. ( 4 9. - * $\mathfrak{W}_{j} l_{\circ}$, Sirall - rj Farb. * fis. ఫ gritu.) 
B. bafenf.: Lippe:

1. lamjettformig,

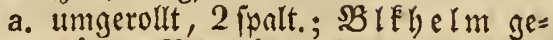
woilbt; $\mathfrak{B}^{\prime} \mathrm{l}$. glăinj, fingerf. = 5 theil.;

Lappen:

a. breitlanjettf., febr Eurzgezabnt; Blmftle unbebant (4 5. 9. * Siraut.)

* 69.

b. lanjettfớrm. 3 fpalt. = zerichnitt.; $\Re$ limitle feimbaarig (4 SE, 8. 70 . - Sirnut.) i

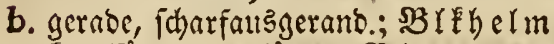

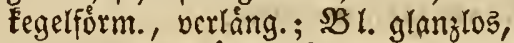
5 theil., mit lángl. = feilf., 3 fpalt., * 71 . gezahnt. Lapp. (4 8. - * Sitaut) + Cammürum.

2. berk. = bergf., altiffeig.; Stgl gewurts ben; Die 5 theil. Bl.:

a. glåkit., mit breitłeilf., 3 ppalt., ge= 72 .

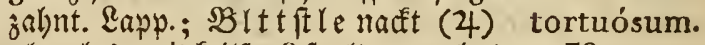

b. glanjlos, mit feilf., 3 ppalt., gejahnt. Iapp.; $\mathfrak{B l t t}$ ftle gewimp. (4 NAs.) volubule.

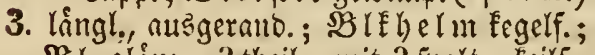
Bl. glánj., 3 theil., mit 3 ipalt., Łeilf., gejalint. Lapp. (4 9.)

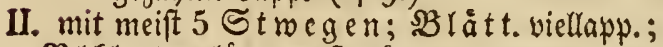
SBlEhelm langergeftred ( $4 \mathrm{NAm}$.)

74. sariegcitum. 75. uncinátum.

\section{ACTÁEA. 413.}

\section{Traubenblithe:}

1. eyr.; Fr úch te beerart.; Blbl.:

a. långet als b. Stgef. (4 4. nebst Bld, w. - 76. ६ ૪ Jarb. (d)warz)

b. Finger ( 4 NAm.)

if (spicáta.) rubra. 78.

2. fehr lang; frủ hote troden (4 NAm. w.) racemósa.

\section{ADÓNIS. 433.}

Blüthen :

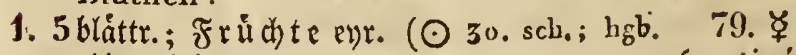
- $\left(1 \mathrm{ln} \mathrm{fr}_{\text {.) }}\right.$ 
2. Sblattrig; Friichte:

80.

a. faft waljenf. ( $\odot$ zo. dr.; hr.; gb.; sch.) (autumnális.)

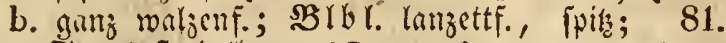
Stgil. ftacherlaat. ( $\odot$ 3\%. r.)

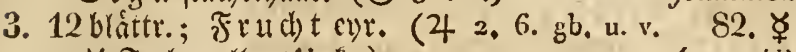

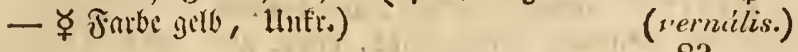

4. $1.5 \mathfrak{b l a ̊ t t r}$; $\mathfrak{F} \mathfrak{r} \mathfrak{u} d \mathfrak{t} t \mathfrak{e y r}$. (4 SE.) apennina.

8. ADÓXA. 322. (366

(4 4. grnch.)

84.

(Moschatellina.)

7 AESCƯLUS. 303. (3\%9:

$\left.801^{x} .804^{x} \cdot 805^{x}.\right)$.

Blätter:

I. 5 u. 7 joibl., mit verke = eyr., langge pipitst., Sopp. gejägt. Bláattd).; Blk.:

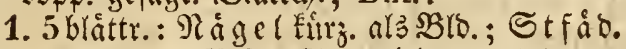
fo rany als SBle.; St aubbeut el baar.; Frit th te ftachl. († 27. w. u. ros. -

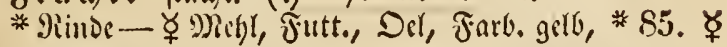
braun, (Serv.) Hippocastănum.

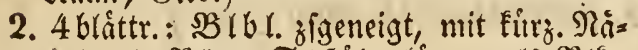

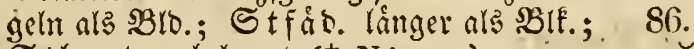
ङ tbeut. umbehaart (ち NAm, r. ) carněa.

II. 2arke 5 zálhlig; Blk.:

1. 4 blàttr.; Blblätter:

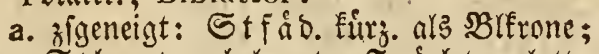
Stbell t. unbebaart; - Frith te glatt; Nägel:

G. Po lang ala Bro.; B̉láttd). oval= lanjettf., langgelpişt, bopp. gefăgt 87 . (ち NAm, r.; ubch. u. r.) Pavía.

b. länger; $B \mathfrak{B} \mathfrak{a} t t d$. langettf. $=\mathfrak{c y r}$.,

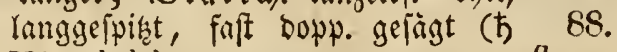
NAm. bgb.)

b. nicht; Stbeutel haar.; Frudte ftad)l.; Nägel: 


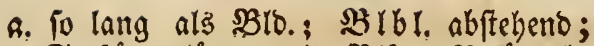

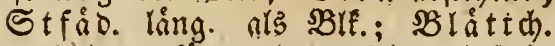
oval = lanjettfurm., langgefpiat, einfach =

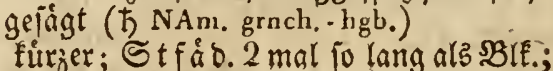
SBlát tch. lanjettí: = lánal., an bevben Enten lomaler, langgeipint, bopp. ge= lägt (ち NAm,

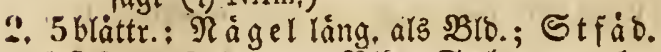
faft 3 mal fo lang als sBlf.; St tbeut. unbe=

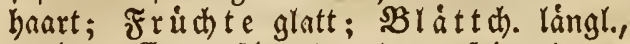
an bevo. Enoen ichmaler, langgeipitat, bopp. gejågt, unten etwas fifg. (ち NAm. w.)

5. AETHÚSA. 201,

$\left(\odot 32,29, w_{*}-\not{t} \operatorname{llnfr_{*})}\right.$

* $92 . \not$

† (Cynapüum.)

19. AGERĂTUTM. 650 .

2Bl. enr., faft berzf.; Stgl haar.; Spreubl. D. Sk rone begrannt, feingejabnt ( $\odot$ Am. gb. ; bl.; w.)

93.

conyzoides,

\section{AGRIMONIA. 390. (319 ${ }^{\mathrm{x}}$ )}

Bld. d. Frucht;

1. had̛rig; Blblätter:

a. 2 mal fo lang als sBlo,;

a. St glbl. gefiebert, mit längl. = enr. Blåttch.; 2l ebren aufr. (4 4. 5. gb. * 94.

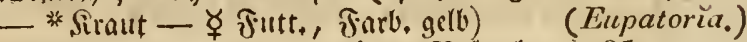

b. $\mathfrak{B l}$. fónmtl. gefięo., mit långl, SBlăttch): bie unt. fleiner (4 SE. gb.)

95. odoráta.

b. 3 mal jo lang; Stglbl. gefiebert, mit längl. Skláttd); X X b ren faft fticllos (4 96. As. $g \mathrm{~b}$, ) repens. 97.

2. glatt; ๔tblbl. 3 zåbl. (4 1. 4.49. gb.) Agrimonoides. 


\section{Agrostemma, - Aiuga,}

10. AGROSTÉMMA. 366.

\section{Pfanze:}

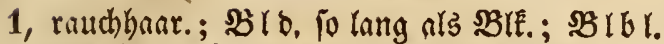
ungeth., nadft $(\odot$ 30, hr, ; wch, -

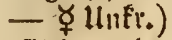

2. filzig; bie ausggerand. Blbl: :

(Githaigo.)

a. gefront, gejågt; $\mathfrak{B l}$. enr, =lanzettf. (o 99 .
SE. w, u, r.; r.; w.; h.)
Coronařa.

b. nicht; Blutben in Doldentrauben 100. (4 hr.)

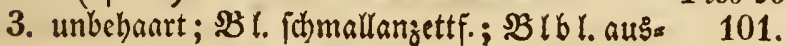
gerano. ( $\odot$ SE. rch.) -

Coeli rosa.

\section{AIILANTHUS, $800,\left(35.52^{\mathrm{x}} .800^{\circ}\right.$.)}

Bruthen in Rifpen; $\mathfrak{B l}$ l. ungepaartgefico.:

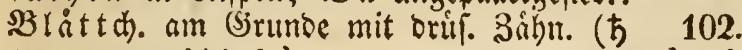
As. grn., wohlriech.) glandulósa.

\section{AIǓGA. 456.}

Bliithen:

I. im $2 u i r l ;$

A. mit friech. 233 zrlfprofien (4 4. bl.; w.; 103.
ก. - * Niraut)
(reptans.)

B. nicht; Blätter:

1. lämmtl. eyr.; $\mathfrak{B}$ lf. gang umgewandt, 104. feinbaar. (4 As. bl. u. w. ; r. u. w.) orientális.

2. Wurzelblätter:

a. febr groß̧; গুo fanze 4 fcit. = pyrami= benf., zott. ( $\sigma^{2} 3$. 1. 14. bl.; w.; 105. భ̧ rch. - * Siraut - $ซ$ Futt.)

b. fo lang als Stglbl.; Stgl eimf. (4 106. 9. r.; l. $_{\text {; }}$ w.) alpína.

c. Eleiner als Stglbl. (43. 6. w. ; bl.; 107. A.)

II. cinjeln in o. SOltwinf.; $\mathrm{Bl}$ :

(genevénsis.)

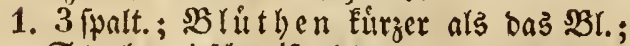
Stgl weitijhweif. ( $\odot$ 19. 21. 29. gb,; * 108. ros. - *iraut)

(Chamaepritys.) 


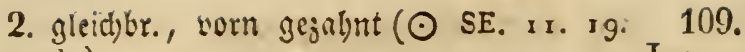
gb.)

\section{AIZÚON. 399.}

Blithen ftiellos; Blätter:

1. feilf.=elyr. $(\odot$ Af. wch.-grn.)

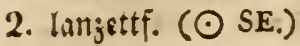

110.

canariénse. :

111.

hispanǐcum.

\section{ALCHEMfLLA, 79." (3.)}

\section{Blätter :}

1. nierenf., (SI lith. in Entsolocntraub.)

a. Tlapp., gejahnt, unten feitenart.; (4 112. As. 23.)

b. T-9 lappig,

6. unten feinthar.=glinj., fuarfgejabnt; 113. (4 8.) montána.

b. unkehaurt: Lappen:

1) ja|t balbefeišruno, ipieggejågt (43.

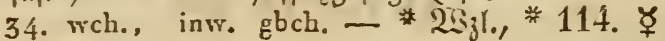
Sîraut - ఫ̧ (jirb.) (vulgáris.)

2) nerE. = evr. . jerjannitt. = gejågt, am (5runce ganjrano., bis jur Mitte 115. eingejanitten ( 4 9.) fissa.

2. fingeri., 5-6jäbl., lanjettr. = feilf., an b. Epike angebrudit = geligt, unten jeibenart.s glanj.; SBlith. in Enbodisentraub. (49. 116. 23. w.)

3. Ganoform. $=3$ ipalt., mit 3 ipalt. Eappen; $\mathbb{B}$ lith. getnauelt in sen $B$ lttwink., mit

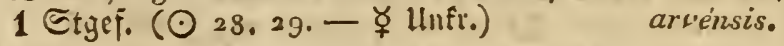

\section{ALISSMA. 302.}

Blätter :

1. cyrunt,

a. ipiţ; Frucht ftumpi 3 jeit. (4 58. 48. ros., dann w.) 
b. Fumpi; $\{B$ Im ft le cinjeln (4 45) natans.

2. hergformig, rumsl., ipif; Skapjel be $=-120$. graunt (4 44. w.) parnassifulia.

3. ichmallanjettiorm.; Früchte fugel.sperr. 121. (4 45.)

(ranunculoides.)

\section{ALLIUM. 273.}

\section{Mit Stengelblättern;}

A. $S t g l b l a ̈ t t e r$ flach;

1. Dolde zwiebeltragend.

Staubgefäfse :

1. mit 3 fcinen Spiencen

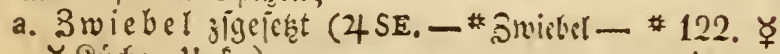
ఫ̧ Silidge, lliftr.)

b. Blattscheide:

satívum.

s. 2 ithneit.; 33 t. feingeferbt (4 3. 5. 123. ఫ

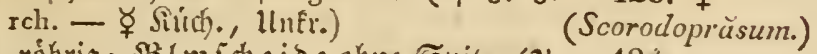

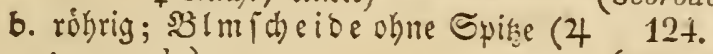
4. $19 . \mathrm{rch}$.)

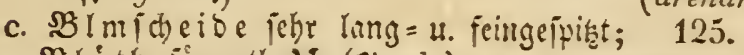
B lủth. iámmtl. il. (4 rch.) controvérsum.

2. piricmenformig; Dolo e etmas abfehent;

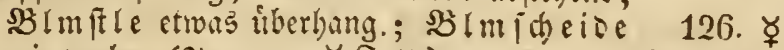
wie vorlher (4 29. - $\Varangle$ futt.)

(carinćttum.)

\section{Dolde nicht zwiebel- tragend.}

Staubgefälse:

1. mit 3 feincr Spinen;

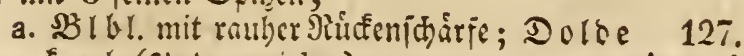
Eugel. (4 As. w。; hp.)

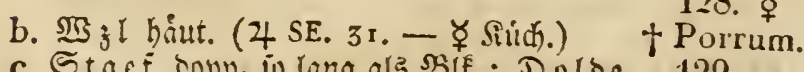

c. Stge f. Sopp. io lang als Şlt.; Dolse 129. fugcl. (24 NAs.)

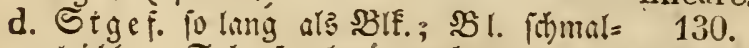
gleidhbr.; $\subseteq$ da f t abwártşgekog. (4) defléxum.

e. Seiterblith. úberbang.; Dolbe faft 131. fugel. (4 dr.)

rotíndium. 
2. piriemenform., bopp. fo lang als siltrone; 132. Dolbe fopff. (4)

suaveòlens.

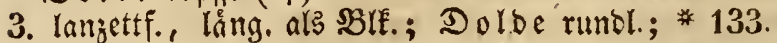

$\mathfrak{B}$ l. ellipt. (4 9. gr. $:$ w. - * $\mathfrak{S B j}_{\mathbf{3}}$ ) Victoriális.

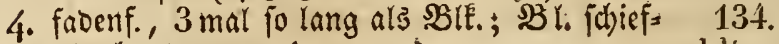
gebrel)t (4 NE, gb, u. grn.) obliquum.

5. einfach;
a. Doldo flach); SBlått. balbwalzenfơrmig (4 NAs. w.) w.; p.)
magǐcum.
b. bejonto. 3weig jmiebeltrageno (4 136.
B. Stglblätter röhrig;
1. Dolde zwieteltragend.

Staubgefälse:

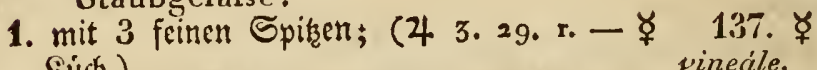
Sitich.)

2. nicht; 2 l. fdyarf, balbualzenf., linten ges 138. furd)t ( 4 3. 5. 8. wch. - 6 Sitich.)

\section{Doldenicht $z$ wiebel- tragend.}

Staubgefälse :

1. mit 3 feinen Spizen, $\mathfrak{u}$.
a. láng. als BlE.; $\mathfrak{B}$ l. balbwalgenf. ( 4 3. ; 139. 19. r.)
b. fưrz. als ß̊lk.; Dolbe faft Eugel. (4 140. hr.)
carnĕum.
141.

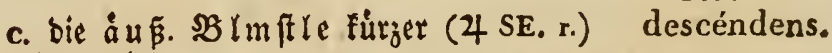

2. nicht, aber

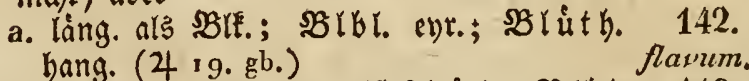
b. Dolde gleichbod, meift 6 bluth.; BIbl. 143. fpit: $\mathfrak{B l}$ l. borftenf. (4 SE. 2. 6.) id) eibe jebr lang ( 4 3. r.) paniculátum.

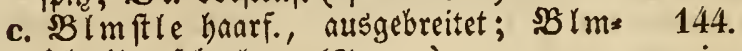

\section{Mit WVzlblätt. u. nacktem Schaft;}

A. $W z l b l$. röhrig.

Schaft:

1. ftielruno; Stgefälse: 
a. mit 3 feinen Spizen; $\$ B l . p$ friemenform.; $145 . \nvdash$ Dolbe fuget. (4 As. bl. - ఫ Sitil).) ascalonĭcum.

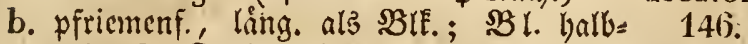
walgenf.; Dolo e bid)t (4 8. w. u. grnch.) sibirǐcum.

c. einf.; $\$ B l$ l. botftenf.:pfriemenf., gewwimp.:

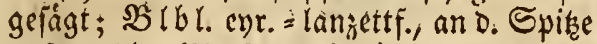
ausigernint. (4. SE: 8. $2 \mathrm{r}_{0}$ )

2. unterbalb baudb), langer als bie ftielrunt. 281. $(4-\Varangle$ Sili(f) $)$

3. nur jo lang als bie ftielruno. Blätter:

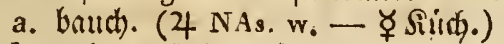

b. priemenfofabenf. (4 9.23. untit.)

147. setacĕum.

148. - Cepa. 149. $\Varangle$ fistulósum. 150. ఫॄ (Schoenoprŭsum.)

\section{B. Wzlbl. nicht röhrig.}

\section{Schaft:}

1. 2 fdyneioig; die gleichbr. Blätter:

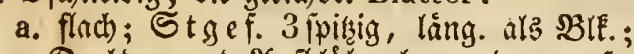
Dolde vor $D$. 2ufbluben hang., bann aufr. 4 NAs. r.; w.)

b. unten gewólbt, glatt; Dolbe runol.; Stgef. pfriemenf. (4 3. . - - §iin.) senescens.

c. rimnenform., unten etwas edf.; Dolde 153. gleich) hoch ( 4 3. 49. bl.; v.)

2. ganz ftielrumo; Stgefälse:

\section{(angulósum.)}

a. pfriemenform.; Dolbe gleidhboch; \$Bl. 154. fchmallanjettf., flach (4 36.)

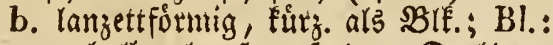

a. Kalbwalzenf., zigebr.; Dolbe gleich)= boch (4)

b. gleichbr., rimnenf., wechi.; Dolbe wenigblüth., gleichboch; $\mathfrak{B}$ Imftle am Sirunde natft; Stgef. halb fo lang als ßif. (4 NAf.)

c. einfach);

illyricum.

a. Dolde :

1) gleichbodt ; $\mathfrak{B} \mathfrak{l b}$ l. gleichbr., febr abs 157. ftebeno; $\mathfrak{B}$ l. fdumallanzettf. (4 SE.) atropurpurěum.

2) balbetugel.; $\mathfrak{B} \mathfrak{l b l}$ abjtebend; $\mathfrak{B}$ I. 158. $\Varangle$ lamjettf. (4 SE. - ఫ Silid).) nigrum.

b. Aspfthen zroiebeltragent (4 NAm, 159. wch.)

3. faft ftielruno; Dolbe vielblut h., gleich)= canadénse. bod); $\mathfrak{B l} \mathfrak{l}$ ft le ' mit Nebenbl. am Srumbe; 
BBl. gleichbr., rinnenf., unten ecf.; Stge piriement., balb fo lang als SBlf. (4 SE. w. u. brach )

4. nur oben fticlrunt; Doloc halbetugel.; SI. gleidbbr., flach), an (Sirunte rimmenf.;

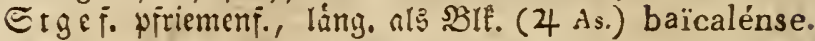

5. 3 Eant.; SBl. lanjettform., geftielt; Dold e gleichboch (4 4.47.w. - ఛ Jutt.)

6. Fuft + jeit.; Dolbe gleibhoch, hbergebog.; 163. Sil. gleid)br., flach; Stgef. wic 161. (4) cernŭum.

7. jaft waljeni.; SBl. lanjettī., ftiellos; Dolbe 164. gleid)god (4 SE, gb.) Moly.

\section{ALNUS. 740. (W. 63.)}

\section{Blätter :}

1. rumbl. = Feilf., ftumpf, an D. Epif̨e etwaz eingeorudft, Elebrig; 2f oern in o. Winfeln etwas langhaar. (ち 48. - ఫ Dil, Farbe (d)warj, retl), (uraun)

2. cllipt., etwas ftumpr, Elebrig; 2 bern in b. SBinfeln unten unbehant (ち SE.)

3. längl., ipis; 20 orn wie 166; Afterbl.: a. lanjettf.; $\mathfrak{B} 1$. unten feinbaar. (5 9. 19.) incrina. b. cyruno=långl.; $\mathfrak{B}$ l. am (jrunde runo; 168.

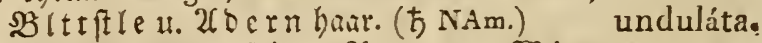

4. verk. =enr., langgejpiţt; $\mathfrak{Z}$ o e r n u. $\mathfrak{B}$ ins Eel terjelb. unten haar.; Ufterbl. ellipt., 169. ftumpi (ち $\mathrm{NAm}$.)

serruláta.

170.

5. herjf., langgeipiest, unbebaart (ち $S E$.) cordáta.

(ghutinósa.)

166.

oblongáta. 167.

\section{ALSIINE, 245. $\left(47^{2}\right.$.)}

Blblätter :

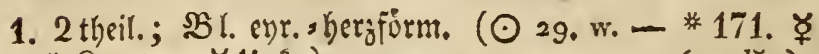
* Siraut - $\not$ utufr.) (medĭa.) 172.

2. ungeth); $\Re$ I. pfriemenf. $\left(\odot 300^{\circ}-\Varangle\right.$ Untr.) segetális. 
16. ALTHA'EA, 549,

\section{Blätter :}

1. filgig,

a. längl. =enr., unceull. 3 lapp., gézahnt (4

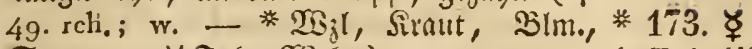

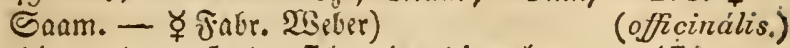

b. Die untern 5 ober 7 lappig: die obern 174 . 3 lapp. (4 SE. p.)

2. filjig=icharf: Die untern handf. = fingerf.: bie oberften 3 jabl., mit fehr lang. Mrit= 175. telbláttch). (4 SE. 5. dr.) cannabĭna,

3. oben unbehaart; berzf., ftumpf, 5 lappig; 176. Stgl raut) (4 26. ha.)

4. uberall unbehaart,

a. 5 ob. 7 lappig $=$ ed. ( $\sigma^{7}$ As, verschiedenfarb. * 177 . — B $3(\mathrm{~m}$.

b. runbl. = 5 lapp.: \&app. mit 3 Rerben; BI 1 ft le georáingt, 1 bluth. ( $\odot \mathrm{SE}$. w.) Ludwighi:

179.

c. 7 lapp. $=$ Gandf., ftumpf ( $\sigma^{7}$ NAs. r.).

ficifolia: 180.

d. runol.=ey)r. ( $\odot$ SE. r.)

pallı̌da:

\section{ALÝSSUM. 506.}

\section{i. Schötchen zsgedrückt;}
A: Stengel staudig.

\section{Blätter :}

1. rdumalfanjettfōrmig,

a. ftumpf, feitenart. glanz.; bie blutbe= 181 . trag. 3 weige born., Saur. (ち $\mathrm{SE}$. w.) spinòsum.

b. Inis, etwaz grau; Stgl nicoerliegeno; Stgef. einf.; ङ bitd. rund., ungeth). (ち) SE. w. wohlriech.)

2. feiff. = lanjettôrmig, frumpf, untert grän; S th ót th. vert.=eyr.; Stgl. u. Stger. wie vorker (ち SE. w. $\triangle$ )

3. verk.=enr., Feilf., gangrano.; Sigl faft

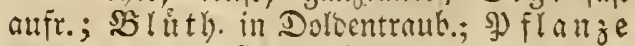
punct. , runoumftathl: (方 SE. 24. gb.)

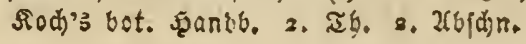

$1 S 2$. maritĭmum.
183.

halimifolium.
184. murále. s3 
4. lanzettf., fekr meich, auggejdweift; $S \operatorname{tgl} 185$. rifpenf. ( $\hbar$ SE. hgb.)

saxatřle.

5. patelf. =lângls, unten grau; Stgl aufr.; Iraub. rifpenform.; Scbótch. evr., grau 186. (ち SE.)

6. runor. = fpatelf., grau; $\mathfrak{Z} \mathfrak{r} \mathfrak{u} \mathfrak{b}$. bolbent.s argentěum. traubenf.; Stgl aufiteig. (ち SE. 9. 24.gb.) alpéstre.

\section{B. Stgl krautartig.}

I. Steng̀el:

1. aufrecht; Blätter:

a. Filzig; Stgbl. lanzettf., etwastpinu.

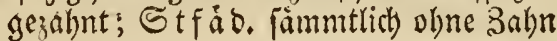
(O' SE. 23.)

b. weifgrau, Innzettformig, ganzrano.; Blutb. in Doloentraub.; B $\mathfrak{b l a ̊ t}$. 2 ipalt. (4 2. 19. w.)

c. Gendes nicht, langettf., etwas gezahnt, pfeilf., umfall.; Stgef. einf.; ङch $\mathfrak{v} t=$ d) en rundl. = kugetig, neţart. = rmjl. $(\odot$ i 5.)

2. weitldyeifig; Blätter:

188

edentǔlum.

\section{(incìnum.)}

190.

paniculcituni。

a. fily., fibnthallonjettf.; Stgef. fánumtl. gezabnt; Schotch. Febr glatt, freis= rumo $(\odot$ gb.)

b. weis̄grau: bie untern cllipt., fjik: bie obern faft lanjettf.; Irauben cinf. (4 2. I . dgb.)

3. hin $u$. her georeht=weitichmeif.; $\mathfrak{B} 1$, weif́s= grau, fâft lamettī.; Enotraub. bolden= traubenf. (4 SE. 13.)

II. Bldecke :

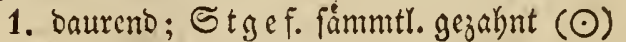

2. abfalleno; Schütchen:

a. mit einf. Saaren ( $\odot$ As.)

191.

minünum.

192.

(montćnum.)

b. mit fternf. geftellten Şaaren; Stgef. umgeben mit e. Shar Borften $(\odot$ Is. gb., w. - $\not$ D.D

193.

tortuósum.

194.

(calycĭnum.)

195.

Draba.

196. ఫ

(campéstre.)

II. Schötch, aufgeblasen, od, in Bld. verschlossen.

\section{Blätter :}

1. lanzettfơrmig, 


\section{7.}

a. entferntgegabut (4 SE, i4. hgrn.; gb.) sinuátum.

198.

b. gangrant., glatt; Stgl aufr.' (4 As. gb.) utriculátum.

2. $\mathfrak{2}$ zlbl. vert. = evr., etivas fitz.; 3iveige 199. ausgebreitet ( 4 8. dgb.) gemonénse.

\section{AMARÁNTHUS. 735. (744. W: 74.$)$}

\section{Mit 3 (zurw. 2) Sigefäfsen u. Knauelblïthen.}

\section{- Blätter:}

1. verferbet = eyrunt,

a. an b. Spibe eingebridé; Stgl 4 edig, cinf.; Snauclblüth. in o. NB!attwint. ( $\odot$ NAm.)

b. ausgeramo.; $\mathfrak{B l i t h}$ lipalt.; Stgl faft fiticluno, aft.; Snauclblutb. wie vorber $(\odot N A m$.

2. eit) = lanjettformig,

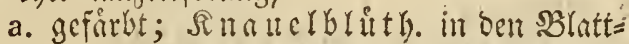
wink. geftielt, rumbl. ( $\odot$ SAs, die ganze Pllanze $r_{0}$ )

b. anל̧getant.; Snallel febr turjåbrenf.; 203. c)r. (๑ SAs.) gangetǐcus.

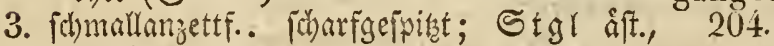
allit. ( $\odot$ As.)

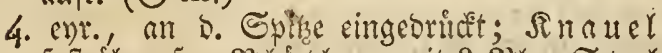

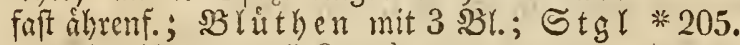
weitichroif. ( $\odot$ - * Siraut)

5. elliptich b),

200.
albus.

201.

graecı̌zans.

a. an b. Spize eingebrictét, Snauel fait ágrenf., runt; Stgl aufrecht ( $\odot$ NAm. dr. $; h_{\text {. }}$ )

b. ausgerand.; am Rante wellenf. ; In auel in o. Slattwink. gepart; M. BY lit then 3 blattrig $(\odot$ SAm. Bl. mit $\mathrm{cch}$, Rande; Stgl rch.)

6. långl., rumbsl., febr ftumpf, ausgeranoet;

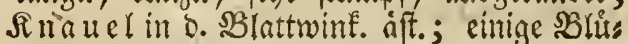
then nit 5 Stgef. ( $\odot S A s$.

angustifolius.

(Blitum.)

202.
melancholicus. 
7. rautenf.=enr., an o. Spitie eingeorueft, $c \dot{c}=$

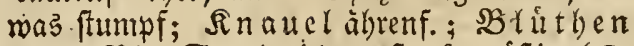

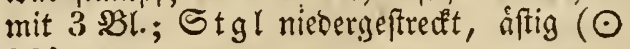
$\mathrm{SE}$.)

8. rautenf. =lanjettf.; 2 ehre fehr furz, wes

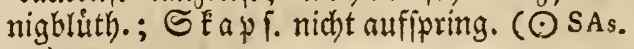
hr.)

\section{defléxus.}

\section{9. \\ prostrátus.}

210.

\section{Mit 5 Sigef. u. Trauben- blïthen.}

Traubenbliithen :

I. zujammengejest,

A. aufrecht; Blätter:

1. eyrunto,

211.

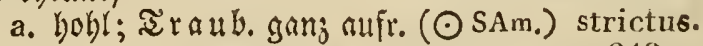

212.

b. ftumpf, ftechenofpis $(\odot)$

Jaetus.

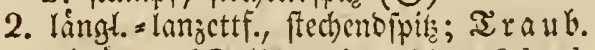
gebrängt $(\odot \mathrm{NAm}$. dr,; Bl. auf beyd. Seit, in d. Mitte r.)

213.

hypochondriă-

cus.

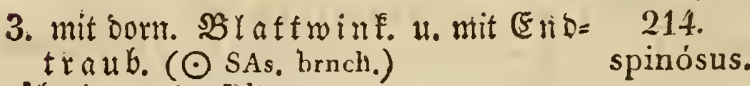

B. uberbangens; Blätter:

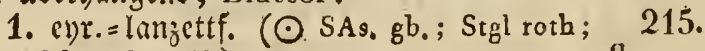
B1. rothstreif.)

Havus.

216.

2. ganz langettf. ( $\odot S A m$. grach.; hr.) chlorostàchys.

II. Soppelt zulammengelekt,

A. alifr., georángt; $\mathfrak{B} l$. eyr. =lanjettiorn.

( $\odot$ NÁm. nebst Stgl r.), hybridus.

B. etwab abfteberto, nacft; $\Re B l$. lanj. $=\mathfrak{C}$ )t. (○ As. dr.)

C. hangend; $\mathfrak{B l}$. wie vorker; Stgl iber. bang. ( $\odot$ As, $r_{i}$ )

III. melbrfach zulammengefeft; Blätter :

1. långl., fpitis; 3 weige abfteberio, unbe=

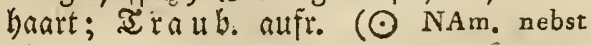
Bl. u. Stgl p.)

2. eyr. =lanj.; 3 weige abfeteno, fcinthat.

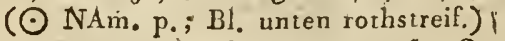
218. cruéntus.

219.

caudátus.

3. ganz ent., am গiante wellenf.; 3 weige

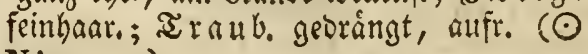
NAm, grn.)

220.

sanguiněus.

221.

paniculátus.

222.

retrofléxus. 


\section{(j. AMARÝLLIS, 268.}

Blumenicheib e 1 blith.; Blithe:

1. ftielthas; $\mathfrak{B}$ I m i d e e ibe ungetheilt, frumpf;

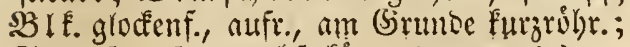
Stgef. aufr., wechi. für子. (4 SE. gb.) lutěa.

2. geftielt; Blmscheide:

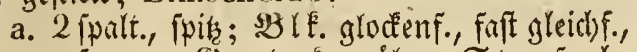
aufr., am Srunbe furzrókr.; Stgef. abs.

wartśgebog., gleidy (4 NAm. w. u. rch.) Atamásco,

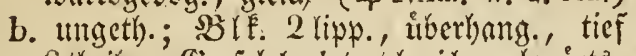

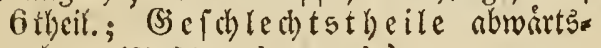
gebog. (4 SAm. dr, u. sch.)

223. luter.

\section{4.}

225.

formosiș̣̆ma.

\section{AMBROSİA, 743. (W. 35.)}

\section{Blätter:}

1. 3 lapp., gefågt ( $\odot$ NAm.)

2. Dupp. fiederformig,

a. 2atie uno
a. wenig bchoart; BRlattifle langges

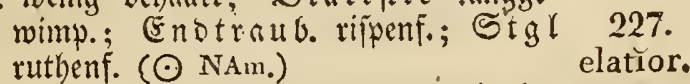

b. untẹn grail, Ftumpf; (Endtráb. ein=

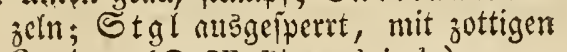
Эึweigen ( $\odot \mathrm{SE}$. Bl, starkriech.)

b. nut bie untern u. unten graulich: bie oberften einfachfieberf; Endtraub. 229.

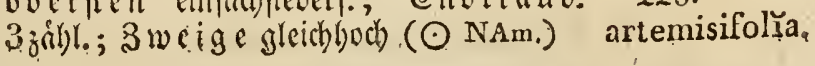

\section{AMETHỴSTÉA, 25.}

(๑) NAs. 8. b!.)

\section{6. trifĩda.}


2. ftielruno, geftreift, unbebaurt; $\mathfrak{B}$ l. bojp.

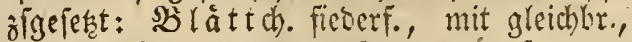
fted)endpip., etwas entfernt. u. geppernten 2.32. Eapp.; Doldenftrahl Datt. ( $\odot \mathrm{SE}$. w.) Visnága.

\section{AMÓRPHA. 562. $\left(543^{4}\right.$.)}

SBl. ungleichgefies.; Bldzähne :

1. Ullelanggeipist; $\mathfrak{B}$ lo. u. SBlatth. pein= 233. haar. (4 NAm, w. - blch.) pubéscens.

2. nur (Einer: L frumpf; o. brifing Bld.:

a. feinhaar.; Şlàt. feinhaar., mit ctwas Durdhidbeineno = punct. SBláttch.; Stfåd. ant Grumbe nuri wentig verwachj. (5. NAm, braun-v. - ఫ̧ Farb. ge(b)

b. unbehaart, mit surbjechento = punctirten Blättho.; Stfido. vom (sirumbe bij jur

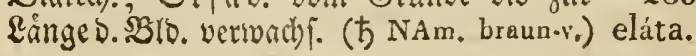

234. 후 fruticósa,

235.

\section{AMSONǏA. 134.}

SB l. obalslanzettform.: Die obern langgeipict; Stglglatt; $B a l g$ lap lóngl. (4 NAm. hbl.; w.)

\section{6.}

latifolıa,

\section{AMYGDĂLUS, 388.}

\section{Blätter :}

1. breitlangettf., gefägt, mit fơmmtl. fpiţen,

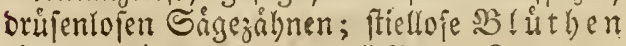

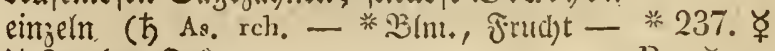
ఫ̧ Trudst, Dir)

2. langettformig, Persǐca.

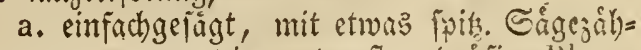
nen, wovon bie unterften oriffi, Blattstiele:

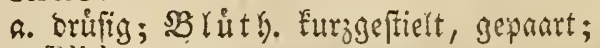
Blbl.:

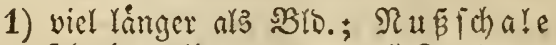
febr hart (ち SE. hr. - * Frudbt- - 23S. ఫ

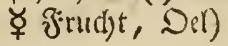
commúnis. 


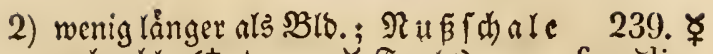
zerbrechl. († As. - ఫ ₹rucht) fragilis.

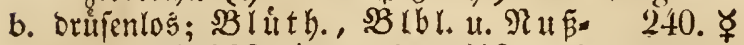

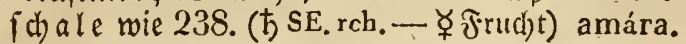

b. oopp. gefágt, nit brilenlofen Sảgezảinn.; 241 . Brit th. langgeftielt ( $\hbar$ NA. hros. $\Delta$ ) pumìla.

c. ganzranb.; baur., filberfarb.; BIttft.l 242. furz (ち As. $\Delta)$ orientális: 243.

3. långl. = lanzettf., gefăgt, unten fir

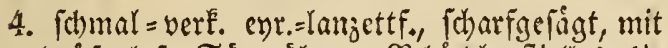

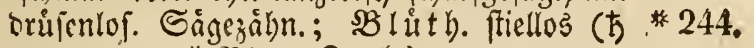
NAs, ros. - * Billt., frudft) nana.

\section{ANACYCLUS. 654.}

Die faft ftielrunben Blätter:

1. Dopp. geffed., grau, hoblpunct. $(\odot)$ SE. 245. $g b$.)

2. Dopp. zigejckt, gleidbbr., mit getbeilt., fpitz. 246 .

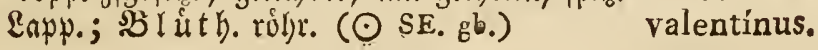

\section{ANAGÁLLIS, 111. (540.)}

Stengel :

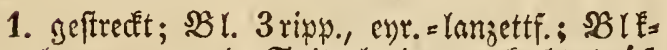
lappen an ל. Spige breiter, geferbt=brif. $(\odot$ 28. sch,; hbl,; w.; Q. - $*$ Siraut - *247. 支 ఫ̛ Uner.) (phoenicéa.)

2. aufifteig.; $\mathfrak{B}$ r. Fchmallanzettf., entgegenger. 248 . so. im Suirt (4 SE. bl.)

3. ctwas alfr. u. geflingelt; $\mathfrak{B}$ l. 5 ripp., evr.: - lanjettf.; BZlELapp. an o. Epire etwas ges

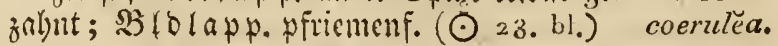

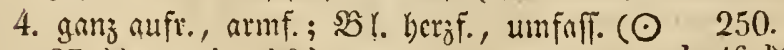
SE. bl. u. rch.; ha.)

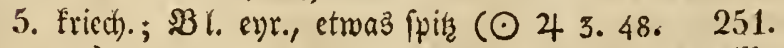
ros.) tenélla. 


\section{ANAGÝRIS. 336.}

B!̣̣

\section{ANARRHǏNUMM. 498.}

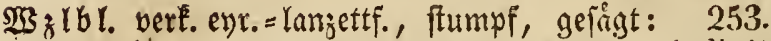

S tglbł. geth., gamzrano. ( $\sigma^{2} \mathrm{SE}$. blch.) bellidifolịum.

\section{ANASTATǏCA. 511.}

Br. ftumpf; Zlehren fehr furz in bett Blattz

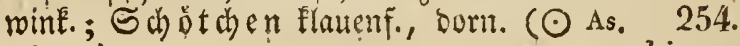
$\left.36, w_{0}\right)$

hierochuntịca.

\section{ANCHÚSA. 94,}

Blätter :

A. lanzettfơrmig,

1. frrieglict),

a, ipiegelglatt, am Siande wellenförnt. ; Stgl aufr., fift.; ; $r$ a ub. gepaart, ausgebreit., rifpenf., mit Mebenbl.; SB lb. läng. als bie faft gleich). SBle. róbre, mit pfriemenf. Eapp. u. pins felf. Silappen $\left(\sigma^{7} \mathrm{bl}.\right)$

b. am Ranbe lad blátt. fobmallanjettf.; SBlo. mit

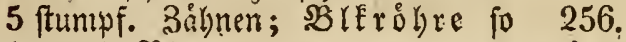
lang als şits.

2. badrig; Nebenbl, enr. =lamzettf., io ochrole'uca: lang als D. 5 fpalt., fpifze $3 B$ lo. (4 14 . 22. 30. p.-vo; w. - * $\mathbb{W}_{3}$ l, Sirkut, $\mathfrak{S}(\mathrm{m}$. -

255.

paniculáta,

3. ftrieglidjt= hadre, groublynt, am Rambe wellenf.; Traub. chibrenf., georangt; Rebenbl. lanjettf.; $B$ ro. zur SBlus thezeit 5 fpalt., fpis ( $4 \mathrm{SE} .3 . \mathrm{hbl}$.) unduláta

B. långlicts,

257. 후

(officincilis.)
258. 
1. hactr., etwas ftumpf, unbeutl. u. feinge= zalnt; : Steng el auffeeig., tweitichweif:;

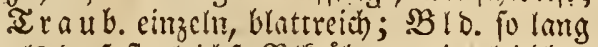
als b. faft gleichf. \$llfrobre, mit gleidjbr., ftumpfen Eappen $(\odot$ ros, ; hl, mit gelbl. Klappen)

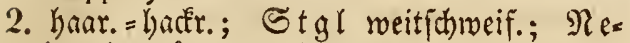

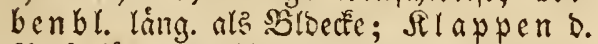
BZlk. kints. als Stgef. (4 SE. blutroth -

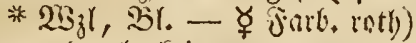

c. eyruno, hactrig,

\section{9. \\ Milléri,}

1. undeutl. = u. Feingezahnt: bie unt. lange geftielt: bie obern ftiellos; Stgl anfr.; sB Intfle in D. BSlattwinfeln, 2 blüttr.;

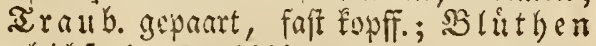
gleidl)f. (24 SE, hbl.)

2. etruas geftielt; Stgl niebergebrudeft, gas

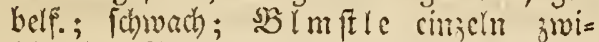

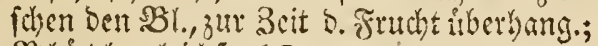

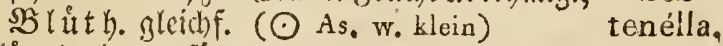

D. lingl. =lamzettförm.

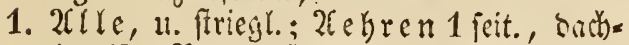

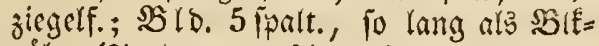
roibre (4-8. 22. r. ; bl.; w.)

2. nur bie untern, unt ausgeichneift = ges * $260 . \not$ tinctorǐa,

261.

sempervírens.

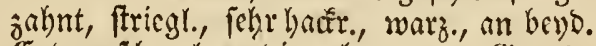
Enden ichmaler: die obern aim (Sirunbe

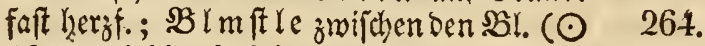
Af. w., Schlund gb.)

verrucósa:

\section{ANDRÁCHNE. 772. $(W, 76$.}

265.

Stgl nieberlieg., frautart. $\left(\odot S E . w_{0}\right)$ telephioídes:

\section{ANDROML̆DA. 328,}

\section{Blätter ábfallend.}

\section{Blkronen :}

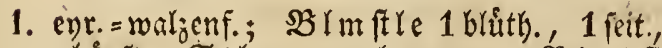

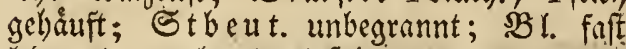
leberart., oval, etwas fpis, gamzramb., un= belyaart, unten bla fer ( $ち$ NAm。 w.)

266. mariána: 
2. glodenf.; BI I mftle 1 blith., in D. Bltt= wink., ober an entblattert. 2eftchen, gehouft; Stbeutel an b. Spifze gepaart 4 grann.; B l. eyr., oD. eyr. slángl., entferntgciágt, unbebaart, unterbalb weipbereift, oocr nicht (ち NAm. 27. w. $\triangle$ )

3. faft fugel., feinbaar.; Stbeut. ftumpf, unbebant; Enotra ub. rifpenf.; \$Bl. verÉ. eyr. =lanjettf., fpilz, faft ganjrand., ctwas feinlaar. (ち NAm. 4. 47. w. $\triangle$ )

4. eyr., feinbarr.; Stbeut. gleichbr., unbe= grannt; mit Enorifpen; 3Bl. oval, lang= gefpist, gejagt, unbelyant ( 5 NAm. 47.w. $\triangle$ )

5. bauch. = waljenf.; $S_{t b} c u t$. an D. Spike ges paart $=4$ gramn.; (5n btraub. 1 feit., cinf., mit 2 Rebenbl., nad) 1 Seite gerwand; $\$ 2 l$.

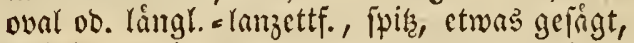
unbehaart (万 NAm. 47. w. wohlriech. $\triangle$ )

267.

speciósa.

268.

paniculáta.

26!).

arborěa.
270.

racemósa.

\section{Blätter daurend.}

\section{Blkronen :}

1. Eugelig; bic leberart., am Rande umgerollt., ganzrand. Blätter:

a. verf́. =evr., meift ftumpf, unten roftbraum=

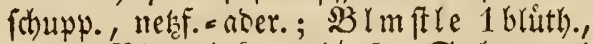
in ben Blattwink.; gebáuft; Stbeutel unbegranit ( 5 NAm. w.; rch.)

b. oval =lanjettf., oo. idjmaflanjettf., furz= u. Fharfgefpist, unbelsaart, unten weisl.; Fnoblumenftiele gelauft, 1 brithig, fleifdfarb.; Stbeut. gegen D. Spitze be graunt ( $\hbar 46$.

2. malzenf.; Stbeutel am (Grunde 2 born.; Itaub. in D. SBlttwine., gebuifdclt; $S B l$. leberart., enrumb, ftumpfgefpift, gallarano., gerintelt, unbehaart ( $\hbar$ NAm. rch. $\triangle$ ) 271. ferruginěa.

3. malgenf.=enr.; Stbeut. grannenlos; sglit= then iberbang.; $\mathfrak{I} \mathfrak{r} \mathfrak{u}$ ben boloentrauben=

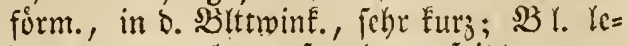
berart., eyr. =lanjettf., langgeipizt, ganjs ranb. ob. unglecichgefägt, unbelaart, glánz: (ち NAm. 1g. 47.w. $\triangle$ )

272. (polifolía.) nitı̌da. 274. acumináta.

4. langlich; o. leberartigen, unbebaart., oben glánz. Bl.: 
a. oval =err., furzgepikgt, gegen Die Spike weitlåuft. gelägt; $\mathfrak{T} \mathfrak{r a} u b$. einfach, in b. sBlttwinf.; SB lat $t$ ). mit 2 Nebenbl. († NAm. w. $\triangle$ )

b. ganz eyr., langgefpint, fein - u. fcharfge= fägt, werlenf; ; $\mathfrak{x} a$ ub. wie vorker, $\mathfrak{u}$. fdupp. = utcbenblättr., nach " 1 Seite ge=

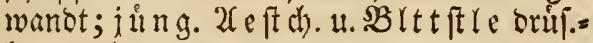
hanr. (万 NAm. 3. $\triangle$ )

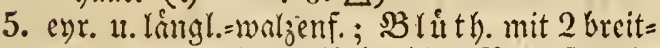

\section{5.}

axilláris.

eyr., langgerpitaten Yebenbl.; $\$ B l m$ ft le in D. SBlattw!nE., 1 brith., nach 1 Eeite ge= want, an ह. Spize D. 2fefthen e. blätrige

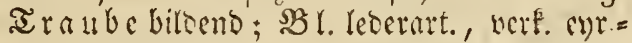
lanjettf., u. vert. evr. = oval, etwas fpits, an Ranoc etwas umgefrimmt, unocutl. gelägt, unteljaart, fidupp. = punct. , unten roftbraun (ち NE. w. - rcb. $\triangle$ )

277. calyculáta.

\section{ANDROSĂCE. 109.}

\section{Blithen :}

I. in Dolven; Blk.:

1. Felje flcin; Pflanze:

a. îberall zott.; $\mathfrak{B}$ làtt. evr. =lángl., $\mathfrak{u}$. nebjt den SBiblapp. gejalunt; Scillen [e hlo groz $(\odot 30$. w. $)$

b. Fehr aftig uno ctwab fojar; 3 weige fehr abjtegend; SBl. längl., etwas ge= zabut ; be jont. BIm ft le verlångert; SZlolapp. lanjettf., ganzranto. ( $\odot$ 23. 2:8. w.)

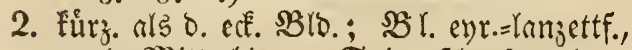
von D. Mitte bis sult Épike idjarfgezahnt;

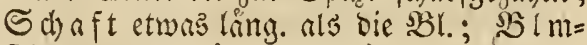

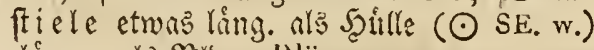
276.

Catesba'ei.

3. Yánger als SBlb.; Blätter:

a. lanzettf., gejallyt, am (3runto fothma=

ler; M flanje ctwas fordar, aufredt); bef. Silm ft le verlángert, ganj gerabe;

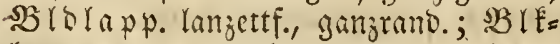
lapp. eyr., imgeth. (๑ 9. 24.w.) 278. maxima.

279. (elongáta.)

280. nana. b. lanjettf. = gleichbr., an $d$. Epitice cinges

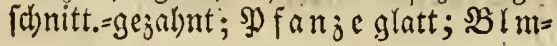


ftiele etwas abftebeno, werlángert; $\mathfrak{B}$ rf 282. lapp. verté. = berjf. (4 NAs. w.) , lactiflóra,

c. ftumpflangettf., gangrano., zott.; Dolde wenigblutt).; SBlo. enr. =glodenf. (4 9 . w. u. gb. od. ros.)

d. gleichbr., glánj., ganzrano., an o. Spifae gewimp.; Stgl glatt; Dolde menig=

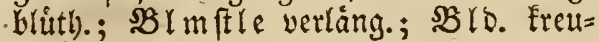
felfórm. (4 9. w.)

283.

villosa,

c. gleichbr. =pfriemenf., etwas gerwimp., zer= ftreut; Stgl feinfrar.; Dolde wenig.

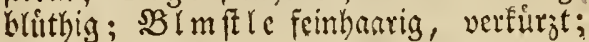
Blo. glatt, livellelf. (24 SE. 9. A.)

285. carnĕa.

f. ellipt., lanzettf., glatt; $\sigma_{\text {ch }}$ aft boloenf. (4 9.)

1I. faft ticllog (4 9.)

284.
laclëa.

III. Fefr furzgeftielt; SBIflappen zigencigt; Stgl alt.; $\mathfrak{B l}$ l. sachjiegelf., lạnzettf.=gleich)= breit, gangrano., oben unbebaart, bobl, un= ten und an Siande feintaar. (4 SE. 9. gb. hernach grün)

288.

Vitạliạna,

\section{ANDRYĂLA. 606 .}

Bl. evr., wollig: sic unt. etwas gejabnt; mit Enoboloentrauben; $B$ Imfticle meift 2 blitth, (4 SE. gb.) 289.
lanàta.

286. 287. helvetica. 


\section{Anemone. - Angelica.}

\section{Saame nicht geschwänzt.}

I. Blithen :

A. am 1 blutb. Stgl; Stglblàtt. 3 find)= 3 jốhl.; Blättch.:

1. cur., gejabnt (4 w.)

292.

2. lamsettf.; Blk.:

a. 6 bláttr.; sBlatt d). 3 fpalt. (4 4. 13. w.; ros. u. p. - ऊiritut, isim.)

b. vielblattr.; $B$ lat $t$ h. jeridunitten= gejalint (4 SE. hbl.)

B. ant 2 blith. Stgl; Saame:

trifolìa.

1. wollig, rmol.; $\$ 3$ l. 3 theit: mit 3 fpalt., gezabut. Lapp.; $2 \mathfrak{a} \mathfrak{a} b c$ baur.; ভ $\operatorname{tg} \mathfrak{l}$ blattr. (4 5. S. w.; p.; grnch.)

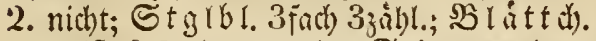
meift 3 ppalt., an ber Spise gejahnt; BIbl. longl., ftumpi, abftebeno (4 3. 4. gb.)

C. am vielbleith., brettr. Stgl; $\mathfrak{B}$ I. 3 theil.,

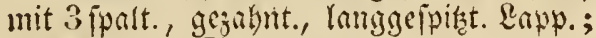
BIbl. pity; Sa a me raut); (sir iff. feldr furg, baltr. (4 NAm, grn.)

D. in Dolben;

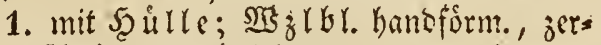
(d) nitt. = gejahnt (4 9. w. u. rch.)

2. obne Şulle; Stglblitt. einf., in

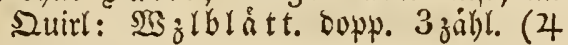
NAm. w.)

II. Wurzelblätter :
* 293.
+ (nemorósa.)
294.
apennína.
295.
(syliestris.)

1. 3 fad) boyp. zigereft, mit ftechenofpitan Båknen; Şürle blăttr.; Sa ame woll. (4 SE.)

2. fingerf., mit 3 fpalt. Sappen: Stglbl. 3 zábl. lanjettf., jigervad)j., ctrons getl.; Sa ame woll. (4SE.)
300.
coronäřa.

297.
virginiána.

f(ranunculoides,

298.

narcissiflóra.

299.

thalictroides,

301.

horténsis:

\section{ANGELÏCA. 226:}

\section{Stengel:}

1. unbebaart,

a. geftreift, ftielrumb; $\mathfrak{B}$ l. bopp. gefiebeit

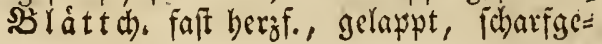




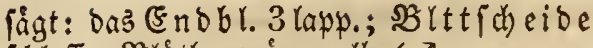

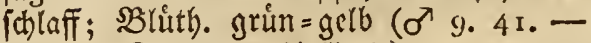
* 2isjl, Sonam. - ఫ (sicr5.)

b. bereift=gefárbt; $\mathfrak{W J}_{z}\lfloor$ bl. 3 zábl., herzf.,

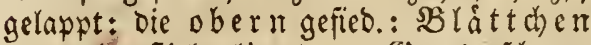
fammtl. geftielt, làngl, am (Sirunbe forma=

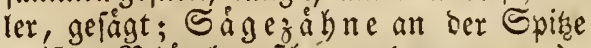

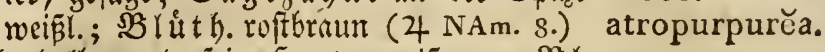

2. oberbalb von D. Fein. Şaaren meisgrau; $\mathfrak{B} l$.

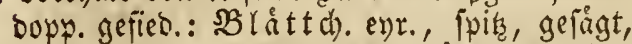
am (Srunde meift 2 lapp.; $\mathfrak{B}$ lüt (4 5.49. - ซ Jutt.)

3. gefurcht,

a. obeir edf., fehr glatt; $\mathfrak{B l}$ l. 3 faci) Dopp.= zigeiefict, unten rauth: SBláttd). etroas ausgejpert, her $F_{0}=\mathfrak{e v r}$., ungleichgejahnt (4 3. wch.)

b. itharfect.; $\mathfrak{B}$ l. 3 fach boppelt = 3rgereft; BB lát tch. 3 fpalt. = fieberf., lángl. = lan= jettf., mit reisk. idbarf. Spisen; ভtaub= wege an b. Frucht ungejolag. (4 3. 5 . wch.)

c. cinfi, meift blattlos; $\mathfrak{S i g}_{3} l b$ l. faft oopp.= fieberf., glatt: SBlåt $t$ d). etwas aufrecht, mit faft ohrf. 2enbang, u. mit gteichbr., ipic. Rapp.; be T. So úle en borftenf., faft to lang als bie bej. Dolden (4 SE. s.) pyrenăca.

* 302. $\Varangle$ Archangrelica.
$304 . \underset{+}{\not}$
(sylvestris.)

\section{5. \\ praténsis.}

306.

Carivifolia.

\section{ANTHĔMIS. 664: $\left(669^{\circ}\right.$.)}

\section{Blüthenstrahl w'eiss od, rolh,}

\section{Blätter :}

I. einfactficoerf., ftiellos: Lappen :

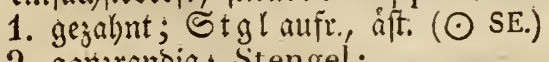

2. ganzrandig; Stengel :
a. aufr., aftig; $\mathfrak{B} l$. gleichbr. (SE.)
b. feinbaarig, 1bluth.; $B$ lattlappen gleichbreit = pfriemenf., fammf., gejadt (4 9.)

II. buchtig = fieberf., geftielt, gezahnt, unten reipgrau; $\subseteq \operatorname{tgl}$ ftrauch. ( $5 \mathrm{As}$.

308. mixta. 309. coronopifolía.

310. alpina.

311. artemisiąefolı̌a. III. Doppeltfieberformig; 
A. 2rle; Lappen:

1. ungeth., lanjettf.; $\mathfrak{B}$ l. unten punctirt; 312 . ভ. mafft; Stgl frautart. (4 SE.) maritĭma.

2. gejabnt, lanjettf: Die unt. 3åkne etwas umgefchlager; berzf., an b. Spike ftictrumo = feinge= 313 . fpirgt $\left(\odot \mathrm{SE}_{0}\right)$ altisšma.

3. etwas gezalnt, fteif; Spreubl. lángl., 314. langgeipitat (4 As.)

4. pfriemenformig = gejabnt, gleidbreit; Spreubl. longl., an b. Spişe ftiel= rund $=$ frechendipitis $\left(\odot \mathrm{SE}_{\mathrm{i}}\right)$

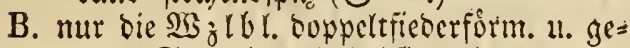
zaknt: $S_{\mathfrak{g}} \mathfrak{i b}$. cinfadficoerf. u. etwas gezabnt (24 SE.)

rigéscens.

315.

Cota.

316.

Chamomilla.

IV. cinfachgefiedert: Fiedern:

1. gleid)breif;

a. ganzrandig,

a. ftumpf; Stgl unbchant, 1 blitb. 317. (4 SE. 9.)

b. etwas feinban.; $\mathfrak{B}$ l. an o. $\mathfrak{B} \mathfrak{l} \mathfrak{u}$

then einf.; 3roeige 1 bluthig (4 318 .

SE. 21.)

b. 3fualtig, etwas ffumpf; Stgl anf saxatilis, fteig.; SBl. feinhanrig; $\mathfrak{B}^{2} \operatorname{lm}$ ftle ver= 319. lâng., nadft, fill. (2.SE.)

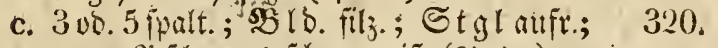
ganje gi fla

2. gleichbr. = pfricmenf., meift 3 fpalt.; $\mathfrak{B} l$.

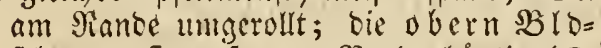

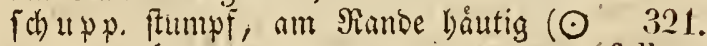
stark riech.)

V. boppeitgejiebert,

A. wollig=jott.; Sglbo. Eegelf.; Sleu= 322 .

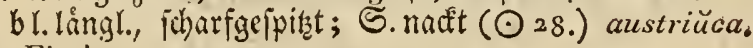

B. Fiedern:

1. gleichbreit;

a. SBlm ft le attgeblaf; S. gefligelt 323 .
( $\odot$ Af. 28.)
claváta.

b. Bldecke:

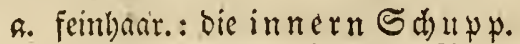
and. Spize wie verbrannt; Stgl 324. aufr. ( $\odot S E$.$) pubéscens.$

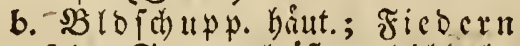
ipis; Stengel ât., gleichbod); 
3weige 1 brith : Seitengwei=

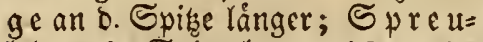

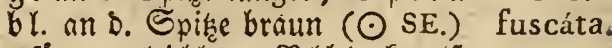

2. lanjettförm. =gleichbr.; $\$ B \backslash b$. Eegelf.; Spreub!. Lanjettf.; S. mit e. Rande gefroont $(\odot$ 29. $-\Varangle$ ünfr.)

3. pfriemenf., 3 thatig; sglvo. Fegelf.;

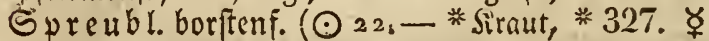

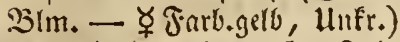

4. gleidjbr. = pfriemenf., 3 theil., etras zott.; $\subseteq$ tgl am (Sirumbe åft. (4 2.34. * 328 . * 3 iblim.)

nobilis.

VI. meift soppeltgejied, gleichbr., fpit; Stgl cinf., etwas aufr., zott.; sBlb l. verf. ey)r., 329. felyr ftumpf, 3 zahn. (4 9.) corymbúsa.

VII. 3 fachgefied.: $2 B(a) t t h$. gleidhtr.; Stgl nicberlieg.; 3 we eige in o. SBlattwinte. 16(úth. * 330 . (4 4. 8. - * Siraut, $23(m)$ ) Pyrěthrum.

\section{Blüthenstrahl gelb.}

\section{Blätter :}

1. cinfach, entgegenger. geffielt, ènr., gefågt, 3 fact gerippt, unten feimbaarig; Strabl vielblitth. (24 SAm.)

2. Doppelt fieberf., gefågt, untent feinhaarig;

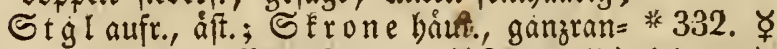

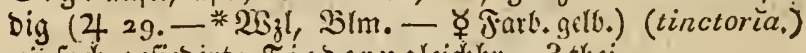

3. einfach gefiedert: Fie berngleidbbr., 3 thei= lig; Stgl iprollend; $\mathbb{B} \mathfrak{l}$ it th. eimzeln, ftiel= los in 8 . 2(ftrine. (广 Af.)

4. Dopp. gefied. gelägt, unbchant; Stgl aufr., åft. SE ro ne båut., gejabnt, an b. e. Seite eingeidunitt. (4 SE.)

331. buphthalmoídes.

* $332 . \underset{\gamma}{\not}$ (tinctoria.)

333.

arabǐca:

334.

discoidéa:

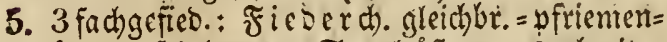
form., feinhanar.; Stgleft., aujgebreit.;

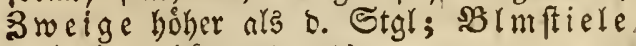
nad' oben biffer (๑ SE.)

335: valentína:

\section{ANTHERICUM. 28s;}

Blätter :

1. flach; Schafft:

336. serotinuni. 
2. åftig; Ş lF. flad); Stweg gerabe (43: 337. 23. w.) (ramósum.)

3. ganz cinfadt); Blk.:

a. flach; S Etweg abwaitsgeneigt (4 5. 338. 8. w.) (Liliágo.)

b. glodenform.; Stgef. abwairtsgeneigt 339 .
(4 SE. 9. w.)
Liliástrum.

II. rinnenf., lanzettf. =gleitbbreit, mit flumpf.,

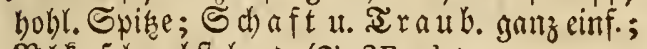
SB IF. fehr abfteheno (4 SE. gb.).

III. fohmerbf.; Stfåo. woll. (4 47.gb.) 340.
sulphurěum.
341.
t ossifrăgum.

\section{ANTHRÍsCUS. 213.}

Stengel :

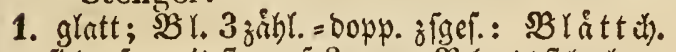
fieberf., mit ftumpf. Eapp.; $\mathbb{B}$ lat tffte baa= rig; Dolben evr. = Fegerf., ben Bl. entge=

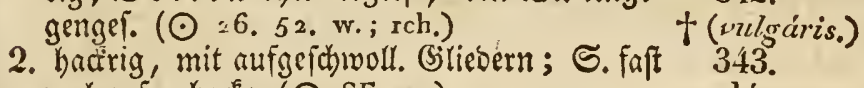

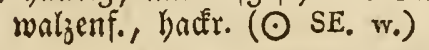

342.

nodósa.

I. Srautartig; Blätter:

17. ANTHÝLLIS, 564. $\left(543^{2}, 59^{4}{ }^{\mathrm{x}}\right.$.)

A. einfachgefiedert,

1. gleichformig;

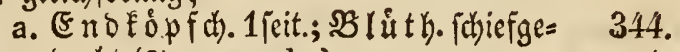
orebt (24 8. 23. dp.) montína.

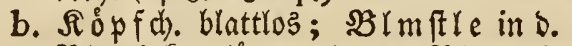
Blttwinte, lång. als bas sBl.; mit

e. Spurvon $2 \mathfrak{f}$ terbl.; 3 weigebl. 3 zónhl. (4 SE. 8.)

2. ungleichfơrmig; Köpfchen:

onobrychioides.

a. Doppelt (4 3. gb.; sch.; rch.; w. - 346. ఈ ఫ్ శึttt., Farb. geto)

b. cinzeln, geftielt; boirlen natenf., 347 . ftumpf, fứr. alz \$Bld. ( $\odot S E$. w.; gb.) cornicína.

B. 4 fach gefiesert; mit Seitenbluthen 348 . $(\odot$ SE. gb.)

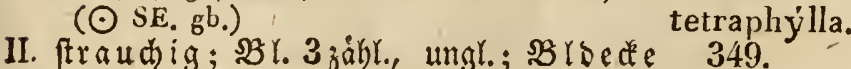
woll., żur Seite ftekeno ( $5 \mathrm{SE}_{\text {: gb.) }}$

cytisoídes.

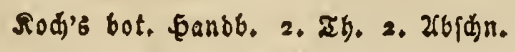




\section{ANTIRKHINUM, 497.}

Blätter :

1. lanjettfórmig,

a. entgegengef.; $B$ lůthen traubenformig;

sB lo a bidn nitt. oruif.baar., enr., ftumpf

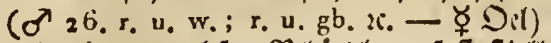

b. Die obern wechi.; $\mathfrak{B}$ lủ then faft fltielfos;

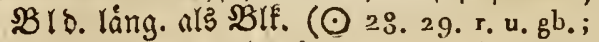
w. ; Skaps, todterikúpiff. - ఫllitfr.)

2. her $f_{\text {. }}$ entigegengef., ungleichgeferbt, etwas lapp., baar.; Stgl niedergeftrectit (4 SE. 23. up.)

350. ఫ્子

(majus.)

351. $\Varangle$

(Orontium.)

352.

Asarǐna.

\section{APARGL̆A. 608.}

\section{Stengel:}

1. blattr., etwas åf., ftachelbaar.; 2Blo. un= behanrt; $2 B l$ lanzettf., färotiägef., ftachel= haar., mit gabelf. Şanren (4 SE. 5. 2 I.)

II. nicht; Schaft:

A. ichuppig,

1. áftig, abroortzgebog.; $B$ l. lanjettf., fabrotiágef. = fiederf., fajt unbebant (4 3. 34. gb.)

2. oben biffer, etwas ftachelharar., 1 bluth).; B l. lanjettf. = lóngl., falt unbel)aart; sBlo. londr. (4 9.)

B. faif nadet,

1. 1 blutthig, u. nebit $\mathfrak{B}$ lo de e

a. ftachelbaar.; $\mathfrak{B l}$ l. lanjettform. am (Srunce gezabnt, mit gabelf. Şanten (4 9.)

b. feinhaar.; $\mathfrak{B}$ l. lanzettf., fpics, et. was feingezalint, grau=icharf, mit vielipalt. Soaren (4 8.)

2. meift 2 bluth., nufi., faft nacft, oben bicfer; $\mathfrak{B}$ ld. haar.; $\mathfrak{B}$ l. lanjettform., 358. f(b)rotjägef.=gejahnt, unbehant (4 SE 9.) Taraxăci.

C. ganz nact, u. nebit $\mathfrak{B} \mathfrak{l}$.

353. aspěra.
354.

(autumnális)

355.

alpina. dubia.

357. incána. 359.

1. unbehaart; $\mathfrak{B l}$. wie vorker (4 3. 48.) hastilis.

2. fachelhanrig; bie ftachelhar. Bl.:

a. ichrotiäge $=$. Fieberf., mit umgefrummt., gejalint. Lapp. u. 3 [palt. Scaren (4 SE. 23.)

360. crispa. 


\section{Apargia. - Aquilegia.}

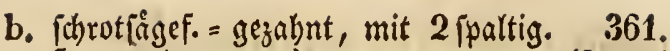
Şaaren (4 3. gb.)

(hisp̌̀da.)

\section{APIUM. 193.}

Der äftige Stengel:

1. gefurdht; $: B$ l. gefiedert: Stglblättchen feilf., zerid)nitt.; Dorben faft ftielloz ( $\sigma^{7}$ 36. 48. wch. - * $\mathfrak{W}_{3} \mathrm{l}$, Siraut, Saam. - 362. ఈ

ఈ̧ Siuch., Futt., llutr.)

2. elf.; $\mathfrak{B}$ 1. Dopp. zigeierst, fpiegelgratt: bie †, graveŏlens.

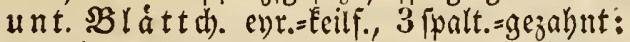
die obern lamzettf., faft ungeth.; beiond.

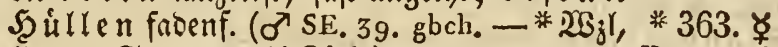
Siraut, Saam. - $\Varangle$ Sitd.). $\quad$ Petroselínum.

\section{APOCY̆NUM. 132. $\left(173^{x}\right.$.)}

\section{B t. unbehaart; Blumenkronröhre:}

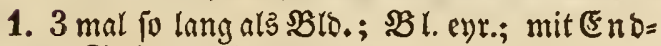
u. Seitenaferbolben (4 NAm, 日.) tandrosaemi-

2. eben folang als \$̧ro.; Blätter: fol̆um $\cdots . . .$.

a. LanzettF, an bend. Enden fpir, $\mathcal{X}$ fter= Dolden rifpenf. (4 NAm. A.)

b. långl., Febr Eurzgeftielt, icharfgépiat, am Srunde ftumpf, faft herzf.; 2ffterdol= ben fúrt. als bas $\mathfrak{B l}$. (24 NAm, f.) hypericifollum.

c. lảngl. = ellipt., icharfgeipist, am (Srunbe etras fdumaler; 2f fterdolden rifpenf. $4 \mathrm{SE}$, A.)

367. † venĕtum.

\section{AQUILEGĨA. 427.}

Nect. Sporn:

I. eingefrímmt; Stengel:

1. blätrig,

a. nebfit 8. $\mathfrak{B}$ I. unbebaart (4 4.21. dbl.;

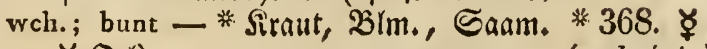
- $\Varangle$ D.r)

$$
\text { (vulgúris.) }
$$


b. vielbluth); $\mathfrak{B l m f t l e , ~} B$ lattfte $\mathfrak{l}_{\text {f }}$ B I. unten etwas feinhaar.; Sporn viel Furry. als \$lffiaum; (siriff. langer als Stgef.; Slapf. zott. (4 bl.)

2. fâft nadt; $\mathfrak{B}$ l. meift 3 lapp.; St engel meift 1 bluth)., Flebr. = haar. (4 SE.)

369. glandulósa. 370. viscósa.

II. gerace, boch an b. Spitze etwas eingebogen u. biffer; Blbl.:

1. To lang alb Rect.; Br rif. u. Stgef. jo 371. lang als $\mathfrak{B l E}$. (4 NAs. dr.; bl.-v.) atropurpurěa.

2. furt. alb शect.; Stgef. eben fo lang als Ble.; (S) riff. 2 mal po lang als $b$. Stgef. (4 NAs. hgrn.)

II. ganz gerabe,

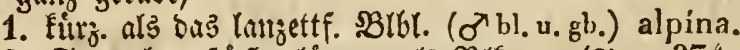

2. Staubgefá NAm, rcb. u. gb.)

372.

viridiflòra. 373. canadénsis.

\section{ARĂBIS. 530 .}

\section{Saam, mit e. Flügel gerändelt.}

\section{Blätter:}

1. umfalfend; bie gleidbr. Schoten:

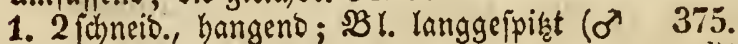
NE. wch.)

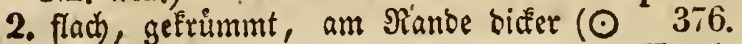
8. 26. gbch.)
Turrita.

II. ftiellos, etwas gezahnt; Wzlbl.:

1. verE. enr. = fpatelf.; Stglbl. långl., fajt

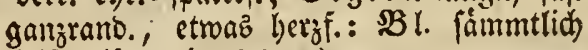
fteif, glän. (4 SE. w.)

2. verf. = eyr.; $\subseteq$ tglbl. lanjettf.; $\subseteq$ tgl bláttr.; Traubenbliithe :

a. aufrecht (4 9.)
b. bangend, wenn bie frucht entfegt, mit 379. 1 feit. Styoten (4 9.) nutans.

\section{Saame nicht gerändelt;}

A. Stglblätter umfassend.

Blätter:

380.

1. berzf., gefågt, jott. (Or $\odot$ SE. 56. p.) verna: 
2. lingl., gegahnt u. nebjt s. aufr. Stgl fta $=$ deelbaar.; Schoten abftebend, mit e. eben io breiten Sriff. als D. Schote ( $\odot \mathrm{SE}$.)

381.

undiuláta:

382.

alpina.

3. längl. = lanzettf., fdharfgejabnt $(49$, w.)

4. [patelf. =längl., etwas weißgrau, gleich) $=$ u. grobgezahnt; Stglbl. preilf. = umfall., am ङ̌runde ganzrano. (4)

5. ftumpf, icharf; bie ganz geraben u. aufrecht. Schoten:

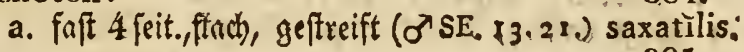

b. Foff ftielruno (4 SE. 5.)

6. Vanzettf., ungl. gejahnt, am Ranbe wellenf. u. nebft 'Stgl feinhaar,; Shoten abfte= bent, unbelyaart (4).

7. Fámmtl. Kadér.: St glbl. in ber Mitte ges jolnt; 2 weige ganj 'gerabe u. aufr. (4 SE. $w_{\text {.) }}$

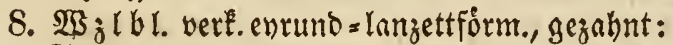
Etglbl. Lanzettf., gezalhnt, pfeilf. = umaall, gewimp. (4 w.)

B. Stglblätter halbumfass、

Stgibl. långl., gezabnt, nebft Stg l badér.,

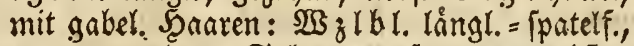
etrwas gejalint; Schote aufr. etrogs neffes beno (o' SE.)

385. aspĕra,

386.

crispáta.

albida.

hirsúta:

358:

stenopetăla:

359.

incina.

\section{Stglblätter stiellos;}

\section{Blätter ungleichförm:}

Stengelblätter:

1. lanżettfo̊rnnig; Wzlblätter:

a. ey)r., etwas gezalint: Stglbl. Fharfges fågt: 2rrle fpiegelglatt, unbehant (2 SE: 3. 3.4. 49.)

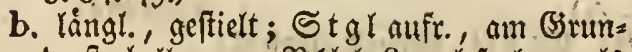
be ftachelthanr.; 316 l: 2 mal po lang als B Ib. $(\odot$ : 9 ; w.)

c. Fârotfágef. = leverf., hadfr.; Stglbr. mit gabelf. J̦aaren; S (4 9. w.)

390.
Allionii.

391 .

(thaliäna.)

392. petráea. 
2. faft lewerf: : 3 weig ebr. lanzettf., zeridnnitt. 393. ( $\left.\sigma^{7} 48 . w_{0}\right)$

3. ganzrano.: $\mathfrak{S B}_{z}$ lbl. undeutl. gezahnt, verE. enr.: Ulle gemimpert; Stgl ganz cinf.; Sdoten aufr. (4 9.)

2. Blätter gleichförmig.

Blätter :

1. ftumpf, gezahnt, bacírig; $\mathfrak{W}_{\mathfrak{z}} \mathfrak{l}$ bl. faft

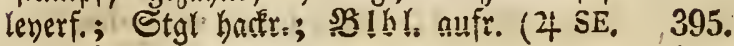
23. w.). stricta.

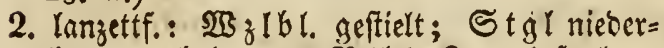
lieg., unbehaart; 3 B Ibl. 3 mal fo lang 396. aโsิ SBlo. ( 4 23. w.) (crantziána.)

3. ellipt., ganzrant., ftiellos; Stgl bin und hergebog., nieberlieg. (O' SE. 23. 24.)

4. långl., - Tpik, ftiellos, ganzranb., unbehaart; Stgl. ftriegl., mit friechemb. S2Bzifprofient; Sd) o ten etras abftebeno (4 SE.)

5. leyerf., rechtwinkl. =gezabnt, bader., mit åft. Scharen; Stgli etwas bláttrig, äftig (ơ v.; wcb.)

397.

serpyllifolı̌a.
394. ciliáris.

\section{Stglblätter gestielt.}

Stglbl. faft cyrunt, mugeidimeift =gezahnt: 400 .

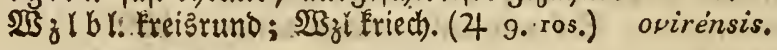

17. ARĂCHIS. 557. $\left(542^{3}\right.$.)

(๑ SAm, gb, - ซ Del)

401. hypoga'ea.

5. ARALLA. 250.

Stengel :

1. baumart. u. nebfit o. Blattch. geftachelt (b 402. NAm. 4. grnch. $\Delta$ ) spinósa.

2. Frautart., glatt; Briąt boppelt jigeiegt;

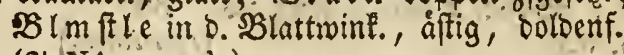
(4 NAm. grnch.) los, unbebaart (4 NAm. grnch.)

403. 404. nudica'ulis. 


\section{ARBǓTUS. 329.}

Stgl. niebergeftrecft; $\mathfrak{B l}$ l. verk, =eyr., leber= artig, ganzrando, neếfírm. = geabert, glánz.

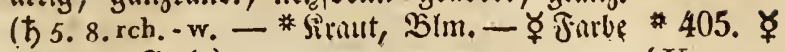
idguary, Berb.)

(Uva ursi.)

19. ARCTĬUM. 634.

Die herzf., geffielt, Stglblätter:

1. feingegahnt; BBlo. unbehaart $\left(\sigma^{7} 22.29, \# 406\right.$. ఫ brn.-r.; w. - * $2 G_{j} l$, Siraut, Saam. — ఫllutr.) (Lappa.)

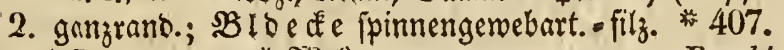
$\left(\sigma^{2} 22.24 .0 * 23 j 1\right)$

Bardána.

\section{ARCTÓTIS. 702. $\left(64^{x} \cdot 7^{x} \cdot 707^{x}\right)$}

Blumenboden:

I. langhnarig; Strahlblüthen;

A. unfruchtbar unb

1. ungeth., lanjettf.; $\mathfrak{B}$ l. fidrot jågefơrm.= fieberf., unten etwas filz. ( $\odot$ SAf. gb. unten rch.)

2. 3.5 their.; 3 I. Lemerf.-fieberf., gezahnt, 409. unten filz. ( $\odot$ SAf. gb., unten grau) hypochondriăca

B. . fruchtbar; $\mathcal{S}$ tg $l$ Krautart., aufr.; Bl.;

1. längl., ftadtelthant.,

a. umfafieno, $\mathfrak{E}$ Kebr., ftechentfpis=ges zahnt; Die a $u \tilde{\beta}$. Brofdup u. et=

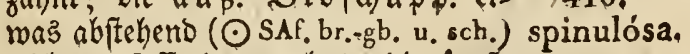

b. nicht umfallent, gezahnt; bie áus. BID.f u up. umgeichlag., gemimp.

(๑ SAf. dr. u. sch.)

2. fieberf., grau, unten filz., mit lanzettf.,

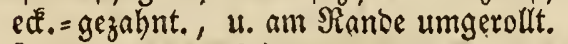
Lapp. (๑ SAf. gb.)

II. fpreuart.; Strahlbliithen:

A. unfruchtbar; Blätter:

1. cinfact)gefied., fil gejahnt (ち SAf.)

2. Dopp. gefieb., gleithbreit, unbehaart; 414. Stgl trautart. ( $\odot$ SAf. v. u. gb.) anthemoídes. 
B. fruchtbar; Stgl frautart.; bie unt. $\mathfrak{S}$ l. geftielt, verf. = enr., auzgeichweift $=$ gezalynt: bie o bern ftielloz̧, lanjettförm., ganżrano. $(\odot$ SAf.)

415.

leucanthemoides.

\section{ARENARǏA. 360. $\left(84^{3}, 305^{3}, 321^{2} \cdot\right)$}

\section{Mit Afterblättern.}

\section{Blätter:}

1. gleidbbreit,

a. Fharfgeipiat; Xfterbl. trodf., icheidef.;

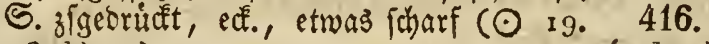
36. bl. - r.)

b. fleifhig; Xfterbl. baitt.; Stgl fein= 417. baar. (๑ 29. 34. w.) medĭa.

2. halbwalgenf., fleifh., obne Spike; $\mathfrak{X} f \mathrm{ft} \mathrm{r}=$ blátt. wie 416; ভ. zigebriadt, geránbelt, 418 . glatt $(\odot \mathrm{r}, 36 . \mathrm{r}$ )

\section{II. ohne Ajterblätter.}

\section{Blätter:}

1. enrumb, fpich,

1. fleirchis (4 36. w.)

2. nicht fleirchig,

a. geftielt, gerippt $(\odot$ 5, w. $)$

b. ftiellos; Blkronea:

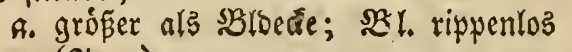
(4.9.)

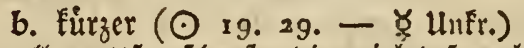

II. fdrmallanzettf., idarf; bie nicht fracht= trag. Stgl ferre lang, riebergeffrectet (4 8. w.)

III. gleidbbreit,

A. Liberall mit baar. Drüfen bebecft; $S$ tgl weitidhueif.; SB lb t. fo lang als SBld.; ङ. geránbelt (4 SAf. w.)

B. nid)t brüfig; Stengel :

1. weitichweif., fabenfơrm., fehr lang; frudtrag. 3weige aufr.; Skapf. cingebriudt $=$ fugel. $(423$.
419:

peploides:

420.

(trinervia.)

421.

multica'ulis:

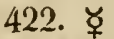

(serpyllifolia.)

423.

montána. 
2. niebergeftrectt; $\Re \mathfrak{Z}$ ld. etmas flebr., mit

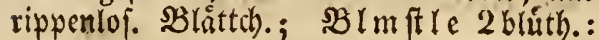
(5noblm ftle aufr. (○, 9.:w.)

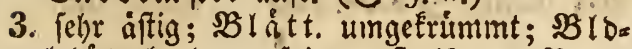

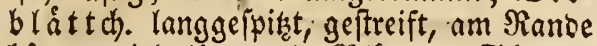
bäut., viel läng. ał's sBlfrone; Skapl. láng. als \$10. (๑)

4. mit aufr. 3 weigen; Enoblmftre fehr lang, 2 zahl.; sBlbl. ftumpf, aubgerand. 428. (4 9.)

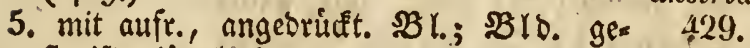
ftreift, längl: (4 9.) striáta.

IV. Galbwalzenf., fleifł., ftumpf; B Blblått. 430 .

lanzettf.; (Enoblm ft le meift 2 zádhl. (4 w.) bavarica.

V. fabenf., gantz gerabe; Stgl einf., aufr., 3 facthgetheilt = rifpenf.; 3 lo. ftumpf, rippen= los, mit Şaut geråno., furry. als bie verE. =evy. $\Re B(b l$. ( 4 NE. w.)

VI. borftenf.; Stgl oben faft nact; 3 Brs. etruas back. (4 8.)

VII. pfriemenfórmig; Stengel :

1. rifpenformig ; Bld.:

a. Comegeipint geftrift

b. leygerna.

b. läng. als b. lanjettf. SBlbl.; Skap 434. aufr. $(\odot$ 8. 22. 23.)

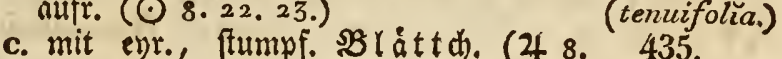 23. $w$.)
435.
(saxatilis.)

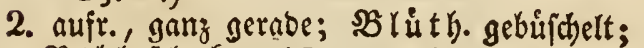
\$B Ibl. jegr Eurz $(\odot$ 19. w.)

3. 1 britth.; $\mathfrak{B l}$. fladh, ganz gerabe: $\mathbb{Z}_{3} \mathrm{z}_{\mathrm{l}}$ blátt. georängt (4 SE. 9.)

VIII. gleidhbr. = pfriemenfótim.;

431.

graminifolǐa.

iaricifolia.

436.

fasciculáto.

437.

grandifìra.

1. umgetrümmt,

a. 1 feit., gefurdt; Stgl niebergeffrecelt, B lmftre meift 2 britth.: Enoblmftre alfír.; BBls. geftreift (4 9.) recúrva.

b. Enoblit th. meift gepaart; B B be de fpis, 3 ripp., fưrz. als sBle. u. nevft 439. o. weitichuveif. Stgln feingant. (4 SE. 9.) rostráta:

2. 3 ripp.; Enoblitthen gepaat; sgib=

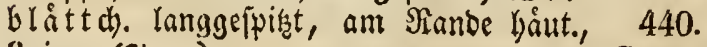
3 ripp. (4 9.)

Geràrdi. 
13. ARGEMÓNE. 418 .

Stap f. 6 [dal.; $3 B$ l. born. ( $\odot$ SAm. gb.) mexicána.

441.

\section{ARISTOLOCHǏA. 723.}

\section{Stengel:}

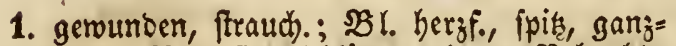
ranb.; $\mathbb{B}$ im ft le 1 blith., mit eyr. Mebenbl.; SBlf. auffteig., mit 3 ppalt., gleidhf. Saum (ち NAm. 8. glbch, - grn, u, tr, - braungeaderl) Sipho.

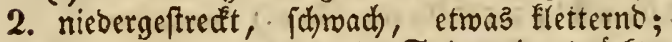
$2 B$ t. herzf., eyr., an b. Spike eingedriudét; B IE. aufr., mit lanżettf., jpis. Sippe (4* 443. ఫ̧ dp. - * $\mathfrak{S B}_{\mathfrak{z}} \mathfrak{l}$ - $\Varangle \mathrm{lunfr}$.) longa.

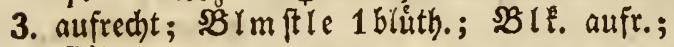
Blätter:

a. faft ftiefulos, hers $f_{0}=e \mathfrak{e n c}$, ftumpf; S $\mathrm{tgl}$ faft einf.; $B$ Imftele einzeln; $B$ lelippe längl., an o. Spitze eingeoriudt (4 gb. u. * 444 .

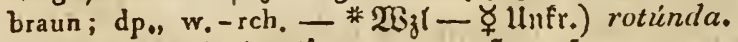

b. gef́tielt, rundl. = herzfơrm., etwas ftumpf;

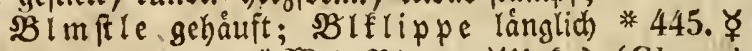

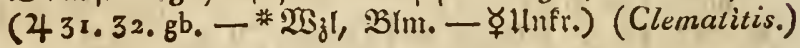

\section{ARISTOTELIA. 376.}

Iraubenbluth. $2-4$ żål. ; $\mathfrak{P}$ l. entgegen= gef., geftielt, enr. u. lanjettform., gejägt, 3 ripy., glánz., baur.; 2 efte zahlreid) (ち SAm. w. $\triangle$ )

446. Macqui.

\section{ARMERǏA. 251.}

Schaft:

I. ftielruno,

A. unbehnart; bie flachen Blätter:

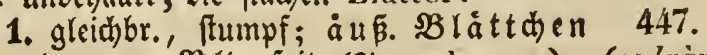

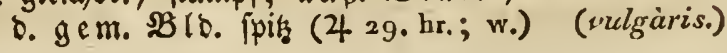




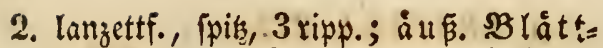
d) en. g. gem. SB ro. ellipt., fiectheno=

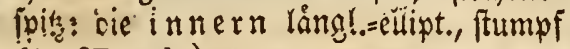
(4 SE. sch.)

B. feinhaar.; $B \mathfrak{B}$ l. gleid)br., flach, ftumpf, am (Srunde getrimp.; B lát td). $0.9 \mathrm{em}$. BB Io. ftumpf ( 49.48 . ros.)

C. etwas Fcharf; $\mathscr{B}$ l. lanzettform., fpik,

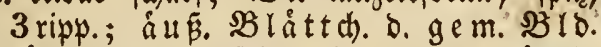
längl. =evrumo, fipiz: bie innern länglo, ftumpf (4 SE.)

II. etraas źgedrúdt, glatt; $\mathfrak{B}$ lått. gleichbreit,

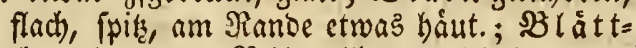
d) $\mathfrak{n}$ o. ge m. $\mathfrak{B} \mathfrak{l}$ b. ellipt.=rundl. (4 9. v.) alpina. III. binjenart.; $B$ I. Fobmallanzettr., ipis, flact), unterhalb idmaler (4 SE. 8. w.)

448. scorzonerifolia. maritíma.

450. plantaginěa. 451. 452. alliacěa.

\section{ARNICA. 679.}

I. Mit nadt., 1 blith. Schaft; $B$ l. geftielt, 453 . verk. =eyr., ausgefchweift $\left(49 . r_{0} ; w_{0}\right)$ Bellidicistrum.

II. mit Stengel; Blätter:

A. ungeth., eyr.: Stglbl. gepaart, entgegenger. (4 3. 9. gb. - * *ajl, Siraut, * 454. ఫ

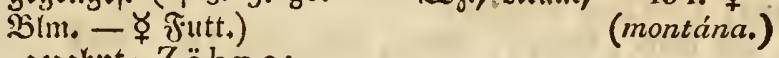

B. gegahnt; $Z$ ähne:

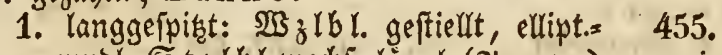
rund.: St glb l. medh), längl. (4 9.49.) scorpioides.

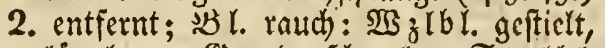
långl., am Sirunbe fdimaler: Stglbl. wedb)., lảngl. = lanzettf. $(4 \quad 10$.

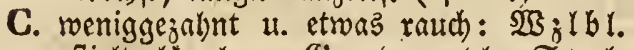
geftielt, langl., am Grunde rundl.: St $g l_{3}$ blát t. wie vorber $\left(\begin{array}{ll}4 & 0 .\end{array}\right)$

456. Doronicum.

\section{ARNOPÓGON. 609.}

Blumendecke:

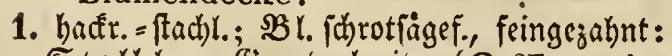
Stglbl. am Srrumbe breiter ( $\odot$ SE. grnch,

2. feinhaar., welhrloz; $\$$ Bl. fhrotjāgef.egezahnt (4 SE. gb. u. rch.)

458.

picroídes. 459. Dalechampị. 


\section{ARTEMISIัA, $655 .\left(662^{x}.\right)$}

\section{Strauchig.}

Stengel:

1. ganz gerabe; bie unt. $\mathfrak{B}$ l. bopp. geficbert: bie obern einfach = gefiebert, baarf.; $2 B$ lo. GalbÉgel., feinhaar. († 2.8. gbch., nebst \#461. Bl. starkriech. - * Sirauti)

Abrotänum.

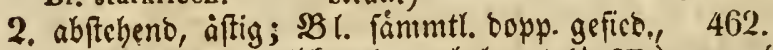
haarf.; SBID. balbfugel., unbehaart ( $5 \mathrm{SE}$.) procéra.

\section{Krautartig;}

\section{A. Stengel einfach;}

\section{Blätter gleichfürmig.}

- Stgl ganz sinf.; fänmtliche Blätter:

1. hanbf.=bielipalt., mit feibenart. meíp. Uebers zug; Blüthen stehen:

a. am Enbe, fitiellos, fugel., gefnauelt (4 SE, ra.gb.)

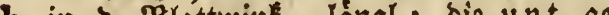
ftielt: bie obern ftiellos $(4$ 1 o.) mutellinas

2. fammf. = gefied, unbehnart, mit gleidbr..* fabenf. Fiebern; $B$ I m f It le 1 blith., in ten SBlattwink.; $\mathfrak{B}$ ID f d u p p. trode., glánjend (O'SE. 27.)

3. Dopp. = fieberf., etwas feinhaar., mit fahmals lanzettf., langgeipizten, ganzrano. Rappen; Enotraub. nacti ; Blúth. ůberí)angeno (4 SE. 9.)

4. 3 fad $=$ Fieberform., haar.; $\Re$ rispe blattloz;

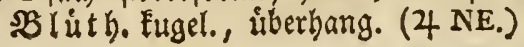

465. pectináta.

2. Blätter ungleichförm.

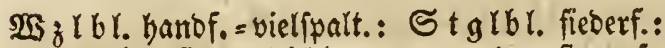
bie oberften gleidbbr., ganzrans., ftumpf; 
2lle mit Feibenartigem weišem Uteberzug; Bliith. in D. Slattwinf.: Die unterften 468. geftielt $(4 \quad 10$.

\section{B. Stongel ästig;}

1. Blätter einfach u.

a. gleichförmig.

2B 1. unbehaart, lanzettf., auf beyden Enoen

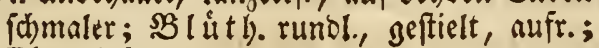
Blmstiele:

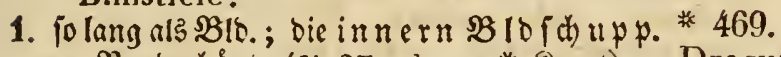
am アianbe boint. (4 SE. gb. - * Siruut) Dracuncŭlus.

2. vielmal linger; $\mathfrak{B} 10$ / $\mathfrak{d} u p$. fámmtl. am 470 .

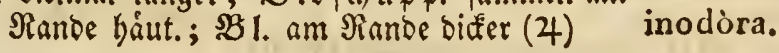

\section{b. ungleichfürmig.}

\section{Blätter:}

1. grau, lamjettf., ganzrand.: $\sqrt{\mathfrak{B}} \mathfrak{j} \mid \mathfrak{l} \mathfrak{l}$. jer= ichnitt.; $B$ l h th. lângl., geftielt, úberhang. (4 SE. 36.)

2. unten filz.; $S_{\mathrm{tg}} \mathbf{l b l}$. fieberf., mit lanjettf., 471. coeruléscens.

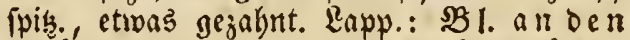

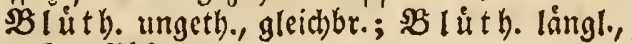
aufr.; Bld.:

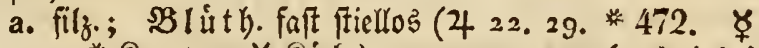
- * Siraut - ఫ Sind.).

b. unbehaart; $\mathfrak{B}$ lúth. gano ffiellos (4 SAs.) (rullgáris.) indica.

2. Blätter zsgesetzt,

a. gleichförmig.

Die jämmtlidh gefieot. Blätter:

1. Jurke feibenart. = grau: Fied. D. Stglbr. 3 theil., gleid)br., langgeipigt; "Etgl auf*

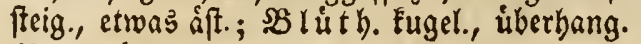
(4 NE.)

2. mur bie Wzlbl., u.

a. mit 3 fpalt. Lapp.; Stglbl. borftenf., unbegaart; Stgl nicocrgeftredt, rutbenf.s äflig; 32 lit tb. eyr., geftielt (42, 29. * 475. grau; rch. - * Eaam. - $\Varangle$ Uufr.)

474. sericěa. 
b. mit vielipalt. Iapp.; Stglblatt. und Bluth. wie vorber; Stgl aufr., mit zertheilt. 3weigen ( $\odot \sigma^{\top} \mathrm{SE}$, 19. Blüthen sehr klein)

476.

scoparı̆a.

b. ungleichförmig;

a. Stglbl. gefiedert.

Andersgestaltete $\mathrm{Bl}$. finden sich:

I. an o. Stgl felbft: 3 palt.: 2llfe Stglbl. fabenf., feinhaar.: $\subseteq t g l$ aufiteig., etwas getlyeilt; $\Re$ ifpe ganz gerabe, faft 1 leit.;

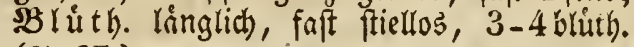
(4 SE.)

II. an b. $\mathfrak{B}_{z} \mathfrak{l}$ u. am untern $\mathfrak{X}$ beil bes Stgls: 3 theil. = vielpalt: $\mathfrak{l} \mathfrak{l} \mathfrak{l e} \subseteq t g l b l$. gleichbr., unbehaart: bie jüngern wei $\bar{\beta}=$ grau; Stgl rifpenf., aufr.; Shlu th. evr.; BBlmftle úberbang. (4 NE.)

478.

neglècta.

III. an ben $S^{B} \mathfrak{i}$ then: ungeth., gleichbr. ob. cinfad); Stglbl.:

1. unbebaart, etwas fleifd., mit einf. od. 2 fpalt., gleichbr., ftumpf. Fiebern; Stal auffteig.; $\mathfrak{B} \mathfrak{l}$ úth. långl., geftielt, aujr. (4 SE. 19. 36.)

2. reifgrau, gleitbbr. = fatenf.; Stgl auf = fteig., fehr áft., rifpenf.; $\mathfrak{B}_{\mathrm{I}} \mathrm{n}$ ft le faft Doloentraubenf.; $\mathfrak{B}$ ID. etwas Eugel.=edf., íberbang. (4 SE. 19. 23.)

477.

pauciflóra.

479.

crithmifol̆a.

480. saxatǐlis.

3. mit feibenart. weiß. Ueberzug u. 3 theil., gleichbr., fpik. Fiedern; Stgl aufrecht; şlúth. runbl., geftielt, úbergebog. (4 NE.)

IV. an o. 3 weigen: einfach, gleichbr.; Alle Blätter:

1. weifgrau; Fiebern b. $\mathbb{Z}$; glbl. 3 theil., u. nebit ben Fiebern o. Stglblått. gleichbr. = fabenf.; Stgl aufr., rifpenf., mit etwas aufr. Sweigen; $\mathfrak{B}$ 'úth. làngl., geftielt, ůberbang. ( $4 \quad 7$.)

2. idhneeweis = filz.; Stglbl. Intit gleichbr., ftumpf. Fiebern; Stgl aufiteigent, mit úbergebog. 2leften; BB lúth. lángl., filz., faft ftiellos (4 36.)

483.

(maritima.) 
b. die untern Bl. gefiedert.

1. Die úbrigen $\mathfrak{B}$ l. einfach), u. nebft Fie= bern b. unt. SBl. idomallanzettf.: Zlle graugrun, feinbaar.; Stgl aufr., rifpenf., gam gerabe; $\mathfrak{B} \mathfrak{l}$ (úth. Eugel., geftielt, uber= bangeno ( $\odot$ NAs.)

2. bie 3 weigebl. 3 theil. ob. einf. : Fiebern 484. glauca.

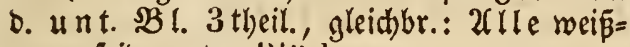
grau = jeibenart.; Bliithen :

a. geftielt, runol., úberbang.; Stgl aufr., 485. rifpenf. (4 23.) austriăca.

b. ftiellos, evruno; Stgl etwas aufrecht, 486 . febr áft. (4) nivěa.

c. Stglbl. dopp. fiederförm.

Lapp. b. Stglbl. langgefpitzt: $\mathfrak{B l}$ a a $b$. slúth. 3 fpalt., od. lamgettf., foft io lang als bie 3meige o. Rifpe: Xlle etroas mei $\bar{\beta}=$ grau; $B \mathfrak{l}$ úth. Eugel., geftielt, úberbang.; BBlo. trodf. (o Ni.)

487.

siversiána.

3. Stglblätter od. unt. Bl. doppelt zsgesetzt.

Aufser diesen:

1. einfache Blätter,

a. an ben $B$ lúth.; Fiebern b. Stglbl.

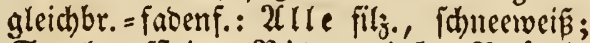
Stgl auffeig.; $\mathfrak{R} i$ spe einf.; $\mathbb{B}$ lúth). längl., ftiellos, aufr. (4 SE. r9.)

b. an b. 3reigen; fieberno. Stglbl.

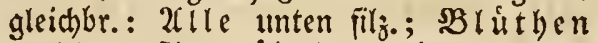
runol., geftielt, uberbang. (4 2. 8. Ix. * 489. gbch. - * Siriut)

2. gefieberte oberbalb, boppeltgefieberte unter= halb, mit gleichbr. = fabenf. Fiebern: 2rre Bl. weiß̈gtau; Stgl aufr.; Blúth. långl., 490. ftiellos (4)

488. vallesiăca.

taurǐca.

4. Wzlbl. dopp. zsgesetzt.

Die gefies. Stglbl.:

1. umjaff., mit gleithbr. \&app.: 3weigebl. einf., gleichbr.: 2Clle sBl. etwas weipgrau; 
Stgl mufitteig., ripenf.; Blo. långl., aufr., 491. geftielt (24 SE. 17. .)

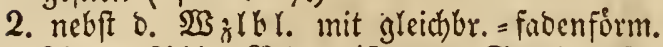

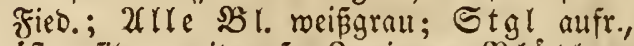
áft., filz., mit aufr. Stweigen; sBluthen lángl., aufr., faft ftiellos (4 SE.)

monogy̆na.

\section{St glbl. 3 fach zsgesetzt.}

Stglbl. fabenf., unbebaart; Stgl aufredit, 493. rifpenf.; $\mathfrak{B l}$ (úth). Fugel., geftielt (4 NE.) herbacěa:

\section{Wzlbl. $3 f$ ach fiederf.}

$\mathbb{Z}_{3}$ rbl. mit lanzettf., gezalynt., ftumpf. Lapp.; Stglbl. bopp. od. eimfachfiederf., mit lan= zettf., etwas fyitz. Lapp.; $\mathfrak{B}$ l. an b.' $\mathfrak{B}$ lit th. ungeth., lanjettf.; 2lle meişgrau; Brut th. Fugel., geftielt, úberbang. (4 22. bgb. - * 494. ఫ * Siraut - ఫ̛ (sirb.)

(Absinthium.)

7. Wzlbl. 3 fach gefiedert.

Die unt. Stglbl. ficberf.: bie obern un= getheilt, gleid)br.: Xrrte unbebaart; Stgl ganj gerabe; $s$ lúth. runol., faft ftiellos, aufr. $\left(\sigma^{\prime} \mathrm{NE}.\right)$

495.

biénnis:

\section{ARUM. 761. $\left(723^{x} \cdot 79^{5^{4}} \cdot\right)$}

\section{Shne Stengel; Blätter;}

1. fußf., ganjrano.; Kolben:

a. Yauj behaart. Satheibe (4 SE.)

b. pfriemenf., láng. nls bie långlo, zigerollt. Scheibe (4 NAm)

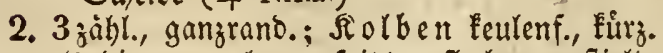
als bie enr., langgeipist., flache, geftielte Stheice (4 NAm.)

3. โpic巨F. zpfeilf.: Lappen:

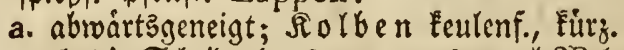
als bie Sheibe (4 SE. w. grnch. — $2 \mathbb{S}_{3} l_{\text {, }}$ Siraut - $\Varangle M e b l$ )

† Dracuncŭlus. 497.

Dracontǐum.

$$
\begin{gathered}
498 . \\
\text { triphýllum. }
\end{gathered}
$$




\section{Arum. - Asclepias}

b. ausgebtcitet, mit ohrf. 2fnhang; Rol 500 . ben rwie vorker; $\mathfrak{B}$ l. weißsnoer. (4 SE.) italǐcum:

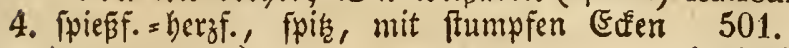
(4 NAm, 48.)

t virginı̌cum.

\section{ASĂRUM. 379 ;}

\section{Blätter :}

1. nierenform., gepaart,

a. ftumpf, unbehaart; $\Re$ rs. 3 ipalt. (4 4. $* 502$.

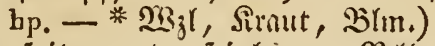

b. Fpis, unten feinharar.; $B$ B r e de 3 their. (4 NAm, r. - braun)

2. Gerzf., ftumpf, unbeharart, geffieft (4 $\mathrm{NAm}$. dr., Bl: weilsgelleckt)

† (europá eum.)

503.

canadénse;

virginıัcumi

\section{ASCLEPIAS. 174:}

\section{Blätter entgegengesetzt.}

\section{Blätter:}

1. oval, unten fitz.; Stgl gand einf.; Dol=

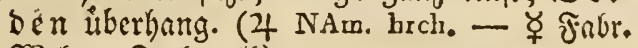
Weber, Finrt. ger6)

2. lángl. =oval, unten etwas feinthaar., faft ftiellos; Stgl einf., mit e. bopp. Sieitie v: feinen Saar.; Endo ord. H. Rect. aufr.,

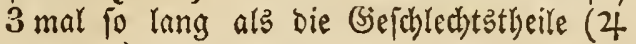
NAm, p.)

3. evrunds

a. unten langhaar.; Stgl eimf.; Dolbe 507. aufr.; Rect. umgebreht (4 NAm. up.) purpuráscéns:

b. unbehaart, runjl.; $\subseteq t g l$ einf.; Dol= $50 S$.

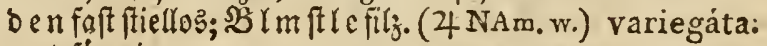

4. ' lanjettfórmig,

a. unten feinhar.; Stgl oberbarb geth.; 509 .

Doiben aufr., gepaart (4 NAm.) pulchra.

b: unbehant; Stgl u. Dorben wie vor $=510$. ber (4 NAm. grn, u. w.) incarnáta:

\section{Blätier wiechselsweisstehend:}

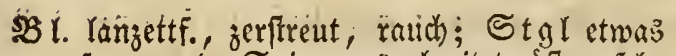
aufr., on o. Spige nusgebreitet =åft., febr

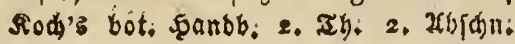


raud); Doldece. abitebenben Enbooldens traub. àbnl. (4 NAm, gb.)

511.

tuberósa.

\section{ASCY̌RUM. 599.}

\$BI. enr.; Stgl ftieltund; Rifpe gabelform. (ち NAm. gb.)

512.

Crux Andréae.

\section{ASPAPĂGUS. 286.}

Stgl trautart., ffictruno, aufr.;,$B \mathfrak{l}$. Gor= ftenf., gebuiftelt ? 2 f $t$ ter bl. paarweis am

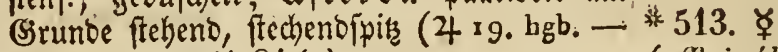
* $\mathfrak{W}_{j}$ lfeim - ૪ Siud.).

(ofricinćlis.)

\section{ASPERÚGO. 103.}

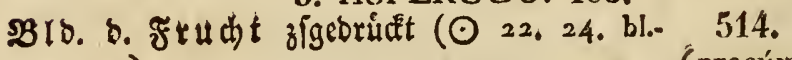
$\left.v_{0} ; w_{0}\right)$

\section{ASPERUัLA. $67 .\left(37^{2}\right.$.)}

\section{Alle Blätter:}

1. 8 zåbl., lamgettf.; Endobroentraub.

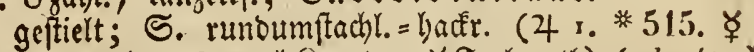
4. w. wohlriech. - * Siraut - ૪̧ふarb. rotf) (odoráta.)

2. 4 zăhlig,

a. eyt. =raifjettf., (3 ripp.); (End britth. 516. gebifchelt (4 SE. w.) taurína.

b. $\operatorname{lan}_{3}$ ettf. = gleidbbr.; Stgl aufr., \&ift., 517. B r úth. meift 3 ipart. (4 SE. rch.) pyrenăca.

c. gleichbr.: bie $u n t$ t. bajdjiegelf.; Stgl am (situnbe febr âftig, niebergeffredt; 518 . Blúth. 4 fpalt. (4 As, 23.) supina.

d. ellipt., unbeutlich gerippt, eben, am Ranbe unbehaart; Frúd te fharf (4 519. 후

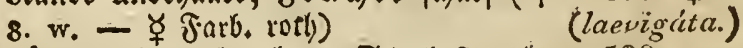

e. Iångl., ftumpf, eben; Stgl frauth. (万 SE. p.)

520 . calabrica. 


$$
\text { Asperula. - Aster. }
$$

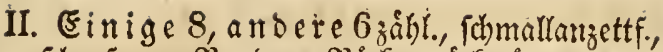

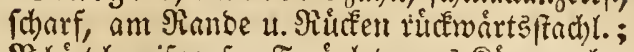

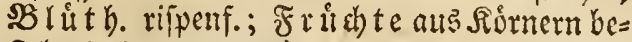
feheno (4 NAs, w.)

III. Die unstersten :

521.

Aparíne.

A. 4 jáblig;

1. verf: ér.: bie oberfiten 5,6 ober

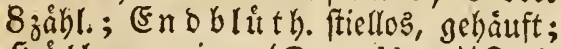

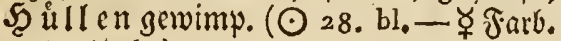
rotl); $\mathfrak{l u n f r}_{\text {.) }}$

2. Ianzettf.; bie obe exit gleidhbr., febri

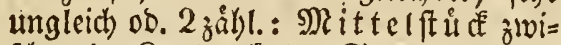
fohen D. Snoten Kurz; Stgl aufrecht; B It. 4 palt.; $\mathfrak{B}$ I m ft le immer zu 3 ge= theilt; $\mathfrak{F} \mathfrak{r} \mathfrak{u}$ d) $\mathfrak{f}$ unbebaart, etwas fnot. (4 3. i $\mathrm{I}_{0} 2 \mathrm{I}: \mathrm{w}_{0} ;$ rch. - $\Varangle$ Parb. roth) (cynanchica.)

B. 6 jäbl: ; bie o berften entgegenger.: bie 522. ४ ४ (arvénsis.) mittlein 4 jóhl.: 2ellegleichbr.; Stgl ichlaff; Blk.:
1. 3 fpalt.; gliatt (4 6. $2 \mathrm{r}_{0}, \mathrm{w}_{0}$, ausw. rch. - $\forall$ rsarb. roty)
2. 4 jpalt., ausw. fcharf (4 SE. w.) montana.

\section{ASPHODËLUS, 275.}

\section{Blätter :}

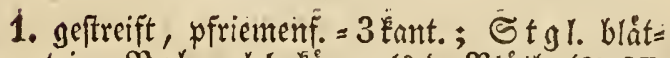

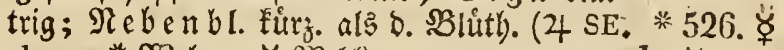

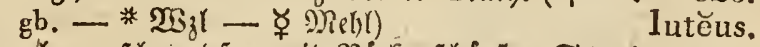

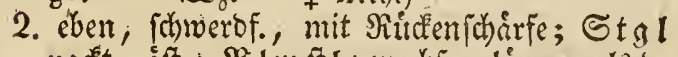

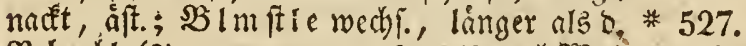

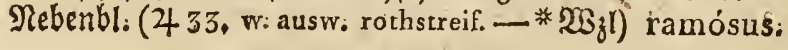

\section{ASTER, 683,}

\section{Sterigel $\mathbf{x}=\mathbf{2}$ blüthig:}

\section{Stengel:}

1. 1 blíth.; ;Bi. ganzrand.; bie faft gleiden Bldschupp:: 
a. etruas ftumpf, lanzettf.; $: \mathbb{B} ; 1 \mathrm{~b}$ !. Ian= zettf. = ipatelf.: Stglbl. lanjettfơrm. (4 8. bl.)

b. langgeipist, gleichbt.; $\mathfrak{W B}_{\mathfrak{z}} \mid \mathfrak{b} \mathfrak{l}$. [pas telf.; Stglbl. jchmallanzettformig (4 As. rch.)

2. meift 2 blutth.; $\mathfrak{B l}$ l. fiellos, lanzettf., ges fágt, ficharf; $\mathfrak{B}$ lo ld up p. badjziegelf., an= georúdt, evr. (4 NAm.)

528. alpinus.

529. pulchéllus.

\section{Stengel mehrblüthig;}

A. Blätterganzrendig,

1. gleichbr, oder lanzettf.,

a. umfassend.

\section{Blätter:}

1. am Rande,

a. febarf, Iamjettf., faft umfafiend, an ber Spitze fomaler; Stgl vom Sirunde an áft.; aufred)t, mit haar. bei. Bweigen; SBIb f chupen iblaff, gleichbreit, fpits, gleich (4 NAm.)

b. gewimp. = hacefr., gleikbr., fteif, etwas

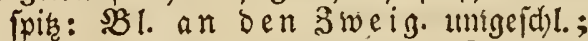
Stengel. áf., ftachellyaar.; 3 weige

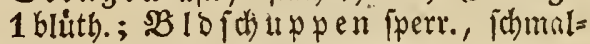
lanjettf. (4 NAm, bl.)

2. unten feinbaar., lanzettf. = herzf., gang'um= falf.; Stglganj einf., feinkaar., mit rib= penf. Spite; $\mathfrak{B}$ Io id) upp. idlaff, oach= ziegelf., lanjettf. (4 NAm.)

3. auf beyben Eeiten haar., langl. = lanjettf., gewimp., berzf., ganzumfaff.; Stg! âftig, ftachelbarr., mit abjteheno., verlang., we=

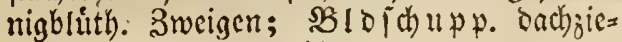
gelf., lanzettf., etwaşabftebeno ( $4 \mathrm{NAm} . \mathrm{hbl}$.) patens.

4. unbehart, lanzettf., ganzumfaffent, am (Srunbe mit ofrr. 2(nl)ang; Die fchloffen, lan= gettf. BIdschupp.:

a. länger als bie Srheibe; Stengel hanr., ganz gernde; Enoblutb. gedrangt (4 NAm. bl.)

b. cben folang; Stgl haar., rispenf., mit meift 1 blith. 3 meigen (4 NAm.)

531.

aestivus.
532. grandiflórus.

533. phlogifolius.
535.

novae angliae. 536. spurius. 


\section{b. nicht umfassend;}

ค. 3 rippig.

\section{Blätter:}

1. am Ranbe fharf,

a. punctirt; Blkstrahl :

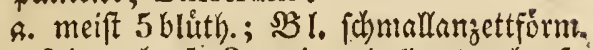
fwis; bef. 3weige bolbentraubenf.= gleid)hoch, zigezog.; \$Blo. bachziegelf., balb fo lang als bie Scheibe (4 NAm. w-v.)

b. meift 10 blitth.; $\mathfrak{B}$ I. gleichbr., entfernt, langgerpint; bef. 3 weige bolbentrau= benf. = gleichbod, ausgefpert; $\mathfrak{B} 18$. wie vorber (4 SE, 1 7. w - v.)

b. nicht punct., fohmallanzettformt., unbeutl. 3 ripp., ftumpf; 3 weige doldentrauben= form. = gleich hoch, $\mathfrak{B}$ I 0 . wic 537; $\mathfrak{B}$ I $\mathrm{D}=$ fd) upp. lángl., ftumpf, angeoruedt (4. NAm.)

2. netwimp.: Stg $\mid b l$. Fomallanzettf., gerippt; B l. a n D. Swe ig. fehr furz., lanjettform., 3 ripp.; Stgl áft, frinbar.; SBlo. Darts. jiegeff., mit jpatelf., fperr. Echupp. (4 NAm.) ciliátus.

3. auf beyd. Seiten feinbaar.=weipgrnu, fdymal= lanjettf.; 3 weige boloentraubenf., ausge= breit.; $\mathfrak{B}$ I 0 . badtzitigelf, balb fo lang als D. Scheibe (4 SE.)

4. unbel)aart, fomallanzettf:,

a: ftumpf, ftechenofpits, am Grunbe aber.; Stgl einf., oben boldentraubenf., feintban= rig; $\mathbb{B}$ I d f f c u p p. Ianzettf., faft gleich (4 NAs. bl.)

b. ber. 3 weige bolbenf.= bolsentrabenf.; B ID. bactziegelf., balb fo lang als bie Echeibe ( $42.8 \cdot \mathrm{dbl}$ ).

\section{7. \\ hyssopifolrus.}

538.

punctátus:

539.
solidaginoides.

541.
canus.

541.
canus.

\section{0.}


2. fomallanzettform., am Sorunde fhimaler: Stglbl.umgeichl.; 3roeige wie vorker:

- be f. Stweiae fabenf., 1 bluth.; $\mathfrak{B}$ lo. oadbiegelf., halb fo lang als bie Scheibe (4 NAm; bl., Scheibe w.)

II. am ?ande fharf,

1. gleichbr., ftechenofpis: Stglbl. ganz gerabe: 3roeigebl. abftebend; Stgl ohen âft.; 3weige 1 bluth., gleichboch; $\Re$ I 0 . bachjiegelf., fo lang als bie Edeibẹ (4 NAm; dbl.; v.)

546.

linariifolusus.

2. Ianzettf., am (Siunde fimaler, Iangge fpigt; Stgl einf., an b. Spize bolben= traubenf.; SBlof thupp. lanjettf., idhlaff (4 NAm, w.)

III. auf beyben Seiten foharf, langl.= lanjettf.; 3 weige bolbentranbenfo; $\$ B$ I s. bachjiegelf., etwas iperrig, mit ftumpfen 2 láttch).: bie innern baut., an $\delta$. Spize gefärbt (46. bl. ; w; r.)

IV. am Ranoe hackigig,

A. [d.mallanjettf.; ber unbehante Stgl:

1. åft., aufr., mit 1 bluth. ber. 3weigen; Bl. an beyden Enden fhmaler; 310 . ondyjiegelf., mit langl., fpits., fchlafi. Schupp. (4 NAm. w.)

2. einfach), oben boldentraubenf.; $\mathfrak{B}(\delta=$ id) upp. lamjettf., ftumpf, gleid) (4 $S E$. $x_{0}-v$. $)$

B. gleid)br., ftumpf, umgefdlag.; $\subseteq t g l$ unbehaart, áft., weitjchmeif., mit 1 bluth. bel. Sweigen; $B$ ID. Dathziendform., mit gleichbr: = pantelf., ftumpf. Sctuppen (4 NAm.)

545.

nemorális.

\section{7. \\ umbellátus.}

548.

(Améllus.)

549.

tenuifolǐus.

550.

pannonicus.

V. auf bero. Eeitem mit meiß̄., frinhane. Ueber= zug, längl.slanjettfơr!n.; S $\operatorname{tgl}$ ganz einf., aufr., feinhar.; mit Endtrauh.; $\mathfrak{B} 1 \mathrm{~d}$. bachsiegelf., mit lanzettf., feibenart., ange三 brúcft. Schuppen (4 NAm, hbl, )
552.
concŏlor.

VI. weis = langhaar., fômallanzettf.; Stgl áf., langhaar.; bef. Sweige meift 1 jeit., 1 bluth.; $\mathfrak{B}$ lo. ichlaff, bachzicgelf. , mit lans zettf., fted)endipif. Sdupp. (4 NAm.) 
VII. unbebaart, gleichbreit,

A. verichiedengeftaltet; Zweigebl.:

1. pfrientenf., angenąbert: Stglbl. ver=

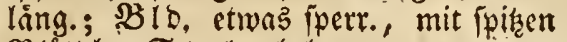
Blâtth); Stglunbehant (4 NAm,w.) ericoides.

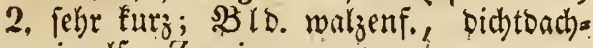
ziegelf.; Zweige:

a. rifpenf. (4 NAm. w.; hbl,)

b. 1 bluth. ; 3 weigebl. ungeid)lag.; Stgl rijpenf., febr aft., foft glatt (4 NAm, w.)

B. gleid)formig,

\section{4.}

555.

dumósus.

5.56.

sparsillórus:

1. fommallanjettf.; Stgl:

a. feinl)ar., rispenf., aufr.; B Y. lang= geipist, an beyben Enoen idhaler; 3roeige menigblith.; $3 B(b$. bach. jiegelf., mit gleichbr., fpis., angex. orudt. Sctupp. (4 NAm. w.)

557.

foliolosus.

b. glatt; $\mathfrak{B}$ lo. bact)ziegelf, , fdlaff (4 558 .

NAm. bl. - R.)

2. gleichbreit,

a. wechlelsmeisfteheno; (5noblüthen einjeln (4 NAm. w.)

b. nicht; $\subseteq \operatorname{tgl}$ feinbaar., febr áftig, weitid)weif., mit 1 jeit. bei. Siveis gen; $S B$ lo. Dachjiegelf., mit lángl., iperrig, ipiz. Schup. (4 NAm.) multiflorus,

3. $23 z$ lbl. lanjettf., ftumpf; Stgl faft nackt, fabenf., etwas ăft,; $\mathfrak{B}$ /mftle 561. madét $(\mathrm{NAm}$,

salicifolǐus:

559.

riğda.

560.

vernus:

\section{2. eyrund.}

SB r. enr., fpit, geftielt, unbehaart, am Siance

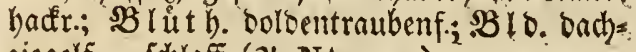
ziegelf., fhlaff ( 4 NAm. w.)

562. huminlis.

B. Blätter ges ägt

1. gleichförmig.

Blätter:

I. ganzumfaffeno,

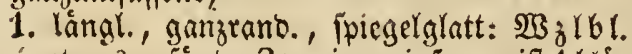
etwas gejâgt; 3 weige einf., meift 1 bli: 
thig; $\mathfrak{B l}$. bachziegelf., mit fajt teilf; fpizen, an ber Spine bidern SBlättch.; Stgl unbehaart (4 NAm. bl.)

2. lanjettiormig,

a. Irle gefagt, etmas fcharf; 3meig. ris= penf.; $\mathfrak{B}$ ld. Fhlofi, läng. als b. Stheibe, mit fidmallanzettf., faft gleieben sBlatt= d)en; S tgl hadfr. (4 NAm.hbl.; dp, ; grn-brn.)

b. nur die unt. etrons gefãgt, unbchaart; BID. pperr., furyer als Scheibe, mit gleichbr. = Feilf. Blåttch). (4 NAm. hv., Scheibe gb., dann brnch.)

II. fait umfanfeno,

1. lanzettformig,

a. 2lI le gefagt, haarig = fdarf; $B$ I fhlaff, mit lanjettf., langgefpist., blatt= art., hackr. SBlattch). (4 NAs, bl.)

b. nur bie untern

A. gejảgt:

1) 2crle unbehaart, am Sande icharf; ని weige etwas geth.; SBID. Io= deroachjiegelf., mit ichmallanzettf. Slattch.; $\subseteq t g l$ ftielruno, unbe= baart ( 4 NAm, hv.)

2) Etgl unbehant; bef. 3weige

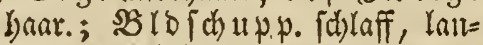

zettf., Dactjicgelf. (4 NAm.) floribúndus.

b. etwas gefágt, unbehart; Stengel einf., an b. Spine rifpenf.; SBI. 569. bichtbactjiegelf. (4 NAm. bl.-v.) concínnus.

2. fomallanzettf., oben fcharf; bie unt. ct=

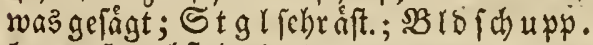
Injettf., abftebeno (4 NAm. hr.)

3. breitlangettf., etwns gelágt; $\mathscr{W B}_{j} l b l$ in D. Mitte gelagt; Stgl febr aft., unbe= bairt; $2 B$ is ich up p. lanjettf., iblaff, Eurry. als Schcibe (4 NAm. w., dann, dv.)

III. ftielloz,

1.: langl. = lanzettf., unbebaart, am Ranbe fdarf; bie unt. gelägt; 3 weig e doloene traubenform., unbehaart: be . Sweige 1 brith.; \&B Io f ch up p. lanzettfo, abftebeno (4 NAm, bl.)

570. bellidiflórus.

566.

sibiricus.

564.

punicĕus.

565.

adulterínus. novi belğir.

568.

567.

, belgir:
571. versicǔlor.

2. lanjettfơrmig, 
a. Xlle geliogt i. unbcharart; Bld.:

a. Dachilegelf.; Stgl ftielrumb, unbes baart; B weige rutbenf. (NAm, w.: dann rch.)

b. fchlaff, mit lanzettf. =gleichbr., faft gleidh., unbebaart, $\mathfrak{B}$ láttch.; $\mathfrak{R} \mathfrak{l}$. am (3runde fomaler (4 NAm, gb,; v.-w.)

b. nur s. unt. gejågt: 2 rle langgeipist; Stgl åft., unbebanrt, aubgebreitet;

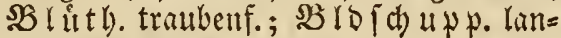
zettf., jhlaff (4 N̦Am.)

IV. etroas geftielt,

1. cllipt. =lanjettf., gejågt, unbehaart; Stgl feimbar.; Zweige:

a. abfteh); $\Re 21 D$. Dachjicgelf. (4 NAm.w.) diffúsus,

b. felyr ausgebreit., bang.; bef. 3 we ige etmaz entferttfelyend (4 NAm. gb., dann braun.)

2. Yanjettformig,

a. ctwas fharf: bie unt. gefigt; 3 we ige boldentraubenf.; $\mathfrak{B}$ loble blattart., faft feilf., etwas fpis, fperr. (4) NAm, bl.)

b. am Ranoc fharf, langgefpiat: $\operatorname{Stg}^{\mathrm{K}}=$ blátt. an b. Spiźe gefảgt: 3 weigebl. ganjrato.; Stgl gatz unbebant, an D. Spite rifpenf.; $B$ lo. Lodfersadyjegelf., mit gleichbr. = pfriemenform. Slattdoen (4 NAm. w.)

3. fchinallamjettformig,

a. langgcipifst, am Rande fcharf: bie un terfe netras gefägt; St gl rifpenf.; bef. 3weige 1 bititb.; sBlo. locer= backjiegelf., mit lanzettform. Blăttchett (4 NAm. hlila)

b. fleifh. 3 frachgeripyt: bie unterften an b. Spitge etwas gefägt; Stghull= behart, boloentraubenf.; $\mathfrak{B} l$ lo. gleidhf. (ठ 17.36. bl.)

578. spectabilis.
573.

Tradescánti.

574.

tardiflórus.

\author{
575. \\ viminčus.
}

pendùlus.

579.
simplex.

\section{2. ungleichfürmig;} a. die obern ganzrandig:

Die obern Blätter:

\section{0. \\ emı̆nens.}

581.

(Tripolŭum.)

\section{J. foft umfanjeno,}


1. längl., faft herzf., haar., am Rante etwas wellenf.: bie unt. berzf., etwaz̧ geiägt, geftielt; $B$ lt tffle geflugelt; Stgl rifo penf., weidfftad)l.; be . 3 weige 1 feit., blattreich), 1 blúth). (4 NAm. dbl. - wch) undulátus.

2. lanzettf., langgeipizzt: Die unt. lanzettf., am Sirunoe fchmaler, gefägt; be F. 3 we i ge - ruthenf:; $B$ lo. Fchlaft, furtz. als Scheibe; Stgl unbehaart (4 NAm, dv.)

583. mutabǔlis.

II. ffiellos; Alle Blätter:

1. eyr., grobgezahnt, gefticlt: Stglbl. am Grumbe feilf.: $\mathfrak{B}$ l. an b. $\mathfrak{B}$ lủ th. lan= zettf., ganzrano.; Stgl. hadfr.; 3weige

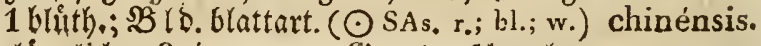

2. lảnglich, 3 ripp., am Grunte fommaler, fpis: Die obern faft ganzrano.; bie unt. gefticlt, gelägt; S tgi einf., an o. Epize Doldentraubenf.; şı lo. Iwaljenf., fperr.; Etrahl firr. als 3Bld. (4 NAm. w.) conyzoídes.

3. Fibmallanzettf., ganzrans.,

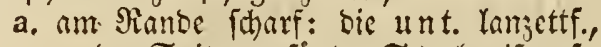
an D. Spise geraght; Stgl rifpenf.,

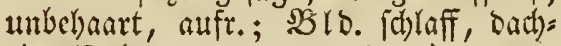
ziegelf. (4 38. w., dann blch.)

b. nicht foharf: Die unter fien lanjettf., etwag gefágt; Stgl âf., weitfhweif., wenig bebaart; 3 It . Dachziegelform. (4 NAm. w., dann v.)

4. Kanzettf. = gleichbreit, unbehaart, fticltos; Die unterften etwnes geiagt: SBl. a 3weig. lanzettf.; 3weige ruthenfórm.; $\$ B$ ls. Dachiegelf.; Stgl menig beljaart (4. NAm, wch, -n.)

III. etwas geftielt:

1. "Stglbl. eyr. = faft berzf., 'Tanggefpist, tiefgejagt, an 0 . Epire ganjrimo.: 3 we $i=$

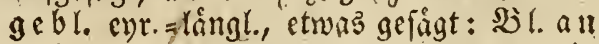
Ђ. bef. Zweigen lanzettf., ganz wenig

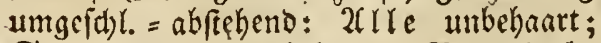
Stgl rifpenf., unbehaart; $\Re(D$. bathe giegelf. (4 NAm. hr.)

2. $B$ l. fâmmetlich fcharf: Stglbr. längl.s lanzettf., ganzrano., ipiz: $\mathfrak{S G}_{3}$ lbl. geftielt, lángl., gefágt: St gläft., boloentraubenf.;

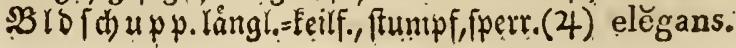

589. heteropliýllus.
586.

(salignus.)

587. lanceolátus.
588. juncěus. 
3, bie obern lanzettf.: bie unt. faft eyr.,

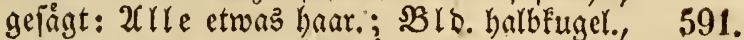

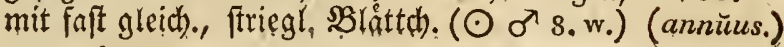

4. $\mathfrak{B}$ l. Fåmmtl. ichmallanzettf. langgeipingt, am Ranto fcharf: bie unterften etwas gefägt: Stglkl, etwab umgeichl.: 3 weige bl. jehr abfteheno; Stgl johlaft, and. Spize rifpenf; Blo. Dachziegelf., mit lanzettF., fpis., an D.

Epike umgejhl. Bláttch. (4 NAm, w.) laxus.

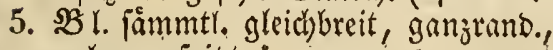

a. Yanggecpirigt:

a. Die unt. fobmallanzettf., etwas gefägt; Siveige boldentraubenf.; $B$ ID. Dachs ziegefform:; Stgl menig behant (4 NAm. w.)

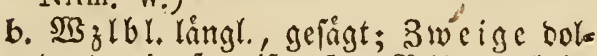

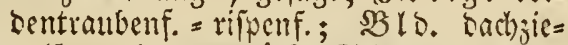
gelf., mit autgeorilut. Sthupp.; Stgl wie vorker ( $4 \mathrm{NAm}, \mathrm{w}_{\text {.) }}$

b. $\mathbb{B}_{3} \mathfrak{l} \mathfrak{b l}$. längl., etwas gejågt; Stgl

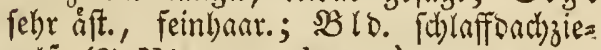
gelf. (4 NAm. w., dann r.)

593.

dracunculoídes.
592.

594.

fragìlis.

595.
polyphýllus,

b. nicht ganzrandig.

\section{Blätter :}

\section{1. eyruno,}

a. langgefpiţt, foharfgejågt: bie unt. ber f., geftielt, nact; $\mathfrak{S}_{\mathrm{g}} \mathrm{l}$ oben boldentralls benf.=gleichlyod); 3weige baar.; SBlo. lángl., Dachjiegelf., mit bichtangebruckten Sd)upp. (4 NAm, w.)

b. geftielt, gefiggt: bie oberften eyr. =herzfo,

596.

corymbósus. friellos: bie unt. berif., geftielt: SBlt fi le etwas geråno.; Stengel áft., weit: ithweif.; $B$ lo. waljenf., Dichtsachjiegelf: (4 NAm. bl.; w.)

2. eyr. =lanzettf., etwas gefägt, geftielt, unbe=

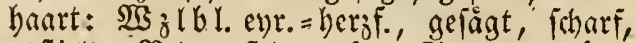
geftielt; $\mathfrak{B}$ lattftle macft; Stgl febr áft., unbehaart; bef. Şweige haar.; Blo lo e e fdlaff, etwaz bachziegelf: (4 NAm. bl.)

597. macrophýllus. 


\section{ASTRAGĂLUS. 580.}

\section{Blattstiele dornig.}

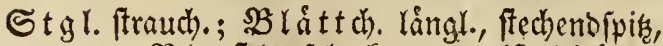

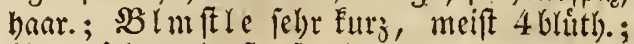

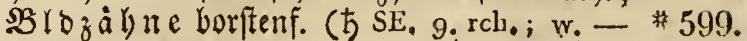
* Soft) aristátus.

\section{II. nicht dornig;}

A. ohne Stengel, mit blofsem Schaft;

1. Blätt. 3 zähl. od. im Quirl.

\section{Blättchen :}

1. $3 z^{2} \mathfrak{a b l}$., gleidbureit =langgeipizat, feibenart.;

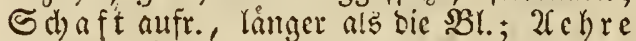

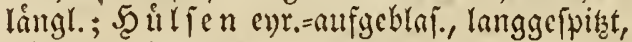
feinbaar. ( $\sigma^{\prime}$ NAs.)

2. 4żabl., im Duirl, pfriemenfơrm., harar.; Schaft rang. als bas $31 .$, mit $\mathcal{X}$ ehien; bie unt. Bluth. entfernt; Şitlen lans zettf., filz. (4 NAs. x9. !1.)

$600 ! !$
oxypliy'llus.

601. verticilláris.

\section{2. nicht.}

\section{Prättchen :}

1. lanzettiơrmig,

a. etwas hanr., fpits; S d) aft alfí., liong.

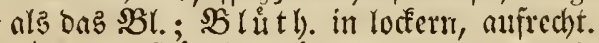
Zchren; Scullfen lingl., fcinthar., mit cinwairtsgebog. Spizge (4 9. rch.)

602.

montánuls.

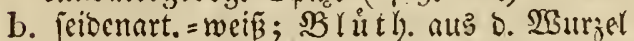

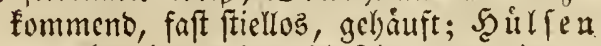
eur., baud).,, mit weif. Filz (4 19.)

603. testiculátus.

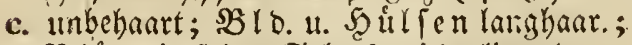

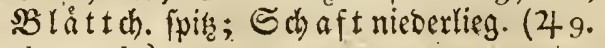

2. eyruno, gh. u. rch.)

604.

campéstris.

a. weipgriunl.; S dh a ft rang. als bas \$Bl.;

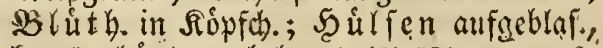
kugel., båut., unbebaart (4 NAs. 17. 36 . rcho; $g \mathrm{~b}$.

605. physódes: 
b. etwas feinhaarig; $S_{\mathfrak{c}}$ aft wie vorher; B I

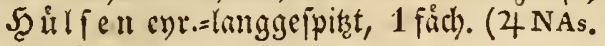
p. - r.)

3. eyr. =lanjettf., feibenart.=langhaar.: S chaft

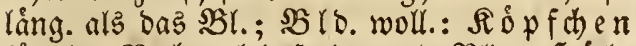

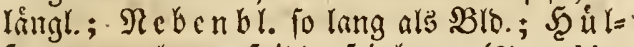
jen enr., langgeppist, fcinlyanr. (4 9. bl.; p. - v.)
606.
ambigŭus:

4. langl.=lanzettf., feibenart.; $S$ chaft noch einmal fo lang als bas $\mathfrak{B l}$.; Shlutben in lockern 2Chren, mit ticfausgerano. Fähnch.;

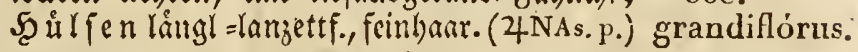

5. gleichbr., flebr.; Schaft ling, als bas \$Bl. u. oben bruif.=baar; 2 e hre wenigbluth.;

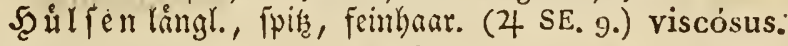

6. långl., ftumipf, haar.; $\mathfrak{B l i t}$ lh. etwa ge

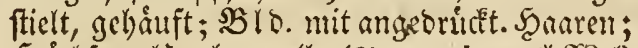

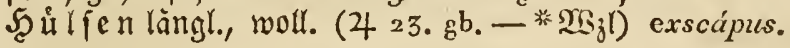

7. ellipt., ftumpf; Schaft traubenf., niebcr $=$ gebog., lång. alz bas $3 \mathfrak{B l}$, mit verlángert.

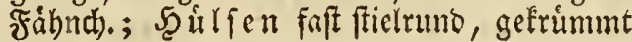
(4 SE. p.; gb.)
607.
uralérisis.

608

609. * 610 .

\section{B. mit blättrigem Sténgel;}

1. Stengel aufrecht;

a. Blüth. in fast stiellos, Aehren.

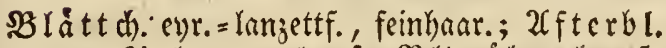

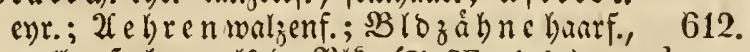
moll., fo latrg als D. SBlE. (4 SE. gbch.) alopecuroides.

\section{b. Blüthen in gestielten Aehren:}

\section{Stengel :}

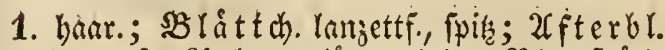

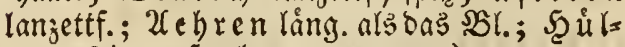
fen pfriemenf., haar. (4 gbch.) 611. monspessulánus.

2. meip̧grat, ftaud.; $\mathfrak{B l}$ l. meift 3 paar., lait= zettf., fpis; 2 fterbl. langl. =langgeipint; Uebren wie vorber, mit ausgerano. Fabnct). Şúlfen lángl., weifgrau (ל NAs: bgb: ; w.) dealbátus. 
3. rauh; sBldtt d. fomallangettf.; 2 ehren ganz gerabe, láng. als tas SBl.; Şîlfen lingl. = lanjettf., 3 fant. (4 SE. gbch.)

4. glatt; Blättchen :

a. langhaar., ellipt., ftumpf; 2 fterblátt. lanjettf.; 2Lebren eyrumo; $\mathfrak{B}$ Io jàbre haart., moll., fo lang als BlE. (4 NAs. f. 19. gb.)

b. feinhaar., langl.; Re ben bl. lángl.; fo lang als SBlo.; Sू ûtfen långl., aufge= fabollen, umgefrummt uno ftechenolpis (4 NAs. 3: 49. gb.)

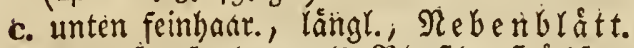

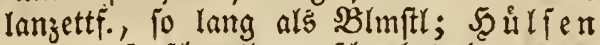
eyr., aufgeidhwoll., gefdnabelt (4 NAm. $\left.g r n_{0}-g_{b}\right)$

615. asper.
616.

vulpínus.
617.

uliginósus.
615.

caroliniánús:

\section{c. Blïtizen in gestielten Traubieri.}

\section{Bliithen:}

1. långer als bas SBlatt; Hiilsen:

a. 3 fant. , 1 fåch.; كtgl geftreift; SBlàt t= b) en ichmallanzettf., glatt; Uf ferblát t. lanjettf. (4 NAs. r.)

b. zigedr. 三3 fant.; fichelf., fpic, bangend; Stgl unbehaart; $B l$ lát th. lanjettform., ipir (4 NAs: gbch.)

2. fatenf. ; Şulfen evrund, rungl., 2 fnopf.;

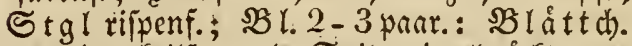
gleid)br. E feilf.; an D. Spite eingcoruckt, un= behaart (4 NAs. 2. 8. r.)

3. åhrenf.; verlång.; Stgl frrauch).; 2 l. meifít 6 paar., ichmallanzettf.; weísgraul.; Şน $\mathfrak{L}=$ fen lángl.; 3 fant. ( $\hbar$ SE. v.)

4. hangeno; Stgl gans gerabe; iubelyart;

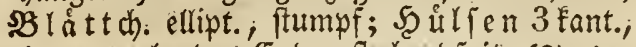
eben, an beyben Enoen ftechendipin (4 $\mathrm{As}_{\text {: }}$ $\mathrm{gb}$ : wch.)

619.

sulcàtus.'

620.

viréscens:
621. melilotôides:

622.

virgátus:
623. galegifórrnis:

2. Stgl weitschweifig od. gestreckt.

Stengel :

1. aufr. =abftebeno, feinbaar.; $S B$ lù th. in bent

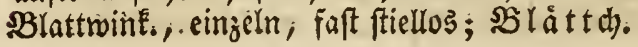


längl., frumpf, ausgerano.; $\mathfrak{X}$ fterbl. lan= zettf:; Şúl fen angelf, pfriemenf., feinhaar. $(\odot$ SE. bl.)

II. auffteig.; Blättchen :

1. längl.; Ent of hren 'gebrangt, geftelt ; Şủ lien långl. = lanjettf., rinnenf. = 3 kant., baar. (4 NAs. v.)

2. eyr. =lanzettfớrm., abwårţ̧gebog., baar.;

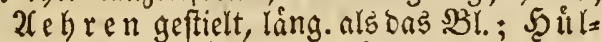
fen lanjettf., hang., 1 fádth. (O' NAs. v.)

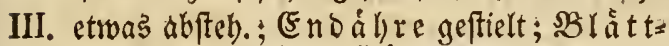

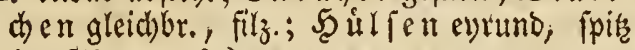
(4 SE. 18. rch.)

IV. weitfdhweifig; Bliithen:

1. in $\mathfrak{x} \mathfrak{x} a$ ben; Hiilsen:

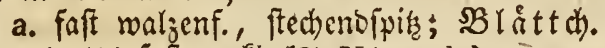
unten faft nacte (4 NAm. gb.)

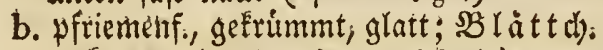
vertf. 三èvr. (NAf, Hülsen rothllecki)

2. in Köpfchen!

a. geftielt,

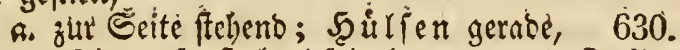
pfrientenf., ftect)entofpis (○ SE. 8. p.) Stella.

b. Dachziegelf., evr., mit aulfr. Bklitt); Scirlien enr., fabmiel., aufgeblajen $\left(\odot \mathrm{SE}_{0}\right)$

b. faft fticllos, zur Geite ftehend; S⿹弋 fen aufr., pfriemenf., an der Epitze 632. umgeidhl. ( $\odot \mathrm{SE}$. blch:; braun).

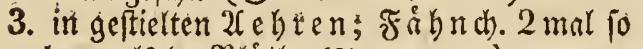
lang nts d. SBlutthe ( 4 3, 11, p.)

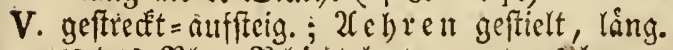

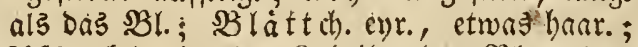
$\mathfrak{X}$ ftectbl. einzeln, 2 theil., ben $\mathfrak{B l}$. entge=

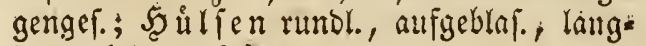
baar. (4 8, gb.)

VI. gariz geftrecff; Bliithen:

$A$. in Uetren; Hiilsen:

1. ènr., aufr., rimenf.; baar.; SBI àtt do. längl., ftumpf: $\mathcal{C}$ e $\mathfrak{l}$ ren eyr., geftielt, lång. als bả 3Bl. (4 I 9, bl; ; rch.) hypoglóttis。

2. lainglich, sesamĕus.

633.

Onobry̆chis:

624.

scorpioides.

625.

adsúrgens:

626.

parvifórus.

627.

taurǐcus.

628.

canadénsis:

629.

ánnuláris.

631.

Glaux:

634.

(microphigllius.)

a. fticlrund, ftacherbaar.; sila ellipt., ftumpf; $2 \mathcal{e}$ f hre $\mathfrak{e}$ lángl., ge= fitielt, láng. al's $\mathrm{D}_{0} \mathrm{SBl}$. (4 9. rch.) leontinus. 
b. 3 feit., ftect)entepip, langhant., mit e. 637. Furd)e; 2 e bre n verläng. ( 4 NAs. blch.) Laxmánni.

c. 3 fant., an b. Spike bafenf. =gefvimmt; $\mathfrak{B l a ̊ t} t$ ch). lángl., ftumpf, ftechenofpir; 2 e hreul geftielt, wenigblith., Eurry. als סаว $\mathfrak{B l}$. (4 SE. gbch. - ఫ Siafifurrogat) 638. ఫ্

B. in $\mathfrak{x}$ rauben; Hülsen:

1. faft fugel. = aufgeblai., ftechendipis, feinz baar.; $\mathfrak{B l}$ lát d). faft unbeliaart, lángl., geftielt, fectsenofpis; 2 f f er bl. lanzettf.;

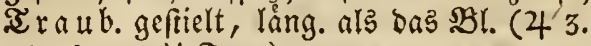
26. gb. -

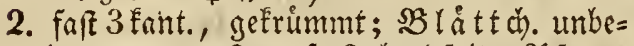
haart, eyr., fumpf, fed)endipis; 2(fter= $\mathfrak{b l a ̊ t t . ~ e y r . ~ = ~ r a n j e t t f . ; ~} \mathfrak{S}_{\mathfrak{L}} \mathfrak{a} u b$. geftelt,

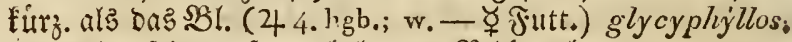

3. angelf., pfriemenf., unbel)aart; $: B$ lát th).

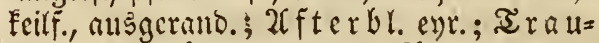
ben menigbluth., seftielt, kưrzer als bas Bl. ( $\odot$ SE. wch.)

4. bogenf.= zigcoreht, feinhaar.; $s g l a ̂ t t c h$. verk. = eyr., ausgerand.; $\mathfrak{z} \mathfrak{r a \mathfrak { u } b . ~ g e f t e l t ; ~}$ DP flanje feinbaar. ( $\odot S E$. I 7.)

5. 3 fant., lanjettf., hangend; Bla a t then gleichbr., abgefturt, auşgerano.; $\mathfrak{T} \mathfrak{r} \mathfrak{u} \mathfrak{b}$. geftielt, lång. als bas $\mathfrak{B} 1$.; $\mathfrak{B}$ lefflúgel 2 ppalt. ( 4 4. rch.)

639. ఫ (Cicer.)

640. ఫ

641.

hamòsus:

642.

contortuplicí-

tus.

643.

austričcus.

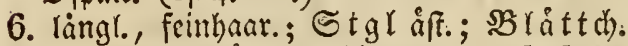
fahmallanzettfơrm., feidenart., zigcfalt.;

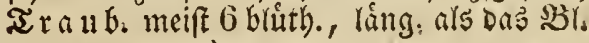
(4 19. bl; ; p.)

7. Fieeruno, ranzettfo,

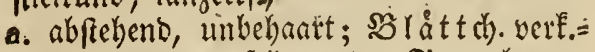
berzf., unten feibenart.; $\mathfrak{E} \mathfrak{r} \mathfrak{a} \mathfrak{u}$. ge= fitielt, faft fo lang als bas $\mathbf{B b}$. (4 wch. $u_{\text {: }} b_{\text {lcbi }}$ )

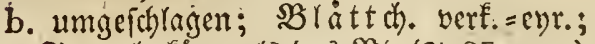
İ $\mathfrak{a}$ u b. fúr. als bả SBl. (4 SE. 9. w.) depréssus:

S. fpik, auf beys. Seiten haar.; $\mathfrak{I} \mathfrak{x} a \mathfrak{u b}$. bantg. (24 SE. 9. v.)

644.

(arenarǔts.)

645 .

leucopha'eus: 646.

deprés
647. alpínus:

C. in Köpfchen:

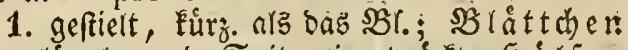

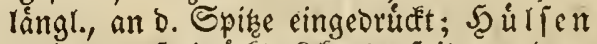
balbeyr., zigeoructett 3 fant., ipt fdupp: = ffachl: $(\odot \mathrm{SE}, \mathrm{rch}$.

64S: pentaglóttis. 


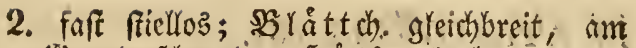

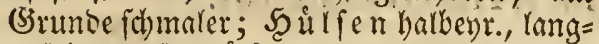
gefpirit, .zigebriclet, umgejchl., feingarar. ( $\odot$ SE. 5. 8. w. ; dp.)

649.

epiglóttis:

\section{ASTRANTIA. 182}

Wurzelblätter:

1. 5 lappig: Lappen :

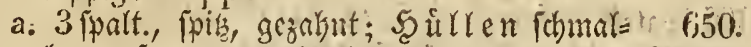
lanzettf., gamzrand. (4 4. 8. wch.)

b. faft 3 part., lingl., etwas pis, frethent= :

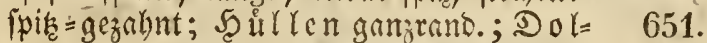
den idhrif, etwas abfetheno (24. $9 \mathrm{gb}$.) carniolica.

2. Fingerf.: S lát tch. meift 7 zábl., lanjettf., 652. fpis', ticfgezalynt (4 9. Hüllen w.)

\section{ATHAMÁNTA. 205 .}

\section{Stengel:}

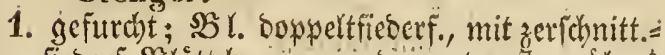
ficoerf. SBlattch), woovin $\delta$. unteriten ubers Sireuz ftehen, und mit lanjettf. Eappen (4 2. 3. II, uw.)

2. fficlruno, unbebarat, bin uno ber gebogen;

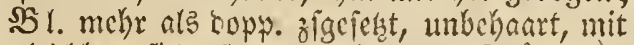
glcichbr. = fieberf., auegrobreiteten SBlâttchen; Scille wenigblitttr. (4 9. w.)

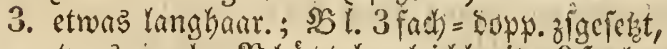

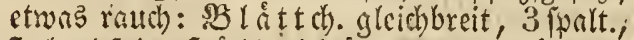

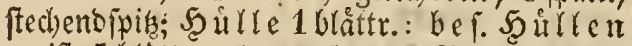
meift 5 blàttr., lmo jettf., an গanee troden $\left(\sigma^{\top}\right.$ 9. wo - * Gaam.)

653.

(Libanólis.)

654. Matthiouli,

19. ATHANASĬA. 645.

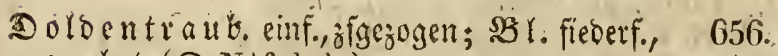
gejalint ( $\odot$ NAf, ligb $\left.b_{*}\right)$

annuัa,

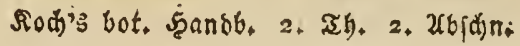

(E) 
- Stgl u. SBl unbehaart ( $\sigma^{7}$ SE. w.; p.) humilis.

\section{ATRAGĔNE. 434.}

Stgl Fletterno; $\mathfrak{B}$ l. bopp. 3 jálil.; $\mathfrak{B} \mathfrak{l}$ mftre 1 blutt).; bic ranggeipirtat.; gefägt. blättch.:

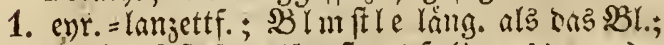
sBlbr. faft fortelf:, ftumiff ( 5 9. bl.; w.) alpina.

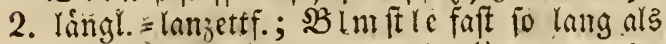

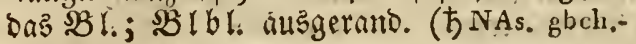
w. $\Delta$ ).

659.

sibirǐca,

\section{ATRIYPLEX. S02. (232.W. 37.$)$}

\section{Strauchig.}

Stgl niederlieg. ; Die ganzrano. Blätter:

1. entgegengef., langl., frumpf, an (5itumbe 660 . ichmaler $(5,36$.

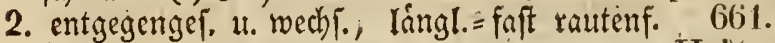
(ち SE. $\triangle$ )

Halimus.

\section{Krautartig.}

\section{Stengel :}

I. aujgebreitet; Blätter:

1. 3 ede., ungleidhgegahut, grau; 3BIb. bor 662. Frud t 4 feit., gezalint $(\odot)$

2. rautenfórm., etwas geczahnt; $B$ lo, Der

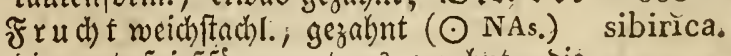

3. bie unt. fpicśfórnt., etwas gejahnt: bie

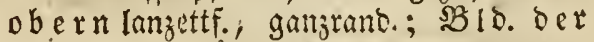

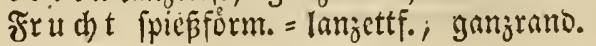
$(\odot$ 22.)

II. aufrecht; Blättter :

1. längl., bucht., änt (s)runbe feilf., unten 665 .

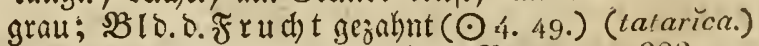

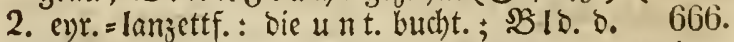

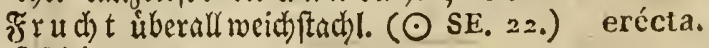
3. 3 feitig, 


\section{Atriplex, - Aucuba.}

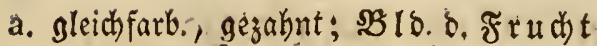

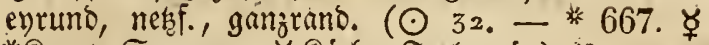
* Siraut, Saam. - ఫ̛ Siuch., Farb. griun) (horténsis.)

b. unten weifl., tiefgejalint; $S B$ ro. ber F $\mathfrak{r}$ ucht $t$ rautenf., 3 ripp., feingejal,nt $(\odot$ 17. 36.)

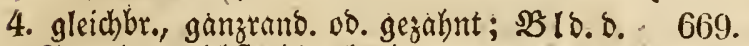

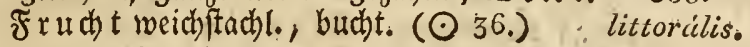

5. 3 jeitig = fpiesfớrmig:

a. 2Ulle; Bld. d. Frucht:
a. 3 feit. =enr.; ganzrand.; $\mathfrak{B}$ l. buictst.= gezahnt, unten jilberwei $(\odot)$
670.
nitens.

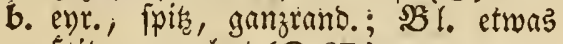 fpich H. gezalnnt $(\odot S E$.
c. bucht. = gezabunt, mit verlang. Mittel $=672$. zahn ; $\mathfrak{B}^{\mathrm{B}}$ logleichfarb., tiefgejahnt $(\odot)$ (hastàta.)
b. nur bie untern: bie oberti lanjettf.,

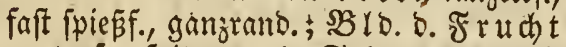

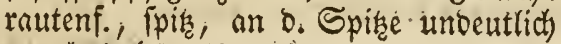 gezabnt $(\odot$ SE. 36.)
671. microspérma,

\section{veněta:}

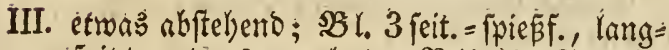

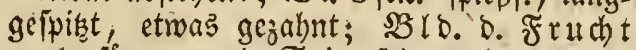
rautenforim., an $b$. Spike feingezahint, in ber

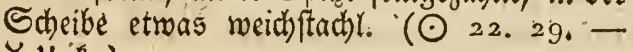
$\Varangle$ luifr.)

IV. Gin u. hergebog., âusgebreitet; $\mathfrak{B}$ l. verté.= entumb, ganzrano.; W. $\mathfrak{B}$ lit then gefticlt, feilf. $(\odot$ 17.36.)

674. $\not$ (patüla.)

675.

(pediuncilláta.)

\section{ATROัPA. 141.}

Stgl frautart.; 3 Y. èrr., ganjtano. (4 5.8. up. u. gbch. - * $\mathfrak{W}_{3} l$, Siraut, $\mathfrak{S}$ m., $\mathfrak{B}$ cer. — ซ̧arb. grim)

* 676. ช

$\uparrow$ (Belládónina.)

\section{AUCÜBA. 736. (W. 13.)}

SB l. entgegengel., geftielt, lángle, gelägt, glånz.;

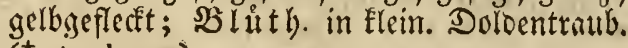
$(\hbar, A s$, braun $)$

$67 \%$ japonica. 


\section{AXÝRIS. 734. (W. 59.)}

\section{Blätter :}

1. eyrund; Stgl aufr.; Aehren:
a. einfach $(\odot$ NAs.)

b. rifpenformig ( $\odot$ NAs.)

2. verk. = eyr.; Stgl etwas geth); SBluty. in Rópfd). (๑ NAs.)

678.

amaranthoides.

679.

hybrǐda.

680.

prostráta.

\section{AZALËA. 131.}

\section{Biätter:}

1. Yanglidl, auf beuben Seiten

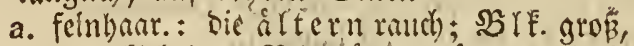

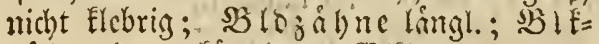
rób re haar., furg. als 5. SBlElapp. (ち NAm. gh. - rch. $\triangle$ )

b. unbehaart, am (Srunde id)maler, gcivimp.; mit Enoboloentraub.; Blk.:

a. auswenbig haar., Eurg. als bie Stgef. (5) NAm. Al.; w. $\triangle$ )

b. Sofbre brif., hat., fo lang als ser ভaum; $\mathfrak{B l}$ l. glail. ( 5 SE. gb. $\triangle$ )

2. längl. = veré. evr., fpifs, nuf bevo. Seit. un= bebart, gleid)farb., an D. Mlittelrippe borft.,

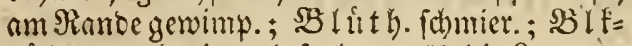
robre noch cinmal fo lang als bie Rapuent; S̊loz ábne febr furj, runol.; Stfảd. Faum lång. als SBlE. (ち NAm, w. u. rcb. $\triangle$ )

684.

viscósa.

3. Initzettform.,

a. an bend. (Enben ipis, gewimp.; mit (5no= Doldentraub.; B lolapp. ipatelfúrm.;

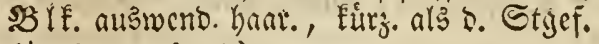
(ち NAm. sch. $\triangle$ )

b. am Sirutbe etwers folmaler, auf benden

681.

calendulacěa. Seit. unbchanrt, unten mit e. borft. S)it=

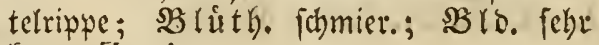
furz; Zweige:

A. badfr.; $\mathfrak{B l}$. fpit, unten graugrim, ant Fande gerwimp.; SB réto bre 2 mal fo - lang als bie Eapp.; Stfito en faft fo lang als bie BBlflapp. ( 5 NAm. w. $\triangle$ ) glauca:

nudiflóra.

683.

pontica.

\section{5. \\ speciòsa:}




\section{Azalea. - Bartsia.}

b. glatt; $\mathfrak{B l}$ l. Flein, etwas icharfgefpiat, leberart., oben gläls., an Riante um= gervllt =gewimpert; S S ling. als dic Eapp.; Stfád. Yervorra= gend ( 5 NAm, w. u. hr. $\triangle$ )

687. niť̌da.

\section{BACCHĂRIS. 658.}

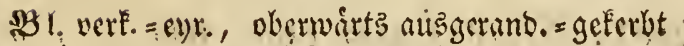

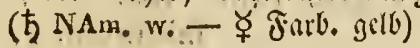
halimifolia.

\section{BALLÓTA. 476.}

\section{Blätter:}

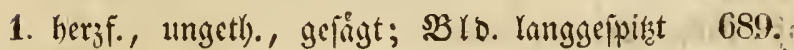
(4 22, w.)

2. banof., gejalnt; $\subseteq t \mathfrak{g l}$ woll. (4 NAs, gbch.; 690 . ausw. yvo)

lantăna.

\section{BALSAMÍTA, 651.}

Stgr frautart.; $\mathfrak{B}$ l. eltipt., gezahnt: bie unt. geftielt: bie obern ftiellos, am Givunoe unit obrf. R(nl)ang; $\mathfrak{B}$ luth. Doloentraubenfưrut. (4 $\mathrm{SE}_{\mathbf{1}}$ - * Siraut)

* 691.

vulgáris,

\section{BALTIMÓRA. 705.}

$(\odot N A m \cdot g b$.

692.

erúcta,

\section{BARTSĬA. 4 S3.}

Br. entgegengeferct, herzform., ftumpfgefägt 693. (4 9. $\left.\mathrm{x}_{0} .4 \mathrm{l}_{0}\right)$, alpina. 
19. BELLIS, 689.

1. Mit etwas blåttr, $S \ddagger g l(\odot$ SE.) 694.

2, mit blattlor., 1 bluth. Schaft; $\mathfrak{B} \mathfrak{l}_{\text {, verf. }}=$ evr., geferbt, mberig (4 2.34. wo u. r.; ver- *695. ఈ

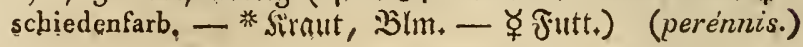

\section{BELLIUU, 677 .}

Stgl blåttr,; baarf.; $\mathfrak{P f l a n z e ~ g a n z ~ H l e i n ~} 696$. $(\odot$ As. 40 .)

\section{BERBĔRIS. 265.}

Blïthen :

1. in einf., bang. Traub.; Blbl.;

a. gangrand., ftumpf; Bl.:

a. vert. $=$ enr., gemimp. $=$ gejågt $(\hbar$ 5, gb. $* 697$. ఫ̧

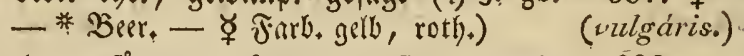

b. lanzettform. =verḱ. enr., faít ganzrans, 698 . (ち NAm. gb.) canadènsis. 699.

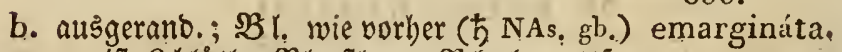

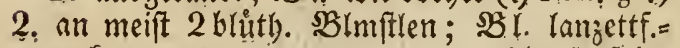
verk. enrunb, sangrano., etwas fohr rgefpist (5 $\mathrm{SE}$.)

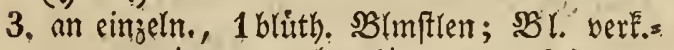
enr., gerwimp. = gezahnt (ち NAs, 9. gb.) sibirica,

\section{\%. BETA. 229.}

Bliithen:

1. gebrängt; bie unt. $\mathfrak{B l}$ l. enr.; $\mathfrak{W G} z l$ bif

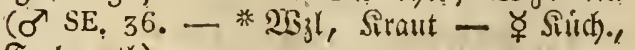
Farb, rott)

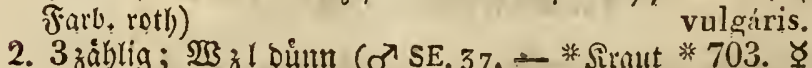
- ఫ̛ sivch., ซ̛utt.)

3. 2 záhl.; Stgl auffteig. (o' SE, 36.)

700. cretǐca. 701.
* $702,-\not$ * 703. ఫ Cicla. 704. maritịma. 
4, in aufr., rifpenf., faft byattlof. $\mathfrak{x} \mathfrak{x} \mathfrak{a} \mathfrak{u}$ b; Bluth). mit 3 . Stwegent, gepant u. cill= seln; șl. bersf., Tipis, am Srutbe ungl:; şio. mcipl. (4 SE.)

705.

trigy̆na,

\section{BETONICA. 460:}

\section{Aehre:}

1. unterbrochen; Blkhelm:

a. ungeth.: Mitter(app.o. urippe auss gerno.; sB 10. foft unbehaart (4 3. 4. * 706 .

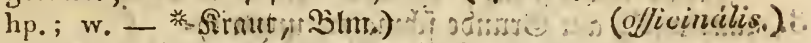

b. 2 fpalt.: Mitterlapp: D. H Hipe gezi

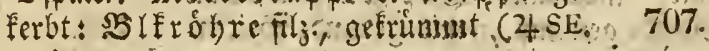
res:)

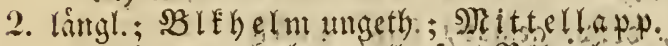

s. Ulippe geferbt = ucllenfi, so id baar (4 NE) -

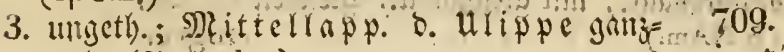
ramb. (4 $\mathrm{As}_{0} \mathrm{hp}$ ) :

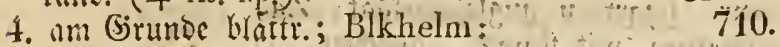

a. 2 ppalt. (4 3. ugb.) alopecíros.

711.

b. ungeth. (4 SE. 9. rch.) - hirsúta.

5. blättr., unterbrochen; SB lo ed e am Sianoe

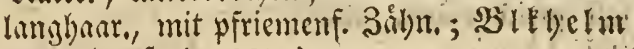
wert: $=\mathfrak{h e r z} f_{\text {f }}$ (4 NAs.)

712.

grandiflóra

\section{1, BETǓLA $\cdot(\mathbf{W}, 62$.}

\section{Blätter :}

A. beltaformig,

1. Dopp. gefågt, fpirs,

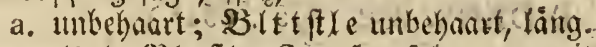
als D. SBImftl; 3 isp fenf

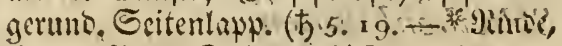

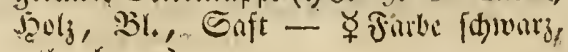
roth), (bratur)

b. unten nebf Ser bef. 3wecigen fein= haar, faft herf. ; apfenf hutpen. swie vorlycr (5 46.48.)

* 713.

(alba.) 
2. ungl. gefågt; langgeipiat. gan unbehaat; BIttifle unbebart - Salfenfol d p wie 713 . ( $5 \mathrm{NAm}$ )

B. eyrutio,

715.

populifolǐa,

1. einfactgefágt, fpis; $B$ rtt title feinlgar., furr. als SBlmftl; Sap fen ichupp. wie 713. ( 5 NAm.)

2. Dopp: gefágt,

a. langgeipişt, mit unten rauch. 20ern 717. (ち NAm.)

b. unbchart; 3apfenfd up p: 'nit gleis chett, nbgeftitist., aber. \&app.; WT:AB $m=$ ?

ftiele a ft. (t)

3. gangrand, ant Sirtmbe fomater, ungleid)=

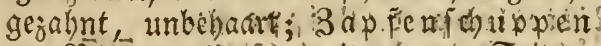

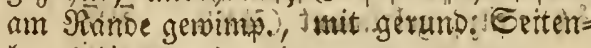
lapp: (ち'NAm.).

papyrácea.

716.

excèlsa,

is. actitisit of

718.

$\mathrm{m}^{-}$

719.

davurica,

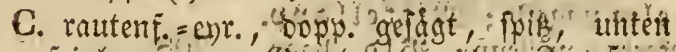

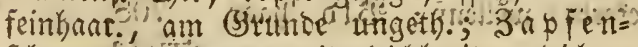
(d) up p, fanghane, mit gleid)breit., gleichen Sapp. (b NAm.) * of

D. berjfo=evr, fharf = u. ungleidgelägt; $\mathrm{Za}=$ pfenschuppen mit ftumpf., gléiden,

1. crbaben=aberigen $\mathfrak{E}$ app. ( $\hbar$ NAm.)

721.

2. aberló , nadt. Lapp.; SBlttif le unbe = baart ( 5 NAmi).

E. runol =eur., fajt gleichgefagt, unbebart; W. 角 ách. lángl. (

F. Ereisumn = verté. enr., geiagt, unten nebit o.

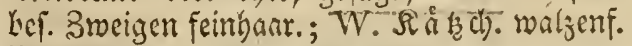
(ち) $\left.N A m_{0}\right)$

G. ganj freišrund, gefertst, unbebanat; inten neef $=$ aoer. (

722. cárpinifolia,
723.

fruticòsa. 
2. enr., auf bend. Seit. $1 ; 2$ zabn.; $5 B$ luth. geftielt; â 1 F. \$Blb. 3 mal fo lang als bie şlitthe; Stgl ftad)elbaar., gabclf. ( $\odot$ As. w. ; gb.)

727

nodiflora.

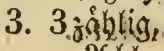

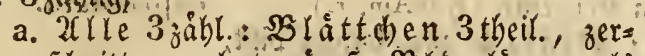

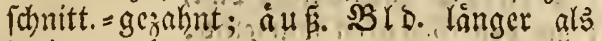
o. inmere $(\odot$ As. $)$

b. mer die obcrn-3 zábl.: bie untern: n.egefiebert; Blâttclı, der obern:

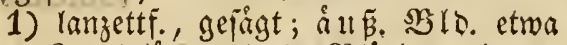
6 mal láng. als b. - SBlutth.; mit am (Siruttoe gewimp. Bláttc). (4 NAm, gb.) gb.)

2) langl.; c5noblát th. lanzettf., nod cinmal fo lang als $b$. itbrigen; ă $u$. B lD. fo lang als D. inmere ( $\odot$ NAm.) pilosa.

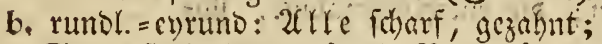
Stgl fact)elbar.; a $\mathfrak{a} \tilde{B}, \mathfrak{B}$ lo. långer als D. Bluthe ( $\odot$ SE. gb.)

729.

frondósa.

\section{parvillora.}

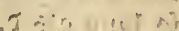

\section{1. mit etwas gestrahlt. Bliith,}

\section{Blätter :}

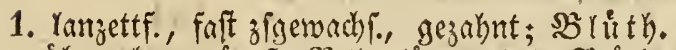

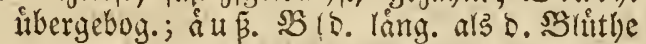

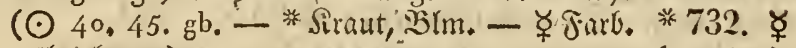
gell, , Grami) (cernüa.)

2. bopp. gefieb., mit ranzettf., fieberf. Blattch. $; 733$.

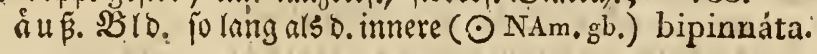

\section{III. mit ganz gestrahlt. Bliith.}

\section{Blätter :}

1. Soppertgeficos., mit Feirf., 3 zahn. SBlåttch.; 734.

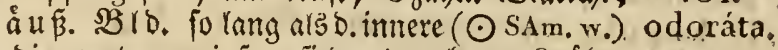

2. Die untern einf. gefico.: $D$, obern 3 zónl.;

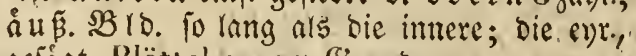
gefágt. Blättchen am Girunde a. glcid) $(\odot$ SAm. w.) 735. 736.

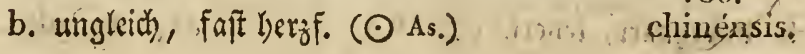




\section{BIGNONIA. 501." (504.}

Blättèr:

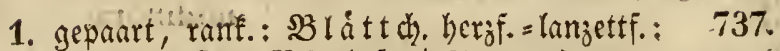
bie unteríten $3 B$ l. cinf. (t) NAm, r.) capreoláta.

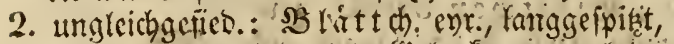
gefágt; Eotgl an ben Gelenfen wiryelno; mitendoverentraub.; BB lerobre 3matfo lang als b. BBID: († NAm. r. $\triangle$ gitle)

738. $\not$ radícans:

\section{BISCUTÉLLA. 510.}

Schötchen:

1. punttirt=idharf; dic entferntgezahnt., fta $=$ d)elhaar: BH. :

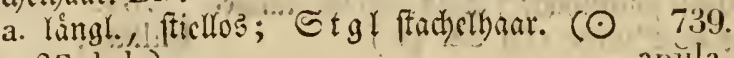
SE. hyb.) apŭla:

740.

b. Lanjettf.; Stgloben unbebant (4 SE.) saxatilis.

2. nur am Rante wark = Fitharf; Stgl ftachel= haar.; bie fticllof., frachelhanro, entferntge= zahnten Bl,:

741.

a. längl:; Stgl aufr. $(\odot S E)$. . didyma.

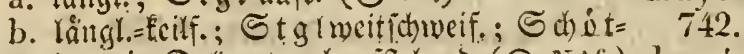
d) en in Dortontraub. ziffeheno ( $\odot$ NAf.) depréssa.

3. gants unbebanrk,

a. geránbelt, mit s. Epitze in e. Griffet ver=

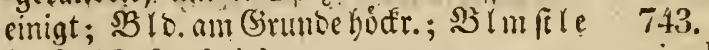
horfif. ( $\odot S E$. hgh.) auriculata

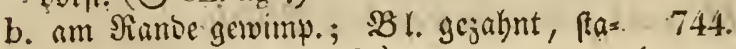

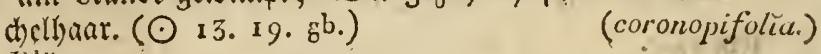

c. Blätter:

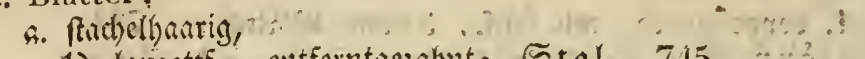

1). Lanizettf., entforntyegahnt; Stgl 745 . unbehaart (4 21.) (laevigáta.)

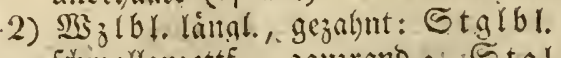
fümallanjettf., gantrano.; Etgl unbehaart (4 SE. 9.)

746. alpéstris. 747.

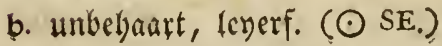
raphanifolǐa. 
$(\odot$ SE. p.) 748. Pelecínus.

4. BLACKBURNĬ, 71,

(ち $S E$.

749.

pinnáta.

\section{BLITUM, 6 .}

31. 3 \{eit.; Blithen:

1. in ăhrenf. EEn bucht. $(\odot 28$. grn - gb. $-\forall$ farb. rotb) $)$ capitáum.

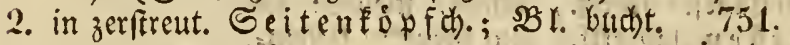
$\left(\odot \mathrm{SE}, \mathrm{z}^{\mathrm{r}}-\mathrm{b}_{\mathrm{b}}\right.$.)

3. in Snaueln, wovon bie oberfen mit Rebenbl., sie meiften bem Blattfil ange $=752$. wachjen; $\mathfrak{B}$ l. ppiesf., etwas feingejabnt $(\odot)$ petioláre,

(๑ NAm. gb.)

753.

19. BOEBÉRA. 688. chrysanthemoídes.

19. BOLTONǏA. 673.

Blätter :

1. ganjtambig (4 NAm. gb. u, fl, - w. )

2. bie unt. gefingt (4 NAm. gb.)
754.

astéroídes.

755.

glastifolǐa,

\section{BORÁGO. 100.}

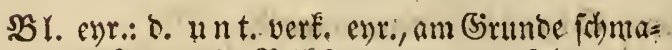

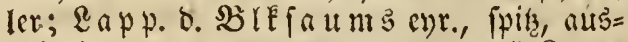
gebreitet $(\odot 22.32$.bl.; w。; fl. - * Siraut, * 756. ఫ

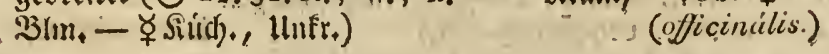




\section{9.! BORCKHAUSİA. 622.}

\section{Blätter:}

I. glcidffơrmig,

1. étr., berzf. = pfcilf., umfaff., gezahnt;

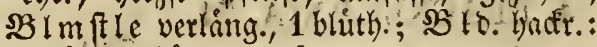

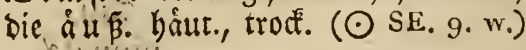

2. fieberform., umfafl., etwas haar.; $B$ ldo blattch. etwas filz. = weip, flach $(\odot)$

alpina.

3. farotjågef. = fiederf., baar. =icharf; Stgl rifpenf.; SBlofchupp. nachenf.=rinnenform., feingaar.; SE rone ftiellos (SE:

II. 1mileichformig,

1. idhrotiagef. = fieberf, idarf, ftiellos: bic oberften longettf., am Sirunoe tiefein= gejd)nitt.; B.ts. cl)r., haar., cef, $(\odot, 5,-760$. gb.u. $\mathrm{P}_{\text {, }} \mathrm{Bl}$, riechen wie bittre Mandeln) foel ida.)

2. fobrotiågef, an (Sirunbe mit ohrf, Znhang: cie obern lanjettf., pfeilf. = ipiesff., am

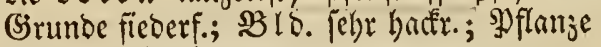
borft. = bacḱr. ( $\odot$ SE.)

761. hispuัda.

3. umfafl.: die unt. lángl., gejabnt: bie obern zerichnitt. = geznhnt; Stgl borft.=

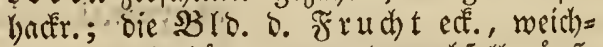
ftachl., mit båut., eyrund., abfall. åuf́. Echupp. (○ SE.)

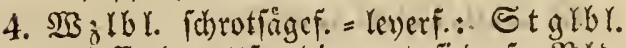
untfalf. Ianzettf.: Die unt. fieberf.; $\$ B$ lo.

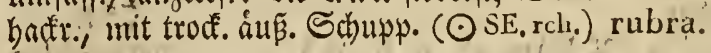

\section{BRACHYSTÉMUM. 461.}

Xehren fopff., dolontraubenf., am Enoe ftebeno; bie ganjrand. Blätter:

1. glcichbr., 3 ripw: $;$ Stge fáá beroorrageno 764 . (4 NAm. w.) linifolium."

2. lanjettform., aberig; Stgef. cinged bloflen 765 . (4 NAm, w., Ulippe v. punct.) lanceolátum. 
15. BRASSǏCA. 529.

\section{Griffel d. Schote etrias stumpf.}

I. Wurzel:

1. ftengelformig,

a. ipindelf.; $3 \mathcal{B}$ l. glatt: bie obern herzf.s lanjottf., umfall.; bic unt. Yeyerform.,

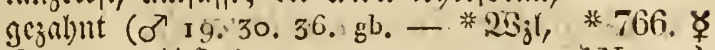
Enam. - ఫ̧⿻il)

b. freisrund, fleifh., oben eingedrifft; (Napus.)

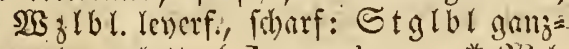

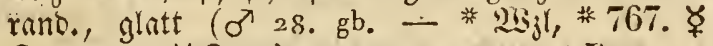
Sanm. - $\ngtr$ Futt.) : Kapa.

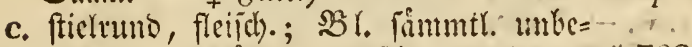
baart, graugrim, altagefchmeift do. ge=* 768. ఫ

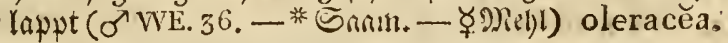
(ธ. ?(nntet. unten.)

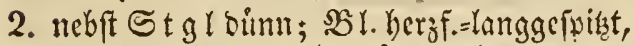
umfaff.: Die unt. keverf., gejabnt, ctwas 769. ఫ hadt. $\left(\odot\right.$ 29. "high. - ఫ Del, llnti.) (campéstris $\left.s_{i}\right)$

II. Blätter:

A. $\mathfrak{Z}$ Ile umfoñent,

1. herzf., tulbellant,

a. ganzrano,; Schote 4 feit., crbaben= 770 . gefurcht $(\odot 30$. gb.; w: $) \quad \therefore$ austriüca.

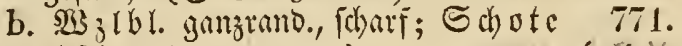
4 feit. ( $\odot$ 19.29. w.)

2. Paterf., ausgechweift: bie oberften 772 . herzf., gamjramo. (4 SE. 22. v. u. dr.) arvénsis.

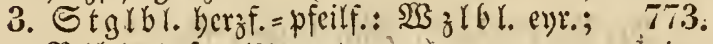
sblbr. aufr. (4 8.2. w.) $)$ aipina.

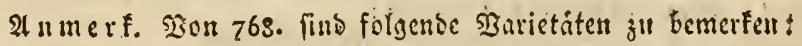

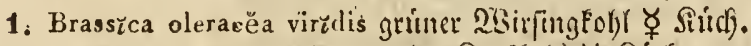

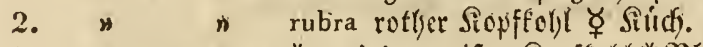

3. " "

4. " "sabrida Gavoverfoly $\not$ Sitidy.

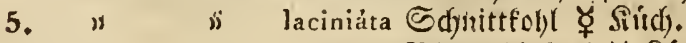

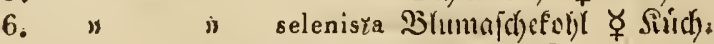

7. " "

8.

9. 1 "

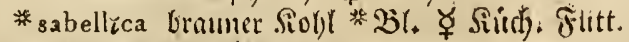
botry tis Dlimineitulfl. $\$$ Sitid).

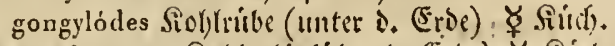

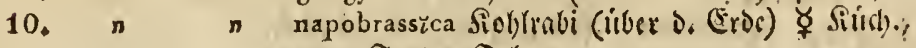
Jiutt, Dil. 


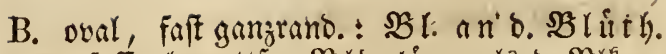
umfâl., lanzettf.; $\mathfrak{B}$ lo. läng. als D. SBlEna= $\operatorname{gel}\left(\sigma^{7}\right.$ SAs.).

C. bie unt. , fihmallanzettf., fieterf. =gezahnt: bie obern glcichbreit, ganjrand.; \$Bld. abitcheno (4 SE.)

774.

chinénsis.

\section{Griffel d. Schole schwerdf.}

Blätter:

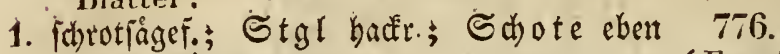
(๑ 2. gb:) (Eruccistrum.)

2. leverf; Stgl rauch; Schote unbehant * 777 .

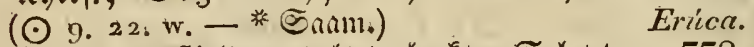

3. fieorerf. gefticlt, gezalint, hadfr.; Sd) ote 778 . Enot., fladi) geld)nabelt $\left(\sigma^{\gamma} \mathrm{SE}\right.$.) , Gheiránthus.

20. BROTÉRA. ร14.

(4 SE, bl.)
779.

corymbósâ.

\section{BROUSSONETIA. 778.}

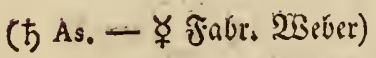

$780 . \not$ papyrifüa.

\section{BRYONIA. 766. (799: W. 7.$)$}

Bł̆̉ then in Ṡraben; bie berzif., 5 lapp., gezabnt., ichwielig = punct. Blätter:

1. (d)arf; mit balbgetrennt. (s) $\int$ d) ledt (4. 26. hgb. u. grn, - 23 zl, Bere, Snam.

2. hatrof.; mit ganz. getrentt. (S) efd)l. (4 26. hgb. u. grn. - $\mathfrak{W}_{j} \mathrm{l}$ )

* 781. ช

$\dagger($ allsa.) * 782 . dioǔca.

\section{BUBON. 209.}

\section{Blätter:}

1. topp. fieberform., puicgelglatt: SBláttch. langl., fieberforntig, ftechenoplyis=gejabut ; 


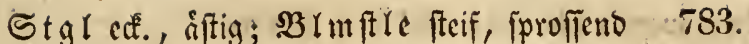
(4 NAs.)

buchtorménsis.

2. meift bopp. gefieb., graugrunl., mit ran= jettf., am (Srunte ichmalern Lapp.; St g't unterbatb unbelgart; 3 weige jebe abfte= bento; Etraglender Dolbe freif, fein= baar. (4 SE. 9. w.) glaucus.

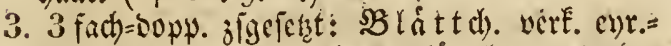
feilf., 3 zahn.: bie obern langl., ungeth.; 785.1

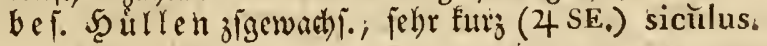

\section{BUFFONIÀ. S4.}

\section{Stengel:}

786.

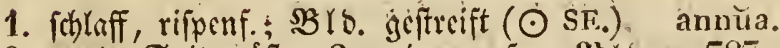

2. an s. Spitze cift.; 3 we ige aufr.; 33 lo. 787. am ranoe trode. (4 SE.) perénnis:

6. BULBÍNE. $2 S 1$.

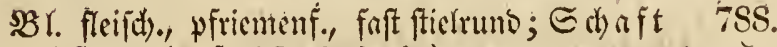

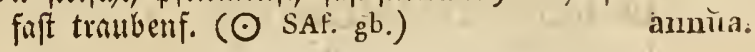

6. BULBOCODĬUM، 274. $\left(69^{\mathrm{r}}\right.$.)

SI. lamzettf. (4 SE. hp.)

789.

vernum.

\section{BUNIAS. 522.}

I. $\mathfrak{x}$ anbenblith). in Domen Libergebeno 790.

$(\odot$ As. wch.-v. $)$

śpinósa.

II. Schötchen :

1. 4 feif., gesah)nt, Blätter:

791.

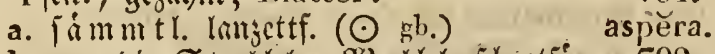

b. mur bie Etglbน: 213 jibl. fhrotfiu= 792 .

2. eyruts, gé. $\left(\odot \mathrm{SE}_{0} 29.49 . \mathrm{hg} \mathrm{b}\right.$.) Erucágo. 


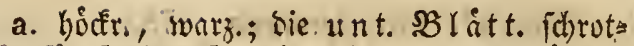
fågef. = leyerf.: Dic obern lanzettfórmig (4 NE. gb.)

b. ctwas runjl. uno ftumtuf; $\mathfrak{W B}_{j}$ lblatt. loingl.: SIglbl. pifeilform.; umfalfeno $(\odot$ SE. w.; higb.)

793. orientális.

3. Fugelig,
a. jharf, faft 3 edf., bafenf. =geidtumbelt; Sil. lanzettf.; fpiz, geftielt $(\odot \mathrm{hp.})$ syričca.
b. hader.; \$l. gefied., mit etwas gezalunt. 796. Blättch. ( $\odot \mathrm{SE}$. gb.)

794. cohlearioídes.

\section{BUNIUUM: 206.}

\section{Blätter :}

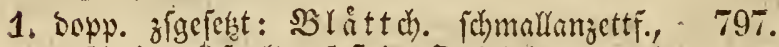

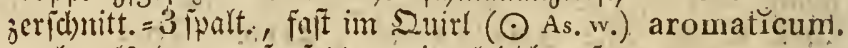

2. melyr als bopp. ofgejest, mit gleid) br. $=f_{a}=$ benf. SBláttch.; D D locu ben MH. entgegen=

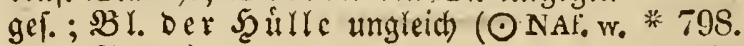
* Gaam.)

copticum:

\section{BUPHTHÁLMUM. 663.}

\section{Biätter :}

I. entgegengeịent,

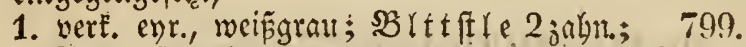
Stgl ftraud). ( 5 NAm. hgb.)

2. eyr., gefingt, 3 fadtyerippt; MIS. blattr.; Stgl trautart. (4 NAm. gb.) heliantlioides.

3. Tpatelf. =långl., feisenart., jebr angená= bert; SBlofth uppe n borftenf., raud); Stgl baumart. ( $\hbar$ Af. gb.)

II. wed) jelsmeisftebent,

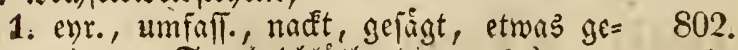
wimp.; Stgl 1 blith). (4 8. gb.) speciosissinnum:

2. långl. = lartzettformig,

a. ganzranto, raach), umfan.; SBrocte S03.

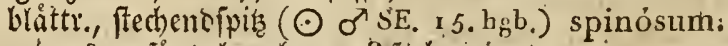

b. etwas gefågt, langhaar., 3 fact)gerippt; B ls. nadt; Stgl frautartig (4 9: hgb.)

S04. saiticifolüunt. 
3. lỏngl., ftummf, Ifaft ignnzrano.; BB to ede fumpfolattr., ftiellos, in Den 2lftwinkeln; 805.

I Stgl gabelf. ( $\odot$ SE, wohlriech.)

4. Ianjettf., etwas feingezalynt, unbebant; BI D. nadt; 5 tgl trautart. (4 9. gb.) grandiflórum.

5. ipntelf.; $\mathfrak{B}$ lo ede frumpfblittr., geftielt; 807. Stgl teantart. (4 SE, 36 . gb.)

6. Die unt. herzf., geftielt, bopl. gejagt: bie obern eyr., ftiellos, gefagt; Stgl traut= art. (4 SE.).

aguatǐcum.

maritı̌mum.

s0s.

cordifolium.

\section{BUPLEÚRUM. 183.}

\section{Stgl krautartig.}

I. Slgne gem. Şưllke; bie ourchbohrt. Blätt.:

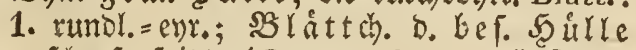
fcharfgeipiat $(\odot$ 3o. gb. - * Siraut, * 809. ఫ Saam. - ఫq Del, Muffr.)

2. eyrr. =rángl., Yeterart.; Dolden 3 fpalt.; Frud te etmas edf.; 'Stgl nieberliegend $\left(\odot \mathrm{SE}_{\mathrm{g}} \mathrm{gb}.\right)$

II. mit gem. n. bef. Şitlte; besondere Hiillen:

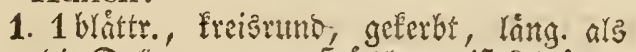
bie Dolsen; gem. 5 inlle meift 3 blatitr.;

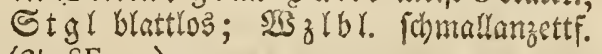
(4 SE, 9.)

2. 5 blåttr.; Blätter:

(rotundifolüum.)

810.

sủbovátum.

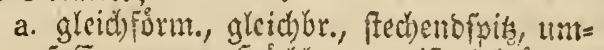
fafl.; gem. Solthen meift 3 blattr.; ber. Soirlyen gleidbr., faft eben fo lang als oic Dolben; Stgl altrectst, rifpenf. ( $\odot$ s. 16. gb.)

b. ungleidfơventig,

a. $3 \mathbb{B}_{3}$ Ibl. geftielt: Stglbl. umfall, längl. =ent.; gemeinfth. So ittre 3-5 blattr.; be F. So itren eyrumb, icharfgeipitit (4 8.)

b. $23 z$ Ibl. Finmallanjettf.: Stgibl.

811. stellatum. umfall., hersf. = längl., geftreift; gem. 5̧utre nreift 3 b́lattr., unt= gleichf.; be f. So illten eyr., icharf= geipirizt, gerippt, abgejonbert (2 SE. 9.) angulósum.

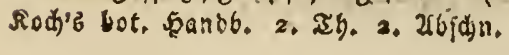

813.

(longifolŭum.)

juncĕıìm. 


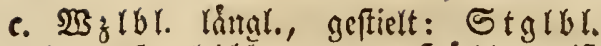
lanjettf. =gleichbr.; gem. Şälle meift 5 blottr.; bef. Şullen fpir; Stgl hin u. hergetog. ( 426 . dgb.)

3. meift 7 blättr., lảngl., fharfgeipiat; $g \mathrm{~g}$ m. Şůlle meift 3 blättr., verlángert: $\mathfrak{W}_{j}$ lbl. gleichbr.; Stgl meift blattlos (4 8.)

4. nebit gem. Scurle jebr flein; B l. geftielt, långl., gerippt, lezerart.; Stgl faft nadt, gabelf. ( 4 5. 8.)

815. (falcàtım.)

S16. graminifoliums.

817. (rigrüdum.)

\section{Stgl strauchig.}

Bl. ftiellos; langl., am (sirunoe fichnaler, ganjrand., leocrart.; Seàllenblát tcben langl., abfall. ( $5 E$. grnch.-gb. $\triangle$ )

S1S.

fruticòsum.

\section{BUTÜMUS. 327.}

(4 44, ros, u. w. - Ұ Futt., Unfrr.)

819. ̧ㅜ

(umbellátus.)

\section{BUXUS, 742. (W. 67.)}

Blattstiele:

1. gerwimp.; Stbelt. cur. =pfeilf.; bie eyr.= lángl., oben glíny. Blätter:

a. leverart., unten glanjlos, an bets. Seit. $\$ 20$. gervólbr ( $5 \mathrm{SE}, \mathrm{gb}$ )

b. faft bautart., auch unten gling., an beyd. $\$ 21$.

Seiten vertieft (ち SE. gb.)

2. unbehaart; $S_{t} \mathfrak{b} u t$. pfeilf.=gleidbr.; $\mathfrak{B} \mathfrak{l}$. långl.=ev)r., lebcrart. (ち SE. gb. $\Delta$ ) balearǐca,

\section{CACALЦА. 652.}

\section{Blätter:}

1. gleich formig,
a. lángl. =lanjettf., gefägt, ftiellos, am (Strunbe feilf., gangrano., berablauf. (4 SE. gbch.-w.) saracenica. 


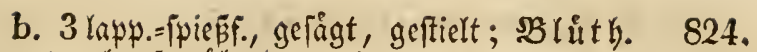
traubenf., iiberhang. (4 NAs. w.) hastáta.

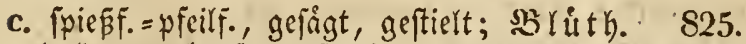
Dotbentraubenf., aufr. (4 NAm. w.) suaveŏlens.

d. herzif., gezahnt, geftielt,

a. unbehaart; SBlttfte nact; Dorben=

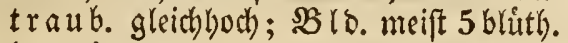
(4 9.)

b. unten grau; SBlttitle am Sirunbe mit ohrf. 2Cnhang; Dolbentraub. u. SBld. wie vorber (4 9.)

2. ungleichfórmig,

a. umfarl., gezahnt: bie unt. Yeverf.: bie obern tanzettf., pfeilf. ( $\odot$ As. dr.) sonchifolia.

b. Feingezabnt: bie unt. vere.eenr., geftielt: bie obern rangl, = lanjettf., pfeilf., um= fall. $(\odot$ SAs. gb. - p.)

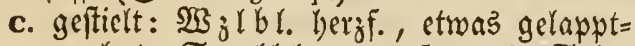
gezahnt: Stglbl. rautenf., ant o. Spise gejahnt; $B l_{\mathfrak{u}} t \mathfrak{l}$. boldentraubenf., aแb̆= gebreit., aufr. (4 NAm. gbch.)

826. alpina.

827. albūfrons.

828 829. agittáta.

830. atriplicifolı̌a.

\section{CACHRYS. 197.}

\section{Hüllen :}

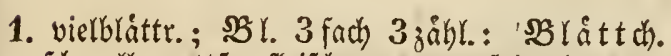
fa)mallanzettf., fleifo., etwas fpic (4 SE. 36. gbch. - ఛ̧ Sitich.)

831. ซ్ maritı̆ma.

2. wenigblattr.; Blätter:

a. mehr als oopp. 3igeject, unbebart, mit vielípalt., borftenf., etras ruşgcbreiteten BBlittch). (4 SE. w.)

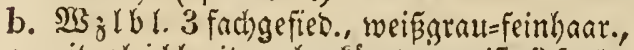
mit gleichbreit., abgeturgt., meift 3 falt.

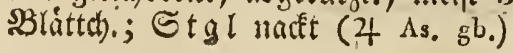

832.

Morinsonı̆.

833. odontalğ̌ca.

\section{CAKǏLE. 521.}

Bl. gefied.: Fiebern gleichbr., etras gejabnt 834 . (๑ -36. p.) maritüna. 


\section{CALGEOLARĬA. 18.}

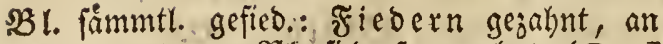
ben untern SBr. Fiederf.=gezabnt $\left(\odot \sigma^{7}\right.$ SAm. gb.)

835. pinnáta.

\section{CALENDǓLA. 712.}

\section{Saamen:}

A. nachenf., weichftachl., gefrümmt:

1. Irle: bie mittlern foft langettf.: bie åu b. runol., foff eben $(\odot$ 28. gb.

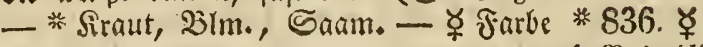
ge(b)

(oficinális.)

2. mur bie innern: die äussern:

a. lanzettf. = pfriemenform., am $\Re$ inden

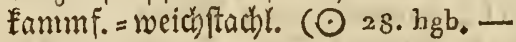
ఫ Farb. gerb)

b. cyr., am Rande băut., gezahnt, am 837. ఫุ (arvénsis.)

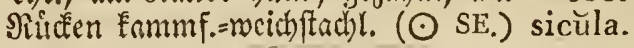

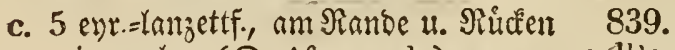
wie vorber $(\odot$ Af. w.; gb.) stellàta.

B. Erugf., vert. =eyr., eben; \$Blo. etwas 840 . meidjftachl. ( $\odot$ As. bgb.) sancta.

\section{Blätter:}

1. fontulyanzettf., bucht.=feingezahnt; Stgl 841.

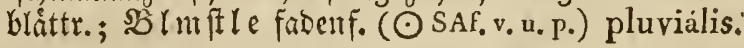

2. långl.= lanzcttf., ftumpf, gezalynt; Stgl Gláttr.; SElmftle oben bifer ( $\odot$ SAf. 842. w. u. v.) hybrĭda.

3. lanzettform., bucht. =gezahnt; Stgl foit 843 . nadit (SAf. hp.)

nudica'ulis.

\section{CALLA. 305.}

sBl. Kerzf.; sBlmicheibe flach; Solben

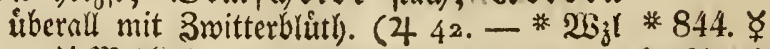
- $\Varangle$ Mell) (palústris.) 


\section{CALLITRICHE. 5. (738* *W. 57.)}

Blätter:

1. 3 ripp., oben gehåuft u. groóper; S. mit et $=845$. was ftumpf. Simbe (45.)

2. 1 ripp., abgeftutht, gleich, an $b$. Stgl zer $=846$.

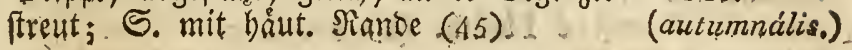

\section{CALLÚNA, 316,}

B r. entgegenger., 4 reibig, gebrångt; $『 n t=$ traub. nach einer Seite gemand, blättr. (ち 13. 29. v.; p.; 月.; w. - * siraut - 847.

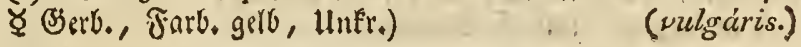

13. CALTHA. 440.

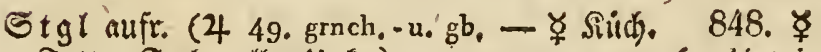

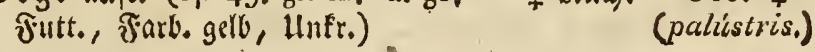

\section{CALYCÁNTHUS, 408.}

B I I lapp. Yanzettf.; bie längl., fpit. Bl.: 849. 1. unten filz. (ち NAm, ur, , wohlriech. $\triangle$ ) florùdus: 850 .

2. unten graugruin=weichbaar. ( $5 \mathrm{NAm}$, braun) glaucus:

3. untefharat (ち NAm. braun $\triangle$ ) 851. laevigátus:

\section{CALYSTEGĬA. 120.}

\section{Blätter :}

1. pfeilf, fehr fpis, binten ftumpf od. abge=

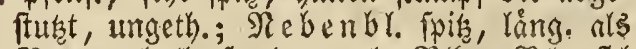

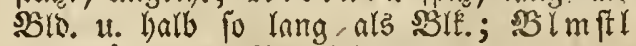
ecf., lång. als s3ltttatiel (4 26. w.; flo '૪्⿱ llutír.)

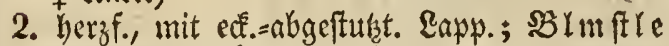
852. ్ (sepüит.) ftietruno, 1 blitth.; Neb enbl. enr., fumpf, 
aufgebla = bauch.; SBlb;åbne ftumpf (4 SE. w. grofs)

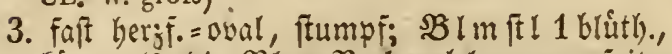
furg. alş bie SBl.; Nebenbl. eyr., ipis; - Stgl aufr., ofen blitbetrag.; Jy flanje feinhaar. (4 NAm, w.)

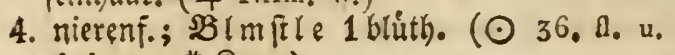
gbcb. - . Jiraut)

853. sylvèstris.
854. spithaměus:

\section{Soldanella.}

\section{CAMELENA. 507.}

Schötchen :

1. vert. = eyr., aufgeblaien; Stgef. cinfach; Stg! aufr.; Die umịn. Blätter:

a. ganzrand., haar. ( $\odot$ 3o. unterm Flachs, * 856. ఫ gbch. - Gaam. - ఛ Dil, Unfr.) (sutiva.)

b. gejahnt, unkehaart ( $\odot$ SE. 3o.) dentáta.

2. runbl., glatt; $\$ B l$. verE. eyr. =lanjettF., ge= jabnt, am Ṡrunie ganjrano. umfallent 858. (4 45.)

\section{austriūca.}

\section{CAMPANǓLA. 146.}

\section{Bldecksaum etwas abstehend;}

A. Blätter scharf;

1. Bliithen in Traub. od. $\mathrm{R}$ is pen;

a. Blätter gleichfürmig.

\section{Blüthen :}

1. an 1 blith., aufr. SBlmitlen in s. NBlatts

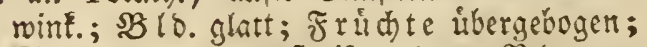
Etgl ftielrumo, geftreift, glatt; $\mathfrak{B}$ l. elyr.= lanjettr., bopp. gejagt (4 8.26. bl.-vo; p.; w. ; grau)

2. 1 jeit., jerftrent, itberbang.; $B$ Is. umge= ichlagen; $\mathfrak{B} l$. Gergf. = lanjettF.; Stgl áfíg

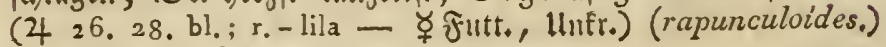

3. in Sifpen; Blätter:

859.

(latifolia.) 
a. cyr.=lanzettf., ftiellos, unten fidarf (4 861. 3. 11, bl, - v.)

b. ganz lanjettf., bopp. gciaght, u. nebft $\delta$. ât. Stgl u. D. SBlo. badtr.; SBlolapp. gefiggt, breiteriverbeno ( 4 As. 9. bl.) lactiflóra;

4. in evr.langl. Endtraube; Stgl ganz einf., SEl. lanjettf. = glcidybreit; $\mathfrak{O} f l$ a n ze badir. (o 9. gb.)

5. abftebeno; $\mathfrak{B l}$ l. eyruno, runjl.; $\mathfrak{B l t t f t e}$ burd) e. Siano breiter, u. gejågt; Stgl cinf., badir. $\left(4 \mathrm{bl}_{s}\right)$

S62.

863.

(thyrsoidéa.)

\section{4.} peregrina:

\section{b. Blätter ungleichförmig.}

\section{Bliithen :}

1. an 1 blútl)., libergebog. Blmftlen in ben Sittivinf.; 5 t gl edf., badtr.; $\mathfrak{B l}$. cyr.= lanzettf., grobgelågt: bie unt. ber jf.; sBlo. hadfr. (4 1. 4.)

2. 3 ipalt.; Stgl eff.; $\mathfrak{B l}$ l. geftielt; $\mathfrak{B} \mathfrak{l}$. gewimp. (4 15, 36, bl.; w.; p. - ఫ Jutt, Unter.)

3. an rispenf. Smeigen; Stgl gefurcht unto nebft ben auj beyd. Enoen fchmalern $2 B$ l. ftas doclhaar.: bie unt. evr. =lanzettf., geftielt: bie obern ftiellos; SBY. umgejthlag., bart. (4 As. 4. 8.)

4. an febr langer Enotraube; Stgl ftiel= runb u. nebft ben $\mathfrak{B l}$. unten fill.; bie $u n t$. sBl. berff. =langettf., gefticlt; bie obern ftiellos; $B$ ID. weisgrau, mit aufr. \&app. (4 6.)

5. uberhang.; Stgl ganj einf., wenigblith); BBl. ftachelhaar.: ' bic unt. berzf. = lanjettf, geftielt: bie obern lángl., ftiellos; sBro. etwas hadr. ( 4 As. bl.)

6. abftelbend, furry. als o. Eapp. o. ftriegl. SBlo.; unt. $\mathfrak{B}$ l. geffielt, cllipt.: bie ober at halb= umifafl., lángl. = lanjettfórm., geferbt $=$ gefägt $(\odot$ SE. $)$

865. urticifolía. 866. ఫ (Trachelium.)

trachelioídes.

868.

(ruthenica.)

869.
collina:

870.
Loréi.

2. Blithen in Köpfchen od. Kna ueln.

Bliithen :

1. in EnSE⿱⺊口) 
a. meift 5zåbl:: Sgltąq. etwas abftes 871. Geno; $B B l$. gleichbr. = priemenf. (4 8. v.) graminifolia,

b. 12 DD. 15 zábl.: $B \mathfrak{B l a p p . ~ u . ~ S k a p f . ~} 872$. aufr.; $\mathfrak{B}$ l. glcichbr. (4 SE. 23. v.) tenuifolra,

c. ftietloz; $S B$ l. fonmallanzettf., am Rande 873. ruellenf.; Sু flanze hader. ( $\sigma^{\gamma}-5 . \mathrm{hv}$ ) (Cervicaria.)

2. in $\Re n a \mathfrak{u} \in \mathfrak{l} \mathfrak{n}$; ber edt., einf. Stgl.:

a. badé; $2 \mathbb{B}$ zlbl. enruno=berzf., geftielt: 874 , Stglbl. hezzf., ftiellos (NAs.) .speciósa. b. glatt; Blätter:

a. ftiellos, långl. =lanzettf., herzf. (4 3. 875. $\not$ Ix. v. - ૪ Unfr.)

b. gefticlt, berzf. =etr., 2 zahn., raudb, attf beyb. Seit. grum; BBlattfile ge= flitgelt; $\mathfrak{B}$ luth. ftiellos; Şullenbl. evr. = berzf., fehr breit; Gir iff. fürz. als SBlE.; Sू flanze rmuch (4) foliósa:

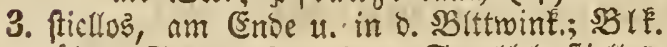
tóbr.; Stgl ed., glatt; Stglbl. ftiellos, gleichgeznhut, am rande wellenf., lmizettf.;

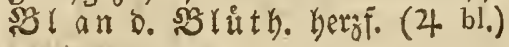

\section{B. Blätter nicht scharf;}

\section{Stgl 1 blüthig;}

a. Blätter gleichförmig.

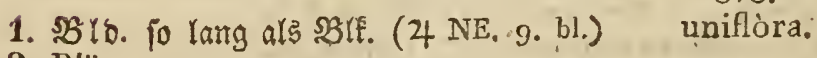

2. Blätter:

a. eyrund,
a. ginzrand.; etwas getwimp. (4 SE. 9 . b.)
b. etmas gef́cuto 8 ro iborgebog. (4 SSO. 9. dbl.) pulla.

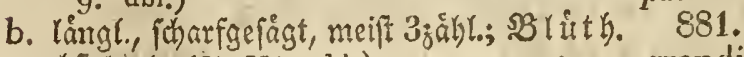 abftebent (4 NAs. bl.) grandiflora.

b. Blätter ungleichförmig.

\section{Pflanze:}

1. feinbaarig; Blätter:

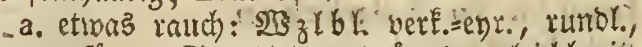
gefägt: Stglbl. georångt, gleichbreit, ganzrano. 0D. fohmallanzettf., etwas ge=

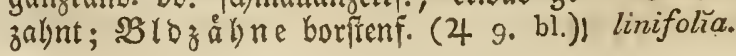


b. glatt,

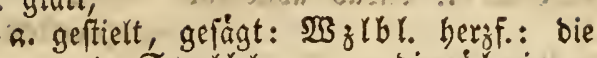
unt. Stglbl. evr.: Die úbrigen lanzettf.; Stgl rauch, nieberlieg., ecf.;

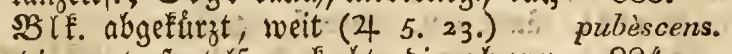

b. bie unt. fpatelf., geferbt: bie obern 884 . Ianzettf.; $\mathfrak{B}$ ID. gezahnt (4 As.) spathuláta.

2. unbehant; Die ü nt. sol. lángl.: S tglbl.

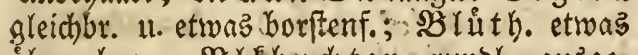

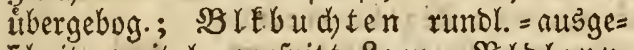
Tdynitt., mit langgefpint. Rapp.; SBlolapp. borftenf., zulekgt umgejotlag. (4 SE. 19.) excísa.

\section{Stgl 3 bliithig.}

\section{Blätter:}

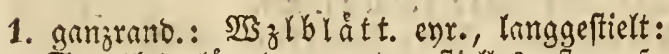
E.tglbl. längl. = eyrund, fftiellos, ftumpf;

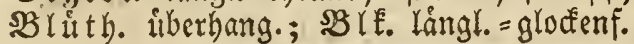
(4 9. hibl.) 886.
Zoysii. obern lanjettf.; Stgl ganz gerabe, ver: lang., åft.; bie untern $\mathfrak{g}$ lmitle meift 887: 3 blitth. (o⿱ 3. 4. 8. bl.; w.)

pyramidális.

\section{Stgl mehrblithig.}

\section{Blïthen:}

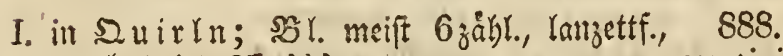
gezalint (4 SE. bl.)

II. in $\Re$ ifpen oo. İva ben; Blätter:

A. gleidefiormig,

1. Yơnglid), feingeferbt, ftiellos, fteif; 889.

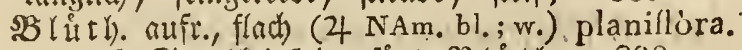

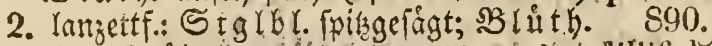
rifpenf., ilberi)ang. (4 SE. hbl, ; w.) " TiTiifolia.

3. rautenform., gejagt; Xehre 1 feit.; $\$ 91$. SB Is. ge galynt (4 SE. 9. hbl.; w.) rhomboidéa.

4. fohmallangettf., feingezahnt; Stgl aft.,. ganz geraoe, ruthenf.; SBrm ftle fac= Denf., verráng.; : Brế. trichterf., 3 u. $\$ 92$. 4 ppalt. (๑ SAf. w. - rch.)

lobelioídes:

5. Kersf., gejagt, geftielt; 3 weige fas

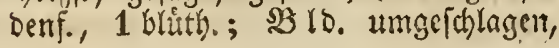




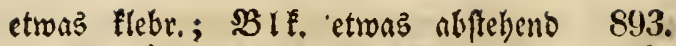
(4 SE. v.) " carphatı̌ca.

B. ungleichfórmig; Bliithen:

1. in Rifpen;

a. $\mathfrak{B}$ z Ibl. lăngl. = u. nierenf., gejågt: ङtglbl. gleichbr., gangranto. (4 4 . 9. 24. 35. bl.; w. - ఫ llufr.)

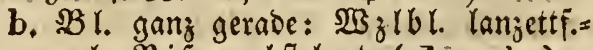
oval; Rif́pe abftebeno (o' 28. hv.)

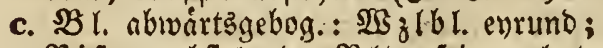
$\Re$ ifpe abfebend; $B \mathfrak{B l b}$., feingezabnt, umgejhlag. ( $\sigma^{7} 28$. bv.) .

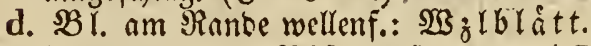
langettf. = oval; Si ilpe jigezogen (o

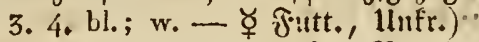

2. ftiellos, in o.shltwinf.; $\mathfrak{B l}$ l. herzf. $u$. langettf., gejägt; $\mathfrak{B l t}$ titl le ber unt. $\mathfrak{B l}$. gewimp.; $\mathfrak{B}$ IE. 5 theil., flach; (Si r iffel láng. als BlE. $(\odot$ NAm. bl.; w.)

3. an zuleht i̊berhang. SBImptten; $\mathfrak{B}$ l. alle

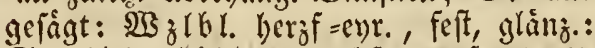
Stglbl. gleid)br., wed) 1. , entfernt (4 SE. 9. hbl.; w.)

4. eyr.=glodenf.; Stgl'éf; die unter= fien $\mathfrak{B} l$. eyr., gelagt: Stglbl. ge= orángt, bart, gejalgnt; $\mathfrak{F}$ rudh then. 10 edE. (4 1x.2x. dbi.)

5. groß, abjtebeno; Stgl edf.; $\mathfrak{B l}$ l. fteif,

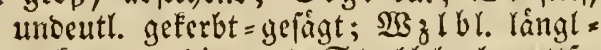
verE. eyr.: die unt. Stglbl. laugettf.= gleichbr: : bie oberften gleichbr., gams ramb., fiellos, entfernt (4 5. 8. bl.; w. -

894. ̧ㅜ

(rotuendifolia, 895. (patüla.)

S96. (neglécta.)

897. ఫ్ (Rapunciulus,)

898. americána.

899. pusilla. 900. cespitósa.

901.' ‘ (persicifulia.)

\section{Bldlappen wechs. umgeschla- gen u. d. Skaps, bedeckend.}

\section{Blithen:}

1. 1bluthig,

a. am hader. Sigl; $\mathfrak{W Z}_{z} \mathfrak{l}$ friech.; $\mathfrak{B l}$ l. vert. evr., gerwimp. = fcharf: $23 z \mathfrak{b} l$. fchmallan= zettf., am Ranbe wellenf., hacti. (4 SE. 9. blch.; ' wch.)

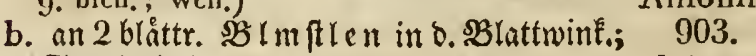
Stgl einf. (4 9. hbl.) alpina. . 


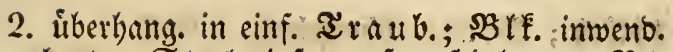
bart.; Stgl einf., aufr., feinbanr.; $\mathfrak{B l}$. lanjettf., geterbt (4 SE. 9. hbl.)

904.

barbáta.

3. in Rifpen; St@l rifpenf., feintaar.; SEl. lanzettf., etroas" ftumpf, am Siande 905. mellenf. $\left(\sigma^{7}\right)$

4. in EnD B l. lanzettf., ftumpf, geferbt; badir. (4 SE:)

5. an meift 3jåbl. Enoftielen; $\$ 2$ l. ellipt.s lo̊ngl., gefógt, haar.; Skapi. nekf. = aber. (o' As.)

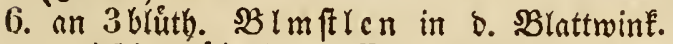
u. 1 feit., úberbang. Enoftielen; Stgl cinf., ausgebreit., feinbaar.; $\mathbb{B l}$ l. lanzettf.;

7. 1 peitig,

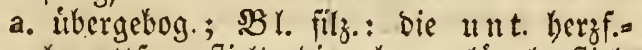
lanjettf., geftielt: bie obern lóngl., ftiels los; Frudt)ten. woll. (4 SE. 9.21. hbl.) gummifĕra,

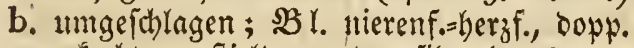
geḱerbt, geftielt, unten fill. (4 SE. 1 . gb. ; w.)

8. wedjelsweisftehent, an e. lodere $\mathfrak{A}$ bre; sil. gleichbr., ganzrano.; sp flanze hadér. (o $8 . \mathrm{bl}_{0}$ )

9. úbergebogen, imwend. punctirt, langlyan.; WBz zbl. geftielt, eyr., fpik, gefägt; Jyflanje 912. ralth) (NE. 2. 8. ausw. w., inw, rothpunct.) punctáta. 10. anfr.; Stgl ungeth., aufr., hadkr.; $\mathfrak{B l}$. 3 ripp. (ठ大 2. 5. hbl, ; w.; fl.) sibirica. lingulata.

907. betonicaefolía.

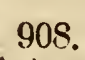

\section{Blk. fast ungleichf.; Narbe fust einf.; Skaps. an der Spilze aufspringend.}

\section{Blätter :}

1. ftiellos: die obern entgegengei., 3 zalnn.; 914. Stgl gabelf. ( $\odot$ SE. bl.; wch.)

2. geftielt, berzf., 5 lapp., unbehanrt; Stgl 915.

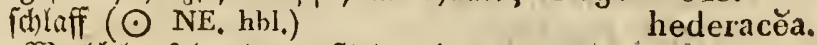

3. $\mathfrak{i g}$ jlbl. febr langgeftielt, herof. = rundel, geterbt: Stglbl. Elcin, faft Tpaterf., fpisz; 
Stgl einf,, 1 blutth.; Bluth. ưbergebog. 916. (4 SE, bl, ) cochlearifolìa.

\section{Skapsel 5 fächerig.}

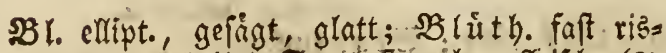
penf., 5 theil.; Stgliftraudy., fleifch. (4 917. Af. $g \mathrm{~b}$.) aurĕa,

\section{Skapsel prismatisch.}

\section{Stengel:}

1. nieberlieg.; $\mathfrak{B l}$ l. lanzettf., weitlåuft. gefågt, 918.

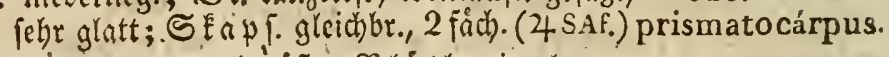

2. weitichmeif., febr âft.; $\mathfrak{B}$ lúth. einzeln;
a. SBl. lóngl., etwas geferbt $(\odot$ 3o.p.・v。; 919.

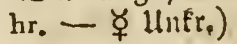
(Specülım.)

b. bie unt. $\mathfrak{S}^{\mathrm{L}}$ l. långl., frumpf: bie obern 920 .

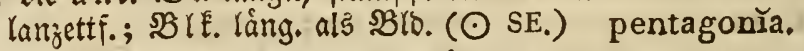

3. aufr., mit abfitebeno., meift 3 bluth. 3wei= gert; 'BR ellipt., umfanl., ftumpf, etwas

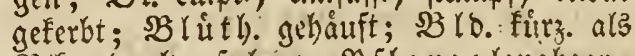
SBre, ob. eben folang; so flanze langhaar.= rauch ( $\odot$ 29.)

4. ganj gerabe; am Girunbe etwas i̊ft.; $2 B 1$. lángl., geferbt; $\mathfrak{B} 1 \mathrm{l}$. gebåuft, láng. alb BZlE. $(\odot$ SE. 3o.v.)

5. cinf.; $\mathbb{S}$ l. herzf., gezalynt, umfati.; sB rut th. ftiellos, gebauft (๑ NAm. bl. - v.)

921. hirta. hybrìda. 923. perfoliàta; :

\section{CAMPHORÓSMA. 77.}

\section{Pflanze:}

1. filz., meisgrau, nit bolz., rafenartigzfge=

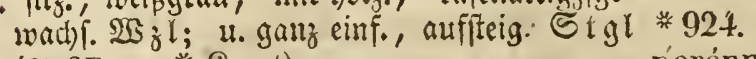
(4 $\mathrm{SE}_{0}$ - * Sirnut) péénne.

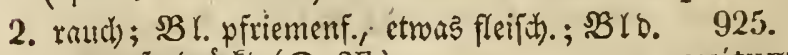
eyr., zigeorudt $(\odot \mathrm{SE}$.) ovátum. 


\section{CANNÄBIS. 780. (W. 52.)}

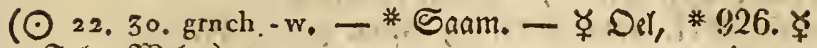
Fabr. 2Geber) satíva.

\section{CAPPĂRIS, 412.}

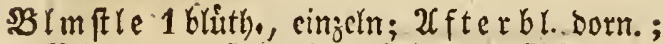
$\mathfrak{B}$ l. rundr., fttintyf, unbehaart; Słap fer

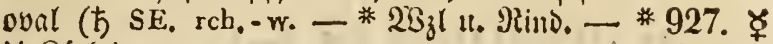
ఛ्र Sirith.) spinòsa.

\section{CAPRIFOLIUUM. 151.}

Stgl gewunben; Blätter:

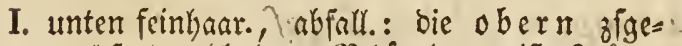

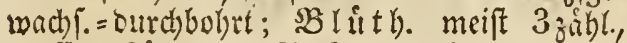

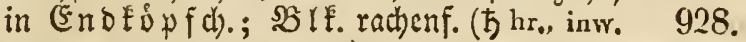
w., zuletzt gb. wohlriech.) etriscum.

II. unbelyaart,

A. baureno, unterharb weisgrůn: bie unt. 3 zábl.: bie vbern entgegengeieft: bie o berften zlgeraddjen = falt burdbbohrt;

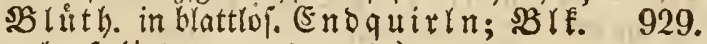
radjenf. (市 NAm, ro, inw, gb.)

B. abfallent ; Blkronen:

1. rachenfúrmig,

a. in End $\mathfrak{E}_{\mathfrak{L}} \mathrm{pfd}$ en; von ben unter= halb weifgrin. Bl.:

a. bie oberfiten. zigemabjen = faft Durchbohrt ( $S E_{0} r_{*} \cdot g b_{0}$, inw, w., zuletzt gb. $\Delta$ )

930.

impléxum.

931.

b. nicht (ち 4. 26. hp., wohlriech.) (Periclymĕnum.)

b. in Endquirln:

a. mit sblått. verjeben; $3 B$ r. unten weis̈grim: bie oberften zige= wacbj. (ち SE. hp., inw. gbch., wohlriech.)

b. blattlos, faft 'Fopff. = gebrangt; BZ K. mie vorber; $\mathfrak{B}$ lE. am Sirunde bodfr. († NAm. p.)
932.

italicum.
933. dioı̌cum. gratum: 
c. in blattloi. . faft fopff. =gyebraingt. $\mathcal{E}_{n} \mathrm{D}=$ quirln; $\mathfrak{B}$ I. roic 932, u. mit e. Enorpl.

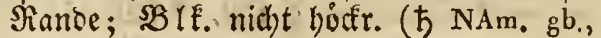
starkriech.)

2. nicht rachenf., mit oben bauch. Mobre u. faft gleid)f., 5 ipalt. Srum; $\mathfrak{B l}$ l. wie 932; sB luth. in blattlof. Enoquirln (ち NAm, r., 935. ohne Geruch)

\section{CARDAMINNE. 53S. $\left(72^{\mathrm{x}}\right.$.)}

\section{Mit 4 Staubgefäfsen.}

B1. gefies. $(\odot$ 28. 32.)
936.

(hirsitita.)

\section{II. mit 4 läng. u. 2 kürz. Sigef.}

\section{Blätter:}

1. cinfach), ganjranbig,

a. runol.=eyr.; Sglttflle feld lang; ber

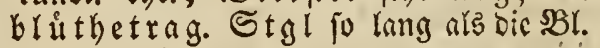
(4 NE. 9.)

b. lángl.=ey)r.; ber blutbetrag. Stgl bober als bie sil. ( 4 9. w. ; hr.)

937.

bellidifolı́a.

938.

alpina.

939.

2. 3 zábl., ftumpf; Stgl faft nadft (4 SE. 9.) trifolia.

3. bie obern 3 lapp. uno gefico.: bie unt. 940. ungetb. (4 9.) resedifolía.

4. Einige einf.: 2 noere 3 zólhl. u. gefies.: Blát th). ftumpf, 5 zabl., 3 lapp. ( $\sigma^{7} S E$. w.; hr.)

5. gefiebert ;

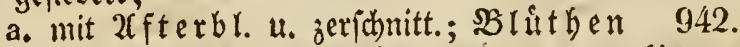
olne sBlbl. ( $\sigma^{7}$ 4. 8: w.)

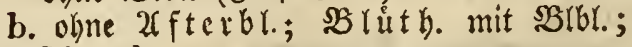
Blättchen:

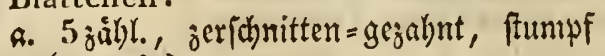
(SE. rch.)

941. thalictroides.

(impatiens.) 
D. rámmtlich 1 umor.; Stgl am Birunbe 946. fprofientrag. (4 4.) amúra.

c. nur die $\mathfrak{S}_{j}$ lb lát tchen runbl.: Die

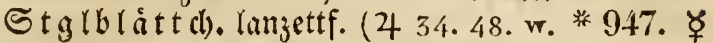

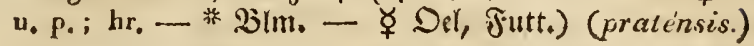

\section{CARDiospérmum. 321.}

\section{Blätter:}

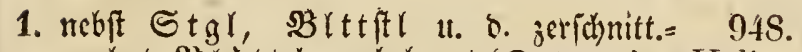

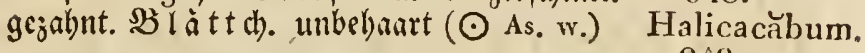

2. unten filf. ( $\odot$ SAm. w.) 949.

Corindum.

\section{CARDǓUS. 639.}

\section{Bläiter ganzherablaufend.}

\section{Bliithen :}

1. am 1 brittly. Blmstiel:

a. Pellir lang, nadt; ; 3 r. gejallint, Dorn.; 950.

Blb. Dorn., eimwärtżgeneigt ( $\odot$ SE.) leucogrăphus,

b. aufr., wehrloz; $\$ 3$ r. Dorn., unbel)aart 951 .

(4 $S E .49$. p.)

c. fil $b_{3} ; \mathfrak{B l}_{1}$. lanjettf., fieterf. = gejabunt, born., unter langbaar.; B lo foupp. piriemenf., born.: bie innern umge 952. frument (o' SE. p.)

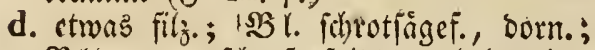

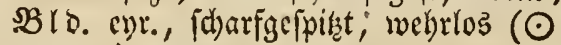
NAf. p.)

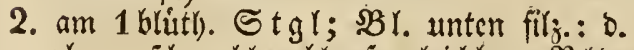
hamulìsus. obern fohmachberablauf. gleidbr.; $3 B$ ro: fch upp. lanjettf., jharfgeipist, abfteleno (4 11. - ఫllifr.)

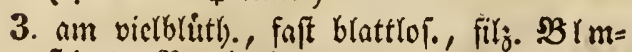
fitiel; 3 r. bucht., born., filz.; 3 1b. 955. born., abfall. $\left(\odot-r_{\text {r. }}\right)$ peregrinus.

4. gefnauelt; 83 r. fieserf., firl., mit hands., 95 tj. born. $\mathfrak{E}_{\text {app. }}$ (4 SE. p.)

carlinoides. 
5. ftiellos, faft gefnault; SBIs. walzenf.; \$3 l. långl., bucht., Dornt., weiß̈nder., un= ton langhaar. ( $\odot$ As. p.)

957. arabı̌cus:

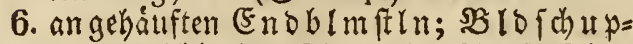
pen gleichbreit = prriemenf., id)arfgeipitat, etwas abfteleno; $S B$ l. langl., bucht., ant Rande Dorn., unten woll. ( $\odot$ 29. p.; w. - ఫ $\mathrm{Ulll}_{\mathrm{f}} \mathrm{i}$ )

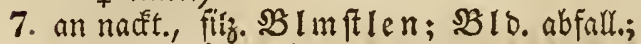

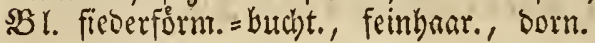

II. Bldecke: (4 SE. p.)

1. Eugel., etwas geftielt, mit gleichbr., um getrummt. Schupp.; $\$ B l$ l. budit. = oorn.

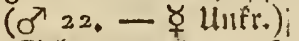

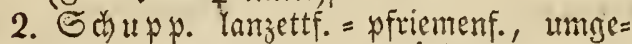

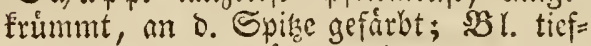
fieberf., mit aufwartzgezabnten, an ber Spitze born., am Sande borftenf.=gewimp. lapp. (4 j.)

\section{8. ఫ્子 \\ (crispus.)}

959.

pycnocephălus

960. ఫ్

(acanthoides.)

961.

arctioides.

\section{Blätter halbherablaufend.}

Bliithen :

1. úbergebog.; SZIofchupp. oben abftekend; $\$ B$ l. Dorn. ( $\sigma^{\top} 22$, rch.; u. Nachts wohlriech. - ఫ llnfí.)

2. foulpp., fiy.; SBID. eyr., mit pfriemenf., gerao. Schupp.; $2 B$ l. lanzettf., fiederf., born., unten filz. (Ot SE. p.)

3. faft fiellos, gebåuft; $\mathfrak{B}$ I frimmt; Stglbl. lángl., ungeth), born.= gezabnt, unten etwas langhanr.: $2 Z_{z}$ lbl. an (simmbe fieberf. ( $\sigma^{x}$ 9.)

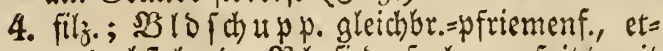
was abitebent; $\mathfrak{B l}$ l. fiederf., langgeipist, mit 2 lapy., gerwimi. = burn. Lapp. (4 9.)

5. an. 1 blitth., fehr langen $331 \mathrm{~m}$ it len; Bl.: a. fieberf. = gejagt, etwas born.= gewimpert,

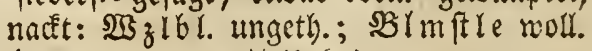
(4 8. p.; w. - ૪ linf r.)

b. ganzrand., lanzettf\%, gewimp.; $B$ I m ft le woll. (4 23. hr.)

c. Dorn. = gezabnt, etwas fleiju, lángl., ur= behaart, unten graigrim (4)

962. $\not$

(nutans.)

963.

candicans.
964.

Personàta.

965.

alpéstris.
966. ̧ㅜ

(deflorátus.)

967.

pannonicus.

968.

crassifolius. 
Carduus. - Garlina,

III. Blätter stiellos.

\section{Blätter:}

1. ficberformig,

a. unten filz., gleichbr., am Sinnoe untige rollt; $S_{t g l}$ faft nackt, 1 blittl.; $\mathfrak{B} i d=$ fth upp. fitz., eyr. =Iangettf., fperr. (4 9.) mollis:

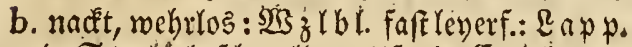
D. Stglbl. jhmallanzettf.: o. Enolapp.

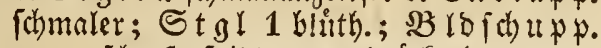
eyr., ficharfgeipiat, angedrudft (4 SE. 8. -

2. fammf. = fieberf., nadet, fharf, mit gleid)br., mebrlof. Eapp. u. ent. Enolapp.: die obern

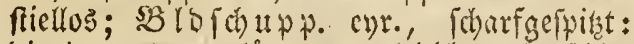
bie innern vertángert, gleichbr., verichie= Denfarb. ( 4 SE. 8. v.)

3. nadt; $\mathfrak{S B}_{\mathrm{B}} \mid \mathrm{b} l$ l langl, gangrand.: Stgl= blåt t. Ianjettform., ctmas gezabutt; Stgl

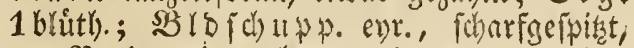
am Ranoe wie verorannt (4 SE, 6. rch.)

970. 후 lycopifolrus.

\section{Blätter umifassend.}

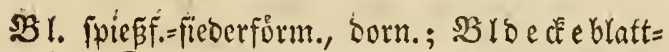
los; Dorn. rimenf., Doppeltborn. $\left(\sigma^{7} 22\right.$. * 973.

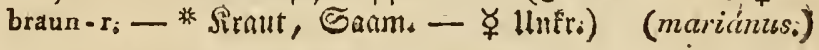

\section{CARLÍNA. 635.}

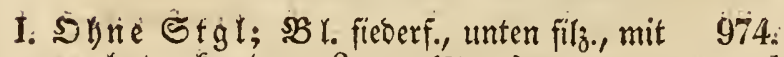
gezahnt = edf., born. $\mathfrak{L a p p s}_{\text {. }}$ (4 9.)

II. mit Stengel:

A. 1 blut thig,

1. einfact; $\mathfrak{B l}$. fieberf., nadet, mit zert=

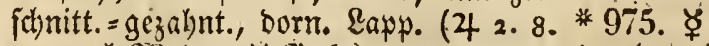

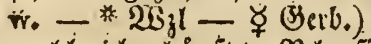

2. zahireidgebåuft ; $\mathfrak{B} r$. Fieberf., unbe= haart, mit fieberf., aubgebreitet., borm. Eapp: (4 SE. 9.)

B. inebreruttyig,

(aca'ulis.)

972.
cerinthoídes.

radiátus, 
1. meift 2 paltig; Nittelbliuthe ftellos; sBl. weiß̈grau, lanjettfơrm.; gejabnt, born. (○ SE. rch.)

2. Doltentraubenf.;

a. feinkaar.; sBLl. lanjettf., gejolint, sorn.; unteir feimbaar. ( $\sigma^{7}$ 8. 19. dr, u. gbch, * $2 B_{3}(1)$

b. faft unbehourt; $\mathfrak{B}$ l. Inimgettf., fieberf.= gejabnt, umbchanrt (4 gb.)

\section{CARPESIั̀UM. 662.}

1. Mit eingelti.; ubergcbog: Enoblutben (4 gbch.)

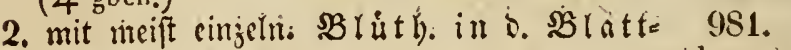
wint. (4SE, I.)

980.

cernuัum. abrotànoídes،

\section{CARPINUS, 748. ( $W_{0}$ 1.)}

Die bopp. geĩagten Blätter:

1. oval=langl., langgefpilit, nabigcrippt $=g \mathfrak{c}=$ abert; Sapfenfdi up. 3 theil:, mit lan=

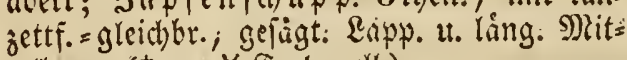
tellapp. (5 -

2. evr., langgeipitist, entferntgerippt genbert; 3apfenfthupp. 3 theil., mit e. ichiefen; evr.=larazettf.; an e. Seite 1-2 zobn. Sit= tellipy: ( 5 NAm.)

3. längl. $=$ evr., , etwas fpici, nabegerippt $=g$ ges abert; $3 a \downarrow f e n[d)$ up p. cyr., am Srunde fchicf, ungetly., etwas ecf.; ungleichgefingt ( 5 SE.)

9S2. ชุ

(Betŭlus.)

983.

americánà:

\section{CARTHĂMÚS. 636 .}

\section{Blätter:}

1. êr., ungeth., born. = gezahnt; $\widetilde{E}$ gl gànj unbehaart; $\mathfrak{S}$. olne srone $(\odot$ As. go.-rch.

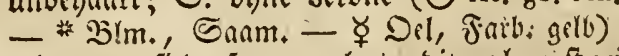

2. Die unt. fieberf., gezahnt: bie oberften umfaffend, fieberf. =gezabnt, born.; Stgl mollig ( $\odot$ gb.)

* 985. భ tinctorusus.

\section{4. orientális:}

986. lancitus. 
3. bie unt. leverf.: bie obern balbumfaft.; ficoerf. =gejabnt, born.; Stgl wernig be= baart; $\mathfrak{B} 10$. etwą woll. ( $\odot S E$ : w.. u. schw.) cretrcus.

4. Stglbl: gleichbr., gefied:; fo lang nls bie 988 . ภ)flanje (4 $\mathrm{SE}_{0} \mathrm{gb}_{\bullet} ; \mathrm{bl}$ ) , Carduricéllus.

\section{CARUM. 186.}

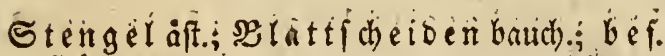

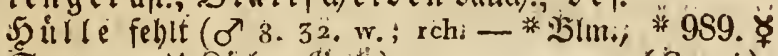
Enam: - ఛ Silid:; (Sitb:)

(Carvi:)

\section{CẢSSİA. 339 .}

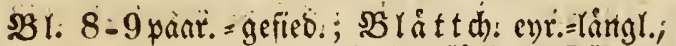
gleid), ftechensipits; init e. Driffe am (sirunde

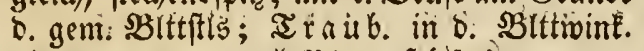

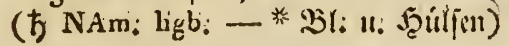

\section{0. marylañdica.}

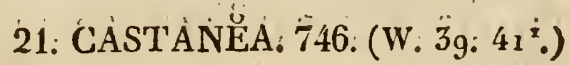

Die ftechenofpich = gefăgt. Blätter:

1. lángl: = lanjettf:; meift fetiwadthergf., lang=

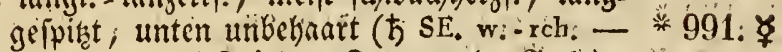

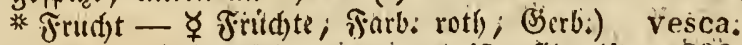

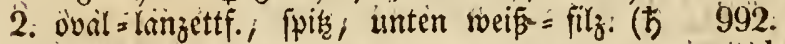
NAm: $\Delta$ ) pumila:

\section{CATÁ́LPA. 22.}

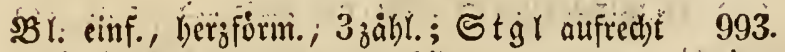
(5) NAm: iv:; uniten $r_{0}$ gead.) syringa efol̆a.

\section{CATANÁNCHE. 600 .}

Die untern Bidschuppen:

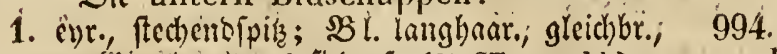
ant Śrunbe cttwas fieberf: (4 SE. 21. bl:) coerulěa: 


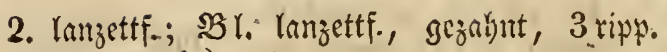
$\left(\odot \mathrm{SE} \cdot \mathrm{g}^{\mathrm{b}}\right.$.)

995.

lutěa.

\title{
5. CAUTCĂLIS. 208.
}

\section{Blätter:}

1. Sopp. jigeletst, ctinaz aufrectht, unbehant:

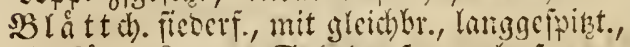
abgefirrt. Rapp.; S.tgl aufr., gabelf., unt= behaart; Szitten 5 Whattr., am @ande troce.

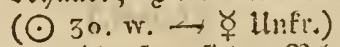

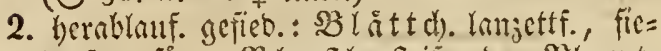
berf. : gejägt; SBtmfte feif, ton BBl. ent=

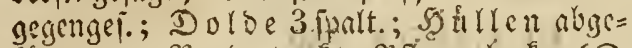

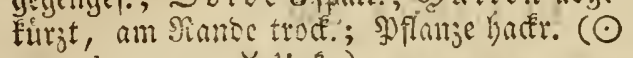
50. rch.; w. - , llnftr.)

3. Doppelfficterform,; gem. Hülle:

a. meif 3 blátr.; ber. Dolde n 5 finm.;

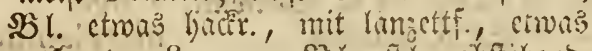

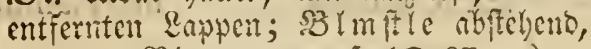
fteif, Den BRt. entgegengef. ( $\odot$ SE. w.) platycárpos.

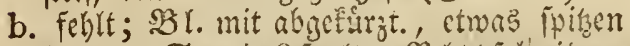

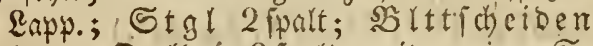
hant.; Dolben 2 part., mit wenigen

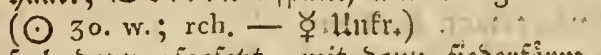

097. ४ (latifolic.).
$996 . \not{\gamma}$
(srandiflúra.

4. 3 fach bopp. jigelest, mit bopp. fieberförm.

\author{
999. ఫॄ \\ (daucoides.)
}

Sláttch.; it. Lanzettf. Rapp.; Stgl rúchw.

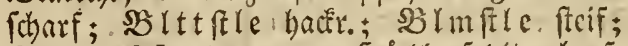

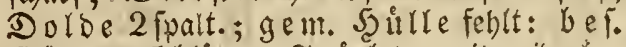
Shürlen 5 blattr.; Fritch te mit wiberba=

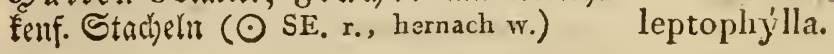

\section{CAULINIA. 726. (W. 44:)}

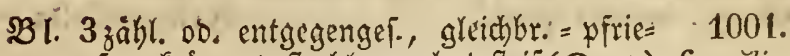
menfo, gefrumunt, ftact)l.=gezabut, fteif $(\odot 44$.$) fragïlis.$

\section{CAULOPHÝLLUM. 266.}

(4 NAm, 1, 8.)

1002. thaliçtroides; 


\section{Ceanothus. - Celtis.}

\section{CEANÓTHUS. $15 \dot{4}$.}

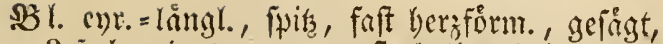
3 fachgerippt, unten fachelgarig = weich; Ri ipen gecringt, in ten langgefficlt. SBitt $=1003$.

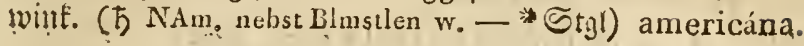

\section{CELÁSTRUS. 161.}

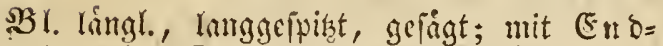
tranb.; Stgl gevumben, vieláft., whue 1004. Dorn!. (ち స̃Am, grnch.)

\section{CELSİA. 492.}

\section{Blätter:}

1. Dopp. gefied. ( $\odot$ As. grau u. hgb.) 1005.

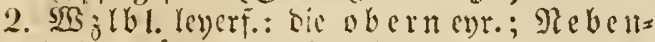
blat t. ling. als o. SBImffte; $\$ 3$ ! b. gleichbr.= lingl., gimjrano. ( $\odot$ SAs.) orientàlis. 1006. coromandeliána.

23. CELTIS. 803. (234.)

Bfätter :

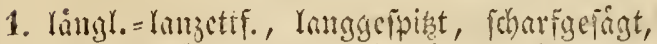
whell forarf, unten feinlgantig, weici), ant

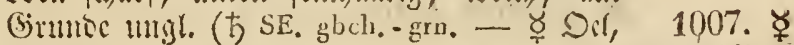
(Sirtb.)

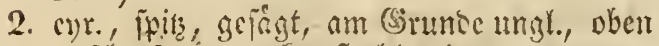

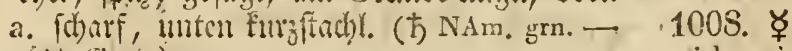
- (

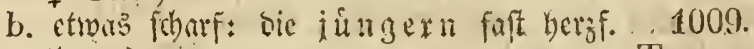
( 5 is.)

Tournefortǐi.

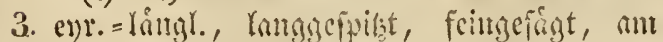

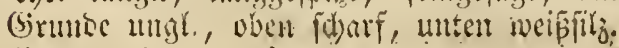
(t) SAs, gbclo, grno)

1010. orientàlis. 
19. CENTAURÉÁ. 695. $\left(63_{2}{ }^{\mathrm{x}}\right.$ )

I. Bldecke gewvimpert, $u$.

A. olune Dornen;

1. mit borstigfederig., gekr ümmt. Wimpern.

\section{Blätter :}

1. gleichfợ̂rmig,

1. längl., ungeth., fharf, fted)endfpits=fein= gelågt; Bld.:

a. freupelf.; Stgl einf. (4 As.)

1011.

salicifolĩa.

1012. ૪

b. micht freuferf. (4 3.4, 8. r. - ఫunfr.) (phryera.)

1013.

2. enr., fharf, ungeth., grobgezalgnt (4) austriüca.

3. evr. = lanjettf., am (Sirunbe gejalnt, $\mathfrak{g e}=$ rippt, feinbar.=(tad)elbaar, ; mit S che $\mathrm{i}=$ benbrumen (4 SE.)

4. FhmallangettF., gam rapo. u. nebjt Stg! 1015, fcharf; $\mathfrak{B}$ !

trichocephăla.

1016.

5. ganz lanzettfo, etwas gezahnt, fitlo. (4 9.) uniflóra.

II. unglẹidf.: Die unt. Stglbl. buicht. = fie= berf.: bie obern u. an s. 3weigen una 1017.

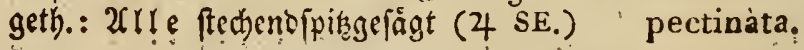

2. mit gewimp., od. gesägt: A nhängen;

a. Blë̈tter gleichförmigr ;

g. Blüthen gelb.

Blätter:

1. längl., gelågt, herablauf., ungeth.; $\Re 10=$ id upp. mit e, idswarg. Saum am Rande (4 As. 9.)

2. ficoerf., unten faharf, mit lanzettf, etwas gezabnt. $\mathfrak{L a p p}_{\text {. (4 As.) }}$

1018.

ochiroléuca.

1019.

țatarı̌ca:

b. Bliithen nicht gelb.

\section{Blätter :}

1. lanzettfórmig,

a. fir子., ftiellos, budht.=fieoerf. (4 SE, 8. p.) Triumf̣́ctti. 
b. faft unbebaart, berablamf., gamgrano.; 1021. ङtgl cinf. (4 9. bl, ; w.)

montána.

2. lángl. = lamgettf., ungeth)., febr fcharf, fpit,

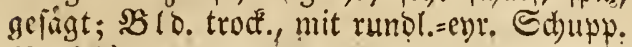
(4 SE.)

3. fieberf., etwas forarf, mit lanjettf., fpific, am (Grunbe fiederf. Rapp.; $B$ ld. feinbant. (4 3. p. - ६ llnfi.)

4. bopk. fiederf., mit lanjettf. Iapp.,

a. auf bevo. Seiten filz. U. \&app. ftumpf 1024. (4 SE. p.)

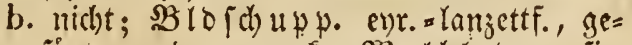
fingt = gerwimp, trode; 233 z 1 bl. Dopy. fies Derf.: Stglbl. gefies. u. fieberf.; Stgl fpinnengewebeart. = woll. $(4 \mathrm{SE}, 23 . \mathrm{dp}$. $)$

Cinerarra.

macrocephăla.

1023. ఫ్

(Scabiósa.)

\author{
Cineraria.
}

1025.

atropurpurĕa.

\title{
b. Blätter u!ngleichfürmigr; \\ A. Bliithen gelb,
}

1. $\mathfrak{B} z$ fbr. bopp. Fiçocr., mit lanzettf. Rapp.: Stgl cinfacblicserf.: 2atle unten fibarf; Stgl eben; $\mathfrak{B} l \delta$. trod; mit evr.=lanjettf., gerägt. =gemintp. Sthup. (4 As, gb, ; rch, $)$ calocephala

2. bie unt. Dopp. fieoerf., lanjettf. =glsid)br.; bic abern einf. fieberf; Stgl aubgebreit.; Blofd) up c cur.=lanjettf., mit o. Spize etwits abftebello (4 As.)

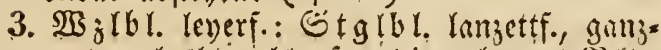
rano., balbberablauf.; bie obern $\$ 3 r 0=$ - (d) upp. an ๖. Spisce trof., gefágt (4 As.) alata.

\section{b. Blithen nicht gelb:}

\section{Blätter :}

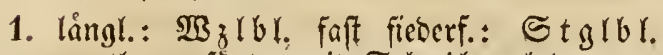
ungeth., gefägt; mit Sheibenb um un (4 4. 8. p. - braun)

2. $\mathbb{M g}_{\mathfrak{z}} \mathfrak{l b l}$. unbeutl. fiederf.: $u n t$. Stglbl. am Srunde ctwas gejabnt: bie ober il ulls geth., ganjano.; die innern 2 Ib (d) trode. (4)

3. meísgrau, lanzettf., berablauf.: bie unt.

1029. (nigra.)

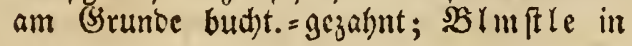

1030. nigréscens. 
ben Slttwinf. u. am Enbe feheno; $231 s .1031$. gejåght (4 8,)

4. gleichbr. , ftiellos, ganzrano.: bie unt. am Grumbe fieberf.; SGlo. gelägt ( $\odot$ bl.; w.; 1032 . ఫ̧

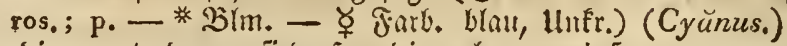

5. Die unt. Dopp. Fieberf. : die obern einf. fieberf.; Stgl rifpenf.; $\mathfrak{B}$ ID. evr., ange= brúlft $(\odot$ 14.22.29. hr. u. wo)

6. etwas filz.: $\mathfrak{Z} z \mathfrak{l} \mathfrak{b l}$ l. fiederf., mit lanzettf., fumpf. Eapp.: bie.obern am (Srunde nach unten hin etwas fieberf. (4 SE. p.)

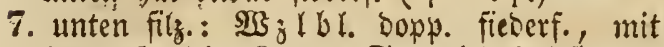
lanzettf., fpic. Laph.: Stglbl einf. fiederf. (4 SE, P.)

8, fiẹberf, f fharf, mit långl. =langettf., fpis. Eapp.: Die oberfen $\mathfrak{W}_{z}$ lbl. am Grumbe

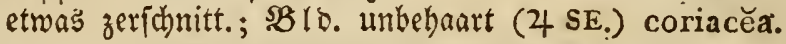

9. långl. = lanzettf., gangrano., jott.ofeinhaar.: Stglbl. berablaufent; $\mathfrak{B}$ ID. bramfledig (4 - v.)

10. auf berd. Seiten fily., freberf.: einige ungeth.; Stgl abmairtsgeneigt; $\Re r^{\prime}$. troâ., miṭ eyr., ftumpf. Schupp. (4 NAs. p.; A.) sibirica,

\section{B. mit Dornen;}

1. Dornen einfach an d. Spitze d. BIdschupp:

a. Blüthen gelb.

\section{Blätter :}

1. gleichfôrm., leverf. =gefied., faift ganzrano., 1039. mit jebr grof́., gezahnt. (Enobl. (4 SE.) centauroídes.

2. 1tngleichformig;

a. bie unt. fieberf.: Die obern gleichbr., ganjrano.; Stgl rifpenf.; mit ভschei= benblumen (○ As.)

b. $\mathbb{E B}_{\mathrm{g}} \mathfrak{b l}$ l. leyerî.: Stalbl. gleichbreit: Ulte weipgrau; SBlo. evr., mit an b.

Epitze umgeidlag. Edupp. (4 As.) parviflóra.

c. nefiedert, mit glcichbr., ftumpr. Raph.:

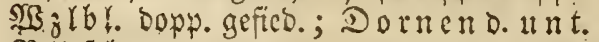
BIofd) upp, umgeid)l. (4 As, sehr wohle riech.)

1040. coronopifolla,

\section{1.} refléxa. 
d. $\sqrt{B}$ z $\backslash \mathrm{L}$ l. Dopp. Fieberf., mit lanzettform. 1043. Lapp.: Stglbl. cinf. fieberf. (4) collina.

e. $\mathbb{M}_{3}$ zlbl. Dopp. gefied., mit gleichbr. = far 1044 . Denf. Fied.: Stglbl, cinf. gefied. (4 SE, rupéstris,

\section{b. Blüthen nicht gelb.}

\section{Blätter :}

1. gleichf., lángl., filz., ftiellos, etwas gezahnt, 1045. am Strunbe fomaler u. tiefgezalynt (4), riğda. 2. ungleị) f., meiв̈grau, fieberf., ganzrand.: 1046.

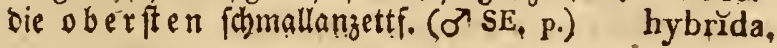

\section{Dornen biischelweis, od.} handf. vereinigt;

a. Blüthen gelb.

\section{Blätter :}

1. gleinformig,

a. lanjettf., fharf, feingezabnt, am Sirunbe fibmaler, berablauf.; sBlo. einzeln, fort 1047. ftiellos, mit geraben Dorn. $(\odot)$ sulphurěa.

b. fieberform., zerid)nitt. =gezahnt, geftielt; 1048. SBlo. ftiellos, geEnauelt ( $\odot$ NAf.). straminěa. 2. ungleidhformig; Dornen:

a. gerabe; Blätter:

a. meip̈graul., fdumallanzettf., herablauf.,

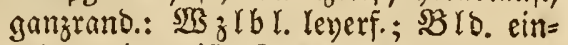
zeln, mit weís. Dorn. $(\odot$ 28.)

b. lanjettf., fharf, herablauf.: bie unt.

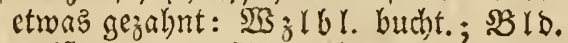
meift gepant, ftiellos ( $\odot \mathrm{SE}$.)

1049. (solstitiális.)

1050. meliténsis.

c. filz., lanzettf., herablauf.: o. unter= ft en feingezahnt, am Sirunbe fieberf.; $\mathcal{B l}$ l. cinjeln: bie innern Sthupp.

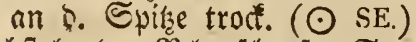

b. abftehent; $2 B l$. fdarf: Stglel. lan= zettf., am Girunde berablauf., feingejubnt: $\mathfrak{M}$ z 1 bl. leverf. $(\odot$ 28.)

1051. Adàmi. 1052. (sicǔla.)

\section{b. Blïthen nicht gelb.}

\section{Blätter:}

I. gleidfợmig,

1. lángl., umfaff. u. Kerablaufeno, 
a, wenig bebaart, auggeictweift = gejabnt, mit born. Báhnen; SBloborn. um= gejul. (4 SE. 36. wch. u. p.)

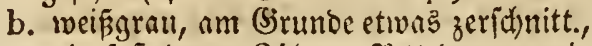
nit faft born. Báhn.; SBloburn. wie vorber (4 SE. p. u. wch.)

2. langettformig,
a. ftiellos, gezahnt; sBloborn. 3 fpitg. 1055. $(\odot \mathrm{SE}$. rch.)
b. faft umfall., fieoerf.=gezabnt; $2 B$ lo. 1056 . einjeln, ftiellos (4 SE. rch.) Isnárdi.

3. leverf, gefticlt; am (Srunde umfaffent, haufr.; SB lo born borftenf.; Re bestol. 1057.

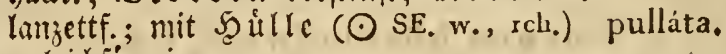
II. ungleichformig,

1. lanjettf., ftiellos, herablauf., haar.=fharf,

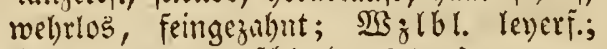
BIboorn. unigefh). (4 SE. r.) romána.

2. Stglbl. lanzettf., gejobnt, herablauf.; 1059.

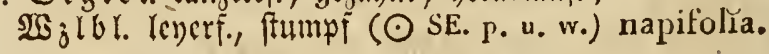

\section{3. mit gefied, dorn, Anläng.}

\section{Blätter :}

1. fieberf., gleichbr., gejahnt; Stgl baar.;

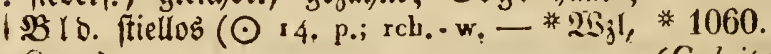
Siraut)

2. Gerablaufeno:
a. 2llte halbherablauf., feingezahnt = born.;

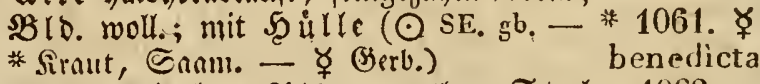
b. einige bucht,: 2(rte ungetb.; Stgl furolfend; $\mathfrak{B}$ lo. woll $(\odot \mathrm{SE}$. gb.)

(Calcitrápa.)

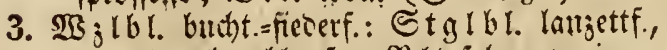
ganzrano., herablnuf.; $\mathfrak{B} \mathfrak{l}$ o $[$ d $)$ up. in e. febr langen, in $D_{i}$ s)litte 2 borit. Sorn ensi= geno ( $\odot$ As.)

1063.

Verútum.

\section{Bld. nicht gerimpert; \\ A. mit dorn, Spitze.}

Blätter:

1. Gerablauf., budbt., born., unten filg. ( $\odot \mathrm{SE}$.) galactites. 
2. fieberf., ganzranto, mit gróp.., gejallynten Enbbl.; $3 \mathcal{B}$ lo. trufe. ( $\odot$ As, wcli, ". rch.) Crocodylium.

3. lamjettf., gelígt: $\mathfrak{I B}_{\mathrm{g}} \mid \mathrm{bl}$ l. leyerf.; Stgl 1066.

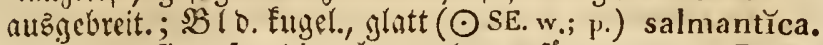

4. bie unt. Fieocrf.: Die vbern hanzettform.; 1067. B Imftre jel)r latig $(\odot, S E$.) muricáta.

B. durche.trockn. Anhang gerundet, oft zerrissen.

Bliithen :

I. gelb; Blätter :

1068.

1. umyetl)., gañ̆rando, herablauf. (4 NAs.) glastifolĭa.

2. etwa fil leverf. ( 4 As.)

babylonǐca.

II. nicl)t gelb; Blätter:

A. gleidffurmig,

1. eyr. =lóngl., feitrgejalint, unten fill.; 1070. $\mathfrak{B}$ lo $\{\mathrm{ch} u$ p. ev)r., jerriाi. (4 SE. 9. p.) Rhapontǐca.

2. gefieb.: Fieb. gleichbr., ftechencipis, ganjrant.; $\Re$ is. walgenf., mit fred)endo= ipits. Situpp. $(\odot$ 19.)

1071.

nitens.

B. ungleichforming,

1. Die unt. Dopp. fieberf., gleidbor.: bie obern gefies., mit gleidbror., etwas geznhnt. Fied.; sBld. enr., meift mit

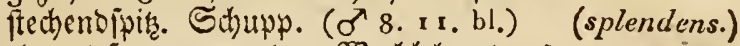

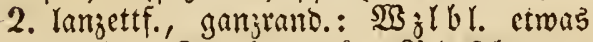
gezalint; 8 weige edf.; 3 loj d upp. eyr., an D. Spitze jerrifil: (4 3. 34. 1073. ఫ̧ b.. - ఫ Futt., Fortb. gelb, llutr.) (Ja६̌̌a.)

3. gefied. =gejalunt: $S_{t g l b l}$ gleichbreit, ain Sirunbe gejalint; sBblo. ungetheilt, '1074. ftechendipit (4 9. w.) (alba.)

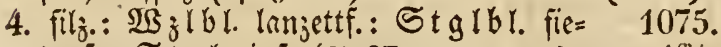
berf.; Stgl einf. (4 SE, 18.21. p.) conifĕra.

C. beydes nicht.

Bldblätter:

I. runolid); Blïthen:

1. gelb; Blätter :

a. leverf. = fieberf; ; $\mathfrak{B}$ ! $b$ id, upp. runbl.= eyro, etroas ftumpf, an o. Spize braun= fleft: $(\odot$ As, wollriech.)

1076. suaveŏlens. 
b. gefiebert, unbehaart: fie bern ganj= rand., an b. Spike unbeutl. geiagt: bie mittlern 2 theil.; Bloidupp. ivr., ftumpf (4 SE.)

2. eoth ob. meisं; Blätter:

a. geficd., unbehaart: bie berablaufeno. Fiellern:

a. icharf u. bopp. gejägt, mit lanjettf. Fnbbl.; $\mathfrak{B}$ lo fdu $u p p$. evr., ftumpf (4 SE. 9. r.)

b. fctarf $=$ fnorpl. $=$ gelägt, mit långl, = e) $r$. (Enobr.; $\mathfrak{B}$ ID id) upp. wie vor= ber (4 NAs. wch.)

b. wenig fiederf., mit faft gangrand, un= terffen $\mathfrak{L}$ appen; $\mathbf{B}^{2} l \mathrm{l}$. feinlyaar., mit

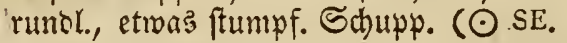
wch.; p. starkriech.)

II. lanjcttfơrmig; Blätter:

1077.

alpína.

1. gefieo., faharf: Fie berchen feingefingt,

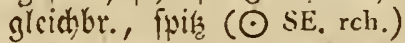

2. raft herablanf., leyerf. =gezahnt; $\mathfrak{B} l d=1082$. fd) up». ftechendpif $(\odot$ NAf, p.) Lippü.

\section{Centauruัum.}

1078.

$107 !$ ruthenǏca.
1080. moscháta.
1081. Crupína.

\section{CENTRÁNTHUS. 1.}

\section{Blüthen:}

1. in Doloentraub.; Befruchtungstheile:

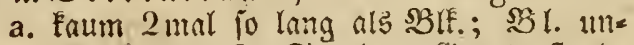
geth., lanjettf.; Stgl am Sirunte ftaul. (4 SE. 22. $\left.r_{0} ; w_{0} ; t_{0}\right)$

b. faft 3 mal fo lang; $2 \mathfrak{l}$ l. gleichbr.; Stgl 1084 . frautart. (4 SE. 9. r.)

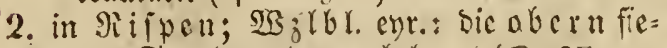
serf.; Stgl gerabe, unbehart ( $\odot$ SE. $2 S$. rch.)

1083.

ruber. angustifolı̆us.

1085. Calcitràpa.

4. CENTUNCǓLUS. 5S,

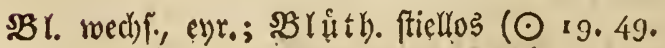
w. ; $x$ ch.)

1056.

(minimus.) 
Cephalanthus. - Cerastium.

\section{CEPHALÁNTHUS. 57 .}

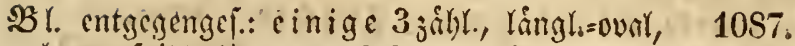

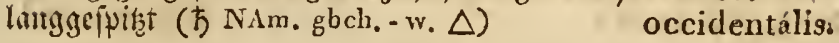

10. CERASTIUUM. 369. (360.

$$
370^{2} \cdot 792^{\mathrm{x}} \text {. W. 83.) }
$$

I. Saamenkapseln länglich.

I. Mit 5 ङtaubwegen; Blätter:

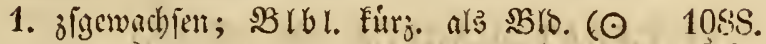
SE. $\left.w_{\text {o }}\right)$ perfoliátum.

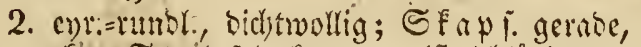

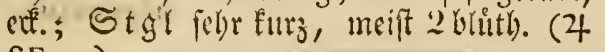
SE, 9.)

10S9:

lanítum. 1090.

3. enr.=lanjettf.; Stgl betl). (4 9.) alpinum.

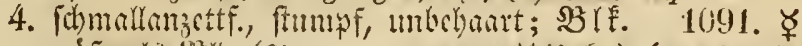
gróp. als sBzlo. (24 8. 14.29. w. - ఫllufr.) (arvénse.)

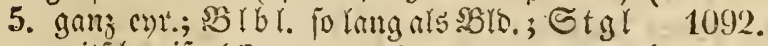

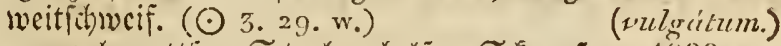

6. gang lanzettif; Stgl gabclf.; Sfapl. 1093. aufr., làng. als $B 10 .(\odot 50$.$) (dichotoomiuni.)$

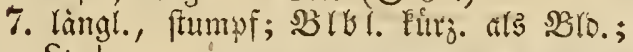
Stgl:

a. aufr., haar. = fĭmict. $(\odot$ 3. 34. w.). (viscószum.) 1095.

Ł. nicberlicg., haar. $(\odot$ 2. 13.) (semidecútdrum.)

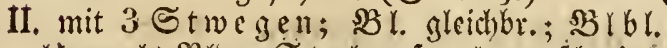

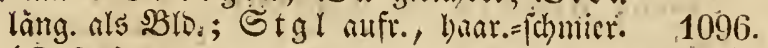
$\left(\odot \mathrm{SE}_{\mathrm{i}}\right)$

anomălum.

\section{Skapseln rundlich,}

\section{Blätter:}

1. Lanjettf.; BImstiele:

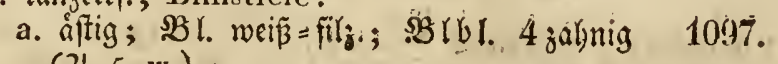
(4 5. w.) ( repens.

b. Fegr lang; Etgl fteifgernde; Sfapiel 1098 . fugel.; of flanze unbehaart $(\odot \mathrm{SE}$.) mantrcum.

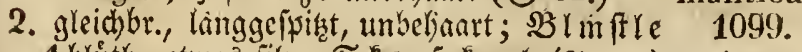

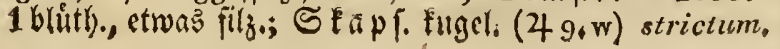


3. fomallanjettf., etrons raud); Stgl baur., 1100. niebergeftredt ( 524 .)

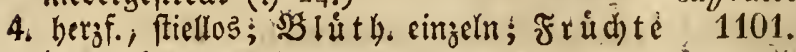
hang. ( 4 43: w.)

(aqualicum.)

5. enruno,

a. etwas filzig; 3iveige meift 1bluth.; 1102. Słàp f. Fugel. (4 9. w.)

b: unbehaart, fpitis etwas gewimp.; Fno= blutben faft boldentraubenf. (49.) latifolüsm.

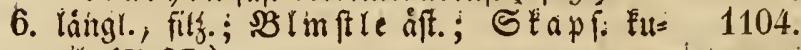
geh. (4 SE.)

tomentớsumi.

21. CERATOCÁRPUS. 728. (W: 55.)

(๑ Ás: 19.$)$

1105.

árenarǐus:

5. CERÁTOCËPHĂLUS: 259.

Hörner d. Fruchthuillen:

1. fithelf.; etraas auffiteig. $\left(\odot 30 ; b_{i}\right)$

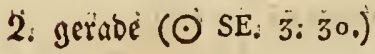

1106. falcátus. 1107. orthocĕras:

\section{CERATOPHÝLLUU: 75S. (W: 41.)}

\section{Blätter:}

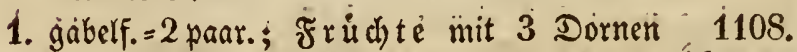
(4 44: 45:)

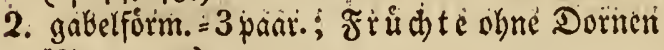
(4 44: 45.)

(demérssim.)

1109.

(sübmérsium.)

10. CERCIS: 337.

Die gaingrano.; inbebaart. Blätter :

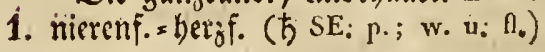

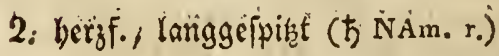

1110.

Siliquástrum. 1111: cariadénsis: 


\section{CERÍNTHE. 92.}

\section{Blumenkronen!}

1. etwas abfelieno, frumpf,

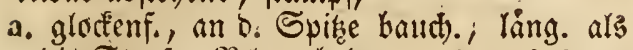
bie Stgef.; $3 B$ l. unbehaart ( $\odot$ 8: 'lob. u. p. - ఫ Farbi blaul )

b. watjeñif; cben fo lang als t. Stgefáke; 1113.

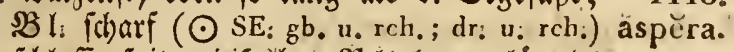

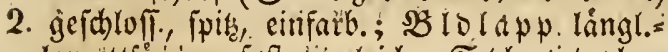

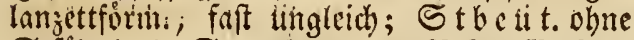
Stfåd. in D. Saumfipalten D: SBlE.; SBlátt:

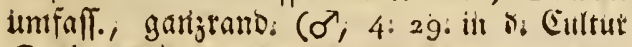
D $\left.g b_{0} ; i_{i}\right)$

1114.

minor:

\section{CHAEROPHÝLLUMM. 212:}

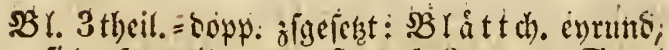

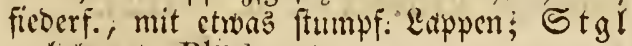
unbel)art; Bliithen:

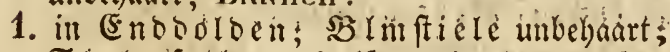
Efgl gefurcht, géftreift, mit strans aufge=

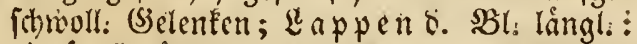
Die álu

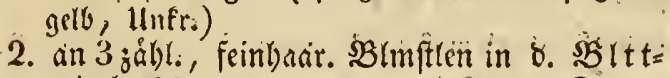
winf: $(\odot$ 28: 29: w: -

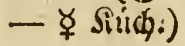

1115: ૪

(syluestre.)

* 1116. ఫุ

(sativiumi.)

\section{1: СНARÄ: $727 \%(\mathrm{~W} 42$.}

\section{Stengel:}

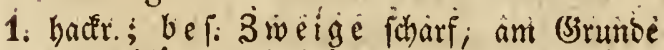

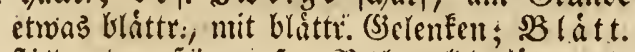

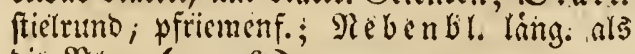
Die Becre $(17: 36$.

2. etrobs bactr., fobwatm.; ber. 3 weige ant

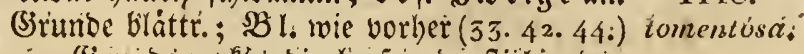

3. am Sirtmoc nact; ; bic be fond. fticlrumoent 3 weige nadt, mit bláftr. (Selené,; $3 \mathfrak{B}$ lát $t=$

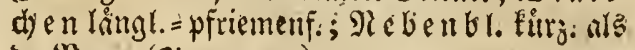
b. SBeere (4 42:44.)

1117

(hispirda.)

1 ils.
1119.: (vulgóris.) 
4. gatt; nadf, Iurhlidt.; bei.. S weige ohme

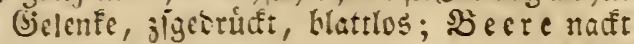
an s. bei. Sweigen $(42.44$.

1120.

Rexilis

\section{CHEIRÁNTHUUS. 532.}

\section{Stengel strauchartig.}

\section{Blätter :}

A. lanjettiórmig,

1. itis,

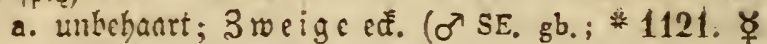
dr., wohlriech. - * $3 \mathrm{~lm}$. - $\Varangle$ Dil) Cheiri.

b. unten meí̄grau, sor gan; cini, ange= trufft, feimen Şaren; 3iveige eff. 1122. (ち SE. gf.; Bld. p., woblriech.) fruticulósus.

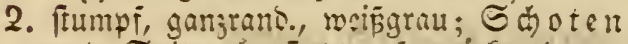
an 5 . Epise abgelust, jlgcirut (ち SE. 1123. 3 6. r.; w. 2c.)

B. gleidbr., ctmas hudt.; S3lủth. fticllos: 1124. \&\bl. mellenf. (ち $S E$. ur., übelriech.) - tristis.

\section{Stengel krautartig;}

A. Blätter ungleichförmig.

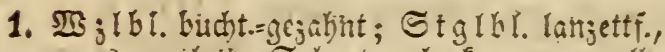
etmas gejahrit; $\subseteq$ dh ot en back., lmgerollt (NAs, v.)

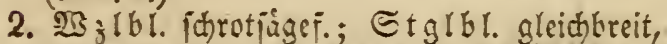
etras gejahnt: Xlle feinthar.; ङ゙diotent aufr. (As. w.)

3. Sie unt. Gucht.: bie 3weigebl. ungeth.:

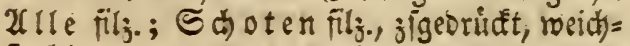
fradgl. (SE, 36, H. u. wchi)

1125.

contortuplicá-

tus.

1126.

leucanthěmus.

B. Bilätter gleichförm, u. 1. lanzettfürmig;

Blätter:

1. gejahnt; Schoten: a. 4 leitig; Stengel: 


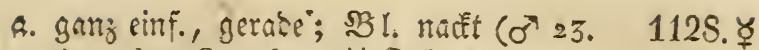
gb., obne Geruch - $\not \subset \subseteq i l)$

(erysimoides.)

. langgejpiat?; 1129. SB 1. nact (o'SE. 9. gb.)

b. breit = 2 idneito., ganj gerabe, topy. io helveticus. lang als o. Dam. Grifi.; Stgl gerne, cirf. ( $\sigma^{x}$ As. hob.)

2. etwas gejahnt; Schoten:
1130.
cuspidátus.

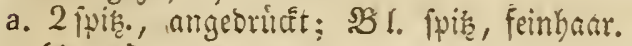
(As. r.)

b. walgenf., am Ente fpis; $2 \mathbb{2}$ l. fumpf, weiв̈grau ( $\odot$ SE. 36.)

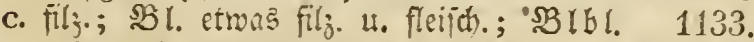
ausgerant. ( $\odot$ SE. 36. P. u. w.)

3. am (jornice entierntgejabnt, unbehaart; Stgl aufiteigent; Edoten jharfgeipibt (o'sE.)

4. Hudft. =gezabut; Schoten ftiellog, horison= tal = u. entferntiteberio, an s. Epitse gabelig $(\odot$ NAf, hv.):

5. ganjrarto, ftumpf; Stgl aufr.; ভtbeut. cingeichlolा. (N.s. r6. p. u. gbch.)

bicuspidátu

1132. annuัus.

littorěus.
1134.
decủmbens.
1135.
parviflórus.
1136.

salinus.

2. nicht lanzettfürmig;

\section{Blätter:}

1. gleichbreit,

a. ungeth., efwas fil

b. ganjrant.; Echoten friellos, lángl., 4 ed̂. (4 NE.)

2. serk. = eyr., acerlos, ausgernt.; S boten

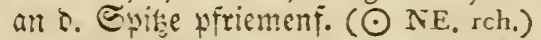

3. cllipt., ftumpf, nact, etras fharf; $S_{t g} 1140$.

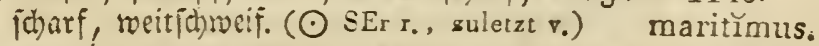

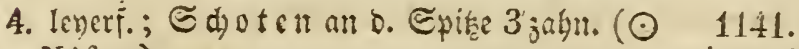
NAf, p.)

1137.

alpinus.

1138.

quadrangŭlus.

1139.

tricuspidátue.

\section{CHELIDONIUM. 410.}

SB rmiti Ye colienf.; Blattfiedern:

1. ctruas gelappt, fumpfgezalunt; $B \mathcal{B}$ l bl. ganj=

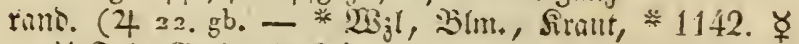

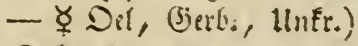

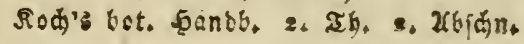


Ir4 - Chelidonium, - Chenopodium.

2. fieberf. =zertifi., fipiggegnlunt; $\mathfrak{B} \backslash \mathrm{b}$. jet= 1143. idtuitt. (4 SE.)

laciniatum.

\section{CHELONE. 502.}

Blätter :

1. fåmmtl. gefticlt, lamettf., gejągt :

a. 2lle entgegengejest (4 NAm. p.)

b. nut die oberfien (4 NAm: w.)

2. nut bie $\mathrm{SB}_{\mathrm{j}}$ lbl. geftiolt u. fpatelf. lanjettf., ganjand.: Stglbl. ftiellos, lamjettorm.;

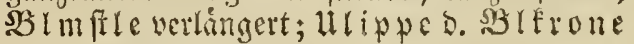
bart. (4 SAm, sch.)

1144.

obliqua.

1145.

glabra.

1146

barbata:

\section{CHENOPODÏUM. 238.}

\section{Blätter stielrund.}

Blätter:

1. froenf., ctwas fpits; Srocige ripenform.,

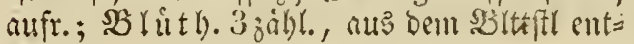
fpring. ( $\odot$ o SE.)

1147.

altissimum:

2. langl., barbftieluno; Stgl matffimcif.; 1148.

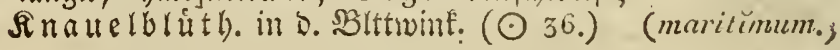

\section{Blätter flach u.}

A. eckig,

1. ungleichförmig:

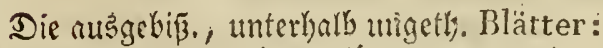

1. fpiesf. budt.: Die obern langl, gamj:ano., 1149. S. punct. $(\odot 22$.

2. rautenf.=enr.: bie obern wie vorther; $\subseteq$. nicht punct. $(\odot$ 29. - ఫ 2 Mell, llnfr. $)$

2. gle ichfürmiğ

Bliithen:

I. gefnaudt, in 5. SYattwinle; Bl. längl., 1151. bud)t.=gejahut, am (jirmbe feilf. ( $\odot$ SAm.) graveŏlens. 
II. ì $\mathfrak{X}$ e $\mathfrak{h} \mathfrak{e n} ;$ Blätter:

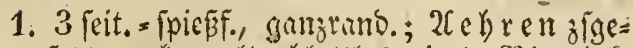
fect, geknault, blattlos; in o. SBlttuine.

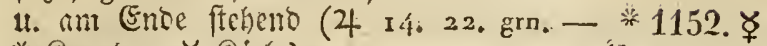

* Siraut - ఫ̧ Sitid.).) (bonus Henricus.)

2. långl.; ausgefthweift; unten graugrün; 2lebren gefnault, nackt, einf., in ben sBlttwint. u. am Ente fteberb ( $\odot$ 22.)

III. in $\mathfrak{I} \mathfrak{i} \mathfrak{u} \mathfrak{b} \in \mathfrak{n}$; Blätter:

1153.

(glaucum.)

1. 3 ectig,

a. etroas gejathnt; íra a ben georingt, ganz gerabe, nabe am Etengel ftebend; febrlang, foft blattlos $(\odot 22.27 .-$ $\Varangle$ Linfr.)

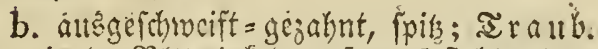
in D: SBlttwinfleln aufr., faft blattlos; Rebenbl. Hlein, einwartsgebog. ( $\odot$ NAm.)

1154. ૪ (urbicuin.)

1155.

rhombifoluum

2. hergformt. $=3$ eat., etwas fttmpf, gezabnt; I $r a$ ub. anfr., zfgecet, ctroas blattr.,

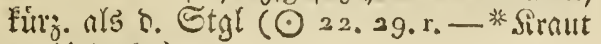
- $($ IInfr.)

3. exrutio,

1156. $\not$
† (rubrum.)

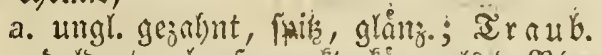
bolbentranbenf:, madt, firtj. alb D. sB!.;

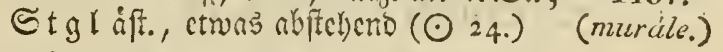

b. ecf. =gezalint, faft berzfo, ranggeipiat, Iraub. ripenf., nadt, ant Enoe uno in oen SBltwinteln ftekent $(\odot 29 .-*$ 1158. * Sirnut - $\Varangle ~ t \operatorname{lnfr}$.

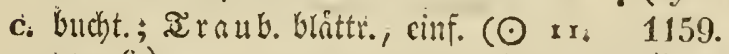
24. 26.) sepüum.

4. belfof., bucht.=gezahnt, rumbl., unbebaart, 1160.

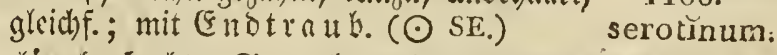

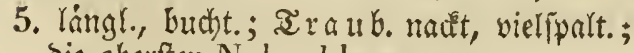
sic oberften Nebenbl.:

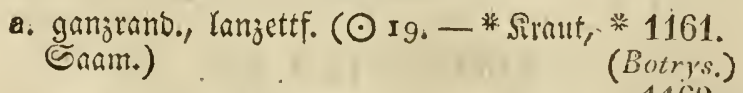

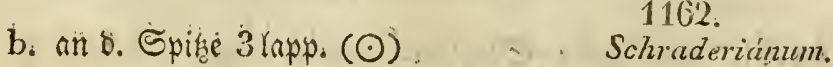

6. lanjettform., entferntgejabnt; 立auben bláttr., einf., in b. SBlttminte ( $\odot$ SE. - * 1163. * Siraut) ambrosioídes $\sqrt{2} 2$ 
B. ganzrandig.

Blätter:

1. rautemf. = evrund; Blithen:

a. gefnutult in \$. Blttwink. $(\odot$ 29. - *1164. ఫ

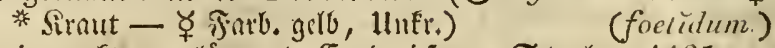

b. in nacte, verlángert. Enorifpe; Stgl 1165. cinf., aufr.; $B$ r. ftumpf $(\odot$ Af.) caudátum.

2. ent.; $\subseteq \operatorname{tglnieberlieg.;~} \mathfrak{X f t e r b o r b e n ~ g a x ~}$ belf., blattlos in o. SBlattwint. $(\odot$ 22. 29.

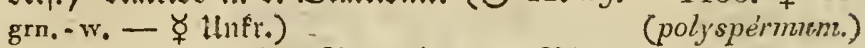

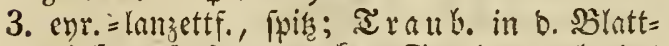
- wink., jigefest, nadt; Stgl aubgebreitet (○ NAm.)

4. lanzettf., ctwas fleift). Dolocentraub.

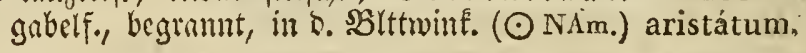

10. CHERLERIA. 361。

(4 9. gbch.)

1169. sedoides.

\section{CHIONÁNTHUS. 10 。}

Mit 3 partt. Enorifpe; $B$ lith. nit furzert,

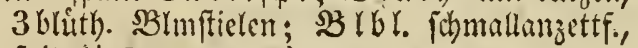
fpic (ち NAm, w. $\triangle$ )

1170. virginı̌ca.

8. CHLORA. 309:

$\left(\odot \sigma^{7} 3 \cdot 8 \cdot 34 \cdot g^{b}.\right)$

1171:

perfoliáta:

\section{CHONDRfLLA. 623.}

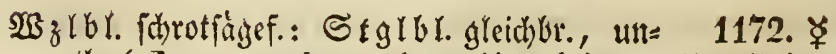
geth. (ơ 15. 18. gb. u. rch. — 


\section{CHRYSANTHĔMUM. $690 .\left(67 \delta^{x}.\right)$}

I. Blüthenstrahl ineist weifs.

A. Blütter ung beichförmig.

1. Die unt. Blätter:

a. Fifmallanzettf., gefight: bie obern fpas 1173.

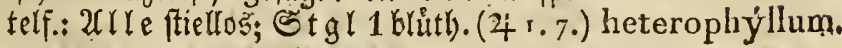

b. Fpatelform., gefight, geftielt: bie obern ictintallanzettform., gefägt; Stgl meift 1174. 1 blitt). (4) montcimum.

c. rumbt.: Die obern eyr.: 2rre geftielt, 1175. gejågt; $\subseteq$ tgl 1 blúth. (4 $9 E .9$.$) rotundifolium;$

d. eyr.=lángl.: bie obern lanzettf.: 2ure 1176. fticlloz (4)

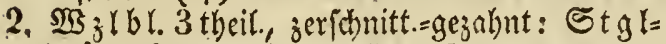
sylvéstre. blâtt. Eeilf., 3 theil., ftumpf (4 NAm.) - arctǔcum,

\section{B. Blätter gleichförmig.}

\section{Blätter:}

1. Keiff. =lónglich,

a, ftumpfgefigt; Stgl ât., weitidureifig 1178. (๑ NAf. 48.)

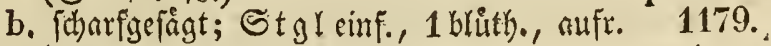
(4 3. 9. Bld, schwarzrand.) atrétumb.

2. Tamzettf., umfâf., gefågt, ain (Sirumbe zets

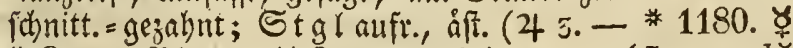

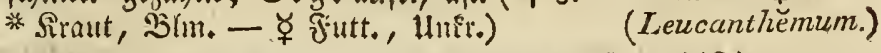

3. gleibbr., faft ganzrant.; Stgl ganz cinf. 1181. (4 SE.)

4. einfact)geficoert, unbelyaart: Fied. Ficherf., 1182.

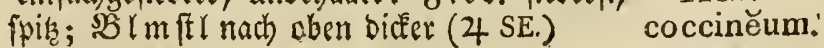

5. Doppeltgefiebert,

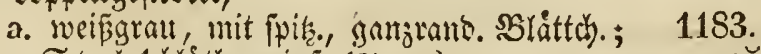
Stgl 1 blitt)., cinf. (4 As.) argentěum?

b. unberbart,

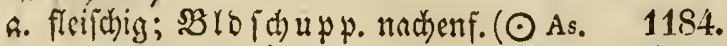
38. w. unten $g b$.)

b. glcidbbr. = pfriemenf.; Etgl aufr., et $=1185$. was off. (๑)

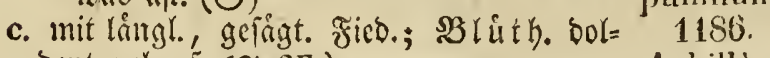
Dentraubenf. (4 SE.) Achillèae. 


\section{Bliuthenstrahl gelb,}

\section{Blätter:}

1. umfaff., oben zerriff., unten geznhnt=ges jägt $(\odot 29 .-\Varangle$ Farb. getb, llufr. $)$

2. zungenf., fumpf, gefägt; $B$ lo f chuppen gleid) $(\odot$ SE. 29.)

3. Dopp. gefied., gefant; sgluth. im Strahl fo lang als in D. Sheibe; Stgt nieberge= frectit ( $4 \mathrm{SE}$.)

4. Dopp. fieberf., fpis, musw breiter; Stgl 1100 âf. $(\odot .22 .30$.

1187. ช్ (seǧ̌tum.) 1185: Mycónis: 1189. italicum. (coronarüum.)

\section{CHRYSOCŎMA. 648 .}

\section{Blätter :}

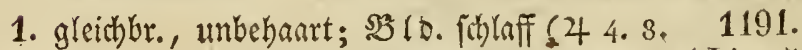
$\mathrm{gb.)}$ (Linosy̌ris.)

2. Yanzettform., 3 ripp., punct., načt; Stgl 1192. rifpenf. (4 NE. gb.; w, u. bl.)

\section{CHRYSOSRLENIUUM. $356 .\left(319^{2}\right.$ )}

\section{Blätter :}

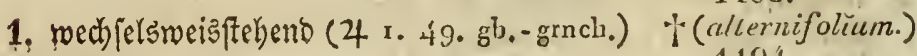

2. entgegengejeftzt (4 $\mathrm{I}, 49 \cdot \mathrm{gb}$.)

\section{3.}

\section{(oppositifolüum.)}

\section{CICER. 582,}

\section{Blumenstiele:}

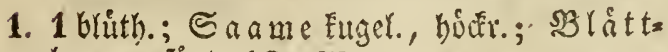

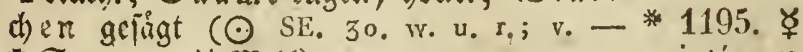
* Saam. - ఛ $M(h l)$ arietínum.

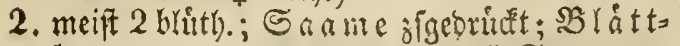

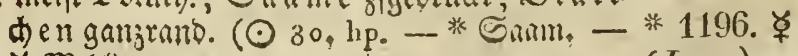
$\Varangle \mathfrak{q}$ (ibl) (Lens.) 


\section{Cichorium. - Cimicifuga.}

\section{CICHORĬUM, 616.}

Blïthen in ben SBlnttwinteln:

1. fticllos,

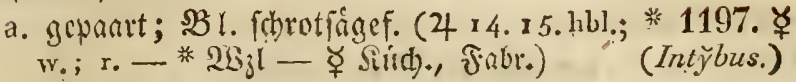
b. eingeln; Stgl gubelf.; 3 weige nuctet,

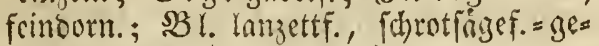 -zahant (o' SE, i 9. 36. libl.)
1198.
spinósum.

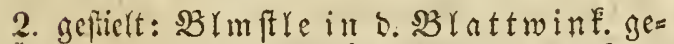
paurt: Der eine 1 blittl., lang: o. andere febre furz $t$ t.
a. nucift 2 bruth.; Stgl guberf; $5 g_{3}$ lbl. fohrotfägcform.: Stglbl. löngl., gezabnt (○ NAf.)
1199. divaricátum.

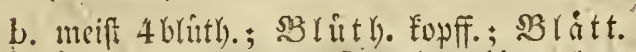
langl., feingezalht; 3 weige bin ul. bers acbog. $\left(\sigma^{7} \odot\right.$ SAs: - * Siraut (jutt.)

\section{* 1200. ६ Endivia.}

5. CICÚTA. 200.

\section{Blätter:}

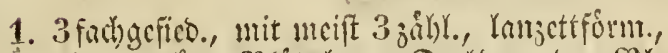

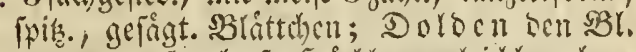
entijegengef.; bef. So ille it gleid)br. = bor= ftenf. ( 4 13.42. w. - * Sirnut)

1201.

+ (virosa.)

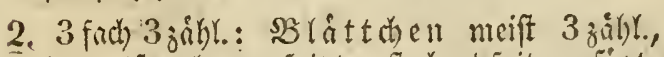
lanjettf., langgeipint, ftech)enofpits=gefägt: bic unt. Faft fusform.; St gl geflect ; mit (5noblumften; ber. Sgillen borftenf. (4 NAm. 48.w.)

1202. † maculáta.

13. CIMICIFŬGA. 426.

(4 NE.)

1203.

foetı̌da. 


\section{CINERARĨA, 685.}

\section{Blätter ungleichförmig.}

Strahlbuithen :

1, 3 zállo, am Ende fteheno; Stgl einfact); $23 z$ zbl. eyr., unbeutl. geterbt: Stglbr. lanzettf., ganjunio.; SBID. braunflect. (4 1204. 9. sch.) (aurcuitiüca.

2. in $\Re$ ifpen; Stgl einf.; $B \mathfrak{l}$. ganz un= beijant, gezabnt: bie unt. beltaf., geffielt: bie obern långl. =lanzettfỏrm., sum sirumbe ichmaler, obrf. =umfail. (4 As. 9.)

3. in Dolbeñ; Stgl curf.;

a. $\mathfrak{Z}$ jlbl. eyr., etwas feingeferbt: Stgls 1206. blatt. Ianzettf., ganzrend. (o' 9.) campèstris.

b. bie unt. SBlátt. ppaterf, gejabut; bie obern lanzettf., ain (E) rumbe ganzranto: D. pberft. uberall gुamzrano. (4 5.9. gb.) (integrifolia.)

4. in boldenf. Doldentraub.; Stgl cinf.;

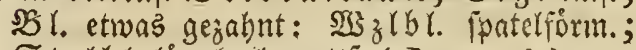
Stglbl. longl. =lanzettf. $\left(\sigma^{5} 5.9 .9 b_{0}\right)$ longifulăa,

\section{II, Blätter gleichförmig.}

\section{Strahlblüthen:}

1. in einf. İraube; Stgl ganz einf.; bie glatten Bl.:

a. berzfơrm., feingezahnt, frumpf; Stgh 1 bianttr. (4 S E. gb.)

b. ipäterf. =berź, gamzrno. (4 NAs.) glauca,

2. in Dorbentrauben; Blätter:

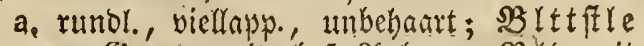
am Grunoe mit ofyrf. 2(nhang; SBlo. nit Zfwhang ( 5 SAf.)

b. breitlanzettform., gezahnt =budt.; ; ङtgl langhaar. (4 47. 48.)

c. gefied.: End fieber fehr grofi, herzf., zeridhnitten = gezalynt: Seitenfiesern

feilf., an $\mathrm{b}$. Spitze gezabut (4 9. gb.) alpina.

3. in Rịp an; Blätter:

1211.

lobáta.

1212.

(palisstris.)

1213, 
a. berzF., geftielt, bopp. geznhnt; Stgl cinf.; $\Re$ ifpe wenigbluth.; $B$ lttffle am (Srumoe etwas gezahnt (4 9. gb.)

1214. (cordifolia.)

b. fieberformig,

6. unten filz., mit ftumpf., faft 3 lapp, Iapp.; Stgl frauch.; $2 B$ lo. firg. (24 1115. ȘE. $36, \mathrm{gb}$.)

b. ctwas langhaar., mit butht. Iappen; 1216. Stgl frautart. (4 NAm.)

canadénsis.

\section{CIRCA'EA. 31:}

Stgl aufr.; Blätter:

1. 'enr., fpis, fcingezabnt, etwas feinfaar.; Stenger feingar. (4 1. 4. w.; rch.; ఫ sarb. ge(b)

2. Gerjformig,

a. gcznhnt, gläit.; Stgl febr åt. u. glatt 1218. ( 4.7, w.) (alpina.)

b. ausgejd

Stgl cinf., etwas glaft (4 r. 8. hr.). intermedia,

\section{CISSUS. 75.}

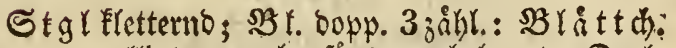
evr, $=$ cllipt., grobgeiagt, unbebaart; $D$ Ol= bentraub. gabelf. ( $ち$ As, gbcb,-w. Bl, übel u. starkriech.)

1217. $\not$

(lutetiána.)

+ orientális.

\section{CITRUS. 597:}

\section{Blațtstiele :}

1. geflúgelt; Blätter:

a. langgelpight; Stgl baumart. († SAs. w:

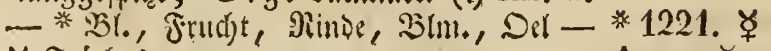

ఫ (rriid)te)

Aurantı̌um:

1222. ఫఫ

b. ftumpf, ausgerano. (ち SAs, - $\not$ frrichte) decumàna,

2. gleidbbr.; SB 1. cyr., langgefpiat ( 5 As. w.-

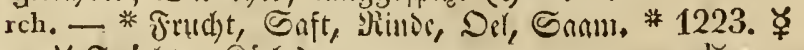
- ซ̧ rridste, Silu.) medǐca. 


\section{CLAYTONIム. 162.}

\section{Blätter:}

1. gleid)br.; $\mathfrak{B} \mathfrak{l} \mathfrak{b}$ l. ungeth. (4 NAm. w. u. r.) virginica.

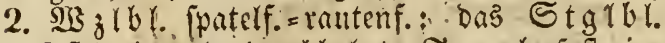
faft freistumo, burchbolyrt; $\mathfrak{I} a u b$. faft cint

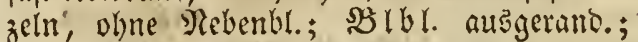
$\mathbb{M B}_{\mathfrak{z}} \mathfrak{l}$ fajer. ( $\odot$ NAm, w: $)$

1225. perfoliata.

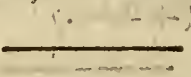

13. CLEMĂTIS. $436 .\left(437^{\times} \cdot 79^{5}, W .9^{2}.\right)$

\section{Stgl liletternd, strauchig;}

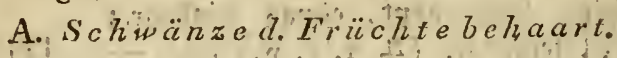

\section{Blblätter:?}

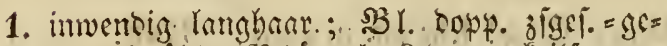
fico., 3 jabl.: sblátt (t). 3 laph., feilf., un=

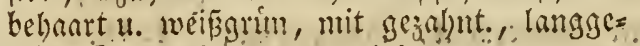
fpitzt. Bapp. († As, hgrn. gh.)

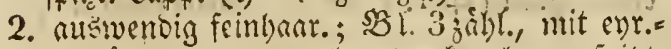
1226. berjform., etwas gelappt =efe, -langgeppigat. solittch.; mit ganjgetrennt. (Gefchl. (ち NAm. w, wohlriech.)

(I) :

3. nuf beyo, Ecit. fit

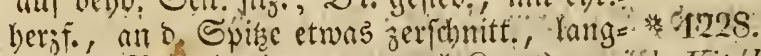
gefpigt. \$Blattch. (ち 26 . w. - * Siraut). *int Titálba.

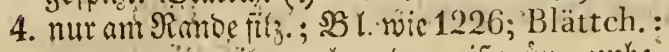

a. cur., zumeilen gelappt, weisgrun, nubc= baort, mit faft frumpf. Lapp.; $35 \mathrm{ln}$ ft le 3 fualt.; Stgl rane. (5) As, uhgb.)

b. einige evr.=lawjettf.: a dio ere 3 lapp.,

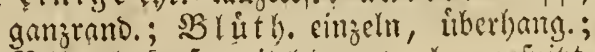
SB IE. glodenf., mit lescrart., langgefwist. \$lbl. (ち NAm, h.)

5. am Sianbe wellenf.; 3 l. wic 1226, Shlát be n evr. u. (ingl., ungeth); $S g l$ lit $t$ ). mie 1229, mit baut, langgcipiat. SBlblatt. (ち) NAm. bl.)

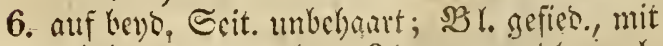
unbehaart., ungeth). 4t. 3 lopp., rumol. oval= lingl:, faft gleichbr., ipis. Blattch). (市 $\mathrm{SE}$. w. $\Delta$ - * Siraut)

1229. glauca.

1230. Viórna.

1231. cylindrica.
** 1232. †Flanmŭla. 
B. niclut veluaartus?

Die 1 brith. Blmstiele:

1. long. nls Das $\$ 2 l$; Blbl.:

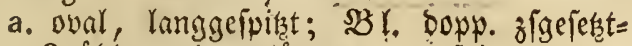
3jial., mit verláng. =evr., fpir., ganz= rand. SBlåttc). (方 As, w. $\triangle$ )

b. verf. = cur., abftch.; $\mathfrak{B l}$ l. bopp. zjfgejetst, gefied. 33 zábl., mit eyr., ungeth. u. ge=

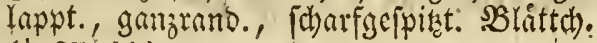
(ち) $\mathrm{SE}_{0}, \mathrm{bl}$.)

2. firr. als bas Slatt; SB Ibl. am (Sirunde

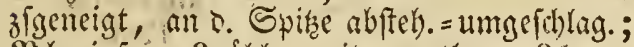

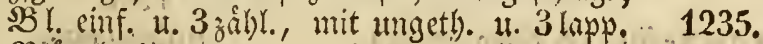
Bláttif. ( ( ) NAm, hv, $\triangle$ )

1233.

florìda.

1234.

Viticélla:

crispa.

\section{Stengel aufrecht:}

Blätter :

1. gefiebert: Blättchen:

a. gleidbur.; Stgl einf.; 6 jeit. (4 SE. 36.) maritima,

b. lanjettfornt., ftumpf: bie unt. 3 theil.;

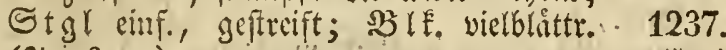

(4 36. w.) angïustifolia.

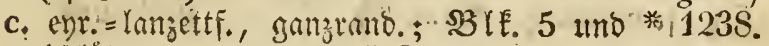

4 blåttr. (4 6. uw. - * Siraut). † (erécta.)

2. einfact),
a. cl)r., feimbaar, ganzrand.; $\mathbb{B}$ (üthen mufr. (4 NAm. gbch.- w.)

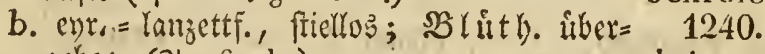 gebog. ( 4 26. dv.)
1239. ochroléuca.
t integrifolia.

\section{CLEOัME, 527. $\left(262^{x} .372^{2}\right.$.)}

Mit 6 St aubgefá $\beta$; Blätter:

1. 2ulle 3 jádblig,
a. lanjettf., ftump; Schoten ipindelform., 1241. (d)mier.=\{charf $(\odot$ As. gb. u. r. $)$

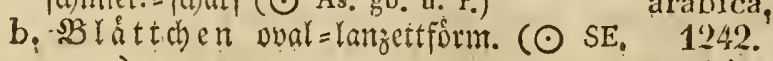

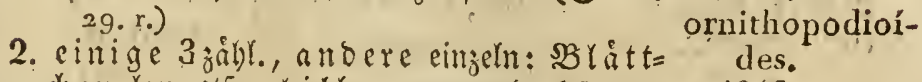
chen lanjettf.=glcid)ḅr., ganzrano, ( $\odot$ SE, 1243. dp. u. gb.) violacěa: 
14. CLEONIA, 452.'

$(\odot \mathrm{SE}, \mathrm{hv}$, rohlriech, )

\section{CLETHRA, 344.}

Die zott. sfify. Fnotrauben einf., mit Rebenbl.; die verk. =eyr., an D. Spize ge= fảgt. Blătter:

1. auf beven Seiten unbebaart ( 5 NAm, w. wrohlriech, $\triangle$ )

2. unten grau = filz. (ち NAm, w. $\Delta)$

1245.

alnifolı̃:

1246.

pubéscens,

\section{CLINOPODĬUM. $47 \pi$.}

Siof

1. burftenf., bactr.; $B$ l. oben baar., entfernt= gezabut; Stg! mcifít einf. (4 23. p.; fl.; 1247. w. - q (Serb., 1 lnftr)

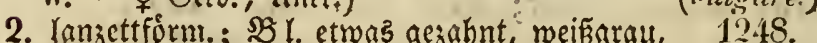
(vulgcire.)
1248 . unten filg, = feinbaar. (4 NAm, A.) incánum.

\section{CLYPEŬLA. 513.}

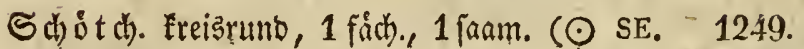

ig. gb.) Jonthlaspi;

\section{CNICUS. 637 .}

\section{Blätter ganzherablaufend.}

\section{Blätter :}

1. Innzettfo, gejahnt, nackt, am Ranbe barn.;

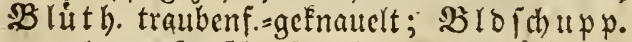
cyr. =lanjettf., fic)arfgefpist, angeoruct. .4 3. 47. $p_{0}$; w. - $\left.w_{0} u_{n} f r_{0}\right)$

2. fiescrf., badtr., mit 2 lapp., anşgebreit., 1250. $\Varangle$ (pailistris.) born. Lapp.; SBID. cyr., fpinnengewebent.: 
feinhanr., mit lanjettf., sorri, abftehenden 1251. ఫ̧

Sthupp. (J 22: p.; ros,; w. --

(lanceolutus.)

\section{Bi. halbherablaufend.}

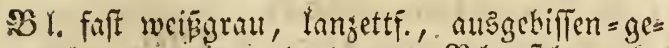

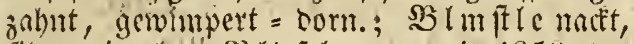

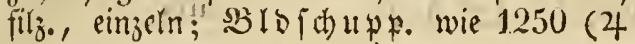
p., Bld. ausw, e. w. Linie)

1252.

camuss

\section{Blätter' stiellos,}

\section{Blätter :}

1. fieberformig; Bldecke:

a. Eugelig,

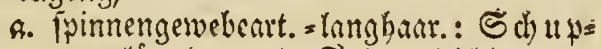
yen Yángl., an $\mathrm{b}$. Spitze glcidjbro, vers= lång., - icharfgeipitat, ungeichlng.; $3 \mathfrak{B}$ l. hadtr., mit gepanrt., aukgebreitct., borrt.

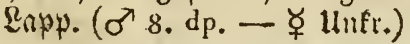

b. fpinuengewebeart. = feinhaar.; $S_{\text {d) }} \mathfrak{p} p=$ pen enrunb, angebridet, an $b$. Spile Dorn.: Dornen abfteb.; $\mathfrak{B l}$ l. ftachels baar., unten fitz., mit 2 lapp., ausges

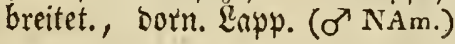

1253.

(eriophürus.)

1254.

discŏlor.

b. mit Mle benblatt. ut. cyr., angeoriaften Sctupp.; $\mathfrak{E} a p p$. b. SB $\mathfrak{l}$. zerfdutt. = ge= fágt, am ßiande born. (2t SE.)

1255. rigens.

c. mit lanzettf., bichtondyziegelf. Sdhupp.; sBl. gezalnt, gemimp. = boin.; $B \mathfrak{l}$ m ftre

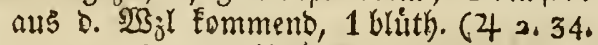
2. Ianzetteurmig,

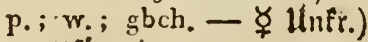

1256. ఫ్

(aca'ulis.

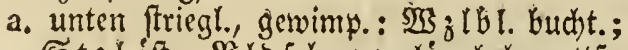

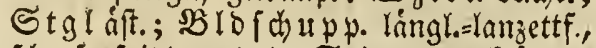
foharigefpist, an \$. Spite ungefrummit 1257. (4)

b. unten fil

serratuloides.

a. ganzramsig;

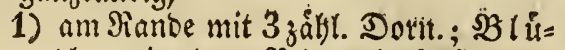
then in ben Blttwink. ftiellos; SBlof I upp. lanzettf., Dorn., ab: fteheno (o' SE.)

1258.

Casabònae: 
2) obne Dorn.; Dorn. in s. Slatt= winfeln am Girunse ctwas áj.; mit Enobluth.; $\mathfrak{B l m}$ lmle in s. $\mathcal{B}^{3}$ lat ts wink. Fujt fitillos $(\odot)$

1259. stellitus.

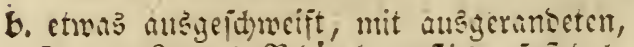
2 corn. Rapy.; $\mathbb{B}$ ! u th. gelticlt, fait ool=

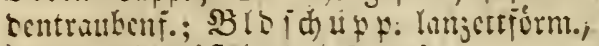
sorn., jebr abfebento (疋 As.)

\section{Bi. umfassend:}

A. gleichförmig.

Blätter:

1. ficerforimig,

a) Guafr., mit 2layp.; ansigcbrcitct., Sortt. Eapp., unten Filj.; SIIS. cur., mit lat= jett., sorn., gemimp., umgejhlig. Rap= pen ( 4 NE.)

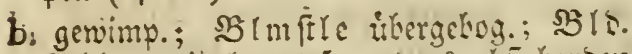
fleter., mit lamjetto, etrots abjelyenteon Єchutp. (4 3. gb.; p.; w.; ugb.)

1260.

afer.$$
\text { . }
$$

c. gcmimp.=iorn., mit 2 lapp., an Sirunbe aufrörtêgejubnt. Rayp.; Stengel meirt

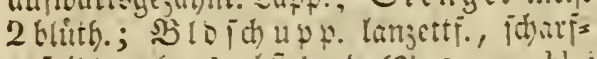

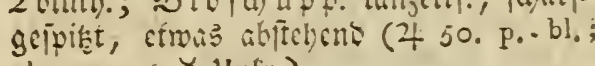
gb.; w. - ఫ

d. gejabint= sornig,

f. Fimbar.; ङtgl sinf.; Enoblutb.

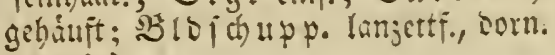
(4 ugb.)

b. inbeburt; Enלbluthen gebauft, fitellos, mit serjchicienjarb., Eorjtig."

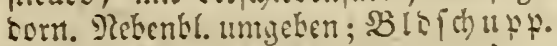

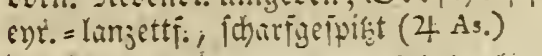

2. berbblatensfiecerf, gewimp.: Fieb. lon= gettf., 3 ripty.: Lie ober it en jigumalen; Blnifte ubergebog.; SBlsiduph. lon= gettf., unigcidlag. (4 SE. 9, ugb.)

3. längl:, gezahnt; Sorn.; weis̄ater.; $\mathfrak{B}$ I ith. ftiellos, mit Rebenbl.; $B 10$ ito up p. lingl.:

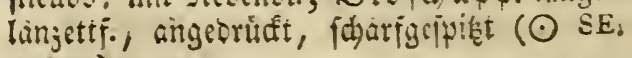
i.: p.)

1261.
ciliàtus.

1262.

Erisithăles。
$1263 . \underset{7}{\not}$
(tuberúsus.)

1264.

spinosissünus.

$12 f i 5$.

obvallatus.
1266.
ochroléucus. 
4. lángl. = lanjettf., gejabnt, gemimp. = torn.;

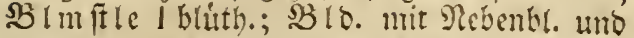
lanjetti., Lorn. Edupy. (4 NAs. w.)

1268.

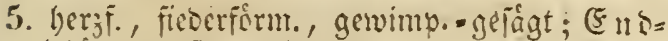
blutb. faijt thubenf., mit etwas gefárbt., cyr. Mebenbl.; BB I D jchupp. lanjettF., sorn. (4 3. 4. hgb. - ఫ Sîud. ; Jutt., Uufr.)

B. ungleichfürmig.

Blätter:

1. hergf., lanjett. ; gelägt, gemimp., unter

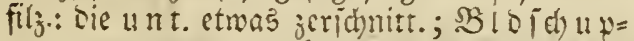
pelt lamjettF., angerrictet, etwas icharfigcipist (4 3. 9.)

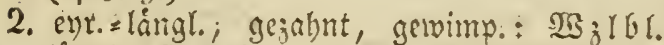
lángl.; ftumpf, bucht., gewinns.; (5noblú then etwas georänt; $S^{3} 1 \mathrm{D}$. mit SJulle, u. formallanjettf., etras abftelent. Echuppen (4 9. ugb.)

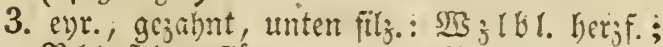
SBlttitle geflug., gejabnt; SB lo. troce, falt cinjeln, bie ain Ente Tebenic ubergebsucn (24 NAs, gbch.)

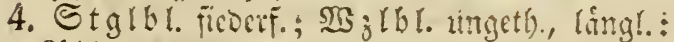
1270. heterophillus. alle gejahnt, gewimp., natt; Stengel

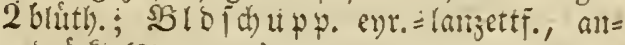
gebrudt (4 3. 49.)

5. Dic unt. u. Skz zlbl. fieberf.: 2Crle wie wor=

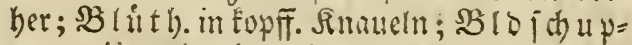
pen wiè vorber (49.)

1272.

cernuัus.
1273: sálisburgènsis:

$127 t$ rivulueris:

\section{CNIDÏUM. 188 :}

Stengel:

1. edefig; Bläiter:

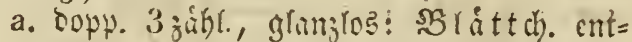
fernt, fieserf., mit jomallanjettf., fivis.

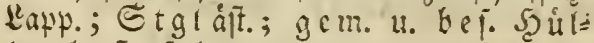

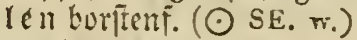

b. melyr als bapp. jigejeft: Fieberch. fie= Serr., mit etwas entfernten, lanjectfourm.; gerippt., faft rimnenf., fitechenofpit: Lapp.;

1275.

Monniéri. 
mit e. Spur v. gemt. Şille (4 3. gb,, ausw. w. - $\Varangle$ Ultfr.)

2. ftielruns, åft.; $\mathfrak{B l}$ l. mehr als bops. zโgef.: B̉ látch. ficberform., mit etwas entfernt., flud)., foft 3 fpalt., etruas ftumpf., geripyt= niver., icharfgeipizt. Lapp.; gem. Sculle viclbláttr. (4 4. 49. gbch.)

1276. ఫ్భ (Silcéus.)

\section{COCHLEAR İA.50S。}

\section{Blätter :}

I. gleidh formig,

1. Deltaf., ipieff. =eứ. $\left(\odot \sigma^{7} 36.\right)$

$12 \% 8$.

danica.

1279.

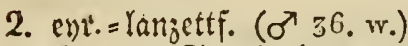

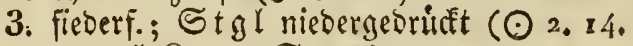
w. - * Sirmut, 巨aam.)

4. Ianzettf., umfafi., gezahnt (4 15.)

5. Stglbl. vere. berzf. = pfeilf., untaffend (व 29.)

II. ungleichformig: Wzlblätter :

1. berzf.=rumol.: Stglol. längl, etwas buchtig (o' $\odot$ 36. w. - 2kil, Siraut, Saam. - ૪̧ Sitidj., Dil)

2. lanjettfơrm., geferbt: Stglbl. zerichnitt.

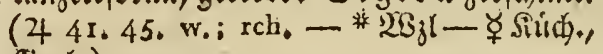
(siertr.)

3. $\mathfrak{l e r}_{j} f_{0}=\mathrm{cyr}$., geferbt; S̈tglbl. lanjettf.,

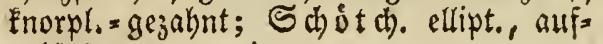
gebla. ( $43.49:)$

\section{GOLCHǏCUM. 297:}

Blätter:

1. flid), lanjettf., aufr. (4 3. 43. ros; ; w: 1286. * Suvisbel)

2. rimnenf;, glcichbr., aufr.; sjifif. Eirrot 1287. als \$BIf. (4 SE. 6. 19:)

arenarium.

1285.

3. am Ranbe mellenf., abfebeno (4 A.s. bunt) variegátum. 
Collinsonia. - Commelina.

2. COLLINSONIA. 29.

\$Bl. eyr. u. nebfi Stgl unbebaart (4 NAm: 1289. 5, ugb.)

canadènsis.

\section{COLUTĚA. 578.}

BHY. ungleidygefied.; Stengel:

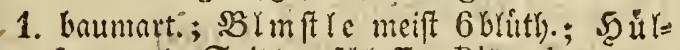
fen an s. Spise gefdlofl.; Blättchen:

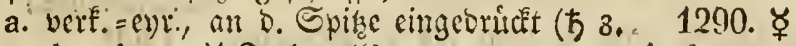

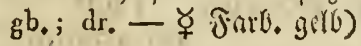

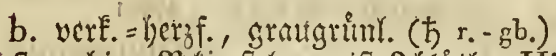
(arboréscens.) 1291. meď̃a.

2. ftraud)ig; 3 tinft le meift 3 Glitth.; Hülsen an b. Spitse

a. Dffen; SBląt tu. rumbl., an b. Spise 1292. abgeftuht, graugrin ( $5 d_{0} ;$ ur. $\Delta$ ) cruéntc.

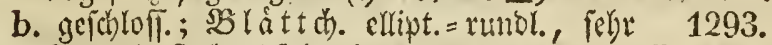

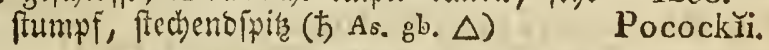

\section{COMĂRUM. 406.}

\section{Blätter :}

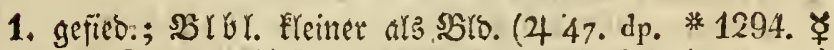

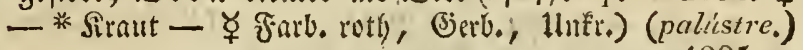
1295.

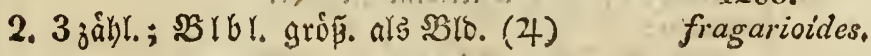

\section{COMMELINA: 39.}

\section{Stengel:}

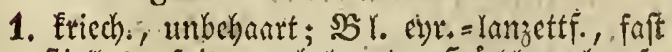
ftiellos, fpis, unbelyatt; 5ूutllen berzfo, 1296.

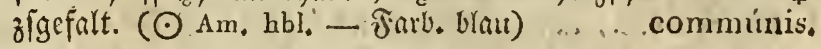

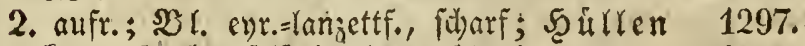
Enppenf., freufelf. (4 NAm. blch.). erécta.

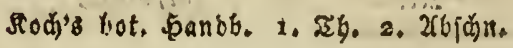


130 Comptonia. - Convallaria.

21. COMPTONĬA. 731. (W. 18.)

(ち $\mathrm{NAm}, 8$.

1295. asplenifolǐa:

\section{CONIUUM. 217.}

\section{Saame:}

1. geftreift: bes. Huillen:

a. Lanzettf., viel Kurr. als bie S̈trablen der

bef: Dolbe (o' 22. 29. w. - * $\mathfrak{W}_{31}$, Siraut, * 1299.

Saam:) (maculátum.)

b. gleidhbri.; eben to lang alls o. Etrablen 1300. $\left(\sigma^{2} \mathrm{SE}\right.$.)

2. gefurdst, Enot.; lángl., zigeoridat; Stgl 1301. gefurdst, gabeff. ( $\odot \mathrm{SE}$ ) ${ }^{2}$ dichotŏmum。

\section{CONVALLARİA. 276. $\left(7^{\circ}{ }^{\mathrm{i}}\right.$.)}

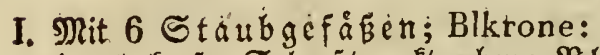

A. gludenf.; $\subseteq$ daft nadt, eben; $\mathfrak{B}$ l. ent. $* 1302$.

(4 3:5. w:; $\mathrm{fl}_{i}$, wohliriech. - * $8 \mathrm{~lm}$.) (majcilis.)

B. trichtereformig; Blätter:

1. in Duirthi łtehend (4 8. grn.-gbch.) (verticilláta.)

2. wechjelsweisftehetro, umfalf.; Stgl.:

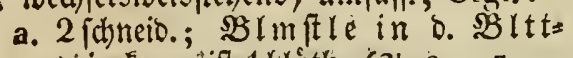
winf: meift 1 bluth: (4 s. 23. w.

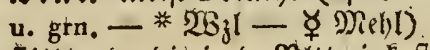

b. ftielruno; bie in o: SBlttwint. ftebend. Blmstle:

A. mèift 2 bihth.; $\mathfrak{B}$ i. èyr., ututeit feinl)aatr.; Stgl mit e. Furche

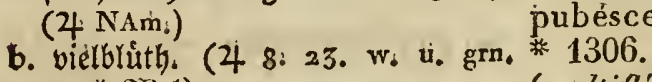
- * 213i) (multiflóra.)

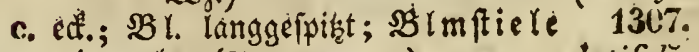
mie vorher (4 5. p. u. w.) latifolía.

C. rabfórm.; bie riechjelsmeisfteh. Bl.:

* 1304. ఫ

(Polygonütum.)

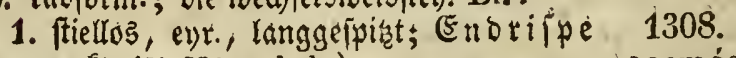
nadft (4 NAm. hgb.)

2. umfaffent, ellipt., etrois fpis; Eno $=1309$. traube einf. (4 NAm. w. -grnch.) stelláta.

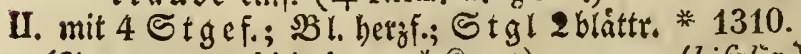

(4 3. 5. wo wohlriech. - * Sirnit)

(bifolia.) 


\section{CONVOLVŬLUS. 127 .}

\section{Siengel geruunden。}

\section{Blätter:}

1. pfeilformig,

a. auf benden Enden fpirz; $\mathfrak{B} \mathfrak{l}$ mftle meift 1 blúth). (4 29. A. - w. wohlriech. — * Siraut * 1311. ఫ - ૪्(lunfr.)

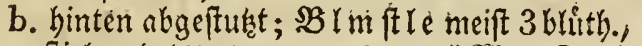
(arvénsis.)

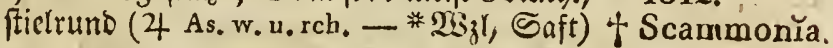

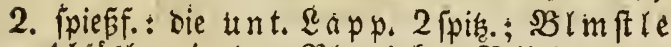
1 blatth., in ben Slitwink:; SBlolappen ftumpf, etwas auşgetano., feinfpikg. (4 NE. rch.)

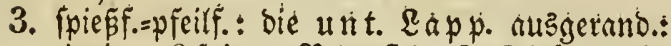
einige 2 fpic.; $\mathfrak{B l} \mathfrak{m}$ ftle $2-3$ bluth., in

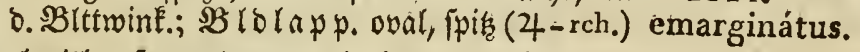

4. breitherzf., gelappt! einige ungeth., gei=

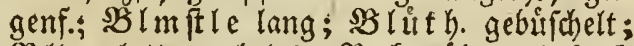
SBlo. glatt, wehrlos; $\mathfrak{B} l \mathfrak{F}_{\text {。 }}$ róbr. = glocfemf. (4 NAm, w, u. r. )

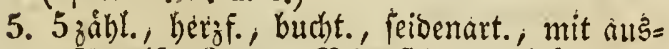

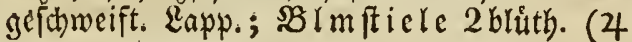
SE. blch.)

6. einfach,

a. Kergformig,

a. unbehaart, langgefpiat, mit am Şrunod ausgebreit. llapp.; $\mathfrak{B} \mathfrak{l m}$ ft le traubenf = boldentraubenf., láng. als bas sl., in b. sitttwink. (4 $\mathrm{Am}_{\text {。 }}$ )

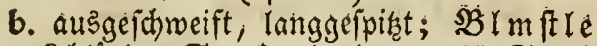
3 blüth.; Stgl mit einen weis. Staub überzogen (4 NAf, f.)

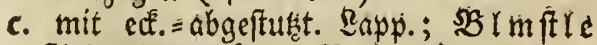

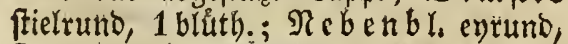

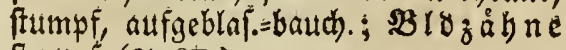
ftumipf (4 SE.)

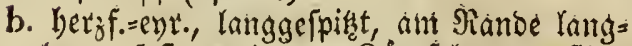

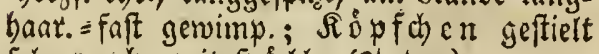

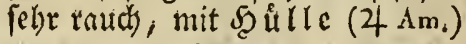

1315.

pandurátus.

1316.

althaeoídes.

bicuspidátus.

1314. 


\section{Stgl nicht gewunden od. niedergestreckt.}

\section{Blätter :}

1. berz $f_{0}=e y r$. ; Blmstle:

a. 1 blüth., furry. als bas $\$ B l$.: bie obert SBl. jpif; Stgl friech. voer etwas ge=

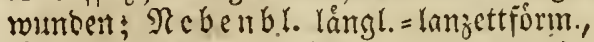

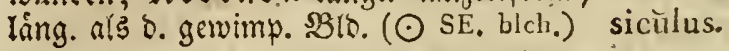

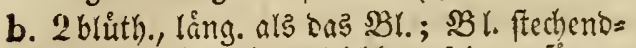
fpis; $\mathfrak{N}$ e $\mathfrak{b} \in \mathbb{n} \mathfrak{b l}$. gleichbr.=p friementurm., fürz. als ber bef. Blimit; B Bro e of e etwab gewimp. $(\odot$ SE. w., inw. gb.

2. geigenfiotnt., ob. ungeth., nusgermb., am Sirunde berzf.; 33 tmple 1 blith.; Stgt fried). (4 SE, ugb.)

3. Kerzf.: einige faft fute fefferm., langhant.; Stglu. Blttfle baar.; SBlmitle vicl= brittl). (As. p.)

4. lanzettf. = cur., unbelgart; Stgl abwairts= geneigt; $B$ lifth. eirjeln $\left(\odot \mathrm{SE}, w_{0} u\right.$, bl.) triculur.

5. ganz lanjettförmig,

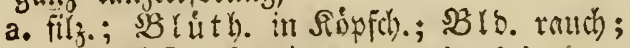

Stgl fâft aufr. ( $5 \mathrm{SE}$. w. od, rch.)

1323.

pseudosicǔlus.

b. Feidenart., geftrichelt, geftielt; $B B$ imft le 2 brítb); SBlo. feibenart., etwas Jláttr. (4 SE. dros.)

c. nadt, ftumpf, geflichelt; 3 we ige 'ab= wártşgeneigt; şlưtben einzeln, batb 5 ipalt. (๑ SE. blch, u. gb.)

6. Pohmallanjettf., ppis; Stgl ait., etwas aufr.; SBld. haar.; sg Imft le meift 2 blitth. (4 SE. H.)

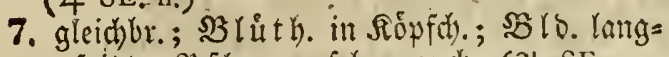
geipiat; sy flanze febr rauch) (4 SE. w., eusw. $x \mathrm{ch}$.)

1324. Imperati.

1325.

hirtus. 1326.

Cneǒrum.

1328. lineátus.

1329. pentapetaloídes.

\section{0. cantabrǐca: \\ 1331. saxatillis.}

\section{CONÝZZA. 659.}

\section{Blätter:}

1. herablaufeno, cyr., jajarfgefpigt, filj.: sie unt. géägt; \$S Iúthen in Dolbentrauben (4 As.)

1332. thapsoídes. 
II. nid)t herablaufent;

A. Stauoig; cie etwas gezahnt. Blätter:

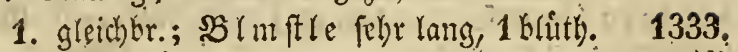
( 5 gbch.) saxatilis:

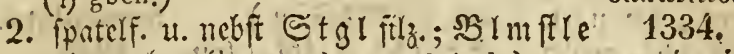

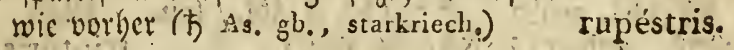

B. frautartig; Blätter:

1. gleitl)formig,

a, ipatelf. =längl., umfaffeno, gejågt, rungl. (4.NAm, hgb.)

1335.

bifrons:

b. formallanjettf., icharf, faft gamjrans., ant Sinmoe umgerollt; ङ $\mathrm{gl}$ tifpenf:;

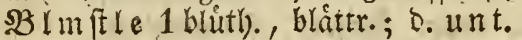

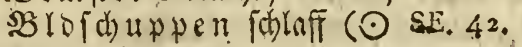
2. Stgl rch.). 1336. sicùla.

2. ungleich.formig,

a. fharf: Stglbl. eyr. =lingl., ges

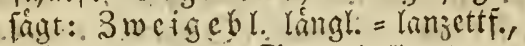
fajt gamgrand.; Stgl boldentrau= benf.; $\mathfrak{B}$ ls. fpertig (ó 4 ז. ugb. 1337. - * Siraut)

b. ftiellós, lanjettf. =långl.: sic unt. (squarrósa.) verf. evr. = lángl., an D. Epita etran gejägt; 3ive ige soldentraukenf.= gleichloch, meif 3 blutbig; S.tgl baar. (๑)

1338.

fastigiata.

\section{COREÚPSIS. 698.}

\section{Blätter: «}

I. gleichfơrmig,

A. gefiebert,

1. im Suirl ftehento, 3 oo. 5 jofhl.: Fie bern gleithbreit, 3 theil., einige unge= theilt; Scheibe d. Blume:

a. veriftedenjurbig (4 NAm. bgb.-u:

$$
\text { (idp.) }
$$

b. gleichforbig (4 NAm.)

2. nid)t im Suirl, geiägt, geftrichelt, un= bebaart $\left(\odot \mathrm{Am}_{4}\right)$

B. Iarijettformig,
1. ganjrano., gerwimp. ( $\sigma^{7}$ NAm.)
1342.
lanceoláta.

1339.

verticillàta.

1340.

tenuifolia:

1341.

coronáta. 
2. gefägt, langgeipikt, etwả geftielt; síluth. Doloentrabenf.; Strahle blutben lanzettf. (4 NAm. gb.)

II, ungleichformig, ganjrandig,

A, enr,: oie unt. 3 zábl. (4 NAm. 8.)

B, $\mathfrak{W}_{3}$ lbl. gefied.: Stglbl. 3 zábl., lan= zettf., geftielt (4 NAm. 8. dgb.)

1343. alternifolía. 1344. auriculàta.

1345. triptěris,

\section{CORIÁNDRUM; 202.}

\section{Stengel:}

1. fielrunp; $\mathfrak{B I}$, bopp. zigefert, pipiegelglatt, mit gleidbreit, etwas ftumpfen SBlattchen;

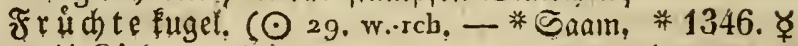
一 ఫ Siuth, , Unfr.)

2, edf.; $\mathfrak{B I}$. Dopp. zigerest, mit fothallanjettf., 1347.

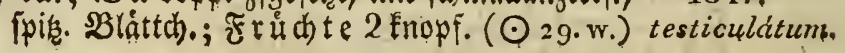

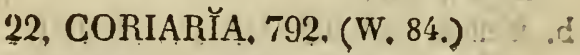

'201, eyr, = larjettf., ganjrand., 3 fachgerippt, geftielt, unbebaart; mit ( $\$ notraub. (ちSE, grach. $\Delta-\not$ (Serb.) myrtifolia,

5. CORIS. 110 ,

$\left(\odot S E\right.$, ז9, 36, x.; $\left.p_{,}-\nabla.\right)$

1349. monspeliénsis,

\section{CORISPÉRMUM, 7.}

Aehren:

1. am Ende ftebeno; ' $B$ r. rippenlos u. nebft D: Rebenbl. wehrlos; Blüthen:

a. von einander abjtebeno (O SE. 19.) hyssopifolrum, b. einanter nakeftehend, 2 blátritig, mit 1351 . 5 Stgef.; S. außgerantet $(\odot$ 19.) canéscens.

2. am Enbe u. in o. Blttwint. fteheno; Bluth.

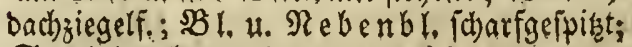
Stgl langhaar. ( $\odot N E, 19$.

1352 , intermedium, 
3. blos in o. Blattwink. ffebeno; $B$ lut then georingt, bact)ziegelf.; 3 t. rippentos u. nebft

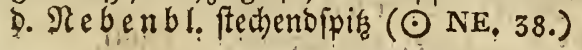

\section{3.}

squarrósum.

\section{CORNUS. 73}

\section{Blïth. in Dolden, mic Hülle.}

I, Frautartig; Zweige ;

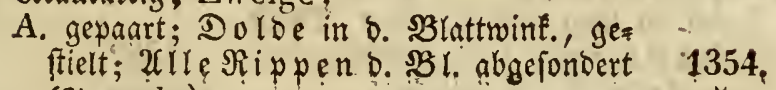
(4.9. dr.) svecica.

B. fellen faft; bie oberften $3 \mathfrak{K}$. im Suirl, 1355. etwas geftieft, nier. (4 NAm. rch. - w.) canadénsis.

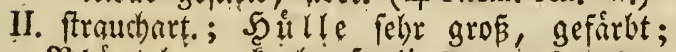

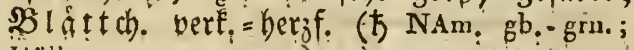
Hïlle w., ausw. ros.)

1356. floṛ̆da.

III. Eaumart.; Ș

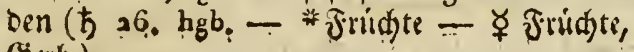
(sert.)

(mascŭla.)

\section{Blüthen in Afterdolden, ohne Hïlle.}

\section{Blätter:}

I, wechf., breiteyr., langgeipight, unten etwas feinhaar., weísgrau; ?tefte gabelförn., falt glatt: bie iúngern v. Den SBlattmarb. fait geringelt; if fterdold niedergeoriudt; $S B I D=$ ád nne fo lang alb d. Nect. Druife; Steinz frud th fuget., fchwarz=veildhenblau: $\Re u \tilde{\beta}$ fugel." († NAm. w.)

II. entgegengefegt; Zweige:

A. gerabe,

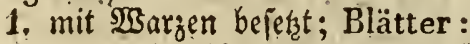

a, runbl. =ellipt., unten graufil

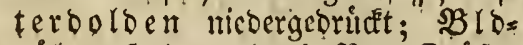
zál ne fo lang als bie Nect. Drifie; Stfruct t nicbergeoritaft = Fugelig,

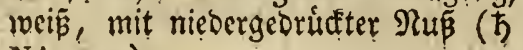
NAm, w.) 1359. circináta.

b. enr., langgefpigt, unbehaart, unten 1358.
alternifolía, wenig blafler; 2 fterbolb. bolben= 


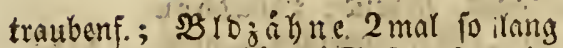
als bie Mect. Druje; St.frud)t nies ocrgeorucfe, fugel., blau, mit cyruno. গuß (ち NAm, w.)

1360. stricta.

2. glatt; $S B l$, eyr. = oval, langgeipięt, $\mathfrak{u n}=$ terbalb etwas blaller u. Feinthaar.; 2f fter. bolden nieoergeoridét; Bldzähne:

a. jo lang als-bie Niect. Srije; Ste in=

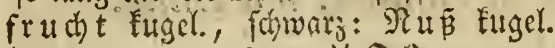
(4 4. 26. w. u. gb. - ६ $\mathrm{Ocl})$

b. 3 mal fo lang als bie Nect. Druile;

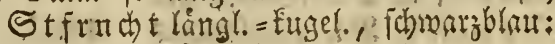

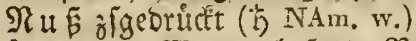

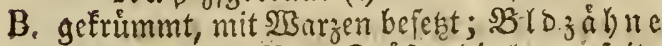
1361. sanguiněa. 1362. sericẹa.

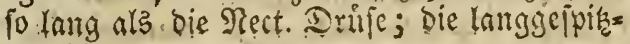
ten Blätter:

1. oval, unten etwas feinlyar, weiforan;

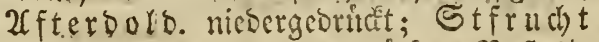

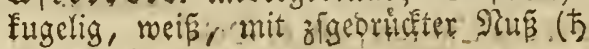
$\mathrm{NAm} . \mathrm{W}_{\mathrm{i}}$ )

2. evr, obent etwas feinhaar., unten unbe= baart, grau; 2fferdold. Dolsentrauben= art.; Stfr. wie vorbar, mit faft fugel. श्रि (ち NAm; w.)

1363. alba.

\section{$136+$. paniculàta,}

\section{CORONÍLLA, 591,}

sBlúthen in Dolsen;

I. Strautbig; $\mathfrak{B}$ triffle meift 3 bithtr; ; $\mathfrak{B l}=$ nágel 3 mal fo rang als sBrb.; - Stgl edf. (ち) $\mathrm{s} . \mathrm{gb}^{\mathrm{b}}$.)

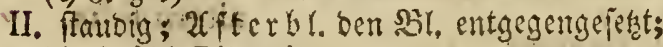
die 9 záhl. Blättchen:

1. ellipt.: bie innein bem Stgl angená= bert; Xfter Q1. Yamjettf. (4.8.gb.) : (coroncita.)

2. eyr.; 2tfterbl ausgerano; Sculfen 1367: cf́,, Enot.; ȘtgI nieocr(ieg. (4 8. hgb.) (miňma.)

III. froutartig; Gliederhülsen:

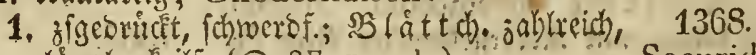
lángl. Ectiff. (○ SE. 3o. gb.) Securidăca,

2. ftieirumb, aufr.; Blättchen:
a. zahlreich), Janzettf., unber)nart $(\odot 26$. 29. p. u, w, - ఫ ซtt, , (Infr.)

1365.
Eměrus. 


\section{Coronilla. - Corydalis,}

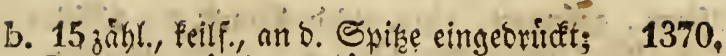

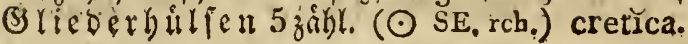

\section{COPRIGIŎLA. 247.}

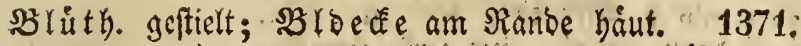

$(\odot \times 9$, w.)

(litcorcilis.)

\section{CORTÚSA. 115.}

$\mathfrak{B r}$, zerfchintt., gelappt, mit fpila $\mathfrak{L}_{\text {appen; }}$

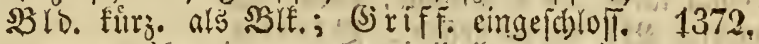
(4 9., ros.:

Matthióli,

\section{CORYDÄLIS. 554. (260.)}

\section{Blk. mit 2 Erhabenheiten.}

\section{Stengel:}

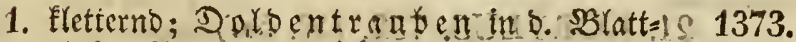

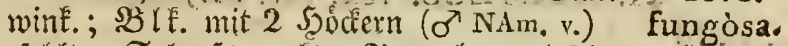

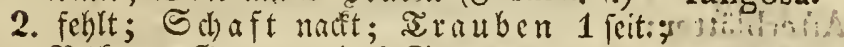
B lé. am (srunde mit 2 Sppornen (4 NAm, 1374. w. u. gb.)

Cuculları̆a.

\section{I. Blk, mit 1.Sporn.}

Stengel:

I. cinfád); mit Enotraub. ; (Nebenbl::

1. langliad!

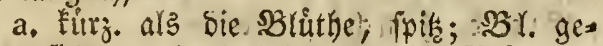
fied., mit.'3 theil. = zerrifl. SBláttchen 1375. (4 NAs, w." u. gb. wahlriech, ) nobîlis.

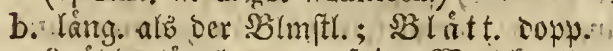

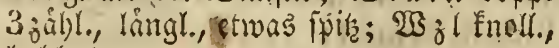
bohl (4 r, 4. p. u, w,; $r_{*} ; w_{*}$; grach. * 1376. - 2 2 33 i)

2. runbr, is fo lang als bie BBhithen; $B$ re $=$ fporm gerabe: $\mathbb{B}$ l, bupp. 3 jăbl.; $\mathbb{Z} 3$ jl 1377. fnoll. (4 I.) 
3. Feilfơrm., gefingert, lång. als o. \$Blmftr;

$\mathfrak{B}$ l. H. $\mathfrak{B}$ z l wie vorber $(4$ ×.) .. Halléri.

II. åftige,

1. aufr.; S屯 oten gleichbr., 3 mal lång. als ber SBlmitl; $\$ 2 l$ graugrun: Stalbl. boup. 3 jabl. (ot NAm, hr. u, gb.)

1379.

2. weitfdrweifig; gleihbreiten Schoten:

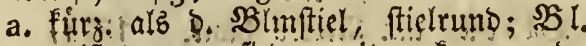
meift oopp. gefieo., mit verf. =evruno., zerifhnitt. SBláttch; ; Stgl ftumpfed. (4 SE, dgb.)

b. länger als o. SBlmitt, 4 jeit.; Stgl idarfect; Stglbl. bopp. 3 zabl: (4 24. ligb, $_{\text {; }}$ w)

c. 3 mal láng. als $\$$ Blmftl, "etwits ge= bogeñ; $\mathfrak{B}$ l. bopp. gefied.: SBlât th). getb)., ichmallanzettf., an beyo. (Enoen ipik (o' NAm. gh.)

3. Flettẹnd; $S$ ch ot en långl., zigcorudet, 2 fanm.: bie untern. 3 l. boph. 3 jobhl.: 3 weigebl. einf. 3 zábl., rantig $(\odot 2 x, 1383$. 4.7.)

sempervírens,

$1380 . !$

lutăa.

(Capnoides.)

1382. aurĕa,

\section{Afterblätter:}

21: CORY̌LUS: 752. (W. 5io)

1. langl: ftumpf; Bld, d, Frucht;

a, glockent., an ber Spife etwas abfteheno, zerrifl. =gejahnt; $\mathbb{B} \mathfrak{l}$. runol., berjfórm.,

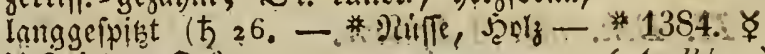
ఫ Frichte, Oer)

b. robr. =walzenf, an b. Spike zigegogen,

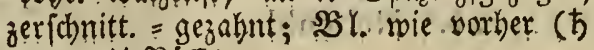

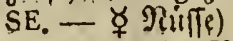

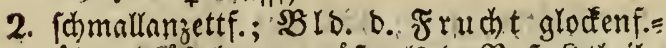

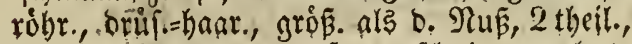
mit am Ranoe wellenf., zerichnitt.=gezabnt.

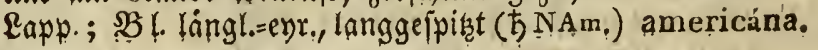

3. Innzettform.; Bld, d, Frucht;

a. einf., febr raud), kugel., an ber Spiźs róhr. = gejunnabelt, mit zeríthnitt. = gezahnt. u. 2mal fo langen Saum, als bie Nús;

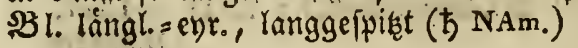

1386. 
b. Doppelt : bie å $u$ F. vieltheil.: bie innere 3 theil., mit handoform. =vielfpalt. Iappen;

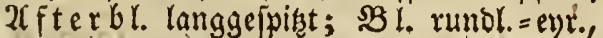
berzf.' († SE.)

1388.

Colúrna,

\section{COSMÉA, 697 .}

BRr. Dopp gefiebert : Blättchen:

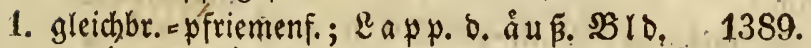
enr. $(\odot$ SAm.)

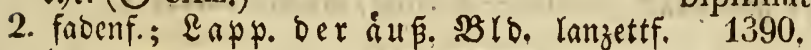
$(\odot$ SAm.)

parvifóra,

\section{COTǓLA, 656. $\left(673^{x}\right.$.)}

\section{Blätter:}

1. gefieb = vielipartig,

a. borftenf:; $\subseteq$ dbeibenblúth. libergebog. 1391.

b. oben breiter; 3 lith. ofme Strahlblumen 1392.

$\left(\odot \mathrm{SE}, g b_{0}\right)$ anthemoides.

2. Lanzett. =gleichbr., umfall, fieberf. =gejabnt;

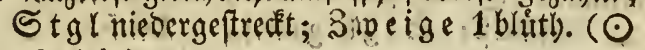
36. $\left.\mathrm{dgb}_{\mathrm{i}}\right)$.......

3. 3 fachgefied., mit ipib Lapp.; Stgl auft coronopifolia Sdcibenblume bolbentraubenf. (๑ SAf.) tanacetifolia.

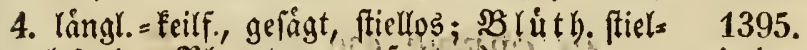
los, ben Sl. entgegenger: (Am.) I' minúta.

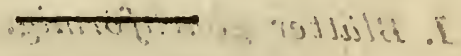

\section{COTYLÉDON, 363 ,}

Blätter:

1. ftierrund; Enotraub. Yanghaar, =fdmier.; 1396, Stgl âft. $(\odot$ SE.) viscósa.

2. gefeerbt =gezahnt: bie unt. faft ífilloförm.; Etgl äbrenf., faft einf.; $\mathbb{B}$ lúth. aufr.; $\mathscr{S B}_{3}$ l Eriect. (4 SE.) 


\section{CRAMBE. 523.}

\section{Blätter :}

1. fieberf, zerichnitt. = gejahnt u. nebft Stgl 1398. unbehaart ( 436 . w. -

2. fieber. = fidrotiageform., långl., fehr fdarf; Stgl faft unbebaart (4 As. w.) marilüna.

3. fieocrf. = leverf., icherf: Enobl. nierenf., ftumpf; Stgl etwas jobar ( $\odot$ SE. w. ఫ̛ Sivio.)

4. vielfoch ziger., zerfornitt. = gezalunt ut. nebft 1401, z

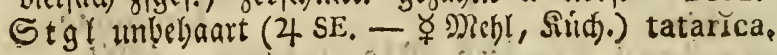

\section{CRASSÚLA. 252.}

Stgl frautartig; Bliithen:

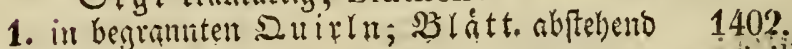
$(\odot \mathrm{SE}, \mathrm{r}$.) verticillaris,

2. in 4 fualt., blattr. 2f fterbolden, u. fticls

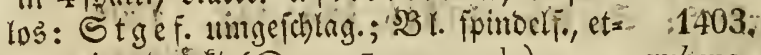
was cingesrifut ( $\odot$ 9. 23. w. u. rcb.) rubens.

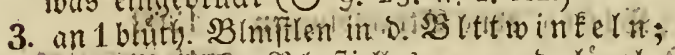

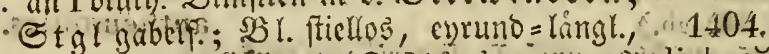

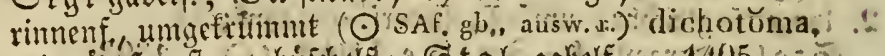

4. Die åu

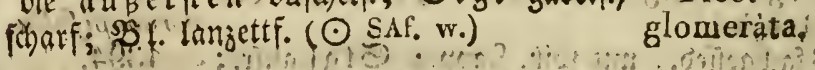

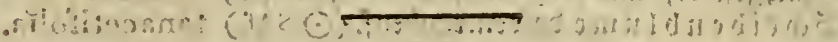

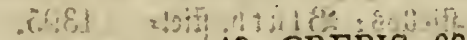

osinirx 19. CREPIS. 620

\section{Blätter gleichförmig.}

\section{Blätter:}

1. id)rotiägef. = reverforin., ftumit, gezabut;

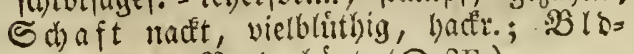

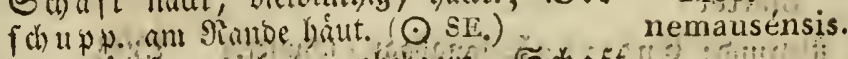

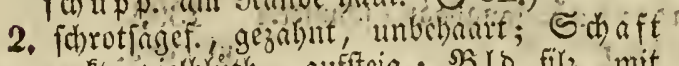

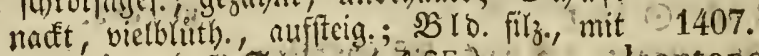

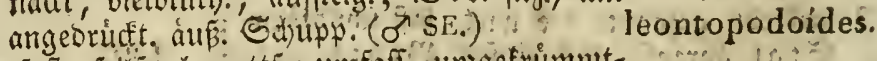

3. falt wfeilf,, lanjettf., umifaff., unigetrummt=

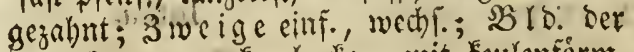
Frud $t$ eyr., ede., batti., nit Eeulenform. Şaaren (○ SE. gb. u. r.)

1408. rhagadioloides. 
4. fiellos, unbehaart, lanzettf., fammm $=$ fie= berf., an b. Spibe gangrand.; Stgl aufr., 1409. åft.; SBlo. feinhuar. ( $\odot$ 15. 29.) pinnalifüla.

5. lángl., umfailent, entferntgejahnt; Stgl

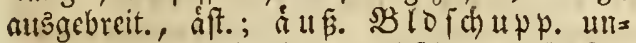
gleich), etwas abjtebend; SIflanje badtr.= id)arf $(\odot \cdot S E$. $)$

sprengeriàna.

\section{Blätter ungleichförmig,}

\section{A, scharf.}

\section{Blätter:}

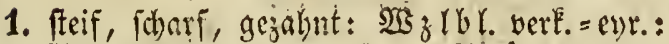
Stglbl. vfeilf., umfall:; S3lith. trau=

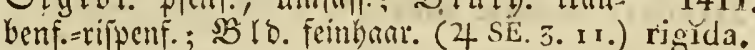

2. hader., farotfägef. fieberf.: sie oberften ftielloz, lanzettform., gryahnt, am Ritten

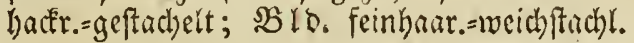
(o' 3. gb.)

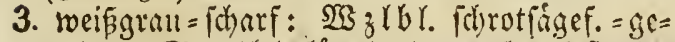
zalint: Stglbl. läingl. =latrzetff., faft um=

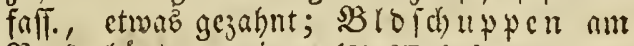

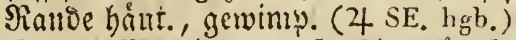

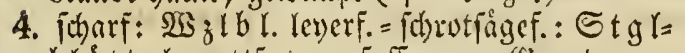
blátt. Lanjettf.; umfafl., ant (S)rumbe ge= zahnt; $\mathfrak{B} 10$. etwas fil

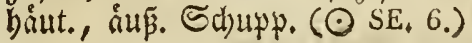

$1+12$.

(biénnis.)

1413. albŭda.

B. $n i c h t_{6}$

Blätter:

1. Die unt. ichrotjägef.=gefict.: bie ober fiteri ganjrand.; 3weige nafti, etwas getbeilt,

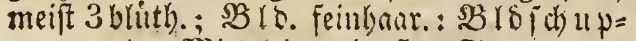
pen and ber Mrittelrippe borft.; Stgl aufr., gefurcht ( $\sigma^{2} S E$.)

2. lanjettf. =id)rutfígef., forf unbehant, nit aufiteig. Eapp. : bie. oberften gleitsbr., fa= benfo. = preilf., tullaff.; $\mathfrak{B l}$ lith. in Dolsen= traub.; $3 \mathcal{B}$ rb. Feinlbaar. ( $\odot$ I r. 25. gb.)

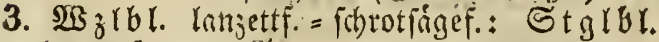
lanjettf., ant Sirutude gejahut, pfeilfürm.;

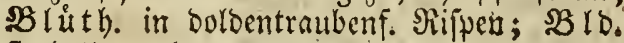

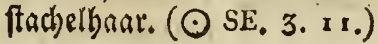

1415. intybacěa: (tectórumn)

taurinénsis.

rangens. 


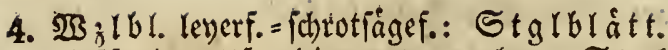
fpief́f.=lanzettf. : Die unt. gezahnt; Stgl aufrecht; 3 weige ausgebreit:; $2 B$ lo. fild.:

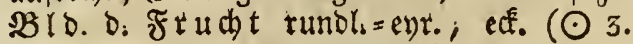
26. zuerst p.; dann gb. u. rch.)

5. fieberf.; mit gleichbr. Lapp.: $\mathfrak{S}_{3}[b \mid$ l. ge= jahnt: Stglbl. ganjtano.; Stgl rifpenf.;

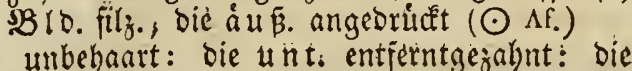

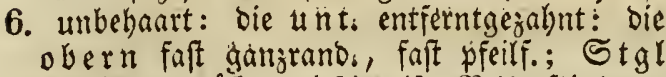

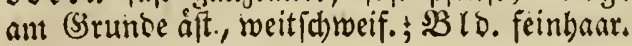
$\left(\odot \mathrm{SE}_{\mathrm{b}}\right.$ 3. gb, u. rch.)

7. lanjettf. = verê. enr., gezalnit; unbehaart: Stglbl. fahmallanjettf.; Stgl eck., unten

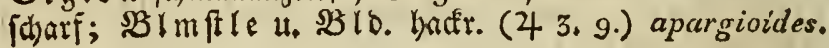

\section{CROCUS, 42.}

\section{Bliuthen:}

I. im Scerbft crjocineno; Narben febr lang, hervorrag., umgeichlag., mit gleichbr., an D. Spite geferbt. 2Ubichnitten (4 SE. v. mit $r$ Adern - * Jarben - ̧ Jarb. gelb)

II. im Frůgling ericheinerto, A. violett u. weis; Blätter:

1. lánger àls 0 . SBlüme;

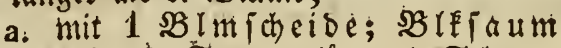
trichterif.; St trueg láng. als Stbeut.; Rarben ro̊ht., geid)ligi (4 9. v.u. w: - *arben)

b. mit 2 Blmscheiden:

a. gleich); 'BS lf jau mt glodéenform. ; Stroeg to lang alis Stbeutel; Narben wie vorber ( 4 w. u., v. mit gelb, Gruid)

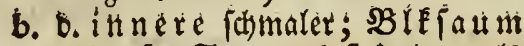
trichterf.; S twe g faft fo lang als Stbeut.; Jarben etwas fady, ferids)ligt (4 w. $\left.u_{i} v_{s}\right)$

2. ebent io lang; mit 2 gleichen $B(\mathrm{~m}=$ * 1423.

(vernus.)

* $1422 . \not{ }$
t sativus.

1418.

(Jioscorüdis.)

1419.

coronopifolra.
1420.
virens.

1421.
1424.
biflórus.

1425.

striàtus.

id) cioen; SBlE $\{a$ u in trichterformig; Stroeg wie vorber; Narben ro̊br.= zerrifl.; $\mathfrak{S Z}_{3}$ lh a ut negf. (4 SE. wo, mit 3 v. Strichen) 
B. gelb;

1. Die $\mathfrak{a} u \bar{b}$. BBIflapp. umgerollt (4 SE.) susianus.

2. Rarben ungleidjlang, viel lánger ats 1428 . Dic Stbeut. (4)

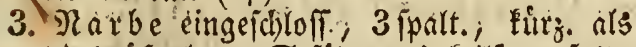
sulphurěus. bie briff: =harar. Etfáb., mit Keilf.; gefalt.,

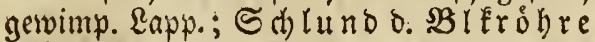
umbelyaart, mit geriben, bohl: Rappen; Stbcut: lång: als bo Ratben (4 SE, 6.) lutěus.

\section{CRUCIANÉLLȦ. 76 .}

\section{Bliithen:}

1. in 2xchren; Stengel:

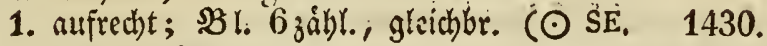
gb.; wch.)

2. nicoergeftredt ; Blätter :

angustifolǐa. 1431.

a. meift 4 záal., lanzettf. ( $\odot$ SE. grnch.) latifolĩa.

b. fpiá: Stglbl. 4 jábl., cyr.: 3 wei= 1432. gébl. meift 6 zálbl., gleichbr. ( $\odot \mathrm{SE}$.) monspeliăca.

II. einzeln in \$. sBlttwinkeln; Stgl:

1. niebergeftredet, ftaud.; $\mathfrak{B} \mathfrak{l}: 4$ zóhl., foharf=

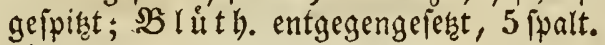
(5) $\mathrm{SE}_{0}$ 36. ugbch., Nachts offen u. wohl- 1433. riechend)

maritima.

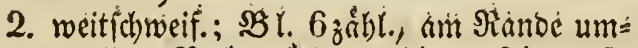
gerollt; $\mathfrak{R}_{\mathrm{e}}$ e $\mathrm{n} b \mathrm{~b}$. gleichbr. = pfriemenf., 1434.

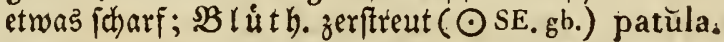

III. gebuifthelt: $\mathfrak{B} I m \mathfrak{f} l \dot{e}$ am Enoce $\mathfrak{u}$. in ben Bglttwinkeln fteheno; $\mathfrak{B} \mathfrak{l}$ É. 5 ipnlt.; 8-12

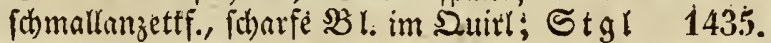
aufr. (4 SE。 wch.-grn.) molluginoídes:

\section{CUCUBĂLUS. 358. $\left(79^{15_{0}}\right.$ W: $\left.68^{\prime}\right)$}

\section{Mit Zwitterblüthen。}

Blumendecke:

I. aufgeblaf., fuger., unbehaart, netgi =gendert;

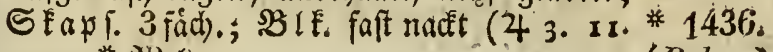
w。 - $\left.2 b_{j} l\right)$ 
II. nicht oufgeblajen; Blïthen:

1. in Rifuen; Blbl.:

a. balb 2. ppalt.; SBIs. Eeulenf.; sifpen

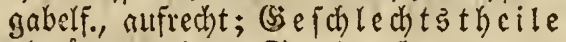
abwartsgencigt; Stgl aufr. (O' SE. w. unten grau)

b. 2 ppalt.; $\Re$ ifpe gabelf., jwifden ben Sinten fomicr.; Stgl u. SBl. fein= haar.: $\mathfrak{B}_{\text {z }}$ lbl. fpatelf. (ơ SE.)

c. 2 theil.; Stgef. lang; sgl. lamgettf.= evr. (4 3. 4. grn., Bld. braun)

2. in vielkituth., entgegengefert. Doldent

trauben; Brbl. 2 fpalt.; Stgl ganz einf.; $3 \mathbb{L}$ ID. fenlenf., geftreift; Bl. fdarf: Etglbl. gleidbr.: SIBzlbl. Fpatelform; ( $\sigma^{\circ} \mathrm{SE}$.)

3. meift in boloenf., blattlofen 2 Ititu; $\$ B \mathfrak{l b l}$ attegerano. (4 NAs. w.)

4. in rochf., 1 feit., faft fticylof. 2(ebren; 1442. $\mathfrak{B}$ lbl. meift 2 theil., undeutl. (24 SE.) reflèxus:

5. einfeit., nieberlieg.; B̉ lblat t. 2 theil.; sBlmftle, cutgegengel., cinjeln, aufr.; Stgl ganj einf. (24 NE. w.)

6. Seitenblutben uberall nieberlieg.;

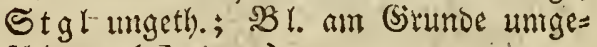
fiblagen (o s. w.)

1437.

italícus.

1438. pilósus. 1439." catholícus.

1440. multiflórus. 1441. sibiřcus. 1443. tataricus. 1444. viscósus.

\section{II. mit ganzgetrennt. Geschlecht.}

Stgl rifpenf.; die gleibjur., ungetly. Bl.:

1. ant Ranbe wellenf., unbehant; $B$ I m ftle * 1445. unbebaart (4 6. 11,gb,-grn。 - * Siratt). (Otites.)

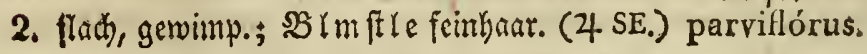

\section{CUCÜMis. 765. (W.6.)}

Kernfriichte :

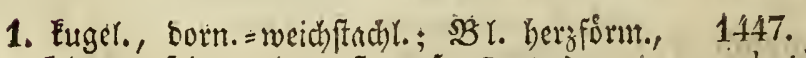
5 lapp., feingezabnt, ftumwf $(\odot$ As. $)$ prophetarum.

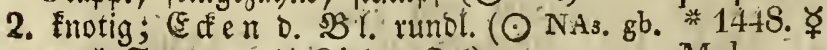

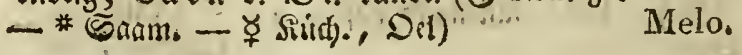


3. långl., fharf; E

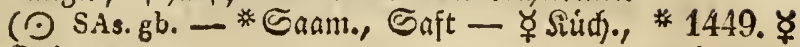
Del) satívus.

\section{CUCURBITTA. 764. (W. 5.)}

Kernfriichte :

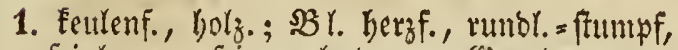
feinhanar., feingejabnt, am Srrunbe unten

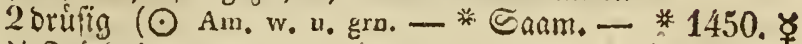

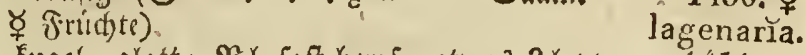

2. fugel., glatt; 3 l. faft her fo, etwas 3 lapp., 14.11.

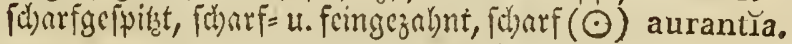

3. verf. eyr., 8. Rånge nad) binbenart. $=$ ge= ftrichelt; S3l. herzf., ecf.= 5 lapw., feingezalgnt, 1452. feinbaar. ( $\odot$ As. gb.) ovifĕra.

4. rumor. od. enr., glatt; SBY. Gerzf., ftumpf, faift 5 lappig, feingegalint $(\odot$ As. dgb. - * 1453. § * Siam. - ४ Sind\%., Oel) Pepo.

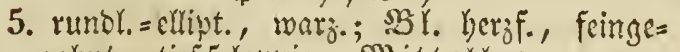
ônht, tief 5 lappig: Mittellappen am 1454. Srunoe fómaler $(\odot)$ verrucósa.

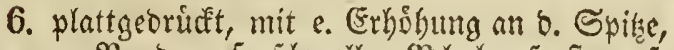

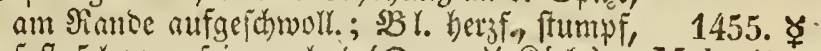
faft 5 lapp., feingezabnt $(\odot-\zeta+$ Sild.). $)$ Melopěpo.

\section{CUMINUM. 215:}

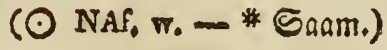
* 1456.
Cymínum.

\section{CUPHÉA. $37 \%$.}

\section{Blätter:}

1. eyr., faft unbehaart; Stgh aufr., fhmier:= 1457. feinhaar. ( $\odot$ NAm. p.)

2. enr., s lanjettf., etras bactr., fursgeftielt; 1458.

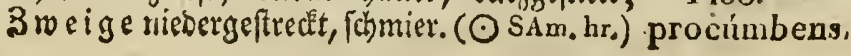




\section{CUPRÉESUS. 767. (W. 22.)}

\section{Blätter :}

1. 4 reibig = bachziegelformig,

a. gemolbt, ftumpf, angebrutet; $3 a p f e n$ Eugel., mit melirlor. Schupp.; 3we ige 1459.

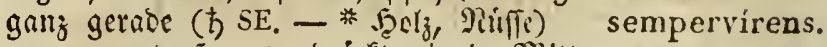

b. eyr. = rautenf., angeorude in D. Mitte

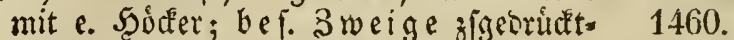
2 ichncio. ( $5 \mathrm{NAm}$.)

2. 2 reibig, abfebend, gleichbreit, abfalleno (b $N A m, \triangle$ )

thyoides. 1461. distı̌cha.

\section{CUSCUัTA, 176, $\left(82^{3}.\right)$}

\section{Bliithen:}

1. faft ftiellos, meift 4 palt.; SBIE $\{$ d I I n o

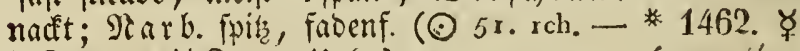
* Siraut - ఛ sutt., linfr.)

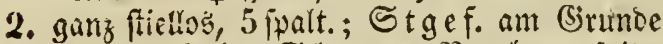
mit e. geferbt. Schuppe; Rarben fpis; Etgl haarf. (广 51.)

3. geftielt; mit $1 \subseteq$ Stmeg (5r.)

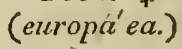

1463.

Epithýmum. 1464. monogỳna.

\section{CYCLÅMEN. 116.}

\section{Blätter:}

1. freišrund, Gerzf, geferst ob. Feingezahnt; B lalapp. larizcttf. (4 5. 8. w. u, rch. * $2 \mathfrak{3} \mathfrak{i}$ )

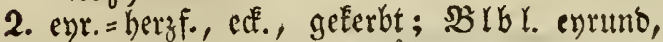
* 1465 . f europá eum. ftumpf; $\mathfrak{B S}_{3}$ I l Fehr grof, ribenart. (4 4. rcb.) neapolitánum.

3. berzf., edf., feingezabnt; $B$ l l lap p. Inmjettf., 1467. lóngl., etwas fpits (4SE. 23. f.; $p ; w_{0}$ ) hederaefolǐm.

\section{CYDONǏA. 395.}

\section{Blätter :}

1. oben etwas jott., unten zott.=fitj., eyr. od.

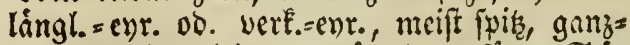
ranb.; Xfterbl. eyr. = lángl., gęágt: $\mathfrak{S}_{\mathfrak{a}=1}$ 


\section{Cydonia. - Cynara.}

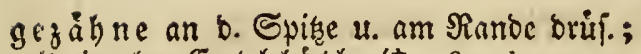
mit einzeln. Endblüth. (方 26. rch. - w. -

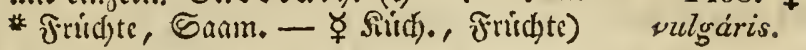

2. oben unbchart, unter $j^{\text {ott. }}=$ feinhaar., verk. enr., lang $=u$. jcharfgejpitzt, mit orufigen

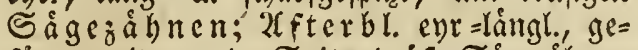
fảgt, mit an D. Spitze brú. Ságejálgnen (5) As. $\Delta)^{\prime}$

3. uiberalf unbehaart, eyr., oval u. verf. eyr., fpis, abgerunoet, am Şrutnoe ctwas łeiff.,

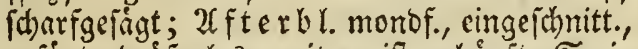

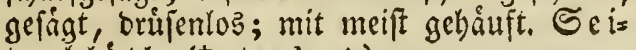
tenbluth. (古。As.dr. $\Delta)$

\section{* 1468. ఫุ} sinénsis.

\section{CYNÁNCHUM. 173.}

Stengel :

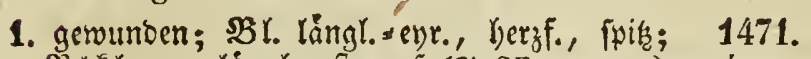
SIElapp. lángl., ftumpf (4 SE. r. ; uw.) accítum.

2. nur oberhalb gewunden; SBle. bart.; bef. B I m ft le ber einf. Dolbe kaum lång. als b. gem. SBlmftr; Nect. $\mathfrak{N}$ ranz balb 10 part. (4 SE. braun.-. r.)

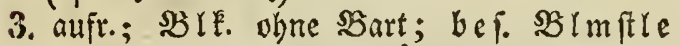
1472. nigrum.

Der einf. Dolde 3 mal lang. als ber gem.

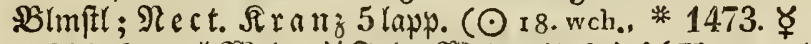

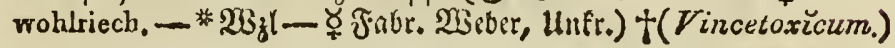

\section{CYNĂRA. 640 .}

\section{Blätter :}

\section{Xlle}
a. fieberf. u. born.; sRrofdupp. eyt. (4 Af. dbl. - ซ̧ Sili,.)

b. gefiebert u. faft glatt, mit gezahnt., born.

Lapp.; Stgl fehlt: bie innern $\mathfrak{B} 1 d=1475$.

(d) upp. an b. Spize troden (4 SE.) pygma'ea.

2. cinige gefied., andere ungeth.: Urre etwas borm.; 2 Iofdu upp. en). (4 SE. bl.

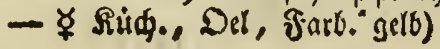

\author{
1476. ఫุ \\ Scoly̆mus.
}




\section{CYNOGLÓSSUM. $10 \%$.}

Stengel aufr.; Staubgefä[se:

I. nicht eingeichlo lien, fondern etwas ling: als

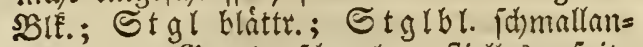
zettF., an Stuno ichmaler, ftiellos, fpis, fabr lang, etwas jott.; $\mathfrak{I} \mathfrak{r} u \mathfrak{b}$ obue $\mathfrak{R e}=$ - benbl., vor D. SBlúben georángt ( $\sigma^{7}$ 9. rch., dann $h v_{\text {. }}$ )

1477.

(apennirum.)

1I. eingefolololien; Blätter:

1. jâmmtl. ichmallangett., langgepipirt, an Grunde breiter, baar.=faft bactr.; İ a dib.

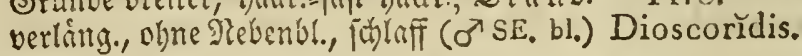

2. die untern:

a. Greitlanjettr., am (srunbe fitmalet: bie obern am Grumbe breiter, pis, fein= baar. =filz; ; I $\mathfrak{a} a \mathfrak{b}$. olne Nebenbl., waikend o. Blithe ripenform.; Stgl * 1479.

bláttr. (ơ $14 . u_{0}$ - $23 j l$, Siraut) (officincile.)

b. lanjettformig,

R. Fivis: Die oberu enr. = lanjettform.; berzi., wenigfilz.; $\subseteq$ tgl brattr.;

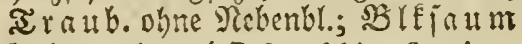

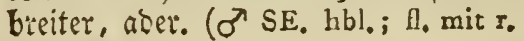
Adern)

b. am Sirunte fchmaler, wenig feinlyar.: bie obern ftellos ob. umfart., faft bersf.; SBlolappen lingl. ( $\sigma^{x}$ w., Klappen r.)

c. oval=länglich, ipis: die obern am (5)runbe berzf., umifafieno, iche raudi): Iraub. Eurs, Doloentraubenf., ogne Nebenbl. (O' NAm. b1.)

d. faft ipatelf.=lanzettf.: bie obern balb= umfâf., eyr. =lángl., fpis, oben fajt unbebaart, fpiegelglatt, unten forarf:

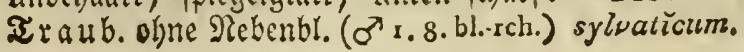

\section{CYPRIPEDĬUM. 722.}

Stgl bldttr.; Rip : zigeorudat, tưrz. als die 2 lbl.; Lappe des Griff.:

1. cllipt., ftumpi ( $4 \mathrm{I}, \mathrm{dr}, \mathrm{u}, \mathrm{hg}_{\mathrm{g}}$ )

1484.

(Calceōlus.) 


\section{5.}

2. 3 peit. , ppíg (4 NAm, ugr. u. braunfleck.) parviflórum.

3. 3 jeit. =lánglich), ftumpf ( 4 NAm. gr. u. r. 1486 . punct.) pubéscens.

\section{CYSTICÁPNOS. 555:}

(@) SAf, wcb.-gb.)

1487. africàna,

\section{CYTǏsUS. 568 ,}

\section{Blätter $3 z a ̈ h l i g$.}

\section{Bliithen:}

\section{A. in Trauben:}

1. einfach, hangent; Huilsen:

a. mit engerrifeten Eleinen Saarer, am Rante bicfer; Sblat t th. obal, gewimp., oben unbehaart, unten feinbaar( 5 9. gb.) (Labírnum.)

b. ganj unbebant, nim obern Ranie faft geflugelt; $2 B(a ̂ t$ th). lanjettf. od. oval= lanjettf., anf keys. Esiten mibebnart:

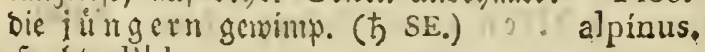

2. aufredit; bld.:

a. frinban., mit e. gleichbr. Alchenblatt 1. Flcin. Sát)nd); mit (Enbtrauben; Blåt d d). vert. cur., oben mbebañt, unten etruas feinlian.; Sy illien. jises oridfé, mit anliegent., fleinen Sanaren (ち 8. gb.)

b. nebft Şilfen foupp. =ichmier.; mit

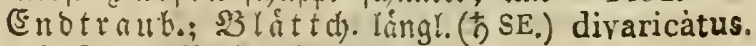

c. mit 3 fad). Rebenbl.; $\mathfrak{B} !$. an den Blutb. fiellos: sie unt. gefficlt; mit

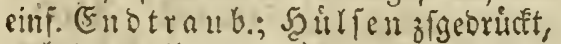
umbebant (5 SE. gb.)

3. 1 jeit., meift 4 bluth., an Enise fickento; 53 lát tch. verl. evr., unten friegl.=feins han:; sblo ede tief 3 theil., feimbanr; Z toeige mit feitren angesrudt. Saaren ( 5 As. hgb. $\triangle$ )

B. in Fopff. Endodolsen; Zweige:

1490.

migricans.' 1491. sessilifolius. 
1. langhaarig; Haare:

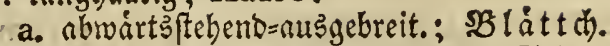
verE. evr., gewimp., auf beyd. Seiten falt zott.; SBID. zott. mit e. gleichbr. Nebenbl.; Șillen zigeorudet, zottig ( $\left.5 g^{b}.\right)$

b. aufr.=abwártsłtehenb; $\mathfrak{B}$ lả ttd). verf́. cyr. = lanjettf., foft angebrúct=gemimp., auf beyo. Seit. friegl.=feinbaar.; $B$ lo. mit angebrúdt. Şanren $u$. e. Lanjettf. Rebenbl.; Sุ ülfen żigeorudt, mit zer= freut. Seaaren (b 8. gb.)

2. Fehr menig feinbaar.; $\mathfrak{B}$ láttch. långl., faft unbebaart; $B$ lo e ce feinbaar. († SE. gbcb.- w.)

1494.

capitátus.
1495.

austriăcus.

\section{6. leucànthus.}

C. an Blumenstielen;

1. cinzeln in D. SBlttwint.: Stgl nieber.

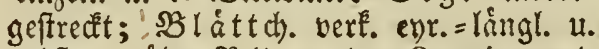

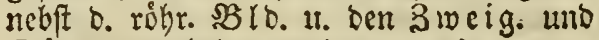
Șílfen unbegaart (b 2. p. $\Delta$ )

2. meift $2 z^{z} \mathfrak{h} h l$ in D. Blttrwink.; Stgl.:

a. nieberlieg.; ; $\mathfrak{B}$ lát $t$ d). verê. cyr =långl., feinhaar. = gewimpert, unten feinhaar.; 3reige mit jebr wenigen aubgebreitet. Saaren; şid. faft bauct), menig fein

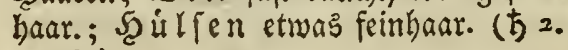
6. gb.)

b. meitfobeifig; 3 weige aufwảrtsgefrummt, mit feinen angeorúdt. Scaaren;

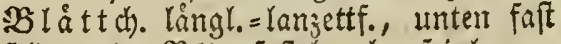
feibenart.; 310 . Faft baudt., Feinhaar.; Şílfe etwas feinhaar. (ち $S E, g b$.) bifórus.

3. meift 3 záblig,

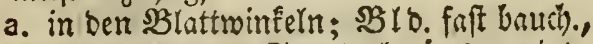
feinhaar.=zott.; Stgl abwántşgeneigt; 3 weige jott., mit abwartsftehenoen Şaar.; $B$ lát tch. vert. enr., zott. = ges mimp., feinlyaar. =zott.; J̦úlfen faft $\therefore$ Fictelf., zott. (ち SE. 8. gb.)

b. am Enbe ftehent; $B$ ls. tief 3 theil.,

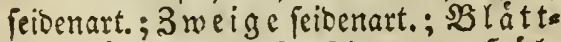

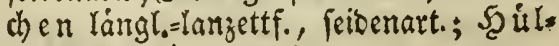
fen zott. (b) gb. $\Delta$ )

4. meift 4 zábl.; Stgl aufr. 3 3weige sers 1498. supinus. 1497. purpurěus. (1) 1499. 
Brattth. vert. eyruno, zott. = feinhaar.; Joulfen etwas jott., mit angeoridften 5ुnaren (ち $\mathrm{SE}_{\mathrm{g}}$ gb.)

5. gehåuft, meift am Enbe fteheno; Bro. rauch, 3 fpalt.; $B$ látt dj. verE. enrund, (d)arfgefpist, unten rauch) (ち SE. hgb.) hirsútus.

\section{Blätter ungleichgefiedert.}

Blát then rundl, = ellipt., grau = fcinbaar.; Er a ub. 1 feit., in ben sBlttwinf. und am Enbe ftelgeno (万 NAs.gb.)

1502. elongàtus:
1503.
1504. wolgarĭcus.

\section{DALĔA. 543. $\left(540^{3}.\right)$}

1. Mit 5 Stgef.; Enbahren walyenf., ges

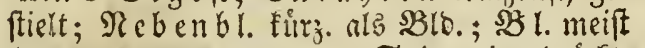
10 parar., ellipt., an Der Spitác eingebridut, 1505. ftechenofipiz $(\odot$ NAm. wch.-bich.).

2. mit 10 Stgef.; Entoibren malizenf; 1506.

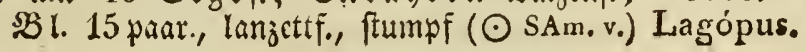

\section{DAPHNE. 317. $\left(788^{2}\right.$, W, 9.)}

\section{Blïthen:}

1. ftiellog,

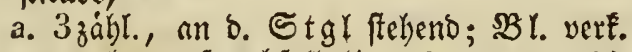
eyr.=lanjettf., abfall. (5 5. hp.; w., wohl-

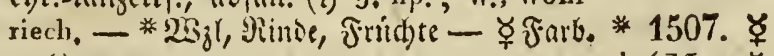
roti)

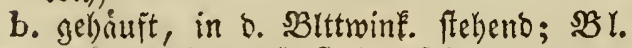
$\dagger($ Mezerěum. $)$ verf. eyr.=langettf., ftecthenofpis, gamzrand., oben faft unbebaart, unten feinhaar., puns ctirt ( 5 9. xch. $^{-}$w.)

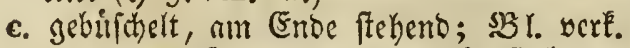
eyr. =lanjettf., gamzramb., nadit, ftechend = fipik ( 5 2.5. hp. wohlriech.)

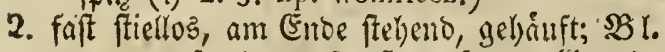
entgegengcr., lonzettf., flumryf, an (S)runte fotmaler, ganjrando, unbehaart (b NE. 9 . w. $\Delta$ )

1508.
alpina.
1509.
+ Cneŏrum.

1510. altaĭca. 
3. in idoppig. Endtraub. mit $\Re \in b e n b l$; SBl. verE.. eyr., ftumpfgeipigt; ganzrand., unbehaart ( $5 \mathrm{SE}_{0}$ grnch, -gb.)

4. in ben SBlattwinf., gepaart; $B_{B} l$. fámals lanjettf., (nit garjgetrennt. (s) $\{$ d d l.) (ち SE. 2. 9. w. - gbch.)

pontrca.

5. in 5 bluth), uberbang. I $x$ a

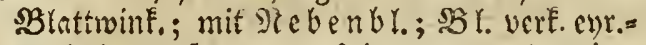
oval = lanjettf., etwas fpits, ganzrano., im= mergrim, - unbel)aart ( 5 5. 8. grn.-gb., übel. riecb. - * *inoe)

1512.

dioǔca.

22. DATÍSCA. 794. (W. 71.)

Stengel:

1. rauch (4 NAm.)

2. glatt (4 As, gb.)
1514:

hirta:

1515.

caumabina.

\section{DATÚRA. 122.}

Fruchthüllen:

A. bornig,

1. aufredit, eyrums:

a. Die oberften Dorn. febr grof, zige= neigt $(\odot$ SAs. w.) ,

† Laureŏla.

b. Die unbelaant., cif.=gezahnt. Bl.:
a. eyruno $(\odot$ 14.22. w. - * Siraut, (Eam.)
b. eur. = faft herjf.; Stgl flect. $\odot$ SE. hbl.; Stgl rcb., mit w. Flecken) + Tatüla.

2. iberbangent, fuger.; SBY. hergf., foft un= 1519.

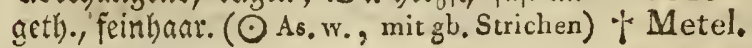
B. webrlos, glatt,

1. nufr.; Sol. eyr., edt. =geanhnt, unbehaurt; 1520. Stgl robr., Erautart. ( $\odot$ Af. w.) laevis.

2. Gangeno, verf. cul.; $S g$ r. eyr. = lamacttf., am Siande wellenf., inten filg.; Stgl 1521. gabelf., Gornf. ( $\odot A_{0}$ grn, u, w., ausw. vo) ceratocáula. 


\section{DAUCUS. 204.}

Saamen hactrig; Blätter:

1. 3 fach gefieo., glanjlos: 'Blát th. fieberf.; mit lanzettf., feingelpizt. Rapp.; gem. uno bef. Sc ullen fieberf., faft fo lang als bie Dolde: Sa amenftacheln gerade jo breit, als D. Frudt $\left(\sigma^{7} 29,4 \cdot\right.$ w, - $\mathfrak{W B}_{\mathrm{z}} \mathrm{l}$, Saan.

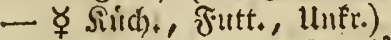

2. Dopp. gefieb., lanzettf.; glanz., unten baar., * 1522. ชุ. (Caróta.) mit evr:=lanjettf., fieberf. BBláttch)., u. eț. was ftumpf. Lapp.; SB (át tc). ber. gem. 11. ber. S⿹ úllen etras breit, faft jo lang als die Dolden, mit umgefrummt. Enppen (ठ্ $\left.\sigma^{7} E_{1}\right)$

1523.

Gingid̛̆um:

\section{DECUMARIA. 387. $\left(372^{z}\right.$. $)$}

231. eyr. = långl., an beyb. Enben ppis, un: 1524. beutt. gefingt (ち NAm. wch., wohlriech,) bartăra.

\section{DELPHINIUUM. 425. ( $417^{\circ:}$}

$$
425^{x} .426^{x} \text {.) }
$$

\section{Mit 1, Scamenkapsel.}

Rectarium 1 brattrig;

1. Stengel:
a. etwas getheilt $(\odot$ 29. db.; w.; n. - $* 1525$. ఫุ

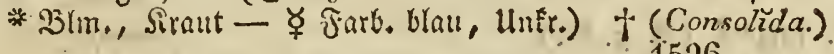
b. cinfach $(\odot$ bl.; r.; w. $)$
2. bef. 3 weige 1 brith.; Pect. vorn 4zahn. 1527. ( $\odot$ SÉ. grau, inw, rch, u. grn.)

\section{II. mit 3 Saamenkapseln.'}

Nectarium:

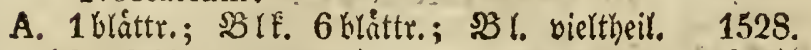

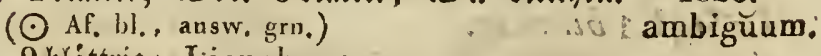

B. 2 blittrig: Lippchen:

1. ungeth.; - Briuth. meit einjeln; $\mathfrak{B l}$. zige - 1529. Fekst, gleichbr. = vielth)eil. (4 NAs. bbl:) grandiflórum, 
2. 2 fpaltig,

a. eyrund: Lappen:

a. eyr.; $2 B$ I. 3 theil., mit 3 fpalt., jer $=1530$. \{chnitt. Lapp. (4 8. bl.). : intermedium.

b. ungl., fehr kuro; $\$ 3$ l. 3 theil., mit 3 fpalt., gleichbr., auf bend. Seit. feinhaar. Ilapp. (NAs.).

b. Yänglich, mit lanjettf., gleict. Iapp.;

(sB I.' 3 theil.: Lappen derselben:

a. 3 ipalt. ( 4 NAm.)

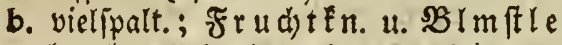
langhanr. (4 NAs. braun-rch.)

3. ausgerand., evyr., mit fehr furz., ungl.

Eapp.; $B$ r. faft fobiltof., 3 their., mit viele fpalt: Lapp. (4 8. dbl.)

1531. hybridum.

1532. exaltàtum.

1533." punicĕum.

1534.

C. 4 bláttr.: Elippden am Sianbe wellenf., to lang als D. sBlbr.; SB Imftele ling. alś Das Rebenbl; ; $B$ l. Ganbf., mit lángl. Lapp. u. haar. SBlttftlen (O'SE. dbl.; p.; w. * Saam. - ૪. Del)

\section{* 1535. ఫ + Staphisagria.}

\section{DENTARIA. 533.}

Blätter:

1. 3 fach 3 zákl., gezahnt,

a. mit e. pfriemenf. Drule in ben $\mathbb{S}$ infeln D. SBlâttch.; Stgef. balb fo lang als b. Bglf. (4 SE. 4. 7. rch.)

b. Ohne D. obne Drúle; St gef. fo lantg als b. BBIE. 1537.

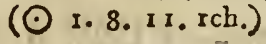

2. fingerf. (4 9. hr.) enneaphilla. 1538.

3. gefiedert,

a. Xlle: $B$ làtt then längl., langgepipizt, gleidygezalint (4 SE. 9. rch.)

b. nur bie unt.: bie oberften einf: ( 4 I 8. hr.) pentaphýllos. 1539. pinnàta. (bulbifĕra.)

10. DIÁNTHUS. 348.

\section{Sigl 1 blüthig.}

Blumenblätter:

1. geferbt; Bldschuppen: 


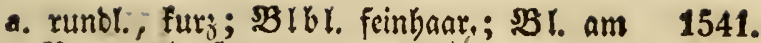
Rande fajarf (4 23. 月.)

b. bie åu ß̉. blattart., fo lang alz NBlbróbre 1542. (4 9. hr.)

2. vielipaltig; Bldschuppen:

alpinus.

a. evr., ftumpf; $2 B$ l. gleichbr. (4 13. 20. 1543. $h_{r_{0}} ; w_{.}$)

b. verk. enr., ftechentipif ; $B$ If. ohne Bart; BS l. pfriemenf., ganzrano., unbebaart (4 SE. 23.)

\section{Sigl meist 2blüthig.}

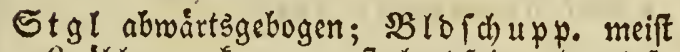
6 záhl., verk. evr., ftechendópin, 4 mal jo

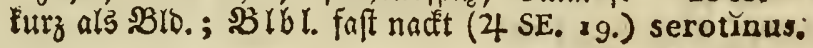

\section{Stgl mehrblüthig;}

\section{A. Blüthen gehäuft.}

Blumenblätter:

I. 2 paltig, mit 3 jahn. Eappen ( $\sigma^{7}$ SE. r..gb., 1546. inw. gbch.)

II. nidft; Bldeckschuppen:

ferrugineัus.

A. To lang als bie sBlorólyre, uno

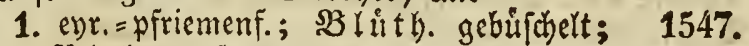

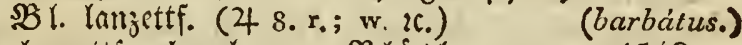

2. $\mathfrak{l a n}_{j}$ ettf., langhaar.; Bluthen ge $=1548$. buifchelt $(\odot$ 13. r. u. w.)

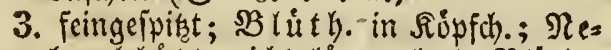
benblát t. nicht läng. als D. Blith.; Stgl glatt; $\mathfrak{B}$ l. gleichbr., glatt (4 SE. r.)

B. länger; Bliithen:

(Armeria.)

1. in traubenf. Doldentraub.; $\$ B(b=$ $\{$ ch upp. eyr.=lanzettf., nach b. Blitthe siel lảnger als \$Bldróbre; Bl. lángl. lanzettf. (4)

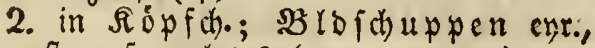
fłumpf, wehrloz (4 13.34, ros.)

C. fintzer;'Bliithen :

\section{9.} diutı̌nus.

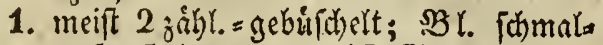
zettf., 5 ripp., u. neblt $\subseteq$ tgl fobarf; Bldschuppen:

1550.

latifolĩus.

1551.

(prolifer.) 

a. eyr. pharfgeipint (4 SE.)
b. evrs slanzettf. (4 8.) collinus, 1553. asper.

1552.

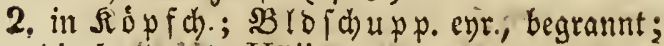
bie begrannte Hiille:

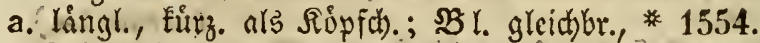
3 ripp. (4 2.13. dr. - (acorthusianorum.)

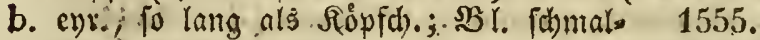
lamzettf., ohnt Mriph. ( 4 SE, 2. dr.) atrorúbens.

$$
\begin{aligned}
& \text { B. Blüthen einzeln, aber meh-: } \\
& \text { reruauf demselben Stgl. }
\end{aligned}
$$

Blumenblätter:

1. geterbt; Bldschuppen:

a. cyrumo, iefre furd,

a. Ulle fpí; $\Re$ Iblåt. bartlos (4 SE. * 1556.

p. 2c. wohlriech. - $*$ Blm.)

b. mur bie ális. fwis: Die innerften fehr ftumpf; $\mathfrak{B}$ rbl. wie vorber (4 9 . r. ohne Geruch)

b. cyr: = lanjettfornig,

Caryophýllus,

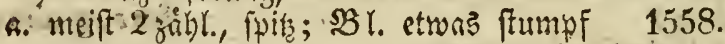

u. Feinbnar. (24 4.34. hr., w. puncr.) (deltoides.)

b. 4 jabl.; $\mathfrak{B}$ r. gleichbr:, ftumpf u. nebft 1559. Stgl feinibar. (4 SE.) glaucus.

c. piricmenf., etwas abfteleno, blattart., to

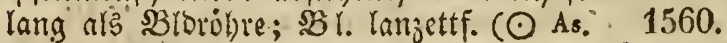
hr. "u, w., yerschiedenfarb.) chinénsis,"

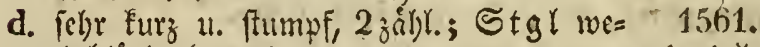
nigblutt). ( 4 f.)

e. ftectientipits, etwas abftelsent, firr. als SBlorobre; 28 rbl. im Schlumbe feinhaar.; Stgl glatt, unbehaart; BBl. gleidbureit, 1562. wenig fharf (4 NE. r. u. gb) versicŏlor.

f, etmas abftehend, mit lanzettf. Spize;

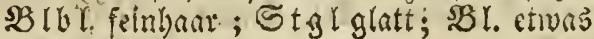
fdorif (4:- rch.) 1563.

Willdenowï,

2. gezalnt; Bldscliuppen:

a. faft fo lang als sbleribre, eur=begrannt; sE l. pfriemenf., gerippt = geftreift, f(t)arf; Stgl rifpenf., glatt (4 As.)

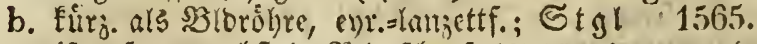
rifpenf., u. nebft D. $\mathfrak{B}$ l. fonarf (4 NAs.) campéstris. 
3. Dopy. gejahnt; SBI it th. faft boldentraubenf.;

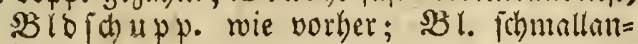
jettf., utocutl. 3 ripp., unbel)arat (4 oben dr. unten wch. - Hl.)

1566.

sylvatǐcus.

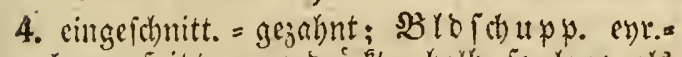
langgefpift, angebricet, balb fo lang als

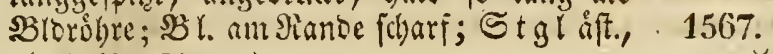
glatt (4 NAs. 9.)

caucašcus:

5. 3erichnitt.=gezabnt, num Ed)Yunbe unbelyart;

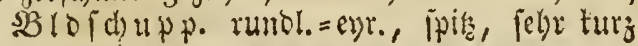
(4 SE.)

1568 .

horténsis.

6. viclfwaltig; Bldschuppen:

a. faft eyr., fegre ftumpf, felgr tiriz, wehr= $103(44.34$. dr. $;$ w.)

b. vfriemenf., gerato, wemig für. als şld= rublere; Stgl aufr. (4 SE.)

7. vielipalt. = haarf.; Stgl aufr.; $\mathfrak{B l}$ lith. in

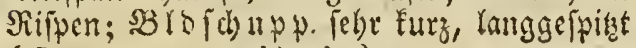
(o'.3. 4. 48. vo; bl.; hr.)

1569.

(plumarüus.)

1570.

monspeliăcus.

1571.

(supérbus.)

\section{DICTÁMNUS. 340。}

Dic am Brunbe ungl. Blättchen:

1. obal; sizlttitiel gerámelt (4 5. 8. wo * 1572.

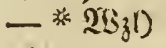

2. långl, am (Brunbe fothmaler; BBlttft! un=* 1573 .

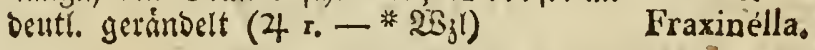

\section{DiERVIÍLLA. 147.}

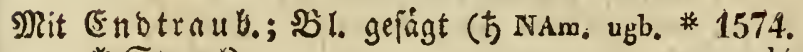
- * Etrugel)

canadénsis,

\section{DIGITÁLIS. 493 ,}

I. Blumendeckblättchen:

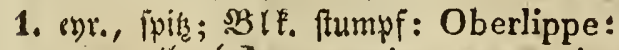

a. ungett). (o' 8. p. u. inw, w.; w. inw, \#1575. mit ringf. Fleck - * Siraut) 
b. meift 2 lapp.; $\mathfrak{B}$ l. Ianzettf., ftiellos, glatt, fajt ganzrano. (4 SE. Farb. wie vorher)

2. lanjettfórmig; Blkronen:

a. ftumpf, mit langhaar. unterft. Iapp.; B I s blattch. ftumpf; $2 B$ l. gleichbreit,

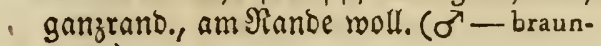
gbch.)

b. ipis, mit 2 ppalt. Slippe (4 × 9 . hgb., inw. braun)

c. am Scetm ausgerand.; $B \mathfrak{l}$. unten feitt= baar. (4 1 , ugb.)

d. Seitenlappp. D. Urippe fpis: N ittellapp. lángl., unbebaart, flach; $\mathfrak{B}$ \úth. Didhtábrenf., georángt; $\mathfrak{R} e=$ benbl. lanzettf., langer alb $D$. SBlo., woll.; $\mathfrak{B}$ ID blät tch. woll. (4SE. 2, 6.) lanáta.

e. geftielt, entferntftebeno, mit evr. SMit= tellapp ber Ulippe; $\mathfrak{B l}$ l. etmas gejabnt u. nebit ben lanjettf. sBloblat then nadt ( $4 \sigma^{\top} \mathrm{SE} .23$.)

3. langlich, felur ftumpf, unbebaart; $S_{\mathfrak{c}}=$ tenlappen D. Ulippe D. BZle. fpis: Dittellappen langlich, raud, bohl; Blútben von cinander abftebeno; $\mathfrak{R}=$ benbl. lanzettf., unbebaart, fo lang als BID. ( 4 SE. braun-gb.)

1582. ferruginěa.

4. fpit; : $B$ If. feinbaar.: Slippe abgeftujt= ausgerandet: Seitenlapp. D. Ulippe ipis: IN ittellapp. eyr. =lángl; $\mathfrak{i} e=$

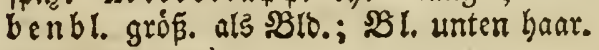
(4 - gb.-ur.)
15S1:
laevigáta.

1577. parvillóra.

1578.

† luť̈e.

(ambigŭa.)

II. Blätter :

1. Feinbaar., weid, umfa (4 SE. p., Scblund blals mit r. Punct.) Thapsi.

2. unbebaart, fhmallamzettf., gangrano., am 1585. (Srunde angeraachlen (4 SE. br. u. gbch.) obscúra.

\section{DIOSCOREA. 786.}

DB L. Gerzf., langgeipist, 9 ripp., unten etwas feinbaar: einige roechl.: andere entge= 
gengef.: nod andere im Duirl; Stgl 1586. faft frielrumo, glatt (4 NAm.) villósa.

\section{DIOSPÝROS. 811. (3๑7.)}

Zweige:

1. unbehaart; Blätter:

a. evr., etraas frumpf, glánj., unbehaart, nesf. = geabert; sB itt ft le feinhaar., init unbehaart. Sinospen (ち NAm, braunlch.gb. $\Delta$ )

b. lánglid, ranggeipikst, unten feinbaar.; Blattfíre feingaar., mit inmento. jott. Inospen (t) SE. ugb. $\triangle$ ) 1587. virginiána.

2. filz.; $3 B$ r. eyr. = oval, ranggeipiat, unten

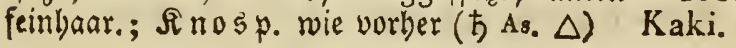

1588.

Lotus. 1589.

\section{DIPSĂCUS. 54 .}

\section{Blätter:}

1. zujammengerwachien,

a. långl., ftumpf, gefägt: Spreubr. lants gettf., fteif, fpis, utmgefrimmt, für. ars

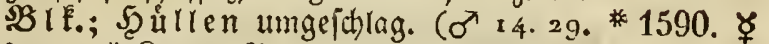

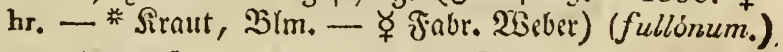

b. evr.=lanzettf., etwas Tanggefpitat; હpreu= brát. borftenf., gerabe, gleidbbr., bieg=

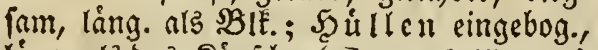

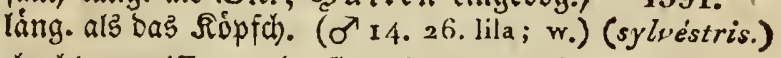

c. bucht.s zerriī., rauh, fieberf., mit wech).

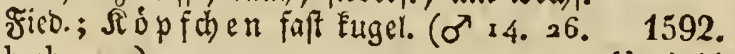
hrch.; w.)

2. geftielt, mit 2(nthang verjeben; $\mathfrak{\Omega} \mathfrak{o} p \mathfrak{f}$. barbfugel.; J Jullen abwartsgebog., fo lang als b. תioppfot. (o' 4. 26. wch.)

3. Stglbl. ftiellos, gleichbr. slängl., fieberf., mit entferntfeheno. Fied.; $\mathfrak{S}_{j} 1 \mathrm{r}$ l. ungeth.; ङpreubl. gerabe; ভtgl u. sBl. Ftadbl. (ON 85. wah.; bfl.)

1591. 1593. (pilósus.)
1594. ferox. 
14. DODARTİA. 499.

281. greidbbr., ganzrano., unbehaart $(\odot$ As. p.) orientális.

\section{DODECATHËON. 117.}

SBl. langl.=oval, ausgefchweift=gejabnt; Dol oen vielblưth., fohlaff, mit fleinen ovalen Rebenbl. (4 NAm, hr.)

1596.
Meadıa.

\section{DOLICHOS. 571.}

Stgl gerwunben; "Sgullen evr. =fábelf.; 5 . eyr., mit gegen fas eme Ende bogenf.=ge= frummt. Slabel (๑ NAf. p.; w.)

1597.

Lablab.

\section{DONDİA. 180.}

Bl. 5 rapp., ftumpf, gefịgt; 5̧ürten längl., ftumpf, gefágt (4 9. gb.)

1598.

Epipáctis.

\section{DORONǏCUM. 686 .}

I. Wurzelblätter:

1. tiefherzf.: Stglbl. umfafl: bie unt. ges fticlt; SBlattfil mit ohrform. 2fnhang;

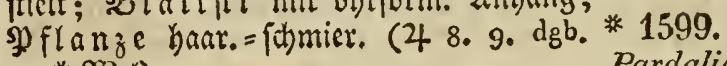

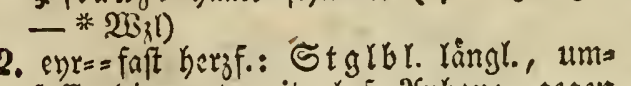
fall.: Die unt. mit ohrf. Uanhang, gegen ben Bruno bin fatmaler; Nflanje etwas rauld (4 8. bgh.)

Pardaliánches:

3. ganz herzfórmig,

a. geftielt: bie oberften lanzettf., unt= fafl.: bic unt. fpatelf. =eyruno: 2alle fcingezabnt (4 5. 9.)

b. tiefgezahnt: St glbl. rongl., umfafi:; St gl meift 1 bluth., (bey ums 2 bis

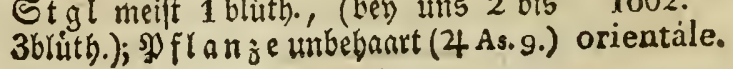


II. bie unt. $\mathfrak{B} \mathfrak{l}$. geftielt, eyr. : bie mittlern geftielt; geflitg.: Die obern umfaff.: 2Alle gezabnt; so flanze feinbaar. (4)

1603.

dentátum.

\section{DORYCNIUM. 590;}

Bl. 5 zåahl.; Stengel :

1. ftrauch.; $B$ l'attch. fohmallanzettf., fpis; 1604: BIOzábne eyr. (ちSE. w. $\triangle$ ) monspeliénse.

2. Ftauto, aufr.; $\mathfrak{B}$ lăttch). verE. $=$ enr., ftumpf; 1605 .

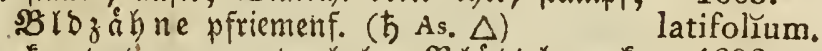

3. Frautart, ganz unten holz.; ; B látt ch. verk. 1606.

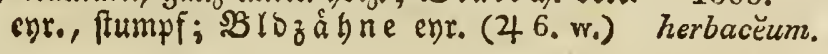

\section{DRABA. 520 .}

\section{Mit nackcem Schaft.}

\section{Blätter:}

1. lanżettfơ $r$ igig,

a. am Ranbe gewimp., fteif, nachenfortm.; 1607.

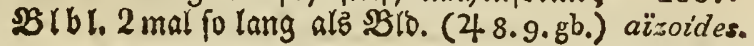

b. ganzranto.; S chaft einf. (4 9. gb.) alpina.

2. långl., etroas fwis u. geiågt u. fatacherhaar.;

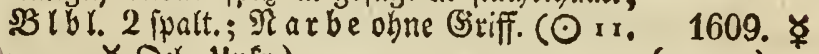
w. - ఛ̧ Del, Unfr.)

3. Feilf., hanbf., 3 lapp. (4 9. p.)

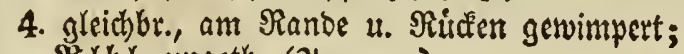
SBlbl. ungetl). (4 9. w.)

(verna.)

1610 . pyrenaǐca.

1611. ciliáris.

\section{II. mit 1-3 blättrig. Stgl.}

Stengel:

1. mit 1 SBlatt; Bl.:

a. lanzettf., ganzranbig, etwas feirbaar.,

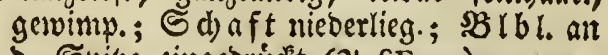
D. Spiae eingeoriutat (4 SE. 9.)

b. lánglide,

1612. nivális.

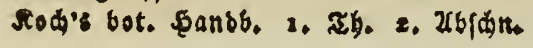


a. etras frumpf, grais, feinthaar., gant: rano., einige gezalint; $\mathfrak{S}_{\text {d) a ft }}$ aufr. (4) 9.) (d)ief, geftielt (4 9. w.)

2. mit 3 Slát ferbt, am SRánbe getwimp.=gezalnt (4 8. w.) androsciča.

\section{III. mit michrblättrig, Stgí.}

\section{Blätter :}

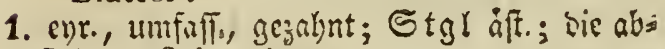
freheno. Schütchen:

a. Feinthaar. $\left(\odot \sigma^{x} \mathrm{NE}, \mathrm{gb}.\right)$

b. unbehaart $(\odot 4 . \mathrm{w}$.)

1616. nemoralis. 1617. (murális.)

2. langettf., gejabunt,

a. grau; bef. Slmftle aufr., feinhanr.; 1618.

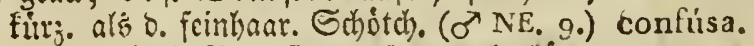

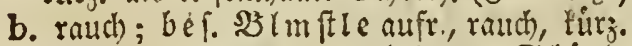
als bie gewunben., unbehaurt: Sdjótds. (ర) NE. 9.)

1619. contórta.

\section{DRACOCEPHĂLÙM. 449. $\left(461^{\circ}\right.$.)}

\section{Blüthen in Aehren;}

A. nuit Nebenblättern.

Nebenblätter?

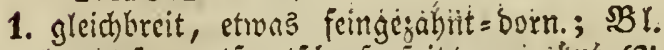
lanjettf., entjernticharfgelpiet $=$ gézalint (4) NAs. bl.)

2. nebit $\delta$. $B$ I. LangettF., ungeth., wehros $(4 \mathrm{bl}$.

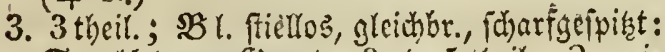
Stglbl. am Grumbe 3 os. 5 theil.: 3 roe gebl. cinf. (4 8. bl.)

1620. peregrinum: 1621. (Ruyschiána.)

1622. austriăcum.

B. olne Nebenblätter.

Blüthen :

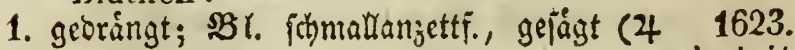
NAm, p.)

rirginiảnum: 
2. entfernt; $3 \mathcal{B}$ l. verk. enr. = lanzettf., oberbalb feingezalnt (4 NAm. p.)

1624.

denticulátum.

\section{Blüthen im Quirl;}

A. mit Nebenblättern:

Nebenblätter:

1. Ianjettförmig,

a. ganjrano.; $\mathfrak{B}$ r. lơngl., ftumpf, gejahnt, gefticlt; Slippe D. SBlo. ellipt., frumpf, ungeth. $(\odot \mathrm{NAs}$, bl. $)$

b. gewimp. =gezahnt; $\stackrel{S}{B}$ I. IanzettF., tiefges

1625.

grandifórum, zalnt, unten punctirt: bie unteriten

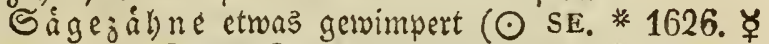
blch. - * Siraut, Saft - ૪̧ ₹arb. griui) Moldavica.

2. lánglict),
a. gewimp.; SBls. geftreift, feinlyar, fints. 1627. als Błltroóhre ( $\odot$ As. bl.; vo $_{0}$ hr. ; gb.) canéscens.

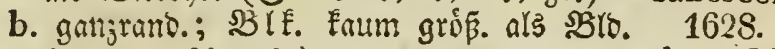
(๑) NAs. bl. - rcb.)

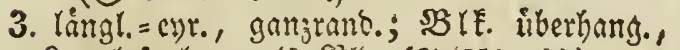
2 mal fo lang als SBlo. (4t NAs. bl.)

4. Ereistuns, gervimp.; $B$ ro. geftreift, unbe=

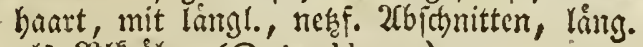

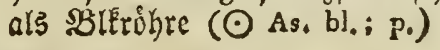

thymiflórum. 1629. nutans.

\section{B. ohne Nebenblätter.}

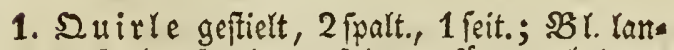
zettf. = Kerzf., langgefpiąt, gefágt, unbehaurt (4 NAs. p., Bl, stinkend)

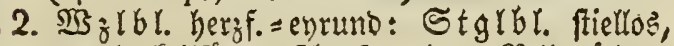

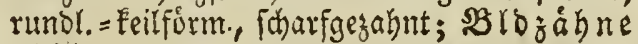
gleich) (4 As. 9. bl. grofs) peltátum. 1631. sibiricum. 1632. altaiénse.

\section{DROSĚrRA. 254.}

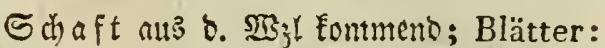

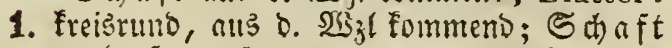
traúvenfo, aufr. (4 47. wo - * Siraut - * 1633. ఫ

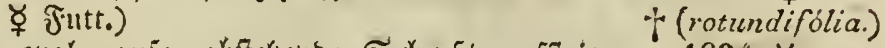

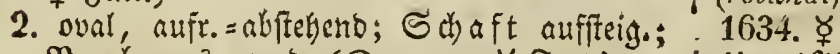

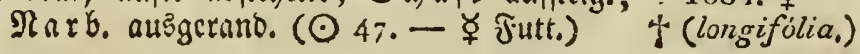




\section{DRYAS. 403.}

SBlủ $t \mathfrak{h}$. einzeln, 8blättr.; Stgl niebergeftrect ;

$\$ \mathcal{B l}$. langgeftielt, långl., ftumpf, gefágt $=g e=$ ferbt, unten reisfilz. (4 9. w.)

1635. octopetăla.

\section{DRYPIS. 242.}

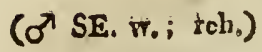

1636:

spinósa.

\section{ECHINOPHOัRA. 179.}

281. bopy. fieberf., mit 3 ppalt., pfricmenf., fteif. Lappen; Șullen ungeth)., Dorn. (4 $\mathrm{SE}_{\text {, }}$ wcb.; rch.)

1637. spinósa.

\section{ECHÍNOPS. 713.}

Blätter:

1. oben feinbaar., unten grau=woll., fieberf.; 1638 . Stgl iff. (4 4. bl.; r.; w.)

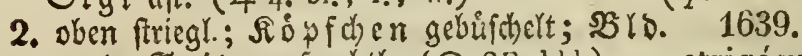

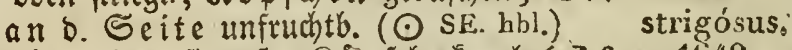

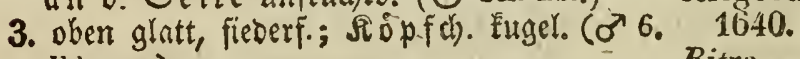
dbl, ; w.)

Ritro.

\section{ECHÍTES. 133.}

Stgl gewunsen; SBlàtt。 oval= langettf., ath (Sirunde pip: Die unterften gleichbreit; $\mathfrak{B}$ lúth). gebujchelt=boldentraubenf. (ち NAm. ugbch.)

1641. diffórais,

\section{ECHัLUM. 98,}

\section{Stengel:}

1. bactrig,

a. aufrecht, 


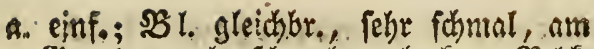

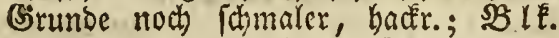
2 mal fo lang als SBld,; Stgef. het=

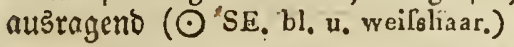

b. rifpenf., punct.; $\mathfrak{B}$ l. Fomallan; ett f.,

1642.

angustịfolium: ftriegl. = baifr.: ioie unt. gerippt; $2 C$ eh $=$ ren verlängert; 38 lit th. etwas ent= fernt; Stgef. noch um bie Scálfte láng. als bie faft gleich). SBlf. (ơ SE. bl., zuletzt $\AA_{\text {.) }}$

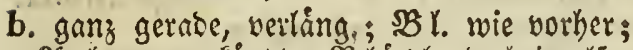
Uchren verkurkt; sBlut th. Dadjuegelf.; St gef. 2 mal jo lang als bie faft gleichf.

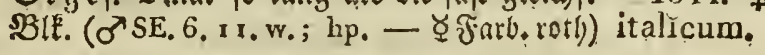

2. beulig = hader.; D. hacér. Blätter:

a. lanzettf.; $\mathfrak{B l}$ le. fo lang ats Brb., u. lång. als bie Stgef. ( $\left.\odot A_{s} \cdot b l.\right)$

1643.

pyrenaĭcum,

b. Flímallamjettf.; Sciteráhren ange=

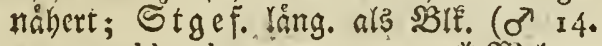
15. 22, bl., dann ros, ; w, — * $\mathfrak{N}_{j} \mathrm{w}_{\mathrm{l}}$ ఛ्(uifr.)

3. raudb, einfach); $\cong$ l. lanzettf., am Ërunde

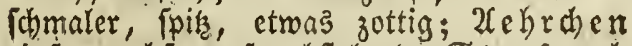
einf., wedh., aufr.abutelicnto; St gef. nodh

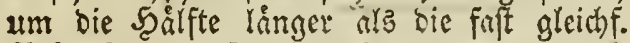
SBIE.; 2 bavon fehr lang (4 SE. Ig. bl., mit v. Strich.)

1647.

lusitanǐcum;

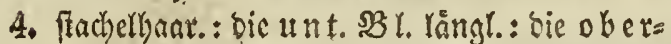
ften lanjettf., am (S)rumbe berzf, umfalf., baar. = hadt.; ; Stgef. fo lang als Bglfrone;

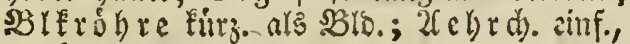
verlinng. $\left(\odot-v_{0}\right)$

16 多8. violacěum.

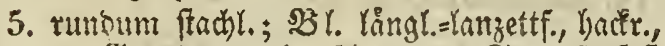
an (S) nunde wenig ithmaler; Stgef. faft

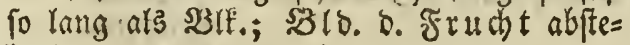

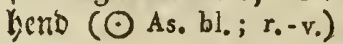

1649. cretı̌cum:

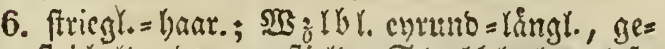
ftribelt=aber., geffelt: Stglbl. Yanjettf., mit langen, weidhen, mflies. Jaat.; S tgef.

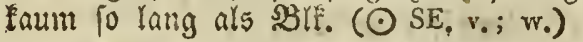

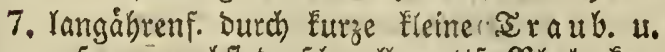
aufr., u. nebjt D. FémallanjettF. SBl. hadét.;

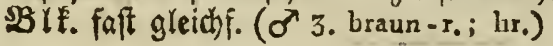

parviflòrum, (vulgáre.) 
8. áftig; Stglbl. enr.; Seitenbluthen 1652. einzeln $\left(\odot A_{s}, b_{b}\right.$, r. gestreift)

orientàle.

\section{ELAEÁGNUS. 81.}

\section{Blätter:}

1. Id)mallanjettf., suf bend. Seit, weisidjupp.;

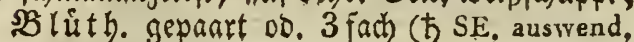
silberweifs, inw, grnch. - gbct.)

2. längl., an bend. Enben pipí, auf benben Seit. weis̄idupp.; SBlủth. meift eingeln, úberhang. (4 NAm. Farbe wie vorher.)

3. enr. =lingl., an bend. Enoen abgerumbet, auf bend. Seit. glanzlos, u., vorz̧ugl. unten, woiffily. (ち As, wie vorher, $\Delta$ )

1653.

angustifolǐa.

1654. argentěus:

1655. orientàlis:

\section{ELATINNE. 323 ,}

\section{Blätter :}

1. entgegengefefit ( $\odot 50$, w, ; ros.)

2. im S2uirl (45, พ.)

1656.

(Hydropüper.) 1657.

(Alsinástrum.)

5. ELLISİA. 13S. $\left(126^{x}\right.$.)

$\left(\odot \mathrm{NAm}, \mathrm{m}_{\text {, }}\right.$ inw, $\left.\mathrm{r}_{\text {, punct. }}\right)$

1658.

Nyctelěa.

\section{ELSHOLZIA. 470 ,}

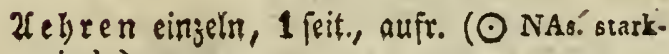
riech.)

1659.

cristàta.

\section{2, EMPĚTRUM. 774. (W. 12.)}

Etgl nieberlieg.; he . 3 meige unbehant;

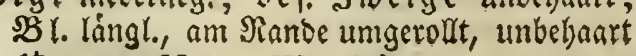
(5 3. 47. M, w。: $\mathrm{W}_{0}, \mathrm{sch}_{0}$ ) 1660. $+($ nigrum. $)$ 


\section{EPHËDRA. 798. (W, 56.)}

Die Sdeioent. (Sirieber 2jahn., ftumpf; Dic geftielten Kätzchen:

1. cinjelit, jerftrẹt ob. entgegengelest, fiur. 1661. als bie Etiele (ち SE. $\triangle$ ) monostachy̆a.

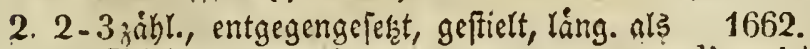
die Stiele ( 5 SE. $\triangle$ ) distachy̆a.

\section{EPIGA'EA. 332.}

Xefte u. Bittitle zott.; $5 B$ r. herzf. = enruno, ganjrano., unten an oer Mittelrippe zotț.; $\mathfrak{B} \backslash \mathfrak{t}$. waljenf. ( $ち$ NAm. w.)

1663.

repens.

\section{EPILOBĬUM. 311.}

\section{Staubgefäfse aufrecht.}

BBibl. 2 palt.; Blätter:

1. Einige entgegengef.: $\mathfrak{A}$ noere wect)

a. faft umfarf., evr.= lanjettf., gefågt, faft unbehaart, nit raudy. 2(bern; Stgl (ef)r 1664. àft., rall (4 49. p.; ros.) hirsútum.

b. finf ftiellos, feingezalynt,

a. lanzettf., auf bend. Seiten feinbaar.; 16 ti5. Stgl einf., zott. (4 3.45.) pubéscens:

b. evr., unbehaart; Stgl ganz cinfach, ftumpfecf.; $\mathfrak{B}$ lit th. falt friellos, in ben Blttwink.; Skap 4 peit., an beys. Enoen bümer ( $\sigma^{7}$ 8. rch,; wch, - 1666. ఫ

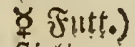

c. geftielt, alpéstre.

a. eyr, = Yanjettf., gefăgt, unbehaart (4 1667. 4. 49.) rosěum.

b. lanjettf, feingejagt, entgegengel.: sie obern wcehlo, unbehaart; Stgl fticls 1668. runo, feinhaar. (4 NAm. Bl, rothader.) coloritum.

2. nur bie unterften entgegengefegt: Alle:

a. Lanzettf., feingejalynt; Stgl 4 feit. (4 1669. ఫ r. 45. 48. brch. $-\Varangle \Im \Im^{\prime} u t t$.) (tetragonulm) 
b. långlo, gezahnt, ftumpf; Stgl faft fticls 1670 . rumb, aufr. (4)

3. Urre entgegengerest,

glabéllum. 1671. ఫ్

a. enr., gezahnt (4 5.rch.; wch. - ఫ̧ Futt.) (montánum.)

b, lanzetrf., ganzrano.; RB Ibl. ausgerano.; 1672. Stgl aufr. (4 42. rcb. - $\Varangle$ Jutt.)

c. evrun =lanjettf., ganzrano.; S friellos; Stgl fried. (4 SE. 9. rch.) alpínum.

\section{Staubgef. abrvärtsgeneigt.}

\section{Blätter:}

1, zerftreut,

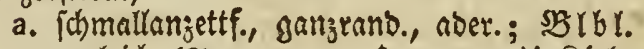
ungleich) (4. 3. 48. p.; A.; w. - ఫ Silid, 1674. ఫ̧ Futt., Fabr. WBeber, Farbe robtylich=braun.) (angustifolum.)

b. gleidbr., unbeutl. feingejabnt, aberlos; 1675. SBIb I. gleich, ganjrand. (4 9. p.) (angustissimum.)

2. einige wechl.: andere entgegenger., lan= zettf.=enr., faft ganjtano., feinhaar., aber=

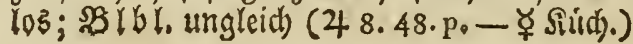
1676. ఫ (latifolium.)

\section{EPIMEDĬUM. 72.}

(4 SE, 9, ur.)

1677.

alpínum:

\section{EPIPACTIS. 720.}

\section{Nectarlippe mit ungeth. Saum.}

\section{Blätter :}

1. enr., umfall; D. unt. Rebenbl. läng. als

o. SBluthe; SBlith. hang.: Ripp. ganz= rand., langgeipiat, Fur. als bie siblatt.; Fru cht $t$ n. Feinthaar. (4 4. P.; G.)

2. Ianjettformig,

\section{8. \\ (latifolia.)}

a. umfaff. ; Rebenbl. Eirr. als D. NGlitthe; Şluth. bang.: Lippe geferbt, ftumpf, io lang als bie SBlbl.; Fru cht tre feins haar. (4 3. 47. uw. u. grn. u. p.)

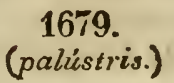




\section{Epipactis. - Erica.}

b. ftielloz, ztwifhen b. Snoten filtr.; Re: benblát t. faft fo lang als o. sglithen; Blitth. ibergebog.: Eippe fpis, fein= geferbt, fo lang als bie sblbl.; $\mathfrak{F}$ rud ts Enot. feingaar. (4 5. 8.)

c. fäf 2 zeil., langgefpist; $\mathfrak{R e b e n b l}$. fehr Elein, pfriemenf.; $\mathfrak{B l u}$ th. aufr.: Lippe ftumpf, balb folang alb D. BBlbl.; $\mathfrak{F} r u$ d t $t=$ Enot. unbehaart (4 4. 8. w.)

d. Rebenbl. láng. als Frudten.; $\mathfrak{B}$ lith. alfr.: R i p pe piph, burd erhabene Stride

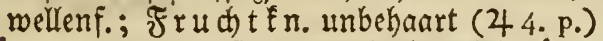

3. långl. = lanzettf., ftiellos; $\mathfrak{R}$ ebenbl. ling. als sBlúthe; BI I furr. als bie Blbl.; Frud tEn. umbehaart (4 4, 8. w.)

1680.

microphyilla.

1681.

(ensifulia.)

1682.

(rubra.)

\section{Nectarlippe mit gespalt, Saum.}

\section{Stengel:}

1. Glattlos, mit Sakibe: Rippe verf. herzf., nodh einmal to lang ats bie fumpf. SBlbl. (4 8.5 r. r. u. gbch.)

2. 2 blättrig; bie entgegenger. Bl.:

a. eyr.: Rip pe gleid)breit, 2 part., $3 \mathrm{mal}$ fo lang als bie ftumyf. SBlbl. (4 3. 49. * 1685. grn, - * Niraut)

b. Kersf.: Lippe 3 fpart., mit fehr kutrzen Seitenlapp.: MRittellappen jeht lang, 1686. 2 theil., gleichbr. (4 4. 8. 49. grn.; r. -gb.) (cordáta.)

\section{ERICA. 308.}

\section{Staubbeutel:}

1. begrannt; $\$ B \mathfrak{l}$ l. 4 jähl., långl., gewimpert;

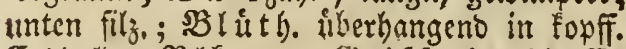

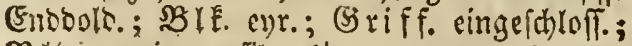
B lb. gewimp., filts. (5 I3. 47. w.; brnch.lila - ఫ Untr.)

2. fanumform.; $\mathfrak{B}$ l. 3 záal., gleidbbr, unbe= 1657. ఫð (Tetrălix.) hanrt; 3 r u th. uhinerbang., an $\delta$. Spizen $\delta$. Zlefte foft e. Enotraub. Kitoend; $B$ Id . Fein= gefägt; $B$ rf. eyr.; (Br riff. faft herborrag.; Raebe fopfi. (b SE. bro; w.)

1684. (Nidus ayis.)

1683. pallens, 
3. benbes nicht, aber nebft (S) riff. beruorrag.;

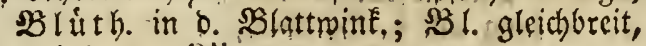
unbehaart; Blk.;

a. glodenf:; $\mathfrak{B} / \mathrm{m} \mathfrak{t} \mathfrak{l}$ fo lang als Blưthe; 1689.

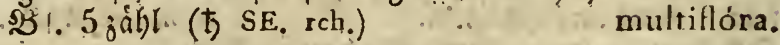

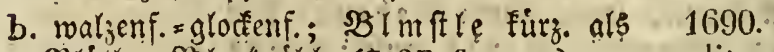

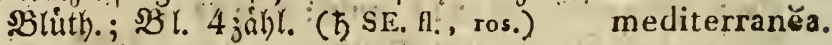

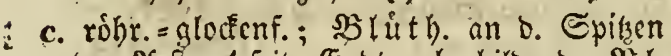
oer 2 efte 1 feit. Enbtraub. biloend; $\Omega^{3} l$. 4 jábl., gleidburçit, unbebanț ( 5 5. 8. 1691. rch.; w.) herbač̆a.

\section{ERIGĔRON, 681.}

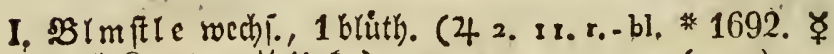

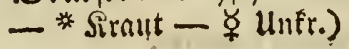

II. Stgl 1 blíth.; $\mathfrak{B}$ Id. baar. (4 9. w.; p.) uniflórunt. III. Blätter:

A. gleichformig,

1. gleid)breit,

a. ganzranh.; EnDblưth. meift cine 1694. zeln; S tgl rifpenf. (4 NAm.) caroliniánum.

- b. 3 weige 1 bluttl.; Stengel ftallo, 1695. (4 SE. gh.)

2. faift gleidbbreit, ganjramb.; Seiten= zweige vie(blitt). ( $\odot$ SE. gb.; Stgl rch.; Bi, wohlriech.)

3. ไanzettf. = gleichbreit,

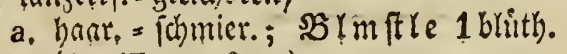
(4 SE. 7. 36. p.)

b. gemimp.; Stgl nebft rifpenform.

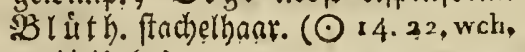
- ૪् $\operatorname{lin}(\mathrm{i} r)$

4. ganz lamzettf., 3 ripp., fdharf, etwas gezabnt, ftiellos; Stgl rifpenf.; $\mathfrak{B l}$ l.

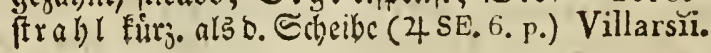

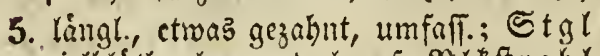

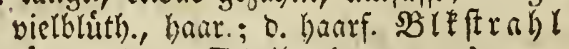
läng. als o. Scheibe (4 Am. p.)

6. ftumpf, unten langhaar.; Stgl meift 2 bluth); 3 MIded e etwas raud) (4 9 . p.; w.) tuberósum.

1696.
grayeŏlens.

1697:

glutinòsum.

1698. ૪

(canadénse.)

1700. purpurěum.

1701. alpinum. 


\section{Erigeron, - Erodium.}

7. Febr lang, fohmal, unbebaart, ftiellos; Stgl febr aft.; Zweige ăhrenf, ; $\mathbb{B}$ los rch upp. lang (4 NAms)

B. ungleidfórmig: Die lanzettf. untern:

1. jerrifl.: $S \operatorname{St} l b l$ gleichbr.; $S$ (úth) in Iraub. ( $\odot$ SAm, rch.)

2. in o. Mitte gezabnt: bie obern gleichbr.: 1704.

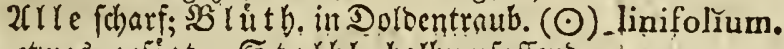

3. etwas gelagt: Stglbl. barbumfallend; Stgl vielblith.; bie baarf. Strabl= 1705. blit th. folang als b. Scheibe (4NAm, p.) philadelphrcum.

14. ERÍNUS, 504.

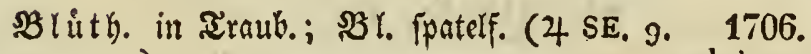
p.; w.) alpinus.

\section{ERODIUM. 541.}

Blumenstiele:

I. 2 blüth.; 3 I. bopp. fieberf., mit gleidbr., 1707.

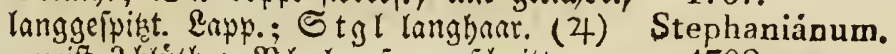
II. meift 3 blitt); g l. berzf., jerichnitt., ges 1708. kerbt, fdarf; Sigl niebergeorildt(4 SE. 36.) maritı̌mum. 1II. vierblitthig; Blätter;

1. herzformig,

a. zeridnitt.; bie oberften 3 theil., mit 1709.

3 theil. = jerid)nitt. Eapp. $(\odot$ As. p.) chium.

b. etwas gefappt, frumpi, gejahnt, un= behaart; Stgl u. B lttitle entrigaar. 1710. $\left(\odot S_{0}, 36\right.$. bl.) malacoídes.

2. 3 jábl., langgeipizzt, jeridunitt. = gezabnt: Mit tcr br, ficoerf., geftielt; Stgl hadr. $(\odot$ SE. bl.)

3. geficbert; Stengel;

a. altzgebreitct; Fiebern fieberf., zera fdonitt = gejahnt; gem. Blttft l jwi= ichen benierben gejalint (4 SE. 9. p.) alpínum.

b. nufferig.; Fiebern fieberf., gezahnt; gem. SBlttifiel wie vorber; SBlbl. 'langl., ftumtpf, 2 zólibl., ausgerand. $(\odot$ SE, hbl.)

1713. ciconŭum: 
c. niebergeftrect, ftachelhaar; ; fieb. fieberf.,

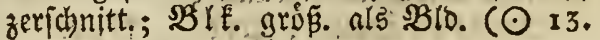
29, r. u. grn. - ఫ üutr.)

d. niebertiegeno; bie zerichuitt. = gezahnten Fiedern:

ค. evr., ftierlus; $\mathfrak{B}$ lE. Eleiner als \$Blo.; (S) $x$ annen unbebaart; Stgl etwas baar. ( $\sigma^{7}$ 13. 19.)

b. längl., etrás geftielt; $\$ B Y$ Y. .l. fo lang als sBlo. ( $\odot$ 8. 34. r.; bl.; Bl. starkriech. - * Jiraut)

e. fehlt; Fiedcrn eyr., fiebcrf; sBIbl. gróß̄. Als Rlo. ( $\odot$ SE. P.)

1714, $\not$

(cicutarium.)
1715 , pimpinellifalĭum.
* 1716.

(moschcitum.)

1717.

románum.

\section{ERVILIL. 575.}

sBlmfte meift 2 bluth. , begrannt, fẹur. als Das $\mathfrak{S l} ; \mathfrak{B l}$ lát $t d$ ). lángl., abgeftuct, un=

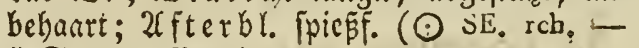
* Saam., Diefl).

* 1718.
† sativa.

\section{ERVUM. 577.}

\section{Blumenstiele:}

1. meift 2 blưth.; 5 îl le r unbehant, 4 faam.;

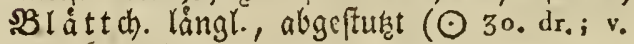

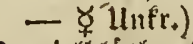

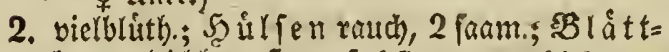

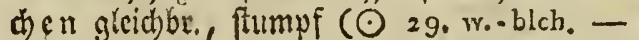
ఫ Untr.)

1719. ఫ্ (tetraspérmum.)

\section{ERYNGIUUM. 178.}

Rippen der Blätter:

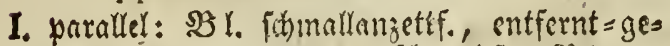

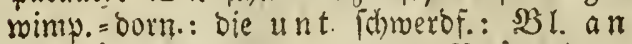

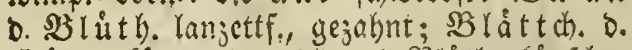
Sơ Epreublát. ungetb.; Stgl faft immer 2 fachgetheitt (4 NAm. hgrne-wch.) II. âftig;

1721. aquatřcum. 
A. Wurzelblätter:

1. ungetheilt,

a. berz $F_{\text {. }}=$ oval, geferbt, flach: bie obern 5 ipalt., gerwimp. = feimborn.; Schullen abftehend, fieberf.; Spreubl. ungeth., ftedhendipis; Stgl faft einf., oben gez fárbt (4 3. bl.; w.)

b. betzfớrmig,

a. Dorn. = gefágt: St glbl. hanbförm., mit olyrf. 2ringang, umgefchlagen; Ri ipe feitr áft, inmer 2 fachgeth.; So opfth. in b. Bblttwine. zulekt ge fárbt; Spreubl. fammtl. 3 fpit:;

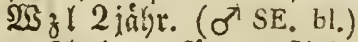

b. zerichuitt.=geiâgt, geffielt: o. obern šjábl., umfall.; Stgl foif cinf., Qben gefärbt; Schlien fieberf., ge= mimp., borft., láng. als bie långl. Sioppthen; Spreubl. ungeth. (4 9. bl.)

c. rundl., gefalt., borm.: bie obern ge= 1722. planum. lappt, hanbr., umfarl., freif; $\mathfrak{b}$ ưllen.

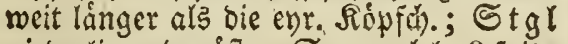
nieberliegeno, åft.; Spreubl. 3 fpis. (4. 19. 36. $\mathrm{wch}_{0}-\mathrm{bl}_{\text {. }}$ )

2. vierlipaltig,

a. meift 3 zábl.: sblåttch. fieberf., mit eyr. Lapp.: Stglbr. mit ohrf. 2in= ballg; Stgl ripenf.; 5 üllen faft unigeth., fámalüanzettf., ftechent, läng. als bie runol. Ropfdten; Spreubl. pfriemenform. (4 13. 28. wch $_{\text {, }}$ hbl, * $2 \mathfrak{z}_{3} \mathrm{l}$, Siratit)

b. 3 theil.; mit 3 ipalt., born. Sappen: Stglbl: hanbf.; Stgl einf.; Shut $=$ Ie n lànjettf., fteciento, aufr., viel lán= ger als bie kegelf., gefárbt. Siópfd)en (4 SE. wch. . übelriech.)

1725:

maritĭmum.

4724.

alpinum.

* 1726.

(campéstre.)

1727.

Bourgátí.

B. S3 1. Dopp. fieterf., etran fraus, mit lan= zettf., born. Lapp.; Stgl aufr., bolsen= traubenf., oben gefårbt; 5 ủ rren lanjettf., fieberf., láng. als bie enr. Riopfchen; die ă menf. (4 8. *.)

1728.

amethystünus. 


\section{ERYSIMMUM. 534.}

\section{Blätter gleichförmig.}

Blätter:

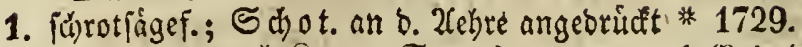

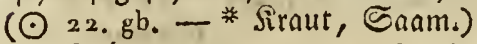

2. berzf. $\left(\sigma^{7} 1,26,29, w_{1}\right.$, Bl, wie Knoblauch riech. - * Sirnit)

3. ranjettformig,

a. gamjant.; ङchot. etwas abjethend $(\odot) 1731$. $28 . \mathrm{gb}$; grn.- w.)

b. etwas gezahnt; sBrbr. ranjettf. = verf́.

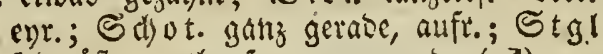
febr aft., rutbenf., gant gerabe ( $\left.\sigma^{7}\right)$

c. entferntgejabnt; S Shoten aufr. $\left(\sigma^{7} 24\right.$. 28. hgb.)

d. ausgefwweift=gezahnt; $\mathfrak{x}$ raub. ben $\mathfrak{B l}$. entgegenger.; ङ th o t. faft friellos, hori= jontalitekeno $(\odot 28$.

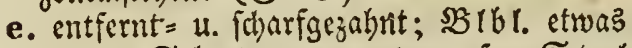

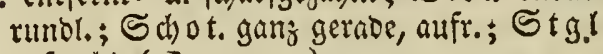
gefurcht $\left(\sigma^{2} 29.3 x\right.$.)

f. haar.; Suljot. an b. Spitze 2 horn. $(\odot$ As. gb.)

4. gleidbreit,

a. gans gerabe, unbehaart; Schot. aufr.= etwas abfteheno; Stgl einfo, an o. Spite $\dot{a} \hat{\mathfrak{f}} \overline{.} .\left(\odot \sigma^{\top} \mathrm{SE} .3 . \times 7 . \mathrm{gb}.\right)$

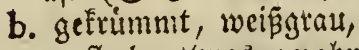

a. flact), etwas gezahnt, fharf; Sdy ot. aufr., abftegerto (on 1.18.$)$

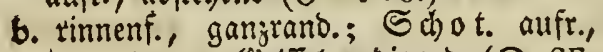
in e. langen Siriffel endigend $(\odot \mathrm{SE}$. tg. gb.)

\section{Blätter ungleichförmig.}

Dic ut t. $\mathfrak{B}$ r. renerf.: bie obern:

1. verE. evr., gezahnt: Nittellapp. o. unt. 1740 . rumol. (4 49. gb.)

2. fieberfớrmo, mit gleidybr. =längl, gangr ranb. Iapp. ( $\sigma^{\top}$ SE. hgb.)

1735.

1736.

bicòrne. (officinále.)

* 1730.

(Alliaria.)

(cheiranthoides.)

1732.

virgatum.

1733.

(hieracifolüum.)

1734.

(repándum.) juncěum.

1738.

diffísum.

1739.

angustifolium.
(Barbaréa.)

1741.

praecox. 


\section{Erythraea. - Eupatorium.}

\section{ERYTHRÁEA, 114.}

Blättêr:

1. fticllos, enruno langettf.; Stgl frautart.;

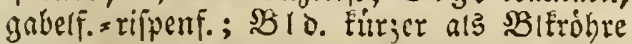

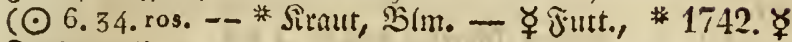
Farb. gelb) (Cenlaurǔum.)

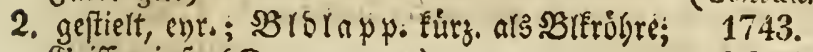
Sriff, einf. (๑.3. 49. ros.) pulchélla.

6. ERYTHRONIUUM. 255.

(4 SE. w.; p.; gb.; A.)

1744.

Dens canis.

\section{EUPATORIUUM. 647. (649. $\left.{ }^{\circ}\right)$.}

\section{Bldecke 3-5blüthig.}

Blätter:

1. umfaffend, abgejonbert, ebr.=länzettf., am (3)rumbe runblid), gefágt, ganz unbebaart; Stgl faft unbebaat ( 4 NAm. p.; w.) sessilifolıum:

2. ftiellos, abgejondert, eyr., fharf, aber.: bie unt. bopp. gerágt: Die obern etrons ges lägt; Stgl rifpenf., feinbaar.; 3 weige gleich hoch (4 NAm. w.)

3. Faft ftiellos, lanzettf., 3 ripp., an beyden Enden fhmaler, feinháar.: Die unt. in ber Mitte gejägt (4 NAm. w.; p.)

4. geftielt,
a. etwas· faharf, 3 od. 4 zåkl., enr., an beyo. (Enoen ichmaler, gefágt (4 NAm. p.)
b. unten feimbaar., 3 jäbl. od. einfach, un.
gleichgelágt; S tgr glatt (4 As. p.) syriăcum.
1748.
trifoliàtum.
1749.

c. unbehaart,

1746.

pubéscens.
1747.

altissimum.

a. evr., langgeipiçt, gezabnt, 3 fachges 1750. rippt (4 NAm. w.) ceanothiforum.

b. 3 theil., mit ranjettf., gejågt. Iappen u. lángern Mittellapp. (4 44, rch.; $w_{0}$

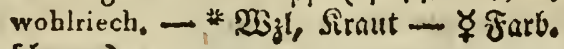
(ditoars)

1751. (cannabŭnum.) 


\section{Bldecke 6-9 blüthig.}

Blätter:

1752.

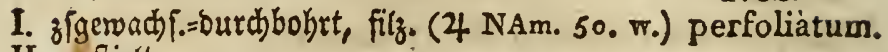

II. geftielt,

1. cyr., fpín,

a. auf beyb. Seiten icharf, gefägt, 4 bis 5 zábl.; St gl bicht, ftielruno (4 NAm. P., Sigl rothgefleckt)

b. unbehaurt,

ค. ftumpfgciagt; - Stgl oben rifpenf.; Brîtben in Doloentraub.; $\mathfrak{B}$ ro. einf. (4 NAm. w.)

b. ungleich = u. grobgefingt; Dorben= tr a ub. vielblüth., ausgebreit.; $\Omega B(b$. faif einf. (4 NAm.w.)

2. berzf. = cyr., etwas ftumpf, 3 fachgerippt, ftumpgelagt; Brath. in Doloentrauben (4 NAm. bl.)

3. eyr.=lanzettformig; Stengel:

a. róbr:; $B$ 1. 4-5 zábr., gerågt, tunzl.= 1757. aber., etwas iharf (4 NAm. wch.) purpurěum.

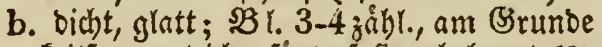
Eeilf., ungleichgeiägt, faft unbehaart (4 NAm, rch.)

4. Gerjformig,

\section{3. \\ punctàtum.}

1754.

aromaticum.

1755.

ageratoídes.

1756.

coelestínum.

a. zerfónitten=gelägt; mit $5 n \delta r i f p e ;$ SBro. vielbritt)ig, pfriemenform., faft ftectieno; Syflanje badf. (4 SAm.) urticaefolium.

b. gejågt, 3 fadhficlirips., unten nebft 1760 .
o. Stgl feinbaar. (4 As.) macrophýllum.

\section{EUPHORBĬA. $350 .\left(35^{ \pm} .805^{\star}\right.$.)

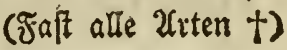

1. Dolde 3 spaltig.

Die bef. Dorben gabelf; bes. Huillen:

1. enr.; $\mathfrak{B l}$. ganzrand., vert. egrund, geftielt 1761. $\Varangle$ (๑ 29: gbch. - ఫ Unffr.)

2. fait herzf., fhatfosefpizt; $\mathfrak{B l}$ r. ranjettf., ets 1762. was ftumpf $(\odot)$

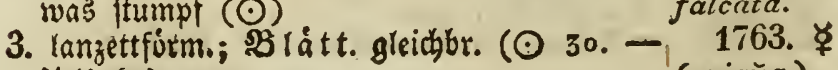
ఫ్ lluft.) 
11. Dolden 4spaltig。

Die bef. Dorben gaberi.; Blätter:

1. entgegengejelzt, ganjranb. (O' 15. gra.-gb, 1764.

2. wechf., gleichbr. = Feilf., ausgeranto, fojarfs gefpiet; b ef. Ş

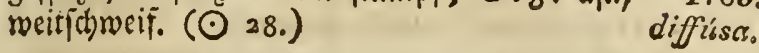

\section{Dolden 5 spaltig.}

Die bes. Dolden:

I. 2 paltig; Blätter:

1. JanzettF; Die ronz. Skapseln:

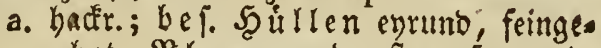
jabnt; $\mathfrak{B}$ r. ganzranb., ftumpf, an b. 1766 : Spire eingebrifdt, unten langhar. (4) epithymoides.

b. baar.; ber. J̦íllen faft eyr., fein= gezahnt; $B$ l. ftumpf, an ber Epite 1767: feingegahnt; SEap f. haar. (4 x. r.) (dulcis.)

c. unbehaart; bes. Hüllen:

a. eyt., ganzranto; bie ganzrans. Bl.:

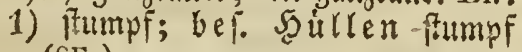

2) (SE.) 3 IEf 5. 9.)

b. rautenf. = cyre, feingegahnt; sz rått. ftumpf, feingegalunt; Stgl gefurdyt 1770. (4)

2. fpatelf. = gleidrbr., ausgeramo., averlos;

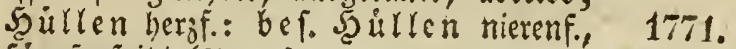
finarfgefpitit (4 21.)

3. gleichbr., aufwarts bathziegelf.; be fond. 1772.

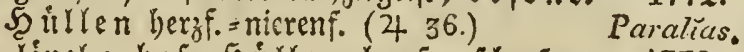

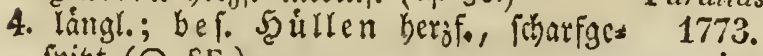
piett $(\odot S E$.$) provinciális.$

5. Keilf., gejägt, unbehaart; S SF a l. glatt; bef. Şullen verk, eyr.; bef. Doldo d) en gakelf. $(\odot 29$, - *2inibebrau, unfr.) (helioscopia.

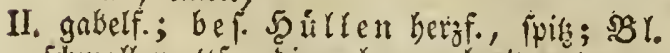
fdimallanjettf.: obie osern breiter $(\odot$ x. 19.30. - ૪ $\left.14 \ln ^{2} i_{0}\right)$

III. 3 Falt. , tie bes. Doldchen:

1. 2 fpalt,; bie Yamijett. BI.:

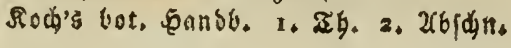

1775. 후

segetális. 
a. etwas haar., an ber Spite feingefägt; 1776. \$B Ibl. ungeth.; Ş îlleneyr. (4 NAs.) pilósa.

b. langhaarig, feingelagt; be . 5̧ullen 1777.

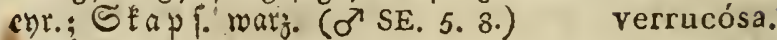

2. gabelformig;

a. Sullleñ am Rudén baar.; 23 l. gejägt; 1778.

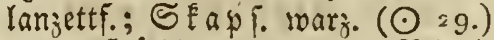
(platypliyllos.)

b. bef: Ş üllen lanjettf.; $B$ l. lanjettf.; feingezahnt, feinbar.; Skap fel warz.l unbehaart $(\odot)$

1779. literáta.

\section{Dolden mehrspaltig.}

Die bes. Dolden:

1. 2 fpaltig; bes. Hüllen:

a. faft bergf.; SBlbl. faft 2 horn.; 3̧weige unfruet)tbat; SBl. gleichf. (4 14.29. gbch. - Siraut - ఫ unfr.)

b. fait eyr.; gem. Dolde meif Sipalt.; SB I. pateli., abftebeno; fleifch., ftechenos ipici, an Rande jouarf (4 SE. sb.)

c. herzf. = runol.; ganjtano.; $\mathfrak{B l}$ l. lanzettf., fd)arfgeipiat, faft leberart. (4)

2. gabelf.; bes, Hüllen:

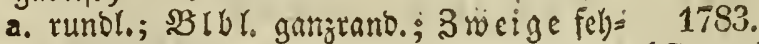
Ien (4 38.)

b. faft herzf.; Bweige unfrudtb.; SBlatt. borftenf.: Stglbl. lanjettf. (4 6. 14. * 1794 . gb. - * * Rimbe D. $\mathfrak{W}_{j} \mathrm{I}_{\text {. }}$ )

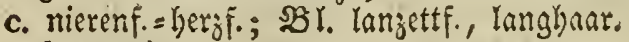
(4 SE.)

d. oval; Nolbe 6 ppalt.; 28 l. gamirand.; 3roige feblen; SEapf. warg. (4 9.) hiberna.

e. Durd)bohit,

* 17S0.

(Esüla.)

1781.

myrsivites.

1782.

niccuénsis.

a. Freisuno; $B$ I. fumpf (4 5. s. gb.) (cimygdaloides.)

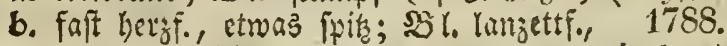
ganjramo. ( 5 ) (sylwatica.)

3. meift 3 palt.: bef. Solocileti 2 palt.; bei. Shitlen enr.; \$31. Tanjett.; 3.veige * 1759. unfruchtb. (4 47. - * $\mathfrak{J}_{3} \mathrm{l}$, Siraut, Mimie) (palústris.)

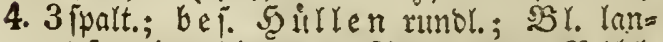

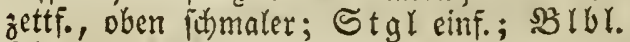
2 borm.; كEap l. unbehart (4 SE. 5. x.) pallida. 


\title{
Euphrasia. - Evonymus,
}

\section{EUPHRASIA. 484.}

Blätter :

1. cyrunt,

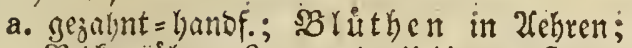

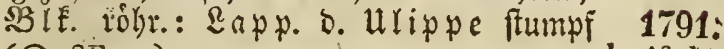
$\left(\odot \mathrm{SE}, \mathrm{r}_{\text {.) }}\right.$ latifolía:

b. ftumpfigezant; Eapp. b. Uriphe ber

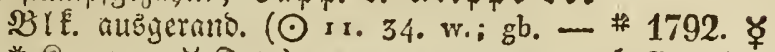
* Sirnut - ఫ futt.) (officinális.)

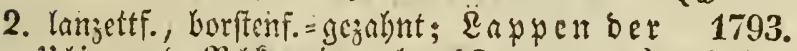
Ulip pe o, $3 \mathcal{B} \mathfrak{H}$, wie vorber $(\odot$ 9. w.; gb.) salisburgénsis.

3. idjuallanjettf.,

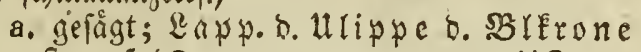

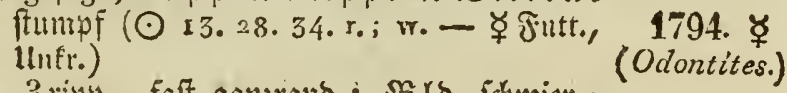

b. 3 ripp., faft ganzrant.; SBrb. fohmier.; Eeitenlapp. b. Ulippe s. SBlerone ausigcranto. ( $\odot \mathrm{SE}_{0}$ g. $)$

4. Keilf. = gleitbbr., 3 ipits.; $\{$ app. b. ulippe

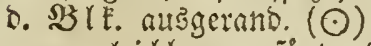

5. ganj glcifbur., gejägt: bic obern ganz= ranb.; Seitenlapy. o. Urippe D. $B$ BF.

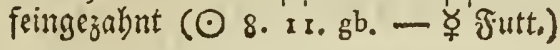

1795: viscósa, 1796. tricuspidáta. 1797. ఫ̛ (lutěa.)

\section{EVONY̌MUS. 163. $\left(70^{2}\right)$}

I. Meift mit 4 Staubgefápen; Zweige: 1. warz.; $\mathfrak{B} l \mathrm{~m}$ ftr $r$ faocnf., ftielruno, meit

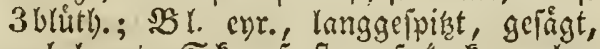
unbehant; SEapf. ftumpf 4 ede, unbehaart ( $\not$ unrein hr.)

2. glatt; dic jigebruddt., frobenf. Blmstiele: a. 3 blúth.: $s B$ l. långl. = lanzettf., unbes baart; SEapf. Ftharf 4 ed?., unbeluart

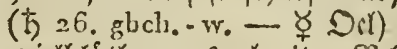

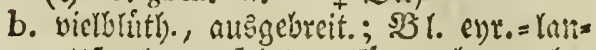
zettê., langgeipint, gefrigt, oben untues baart, unten int jungern Bufanoe fein= baar., hernath fatarf; ERapf. ftumpf= 4 eft., unbebact (ち NAm。 dr.)

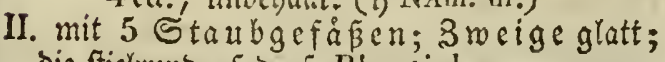

1798.

verrucósa.

\author{
1799. \\ + (europá ea.) \\ 1800. \\ atropurpurěa.
} bie ftieYrumb ${ }_{0}$ faoenf. Blmstiele: 
1. meift 3 blüth.; $2 B$ l. längl., langgeipiat, ges fảgt, unbehant, febr furgeftielt; Ska j. 1801. runol. $=5$ cké., warj. (

2. 5 u. mehrblith); $\$ B$ l. enr. = långl., lang= geipigt, gejägt, unbehart; S 1802. unbehaart, mit geflugelt. Janten (ち gbch.-w.) latifolia.

\section{EXÁCUM. 60.}

3If. 4 palpalt, mit abjtebend. Saum; Stgl

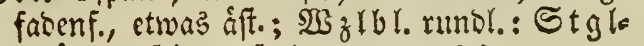
blát t. pfriemenf. (๑.3.49. gb.)

1803.

(filiforme.)

\section{FAGUS. 757. (IV. 33.)}

Die fhiefenr., am Rande gemimp. Bl.:

1. unbehart, fpic, unoeutl. gejabnt (万 4. - * 1804. ఫุ * Riulfe - ૪ Dir, Gierb.)

2. unten feinbar., langgefpiest, gezabnt ( NAn.)

(sylvalica.) 1805.

ferruginěa.

\section{FEDĬA. 36. (30 4$)$}

\section{Mit 2 Staubgefüfsen.}

B lủ th. rachenf.; $\mathfrak{B l}$ Lerippt, etwas gezahnt; bie unt. eyr, geftielt: bie obern lángl, 1806. ftiellos (广 SE. $\mathrm{T}_{0} ;$ w.)

Cornucoprae.

\section{II. mit 3 Staubgefäfsen.}

\section{Blätter:}

I. gleichformig,

\section{Lanjettformig,}

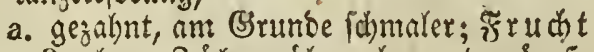
3 zahtr.: 2 ábne úbergebog.: ber åu. grive. ( $\odot$ SE. wch.)

1807. echináta. 1808.

b. Frucht aufgebraf., fugel. $\left(\odot A_{s}, w_{0}\right)$ vesicarĭa. 
2. fleberf., mit lanzettf., fpit. Rapp.: Eno. bláttchen felyr groṕ; Śtgl glatt (4 1809. NAs. gb.)

3. ganzrano., ftumpf; Stgl:

rupèstris.

a. glatt; Fruch t undentl. 3 jabn. $(\odot 30$. w. - rch. - ఫ Silith.)

b. 倘arf; Frutht 3 zabn., wowon 2 feldr fury fino; die obern $\mathfrak{B l}$. am Sirunbe

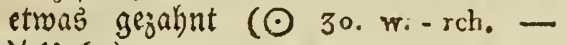
ఫ llner.)

II. ungleidhformig,

1810.

(oliztorïa.)

1811. ్ㅜ

(dentàta.)

1. ranzettfo, ftumpi: die obern ant (3)runbe fieberf. =gezabnt; Frucht:

a. mit 6 geraden 3̇ígnen ( $Q S E$. wch.) coronáta.

b. mit 12 bafeñ. Băhnen ( $\odot$ SE.) discoidéa.

2. $\mathfrak{W B}_{3} \mathfrak{l b l}$. fpatelf.: Stglbl. fieserf., et= was gezahnt, nebfi Stengel feinbaar.;

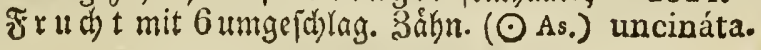

\section{FERŬLA. 221:}

SBr. mehr als bopp. zfgeiest; Stengel:

1. ftielrumb, unbehaart; $s B l a t$ t. ipiegelglatt; Hüllen :

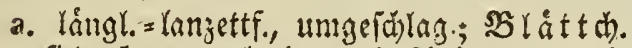
fieberf., aușebreit., mit 2 tubang u. mit gleichbreiten, fted)enofpits., etwas geránd. Eapp.; Sertiefungen flaci) $(436 . \mathrm{gb})$

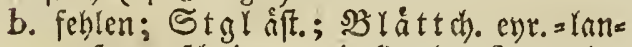
jettf., zeridgnitt., mit 3 zakur. Enpp. (o' SE, gb.)

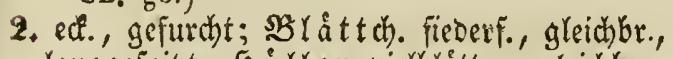

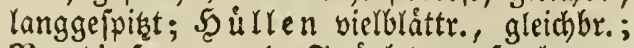
Sertiefungen 8 . Fridide gefurcht (4 1817. $S E, g b$.

1815:

Ferulágo:

1816.

tingitàna: nadiflóra.

23. FICUS. 808 . (40.)

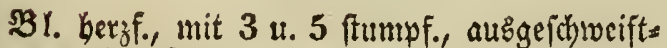
gezahnt. Eapw., oben fibarf, unten feinlyaar.; 
Frud)tbod. birmenf., unbehaart (ち SE. $\triangle$ * 181S. $\Varangle$

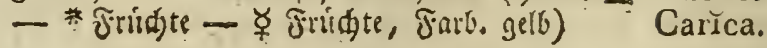

19. FILÁGO. 703.

SBluth. ftiellos; meift ohne Etgl; \$3l. an b. 1819. ग̊lúthe gróper ( $\odot$ SE.)

\section{FONTENESİ. 30.}

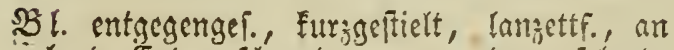
Eeno. Ensen idmaler, ganzratto., auf beys. Seit. unbehaart; Soweige entgegengefert; nit gepaat., 1 blith. SBlmiflat in S. Sbltt= minf., or. Eurg. (Endtraub. ( 5 As. w. $\Delta$ ) phillyraeoídes.

\section{FOPSKOLLั̆ 725, (W. 45.)}

Pflanze:

1. baar. = badr.; $\mathfrak{B} 1$. ellipt., wehrlos; $\mathfrak{Z} I \mathbb{l}=1821$.

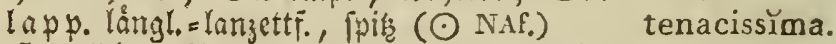

2. frieglicht; $\mathfrak{B} \mathfrak{l}$. lanjettf., mit corn. $=$ bor $=$

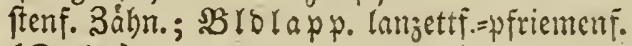
(O As.)

1822. angustifolia.

\section{FOTHERGILLA. 423.}

(

1823. alnifolia:

\section{FRAGARǏA. 404.}

\section{Bldecke der Frucht:}

1. umgeichlagen; das feine Haar:

a. an ten $\mathfrak{B l m}$ ftlen angebrudt $u$. an $\delta$. BIttftlen febr abitebeno (4 5. 1x. 13.

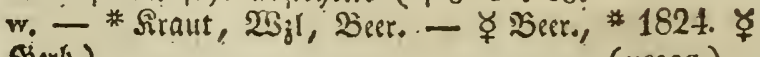
Sierb.) 
b. an beyben febr abfebend (4 Am, ఫ Fridite)

2. abfetheno; das feine Haar:

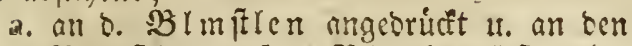
SBlttftlen aufr.; SSl. oben foft unbe= bant (4 NAm. - ఫ Jrid)te)

b. an beyden aufrectit; $\mathfrak{B}$ r. einf.; $\mathfrak{B}$ ts. 1825. ఫ elatìor. to. Frud t gezalint (2t Frucht sehr sauer) monophýlla.

3. aufrecht; das feine Haar:

a. an b. SBImften aufr. $u$, an b. SBItte fiten iehr abjtebeno; $\$ 3$ l. mif beys. Eeit.

b. an beyden:

A. Fehr abitebend: ŞL auf beno. Eeiter langhanr. (4 SAim. - ૪̧ Fridgte)

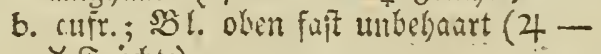
ఫ ซrrid)

1826. ఫॄ virginiána. 1527.

\section{FRANKENIÁ 263.}

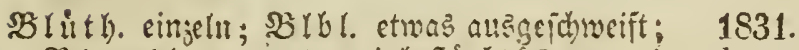

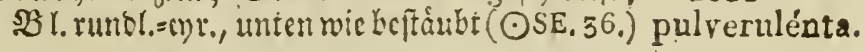

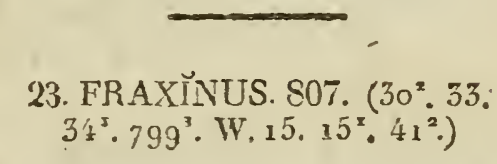

1. Knospen graubestaubt.

S5. ungepanrtgefico., mit 7 oo. 9 lang=

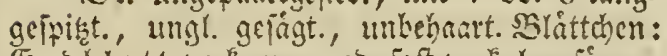
Endblatt ver?. eyr., ob. faft vert. hersform.; Bł rith. volftåndig; Blättchen:

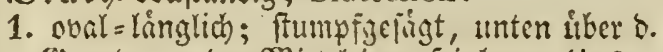

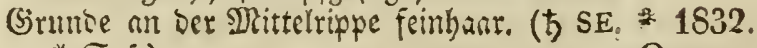

- * Saft)

Ornus.

1833.

2. eทx., fajarjgefägt (ち $\mathrm{NAm}$.) paniculáta.

\section{Knospen braun-gelblich.}

BZl. ungepaartgefieó; Blättchen:

1. unbehanrt, 
1. runol., 7 oo. 9 zåbl., faft ftiellos, ctwas fpis, ungl. gefägt, am (Sirunbe ungleich) Eeilf.; $\mathfrak{B}$ l a tt ft l halbftielrumb, rinnenf.,

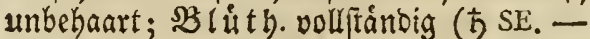
ఫ̧ హarb. gelb)

2. ent., langgeipizt, 9-11-13 zábl., ftiellos, ftachelipis = gejägt, am (Sirunoe Eeilform.;

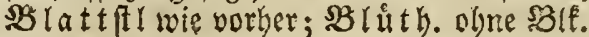
(b As.)

3. eyr. = lạngl., langgelpitgt, $9-11$ (felten 7) zoibl., ungleidgefingt, auf bero. Seiten glan;loz; SBlttitl mebit jung. 3 weig.

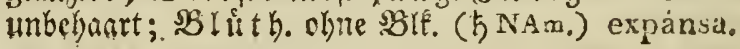

4. lanzettf., langgefpight, 7-9 zål)l., wont D. s)litte an gegen Die Epize ungl. gefägt, oben fpiegeliglatt, unten blaffer; 23 lt tit

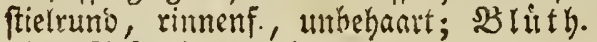
obne SBIE. (

5. långl. = lanzettf., 9-11-13 zåhl., geftielt,

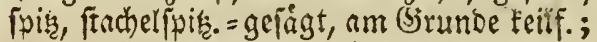
B lattfil wie vorber ( 5 As. $\triangle$ )

6. langettf.=enr., faft fiellos, ipia, 5 bód $)=$ ftens 7 jákl., auperbalb gefagt; $B$ ltt tat wie vorber; frlugelfr. lamjetti = obal (b NAm, )

II. mebr ob. weniger behaart,

A. enrumb, 7 zóhlig, u. unten:

1. Gaar., ftacholfpis., jumeilen nur fduachs geiagt: Enobl, fegr grop u. gerunoet (ち NAm.)

2. etwas feinbaar., fpits, gleidygefagt, firit fcharf: (5nobl. Fetar grop ( 5 NAm.)

3. nebft 8.53 latt ft le n fammetarto, lasig= gepipint, ungl. geiágt, blaß̧grin (ち NAm. - $\gamma$ Farb. geíb)

4. fhwach feinbaarig = seip̈grin uno am

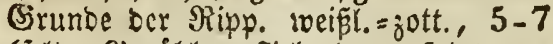
(jelten 9) zåbl., geftielt, langgeipint, ges lágt, glanjloz; $\mathfrak{B}$ lat $t \mathfrak{f} !$ fitelruno, et= sas feinhaar., mit e. feinen Rinne; SB lúth. obne SBIE. (ち NAm.)

5. an ben アrippen feinl)aar., fpis, gefågt, bunfelgrum; SB lattifl faft fticlrumb, rinnenf., unbebaart ( $\hbar$ Am.)

$183 \%$

caroliniána.

1834. ్ rotundifolia.

1835. parvifolia.

1836.

$$
\text { expansa. }
$$
caroliniana.

1838. lentiscifolra. 1839. platycárpa.
1840.

elliptřca,

1841.

ováta.

1842. $\Varangle$ americána:
1843. juglandifolia.

1844.

Richárdi. 
6. an ben Rippen etwas filł. น. fehr blaß,

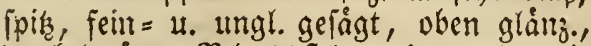
sunfelgrim; solattitle wie vorber ( NAm.)

B. oval u. eyr.=längl., $7-9$ jábl., geftielt, lang= gefpist, faft ganzranto, fpiegelglatt, ounfels grum, unten etwas weisgrim, 11. an (s)rube

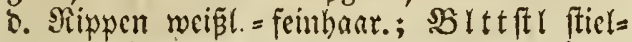

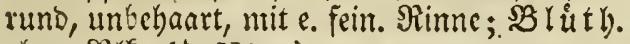
ohne SBlf. († NAm.)

C. oval=eyr. , 7-9 jábl., langgefpibt, geftielt, gelägt, oben unbebaart, fpiegetno, unten, bejonders alt o. Ripp., feingant. ob. ctwas fill.; SBlattit mebft o. jung. 3 weig. ct=

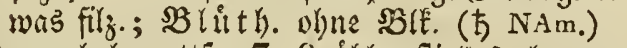

D. oval =lanjettf., 7-9jábl., ftiellos, langgefpist, ftumpfgefägt, oben etwas rungl., fpies gelno, unten an ber Mittclrippe $\mathfrak{u}$. in ben

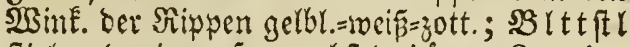
ftie!rund, rimenf. u. nebft o jung. 3 weig. umbebaart; $\mathfrak{B l}$ lath. nadt (方 NAm.)

E. lanzettfórmig, 7 jảhl.,

1. Felat ftark $=\mathfrak{u}$. ungleichgefågt, helfgrum, unten nebft $\delta$. B lattif $\mathfrak{l}$ mit weís., ftei= fen Scanr. befegt (ち NAm.)

2. Febr weitfâft. gefägt, gegen o. (5iruno ganzrano., fehr fpis, oben bunfelgrum, unten blaşgrim, etrwas lyaar. (ち NAm,) lancĕa.:

F. lanjettf $_{0}=$ eyrund,

1. 5-7 zál)l., fpib, focharfgejågt, oben bells grin, unten feinbarig; bef. 3 reeige 4 feit. ( 5 NAm.)

2. 5-7-9zábl., fait ftielloz, fickelf. $=$ juge $=$ ipist, getrummt = fibarficfagt, unten am

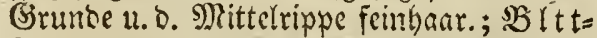
ft i el rimenf., an ben (Belenten feimbar.;

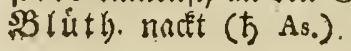

1847.

pubéscens.

virŭdis.

1846.

acumináta.

1848.

sambucifolra.

1849. alba.

1850. ,

1851. quadranguláta.

\section{Knospen schwarz.}

\section{Blätter:}

1. ungepaartgcfiebert; Blättchen :

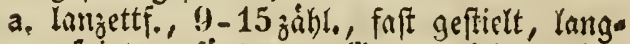
gefpigt, gefägz, am (Ssutmbe łeilf., obeit unbehaart, unten uber ben Sirunde an 
ber Mittelrippe feinhagr, $3 \mathbb{Z}$ l a t $t$ ft $\mathfrak{l}$ halb. frielruno, unten unbehaart, oben feinbaar.,

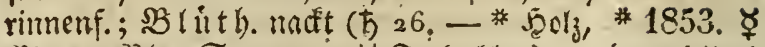

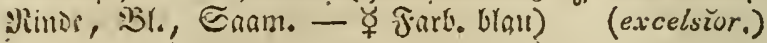

Sciervon giebt es folgenoe \$arietaten:

I. mit aufwartsgebugenen 3 weigen

5. mit hangenden commùnis.

3. mit selkeit, functirter " 4. mit fleinern, am (5runde rundl. feilform. Bláttcier

5. mit frastertr

6. mit margiger Rituse

7. mit giltbunten 31átth.

8. mit weibbunten a

jendǔla.

aurěa.

nana.

crispa.

verrucòsa.

flavo - variegùta:

albo - variegàta.

b. lángl. = eyrr., 9 (ieltcn 11) jơhl., ftiellos, berablaut., langgeipist, gleid) $=$ u. feinge $=$ fógt, umbehant; $\mathbb{B} l+t$ ft $l$ geflugelt (b) nana.

c. verE. enr., $7 \dot{j} \mathfrak{a} b l_{\text {, }}$, faft fticllos, fpis, am obern siande gelagt, etwas butteigrun! (

2. meift cinf., zumeilen 3 żohl., breitebr., fpis, copp. gejägt, oben unbelaat, unten über Dem Sirunce on ben Sippen ctwas jott.; Blttft foit ficlrumb, riment., unbelianrt;

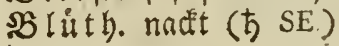

\section{4.}

1855. pallǐda.

\section{FRITILLARIA. 283.}

\section{Blïthen:}

1. in ciner Traube:

a. mit e. $\subseteq$ dh opf, unterbalb nadt; $\mathfrak{B C}$, ganzrans. ( $\left.4 A s_{0} r_{0} ; g b.\right)$

1856. simplicifolĭa.

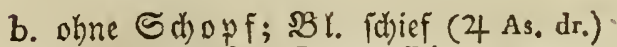

2. einzeln am 1 blith. Stgl; Blätter:

a. im suirl, idhmallanjettf. (4 NAs.)

b. wech jelsweisfteheno,

R. gleichbreit,

1) rinnellf. (4 r, u. w: wie e, Schach. brett)

2) flach, gerippt $\left(4-d r_{0}\right)$

b. långl. = langettf., flach: bie oberften entgegenger. ( 4 - dr.)
1857. t imperiális. 18.58. peršca. 185." verticilláta.
1860. † Meleágris. 1861. nervósa. 1862. latifoľa. 
3. cinige butch e. SBl. getrent; bie unter= 1863. fte ar sBt. entgegengej. (4 SE.) pyrenaǐca:

\section{FUMARIA. 553.}

Der åftige Stengel:

1. aufrecht; Blätter:

a. bopp. 3 jåkl., mit eyr. = herzf., ungeth.; 1864. ipik: Bráttch. (4 2 r. gbch. u. w. u. p.) enneaphýlla.

b. mely als bopp. aigerect, mit 3 theil., fa $=$

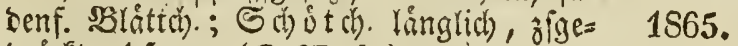
orictet, 1 [ạm. ( $\odot \mathrm{SE}_{9} \mathrm{dr}$ )

2. weitfibmeifig; 32 r. melyr als bops: jigefetat;

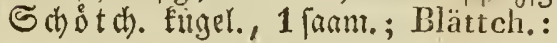

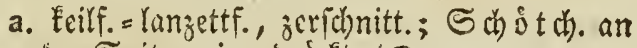

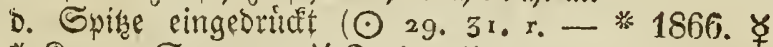

* Siraut, Samm. - ఫ Farb. getb, linfr.) (officinális.)

b. gleidbbr., rinnenf., 3 theil.; ङ

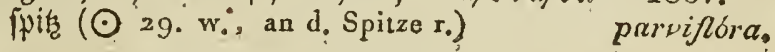

3. Eletterno; Blätter;

a. 3 fad 3 záhl., mit verk. eyr. = feilf., jer=

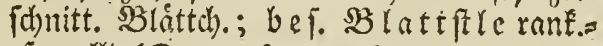
zigerolft $(\odot$ r. wch, an d. Spitze r.)

b. bie unt. Dopp. 3 zólgl.: 3 weigebl. einf.= 3 jólhl. ranfentragend; S b o it th. lángl.,

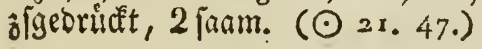

1868. capreoláta.
1869. claviculáta.

\section{GALÁNTHUS. 270.}

(4 3. 5. 48. w. u. gro, od. gb.)

1870.

(nivális.)

3. GALAX. 129.

(4 NAw.) ,

1871. aphýlla. 


\section{GALEGA. 584.}

S21. gefted.: Die unbebaart. Blättch.:)

1. Ianzettórm., fcharfgépict; 2lfterblátt. lanjettfórm., wfeilform.; Sc illfen aufrecht, ganj gerabe (4 15.bl.; w.; r. 2x. - * Sraut * 1872. ఫ - ૪ Jutt.)

(oficinális.)

2. eyr. =langttf.; Xfterbl. eyr.; Sluthen 1873. ůbergebog. ( 4 As. bl.) orientális.

\section{GALEOBDOัLON. 466.}

(4 4. gb.)

1874.

(lutě̌um.)

14. GALEÓPSIS. 463.

Die Glieder żwifhen ben Inot. §. Stgls:

1. gleid); B lúthenquirle fámmtl. entfernt; SBloz ád ne piriement., begrannt, etwas abs ftebeno; Stgl etwas feinbaar. ( $\odot$ 1 2.23. 29. p.i w. u. gb. u. r. —

1875. ४

(Ladŭnum.)

2. oben bider; Bliithenquirle:

a. Fơmmtl. entiernt; $\mathfrak{B}$ lozáhne lanjettf., fcharfgeipist, aufr.; $\Xi_{\mathrm{g}} \mathrm{l}$ mit fein., Driif.

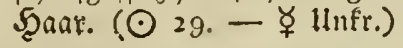

1876. ఫ grandiflora:

b. Die obern einanoer naheftefyens; $31 t$. ftedjeno; Stgl ftadeethaar.; Blk.:

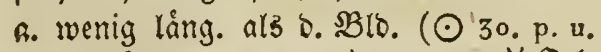

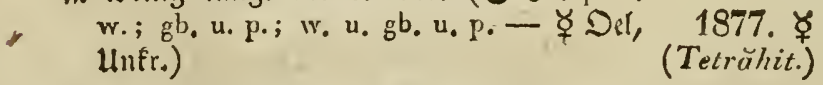

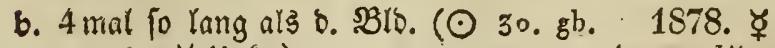
u. p. $-\Varangle$ unfr.)

(cannab̆̌rsa.)

\section{GALINSOGÉA, 667.}

1879.

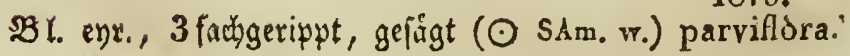




\section{GALIUM. $65 .\left(37^{*}.\right)$}

I. Mit Nebenblättern.

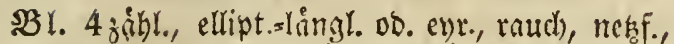
3 ripp.; MY Imfte sift., 2 blattr.: Seiten= blmftle mit 2 långl. Pebenbl.; Stgl uben einf., haar. (4 1.26. gb.)

1880.

Crusiáta.

II. ohne Nebenblätter;

A. Blätter im Quirl.

Frucht:

I. unbehaart, A. glatt ; Blätter:

1. gleithbreit; Blmstiele:

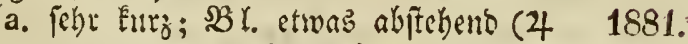
28. up., ausw. blasser) rubrum.

b. gabelf., an bem oberften:igeil bes 1882 . glatt. Etgls blumentrag. (4 2. 8.w.) (glcancum.)

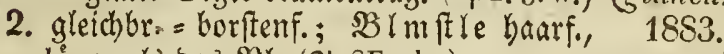
lång. als bas SBI. (4 SE. dp.) purpurĕum.

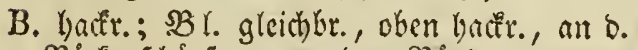

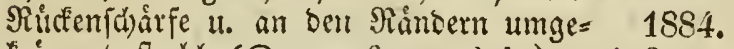
frimmt $=$ frä)r. $(\odot$ 14.26.29. gbch. $)$ inféstum.

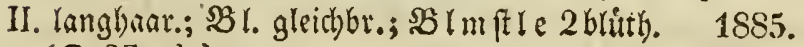
$(\odot$ \&E. gb.) parisiénse.

B. Blätter nicht im Quirl;

1. Frucht unbehaart,

a. slatt;

a. Blätter in gleicher Anzahl.

Blätter:

1. 4 záallig,

1. meilt 4 zábl., gleichbreit, glatt; Stghl fchwach), icharf (4 5. 3. w., vor d. Auf- 1886. ఫ̧ blühen ausw. rch, - $\lcm{乛}$ foutt.)

2. 2urke 4zádrlig, (montárums)

a. eyrr. = lanjettf., 3 ripp., unten fojarf;

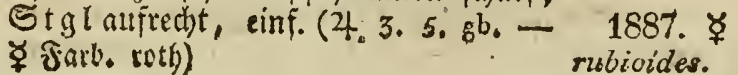


b. vert. cyr., ungl., ftumpf; Stengel meitifbmeifig, obcn áftig (24 47. w. ఫ̧ J'utt.)

18SS. ఫ

(paliustre.)

c. gleichbr.; Stgl nieberlieg.; fharf; 1889 . SBlf. 3 ipalt. (4 42; 45. เv.)

II. 6 zálig, gleichbreit, forargepiat,

1. etwas fcharf; $B$ lmftiele meift immer 3 theil.; Stgl niecergeftredit, weitichweif., 4 [eit., etwas geflugelt, áft., unten ftachel= haar., oben glatt ( 4 5. 8. w.)

trifidum.

1890.

Boccóni.

2. glatt; Stengel 4 feit., weitfuneif. (4) 23: w.)

1891.

austriŭcum.

III. meift $7 j$ áblig,

1. gleid)br., hadr.; St gl rifpenf., oben mit

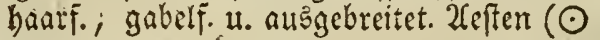
$\mathrm{SE}_{\text {; }}$ 19. 2I. w.)

2. eiltige gleidbrt., anoere lanjettf., etwabs abftebeno, am vortern Sande ftachl.=icharf; Bluth. nit vermengt (Sejhl.; B lmftle in b. SElttrint., 3 bluth.; Frutb t warz.

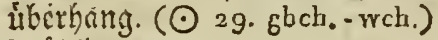

IV. 8 zánlig,

A. meift 8 jår)ịg,

1. gleidbureit,

a. ichari, fitechensipif; Stgl aufiteig., ain Srunde áft.; sie blit thetrog. 3 weige meift inmer 3theil.; $\$ B(b !$. (d)arfgefpift ( 43.8 . w.)

b. fteif, glånz.; Stgl aufr.; $\mathfrak{x} \mathfrak{a} \mathfrak{b}$. furz (4 SE. w., wohlriech.)

1592.

divaricátum.
1893.
saccharàtum.
(n)




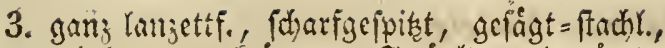
unbegaart, gef́rimmt; Fru dj te, einwarts= gebrog. ( 4 NE. gb.)

1900.

minútum.

4. gleidubr., gefirtht, u. nebft $\subseteq \operatorname{tg} l$ glatt all= zufuhlen, biegiam; bie bluthetrag. Ulefte Eur

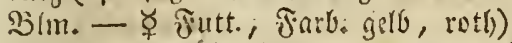

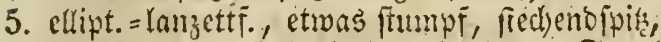
(ierum.) num Siande idjarf, horizontalabfélyeno; Stgh

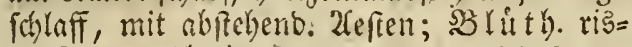
penf., miggébreit. (4 26: w.., wohlriech. - * 1902. ఫ * Bim: - ఛ Futt., Farb. rett)

6. unten rimienform.; Stglaufr, ffictruno; 1903. B İ́. glodenff. (4 8. 2I, wis)

camparsulietum.

\section{b. Bi. in verschịedener Ánzahi.}

\section{Blätter:}

1. 8 u. 6 záal., verk. eyr. $=$ YanzettF., fitharfiges

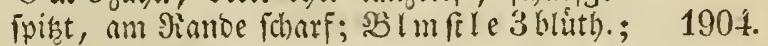
SB IE. begraunt; $\mathbb{S}_{\mathrm{g}} \mathrm{l}+$ jétí, glatt (4 9. wo) tyrolénse.

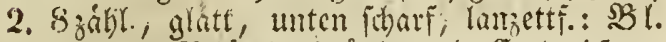
an Den $\mathfrak{B}$ (úth). 2 záll.; mit Endrifpe; SBrmfte haarf.; Stgl fieltumo, glatt 1905. ఫ̧

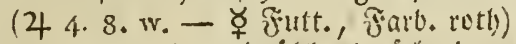

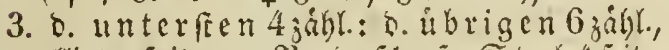

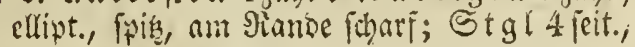
aufr. $(\odot$ As. ugb.)

(syluatĭcum.)

1906.

campéstre:

\section{b. Frucht scharf.}

\section{Blätter:}

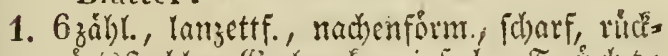
wortsfanchl.; (Selente einfach; Früte glatt $(\odot$ 29. wch. . brnch. $)$

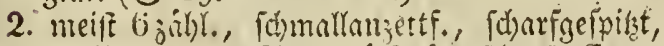

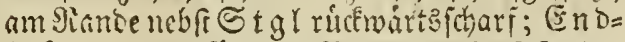

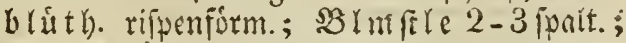
Frucht férn. ( $\odot$ SE, 19. 24. gb. - grnch.) anglicum.

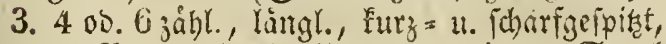

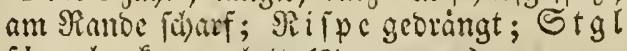
[dhwad), flurz, glatt (4 4.8. w.)

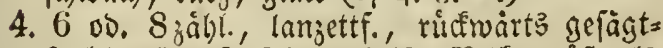

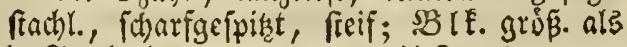

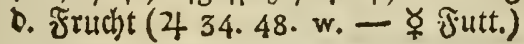

\section{7.}

(spurน̆um.)
1909. (saxatile.) 1910. ఫ (uliginbsums.) 


\section{Galium. - Gaura.}

\section{c. Frucht hackrig.}

Blätter :

1. 4 záhlig,

a. laniettf., 3 ripp., unbebaart, am Ranbe น. Sirden badr.; Stgl aufr. (4 8, 2 I. 49. w. - ఫ ₹arb. ratb) 1911. ఫ్ (boreàlc.)

b. runbl. = enr., 3 riph., bacḱr.sgemimpert; 1912. StgI nicoerlieg. (4 4. 8. w.) rotundifolǔm.

c. verf. enr. =längl., arerlos, etwas jcharf; M. SBlithe 3 ipalt., auf $\delta$. bactiv. Fructsts fnot. Der Z. Bluthe febend (4 3, 26, wch.) hispüdum. .

2. 8 zábl., lamzettf., an bem Rliden u. Sanoe fdharf, rudfwarts ftachl.; Stgl ichlaff; (Se $e=$ lente zott.; 20 lé. Eleiner als o. mit bafenf. Şaaren bedectite Frucht $(\odot$ 22, 26. wch. ఫ. Jutt., Fart. rotl), unfr.)

2. Frucht langhaarig."

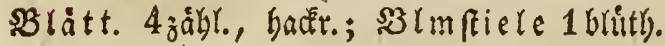
(4 SE. P.)

1915. maritŭmum.

10. GARIDÉLLA. 362.

(๑ SE. wch.)

1916.

Nigellástrum.

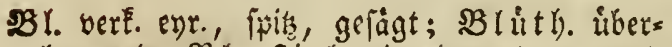
bangent; Sllmfticle cinjeln, oo. gepaart, 1 bluth., in o. Slattwink.; 3roeige unbebaart, nieberlieg. (ち $\mathrm{N} \Lambda \mathrm{m} . \mathrm{w}$.)

1917.

procúmbens.

8. GAURA. 313.

$\$ B$ l. lanzettf., gezahnt; $\$ B l b$ l. verf. cyr., aufo feig. = etras abfelyent; (5) riff. u. Stgef. 1918. abwätsgebog.; Stgl froutart. (O' NAm.s.) biénnis. 


\section{GENISTA. 558. (5422 $)$}

\section{Ohne Dornen.}

\section{Blätter:}

\section{3 joblig,}

1. länglid), unten mit etrwas abfelyenben, fein. Saaren; (Enoblmfte vielblitt).; 3 we ige eff. († SE. w. u. gb., starkriech. * 1919. - $\left.2 \mathrm{j}^{2} \mathrm{j}\right)$

2. verE. eyr., mit fein., angebriečt. Scaar.; (5noblmftle vielblith); 31veige eff. 1920. ( 5 SE. $g b_{r} \Delta$ )

II. einfach,

\section{canariénsis.}

candicans.

A. längl. =ent., u. nebft b. Shitlen hant.; Stgl nufivàrtzgcbog.; 3roeige ftiel= runb, gefteift ( $\hbar \mathrm{SE}_{0}$ gb。)

1921: ováta.

B. oval, meift fumpf; Stgl geftrect, Enot,

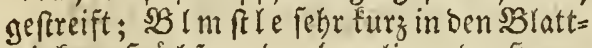
winte; Solifien burch anliegende Sante ctroas fdaut (5 6. 19. gb.)

C. evrumb=lamzettf., haar.; Sू filfen jott.; Stgl geftredt; swe ige 2 fduncis., auf= wárţgebogen, báut., gegliebert (b 13. 19. gb.)

D. ganz Yanzettorumig,

1. unbehant; Zweige:

a. 3tant., auffeig.; Stgl geftrect;

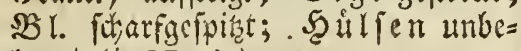
haart (方 SE. gb.)

b. fticlruno, alifrecht,

a. geftreift, unbebant; Stgl auft.; Sollien mie vorker (b rg. dgb. — Farb. gelb, lliffr. - ̧ Futt. Sirant, Bim., Sanm.)

b. Gefurcht, unbebart; Stengel * 1925. ४ (tinctoria.) aufecht, rutbenf.; bie oberften \$ol. fâmallangettf.; Şúlfen mie vorber ( $5 \mathrm{SE}, \mathrm{gb}$.

1924:

trianguláris:
$1922:$
(pilósa.)

1923:

(sagittàlis.)

c. bevoss nidht ( 5 NAs.)

1926. virgáta. 1927. sibirı̌ca.

2. Feibenart. = Feinhanr.; Zweige:

a. Friclruno; Bliithen:

ค. in 1 peit. I犬nab.; 3 weige ge= 1928. frreift (方 $\left.S E_{0}, g b.\right)$

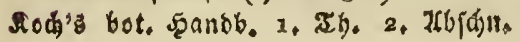

florida.

In 
b. in Endtra b.; 3meige aufr.; SBt. 1929.

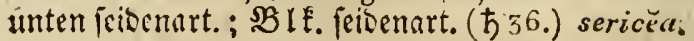

c. an 3 jabl. Blmftien in ס. SBlattwink., faft v. D. Lánge bes skl.; Stgl ge: ftreft; $\mathfrak{B}$ l. ipits, am Panbe u. unten

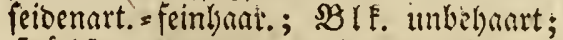
SS úlfen etraa zott. ( $5 \mathrm{SE}$. gb.)

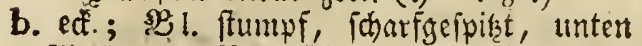
feibenart.; $\mathfrak{B}$ Imftiele in $\delta$. sBlttwint.,

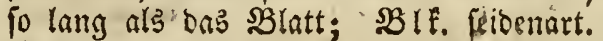
(ち SE.)

1930. procimbens:
1931. decúmbiens.

\section{1. mit Dornen.}

\section{Dornen :}

1. meif einf.: Die blumentiag. Sineige obne Dorn.; \$Sl. lanjettf., fdarfocipint;

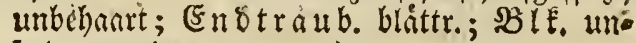
bebaart ( $5 \times 3.49 . g b$.

2. oulammengelest,

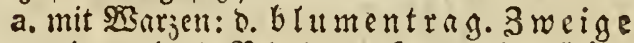
wie vorher; $\Omega$ l. laizettf., rauch; Shil= fen baar.; Enotranben obne selatt.; Sdiffd). D. $2 B(\mathfrak{k}$. frinbaar. (ち 4. 6 . 21. gb.)

b. obne $\mathfrak{B} a r z e n$, biegiam; $\mathfrak{B}$ l. Whmallan= zettf., unten Feibenart.; (Endtiauben verlángert ( 5 8.)

c. ftechento; Shl. lanzettf., langhant.; En o. traub. faft topff. (ち SE. gb.)

1932.

(anglica.)
1933. (Germaň̌ca.)
1934.

sylvéstris: 1935. hispañ̌ca.

\section{GENTIÅNA, 177. $\left(57^{2} \cdot 82^{a}\right.$ b)}

\section{Blk. radförmig.}

Şr. meift 5 ipalt.; Blumenstiele:

1. in fait afterbolienf. Suirl ftebeno; $\mathfrak{B} \backslash \mathrm{b}$.

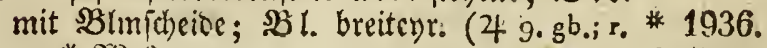
- * 2331)

2. febr lang; shlt. in Salunde bart.; Stgl gabelf.; $\mathfrak{B l}$ l. elut., ftumpf $(\odot$ 10, oben hbl., ausw. wch. u. v.) lutěa. 1937. carinthiñca. 
II. Blk, nicht radförmig, und

A. im Schlunde mit e. Bartw. haarf. Schuppen:

Blkrone :

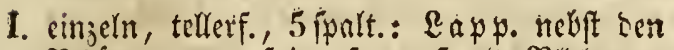
B hát t. cr)r., ipizh, io grón als Boldolapp.; Stgl ganz cinf., meift 1 blitty. $(\odot$ 9. 48.v.) uniflóra. II. melirfarly,

A. glotenf., 5 (Felten 4) fpalt.; StgI meift gabelf.; SB I m ft le verlang., Fanm fo lang

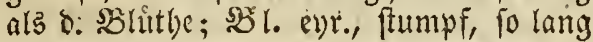

als ¿. Єtgl $(\odot$ I o. wch., mit r. Strichen) nana.

B. tellerformig,

1. 4 paltig; Bldecke:

a. faft gleichỹ., 4 theil.; SBI nu ft le fefti

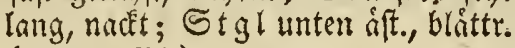
$(\odot$ го. dbl.)

b. mit 2 åu BIb f. ftumpf $(\odot$ 3. x r, x4t dbl.; bl.-vo; rch. u. w.)

2. 4-5 fpalt.; Bléróhre Furrz. als bie faft gleich). SBlo.; $3 B$ l. Yanzettf., ftumpf (๑ 8 . hgb., dann blch.)

1939.

3. 5 phalt., mit ipis. Eapp.; Bl.:

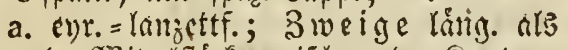
D. Mittelftude zwifd)en; $D$. Snoten; B lolapp. cyr. $(\odot$ 3. 8. dbl, - 1943. * Sirmut)

b. lanzettf.; 3weige thirzer ats bas 1940. glaciális:

1941. (campéstris.)

1942. obtusifolia. (germanick.)

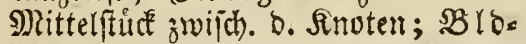
lapper langettf. $\left(\odot-p_{0}\right.$ u. wch. * 1944. * Sirant) Amarélla.

4. meift 5 ipalt., fpis ; Sgls. glcichform.; B l. cort. = Yanzettf.; $S \operatorname{tgl}$ áft., 4 peit.; ant Sirunbe etwas geflugclt $(\odot 34$. 48. hbl.)

1945.
(uliginúsa.)

\section{B. Blk. ohne Bart.}

Blkrone:

I. einzelin,
A. glodecrif., 5 fablt., faft to latth als bet 4 feit. Stgl (4 9. dblo; wrch.)
1946. aca'zllis. 
B. triơteriformig,

1. 10 ppalt.; Stgl unten åft.; 25l. lan $=1947$. jettf. = gleichbr. (4 SE. 9. dbl. u. hgrn.) pyrenařca.

2.' 5 fpaltig; Blätter:

a. Zurle gleitit,

a. lanjettf =gleichbr.; SBlé. etwas 1948. gefägt (4 9. bl. u. wch.) pumila.

b. ganj lanzetti., bachjiegelf.; Stgl 1949. 1 bluth). ( $\odot$ Ix. dgb.) Java.:

c. oval; $B \mathbb{B}$ f. mit ipiken, 2 ppalt. 2Anbången; $\mathfrak{B}$ ld. bauch.; Rarbe (at)nf., feingefägt (4 3. 8. Lld. gefärbt)

o. eyrumb, ipits, am Sianoe fdjarf,

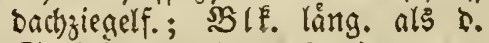
Stgl ( 4 io. dbl, u, hbl.)

b. ungleidh),

a. eyrund,

1) etwas.jpits: 23 z 16 !. nbftebend,

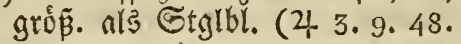
hbl. u. wch.)

2) ftumpf: $\mathfrak{S}_{j}$ lb latt. gebauft, oachjiegelf., fleiner als Stglbl. (4 9. dbl.; w.)

b. ftumpf; georangt: Die unter= ften ellipt., fleiner; $S B l$ le. foft fo lang als $\delta$. fabenform., ganjrand. Eapy.; SBlo. ohne शebenblatt.; 1954:

II. mehrfact),

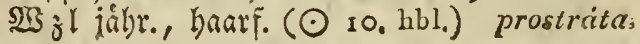

A. glocenformig,

1. in 2 uirln febeno,

a. meift 5 fpalt.,

A. geftreift = punct.; $2 B$ 18. baut., jchsibcr. (4 SE, 9. braun-r. - * 1955. * 2330 purpurĕa.

b. nicht; $\mathfrak{B l}$ l. eyr.; Stgl 4 jeit., 1956. unten aft. $(\odot$ NE. 9. gb.) aurĕa.

b. meift 6 ppalt., febr punct.; o. abge = ftugte Bld.:

G. leberart. (4 g. p. u. gbch., mit 1957. dunckl. Punct.)

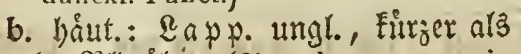
c. Blerobre (4 9. braun-r, mit schw. Punct.) 
c. 7 palt., geftreift, nicht punct.; ß3 Io. 1959 : furz, etwas abgeftufte $\left(2+9 . \mathrm{gb}_{0}\right)$ campanuldta.

2. cm Enoe ftebeno, 5 ppalt., fiticlos, 2 zábl., nit ftumpf. Lapp.; $\mathfrak{B}$ l. glcichbr. = langl., 1960. rippenlos (4 Io. wch., braunstreif.) frigüda.

3. am Enbe 1. in Den SBlattwinf. fteheno,

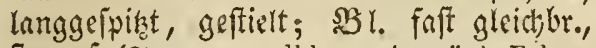
ftumtpf (4 34.49. dbl., mit grünl. Falten * 1961.

\section{- * Nirnut)}

4. entgegenger., am Enbe u. in ס. Blttwint.

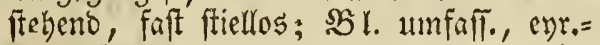
lanjettf. (4 As. 9. bl, mit dunkl. Punct.) asclepiadéa:

B. tellerformig,

1. 4 partig; Stgl:

2. zwententibig, am Grumbe bunner; $\$ B$ If. in Suitl, in ban sBlattwinf. u. eingeln, meifi fiellos (4 2.8. hbl, u. wch., mit grïn. Punct.)

b. edf., bin 11. bergebog.; SBlelappen gejâgt, in o. 9) itte zerichnitt.=genump.; $\mathfrak{B}$ l. lanjettf., cinige gleid) [r. (2 6. 8. bl.)

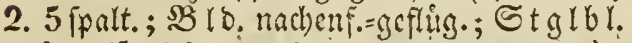
ppatelf. $(\odot$ 3. 8. bl., wch.-bl. gestreift) utriculósa: C. trichterform.; 3weige wechif., 1 blitt).; 1966. Stglbl. lnmzettf. ( $\odot$ 9. hb!., ausw, ligrün) nivàlis. D. baudh., meift 10 ppalt. , geichloff., ftiellos,

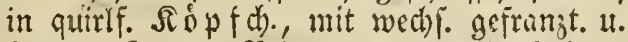

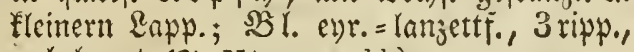
unbelgart (4 NAm. 48. bl.)

1963.

(crucicita.)

1964.

ciliàta.
1967.
Saponaria.

\section{GEORGINA. 669.'}

Etgl nadt; Stglbl. geficb. (4 SAm, Farbe 1968: sehr verschieden) variabilis.

\section{GERANIUUM. 544. (346 $: 367^{z}, 54^{2}$.)}

\section{Blmstiele 16lüthig,}

\section{Blätter:}

1. 5 lapp.shanof., mit långl., zeriđnitt.=gezabnt. 1969. Lapp.; St $\mathrm{gl}$ aufr., åfit. (NAs. W., r. gestreift) sibiricum. 
2. 5 theil., 3 fpart., freiștuno (4 1, 3. 11, 1970.

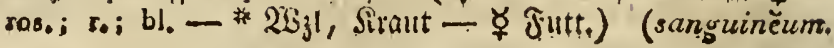

\section{II, Blmstiele 2 bliutligig;}
A. Perennirende.

\section{Blumenblätter :}

A. ungetbeilt; Blätter:

1. 5 theil., zetichnitt.: bie ober ften ftiels loz; $\Xi$ tgl gabelf., aufr. (4 NAm. ubl.; br.)

2. 5 lapp., gezallint; Stgl:

a. gabelf.; SElo. Eligel. = aufgebla jen (4. - p. wohlriech.)

b. nieberlieg. = alfiteig.; $\mathfrak{B I}$ I fitle fibr lang, abluartsgencigt (4 3. 45. 48. dr., unten braungestreift - $\Varangle$ llnf ${ }^{\circ}$.)

3. faft fobiltef., vieltheil., rultzl., fpik (4 3.

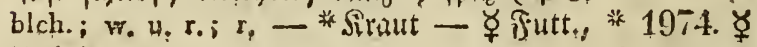
1!ntir.)

B. 2 lappig,

1. negf.egenb.; bie oberfte n $3 B$ l. gezahnt, mit 3 evr., fpit. \&app.; Stgl ftielnuno: Sa amendeden' faft lambebart, am Brunbe zott. (4 SE. w. u, r.; w, u. bl. gegittert)

2. mit bart. Riggeln; Stgef. langhnar.; Stgl etwas jigeor.; 32 l. mcift 7 lapp.;

Saamendecéen faít mafte (4 SE. r.) umbrósum.

C. 3 lapy. = auşgerne.; $\mathfrak{B} l$ l. meift 7 lapp., zer= fchnitten = gejahnt; $S$ țl linghaar, (4 SE. bilk, sehr grofs)

D. ausgeranbet; Blätter :

1. 7 theil., auf beyo. Seit, feibenart. =filz., mit glaichbr., 3fpalt. Enpp.; $B$ I m ft le

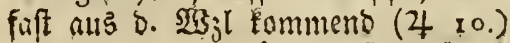

2. nielellf., mit 7 lóngl., ftumpf., 3 fpalt.

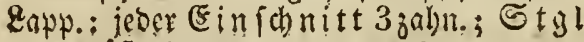
aufr., śft. (4 SE. bl.; p.; w.)

3. falt foliof., 5 ropp., jerichnitt. = geingt; Stgl aufi., bulbentraubenf: (4 5, w:;

w. u. rothatreif. - $\forall$ Fintu. blaui)

4. Die oberfiten 5 lapp., gejabnt: 20 z $l b l$.

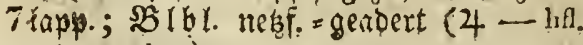
u. dr. geadert)

(praténse,

1971.

maculátum,

1972. macrortizzum.

1973. ఫ (palistre.)

1975 striátum,

1976.

1977.

ibericum.

1978 , argentěum.

1979. pyrenăcum, 1980, $\Varangle$ (syluaticum.)

1981. angulátum. 
5. Stglbl. 3 lapp., geiảgt, mit långl., langgeipizt. Lapp; $\mathbb{S}_{\text {t: }} 4$ jeit.; Sa ac meno effen úberall bant., gleich) (4 SE. 1982. 11. mit $r$. Streifen)

nodósum.

E. am Rambe mellenf.; Blätter:

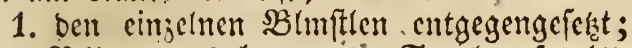
B 10. etwas begrannt; Stgl aufr. (4) SE. 9. dr.. Bl, rothsaft.)

2. balb 7 lapy.; $\mathfrak{B}$ lo. einf., baar.; $\mathfrak{B}$ Ibl. flad) (4 SE. 9.)

F. umbchthlig., jerrill., fo ling als b. webre loie sBlo.; SBlmfte H. SBl. wedhi. (4 SE. r.)

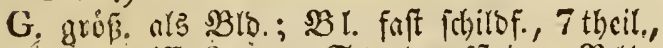
imit jerrifi. Rapy.; Stgl aufiteig.; RID. unto SB limftle langhaar. (4 SE. 2. w. u. rothsireif.)

1983.

(phaeumb

$19 \& 4$.

liv̌̃dum.

1985. refléxum:

1986. aconitifollum.

\section{B. Einjährige.}

Blätter:

1. 5 lappig,

a. Gejabnt; bic ausgeruno. Blblätter:

a. To lanty als o. Blo.; Saameṇbe 1987. de en rauch $(\odot 3.26, \mathrm{bl}$.

bohemictem.

b. Fưrz. als d. SBlo.: ber e. Seitenc la pl. b. BB l. láng., alb o. ubrigen; Sammenteden harf, oben aber.= runjl. ( $\odot S E$.)

b. geruntet; şordecte in o. Iuer runjl.;

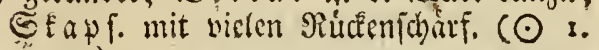
23.p.)

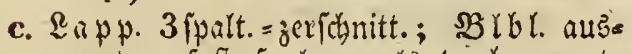
gernitc., faft fo lang als d. begrannte SBlocte; Sarmento deal langhaar.; Stengel:

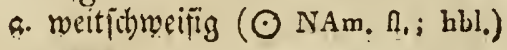

b. aufr., raud) $(\odot)$

2. 5 theilig: bic gleichbr. Lappen:

a. 3 ipalt.; $\mathfrak{B}$ I bl, ansgerano., fo lang alz - b. begramte SBID.; S a a mendeden mit brujentrag. \$aaren $(\odot .3 .4$. r. $)$

1938.

divaricátum.

1959.

(lucĭdum.

1990.

caroliniánum.

1991.

lanuginósum.
1992.

(disséctum.) 
b. vielfpalt.; $\mathfrak{B} I \mathrm{~m} \mathfrak{f t l e}$ lång. als Das $\mathfrak{B l}$; ; SBlbl, wie vorber; $\Xi_{a}$ amendeden unbelaart, glatt $(\odot$ r4.26. ros. - blch.)

3. mcift 7 lapp.: $\mathbb{L} a p p .3$ fpalt.; $\mathfrak{B}$ lat then mit 5 Stgef.; $\mathfrak{B}$ ibl. wie vorher; $\subseteq_{a} a=$ mendeden feinbant. ( $\odot 22.29 . p_{0} ;$ bl.) (pusillum.)

4. nierenformig:

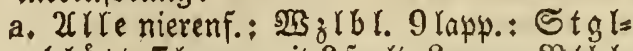
blátt. 7 lapp., mit 3 jpalt. Lapp.; SBlbl. 2 palt., fo lang als b. unbegrannte Slo.; Sa amendeden unbebart, in $\delta$. Suet runjl. $(\odot 27 \cdot$ rch. $)$

b. nur bie $\mathfrak{g} g$ f Stglbl. runol., am Sirunde ab́geftutat, 5 lapp., mit 3 fpalt. Lapp.; $B$ lbl. un= geth., fo lang als o. begrannte Blo.; Eaamendeden raud) $(\odot$ 29. bl. * Si:nut - ఫ Jirb. grib)

5. Cinige 3 , andere 5 joibl.: 2arle 3 ipalt.s flederf.; $\mathfrak{B}$ (b). ungeth., 2 mal fo lang als o. begrannte BRlo.; Sa a mend de den metgf.

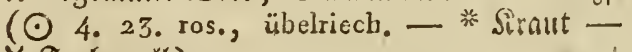
ช. Tarb. gerb)

1993.

(columbinum.)
1994.
1995.

(molle.)

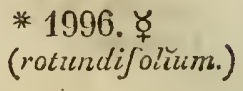

19. GEROPÓGON. 604. $\left(616^{x}\right.$.

Blätter :

1. Gapr. ( $\odot$ SE, rch.)

2. unbebaart ( $\odot \mathrm{SE}$, hI, )
1998. hirsútus, 1399. glaber,

\section{GEUM, 407 ,}

\section{Bliithen:}

I, einzeln sm Stgl; Saamengránnen:

1. gerabe, langhaar.; \$ll. gefieo., baar.; mit e. Febr grof., rumol. Enobl.: bie unt. nacb u. nach fleiner $(49 . \mathrm{gb}$, , El. rothader.)

2. gewunben; bie unt. $B$ l. gefiebert, mit berzf., felbe grop. Endficber; Stgl meift 1 bluth.; ซrt langhaar. ( 4 As.)

2000. montánum.

2001: atlantǐcum. 
1I. meift 2 zábl.; Saamengrann. gerabe,

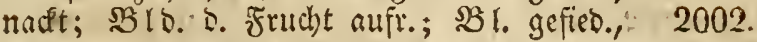
gejalint (4 NAs, gb.)

III. mebrąáblig,

A. aufrecht; bie bafenf.; nadt. Saamengrannen:

1. an D. Spise haar:; Wzlbl.:

a. nebf c. unt. Stglbl. 3 gial.: die obern lanjettf.; $B$ Ibl. túr. alı BBY. (4 NAm. w.)

b. gefiebert: Stglblatt. 3 zád.: bie

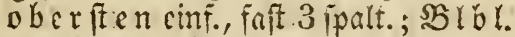

fo lang als SZlb. (4 NAm. w.), album.

c. Yewerf. = gefico, mit berja. Enofies ber: Die obern ङtglbl. rundr.= 3 lapp., ftumipf (4 NAs.)

potentilloídes;

. nicht haar.; Stglbl.:

a. gefico.: B $B(a+t b e n$ u. Xfterbl. zerichnitt. =geipalt.; $3 B \mid b l$. langer als SBlo. (4 NAm, gb.)

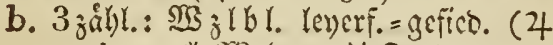
r. gb. - * smist (3)

2005.
macrophýllum.

2003.

virginiánum?

2004 .

strictum.

\section{* 2007. ૪ \\ (urbánum.)}

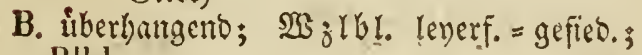

Blbl.:

1." polang als D. SB.s.; Saamengrannen:

a. fecer., in b. Nitte geovett; Etgls b!át t. sinf., 3 jualt. (4 3. 49. p. - *263l, Sirnut)

b. nadt, hafenform. : Sas me baar. Stglbl. 3 jingl. (4)

2. länger als BRto.; Ga amengrannen banr., am Grunce georebt; StgIbl. 2010. cinf., meift 3 fpalt. (4 SE.)

* 2008.

(rivále.)

2009.

intermedrum,

pyrenặcum.

\section{GLADIOัLUS. 44;}

\section{Bliithen :}

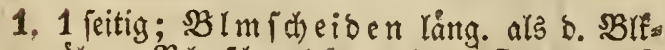

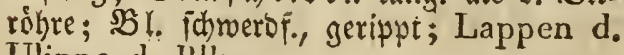
Ulippe d. Blk.:

a. gleid): bie á úf. frumpf (4 3. 4. v.; ros.; 2011. p.; w. $-* 2\left(\mathfrak{b}_{\mathfrak{j}} 1\right)$ 
b. ungleich: bie å abjtelient (4 SE. hr.)

2. in 1 feit. 2lehren; ber oberfte slelapp. ausgefpert: bie 3 innern. faft gleid), mit e. weis., gerino., fohmallangettf. Fleck bes zeichnet ( 4 SE, rch.)

negléctus,

2013.

seğtum.

\section{GLAUCIUUM. 411.}

\section{Stengel:}

1. hactr.; Stglbl. fiederf., zerfa)nitt.; Sdgote 2014. borft. $\left(\odot r_{0}-g b_{0}\right)$

2. glatt; Blätter:

† phoeniciersm.

a. Dopp. fieberform., gleidjbr., unbebaart; 2015. S doten 3 idal. ( $\odot$ SE. v.) violacěum.

b. Stglbl. ausgefchweift; $\widetilde{G}$ d) ote fnot.= 2016. భ̧

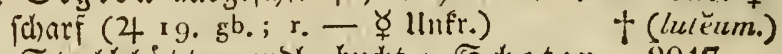

๔. Stglblatt. rund. =bucht.; $\subseteq$ doten 2017. fharf; 23 lath. falt ftiellos (4 SE. dob.) fulvum.

\section{GLAUX. 169.}

(4 17: 36. grn., d̦ann w. oder rch.)
2018.

(maritüma.

\section{GLECHÓMA. 468.}

\section{Pflanze:}

1. rauch; SBiolapp. lanzettf., ftechenofpio 2019. (4 SE. H. u. r. gefleckt) hirsútum.

2. glatt; $B$ lolapp. cyr., fpik (4 26. hbl. 2020. ఫ

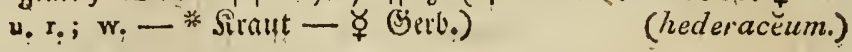

23. GLEDITSCHĬA. $\$ 10$,

$$
\text { (262. W, 17:) }
$$

פ̧ fanze bornig; $\mathfrak{x}$ rab. einf,, in ben Blattwint.; Hiilsen:

1. 1 fanm., oval, ftectienofpis; Dornen einf. 2021.

u. 3 ipalt., fticlruno ( $\hbar \mathrm{NAm}$. grnch.) monospérma. 
2. vielfaum., flad); Dornen:

a. an ben Zeften fteheno,

a. 3 fpalt., faft âft., ftictrunb, gerabe; 2022 . W iil fen fobmerof. (5 NAm. grnch.) triacánthos.

b. cinf. u. 3 fpalt., Feulenf., Jigebruat; 2023. Ş iilicn gefrummt (†) NAm. grnch.) macracántha.

b. am Stamme bujichelf., affr., febr lang 2024. (ち $A s, \Delta)$ horrüda.

\section{GLINUS. 382.}

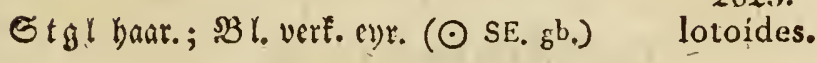

\section{GLOBULARIA. 53.}

Stengel :

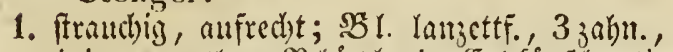

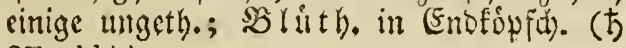
SE, hbl, )

2. Frautart.; Blàtter:

2026.

Alýpum.

a. ganzranto, lanzetif., lång. als b. 33 lttfft; 2027.

Stgl nadft (4 8. b., v.) | mudici'ulis.

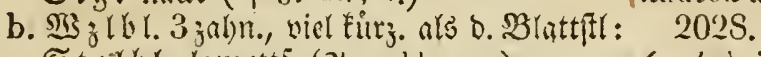
Etgibl. lanjettf. (4 2. bl.; w.) (vulgaris.)

c. $\mathfrak{2 B}_{z}$ lbl. Feilf., an Der Spitze 3 zahn., mit febr Eleinem mittl. Bafyn (4 8, hbl.. 2029. dann, tila) cordifolía

\section{GLỴCÍNE. 569.}

\section{Blätter:}

I. 3 jálltig,

1. unbeyaart, eys.; Blithen:

a. in fatenform., meift 3 blinth. Irroub.,

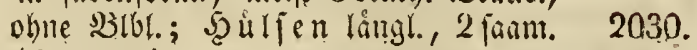

$(\odot$ NAm.)

b, an dortenf. Bhtmftlen, wethe loing. als

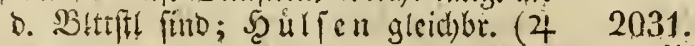
NAm:)

2. rauch); Bhithen in Ecitentrauben (NAm, bl.)

sarmentósa.

umbelláta.

2032.

comósa. 


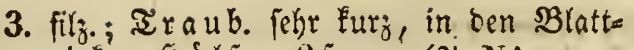
wink.; \$cílfen 2 fanm. (4 NAm. w.- 2033. gbch.; mit e. braun. Fleck im Fähnch.) tomentósa,

II. Ingepaartgefied.,

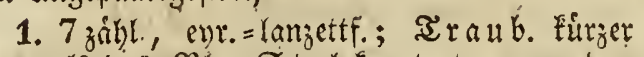
als bả sil.; Stgl Erautart., gewumben (4 NAm, dr. . wohlriech, - ఫ Sîtc.) ApIos.

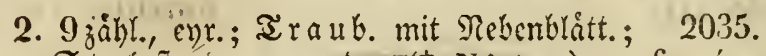

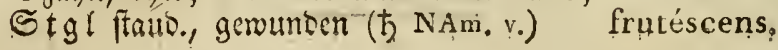

\section{GLYCYRRízA. 567.}

\section{Huilsen:}

1. runoumftachl.; 2 fter bl. lanjettf.; Blith:

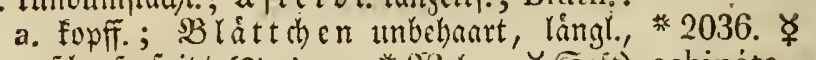
id)arfgefpist (4 As. - * $26 j l-\Varangle$ Gaft) echinata.

b. traubenf.; $\mathfrak{B l a t t c h e n ~ u n t e n ~ f c h u p p . , ~} 2037$. lángl, forarfgejpist (4 As. hgb.) foetĭda.

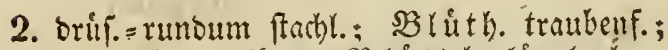
2f terbl. welfend; $\mathfrak{B} l \mathfrak{i} t$ tch. léngl. = lan= zettf., ausgerano., unten flebr.= fcinbar. (4 SE. v.)

2038. glandulifěra;

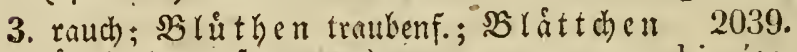
lángl. = lanzettf. (2 As.) hirsúta.

4. glatt; $\mathfrak{B}$ l i tb: traubenf.; oldne 2 fter $b l$.; sBláttch. eyr., an b. Spitze etwas einges Drude, unten etwas klcbr. (4 hbl.; r. - 2040. ૪ * $23_{j}\left(-\not+\sigma_{\mathrm{aft}}\right.$

\section{GNAPHALİUM. $657 .\left(65_{9}^{*}\right)$}

\section{Strauchig;}

A. Silberhaarig.

3B. umfaff., enr. =laingl., 3 ripy., oben moll.,

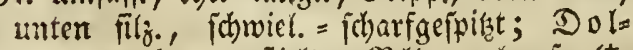
bentrauben geftielt; sBIs. walzenf. (5 SAf. w.)

2041. grandiflorum. 
B. Goldhaarig.

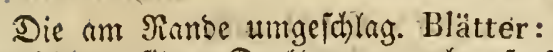

1. gleidbr., filz.; Doloentraub. zigefect,

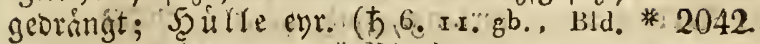
w. , hernach gb. $\triangle$ - * islm.)

2. gleidybr. = Fabenf., unten filz.; Dorben= traul. ăuggebreitet; \$̧îllie maloenf. (ち) $S E$. gb., starkriech. $\triangle$ )

(Stoechas.)

II. Krautartig;
A. Goldhaarig:

\section{Blätter:}

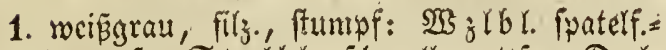
lanjettf.: Stglbl. id)mallanjettf.; Dol= oentrauben jigefert (4 I9.29. dgb. * $\mathfrak{i}(\mathrm{m}$.)

2. auf beyo. Seit. feinbaar., halbumfaffeno, fobmallanzettform., etwas altsgefit)weift: bie unt. ftumpt: bie obern fpit; Dolden=

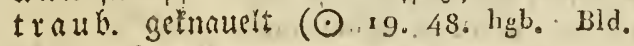
gbch.- w.)

\section{* 2044 . \\ (arenarüum.)}

2043.
italícum

2045.

(luťo-album.)

B. Silberhaarig;

1. mit ganzgetrénntem Geschlecht.

หan en nicberlieg.; 'Sigl gan einf.; Dolbentraub. verengt; die iunern $B$ I (c) uppen verlängert, fiumpf, weís geförbt; Wzlbl.:

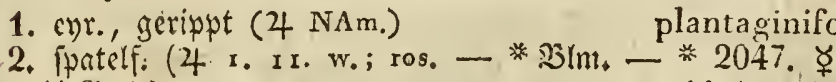
૪̧ (jerb.)

2046.

(dioǔcum.)

\section{2. mit Zwitterbluithen.}

\section{Blätter :}

1. lanjettfơrmig:

a. 2lle lanjettf, u. unten filg., fpis,

a. umfall., gangrano.; Stgl áft. ( $\odot \sigma^{7} 2048$. SAf, Bld, w. od, gb., Bl, stink.) 
b. berab́lauf., am Ranbe mellenf.; Stgl 2049. wie voibler $(\odot$ Af. w.)

undulátum.

- b. nur bie 23 jlbl. lanjett.; Stgl ganj einf.; Enoblutb. gebanft, ftiellos; inn.

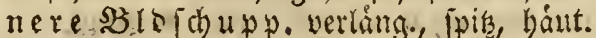
(4.9. braun u. w.)

2. fdimallanzettórmig,

a. etwas.jpib, oben unbel) art, unten feinbant.; Stgl aufro, rijpenf., fils.; EnDooldens. 2051.

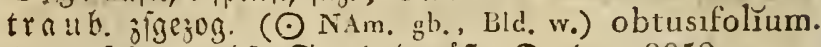

b. langgepipt, wechi.; Stgloben sift.; DoI 2052.

Ientraub. gleichlood) (4 s. peilfarb.) margaritacěum.

3. gleichbr. = ipatelf., lunteit fils.; Stgl autr., ganj einf.; tie ftiellojen $\mathfrak{B}$ lit u. in o. SBlattwinf. Ftehend (4 NAm.)

2050. alpinum.

\section{Fadenkrautartig.}

Stengel :

1. ganz cinfach; Blätter:

a. lanjettF., an beyb. Enoen fismaler und woll.; Stgl aufr.; bic abrenf., ftiellof. SBliitl): aut (Enbe u. in ร. SBlattwinte. freheno ( 4 9. w., Bld. schw.)

b. idmallanjettf., unten fily.; Enbblưth. gebauft, ftiellow; $\mathfrak{R}$ c benbl. langett.,

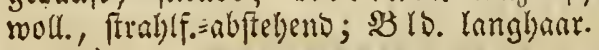
(4 9.)

e. gleid)br., [pile, fil ; ; Stgl:

a. foft aufr. u. 3 bluth.; $\Re$ an berlieg. ( 4 9. Bld, brauu)

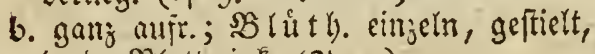
in b. Blattwink. ( 4 9.)

$20 \% 4$.

(sylvalicum.)

2055.

Leonlopodium.

2. einfich); Blätter:

a. Fd)nallanjetti., unten fribenart.; Étgl anfr.; SD lit th. åbrenf., friellos, am Ende น. in ร. SBlattwinf. ( 4 5.)

b. glcich) breit,

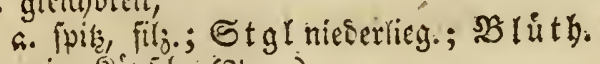
in Siopfoh. (4 9.)

b. ranggelpitat, 3 ripk. , unten weisgrau= ficenart.; Enoblut gethanelt $(\odot$ Austr.).

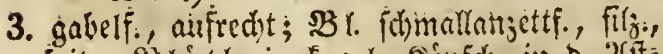
purpurĕum. 


\section{Gnaphalium. - Gymnocladus.}

minl. u. am Ente ftebeno $(\odot$ 18. 28. 34. 2061. gbch.) (germariǔcum.)

4. Faft gatberf., aufr.; SB ritth. getraitigt in Den 2litwinte. 11. am Enor; 131.: . - '2062.

a. glcichbr., langgeppint, weip̈graul. $(\odot$ 18.) gallicum.

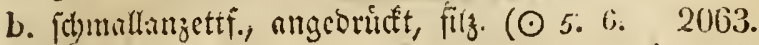
19. wch.-grn.)

(montànum.)

2064.

c. Ianjettf. = fpatelf., féinbarar. $(\odot 28.29)$ pyramidàtum.

5. âftig,

a. aufrest; SB I. verf. = eur., forterf., unten frinbant.; 2 l ( $t$ b. getnanelt, abrenf., in

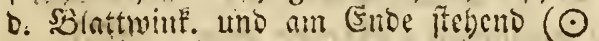
NAn., Bid. gbch, u. grn.)

b. weitiducif, wull.; $B$ I. fdimallangettf.,

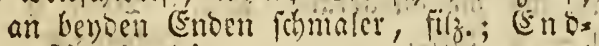
brutb. gerímgt $(\odot 42.4$. dbraun) (uliginosum.)

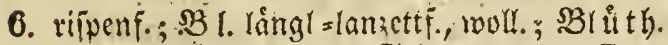
gebáuft, fild., an b. Seite uno an Enbe 2067. fitebeno (๑ 19.29. w. grn.)

2065. pensylvanǐcum.

(arveinse.)

16. GORDONIA. 546.

SB I ủth. langgeftielt; $\mathfrak{B}$ I. lebcrart:; unbebaart; 2068. Elapf. (y). (ち NAm. $\triangle$ )

Lasianthus:

\section{GRATIOัLA. 14.}

21. Intrettf., an b. Spirzi gefägt, 3 ripp.; D I m ftre 1 bluth.; $\$ B$ lo. mit 2 Rebenbi. 2069.

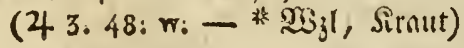

+ (offocinditis:)

22. GYMNOCLÁDU̇S. 791.

(5) NAni, พr. $\triangle$ )

2070.

canadénsis. 
2B Y. gefico., mit gleithbreiten, vielfact)gelappt., 2071.

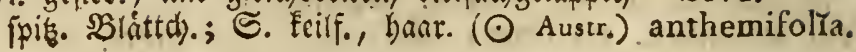

\section{GYPSOPHILA. 350. (W. 48.)}

\section{Blätter :}

1. Iangettformig,

2072.

a. Foft 3 ripp.; $5 \operatorname{tg} l$ aufr. (4 NAs. w.) altissima.

b. glatt; Stgl meit (id) weif.; Strueg ling. . 2073. als bie glodfenf. SBlfe. (4 9. w. 'u. p:) prostráta.

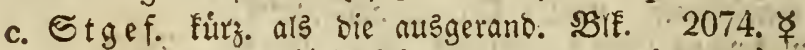
(4 8. w. u. Al. - ซ̧ Ulufr.)

(repenis.)

2. Fめmallanjetti.: bie unt. fharf; Stgef. 2075 .

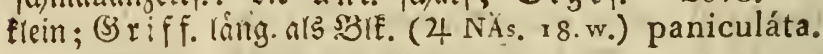

3. ent. = lanzettf., glatt, ant Ginune herjf., unifall; Zlveige jwi ithen o. Stnoten in b.

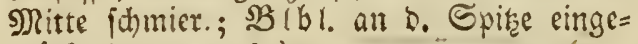
bridat $(\odot$ As, rch.)

2076.

viscósa.

4. gleichbreit,

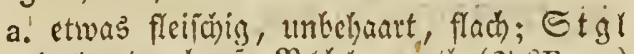

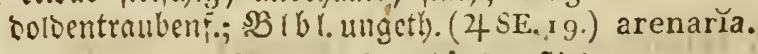

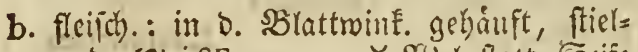

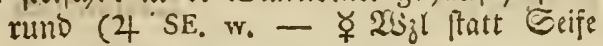 beim 2 afd den)
2077.
2078. ఫ్ Struthüm.

c. flact),
a. Fưry. ars D. Mitterffurd zwifhen ben Snoten; SBib. blattros; Stglgabelf.; Biblátt. geferbt. $(\odot$ 14. wch。-ro ఈ Unf $\mathrm{r}$.)
2079. ૪ॄ
(muràlis.)

b. langer; $B$ rb. H. Stgr wie vorher; 2080. ఫ

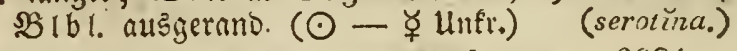

d. BB 18. eff., mit 4 Edupp.; SBIE. aus= 2081. gerano. ( 4 29. bfl, u. r.)

(Saxifrŭga)

5. Tanjettif. = gleid)br., unoeutl. 3 Eant., glatt, ftumpf, 1 icit. (4 23. ros.; w., Bld, blch.

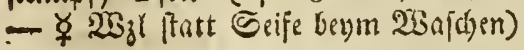




\section{HALESIA. 372. (544²)}

Blätter:

Mit langgeftielt., iberbang. Seitenblitth.;

1. eyr., langgeipizt, gejigt, unten an ben 2roern feimbar.; $\mathfrak{B}$ luth. 2-4jåhl., mit 12 Stgef; Flugel ber Frudbt gleich (ち NAm, w. )

2. långl.=evr., ftumpf = langgefpiat, gejågt,

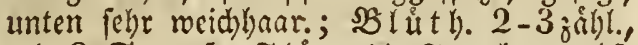
mit S Stgef.; Fligelo. Frudt weed). gróper (ち NAm. w..)

2083.

tetraptëra.

\section{HAMAMÉLIS. 82 ;}

(与) NAm, gb.)

2085.

virginica.

\section{HASSELQUISTIIA. 195.}

Blätter :

1. gefieb.: şi latt th. fieberf., mit ftumpfge= zahnt. Lapp.; Stgl hadtr., åt.; Şitle fehlt faft $(\odot$ NAf. w.)

2. Die unt. 3 zåhl., mit faft herzf. =enr., ges

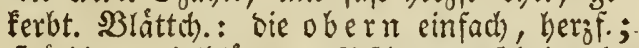

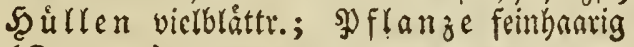
$\left(\odot-w_{0}\right)$

2084.
diptěra. 
19. HEDYPNÖIS, 626.

Stengel:

1. aufrecht,

a. áftig; $\mathfrak{B l}$ l. langl., etmas gejahnt u. herjof.,

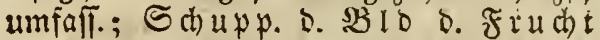
wed)f. an b. Spitze etmaz burft. $(\odot-g b$.) mauritanica.

b. rifpenf.; $\mathfrak{B l}$ l. lángl., hadfr., tiefgezalynt;

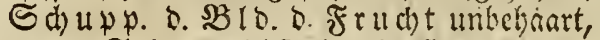
an o. Spite weithftathl. = borft. $(\odot$,

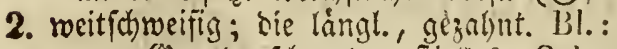

a. am Srumoe idmmaler, ftiellos; Schupp.

d. Bld. d. Frucht:

a. unbehaart $(\odot \mathrm{SE}$.)

b. ftacthelfaint. ( $\odot$ SE.)

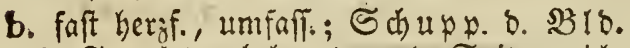
D. Frudit unbehaart, an D. Spige weich)= ftacbl. = Borft. $\left(\odot A s_{\text {s. }}\right)$

\section{2.}

monspeliénsis. 2093. rhagadiǒlus. pendüla.

2094.

cretica.

\section{HEDYSĂRUM. 588 。}

\section{Bl. 3 zählig.}

\section{Blätter :}

1. längl. slanjettfórmig; Bliithen:

a. in $\mathfrak{I} \mathfrak{x} \mathfrak{a} \mathfrak{b}$.; $\mathfrak{A}$ fterbl. fabenf.; S5 tie ber b. (3) liederbúlfe ftumpf 3 ecfig, hacfr. (4 NAm. r.)

b. in Enorifpe; sBl. unbehaart; (5) lie= ber D. Silieberhúlie rautenf., fein= haar. (4.NAm. P.)

2. runbl., unten feinfant.; 2 fterbl. evrund,

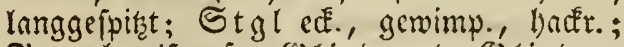

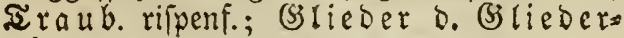
hiulfe 3eff., hactín. (4 NAm. w.)

3. lanjettf. =lingl., ftumpf, unten fribenart.; Ufterbl. pfriemenf.; $\mathfrak{Z} \mathfrak{r} \mathfrak{u} \mathfrak{b}$. in $\delta$. BBlatt= winke, eyr., furr. als bas \$3l.; (s) ficber=

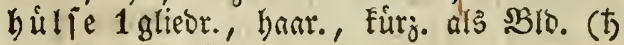
NAm. r. - ghch.)

\section{5. canadénse.}

2096.

paniculátum.

2097.

canéscens.

2098.

trutéscens.

4. gleidfbreit, ant (j)runbe fdhmaler, unten frriegl. = feingaar.; T⿱乛龰auben in b. SBlatte 
mint.; (s) lie b exbillfe 1 glieor., eyr., glatt, 2099. fo lang als SBlo. ( $\odot$ NAs, w.)

5. cllipt., ftumpf; $\mathfrak{I} \mathfrak{x} a \mathbf{u}$ b. boldonf., fo lang

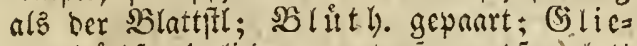
ocrbulfe 1 glicor., rautenf., nesgf., glatt (ち NAm. v.)

6. rumbl. = ellipt. autsgerand.; $\mathfrak{x} a u b$. in $b$.

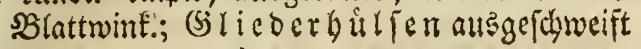
(4 NAm, w.; r.) juncěum.

\section{Bl. gefiedert.}

\section{Stengel:}

1. aufrecht; bie umbehant. Blätter:

a. enr. =lanzettF.; 疋 $r$ a $u$ b. verling., in $\delta$. Blattwint.; Siebenblät. fury. als ber

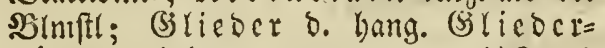

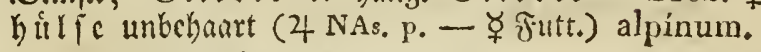

b. Feif.; Blkflitgel, fo lang als Blb.; (silieberbillfen mbehart, 1 faam., geftach.=gezahnt (4 2. x3.hr. - ఫ̧̧utt.) (Onobrychis.)

c. lángl. = lanjettf.; SBlEflúgel 2 mal fo lang als SBlo.; SH liebcrhillen feins haar., 1 famm., gejabnt (4 As. 2i. w.) petráeum.

d. langl.; (S) lieb crhitl. 1 framt., frachl.; a. Solblát. faft gleidlang; Sabue b. Gilieberluilfe langettF., feingejahnt (○ SE. r.)

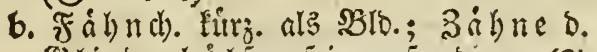
(siliederbulle pfremenf, born. (4 SE. rch.)

2. bin u. hergebog.; $B \mathbb{B}$. eyruns, umbehaart;

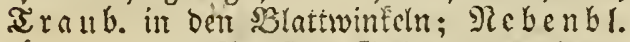
lánger als D. Shlmft; Glieber b. Gang. (5) lieberbillie unbebart (4 9. p. - - ఫ $\delta$ utt.)

3. weitjdhroeifig; Blätter:

a. rullol. =ellipt.; (Silieder o. Gr lieder= búlfe runol., ftad)l., nadi ( $4 \mathrm{SE}_{0} \mathrm{p}_{\text {.; }}$ w. -

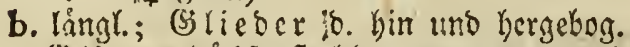

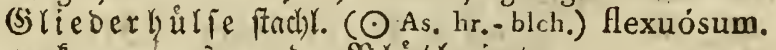

c. verE. eyi., ausgerano.; sBlith. in trau= 2105.

Crista galli: 2106. Caput galli:

2100. violacĕum. 


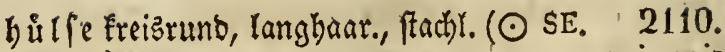
w. $-\mathrm{rch}$.)

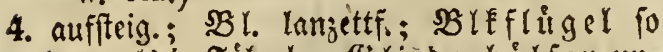
lautg als b. Fáfnch.; (S) lieberbulfen un= 2111. bebaart, 1 faam., ftachl.sgezabnt (4 8.) carpaticum.

19. HELENIUM. $67 \%$ :

2112.

281. gejågt, ganz unbehaart (4 NAm.49.gb.) autumnáles

\section{HELIANTHĔMUM. 416 ,}

\section{Mit Afterblättern;}

A. Staudig.

Stengel :

A. niebergeftreaft; Blïtter:

1. Iánglich), am Manbe

a. ungerollt, etwas baarig; 2fterbl.

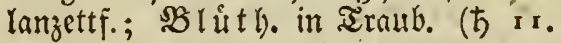
34. gb., d. 3 gröls, Bldbl, gbch. u. grn. 2113. - Siraut)

b. eben, unbehaart; 2ffterbl. u. SBł (uth. 2114. roie vorker ( $\hbar-r_{0}$; w.; bgb. ic. $\Delta$ ) mutabile:

2. Lanjettf, an Sante eben, mit feim., fternf. Şar.; Enot taub. wenigbluth. 2115. (ち, SE, $\triangle$ )

3. ellipt., geftielt, etwas fharf, baar., auf 2116. beyo. Scit. grû́n ( $ち$ SE. gb. $\triangle$ ) obscúrum.

B. etwas aufr.; $3 B$ l. lanjettform., am Ninnoe eben, ftachelbaar.; Uf fterblátt. Febt Flein,

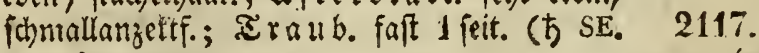
w. $\Delta)$ apennínum.

B. Lrautartîg

Stengel :

1. aufit.; Blumendecke :

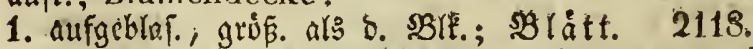

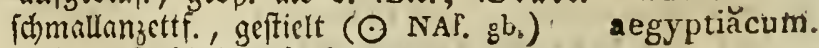

2. nicht aufgeblar.; Bliithen: 
a. in ciner Emotraube; Stgl feinfanr.; BZl. entgegengefeçt, långl.: Die unt. 2119. verf. enr. ( $\odot)$

b. in o. BRlnttwint; BImfte aufr., 2120. fúry. als D. \$Blo. ( $\odot$ SE, gb.) ledifolüum,

II. ctwas abftebeno, langlyanr.; $B \mathfrak{Z}$ íth. wie vorbat; sB I m ft fe borizontalfebeno, lang.

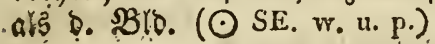

2121. salicifoḷum,

\section{1. ohne Afterblätter:}

A. staudig.

Stgl niebergeftrect; Blätter:

1. wechferszeisftehend, gleichbr., am Ranbe 2122.

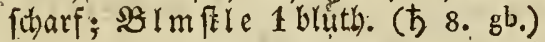

Fumána.

2. entgegenger., geftielt, lingl.s.

a. unten graufilz., ftumpf; $\mathfrak{x}$ rab. wes nigbluth. ( $53 \mathrm{r}$. gh.)

b. auf beno. Seit unbehart; Blattfle 2124

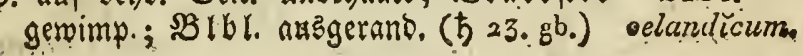

\section{B. krautartig.}

Bl. entgegenger., långl., 3 ripp., ftachelhanr.:

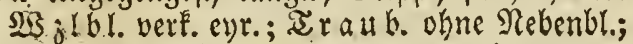
SB (K.I. ungeth. ( $\odot$ SE. r. gelleckt).

2125. guttạtum.

\section{HELIÁNTHUS, 694.}

\section{Blätter einander gleich.}

\section{Blätter:}

1. Kerzformig,

a. 3 fachftelriypig; Blmstiele:

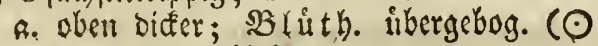 SAm. gb. - - ซ Del)

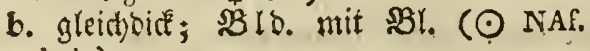 hab.)
2126. $\not$ annǔus. 2127. indǐcus.

b. 3 factgerippt, ranghaarig, am (Strunbe feilf.; $B$ Imftre oben bif́,, robr. $(\odot$ SAm.)

2128. tubaefórmis. 
2. berzf. =eyr., 3 fachgerippt, umfall., angec

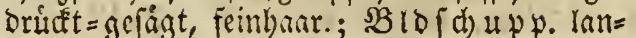
zettf., langhaar. ( $\sigma^{7} \mathrm{NAm}$.)

3. ganz.cyr., langgefwist, 3 fachgerippt,

a. auf berb. Seiten icharf, entferntgefingt;

SB r ichup.. lanzettf.; foft gleich, etwas getwimp.; 10 po. $12 \mathrm{SB}$ If. im Strabl (4 NAm, gb.)

b. nur unten (d)arf, gefägt; $B$ I $\delta$ [d upp. fomallanjettf., an Sirunde gervinip. (4 $\mathrm{NAm}, \mathrm{gb}$.)

c. oben icharf, unten weisfich = Feinbaar.; Bldscluppen:

a. glció)br., fperr.; SBI. gefógt (24 NAm.) macrophýllus.

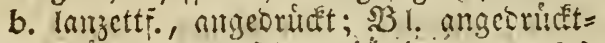
gelingt, unten febr weid) ( 4 NAm. gb.) mollis.

4. ent.= lanjettf., langgefpist, gejagt, 3 froti $=$

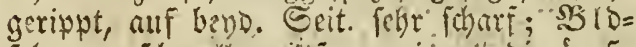

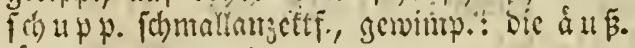
langer (4. NAm.)

5. enr. = lángl., entgegengef., ftiellos, 3 ripł.; Silipe gakelf. (4 NAm. hgb.)

6. Iangettf., wechf., gelingt, fcharf, 3 fachgerippt, a. an beyoen Enocn finmaler, faft ftielos, am (3rumbe

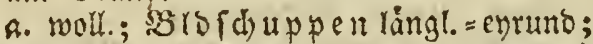
Stgl in 2 Sieiben langhanr. (4 SAm.) b. gervimp.; SBlo ich «p. lanjettf., ge= roimy. (4 NAm. gb.)

b. nur an D. Spire ichmaler, gefticlt; Brtt ft (e gewintp.; $B$ Io $f$ (b) upp. wie vorker (4 NAm. gb., Stgl rch.)

7. Thatelf., entgegengef., geferbt, 3 fachgeripht,

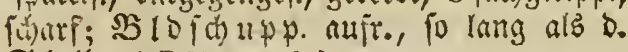
Edyeibe (ơ NAm, gb.)

S. gleichbr., mechi. (4 NAm, dr, Stgl rch.)

2129.

pubéscens.
2130.

decapetălus.

2131.

strumósus.
2133.
2134.

trachelifolius.

2135.'

divaricátus.

\section{Bl. 'einander ungleich.}

Die 3 fail)gerippt., fhharf. Blätter:

1. Kampettf., gefágt: bie obern ganjanto.; BB rofch upp. In jettf., gewimp.; Stgl niederlieg. (4 NAm.)

2. bie untern:

2136.

excélsus.

2137.

gigantěus.

2138.

altissimus.

2139.

atrorúbens.

2140.

angustifolius.
2141. prostrátus. 


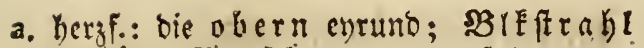
vielblith.; SBid f ch upp. lanjettf. (4 NAm. gh, - $૪ \bigcirc(1)$

b. berzf. - eyr.: Die ob exn ganj eyr., lang=. gelpirt, wechi.; sBlttfie all (Sirunde gerwimp. (4 SAm, - ఛ Silid.)

2142. ซ్ multiflórus,
2143. $\varnothing$ tuberósus.

\section{HELIOPHILLA. 539.}

\section{Blätter:}

1. แmafa I., Ianzettf., ganzrano., unbebaare; 2144. ङ (h) o t. rofenfranzf., bang. (ち SAf, wo; r.) amplexica'ulis.

2. nict) umfanterto,

a. latjettf., ungeth). (○ SAf, bl.)

b. hersf. ( $\odot$ SAf.)

c. geficbert: Blättchen :

2145.

integrifolia, 2146.

Circaeoídes.

a. frocnf., unbebaart; $\subseteq$ do ot rojen= 2147. tranzf, hang. ( $\odot$ SAf. hgb.)

b. balbfictrmb, oben rimmenf. ctwas fleifh.; $\subseteq$ do ot. gleidbr., diberbang. ( $\odot$ SAf, bl, , dann w.) pendüla. 2148. crithmifoly.

\section{HELIOTROPIUM. 106.}

\section{Blätter :}

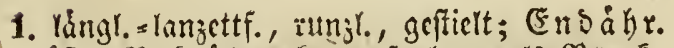
ât.; SBIEróbre frum fo lans nis Bibeci? (ち SAm. blch., wohlriech.)

2. enr., gangrano., flad), ge(trich., f(d)mad)fils.; - Etgr aufr.; Eeitenábr. eimjeln: Eno= álen gepaart; $B$ ro. b. Frucht fternf. abjeheno $(\odot$ 29. rv.)

3. oval, ftumpf, gefalt., am ranbe ctroas ungejchl., monbeutl. feingeferbt, unten grau, ranghaar.; Etgl nicberlieg.; $\mathcal{X}$ e hren meift. cinjeln; $\mathfrak{B} 18$. gefdloflen, mit $\delta$. Fructst abe falf. ( $\odot S E, w_{1}$ )

2149.

perłuviánum:

2150. europá eum. supínum. 


\section{HELLEBÖUUS. 438.}

\section{Blïthe :}

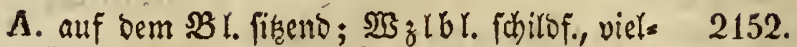

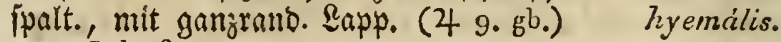
B. am. Schaft:

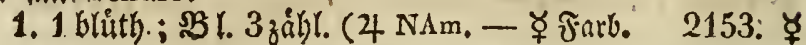
gefb)

2. meift 2 bluth., faft nodt; $\$ B Y$. furfiorm.

(4 8.'w.; rch., wohlriech, - $\mathfrak{W}_{j} \mathfrak{l}$ neblt 2154. Wiglfaperm)

C. am Stgl do. Sen 3 weigen; Blätter: trifolius.

1. gefingert,

a. unten feinhaar.; Blättch.:

s. getheilt; Stengel meift 2 ppartig, 1-2 blitt)., mit blăttr. Sweigen (4 SE, nebst Bl, grno-rch.)

† niger.

b. ungeth., am Sirunbe ganjrandig;

.enir Etgl2-3 palt., mit bláttr., 2 blutth. 3rweigen (4 SE, grn.)

2155.

purpuràscens.
2156.

odórus:

b. unbehaart; Stgl 2 palt., mit 3mei= * 2157 .

gen wie vorber (4 8. grn, - * $\left.2 \sum_{j} 1\right)$ † (virüdis.)

2. fufformig; Stgl:

a. 2fjaltig, mit 1-3 blith., blättr. 3ivei= 2158. gen; $B \mathrm{~B}$. unbehaart (4.SE:)

b. vielblith., blattr. (4 5. s. grach, u. p. - * Sraut) 3. 3 gabl.; Stgl wie vorther (4 SE.) livĭdus. dumetórum. * 2159. Ifoetüdus. 2160.

19. HELIMINTİA. 629.

$\left(\odot \mathrm{SE}, g^{b}.\right)$

2161. echioídes,

6. HELONIAS. 298.

( d) aft meift brattlos; $\mathfrak{B}$ lát t. Lanzettfơrmig, NAm, 47.): 


\section{HEMEROCÁLLIS. 280.}

\$B1. gleidbbr., nadjenf.; Blblätter:

1. flad), fpis, mit ungeth. Rippen (4 SE, gb., 2163. wohlriecb.) Hava.

2. bie 3 innern am Mandewellenf., ftumpf, 2164. mit âft. '̊̊

\section{HEPATICA. 429.}

* 2165 .

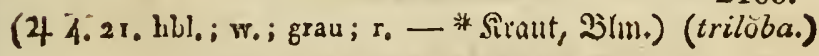

\section{HERACLÉUM. 225.}

Blätter:

1. einfact), herzf., unbehaart, ftumpfgelappt; Stgl faft cinf., rauct; Strablblüth. weislidy (4 9.)

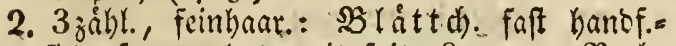

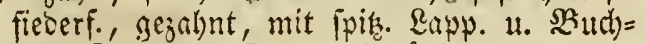
ten; Dolbe gefrahlt; fro $r$ id te ellipt., in b. Sdeibe ctraas langhant. (SE.)

3. 3 záblo = fieberf., unten filz., mit bucht., ge= lappt. Enobl. u. ftumpf., gejahnt. Lapp.; 3 weige meift im Duirl; Jूulten baur:;

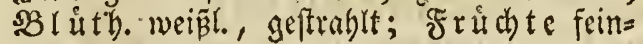
haar. (4 SE.)

4. gefiebert,

a. berablauf. gefiebert, faft freuzf. fteheno,

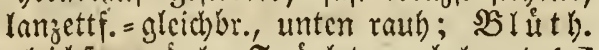
gleichf., gruml.; Fr úd) te unbelgant (o' 3. 8.)

b. Dopp. gepaart,

a. forarf=raidb, gejabnt: Seitenblát to d)en budht.: (Enobláttd. 3 lapp.,

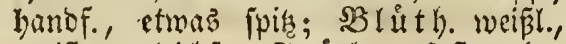
meift ungleithf.; $\widetilde{F}$ rudchte faft unbe= hant (ơ 3. - * Siraut - $\Varangle$ Futt.)

b. raudc): Seitenblát th. evr. =lángl., bopp. gejâgt, ungeth.: (Enoblát th.

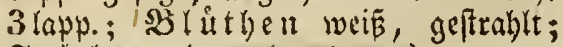
Frath te wic vorber (4 9.)

2166. alpinum. 2167. pubéscens.
2168. pyrenă̌cum.
2169.

(angrustifoliüum.)
* 2170.

I (Spondyluum.)
2171. austriăcum. 
c. unten fils.; ; (5.nobla tat ch. bucht., ge $=$ lapst, mit langgcipifzt., gejägt. Iapp.;

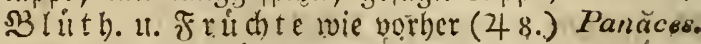

o. umbethaert: Błlát th). etrabs entfornt, bucht $\bar{t}=$ gclappt; $3 B$ lu th. 'grunl., glcich = forme; fru hu te vert. berzf., unbehant (2) NAs.)

5. bopp. jigcient, meift 3 iaflel, faft unbelbaart:

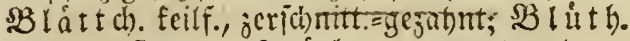

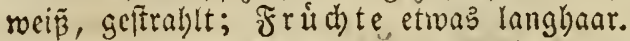
$\left(\sigma^{7}\right.$ NAs.)

\section{3. sibiricum.}

2174. ligusticifolym.

\section{HERNIALIA. 230. $\left(82^{5} .354^{\mathrm{x}}.\right)$}

1. Unbebant; $3 \mathfrak{3}$ íthentnauel vielblith. ** 2175.

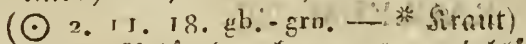

2. raud); $B$ líthent nauel wenigbluth. (4 13. w.)

\section{HESPĚRIS: 331 .}

\section{Blätter:}

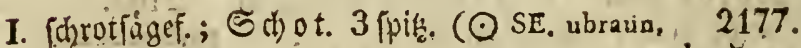
wohlriech.)

lacĕra.

II. nitht; Stengel:

1. eimf, nufr.; Blätter:

a. cyr. =lanjettf., feingejabut; $B \mathfrak{b} \mathfrak{b l a ́ t t . ~}$ mit e. id)arf. Spise ausgerano. ( $\sigma^{x}$ I. 4. w.; $p_{0} ;$ A., $_{0}$ wohlriecl.)

b. fait fpieff., gezahnt; $23 l b$ l. ftumpf

2. afitig, $\left(\sigma^{7}\right.$ 22. wo; p.i giün, wohlriech. - ชุ
2179. ซ inodóra.

a. abftcheno, Gadir. (J゙28, ubraun, Naches wohlriecls.)

2178.

(matronális.)

\section{tristis.}

b. meitidbreif., fehr áft.; $\$ \mathfrak{b l}$ lanjettf., geftielt, ipisgezalnt, idharf; $\mathfrak{G}$ chot. 2181. ftiellos ( $\odot$ SE. f.) africána.

c. Feinbanr.; $\mathfrak{B}$ l. lanjettform., fpir: bie unt gejabnt, ftiellos; $S$ thot. fnot., 2182. priemenf., ftiellos (NAf. v.) arenarĭa. 


\section{HETEROSPÉRMUM. 687.}

Etgl glatt; \$zl. gefies., mit glcichbr. =p prie= 2133. menf., ganzrand. sBlátt(t). ( $\odot$ SAm.) $\because$ pinnátum.

\section{$\therefore 1 \div$ \\ 19. HETEROTRÍCHUM. 638.}

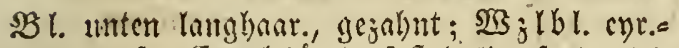
lampttf.; (Enoblutb. faft Doldenf. (4 SE. 2184. 9. p.) alpinuas.

\section{Stengel:}

\section{HIBÍSGLTS. 547.}

I. ftrauchart.; $\mathfrak{B}$ Y. rautenf., foft 3 lapp., une gleich jerichnitt. = gejaigt; a $11 \tilde{\beta}$. \$3 lo. meift Sblittr., fo lang alb-o. innere; Stapl., 2185. langhaar. (4 As. w. u, r., v. x. $\Delta$ ). syriăcus. II. Fyutitartig; Blätter:

A. glcidiformig, evr., faft 3lopp., unten filz-; Stgl ganj cinf.; $\mathfrak{B}$ luth. in o. " 2186 . Blattivint. (4 NAm. po sêhr grofs) palústris. B. ungleid)formig,

1. langgeipizt, ungleid̆gejal)nt: bic un t. ungeth., bergf.: die obern hergform.= lánglich), 3 hrpp.; mit (Enotraube; Blut th. úbergebog.; Stwege über= bang. (4 NAm.)

2. bie obern. 5 lapy., mit lângl., fait gleich., ftumpf. Lapp.: 2tle gezabnt;, $218 S$. $\mathfrak{B} \backslash$ b. aufgcblai., báut., gcrippt ( $\odot$ Af.) vesicarius.

3. bie obern 3 theil., mit lanjettf. Lapp., 13. Fel)r langem Mittellapp.: $\mathcal{X}$ lle ge= zabnt; 3 IO. wic vorber ( $\odot$ SE. hgb. ข. p.)

2189.

Triónum.

\section{HIERACIUUM. 614.}

\section{Mit 1 bliithigem Schaft;}
A. Strauchig.

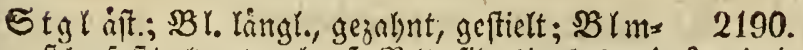

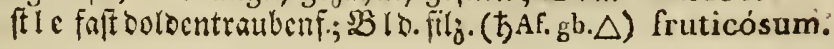




\section{B. Erautartig,}

\section{Blätter :}

1. Iangettf. = ppatelformig,

a. idhrotiágefórm. geznhnt, wenig behaart; Sd) a ft faft nacti; $2 B$ lo. bacír. (49. dgb., unten r.) -

2191.

b. ganjrand., ftumpf u. nebft $\mathfrak{B}$ lb. haar,; 2192. Schaft 1 blattr. (4 9.)

pumiłum.

2. lanzettformig,

a. ichrotfágef. gezabnt, etwas feinghar., mit umgefrummt. Bâhn.; Schaft 1 bláttr.; Bis. ftachelhaar. (4 8. gb.)

b. feingejabnt; ङ̦ aft 1 blattr., oben fil 3 ; BZlo. waljenf., filj. (4 3. 9. gb.)

2193.

rupéstre.

2194.

alpe'stre.

c. ganjrand., ipis,
a. langhaar.; S chaft faft nact, lang= baar.; $B 10$. felor langhaar. (4 9. hgb)
b. ganj unbehaart; $\subseteq$ d a f $t$ nieift 16 luth., bláttr.; oben baar.; $2 B l b$. langhaar. (4 9.)
2195. (alpinum.)
2196. glabrátum.

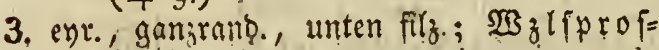
fen frieds. ( 4 I0.11. 34. gb., ausw, rch. * Siraut, SIm. - ૪ F゙utt., Llıfr.)

4. fahrotiäigf. = fieserf., auf beyo. Eeit. lamg=

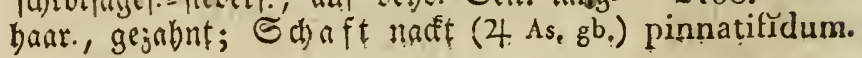

\section{II. mit melurblüthig. Schaft;}

A. Blätter ungleichförmig,

Edaft bláttr.; Bliithen:

1. bolbentraubenfórm.; $S_{\text {d }}$ a f etwas forarf; 23 l. beflelben lanz., ganjrano., Gerablauf.: 23 zlbl. geftielt, lángl., ftumipf, faft ganz= rano., fcharf (4 3. 9. gb.)

2. Dolbentraubenf. = rifpenf:; SBls. Feinbaar.; $\mathbb{X I}_{\mathrm{z}} \mathrm{lb} l$, ganjranto, vert. eyr., ftumpf, ge= rimp. ( 4 NAm. gb.)

\section{* 2197. (Pilosélla.)}

2198.

B. Blätter gleichförmig.

Bliithen:

1. meift 3 zábl. am હdjaft; die fdgmallangettf. Blätter: 


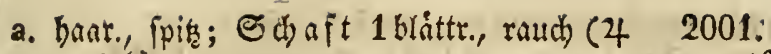

b. unbebaart, feingezabut; $S_{\text {d }}$ aft faft 2202 . nact, áft.; $\$ B \mathfrak{l}$ ft le fulupp. (4 9. hgb.) staticifolüum.

2. meift + jabl.; $S$ d a ft nadt; $\mathfrak{B l} l$. langl., ftumpf, ganjrano.; $\mathfrak{W}$ glfptoffen Eried. (4 3. 4.9. gb.)

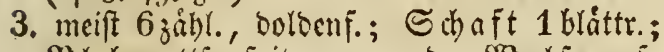

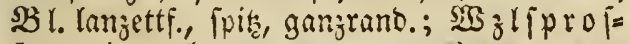
fen wie vorber (4 11. 34. gb.)

4. Doldentraubenf.; ber blíttr. Schaft:

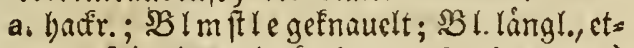
was ppif, hanr.=baftr. (4 5. dgb.; dr., braun)

b. am Srunte barr.; SBIm file fir

lanzettf., fpits, fajt gamzranto, haar. (4) fallax.

5. ooldentraubenf =rifpenf.; ber blättr. Schaft:

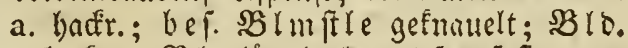
badfr.; $\mathfrak{B l}$ l. langl. = lanjettf., faft ganz= rand., ftachelhar. (4 8. 34. gb.)

b. Faft glatt; be $\Omega^{2}$ lmftle ausgebreit.; $\mathfrak{B}$ lo. ftachelhaar.; $\mathfrak{B}$ l. lamjett gangramb., faft unbel)aurt (4 2r.)

6. traubenf.= Dolocntraubenf.; S baft nact, am Srrmbe foharf; SBlàtt. lángl., ftumpi, feingezabnt, ftachelbaar. ( 4 9. Al.)

7. traubenform.: bie oberu juerft ericheinento;

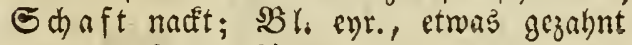
(4 4. 8. g $b_{i}$, grols).

2203.

(du๖žum.)

2204.

(Auricŭla.)

2205.

aurantiücum:

2206.
2207.

(cymósum.)

$220 \mathrm{~s}$.

foreintinum.

2209.

incarnátum.

2210.

(praemórsim.)

\section{Mit blättrigem Stengel;}

A. Blätter leyerf., fiederf., stark gespalten.

\section{Blätter :}

1. lánglich,

a. am (Srunbe ctwas fteberf.; Śtgl àuft.,

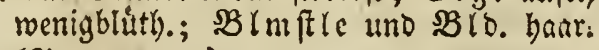
(4 9.23.24.)

b. am (S)runbe fchmaler, ichrot jägef. gezabnt, unbebaart: St glbl. umfall.; Stgl einf.; $\$ B$ lúth. ripenf.; $B$ ld ed e badtr. (4 4 . 47. $\mathrm{gb}_{6}$ )

2. eyr., am Sirunbe tiefgejabnt; Stgl aufr., einf., baar.; Blúth. rifpenf. (4 x. 23 . $g_{6}$ - * Nirnut)

2211.

humüle:
2212.

(palıdósum.)
* 2213.

(murórum.) 


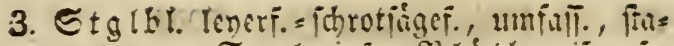
delbar.; Etgl cmī.; Şlúth. rijpenf.; 2214. $\$ B$ เD. Güfr. ( $4 \mathrm{SE}$.)

lapsanoides.

B. Blätser mehr ungetheilt, 1. gleichfürmig.

\section{Blätter :}

1. Iamjettiormig; (ङtgl Mifr.)

a. frriegl.=biter., faft ganjems, om Girunie

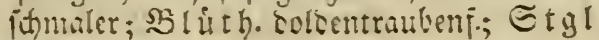
frriegl.s badtr. (4)

b. hadtr, gejabnt, fitillos, an beys. Enien iamaler; Etgl alt., bater.; SBlmfile oban riffer; S310. id)laர̃, badt. (49. nch.)

e. baur., graugrim, ftiellos, ctmas gejobnt, an bevo. Encen idmaler; $\subseteq \operatorname{tg}\{$ ajt. (4) SE. 2. ab.)

d. unbchart, gejahnt; Stgl vielbluth.; sb Im lit le Filj. (NAm:)

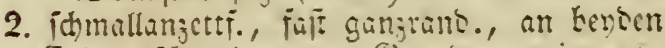
Encen idomaler, am Grume gewimpert; Etg! aujr., afft. ( 74 23.)

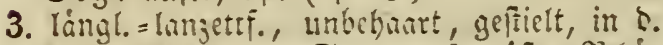

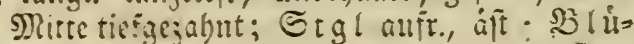

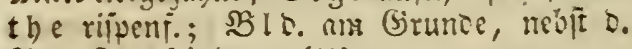
$\mathfrak{B}$ I m ft le feing aur. (4)

4. evr.=langettī., iaingejuhnt, ftiellos; Stg! aufr., einf., blattr, 1 blüth). (4 9.)

2215. eclioides.

2216.

intybacěum.

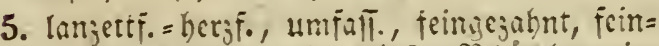
baarig; ङtgl aufr., cinf; SSlutben in traubenf. Dolsentraub. (4 4. s.)

6. linglich,

a. gejahnt: cie unt. 3áhne lánger, lang=

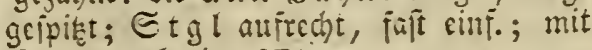
Enotraub. (2t SE.)

b. ctruas gejabnt, langhar.; Etgl blättr.,

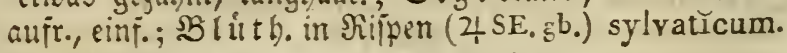

c. nur am Grunce gejabnt, geftielt, haar.; Etgl nadt, wenightuth.; 33 I m itle $u$.

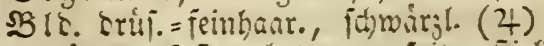

glaucum. 2218.

Kalmii.

2219. saxacile.

2220.

la evigátum.

2221.

montánzsm.

2222.

prenanthoides.

2223.

racemósum.

2224.

2225.

uigréscens.

7. enr. =lángl., fajt untebaart, fpis, friellos, faft umiañ., gegen ben Erruno ju gejabnt; 
Otgl anfr., cinf.; $\mathfrak{B !}$ ! ther in Dotven= 2226. traub. (4 4. dgb.)

(saba'iidum.)

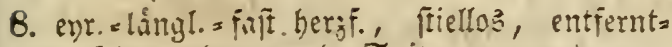
u. Feingegabnt, an 0 . Epitae ganjrano., un= ten haar: Sie unt. am sirmbe finmaler; Etgl aufr., har., ripeni. (7 SE.)- cydoniaefolrum.

9. verf. evr. =langl., jegen ten (Srund ju ge= jabnt, msis = Feinbaar., mit fererig. Şakr.; ङ $\operatorname{tgl}$ aufr., á|t. , mciöl. (4 SE.)

10. ganj enrmo,

a. Fcingejullnt, sim (Sirmbe ungl., gë̈iclt; SElttitle gejahnt: $E$ tgl nieberlieg., ant (Grume ajt., menigblith.; SB Imitle u.

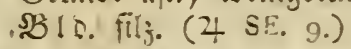

2229. 2228. undulátum.

b. am (Sirunce ticighiahnt; $E$ tgl aufrectit, 2230 . rijpeni = àt.; $\$ B$ lutb. ripipị̂. (4 SE.) ramósum.

c. ftechendipit = gejulint, graligrun, ofen un= bebuart; હtenge! aufr, meifí 2 blattr., haar., meift 3 bluth). (4 SE. 9. 33.) palléscens.

11. enr. = bergfo, umfilfent;

a. feingcjabnt, gewimw.; tgl auTr., cini.; 2232.

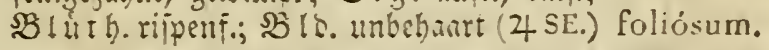

b. gegen ten (jrunt gejalnt; Etgl aufr., 2233.

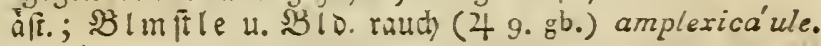
12. gleichbreit,

a. gaujranb.; હtgl aufredt, áfr., blattr. 2234. (4 9. gb.) porrifolüum.

b. etraas gejathnt; Stengel aurre, eini.;

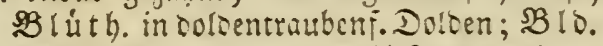
pperr. (4 11.34.gb. - $ఫ$ Jurb.gelb)

2235. ఫ̧ (umbellcitum.)

2. ungleichförmig.

\section{Stengel :}

I. aufiereig., einfüh, gefurdt, baur. = fthmier.;

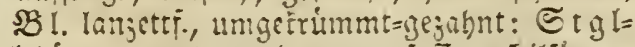
biátt. ctwas gejabnt, minall., preilform.s

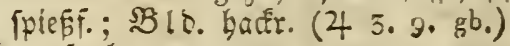

II. nuirecht,

1. huar.; StgIthl. langl. slanjetti., um=

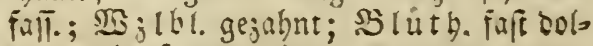
rentraubent. ( 4 9.)

2. langharig; Stglbl.:

2236. grandiflorum.
2237. molle. 
a. langettf., ftiellos: $\mathfrak{B}$ z rb.l. langl: : 2crle baar., gefnbnt; Stengel meift 2 blith.; $\mathfrak{B}$ ro. langhaar. (4 9.)

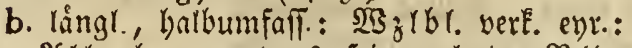
arre baar., etmas feingezabnt; $\mathfrak{B} 1 \mathrm{~b}$. rauch $(4$ SE. gb.)

c. ent. = hergform., umfaft., etwas gezahnt: $\mathfrak{W}_{\text {z }}$ lbr. lángl. = lanzcttf., gezahnt: 2arle langhaar.; Stgl etwaś áft., langhanr.; $2 B$ is. feldr langbar., etwas abfebeno (4 9. gb.)

2238. Halléri.

2239. cerinthoides.

3. Feint)aar., rifpenf., gefurdt; $23 r_{\text {. }}$ rungl.: Stglbl. umfall., am Srunde ungl. = und ticfiezalynt: bic oberfien langettf.: 203 z $l=$ blát t. lángl., gezahnt; $\mathfrak{B}$ ID. jchlaff, hacḱr. (4 NAs. 9.)

4. umbehant,

a. iberall umbehant; Stglbl:

a. langettf. = id)rotingefirm., langgeppięt: SIJ z rbl. rangl. = lanjettform., ungeth.; Stgl wenigblitt). (4 8.gb.- - ซ Futt.)

b. YanzettF. = fpie sif. = pieilif., umfaff., ge=

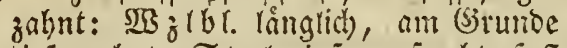
tiefgejalgnt; Stgl cinf., gefurcht, fafe unbchant; SBIb. folaff, baar. (4 9.) pyrenăcum.

b. nur unten unbelaart; $\mathfrak{B}$ r. ctwas lang= baar, Tanjettf., fpit: $\mathfrak{B Z}_{3}$ z l $\mathrm{b} r$. feingejabnt: Etglbl. ftiellos, foft umfalf.; sB roede ranghaar. (4 SE. 9.)

c. nur oben unbebant, etwas foharf; $\mathfrak{B Y}$. rauch, etwas feingezahnt: Stglbl. langl.=

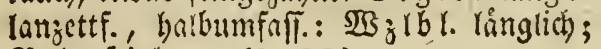
23 10. feinbaar. (4 SE.)

2242. ఛ̧

(chondrilloides.)
2241. sibirı̆cum.

2240.
villóstim.

2244. flexuósum.

\section{HIPPOCRÉPIS. 593.}

\section{Gliederhülse :}

1. fitellos, eingelti, faft gerabe, unbelyant:

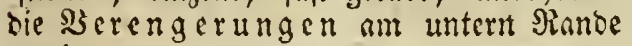
gewintp. ( $\odot \mathrm{SE}$, gb.)

2. gefitielt;
a. meift 4 zákhl., bogenf. getrummt, unbehaart, an untern Sianoe gleid) $(\odot$ SE. 13. gb.) 2247. multisiliquósa.

2246. unisiliquósa. 
b. meift $6{ }_{j} \mathfrak{a} h l$ l., an benb. Siånbern unbe= haart, am untern butbt.: Serengerun= gen forfor; 2 fterbl. evr., fpitz (4 r3. gb., wohlriech.)

$\left(\begin{array}{c}2248 . \\ (\text { comós } \alpha .)\end{array}\right.$

\section{2. НIPPOРНӐЕ. 776. (W. 27.)}

\section{Blätter:}

1. fontallanzettf., oben unbebaart, unten mit 2249.

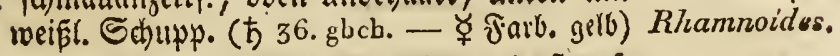

2. ent. = långl., etwaz. fpit, oben mit fternf.

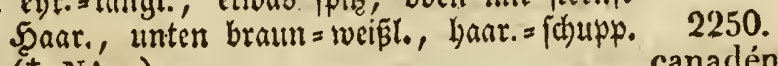
(万) NAm.)

\section{HIPPÚRIS. 3.}

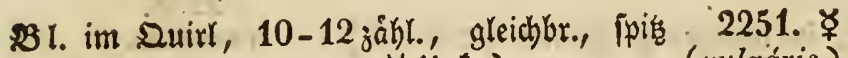
(4 40.42.45. uw. - ६ứfr.)

\section{HOLOSTẸUUM. 49.}

Br. ellipt., graugrim, nebft Stgl unbehaart;

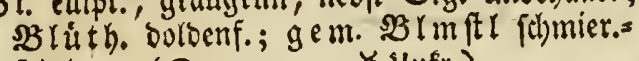
feimbaar. $(\odot$ 28. wo - $ఛ$ unfr.) canadénsis.

2252. భุ

(umbellátum.)

\section{HORMINUM. 454.}

31. eyr. = runol., geferbt gegahnt; $2 B$ rutb. im Suirt: bie u nt. entferntfiet)eno (4 10. 2253. $r_{\text {. }}$ bl.)

pyrenaicum,

\section{HOTTONIA. 118.}

DBrithen im Suirt, geftielt (4 42. 45. A.?

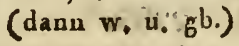

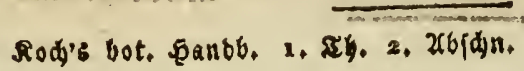




\section{HOUUSTONIA. 63. $\left(62^{x}\right.$.}

DE l. enr.slanzettf.; $\mathfrak{B}$ lủth. in Endooldentials 2255.

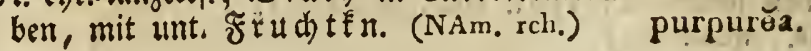

\section{HUMŬLU்S. 781. (W. 54.)}

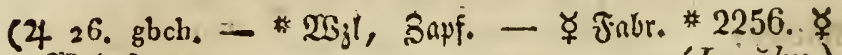
Weber)

(Lipülus.)

\section{HYACINTHUS. $27 \%$.}

\section{Blumenkronen:}

1. glocfenformig,

a. 6 thcilig,

A. an D. Spifge uimgerollt ( 4 SE. 4. bl.; 2257. พั. ก.)

non scriptus.

22.58.

b. in übergebog. Iraub. (4 SE. n.)

b. balb 6 ppaltig,

a. am (Srunde malzenf. (4 NE. dbl.) amethystinus:

b. in Zraub.; StgeF. baut. (4 SE. 29. 2260. 32. w.)

2. tribterf., bals 6 palt., an (srunoe bauch. (2t As. w.; bl.; p. 2C.)

románus. 2261. orientális.

\section{HYDRÀNGĔA. 354. $\left(38^{2}\right.$.)}

\section{Siluthen in Endafterdolden:}

1. geftrablt: Strablblut then geiflechtjlos;

\section{Blätter:}

a. unbebarart, oval, an bevo. Enoen frifi, 2262. ungleichgelágt ( $\hbar$ SAs. hr. $\triangle$ )

horténsis.

b. unten feinbaarig,

ค. evrino, langgelpist, icharfgelägt ( 52263. NAin. w.) radiáta.

b. breitènrund, meiti 5 lapp. = buct)t, un= gleichgefagt; 2 fteroolo. ftraupart.: 2264. rijpenf: (ち NAm: w:, Strablblüth, rch. $\triangle$ ) quercifulia.

2. nicht geftrablt, glcichf.; $\mathfrak{B} l$ l. eyr. u. oval= cyr., langgêplekt, ungleidgejågt, unbebaart (5 NAm. w.) 


\section{HYDRÁSTIS, 435 .}

(4) N.Am, 44, w。)

2266.

canadénsis.

\section{HYDROCHĂRIS. 790. (W, 87.)}

(4 44. $\mathrm{vr}_{d}$, wohlriech; $\mathrm{Bl}_{\text {; }}$ im Wasser schwimmend)

(Morsus Ranae.

\section{5: HYDROCOTYัLE. 1Sร.}

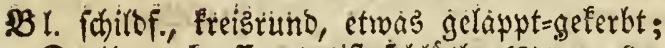
Dolden fopff,; meift 5 bluth): (4 50 . 1. .
$w_{0}$ - ఫ̧ Ulifr.)

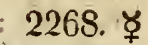
† (vulgáris.)

\section{HYDROPHÝLLUM. 107.}

\section{Blätter :}

1. 2rre gefied., nit ungeth.; an Rande wel= lenf., ungleich. Blättch); $\mathfrak{P}$ flanze, filz $=2269$. woll. (4 SE. hil. - wch.) magellanücum.

2. einige gefies, andere ficoerf. od. geloppt, init evr. =lanjettf., ócrfchnitt: =geiágt. $\mathfrak{L}_{a p p}$;

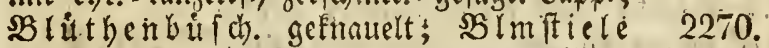
lång: als $\delta_{0}$. Blittftle (4 NAm: wch.

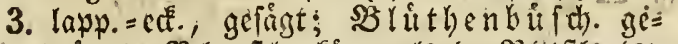

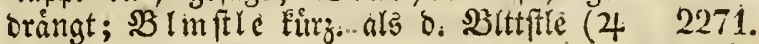
NAm, w. u. rch.) canadénse.

\section{HYMENOCÁRPUS. 586:}

Die nietenfị̂. Hülsen :

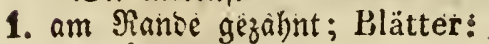

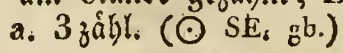

2272.

b. gefitco. $\left(\odot S E_{i}\right)$

radiátus.

2273:

circiinnátus.

2274 .

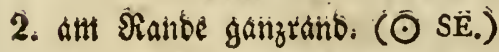
nutnmularǐus. 


\section{HYOSCYĂMUS. 123.}

\section{Blätter :}

\section{I. geftielt,}

A. gleid)formig,

1. eyr., fharfect.; $\mathfrak{B}$ lo. rectrlos; $\mathfrak{R}=$ ben bl. ungeth). ( $\sigma^{\top}$ SAf. ausw. grnch, 2275. dann dr. u. wch., zuletzt w.)

mutřcus.

2. eyt. = faft berzf., ganzrano.; shluth. einzeln, geftielt, in 0 . SBlttwinf.; (5) $0=$ blutb. Doloenf.; $\mathfrak{B}$ ro. aufgeblaj., faft fugel. (4 NAs, rch.)

3. lainglich =lanzettfơrm., etwas gezahnt;

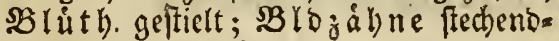

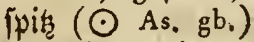

B. ungleichf.: Die unterften Ereistunt, ungeth).: Die úbrigen herzf. =am (Srunde

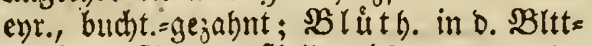

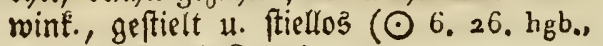
dann w. - \# Siraut)

II. nid)t geftielt,

1. halbherablauf., bucht. =gezabnt, faft un= bebart; Stgl einf., feinbaar.; SBlúth. fticllos ( $\odot \sigma^{\circ} 39$. gbch. - w.)

2. umfalfent, edt.: $2 \mathfrak{z} z \mathfrak{b l} l$ ed. =gezahint;

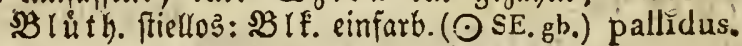

3. $\mathfrak{N B}_{z} \mathfrak{l b l}$. Fieserf. = bucht. : Die obet $\mathfrak{n}$ ums

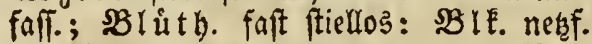
$\left(\odot \sigma^{\star} 14.22\right.$. gb., mit v. Adern - * $\mathfrak{2 b j l}^{2}$ *2281. Staut, Caam. - $\not$ Del)

2276.

† physaloídes.

2277.

pusillas.

$$
\begin{aligned}
& \text { * } 2278 . \\
& \text { t albus. }
\end{aligned}
$$
2279.
agréstis. 2280. + (niger.)

\section{HYOSĔRIS. 625.}

I. Dhne Stgl; o. 1 blith. Schaft:

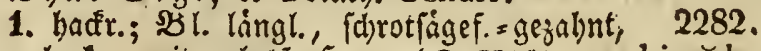
badfr., mit gabelf. Şaar. (๑ NAf.) hispida.

2. nadft; Blätter:

a. unbelyaurt, leverf = ¡ đrotfågef., 2283.

a. gezabnt, mit 3fpalt. Enolapp. (4SE.) radiáta.

b. etwas, fleijh), mit eff., faft bach)= 2284. jiegelf. Eapp. ( 4 As. Schaft rch.) lucĭda.

b. etwas fharf, gewimp.; leverf. =fieberf.,

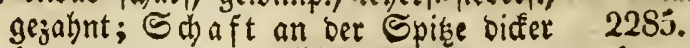
(๑ SE.) 
II. mit ål., blåttr:, meitfdymeif. St $\mathfrak{g l}$; $\mathfrak{B}$ l. umfaff., lángl., sejabnt, iłharf, am Ranbe 2286. gemimp.; SB Imitite oben biffer $(\odot$ NAf.) arenarăa,

\section{HYPECŎUM. 83. $\left(84^{x}\right.$.)}

\section{Schoten :}

1. bogenf. gefrummt, źfgeor., gegliebert; Blbl.:

a. ungeth.: bie $\alpha^{\alpha} u \hat{\beta}$. langer, gleichbr. = fpe 2287. telf. ( $\odot$ 19. 36. hgb.) littorále. 2288.

b. Die $2 \mathrm{gro} \bar{\beta}$. ftumpf 3 lapp. ( $\odot$ SE. gb.) procúmbens.

2. vibergebog., ftielruno, walzenf., faum ge 2289 .

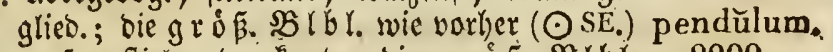

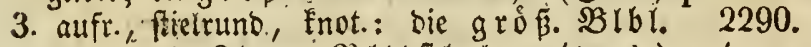

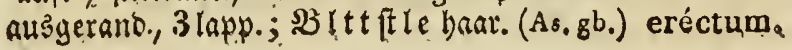

\section{HYPERĬCUM. 598.}

\section{Mit 5 Staubwegen;}

A. Strauchig.

Endblüthen:

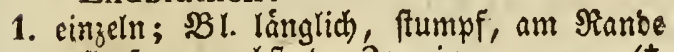
wellenf. u. nebft D. şweige a warz. (5 SE. $\left.g b_{4}\right)$

2. Doldentraubenf.; $\mathfrak{B}$ r. ichmallanzettform. (5) NAm, gb.)

2291. balearĭcum. kalmiảnum.

B. staudig.

Endblủth. einzeln; $s g r_{\text {. }}$ eyr. = oval u. ovale lángl., ftumpf, burchidecin.=punct.; 3 we e ge 4 feit.; $B$ lo lapp. verf. eyr., fehr ftumpf (b SE. gb. $\triangle$ )

2293. calyč̆num.

\section{Krautartig.}

Die ftiellojen Blätter:

1. lángl., fpit, unbehaart; mit Enoblit th.; (3riff. futz. als b. Stgef.; b. 4 edte Stgl: 


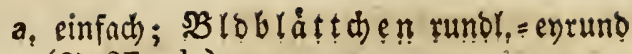
(4 $S E_{0}, b^{b}$ )

2294.
Ascy̆ron. 2295.

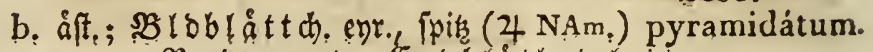

2 enr., an Ranoe punct:; (5noblutb. bol= Sentraubenform.; $B / b$. germimp, =gezabnt; Stg! aufr., ganj pinf. (4 SE.) 2296. fimbriátum.

\section{II. mit 3 Staubrvegen;}
A. Bld, u. Nebenbl, gesügt- drüsig:

Blätter:

1. långliç̣,

a. umfafi, nicht punct., am Ranbe formargs gefledt; Fnoblut th. Dolbentraubenform.

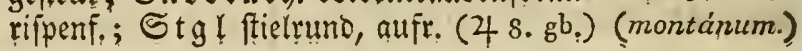

b. am (Sirunde famaler, ourchficht. punct., unten feinhaar.; Stgl ftielrumb, aufr., fẹinl)aar, (46,8. gb, u. schw.)

2298.

(hirsiútum.)

2. 'evt.=långl., umfaff., fild:; $\Re$ Is. ftumpf, 2299. Stgl ftielruno, aufr. (4 As.) lanuginósum.

3. rundl, =evr., ftiellos, feinbanr; $\mathfrak{B}$ lo. $u n=$ bebaart; Stgl ftielruno, Eriecheno (4 46 . 47, gb,)

4. hersf., umfall., unbehaart; Stgl ftielrumb (4 4. gb., ucten rch. punct.)

5. eur. = lanzettf., fajt berzf., langgefpitat, um= fafl.; Stgl faft ftielrumo, aufr., rippenf.;

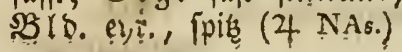

2300.

elódes.

2301.

(pulchrum.)

2302.

elĕgans.

\section{B. nicht;}

\section{1. strauchig.}

\section{Blïthen:}

1. in 4-6 ftraht. Enobolden; SBl. enruno, ftumpf, ftectenofpits, fehr fein ourchidein, punct.; 3roeige 2 fdneid.; BS lol appen eyr., ftumpf; Frụchte beerart, (ち) SE. 49 . gb., Bl. stink. $\triangle$ )

2. in wenigbluth. Doloentraub, am Enoe u. in o. Blttwint. ftehend; o. Fein ourd $=$ fheinents punct. BI.: 
a. längl.senr., fpitz; 3 weige faft 2 fonneib.,

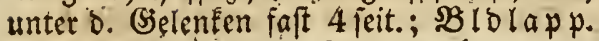
lanjettf. =evr, , piś; Stgef. långer als Ble. ( 万 NAm. gb. $\triangle$ )

2304.

elátum.

b. fomallanzettform., furzgeipitst; 3 weige 2 fchneib.; Doldentraub. bicht, flach, mit jebr kurzgeftielt. mittleren Bluthe ( NAm: gb.)

\section{5. prolifícum:}

\section{2. st a dig.}

Blüthen:

1. an 3 blüth. Blmitlen in Den Blttwinfeln; (3) $x$ iff: zisemachf.; $3 \mathrm{Bl}$. fdymallanzettf., am Srunoe ichmalir, geftielt, im Suirl ( $\hbar$ NAm.) fasciculátum.

2. in wenighlutb. Dolbentraub., am Enoe u. in ben SBltwinf; ; Stg! faft fticlrumo: 3rveige 2 jetreib., unter $\delta$. Sielenfen faft 4 jeit.; $B$ l. lángl., etwaz ipir, febr feine ourchichein. punct.; $\mathfrak{B}$ lol a p pen lanjettf., fpis; Stgef. lạng. als $\mathfrak{B l f}$. (ち SE. gb.) hircinum:

\section{3. krautartig:}

\section{Stengel:}

1. 2 f̧nneioig; Die ftumpf: Blätter:

a. Durchichein. punct.; $\mathfrak{B}$ loblát th. lan= zettf. (4 3. gb., Stbeut. mit. e. ochw. Punct - Siraut, Żlm. - ఫ Jutt., Jarb. gelb, \#2308. (Serb.)

b. nid)t punct., langl.; Enbbluthen faft afterboldonf.: $\mathfrak{B}$ luth. in $D$. Blattwint. einjeln (4 28. gb.)

2. 4 feitig; Blätter:

a. gleid)br., am (Grunbe idymaler, ftiellos; Stgl oben gakelf.; $\mathbb{B} \mathfrak{l} \mathfrak{u} t \mathfrak{t h}$. in $b$. $2 \mathfrak{f t}=$ wint. geftielt, einzeln ( $\odot$ NAm. $z^{\mathrm{b}}$.) canadénse.

b. Durchidbein. = punct.; $\mathfrak{B}$ loblát td). lan= zettform. (4 3. sb. - * Staut, $2 \mathrm{~lm}$. Futt.)

c. nicht punct., ftumpf; Stgl unbeutlich 2312. 4 eff.; 2810 blåt th). élipt. (4 14.26. gb.) dubǔum.

3. ftielruno"; oie ftuntpf. Blätter:

a. ellipt.; ftiellos; Enobluth. Doldentrau=

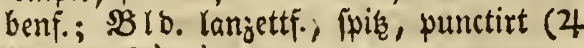
NAm. hg̈b.) 
b. långl.=lanzettf., punct.; $2 B$ lå th. rifpenf.; Stgl cinf.;. B ID. gerwimpert = gejabnt, punct. (4)

2314.

barbátum.

\section{HYPOCHOERIS. 602.}

\section{Blätter :}

1. gejabnt,
a. lantzettf.; Stgl einf., blättr., 1 blưthig

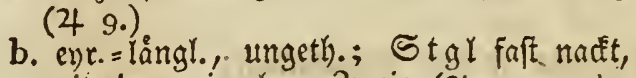 mit einem eingelnen 3weig (4 3. 2 r. gb., * 2316.
Bldschupp. schwch. - Firaut, $21 \mathrm{~lm}$.) (maculáta.)
c. etras idarf; $B$ lo. hactr.; Stronen D. Stueibe geftielt, feber.: D. Strahlz 2317. ftiellos, borft., am. (Srunde feoer. (4 NAf.) minima. 2315.
helvetica.

2. gezahnt =bud)t.; $\$ 2$ l b. lángl., bachjiegelf.; ङtgl-åft., nadt; sp fla nje unbehaart $(\odot$ 19.. gb.)

3. gefägt, fadrotfågefơrm., ftumpf; Stgl åft., nadit, glatt; $\mathfrak{B l m}$ ftle fhupp. (4 34. gb. - Siraut, $\mathfrak{B} / \mathrm{m}$.)

\section{8. (glabra.)}

* 2319.

(radicáta.)

\section{HYSSÓPUS. 464.}

\section{Blithen:}

1. faft quirlfórmig,

a. in 1 feit., wenigblith. Doldent raub. ( in D. SBlttwint.; M itt elfapp. D. Un ne terlippe b. $\mathfrak{B l k}$ lapp., ganjrano.; Bldzähne:

a. aufr., faft gleid); $\$ B$ l. lanjetti. ( 522.

24.bl.; w.; r. - Siraut, Saain. - * 2320. $\not$ ఫ (Serb.) (officinális.)

b. etrab abjteheno, ungleid); $52 l$. famal= langettf. (ち As. bl. $\triangle$ )

2321.

orientális.

b. in walienf. 2léfen; Mittellapp. b.

$\mathfrak{B} \backslash$ f. geferbt; Griffel:

A. fitr. als b. SBlE.; $\mathfrak{B}$ l. faft herzfo=enr., 2322. langgeipist, icharfigeiágt (4 NAm. wch.) nepetoides.

b. lång. als $\mathfrak{B l E}$; $\mathfrak{B}$ l. bcrif. = ent., lang= 2323.

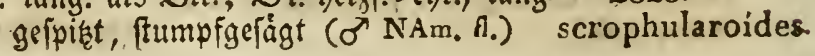


2. an afterborbenf. Bimften in b. Blttwinef; $\mathfrak{B} \backslash \mathfrak{F}$. umgewanot, mit gekerbt. SRittellapp.; B I. lángl. = Kerzf., gefágt (4 As. gb.) Lophánthus.

\section{JASIÓNE. 164.}

\section{Blätter :}

1. idhmallanzettf., am Grunbe fammaler, badr., 2325. weltenf.xł́raus $(\odot 6,11$. r. bl.; siarkriech.) (montána.)

2. gleid)breit, faft glatt, flach, etwas frumpf 2326 . (4 SE. bl.)
perénnis.

\section{Blätter:}

\section{JASMINUM. 13.}

1. 3 zåhl., wechj.: $s$ r åt tdy. verk. eyrumb u. Eeilf., ftumpf; 3 weige ed.; B Blblapp. piriemenf. (5 SE. gb. $\Delta$ )

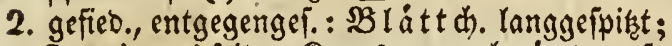
3 weige 4 feit.; finospen abwáts=aufe * 2328. retitiftebeno (ち.SE. w. $\Delta \div$ * $\mathfrak{B}[\mathrm{m}$.) officinale.

\section{IBÉRIS. 512.}

\section{Strauchig.}

Die gangrano. Blätter:

1. fpatelf., unbehaart, ftumpf; $B$ lut the in

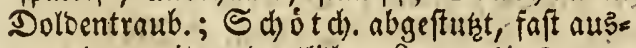
geranb., mit unbeutlitien \&app. († SE. w. wohlriech.)

2. gleichbreit,

2327.

frutǐcans.

a. 2crle, น. ftumpf, am (s)runbe fohmaler, unbelbart; 2 lú then in boldentraubenf. Ziraub.; S ch o t th. Durch e. (dhmale \$3udt) ausgerand. (ち SE. 23. w.)

b. nur bie unt.: bie obern Ianzettfórm.: Ulle feinhaar., fwik; $B$ lúth. in Dol= Dentraub. (ち SE. w.)

2329.

semperfórens. 


\section{II. krautartig.}

\section{Blätter: 1}

I. gleid) förmig,

1. gleid) brẹit,

a. am Sianoe gersimpert =gezahnt, fpik, etmas fleifo : unbebaart; Stgl aufe fteig. ( 4 As. sch.)

2332.

b. gejägt, oben breiter $(\odot$ SE, 9. ․ u, 2333. w.: wohlriech.) odoráta.

2. Eeilf, fpik, etroas fleifd., an D. Epitse 2334. gejabnt ( 49, rch.) cepeaefolia.

3. fpatelf., ganzrano., ftumpi, etwas fleifob. 2335. (4 SE, w.) carnósa.

4. lanzettF., fpic, etwas gezahnt; \$2lúth. 2336. in Iraub. ( $\odot 3^{\circ}$ w.) (amára.)

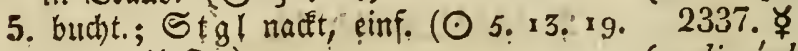
w. - ఫ Dị)

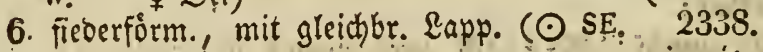
36. w.) ... pinnáta.

II. ungleid formig: 0 . untern:

1. geftielt, runol =evr.: $5 \mathrm{tg} l \mathrm{bl}$. länglich, umfait: : 2rile etras fleijh., ftumpf (4 9. rch.)

2. gejägt: bie obern ganjtand.: 2Cl te lane 2340. zettf., langgefpist $\left(\odot \mathrm{SE}, \mathrm{r}_{\text {; }} ; \mathrm{w}_{\text {. }}\right)$ umbelláta.

4. ILEX. 85 .

Blätter :

1. immergriu, leberartig,

1. evr., fpis, born.,

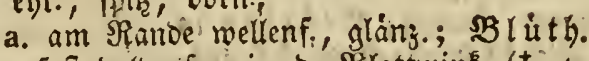
faft oploerif., in D. Blattminte. ( 5 4. 2341. uiv.; rch. - * Fraut', Beer.) (...t (Aquifolium.)

b. flach, unbehaart; SSlit then zerftreut

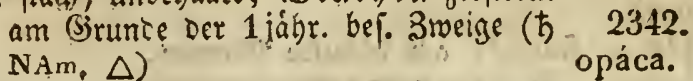

2. lanzettef, an beyb. Enden ictmaler, an o. Spize etras gezahnt, wechl., entfernt= ftehento, unten am Sande $\delta$. Nittelrippe ftachcllaar. ( $\hbar$ NAm.w.)

II. jấbrl, abfall., lángl., langgeipitert, an ber 2343.

Cassine. Spize etwab gefágt; $2 B l$ mitt iele 1 bluttb., 
lang, in Den Blattwint:; $\mathfrak{F} \mathfrak{r} \mathfrak{d}$ d fugel.,

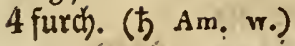

2344.

canadénsis

\section{ILLEECĔBRUMM: 169.}

\section{Blïthen :}

1. im Duirl; Stgl fabenf., nicberlieg., un

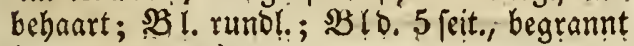
(4 34. 48. w.)

2. in 2 fterbolben; Stgl ăft., aufr, (aušs 2345. (verticillàtum.)

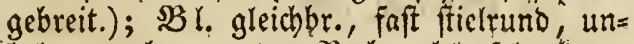
behaart, begrannt; Nebenbl. fébr furz;

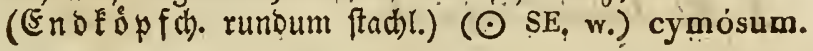

\section{IMPATIENS. 159,}

\section{Blumenstiele;}

1. 1 bríth., geháuft; $B$ r. lanjettf.: D, obern

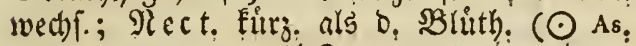
w.; r. blch. $2 c_{0}$ - $\Varangle$ Farb, roti)

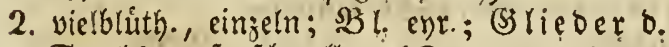
Stgl's aufgefdhmollen $(\odot$ 4, 49. gb. ఫ̧ sarb. ge(v)

2347. $\not$ Balsamuna.
2348. ซ̧ † (Noli tangĕre.)

\section{5, IMPERATORǏA, 224,}

\section{Blätter:}

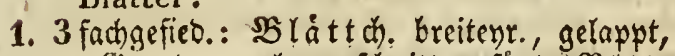
am Srunde ungl., o eridonitt.=gefagt ; B 1 t t focioen meit; Stglftieltuno, unbebaart, * 2349. faft einf. (4 7. wch.; rch. - * $\mathfrak{2 S}_{31}$ )

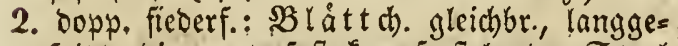
fpiet: bie unt. faft Freuzf. ftebeno; Stgl faft ftielrunt, geftreift; $3 B$ Ittidecioen ver= láng., welf (4 4: wcli. u, rch.)

(Ostriulhium.)

2350.

(Chabra'ei.)

\section{Blätter:}

\section{INDIGOFĔRA, 587.}

1. einf., verf́. =eurund, unbehaart; Şůlfen 2351. fithelf., rundum ftad)l. $\left(\odot \mathrm{Af} f_{0}\right.$, echináta. 
2. gefiebert,

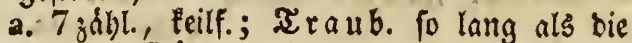
Bl.; Şúlien 4 jeit., 2 faam.; Stgl nieberlieg. ( $\odot$ As. dr.)

b. vielpaar., lanjettf., unbehaart; I raub. fabenf., láng. alż bas Bl.: F́ábnchen ftriegl.=haar.; Sूulfen aufr. ( $\odot$ Af, ros.) dendroides.

2352. enneaphýlla.

2353.

\section{INǓLA. 680 .}

\section{Bl. herablaufend.}

B1. evr. = långl., gezahnt, an b. Spike ganz. ranb.; Slúth. Doloentraubenf., georángt 23.14. ( $\sigma^{7} \mathrm{SE}$. gb., PH. starkriech.)

bifrons.

\section{Bl. umfassend.}

\section{Blätter:}

I. ungleichf.: $\mathfrak{Z}_{\mathfrak{B}}$ Ibl. evr.: Stglbl. lanzett= form.: 2clle gezabnt, (e)r rauch; Stgl wenigb(utt). ( 4 8. gb.)

II. gleichformig,

1. eyr., rumgl., unten fir.s.; SBIb fhupp.

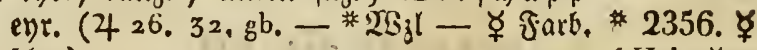
blail)

2. her $\tilde{F}_{0}=$ lỏngl., nadt', gelågt, unten baar.;

(Helenı̆м.) Stgl feinbaar., rifpenform.: Seitens zroeige an b. Spitse etroas abitebent; $\stackrel{B}{*}$ (bid) up p. borftenf. (4 45. 49. gb. - * 2357. ชุ. * Siraut -

3. ganj långlich),

a. raudh, ungeth.; $\mathbf{S} \operatorname{tg}($ haar., bolben= traubenf. ( 4 × 1 . 14. gb.)

b. langhaar., am $\Re$ nnoe mellenf.; Stgl aufr., rifpenf.; $\mathfrak{B l} \mathrm{l}$ ft le 1 bluth., ben

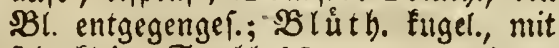
febr flein. Strabl ( $\odot$ 14. 27. gb.)

(dysenterica.)

2355 odbra.

2358.

Ocülus Christi.

2359.

(Pulicaria.)

4. lanzettf, am Srunbe geiágt, unten haar.; Stgl boldentraubenf., langhaar.; Se i. tenzroeige an $D$. Epize láng. (4 48 . dgb.)

2360.

(britannica.) 


\section{Bl. siciellos.}

\section{Blätter:}

1. oval, fteif, feingefägt, fharf, nekff-aber; 2361. B 10 ich upp. evr., umgejd)l. (4 3. hgb.) squarrbsa.

2. länglich,
a. ganztand., jpik, fcharf; Stgl oben åft.; Slit b. . in Dolbentraub. (4 4. 8. gb. 2362.

- *iraut)

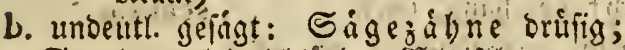
Stgl raud), 1 bluth.; SBlojd) uppen lanjettf. langhaar. (4 As gb.)

c. Enorpel.: =feingejabnt, fdarf, fteif; Stgr glandulósa.

(germanǐca.)

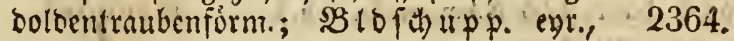 ftumpf, iperr. (4 $36 . \mathrm{gb}$.)
Bubonüum.

3. lanjettfóminig,

a. gefagt=icharf, gefrummt; 3weige edt.; 2365.

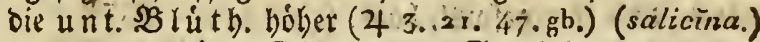

b. unbeutl. gelägt, fteif, baar.; Stgl et= was getbeilt, Janghaar.; meift 1 blith,; BID (d) Upp. lanjettf. (4 3: 34. gb.)

4. ไångl. = lanjettF., gefágt, unten fil rifpenf.; (5noblủth. geftielt, meift 4 zábl., fait colbenf. $(4,9$.

5. gleitb)breit, 1 is

$$
\begin{aligned}
& 2366 . \\
& \text { (kirta.) }
\end{aligned}
$$

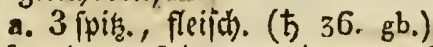

b. langgeipist, gerippt, unbegaart, zers 2369.

ftreut; S tgl meift 1 blutb. (4 5. gb.) ensifolia.

6. gleicbbr. = fpatelfơrm., ganjranb: : die unt. ftumpf: Die obern etwas fpiá; 3̂weige 2370. meift 1 blüth. ( $4 \mathrm{SE}$ )

\section{Bl. etwas gestielt.}

\section{Blätter:}

1. ellipt., am Ssrunbe fdmaler; baar.: bie $237 \mathrm{p}$. unt. gejabnt; $\mathbb{S}_{\mathrm{g}} \mathfrak{l}$ vielblith. (4. $\mathrm{SE}$.) svaveŏlens.

2. lanjettf., raudh, gangrano.; Stgl'1blutb.; 2372. 8310. furz, bachziegelf. (4 gb.) montàna.

\section{IPOMO'EA. 126.}

Die Łcrź. Blätter:

I. gelappt, 1. 3 lappig; Blumenstiele: 
e. 1 bluth.; Seitenlapp. ร. SBl. ppis: Nittcllapp. langgeipizt; $B$ I d e de rauch, mit lanjetti., langgeipitzt., pfrics

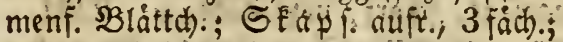

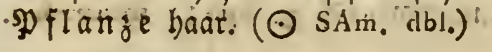

b. vielbluth, träubenf. (๑ Am. v.) 2373. hederacěa. 2374.

c. lang. ars bet sittitl; Etgl gerwun= cen; $F \mathfrak{r} u$ cht tuberhang. (w.)

hedérifolia. $23 \frac{4}{7} 5$. scábra.

2. unten uniteutlict) ausgerchineitit ob. edf.;

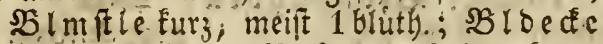
baur., gevimp.; $\mathcal{B}^{3}$ lE

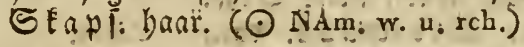

II. uttgetheilt; Bliithen:

1. getrángt; $\mathfrak{B}$ IE. ungetb; ; $\mathfrak{B l}$ l. ganjrano. (SAm. p.)

2376. lacunósa.

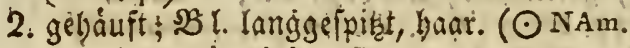
b., dann schwch.)

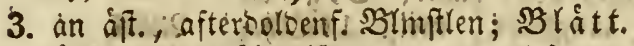

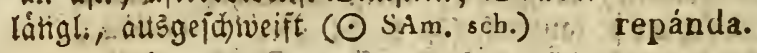

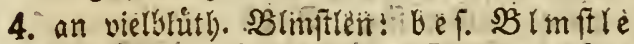

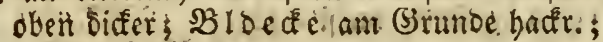

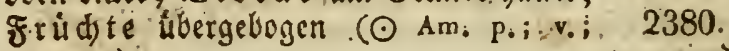
tos: : w.)
3. IRIS 45.

purpirěa.

I. Die abstehend: Blbl oben hart:;

A. Schaft länger als die $B l$.

Wi. Thwertị:; Schaft:

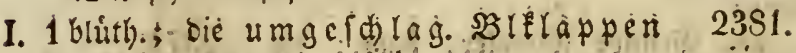

fleiner ( 4 SE. uw. u. dr.)

$23 \pi \%$ violacěa. . $237 \mathrm{~S}$. tamnifolia. $237 \%$. 2 bluth:; Blmscheiden:

1. to lang als bie Biftróte (4 NAs, gb, 2382. braungeadert) susiána.

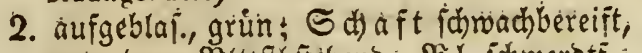

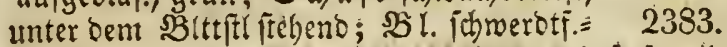

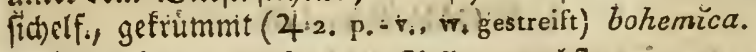

IIt. meift 2 bluth: ; $B$ lủ then ftiellos, neblt

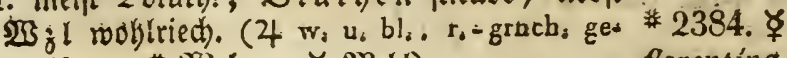
otreift - \# 2: florentina. 
IV. 3 bluth.; bie gróß., umgefd)lagen.

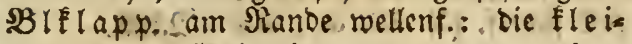
nern ausgetano. (w. u. grn., an Rande v. punct., mit v: Adern)

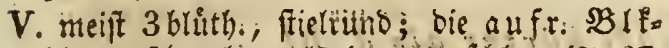
lápp. jomalèr, alb o. ümgejhlag. (4 SE. p.-v.; Scheidén grn.-v.) 2386. biflóra. V1. mebrblithig; Blkscheiden:

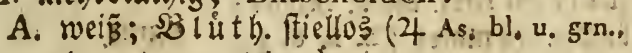
mit v. -braun. Adern)

B. nidt; bie à ufrecht. Blklappen:

1. Hund. = vetE. evr.; bie unt. $\mathfrak{B}$ lúth. gefticlt; sie $\mathfrak{R}$ I mfd sioen zuleht ges

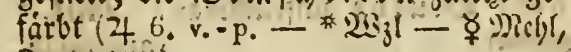
Farb. blaii)

2. flach): 2clle auggcrano: (4 SE. hbl., miit v. Adetn) sos sif sumberna.

3. etwas gebdg., etwas ausgerano. u. am, 2390 .

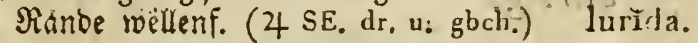

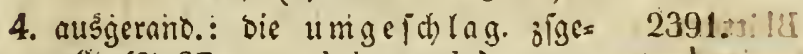
rollt (4 SE: grn. - gbch: u. xch.)

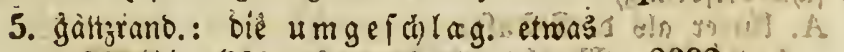

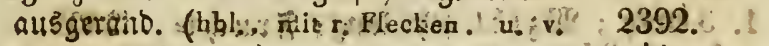
- i. i. w. . / wohlriech.) (.ivi neglécta?

6. breiter, als bie andern: 2 lle wellenf. gefalt.; Stgl ftieltuno (4.w. u. hbl.;. mit $r_{\mathbf{d}}$ Adern) 2393. plicáta.

\section{B. Schaft co iang als di $\dot{B} l_{\text {. }}$.}

Schaft:

1. meift 1 bluth., zigeoruffet; s B lol a p p gleida,

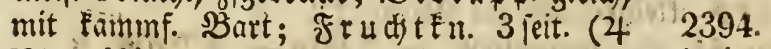
NAm, bl:)

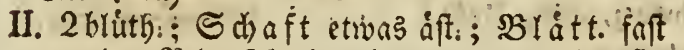
gerabe; $\mathfrak{B} I m[d$ c i be blattart. : bic obcrfte 2 blưtb., $S B$ Ibl. unten zigeneigt (4 SE. $w$ : u. v.)

III meif 2 brith

cristáta.

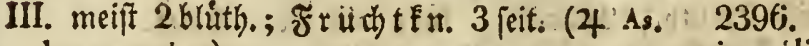
braungeadert) IV. mehrblitthig;

1. Die umgefd. \$B IElapp. ausgetand.: bie aufr. láng!. (4 gra, - gb, u, wch.. mic ,p. : v. Stricb.)

2397.

variegáta. 
2. 2 lmidheiben aufgeblajen: bie oberfte 2 blith.; 3 l. jaft fichelf. (4) SE, dv.; mit w. Stricb:)

2398.

hungarǐca.

\section{Schaft kürzer als d. Bl.}

Schaft:

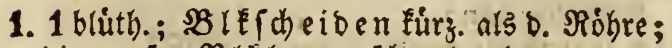
bic aufr. Blelapp. fdimaler (42.6. p.; bl, ; w.)

2. meift 1 bluth.; $B \mathfrak{l}$ m fdeibe aufr., fo lang 2400 . als b. Blfrébre (4 8. w..gbch,, a m Grunde v.) lutéscens.

3. 2 blùth.: Die obere 3 lit the unfruditbar 2401 . (4 SE. gb., v. geadert)

II. die abstehend. Blbl, nicht bartig.

Blätter:

I. 伤werbtfórmig;

A. Eúrzer als D. Schaft:

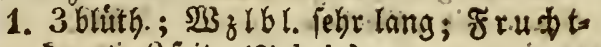
Eno.t. 6.feit. (4 bgb.)

2. mel)rbluthig, ?

a. 1 edfig (4 SE. ubl. $\rightarrow$ * Wigl) arenarĭa:

2399. pumila.$$
\text { areme }
$$

b. 2 fळmmioig (4 NAm. blch, , $x_{0}$ ader.) virginǔca.

B. eben fo lang als d. Schaft:

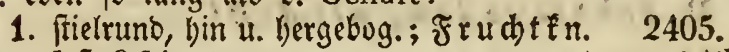
faft 3 feit. (4 NAm. grn, u. w. u. p.-v.) versịcŏlor.?

2. nicht; $\mathfrak{B l}$. flach; bie innern $\mathfrak{B l}=$ lapp. Fleiner als die Rarbe (4 38. 42. 45. gb., braunstreif. - \# $\mathfrak{2}_{\mathfrak{j}} l-* 2406$. $\not{q}$

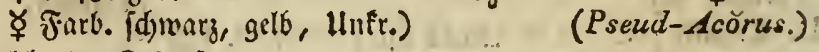

II. glcidbreit; Schaft:

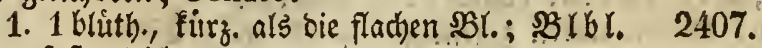
faft gleich) (4 NAm, p.-vo, wohlriech.) verna.

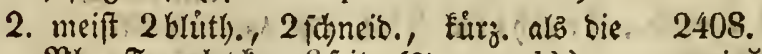

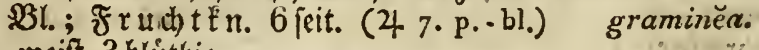
3. meift 3 blutthig,

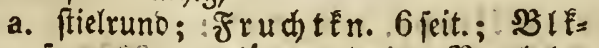
fau m enr., láng. als ber. Slagel (4 As. gb. u. w. )

2409. ochroléuca. 
b. rókr., bóber als Dic $\mathfrak{B l}$; Fruth tf n. 3 jeit. (24 3. bl. u. gb. u. wch. — ఫ fँutt., linfr.)

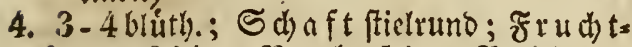

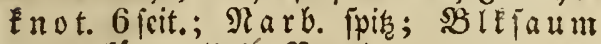
runo, fưrs. ałs b. Naget (4 3. bl. - v. u. gbch.)

III. rimnenförmig; Schaft :

1. 1 blitth., viel furr. als 8. gleichbr.=pfrie= menf. SBl.; die in nern SB IE (app. jebr furz, febr abftejeno (4 As, w. mit gb., 2412. v., bl. u. r.)

2. 2 Glitth.; $\mathfrak{B l}$ l. rinnenf. = pfriemenförm.; Blklapp.:

a. viel breiter als ๖. Narbe; Frud)t fdarfect. (4 SE. w.; v.; bl.)

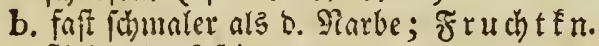
ftielrumb, 3 feit. (4 SE. gb.; bl.; v. ic. - ૪ garb. griwi)

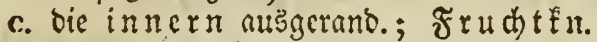
falt eingeichloll. (4 SE.)

3. meift 3 bluth., lang. als o. SBl.; $S B I L$

- róbie fadenfórm., fébr lang, mit wedji. aufr. Saumlapy. (4 SE, hbl, mit e. gelb. Fleck)

IV. vicreitig (4 SE. braun-v.; am Rande grn.peršca. 2410. (sibirica.) 2411.
spцría. 1

2413.

Xiphioides. 2414. ఫ Xiphrum. 2415. lusitanica.

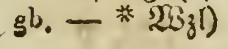

\section{6.}

Sisyrinchĭum.

* 2417.

tuberósa.

\section{ISĂTIS, 515.}

\section{Schütchen :}

1. verf. feilf., an $\delta$. Spike cingeorudft, unbe=

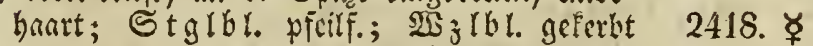

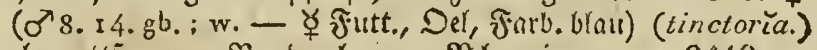

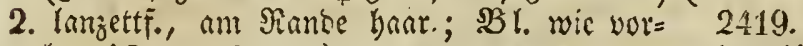
her $(\odot$ As. gh.; w.)

3. Ger (O A

3. her form.; $\mathcal{B}$ \%. ganzrano., hergf., binten' 2420 . ftumpf (As.)

arména.

\section{ISNARDIA. 80 .}

SB 1. gefticlt, eyr., fpis $(\odot 37.45$. grn. $)$ 
242 Isopyrum. - Iuglans.

13. ISOPÝRUM. 439. (422. $424^{x}$ )

Afterblätter:

1. piriemenf.; $\mathfrak{B} \mathfrak{l b l . ~ f p i s ~ ( 4 ~ N A s . ~ g b . ) ~}$

2. eทr.; SBlbl. ftumpf (4 r. 9. w.)

3. unoeutlich ( 4 SE, 9.)

2422.

fumarioídes.

2423.

thalictroides.

2424.

aquile gioides.

5. ITĔA. 160 .

Bl. gefticlt, wechi., oual =enr., fpir, gefigt; Xefte fticlrumo, feintiffig; (5notrauben aufr. (ち NAm. gbch. - w. $\triangle$ )

2425. virginica.

\section{IUGLANS. 751. (W. 2.)}

1. Mit vielen Stgef. u. einfach. M. Kätzchen.

RI. ungeparitfgefiebcrt: Blättchen:

1. 5-9 jåbl., oval, etwas gefágt, linbeljaart, faft gleidj; Frifch t e Eugel.; punct., glatt

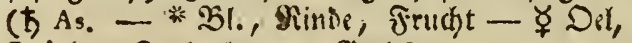
Frudgte, Farb. braum, (Serb.)

2. 9 ob. 11 zábl., eyr. = lángl., langge finit, ges fágt, unbebart, oben an $\delta$. Mittelrippe u. unten an ben Coocn feinbanr., am (Srunde ungl. ( $\hbar$ NAm.)

3. 11-17 zábl., langl.=eyr., langgeipicat, ge= fágt, unten féinl)ar. = weith; SB lattfitle

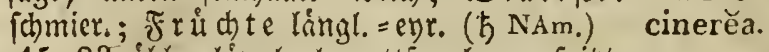

4. $15-23$ zabl., lángl. =lanjcttf., langgefpiact, gefågt, linten feirhaar.; $\mathfrak{F} r$ ud d t e fllgel.,

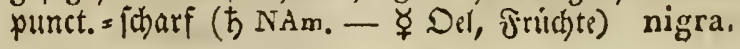

\section{II. mit 4 Stgef. u. zsgeselzt. M. Kätzchen.}

B lơt t. ungeprartgefieo ; Blättch.:

1. lanjettf., langgeipikt, feingelägt, unbebaart, faft ftiellos, 11-17 jábl., mit e. werf. eyr. 
u. langgefticlt. Enobl.; $\mathfrak{F}_{\mathfrak{r}} \mathrm{u}$ b te längl., 4 cit. ( 5 NAm.)

2430.

olivaefórmis. .

2. lángl., langgefpint, gefógt, unten feinhanr.= weich, 5 oo. T zábl.; mit felyr furzgeftielt. Enobl.; Blattfle feinlaar.; Frubte

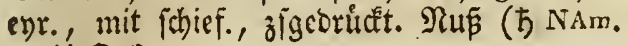
- ૪्⿱一⿻上丨)

3. oval = lángl., ftumpfgefpist, yon b. Mitte bis zur Spike gejågt, unten feinbaar.=weich, 7 zall., mit geftielt. Enobl.; SBlattfte feinbaar. ( $ち$ NAm.)

4. eyr., langgefpift, lunbebant, unten barjigs= punct., 7 johl., in D. MBSint. Der Ripp. hauts art., mit fajt ficlloiem Enobl.; Fru

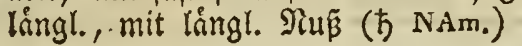

\section{1. $\Varangle$ \\ compiéssa.}

2432.

pubéscens:

\section{3.}

glabra.

\section{IUNIPĚRUS. 796. (W. 65.)}

\section{Blätter:}

1. 2rlle angeoridft: bie åltern meift entge= genge F., rautenf., langgeipitst: bie jüngern entgegenger., 4 reibig Dachjiegelf., ftedhend $=2434$. ఫ ppik (ち SE. $\triangle$ - Sraut - ఫ (Serb.) Sabina.

2. entgegengef., od. 3 jålll.: bie ålter n aufr.= abftebeno, lanjettf., ftechendipis: bie juns gern 4 reil)., meift bachsiegclf., frechenolpis (b NAm.)

3. 3joblig, abftebend, ftechentipic,

a. lánger als D. SBcere, rinneni. = gleichbr., gegen die Spibe fd)maler ( 5 5. - * . Dolz, Beer., Şarz - ఫ̧ Sierb.)

b. To lang als bie Sbcere, rinnenf $=$ gleidjbr. (ち) 9.)

c. Hurger als bie Beere, fdmallamgettform. ( 5 SE. $\triangle$ )

* 2436. ఫ

(commúnis.)

2437.

nana. 2438.

Oxycědrus.

\section{IUSTÍCIA. 16.}

Die singelnen, ftiellofen Blithen in ben Blattminfeln:

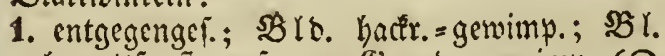

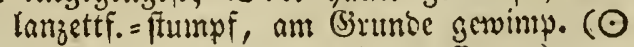
w.-u. grn.- gbch., mit 2 braun. Punct.) 
2. im Quirl; Nebenbl. \{hmallangettform.; $(\odot$ l. lanjettf.; Ulippe ber $\mathfrak{B}$ !f. 3 lapp. 2440.
lithospermifolìa.

\section{IVA. 708.}

Stengel :

1. frrauch.; $\mathfrak{B l}$. Ianjetti., tikfgejågt, punct.s

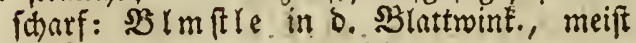
1 bluth. ( $\hbar$ NAm. gbch. $\triangle$ )

2. Erautart.; $B$ l. lanzettF. = evr.; Rebenbl. lanjettf. u. mebft $\mathfrak{B}$ lt tit le n feinborar. ( $\odot$ SAm. hbl.)

2441:

frutéscens.:
$24+2$.
annuัa.

\section{IXIA. 43.}

\$Bl. flach, glcid)br. = p friment., viel furb. als ber 4 blith. Sdhaft ( 4 Nim. bl.)

243. coelestína.

\section{KALMĬA. 331.}

Doldentrauben:

1. am En be ftebens; dic unbehaart. Blätter: 1. evr. =ellipt., 3 jågl.; cinige jerftreut, am Ranbe cben; jüng. 2 2 eftch. frieltund (ち NAm. hr.)

2. lángl., entgegengel., am Ranbe umges

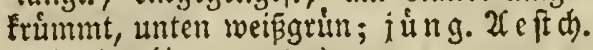
2 idjneio. ( $\hbar$ NAm. br.)

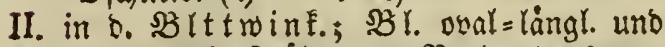
oval=lanjettí., 3 záhl., am Sinnde etroas um= gefrummt; iu $\mathfrak{n g}$. $\mathcal{U}$ e ft d). ftielrumo ( $5 \mathrm{NAm}$. $r_{0}$, dann $v_{0} \Delta$ )

\section{4. \\ latifolǐa.}

2445.
glauca.

2446.

angustifolía.

16. KITAIBELIA. 550.

2447. vitifolra: 


\section{Knautia. - Krigia.}

4. KNAUTİA, 55.

Blạ̈tter :

1. zerichnitt.; b Bglo. ( $\odot$ As, hr.) orientális.

2. bie obern gefied.; $B$ rb. 10 brättr.; S. mit 2449. Sftrone (๑ As. hblch.)

plumósa,

5. KOCHǏA. 237.

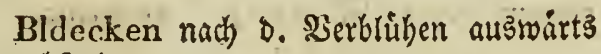
aแșadtjento

1. in 5 trodine $\mathcal{X}$ (n) antnge; Stgl:

a. ftaub., wweishbarr.; $\mathfrak{B} \mathfrak{l}$. gleidb)r., flat);

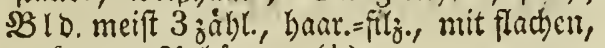
verf. = eyt. 2furbángen (

b. Erautart.; Blätter:

a. gleichbreit, etwas fieiity., feinhaar.;

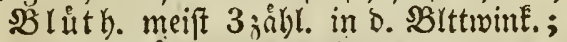
Bloanbånge ftumef $(\odot$ ะ. 9.$)$ arenaría.

b. Pifmallanzettf., gewimp.; $\mathbb{B}$ id. ges paart, mit felyr kurrz., (ppiź. 2Unbången; Dg flanje feinhad. ( $\odot \mathrm{SE}$.)

2. in 5 ant Nitfen bafenf. Stadyeln; Stgl frautart., feinhar.; $\mathfrak{B l}$ l. gleid)breit, flady; BID. gefnaue!t, woll. ( $\odot$ SE. 17.)

$24500^{\circ}$
prostráta. 2452. Scoparĭa:

\section{3.}

hyssopifolla.

\section{KOELREUTERĨA. 310.}

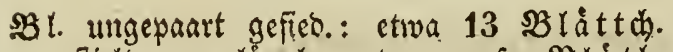
geftielt, evr.: långl., entgegenger.; $\mathfrak{B}$ lúth. in Rifpen, am Enoe u. in D, SBlattwinkeln fteberto (万 As, bgb. $\Delta$ )

2454. paniculáta
19. KRIGIA. 612 .

$(\odot$ NAm, dgb.)
2455. virginiัca. 
19. KUHNIA. 649.

Br. gleidgbr., faft gangrant.; (5noborben= traub. ausgebreitet ( $4 \mathrm{NAm}$.).

2456. Critonı.a.

\section{LACHENALǏA. 290.}

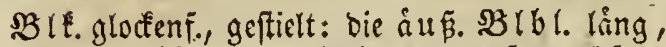
ctmas abjtebend: die innern zigemachjen;

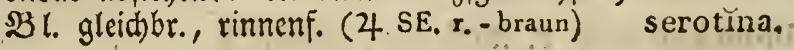

\section{LACTÚCA. 613.}

\section{Blätter gleichförmig.}

\section{Blätter :}

1. bucht. =geferbt, gejabnt, am Rande wellenf.,

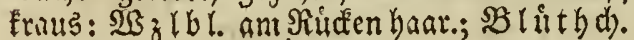
5 theil. (๑)

2. (d)rotfägef. = fieberf., am Shuden ftad)l., am Enoe fpits, an Grunbe pfcilf., verticalfte= beno ( $\odot$ 15.22. gb. - *iraut, 巨aam.) † (Scuriöla.)

3. langl. =lanjettf., gewimp.= feingejabnt, am 2460. (Sirunbe pfeilf., unten glatt ( $\odot$ SE. r g.) augustána.

4. fipoerf., mit gleidjbr., aufwårtsgezabnten Lapp.; $\mathfrak{B} \backslash$ i th. in ooldentraubenf. Rippen (4 4.8.3 3 . bl,; w.; th.)

2458. crispa.
* 2459.

2401. (perénnic.).

\section{Blätter ungleichförmig.}

\section{Blätter :}

1. rumbl.: Stglbl. berzf; ; Stgl bolbentrau= benf. $(\odot-\mathrm{gb}$ - - Straut, 巨nam, - 2462 . ఫ ૪ Der, Sitid.) † sativa.

2. bie unt. 3 theil.=fieberf., mit langl., frumpf. 2463. capp.: die oberfien berzf. $(\odot)$ palmata.

3. bie unt. lanzettf., fujrotjägef.=gezahnt, am (Srunde fdimaler, ftiellos: die oberngleid $=2464$.

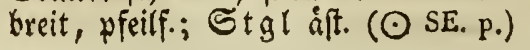
segusiána. 
4. Thtrotfågef = fieberf., ungleidgrazahnt: $\mathfrak{B Z}$ l.

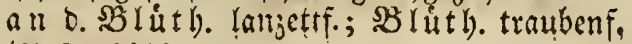
(4 SE. hbl) )

5. am Rilden ftatblig,

a, lơngl. = langettf., feirrgezabut, borizontal= ftehend, an Ende ftumpf, am Bramtie pfeilf.: bie unterften bud)t. $(\odot 26.45$; - Siraut, Saam.)

b. $\mathfrak{S B}_{\mathfrak{Z}} \mathfrak{l b l}$ lanzettf., fieberform.: Stglbl: gleidbr., ganzranto., pfeilf. $(\odot, 7$.

6. unten glatt:

a. bie untern fartotiagef.

a. Peingezahnt, am Esunde Greiter umb freilf.: bie obern lanzettf., pfeilform:; Siifpe ganz gerabe (4 4. 8.)

b. ganzrans., umfall.: bie obern lan= gettf., fticlios: Die unterftengezahnt; B lúth) in torbenf. Rifpen (NAm.) elongáta.

b. Walblätter:

a. Leverf. = fchrotfiggef.: Ștg glbl. lanzettf., gezalynt, am Gsumbe pfeilf., mit $a b=$

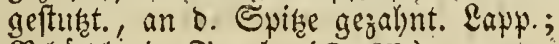
SBluth. in Tratb. (O SE.)

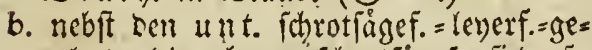
zalnt: bie obern fotroffägef = fieberf.,

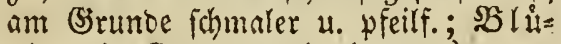
then in Dolbentraub. (ơ.SE.)

* 2466.

† (virósa.) 2467.

(saligna.)
2465.

sonchifolĩa.
2468.
(quercina.)

2470.

Chaíxi.

2471.

stricta.

\section{LAGOECIA, 167.}

$(\odot \mathrm{SE}$. grnch.-gb.)

2472.

cuminoides.

\section{LAGOSĔRIS. 621.}

\section{Blätter:}

1. fieberf, geferbt; S dh oft nadt; menigblutth, (4 SE.)

2. $23 \mathfrak{z} l b l$ u. bie unt. Stglbl. gefiebert, lenerf.: Die obern Stglbl. lanzettf., ums

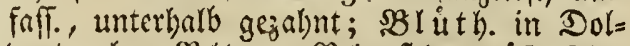

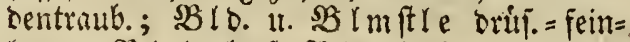

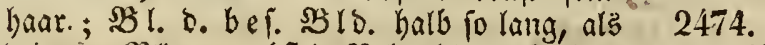
๖. gem. SBlo. U. nebft o. Aebenbl. trof. (4) raphanifora. 


\section{LAMIัUM. 462.}

I. Die hetgf. Blätter:

1. ungl. = u. 伤arfgejägt; $B$ lf. mit aufge= Whe. Edyluno u. an bend. Seit. 3 jain. urippe; $\Re 10$. gefárot (4 SE. p.)

2. feinbrar.; $\mathfrak{B}$ lke. nit aufigeblaf. Echluno, getro. Robre, u. Ulippe wie vorber (4 8., w. - rch.)

3. 'anggefpitzt; Blüthenquirle:

a. 10 blith). (4 14.26. p.)

b. 20 blitt).; 33 l. gefógt, geftelt (4 29 .

w.; f., Bld. braunfleck, - * Siraut, * 2478. Bim. -

4. gleich $=$ u. ftumpfgejabnt, geftielt, ftumpf 2479.

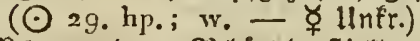

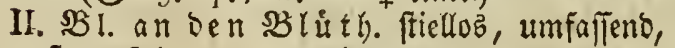
frumpf $(\odot$ 29. - ఫ unfr.)

2475.
Orvăla.

2476.

garganicum.

2477 .

(inaculàtum.)

(album.)

(purpurěeím.)

2480. ఫ్

(amplexicáule.)

\section{LAPSĂNA. 627.}

I. Shme Stengel; Schaft:

1. 1 blitth.; $\mathfrak{B} \mathrm{I}_{\text {. }}$ ichrotfágef. = fieberf., mit 2481.

e. rautenf. Endlapp. (4 9.) foetĩda.

2. meift 3 blutth); $\mathbb{B} I_{m}$ ft le robr., oben

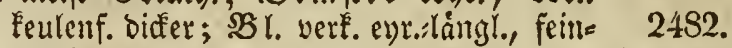
gejabnt $(\odot$ 2.28. hgb.) (pusilla.)

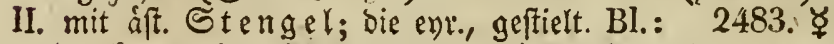

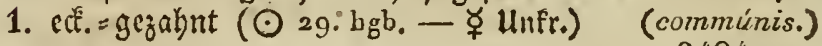

2. bopp. gezabint $(\odot)$

III. mit rifpenf. Stgl; Stgl unterhalb feins 2484. crispa.

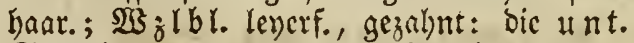
Stglbl. eyr., gejabnt: Die obern lan= zettf., ganjtand. (4 As.)

\section{5. lyráta.}

\section{LASERPITIUUM. 223.}

\section{Blätter:}

1. Dopp: gefiebert,

a. glant. leserart., nit idjieflherjf., fte= d)enropingezahnt, 'Bláttch.; Flúgel ber $\$ 2486$.

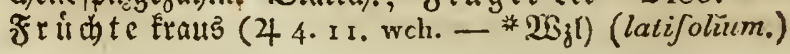




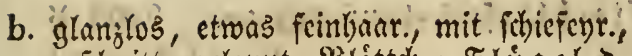

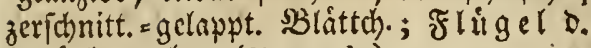

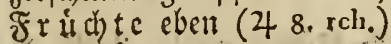

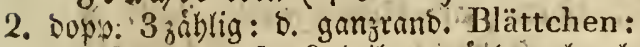

a. voal=lanzettf., 3 theil., geráno., glanjlos * 2488 .
(4 9. w.
* Grant.)
Siler.

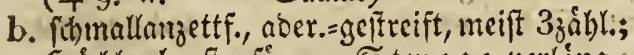
Scủrle borftenform.; E twe ge verlảng.; 2489. baut. (4 9. w., ausivi. rch,; dann w.) peucedanoides.

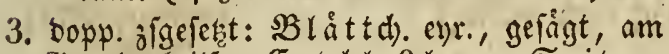
Grunbe kcilf.; Fnobl. 3 lapp: : Seiten= blátter 2 lappp.; Stgl gefurdot, gefledt; Bltticheiden weit; \$y flanze rauch) (4 23. v... "anfangs rch.)

2487.

Libanótis.

4. 3 fad)oopp: gefied.; Blättch.:

a. enruns, fieberf. = gejahnt, Eeilf., medif.; Stengelcinf., faft ftiefrund; gl franze ftad)ethaar. ( $\left.\sigma^{7}\right)$

b. eyr. =lanzettf., fieberf., mit lanzettförn., f(h)arfgeipift., aber. Lapp.: 2(rle unten raud); Stgl faft einf., etwas edf., mit umgeidug. Saar.; Şülten. gemimp., umigeid)lag. (4 4. 8. w.)

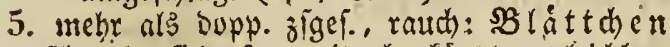
facmal, fiederf., mit abgetiuret., gleichbr., fcharfgeipist. Rapp.; Etgl fitelrumb, ge=

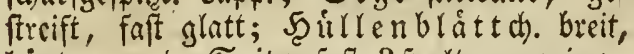
biutt, an b. Spike faft 3 fpart., gewimp. (4 SE. 9. w.)

2493.

hirsútum.

\section{LATHRA'EA. 481.}

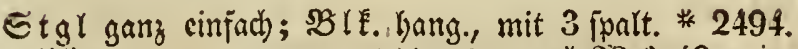
Ulippe (4 1. p. u. w., Bld. rch. - * $2_{3}{ }_{j} l$ ) (Squamaria.)

\section{LATHY̌RUS. 572.}

\section{Blmsticle 1 blüthig.}

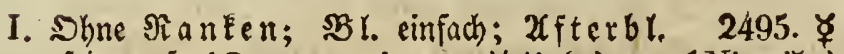
pfriemenf. $\left(\odot 29.30 . \mathrm{hr}_{0}-\Varangle \mathrm{unft}_{0}\right)$ (Nissolia.) II. mit Ranken: 


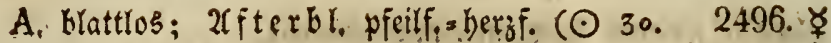
dgb. - - $(\mathrm{ln} f \mathrm{r}$ )

B. Ifle 2 blåttrig ;

(Aphäca.)

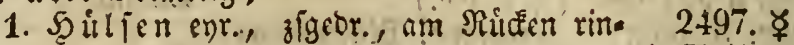
nenf. $(\odot$ SE, $r,-\gamma$ Jutt. $) \quad+$ Cicĕra.

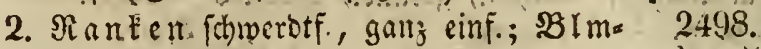
ftle begramit $(\odot$ SE, sch.) .... sphaeřcus.:

3. Blättchen:

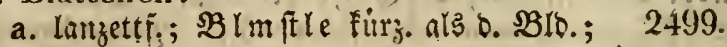
$\Re$ anf. ẹnf. ( $\odot$ As. hr.)

inconspicŭus.

b. borftenf. $=$ gleidfbr. $(\odot$ SE, p. $)$

2500 .

c. längt: = ellipt., ftumpF, mit e. fleirt.

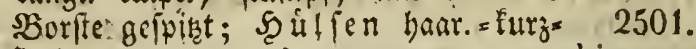
ftachl. $\left(\odot-p_{0} \cdot v_{0}\right) \quad \therefore$ hirtus.

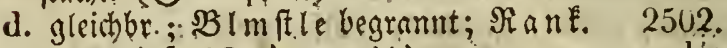
ganz einf ( $\odot$ SE, r.: bl.) angulátus.

C. einige 2:, anbere 4 blâttr.; Hiulsen:

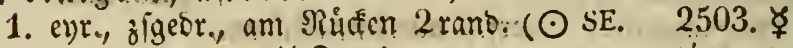

bl. u. r, ; w. - $\Varangle$ filtt.) sativus.

2. aufgeidnwoll., langhact., aufr.; SEIm= 2504 .

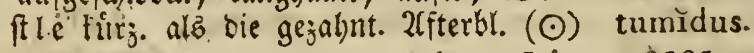

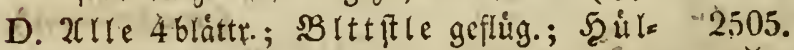
ren jigeor. ( $\odot$ ) spurĭus.

E. vielbláttrig; Blättchen;

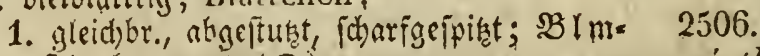
ftle begrannt $(\odot)$

moncinthos.

2. langettfirm., wedf.; $B 3$ lttifle geflug.; 2507. BImfle meift 1 bluth. $(\odot \mathrm{SE}$. p. u. w.) articulátus.

\section{Blmstiele 2 blüthig.}

\section{Ranken:}

1. 2 blåttrig; Blättchen:

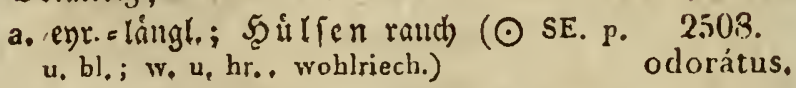

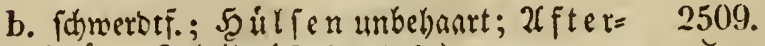
blát t. 2 theil. ( $\odot S E$. bgb.). annuัus.

c. Iamjettf., unbebaart, wed)F; 2 (fterbl. 2510.

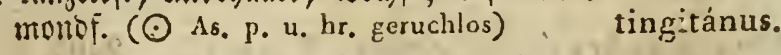

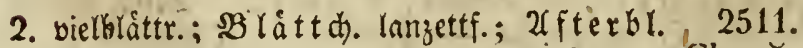

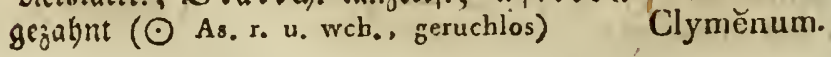


III. Blmstiele meist 2 blïth.

Blattfiele geflig.: bie unt. blattlos: bie

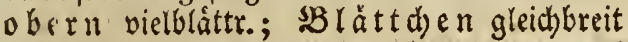
(NAf. bl.)

2512. ténuifolius.

\section{Blmstiele meist 3 blïth.}

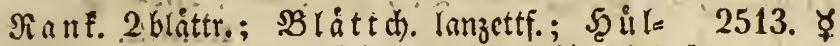

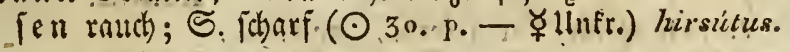

\section{Blmstiele vielblüthig.}

\section{Ranken:}

A. 2 Glåttrig; Blättchen:

1. gleidbreit; Ranken cinfach, febr furz; 2514. Ufterbl. po lang als bie. $\mathfrak{E l}$ l. (Am.) sericěus.

2. oval, ziviftien Den Jinoten nid)t häutig

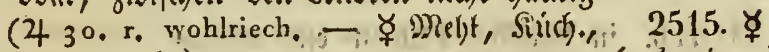
Futt., Lufr.)

3. runol.; เzwifhen ben Sinoten báut. (4 2516 . As. r.) rotundifolrus.

4. lanzettformig;

a. SianE. gan einf. (4 3. gb., wohl- 2517. riech, - ఫ政t.) (praténsis.)

b. Bráttchen zmifothen o. Snot. båut. 2518 . ఫ

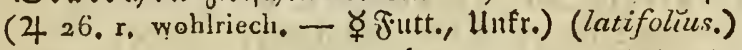

5. ichwerbtf, , zwifhen D. Snot. baiut. (4 3. 2519. ఫ

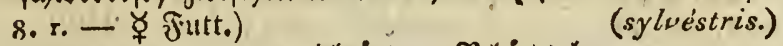

B. einige 2 , andere 4 blattr.; $B \mathfrak{B} \dot{a} t \mathrm{t})$.. lanzettf., jwifhen D. Sinot. båut. (4 8: r, 2520. u. wch.)

heterophylllus.

C. vielblåttrig ; Blättchen :

1. fdmallanzettf., fwis: : ffterblatt. balb= pfeilf., lanjettf., zwifchen o. Jnoten báut. (4 34. 47. bl.-rch.; hr., wohlriech.)" (palisstris.)

2. lanzettf. = langlich, ftumpf, fabarfgefpist, zwijhen ben Snot. båt.; Şน̉lfen ge= frimmt (4 NAs. p.; p. u. w.)

3. ellipt., ftumpf; 2 fterbl. balkpfeilförm., cnr., breiter als bas $3 l_{a}$ attchen (4 bl.; wch.' u. p:)

2522. incúrvus. 2523. pisiformis. 


\section{LAURUS. 325. $\left(275^{x} \cdot 788^{3}\right.$ ?}

$$
\left.79^{5} \cdot \mathrm{W} \cdot \mathrm{x}^{\mathrm{s}} \cdot \mathrm{l}\right)
$$

\section{Blätter :}

1. longlid)= lantzettf., langgefpigzt, ganjramb., ripp. = aber., immergrún; $\mathfrak{z}$ raben in ben Bittwinteln faum láng. als bie SBlattftiele; Bbluth. 4 fpalt, mit gangetrenntem.

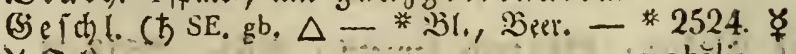
$\Varangle$ Dir) nobilis.

2. verf: eyr:=lánglich, fur;geipist, gantrano., entferntrippp.=aber., abfall.; Seitenblith. * 2525. 4 u. 5 záhl. (b NAin. gb: $\triangle$ Gaft) Benzüin.

3. ungetl). : einige 3 lapp. († NAm, zb. $\triangle$. -

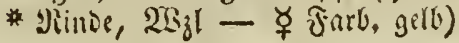
* 2526. ఫ Sassăfras:

\section{LAVANDŨLA 444}

SBluth. in blattloi. Suirln cinte unter= broch. Tebre bilbent, mit-langgejpigt. Reben= blátt.; Die ftiellor. Blätter:

1. "Thmallanjettf., am Sambe lumgerollt; bas unterfesebenbl. 3 fpif.: Die obern

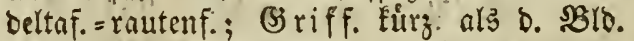

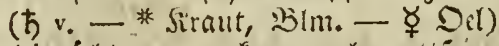

2. Die áltern verf́. cyr. = langettfi, am Ranbe faff eben: bie jünern wie vorker; Urte Rebenbl. 1fpis.: Das unterefaft rautenf.= cyr.: bie obern eyr. =rautenf.; Bitiffel láng. als D. SBlo. (ち SE.: vorch.)

* 2527. Spica.

\section{LAVATÉRA. 548.}

Der Erautart. Stengel:

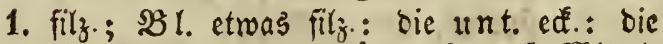
obern 3 lapp., mit láng., ftumpf. Mittels lapp.; $B \mathbb{l}$ Inf fte einzeln in $D$. SBlttwinteln (4 26. p.)

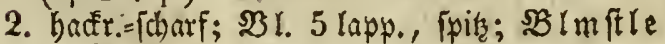
gebauft in 0 . BBlttwint. (SE. glo, grofs)

3. punt. = fđ)arf; $\mathfrak{B}$ l. fternf. zfeimhaar., längl.s langetti., gelägt: bie obern ganjtandig;

2529. (thuringiăca.) cretǐca.

latifolia. 


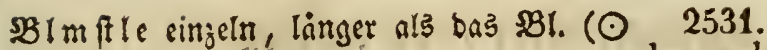
hr. 'grofs)

lanceoláta.

4. nur sben bactis:scharf; bie unt. $2 B l$. runbl.s berzform.; bie obern edf.: ' die oberften

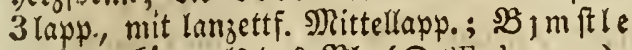

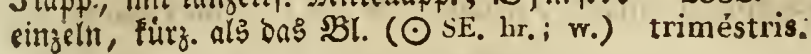

\section{LEDUM. 341.}

Mit ङnobolbentraub.; bie ain Fanbe umgerollt., unten roftbraun = fil

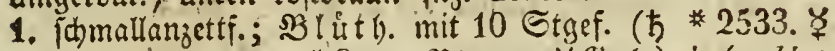

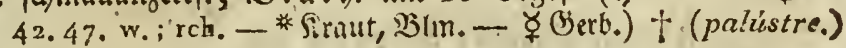

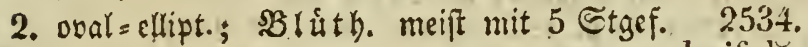
(b NAm, w.) :

latifolrum.

\section{LEMNA. 34. (73o. W. 32.)}

\section{Blätter :}

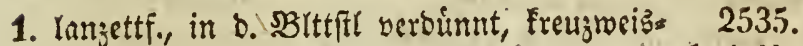
ofluang.; $\mathfrak{N B}_{3}$ l einzeln (4 44.)

2. cuiprija); Wurzel: a. eitrjeln; Blätter:

a. unten blni. =geroilbt $(4$ 44.)

b. auf beyben Seiten frach, aim (Srumbe jibang. (4 44. - Sitaut) (trisúlca.)

2532.

triméstris: 
3. aufr., angebrưft; $B$ lojdupp. cyr.; bie glatten BI.:

a. lanjettf., budjt. = gejabnt (4 3. 48.) (lividus.)

b. fibrotfägef. ofieterf, gejahnt ( 4 SE.) laevigátus.

\section{LEONÚRUS. 459.}

\section{Blätter :}

1. 3 od. 5 lapp., zeridnitt.=gezalynt, am Rambe

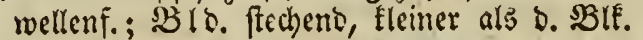
(4 NAs.)

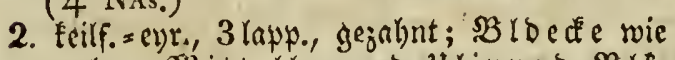
vorber; Nittellapp. D. Ulippe b. BBlE. \#2545. ipira (4 22. rch. - * Siraut)

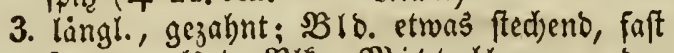
fo lang als D. SBlE.; Mrittellappent oer 2546. UIip \&e D. $\$ 2$ IE. runol. (4 22. 26. rch.). (Marrubiástrum.)

4. 3 theil., mit etwas zetidhnitt. Iapp.; BId. nidst ftecheno; Olippe d. Blk.:

a. flach), aufro, umgejhlag. (ơ NAs. 月.) tatarǐcus.

b. ausggebdglt, gerabe ( $\odot$ NAs. r.) sibiricus.

2544. crispus.

\section{(Cardiüca.)}

\section{7.}

2548.

\section{LEPECHINIA, 453.}

Uehren geftielt, mit Rebenbl.; 33 r. eyrumo, geferbt, am (jrunbe abgefturigt (4 SAm.) spicáta.

15. LEPIDǏUM. 514. $\left(30^{3} \cdot 32^{2}\right.$.

$$
\left.39^{x} \cdot 262^{2}\right)
$$

\section{Blätıer gleichförmig.}

1. Nit 2 Stgef.; $B$ latt. ficberf.; $\mathscr{F}$ rud te 2550 . 2 Enopf. (๑ WE. w.)

II. nit meift 3 Stgef.; $\$ 2$ l. gleidbr., gefied; 2551 .

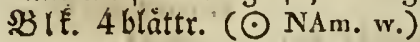
virginücum.

III. mit $4 \mathcal{S}_{\operatorname{tg}}$ ef.; ; $\mathfrak{s}$ l. gefied., ganzrand., unbehaart; b. but thetrag. Stg! nadét, 


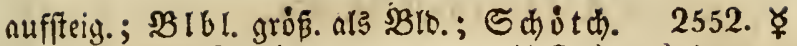
lanjettf., jharfgeipitgt (4 9. w: - ૪ Del) alpinum.

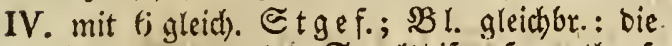
obern gangrand.; Etgl rifpenf.; rutbenf. 2553. (4 26, w. - $\left.\boldsymbol{\gamma}^{2} \mathrm{Dil}\right)$

graminifolüum.

V. mít 4 làng. u. 2 fútr. Ș tgef.; $B \mathrm{Bl}$ :

1. gefied., gangrano.; $2 I^{2}$ l. allsgeranb., 2554.

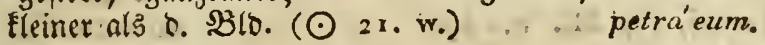

2. Yángl., vierikalt. $\left(\odot-w_{0}\right.$ - Siraut, *2555. ఫ̧ Enam. - ૪ Del, Silidy.) satívum.

\section{6.}

3. Yeyerf., fratts ( $\odot$ "As.)

lyrátum.

4. eyr. = lanjettF., ungetb., gefägt (4 SE. w. - $\not D_{(1)}$

2557. $\not$,

latifolium.

\section{II.' Blätter ungleichförmig.}

I. Mit 2 Stgef.; Blk.:

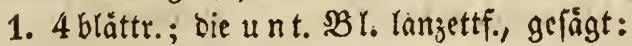

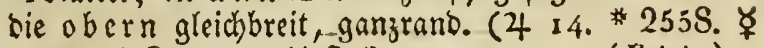
w. - * Siraut - $\not \ngtr$ Det) (Iléris.)

2. Fchlt; $\mathfrak{B}_{3} \mathfrak{l b l}$ gezahnt =gefied.: 3roeis gebl. gleichbr., ganzranto $(\odot$ × 4. 22. w.. stink. - $\ngtr$ Del)

II. mit 4 lóng. $\mathfrak{t l} 2$ furz. Stgef.; Bl.:

1. bie unt. u. $S_{3}$ glbl. bopp. fieberf., mit gleidbr., etwas gejagnt. Rapp.: D. obern berzf., umfall., ganzrano. $(\odot-\mathrm{sb}$.) perfoliátum.

2. bie unt. gefieb.: bie unt. B̉láttchen balbeyruno, oben gezalnt: bie oberfiten f́chmallanjettform., verlang.: bie obert Stglbl. lanjettfo; $\left.\mathbb{S}_{d j} \dot{t} t\right) 2$ zabn. (๑ As, w.)

3. bie unt. fieberf.: bie obern lanzettf.,

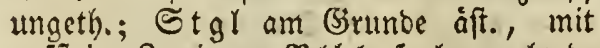
aufiteig. ameigen; BBlbl. po lang als b. BID. ( $\odot$ SE. w.)

4. ungethcilt, ganzrand.: $\mathfrak{B B}_{z}$ lbl. geftielt, enr.: Stglbl. ftiellos, lanzettf.: einige pfeilf. (4 SE, w.)

2559. ఫุ

(ruderále.)
$25 f j 0$.
perfoliátum.

2561.

spinósum.
2562. procúmbens.
2563. crassifolium. 


\section{LEUCÓJUM. 271.}

Blumenscheide :

1. 1 blüth.; (5riff. Eeulenf. (4 x. 3. 48. w. 2564. u. gb.- grach.)

2. mehrbiuth.; Griffel :

(vernum.)

a. feulenf. (4 3. 8. w. u. grn. u. gb. gelleckt) aestivum.

2566.

b. fabenf. (4 SE. w.-n.) autununále.

\section{LIÁTRIS. 642.}

Die gleichbr., ganzrano. Blätter:

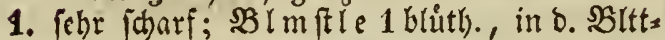

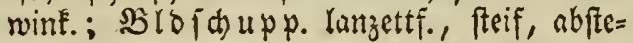
henlo (4 NAm. p.).

2. glatt, am (5irunoe gewimp:, gerippt uno

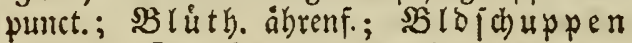
gleid)br., ftumpf (4 NAm, p.).

2567. squarrósa.

$=2568$. spicáta.

\section{LIGUSTǏCUM. 218.}

Blätter:

1. boup. 3 jăblig,

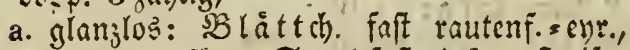
gejahnt=gelägt; Stgl faft cinf., geftreift;

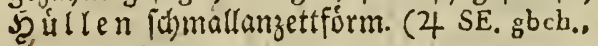
ausw, rch.)

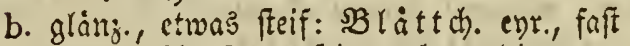
ftiellos, icharf $=$ u. feingejabut: bie unt. am Sirunoe fiederform., mit ungeid)lag. Lapy.; $\mathfrak{S} \pm g l$ fajt einf. (4 8. w., ausw. ich.)

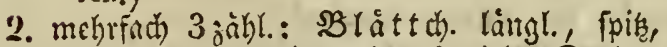
tiefgeiagt; 3weige im Sutrt; Dolde rippenf., jehr zablreid); Şúllen.glcicbr., nbgefintht (4 SE. 9. w., vor dem Aufblühen rch.)

3. Dopl. getieb.; $\mathfrak{B}$ lát t ch). entferntfieberf.: $\delta$. unterfien abgeftukt, mit meift 3 ipalt., foift fichelf., am ßance idharf. Rapp.; Stgl gefurcht; $S_{2} \dot{u} l$ le fiederf̣. (4 ghch.-w.)

2569. scotǐcum.

(Cervarǐa.)

2571. nodiflórum.

2572. athamantoides. 
4. mehrfach bupp. zigcicet; Stengel:

a. gefterift, fierrumb; SBratt. 3 froch. Soph.

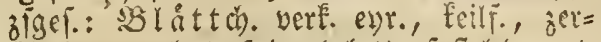
idnnitt.=gesulnut, ipiegelglatt, faff lcoerart. (4 9. gbel.. - * Sirnut, 26?l, Enam.) Levistĭcum.

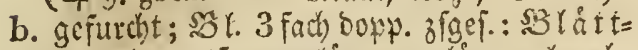
chen lanjettf., verlätg., Glanz., berab= rauf. gefied., mit fchatfficoerform., an ber Spitse fomalern capp.; Seitendoro. 2574.

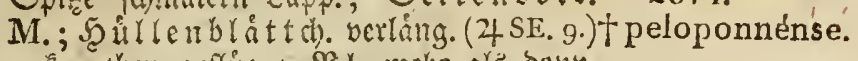

c. eat., dben geflitg.; 33 l. meth als bopp. ófgef.: SBláttch. geftictt, cyr., berablauf. gefico., mit zerichnitt. = Geforgt., icharfige=

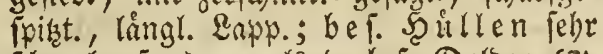
fitmal, fo lang als b. bef. Doloen (4 As. ros., dann w.)

2575. alátum,

\section{LIGÚSTRUMM. 11.}

:B r. ellipt. Yanzettf., umberkart; $\mathfrak{I} \mathfrak{r} \mathfrak{a}$ ben

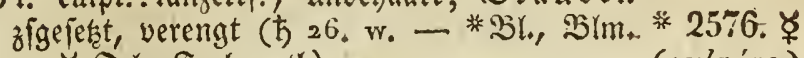
- ૪ Del, Jarb. rotly)

\section{(vulgáre.)}

\section{LILIUUM. 282.}

Blätter:

I. fåmmtl. in Suirrm; Bliithen:

1. umgeíhlagen; Blätter:

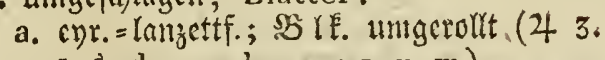

5. 8. dr. u. sch, ; w.; r. u. $w_{\circ}$ )

b. Innzettf.; $B$ IE. glodenf., mit umge= fhlag. Eaum, inm. punct. (4 NAm. r. u. schw. punct.)

2. aufrecht; ş IE. glockeñ.: Blbl.:

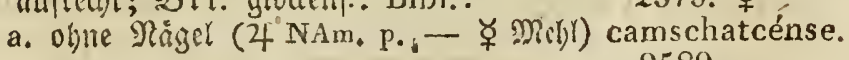
2580.

b. mit Ningeln (4 NAm. r.-gb.)

II. nur die unt. im surut: D. itbrigen zer=

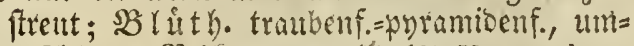
gefdlag.; $\Omega^{3}$ lf. umgerofft (4 NAm。gb. u. schw, u. orange)

III. 3jofbl, einige zerftreut, gleichbr., fait 3 ripp.: bie obern am Nanbe etroas fillz;

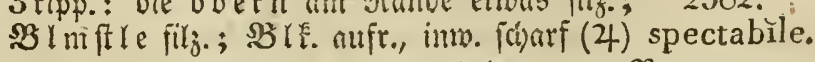

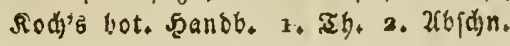
philadelphrcum.

2581.

supérbum.

(Martägon.)

2578.

canadénse. 2579. ఫ్ 
IV: 2crle zerftreut; Blk.:

1. glocfenformig, invendig

a. glatt; $\mathfrak{B}$ l. langettf., am (Sirunse fdyma= Icr (4 SE. w., wohlriech. - * $\mathfrak{I S Z}_{3} \mathrm{I}$, * 2583. Dilm., Cotbeut.)

candĭdum.

b. fabrf, alfr. (4-3.22, r.:gb.)

2584.

bulbifïrum.

2. aufr., mit langen Rảgeln, am Rande wellenf., an b. Spitze umgeichlag.; $\mathfrak{B}^{2} \mathrm{Y}$. famallanjettf.; Stgl 1 blitt). (4 NAm. r., schw. punct.)

3. umigeitalag., mit umgerolut. BIbl.:

a. inment. warz. =gezabnt; $\mathfrak{R}$ l. glechbr. pfriement. (2 SE. 9. r.; gb. punct.)

2585.

Catesbáei.

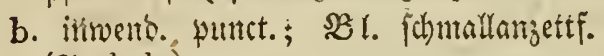
(4 $\left.\mathrm{dscb}_{0}\right)$

2586.

pomponIum.

2587.

chalcedonicum.

c. inw. mar's:= punct.; SB lâtt. gleichbr.; $258 S$. SBImftle verláng. (4 SE. 9. r.) pyrenarcum.

d. inw. warg.; $B$ l. ftiellos, 5 riph.: die obera berof. =evruno (4 As. r, schw. punct.)

2589.

tigrínum.

\section{LIMODÓRUM. 715.}

Stgl blattlos; Shaft mit Sheibe;

1. SBbl. aufr.: Rippe eyr., am Rante wel= Icmf.: 50 orn priemenform., fo lang als 8. Fruch! En. (4 r. 5: v.)

2590.

(abortivum.)

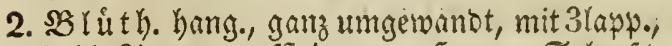
hohl. Ripp. u. auffeig., cyr. Şorn; Sdhaft mcnigblutb. (4 9.)

2591.

Epigogiunı.

\section{LIMOSÉLLA. 488.}

2Bl. lanjettf. = patelf.; Sd) aft furiz. als bie BI. (4 50.)

2592.

(aquatica.) 
14. LINAR 496.

(Die meisten Arten $H$ )

\section{Saamen eckig;}

B. Blkspornläng. als Blk.; od. eben so lang.

\section{Blätter:}

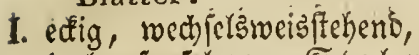

1. leerzf.; 5 lapp.; Stgl nieberlieg, (4 23. 2593. Ұ 24. v.; w. - ఫ Unfr.)

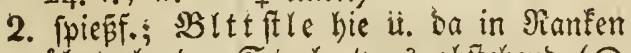
iubergchent; Stal etwab abjtehent $(\odot$ NAf. hbl. u. wch. u. r.)

3. runol.=evr., haar.: bie itnt. unocutlith gezabnt: Die obern faft ftiellos, gan $=$ rano.; Stgl nieberlieg. (4 2S, gb. u. p.

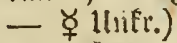
cirrhósa.

II. nicht ectig,

A. 2ulle entgegengelest, 4 jôhl.,

1. gleidbr.; Ier, blutbetrag. Stgl aufr., ábrenf. (4 SE. grau.bl., wohlriech.)

2. 仿mallanzettr., glanjlos; Stgl weit= (c) weif.; sblut th. traubenf., mit geras. Sporn $(\odot$ 9. dbl. u. gb.; p.)

2595. ૪

(spzcrŭum.)

2596. purpurø̆a.

\section{7. \\ alpina.}

B. nur bie unt. entgegengef.; Bl.:

1. fel mallangettiorm.: bie unt. 3 zál)l; Stgl aufr., åbrenf. ( $\odot$ SE. bl.; gb. mit. bl. Sporn)

2. pfricmenf., rimenf., fleifo).: bie unt.

- 3jåbl.; Stgl rifpcnf. U. nebit S3lé. ganz unbebaart ( $\odot$ SE. gb.)

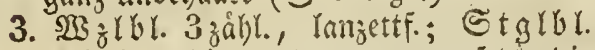
gleidbr.: bie unt. entgegenge pert; bie obern wechlo; s5 (égelm aufr., flach, 2 their. ( $\odot$ As. bl.)

C. Ucte med) felsmeisfteleno,

\section{8. versicŭlor.}

2599. spartěa.

bipartíta.

1. langettf., Yanggefpint; Я i fpe ruthenf., bin u. hergctog. (4 gb.)

2. fomallangettf.; $B$ lútledt traubenf.; SBlb. laing. als o. sBle; Stgl aufr. $(\odot$ SE. w. u. v.)

3. langettf. = gleidbur., getrángt; Stgl

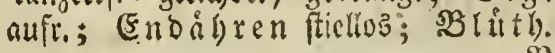


backjirgelf. (4 22. gh., Gaumen langhaar., feuergelb - * Siraut - ૪ Tarb." 2603. ఫ getb, Ilnfr.)

(2) $\mathrm{m}$. Eime monfrofe 2 batt bierbon if sic Peloria; $\mathfrak{A}$ e hre $9-18$ blith.; $\mathfrak{b} 10$.

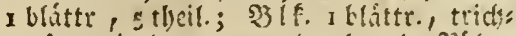
terf., mit lang., geras., bauch. Sithre 11. 5 fpalt, Saum: 5 siect.: au Der

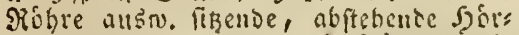
ner, fapt fo lang als tie 9iobre; mit 5 glcidlangen $\subseteq \mathrm{tg} \mathrm{c} f$.)

B. Blksporn liüz. als d. Blk.

Blätter: .

1. im Şuirl oo. zerftreut, gleichor., graugrin; Stgl ripenf.; $\mathbb{B}^{2} \backslash \mathrm{d}$. unbebant (4 $\mathrm{SE}$. w. u. bl: gestreift, geruchlos)

2. entgegengel, verE. enr.: Die Bl. an ben B lutb. med) f.; Stgl aufiteig., feinfar.; Blfipor a gerabe (4 SE. bl.)

3. 2lle wechfelsweisftehend; simfte in 5 . Blttwinf.; Bl.:

a. fohmallanzettī, ftumpi, u. nebft s. aift.,

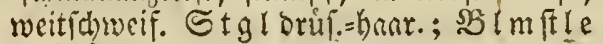
vicl läng. als SBlb. (

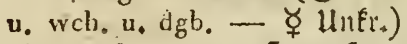

b. lanzettf., etwas ftumpf u. nebit o. aift.,

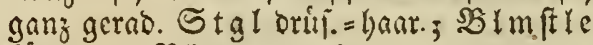
tury. als S5lo. ( $\odot ~ 36$.

2604.

repens.

\section{5. \\ origanifolía.}

\section{Saamen nicht eckig.}

\section{Blätter:}

I. XrY e entgegengefest,

1. 3 joibl., 3 rippig,

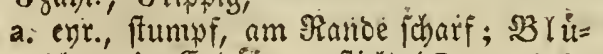
then in Endábre geftielt $(\odot$ SE. hgb. u. dgh.)

b. enr. = tam;ettf.; Brơthen in Endibre ftiellos $(\odot$ NAs, gb.)

2. 4 jåhl., lanjettfơrm.; S $\operatorname{tgl}$ aufr., åft.; B luth. geptictt (4 SE: p.)

3. 5 jadhl. gleidhbr., fleifd.; 3 rit then in אifipfd): ( $\odot$ SE. wi, Sporn bl.)

2608
triphyilla.
2609.

latifolǐa. 2610. triornithopophŏra 2611. multica' ulis. 
II. nur bie untern:

1. entgegengejefft, bie ibrigen jerftreut:

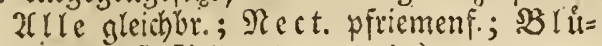
then faft ffiellos (4 SE. dr.)

2. 4 zallig: 2flle faft gleid)bi.; S5 Is. baat.=

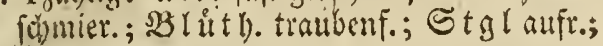
Biksporn:

a. gerabe ( $\odot$ SE. bl.

b. gefrumnat $(\odot 28 . \mathrm{gb},, \mathrm{bl},-\Varangle$ lnftr.) (arvénsis.) III. 2T the wedteläweisftebeno,

1. berzf., umfaff. (○ SE. gb.)

2. lanjettf., ftadielhaar.; ş luth. ibrenf:; bas oberfte $\mathfrak{B}$ loblát to en jelyr gruás (๑ SE. gb.)

IV. mur $\Xi t g \mathfrak{l b l .}$ เoch) 3 jabl., lanjettf.; $B$ rit th. in Dolcentraub. ( $\odot$ SE. bl.)

2612

tristis.
2613. simplex. 2614. 후 2615. dalmaț̣ca. 2616. hirta.

2617. pelisseriána.

\section{LINDERNIA. 489.}

BI Infle 1 blith). in belt SBltmint.; bic ftielloi. Blätter:
1. laugl.=evruno, ganjmano., 3 ripp.; Stgl niederlieg. ( $\odot 42.50$.hp.) As. brnch.) japonica.
2. vert. evr., gefingt; mit Enotraub: $(\odot .2619$.

14. LINNA'EA. 479.

sBluth. gepant (ち 1. 5, 9. 13. w., rch.-w. * 2620. - * ל̇.) borẹalis,

\section{LINUM. 256. (88 $\left..541^{2} \cdot\right)$}

7. Blätt. wechselsweisstehend od. zerstreut;
A, Blk, bbau, roth $\left(z u w_{q} w.\right)$

I. Bidecke:

A. langgeppikgt; Blätter: 
1. wedf.: 3weigebl-entgegengerest; $\mathfrak{B l}$ lo. rauch), ffieitos, wed)j. (4 8. 33 . bl.; fl.)

2621.

Tirsítum.

2. lanzettf. zerftrent, ganz gerace, icharf; Stgl fielrund, an cirunbe sif. (24 19. bl.)

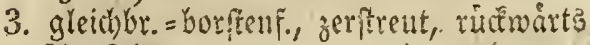
icharf (4 33. ros.; p.; w.)

4. Feingar.; bie unt. 5 ripp.: D. obern

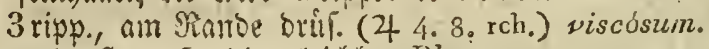

B. runol., ftumpf; dic gleichbr. Bl.:

a. etwas fpit: Stengel abmairtsgeneigt (4. 9. hbl.)

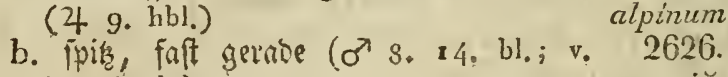
Nagel gb.)

II. Bldeckblättchen:

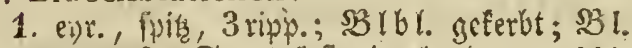
lamzettf.; Stgl faft cinzeln $(\odot 30$ hbl。* 2627. భ

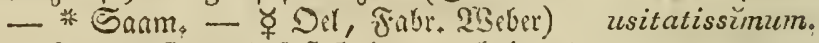

2. verf. eyr., ftumpf, faft 5 ripp., unbebarart; Stgl zablreid), aufiteia. (4 3. 1 r. hbl.;

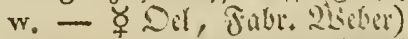

3. ellipt., fait 3 ripp., nebf Skayf. lang= geipiat; $\Omega^{3}$ r. fibmallanjettform., 3 ripy.; Stgl jablreich) (2 SE, hr.)

2628. ఫ̧ perénne. narbonénse.

2623.

(tenuifolüum.)

2626.

austriŭcum.

\section{B. $B l k, g e l b$,}

\section{Blätter :}

1. am Grunoe auf beyd. Seit, punct., orif. 2630. (4 SE.)

2. nicht; BIdecke:

$a_{9}$ pfriemenf.; Bl.:

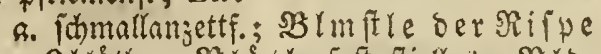
2 bluth.; $\mathfrak{B}$ lut th. faft ftiellos; Bro. pith $\left(\odot \mathrm{SE}_{*} \mathrm{gb}\right.$, , dann wo)

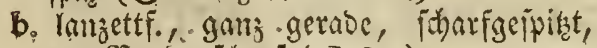
am Sande joutf ( $\sigma^{7} \mathrm{SE}$.)

b. evr., fpí, webrlos; $\$ 3 l$ l. lamjettf.: sie untern entgegengef. (4 53.)

c. pits, wechi.; Stapi. mebrlos; Stgl ripenf.; $B$ l. lanzettf.; $: 20 ; l b l$ enrumb (๑ NAm.)

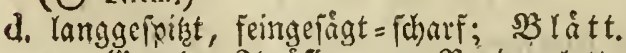
campanulátum.

2629. angustifolıum. am Srunbe 2 orufitg, a!n Name glatt; 
Linum. - Lithospermum.

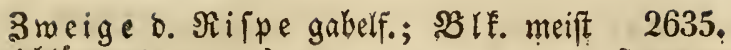
1 bláttr. (4 2..3.) Jlavum.

II, Blätter entgegengesetzt.

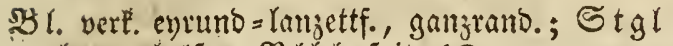

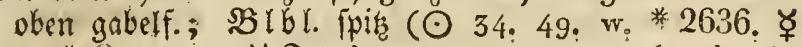
- * Siraut, - $\Varangle$ jutt.) (catharticum.)

\section{1, LIQUIDÁMBAR. 754. (W. 6o.)}

Blätter hantof. $=$ gelappt, unterlyalb in ben 2 Binfeln Der 2Cocrn am Girmnde

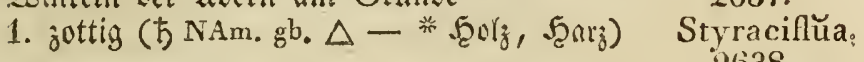

2. unbehaart (ち As. $\Delta$ )

* 2637. 2633. imbérbe.

16. LIRIODÉNDRUM. 430 .

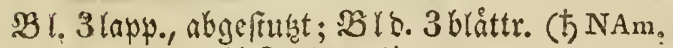

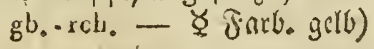

2639. 후 Tulipifera.

\section{LITHOSPÉRMUM, 99.}

Blkrone:

1. getb oo. weis; Blätter:

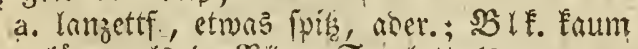

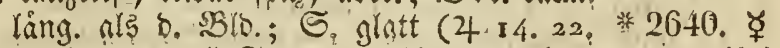
ligb.; w. - * Saam. - ఫ Mien, Der) (olficinále.)

b. Innzettfórm.=gleichbr., ftumpf, aberlug,

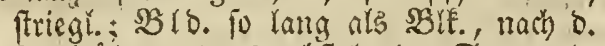
Serblitien etroas abftelent; S. rumzl.,

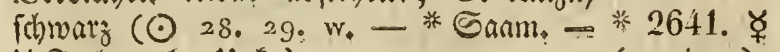
ఫ̧ Farb. roth, Unfer.)

c. Thmallangettfo, fpir: Endo a huen 1 feit.,

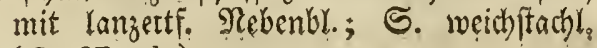
$\left(\odot \mathrm{SE}_{\mathrm{g}} \mathrm{gb}\right.$.)

2. blau ob. roth; Blätter: (arvénse.)

a. Yanjettf., aberlos, an beno. Enden fpir;

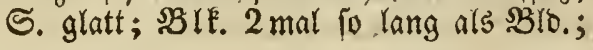




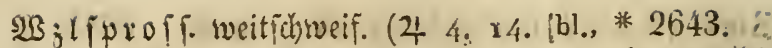

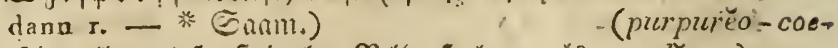

b. fdmallanjettf., frriegl.; $\Re$ lo. fo lang als ruleum.)

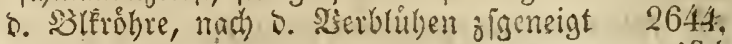

$(\odot$ NAf. bl.)

c. gleidbit; S. glatt; $2 B 18$. abftchento, ein= isårtsgefrummt ( $\odot$ SE. hr.)

tenuiflórum.

264.).

dispérmụum,

21. LITTOPELLA. 739. (W. 10.)

(4 43.)

\section{6. lacustris.}

\section{LOBELIA. 148.}

\section{Stengel aufrecht.}

\section{Blätter:}

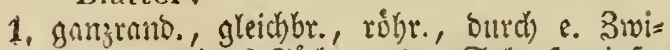

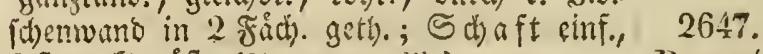
fâft nacăt, åfít. (4 38. 42. lili) Dortmánna.

2. nimbt ganzrandig,

a. ichnallanzettf., fcingezahnt, am Sanse umgerollt, u. nebft Stgl feinlyar.; mit (Enotia $\mathfrak{u} \mathfrak{b}$. (4 SAm, sch., ausw, feinhar.) fulgens.

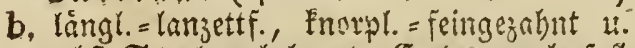
nebft Stgl unbebaart; Enotranb. faft 1 ieit., etwas blattr. (4 NAm. sch.)

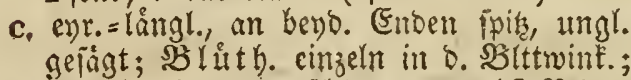

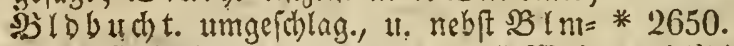
ftren frachelbaar. (4 NAm. bl. - * $2 \mathrm{~S}_{3}$ l) syphilitica;

d. hergf., unoeutlich gejalont, gefficlt; mit 2651. EnDórbentraub. (○ NAm.r.) Cliffortiána,

e, bie unt. entr. =lángl.; bic obern enr.: 2ulle gezabnt=gefágt; Stgl ftachellhaar.; SBlm triefe cinjeln in ben Silttwinfelln;

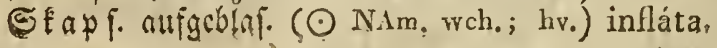

\section{Stengel nicht aufrecht,}

Stengel:

1. etwass abftehent: $B 3$ r. etwas gejabnt: bie unt, ellipt., geftielt: bie obern fchmallan= 
zettfo, faft friellos; B S I m ft le 1 blüth., ling. als bas SBl. ( $\odot$ SAf. bl.) 2653.

. niebergerínus. gegater

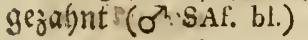
2654. Erinoídes.

\section{LOEFFLINGIA: 38.}

SBr. pfriemenf., finarfgefpift; 3 r hith. ftielloz in b. SBlattwint. $(\odot \mathrm{SE}$.)

\section{Beeren:}

\section{LONICËRA. 152.}

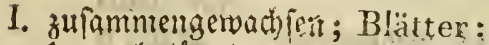

1. onal=längl., langgefpirt, weirig gewimp.; BImftls 2 blith., viel ling. als s. SBlit= then; ; Beeren 2 fnopf. (5) 9. braun - lila - r.o Firucht r.)

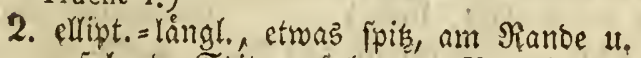
auf beyd. Seit. unbeharert: Blmitiele 2 blút)., Eürzer als D. SBluthen (b) ugb., Frucht bl.)

3. rundl.=l)ergf., fill.;, $\mathfrak{B} \mathrm{l} \mathrm{m}$ ft le wie vorher (ち NAs. ugb., Fr. r. )

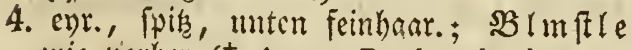
wie vorker († As, - Frucht schw.)

5. fingl. u. oval elr., unbehaart; $s \mathfrak{B}$ inftele 2 bluttl., lang. als die Shlitbe; $B$ eeren foft inmener zigeryadbjen (5 8 , brnch,.- lila, Frucht schwarz)

II. abgefondert; Blätter :

2656.

alpigènạ.

coeruléa.

2658. iberica. 2659 . orientális?; 2660. (nigra.)

1. eyrumb,

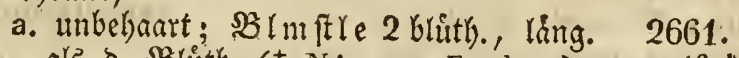
als b. Bhluth. († NAs, w.. Frucht r.) parvifolǐa.

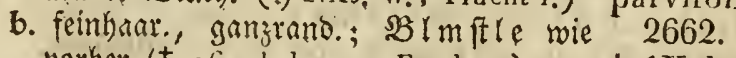
vorher ( 526. gbch, - w., Frucht $\left.r_{0}\right)$ + $+\left(X_{y}\right.$ losiěcum. $)$

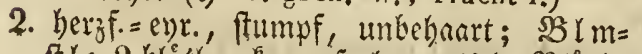
ffle 2 britt., Eaum fo lang ats D. SBluthe 2663. ( 5 NAs. r., Frucht r.) tatarica.

3. verfe. enruno=lanzettf., unbehaart, unten 2664 .

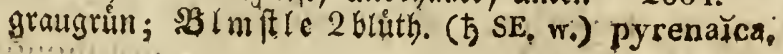




\section{LORÁNTHUS.784. (W. 19.)}

Mit rinf. Enotraub.; SBlith. mit ganzgec, 2665. trennt. (Siejchl. († 5 I. auf Eichen) $\therefore$ europa'eus.

\section{LOTUS. 589 .}

\section{Blumenstiele 1-4blüthig.}

\section{Huilsen:}

1. einzeln,

1. báutig = 4 ectig; Blätter:

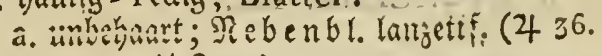
tb. - ૪ (jutt.)

b. unten feinbant; S.tgl nieberliegers

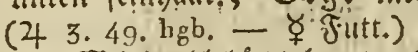

c. Das Nittelblattch. etroas gezahnt; giebenbl. eyrumb; Stgl wie vorber $(\odot$ SE. r. - ఛ Mebl, Hufr.)

2. getrummt, yơftr., nteifi einjeln $(\odot$ SE, 2669. ఫ̧ gb. Ұ Ұ Үichl)

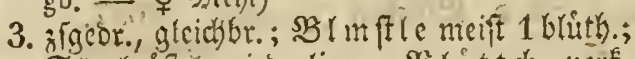

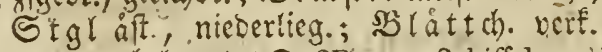
ey)r., unbehaart $\left(\odot \mathrm{SE}, w_{0}\right.$, Schiffch, an d. Spitze v, Fähnch, r, gestreift)

2670.

coïmbricénsis.

1I. 2 zállig,

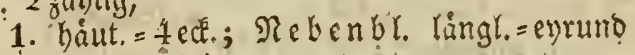
(○ SE. gb.)

2. etruas jfgebr., gleid)br., ilbergebog., meift 2 jåbl, ; Slátt d)en vert. cyruns, baar.; Stgl nieverlieg. ( $\odot$ SE.)

3. gleichbr., ganj aufr., meift 2 jofhl.; $\subseteq$ gt

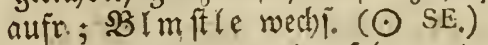

4. ftielrumb, ganz gerabe, fehr zart; $\$ \mathfrak{Z l} \mathrm{l}=$ ftiele meift 1 biuth.; $S$ t gl fehr át., nie= berlieg.; $\mathfrak{B} l$. U. $\mathfrak{B}$ Io. ganr. ( $\odot$ SE.) diffúsus.

III. meift 3 jạhlig,

1. walzenf., begrannt; Stgl niebergeftredt; SBlmfti 3 blith); Rebenbl. 1 blîttr: (4 As, bbraun, Fähnch, r. gead.)

2. jfgeor., bogenform.; Stgl reitidneif. (○ $S E, 48, g b$.

2671. conjugátus. 2672. peregrínus. 2673. angustissimus.

2674.

diffisus, $2667 . \not{\zeta}$ (siliquósus.)

\section{)}


3. fietrunb = pfriemenf.; gerabe; SBlb. gee vimp.; $B$ láttd. lángl.; Stgl aufredbt $\left(\odot S E . g b_{0}\right)$,

IV. meift 4 jabl. $i$ ftictrumo, begrannt; Stgl

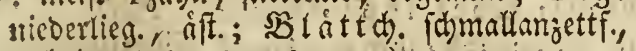
unbebaart (4 SE. gb.-grno)

2677. gracùlis. 2678. tenurss

\section{Blumenstiele mehrblüthig in Köpfchen.}

Küpfchen:

1. rumol.; Stght aufr., ftacherbaar., mit ficts. runo. 2leften; $\mathbb{B} \mathfrak{l}$. 3 zóhl., mit oval., jott.

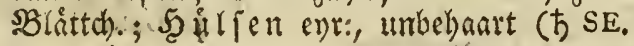
rch. $\triangle$ )

2. Foft Euger.; Stglunifr.r, ftierruno, unbe=... . . haart, mit unbeutl. 4 feit, etwas frachelfaar.

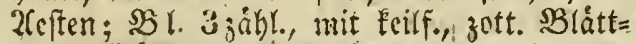
dent : SS ir f en.ftielrumb, gerabe, umbehaart (5 SE, rch, $\triangle$ )

3. balbfugelig;

a. Shilficn langl., figebr.; SB is fo lang 2681. als b. SBle; ; Nebenbl. 1 bláttr. ( $\odot$ NAf.) parviflórus.

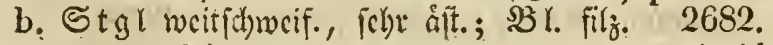
(4 36. gb.)
cytisoides.

4. viebergeoruat; Stgl mieberlieg.; Șillen walizenf., abfertent (4 14. 19. gb., wohl-

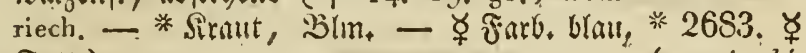
Fitut.)

2680 (corniculátus.)

\section{LUNARIA, 516,}

\section{Blätter:}

1. berziórnig,

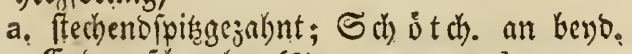

Enten ibimaler (4 4. 23. 48, braun-r., woluliech.)

b. ftumpfgezahnt; S b b id th. ellipt., an beub. 2684. Enben ftumpf (ơ 4. 3. p.; w:, geruchlos), (annüa. )

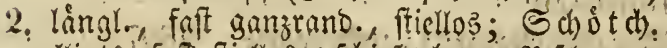
cllipt:, faft fticllos'; fetiefgebog.; won fternif. gefterlt., feinen Jaaren weikl: (4 St. gb.)

2686 . cañéscens. 


\section{LUPÍNUS, 559. $\left(542^{3}\right.$.)}

Blätter :

1. cillf.; langl, langhaar,; 30 ro, in e. balb.

auirl, mit Unlang: Soberlippe 2 palt: 2687. ulip pe ungett). (NAm, rch., inw. e. v. Fleck) villósus,

II. fingerformig; Bldecken:

A. mit 2ungang,

1. in e. baltin sutul: Slippe 2 palt.: 2688.

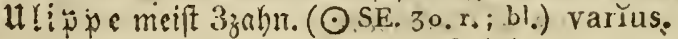

2. in e. gatjen suirl: Slippe 2 theil.:

Unterlippe:

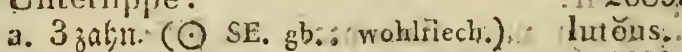

2689. 2690.

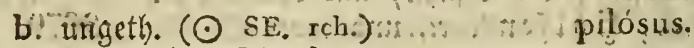

3. medyelsincis; Oberlippe:

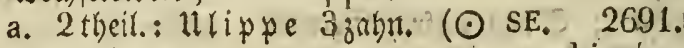
blch.)

hirsútus.

b. 2 jpalt:: Unterlippe:

- a. faft 3 palt:; Slattch. 9 jábl., 2692.

gleidbor., rimnenf. $(\odot)$ linifolĩus.

b. ungetbeilt; B lát tben 7 zåd., 2693.

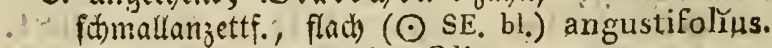

B. ohne 2(nhang u. wedfielsweis: Olippe:

1. ausgerano.: urippe ungeth. (4NAm. bl. v. v.)

2. ungeth.: Ulippe 3 zahn. ( $\odot$ As. w. 2695: ఫ

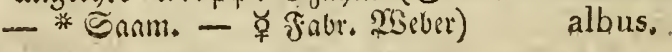

10. LYCHNIS, 365 . ( $358^{2}$. $362^{4}, 791^{3}$. W. 81 .)

\section{Mit Zwitterbluthen,}

\section{Bliithen :}

1. gebuifhelt, gleich)och (4 NE. sch.; w.; 2696. gb., f.) chalcedonica.

II. nicht; Blblätter :

1. 2 fpaltig; Bliuthen:

a. cinzeln; $: B$ l. fohmallanzettf., unbehaart; 2697. $\Re$ Bo, mit 10 Rúffeniỏárfen $(\odot, S E$.) laeta.

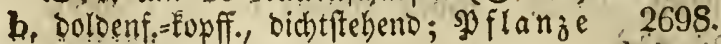
unbehaart ( 4 o' SE. 9. r.) alpínà. 
2. 4 zahnig; Stgl gabelf; ;Bl: unbelanat, 2699. umgeftrimmt. (๑ 8. w.) (quadridentáta.)

3. 4 fpalt.; $₹ r u c h t$ rumbl. (4 49. p.; w. - 2700 . $ఫ$

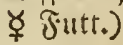

(Flos Cucǔli $)$

2701. ఛ

(Viscaria.)

4. unget). (4 3. x 1. p.; w. -

IIs mit ganzgetrennt. Geschl.

SBlE. 2 palt.; $B$ l. feirhgar.; Stengel:

1. feinhaar.; ভEa

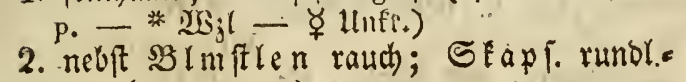
eyr. (4 4. 49. r.)

(diǒca:)

2703.

sylvéstris.

\section{LYCIUM. 143.}

3 iveige borr.; Staubgefälse:

1. To lang als b. SBlffaum.; 3 weige mieter= gebog.; B Brmftre láng. als $\mathrm{D}$. SBlo.; die unt. B r. gebilichelt: bie obern wechf.: Alle Bl.:

a. idmatlanjettf., etroas fleifh., am Srunte fojmaler (古 NAs. lila - $\mathrm{r}_{0}$ )

b. lanjettf., am Briunde fojmaler; Bro. $2-3$ part. (ち $S E$. lila-r.)

2. Hủzzer als ber Blefaum; 3 weige fhlaff; Br. fothallanzettf., ftumpf, foriefgebog. (5) $S E_{0}$ gb, ; t. $\Delta$ )

2704.

ruthenicum.

2705.

barbărum.

2706. europa'eum.

\section{LÝCÓPSIS. 93.}

Stengel:

1. auft., aff.; $B \mathfrak{l}$. tangettf., harbumfail., et=

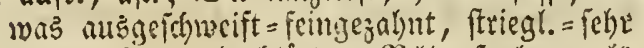

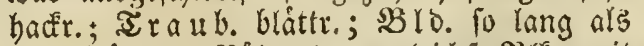

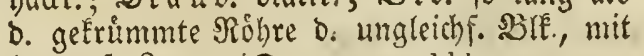

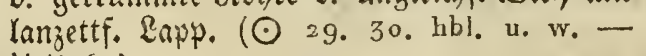
ఈ llufr:)

2. auffeig., faft cinf; ; 3 1. ent. = lämln, balb=

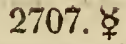
(arvénsis.) umfaff., warr., hacfr., musgeichweift = ge= zahnt; $\mathfrak{x}$ raub. einzeln, unterlyalb nit gie= 
benblatt.; BZlf. wie borber, mit feinbaar. 2708. Silappen ( $\odot$ SE. bl. u. w.)

variegáta.

\section{LYCŎPUS. 23.}

Biätter :

1. eyruno =lanjettf., langhaar., bucht. = gejagt (4 38. 45. w. u. x. punct, - ૪ Farb. [dhroarz, Futt.)

2. garj lanjettf. : bie unt. àm Sirunbe fieberf. : Die obern entferntgejägt u. nebjt Stgl inbebaart (4 SE. w.)

.

3. 2lle fieoerf., ftad)elfaar., nith langl., et = 2711.

ras. gezalynt. Eapp. (4 SE. w. u. r. puuct.) exaltátus,

\section{LYSIMACHัA. 112. $\left(540^{\circ}\right.$.)}

\section{Bimsitele 1 blüthig.}

\section{Blätter :}

1. $3-4$ jábl., eyr. of. eyr. lanzettf., etwos ge= fielt, unten feinlbaar. u. braumpunct.; $9 B / \mathrm{m}=$ fticle im suirl in o. Blttwint.; SBlbl.

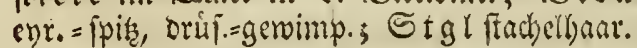
(4 4. 48. gb.)

2. entgegengejest;

a, eyr., fpis; sBluth. cimjeln; Stgl nic= 2713. oerlieg. (4 4. Is. gb.) (nemorrum)

b. IanjettF., fticlos; $\mathfrak{B l}$ Imfte in $\delta$. Blatt= wint. entgegengel.; Stgl jehr åf., unbe=

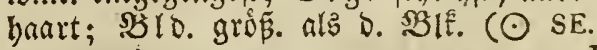
w. - grnch.)

2709. ఫฺ

(europá eus.)
2710.
2712. püinctúta.

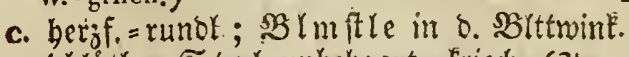
1 blutb.; Stgl unbebaart, frich. (4 I. 15. hgb. - * Siraut - ఫ jutt.)

\section{Blmstiele vielblüthig.}

\section{Traubenbliuthen:}

1. am Enoe ficherto; Blätter:

a. lángl. =lanzettf., 3-4 jáal., entgegenger.; Irauben zigefetet; (4 386 42. gb. - 


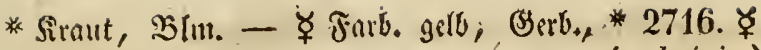
Futt.)

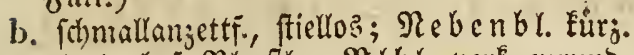
als \&. ber. Bimftle; B̊lbl. verf. evrumb, ctwas abfteheno ( $\odot \mathrm{SE} . \mathrm{dr}$.)

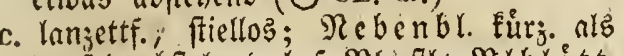
ber febr abjebend. bef. SBlmftl; sBlblátt. lanjettf.; etras abftebeno ( 4 NAm: gb., $\mathrm{r}_{\text {: }}$ punct $_{\text {) }}$

2. in o. SBlittwink., gefielt; evr., zigeoriangt, fúrz. als tas $\mathfrak{B l}$.; $\mathfrak{B}$ l: entgegengel., lan= zettf. (4 42 ; gb.; r. punct.)

2717.

Epheměrum:
2718.
stricta.

(thyrsifiora.)

\section{LYTHRUM. $378 .\left(30^{x} \cdot 2638\right)$}

I. Nit 12 Stgefínen แ. entgegenge\%. Bl.:

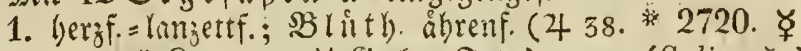
p. - * Sirmut - ఫ̧ (jorb., Fittt.)

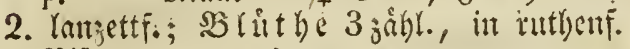
Rippe (4 48. r.)

3. etwas filf: 11 . geftielt; $\mathfrak{B} \backslash \mathfrak{u} t \mathfrak{h}$. in Sei= tenquirti ( 4 NAm, hp.)

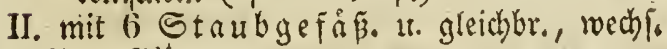
2Bl.; Blk.:

1. Gbrâttr. ( $\odot 50$. p. u. w. $)$

2. 4 bláttr. $(\odot$ 47.)

(Salicaria.)

2721:

ritrgétum.

2722.

verticillátum.
2723.

(IIyssopifolia.)

2724.

( 7 hymifolia.)

19. MADЦА. 692.

Die lanzettf., fot)micr. Blätter:

1. umfaffeno $(\odot S A m$.)

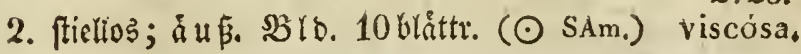

2725.

mellósa.

2726.

\section{MAGNOLIA, 431.}

\section{Blätter:}

I. Daureno, vert. c)r. ob. oyal=långl. ob. längl.s langettf., leocrart., oben glånz., unten grun, 
zumedlen roftbraum; SS IE. auit., 9-12blittr.,

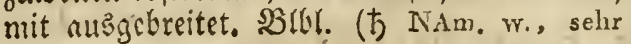
grofs $\Delta$ )

II. abjalleno,

2727.

grandiflórá.

1. obal u. långl., unten graugrůn; SBle. 9-12 blättr., ntit jigeneigt., eyr., ver=

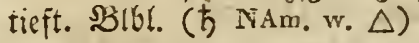

2. oval it. lanzettf., ausgebreit. =abftel $e_{e n t}$; bie áltern unbehaart: bie jüng. fein= baar.; 35 If. 9-12 Glattri., mit berabbang.,

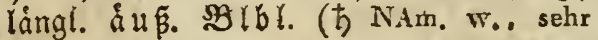
grois $\triangle$ )

3. oval, langgefpict, unten feinhaar.; $\$ B l E$. 6-9 blåttr. ( 5 NAm. grn. - gb. $\triangle$ )

2728.

glauca.

4. Fatelf. = vert. eyr., am Girunoe herzform: unbehaart,

a. unten faft grangruin, mit ftumpf., an= genáberten 2fnhången; 35 lo. 3 blåttr.; B̉ If. 9 blàtti.: $\mathfrak{B}$ lbl. làngl. (ち NAm. w. $\triangle$ )

b. auf bens. Seit. gleichfarb., mit aus= gebreit. 2unbången; $3 \$ 31061$. abfebend;

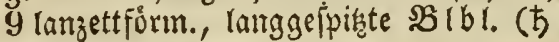
NAm. $\triangle$ )

5. längl, = verk. eyt., falt feilf., am Grunde herjf., febr gros; 3 SB lobl. akftebend; SBlE. $6-9$ blattr.: 3 I $b$ l. wie vorber (b $\mathrm{NAm}$, wo inw, r. Reckig $\triangle$ )

2731.

auriculáta:
2729.

tripetăla.

2730.

acumináta.

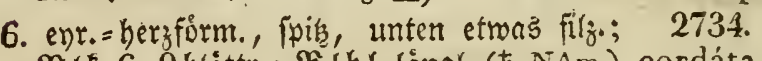

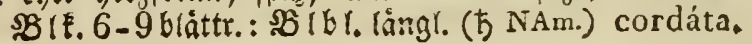

\section{MALÁXIS. 721.}

\section{Blätter :}

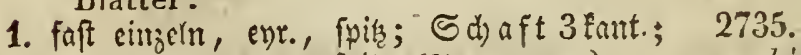
Eippe bohl, langgeipizt (4. 5. 9. 42.) monephyllos.

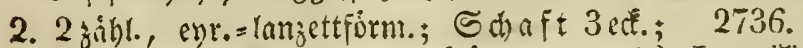
Rippe an D. Spike evr., gefrummt ( 4 46.) Loeselü.

3. meift 4 zábl, an D. Spite itharf; Sabaft 5 cấ; \& ippe bohl, fpis (4 42. 46. grn.gbch.)

2737. (paludbsa.) 


\section{6: MALOัPE. 552.}

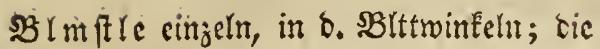
lingl. BI.:

1. thumpf, ungeth), geferbt, obert unbelbart $(\odot$ SE. p.-v.)

2. langgerpikgt, harb 3 rappl., gezahnt, ủberah unbegaart ( $\odot$ Af. p. grofs)

2738 .

malacoídes:

\section{MALVA. 551.}

\section{Acuissere Bld: 2 blätrrig.}

\section{Stengel :}

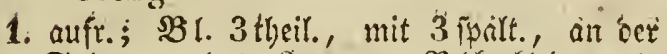

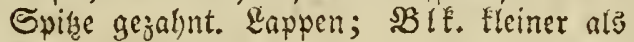

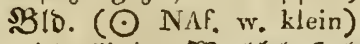

2. niebertieg.; $2 Z_{3}$ I 16 t. 5 theil., 3 lapp., gleid) $=$

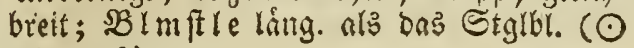
SE. grofs)

2739.

trifída:

\section{Acussere Bldt. 3 blättrig.}

B $\mathfrak{l}$ : ecfig; Stengel:

I. aufredut; Biätter:

A. ungleidfơormig:

1. bie unt. eat.. bie obern 5 theil., ct=

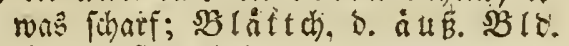
làngl., ftumpf (4 8, 26. ros.; w. - * 2742 . * $2 s_{3}$ i)

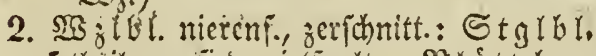

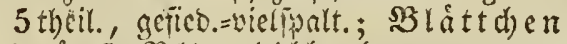

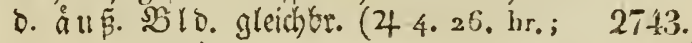
w. starkriech:)

B. gleidfoirmig,

2740. aegyptřas

$27 \pm 1$. tournefortiána.
(Alcća.)

(moscháta.)

1. banof.; 2Chren 1 feit., in b. Blatt=

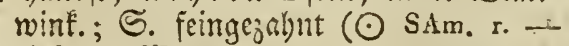
ఫ Tabr. 25eber)

2. gelappt; $\mathcal{A}$ elren mie vorker;

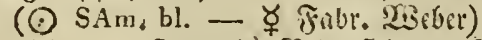

35 lapp., frumpr; $B$ limftíe ti. $B$ lt t= fitielc foft unbehant $\left(\odot 2_{2}\right.$ d dr.; bl.;

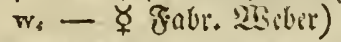

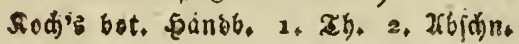

$2744 . \zeta$ peruviána. 2745 . liménsis.
2746. (mauriticina.) 
4. Fraus; SBlithen in ben sittrinf. ge $=2747$.

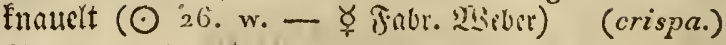

5. Tlapp., iptis; SBlmftle uno SBIttftle haar. (o'. 29. blch.; rch.; w. - * Sirrut, * 2748.

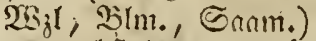

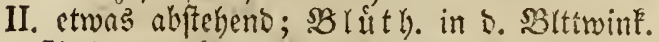
ftiellos, gefnauelt; $\mathbb{B}$ ts. glatt, abftcheno $(\odot$ NAf. bl.)

11I. niebergeftreçit; Blmstiele:

(syluéstris.)

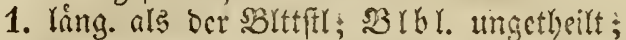

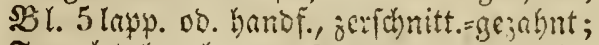
Frud t lamghanr. (○ NAm. r. ; gbch.)

2750.

caroliniána.

2751.

2. aufr.; $\mathfrak{B}$ ld. haar.; $\mathfrak{B} l$ f. fpir $(\odot S E$. nicaeénsis.

3. gebog., cingeln, 1 britll.; $\mathfrak{B}$ l. Excišruno; 2752. gefalt.; fil $\jmath_{\text {. }}$ geferbt ( $4 \mathrm{As}$, rch.) sherardiána.

4. Die fitll tht trag. niecergebog; ; Shlatt. herzf. = Freibruno, unocutt. 5 lawp. (4 14.

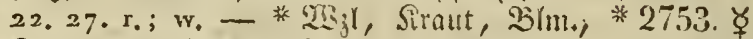

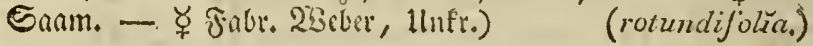

5. MANDRAGÖRA. 142:

(4 2. 9. w.; rch.; bl. - 22 sl)

* 2754.

† (oficinális.)

\section{MANULËA. 500 .}

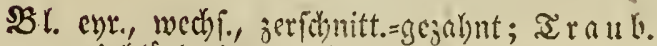
wenigblittl. in Den SBittwint. OD. am Ente 2755. ftekeno $(\odot$ SAf.)

foetùda.

\section{MARRUBIUUM. 473.}

Blumendecke:

I. 10 john.: SS Id

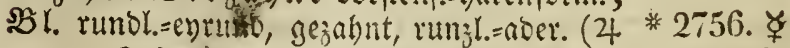

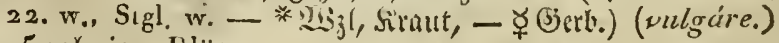

II. 5 jahnig; Blätter:

1. Feiff., 5 jahur, gefalt.; Brithenquirle, 2757. ohne Şuille (4 SE. p.; bl.)

Alýssum. 


\title{
Marrubium. - Medicago.
}

2. länglich, grau, runglig = aberig, gejahnt:

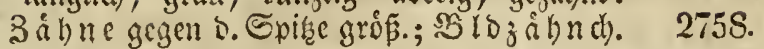

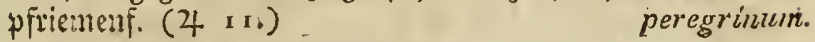

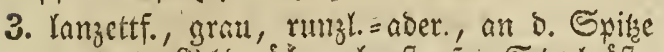

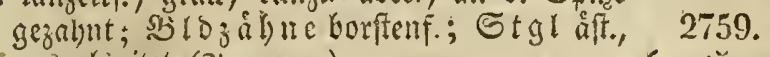
nusgevereitet (4 22. w.)

4. cy)rums,

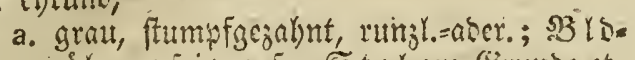

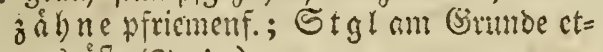
was åf. (2- As.)

b. vben grun, unten braffer, ticfgeterbt; sB lo jabue pfriemenf., unbeibart, abs ftebeno (As. w.)

(crelicum.)

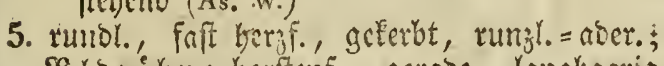
sBlojénge borftenf., gerade, langharig (4 9. w.)

\author{
2760. \\ candidisšmum. \\ 2761. \\ catariaefolıum
}

2762.

(supinumn.).

\section{MATRICARĬA. 691.}

\section{Blätter :}

1. cinfactgefied., etruas ficcija., mit gleidjbr.;

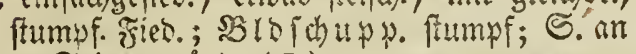
e. Seite gerimb. ( $\odot)$

2. oopp. gefies.: Sglof ful up. etras ftumpf

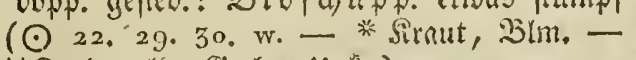

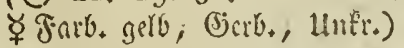

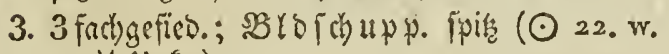
- ఛ $\left.\ln f r_{\text {. }}\right)$

\section{3.}

pusilla.

\section{* 2764. 후 \\ (Chamomilla.)}

2765. ఛ्⿱

(svaveŏlenis.)

\section{MEDICÁGO. 585.}

I. Hülsen schnectienförmig ge wunden $u$.

A. nicht stachlig.

Hiilsen!

I. Freizrumo; Blmstiele:

1. meift 2 Grittbig; Huilsen:

a. ctwas gewibt; 2 fterbr. borftenf.

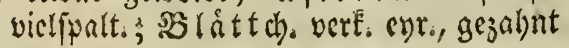
$(\odot$ 28.)

2766. orbiculáris. 
b. ganz flach, an Nanbe in b: Nuer rumb!; a fiterbl, gezabnt; $B$ lat td. ratutenf.= verf., enr., gezalint $(\odot \mathrm{SE}$.)

c. am (Sirunde gewólbt, oben flach, mit concentrifoen $2 b_{\text {sindungen; }} 2$ fter $b l$.

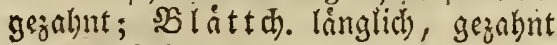
(O SE. hob.)

2. vielblüth.; Ş îlien flach), mit weiten Minoungen; 2 fterbl. gewimp. =gegnhnt;

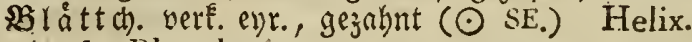

II. malzenf.; Blmstle:

1. meift 2 bluth.; Hiilsen auf teno. Sciten:

a. gewólbt, nit oachziagelf. sisindungen; Uf ferbl. geznhnt; $\mathfrak{B}$ låt tcb. rautenf.= bcrf. evr., gejabnt ( $\odot \mathrm{SE}$.)

b. faft flact, mit beuligen Sisinoungen;

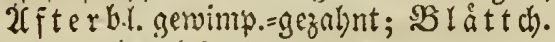
wie vorber ( $\odot$ SE.)

2. vielblittl.; Şulfen auf beysen Eciten flad), mit meiten Winoungen; 2f fterb $\mathfrak{h}_{2}$ wie vorber; $\mathfrak{B l a t t}$ b. verk. eyr., gejabnt (๑) SE. gb.)

2770.

turbináta.

$277 \mathrm{i}$ :

tuberculáta.

\section{B. ineichstachlig.}

Blumenstiele:

1. meift 2 blüth.; Şůl fen wargenf., auf bet) Ecit. faft flach, mit am rande weidyftachl.

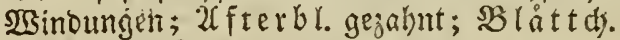
rautenf. = verf. evr., gezabnt $(\odot)$

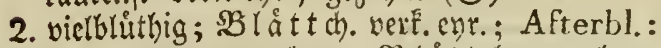
a. geromp.=gejabut; $\mathfrak{B l}$ látt d). gezalint; Şúlfen auf beyben Seiten flact), mit

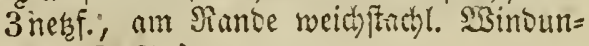
gen (๑ SE.)

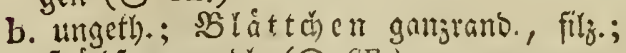

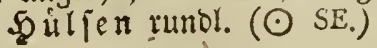

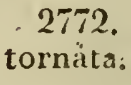

2767.

elěgans:

2768. scutelláta:

\section{Ihartstachlig.}

2774. apiculáta. $277 \dot{5}$. marína:

1. BImstlemeist 2 blüthig:

Stacheln:

1. borftenf., feinbant., 2 reihig, angebrudct; Shilferi oval; 2Afterbl. gerwimp.=gezahnt;

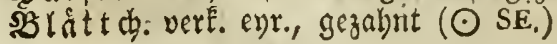

2776. intertéxta: 
2. pfriemenformig; Hülsen:

a. owal: Stadeln feinthaarig, gerate;

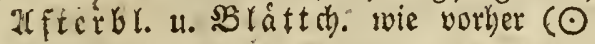
S.E.).

b. nuf beyd. Seit. offgebrideft; Stacheln

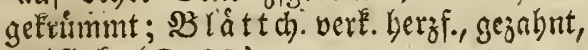
rotfflect. ( $\odot$ SE.)

c. watlenf.; Stachèln :

a. 2 reih., angeoriuct; 2 ffterbr. fieserf.;

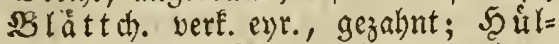
ien auf beys. Seit. flad $(\odot \mathrm{SE}$.)

b. gerabe, an D. Spiac hatenf.; Afterbl.:

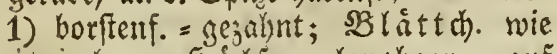
vorher; Shurfen langhaar., all

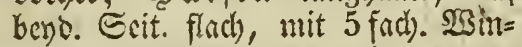
Dungen $(\odot$ SE.)

2) gervimp $=$ gejalint; Blättch.:

a) vert. ent., gezalunt; şa in o un= gen b. Ş ill ie naffeftheno ( $\odot S E$.) nigra.

b) glciübr., jerictuitt. =gezahnt, abs. geftutit $(\odot$.SE. hgb.)

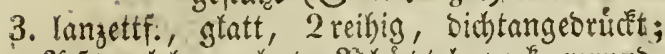

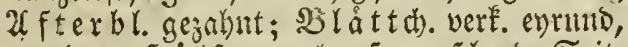
gezabnt; Şullen walgenf., auf bers. Seit: flach ( $\odot$ SE.)

4. fegelf. 2 reiligi, umgeichlag.; Xfterbl., 2784 . B!átth. u. Șúlfen wie vorher $(\odot S E$.) tribuloides.

\section{Blmstiele vielblüthig:}

\section{Stacheln :}

1. priemenfơtmig,

a. gerabe; Shulfen anf beno. Seit. afge= oridat; af ferbr. grablut; SBlateben ciur, gezabnt; Stgl aufr. (4 9.)

2785 :

carstiénsis.

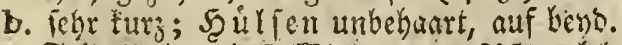

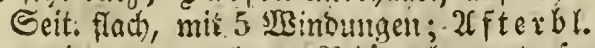

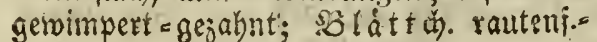
verf. ent., gejalgnt $(\odot$ SE.).

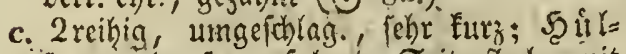
fen walgenf., auf beys. Seit. flach, nit 5 Binoungen; 2 fiet 6 la

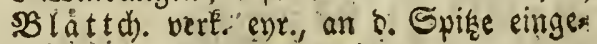
bruett, gezahnt $(\odot$ SE.)

d. Gafenfórmig, ciliáris.

2778.

maculáta.

2779.

granadénșis.

2780.

Gerárdi.

laciniáta.

\section{3. \\ tenta culáta.}


a. umgefdlag., 2 reibig; 5ूulfen rall= zenf., auf beyd. Eeit. flach, mit weiten Winoungen; Afterblátter gezabnt;

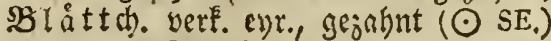
b. gerabe; Afterbl.:

1). ganzrond.; $\Omega 3$ lát d). wie vorber; Sุílfen bam. $(\odot$ 29.)

2) undeutl. geiảgt; S3låttchen verf. erro, an s. Epite gezabnt, auf beys. Seit. langhanr.; Şülien etrał haar. ( $\odot$ SE.)

e. sidtangeoridt, 2reibig; 2 fterbl. am Grumbe gejahnt; SB lattch. verE. herzf., gejalgnt; Soulfen auf bend. Ecit. flach, feinbanr. ( $\odot$ SE.)

2. borfenf., ausgebreit.; Soulfen auf beyoen Seit. flach, mit 2 neşf., am গande ftachl. sginoungen; 2f fterbl. gemimp.=gezabnt; S. lattch. vert. ent. gezabnt ( $\odot$ SE.)

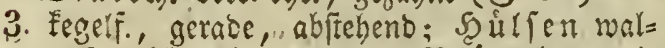

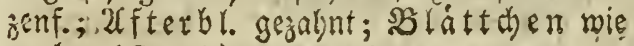
vorber $\left(\odot \mathrm{SE}_{\mathrm{i}}\right)$

2788.

uncináta,

2789.

(minǐma.)

2790.

graeca.

2791.

coronáta,

2792. denticuláta.

2793. rigidŭla:

\section{II: nicht?}

\section{Hülsen:}

1. nicrenfurmig,

a, 1 fant.; 2febren owal; 2 f terbl. gant=

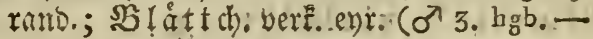
- ఫ Futt.)

b. 2 janm.; $2 \mathbb{I}$ mftle trabenf.; 2 fterb t. . 2795. gezahnt; $S B l$ át $t c h$. rautenf.=enr. ( $\odot S E$.) obscúra,

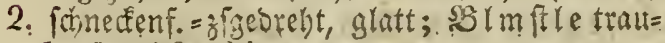
benf.; Afterbl.:

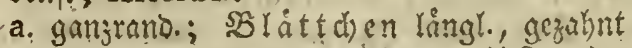
(4 SE. p.; v.: bl.; gb. 2c. - ఫ Jutt.) satíva.

b. am Grumbe gejabnt: SB lát tch. gleichbr., an o. - Spitge gejobut (4 SE. gb.)

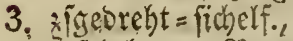

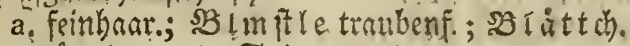
langl, an s. Spite gejabnt, (4, 2. 3. x x:

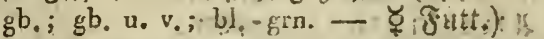

b. etras feinbar.; Steng el füf nufrecht;

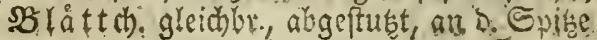
fengejahnt; 31 m fị ! e wie vorber ( 4 SE.) glomeráta. 


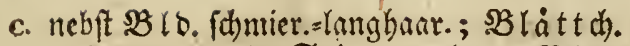
verf. evr., an b. Spitge gejabnt; $B$ lm= fitiele wie vorher ( $4 \mathrm{SE}$. grs.-gb.) glutinósa.

\section{MELAMPODIUM. 699.}

SBl. fticllos, lanjettf. = fpatelform., etruas ed, 2801. (○ SAm.) longifolium:

\section{MELAMPÝRUM. 486 :}

\section{Bliithen:}

1. in Aehren:

a, 4 peit.; Rebenbl. herjf., feft, feinge, 2802.

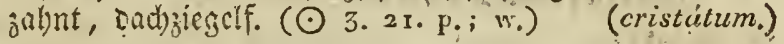

b. fegelf., fdlaff; Nebenbl. gejahnt=bor=

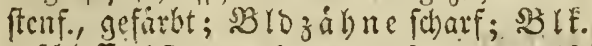

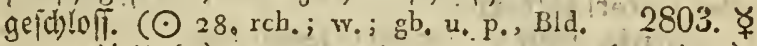
$\mathrm{P}:$ - ఛUnfr.) (arvénse.)

2. 1 feit. an o. Scite ftelend; Rebenbl. ge = jahnt, berjf =lanjettf: bie obern gefarbt, unfruchtb.; 23 ls. woll. (4 4: gbch.; bl.; * 2804. v. - * $\mathfrak{2}_{3} 1$, Sitraut)

3. in o. SRlattwinté, 1 reit.; Blk.:

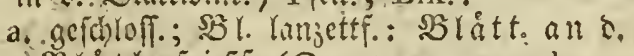

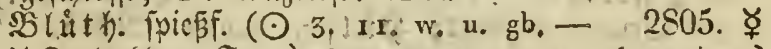
ఫ Jarb. blau, Tutt.) (praténse.) 2806.

b. offen; $B \mathfrak{l}$. alle langettf. $(\odot 5.8 \cdot \mathrm{gb}),.($ sylialicum.)

17. MELIIÚTUS, 596 ,

'I. "Mit nackt. Hülsen u. traubenf: Blüthen;

A. IL Ïlsen Iscamig:

Stengel :

I. aufred)t; Huilsen:

1. rumglidi), 
a. runol., (b. trodt nen zfgebridft), ftumpf, Bláttchen fadmathanjetti., abgefubst, 2807. gejägt $(\odot$ As. gb., klein)

b. verk. exr., fpiç; Xfterbl. borfenf. 2S08. ఫ్ (o 29. w. - ఫ Fitit.)

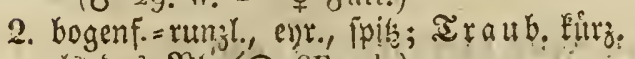
als Das $2 B 1$. ( $\odot$ SE, gb.)

(wulgáris.)

2809.

II. auffeigent;

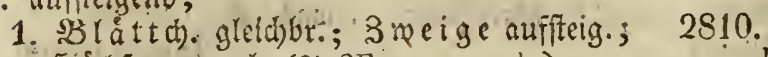
Sुúlfen rumzl. (2! SE, 17.49. gb.)

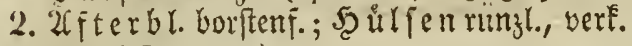
ci)r. $\left(\sigma^{2}-w_{0}\right)$

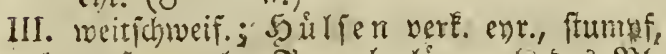

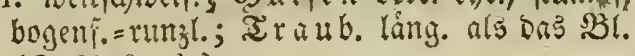
(๑) NAf, gb.) macrorhíza. 2811. Petitpierredna.

B. Hülsen 2 saamig:

Stengel :

I. aufredit;

A. mit Afterblältern:

1. Ianzettf, båut.; $\mathfrak{i} \mathfrak{r} \mathfrak{a}$ b. långl., ge

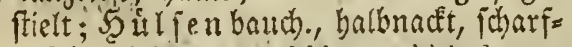
geipitat $(\odot$ 8: 32 . Lbl., wohlricch. * Siraut)

谈 2813 .

coeruléa.

2. piriemenfơrm.; Şúlfen etwas rumjl., eyr., frift jfgeoritest, ipip $(\odot 29 \cdot$ - gb.. braun gead. - - Siraut, 3lim., Eaam. * 2814. 후

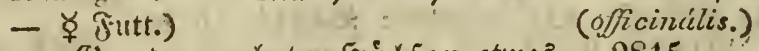

3. am Sirumbe gezahnt Soulfen etwas 2815. rumbl, fajt fpits (4 SE. gb.)

dentáta.

13. oline 2ffterbl.; Hülsen:

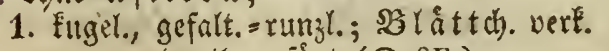
enr., unceutl. geiágt ( $\odot S E$.

2. ellipt. = rundr. , jfgevruct=flah, biat.,

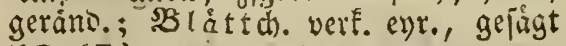
$\left(\odot \mathrm{SE}_{\mathrm{q}}\right)$

3. lanzettf. (○ SE. hgb.)

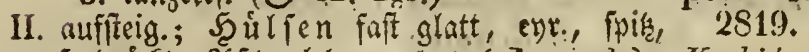

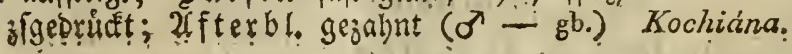

C. Hülsen meist saamig: Shillen lángl., runzl.; Bláttch. cllipt., gea 2820. zafnt $(\odot$ SAs. w.) rugulósa. 


\section{Melilotus. - Menispermum.}

II. mit kopff. Hülsen, ineist

8 saamig.

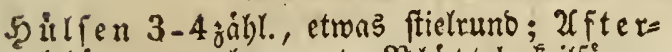

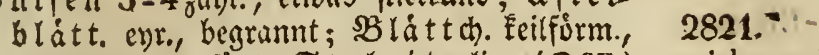

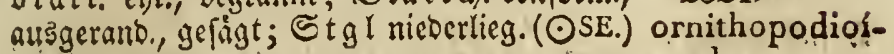
des.

\section{MELISSA. 451.}

\section{Bliithen:}

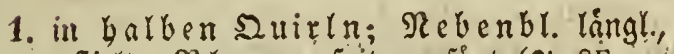

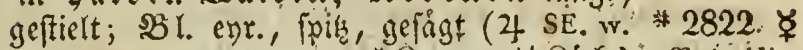

Bl. mit Citronengeruch - * Siraut - $\not$ Silidj.) officinális.

2. in Den SBIttwink.; Blmstiele:

a. 3-4blitt).; Rebenbl. linjettf., ftiellos; 2823 . ş 1. enr., fpich, fọarfgefágt (4 8. p.) grandifóra.

b. vię̧blüthig,

a. gabelf.; $\Re$ l. el)l., ftumpf, fharfaciågt, auf keyo. Seit. ratth); Stgl itacherhaarig (4: 8. 21. $r_{0}$; r., Bld, rch: 2824 : * Siraut)

b. gabelf. = Dolbertraubenf.; $\$ B l$. eyrumb, ftummp, etivas gefágt uno unbelyaart; Stgl etwas raud) (4 SE. blch. u. w. * 2825. - *itaut)

Nepětą;

\section{MELITTTIS. 450.}

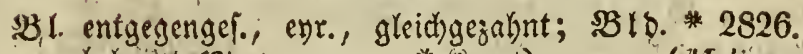
unbebaant (4 4. 9. $x_{0}$ - * * Siraut)

(Melissophyillum.)

\section{MENISPÉRMUM. 793.}

$\left(79^{3}\right.$, W. $\left.49,70^{x} \cdot\right)$

Die. fchillof., herzf. Blätter:

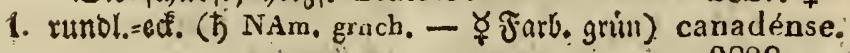

2. gelappt (5 NAm. grnch.)
2827. ఫ్

2828. virginụcum. 
14. MENTHA, 472.

\section{Blüth, in walzenf, Aehren.}

\section{Blätter:}

\section{I. foif ftiellos,}

1. Lanjettf., am Brunbe feilf., fharfgejägt, auf bend. Seit. unbehaart; 2 f $\mathfrak{h}$ ren un= terbroch.; $\mathfrak{B}$ lozábne etwas roud) (4 4. 48. 月, - Sirall, $\$ 3(m$.)

2. enruno = länglich,

a. meibgrau, unten filg., fdarf = u. un= gleidgelagt; 2 ehren an (Sirunde un=

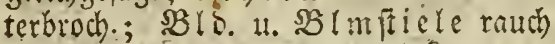
(2) 45.48. blch.; w: - Siraut (8) 8 utt.)

b. auf tend. Eeiten meiñ = 1t.-feinhaar., fted)enofpis =gefägt, am rande wellenf.,

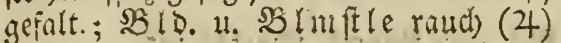

c. unbebanrt;

ค. ftechenolpif = gejagt, 'an Siande wel= lenf.; Xebren unterbrot); $35(\mathrm{r}=$

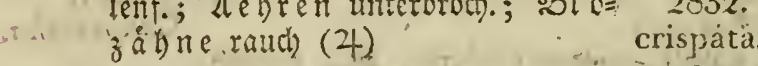

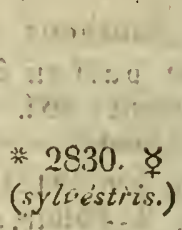

(virülis.)

$2831:$

unduláta.

¿1...ti. .

2127

b. entferntgejagt; $\Omega$ lo.e de unbelyart; 2833. A ebren wie vorber (4)

3. långlich, faft berzf, gleivigeiagt, unten

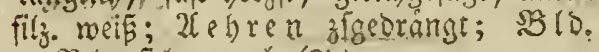
4. 39 I m fit te rauch (24)

làevigáta.

4. eyr. = ellipt., an D. Exise rutol., gelägt,

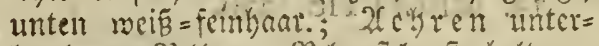

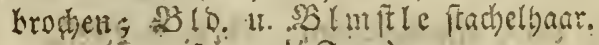

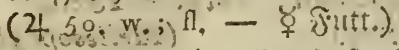

II. ganj fticllos, fobmalfanzettf., faft gasizano., zfgefalt., auf beno. Esit. sueiffitz.; 2 e gre rt

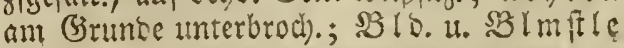
langbaar. ( 4 SE.) 2834.

(neinorósa.)

III. gefticit,

1. eyr., gefägt, unten feinbäar. = Eurgftadil.;

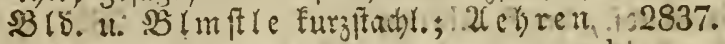
- wic vorker (4):

\section{8 \\ (rotunidifolia.)}

2. eyr.=langettf, forfigejagt, am (Sirunce ganzrano:, unten wenig feinbaar:; $: 52 \mathrm{~lm}=$

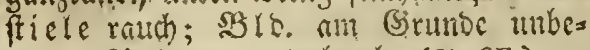
baart: 2ebren unterbrod). ( 4 SE.) balsamëa. 


\section{Blïth, in längl, Lehren.}

\section{Blätter:}

1. faft fiellos, unten weişeinhaar.,

a. langl, $=$ langettf., entfernt = uno forarfgea foigt, an Brumbe ganjrano.; 2 Uehren am (Srunde unterbrod).; $\mathfrak{B l b}$. 4 . $\mathfrak{B l m}$ ftiele rauch (4 NAf.)

b. eyr.=ellipt., ant b. Spike rumol., gejägt; 2lebre unterbrochen; $\mathfrak{B}$ lmfte u. $\mathbb{B}$ id. furbftachl: (4 50, w.; hf.)

2. gan! fitiellos, làngl, zrgefalt., ftumpfgelägt,

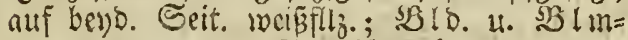
ftiele lamghar.; Stglielyr áft. (24)

3. geftielt, gelägt,

a. eyr., unten feinbaar.=10eíp; 32 !s. und SE Imfte furftad)l.; Stgl febr aft. (4 Bl. "stink.)

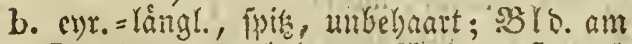
(Siunde ganz unbehaart; 2 Tebren ftumpi, am Sirmbe unterbroden (4 WE, dp., El. * . 2843. wohlriech. - * Sirnut)

piperíta.

III. Blüth, in koplf. Aehren.

\section{Blätter:}

1. folt fitellos, eyr. = berjfo, frechentipingefight,

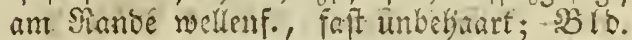
am (3)tunbe unbehant; $2($ ebrent unterbrod). (4 45.idrio ; Blo starkriechi - * Stratt). (corispa.)

2. geftielt, entw, gefigt,

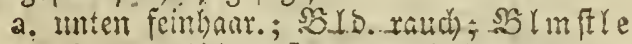
mit umgeifhlag. Scaaren; Xehren etwas unterbrocken (4 43.45. A. - * Ninut- * 2845. ఫ్ $\Varangle$ gitt t." (aquatica.)

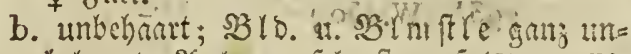
behart; 2febren felyr ftumpf (2F 48. Bl. :2846.)?

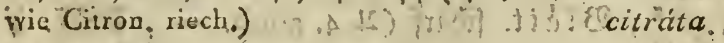

\section{Blïthen in Quirl}

Blätter:

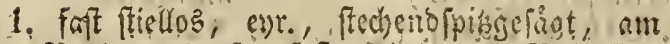

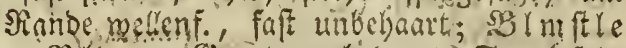

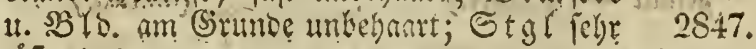
ift. (4)

dentáta. 
2. garig ftiellos, lanzetti., faft gangranb., un= 2848 . bebart; Tlebenbl. hansi. (4 SE. w.; fl.) cervina.

3. gefticlt, evruno,

a. unbehaart,

a. gelägt, am Girunbe ganzrano.; Bro 2849. ofibne riucth (4 SE:) rubra.

b. utberall gefágt, fpik; Stgef. furracer * 2850. als o. SBlE. (4 45. 47. - * Sirnut) (gentilis.)

b. oben icharf, etros ipis, gejägt; Stgef. láng. als Blté (4 3. 49. rch, — * Siraut * 2851. ఫ $\left.\therefore-\Varangle 1 \ln f r_{0}\right)$

c. ftachelgarig; Stengel:

(sativa.)

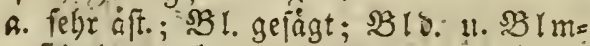
fricle raub) (4 29. blch. - * Siraut * 2852. ఫ - $\Varangle$ lliffr.)

b. aufr.; $\mathfrak{B l}$ ! gejágt; $\mathfrak{B}$ lb. rauch; $\mathfrak{B} I \mathrm{~m}=$ ftiele untebart (4 43. 45.)

(arvénsis.)

2853.

alestriüca.

c. nicberlieg.; $B \mathbb{L}$. Itumpf, weniggelägt;

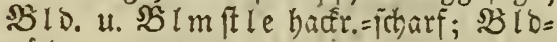

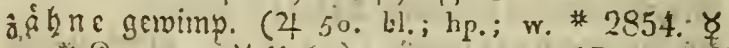
-** Sirant - ఫ linfi.) _ (Pulegüum.)

\section{MENYÁNTHES. 119.}

Blätter :

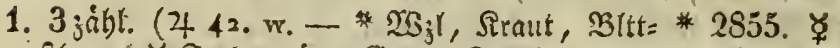
ftle - ఫ Farb. grim, (Jirb., Futt.) (trifoliáta.)

2. hergi., ganjrano.; $\mathfrak{B}$ IE. gewimp. (4 44. 2856 . 45. hgb.)

nymphoides:

22. MERCURIÁLIS. 789. (755\%: $79^{3} ;$ IV. 50.)

Stengel:

1. ganz cinf.; Rlátt. fharf (4 4. grnch. - 2857. ६ $\Varangle$ Farb. blaii)

2. armförmig; bie unbehaart. BI.: † (perénnis.) a. enr.lángl., gemimp.; $B$ litth. im Nuirl, 2858. iM. u. W. vermilgt ( $\odot$ SE.) ambigŭa. b. långl.; M.'sBlúth. åhrenf. ( $\odot$ x. grw. 2859. ఫ - Siraut - ఫ̧ Un(tr.) (annйа.) 


\section{MESEMBRYANTHĔMUM. 397.}

$\left(392^{5} \cdot 399^{x} \cdot 401.\right)$

1. Faft ohne હtgl; şl. punct., gleidubreit= ipatelf., öigewad) pen, unten gewólbt, oben mit e. Furd) $(\odot$ SAf. r., unten w.)

iI. nit Stenger; Blätter:

1. flach,

a. längl, fieberf. ( $\odot$ sif. w.)

b. eyt., warz., wedt.; $\mathfrak{B}$ lúth. ftiellos;

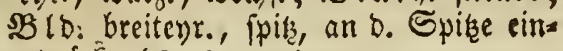
gebriuât $(\odot$ SE. w: $)$

2. unter gewólbt, abgejonbert,

a. umfait., gleidbr., zwifden ben läng:

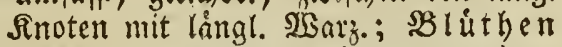
geftielt; 3 ID. 5 ipalt. ( $\odot$ SAf. w.)

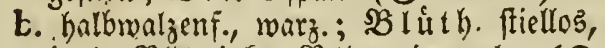

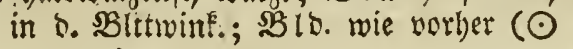
NAf. w.)

3. ftiefrumb; ftumpi; am Brunbe gerwimp., wedy. ( $\odot$ SE. w.)

2860.

tricŏlor. pinnatifrdumi

2862. crystallinurh.

2863:

apetălum:

2864. coptǔcum: 2865. nodiflórumi:

\section{MESPILUS. 393. (384

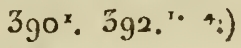

\section{Mit Dornen.}

Blätēer :

1. auf benoen Seiten haarig,

1. zott. = feinhaner., ficoerf., mit abfetelento,

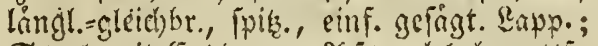
Stgl mit Enovorn.; X f ferbl. lanjetff., orili.=gemimp.; s及luth. in Enboolocn=

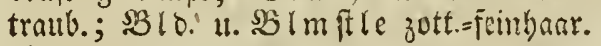
(5 As, w.)

2. oben ftadje!haar., unten an b. Mipy. Fein= baar.: Die al tern unbehaart: 2C tre rundl.=

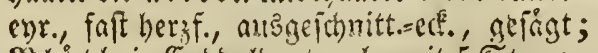
SBlith. in Enodolbentraub.; mit 5 Etweg.; SB to efe u. feinhant. szlttfte brif. (5 NAm. - ఛ જriidjte)

2866.

orientális.

II. nur unten

1. Feinhaarig, oben fpiegelglatt, feilf. =verf. enr., zerichnitt. = gejägt, faft lapy:; Bld, : 
a. nebf SBlttflen u. Xfterbl. orif.; S马! Utb. meift einjeln; B B cr. Ereufelf.,

4 fanm.; Stgl oorn. ( 5 NAm. w.) Alava.

b. mit lanjettf., zerfibnitt., 11. fo langen Rapp., als bie faft treufelform. BBectc; SZl. faft ftiellos; $\mathfrak{B}$ l u t b. mit 5 Stueg. (b) NAm, w。)

2. cir $D$. 2roern jott., faft ficberf. $=5$ rapp.; mit ausgcbreit., an D. Spize jerfdunitt., ungl. gciagt Rapp; $\Re$ I i tr). mit 5 Etweg., in Enosoloentraub. : SB 10 . frinbart, mit febr Eurz. , runol. - Inpj. (b) SE. w.)

2870.

III. auf bryo Eeit. Iintblynart,

pentağ̌na.

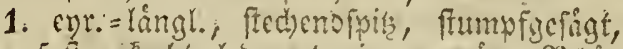

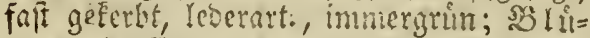
tben in Gnosolocntraub.: an ben jut $n=$ geru in D. Dorn. 2(ftrointe. (i) SE. w.)

2. betof.=cyl., auEgefdnitten=ed., gelägt; BI I then mit 5 Sturg. in Enobolocn= traub.; SY I. L. unbebrart. SYltifte olne Druifu (万 NAm。)

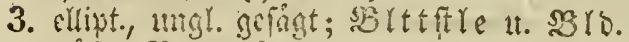
oruf.; BEer. fuscl., 5 fasm. (

2868.

\section{9. \\ parvifolia.}

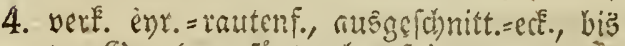

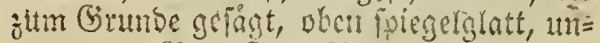

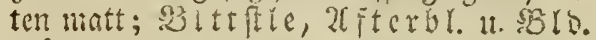
orit.; SBceren obal, 5 fanm ( 5 NAm.w)

5. vert. car. = feiform, geiggt, am (s) runde ganzrund., glåms., faff ftiellog, lescurt. $\frac{3}{3}$ Bluth. meit mit 2 Etweg. in Gnobol= Dentraub. ; 2310 . unbehnart, mit langettF. Rappen, SBeeren foff fugel, bang. (5) NAm. w: - ఫ̧ Srichte)

6. longl.= lanjettf. 4. routenf., lungleid) ge= fägt, an D. Spite ctwn fcilfurm., meift nanjrano., oben friegelglatt, linten matt; SEluth. wie vorber; siste. múchaart, imit jerichnitt. Rapp. (' NAm. w.)

7. 3 lapp. (felten faft fieserf. $=5$ lapp.) mit

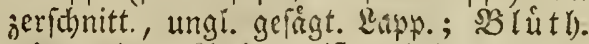
wie vorber; sBls. meift unbebart, mit abftehero., ipicis. Rapw. (5 4. 26. w, - 2877. * 31., $231 \mathrm{~m}$. ; Betr.)

8. faft fieberf. $=5$ lapp. (iclten 3 lapp.), mit 2S75. ૪ Crus galli.

2871.

Pyracántha:

2872.

cordáta.

2873.

elliptrica.

2874.
glandulósa.

2876. heterophyllla. ausgebteit., ant D. Spişe zerfdynitt., ungl. 


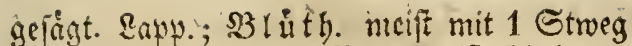
in Endoolosntraub.; $\mathbf{B} / D$. meift feinhanr., mit umgeiblag., langgejpitaten Rapp. (ち w.; r.)

9. foft 3 paltig, gegen o. Expise gefägt, am Grunbe etrons pis; Slthen meif mit

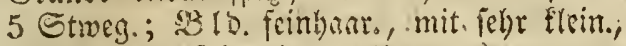
untgeidjlag.; fipis. Lapy. ( (ち NAs.)

\section{8. \\ monogy̆na.}

2879.

melanocárpa.

\section{II. ohne Dornen.}

\section{Blätter:}

1. oben 4. linten feinbaar., langl., arr Grunde (d)maler, ioft gonzrano., gegen 5 . Epitue ungl. = zerichutitt., fift 3 lapp. Do. ungethcilt, ungl. gefingt, faft geripst=aser.; $B$ litth. mit 3 Etmegin, an Ende ftebend, 1 bis. 3 zábl. (ந)

2. nutr uinten fils.; ganjtand;

a. ellipt., os. elfint. = onnl, ftumpf; Sgluth.

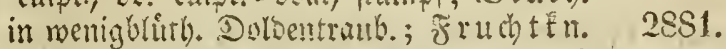
woll.; sBeeren 5 farm. (古 SE. sch. - w.) tomentósa.

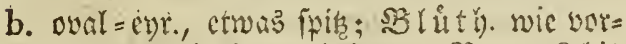
ber; Fru th tEn. unvehast; BSecr. 2 bis 3 faam. ( $\left.52.6, \mathrm{rcl}_{\circ} \cdot \mathrm{w}_{0}\right)$

3. anf bensen Seitert unberbart, obal, fpis, bopp. gelägt; SBlúthen in fopfi. Dolven= traub. (5 9. w, u. rch.)

\section{0. grandiflóra.}

\section{2. \\ (Cotoneáster.)}

2853.

Chumaemespilus.

\section{III. mit $u$. ohne Dornen.}

\section{Blätter :}

1. unten fils., langlich = lamjettr., neift ganijs rant.; SBluth. cinjoln, fiellos, am Enoe

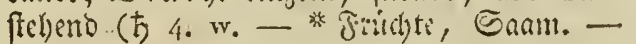

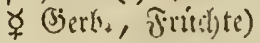

* 2S84. ఫ (germanica.)

2. oben unbebant, unten feinbanr.,

a. vert. cur., langgefpit, gegen D. Spice

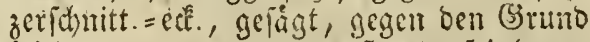
feilf., ganjtano.; sistt fitie le feinbaar.,

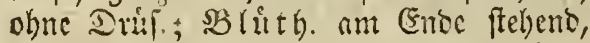
cinjeln, gepart os. in Dolsentrauben; $\stackrel{B}{ }$ eer. frugi. ( $5 \mathrm{NAm}$. w.)

2885.

pyrifolǐa. 
b: vert. eyr. = feilf: , bopp. gei̊agt, gegen bett (5iruno etroas einf. gefágt; $\mathfrak{B}$ lut then in Enoboloentrauben; SB Id. fajt jott., mit gleichbr. = pfriemenform. Lapp; SBeer en faft Eugel., mit niebergeoridft. Rabel ( NAm. w.)

c. faft Exilf., meift 3 ipalt:, an ber Spige meift gezabnt; sololápp. evruno (ちఫ̧ (richte)

2886. punctáta. 28S7. $\not$ Azarólus.

3. oben feinbaar., unten jott. Efeinbaar., faft fieberf. =lapp., mit abftehend., fpic., bopp. gefágt. Lapp.; 2 fter fl. halbmonof., ge= fágt; $\mathfrak{B}$ lùt $\mathfrak{h}$. mit 5 Streg. in Endoolden=

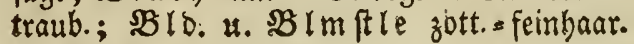
(ち SE. w.)

\section{8. nigra.}

\section{MEUM. 190.}

\section{Stengel :}

1. faft einfach;

a. mit meift 1 blattr. gem. Şulle: be Şullen faft halbungeb.; $\$ B l$ la fammit. mebr als boppelt origejest, borftenform.: B lát d). fait im Suir'; Scbeiden ber

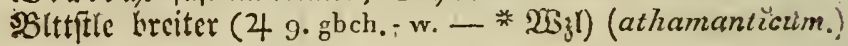

b. olne gem. Şúlle; bef. Şullen lan=

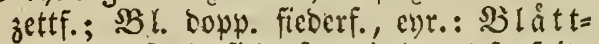
i) en vielipalt. fieberf., mit lanzettf., fpis. Eapp.; Stheiden b. Bglttit le breiter, båut. (4 9, $x_{0}$, starkriech.)

2890.

Mutellina.

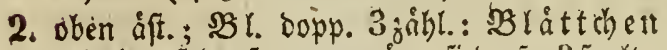
gleichbr. = fadenf., graugrim, fieber.$=3$ palt.; olue bef. Şüllen (4 14. 29. bgb. -

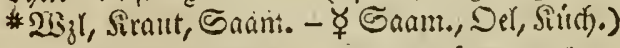
* 2891. ఫ (Foenicülum.)

3. febr âft., meitichmeif.; $25 z$ z lolát t. gefico., mit runol., zerfduitten = gelappt., gejägten SBláttch. : Etglbl. gleid)br. = favenf.; obne gem. Sculte (o' SE: 9. w.) 2892. heterophýllum.

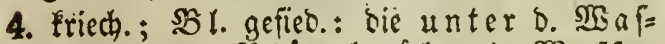
fer baarf.: SBlattd. úber o. Malf a jer feilf., zerichnitt. $=3$ ppalt.; Dolsen 2 fpalt: $\left(442, r_{0}\right)$

2893. inundátum. 
Micropus. - iMitchella.

19. MICROัPUS. 706.

Blätter:

2894.

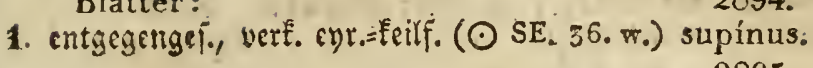

2895.

2. red) f., lanzettf.; $\mathfrak{B} \backslash$ ưth. woll. $\left(\odot S_{E}\right)$ eréctus:

5. MICROTÉA. 233.

$\left(\odot S E_{0} g r a_{i}-\dot{w}_{b}\right)$

2596.

debìlis:

14. MIMÜLUS. 494 :

Blätter:

4. Lanzettf., länggeipist, untochant, fliellos; sB I m ft le länger als b. SBlitt)e (4 Nam. bl.; r.)

2. runst.=eyr., gerippt: bie untern geffielt; Etgl frieth. (4 SAm. $b^{b}$ )

$289 \%$. ringens: 2898 . lutĕus:

\section{MINUARTIA.}

Biätter:

1. fabenf., am Grunbe breiter, 3 meige am

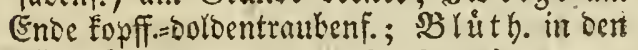
2(ftrine. ( $\odot$ SE. Pflanze fast braun)

2899. dichotouma.

2. haarformig;

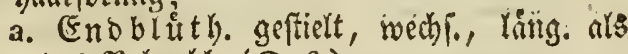
Das Miebenbl. $(\odot$ 6.)

b. Dolbentraub. blattr., in s. BBttrine.

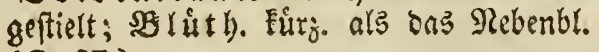
$(\odot \mathrm{SE}$.

2900. campéstris. 2901.
montána.

4. MITCHÉLLA, 62.

(b NAm. m.)

2902. tepens。

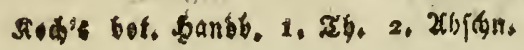

$\widetilde{x}$ 


\section{MITÉLLA. 351 .}

Bl. berzform.; faft 3lapp., gezabnt; Schaft 2903. 2 blattr. (4 NAm; wch.) diphýlla.

\section{S. MOEIRINGǏA. 319.}

\$3l. gleid)breit, flach; fo lang als die (5ilieber 2904. zwijhen D. Sinotent (4 9.39.40. w.), (muscósa.)

\section{MOLLÚGO. 52.}

2Bl. im Suirl, feilform.; fpis; Stgl ctwis 2905. getb., nicoerlieg.; $\mathfrak{B}$ I m ft le 1 bluth). (○NAm.) verticillátà:

\section{MOLUCCÉİA. 474.}

SB lo. glocfenf.; mit 5 gleich., wethrlui. Sillunen; B l. geftielt, rumbl.=evr., gezolbut $(\odot$ As: $2 ! 06$. w. u. r.) laevis.

\section{MOMORDİCA. 762. (W. 3.)}

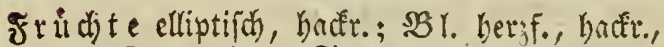
ftumpf, gejabnt; $\subseteq$ tgl obne Sanfen $(\odot * 2907$. SE, gbch. u. grnch. - frudde)

+ Elaterŭum.

\section{MONÁRDA. 24. (463º)}

Blätter:

1. langl. = lanjetti. = herzfo, feinlonar.:

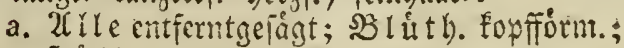
Scullen roth; Stgl bicht (4 NAm, r., punct.)

2908.

fistulósa.

b. nur bie untern entferntgefigt: to ober= ften faft gangrane.; $\mathbb{B}$ lit th. wie vorber; Rebenbl. Glafler; Slippe D. SBlf. an ๖. Epifie bart. (4 NAm. dila) 
2. långl. = lamgettfơrm., am Birunbe runol. u. (h)maler, jott., flach); $\$ B$ (E. punct. (4 NAm. hr., Hüllen unten grn.-blch.)

3. ganz lanjettf., entferntgefígt; unbebaart; Bł lutb. im Duirl; Nebenbl. blafer ( $\sigma^{\prime}$ NAm, gb., r. punct.)

2910.

oblongáta. punctáta.

4. enr. -lanjettf., am (Sirunbe runbl., unğleich,

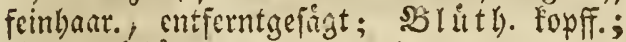
Rebenblătt. wie voller (4 NAm. gb, r. Heck. Nebenb]. p.-v.)

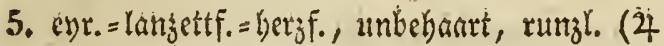
NAm. w:)

6. enr. = lingl. = herif.; feinhaarig, grobgelägt; SBluth. Fouff.; Şullen roth; Stgl rơll: (2) $\left.A m_{0}\right)$

7. ent.= etwots herz. , langgeipint, bichtgeingt, fait inbehanat; 3 I 1 th. in topff. Suirln; Soullen roth (4 NAm. p., wohlriech. * Siraut)

8. ganj euruto,

a. am (3runde runbl., gleid, ftachelkatis.,

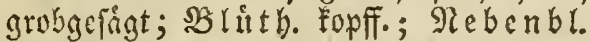
blnfier (4 NAm. lila)

2912. clinopodia

2913. rugósa.

2914. medǐa.

b. an (S)motiof fomaler; Stgl u. Suirle raud); Nebenbl. cyr., fo lang als bie \$Bis. (4 NAm, bl., r. od. sch, punct.) ciliáta.

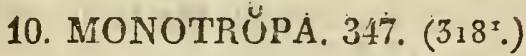

Seitenbluth. mit S: Enoblutben mit 2918. 10Stgef. (4 $5 \mathrm{r}$ )

\section{MONTİA. 47 .}

Stengel:

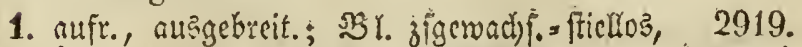

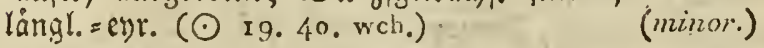

2. (d)rach, gabelf.; SBl. entgegengef.; fiellos, 2920.

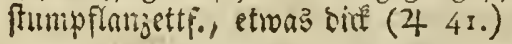




\section{MORA'EA. 46.}

1. Sha fit cinf.; $2 B$ l. fohwerbtf.; SBImftle 2921. faft orloenf., meift gabelf. (24 As. gb) chinénsis.

2. St gl geglieb., efwas aft.; $M I^{1}$. limgejulag., am Ranoe etwả wellenf.; X c bre hin u. hergebog.; 3 l l lapp. faft gleich, lángl., $a b=$ ftekeno; St fáb. am Grunoe jigewadj. (4 SAf, gb. u. grn.)

2922.

flexuósa.

2. MORÍNA, 32 .

(4) As, r. u. gbch.)

2923.

persica.

\section{MORUS. 741. (779. W. 58.)}

Blätter :

1. unbehaart, glatt, ungeth. u. gclappt,

a. ey)r., ani Grunbe fobiefberof., ungleich)=

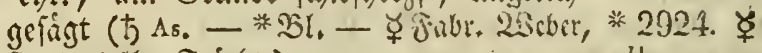
Farb, $\mathrm{gel}$, Friditi) alba.

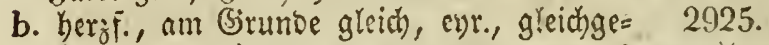
fágt ( 5 NAs.)

2. oben fichat, unter ftachelfaar., etroas ficharf, berzf., cyr:, ungeth. u., gelappt, dit 5 lapp., ungleichgefágt (4 SE, $\triangle-$ * Becr. - *2926. \&

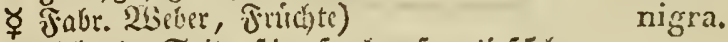

3. autf beyd. Seit. jharf, berzf., tief5 lapp., mit langgefpisten, am (Sirunbe fchmalern, 2927. gleid)gefágt. Lapp. (ち NAm.) scabra.

4. oben idjarf, unten feinbaar.= weich, herzf.,

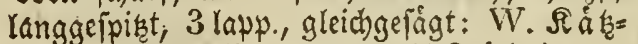
den waljenf. (ち NAm. - ఫ Friddit) rubra. tatarica. 2928. $\not$

\section{MUSCÁRI. 278.}

Blumenkronen :

1. waljenf.=ent:, gleich., horizontalftebeno, faft 2929. ftiellos. ( 4 As. ugbeh., sehr woblriech.)

2. ganj walzenf., eff., langgeftielt: bie ober= fiten unfruchtb., fehr langgeftielt (4 29. p.; 2930. w.; H.; bl. 2c.) 
3. ganz enr., gleidf., gebringt: b. oberften

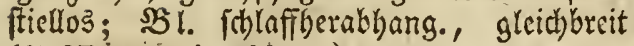
(4 SE. w.; gb.; bl. 2c.)

4. fugel., gleibhf.; bie unt. entfernter; $\mathfrak{B l}$. gerabe, gleidbbr., am Brtumbe fotmaler, rint= nenf. (4 8. 33. bl.; w. 2c.)

2931.

racemósum.

2932.

(botryoides.)

\section{MYÁGRUM. 519.}

Schötchen:

1. 2 glieor., 1 faam.; $\mathfrak{B l}$. auşw. budst., feit= 2933. gejafint (4 14. 29. gb.)

2. gefurtit,

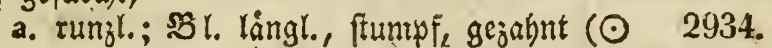

- gbch.)

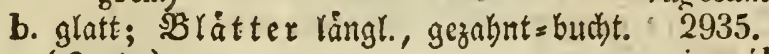
$(\odot$ As.)

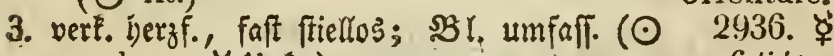

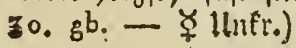
perfoliátumm:

\section{MYOSÓTIS. 101.}

Saamen gratt; Blumendecken:

1. 5 z chnig; Die fifumpf. Blätter:

a. lanzettf., faft glatt;, 3 Ib $^{2}$. faft unbebaart:

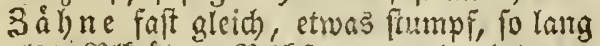

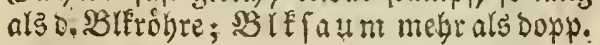
fo lang als. D. SBld. (4- 48. 月., dann hbl. u. gb., ros. u. w. - $\Varangle \operatorname{linftr.)}$

b. verf. eyr.=lanzetef., etwas folsarf; $\mathfrak{x} \mathfrak{r a u =}$ 2937. ఫ్

(scorpioides,)

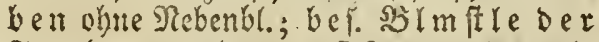
Fr ut th $t$ ausagchereit., foft 2 mal fo lang als

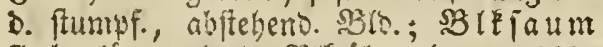
fladt, láng. als b. Sflthrobre (2+ 48. hbl. u. gb. - ఛ̧ fiutt., Untr.)

2. 5 ipaltig; Stengel:

a. faft einfach,

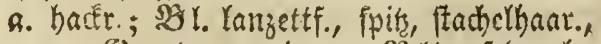
am Bintube gevimp.; SGrb. Fehr ab= fteberno (4 SE. 9. dbl., wohlriech.) suavcơlens.

b. fiachelfaat.; Traub. ofme Rebenbl.; sols. geithtofi.; bef. sgrmftle der

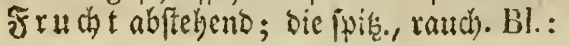

2939. (palístris.). 


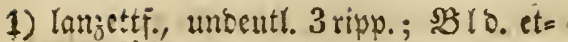
was furr. als. D. SIImfíc D. Frutht; SLIEjaum fiach, ling. alb b. BlE= róbre (4 9. dbl.)

2) langl = lonjettF; $\$ 318$. Kalb io turz alb D. SBlmftle D. Frucht; SY L f i a u m nufr., abftel)ent, foft jo lang als bic sBifróbre ( $\odot$ 28.29.30. l.bl. u. gb.; w.; xch. - ఫ llinfr.)

b. åf., weitf̣meif:; Blätter:

2940.
alpéstris.

2941. ్ㅜ

+ (arvénsis.)

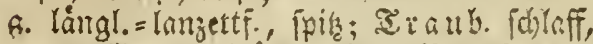

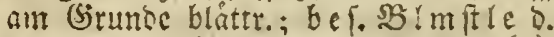
Frucht abmàttsgesog, sid läng. ais bie abfebente, pipe sBls.; SBIE. Elein (○ r.)

b. langl.=ersuno, ftumpf; Srocige ts. SI I m ft te ganj gerabe, viel furz. als D. SBlD. (๑ 29.)

c. verlangert, baar.; $\mathfrak{B l}$ lanzettF., etwas frumpf, raud); 疋aub. oljtic Nebcnbl.; bef, SB lmfte o. Frust aufr. =rbfteheno,

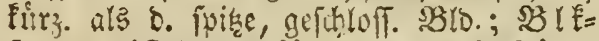

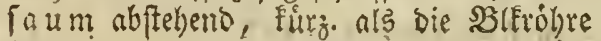
$(\odot$ 4. 19. gb. u. bl.; gb.)

2944. sersicülor:

3. 5 theilig; SBl. longl.slangettf., etrots ftumpF, rocib)=rauch; $\mathfrak{T} \mathfrak{r a b}$. Febr lang, fchlaff, obne Nebenbs.; bef. S3Intfte o. Frudt aus= gebreit. =abjtebent; s.zlo. gcictloll., ctwas Eirr. als jene; $2^{2}$ le faum flach, láng. als D. SBlfrobre (4 4. 8.)

2942.

sparsiflóra?

2943. stricta.
2945.

sylvatǐca:

\section{MYOSÚRUS. 258.}

2946. ̧ㅜ

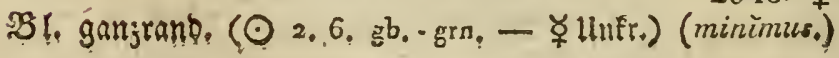

\section{2, MYYRICA, 779, (W, 6 1.)}

Die an b. Epike etraas gẹågt. Blätter:

1. Vanjettf., am Sirunde feilf:; S chupp. b.

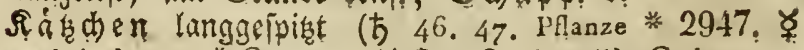
starkriech, - * Siraut - $\forall$ Del, ₹rarb. gerb) Gale. 


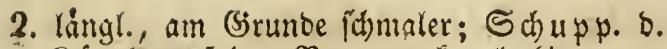

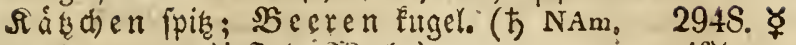

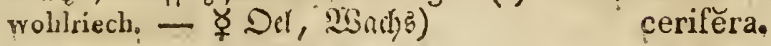

\section{MYRIOPHÝLLUM. 756.}

Blätter:

$$
\left(322^{x}: W, 88 .\right)
$$

1. Ulle gefico., baarf.; End å $\mathfrak{b} \mathfrak{r}$ quirlfớrm., nadit (4 44. grnch.)

2. Die obern Eammf. = firoerf.; Brath. in o. \&lttrwinté, quirlf. (4 50. grnch.)

2949.

\section{(spicátum.)}

2950.

(verticillátum.)

\section{Stengel:}

\section{MYRRHIS. 211.}

A, geflectit,

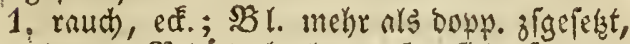
baar.: $3 B$ I a tt ch. lanzettf., fieberf., zers id)nitt. = gefágt, an $\downarrow$. Spiţe fohmaler, ver= läng.; Frud te webrlos, geforbt (48. 22. 26 . gbch, - w, , dann sch.)

2. fdarf, mit aufgerthwofl. (s)lenfen; $\$ B r$. 2951.

(aurĕa.) faft bopp. gefied, nuf beyd. Seit. etmas

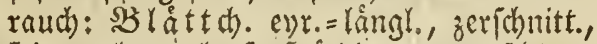
Feingezalhnt; be f. Şưlten umgejalag., gewimp.; Dulden yor b. SBlithe ume

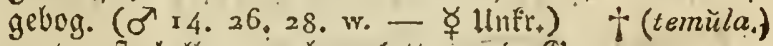

3. unten ftachelbaar., oben glatt, ant $\delta$. (Se $=$ Yenten aufigeichwollen; $2 \mathfrak{s}$ ol Enoll.; $\mathfrak{B l}$ l. megr als bopp. zigeferet, faft unbeganrt:

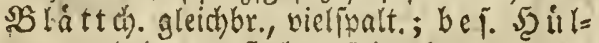
tẹ untebart, frectenofpis (o' 14.26 . w. -

B. nicht geflectit,

1. ctwos jott.; 25 1. 3 fach bopp. ofigeiest: B líttch. eyr. = lanzettf., fieberf., mit ges

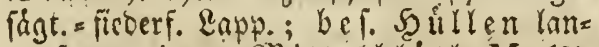

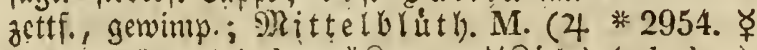

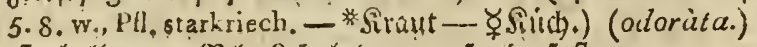

2. ftadjelhaar.; B I. 3 fact Dopp. sigel., faft

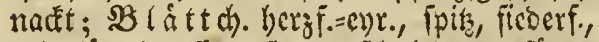
mit langl., fieberf., zerichnitten=gerügt.,

2953.

* (bulbósa.) 
gewintp. Eapp.; ber. Şullen faft unbe= baart, ungeidlag.; Stroege Daur.; aus= gebreit. (4 9. w.; r.)

3. unten ftachelbaar.; $\mathfrak{B} l$ l. 3 zóhl., faft bopp. gefied., etrob tauch: SB lát tob. am (Sirunde

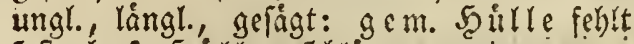
foft: bef. Scitlen 5 bráttr., gewimp., um= geichl.; (5) riff. Dautr. (4 r. 3. w.)

2955.

(hirstita.)

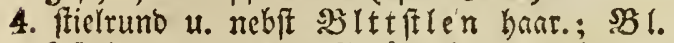

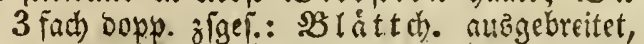
mit ctwas entfernten, fieberf. Bapp.; bef.

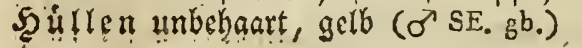

\section{6. \\ aromatžca.}

12. MYRTUS. 385.

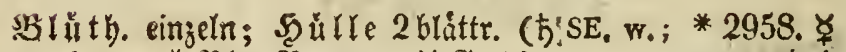
rch. - B., Ber. -

21. NAIAS. 737. (W. $43.44^{x}$.)

2959.

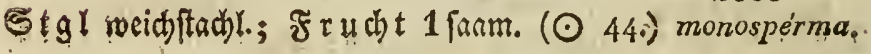

\section{NARCÍSSUS. 267 ,}

\section{Nect, Kranz länger als $d$, Blkscumo.}

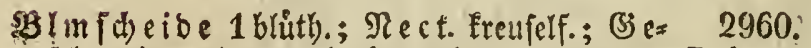

他

\section{II, eben so lang.}

SBImidheibe 1 bluth ; Nectar, ?

1. glocécenformig, fraub,
$a_{4}$ aufr.; Błérapp. en), (4 3. 32, gb,; 2961.
iv, u, sb.)
Pseudo - Nar- cissus. 2962.

b. am Fiande etraas abftebetto (4 SE, gुb.) bicŏlor. 


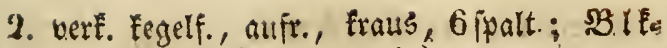
Iap p. lamjettf. (24 SE. gb.)

2963.

3. walyenf., abgeltukt, ctwas nus̉gejdyreift;

SBI lapp. längl. (4 SE. w.)

minor.

2964.

moschátụs.

\section{Kürzer.}

\section{Nectar. Kranz:}

A. balb lo lang als'o. BBlffaum; Blinscheide:

1. 1 blutb.; Nect. glodenf., gefalt., fraus; BRI. flach ( 4 SE. w. u. gb.)

2. meift 2 bluth.; $\mathfrak{R}$ c t. glocfenf., 6 fpalt.,

glatt; $\mathfrak{B l} \mathrm{l}$. halbivalgenf. (4 $\mathrm{SE}_{\text {: gb.) }}$ odórus.

3. meiti 3 bluth.; - Rect. glodenf., ausge=

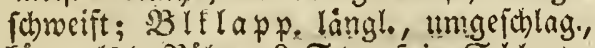

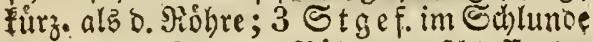
ftebeno u. 3 in b. Siobre verichlofi. (4 SE. w.)

4. vielblith.; Rectar. Fehr furz, 6 theil.;

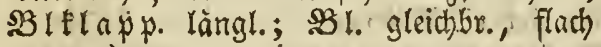
(4 $\mathrm{SE}$.)

B. cin Drittel fo lang als o. MlEfaum; Blmscheide:

1. meift 3 bruth.; Nectar.:

a, glodenf., 3 fpalt., attagernmo.; sBI $=$ lapp. lóngl., läng. als d. Siobre (4 As. v. u. gb.)

b. gefalt., geferbt; 8 relapp. langettf., fpis; $\mathbb{S}$ baft 2 idneib., faft b feit. (4 w.)

2965.

incomparablis.

2966.

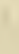 \\ 2967. \\ triándrus.
}

2968.

longiflórus.

2. vielblith.; Rect. glodfenf., abgeftugt; Blbl, :

a. gleich, anr. (4 SE. w.)

wechi. breiter,

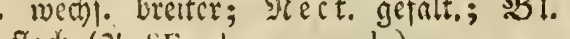
flach ( 4 SE. gb.; w. u. gb.)

C. noch füger; Blmscheide:

1. 1 blith,; Stect. riof., ganj traf., fein: geferbt (4 3. 32. w. u. p.)

2. '2 blutljig; Nect. :

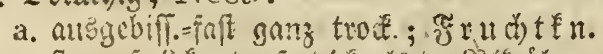

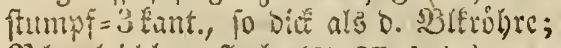
${ }_{B}$ l. gleichbr., fach (4 SE. hab) biflórus.

b. gefalt., rabf.; Frtath thot, langl., ftunpf 3tant.; $\$ 3 !$ gleidbr. =piricmenf., flaci) (4 w. u. gb.)

$296 \%$.

orientális.
2970.
nivěus. 
3. vielbluth.; Nect. wie vorber; Fruct ttu: fcharf 3 Eant., cyr.; $B 3$ l. pfriemenf., 3 fant. (4 SE, gb.)

2976.

Jonquílla.

\section{NEOTTĬA. 719 :}

Wurzelblätter :

1. länl., etwas geftielt; $\mathcal{X}$ elre gemumben; $2 B$ ùth. 1 feit., mit eyr. Rippe (4. 3.,34. w., wohlriect, )

2. err., geftielt, nekf.; S d a fi fheibef uno neblt Blith. Feinhaar.; Blutben 1 leit., 2978. mit lanjettf. Eippe u. $\$ B l b l$. (4 5. 13.w.:gbch.) (repens.)

2977. (spirális.)

\section{NEPËTA; 465}

\section{Blithen:}

I. in Uebrén; bie fieberf. Blätter:

1. ganjrano. (4 NAs. bl., sehr klein)

2. mit gleid)br., falt gleich Eappen; Se i= tenlapp. o. sole. etwas abjtel)eno ( $\odot$ NAs, w.. sehr klein)

II. in quirlf. 2lebren:

1. Iuirre etwas geftielt; Blatt. geftielt, berjí, gezabnt=geiagt (4 14: 22: w. u: $r_{.}$- * Sraut)

2. Suirl unterbroch.; $\mathfrak{B}$ !. Kerzf., gezahnt, rumzl, am siande wellenf., geftielt, grau (4 As. bl.)

3. $\mathfrak{B}$ เ ut th. fliellos; Quirle:

a. mit e. ซُfil子 verbullt (SE. w.; wohlriech.) hirsúta, b. nicht; Re ben bl. lamettf, ro lang als

D. sBlo.; Die geftielt. Blätter:

h. an o. Epibe ganjrano.; Suirle 2984. entferntitebent, fopff. ( 4 - ur.) marrubioides. 2985.

b. nicht (4 NE. w., r. punct.) .

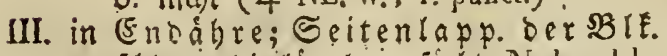
italica.: umgeichlag.; bie lángl., gefírbt. Nebenbl.:

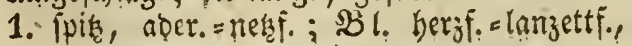
feinbaar. ( 4 As. hr.)

2. langgefpiat, gerippt = geftrichelt; $\mathfrak{B} \backslash \dot{a} t t$. berzf., feinbaar. (4 SE. v.)

2979. multifĭda: botryoides:

(Cataria.)

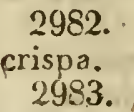


IV. in geftielten 21 fterooloen; Blätter:

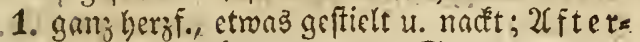
bold. vielbhith., baar.; Seitenlanp.

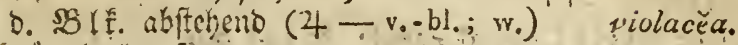
2.' loungl. = berof f,

a. zott., faft ftiellos; 2fferbolb. viels bluth:, ftathelhaar.; Se it entapp. D. $\$ 3$ tł. umgeidjlag. (4 bl.)

b. geterbt, fild; ; if ter bold, vielblintb. (o' As.)

c. gefágt, unten grau, rumblig aber.; 2Cfteroold traubenf.; Bld.:

a, bauch)=lwatzenf., ftadhethan ro; s lippe

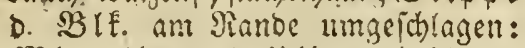
Mittellapp. o. Ulipye bobl, am Grunde etwas haar. (4 As.) b. valzenfurm., feintane.; Slippe b. Slfrone. flad); Mittellapp. o. ulip pe flad), am(sirunde bart. (4As.)

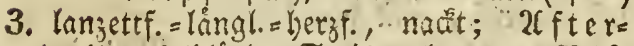
borbe vierbluth.; S eitentrapp. D. sgré. - umgerthlag. (4 - bl. ; p.; w.)

4. berzf. = lángl. = lanjettf., tiefgejågt, filz: (4 SE, rcla.)

5. längl. = lanjettf., am Srrunbe feilf.; faft

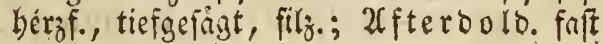
1 ieit. ( $\sigma^{\top} \mathrm{SE}$.)

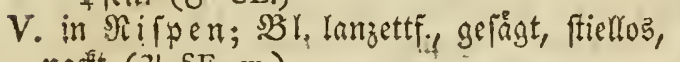
madft (4 SE. w.)

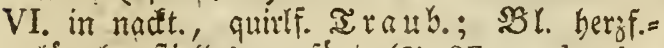
långl., ftieflos, gefágt (4 SE: $w_{0}$ - brach:; bl, u, w.)

2991.

longiflóra.

2989.

coerulěa. 2990.

incána.

2992.

cóloráta.

2993.

pannonica.

2994.

Nepetélla.;

299.5.

graveŏlens.

2996.

ucrančca.

2997.

nuda:

\section{5, NERĬUM. 135,}

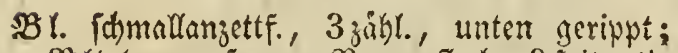

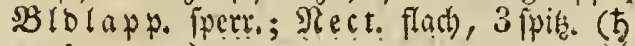
As, hp.; uw.)

2998. tiOleánder.

\section{NICGOṬIÁNA 124.}

\section{Blätter:}

1. umfafl., eyr., fpis, am Brutbe mit 2ins

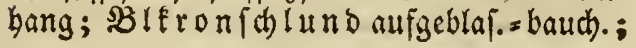


Saumlapp. Furs, langgepipigt $(\odot \Delta m$; gro. u. ros. - ఫ Fabr.)

2999: ఫ

macrophýlla.

2. ftiellos:

a. Ille: $\mathbb{2} z$ zlbl. lángl. = oval; Stgl ga= belf.; SSID. Fehr Eury; SBIEróbre falt ralzenf., 3 mal fo lang als Sild., mit evr., fpis. Saumlapp. (4 Am.)

3000.

pusilla,

b. nur tie obern u. längl.slanenttf., lang=

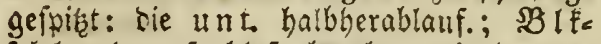
falund aufgebla = = baum., mit langge. ppitht. Saumlapp. (ค Am. ros: - - Siraut - $\Varangle$ Del, (jerb., Jabr.)

\section{3. geftielt :}

a. Zhle geftielt u. ganjranb.,

a. berjF.; Blk.:

1) faft rachenf., mit eyr., fpis. Saumk lapp.; Blith. in 1 feit. Irauben; B 10. falt lippenf., mit fefr lang. oberften $\mathfrak{L}_{\text {app. }}(\odot$ SAm, r.-gb. $\Varangle$ Jab:.)

2) mit keulenf., feinhaar.; viel länge= ret Midyre als D. SBtb. u. mit ganj furg., pit. Saumlapp.; ङtgl $\mathrm{cm}$ Srunce áf. $(\odot-$ gra. - gbch. $)$

3002. $ซ$ $t$ glutinósa.

3003.

cerinthoídes.

b. evr., şltiogre maljenf., lang. als D. Bgrb., mit rumol., ftumpf. Saum= lapp.; Stgl ftielrund ( $\odot$ SE. grn.. stach. - * siraut - ఫ Fabr.)

* 3004. † rustǐca.

c. eyr. =faft borzf; B Blerobre teulenf., ganj glatt, viel läng. alb o. $3 !$ !d., mit furz, ipia. Saumlayp; Stgl foft einf.

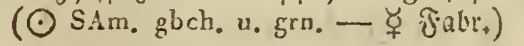

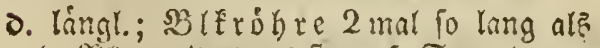
o. SBlo., mit ctmas ftumpt. Caumlapp.; Stgl ât.; SEa f. 4 fdal., faft tugel.

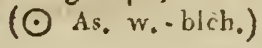

b. nur bic unt. gefficlt, eyr., ftumpf: Dic

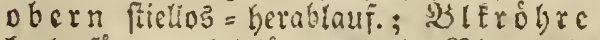
fculenform. viel lóng. als D. SBlo., mit fumpf. Eaaum (S.An, grn.)
3005 . 후

† paniculáta.
3006.

quadriválvis.
3007. Langsdorfii. 


\section{NIGÉLLA: 428. (438')}

1. Mit 5 Staubwegen; Blüthen:

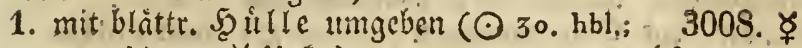
w. u. bl. - ६̧ Luffr.)

2. nid)t; Saamenkapseln:

(damascána.)

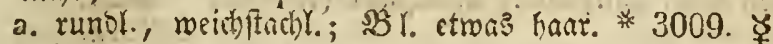

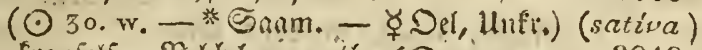

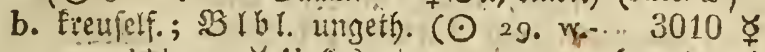
grn : hbl. - ซ 4 (nter.)

II. mit 10 Staubwegen:

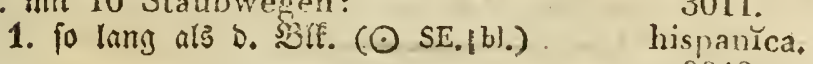

2. långer $(\odot$ As. gh.) 3012. orientalis.

11. NITRARIA. 374.

B. ganzrato., fitumpi (

3013 .

Schobéri:

\section{NYMPHA'EA. 421.}

2B 1. Gergf.; Bldecke:

I. 4 bråttrig; Blätter:

1. gantrambig; Lappen Derjeftern:

a. Dad)jiegelf., rumo (4 44. w.. Bl, w. wo * 3014.

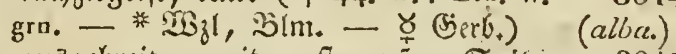

b. alsgebreit., mit e. ftumpiten Epiff; 3015. \$1. nusgetranto. (24 NAm.) odoráta:

2. gejabnt, fehr glatt; $\mathfrak{L}_{\mathfrak{a}} \mathfrak{p}$. antgenábert $3 \cup 16$. (4 SE. w.)

II. 5 blăttr., lång. als o. sBibl:; a appen b. ganzrand. 38 . angenáhert (4 43. 44. gb., * 3017.

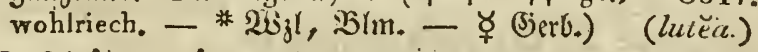

III. 6 bråttr., lårrg. als o. BBrbi.; Rapp. b. 3018.

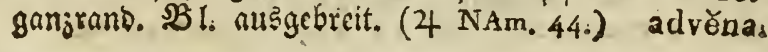

23. NYSSA. 809: (171.).

Blumenstiele d. W. Blüthen:

1. 1 blưtth.; bie långl. Blätter: 
a. Tebr furzgeftielt, faft ganjtand., am Sirunde 3019. feilf., unten weifl. ( $\hbar$ NAin. $\triangle$ ) candǏcans.

b. langgeftielt, langgeîpist, weitläuft. ge 3020 . fägt, auf bevb: Seit. unbehaart ( $ち$ NAm. $\triangle$ ) denticuláta.

2. 2 blintl):; $\mathfrak{B} l_{\text {: }}$ geftielt, eyr.=lángl.; an beyd. Enben pis; ganzrand.; unbehaart (b NAm. 44: grnch: $\triangle$ )

3. 3 blütf; ; $S B$ l. geftielt, laitgl., an beys. En= ben fpit, ganzrano., am Silttftiele, D. Mit 3022 . telrippe u. Rande jott. (ち NAm, grnch. $\Delta$ ) villósa.

\section{OCY̌MUM. 455 .}

Die eyr. Blätter:

1. etrous gejägt, unbehant; $\mathfrak{B} 18$. gemimpert

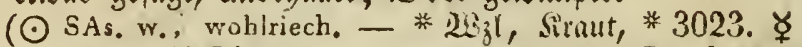

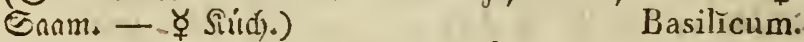

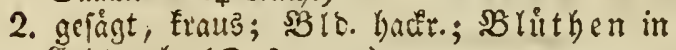
Enotraulb. $(\odot$ SAs. w.)

3. ganzrano. ( $\odot$ SAs. rch, u. wch., Plauze wohlriech.)

3021. biflóra.

crispum.

3025.

miňnı um.

\section{ODONTITES. 154.}

\section{Hiillen :}

1. Yånger als b. Dolbert,

a. 5 bláttr., lanjettf., abet., bånt., labarf= geipigt; sgl. gleichbr., 3 ripp.; St engel rifipenf. ( $\odot \mathrm{SE}, \mathrm{Hüll}$, gefärbt)

b.'fdymal, langgejpist, geftreift; Dolden

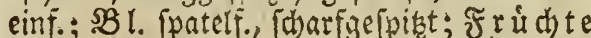
icharf $(\odot \mathrm{SE}$.)

2. furzer, borfenf.; Dolbe meift 3 bluth. int ben SBlttwint.; SBl. gleichbr.; Stgl lebr

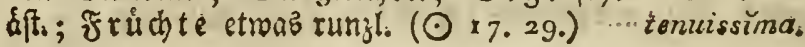

马. OENÁNTHE. 191.

\section{Blätter:}

1. Copp. gefiebert; Wurzel: 
3. atręgeichroift=gezahnt; feinbaar.; Stgl: 3040 . ftieltund, feinthar. $(\odot$ SAf.gb.) i noctúrna.

4. am (Grmbe fiederf. = grjabnt; Sfapr. furjgeftielt, vert. eyt., 4 flugl. (4 SAm. 3041. w., dann p.)

5. ganjrano., frumpf; tetraptŏra.

a. an bevo. Enoen it)meler, graugrül., umbetrant; SEapi. fticllos, cy)r., edf. 3042. (○ NAm. p.)

b. Sfaџi. etroas gefficit, ellipt. = vert. 3043 . evr., cet. (4 NAm.gb.) pumìla.

6. etwas gezahnt, fpis; كkapi. geftielt, 3044. lángl: = f́culenf., ect. (4 NAm, w.; gb.) frulicósa.

III. breitlangettf., gcjahnt=bucht.; Stap 3045 . prismat. ( $\odot$ NAm. gb.). sinuáta.

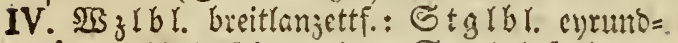
lángl.: 2 lle feingejabnt; Stgl einf., banr.; SBlbl. von cimanoer abftelent, 2 laps. $(\odot$ ơ Am. gb.)

3046.
longiflóra.

\section{OLËA. 8 .}

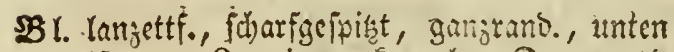
weip̈grau; 3roeige cE., ohne Dorncn (ち * 3047. ఫ SE: w: - Frichte, Dal-

\section{ONÓNIS. 563. $\left(543^{\star}\right.$.)}

1. Stengel strauchig.

SBIm ftle meift 3 blith.; dic 3 jerb!. Blätt.:

1. lanzettf., ftiellos, gelägt; 3 weige feir =

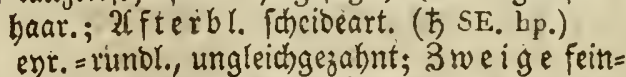

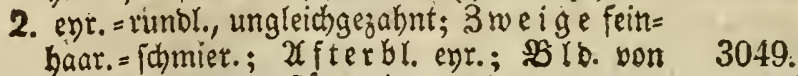
3 Rebenbl. unterftust ( 5 9: p.)

3048. fruticósa. rotundifolia.

\section{II. krautartig;}
A. Biüthen meist stiellos

\section{Bliuthen:}

1. eingeln,

a. grós. als dàs sBláttă.: die unt. sỉ is 3 zábl., lanjettf., an D: Spize gejabnt, 
4. mebft D. 3 weigen born., unbehart 3050. (4 SE.)

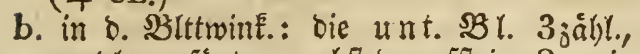
runol., gefágt, $u$. nebft $\delta$. nuffeig. 3 wei= ge II borm., zott. (4 SE. 36. p.)

2. meift gepant, in ben SBlttwint.; die unt. 3051. repens. 3 zábl., gejägt. Blätter:

a. evr.=lanzettf.; Siweige bort., zott. (4 *3052. ६

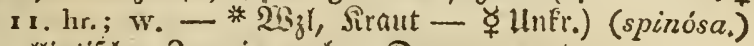

b. elliptifh; 3 weige obne Dornen, gott.=

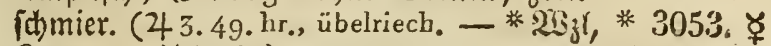
Siraut - ఫ llif́r.)

3. faft ftiellos, jur Scité ftebeno; Blätter?

(hircina.)

a. fámintl. 3 zåhlig,

a. Feilf., unbehaart; 2 fterbl. borftenf., gangrand.; B Ib. truck., láng. als die SBlf. ( $\odot$ SE. gb.)

b. lángl,, feinhaar.; 2 fterbl. lanzettf., feingezahnt; 3518 . wie vorher ( 423 . gb.)

b. bie unt. 3̧joll., evr., fpit u. nebft Stgl

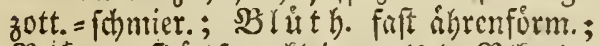

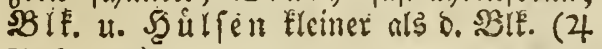
NAf, ros.)

c. einf., evrumb, ftumpf; iffetbl. oben

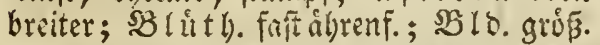
als b. unbegaarte siff. ( $\odot$ SE. p.)

4. ganj friellos in 2fobren; Siebenbl. after= hlattatt., eyruno, bautb., trode., backjicgelf. (○ SE. bp.)

3054.

minutissıma.

Colímnae.

B. Blütheng gestielt;

1. Blmstle ohne Grànnen:

SBrmftle 1 brith.; Br. 3 záhl., runol., ge= 3059 .

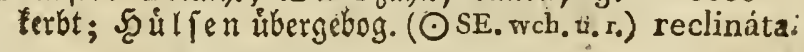

\section{Blmstiele begrannt。}

Blumenstiele:

1. 1 blútthig,

a. fo lang als o. $381 . ; 3 \xi$ l. einf., lơngl.; gefågt, fdumier.: bie unterften 3 jŏhl. $\left(\odot \mathrm{SE}_{*}\right.$ hg $\left.\mathrm{b}_{0}\right)$

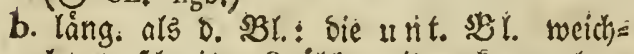
haar. =fojmier., 3 zályl., mit vert. entr.=lan=

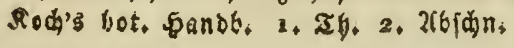


zettF., gegen D. Epike gefingt. = gezabnten SElóttch.; 2 Fterbl. längl. = lanjettfornt.; 3 weige feinbar. =zott. (4 3o. gb.). 3061. Natrix.

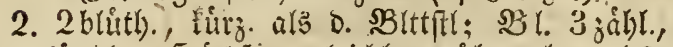
langl.; Schlifen gleidbr.; ubergebog. $(\odot$ SE. gb.)

3062.
ornithopodioi-
des.

\section{ONOPÓRDŨM. 641.}

1. Mit S̈tgl; Bldeckschuppen:

1. etwas abfteleno, eyr. =langettf., forarfge, fpist; 31 . berablauf., fils., etwas budt., gejabnt, Lorn. ( $\odot$ As.)

2. jebr ahftehent;

a. 2lile; bie berablauf., bucht., gezalnnt., born. Bl.:

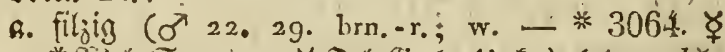 * gisjl, Saam. - ఫDil, (birb., Unfr.)

3063 .

graecum.

b. auf berd. Geit. unbehart ( $\sigma^{\star}$ As.) tauricum.

b. nut bie obern: die unt. unigejding.; BZl. Gerablauf., filzo., buctst., gezańnt, born. $\left(0^{7} \times 4.24:\right)$

3. angedrudt; et)r., fharfgefpitst; $B$ !. ber= ablauf., etruas filfo; budit., gejahnt, Dorn. (ठ $\sigma^{\top} \mathrm{SE} . \mathrm{p}_{0}$ )

II. obne Stgl; SBlo. Fugel., faft ftiellos: Sd) upp. lanjettf., born., febr abftebend; BI. geftielt, fieberf.; gezabnt, corn., filz., $\left(\sigma^{\top}-\right.$ wch. $)$

3066.

(illyricum.)

\author{
3067 . \\ arabicum.
} $306 S$.
acáulon.

\section{ONÓSMA. 97.}

\section{PHanze:}

1. warz. = badir., mit aufr., jerftreut. Soar.; Stgl áft.; $\mathfrak{B l}$ l. Ianjettf.; Stbcut. po lang als D. Stfío. (4 23. w., dann gbch.)

2. rauch; mit nieserlieg.; zerftreit. Seanen; bie blumentrag. Stgl. cinf., gebruft; BZl. gleichbr., etwas ipiá; als t. Stfád. (4 As, gbch, - w.)

3069. echioides.
3070. simplicissimum. 


\section{OPHRYS. 717.}

I. Mit nadt. $\sigma_{\text {d }}$ aft; Nect. Lippe:

1. 3 theil.: $\mathbb{R}_{\text {ap }}$. glcidbr., an D. Scite auss= gebreitet; Ne benbr. fo lang als b. Frutcht= Enot. (4 3.47. gbch. - grn., wohlriech.)

2. ungeth., lanzettf.; ftumpf, auf beyo. Seit. 1 jahm.; Nebenbl. láng. als o. Frubt= fnot.; SBl. gleidbr. = boiftenf. (4 SE. 9.) 3071. (Monórchis.)

3072.
alpína.

II. nit bláttr. Stgl; Nect. Lippe:

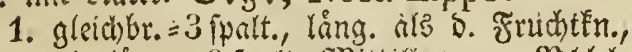

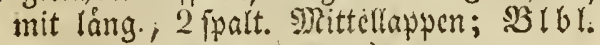
zigeneigt ( 4 - gb. u. r.)

2. 3 theil., feinbaar., mit fünallangettform.

3073. anthropophöra.

Seitenlapp., ut längl., 2 lapp., langerm

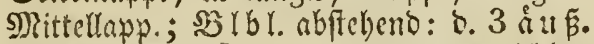
lampttf., frumiff; bie 2 innern gleid)bro, felis furg $(43.44$,

3. 3lapp., langhaar.: \&. verk. ent. Mittellappen:

a. Jlapp., verlong.: Enolapp. pfrie menf., ungefrummt: Seitenlapp. lángl.; SBlbl. ablteheno: Dic 3 à $u$ B. ellipt.; ftumpf: die 2 innern lanjettf.; 3075. fehe furr (4 3. 34. p. u. gb. u. grncl.) apiféra.

b. an b. Sppise febr Eur 3 lapp.; Ss lbl. abfekend: Dic 3 å bie 2 inern fidma!langetf., febr furz (4 34. grn. u. dr. w. bl.; w, u. r.)

3076.

(aractinites.)

\section{OKCHIS. 716 .}

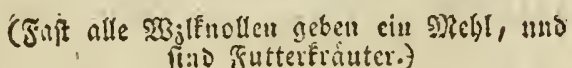

\section{VValknollen gepaart.}

Nectarlippe:

1. ganzant, gleidbur. ftumbr: 5ूorn 2 mal

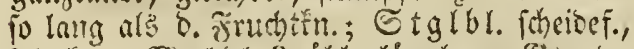

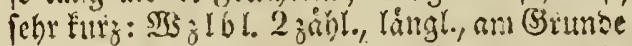
fanmaler (4 25. 34. wch., Nachts wohlriech, * 3077.

II. ungeth.; vetE. cyt.; gejabni, aitsgerant.; 
fưrz. als bet Fruchten.; Rebenbr. baut., 3078 .

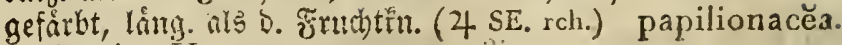
III. 3 theilig; Horn:

1. Fât jo lang als b. Fructifn.; Sectar Iippe oben am (Strunde '2horn., mit gleid)., ganjrollo. Rappen; 2 (ebre bidt, lingl. * 3079 (4) r $9 . \mathrm{rch}$. - *23j!) (pyramidális.)

2. fehr Eutr, Fegelf., fadf.; Seitenlapp. o. Rect. Lippe gleichtercit = prriemenf.: Mittellapp. verlang., 3 mal fo lang alb b. Frud)ten., gleichbr., 2 palt.; $\$ B$ I 1 . of figencigt ( 46 , grnch. u. gb.u. r., übelriech.)

3. 2 mal fo furj, als o. Frucbtfn.; Nectarlippe :

a. punct.=[ant: Scitenlap». glcichbr.: Mittellaph. 2 lapp., frumpf, mit c.

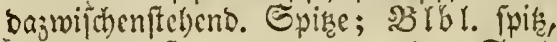
zfreineigt; Sorn gcrace; mit e. Spur v. Rebenbl. (4 3. w.; blch. u. p. * 2 (2)

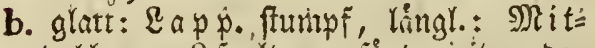
* 3081 . (militáris.) tellapp. 2 fpalt., geiagt, nut e. $5 a=$ zwifhen fiebenden Exite; Blbl. wie wother; 5orn gerabe, pfriemenform.;

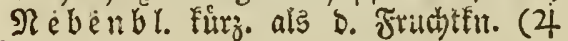
3. 8. bunt)

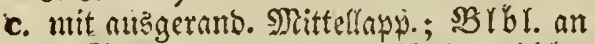
b. Spise fdarfocipitst; 2lare bicht, ev)r.; 3 l. lanjettr. (4 i. 3. rch.)

4. 3 mal io furz als D. Fructitit.,

a. Fegelf:, aufiteig.; $\mathbb{L}$ app. D. Nectar= lip pe ungl., geterbt, herabhangento; SBlblátt. jfjencigt (4 34. grn.-rch.; braun, stink.)

b. bafenf.; Lappen b. Rect. Rippe gleichbr.; punct.=fcharf: In ittell ap\%. 2 theil.; sBlbl. aufr., pipis; Nebenbl. faft io lang als b. Frudbten. (4 3. w. u. $r$.)

c. faft gerabe; Rectar Rippe punct.= fcharf: Seitenlayp. longl: SNit $i$ tellapp. grops, 2 lapp., geterbt, mit

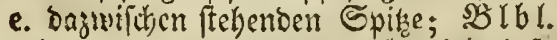
fpis, ofgeneigt; Nebenbl. 4 mal jo furz als o. Frudotfn. (4 3. 8.)

3084. (coriophöra.)

3055. (ustuláta.)
3086.

fusca. 
IV. 3 lappig; Horn:

1. fint: als b. Fruchttn. Kegelf., auffteigend; sapp. o. Nect. Rippe geferbt, ftumpf: Dittellapp. allgermo.; Mํbl. anf= jiteig., frumpf (4 5. 2x. p. u. grn。; w. * $24 j l)$.

2. 2 mal fo furz als o. Frudtên., aufiteig. ; * 3087. (Morio.) Rect. Lippe vert. elyr, forwach 3 lapp.: sapp. foft glecth; $\mathfrak{B} \mid$ bl. abfeheno (4 3. 47.)

3. To lang als o. Fruthtent., fegelf.; Nect. Lippe:

a. feingeferbt, ftumip , mit 2 ipalt. Mlit=

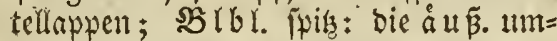
geidlag.; Sorm auffteig. (4 4. p. $* 2(3 ; 1)$

b. (chwach 3 rapy., mit an o. Spitze eitl=

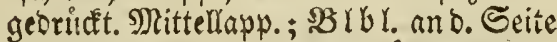
abfebeno; Xehren zfigerangt (45. gb., übelriech.)

4. 2 mal jo lang ats o. Fructetenot.; Nect. sippe runol., ftumpf 3 rapp.; o. obern SB Ibl. zrgeneigt: Dic an D. Scite $a b=$ ftebeno (4 9. w., sehr wohlriech.)

* 3089 .

(masciula.)

3090.

(pallens.)

\section{-11. Wzlknollen handförmlg.}

Nectarlippe:

1. ungeth., eyr.=langgeipitat; SBlbl. abfethent Şorn fumpf, fadff.; Xehre bidt, eyr.; SBl. glcichbr. (4 1o, dp:s sehr wohlriech.) nigra.

2. an b. Spitze 3 jabn., gleichbr.; $\mathfrak{B}$ lbl. ziges neigt; Seorn wie vorber; $N$ ebenbl. noch batb mal lenger als bie slittbe (4 3. 9: bgrri.; dp.)

3. 3lappig; Horn:

a. Kurg. als b. Frutchttru.,

a. walyenf.; SRect. Lippe flady, geterbt; bie obern SBlbl. zigeneigt: sie ant b. Seite abfekeno; Ote benblatt. po lang als o. Fruchten. (4 3. 4. ri: w. 3094. ఫ़ - * 2 ijl

b. Kegelformig; Nebenbl.:

3091.
ornithis 3092.

3093. (vịuldis.)

1) lång. als $\delta$. Slutbe; Rect. Rippe folwach 3 lapp., an b. Seiten um= 


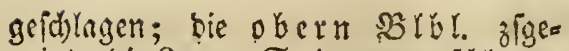
neigt: bie 2 zur Seite umbeidhlagen * 3095.

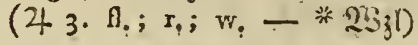

(lalifolia.)

2) eben fo leng; Nect. Rippe unbeutl. 3 rapp., geterbt; Blbl.:

a) Innggeppiht: bie 2 å ú. un!gejib!ag.

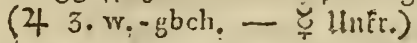

3096. $\Varangle$ incarnáta.

b) ctwas ftumtpf: bie 2 å 1 ह. wie vora her (4 8. grn.-gbch; ; r.)

b. 2 mal fo furz, ungefrimmt; Rect. Rip pe ftumpf 3 lapp.; SBIbl. alt D. Seite abftelieno (4 6. xx. p.; w., wohlriech.)

c. 2 mal jo lang, pfriement.; Rect. Ripwe u. SBlbl. wic vorker (4 3. 8, r. - ̧llitfr.)

3097.

(sambuč̆na.)

3098.

(odoratissima.)

3099. ఫ

(conopš̌a.)

\section{TII. TV zlknollen gebüschelt.}

Rect. Rippe 3 theil.: Lappen:

1.' glcid); Sूor n 4 friemenf., furz. alङ D. Frucht= enot.; Stgl feinbaar.; $\mathfrak{B}$ l. eyr., geftielt (4) Am.)

2. gleichbr., fpis, mit ftumpf. Mittcllappen; SIbl. afgeneigt; 50 rn 3 mal firy als o, Frudten., ftumpf $(43.5 .8$, wch.-gm.)

3100 . hirtélla.

\section{1. (albüda.)}

\section{ORIGĂNUM, 478}

\section{Aehren :}

1. prismat., laitg, gerabe, gehiult ; Nebent. båut, 2 mal po lang als D. BSlo. (4 SE. w. * 3102. ఫ - * Siraut, शlefren - ఫ̛ Sirch.)

2. lang, geftielt, gebiuft; Rebcnbl. fo lang als D. SBlD. (4 23. A.; w.; bl., wohlriech;

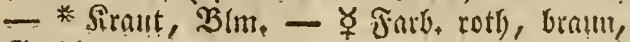
(E)erb.)

\section{* 3103. ఫ్}

(sulgáre.)

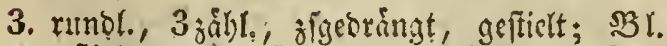

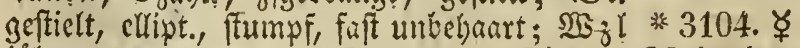

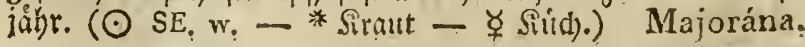




\section{ORNITHOGĂLUM, 289.}

\section{Blk; gelb.}

1. Blätter:

1. gleichbra =fabenf., gerabe; $\widetilde{S} \mathfrak{d}$ a f $t$ faft fticle rund, 1 bláttr.; $\mathbb{B}$ lm fit $l_{e}$ meift 3 jộlyl., boldenf., mit 3 bláttr. $5 \mathfrak{L}$ If

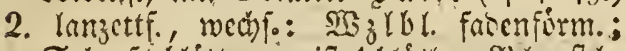

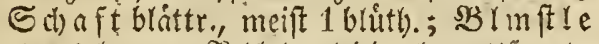
etwas baar.; \$S Ibl. gleid), lamzettf., ct= mas ftumpf (4 21. 48.)

3106.

bolseinicum.

3. Die unt. $\mathbb{B}$ l. an. o. SB (ut h). entgegen= geโ.; Wzlbl.:

a. geparrt; $\mathfrak{B} I m$ ftiel cáfit., bolbentrau=

benf.u. nebft b. SB lt t ft le n rauch (4 29.) (villósum.).

b. cinjeln, flach; $\$$ Im it le einfach, faft bolbenf,; SBlbl. ftumpf, unbebant; Zwiebel:

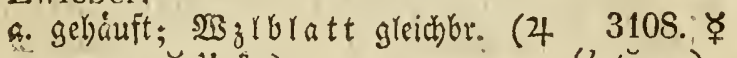
29. - ૪्⿱ llnfi.)

b. einjeln; $\mathfrak{W B}_{j}$ lb latt fdhallanzettf. $(45$.

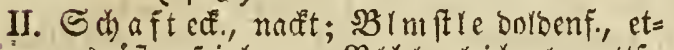
was äf., feinbaar.; $B B \backslash b l$ gleich, lanzettf., fpits (4 29.)

(luiť̌um.)

Зँ109. sylvaticum.

\section{Blk, weifs od, griin.}

\section{Bliithen:}

1. 1 fcit., bang.; Rect. ftaubfadeitart., glo= 3111 , denf. ( 4 29. inw. w., ausw. grnch.) (nutans.)

2. in menigbiuth. Doldentraub.; $\mathfrak{B}$ (m fitele lang. als o. Mebenbl.; Etfab. pfriemenf. (4 29, w. u. grn. - ఫ llnfi.)

3110.

(miniัmum.)

3. in e. Traube:

a. feb: lang: Stfäden:

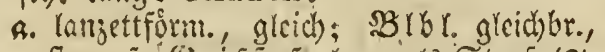
fumpf; (3) iff. fo lang als Etgef. (4 3. 9. w. u. gru.)

b. brcitlanzettform., wech) batb fo lang; , 316 1. Innjertf.:lóngl. (24SE. w. u. grn.) stachyoides.

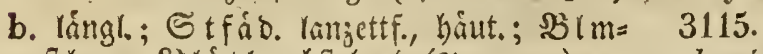
ftle u. ßlúth. abjtebeno (4 23. w.) narbonénse. 


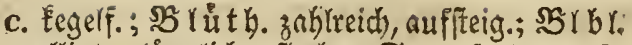
elript. =långliti, , flach; Stgef. ranzettF., 3116. gleict); (S riff. fel)r fur ( 4 SE. w.) pyramidále.

d. Febr furz; sBibl. ftumpf; Stfäb. pfrie= menf.; Rebenbl. lanzettf., fo lang als D. 20 luthe $(4$ 1 7. w.)

3117.

comósum.

\section{ORNITHŎPUS. 592 ,}

\section{Blätter :}

A. gefiebert:

1. 2rre gefiebert; Blithenküpfch.:

a. mit e. Rebenbl; ;ie getrummten Gliederhiilsen :

6. foft ftielruno $(\odot$ > $9, \mathrm{gb}, \mathrm{u}, \mathrm{r}$.

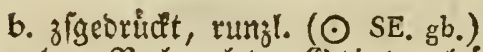

b. ohre Rebenbl:; (sliederbulfen fierruns, gefrimmt (4 SE.)

2. nur pie Stgrbr. gefieo.: D. unterften einf.: bie oberften 3 jábl.; 2 fterbl. verf. herzf., Den SBI. entgegentiger.; (S) I i e= Derbúlfen ausgefduweift $(\odot \mathrm{SE}$.)

B, 3 żábr., faft ftiellos: bas Mittelbr, febr 3122 . gró $\left(\odot \mathrm{SE}_{\mathrm{g}} \mathrm{gb}_{\mathrm{e}}\right)$

3118.

(perpusillus.)

3119.

compréssus,

durus:

3121 , heterophy'llus, scorpioídes,

\section{OROBÁNCHE. 482. (488\%}

\section{Blumenk̦ronen;}

1. 4 ppartig,

a. aufgeblajert; Staubgefälse:

A. unten nadat; Rarbe mit 2 abftehene. Eapp.; Es riff, oben feinhaar.; Etgl gant einf. (4 4. xr. 5 r. grnch,; gb., 3123. ఫ్ Pflanze wie Gewürzaelk. riech. $-\Varangle+1$ lufr.) (major.)

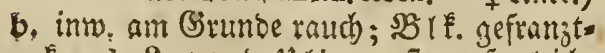
fralts: $\mathbb{E} a p$ p. o. ulippe ftumpf, greid); 3124 . Stgl einf. (4 4.29. starkriech.) caryophyllač̌a.

$b$, nicht aufgebrajen; Staubgefälse:

a. unten baar.; Narbe verk. herfoorm.; (5) tiff. ober unbebant; Stgl ganz einf. (4 15.29.)

3125, elatīor. 


\section{Orobanche: - Orobus,}

b. unbebaat; siriff. oben haar. =brif.; SB rb. 2 poult., mit ungeth. Eappen; Stgl ganj cinf. (4t NAs, w.)

2. 5 palt.; 2 c benol. 3 jabl.; Stgl:

a. Fajt cinf.; SS to. rọbr., balb 4 jpalt. (4 3127.

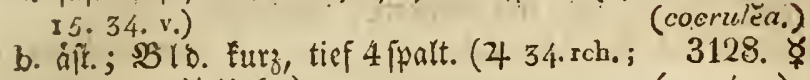

blcb. - ఛ Ulufr.)

3126.

alba.

(ramósa.)

\section{Blätter :}

1. gepnart,
a. foft ftiellọ; 2 fterbr. gezahnt (4 NAs. bl.)
3130.

\section{ORŎBUS. 574 .}

b. geftielt; $\{$ fterbl. ungeth. (4 As. blch.) hirsútus.

2. 2 paarig; Stgl :

a. cinf.; $\mathfrak{B} l$ l. fidwerdtf.; Afterbl.:

R. einf.; $\mathfrak{B} l_{\text {: }}$ geftielt $(4-w)$.

3131.

albus.

3132.

b. pfriemenf. (4 NAs. gb.)

angustifolius.

b. aft.; \$Bl. gleidbr.; 2 f fterbl. balbpfeilf., 3133 .

pfriemenf. (24 SE, w. -bl.)

3. meift 4 paar., fdomallanzettf.; Xfterbl. balbpfeilf, gangrant.; Stgl geflug., oben etwas áf. (4 SE. r. u. ugb.)

4. 6 paar., cyro = linglich); Ștengẹ ạff. (4 8. $p_{0} ; b_{0}$ )

5. geriebert,

canóscens, .

a. 3 paar. eyruno, ranggefpitgt; 2 fterbl. balbpfeilf., ganzrand.; S I gl einf. (4 4 . r. u. bl.; bl.; w. - ${ }^{*}$ Xiclli)

b. 3 ob. 4 paar., lanzettf.; Afterbl. wie vorber; Stgl geflug. (4 3. 5. p.)

c. 4-5 parar, langlich, unten graugrún; Xf fer bl. balbpfeilf., am (Sirunoe gejabnt; Stgl eimf. (4 SE. 9. gb.)

d. vielpaarig,

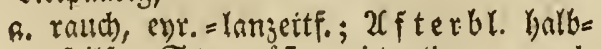
pfeilf.; S $\mathrm{tg}$ !. åft., nicberíieg:, rauth (4 4.26. p.)

b. unbehaart, ellipt.; $\mathfrak{A}$ fterbl. enrund = langettf.; Stgl ât., aufr., raut) (4) \$E, ugb.)

3134. varius.:

3135, (niger.)
* 3136,
(vernuss.)
3137.
(tuberósus.)
3138,
lutĕus, 


\section{9, OSTEOSPÉRMUM. 711.}

\section{Blätter:}

1. verf́. eyr., gefägt, geftielt, faft herablauf. 3141 . (万) SAf. gb. $\triangle$ ) gefágt. Yapp. (ち SAf, $\Delta$ )
3142.
coerulěum.

\section{OSTRY̌A. 750. (W. 24.)}

Die gerippt $=$ gent. Blätter :

1. eyr., fpis, ungleid)geígt; 3 apf. eyrund, 3143.

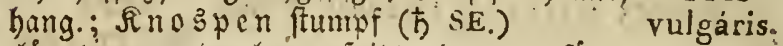

2. långl = eryrumo, langgeipint, bopp. gefágt; 3apf. lángl.segr., aufr.; నnospen fpis (万 NAm.)

3144.
virginuca.

\section{OSÝPIS. 775. (W. 28.)}

Bl. ihmallanzettf., ganzranb., wedt) F, fehr

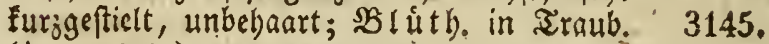
( 5 9. gbch.) alba.

\section{OTHÓNNA. 709. $\left(710^{x}.\right)$}

\section{Blätter:}

1. tieffieberf., anbehanart, nit glcidbr., ctwas

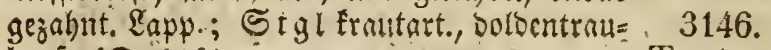
benf: $(\odot$ SAf.)

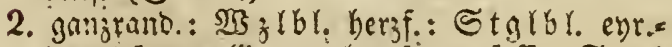
lanjettf., ant Sirumte herifo, umfafl.; Stgl 3147. fdilaff, fadenf., aff. (24 SAf.) Tagétes. filica'ulis.

\section{1, OXĂLIS. 36S. (544x)}

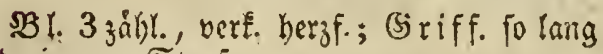
als o. innern Stgef;

1. ohne Stgl; S thaft 1 bluth., läng. als

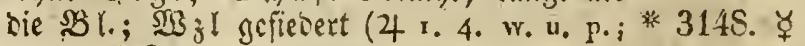
bl. - * Siraut - ఫ̧ Futt.) 
2. mit aif. Stgl:

a. aufr.; $B$ Im ftel boltentrag, furz, ats b. 3149.

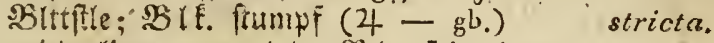

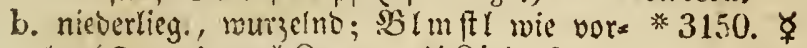
ber $(\odot$ - gb. — * Siraut — ఫ̧ Sitif., Jutt.) (corniculáta.)

\section{PAEDERÓtA. 19.}

Stgl blittr.; Blätter:

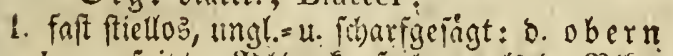

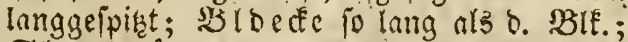
Seapi lingl, langsinitht (3t 9.23 .

Etapl. langl., langgeipitat (4 9. 23. gb) Agcrěa.

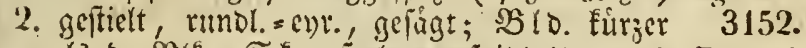

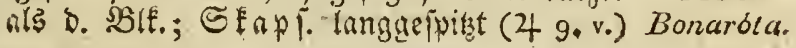

13. PAEONİ. $422 .\left(423^{2}.\right)$

Blätter:

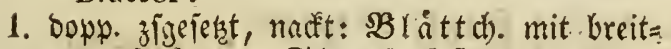
lanzettf. Lapp.; Stapi. fait gerabe, filts.

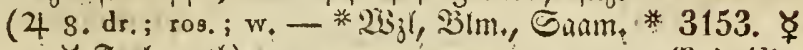
- $̧$ farb. roth) officinális.

2. Doppelt 3 zoldlig: Blättchen:-

a. eyr., ungetl.; naft; SEap F. getrinmt, 3154. fillo. (4 SE. p.)

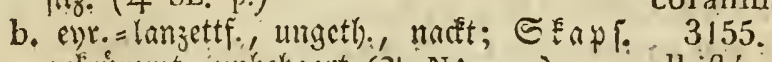
getrumme, unbelgart (4 NAs. w.) albiflóra:

c. 3theil.=3errifî., unten etmis zott.; SEap F. 3156. faft aufi., baur. (4 SE. P.) h huṃ̣lis,

d. vicltheil., nacit: Lappen:

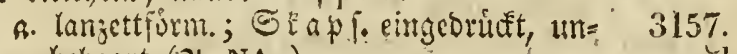
bebaart (4 NA.) ) anomăla.

3158.

b. gleidbreit; Słap 1. firz. (4 NAs.) laciniảta.

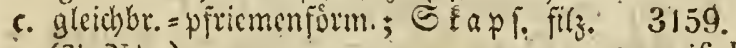
(4 NAs.)

tenuifolía:

\section{PALIÚRUS. 156.}

Stant nt faif flettcrno; 3 weige mit gepaart., afterblattart. Etadjelit, wovon b. e. getrummit 


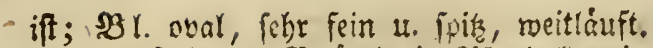
gefingt, 3 ripp.; $\$ B(\mathfrak{B} t h$. in Ufteroold, in D. So lattwinf., nidht viel ling. als D. SBltt= ftiele (5\&.grn.-gb. $\Delta$ )

3160. austrulis.

\section{PANCRATIUM. 269.}

Blumen

1. fdumallanjettform.; 12 Rect. 3 áh ne die 3161. Stgef nicht trageno ( 4 SE. $\omega_{.}$)

maritr̆mum.

2. lanzettf.; 6 Rect. 3ahne ipis, 2 pualt., bie Stgef̂. nicht ţagend (4 SE. w., sțark. riech.)

3162.
illyrịcum.

\section{PAPAVER. 409.}

Saamenkapseln :

I. bactrig;

1. mit blåttr., vielblüth. Stgl; Skapseln:

a. foift fugelrund, fnot. ( $\odot$ 3o. hp. - 3163. $\Varangle$ ఫ Unfr.) (hybrüdum.)

b. Eetrlenf. $(\odot$ r9. 29. hr, , unten schw.; 3164. dbraun - $\Varangle$ llifi.)

2. mit 1 bluth;, nadt., hadr. $S$ d)aft; Bl.: 3165 .

a. Dopp. gefieo. (4 9. w. u. gb.) alpinum.

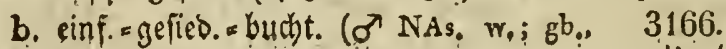

I1. glatt; Blätter: wohlriech.)
zerichnitten,

1. fiederf.; bielblüth. Stgl:

a. baar.; SEapi. Fugelruno $(\odot$ 29: 3o. sch.; w.; r. u. w. - Blm., Siraut - ఫ Farb, roth), Hufre.) pụdicáule.

b. mit angebrucft. Sorften; SEał

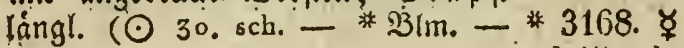
$\Varangle$ unfr.j

2. umfaff.; $\mathbb{B}$ lo. glatt $\left(\odot=2 .{ }^{29 .}\right.$ w.; v.; p. 2 . - Eam+, Stapl., Saft *3169. ఫ - ૪ Del)

3. gefied.; Stgl vielblưth., glatt; Słapi. långl. (4 SE, gb.)

- 3167. ఫ

(Rhopas.)

(dubium.)

$\$ 170$.

cambrǐcum. 
B. gefägt, gefies.; Steng cl 1 britty; f fuar, * 3171 . blîttr. (4 As. sch. - * Enft (Dpium) forientále.

\section{PARIETARIA. 801. (78.)}

\section{Blätter :}

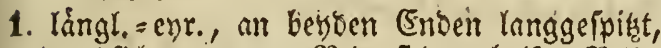
Durdficht. = punct.; $B \mathfrak{l m}$ ftle gabelf.; $3 B$ lo. * 3172 . 2 blättr. (24 22. wch., Pflanze salzig - "Siraut) (officinális.)

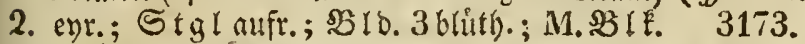
verláng. = waizenf. (24 - grnch. $)$ judaǐca.

3. lánglich $=\operatorname{lanj}_{3}$ ett $F_{\text {. }}$ aber., nicht Durchficht. $=3174$.

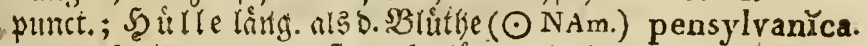

4. rautenf. =cyr., etwas ftumpi, lang. als ber

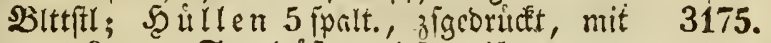

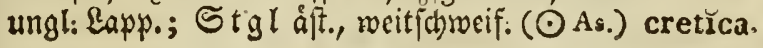

8. PARIS. 324. $(324 x$ :

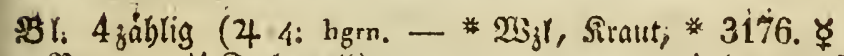

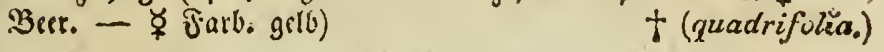

5. PARNASSĬA. 249.

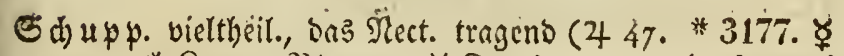

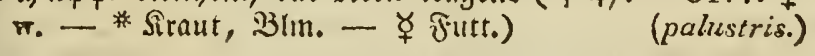

19. PARTHEENIUUM. 704.

31. ungeth., lăngl., gezahlynt (4 $\mathrm{NAm}$ ) integrifolxum.

\section{5: PASTINÁCA. 227.}

Stengel:

1. gefurd)t;

a. $\mathfrak{B} \mathfrak{l}$ : gefiesert, glonn., unten feinhaar.: $s B$ lit $t$ tch. lángl., frumpf, geferbt=gezahnt, am Brunoe jerichnitten: Das áu 


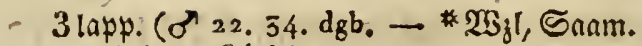
- * Der, Silid.)

b. $\mathbb{W}_{3}$ Ibl. einf., herzf.: Stglbl. 3 uno 5 zábl., am (Srimbe fdmaler: 2 lle fiec= gelglatt, geferbt; Stgl jebr dift.; Dol= be it fehr zabtreich, rilpenf. ( $\sigma^{\top}$ SE. gb.) luč̉da.

2. einf., fielruno; $\mathfrak{B} \mathfrak{l}$. Dopp. zfgejeft, unbe=

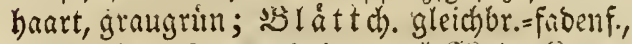
ganjramb. $\left(\odot 50\right.$. hgb. - * $2 b_{j l}$; - Siraut, ilm., Eam. - $\Varangle$ Dil

* 3179, ६ $+($ sativa.)

\section{0.}

\section{lucrua.} * 3181. ఫ Anélhum.

\section{PEDICULÁRIS. 495.}

\section{Mit ästigem Stengel.}

BIfbelm flumpf, abgeftuat; $\mathbb{B} \mathfrak{l}$. ge= fiecert: Fiedern:

1. fieberf. = gejahnt; $B \mathfrak{l}$. eyr., aufgeblaíen, 2 theil., tammf. ( $\odot$ 42. p.; w. - * Siraut - ఫlunfi:)

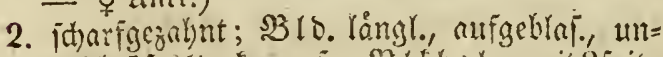
gleid 5 palt., fammif.; $\$ 31 \mathrm{tb}$ elm mit 2 fpis.

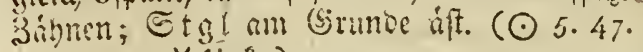
p.; w. - ఫ (lufr.)

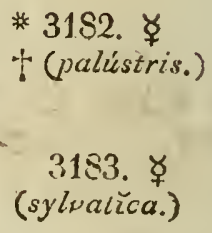

3183. ఫ్

(sylvalǐca.)

\section{II. mit einfachem Stengel.}

\section{Blätter:}

A. cinfach = fiecerformig: Fiedern:

1. Feingeterbt, ausgeichmeift; $\mathfrak{B}$ I o. 5 ipalt., fammf.; SBlb. gejoldll. (4 9.gb.u.dr.) Sceptrum Caro-

2. unterbalb gejabnt, lángl.; $\mathfrak{B} 18.5$ ipalt.; faft fanme.;, $\mathbb{R}$ ! El e e m hafenf.; langge=

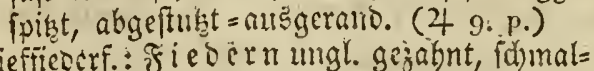

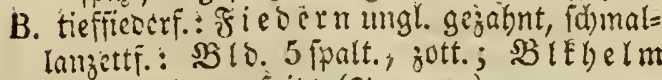
bafenf. langgefpitet ( 4 ro.p.)

c. Etglbl. fieberf, 4 zalgh.: Fi co ern längl.; ftumpf, gejalont; 2f ely re fopff.; 8 broc de rauci), 5 jabn.; siblebeln febr ftumpf (4 3. 9. p.).

D. bopp. fiecerf.; Fie be cu längettf.; gezahnt;

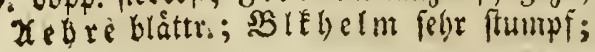

\section{linum.}

3185. asplenifolia.

$3186 j$. incurnáta.
3187. verticillátá. 
1. Zerte 3 l. tieffieberform.; $\mathfrak{A}$ ehre biht; 3188. Blo. 5 zahn., unbehaart (4 1o. dr.), recutíca.

2. nur bie Stglbl. tieffiederf. : Fiebern langgeipist; voh d. 5 B bldjąhn. ber obere 3189. Febr grofí (o SE. 9. ugb.; p.) foliósa.

E. gefiebert: Fiedern:

1. oupp. gejahnt, eyt.; ftumpf, bachjiegerf.; Sblo. 5 jahn.; şlebelm ftumpf (4 9. 3190. gb., mit e. sche Fleck)

2. Fieocrf.; SB Io. 5 part.; Bikhelm:

a. ftumpf; Fiedern gleidybreit; fpis; 3191. ßls. ratich (4 10. p.) roséc.

b. bafenf. =langgeinitat,

a. abgeftutgt, Stgl aufiteig.; Fico. 3192.

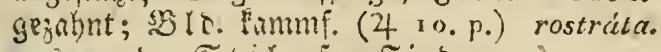

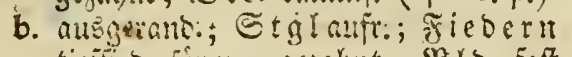
tiefiteoneform., gezahut ; SBlb. faft 3193.

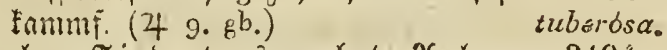

c. 2 zahn.; Friç. ctwas gezahnt; 2xehte 3194. blăttr. (4 9. hgub.; lir.) comósa.

III. ohne Stenget.

S1. gefied.: Fico. evruno, ffumpf, gejahnt ;.

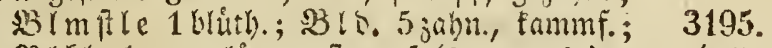
Shlatelm nertíng., ftumpf (4 9. wch.) aca'ulis.

10. PEGĂNUM. 375. (376. $\left.{ }^{x}\right)$

3136.

Bl. villpalt.; Stgl frautart. (4 $\mathrm{SE}_{0} w_{0}$ ) Harmăla.

16. PELARGONIUM. 542.

(303. $540^{4}$.)

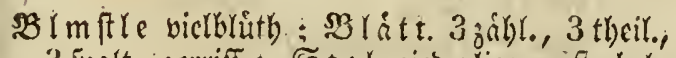
3 pwalt. =3errifi.; Etgl rieserlieg., ftadjelz buar. (4 SAf.) 3197.
columbinum.

15. PELTARĬA. 524.

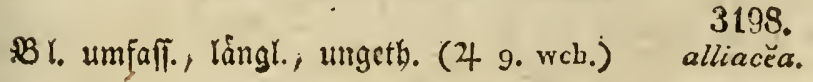




\section{PENTHŎRUM: $367 .\left(36 \eta^{\mp}\right.$.)}

(4 NAm, grn, g gb.)

3199.

sedoídes.

\section{PENTSTÉMON. 503 .}

Stengel:

1. Fenhaär.; ber unfrudbtb. Stfas. v. b: Epise bis unter D: Mitte bart. (4 NAm. 3200. v.; wch. - rch.)

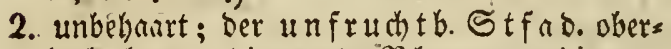
balb bart.; bie unt. $\$ 3 \mathfrak{l}$. ganjranbig (4 $\left.N A m_{0}, v_{0}\right)$

\section{PEPLIS. 264. $\left(292^{3}\right.$.)}

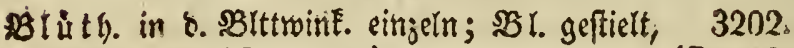
runol. = engr. $(\odot$ 50.' p.)

(Portüla

\section{PERIPLŬCA. $17 \% 2$.}

Enbooloentrabub. imbent. rautch; Br. gee fticlt, entgegengei., ent. u. Lamjettf. (ち As. 3203. - bra..r., Milchsaft) graeca.

\section{FEUCEDĂNiUM. 220.}

Blättèr:

1. 5 fach 3 theil.: $\$ B$ Ia $t$ t dh. gleidhbr. = fabenf.,

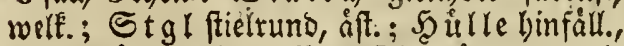

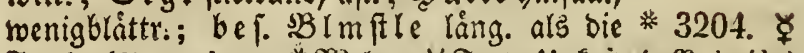

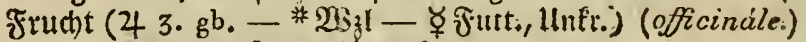

2. 3 fach Dopp. 3 fige fegt ; Blättch.:

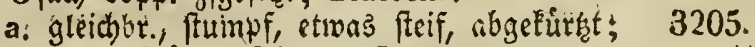
gem. Scillte fehlt meift (4 SE. gb.) arenarĭum.

b. 3 fpalt. $u$. fieoerf., mit lanjettrórnt., faft fichelf., aber., ftechenofpits. Lapp.; meift olge geñi. Şülte (4 SE. wch.)

3206. alpéstre. 
17. PHACA. 583.

D8 Y. gefieocrt: Blättchen:

1. obal, ipis; Stgl aufr., haar.; 2lfterbl.

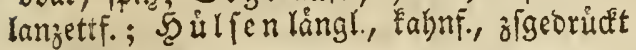
(4 SE.)

2. lángl. =lamzettf., ftumpf, viclpant. S Stgl auft., aff., feinluar.; Jeilfen halbenrunto, fpit (4 9. ligb.)

3. langl., frumpt, etrias geminip., nieift 5 paar.; Stgl aufr., ungeth); Se illien !ängl, ouf= geblat. (4 In. gb., mit 2 v. Fleck.)

4. lanjettf., meift 8 paar.: (5nobl. faft fticl= frigìda.

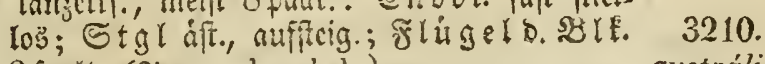
2 ppalt. (4 9. wch:-zbch.) austrális:

5. PHARNACËUUM. 248.

Eeitenblumenftiele faft solvenf., formg als bie gleichbr. SB!. $\left(\odot-w_{0}\right)$

3211.

Cerviána:

\section{PHASEOLUUS. 566;}

Stengel:

I. gerwinberl;

A. Blüthen :

1. it e. cingeln. Biratbe an gepacirten

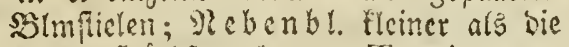

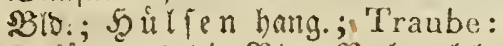

a. Eirs. als Die SBl.; Rebenbl. abe fathento (C) SAs. w.; dsch.; gbch. * Eram. - ४ Yiehl)

b. eben fo lang at bie SBl.; Rebenbl. angetrudét ( $\odot$ SAm. sch.; w.)

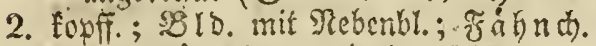
furz: F́f

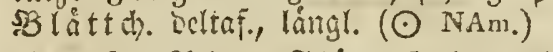

3. in roder. 2lebre; Flíg. ;o lang als

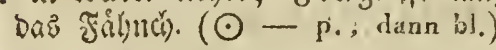
* 3212. vulgáris.

3213. multifórus

3214. helvolus:

3215. alátus.

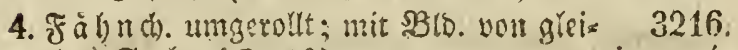
cher frarhe ( $\odot$ Af.)

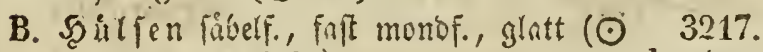
As. grn. v. w.- gbcli.)

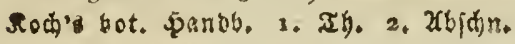
lunátus: $\mathfrak{x}$ 


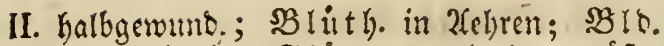
obne Rebenbl.; Flug. ausggebreit, gróß.;

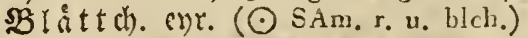

III. nur etwas gerwuno, glatt; Scitenblat te dien 2 laps.: Enobl. 3lapp., mit ersumb.

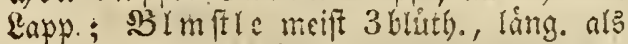

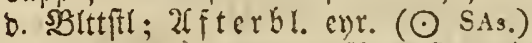

IV. nicerlieg., farenf.: $B$ ( m fthe 1 blith); sBláttd). lanzettf. (SAf.)

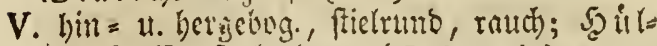
len fopff., ftatbelbaar. ( $\odot$ SAs. gb.)

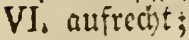

A. Hülsen :

1. slgecridt, runjl., hang.; Stgl glatt;

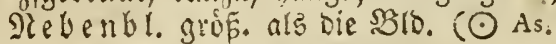
w. u. schwch, - צ Met)l)

2. ftadchanr., bang.; Stgl erfe, backr. (○ As.)

3. fticlune=pfricmenf.; $\mathfrak{B}$ lát thb. långl., langgeipitzt; $\mathfrak{B l}$ I flt le berlángert $(\odot$ SAm. sch.)

B. Sa ame Eugelmuno, mit geparubt. Nabel $(\odot$ SAnt.)

3222. ఫ

nanus.

Max.

semieréctus.
3219.

trilǒbus.

3220. capénsis. 3221.

Mungo. 3223.

322 lathyroides. 3225. sphaerospérinus.

\section{PHILADÉLPHUS, 384. (386.)}

Die langgeipint. Blätter:

1. gefágt=gezabnt, ey)r.eflipt., unten in $\delta$. Xres= wink. unbel)áart (ち) 26. w., wohlriech. - 3226. ఫ

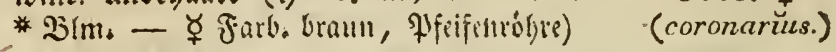

2. gejabnt, eyr., linten in o. 2rocrmink. mit 3227. bujduelf. ftebenben Seraren (ち NAm. w.) grandiflórus. 3228.

3. ganjtano., eyr. (古 NAm. w., geruchlos) inodórus.

2. PHILLIRËA. 9.

2Bl. famallanjettf., ganzrand., unoeutl. aber. 3229.

(ち $S E$, gbch, - w. $\Delta$ ) angustifoľa. 


\section{PHLOMIS. 475.}

\section{Blätter gleichförmig.}

\section{Blätter:}

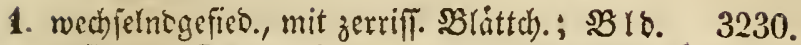
rooll. ( 4 o' As. up.)

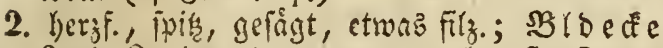

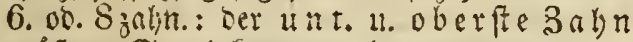
gróßer; Stgl frautart. ( $\odot$ SAs. sch.) nepetifolra.

3. enrunb =làngl., geiảgt, untent ftachế(haar.;

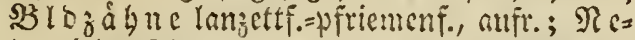
benbl. wfriemenf., u. nebjt Stg! ftachels baar. (24 SE' rcli.).

laciniáta.

3231.

4. Ianzettf., etras gefägt; mit End SB ID S zalju. ( $\odot$ SAs. w.)

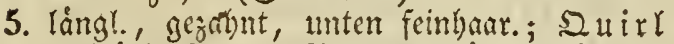
vierblütb., fugcl.; \$3 I b. cinmórtşgetrummt, Szabn: : ber oberfie Sabn febr lang $(\odot$ SAm.)

3232.

Herba venti.
3233.

zeylanǐca.
3234. martinicénsis.

\section{Blätter ungleichförmig.}

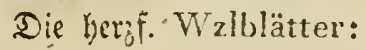

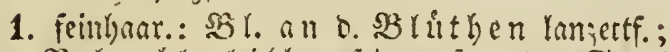
Stebenbl. gleidlbr.=pfriemenf., jott.; ङtgl feinhaar. ( $4 \Lambda s_{\text {s. }}$ )

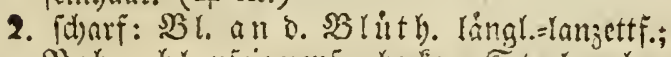
Pabenbr. pfriemenf., bactio; Stgl umbes buart (4 NAs, hp.)

3235. alpina.
3236.

tuberósa.

\section{PHLOX. 128.}

\section{Stengel:}

\section{1. auf̣tecbt; Blätter:}

1. Tangetfórmig, flach, am Ranbe fdari; Etgl glatt; Doldentraub. rifpenf.; SBlelapp. runbl. (4 NAm, rch.)

2. lånty!.. = lamietr F.; Stgl:

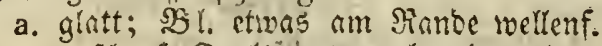
u. [üarf; Dolecuteaub. wie vorber; Blétapp. ant'b. - Spilge etwas einge= briaft ( $\left.4 \mathrm{NAm} . \mathrm{bl}_{0}\right)$

3235. unduláta.

\section{7. \\ paniculáta.}


b. Tharf, geflectt; $\$ 3$ Y. unvebart; am Siande raub; siipe laingl.= gedringts blitth.; sBitlapp. rumbl.; Bidojabne fpis, umgefrimmt (4 NAm. p. dann v., Stgl rch.)

3239.

maculáta.

3. eyr.=lanzettfórm., auf beyo. Geit. glatt; 1

SElt lapp. runol.; Stgl:

a. Fegr glatt, olyne flectem; Ir auben

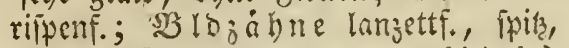
etroas aufi. (24 NAm. w., wolliriech.) suaveólens:

b. feinbanr.; Do loentraub. faft gleith= hodi); bei. 3ureige meit 3 blithig; SIDjábue unbebant, lamzettf., auls. (4 NAm, rch.)

4. enr.; SB (it th. cingeln (4 NAm. hp.)

II. auffteig., unbebnart; $\$ 8 l$ l. ichnollanzettf., glatt; (Endooldentraub. foit gleidboci); sB!D. faft welgrlos (2 NAm.hp.)

III. nieberliegeno; Blätter:

1. eyr.=lanzetfform.: Dic meiften werilfo; Stgl niebrig, feinhant:; be 1 . 3meige mentgbluth.; SZld. p priemenf.=gleichlur.; sBlEYay\}. faft berzf. (4 NAm. hblch.)

2. fomallanjetti, furz, etwos raul); હtgl wie vorber; Enbbitichel foft friellos:

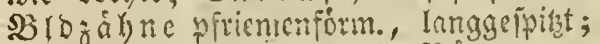
self lapp. rumbl. = fumpf; sidbre ge= rabe, glatt (2+ NAm. hrch.)

3. gewimp.: bie unterften borftenf. =pfrie= menf.: Die obern fintallametti.; 3 we i=

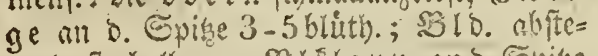

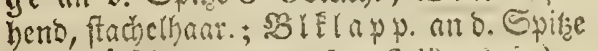
eingeorucớt ( 4 NAm. rch., Sclilund dr.) setacĕa.

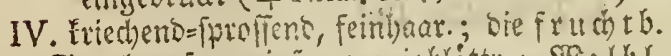
3244.
divaricála:

3243.

glaberrima;

3241. carolina. 3242.

ováta.

Stgl aufr., cinf., wenigblättr.; $\mathfrak{N Z}_{3} \mid b l$.

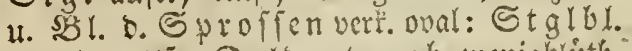

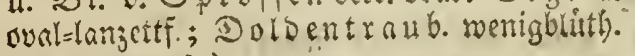
(4 NAm, rch.)

V. Elcin; Blätter:

1. jfriemenf., frechens, geming., gebuldbelt; Etgl weiffeinhanr.; wenige ber. \$B lm: ftiele am Ente ftebeno ( 4 NAm. bro ) subulata. 3245
pilósa.

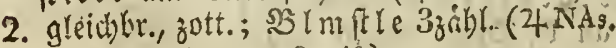
rch,; w. mit xo r. Streif.) 


\section{PHYSĂLIS, 140.}

\section{Perennirende.}

\section{Blätter:}

1. gepaart, ungeth., fpis; Gigl unterhalb etracs áf: (4 8. 14.26. uw. - * Siraut, * 3250. ఫ

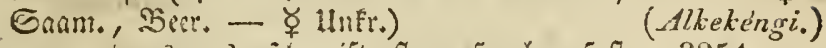

2. evr., etroas allsgeidmeift, ftumpf, oben foft 3251. nackt; $\$ 3$ lit

\section{Jährige.}

\section{Stengel:}

1. edf.; SBl. gepaart, herjfortm., faft ungeth.,

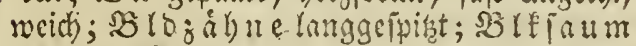
inw. am Grumbe wit 5 Flecte; Sl flanje feimbaar. ( $\odot$ NAm. gibch, u, vo)

2. Fełr \&fftig; Blätter:

3252.
pubéscens;

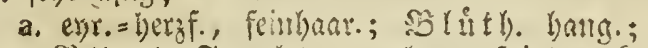

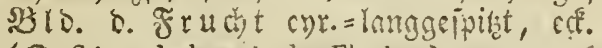

$(\odot$ SAm. logb. mit dv. Fleckein)

b. eyr., geşahnt; 3 weig \& caf., unbehart (○ As, uw.) c. zott.; $B \mathrm{Im}$ frle gimz gerabe $(\odot \mathrm{Am}$.) pruinósa.

3253. barbadénsis.
32.4. anguláta. 3255.

3. Deerhalb rifucmi.; s3t. gepart, sezahnt=

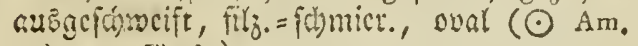
5 braune Fleck.)

3256. foetidissima,

\section{PHYTÉUMA. 145.}

\section{f. Blälter gleichförmig.}

Bliithen:

I, in Siọpfä) en; Blätter:

1. Kanjettförmig : Nebenbl.:

a. rund. = Kersf., gcwimp.; fidpfthen $325 T$. etwas bláttr. (24 10.bl.) pancifbirum.

b. Glecd) br., länger als s. ettwas blittr. 3258 . Riopich.; 98 l. geznint (4 9. bl.) Scheuchzéri.

2. verE. enrund, in D. SBrtffer herablenfent;

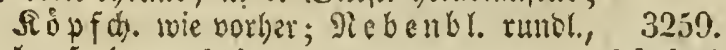

herzf. (4 9. bl.)

globulariaefolium. 
3. gleidybr., faft ganzrant., Faum firtz. ars

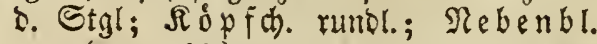
eyr. (4 9. bl.)

II. in o. Blttwinte zerftreut; 3 weige ruthenf; $\$ 3$ l. lanjettf., nuf beyd. Enden ipis, ungl.

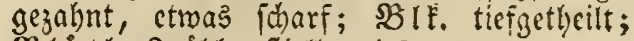

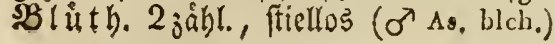

3260.
hemisphaericum.

3261.

virgátum.

\section{Blätler ungleichförmig.}

\section{Bliuthen:-}

I. in runol. Küpfchen:

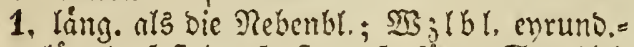
langl., faft herzf, frumpigciägt: Stglbl. fodmallanjettf., am Srunbe gejagt (4 9.* 3262. r. - * $\left.2 \mathrm{~S}_{j}\right)$

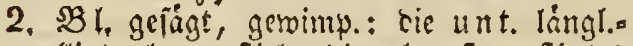
ellipt., langgefielt: bie oberften fliellog (4. 9. bl.)

II. in Aehren:

(orbiculáre.)

3263. ellipticum.

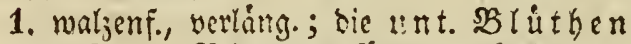
entfernt; SBl. Lamgettiorm., geferbt: bie obern gleiderer. (4 SE, bl.)

2. wabreno s. Szrit the oval, jur seit ter

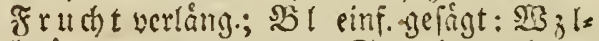
blátt, unb bic unt. Stglbl. geltielt, herzf.: Die oberften mafall., Imongttf.; Nebenbl. borftenf. (2 NAm, schw.)

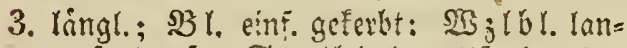
jettf. = berjf.: Etglbl. lamjettf. (4 SE, hbl, ; w.)

4. verlång., länglich); (5) riff. etwas yaar.,

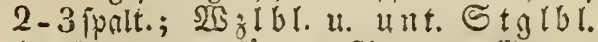
bersf., bopp. gelägt, geficict, ant Brunbe roftbraungefledt: Die ober ften ficllos, fohmallanzettf., weniggerígt (4 3. 9. ugb.; bl. - - * 2531$)$

** 3267.

(spicátumb)

5. eyr.; (5) riff. rauth, lung. alb t. SBlithe, ausgerandet $=2$ ipalt.; $\sin$ \& Ibt. u. unt. Stglbl. heriff, boyy. gefugt: \$. obern lanjettf., einf. gefúgt (24 3.8. dv.)

3264.

acorzonerifolium.

3265. nigrum.

3266.

betonicifolium.

III: in ftiellof. (Enobir fold sBl. gejalnnt:

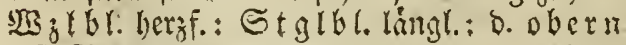
faft fticllos (4 23. unten hbl, oben dv.)

3268. Halléri,

3269. comósum. 
IV. in $\mathfrak{x} \mathfrak{a} \mathfrak{b}$. in b. \$3Inttwink.; $\mathfrak{B}$ I. ftiellos: bie unt. verk. ebruno, geferbt=gefägt : oie obern lanjettf., gamzrano. (4 SE, bl.)

3270.

canéscens.

\section{PHYTOLÁCCA. 371. $(324 \%$ : $370^{x} .408^{x} \cdot 79^{2}$, W. 89 .}

Mit 10 Etgef. u. 10 Stiveg. (4 NAm, p. 3271 . ఫ w. - Siraut - ఫ Farb. (lau, rotb) decándra.

\section{PICOTIA. 105.}

\section{Blätter:}

1. länglich=lanjetti., gerippt, oben unbebaart, glánt., unten feinhaar.: bie unt. langge $=$ ficlt: Sie obern ftielloz (4 SE, bl. u, ob. u, w.)

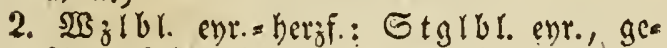

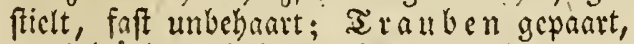
venigbluth,; mit frich. 25 olf tofien $(4$ 4. 7. hbl, u w.)

3. $\mathfrak{M S}_{j}$ lbl. feilf.: Stglbl. fomallanjettf; unbebaart, am Ranbe feingezabnt=ld)arf: Sa a m. Érugf., gejabnt, runjl.; $\mathfrak{x} \mathfrak{r} u b$. olne Nebentl. ( $\odot$ SE. w.)

4. $\mathfrak{B}$ l. Lanjettf., ftharf: bic unt. ppatelf., ent. gegengel.: Die ub bigon wed $)_{\text {. }}$; Stgl nies bergeftredt, gabelfo, faft 4 edf.; 31 Imlt le

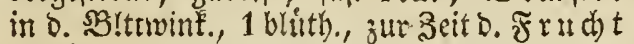
abwairtzgebog.; S. genabelt, glatt $(\odot 1$. 4. 48. hbl. u. gbch,)

\section{2. niț̌da.}

3273.
verna.

3274.

linifolıa.

3275.

scorpioides.

\section{PICRIS. 619.}

Der aufr. Stengel:

1. idharf; $\mathfrak{B l}$ l, mufofl., lanzettf., gezahnt; Blut then dolentrauberf.; bic áu fd)laff (4 15. gb. - ఫllufr.)

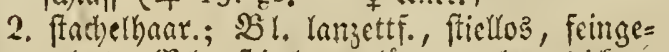
zahnt; SBlimftiele yerláng., oben bidfer; 3277.

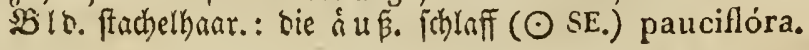




\section{PIMPINELLLA. 192.}

\section{Blätter:}

1. gefiesert; Blättchén:

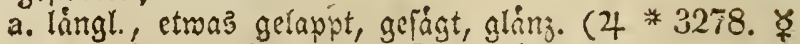

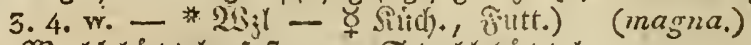

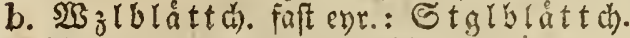
fd)maler, lanzettf. = gleichler. (4 ir. 14 . 34. w. ; rch. - * ijji, Sirnut, Caam. * 3279. ఫ̧ - ఫ Silidt:, futt.)

2. bopp. Jijefeft, gleicibr.; Stgl felyr aft., rifpenf.; Dolden febr jablecith (4) 3.3 . wch.)

\section{(Saxifrŭga.)}

\section{0. diǒ̌cs.}

\section{PINGUICǓLA, 20\%}

\section{Nectarium :}

1. piriemenf., faft gernde, jo lang alb bas \$B!bl.;

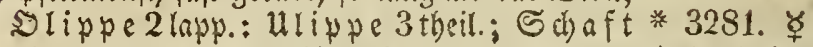
glatt (4 47. v. - ל Uluf́r.)

2. Eegelf., finz. als b. SBlfloppen, gefrummt (4 9: wch., mit gbch. Streifea)

+1 (vulgáris.) $32 S 2$.

(alpina.)

21. PINUS. 770. (W. 26.)

\section{Blätter gepaart.}

\section{Zapfeñ:}

1. enr. = fegelformig,

a. meift gepaart, am esrunbe rundl., fo lang

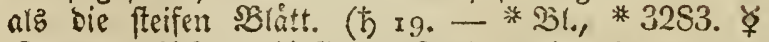

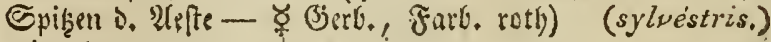
b. einzeln,

S. am (S)runde rundr.,

1) ba!b fo lang a!s bic SBl., mit webre 3284. Iof. Solupp. (t) Ninu.) resinósa.

2) fait Furr. als bie febr lang. $\$ 31$, mit 3285. fumpi., glatt. Schupp. (b NAs. $\Delta$ ) halepénsis:

b. Febr glatt, gefitieit; \$3i. fehr frin (i) 3286. SE.) mariț̣ma,

2. e๖runo; Blätter: 
a. Febr lang, ungreidjf.; 3apfen fold am (Simnde fommaler, an D. Spibe fehr Did u. obne Edfen ( $5 \mathrm{SE}_{\text {. }}$ ) 3287.

b. Die erftern geroimp.; 3 apfen frumpf,

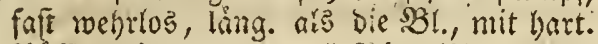
slüfen (ち SE. $\triangle$ — Milfe (Pinicn) ఫ $\mathrm{Del}$ )

Laricio:

3. rumol.=enr., ftumpf; mit wehrlof. Schupp.;

Stainm auffteig.; SB l. angeoridft (5 9.) Pumilio.

4. ไangl.=fegelf., aun (Stunibe runol., mit frachl. Solupp., I.

a. fing. als bie SBl. (方 SE.)

b. To long als die SBl. (ち NAm.)

3290.

Pináster. 3291. inops.

* 3288. ధ

Pinĕa. 3289.

\section{Bl. gepaart u. $3 z$ ählig.}

R1. fteif; $3 a$ pen longl., mift gepant, am (5)unde rumbl. (5) 9.)

3292.

Mughtus.

\section{Bl. 3zїhlig:}

\section{Zapfen :}

1. enr., gchăuft: S chupp. mit zurudgefchlag. 3203. Dornet; $\$ B ! t t f b$ e io en abgetimgt ( 5 NAm.) riğda.

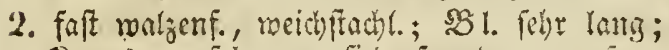
snospenfdulp. fieoerf., Daut., afrer= 3294 . blattart. (b NAm.)

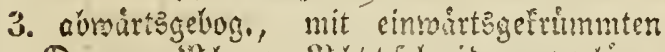

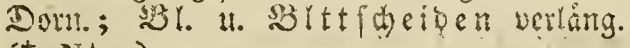
(ち NAm.) palústris. 3295.
Taeda:

\section{Bl. 5 zählig:}

\section{Zapfen:}

1. enr., ftumpf, mit angeoruft. Sthupp. u. bart. Núfien; bef. 3 weige bidjt u. ftare genarbt, etwas filz. (5 9. - * ¿arfam ૪ Der, Barb. roti)

2. walzenform., fdylaff, an o. Spike bumer,

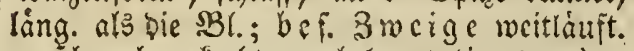
u. ichnach geferbt, unbchaart (b NAm.) Strobus.

3296.

Cembra.
3297. 


\section{Bl. in Büscheln.}

\section{Blätter :}

1. baurend, fteif, fpik; 3 äp f. faft fugetruno, 3298 . mit abgeftukt., angeorúdt. Sđupp.(ち) As. $\Delta$ ) Cedrus.

2. abfalleno; Zapfen:

a. långl: $\mathfrak{S}_{\text {ch }}$ pp. mit eingebog. Rand; Nebenbláttch. geigeñf., mit (chmaler Spitze (ち NAm,)

b. rundl., menigictupp.: $\subseteq_{\text {chupp. umges }}$

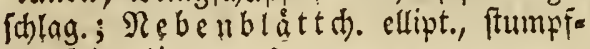
zugeipict ( $5 \mathrm{NAm}$.)

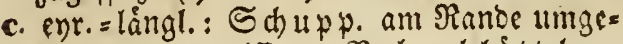
foblag. ul. zerriften; Rebenblattchen geigenf. ( 5 9. - ${ }^{*}$ Ierpinttin)

3299.

pendūla.

\section{NI. Bi. einzeln, am Grunde abgesondert.}

\section{Blätter:}

1. flach,

a. 2 jeilig, ganjrano, aliggerant.; Zapfenschuppen:

ค. Febr ftumpi, angeoricêt († 9. pentbin - ఫ (5ierb.)

b. am blibeno. 3apf. langgeipigt, unges fd)!ag.; B (. obcrbalb faft aufr. (ち NAm, - Terpentlinil)

b. faft 2 zeil., feingefägt, etmas fpiç $3 a=$ pfenichupp. runol., gerade (b NAm, 3304.
- Caft)

2. 4 fantig,

a. 1 feit., getrummt; $3 a p f$ faft walgenf., folluff, hang., mit rund. = ipatel $\overline{1}$, ganjz rant. Schupp; bef. 3 weige unbehaart ( 5 NAm.)

b. faft 1 leit., aufr, ganj gerabe; 3apfen eyr., mit aufr., ellipt., am Ranie wellenf. Sthupen; bef. 3weige feinbaar. (b NAm.)

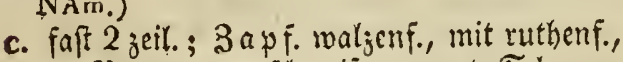
ant Rande ausgcitumeift=zernagt. Echupp; beiono. 3weigenubebart (t) 49. -

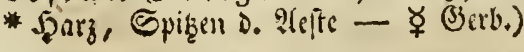

3305. alba.

3306. nigra.

* 3307. ఫq (Ab̆̌es.) 


\section{Pisum. - Plantago.}

17. PISUM. 573.

Blumenstiele:

1. 1blithig; Blattstiele:

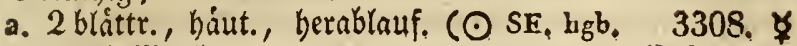
- $\forall$ Mefi) ${ }^{\circ}$ Ochrus,

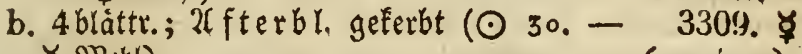
ఛ्र פibl)

2. vierblitthig: Blattstiele:

(arvense.)

a. ftielrumb; $\mathfrak{A} f$ terbl. unterbarb rund, gea 3310. ferbt $(\odot$ 29. w, u. ghch. — $\Varangle$ Mefh, Siuid.). sativum.

b. oberbalb etwas flact); Stgl ect.; 2 fter= 3311. ళ̧

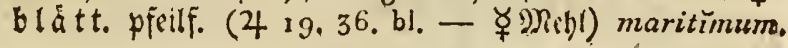

\section{PLANÉRA. 231.}

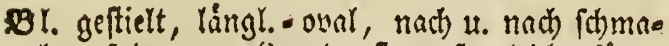

ler, fpis, am sirunoe ftumpf, gleidgejágt; 3312.

Stapf. idarf (ち NAm. braun-grn.)

aquatı̌ca.

\section{PJAANTÁGO. 59.}

\section{Mit nacktem Schaft;}

A. Schaft stielrund;

1. Blätter eyrund u.

a, zonbehaart.

Jebre walgenf.: Blïthen:

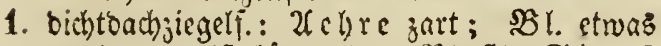

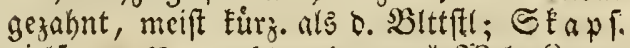
vielfam. (4 r 4 , bl. -rch, - * $233 \mathrm{l}$, Siraut, * 3313. ఫ Gaun. - $\nsucceq$ Futt., Birb., llukt.) (major.)

2. am Brumbe entfernt; $B$ lát t. etroas bid"; selttifle fegr lang, eff.; Sdjaft ver= 3314 . làng. (4)

exaltáta.

$$
\text { b. behaart. }
$$

\section{Blätter:}

1. ganzranoig,

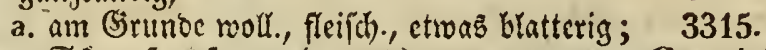
Etapf. 4 fám. (4 SE.)

Cornúti. 


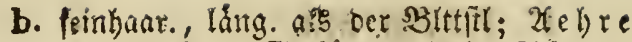
walzenf., furz; St fád. Yilafarb.; Stap f.

2-4 jaam. (43.14.34. - ఫjutt., linfr.) (media.)

2. feingejabnt,

a. 9ripp., fsinhaar.; Xehre wnaljenfơrm., 3317. Dachjiegelf.; $\subseteq$ aft lod (4 NAm.) maxima.

b. 5 ripp., hanr.; 2 Cehre malyenf.: $\mathfrak{B} l u=$ then etwas entferntftchens: bie unter=

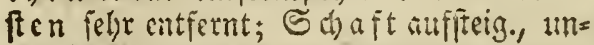
ten fificlrund (4 - hbraun)

$3316 . \not$

\section{Blätter lanzettförmig:}

\section{Blätter:}

1. ganj lanzettf., 5 ripp., undeutl. Fringezabnt

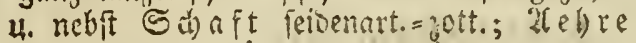
enr.; $\mathfrak{B Z}$ ! 3 biattr. (4 SE.)

3319. sericěa.

2. eyr. = lanzettf., faft ungeth.; $\mathfrak{X}$ chle lang, zart; \$Bluth. einjein 1. jerffreut, unbe= banț $(\odot \mathrm{NAm}$.

3. fohmallanjettif.,

3320.
sjarsifióra.

a. feinlyantig,

a. ganzrants.; Sobaft Finhanr.; $2(e ̣)=$ ren eyr.; Rebenbl. breit, trof., an

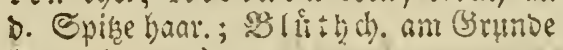
baar. ( 4 SE.)

3321.

saxatìlis.

3322.

b. gezalunt $=$ gefiggt, 5 ripp. (4 SE.) Serrarì.

b. rauch); $2($ el) $r$ e fugel., $12-20$ blutt)., am (Srunde etmas rauti); Reberibl. Hurjer als bie sBluthe; Solit aft frinhar., am Grunte mit brounlich. Santen umgeben (4 SE.)

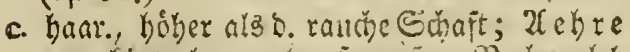
evr., binterlier walgent., auft.; פebcnbl. lanjettf. ( $\odot$ SE.)

3323. victoriális.

3324

Bellárdi.

3. Blätter schmaler.

\section{Blätter:}

I. gleid)breit,

1. etroas gejabnt; A ehre:

a. malzenf., unbebant; Nebenbl. fo lang als b. Blo.; $\mathbb{G}$ (h) aft rauch); $\$ 3 !$ unten etras gemolbt, unbebaart ( 4 SE.) salsa. 
b. longl., aufr.; Rebenbl. enr., folf, am Yuñde båut.; $\mathbb{2}$ !. all D. Enden (4)maler, entferntgezabnt; Sd)aft

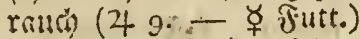

c. faft eyr.; Rebenbl. nadjenf., bát. (○) SE:

\section{6. \%}

alpina.

3327.

Loeffing II. 3323.

2. fieverf. = gezaknt $(\odot$ r. 8 .)

Coronópus.

3. gamjranoig,

a. flach,

a. fleifing, frumpF, fort gamzronoig;

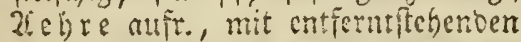
SElith.; SBYE TaUm aUfr. (O SE.) tenuiflóra. 3330.

b. 3ripts., an bego. Eno. jomalcr (4) b. rimenformig,

a. getrimnt, naft ( $\odot$ SE.)

Wulfénz. $\therefore$

$3331 \cdots$.

b. dunterhars mit, feif. Simporn, am (Sirunde bact.; Sch a ft pringonr.;

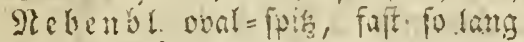
alB. D. SBtuthe ( 24 SE.)

recurváta.

II. Garbwalzenf., ganzrano., ani Gerunce wodl.

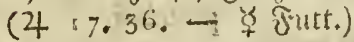

3332 :

subulata,

III. barbwarjenf. $=3$ fant., feingefingt $=$ gemimip., am (Srumbe woll.; Sd) a ft zott; ? 2lebre

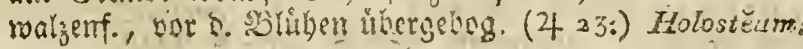

B. Schaft ectig.

\section{Biätter :}

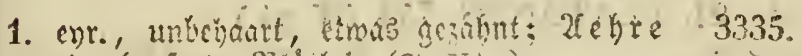
nit abgerons. Brathot). (24 NAs.)

asiatica.

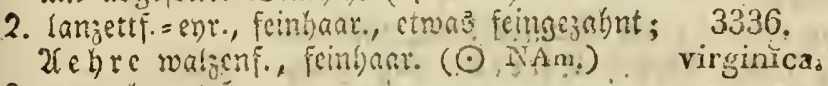

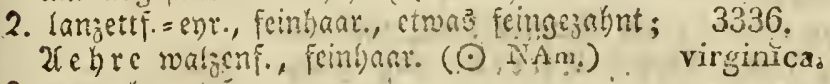

3. gand lamjett..,

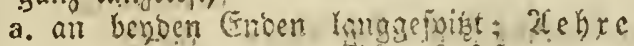

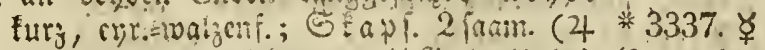
13. 14. wch - * Siraut - ૪̧Gerb., Unfr.) (lanceoláta.)

b. 5 ripp., ganzmano. u. gezabnt, unbebanrt;

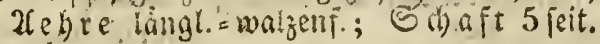
(4 48.)

4. breitlangettf., 3 ripp., etroas gejahnt und baar, Z A Chre langl., raith (4 SE, w. gbch.)

3338.

altissüna.
3339. lusitañ̌ca. 


\section{I. Mit Stengel;}

A. staudig.

Etgl airt., etmas ithari; 3 l. fubeni., faft

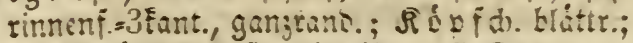

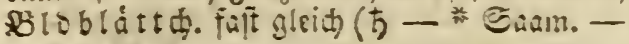
$\checkmark$ LuEr.)

- 3340.

(Cynops.)

\section{B. krautartig.}

\section{Blätter:}

1. ganjiantig,

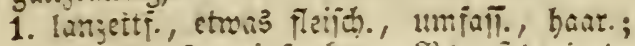

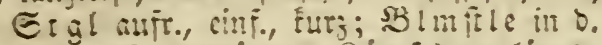

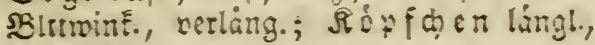
Elattlos $\left(\odot S E_{0}\right)$

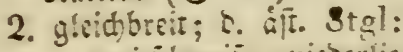

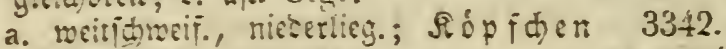

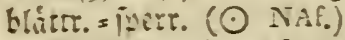

b. aufr.; sB!. tinneni.; $\mathfrak{A} \delta$ pid. blatt= log $(\odot)$

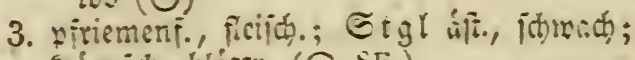

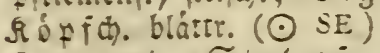

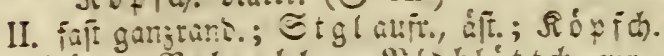
bláttr.; Mebenbl. u. șleblátt d. ent.;

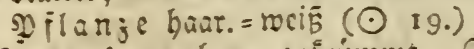

3341.

amplexica'ulis. squarrósa. 3343. stricta.

(Orenaria.

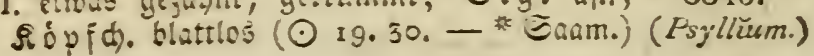

\section{PLATĂNUS. 753. (W. 94.)}

Blätter:

1. 5 lapp. - bantó., am Stunce feilf., nit lans jetti., bucht. Lapp.; 2afterbl. iaft ganjs

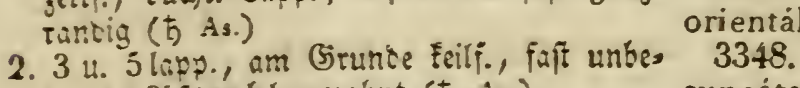
baart; 2L fterbl. gejahant (5 As.) cuneáta.

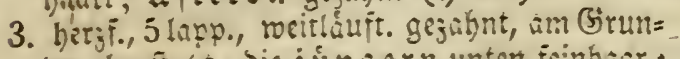
te abgeftust: Lie iungern unten feinhuar.; $33+9$. Uf ferbl. gejahnt (万 As.) acerifolia.

4. 5 cff., unteutl. gelaypt, gezăhnt, am Grinte feiff.: bie jüngern u. df ferbl. wie ror= bet (ち Nom.)

3350. occidentális. 


\section{PLEUROSPÉRMUU. 196.}

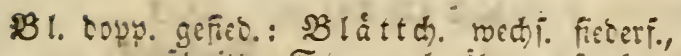
eyr., 3erithnitt.; Etengel rébr., gefurbt; 3351. Sullfe klittart. (4 9. w) ) auscriäcum.

\section{PLUMBÁGO. 91.}

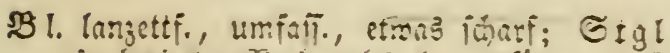
ausgebreitet; Necrent. langetrơrm., fant nadt; bel. 3weige glatt (4SE. p.; v.; 3352 . w. - 2ijil) teuropa'ea.

\section{PODALYRĬA. 335.}

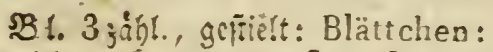

1. runol. = verf. cyrunt, fiumrf, auşgerant.; 3353.

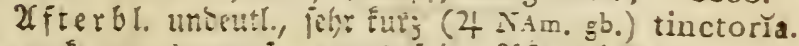

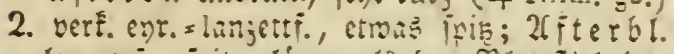

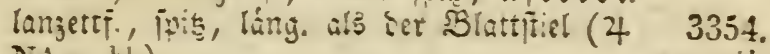

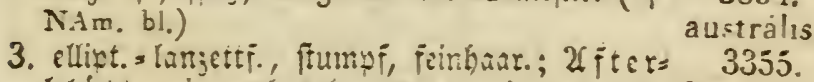
blát t. roie sorber (4 Nis. gob.) lupinoides.

13. PODOPHÝLLUM: 420 .

BI. Pdift., gclappt (4 NAm. พ.)

3356. peltátum.

\section{POLEMONIUU. 130.}

S2 1. gefietert; Blüthen:

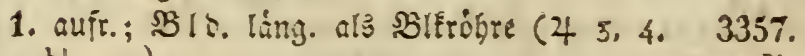
bl.; w.)

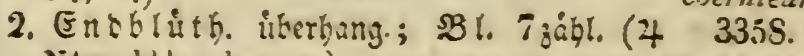
Namo bbl., danz w.) reptans. 
6. POLYÁNTHES. 279.

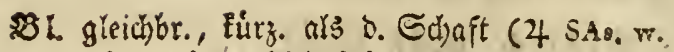

u. rch.. sehr' wohlriech.)

3339.

tuberósa.

3. POLYYÁRPON. 48.

3360.

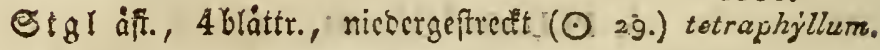

\section{POLYCNEMUM. 41.}

Blätter!

1. pfricmenf.=prisntat., an o. Spite feintoornig 3361. $\Varangle$ (๑ 28.)

2. pricmenf., jerftreut, ctrias abfergend, abs gefonbert, etwas getrinmt; \$310. faft jo 3362.

lang alb bie Giapi. ( $\odot$ SE.) recúrvum.

\section{POLYGĂLA. 556. (542\%)}

\section{Blithen:}

1. mit pinfelf. 2anbang, traubenf.; Bldnügel:

a. 3 ripp., ftumpi,

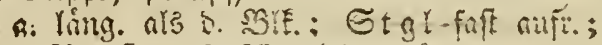

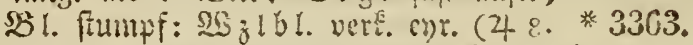

9. hbl.; v.; f.; $w_{0}$ - * $2 W_{3} l$, firaut) (anára.)

b. eben fo lang a!s o. Br. ; Stgl nic=

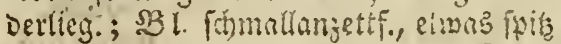

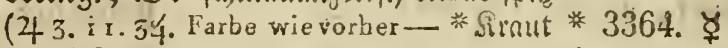

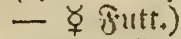

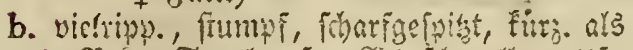

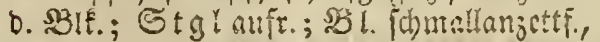
ipity (24. 3. .)

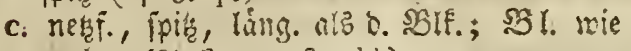
vorber ( 4 6. 1 3: uी.; bl.)

2. nicht; Stengel firruth.; BSI. oväl=löngl., (wulgáris.) fpis, ganjrano, am Sande umgetrummt;

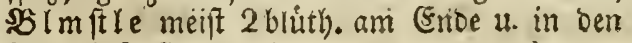
\$Blttroinf. ftebento (

3365.

major. 3366. (monspeliäca.) 3367. Chamaebríxus. 


\section{POLYGŎNUM. 320. (274}

$292^{3} \cdot 305^{x} \cdot 362^{x}$.)

\section{Strauchig.}

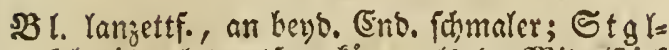
(b) e i De lanjettF, furg. als D. IMittel fut

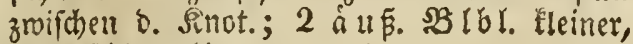
umgcichlag. (衣 NAs. rch.)

3368. frutéscens.

\section{II. krautartig;}

A: mit 5 Staubgefäfsen.

Nit 1 balbgefpalt. Stmeg; Blätter:

3369. 1. eyr.; $\mathfrak{B}$ IE. 4 fialt., ungleidf. (4 NAm. w.) virginiánum.

2. långl. od. lambett:; $\mathcal{A}$ ehren evr.; Stgls ide iden am Ranbe ofrriff. (4 3. 44. 11. 3370. ఫ

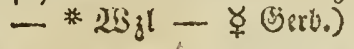

B. mit 6 Staubgef äSsen.

I. Nit' 1 Stiveg; 3 I. Fömallanzettforis.; 3371.

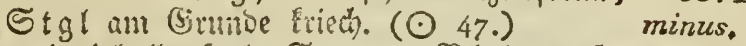

II. mit 1 balkgeipalt. Stıe $; \mathfrak{B}$ l. lanjettf.; Etglfecisen bortit: = gemimp.; Uehren

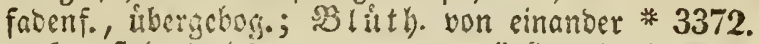

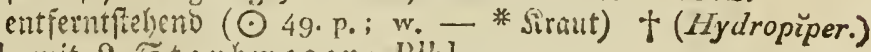

IIl. mit 2 Staubregen; Bibl.:

1. meitit 3 ripp.; 2 2 c bren eyr. =lengl.; 38 t. lan;etf., umbebart; Siglfcheiben ab. gefurzt, jecrifi., gervimp. ( $\odot$ 14. 48. p.; * 33\%3. ఫ

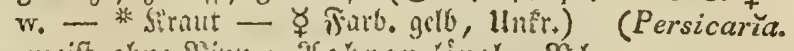

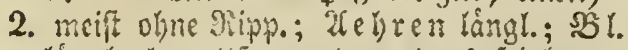
lángl. =lanjetif., I unten etwas frinbaar.; Stglfde iben wie vorber $(\odot)$

IV. mit З Stweg.; 2fehren ruthenf.; Stgl=

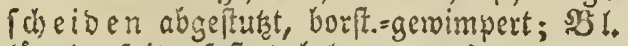
langl., fivis, faft unbebaart (As.)

3374.
incánum.

3375.

barbátum.

\section{C. mit 7 Staubgef äfsen.}

Mit 2 Stweg.; $3 \mathfrak{B}$ !. eyr.; Stgl auir. unb nebft Stglicheioen ftachelluar.; 2(ebren überkang. ( $\odot$ As. r.)

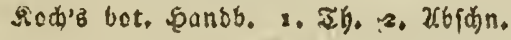

3376. orientále. 


\section{D. mit S Staubgefä $\int s e n$.}

Nit 3 Staubwegen; Stengel:

1. nicht gewunoci, ganz einfach; Bliithen:

A. in $1 \mathfrak{2}$ e h $\mathfrak{e} \mathfrak{e}$, Blätter:

1. evt., in D. SBgttfitel bernklauf. (48.

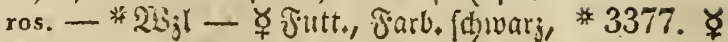
(Bert.)

2. lanjettf. (4 9.34. w.)

B. in trauburf. sicipen; Blätter:

(Bistórta.) $337 \mathrm{~s}$.

vivipărum.

1. am (Sirumbe id)maler,

a. fdymallanjettf., unbebaart; Stglscheiden:

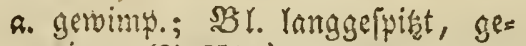
tvinip. ( 4 NAs.)

b. nid)t gemimpert; Stengel áft. (4. NAs.)

b. gatij lanzettfo;

A. glatt; Stgl meitifomeif., etwas abfteheno, umbelanrt (4 NAs. $r$. - ૪

b. oben fdjarf, unten feinkanr., an Siande wellenf.; $\mathfrak{S} \pm g l$ áf., lut terbult fcinbant., mit gemimpert. (Selenteit (4 NAs.)

c. eyr. =lanjettf., unbebaart, am গante gervimy.; Stgl dift., mit bart. (B)e= lenfen (4 SE. 9. w:)

2. am Grumbe herzf. =pfeilf.; Stgt auft., 3381. ఫ divaricátum.

salignum. 3350. acidǔlum. -

3382.

undulátum.

3383.

alpínum. melirlos; Saamen:

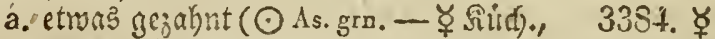
Futt., Fartu. gerú)

tatarı̌cum.

b. an b. Spise ahgeftuht, auşgerano., 3385. mit fncrpl. Fłingein $(\odot \mathrm{As}$, w.) emarginátum.

c. nit gleid). Edéen $(\odot 30$. w. - rch. -

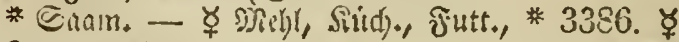
Farb. blait)

II. gewunben; Blätter:

(Eagopyrum.)

1. ellipt., frumpi, etwas fleifh.; Stengel

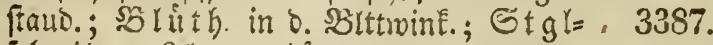

iheiden 2 laps., hăut. (5 19.36.) maritǐmum.

2. lanzettf.; Stgl fralitart, nieberliegeno;

\$Blủ h. mie vorker $\left(\odot 22.29 \cdot r_{0} ;\right.$ w. * Siraut - ఫ gutt., unfr.)

3. Gerzf.; Stgl.: 
a. edig; $B$ litthen ftumpf $\left(\odot 29 . w_{0} \cdot 3389\right.$. grnch.) (Convolvŭlus.)

b. glatt; $B$ lúthen nachenform. = geflugelt 3390 . $(\odot$ r. 4.)

(dumetórum.)

\section{POLYMŇ̃. 707.}

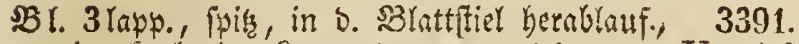
mit ed. =bud)t. Eapp. (4 NAm. gb.) Uredalia.

\section{POPULLUS. 789. (W. 78.)}

\section{Blätter :}

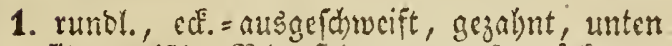

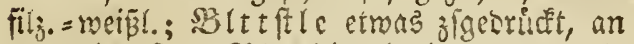
ben oberfen S3l. nicht balb jo lang als

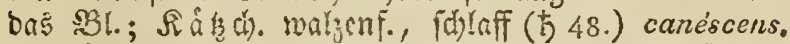

2. hergform. = runol., gelapst, gejahnt, tunten

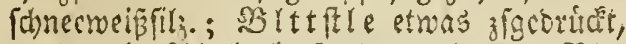
an D. o J. S3\%. bull fo lang alb das \$3r.;

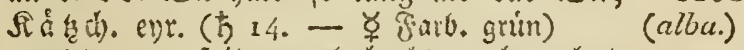

3. runbl. =egr., phits, ungl. bucht.=grobgezalont, unbekaut: bie jungern oott.; SBlttifle oben jigeorid $\left(\frac{5}{5} \mathrm{NAm}\right.$.)

4. runol., mit einem mal langgefyist, gezabnt am. (Srumbe oben 2 oriif., unbebaart: bie jifngeru pionart. (方 NAm.)

5. Ercistuno, firis, gesabut=cef., auf beysen

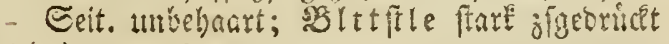
(b) 4. 26.)

6. rund. = enr., ctwas berzf., gleid' 'u. anges exidt =gefágt, unbehacrt, an Ranbe etwas

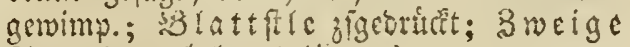
frieltuno, untebant ( 5 SE.)

7. ocitafiomig, langgepint, gelögt, auf beyo. Scit. unbeiacrt,

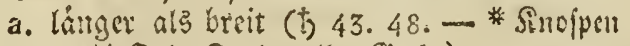 - $\Varangle$ Dir, Jarb. gerb, Gerb.)
b. breiter als lang ( 5 SE.)
(nigra.) 3399. dilatáta.

3394. grandidentáta.

3395. trepida.

(tremüla.)

\section{7. graeca.}

(J)icrwon giebt $\mathrm{cb} 2$ Tibarten:

r. mit falt frieltumen bef. Stwsigen:
3. mit etwas edf.

italkca.

carolinèngis.) 
8. rautenf., langgerpist = verlårtg., gefig̨t, anf bens. Eeit. unbelaart: Die jing. bejond.

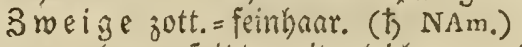

9. eyti., langgepist, mit gleich., engeoridten

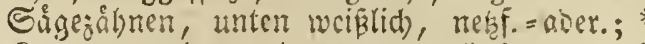
Inospen harg. (5 NAm. - * Warg)

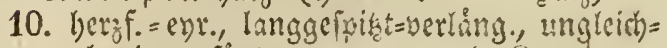
u. bogig = gefägt, unten, und sinospen wie vorhe: (5 NAm.)

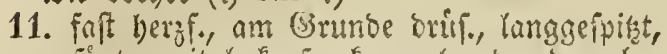
gefugt, mit hafenf., fnorpelrano., dem $b c=$

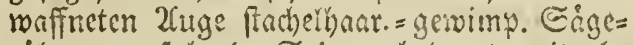
zånhen, nuf beys. Seit. unbebnart, mit ab=

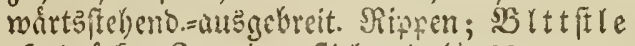
zigebrioút; 3 weige ftielruno (ち NAm.)

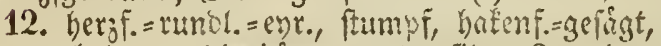
unbehaart: bie jưng. unten fild.; S weige fitelrund ( $5 \mathrm{NAm}$.)

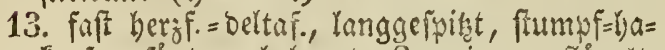
fenf. =gefảgt, unbebart; 3 weige gefligelt= ed., (b NAm.)

\section{0.}

hucisonica.
* 3401 .

balsamifěra.

3402.

candicans.

\section{3. \\ monilifĕra.}

3404. heterophýlla.

3405 . anguláta,

\section{PORTULÁCA. 373. $\left(262^{3}\right.$. $\left.366^{2} .381^{3}.\right)$}

Bl. Eeiff.; Szluth. fthellos; Stgl nieberge= frectit $(\odot$ 2r. 24. 29. gbch. - * Siraut, *3406.

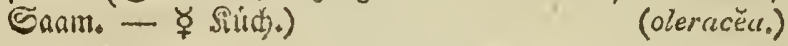

\section{POTAMOGÉTON. 89.}

\section{Blätter elwas breit.}

\section{Blätter :}

\section{1. elliptifoch,}

a. Urle ellint., geffict, fobroimment: bie

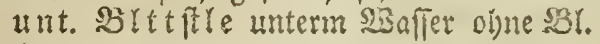
(4) 37. 44.)

b. bie obern ellint., geftelt, an kend. End. fdimaler: bie unt.'gleidbre, ftiellos, ge= biuft (21 42.45.)

2. herzf., umfaff: 2rrle unter ftietruno $(437.42$.

3407.

(natans.)

3408.

helerophy'llus.

(perfoliátus. 


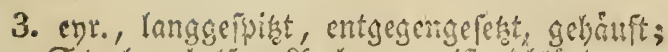
Etgl gabelf.; 2felgre meift 4 bihth. (4 42. 45.) 3410. densus.

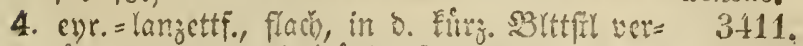

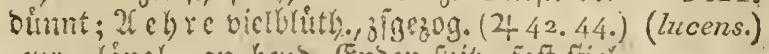

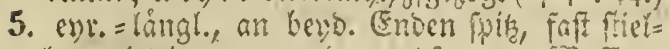
los, vicltipy., ungeth, wechf, unters Saffer; 3412 . 2fterbl. beritianjett. (4 37.) obscírus.

6. Die fad witumenoen lanjettf.=eyro, lang= geffielt, an herove Enoen fomaler: o. unt.

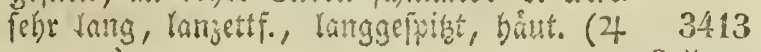
37. 42.)

fluťtans.

\section{Bl. sclzmaler.}

\section{Blätter:}

1. Kanzetterornig,

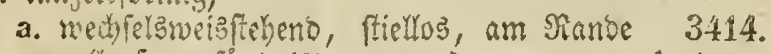

wellenf, gefäst (4 4r. 42.)

b. entge crigcicht,

(crispus.)

3415.

a. am Sianoe etwas wetrenf. $(437.4 x$.) serrítus.

b. lamggepipiat ( 4 42.45.)

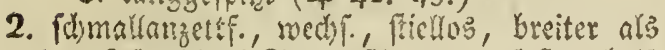

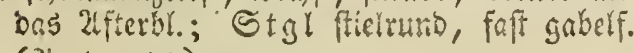
(4 4. 45.)

3. gleicioberit,

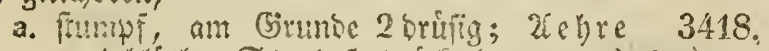

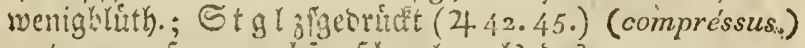

b. entigesengef. u. wechi., fchmaler are bas 2ffterbl., am Grunte Geffrkeno; Stgl 3419. fitietruno (4 4.2.) (prcsillus.)

4. Yanzettf. glcichbreit, flach, ganzäando, in e.

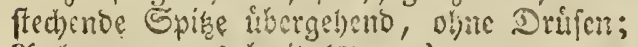
2lebren etwas breit (4 42.)

5. borfenf., parallet, angenábert, 2 zcit., an Girunde idjeidef. (4 42.45.)

3416.

( $($ setacěus.)

3417.

(graminěrıs.)
3420.

acutifolürs.

3421.

(pectinátus.)

\section{POTENTÍLI.A, 405.}

\section{Sirauchig.}

B1. gefict., feinhanr., mit longl. = Inmzettf.,

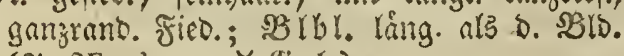

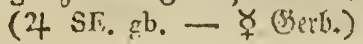

3422. ఫ๐ fruticósa. 


\section{II. krautartig;}

A. Bl. sümmtlich grefiedert.

\section{Stengel :}

1. fried); $\mathfrak{B}$ r. unterbroch. gefico., fetbenart.:

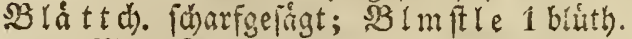

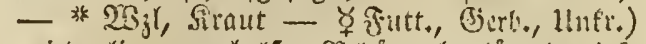

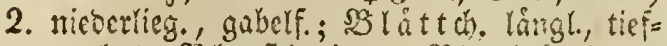
gezahnt; SYImfte in ben SBlttwint. cinjeln (๑ - hgb.)

3. aufrecht; Blätter:

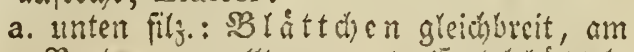

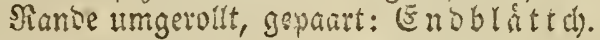
3 fwalt. (2. NAs. gb.)

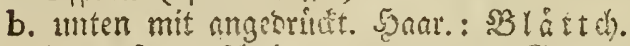
lonjettf., jerfornitt. = gezahnt, am (Strunoe berablauf.; 20fterbl. migeth.; $\$ 316$ l.

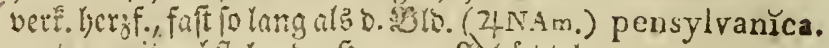

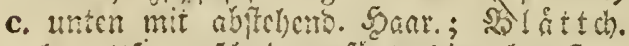
langettf., jerfonitt.=gefagt: Die b berfen an Grumbe herablanf.; ifterbl. jet=

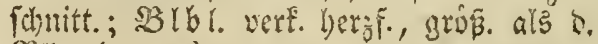
SBlo. (24 $\mathrm{As}_{0}$ )

nis 3427.

$$
\text { B. Bl. gefied. u. } 3 \approx \ddot{a} h l i g \text {. }
$$

Stg! sufrectit;

1. 3weigebl. 3 jahl.: bie ubrigen gefies.

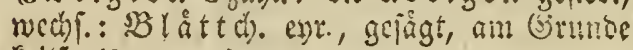
failf. (4. 8. w.)

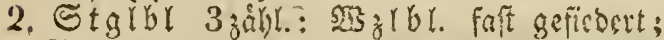

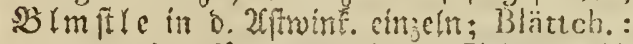

a. eyr., ticfgefigt, anf bryo. Seiten nebft Etgl angeoruckt=bant. (4 NAs. ob.)

b. Ianzettf., unglrich = u. grolugefagt; auf beno. Seit, nebft Stgl abizehent=bant. (4) diffúsa.

$$
\text { C. Bl. dopp.fiederförmig. }
$$

Stgl niederlieg.; Blätter;

1. Laar., obear mubgart, unten fil entfernt. 2fGichnitten (4 SE.) multifüda.

2. vicłpar., auf bent. Esit. fitz., mit parallel., 3432. angenábert. 2abidntitt. (4 NAs. gb.) sericěa. 
D. $B l . \ddot{a} m m t l .5=\ddot{a} h l i g$.

Stengel :

1. aufrecht; Blättch.:

1. verE. eyr., grobgezahnt; SBlbl. an Der Spise etwas cingeoridet, fleiner a's bie SBlo. (4 SE, gb.)

2, feilf., zerfounitt., unten fils. (4 22. gb.

- - Farb. (f)

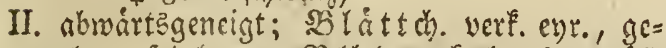
zahnt, fcinbaar.; \$Ibl. vert. Lerzf., grób. als D. SBlo. (4 1 . 34. gb. — ఫ (5itb.) 111. aufiteigeno; $\mathrm{BI}$.:

1. zott.; Stgl ratt) (4 NAm. gb.)

2. 293 j lbl. länl., gejalnt: bie oberfen

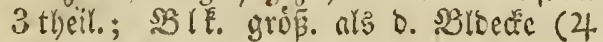
NAs. ligb.)

IV. nieberlicgent; $B$ Y. an D. Spitse zigeneigt= gefágt; SBlbo. rauch); Stgl:

1. fuecnf. (4 5. 9. w.)

2. viclblittl.; Blbl.:

a." vert. eyr. ( 4 9. wo)

b. runor. (2) 9.)

V. niebertieg. u. fproffeno; $\$ B I n$ ft te 1 btuth., in D. SBlttwine ; Bl.:

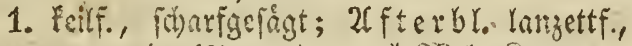
ganjranb. (42.gb. - $25_{j} l$, Siraut ఫ (ङ)

2. vert. ejuns, grobgerägt; 2fiterbr. zer = (b) nitt., 2 ipalt. (4 NAm.)

3433.

pilósa.

3434. ఫ్

(argentěa.)

3435.

(verna.)

3436 .

canadénsis.

3437.

astracanı̌ca.
3438.

(alba.)

3439 .

cauléscens.

3440.

clusiána.

\section{E. $B l .5 u .3 z \ddot{a} h l i g$.}

Stengel:

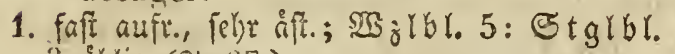
3 zóliglig ( 4 SE.)

3443.

intermedia.

2. auffireigeno;

a. Srocigebl. 3jágl.: die ubrigen 5 jäbl,

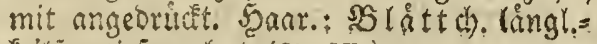
feilf., ticfgezahnt (4 SE.)

b. $\odot t g l b 4.3$ joblig:

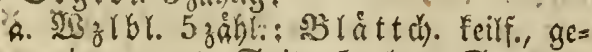
roimp., an o. Spine 5 zabn.: Stglbl. falt ftiellos; SBlbl. vert. berif., gros.

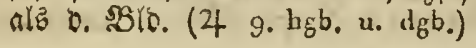

3445.

(aurěa.) 


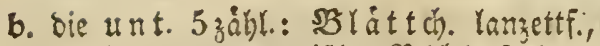
gezaínt, unten meiß̄l.; SB Ibl. jolang alక D. Sglo. (4 SE.)

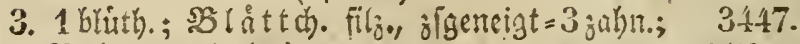
.3Y60. vo:l. (4)

3446. inclináta. nitida.

\section{F. $B l .5 u .7 . \ddot{\alpha} \hbar l i g$.}

\section{Stengel:}

1. nicterlieg., fatenf., fiachelgaar.; $5 \mathrm{j}^{-1}(\mathrm{~b})$. 7 น. 5 zohl., gleichor. Feilf., gezahnt; Ş lbl.

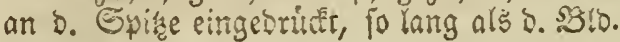
$(4-\mathrm{gb}$.)

2. nufredt; Blättch.:

a. Ecilf., zerjobnitt., baar.; Stgl ftadyel= 3449 . brar. ( 4 9. gb.)

\section{hirta.}

b. Yanzettî, grobgezahnt; die vert. Gerzí. Blibl.:

a. grōj. als ๖. 31. . (4 15. 24. hgb.)

b. To lang ars D. \$3lo. ( 4 NAs. gb.) 3450.

obscúı.

\section{G. Bl. sizmmtl. $3 \approx \ddot{z} h l i g$.}

\section{Stengel:}

1. aufr., åf.; $3 \mathfrak{I m}$ fte iber D. Belente fte 3452 . bent $(\odot$ SE. gb.; w.)

2. gabelform.; BImftle in D. SBlttwint. $(\odot$ NE. 'gb.)

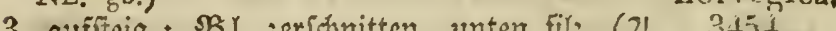
monspeliénsis. NE. gb.)

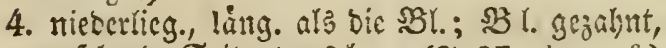
auf beyd. Seit ctwaz haar. (4 SE. gb., rofs) grandiflóra.

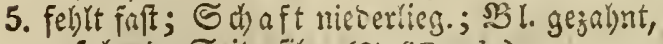
auf beno. Seit. filj. (\% SE. gb.)

3456. subaca'ulis.

\section{POTERIUM. 760. (W. 47.)}

Shre Dornen; Stengel:

1. etwas ect. $(242 x$, grn., dann p. - * Siralt * 3457. — ఫ Silich., Futt., Sierb.)

2. ect.; Enobluth. W.: Die unterften $M$. u. bie zroifden beyden Z. (4 SE.) polygămum. 
3. ftietrunt, ganz gerabe (4 SE.)

3459.

hybrüdum.

19. PRENÁNTHES. 624.

Blätter:

1. gleichtor., gantzrandig,

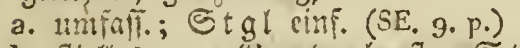

b. Fiellos, ant (s)unoe borfit.; Stgr aff., rutbenf. (4. NAs. bgb.)

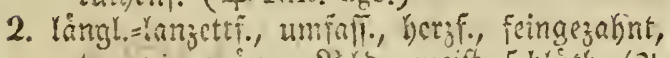
unten ginugrim; 310 . meift 5 biuth. (2) 4. 8. p.-bl. u. wch.)

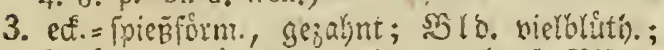
şluthen itberyeng. in trabenf. Sifpen (4 NAm, w.; hp.)

4. 3 Lapp., geftielt, pef̃., feingegahnt, am Sanbe

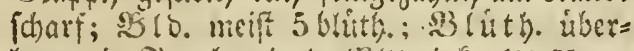
hang. in Irauben in b. sBittwinte. (4 NAm. bgb.; hp.)

5. berablauf.: bie unt. ficucef., atsbartşge= óbnt: bie oberfien gleidbr.; Stgl âf., ausgebreit. $\left(0^{7} 4.8\right)$

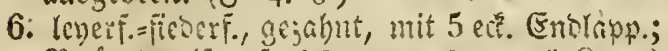

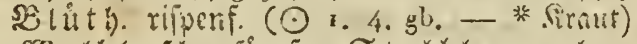

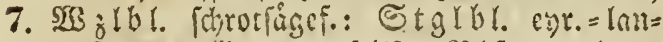
zettf., am sirunte pfeilf.; Rifpe orloen= traubenf., ausgeppert, nacit $(\odot \mathrm{SE}$.) hieracifolla.

3460.

tenuifolıa. 3461. hispìda.

3462.

(purpurĕa.)
3463.

alba.
$3 \frac{4}{4} 64$

altissıma,
3465.

viminĕa.

\section{(murális.)}

\section{7.}

\section{PRIMUัLA. 108.}

\section{Blätter :}

1. geferbt,

1. Dopp. geeerbt, herzf., geffielt, unbehaart, unten an o. 2roern baar.; SBlt t file zott.; Dolbe vielblitth., nufr. (4 NAs, rch.; w.) cortusoides.

2. einf. geterbt, auf beys. Eeiten

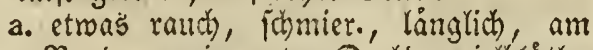
Rante gewimpert; Dorbe vielb(utth., aufr.; S dhaft u. 23 lo ede feinhart.= fofmier. (4 9. r. - braun u. gbch.)

b. unbetaart, am Ranve meslig; 2318 . febr furr (4 SE. 9. rch.).

3469.

pubéscens.

3470.

crenáta. 
II. gejabnt,

1. geferest =gegabnt, unten mit bidt. Megl beftreut, Eeil $\bar{f}_{\text {. }}$ lanzetti., rumzl.; Dolbe vielbluth.; $S B l m$ ftiele abfrehello; $5 B$ l rógre an $\delta$. Naundung orir., $u$. etwa po lang als D. flache Saum (4 3. 9. 48. A:; v.; w. u. gebch.)

2. 5 zabnig,

a. unien

क. rau(h), rungl.; Dolbe vielblüts.;

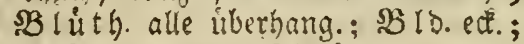
BIElaum hobl, febr furz (4 3. 4 . gb.; w.; sch., wohlriwch. - * 20jl, * 3472.

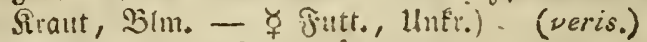

b. etroả jott., verk. err =längl., runglt.; Dolde aus o. $\mathbb{B}_{3}$ l fommeno; be

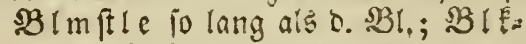
foum flach (4 4. 5. hgh.)

3471. farinósa.

c. faft nactit, lanjettf.= verte. eyt., etrwas geftielt; Dolo e wenighritty., ganz aufr.; 3 latt h. D. Sc ulle lanzettf.;" BID baut)., feinrunzl.; Eaum= lapp. verE. eytr., halb jo lang ald 0 , Robre (4 g. rch. u, gb.)

b. auf beyden Seiten

a. rauch), runjl.; Dolde vielolitth. : D.

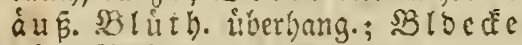

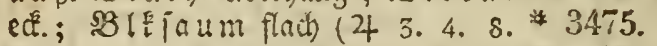
hob.; w.; r. 26. gertichlos - $B\left(m_{4}\right)$ (elatior.)

b. etmaz feintjant. u. Fletrig, feilf., an o Spirc atgefuct; $C_{\text {ch }}$ aft 1 bis 2 bluth., ling. als bic SBl.; 351 I $=$ lappen in 2 glcichor., akgeftute Stufe getheilt, mit nact. Soblund (4 9. 1..)

3. an 0 . Epike vielzatin., feifform., glånj.; Sol aft meift 1 bitith., furz. als bie \$St.;

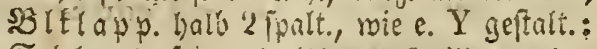
Sd) luno feinjott. (4 10. Al.-lila; w.) minzma. III. gejägt,

1. getertst=gefägt, vert. ent. = Eeiff., etroas fithier., nuf beyb. Geiten.

a. Feinjott., glanjlos, grolige erbt=gelägt;

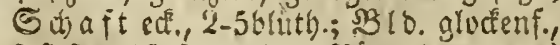

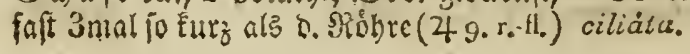


b. unbebart; S haft 2-3bluth.; $B$ lát to d) en b. Seulte vert. eyr. =langl., ge= fárbt; SBlo. ctwa jo lang als o. SRobre; :3luth. faft friclós (4 9. p.)

2. feingelägt, jott., blapgrü, lángl. = oval; Schaft 2 -3 bluth., aufrecht, ftielrunt; SB ID. fugel., 3 mal fo furz als 0 . Robre; SBlEtóbre feinzott. (4 9. r.; b). u. w,; v u. w.)

3. etrab gejägt, ellipt, unten mit Jekl be= ftreut; Dolo uberbang.; 3 lo e e e tief 5 fwalt., 3 mal jo fury als bie jebr lange Siugre (4 9. hr. u. gh.)

4. nur oben gefingt, unbelgart, iamierig= bruii., lång!.:lanjettf., ftumpf; sB látt th. D. Sू IIlle gefärbt, faum fo lang als die ftiellof. Bluth. (4 ro, v. u. w. u. gbch, wohlriech,)

IV. gefágt u. ganjrano., ficifà., reré. crrund; S. th a ft aus. Der ylitte fo lang als D. SB1.;

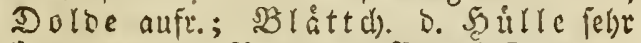

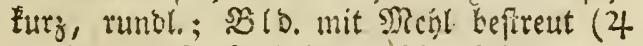
9. gb.; verschiedenfarb., wohlriech.)

V. fait ganjandig, elript.,

1. unbebaart; Dolde merighlith., aufr.;

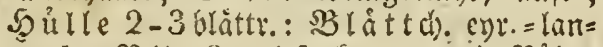
gettf.; salo. 3 mal jo futr als bie giogre (4 6. 月. - v. u, wch.)

2. etruas dice, min Rande fnorpl. ob. etrass

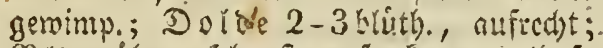
$S B$ lo. róbr., itgr frumpf, faum balb jo

- lang als bie Sífire (4 10. 11. "., wch.; w.) integrifolia.

3479.

Flloerkecina:
3480, villósa.
3481. longiflora.
3482. glulinósa.
3483. Auricüla.
5484. carniolica.

3485.

\section{PRINOS. 260. $\left(303^{3} \cdot 307^{2} \cdot\right)$}

Blmstiele in b. $\$ 3$ lttwinté:

1. $5-7$ bluth., falt fo long ars o. SBlattficle;

SBluth. Faft borbenf.; bie abfollend. Bl.:

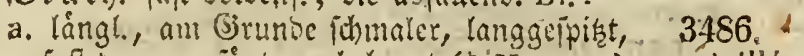
faft bopp. gejånt, unbebaart (

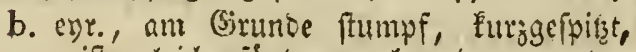
meift gleichgeiingt, run $r_{0}=$ aber., untert 3487 . weid)lgar. (5 NAm. w.) padifolĭus. 
2. 1-3 blith.; 53 ! cour., Yanzttf., cm (Brunte fhmaler, an b. Spitse tuno? u. gerigt, gang= 3488. rano., unbebaart, leoerart. ( 5 NAm. w. $\Delta$ ) glaber.

\section{PRUNÉLLA: 447.}

\section{Blätter:}

1. geftiolt, am Cirunoe gezahnt; Stgl auf= fîeig.; Blärter :

a. längl.=eyr.; Olippe d. Bld.:

a. 3 fpalt. ( 423 . bl.; เ.; fl.)

(gruendifóra.)

b. 3 grann., abgeftute (4 34. w.; gb.; *3490. ఫ

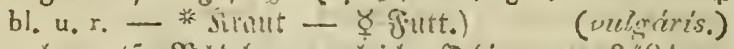

b. eyr.=larzettf; SItolapy. greid): Slippe 349l.] mie oorber (o NAm, bl. u.w.) pensylvanica.

2. fficllos, lanjchif., ganjanto, fäanf; Stgl 3492.

aufr. ( 4 SE. hbl.; p.-blch.) hyssopifolía,

\section{PRUNUS. 389}

\section{Stamm aufrecht.}

\section{Bliithen:}

1. in $\mathfrak{I}$ raben; Blätter:

A. ahfalterio,

1. bopp. gefägt; Blattstiele:

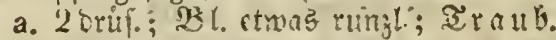

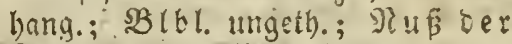
St frudt gruóig to 40.26. w. - * 3493. ఫ * Urinie, Beer. - ఫ̧ Der, Tart. griir) (Padus.)

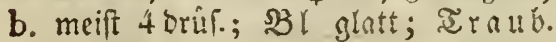
ficlaffiberbang ; $3 B$ Ibl. ungetheilt; 3494.

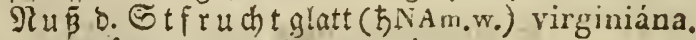

2. E nfachigelägt, glett; Blanstiele:

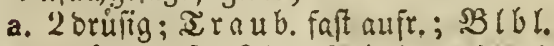
verḱ. =herif., 2 lapp. (

b. obne Drúlen; BI.:

A. eyr., am Grunte 2 brilf.; Tr $\mathrm{r}$ a us ben bangento, blättr.; \$Slo. ge= fågt ( $(\hbar)$

b. faft leberart., mit meifít orui. un=

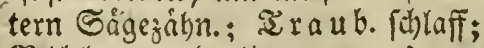
B Ibl. ungeth. ( $\hbar$ NAm, w.)

3496.

semperflórens.

serotina. 
B. immergrin, gefrigt,

1. am Sirunbe meift 2 brilf., leoerartig, sben fiegelglatt, unten matt ut. blafler;

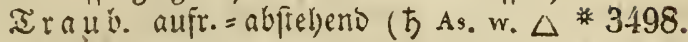
- *3im.)

2. ohne Druifen, lederaut., g!att, mit ftumpion Sagejábn.; $B$ It tifle obne Drifr.; Iraub. auTr.; ; Bl. :

a. evr. lanjettf. (尚 SE.)

b. langlich=lanjettform., forarfgeipigt ( $5 \mathrm{NAm}$.)

+Lauro-Cerăsus.

1I. in (5nDDoloentraub.; sEl. eys:=runol., faft herof., gefágt ( 5 4. 8.w.)

III. in Dolben; Blätter:

1. eyr =obal, gefógt: bie iủngern unten feinbaar.: bie át tern unbehant; $3 \mathfrak{B} 1 t$ =

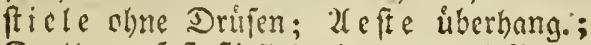
Dolden paft fticllos (t) w. - D Blm., * 3502. Friddste, Sajt - ૪ Trud)te, Del)

3499.

lu-itanica.

3500 .

carculiniána:

3501.

Maháleb.

2. verf. eyr., geiagt; 2 the unten feinbarar; B It tifle meift 2 oriil; 2 2 efte abfteheno, gerabe; Dolden mie vurber (5 4. w. -

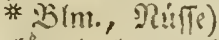

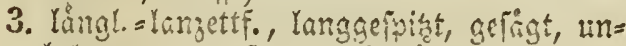
behant, an (Stunbe 2 orif.; BB It ffle obne Drufer; Dolden gebafut, viel=

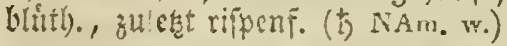

4. elliwt=bere. eyr., langgefoint, fitharfgefögt, am Sirunbe 2 orif., oben ipiegelglatt, un=

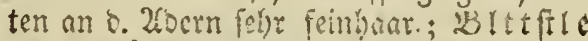
obne Druf.; Dolo. 4-6blith. (5 NAm.) pygmáea.

5. ent., langgepint, an Grumbe formarel, ungl. sopp. Selogst, mbehourt: bie $j$ ín gern jebr feingeromp.; SB It tif ! e 2brui; Doloen eingetn, 3-4brits., freflos; B luth. zumeilest nur gepacit (b) NAm。 w。) nigra.

6. långl=ovar, langgelpist; 2f fterbl. bor= ficnf. = jigeoruet; Dorloen fiteros, ge= båkft, uenigblith.; 2 lolapp. langett. (b) NAm.)

IV. in ficllof. Seitentorben; Blätter:

(Cerŭsııs.)

* 3503.

(aviัuมn.)

3504.

pensylvanica.

1. verf. enr. = lanjettf, meit!áuft. gefảgt, am (Gitutice feilf. U. ganzrond., unten meiss grim (ち NAm, w.)

3506.

3507. hyemális.
3508. punìla. 
2. wie vorber, nur vetle, eyruno = lánglich (b NAm. w.)

3. einige vert. ent., anbere långl., etraas ftumpf, oruj. = gefágt, unbebant; $\mathfrak{B} \backslash t \mathrm{t}=$

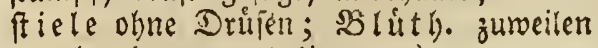
einzeln od. gepaart (5 9. w.)

V. einjeln oo. gepant,

A. Ftielíos; Die langgeipigt. Blätter:

1. lingl.=eyruno, faft bersf., feingefigt; Słluth. meift eimeln (b As. rch. ఫ Tridte, Del)

2. gam cyrund,
a. einf. Geirgt: SBIttfte okne Dris iet (t) NAs.)
b. Doph. gefigt; Bzlattfielc orilf.;
- $\mathfrak{B}$ lútb. meif́t cinzeln, falt ficlios (ち SE. w:)
3512.
sibirǐca.
3513.
dasycárpa.

-B. gefticlt; Blätter:

3509.

Susquehánae.

3510.

Chamaecerăsus.

1. eyruno,

a. langgeppist, am (Sirunde vunol., uns gleich $=$ u. bopp. geiogt, unbehaart; Bittit ie 1-2orúf; âtere 2lefte Dorn.; SBlith. gesanet od. cinjeln (万 SE. w.)

b. ctwag ftumpf, gefigt, nuten fein=

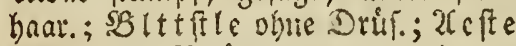

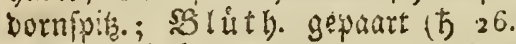
w. - ఫ frichte)

2. oval=eyr., ranggelpight, gclagt, unten feinbaar.; $B$ lat friele obne Drifen; Xefte ohne Dorn.; SBlithen einjeln Do. gepaart (b 26. w. - Fridute ఫ Fruidere, Del)

3. faft rautenf.=Dual, ftunipf, gejägt, oben umbehaart, unten wenig feingaarig; Bittifle obre Drifen; afe meift obne Sorn.; SBlution wie vorber;

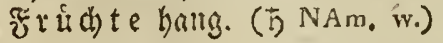

3511. ช్ Arneniăca.
3514.

brigantı̆ca.
3515. ఫ్

(insiticia.)
* $3516 . \not$
(domestica.)

3517.

cerasifěra.

4. eyt. = lánglici), langgefpitat, unbelyaart, Dopy. geiagt, mit ionargeipist. Sage=

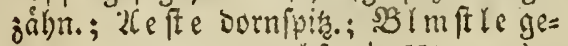
paart, unten verwaci) j. ( 5 NAm. w.)

3518.

maritı̆ma.

5. oval=lanzetti. , fwis, gejägt, oben uns bebaart, unten fcinbaar.; BS lattitle

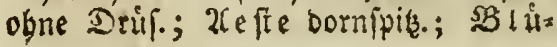


then einicln (5 2. wo - * Minne, Blm.,

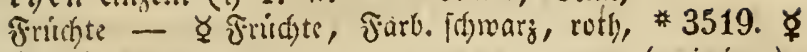
(sibrb.)

6. oval =evr., ftumpf, gleid $=\mathfrak{u}$. feingejägt, un=

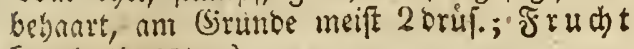
Eugel. (ち NAm.)

(spinósa.)

\section{Stamm niedergestreckt;}

Br. jerichnitt. =gefingt, ohne Driforn, unten reeisfilo. (尚 As.)

3520.

sphaerǐca.

\section{PTELLัА. 70. $\left(777^{2} .800^{2}\right.$.W. 14.)}

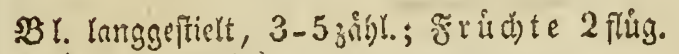
(b) NAm, wch.)

3521. prostráta,

\section{PULMONARĨA 96.}

Blumendecke:

1. po larig als b. SBzterótie; Bt:

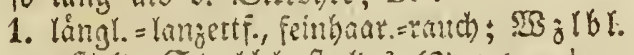

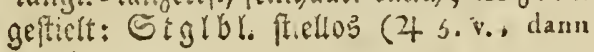
blch.)

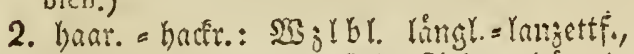
3523. (angustifolia.) langgejpist, in Den SBSattfiel yerounnt: St grbt. Geraubram.; 3 IE faum glocenf. (24 - bbl. $\left.14, \mathrm{rch}_{0}\right)$

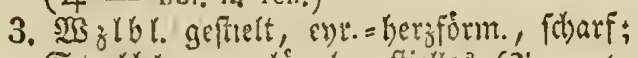
Stgrbl. ent. =lángl., fitillos (4 1. 4. ros., darn v. - * Siraut)

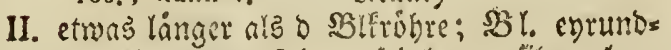
lanjectf., langgripist, feinharar $=$ filfo, ber $=$ abrauf.: $2 \mathbb{B}_{\mathrm{g}}$ lol. geftielt (4 SE.)

3522.

trifoliáta.

III. E⿱⺊口𧘇r

1. ey)e, graugrim, fleift.; Stgl iffo, nic= 3527. Derlieg.; 15 fla als $\mathfrak{e}$ unbehant (4 36. p.bl.) maritima.

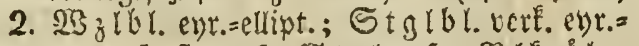

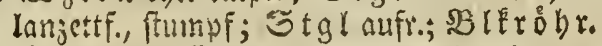
lang. als b. Saum (4 NAm, bl. -v.)

3. $\mathfrak{S Z}_{3}$ Ibl. hergf. (4 NAm. p.)

\section{* 3525. \\ (of́a cinális.)}

3526.

mollis. 
13. PULSATÍLLA. 441.

SEImft I mit Seulle; Blätter:

1. fingerf. ( 4 4. dbl.; gb.; v.; w.)

2. einf. gefiebert; bie 3 theil. Blättch.:

3530.

patens.

a. ftumpf, faft ganz unbebant; SBluthen 3531 . aㄷfr. (4 5. 13. w. u. p.. mit gb. Haar.) vernális.

b. langgeipifgt, jott.; SBluth. faft aufrecht 3532. (4 SE. 9.)

Halléri.

3. Dopp. gefiebert; Blbl. :

a. an 5. Spinge umgeichlag. (4 2.3. grnch. * 3533. u. w. u. dbl., mit w. Haar. - * Siraut) † (praténsis.)

b. gerabe (4 2.6. bl.; w. - * Siraut) f(vulgáris.) -

\section{PUNICCA. 386.}

321. lanzettf.; Stgl Faumart. (ち SE. hr, - * 3535. ఫ

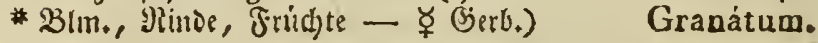

\section{PYR ETHRUM. 678.}

\section{Mil weifsem Blüthenstrahl.}

Blätter :

1. lanjettformig,

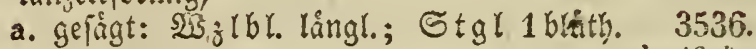
$(4 \rightarrow E$.)

latifolium.

b. Die unt. an D. Epite griagt: D. obern gangrano.; 3roeige bolocntraubenfürm. (4 NAm.)

c. 2rre tiefgefagt; Stgl mufrect, an ber Epine åt. (4 SE 42.)

d. Stglbl. tiefgejabnt: $\mathfrak{W}_{3}$ Ibl. fieberi.; Stgl 1 bruth. (4 SE. 9.)

2. fiecreriormig,
a. faif fiellos, gezahnt, fimmp, ftachellyar.; Enodoibentraub. sigciest ( $4 \mathrm{SE}$.) macrophyllum.
b. bie $\mathfrak{L} a p$. D. unt. SBl. finmallanjettf., gangrano. ob. 2 fpalt.: D. obern gleichbr, 3541. gangraris.; Stgl 1 jhith). (4 SE.) ceratophylloides

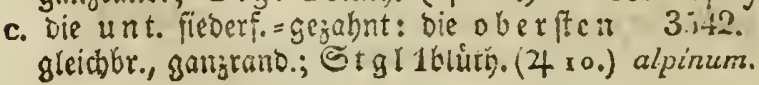

\author{
3540.
}

3537.
sernirnum.

3538.

uliginósum.

Haliéri. 
3. Teverf. = fieberf., ungl. gejahnt, faft friellos,

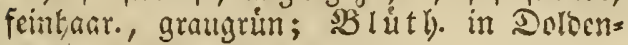
traub. (4)

4. einfactgefiebert; Fiedern:

a. Ianjectf., fieberf., fharfgelägt: die ober= ften offliç.; B lu fticle od docntraubenf. (4 5. 3.)

b. långl., futmpf, fiescroorm. gezabnt: bie

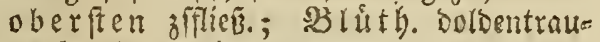
benf.; Stengel:

Q. dift.; S3lutbenftrabl faft bopp. fo lang als die Blo.; Sirolte gezabnt 3545. (422.29. - * *irnut, Dilm.)

b. ruthenf.; $B$ luthenftrabl 3 mal fo 3546.

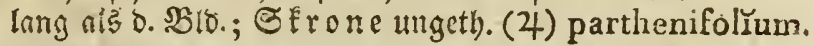

c. einf. ficberf., ftumpf, gejohnt, mit weifl. Megl bereift; $s B$ l m fit le faft boldentraus benf.; Sktone gezabnt (4 As.) - pulveruléntum.

d. bopp. u. einf. fieberf., mit fpit., ausge= brcit. Sapsen: 20lf unbehart; Stgl

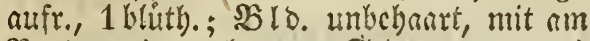

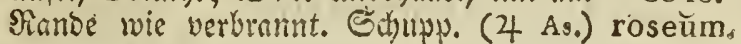
5. bopp. geficoert; Fiedern:

a. fieberf., gleichbr.: Stglfiebern bopp. fieberf., mit gleichbr., fafit gangrano. Lapp.;

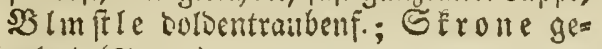
falint (24 As.)

b. gleichbt: = wriemenfirm.; Stgl 1 blith. (4 As.)

c. glcichor.=fabenf., 2 -3 theil.; Stgl weit= funreif., áftiz; SErone gangrano. ( $\odot$ 32. 28.)

3544.
(carymbósums.)

pinnatifüdum.
(Parthenüum.)
* 3545.

3547.

3548.
roseŭm

3549.

tenuifolı̆um.

3550.

caucasǐcum.

d. gleichbr., ftuminf, fleifaj., 3 palt.; $5 \operatorname{tgl} 3552$.

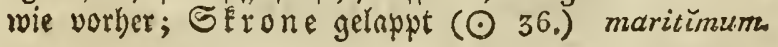

\section{I. mit gelb. Blüthensirahl.}

B. I. Dopp. gefieso, gleichbr., ftumpt; Stgl:

1. foft bolbentraubenf., auffteig.; $\subseteq$ tragl fo lang ats o. SBlbichutpp. (4 NAs.)

3553.

millefoliảtum.

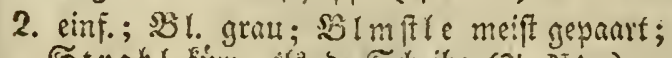
Strabl furr. ats o. Schcibe (2 NAs.) 355 .

bipinıátum. 
Blïthen :

\section{PYRUัLA. 345. $\left(346^{2}\right.$.)}

1. in Trauben:

a. 1 feit.; Stgef. u. b. bervorrag. (5) riff. gerace; $2 B 1$. cyr., zum. oval = eflipt., ob. ellipt. = runcl., furzftadbelipis., aber. (ち) 5. w.)

b. nicht einfcitig; Staubgefäise:

3555.

(secuinda.)

a. aufwártzgebog.; (S) riff. niebergebog.; B l. runol.; ader. (4 4. w. - * Siraut 3556. ૪ - ఫ (serto.)

b. jigeneint; Griffel:
1) nictergebog.; $\$ \mathrm{Bl}$. runol., 5 factgge= rippt ( $ち$ - bgbch.. grn.)
2) gerate; $\mathfrak{B}$ !. rumol., ellipt. u. eyr., aber. (b 5. rch. - w.)
$355 \%$.
chloránthá.
3558.
(minor.)

(roluendifolúa.)

2. in boloenf., langgeftielt. (Enoboldentraut=

ben; $S B$ l. lanjettf. = verk. enr., am Sirunbe Eeiff., ganjiano., weitláuft. gejägt, im Suirl; Stgef. zlgeneigt; (5) riff. im Fruchtfnot. verid) loif. (5 5. rch.. w.)

3. am 1 bluth. Sdaft; $B$ l. enruno = runcl., aber. ( 5 5. w., wohlriecb.)

4. an 2 blitth. BImftlen ( 5 NAm.)

3559.

(umbelláta.)

3560.

(únifiora.)

3561.

maculáta:

\section{PYRUS. 394. (795*. W. 82.)}

\section{Blüthen in Doldentrauben.}

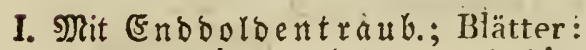

1. eyr., gefágt, unbehaart: bie jủngern unten zott. = Feinhaar.; Doloentraub.

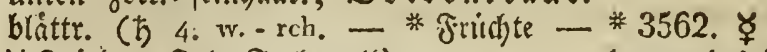
ఛ̧rridite, Det, Farb. getb) (commúnis.)

2. oval, ftumpf, ganzrano. od. an o. Spitze

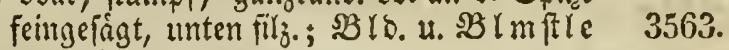
zott. (ち 8. w. - sch.)

3. ent. =lángl., einige lanjettF., ungleich) $=3564$. meift bopp. gefágt, unten filz. $\left(\hbar-w_{0}\right.$ ) Pollvería.

4. evr. =lanzettf., zerfodnitt.=gelappt, gejahnt, oben meift unbehaart, unten weisfili B Iolapp. faft unbehaart; $\mathfrak{F}$ rudten. u. $\mathfrak{B I m}$ Itte fill. ( $5 \mathrm{NE}$. w.) intermecisa. 


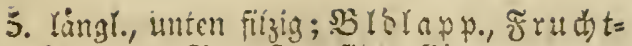
f not. u. SEI Imitte fils.; Bl.:

a. an keso. Enton fpin, gleidrgejägt (b)

b. aln Cinnoe feilf., ungl. u. Sopp. gefágt (†) SE, w.)

6. rmol.esmi., jerifunitt. =gejolgnt, unten

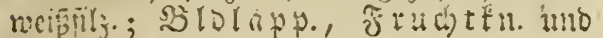

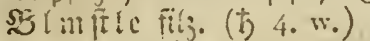

7. vert. cyr = langl., langgcipiat, fringeiagt,

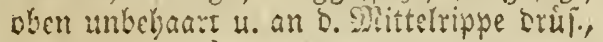
unten

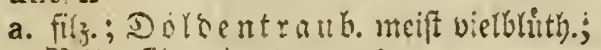
$5 B$ 10. Filis.. (方 NAm.w.)

b. Ineniy finbar.; Doldentraub. we: nigbluth.; 310 . unbehnart (ちNAm. w.) melánocárpa.

3. berzf.; ocrfynitt. = gefigt, ed.; mibshaart; Dolbentraú. 4-6bluth.; SBlo. auser. baló neyie ssimfilen untebant: imerbalb

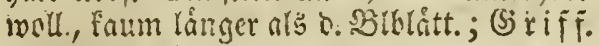
lubbebaert ( $\hbar$ NAm. liros.)

9. ungl: geficto., nit längl. = runol. Blättch., u. grós. Entbl., gejágt, unten feinbaar. (einige einf. u. ungeth., anoere fieserf.) ( 5 - w.)

10. herz $\tilde{T}_{0}=$ cyr., zeridunitt. = gelappt, gelägt, unbeganart: $\delta$. jüngern unten feinbant.: bie 2 unt. \&app. gro̊ß. u. ausgebreit.; sBlolapp. faft unbehant; Fruthten.

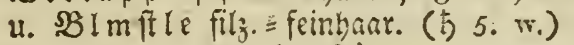

II. mit Doldentraub.; Bl.:

1. cyri. =långl., faft ganjrand., unten weipl. ( 5 As.)

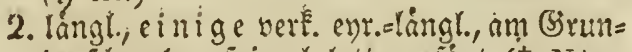
be fomaler, ipicgelglatt, gelagt († NAm. $\Sigma_{\text {., }}$ wohlriech.) ?

\section{1.}

coronarla. alpina. 3567 . edúlis.

3568 . (Aria.)

3569.

arbutifolia. 3570.

3572.

hybrìda:

3573.

(torminális.)

3574.

sină̌ca.

3575. angustifolia :

\section{Blüth, in Traubेen.}

\section{Blätter:}

1. längl. = cllipt., ftechenofpis, unbebant; bie jư traubenf.; SIEI apy. jott.=gewimp.; SBlbl.

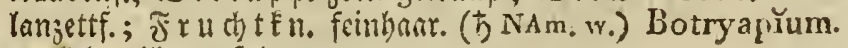

2. ruribl. sellipt., fpit, 
a. unbehaart; SRrorapp. น. Fruthtin. 3577. feinhaar.; $\$ B \backslash b$ l. xark. cyr. ( 5 NAm. w.) ovális.

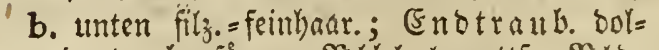

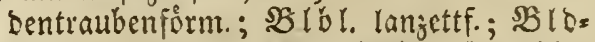
lapp. unbehaart; Frudten. fit $=$ Fein= baar. ( $523 . \mathrm{w}$ )

3578.

(Amelanchǐer.)

\section{I1. Bliuth. in Dolden,}

\section{Blätter:}

1. enr. = lángl., langgefpist, gefägt, unbehaart;

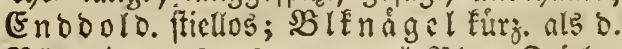

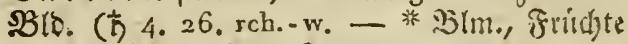

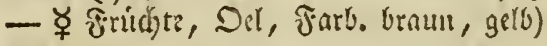

* 3579. ఫ (Malus.)

2. voul=längl., gejägt, unbehaart: bic jîn= gern unten am Sirunce u. an b. Mittelrippe

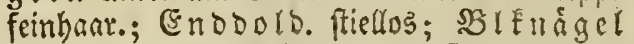
läng. als b. SBlb.; (Si riff. am Girunbe woll. (方 As.)

3. eyr., langgeipitst, geiagt, oben unbehaart, unten an b. 2loern renig feinkaar.; Doloen ftiellos, ant Enoe u. zur Scite; SB Imftiele feinhaar. \$ SB (5) $r$ iff. wie vorker ( $ち$ NAs. w.)

3580 . spectabilis.

3581. prunifolia:

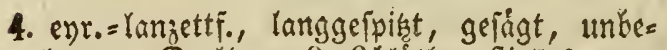
baart; Dolsen 3-6bluth., fiellos, zur Seiteftebeno; $\mathfrak{B}$ lénágel wie vorber; $\mathfrak{B}$ lD.

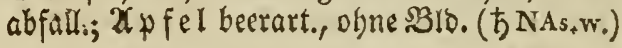

3582. baccáta.

\section{Blüth. cinzeln.}

\section{Blätter:}

1. oval, langgeipist, bops. gejägt, unten mes nigfilz.; SBlüt h. mit ganjgetrennt. (Siejhl.

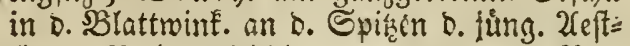
d)en; $\mathfrak{B} l b l$. gleichbr., fo lang als D. SBlo. ( 5 - gbch. - grn.)

3583.

2. ichnallangetti., foit, gangrant, oben idswact) filz., unten'meiffilly; $\mathfrak{B}(\dot{\mathrm{u}} \mathrm{t} \mathfrak{h}$. in D. SBlatt= winf.; foft ftiellos (5 As. w.)

3584. salicifolĩa, 
21. QUERCUS. 745. (W. 53.)

\section{Blätter ganzrandig.}

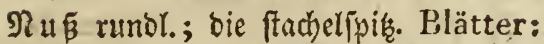

1. fomalfanzettf., an beyben Enden fomaler, 3585 ; unbebaart, abfall. ( 5 NAm.) Phellos:

2. oval =lángl. u. obal = lanjettf., unten feins 3586 . baar. (ち NAm.) imbricaria.

\section{Blätter gezahnt.}

\section{Blätter :}

1. auf bend. Seit. unbebant, längl., ftachele fpit. =gejalbut,

a. am Srunbe runol., etwas weişgrin (衣) As. $\triangle$ )

b. am Brunde faft feilf. ( $\hbar$ As.)

3587.

infectoria.

3585.

Turnéri.

2. unten bebaart,

a. feinbaar., verf. eyr., fpis, mit faft gleith., groben, an ber Spitye idwwiel. Sáhnen; SBlo. Der Frucht an (Srunbe fonnaler; Ru B. eyr. (ち NAm. - ఫ Farb. roth)

35S9. 후 Prinus.

b. weiffilz., faft rautenf. =obar, fpic, mit

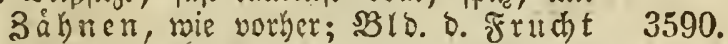
faft balbfugel., mit cyr. $\mathfrak{R} u \tilde{\beta}$ (ち NAm.) montána.

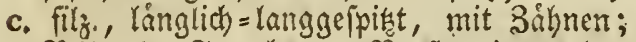

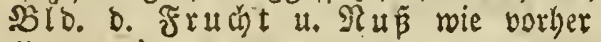
(5 NAm.)

3591. Castanĕa;

\section{Bl. an d. Spitze gelappt.}

31. Aeif., unten meiffily, an 8 . Spise mit

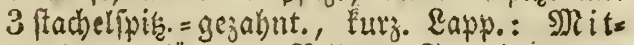
tellapp. lánger; $B^{\prime}(D . \delta$. Frud t einges

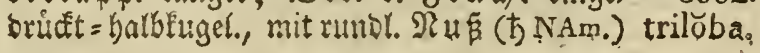

\section{Blätter buchtig,}

A. mit scharfogespitzt. Lapp;

\section{Blätter:}

I. auf beyd. Seit. unbebaart, lángl., tiefbucht.; mit långl., abftehend., fpis., borft. = geipist., grobgezabnt. Lappen: 
1. auşgebreit.; F็ 1 . D. Frutht am (Srunbe 3593.

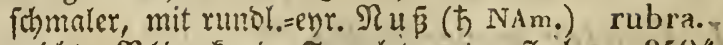

2. nicht; SBloede D. Frutht unten flach: 3594 . $\mathfrak{R} u \bar{\beta}$ wie vorber (ち NAm.) : coccinĕa.

II, unten Gebaart,

1. feinbaarig?

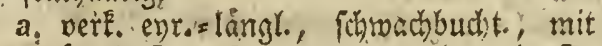

(s) lángl., ftumpf., unbeutl. gezahnt., borft.= idharfgeipint. Eapp.; $\mathfrak{B}$ ID. D. Fra dit unten fladh, mit runol. Si uB (5 NAm.) tinctorra.

b. långl., fieberf. = bucht., mit \&npp. wie vouber; BBto. o. Frutht am Grambe

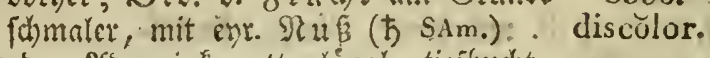

2. in ben 2coerminf. zott., lángl., tief̧but)t., mit ausgebreitet., gezalurt., fwis., borft.= foharfgeipit. Eapp.; BBls. Der Frud t am (Sirunoe flach = Ereujelr., mit rundr:

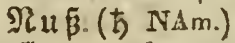

3. Filz., verk. enruno = Fallf., 3-5 lapk., mit borft=icharfgerpitat. Lapp.; B lo ede ber Fru $\mathrm{r}$ t rie vorber, fwaterhin faif fugel., mit fuger. $\Re \mathfrak{R} \bar{\beta}$ (

\section{B. $n i c h t$}

\section{Blätter:}

1. auf bevo. Ceiten unbehant,

a. lángl., runblapt.,

B. geftielt, an (sirumoe runolid ob. fait herzf. Feilf.; $\$ B$ ! D. D. Frud t faft balb= fuget.; roars. = fohuppig, unbebaart; Frud te friellos: গ⿴囗十 (5 4. - * 9iside, Doto, Fridite -

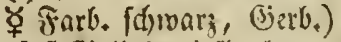

b. foft fiterlos, tiefouct., am Grunoe um=

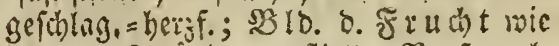

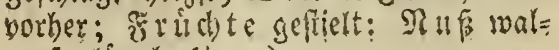
zenf. $=$ lingl. (b 4.)

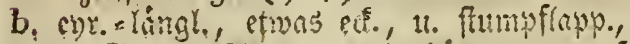
am (S)unoe formaler: wie jungern allf

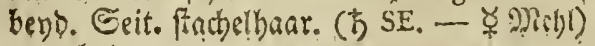

2. unten behart,

a. ftachelbaar., lingl., fieberf.=bucht., am (3)runbe fó)maler it. faft rundl., mit läigl.=

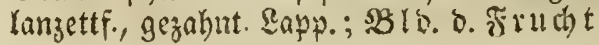

\section{9. 후 (Robur.)}

3600 .

(pedunculcíta:)

3601. $\not{q}$

Escǔlus: 


\section{Quercus. - Radiola?}

balbfugel., runoum ftachlo, mit wargenf.=

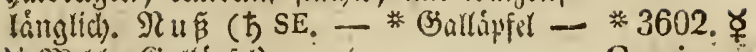

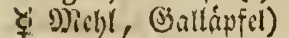

Cerris.

b. an o. untern 2rpern haar., langl., tief= bucht., mit lingl. Eapp., wovon D. obern breiter, eft., u. an D. Spize faft 2lapp. fino;

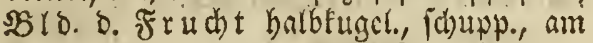

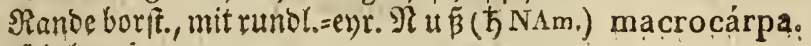

c. Feinfaarig,

6. långlits.

1) fieserf. =bucht., mit idomallomjettf. frumpien, ganzrando, am (Sirutioe

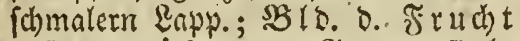

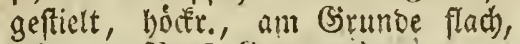

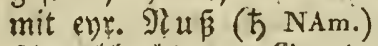

2) fiblwachbutht., am (3runbe ichnaler, mit folte kurz., verk. evruno., etwas

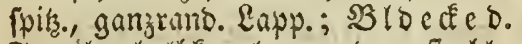
Fru cht balbfugel., runoum ftadbl., mit eyr. $\Re u \tilde{B}(\hbar)$ 3604. alba.

3603.

\section{alba.}

3) bucht:=5 lapp., mit ftumpf. Iapp., movon bie obern breiter u. 2 lapp.

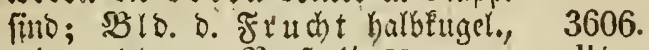

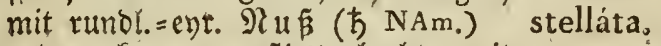

b. längl. = berk. cyr., geftieft, bucht., mit ftumpf., eff. Iavp., am Srrumbe herzf., ungleich; $\mathfrak{F}$ rúd te falt ftiellos; B 10 .

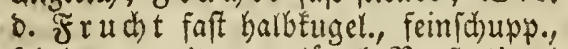

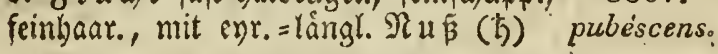

Blithen:

\section{QUERIA 50 ,}

1. gehiuft, am Enbe ftehent; 31 l. entgegen= 3608 .

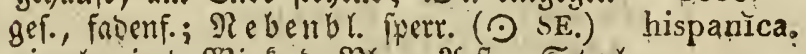

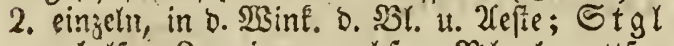

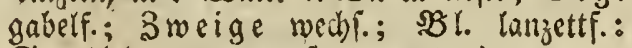
Eัtglbl. entgegenger. (4 NAm.)

3609 . canadénsis:

\section{RADIǑLA. 87.}

(० 19. 50. w.)

3610.

linoides. 
5. RAMONDǏA. 121.

(4 SE, rch.)

3611.

pyrenăck.

\section{RANUNCŬLUS. 432.}

(Meistentheils $\dagger$ )

\section{Blk, am Grunde ohne Schupp.}

\section{Blätter:}

1. gleichf., runbl., 3 lapp., gangrand.; Stgl 3612. friech. (4. 50. w.) (hederacěus.)

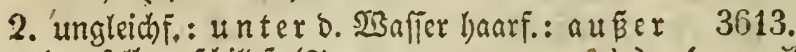
bemperben fabildfo (4 41. 45.50. wo u."gb.) (aquatilis.)

II. Blk. am Grunde schuppig;

A. Blk. weifs;

1. Bl. einf, ungetheilt,

Blätter:

1. gleid)fórmig,

a. gleichbr.; ভigl aufr., geftreift, meift 3014 . 2 blith. (24 9.)

b. evr., langgepipist, umfaili.; Stgl viel= 3615 :

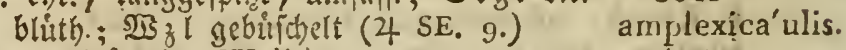

2. ungleid) förmig: Wzlbl.:

a. runol. =ent., faft berzf.: Stglbl. eyr.= 3616 . lanjettf., ftiellos: 2urle gcrippt (4 9.) parnassifoliuse

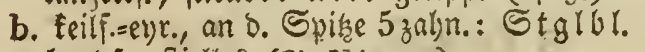
banof., ftiellos (4 NAs.9.)

frigĭdus.

$$
\begin{aligned}
& \text { 2. Bl. getheilt, gelappt, } \\
& \text { zerschnitten, }
\end{aligned}
$$

\section{Blätter:}

1. gleiḑfơrmig,

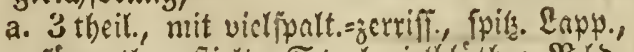
fánmet!. geftictt; Stgl viclbiutt); $\$ 3$ ls. 3618. unbehaart (4 9.). Seguiéri.

b. gabolf. = haarform.; Stgl fohmimmeno (4 37.)

2. ungleiăfớrmig, (fuviatilis.) 
2. 5 lapy., gejahnt: Lappen:

h. langgepint: NR i t tellappen 3 palt.; Die obertien $\$ B$ l. an 0 . SBluthen fingerf., friellos, lanjettf. (4 9.)

b. ftumpf: Nittellapw. wie vorber; b. oberfen $\$ 3$ l, an $\$$. SElitb. fingerf. ftiellos, gleid)br. = priemenf: $(49$.$) platanifolius.$

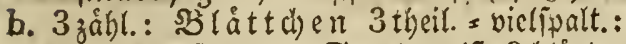

Stglbl. ftiellos; Stgl meift 3 bluth.; B 10 . rauch ( 4 9.).

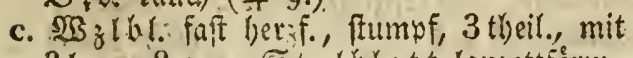
3 lapp. Eapy.: Stglblat t lanzettform., ganjrand.; Stgl meift 1 blitth. (4 9:) alpéstris.

\section{B. $B l k \cdot g a l b$;}

\section{Bl. einfach, ungetheilt,}

a. gleiohfürmig.

\section{Blätter:}

3624.

1. curumb, geftelt; $\$ 3$ lúth. ftiellos (4 SE.) nodiflórus.

2. eyr., ob. faft bergf., an ber Evite 3 oder 3625. 5 zahn.: Stgl frief.). (4 NAs.)

3. egr. =lnngettf, geftielt; "Stgl aluirtsge=* 3626. ૪̧ reigt (4 34. 48. - * Siraut - ఫluıtr.) (Flammüla.) $3027 . \nvdash$

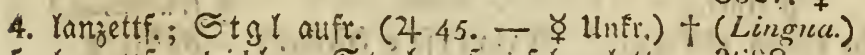

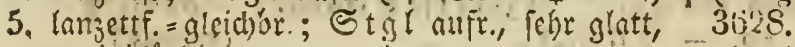
menigbirtth. (4 44. 45.) graiminěus.

6. bergf., edt., geftielt; Stgl 1 blith). (4 r. 3620 . ఫ

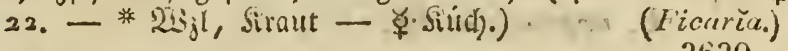

7. gleid)breit; Stgl friech. (4 38.) 3630. repians.

\section{b. ungleichförmig.}

\$B l. nicrenf., foft 3 lapp., gelerbt: Stglbl.

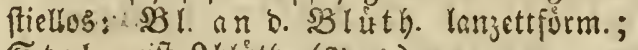
Stgl meift 2 blitt). (4 j.)

3631. †. Thora.

\section{Bl. getheilt, gelappt, zerschnitten. \\ - gleichförmig。}

\section{Blätter:}

1. $\operatorname{cinf} a$, 
a. gelappt, ftumpf; unbchant; Stglweits 3632.

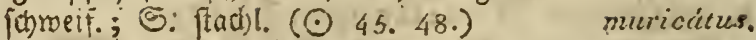

b. jerriff., fiph, raud); Stgl wie vorber; 3633. S. weichftadhl. (○ SE)

2. 3 ikalt., gelappt, geferbt, ganj feibenart.; SB I D. etroas abféfeno; SBl m ft le ftieltumo; Stgl u. sblttifle raud) (4 8.)

3. 3 jónlig,

a. Feidenart=zott:; $s B$ latt d). 3 ppalt., etros zeridnnitt., ganzranto; mit lanzettf. \&app.; Bg 10. umgeisthlag. (4 8.)

b. untert haar:, 3 palt., zeridnitt.; Stgl

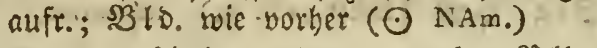

4. 3 theil., zerithnitt. = gelapst, rauch; $3 B$ is. julest umgeichlagen, bortt.; $B(\mathrm{~m}$ itle ges furcht; Fruot: faft fugel. ( () .4\%.)

parviflórus.

3634.

(lanuginósus.)

5. vieltheil.; $\mathfrak{B}^{\text {to }}$ etras abfelgend; $\mathscr{B}$ Im. ftiele pie vorber; Etgl aufr. (4 33. ఫ lluft.)

6. 3ufammengefest; Brmet le gefurcht; Bld.: a. jurudfigerog.; Der aufr. Sigl:

G. 1 bluth.; $B B$ lát t. gleictbr. = vielfpaltig ( 48 .)

b. vielbluthig (4 3. $340^{\circ}$ - $\mathfrak{W B}_{\mathfrak{f l}}$ $\Varangle$ Uufr.)

b. etras abftekend; SIJglreffer triechend (4 29: - ఫุ Unf.)

3635:

(illyriacus.)

.3636:

perisylvafiǔcus.

3637.

(Philonótis.)

3633. 항

(polyanthërnos.)

3639.

chaerophýllus.

* 3640. ఫ్

† (bulbósus.)

3611. ซ

(reperis.)

\section{b) ungleichförmig:}

\section{Blätter:}

1. 3 theil. = vielpalt.: bie ober ften gleidbr.; B lo. etwas abftehend; $B$ Imft le friclrund * 3642. (4 3. 3.4. - * Siraut, $\left.231 \mathrm{~m} .-\Varangle \mathrm{Unfr}_{\text {r. }}\right)+$ (acris.)

2. 3 záhl. u. bopp. 3 zábl.: 8 láttch. 3 fpalt., 3643 . jerichnitt:; S $\mathrm{gl}$ unten åf. (4 As.) asiatreus.

3. 3 jabl. u. gefied.: $B \mathfrak{l}_{\mathfrak{a}} \mathrm{t} t \mathrm{th}$. 3 theif. = viel=

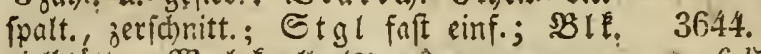
vielb!áttr.; $\mathbb{N B}_{3} \mathfrak{l}$ : Enoll. (4 9.), rutcefolǔs.

4. vielpalt.: Stglbl. wedti, ftiellos;, Stgl 3645 . aufr., 2 bláttr. (4 As,)

5. 5 lappige
a. gantrond.: Stglbl. friellos, fingerförm.; Stgl 1 bluth. (24 9.)

grandiflórus. 


\section{Ranunculus, - Raphanus,}

b. gejalynt; Stgl 1 blitth; has ftellofe Stglblatt:

A. fingerf., mit fohmallanzettf., ganzrand. Eapp. (4- 0.)

b. banbF., mit lanjettf., gejalynt. $\mathfrak{L} a p p$. (4 SE. 9.)

6. gefieo., feibenart.u. zott. : jy i b b rn ichmal= lanzetff: gunzrand.: Die unterften âtheil.; 3649 . 3 10. umgerólag. (4, Ass)

7. Wzlblätter:

a. runbl.hereff., geferbt: S.tglbl. fingerf., 3650 . gejalnt; Etgl vierblitt). (4 1. 4.) cassibrcus.

b. nierenf., geferbt, jerict)itt:: Stglol. fingerf. gleidyr.; Stgl wie borher (4, 3651 . 34. 49.$)$.

c. her f. u. 3 joill.; Stglbl. 3 joll., faft fticllos; ganzrant; Stgl wie vorker; BIVl. Furr. als D. SBlo. (24 NAm.) abortivus.

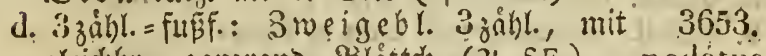
gleichbr., ganzrano. SJlâttch. (4 SE.) pedátus.

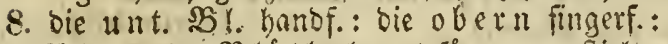
Bl. an D. $B$ líth. lanjettfórm., geftielt;

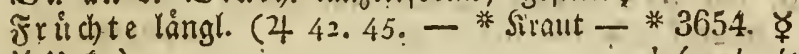
ఫ̛ Untér.)

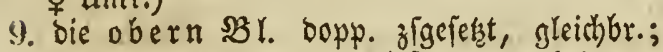

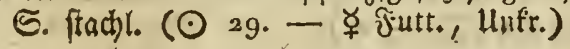

\section{RAPHĂNUSS, 526.}

\section{Blätter:}

1. ไenerfórmig; bic fticlruno. Schötchen:

a. Enot., 2 fich. $\left(\odot \sigma^{7}\right.$ As. ros.; p.; wo - * 3656. ఫ

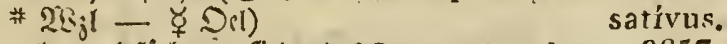

b. glatt, 1 fäch), gefiedert $(\odot 30$, w,; rch,; 3657. ఫ gb. - $\forall$ Der, llifr.)

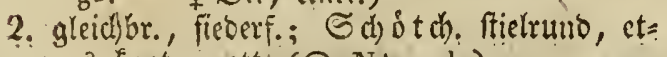
was Enot,: zott: ( $\odot \mathrm{NAs}, \mathrm{gb}$.)

(Raphanistrum.) 3658.

sibiṛ̆cus.

3. Ianjettf., gejahnt, untehaart: sie unter=

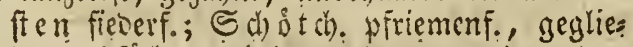
Dert, 2 finc, unbehart $(\odot$ As. p.) .. tenéllus.

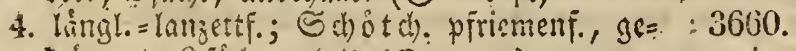
f̧rummt, 2 fấct), glaft $\left(\odot-v_{0}\right)$ arcuàtus. 


\section{1, RESEDA. 381: (3582, 362": \\ $381^{x} \cdot 382^{2} \cdot 423^{x}$.)}

\section{Blätter:}

I. gleidfformig,

1. Iangettfurmig,

a. ungetheilt,

h. am Srunde allf beyo. Eeit. 1.zahn.;

B10. 4 ppalt. (4 r 4. hgb. - * Siraut, * 3661. ఫ

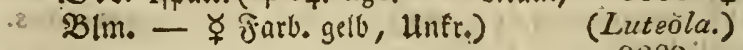

b. Frủchte fternf. (SE.) 3662.

sesamoídes. 3663.

b. am Ranoe wellenf., baar. (4 SE, w.) canéscens. 2. gleichbrit, am Sirunbe gejabnt; mit 3664 , 4 Stroeg. (2t St. 9.)

3. gefiebert;

a. mit 4 Strogen; Bld.:

a. 6 theil. $(\odot-6.2 z$. w. $)$

b. 5 theil., abftebeno; $\mathfrak{B}$ l. an b. Gpitge umgefrimmt; $\widetilde{S}_{\mathrm{tg}} \hat{i}$ am (Srunde fraud). ( 4 SE. w.)

b. mit 3-4 Etweg.; $\mathfrak{B l}$ l. am Rande mellenf. (4 SE. w.)

II. unglcichformig,

1. ungeth. U. 3 lapp.; Blll.:

a. jebr groß̈, bitbeif. $(\odot-$ w.)

b. fuirg. als o. Bzlitthe ( $\odot$ As. w.)

c. eben fo lang als o., sBluttye (o' NAf, wch., wohlriech )

2. fémmtl. 3 ipalt.: bie unt. gefíed. (48. 34. 22, bgb.)

gláuca.

... 3665.

(aba.)

3666.

fruticulósa,

3667.

undáta. 
5. RHAMNUS. 153. $\left(72^{2} \cdot 777^{3}\right.$

$$
779^{4} \cdot 807^{x} \text {. W. 29.) }
$$

Blíts. 4 ppalt., mit ganzgetremut. (S)es f(d)led)t;

I. SMit Dornen:

1. am (5nde D. 2effe ftebend; Blätter:

a. loingl.=evr., geifigt, mit einwortsges

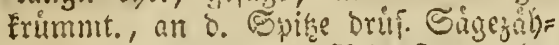
nen, 7 fach gerippt; ssittifte linbe= haatt; ș I mift le ling. als o. SBibecte;

Beeren Engel. (5) 26.48 , grnch. * Beer., Minde - ఫ Jarb. gelo, grim)

$36,4 . \%$

(calharticus.)

b. enr., geiágr, mit Eagezahtr. wie wor= 'ber, 7 fach geripyt; sis It ftiele iott.; SOImftle kaum io lang als bie Bib.; SBeer. meift bergf. (占 SE. grnch.) tinctorys.

c. Ianjettf. t. evr., am Grumbe fömaler, feingejägt; 2fefte aufr. und geftredt; W. SBluth. ohre SBlbl. (5 _ 8. 23.) saxatilis.

2. am Ende ber Refte u. zur Seite fte=

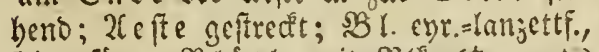

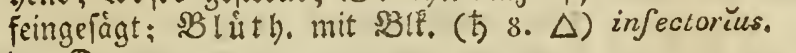
II. obne Dornen;

1. mit 3 witterblutben; Aeste:

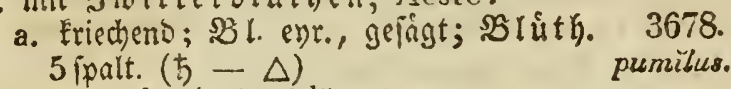

b. nicht Eriechend; Blätter:

a. elliptif(h),

1) am (siunde gamgrand., u. fáma. ler, mit einwárţ̧gefrummt., an o. Epite oruip. Eaggeáliner, ets was gerippt $=$ genteit (b NAm.

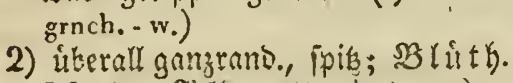

5 ipalt.; S\$D ID. zott. ( 5 As. $\triangle$ ) latifolius.

b. eyr. = langlich, faft oval, ganjuno., gerippt =geab.; mit $1 \mathbb{S}^{\mathrm{t}} \mathrm{weg}$ (t) 4. 49. w. - * Ninde - ఫ Jarb. * 3681. ఫ gelb, griu, rotty)

2. mit balbgetrenniem Beidh.; SBl. elliptifi $=$ oval, leverart., mit einwártsge= frummt., an \&. Spize brif. Sảgezályner, bie am Grunbe meiter abftehen, als an b. Spige $(b-\Delta)$

3679.

alnifolxus.

3680.

\section{* 3681.
(Trangǔla.)}


3. mit ganjgetrennt. (Ge fob!.; B1.:

a. oval =ellint. =am Girunbe faft hersf., mit

- cinwartsgerrinmt., can Der Exilze oruir.

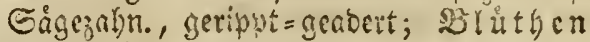
4 ipalt. (b) 9. wch. - grir.)

3683.

alpinus.

b. oval, frocrart, immergrü, fwifs, gefơgt, am Sirunoe meift funtuf; SR arbe 3 fach

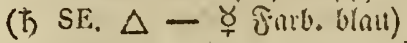

c. laingettf, an bevo. Enoen foik, ftachel= fpis = gejahnt, lecerartig, immergrůn; Ra a b 3 fact) ( 5 SE. $\triangle$ )

3684. ధ๐ Alatérnus. $368 j$.
Clusĭi.

\section{RHEUM. 326 .}

\section{Blätter :}

1. ganj linbebant,

a. etnas gelappt, ielr jtumpf, ipiegelglatt, 3686 .

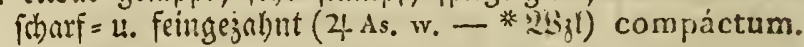

b. ungeth., hersf. =ei)t., flach; sBlttftiele 3687. balbitielruns, eff.; গif ifpe gefurd)t (4As.) tataricum.

2. oben unbebart,

a. unten etwas bnar., faft gelappt, fpib, mit ichmaler SBlicht am (s)tunoe; SBlt t= fi iele oben umbeutl. gefurcht, am Ranbe runol. (4 As. - * $23 ; 1$ )

b. unten on D. Xeern etwas banr., ftumpf, mit breiterer Budt) an (Situnbe; $B$ lt $t=$ file oben gefurcht, an Rande runb (4 * 3689.

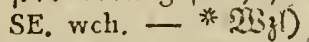

* 3688 .

hybridum.

3. iberall etwas zott., am Sinnoe wellenf., mit

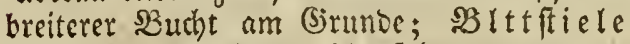
obcr flach, am Siande fdyarf (4 As. grno-gb; 3690. - * $\operatorname{Nij}_{3 i}$

4. ctras forarf, banbF, langgcipitat, mit brei= terer Butht am Srimbe; Bblttfile oben unbeutl. gejurd)t, am Siande runo (4 As. bfl. - * $2(2 j \mathrm{jl})$

Rhapontřcum.

5. etwas feinmar ${ }^{\circ}$; jehr ftumpf, mit unten feinoorn. 2roern; sBlttfile oben flach, am গiande runde (4 As.)

\section{undulátum.}

\section{* 3691.}

palmátum.

3692.
Ribes. 
Rhinanthus. - Rliododendrum.

14. RHINÁNTHUS. 485 .

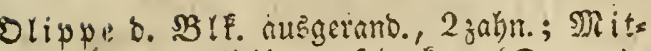
tellayp. o. Ulippe febr furz $(\odot$ 3. gh. - ఫ Jutt., Uuft.)

3693. ఫ

(Crista galli.)

\section{RHODODÉNDRUM. 330 .}

\section{Blk. trichterförmig.}

Die immergrumin Blätter:

1. auf beys. Seit. unbebáart u. faft gleciujarb., etwas leverart., vual=lo̊ngl. u. oval =lans

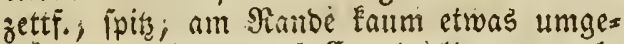
frument; nuit boldenf. Endodolbentraub. $(\hbar-\triangle)$

2. auf beys. Seit. harg. = punct., faft Yeberart., oual, fpits, am Sianoe geferbt, ourch ent= fernte, lange SBorfen gewimp.; Bluthen 3695 . langseffielt in (Enbooldentraltb. (ち 9. hp.) hirsútum.

3. oben glatt, $\mathfrak{k}$. unten

a. rofftraun burd) faupls. STarzpuncte, les

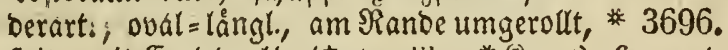

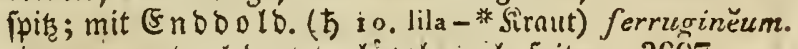

b. barz. = punct.; leberarto, längl.=obal, pipit, 3697 . ganjrand.; mit Endoold. (5 NAm. lila) punctátum.

\section{Blk. glockenförmig.}

\section{Blithen:}

1. in Endbolden; BBr. furjoyal, an berb. End. gerund. = ftumpif, unten blaffer; $B \mathbb{B} 10$ lapp. verlång. =längl. (方 NAm.)

2. in EndDoloentrauben; SBl. Tederart.; immergrim, oval ú. veit. cตr. = Kángl., Furz= geipiget, am Rande etwas unigefrimmmt, un= bebaart, unten blaffer; sB lolapp. ipis ; $\mathfrak{B}$ lélapp. oval långl. (†) SE. v.)

3694. azaleoídes: 
a. verk. enr., fpis, gerbimpert, auf beysen Seiten glatt; $\mathfrak{B l u t}$ luen geftielt: SBrbl. ipis ( 5 NAs. ros.)

b. IGingl., unbebant, oben bitstuunct., un= ten bicht rofturaumpunct. ( 5 As. r.)

2. einjeln, ob.'2-3 zålyl., am Enoc febend; BS r. faft leocrart., immergrü, ovat, ipit, gangrano., mit entfernt., Eruf. Şaarent ge=

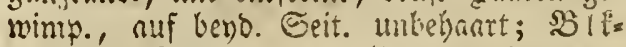
rapp. vert. enr。= rumbl. (i) 9. ros.)

3702.

Chamaecístus.

3. in Endodben; die lescrirt., immergrun., oval =lóngl, am fianos etras umgetrumms ten Blätter:

a. fpis, unbehaart, unten braffer ob. etwas

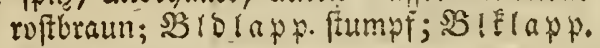
runol. (ち NAm.. rclss w. $\triangle$ )

b. nm Ende fumpi, am Srunbe idomaler, oben unbebaart, unten rofifarb. = fits., nit fic) ablófentem Filz; SBIE. rumbr. (ち) As. 10. $\Delta$ )

4. in menigbluth:, Doloenform. (5nodolsen= traub.; $B$ l. lingl, an beys. Eno. jobmas ler, am Rande ungefrummt, jobrif, unten blaffer (ち NAs, gb. - * Siraut)

3703.

maxĩnum.

3704.

caucasǐcum.
* 3705.

Chrysánthum.

\section{RHODÓRA. 338.}

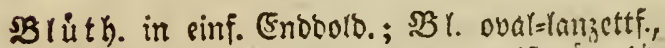
am $\Re$ Rance umgerollt, unten weisgrin ( $\hbar$ NAm. lila-rch)

3708. cańadénsis.

\section{RHUS, 241. $\left(781^{x}\right.$. W. 70.$)$}

\section{Bl. gefiedert.}

\section{Blättchen:}

A. Ganjranoig, langgeipitat,

1. auf beys. Seiten unbebaart, eyr.; gem. sBlttfil ungefligelt ( 5 NAm. grncls.gb. $\triangle-\Varangle D \cdot !)$

2. oben unbebaart, unten etwas feingaar., ranzettf.; $g \mathrm{em}$. $\mathfrak{B}$ lattfít iel geflugelt ( NAm, grach, - gb. - * \$arz)

3707. † Vernix.
* 370s.

Copallinum. 
B. gejägt,

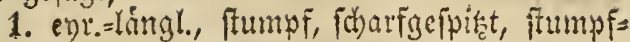
gefígt, oben idorf, unten zott.; gem.

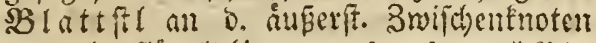

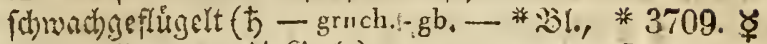

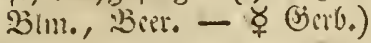
Coriaria.

2. Ianjettformig, langgeipicht,

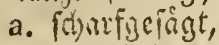

क. unbehaart, mur an ber Mittelrippe feinbanr., unten binfer; gem. SB It t= fiel jott. ( $5 \mathrm{~N}^{2} \mathrm{~m}$. grr.)

b. unten har.; gem. Slattft wie

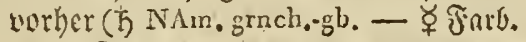
gers, (Serb.)

b. enggejägt, fwis, unbehant, unten meisl.; gem. sB It teft unbelyant. (方

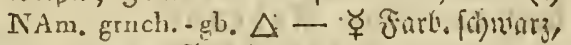
getle, inth), (Sistr.)

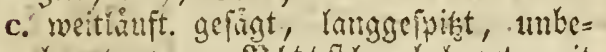
baart; gem. SB Itt fit unbehart; mit ganzetrennt. (s) sid)!. (5 NAm, scb.)

3710. viridiflóra. t)phn typhǔna.

$3712 . \not$ glabra.

3713. elĕgans. :

\section{B1. 3 zählig.}

\section{Stengel:}

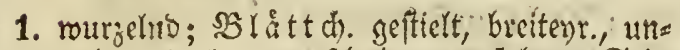
seth., oo. lapp. 3erichnitt, auf beyb. Eeit. unbelyant os. unten feinganr. († NAm. grach.

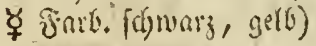

3714. ఫ $\uparrow$ Toxicodéndrum

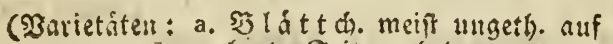
beys. Eeit. unbebart rulgáre.

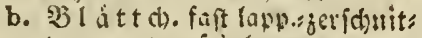
ten, Huten frinbaar. quercifollum.)

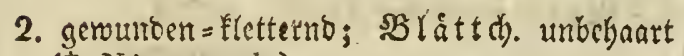
(万) NAm, grnch.)

3. aufiecht; Blättchen:

a. unbelyart, cyr. = rautenf., gerifonitt. $=g e=$ jight: Die ander Seite faft ftiellos (5 NAm. grnch., wohlriech.)

b. unten fringar., tautenf. = oval, gezahnt; B (üth. mit ganjgetremnt. (Seichlecht in cimzelt., os. 3 fact., wor 8. sBl. cricteci=

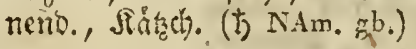

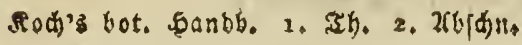

3715.

volubilis. 
III. Bl, einfach.

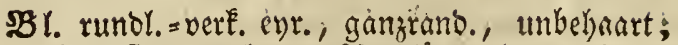

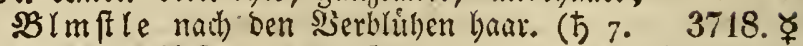
grnch. - ఫ̧ ซarb: gitb; Gerb.)

\section{RIBES. 166. $\left(779^{3}\right.$. W. 16.)}

\section{Ohne Stacheln.}

\section{Blätter:}

I. auf beyd. Seiten nicht punct.; Traubenbliithen :

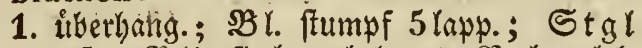
aufr.; $B$ lo. flach, unbehaart; Rebenbl. fultz. als §. bef. sBimftle; SBeren unbe=

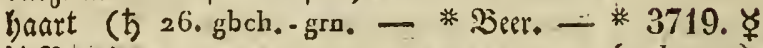
$\Varangle$ Berr:

2. aufred)t; Bld.:

a. etrads flach;

ค. unbehaart; $\mathfrak{B}$ l. langgefpiat $=5$ lapp.; Rebenbl. Eurry. als b. bef. Shlmfte, feingenimp.; Seerent unbebaart 9. $\mathrm{rch}_{\text {.) }}$

b. baar. = orioj.; 2 efte zuridfigelegt $=g e=$

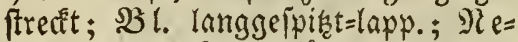
benbl. Febr Elein, fürz. als b. bef. SBlmfte; $\mathfrak{s}$ eer. fteiflaar. (ち $\mathrm{NAm}_{6}$ (万) rch.)

b. glocenf., intechaart; $\$ B l .3$ lapp., un= ten fipiegelglatt; mit ganzgetrennt. Ge f chl.; Mle benbl. folang od. láng. als b. SBlitt).; SBeer. unbehaart ( 58 . gbch..grn.)

3. fohlaff; $S B$ l. 3 rapp., mit ftumpf., wenigs 3720. petra' eum.

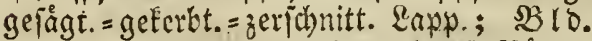

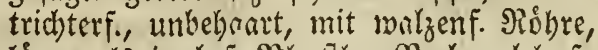
laing. als o. bei. SBImpte; Rebenbl. po lang, als b. bef. SBImftle; BBeer. unbe= baart (ち NAm. gb.)

3721.

prostrátum.
3723. aurěum.

II. unten punct., fpic, 3 do. 5 lapp.; $\mathfrak{Z} \mathfrak{r} \mathfrak{a} \mathfrak{b}$. ichlaff, niebergetog.; $\mathfrak{B}$ rs. glodenf., unbe=

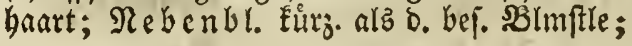




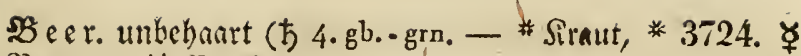
Becr. - $\not$ bier.)

III. alf benden Seiten punct., fiph=3 3 lapp.;

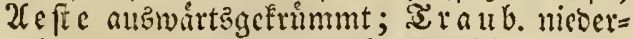
gebog. =bang.; s. lo. gloctenf. = tridhterf., un= behaart, mit bauth. Robre; Rebenbl. lantg. als D. bef. SBlmftle; $\mathfrak{B}$ eeren unbelyaart (ち NAm. grnch. - gb.)

(nigrum.)

\section{Aeste stachlig.}

Stacheln :

1. einfinch); $\mathfrak{Z}$ efte ungeftiummt; $\mathfrak{B}$ I. 3 Yapp.; BIm ftle 3 blutth.; Rebenbl. idecibeart., meift 2fpalt.; 3 eer. unbel)art (5 NAm. grno u. w.)

2. gepaart, afterb(attart.; $\$ B$ l. Eeilf., fdumad) 3 lapp.; 3 líth. tranbenf.; $\mathfrak{B}$ eer. unbe= baart († NAs, gb.-grn.)

3. cimjeln, gepaart, od. 3fach; Xl efte gerabe, of. etwas umgctrummt; $\$ 2 B$ l. 3 lapp. ; Beeren: a. rauch; SBlttftle hanr., olgne Drúpen; sRlmfte 1 blutth., mit 2 abgcionterten

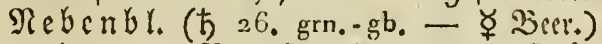
3726. triflórum. 3725.
florìdum. b. unbebaart; $B^{3}$ ttiftle haar., mit Dri= fen; solmfitiele mit 2 verwady fen=robr.

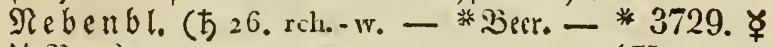
ఈ̧ Berr.)

372S. ఫุ

(Grossulariáa)
3727.
Diacántha. 


\section{ROBINIA. 579.}

Blätter :

I. Ingepaart gefiebert; S⿱乛⿻上丨ub. inlo. SBlatt= winf., mit 1 bithth. bejond. SBlmften; Aestchen:

1. obne Driffer u. Snare; 2fferbr. Dorm; Scillen nadit (h) NAm, w., woblriech. - - Der, Garto. geto)

3733. $\not$

Pseudacacǐa.

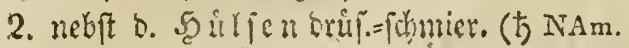
rch., geruchlos)

3734.

viscósa. 3735.

3. fteifbaar. (尚 ros, geruchlos)

II. gepartgeficbert,

1. 2 part.; Xeft chen Dutect) D. Daur. SBltt= fticle D. vorigen Sabres born.; Biätteh.: a. Fibenart.; SBl mfile 3 blittl., in ben

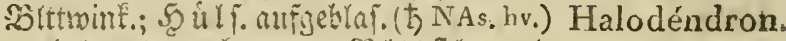

b. unbeharat, vert.=evt.; $B$ lttfle uno

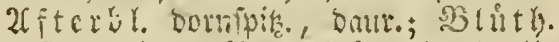

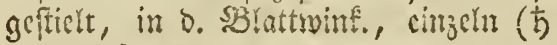
As. ugb.)

2. A paar.; D. geftelt. Blïth. in D. Norttwint: hispı̃da.

a. meift einjoln; 2feft ib. wie vorber $\mathfrak{t}$.

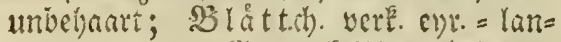
jettf, igegent o. (Sivuns Eeilf., untbehant; SBlattifle u. 2ffterbl. wie vorber; Sciflien frielrund (ち) NAs. gb.)

b. zablreid); 2ffterbl. nbfallento, ohne Dorn.; Şutfen walzenf. (节 NAs, gb.)

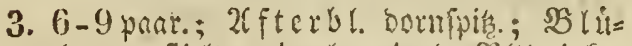
then gefticlt, einjeln, in o. Bolttwint.; Seillen zfgerudit (b NAs. gb.)

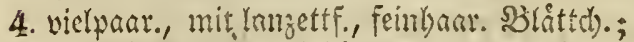

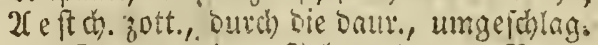
Bittifle D. vorigen Snlyres born.; SBIt $1=$ ftle fobenf., dounipifs., baur.; sslutb. mie vorber; Solifien ftielrumo (b NAs. $\Delta$ ) jubata.

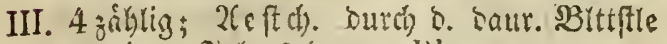

s. vorigen Sahtes borm.; Bl.

1. furjgeftelt: sie vert. eist, ftuchelpipis. Blättch.:

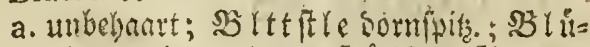
then wie vorber; So ulfen fictruns, น. meft $\mathfrak{B l m f t}$ le 


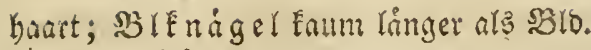
(ち) NAs. ${ }^{\circ}$.)

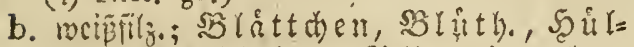
fen, solmfticle u. Sols. wie yorther;

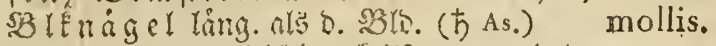

2. faft frietros, mit gleichbr. = Feilf., geruntet.,

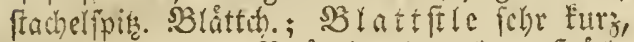

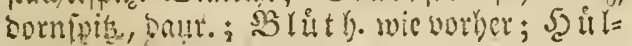
fen fieiruno (ち NAs.gb.)

\title{
3744 \\ pygma'ea,
}

\section{2. \\ frutéscens.}

\author{
3743.
}

\section{ROCHELIA. 102.}

Frif de te nfr.: Saamenstacheln:

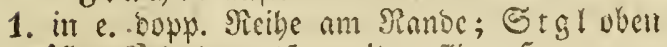
. aff.; $3 B$ r. tantecttF., mit nuflieg. Saar., gg= wimp.; SIE. Elein, mit aufro, abjtethenoent Saum $(\odot$ 22. 24. hbl, mit w, od, gb.)

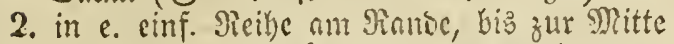
z)gewachf.; sBl. lángl, ftumpi, mit crwas

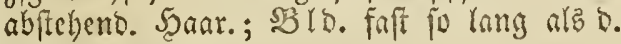

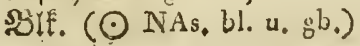

\section{5.}

(Lappüla.)

3746.

squarrọsa.

\section{ROSA. 401.}

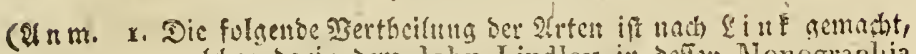
welder Darin Dem John Lindley in beflen Mlonographia Tosarum. London, 1820. gefolat ift.

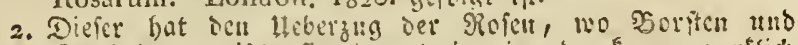

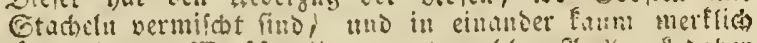

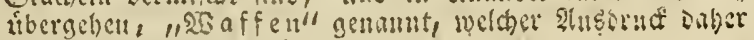
aud bler benbebalter ift.)

\section{Heberfidyt beg Ganzen;}

A. A e ste nit daur. Filze bedeckt; Fruclit nackt

B. G riffel in 1 Säule zshang.; Afterbl. angewachsen

I.

C. heydes nicht; liand d. Bid.:

x. schmal; Blätt ah. lanzettf. od. längl., olne Drüisen; mit Nebenbl.; Pflanze stachelborstig od, wehrlos

2. kaum beme:klich; Bldblättch. daur., zsgeneigl; Bl. eyr. od. längl., selten mit Nebenbl.; P flayze stachelborst. nit dichtstehend., fast gleichf, WVafien, od. unbewaflinet

3. vardicht, den Schlund schliessend; Bldblätchon:

II.

III. a. abfallend; die gröss. A este bogenf.; Bl. eyruml, 
ohne D rüs en, mit zsgeneigt. Sägezähn.; St a cheln gleich, zurückgekrümmt

b. daurend; Blät tch.:

a. runztich, längl. od. eyr.; Bldblät tch. zsgesetzt; mit Nebenbl.; Pflanze stachelborstig mit un. ungleichif. Waffen

b. mit ausgebreit. Sägezähn., eyr. od. längl.,

1) drïsig; A este bogenf.; Stacheln ungleich, nicht borstenf., selten fehlend

2) nicht drüsig; A este lang $u$. ganz gerade; BIdblät tch. zsgeneigt

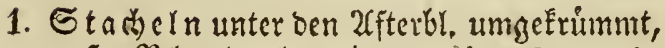

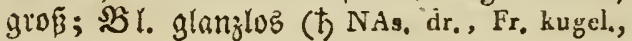
sch.)

3747.

camtschatǐca.

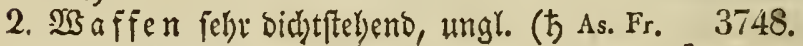
kugel., sch.)

\section{II. .}

\section{Blättchen :}

1. evr. u. 'eyr. =lanjettfórm.; $\mathfrak{L}$ f fte auffercig.; 3749 . Stadeln felyr ungefrummt (ち SE.) systy̆la.

2. unten weif́grim; Bliithen:

a. meiff einjeln; Uf efte rankenf.; Stadeln. ungl., oggefrummt ( 526 . w., Fr. längl., nackt)

b. in vielbluth. Dolbentrauben; $\mathfrak{B}$ lb. blatt d). jigejeft, langgeipist; 2 eften en faft unbebart; SBlátt ch. oval, langge ipift, mit eingebog. Sagezábn.; Ufter bl.

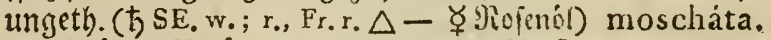

3. immergrun; SBlitth. faft boldenf.; $\mathfrak{A}$ efte Eletterno; Stacheln faft gleid), zgefrummt ( 5 26. w., wohlriechend, Fr, blein, rundl,, dgb. $\Delta)$

3750.

(arvénsis.)

3752.

sempervirens.

\section{III.}

A. Blättchen lanzettf.

Blättchen :

1. langettf., faft unbebaart, icharfgefägt ; $\$ 2$ r 8 . fhmier.; Xfterbl. gleichbr.; Stacheln afterblattart., nabelf. ( 5 NAm. hr.)

3753. parviflóra. 
2. fahmallanzettf., flach, glån

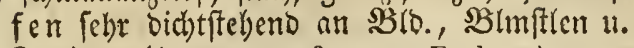
3meigen (ち NAm, plirs: $r_{9}$, Fro kugel, , stachelborst, , sch.)

3754. ṇitǐda.

3. Greitlanjettf.; $\mathfrak{B} l$ it th. Doldentraubenform.;

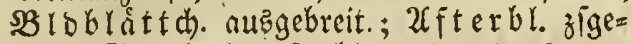
rollt; St a cheln afterblattart., ctwas jae= frumtmt ( $5 \mathrm{NAm}, \mathrm{r}_{0} ; \mathrm{Fr}_{0}$ kugel., an d. Spitze niedergedr., stachelborst, $\mathrm{dr}_{\bullet}$ )

3755:

carolina,

\section{B. Blättch. länglich:}

Blïthen :

1. eingetn; Blättch.:

a. flad); $\mathfrak{B}$ Ittfti haar.; $\mathfrak{S}$ affen abfall. (5 NAm. hro, grofs; Fr. r.)

3756.

blanda,

b. runzl., unten filz.; $\mathcal{X} \mathfrak{f t e r} \mathfrak{b l}$. crweitert, wellenf.; $\Xi_{\text {ta d }}$ el $\mathfrak{n}$ afterblattart., faft gerabe; Xefte gam gerade ( 526 . hr., Aeste zimmetbraun ; $\mathrm{Fr}_{0}$ eyr, - rundl scb.)

2. meift gepaart; $\mathfrak{F} r u$ dit fugel., an $b$. Spige eingebridft; Die afterblattart. Stacheln:

a. gerade; sib átt d) en Dachziegelf., flach),

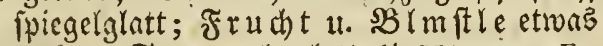
badr.; Stengel glatt († NAm, $r_{0}, F_{\text {: }}$ stachelborst.; hr.)

b. an b. Stgl gepaart; şlattch. Tpit; Xfterbl. bruf = sewimp; SBlttifle u. Brattabern unten feinhan.; ${ }^{\circ}$ rud $t$

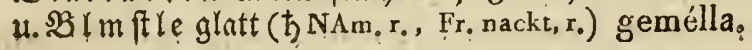

3. in wenigblitth. Xffterbold.; Şlatt then wellenf., glanjlos, unbehant; $\mathfrak{A}$ eft e ganz gerave, etwas weisgrim (t) NAm. r., klein, Fr, rundl. od. eyr., klein, $\mathrm{hr}_{\text {? }}$, nackt)

3757. cinnamoměa:
3758. lucǐda.
3760. fraxinifolla

\section{IV,}

Stengel:

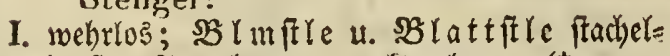

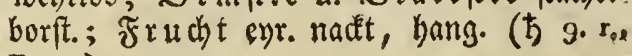
Fr. r.)

3761.

II. mit Waffen:

alpina. A. gleit); Frucht: 
1. cyr., mit zfgencigt. SBloblattd). gefrónt, verláng., bang.; Sुaffen bichtfelyeno ( $\hbar-$ hr., Fr. sch.)

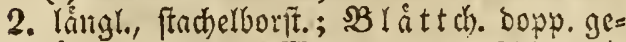

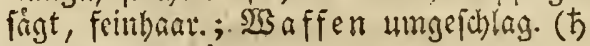
SE, ros., Fr. r.)

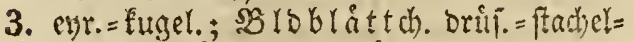

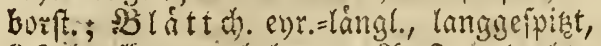
3 frichgejagt, umbehant; 2 efte gcorebt: die jungern bereift (5 15. T., Fr.'P.)

B. ungleich; Blättchen :

1. oval, meişgrin; 2 fiterbl. glcich br., an D. Spitze ausgebreitet; Sulo. balbtugel. (ち As. hgb.)

2. einf. gefigt, flati), unbebant; $\mathfrak{g}_{3}$ affen ber a fefe febr sichtfeberio, fatant, um= geichlag.: bie ber 2 eft then felbr flein, foft gleid) (ち NAs, hgb, , Fr, rundl., nackt, schw.)

3. långl.=oval u. ellipt., meift bopp. gelägt, flact), glanjlos, unbehart; $\mathbb{S B}_{\mathrm{s}}$ aff en fehr bichtitebeno, gerabe, ausgebreitet (i) NAs, gbch.- w., grols, Fr, kugel., an d. Spitze eingedrückt, r.)

4. runol. u. oval= runol., cinf. gefägt, flach), unbehaart; Fru (b)t tugel. (b 26.5 r. schw.)

5. oval $=$ rutrol, einf. =gelagt, unbehaart, an

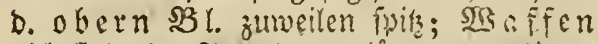
sichtfiebeno; forud)t vertáig. =cur. (方Fr. sch.)

6. faft freišruno, \&ru․, unbebant; cie gróp. Sgr affen boldff. (b SE. w, - rch.)

3762.

rubélla.

3763. revérsa.

3764.

(margináta.)

3765.

sulphurĕa.

3766.

lutéscens.

ochroléuca. 3768.

(spinosissima.)

3769. suavis.

3770.

myriacántha.

Blättchien:

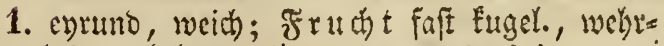
los, unbebaart ( $\hbar$ As. hros, , Fr. dr.

3771. caucasica.

2. eyr. = oval,

a. unten etroas wcifggrin, unvelgart, facif; Frucht eyr., webrlos, unbehaart (b 26 . ros.; w., Fr. $r_{0}$ - $*$ Bim., Friristi, Saam.,

Edfwamm - ఫ̧ Frid)te, (Sirb.)

Iarietáten; Blättchen :

1) cinfadigelágt ; Blttstle:

(anina.) 
a) fachl., unbehaart; Afterbl. ant Ramoe etious biuff. vulgáris.

b) am (3rmubc faitwebrios; afterbl. oben oruf.sgetwimp.: : $\mathfrak{V}$ lát th. faurfs gefágt nit laugneinik̨t. Sägejábu.: ile fit de febr oicht ramusissima.

2) Sopp. gefágt; Blttstle ;

a) ftadsl., orif.sptachelborft., infond. an D. Grunde; defterbl. orif.: ges wimpert ... glandulósa.

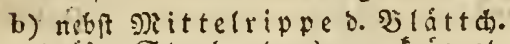
Drif.; St a ch. etwas umgefrimmt, ftarf, febr didutitebello squarrósa.

b. auf beys. Seit. gleidfarb., feimbanr, ete was fteif; Frud) t wie vorber ( r., Fr. r.)

3773.

dumetorum.

Sarietáten : Blätichen :

1) cinf. gelúgt, mit ąewimp. Sígezătn.t anf bevo. Ecit. etwas jott. seplum.

2) Dopp. gelahat. mit oruif. Gagejabn., oben feimbar., untell etwas jott. vulgánis.

3. oval = lăngl., glan\} was rothgeab.; A fefte bellblaubereift; Sta. d) eln Eletii, entferntffekeno (5 9. dros., Fro surdl, . langl., welulos, nabehaart, dr.).

3774.

rubrifolia

\section{VI.}

Waffen:

I. ungreid): Dic grón. umgefrimmt;

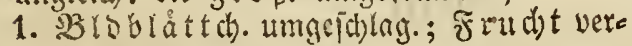
limis: (t) SE, r, ; w.)

2. SBlattd. brif. = gewimp.; SPlut then íbergebog.; $\mathfrak{B}$ ls. fismicr., etwas bruf.=

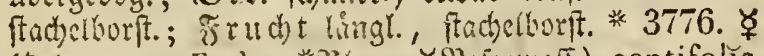

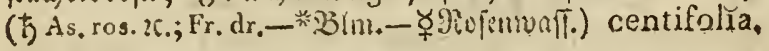

: Barietâten: Blmstle u. Bld.:

1) mit eitrf., briffutragend. Stachelfors ftet! provinciális.

2) Durh âf., brífentrag. Stubclborftea moofidet muscósa.

3) it allen Theilen fleiner pomponia.

4) mit oоpp. gefico. $\$ 1$. bipinnáta.

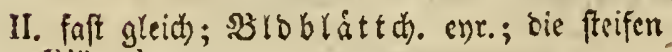
Blättchen : 
1. ellipt. u. runtr. ellipt.; $\mathfrak{B}$ lùt h. aufrect)t; 203 affen gleichi., jowach; Frucht falt * 3777.

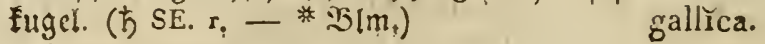

ßarictáten:

1) mit 3-4 Fuf bobem Stgl elatior.

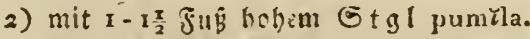

3) mit fleinern gefillt.

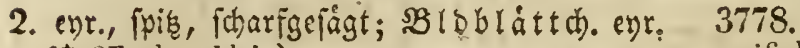
(ち SE. dr, klein)

parvifolǐa.

\section{VII,}

Blättchen:

1. flad), vertieft; Stacheln gerabe; $2 B$ lo. webrlos, mit ungcth. Iapp. (ち 26. gb.; gb. u. $r_{0} ;$ Bl, wohlriech.; Fr, eyr,-kugel., an der Spitze eingedrückt, sch.)

2. rum;l., glanjloz; ङtacheln umgefrummt; 3780 .

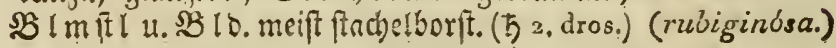

Narictáten:

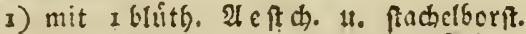
ร) 10. vulgáris.

2) mit mrift 3 blúth. $\mathfrak{A} e \mathfrak{f t}$. triflóra.

3) mit 1 blitth. $\mathfrak{A} e$ it d. , balb fo aros.

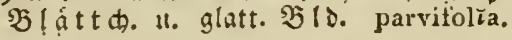

3. auf beys. Ceit. bereift: die obern faft im 3781 .

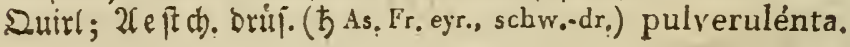

\section{. VIII.}

Bldecke:

i. freuielf. (ち $S E, x_{0}$ )

II. nicht; Blättchen:

1. ellipt., ftumpf; $\mathfrak{F} r u d t$ fehr groź, nit fteifen, oichtitebeno. SBaffen; NBlo la p p. fomier., fracbelborft. ( 5 - dr.; Fr. fast kugel., dr. - ఫ (̧rud)te)

2. eyr., etmos fpis; Frucht ftacheltorft., 3784.

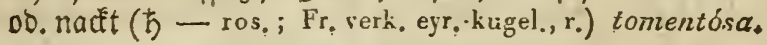

3. lángł., einf. gelägt, wcis̄grun, oben faft

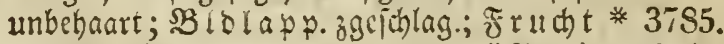

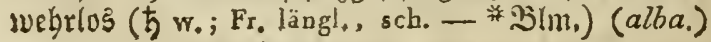


Rosmarinus. - Rubus.

2. ROSMARINUS. 27.

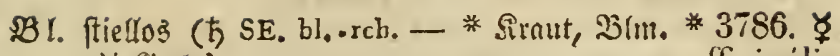
- $\Varangle($ Sirtb.) officinális.

\section{ROTHIА. 605.}

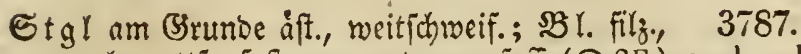

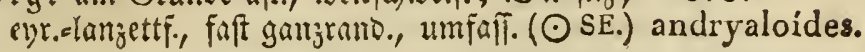

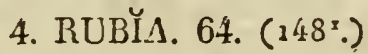

Dic lalizettf. Blätter:

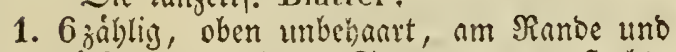
Ruden unten icharf; Etgl frautart., ftachl.;

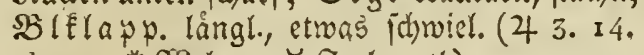

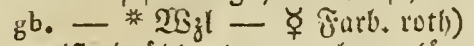

*3788. tinctorum.

2. meift 4 zábl., baut., bben glánz., glatt, an Rambe $u$. an $b$. Nittelrippe unten idjarf;

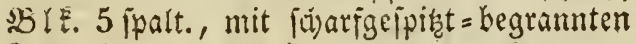
Eapp. (4 SE. gbch.)

3789. peregrina.

\section{RUBUS. 400. $\left(795^{3}\right.$, W. go.)}

\section{Krautartig.}

\section{Blätter:}

I. 3 zåál.; Bliithen:

1. einjeln, am Enbe fteheno; !Stgl anfr., feintaar., welytros (4 NE. ros,; Fr. dr., *3790. ఫ sehr wohlschmeck. - * Beer, - $\not 33 \mathrm{icr}$.) arctǐcus.

2. an $3-5$ blüth. SB Imit len; Stgl aufr., eff., feinlbanr., mit febr feimen, geraben, auşgebreitet. Stacheln u. Frieh. Sanfen (4 21. w.; Fr. r., mit sehr gुrols. Beerch.) (saxatülis.)

11. cinf., geloppt; $\Xi_{\mathrm{tgl}}$ wehrlos, 1 blitt). (4* 3792 . 46. 47. - * Siraut)

Chamaemórtes.

\section{II. strauchig.}

\section{Blätter :}

I. Ulle 3 jáblig: Blättchen:

1. un(er)aart, 
a. ungl. gejägt: das mittlere eyr. : sie Seitenbl. rautenf.; S3ls. frimbar.;

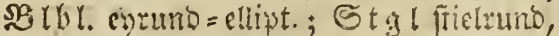
gefted, mit umgefrimmt. Stad)ein ( 5 NAm. w ; Fr, schw.)

b. zelidnitt. =geîgt: Das mittlere gic=

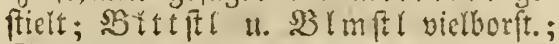
Stgl niesergeftredit, mit vielen, fteif. Borfien ( 5 NAm.)

2. unten an Den 20ern etwas ftrdyelbar., runol. =evr., lariggefpitst, fachelpifs. = ges fágt; SBlttfi, SBlmfte u. SBlo. fachl. u. orui.=borft.; $\mathcal{u}$ eft e edf., ftachl. u. oruif.= borff. ( 5 - Fr. $8 \mathrm{chw}$.)

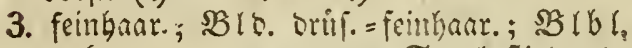
3793.

flagelláris. vert. evr., altogeramo.; Gtgl ftielrumb, blanbereift, mit etwas limgefrummtent Etad)eln ( 5 4. w.; Fr. schw., bl. bereift * 3796. $\Varangle$

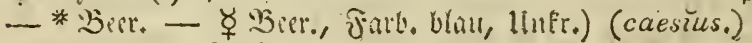

4. unten filsig; Stgl:

a. mit gerad. u. umgetruumt., pFriemenf., am Grunde breitern u. oigect. Etacheln; SBlattd). langgelpint; $B$ lttft falt fticlrund, mit e. Fithmalen SRinne ( 5 NAm. Fr. schw.)

b. obne Stacheln, frieltunt., etwas filt.; BIattib enr., ung!. gelägt, die an o. Seite etmons zerithnitt.; $B$ Itt ft le u. $\$ 3$ Imfte webrlos (ち NAm.) , 3794. hisprัdus, 379:; glandulósus.

3797.

occidentális.

5. auf beub. Seiten filz.. cyr., etroas zer=

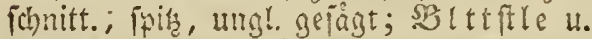
B lmitle fradt.; Etgl cer., mit umge= frummf. Etacheln ( $523 . w_{0}$; Fr. schwo)

II. Ulle einfach): bie unt. 5 tayp.: bie ober= ften 3 lapp.; Stgl cone Etndeln; $\Omega$ ID.

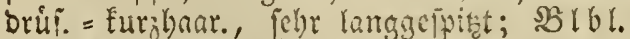
rumbl. (占 NAm,dr ; Fr klein, Hach, r., säuerl.) odorátus.

III. 3 åń). u. cinf.; SBlatthen runot. ob.

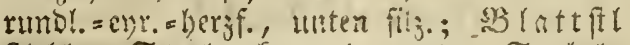
ftruhl.; Stgl eck., mit gernoen Stacheln (万) As, Fr, s hw.)

3801. obtusifolius.

IV. 3 u. 5 jåhlig,

1. zottig: B i. an unfuchtb. Stgl 5 ,

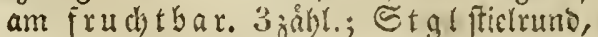
fott., mit faft gernosn Stackeln; 2810 .

\section{9.}

tomentúsis.

\section{0.}


orúf. = feinhatat.; \$B (bl. eyr., utgeth. u. aud ausagerand. (5 4. 49. ros.; w.; Fr. schw.)

2. ftacheithant, ent., langgefipht, ungl. ge fiogt; SBlmftiele wehrloz, brifl. = haar.; Stgl nieserfteg., brif.=fteifban., mit $11 \mathrm{~m}=$ gefrummt. Etad)elu ( 5 SE. Fr. schw.)

3. etwas feinlaar.; Stgl mit umnètrummt. Gtade!n: die niescilles. ftiefrumb mit

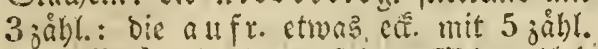

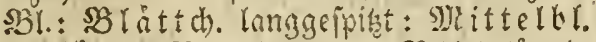

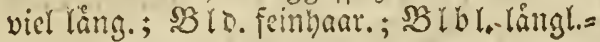
eyt. (ち NAm, w. ; Fr. schw.)

V. 3 u. 5 zêl. = geffebert; St $g l$ :

1. mit getrien, borft. = pitientienf. Stachelt ob. webrlos; SBlatt ch. furzgeipigt, unten filj.; SBltitl mit e. breiteli Rimne (衣

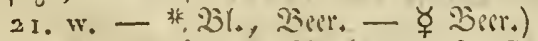

2. mit umgefrummt. Stacheln, ect., frachels

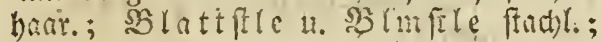

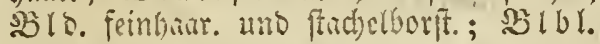
långl., jerichnitt. (ち) - hros.; Fr, schw., säuerl.)

3. mit oict)tfethent., roth. SBorften, ftielrunb;

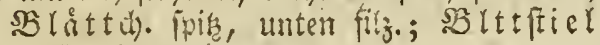
borf. ( 5 NAm.)

VI. 3 แ. 5 jofhl u. einjach; Stgl mit umge= Erimmt. Staciseln 1 .

1. ftumpfect, nieberliegr.; 2 efte anfr., fticl= runo, unten ftachl., oben feinhaar.; $\$ B Y$. unbebaart: die am Stgl 5 zábl., an b.

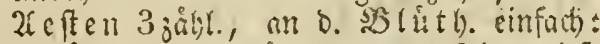
SBlått d. cyr. =långl., langycipiat, tic $F_{3}$

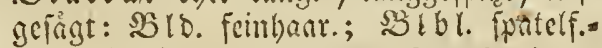
lanjettf. († NAm. w.; Fr, rch, -schwo) heterophýllus.

2. 5 edigh,

a. fait unbehanet; $\mathfrak{B}$ !, jett.; $\$ 3 / 8$. faft umbehant: fruchttrag. umgefchlagen;

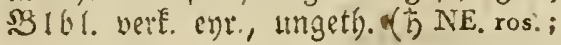
w.; Fr, sclow.)

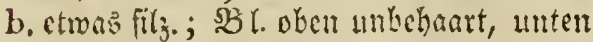
fits.; $\$ 3$ is. wenisfily.: frud)ttrag. um= geichlag.; $\mathfrak{B} 16$ l. wie vorber (t) 26.36 . wv., ros.; dr.; Fr. schw. - * Wier. $\Varangle$ Bect, Girb., Hufr.)

** 3805 .

(Ida' eus.)

3803

hirtus.
triviális.
.

\section{(nemorószus.)}

\section{6. \\ laciniảtus.}

3807.

strigósus.

\section{9. \\ corylifolrus.}

*3S10. ఈ
(fruticósus.) 


\section{RUDBECKIA. 693. $\left(69^{4}{ }^{2}\right)$}

\section{Blätter:}

1. gleichoormig:

1. gleicbbr., ganjrand., entgegengejest (4 3811. NAm. gb.)

2. (patelf. $=$ evr., ungeth., 3 fach gerippt, ge=

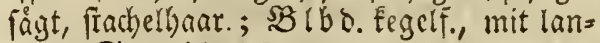
jettf. Spreubl. (o' NAm, gb.)

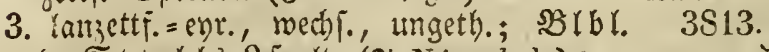
D. Strabls 2 palt. (4 NAm. hgb.)

4. lángl. =lanjettf., am (Sirunde (t) malcr,

a. fajt berzf., bater.; $\mathfrak{B} \backslash$ b. backr.; $S_{a} a=$ mentroule feingeferbt ( $4 \mathrm{NAm}$.)

b. berzf., umfaff : D. unt. gejágt; $S$ che $i=$ be walzenf. = fegeli. $(\odot$ SAm.)

5. gefiebert: bie c. od. anbere ber untern 2 theil.: biu ⿺̊丶 brigen ungeth.; Strone ganjrans.; Stgl gefurd)t, baffr. (4 NAm.) pinnáta.

II. ungleichformig: oic untern:

1. gefied.: Fiedern:

a. 3 lapp.: die oberften cyr.; Serone 3817 . geferbt; Gtgl unbehaart (4 NAm.) laciniáta:

b. fieberf.: bie obern sinf. gefies.: bie oberften 3 ppalt.; Skrone geterbt; Stgl glatt ( 4 NAm.)

2. 3 lapp.: Die obern ungeth.: 2rlle fpa= telf. (4 NAm. gb.)

3818. digitáta. 3819. trilǒba.

6. RUMEX. 296. $\left(29^{2} \cdot 7^{44}\right.$. $785^{x}$. W. 66.)

\section{Mit Zivitterblüthen;}

A. mit 3 staubwegen;

1. Blbl. mit e. Körnchen versehen;

a. Alle Blblätter.

Blätter:

I. lanzettformig,

A. Ulte langettf.; Blbl.:

2. ganjuanoig,

a. eyr., aberlos; die fruchttrageno. 3820.

$\mathfrak{B} I m$ ft le bang. (4 45.) 
b. 3 ect. $=$ eyr., geab.; $3 B$ l. am (sirumbe 3821. $\not{q}$ fchmaler (4 38. 45. - ఛ (Sierb.) Hydrolapăthum.

2. ungeth.; $B$ I. am Ranbe wellenf., fipis 3822. $\Varangle$ (4 14.45. - ४ unfr.)

(crispus.) 3823.

3. gezahnt $(\odot$ NAm.)
B. mur D. obernlamzettf. : bie unt. herzf. persicarioídes.: lanjettform.; SBlbl. ganjramb., glectbbr.,

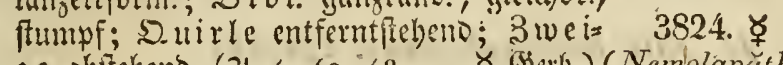

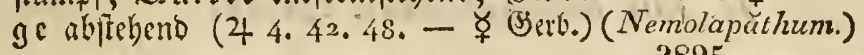
3925.

II. gleichbr.; ; 3 rbl. gegahint $(\odot 36 ; 48$. III. herłf. = lângl.; 3 I Ibl. gejabut; Blätter:

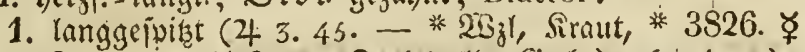

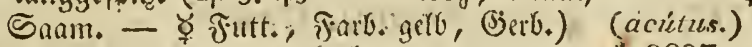

2. etwas ftumpf; feingeferbt (4 22. 45. - *3827. * $233^{\prime}$ )

3. gan ftumpf, fcinbaar. ( $\sigma^{\prime} S E$.) (obtusifolús.) 3828. divaricátus.

\section{b. nur Eins,}

\section{Blätter:}

1. Yanzettf.; $B$ rbl. róngl., ftumpi, ganzranb. * 3829 . $(4-* 23 ; 1)$ neinorósus.

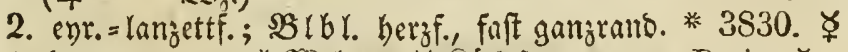

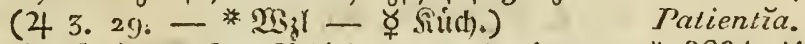

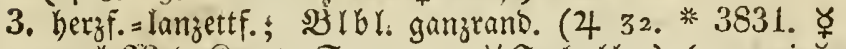

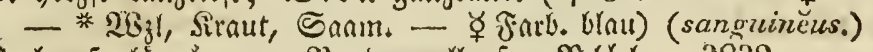

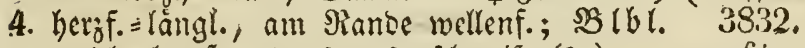
rund. = herz $\tilde{f}_{0}$, ctwoas ausgeichweift (4) confértus.

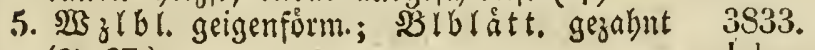
(4 SE.)

pulcher.

\section{Blbl. ohne e. Körnchen。}

1. bef. $3 \mathfrak{I m}$ ftre find), umgejalagen, oben 3834 . biffer; 3161 . gezahnt $(\odot S E$.)

2. Blätter: rus.

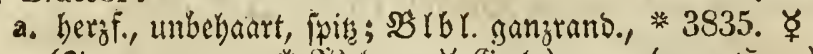
(4 35.42. - $333 l-\Varangle(5 \mathrm{crb}$.) (aquaticus.)

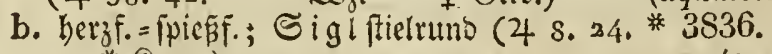
- * Sirnut)

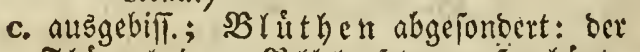

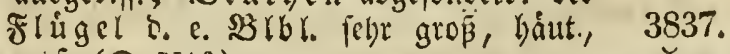

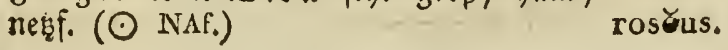




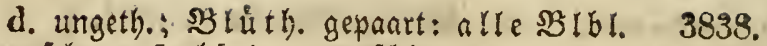

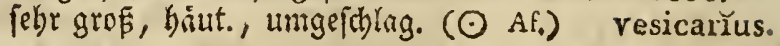

B. mit 2 Staubwegen.

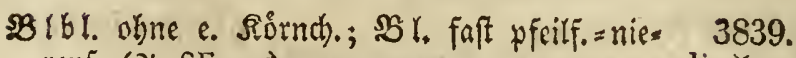
renf. (4 SE. 9.) digy̆aus.

II. mit halbgetrennt. Geschl.

W. Blo. 1 bråttr.: bie åu $\mathfrak{\beta}$. Błbr. umges 3840.

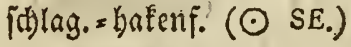
spinósuis.

III. mit ganzgetrennt. Geschl。

Blätter:

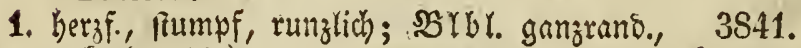
nacte (4 SE.)

2. lánglith = pfeilf. (4 34. - * 2.

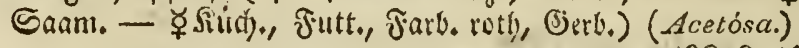

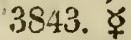

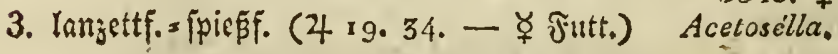

4. RUPPIA. 90.

(4 35.41.)
3844. maritžma.

\section{RUSCUS. 797. (40.)}

1. Mit 3witterbíth. in Enotraub. (ち SE. 3845. gbch. $\triangle$ )

II. mit ganjgetrenntem (5) efol.; bie Bl. tragen Bliithen:

1. Dberhalb,

a. unter e. bejonbern Bráttti). (ち SE. $\triangle * 3846$.

- Siraut) Hypoglóssum.

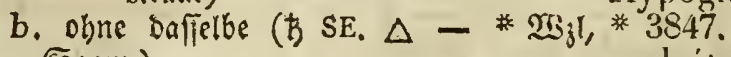
(sam.)

2. unterbalb ohne e. ber. SBlittch. (ち SE. * 3848 .

$\Delta-*$ Siratit)

Hypophýllum. 
10. RUTA. 343. $\left(812^{x}.\right)$

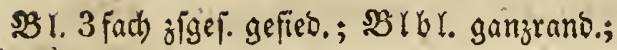
Blättchen:

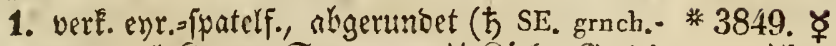
gb. - * Sitaut, ๔anm. - ఫ̧ Siúd., (jer\%.) graveŏlens.

2. gleid)br.; fpicis (ち SE. $\triangle$ ) 3850. montána.

\section{SAGÍNA. 88.}

Stengel:

1. mit nieserlieg., unbehaart. 3weig.; \$2lbr. 3851. ఫ్

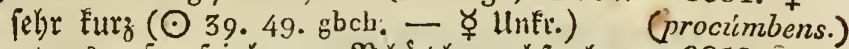

2. etmas aufr., feinhaer.; $\mathfrak{B}$ (uth. wechjoghe 3852. SB!kl. $(\odot$ 19. 49. w.) apetüla.

3. aufr., meift 1 blitt).; $B$ loblatt出. fpis; 3853 . sblbl. ungeth. $(\odot$ 23. w. $)$ (erécta.)

\section{SAGITTAPIA. 759. (W. 91.)}

Dic pfeiff. Blätter:

1. Ianzettf., liniggeipitigt, mit lanzettf., gerab:

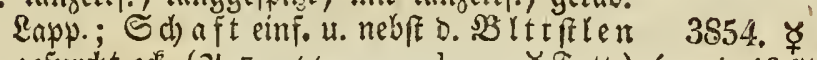

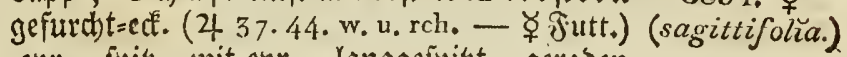

2. eyr., fpirs, mit enr., langgejpist., geraben

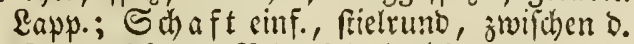
Sinoten 3Fant.; $\mathfrak{B}$ ltt fifle halbfiielrund, glatt (4 NAm.)

\section{5. latifolì:}

\section{SALICORNIA. 2. $\left(3^{x}{ }^{x}\right)$,}

\section{Stengel:}

1. fraud.), aufr.; (3rieb. b. ber. awcige

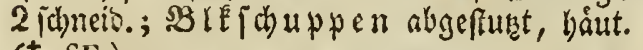
(5 SE.)

2. Erautartig,

a. etwas abfeheno; Crlieb. an ber Spise

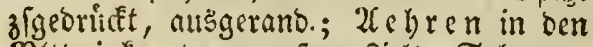

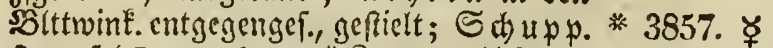

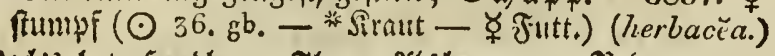

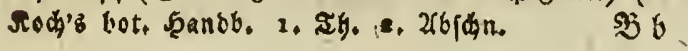


b. niescrlieg.; (5) lieb. verE. fegelf.; 3 we i= ge einf.; 2 ebren faft gleid)boch; mit 2 Stgef. $(\odot \times 7: 47$.

3858.

procúmbens.

\section{SALISBURYÁ. 747, (W: 34.)}

(5 As.)

3559.

adiantifolra.

\section{SALIX. 773. $\left(34^{3} \cdot 77^{3}\right.$. \\ $779^{2}$. W. 21.)}

\section{Mit 5 Staubgefäfsen.}

Bl. verf. eyr., langgeipiat, unter 8 . Mitte ets was zigrzog., gefägt, unbehaart; szitt ffte

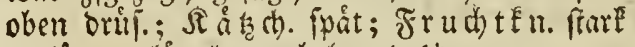
verlaing., lângl., untbehaart († 38. 43 . -

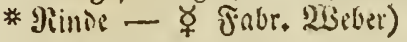

* 3860. 후 (pentíndra.)

\section{II. mit 4 Staubibgéfäfsen.}

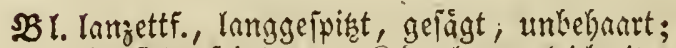

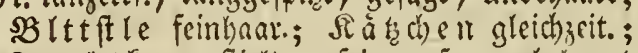
Frud tEn. geftielt, pfriemenf., nmbebant (ち. NAm.)

3861. nigra.

\section{III. mit 3 Staubgefäfsen.}

Fruchtknoten:

I. unbehaart; Blätter:

A. lanjettformig, gejugt,

1. unten weisgrun;

a. nit vermengt. Seichl.; $\mathfrak{B l}$ l. an

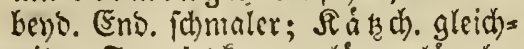

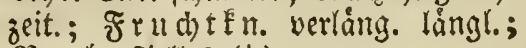
Rar . Fiellyos (b)

3862.

Hoppeána.

b. nit ganzgetienntem (5) eidl.; $\mathfrak{B l}$. am (S)runde ftumpf; $\mathfrak{F} r u c h t=$

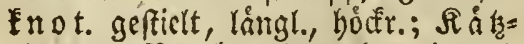

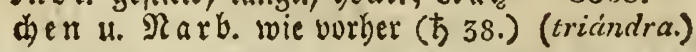


2. nid, ; $\mathfrak{B l}$. langgeppigt; $\mathfrak{F} \mathfrak{r} \mathfrak{d}) t \mathfrak{E} \mathfrak{n}$.

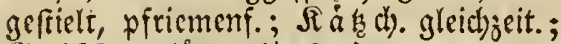
(S) $r$ iff. verlång. (

B. lång! = lanzetî., langgefpiat, unbehanrt;

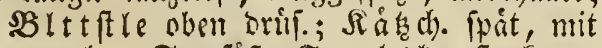

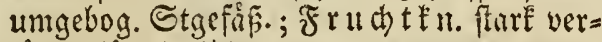
lång. lảngl. (5)

II. feinboar., gefticlt, verlång. lỏngtich; $\mathfrak{B}$ l. lanjettf., langgeipifat, am Girunce.ftumpf,

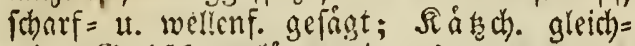
zeit.; (5) riff. verlang. (†) 38.)

\section{4. \\ Russeliána.}

3865.

(Meyeriána.)

\section{IV. mil 1 Staubgefäfs.}

Frudittu, bebaart; bie gefägt., unbe= hanrt. Blätter: -

1. verk. evr. =langettform.; (5i riff. felor furz; Narb. eyr. ( $b$ 38. Aeste braun-r.)

2. lanzettf., langgeipizt, feingeingt; (5iriff. verlang., favenf.; Jarb. gleichbr. (方 44 . Aeste g!änz, , aschgrau od, rch,-braun)

3867. (purpurĕa.)

\section{V. mit 2 Staubgefäfsein;}

A. Fruchtknot. unbelaart;

1. Bl. 3 od. mehrmal so lang als breit, lanzettf.

\section{Blätter :}

I, unbebaart, gefögt,

1. gleichgefigt, langgepisht, unten weipgriun; Si åt) (d). gleichjeit.; Fruchtkn.:

a. ftisllos, ftark verläng. lângl.; $\mathfrak{B} \backslash t t=$ ftiele etwas barr.; saaben ftiellos, 2 lapp.; 2 lefte eygerb, fpiegelglatt ( 38. - * Minde)

b. geftielt, eyrund; 2 efte hang. († As. Aeste oft bis zur Erde herabhang.)

2. id)arfgefígt, langgeipist, glínz., unten bleşrim; 2lfterbl. balbherzf., gefägt, abfall. (方 SE. Aeste braun, jüng. feirhaar.) lanceoláta.

3. ungl. = u. ftumpfgefägt, longgefpiat, tun= ten etmas weisgrun; Ŝ̉ ách. frubzeit.; 3872.

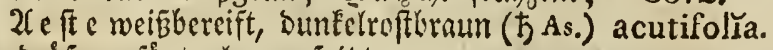
4. oru. = gefägt, langgefpibt, 
a. glcidfarb.; S it o. M. Blith. Dopp.; Flud)t not. foft ftiellos, ftrote vorláng. longlich (b) 48. Aeste braun, leicht zerbrechlich * Minse - ఫ farb. retf),

b. unten wcişgrim, breitlanjettf.; $\mathfrak{\Omega} \mathfrak{a}\{=$

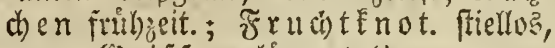
evr.; (Siriff. verlangert ( 5 38. Aeste dbraun, zerbrechl.)

II. bebaart,

1. faft jott., unten weisgrion, langl., lang= gefpict, gelágt; SBlttifle jott.; 2 f fter= blet t. halbberzf, geragt, abrall.; תap verlång. = evr.; Giriff. verláng. (古 NE. Aeste rostbraun, w. bereift)

*3873.

(fragilis.)

3874.

praccox.

2. oben feintianr, unten filz, runjl=abcr.,

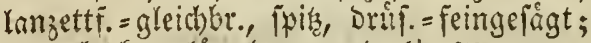

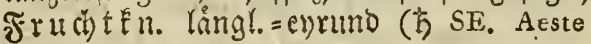
schw。-braun, zuw. gbch.-braun)

3. auf beyo. Geit. feinbaar. = feibenart., mit Ságezábnen, wowon sie unt. orillig

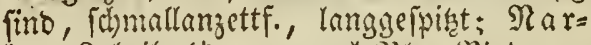
ben 2 theil. (5 27. - * $31 .$, אimbe ఫ̧ jarb. getb, roth)

3875.

cinerěa.

\section{Bl. längl., eyrund.}

Die unbebnart. Blätter:

A. geiågt,

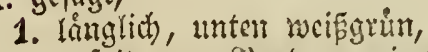

a. Ipit, an Stante gerwintp., in o. Nnitte gefigt, unbel)aart, oben glan!z., netif.=

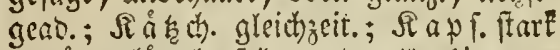
ver'lang. långl., feibenart.=jott. ( 5 Atste rch. - braun)

3876. riparìa.

b. an beyd. End. Fpis, am Rante um= gerollt, in ber sgitte etwas gefägt; Fruth te $n$. ftarkenertang. langl., unbe= bart ( 5 NE. Aeste gb.-braun)

3875.

formósa.

3577. ఫ্

(alba.)

3879.

Ginmarchica.

c. langgefpiçt, wellcnfogefingt, unbefant:

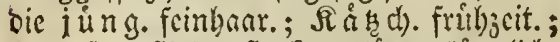

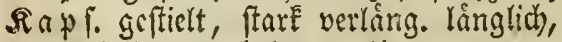
langgcipitit, unbegnart († NE. Aeste echwch. - braun)

3880.
phylicaefolia. 
2. oval, unbebant, unten weiggrim, an $\delta$. Epize etmả abgerumoct, gefigt; $2 B$ It $=$ ftle lang, feinbaar.; $\mathcal{A}$ iterbl. eyr., ge=

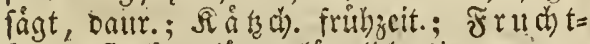
f not. ftart verláng. långlich (h. 9. Aeste dbraun, Aestchen feiuhaar.)

3881. Amaniána.

3. evr. =ounl, am Sirunde etros abgerumb. illecall feingefågt, unten unbebart, gläns.

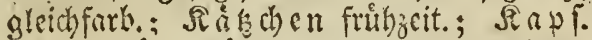
frarê verling. längl., jeibenart, = feinbaar. ( 5 SE. Aeste braun, liaar.)

4. evr., ipis, unbchant, unten rocipgrion; bie gejågt., baur. Afterbl,:

a. halbherzf.; $\mathfrak{B r}$. an (S)runde berzform.; SBratifiele febr furz, umbelyant;

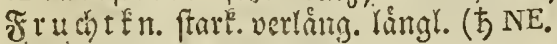
A'este dbraun, feinhaar.)

b. enr.; St. gefigt; Shltfle wie vor=

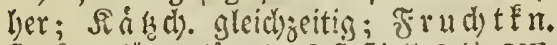
frare berläng. langl. faft ftiellos (ち NE. Aeste dbraun)

5. faft eltint. ob. vert. cyr. = oval, zutweilen furzgefpigt, gefigt, unuchaart, anten et=

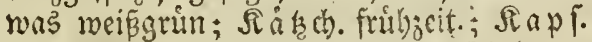
enr., furgeftielt, anfangs etwas feinbaar.,

nacbl)er unbebant (5 9. Aeste dbraun) IVulfeniána.

B. ganzrandig,

1. verE. eyr.=ellipt., frumpf; Stamm aufr. (5) Aeste gb. - braun)

2. vert. eyrums, ipic ob. ausgerano., oben

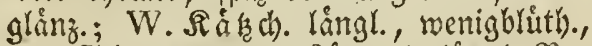

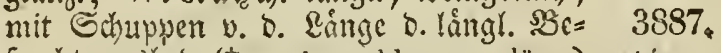
frud)tungsboo. († 9. Aeste hbraun, glänz.) retusa.

B. Fruchthnot. behaart;

\section{Bl. $3 \mathrm{mal}$ so lang als breit.}

Blätter :

I. Yanjettformig,

1. unbehant, gejagt, unten weipgruin; Frudtenot. geftielt, eyr., jeibenart.; $92 \pi r b$. 2 lapp., ohne Griff. (ち SE. Aeste r. braun, schlank, biegsam)

3882. myrsinítes,

3883. hastáta.

3884. serrulátą

3886.

refléxa,

3885.

2. bebant,

a. oben unbebaart, unten fcioenart., 


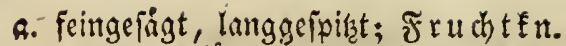
feidenart., långl.; $\mathfrak{A} a r b$ obne Sriff. (ち NAm. Aeste braun, ijüngere wcb. feinhaar.)

b. gamzrano., an beyb. Eno. Fanmaler,

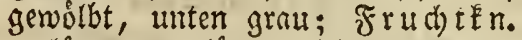
verláng. eyr. = rîngl., jeibenart., ge=

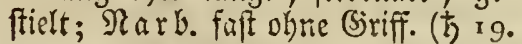
48. Aeste hbraun, Aestchen rch.-gb.) (incubacěa.)

b. oben faft unbebaart, unten fehr meichilit?.

3889. grisěa.

u. rungl. aber., langgefpist, an o. Spise

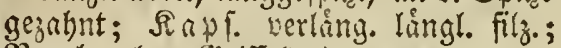
$\mathfrak{R a r b}$. ohne (Sriffel (⿳亠口冋丁. 58 .)

II. lănglo = lanjettf., meitliaift. gelight, oben un=

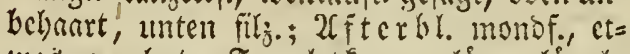
was gezalint; $\mathfrak{F}$ ructhten. verläng. längl., zott.; (S r iff. verlíng. (ち NAm. Aeste dbraun) IIl. ichmallamjettf.,

1. unbehaart, fpici, feingefingt ob. ganzrand., gleichfarb.; Stfáden anfangs bis zur

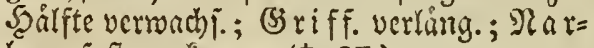
ben foft verfe. eyr. († SE.)

2. befaart,

a. inten felyr zartfilz., grim, fait gam

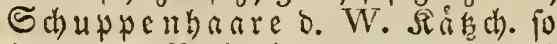

lamg als \$. Narbe (ち) 38. Aeste weichfilz.) mollissima:

b. oben fcinbaar., unten jeiscrnat., [pits,

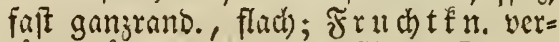
läng. länglidy, zott., geftielt; (S) riff. verlång. (5 19, 46. Aeste hbraun)

c. obén feithaar., unter bideffilz., felhr lang, an D. Spitze unbeutl. feingezalint; $2 f$ ferbl. lanzettf., faft jo lang ais 8 . BBlttfft ( $\hbar$ NAm. Aste r. •braun, an der Spitze mit e. sich ahlösend. Filze)

IV. Ianzettf. = gleidhbr., fehr laurg, faft ganz= rano., unter weisfeibenart.; 2f efte ruthenf. (万 38.)

3891.

holoserič̌a.

\section{2. \\ conifĕra:}

3893.

rubra.
3894. 


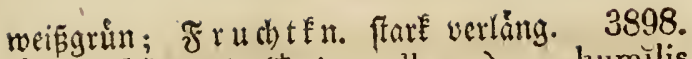
laitgl., feioenart. (†) Aeste dbrạun) l̦umilis.

2. oval, ipit, unter weipgruin,

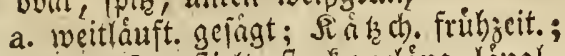
Sapl. geftielt, ftart verlang. lämgl., langgefpigt, feibenart. ( $ち$ SE. Aeste ochwch, braun, brüchig)

3899. nigrĭcans:

b. weitlåuft. = u. Feingciägt: bie jịng.

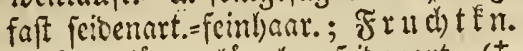
fark verlång. lángl., jeitenart. (ち) $\mathrm{SE}$. Aeste dbraun, etwas brüchig)

c. am Sirunbe ganzrano, gegen o. Spike gezahnt, unten ctwas weifigriin;

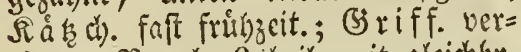
lang.; $\mathfrak{i}$ r b. 2 theil. mit gleichbr. \&apy. (ち 9. Aeste braun, glänz.)

3901. Wִ eigeliána.

B. ganzrandig,

1. verk. enr. = läng!, fpí, zuweilen an b. D)itte ftumpfige Fru cht tétr. zott. (ち) A este r. - braun) violaç̌a.

2. Faft Ereisrund, oocr ellipt. = freizruno, meift unbehaat, unteit neş. = aber.;

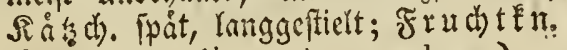

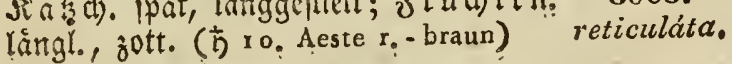

II. behaart,

A. lángl. = lanzettf.,

1. auf beyd. Scit. Feibenatt., ganzranto., ftumpf; obne 2(Fterbl.; Sapf. ebrund = längl., zott,, ftiellos ( $5 \mathrm{SE}$. Aeste braun, glänz.)

3904: sericěa.

2. unten filgig,
a. ipis, and Sirumoe ftumpf, oben grum, rungl.=aber., feinbaar.; 2afterbl.

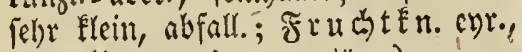 jott. (ち Aeste braun, glänz.)
3905. leucophýlla.

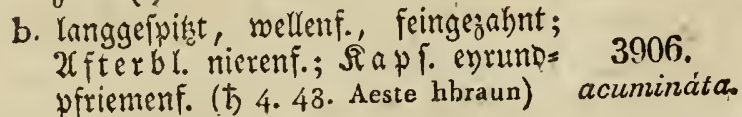

B. oval = langettformig,

1. oben unbehaart, unter feibenart. = fil an bevoen Enden fowmaler, fpis, fait ganzrand.; 2 f terbl. lanzettf. =pfrie=

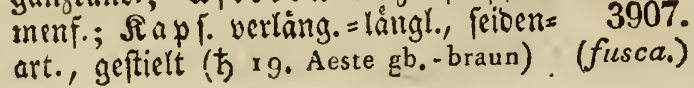


2. oben feinhaar., unten foft jeibenart.; 1 tm.

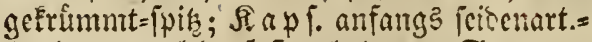
feinhaar., nachlyer faft unbehaart; S $t$ a m m niebergcoructet, afterblattloz (h r. Aeste dbraun)

C. ganz oval, ganzrano., umgefrummt = fyis, oben feingaar., unten feibenart., filberweiß̈;

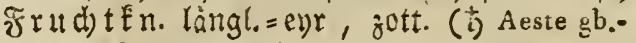
braun, dichtstehend)

3908. (repens.)

D. eyr., langgeipicet, geiägt, wellenf., unten filz.; 2ffterbl. faft monof.; Anpi. bauth. (ち 1 . - ఫ jarb. ifjwary, Gab.)

E. verk. enr. = oval, ipif, oben feinhaar., grü, unten etwas baar, meisgurin; 2 f ferbl. rumbl., halbherž., gezahnt; Fruchten. ges fticlt, werläng. lăngl., jott.; Rarben obne (Siriff. ( 5 4. 26. 49. Aeste braun, gegen die Spitze wch, - feinhaar.)

3911:

aquatica:

F. verf. eyuttmo, oben grim, feinbacr., unten runzl. = aber., umgetrummt = Eurjipipižs.,

1. etroas gejágt, am Sirunoe ichmaler, fta= d)elipit., unten grau=weid faar.; 2ffter= blätt. Galbăerzf., faft gangrand.; Narb. ofne (Srifi. (5 4. 48. Aeste braun, oben feinhaar.)

3912.

(aurita.)

2. gegen bic Spife mellenf. =gezahnt, unten graujott.; 2 f f terbl. balbhergf., gezahnt; a a p . verläng. lángl., geftielt; Marb. obne Briff. (b)

3913.

uliginŏsa:

G. Ianjett $\bar{\sigma}_{.}=$vert. eyr., ungetrimmt=langge= jpiat, gegen $\delta$. Spike geiägt, ofen feinbaar.,

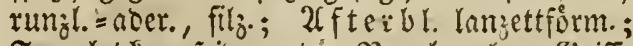

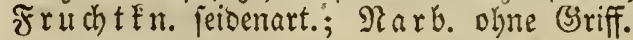
(5 Aeste braun, nach oben wch. - feinhaar.)

3914.

spathuláta:

H. runol. = verf. eyr., am Finunbe etwas herzf., umgefrimmt = furjgejpist, ftachelipifo, wel= lenf. gezabnt, oben feinbaar., grint, unten rumbleaber., graufeingaar.; 2 fter bl. fobief=

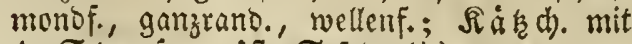
1. Stgef. u. ât. Stfad. (ち)

3915.

(cladostémma.) 


\section{SALSǑLA. 236.}

B 1. pfriemenf., fharfgeipiat=oorn.; 3 ro. nact) Der sBlithe attswentig crweitert in 5 tro= dinc, flache Fortsätze:

1. gefarbt; BB Is. cingelin; Stgl. ftadhelhaar. $(\odot$,6.36. grn. $-\forall$ potnifhe)

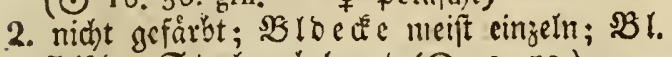
fleifd. ; Etgl unbegaart $(\odot 28.38$.)

3916. ఫ్

(Kali.)

3917.

Tragus.

\section{SALVIัA. 28. (34:.)}

\section{Bld. 3 lapp., erweitert.}

\section{Blätter:}

1. enr. =lanjettf., runjl., feingeferbt = am $\Re a n$ te wellenf.; SBIb. ftumpfáabn., lång. als bie eyr. Neôenbl. († As. hbl., mit e. w. Yleck auf d. Ulippe)

2. gefied., ganzrand.: B råt tch. lanjettf., faft

3918. pomiferra. 3919. Habliziána.

\section{Bld. 3 zahn., meist walzenf.}

\section{Blätter :}

1. YanjettF., ftumpi, entferntgefägt, unten feine haar., geftieit; $2 \mathcal{A}$ eb re traubenf., in b. $2(f \mathrm{t}=$ mine.; Suitle 2 bluth.; Rebenbl. lans jettf., fo lang. als o. SBimftl $(\odot-b$ bl.) lanceoláta.

2. laingl.=cyr., gekerbt; $\mathfrak{B}$ lu the $\mathrm{en}$ in abrenf.

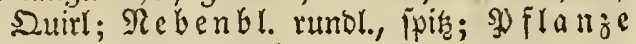
raud $)(\odot$ SAm. bl.)

3921.

hirsúta.

3. eyr., gefägt; B̊lttffle auf benben Seiten

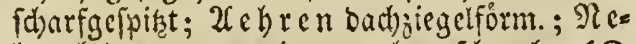
benbr. cy)r., gerwimp., oben febmaler $(\odot$ SE. hbl. - $\forall$ D (I)

4. herzfórmig; Bld.:

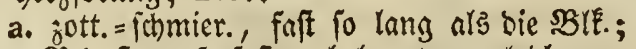
$S_{\mathcal{B}}$ r. ftumtpF, faft unbehaart, ungleid $=$ u. ftumpfgefägt (As. blch.)

b. faft umbehaart; $\mathfrak{B} \mathfrak{l}$. runzlich, geferbt= gleid)gefígt, ipit (4 SAus. bl., snit 2 w. Strichen)

3922. ఫ hispanı̌ca.
3923.
serotĩna.

3924. tilia eforía. 
5. herzf. = pfeilfónm., grobgelägt, langgeipicht;

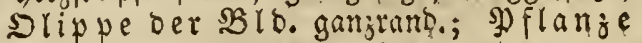
jott. = fchmiet. (4 SE. ugb.)

\section{II. Bld. 5zahn.: Olippe meist$$
\text { 3-, Ulippe } 2 \text { zahn.; }
$$

A. Bl. ungetheiltu.

1. am Grunde schmaler.

\section{Blätter;}

1. 伤mallanzettförmig;

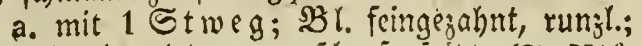
Rebenbl. ent., icharfgeipigt (4 NAf, $w_{0}$; ugb. . mit v. Punct. . kleın)

b. mit 2 ङ twegen; $B$ ro. tief 2 theil. ( As, hbl,)

2. langettf: =enr., feingeferbt: 2 uirle wenig= blittl).: Sie unt. blattr.: bie obern neben=

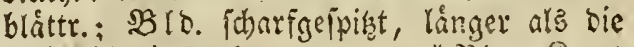
Rebenbl. (ち SE. b!.; w., r. - * Blm., Siraut - $\Varangle(5 i 26 .$, Silid).)

3. eyr = lingl., gezahnt, am shlttift herablauf.; sy franje zott. = iümier. (4 NAm. bl.)

3926. aegyptiăca. 3927. cretrca.

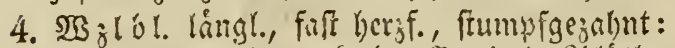
Etgibl. gejaint=geterbit; Suite 6hlitt): B luth. borizontal: Enoblmft 16lith. (4 As. b].; r.)

\section{* 3928. ఫ officinális.} 3929. urtica efolıa.

2. am Grunde herzförm.;

a. Nebenbl. kleiner als d. Bld. u. welkend.

\section{Blätter:}

A: ganj herzforermig,

1. geferbt = gezahnt; Suirle faft nadet, faft 1. peit.; (S) riff. auf o. Ulippe D. SBlE. lieg. (4 SE. rch.)

2. feingckerlt, unten grau; Stgl molletrag.;

bie obern Szuirle gebåft, ftiellos;

slippe b. SBIE. abgeturgt ( - bl.) crassifolla.

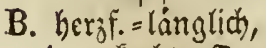

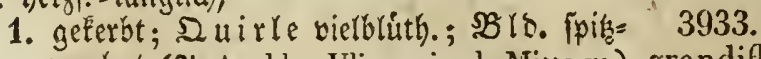
gejannt (4 As. bl., Ulippe in d. Mitte w.) grandiflóra. 
2. geferbt ob. jerfidnitt.: bie oberften $u m=$ faff.; SR e benbl. faft jo lang als şlo.; BlEhelm fit)elf., fathier., lang. als b.

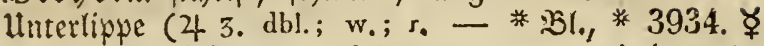
Sram. - ఫ Jutt., IInfr.)

3. gezahnt=geferbt, rum; l.: Sglbl. geftielt;

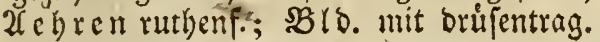
Saar. (4 SE. bl.)

4. feimgeferbt, rumjl., etwas fpis; $\mathfrak{R}$ e ben= blát ter herzform. = runol., langgelpist; Sg filange zott. = fit)micr. (4 SE, wo u. dr. u. hr.; w.)

5. altsggbillen, bucht.; Stgl faft blattlos; Suirle jebr jott.; Stgef. jegr lang (4 ugb., mit v. drüs. Haar.)

3935.

variegáta.
3936. viscósa.

C. längl. = Gergf.; Stgl gan; cini., faft blatt=

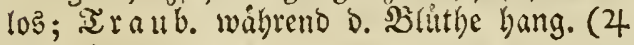
$S E$. v.)

D. herif $\tilde{F}_{0}=$ lonjettformig,

1. ungleibgeterbt,

a. barbumfafl.; Rebenbl. berzf., lang=

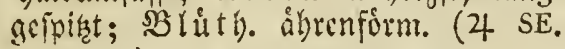
blch.; w.)

b. nicht, ater mit am (Brunbe gectlofi. SBucht; Stglbl. faft fticllos; Stg! 4 feit.; D. zigefeste Endtrauk. Faft nact, ubergebog.; Nebenbl. gefirbt, gemimp. ( $4 \mathrm{NE}$.)

2. gleichgekerbt, mit ant Grunde offenter Sutht; Stgl 4 fant.; פ. einf. (5no= traube vor ber Blitthe uberbang.; Te= benbl. wie vorber $\left(4-v_{0}\right)$

3939. amplexica' ulis.

\section{b. Nebenbl. gröfs, als d. Bld. u. daur.}

\section{Nebenblätter:}

I. gefårbt; Blätter:

1. her ff., rumzl., bopy. gefägt; Nebenbl. furr. als D. SBlitth., langgeipiat; Şanre an Stgl u. $\mathfrak{B}$ lo. einf. (4 29. 3 I. b.; rch.; w., Nebenbl. p.)

3940. betonicaefolia.
3941. pendŭla.

2. berz $f_{0}=$ långl., runzl., zott., gelågt $\left(\sigma^{\top}-3943\right.$. wch.- blch., starkriech. - * điraut)

3942.

(syluéstris.) Sclarěa. 
3. ftumpf, geterbt; bic oberitcn 27 cbc $n=$ blátt. unfruchtb., gro̊á., gefáb́t (○ SE. bl.; r., Nebenbl, v, od, ros. u. grn.)

3944

Horminum.

II. nicht gefärht; Blätter:

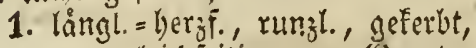

a. ungleicheitig, am (S)runde mit e. $2 \mathrm{fn}=$ bang an o. Butbten (4 Af. bl.)

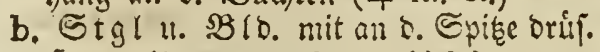
Scant. (4 As. w ; hros.; blch.)

2. hersf. = langl., bopp. geferut, etras aus=

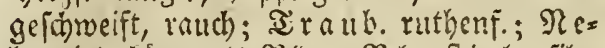
benbl. Eury. als Bro, ; S r mftiele filz. (4 SE. bI.)

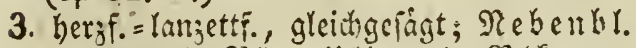
fo lang als SBlo.; Ulipp. o. SRlE. um= geliblag. ( $4=9$. bl.; ros.; w.)

4. lánglich,

a. ausgcichneift; NG ID. Dorn.; Nebenbl.

berzf, fcharfgeipict, hobl ( $\odot$ ơ NAf. w.) spinósa.

b. ausgchifi. uno nebit o. 2 u irl. moul.; Rebenbl. umgefrummt, etrab born. $\left(\sigma^{\pi} 11, w, u . c h.\right)$

e. ftumpf, gleichgeferbt, geftielt: $\mathfrak{B} \mathfrak{l}$. a $\mathfrak{n}$ b. SBluth. umiall.; oie unt. Duirle entfernter; $B$ l G belm ftumpf, verling.;

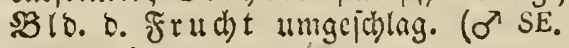
ho, u. v.)

3950.

Aethiöpia.

$39+5$

3946.

virgáta.

3947.

campéstris.

3948.

(nemorósa.)

3949.

Aechiogia.

3951:

virùdis.

B. buchtigr, getheilt.

Blätter:

I. ungleidfórmig,

1. Die unt. leyerf.: Die oberften hergf.; Blůth. im Szuitl; $B$ lo. (d)arfgerpist, gemimp. (4 dr.- vo $_{0}$ )

2. bie unt. fipiesf,: einige lencrform.: die ibrigen berzf., autgeichweift=gezalynt; SUlirle faft nact; S Slippe D. SBIE. furzer, herzf., geránd.; B3lo. ctwas abs fteheno (4 SE. lila)

$3 \mathfrak{2 S z}_{3} \mathrm{lbl}$. berzf., handform. of. ungeth.: Stglbl. pfeilf =lanjettf., ungleid)gezalint; Rebenbl. umgefolag., Eur. als לे. uber= bang., mit Drüj. Scaar. verfeben. SBlocfe (4 NAf, Helm bl.v., mit gb, Punct., Ulippew.) bicŏlor.

3952.

abyssinica:

3953. napifolra. 
II. gleidüörmig,

1. buchtig,

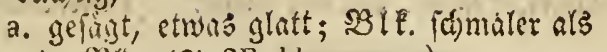

D. sSis. ( 4 SE. bl.; r.; w.) -

3955.

Verbenăca.

b. geferbt = gejabnt, ecig; Szld jabne cornt, mit in D. Eten u. am Nande gewints. Estumo (4 NAf. bl.)

2. fait 3 laps., geflielt, rumbl, mit long

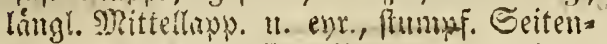
lapp.; Ofianje filz. (节As. r. -v. $\triangle$ ) triloba.

3. $\mathfrak{2 B}_{3}$ lb l. lever furis; Stgi foft blattlos, mit richworte. ftebeno. Sgaar. (4 N.tm. p.-r.)

3956. nilotica.

3957.
trilŏba.

3958. lyráta: :

\section{SAMBÚCUS. 240.}

SBl. gefieiert;

I. mit Erautart. Etgl; $\$ \mathfrak{b}$ lathen in 3 their.

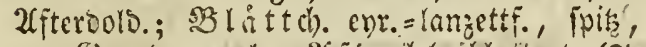
am Girurbe ungl.; 2lfterbl. blattart. (4

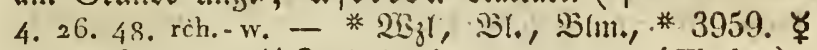
Beer., Simoe - ఫ Farb. (taul)

II. mit framch. Stgl; Bliithen:

(Elülus.)

1. in 5 theil. 2fftero old.; SBl. auch sopp.

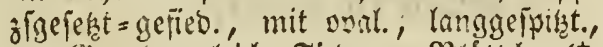
am (Sirunoe gleich. Fico. It. Blátth. († NAm. gbch.. w.)

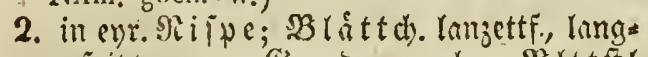
geipitat, ant Grunbe ungl.; SBlttift frachelhant. (点 NAm, uw, $\triangle$ )

III. mit butmart. Stamm; Bliithen:

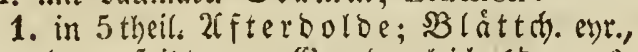
langgeipist, am (sirunde gleich) ( 5 4. 26. w. - * 21., B3m., Beer., Shinie ఫ̧ Fribte, Dil, Farb. Glaul)

2. in evr. Rifpe; $B l$ lattch. linglid) ober längl. = langctt., langgeipilat, am Sirunde ungleid); $\mathfrak{B}$ It tfit lunbebaart $(\hbar-$ gbch.) (racemósa.)

(nigra.)

\section{0. \\ canadénsis.}

\section{SAMŎLUS. 144.}

Stgl aufr.; $\$ B l$ fämmtl. verḱ. eyr. od. längl., fumpf, båut, ; $\mathfrak{x} \mathfrak{a} u$ b. boloentraubenfórm.; 
bсі. $2 \mathbb{l}[\mathrm{mft} / \mathrm{c}$ in D. Nitte mit Rebenbl.; 3964. Skapi. faft fugel. (4 36. 40. w., klein) (Valerándi.)

13. SANGUINARIA. 419.

(4 NAm. Saft gb.)

3965. canadénsis.

\section{SANGUISÓRBA. 61.}

Aehren:

1. evr.; Stgef. faft io lang als o. SBlfrone;

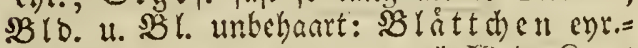
faft berji. (4 3.48. dr. - * ISijl, Sirant

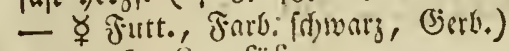

2. waljenf.; Stgefälse:

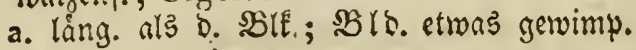 (4 NAm. r.)
b. vielfach langer; 2 ehren fehr lang (4 NAm. wch.-r., Bld. w.-grn.) medřa. 3968. canadénsis.

* 3966. ६
(officinális.)

\section{SANICǓLA. 181 .}

Blätter:

1. Fåmmtl. fingerf., mit längl., jerid)nitt. =ge=

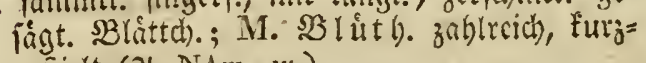
geificit (4 NAm. w.)

2. sie unt. Ganbf, mit 3 part., jeridnitten=

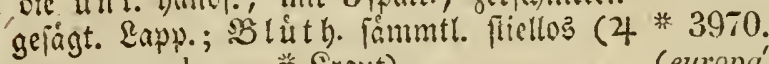
5. 8. $w_{0}$; rch. - * Siraut)

3969.
marylandica.

\section{SANTOLINA. 646.}

Brittb. einjeln, am Enoe ftebeno; $B$ l. 4 reibiggezahnt; Aeste:

1. filz̧̧⿻;丨子 Blattzähne:

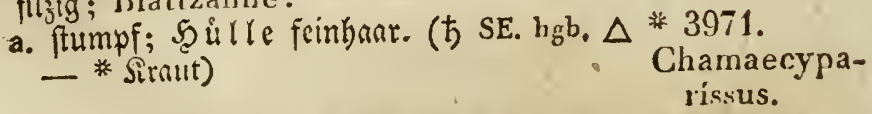

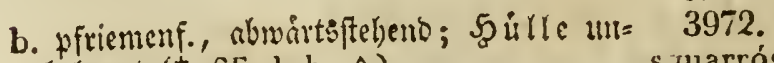 bebart ( $\mathrm{G}$ SE. hgb. $\triangle$ ) s.juarrósá. 


\section{Santolina. - Satureja,}

2. unbehanrt: $B$ latt $z$ a h ne pfriemenf, gerade; Şưlle wie vorker (ち SE. bgb. $\Delta$ )

3973. virĩdis.

\section{SANVITALIัA. 666.}

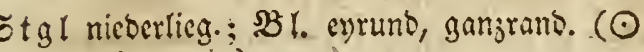
SAm.gb. u. dr.)

3974. procúmbens.

\section{SAPONARIA. 349.}

Bldecke:

1. maljenfirmig,

a. unbehaart; B1. eyr.=lanzettf. (4 - w.. * 3975. rch'. - * wist, Siraut)

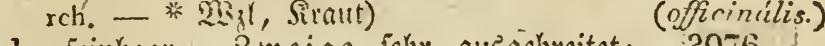

b. feinfarr; 3weige febr ausgebreitet; 3976 . Frúchte hang. ( $\odot$ As. rch.) porřgens.

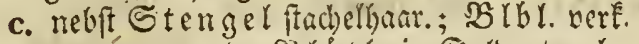

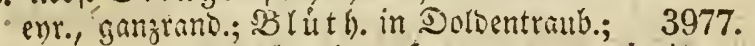
$\mathfrak{B l}$. id)maflanzettf., rinnenf. (4 SE. 9.) lutěa.

d. brúf.: Gaar.; Etgl gróelf., nicoerlieg.;

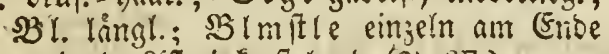

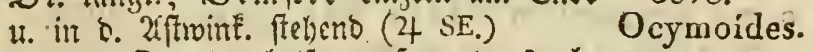

e. baar.; Stgl gabelf., aufr., etwas ab= fteheno:; Sto langl. = lamettf.; SBlniftle in o. 2ffrwint. $(\odot-$ p.)

2. pyramiocnF., 5 feit.; 33 1. cur., langgeppist; 3980. ఫ

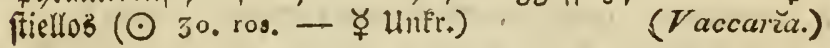

\section{SARPACENIA. 417.}

\section{Blätter:}

1. rơbr., aufr., mit ant Shnlie zigezog. u. an 3981. b. Epize flach., aufr. Schate (4 NAm, gb.) Hava.

2. Eappenf., bauch., etwas abftehent, gefrủmmt 3982. (4 NAm, rch.) purpurĕa.

\section{SATURÉIA. 458.}

SBImftre afterborbenf., in D. sBittroint.; Bl.: 1. gezalunt, runtol. =aufi., am Girunde fämas

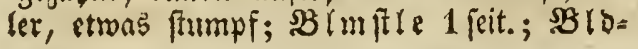


a bidnitte fumpf, obne Etadetpige (b, 3983. 23. hv.) rupéstris.

2. ganjranoig,

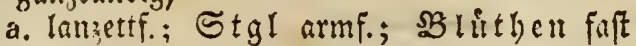
afterdolienf. (๑ SE. rch., Blätt, staskriech. - Siraut - ఫ (berb.)

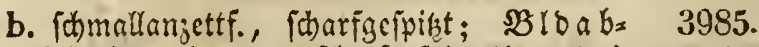

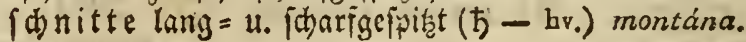

\section{SAXIFRĂGA. 353.}

\section{Blätter ungetheilt;}

A. Stengel ganz blattlos:

Blätter:

1. lanjettiórmig;

a. mit 2 blưtl. Stgl; \$Sl. fumpf, haar. 3986. (4 9. wch.)

b. nuit gefticlt. SBlütbe; B̉latt. gehåuft, rechi. u. entgegenger., falt lanjettf. (4 9. sbch.)

androsacěa:

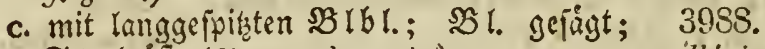

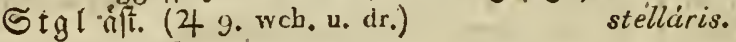

2. långl. = lanjettí.,

a.:etwas haar., feingegahnt; $32(\mathrm{~m}$ ft le wedhr., 3989. boldentraubenf. = rnopff. (4 N.Am. grn.) pensylvanica.

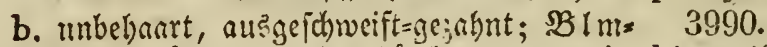
ftle 1 bluth)., wed)i. gebouit (4 SE. 9.) hieracifolia.

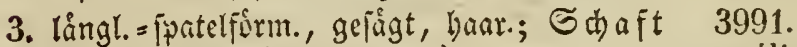
rijpenf., haar. (4 NAm. w.) vernális.

4. ellipt., ftumpt, ant (Sirumbe feilf., groós $=11$. fumpfgejahnt; SBlttfile gewimp:; Stgl rifpeni. (4 SE.)

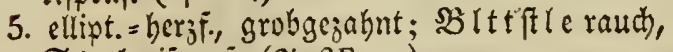
Stgl rippenf. (4 SE. J.)

3992. umbrósa. 3993. hirsúta.

6. enr., an b. Spite cingeoruffet, unoeutl. ge= jâgt, geftielt; Rijpe jigefnauelt (4 NAs. 9. r.)

7. gleidher., burchbol)rt = punct., gcbaltft, ums gctrummt; Stgl vielblúth). (4 9. w.) caesia.

8. Feilf., febl fumpf, ausgefhiveijt; Stgl 3996. ripeni. ( 4 9. w, u. och.) cuneifolia. 
9. nierenf., grobgezahnt, auf beyb. Seit. nebft 3997:

D. B Ittiften raud); Stgl ripenf. (4 9.w.) Geum.

B. Stglfast blattlos

Blättch.:

1. lanzettf., forarfgeisitzt,

a: am Grunbe geroimp., Dachiegelf.; Stgl 3998.

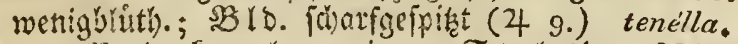

t. am Stande froipl., gemimp.; Stgl noie 3999. verber; SSID. ftumpf (4 9. gb. u. r.) bryoides;

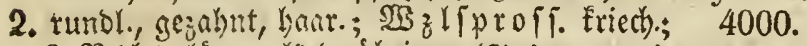

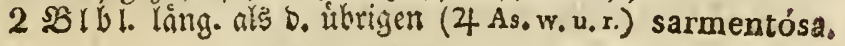

\section{C. $\operatorname{sig} l$ blättrigg。}

1. Wzlblätter:

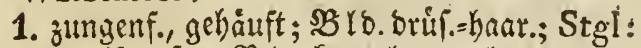

a. ripenf.; $\$ B \mathfrak{l}$ l. Enorpl. = gezabnt (49. w.; w. u. r.)

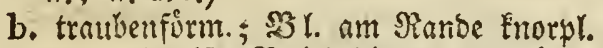

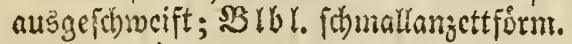
(4 9. gb.)

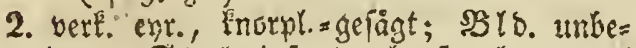
hart; Etgl cinf., traubenf., oben etwas baar. =[(t)micr. (4 9.)

3. lanottf = yert. ctur., fnorpl. gejägt, ges

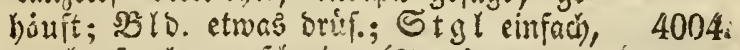
traubenf., baat.=fämier. $(4$ 9.) intácta.

II. Stglblätter:

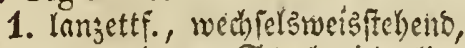

a. gemimp.; Stg! nicoerlieg. (4 SE. 9, 4005 . hr.; wch. u. gb.) aspĕra.

b. nidt gervimpert; Stgl aufr. (4 42. 4006 . gh. u. r.)

2. eyt., entgegenger., sathziegelf.: $\delta_{0}$ obet ften gevimp. (24 SE. 9.23. dbl.; rch.) oppositifolia.

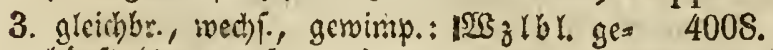
biuft ( 4 49. gb. u. r.)

Cotylédors:

4002.

mutáta.

(Hircüllus.)

4007.

autumnális.

4. gleidbbr.=pfriemenf., jerfteit, nad, webr= 4009 . los; St gl nietertieg. (4 9: gb:) Aizoídes:

5. nierenf., gezalnt, geftielt; Stgl rifpenf. 4010 . (4 9. w, r. gefleckt)

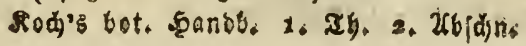
rotundifolia. G $\dot{c}$ 
11. Blätier gelappt,

A. gleichfürmig.

Stengelblätter:

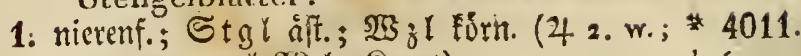
w. u. grn. - $* 2 E_{3} I$, Sirnut) † (granuláta.)

2. Feilfơrmig,

a. 3 lapp., gejafnt, brifi. = raudh; sBIm fitle 1 brutt)., feltr lang; Stgl auffteig., am (5rumbe aft. $(\odot \%)$

b. 3 fipalt.; wedbf.; Stgl aufrecht, áft. $(\odot$ 9. $w_{0}$ )

c. Gallof. = 5 theil., firirz. als ber gleidgbr, ge= mimp. SBlttel; D. bluthetrag. Stgt. aufficig., âft.: bie itbrigen weitichmeif., 4014. bichtgeorángt (4 3.)

d. hantof. $=5$ fpartig,

G. gewimp., laing. als ber gleidtbrt. SBrtt= fitel; o. blutbetrag. Stgl allffeig., einf: : Dic úbrigén jefri kurz, Dichtge= brangt (4)

b. zott., foft v. D. Range D. glcichbr. SBltt= fticls; $\mathrm{D}$. b h luthetrag. Stgl aufireig., aft.: bie it brigen weitfátweif.=bid)tge= brangt (4 $\mathrm{SE}_{\text {. }}$ )

(adscéndens.)

4012.

rupéstris.

4013.

(Tridactylites.)

4015.

Sternbargü.

4016.

villósa.

\section{B. ungleichförmig:}

I. Stengelblätter:

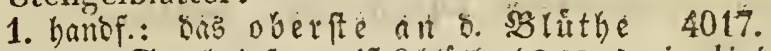
eyr.; Stgl einf., meift 2 blitth. ( $\odot$ NE.) rivularís.

2. gleichbr.: Wzlbl.:

a. niercnf., 5 lapp., vielipalt.; Stgl faft 4018. natt', aft. (4 $\mathrm{SE}$. w.)

b. hanoform. =5 theil.; Stglbl. ungeth; 4019 . Stgl alfifteig., vielbeith). (4 SE.)

3. gleidbreit: cinige ungetbeilt: andere 3 fpalt.; $\mathbb{I}_{3}$ gliprofi. nicoerlieg.; $E$ tgl aufr.; faft nacedt (2t 9. uw.)

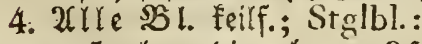

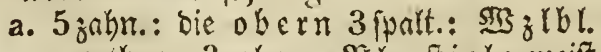

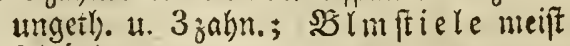
3 bituth. $(\odot 9$. w.)

ajugifolía.

b. 3 theit $=$ gleithbr.=fabenf. : bie itbrigen furr. als D. SBlttftl, 3̈theil., mit gleid $=$

4020.

hypnoides. 
breit., 3 ipalt. Rapp.; Stgl cinf., auf= 4022. II. Wzlblätter:

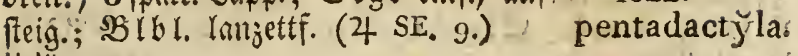

1. gleidbbeit, geháallt,

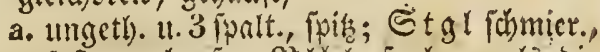
faft traubenf.; 3 rtbl. fo lang als bie 4023. \$Blo. (4 9.) moscháta.

b. 3 palt. gerientitt, frumpf; Stenger

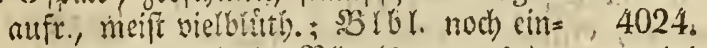
mal io lang ats b. SBlo. (4 1 o, gb.) caéspitósa

2. kingl., frumpt, ungeth). u. 3 fpolt.; Stgl

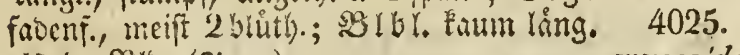
als b. Şlb. (4 9.) , is

\section{SCABIÓSA. 56 .}

\section{Blboden mit Spreublättchen;}

A. Bld. dachzieggelförmig;

1. bes. Blk. 4 spaltig;

a. Bl. gleichlförmig.

Die bes. BIkronen:

1. gleichfurmig; Blätter:

a. gefics.: sh 1 at the. Yamzettf., herabramf.,

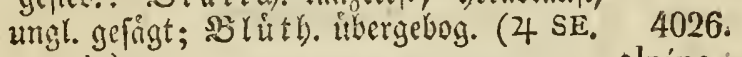

9. ugb.) alpína.

b. lanjettf.; :Bro. u. Spreubl. begrant; 4027.

Etgl gabelf. (4 As. w.) syriăca.

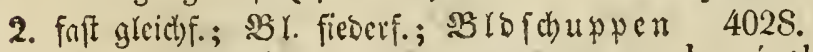
cyi. (4 $\mathrm{SE}_{0}$ w.)

3. geftrablt; B 3 l. lamette, firoerf., mit faft 4029. Dact)sicgelf. Rapp.; Stgl bractr. (4 NAs. gb.) tatarǐca.

\section{b. B3. zungleichförmig。}

Die bes. Blkronen:

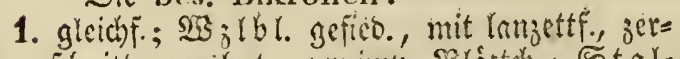

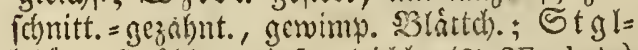

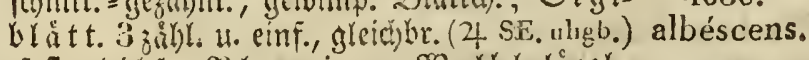

2. faft glcichf.; $\$ 3$ r. gewinus.: $\$ 73$ z 1 b l. lingl., ganjrano.: bie unt. Etglbl. fiederf. (4 SE. hgb.)

4031. centauroídes. 
3. geftrablt; Blb: นt. Spreubl. begrant;

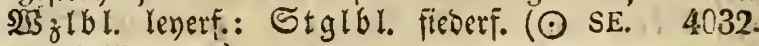
bl, od. lila u. w.)

transsylvanYca.

2. bes. Blk. $5 \mathrm{spalt.,} \mathrm{ungl.}$

\$Bl. fieberf.; Stgl frautart., aufr.; ऽ. bes grannt u. mit e. feser. Sfrone; Spreub! oben breiter, -begramt, ftadhelban. ( $\odot \mathrm{SE}$. wch.)

4033.

pappósa:

B. Bld. nicht dachziegelf., vielblättrig;

1. bes. Blk. $4 \mathrm{spaltig}$

Sie bes. Blkronen:

1. faft gleich); Blätter:

a. gleibf., lanzettF., ganzrans.; MYo. Furso.

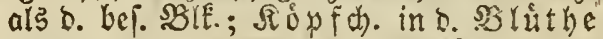
Fugel.: zur aeit b. Frud t lang! = cyr. (4 42, gb.)

b. ungleid) f: : Stglbl. gattgrand., faft ftic!= los: MJatblatt. gejabnt, gefficlt: Xlle långl.; Stgl gatelf.; Sis $\$$ f Srocigewint. Frift ficlloz: o. an Enbe ftehenoen geftielt ( $\odot$ SE.)

2. ganj gleich; Stgl einf., mit angenélyert. 3̂meig.; $\Omega \dot{\delta} \uparrow f \mathrm{~d})$. Eugel.; Bl.:

a. lanzettf.=eyr., feinbaar.: $5 \pm$ glb l. glcich $=$ breit, faft gamgranto; vef. 38 10. 4 borft.; Spreubl. fo lang als SBld.; S. Gaar.

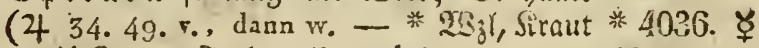

구 अutt., Fart. geto, grim)

b. långl., urbehant: Stglbl. lanzettf, am (S)unte tiefjerfdyitt. = gejabnt: Lie ober= ften gleichbr., garijrant. (4 9.)

$403 \%$.
austrális.

4035.

dichotŏma.

(Succisa.)

4037.

glabráta.

2. bes. Bik. 5 spaltig;

a. Bl. gleichförmigi.

Die bes. Blkronen:

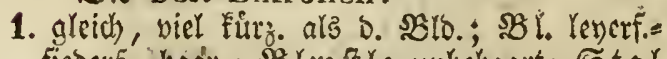
fieberf., baar.; $\mathfrak{B} \mathrm{Im}$ fifle unbehant; Stgl aff., auggebreitet ( $\odot \mathrm{SE}, \mathrm{rch}$.)

4038. sicŭla. 
2. geftinflt; Blätter :

a. zerichnitten; Blbaden:

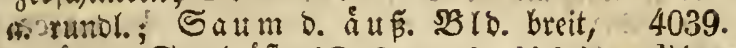

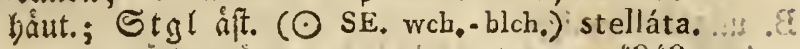

b. pfriemenf. (๑ As. dr.) 4040.

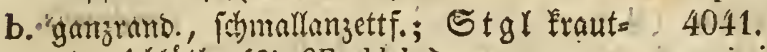
art., 1 blitt). (4 SE, blch.) . graminifolla.

c. fait gamzrano., längl.=lanzettf., feinhana.; Stgl gaberf.; soluth. fait fitlios in o. 4042.

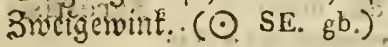
prolifura.

d. fieberf., mit gleichbr. Eapp.; $\mathfrak{B} \mathfrak{I} \mathfrak{m} f \mathfrak{t} \mathfrak{l}$ Febr lang; Stgl Fitruno (4 SE. wch. ;.. 4043 . sos.)

argentěa.?

\section{$-2.04$ \\ b. Bl, ungleichförmig:}

Die bes. Blkroner:

I. gleidjformig; Wzlbl: :

1. långlid), geterbt: bie obern Stglbl. bopp. ficberf., mit gleichbr.=fabenf. Eapp: ; SErone 5 borft.; sy flange weisfitz. (4 4044. SE. rch.; bl. -r. )

2. laitgette, gefagt: Stglbl. ungepaart=

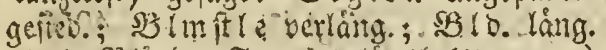

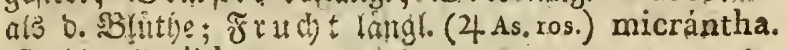

II. geifrainlt; Stglbl.:

A. gleithformig,

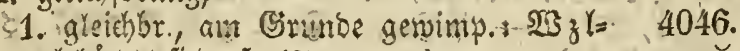

(... bratt.) fieserf. (4 As, w.) pyrenaǐca, if

2. cinf. geficoert; Wzibl.:

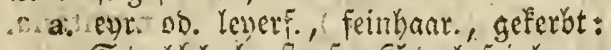
Stglbl. borfenf.; Stgl feinbar. ucraňca.

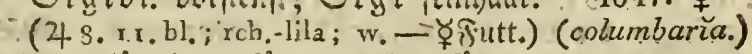

- b. evtr. lơngl., gefígt oo. lenerfơrut.;

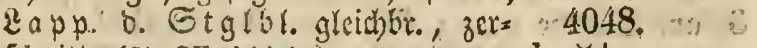
fopnitt. (4 $\mathrm{SE}$, hblch.) Iucịda.

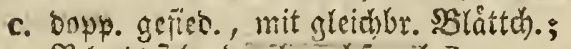

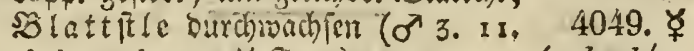
gbch.-wch: (ochrole'icca.)

d. lanzettf., geftelt, gangrand.; "Etgl . 44050.

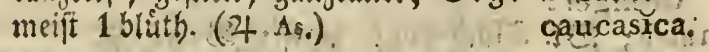

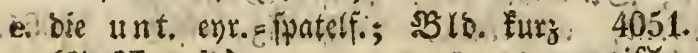
(as) (24) SE: wolic) setifera. 
3. meift boup. gefied.: $\mathfrak{M Z}_{\mathfrak{l}} \mathfrak{l}$ bl. Leverfirm.;

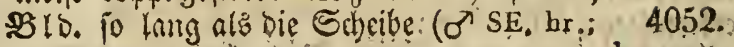
ugb. u, rch.) .

banatica,

B. ungleid)formig,

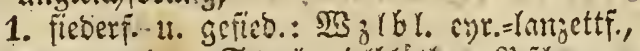
iganirano.; Etgl viclblith.; sp flanje

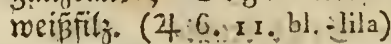

2. $3 f(d)=u$ cinf. gefteo, mit gleid)breiter.

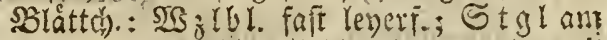
Sirumbe, 4. SBlmfiele ohen routib, mit rüfio. getefrt. Sgaar. (o SE. bliia)

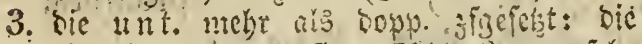

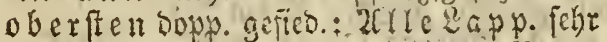

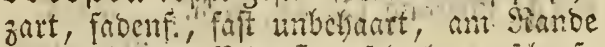

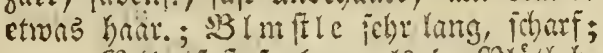

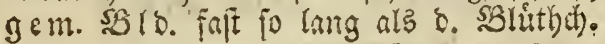
$\left(-v_{0}\right)$

4053.

canescens. d

11. Blbd. ohne Spreubl. z. häarig; Blüthe gestrahlt; bes.oin. Bil: 4 spalt.

Stẻngèl:

1. badtig; Blätter?

a. ungetheilt,

A. eyt. = ringl.; die unt. gefägt (4 5. P. m. v. ; lila; w., wohlriech.).

4056.

b. einige fiederf., andere jerifhnitten (4 3. I 8. 50. hr.; bl.; w. - * $\mathfrak{W}_{j}$ t,

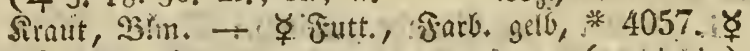

grin, llufr.) (arsénsis.)

b. gepagt, langl.: sie unt. ungetio. ob. jer = 4058..? rifl.; Stgl armf. (更 As. w.)

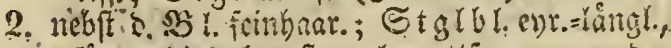
igejagt: bie oberfen lanjettf., gnminano.: Sis $\mathrm{z}$ !bl. leverf. = fieber. $(4-$ ugb. $)$

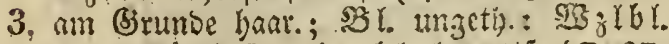

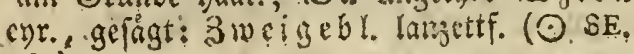
rch.)

montána: capilláta, sylvatica.

4059. pubéscens, 4060. integrifolia, (a)

Stengel:

3. SCANDIX. 210 ,

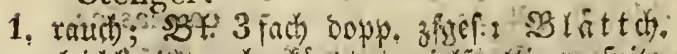

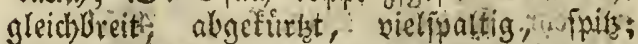

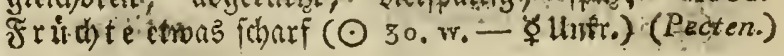




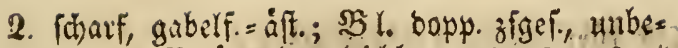
baart:- SB látt do. Slcichbr., etroas ausgés perrt, verling,; Dorben gebuilchelt, mit e.

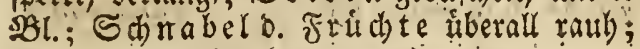
(5) riff. zfgencigt ( $\odot$ As. w.)

4062. pinnatifìda.

\section{(4) 47.)}

6. SCHEUCHZERĬA, 300,
4063.

(palistris.)

4064.

abrotanoídes.

(○ SAm.)

\section{SCHKUHRIA. 676 .}

6. SCILLA. 287 ,

\section{Blüthen:}

1. in Tratiben:

a. เoenigblíthig,

$\therefore$ R. mit Rebenbl.; SBlE. glocénfơrm.;

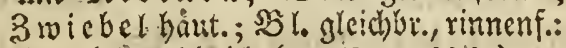
2S z Ibl. jablreith (4 SE, wo - blch.).

4065 verna.

b. ohne Rebenbl.; Br. ramettf., an D. Sosen georuct; 3 wiebel idupp. (4 SE. bl.; W.; p.)

4066 .

Lilio - Hyacínthus. 4067.

b. fegelf., långl. ( 4 - w.; bl.) ịtalǐca:

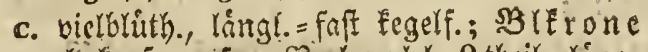
glodenf., aufr., sie b en bl. 2 theil., láng. als ber sBlmfit; sBt. ranjettf.; 3 wiebel. bid)t (2! SE. dbl. - y.)

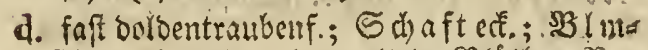
ftiele 2 mal po rang als b. Shlithe; Pe Genbr. unbeutt. (4)

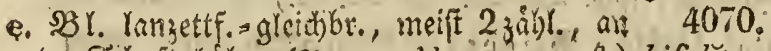

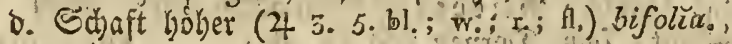

2. Doloentraubenf.; sig im fite nadt, auffeig.,

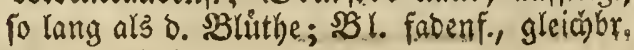
(4 $\mathrm{SE}, \mathrm{hbl}$.).

4071. autumnális. 


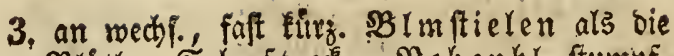
Bluthe; Schaft ed.; $\mathfrak{A}$ ebenbl. Ttumpf, 4072: febr furg (426.32. vi, u, wch.; $\left.r_{0}\right)$

10. SCLERÁNTHUS. 355.

Bldecke d.'Fracht: 4073. ఫ

1. etwas abftebend ( $\odot$ 19.28. grn. $-\not ̧$ unfr.) (annüuis.)

2. gefilofien (4 2. 19. 29. wcb, - * Sraut * 4.074.

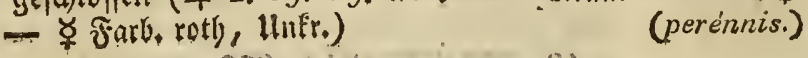

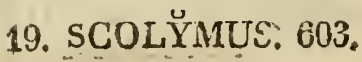

Blüthen:

1. cinzeln,

a. an b. Seiten friellos; BB K Ketrbtaufeno; 4075 Etgl faft cinf., zoit., aufr. (4 As.). grandiflórus;

b. 3 l. etwas fdarf, glatt; Stgl gefligalt, 4076 : is gezalynt ( $\odot$ SE. gl.)

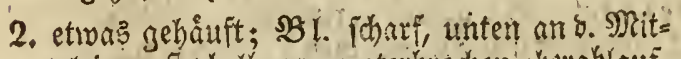
telrippe fratherbaar., unterturoden = Verablauf. $\left(\sigma^{\prime \prime} S E_{0} g^{b}\right.$.)

4077. hispanĭcus,

\section{SCORPIÚRUS, 594 。}

\section{Blumenstiele:}

1. 1 bluth.; ( S rieberbúlfen gang mit ftumpf. 4078 . Schupp. bededf ( $\odot \mathrm{SE}, \mathrm{gb}$.$) , .. vermiculáta.$

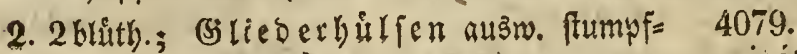
fachl. ( $\odot$ SE. $\mathrm{g}^{\mathrm{b} .}$ ) : muricáta:

3. meif 3 brith.; (s) fieberbirfen ausw. mit 4080. abgefond., fipi:. Dornen ( $\odot$ SE. gu.) " sulcáta.

4. meit 4bluth.; (s)lieberbirlfenausw. mit 4081 , gramingt, fpic. Dorrien ( $\odot \mathrm{SE}, \mathrm{gb}$.) subvillósa: 


\section{SCORZONERA. 611 ,}

\section{Blätter gleichförmig.}

\section{Blätter:}

1. Lanzettf., Limfoif., ungeth. am Grunte etwas

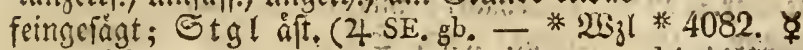
- ช̛ Silid.) hispånŭca.

2. ichmallanjettifo,

a. langgelpist, unbehant, gerippt, flach; 4083. Stgl meift 1 hluth., blittr. (4 6.) glastifolia.

b. Stgl fait mackt, 1 bruth), u. ncbit ben 4084 . SB ro jü up p. unbehaart $(4$ 2. 3. 6.) austričca.

3. långl.=lanzettr. , gerippt, flach); Etgl falt

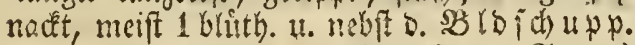

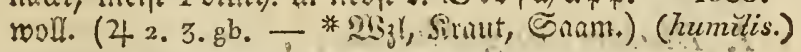

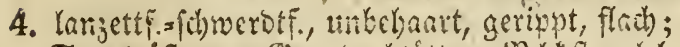
Stgláft., am Sirumbe blattr.; sbleftrabl

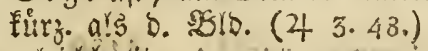

5. Gleict) Ereit,

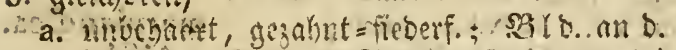

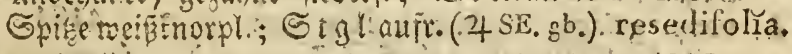

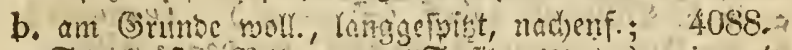

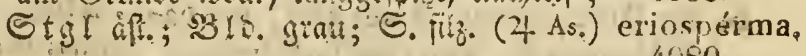
4089.

c. nebft D. 1 brith. Stgl ratch (4 As. gh.) hirsíta.

6. pfriemenf., ungetl); Sirnfit oben bicker; 4090 . Etgl ant Erunde jott. (24 6.21. gb, u. rch.) (angustifolia.)

7. gleichbi. = futwerotf., langsefpiat; fteif, $\mathfrak{g} E=$ ripyt, nachenf:; SElo. jott, am Eivunos blattr:; كsgl etmas aif. (44 4. 3 . hab.) graminifolia.

8. gleichbr. =p friment, rimenf. = 3 fant,; Stgh 4092 . âf. (4 3. 8. bl:-1.) (purpurę̧a.)

9. 估rotfingeformig,

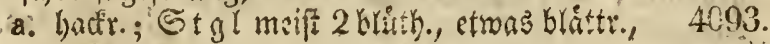 hadr. (4 As.)
b. glatt,
asperịma,
Q. ftumpf; ङdiaft blattlos,
b. S daft nadt, 1 blitio. $\left(\sigma^{7}\right)$

10. bicht feingerant, fivis Stenget meif ach alis.

1 bluth. ( 4 As.igb.) 


\section{Bl, ungleichförmig !}

\section{Blätter :}

1. Ianzettf.=gleid)br., flafh): Stglbl. rimenf.e 4097 , gleidhbr.; 5 tgl 1 bluth. ( 4 4. 9. blch.) roséa.

2. bie unt. fieberform., gleid)br.: bie vbern gleichbr.; $B$ lo. unbehaart, mit etwas $a b=$

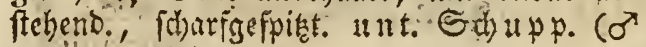
3. 8. $14 . \mathrm{gb}$.

3. Die unt. berablauf. =fieserf., lanzettf.: die

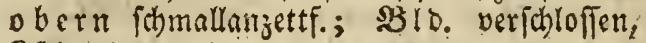
8 [eit. $\left(\sigma^{7} \mathrm{SE}.\right)$

4098.

(laciniáta.)
4099. octanguláris.

\section{SCROPHULARL̆A. 491.}

\section{Blätter ungetheilt.}

\section{Blätter:}

1. berzformig,

a. einfachgejagt, fpic, am Sirunbe runbl.; Stgl ftumpfect. (4 NAm. grnch.)

b. bopp. gefingt; feinbaar.; Kispen:
a. in o. Sittwint. gatelf. $\left(\sigma^{7}-\mathrm{gb}\right.$.)
b. am (5nde ftelseno, 3 fact) geth., mit tac

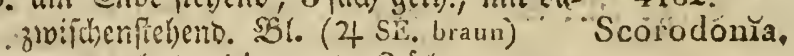 (vernális.) 4102.

c. bopp. ge onint: bie litto.3ahne gegen. o. Grumo geribtet; En BImfícle $2-3$ blith), weit). (4 NAs. 9. hgb.) 4100.
marylandica.
4101.

d. herab!nuF, geffielt, ftumpf: $\widetilde{t g} 1$ ourd) Şaute et.; mit Endtraub. (N.SE. - 4104. * Sirnut)

e. mit 3 Stictripper;

R. 'auf. bend. Exat. feinhaar.; Brttfle geroimp. : Stmftiele it. - Rebenbl. bant.= orü: (4 SE:)

glandulósa.

b. mit fitmpied. Stgl (4 r. 43. ro-braun 4106 . u. grnch., übelriecl. - * $23 y$ (nodósa.).

f. geftrichelt, fwiegelglatt; 231 mifte in ben 4107.

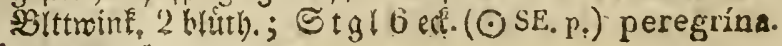

2. langl. = berifo,

a. unten raub), bopp. gejalint, am (Srunde mit 2(nhang; Sittfte gleiti); mit Enb= traub. (4 SE. p. u. gb.-grnch.)

4108. auriculáta. 
b. auf berdo. Seiten unbegant, am Ranbe gelappt; Blattfle wie vorker; End

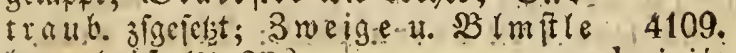
(buar. =orif. (24 SE.)

3. Jersf. =lángl., gezalnt: 3 án ne gamran laciniáta. am (Svumoe tiefer (4 SE, ur. u. grnch.) betonicifolia.

4. lanzetti., gefägt, gefielt: S tglbl. 3 jåkr.: 4111. a weige bl. entgegengef. (4 As, braun) orientạ́liq.

\section{Blätter gefiedert.}

Blätter:

1. 2rre einf. getico; mit Enorifpe; Brma filc gabelf.; Seitenlappen D. Uliphe

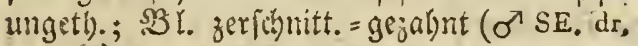
n. grin.)

2. Dic.unt. 3 fachgefied, ftumyf: bie obern

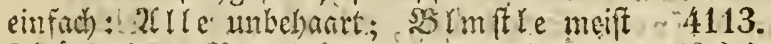

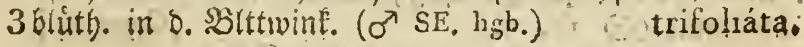

\section{SCUTELLARIA. 445 .}

\section{Blätter:}

1. zerichnitten, unten fils.; Rebren undre 4114. 4 fcit. (4 As. gb.) : orientális.

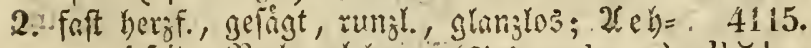
ten 1 feit.; Jte benbt. ent. (4As, wch,; va) albǐda.

3. gant herzifo:mia,

a. gefigt, 1unbebaurt; 2 e eren vertangert,

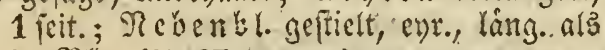

ร. SBR. (4t SE. p.; w.)

3. zerichnitt. = geiógt;

s. geterbt; $2(e b i e n$ bathziegelf., rundr.= 4 feit.; Tebenbl. batis fa lang als b. SBlütlie (4 SE. .g. v. u. wch.)

b. Fiph, unbehaart; Xebien wie varber; Rebenbl. To lang als b. Şluthe (4 $A_{8}, w_{,}$, grafs)

4. berzf. = YanzettF.,

4116. . .

peregrína,

a. an 8. Rúctenichảje icharf; Seitentrau= ben blättr. (4 NAm,

4117 alpína.

4118. lupulina;

4119. laterillóra,

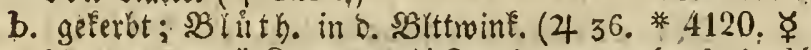
bl. u, w. - * Siraut - - Ұ Jutt.) (galericuláta.) 
3. bergf. = enr., faft ganzrant.; SB tưt hen wie norber ( 4 42. rch.)

6. bie unt. fpiç̧f.: Die obern pfeilf.: Jhle 4122 ganjano. (4)

10. SEDUM. 364. $\left(382^{x}\right)$

\section{Blälter flach,}

A. gleichförmig;

1. $\operatorname{mit} 10 \mathrm{Staubgefälsen.}$

Blätter :

1. langettformig; Bliithen:

a. in e. ftiellof. Endafteroorbe; Stgl allfr.; 33 l. gejägt (4 NAs. gb.)

b. in Rtipen: Stgl åfrig; Sb̉lbl fuib begranut, $(\odot$ I 7...w

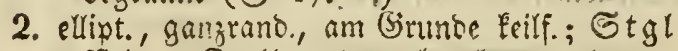
auffeig.; Doloentraub. offgezor. (4.9. 23. $\mathrm{p} .-\mathrm{v}$.)

3. längl., gefägt; : Dolbentraut. zfgefegt, bláttr.; 巨tgl aujr. (4 I. p.; gbch.; w.

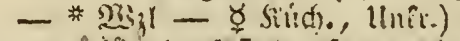

4. err. =longl, fafi berji, ungl. gezalunt; ge= ftielt; Endosloentraub. jfgejegt; Etgl frraud). (t) NAs, w. )

5. vert. ent. = rciff, lobl, an s. Spine gezahnt; Stgl friech.; bít thetrag. Sneige auri.; a fterdolo. nusgeiwert ( 2 - As.)

6. rlinel. =net. enr., an (Sirunde teilf., an 0 . Spize geterbt, am Ronoe inorpl.=beichftachl.;

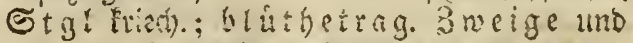
2fterdolse roic porfer (4 As.)

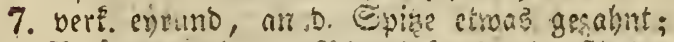

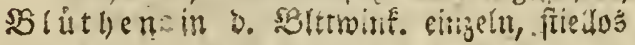
(○ SE.)

4123.

Aizóon.

(Cepáea.)

\section{5. \\ (Ainacampsĕros.)}

* 4126. $\Varangle$

(Telephüun.)

4127.

potiulifolịum;

4129.

hybsitum.

4129.

spuizum.

$\therefore 130$.

stellatum.

2. mit $S$ Staubgefä $[\operatorname{sen} u$. gauzgetrenat. Geschl.

SBl. långl. = teirf., an o. Spitac gefägt; Stgl aufr.; Doloentraub. zfgezog. (4 9. gb. \#4131.

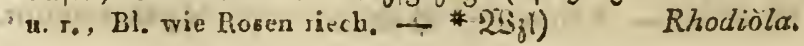


B. ungleichförmig,
1. mit $S S$ taubgefäIsen:

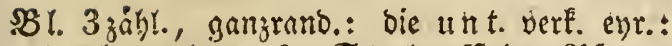

D. obern ranjettf.; Stgl auffeig.; Xf ter. 4132.

bolbe ausigefpcrtt, blíttr. (4 NAm.w. u. A.) portulacoídes.

\section{2. mit 10 Staubgefälsen.}

\section{Sterigel:}

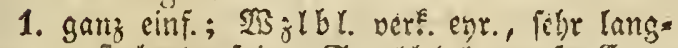
u. Fechenogeppist: Siglbl. Langetti.; End

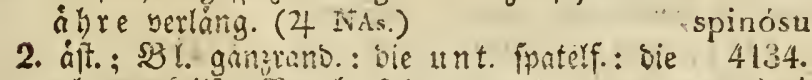

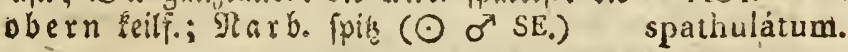

\section{Blätter halbstielrund.}

\section{Blätter:}

1. im Duirl, 43. wistut; Stgi triett. (4 SE.)

2. nidit im suitl,

a. lángtín, oben strwas flach, foinfunc.;

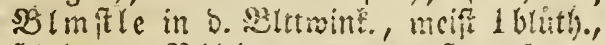
feingar.; $S B ! b l$. cyr., ctrons ftumif $(\odot$ 3. 42. hr.)

monregalénse.

be

nicoerfieg. $(\odot 9.23 . \mathrm{gb}$.)

\section{Blätter ganz stielrund;}

A. mit 12 Staubg of $\int_{\text {sen }}$

B). faft fitielrunb, etwas graugrim; Stgl

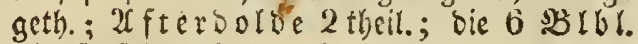

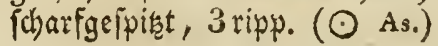
4138.
sexfìdum.

B. mit 10 Staubgefäfsen.

Blätter:

1. entgegengejebt, enr., fiumpf; Stgl fobadi); 4139 . Bluth. zerftreut (4 SE, rch.) dasyphýllom.

2. medhelsweisfeinento,

a. längl., ftumpf, am Stgi lofefangento; 4140 . 2f ftero olbe 2 ipalt., unbebart (NAf, bl.) coeruiĕum. 
4if $\therefore$ Sedum. - Selinum.

b. verf. eyr., angewach) = =ftiellos, bodfr., et= wả aufr.; 2f fterbolde 3 palt. (4 x $x$. 4141. 13. gb. - * Sitaut)

c. eyr., ftiellos, yodetr.; Stgl aufr., ein= zeln, jấrig; $\mathcal{X}$ fteroo bo umgefrummt $(\odot$ NE. gh.)

(acre.)

3. benbes nicht,

a. lánglich); Bliithen!

a. in áft: 20fteronloe; Şlâtt. ftumpf, 4143. fitillos, abfebeno (4 23. w.)

b. in glcibboh. Doldentraub.; Stgl 4144 . aufr. ( $\odot$ 9. gb.-grn.)

4142.

annǔum. (album.)

atrátum.

b. gleithbri, ftiełumb=eingedrürt, zerftrent; Ufterdorde etruas aufickeno; 3 rf. mit 6 BBIUl. (4 9. wch.)

c. pFriemenf., zerfirent, loje am Stgl hang.,

a. graugrion; SBlith. afteriolsenform.; SB Ibl. 2 mal fo lang aís o. Blo. (4 7 . gb. - $\Varangle$ Sitid.)

b. Die unt. ungefrimmt (4 $7 . \mathrm{gb}$. ૪ Sitid).)

d. faft eltuno, angemad)f. = friellos, bocer., faft aufr., in e. 6 facti. Naibe Dadisiegelf. (4 2. Ix. grn.-gb.)

$41 \frac{4}{4} 5$. hispanicum.

\section{SELININUM. 219.}

\section{Stengel:}

1. ftielruno; Blätter:

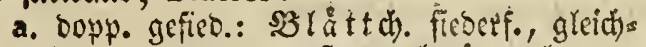
breit: Die unterfiten abuartşgebogen; Stgl oben eff., febr aft.; SBlittchen b. vielbl. gem. Scurle zallreid), etmas feingezabnt: bef. Shulle n $\mathrm{mm}$ (sirmbe Jibang., làng. als o. ber. Doloen ( $4 \mathrm{SEw}$ ) baicalénse.

b. 3 fachegefied.; B̃ lattfite umgebrodten;

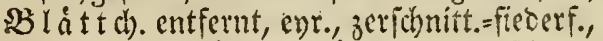
ausgeipert, gláns.; Şüllen umgelblag. (4 2.6. w. - * $\mathfrak{W}_{j} \mathrm{l}$, Siraut, Eaam.)

* 4150。

(Oreosclinum.)

2. gefurtht; Blätter:

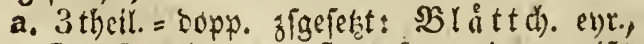
fieberf., mit etrons ftumipf., u. in c. weipe

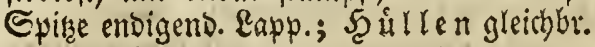
(4 8. w.) 


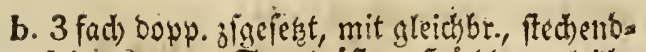

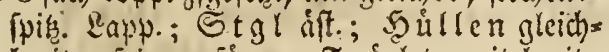

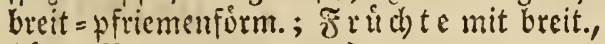
biat. Rande (4 - w.)

3. geftreift; Błlátt. gefféc., etwas lederart.:

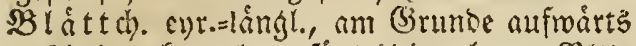
zeridunitt., fnorpl. = gelägt; bie obern 3 Bttts ideiben weit, blattlos (As. w.)

4152:

lineáre.

\section{SEMPERVÍVU̇M. 383. (382.)}

\section{Blätter:}

1. gewimpert: Fortsätze Derietben:

a. etwas ciffelycrid mit 12 Sigef. unt

12 Etreren; Nect. Feiff, wargenart: 4154 .

(4 6. 25. grno-rch. - * Straut) (tectórem.)

4155.

b. Eutuel. (24 6. 24. 25. gb.)

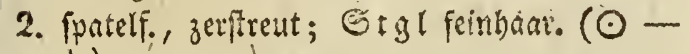

gb.) globif̌rum. Fortiácin (4 SE. 9. p.)

4. nebft Stengel a. D. Epianen b. BBtbl: 4158. factijeligarr. (4 - grno-gbch.) hirtum.

5. ganzrant., mit ctwas abfictento. Fort fó 4.159 . een (4 23. ros.). montánums

6. bie unt. frictrumb: bie obern cingedrúdet: 4160 . alle zerfit "t (4 SE.) sedifórme.

\section{SENECIO. 660. $\left(686^{3}\right.$.)}

\section{Blïth. nicht gestrahlt (röhrig.)}

Blätter:

1. långl., umfaffeno, ungleidj= u. tiefgezalgnt \$ 4161 .

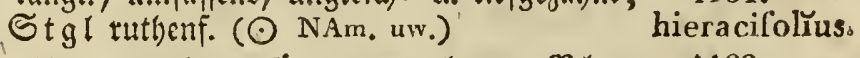

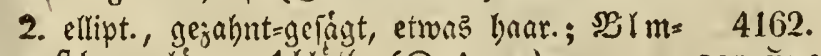
ftle verláng., 1 britth. ( $\odot$ As. v.) cernŭus.

3. verf. eyrumb, geftielt, gerfdjittten = gejahnt; 4163. $\mathfrak{B}$ I mftie fabenf., 3 britth. ( $\odot$ Naf.)

rerbenaefolius. 
4. fieberf., umfaff, gezahnt; $\mathfrak{B l}$ luth. in zige= "jog. Dolbentraub. ( $\odot$ 22. 29. gb. — \# Siraut - ซq lintr.)

\section{Blïthen gestrahlt:}

A. Strahl umgerollt.

\section{Blätter :}

1. Yamzettf., umfalf., gejahnt; Brofdipupen 4165 . (el)r furz $\left(\odot S^{2}\right.$.)

2. Yánglich, geffielt, butht.; SBIntficle meift

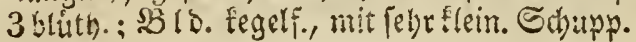
$\left(\odot N A f_{\text {, }}\right)$

3. fieberformig,

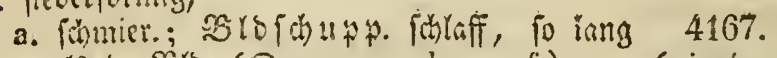
als b. Sgict. ( $\odot 22.27$. gb., grol's) (viscósus.)

b. Feingezahnt; Etgl Dolbentraubenf., aufr. 4168. $(\odot$ 5. gb., kfeiu).

(sylvaticus.)

B. Strahlabstehend;

1. Bl. Alle fiederförmig.

Blätter:

1. gan? umfaînent,

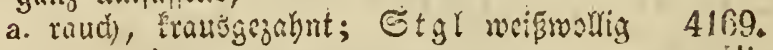
$(\odot \mathrm{SE}$.

b. obfn unbehnart, mit eck., grabart. Lapp.; 4170 . Etgl it. 2 Is. unbelinart (4 SE.p.) rupéstris.

c. auf beys. Scit. unbehart, Ieryerf. ficterf., 4171. gefalynt, flach); Stgl unfeganet (4 S.) montánus.

2. balbumfofi., mit glcichbur., etrass gezabní, entferntfethend. Lapp.; Stengel aufr. $(\odot$ 24. 29.)

3. nicht umfaffeno,

a. etwas frablitgar., gezahnt; Ëtgl aufr. (4 34. gb.)

b. auf beno. Seiter fitz., fohneerweis, mit gleichbr., ftumpfo, etwas gejahnt. Lapp.; Doldentraub. zigegog. (4 9. gb.)

4172.3 squalídus.

4173. (erucifolüus.). 4174. incánus.

c. unten grau, lanjettf., faft fieberf.; Dol= bentrallb. wie worker (4 9.)

d. unbehaart; leverf. = bopp. fieberf., ausge= fpert, gegabat; Stgl aufr.; Strabl 
abfebent (4 34. 49. gb. - Staut, 4176 . 23lm. - ఫ forb. griui)

(Jacoba'ea.)

2. Bl. fiederf. u. ungetheilt.

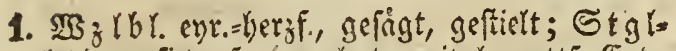
blatt. ficoerf., gezalnt, mit lanzettf. Ento $=4177$. lapp.; $\mathfrak{s}$ I mftle oben bifer (4 NAm. gb.) aurĕus.

2. Dic unt. vete. evr., ungeth.: die îbrigen

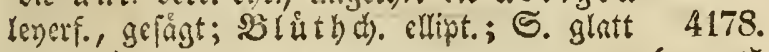
(4 47.)

(aquať̌cus.)

3. Bl. gefiedert.

Die gleiłhbr. Fiedern:

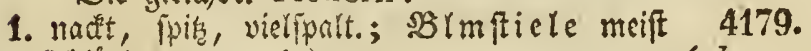
2 bluth) ( 4 9. gb.)

2. unten feinlaar., pfriemenf., ctwas zerfohnitt.; 4180. Stgl etwas raudi (4 5.)

(tenuifoliuss.)

4. Bl. Alle ungetheilt,

a. ungleichförmig.

Bläiter:

1. Sie unt. cyr., in D. Gticl herablauf.: bie (4 SE.)
(4) SE

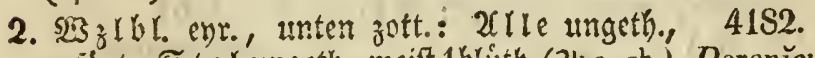
geiägt; Stgl ungeth., meift 1blith. (4 9.gb.) Dorončcum.

b. gleichförmig.

Blätter :

1. lanjettiörmig,

a. baibuminil., fharfgefägt, unten etwas zott.; Fid oboldentiaub. etmas ausge= perrt; Stgl gand gerade (4 36. 42. gb. u. w.)

b. foft herashur., unten etwas zott., gefigt;

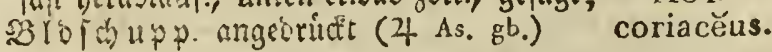

c. faft fiellos, faft unbebart, am (sinnos 4185. feilf., fichigefigt (4 4. 8. hgb.)

2. eyr. = lanzettformig,

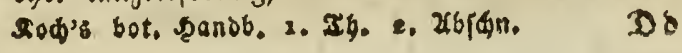

4183.

(paludósus.)

4184.

(saracenicus.) 
a. unten fefr żartfeinlbaar., gefågt, am Ranbe getrimp., ftiellos; an Sirunbe ungl. (4 4. gb.)

b. auf beys. Seit. unbehant, fharfgefo̊gt, faft flielloz (24 4. Stgl dro)

3. Kångl. =langetff., foft Gerablanf., etwas grau=

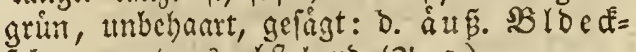
f由upp. etwas abfebeno (4 5.)

4186. (nemorénsis.)

4187. ovátus.

4188.

(Doria.)

\section{SENNEBIÊRA. 525.}

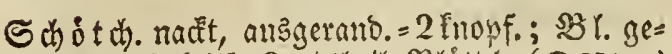
4189.

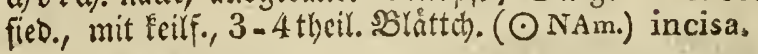

\section{SERAPIAS. 718 .}

Rectarlippe 3 theil., mit ftumpfen, aufr., zfgeneigt. Se it enla a p. u. långl, lanjettf., faft unberbart., lyerabbang., etwas fpiten Mitterrapp. (4 - rostbraun)

4190. Lingua.

19. SERIǑLA. 601.

BI. verf. eyruno, etwas gezahnt; $\$$ flanje 4191 . haff́r. $(\odot \mathrm{SE}$.)

\section{SERRATUัLA. $633,\left(637^{x} \cdot 639^{x}\right.$.)}

\section{Blätter :}

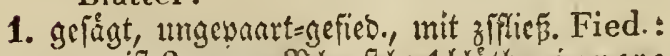
a. meift 2 paar.; BSImft le 1 britt).; innere SB Iofoh upp. verlángert, gefirbt (4 As.) quinquefolis.
b. meift 5 parr.; $B$ lmfitele wie vorber; B rutb) geftrablt $(44$.
4192. 4193. coronáta.

2. Fharfgefägt, ant (Stuntbe fieberf.; mit gleid)= boher Dolbentraube (4 3.4. 4.; w. * Nraut - ૪ Futt., Farb. gelb)

3. feingelägt, långl., unbehanart, borft. =gcrimp., F̂tumpf, Fharfgeipiets; Stgl bolbentraubenf. ( $\left.\sigma^{7}\right)$ 4194. $\Varangle$
(tinctoria.)

4195. setósa. 
4. gezahnt, unten fill.: $\mathfrak{S Z}_{3}$ lbl. chr., faft herzf.: Etglbl. curr. =langettf.; ENnoblutth. faft Dolbenf. (4 9.)

5. fiederf. =gezalynt, lamzettf., am Manbe wellenf., born., unbeibart; S Stgl rifpenf. (ơ' 28. hr.; w.; bräunch., wohlriech. - $\Varangle \mathfrak{U}_{11 f} \mathrm{f}_{\text {.) }}$

6. Ficoerf., ichief, fwis, unbehaart, ofne Dorn.; sB lo fduppen fobarfgeppist: bie innern troct. (2t NAs. p.)

7. am Ranise ungerollt, fribmallanzettF., rauth;

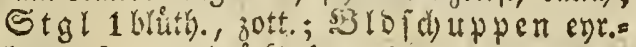
lanzettf., angeoridat (4 9.)

4196.

discōlor.

4197. ఫุ

(arvénsis.)

4198.

centauroídes.

4199.

pygmáca.

\section{SESĔLI. 189.}

\section{Blätter gleichförmig.}

\section{Blätter:}

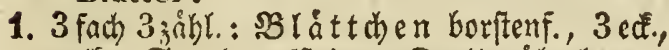
welf; Stgl auffiteig.; Dolt. itberhang., mit vertüng. Strablen (4 SE. gb.)

2. Soppeltgefieoert; Stgl:

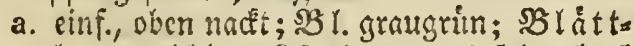
chett gleidbr., 3 fpart., ctwas pipis; ber. Şülten 1 bláttr., beckenf., geterbt (4 2r. w.; wo u. rch. - $\Varangle$ futut.)

b. ganz gerabe, oben áft., glatt, focheinef., mit fohloff. SSltticheiten: bie uniter ften bang.; SBleitt th. gleichbr., meif 3 part., etwas fwis; 5 úllen borftenfórm.: ge m. Şille weniggetheilt, vielbláttr., fo lang als b. Dolben (4 3. 59. wch. u. v.)

c. etwas bolz. , geftreift, ait., fnot.; $\$ 5 l$. 4200. gracile. etwas freif, abgetiurst, mit gleit)br., lang= geipizt. SBláttw); DolDen meift in bem * 4203 . SBlttwinf. (4 3.4. 8. gb. - * Saam.) (tortuǒsum.)

d. hin $=$ u. hergevog., fnot.; $\mathfrak{B l}$ l. gleid)br.= fabenf., rimmenfo, etwas fteif; Sculle feldt faft; F⿱乛龰úd te yor b. Reife raub (4 4. 8. w.)

3. Dopp. jigeferęt; Stgl:

\section{1. ఫ̧ \\ (Hippomară- \\ thrum.)}

a. aufiteig., fait einf., bichtgebrångt; $\mathfrak{B l t}$ t=

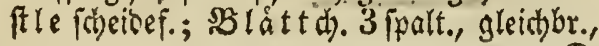


etwas ftumpf, faft zigeneigt; Frúchte 4205. faft prismat. (4 SE. w.)

b. aนfrectich,

montánum.

a. geftreift, faft einf.; sglttfíte fuscioef., ganj gerabe u. nebft o. 20 l. aufrecht: $\mathbb{B B}(a) t t c h$. gleiubbr., 3 theil., ftecthent. fpin; bef. Sृưlle vielblattr., faft lans jettf., firiz. als 8. Dolden ( $\sigma^{7} 3.4 .8$. wch. u, r.)

- b: bin u. bergebog.: $B(a+t$ d). borftenf., rinnenf.; Sullte meift 1 blättr.: bef.

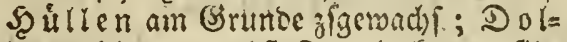
bent bicht u. neiff $\mathfrak{F}$ rud tenot. filz. (4 SE. w.)

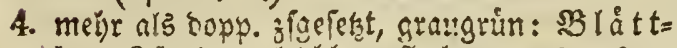
d)en 3 parti, gleichbr., flach, etrab atigge= fpert; Stgl jebr åft., Enot.; Frůchte un= bebaart (4 I1. rch. u. w.)

\section{Blätter ungleichförmig.}

1. Die unt. bopp. fieberf., mit auszgefpert., entfernt., fchmallanzettf. Rapp.: bie obern

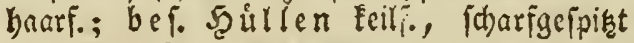
( $\odot$ SE. w.)

2. bie unt. abgefurtst, faft im Suirl fekend: bie obern verláng.: Ull haarform.; bef. \$ุüllen borftenf. ( $\odot$ SE. w.)

4209.

ammoides.
4207.

leucospérmum.
4208. glaucum.

\section{SHERARDIA. 68:}

Die unt. 3 T. 8 u. 4 jåhl.; mit (Enobนำth.;

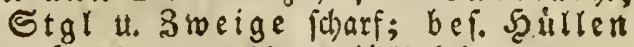

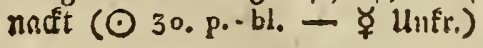

4211. ซ్

(arvensis.)

\section{SIBBALDǏA. 255.}

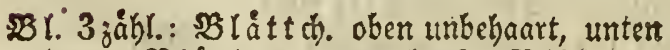

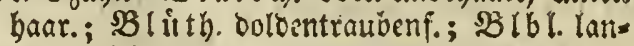

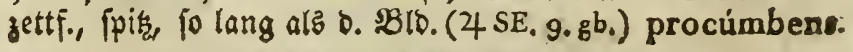


14. SIBTHORPİ. 490 .

4213.

(4) $\left.\mathrm{SE}_{1}, \mathrm{x} \in \mathrm{b}.\right)$

europa'ea.

\section{SICY̌OS. 763. (W. 4.)}

Die Kerźf. Blätter:

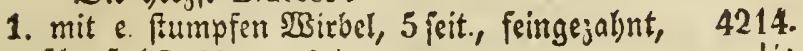
icharf ( $\odot$ NAm. gb.)

2. mit e. rundr. Buddt, 5 lapp., gezahnt, baar.s

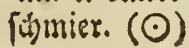

anguláta.

4215.

vitifolla,

16. SIDA. 545. $\left(79^{8} \cdot\right)$

\section{Blätter gelappt.}

\section{Blätter:}

1. meifi 5 lapp., unkebaart, mit långl., lange

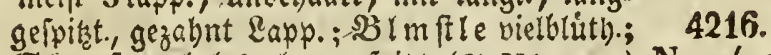
Skapf. rebrlos, lamggepist (4 NAm. w.) Napáea.

2. 7 lapp. = banbf., fcharf, ntit lanzettf., jer = fohnitt.=gejahnt. Rapp.; SB lúth. Dotoentrau= benf, mit Rebenbr. u. ganjgetrennt. (B)

\section{7. dioǔca,}

II. Blätter nicht gelappt,
A. sleichförmig.

\section{Blätter:}

1. 3 ect. = faft rautenf., gejalnt, am Scrunbe $u$. an b. Spise ganjtano.; ss imfte 2 mal jo lang als d. SBittitt ( $\odot$ S.Am.)

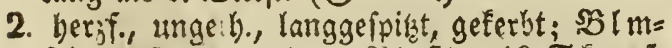
ftiele fo lang ars D. Sbittfte; 10 Skap 3 faom. ( $\odot \mathrm{Am} . \mathrm{gb}$.)

3. rundtich = berzf., langgefpist, gezahnt; bie eingeln. Blmstle:

2. Eurzzer als D, SGlttflt; Blätter:

6. feingaar.: \&app. ant (5runde angenă: bert; Stap f. abgeflutst, mit 2 hori=

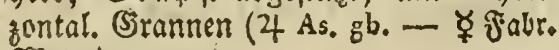
DSeber)

4220. 후 Abutălon. 
b. feinlanr. s weich: $\mathbb{E} a p p$. am (Sirunbe ausacbreit.; Stapf. mit 2 aufredter Grannen ( $\odot$ As.)

b: Yång. als bas SRlatt; Skapi. abgeftust, ftumpf, gróß. als D. SBlo. (SAf,)

4. enr. = berzf., gezahnt, etmas edt. It. ftumpf; B lmftle einzeln, etwas kúr. als b. SBltrft;

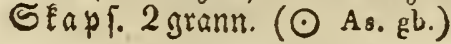

4221. tiliaefolia. 4222. Sonneratiáas. cordifolıa.

Die unt. Blätter:

1. herzf., langgeipist $=5$ ect., etwas gejabnt, fumpf: bie obern fiepsf., langgeipist, am Grunde etwas gezahnt; S3lm fil e einjeln in

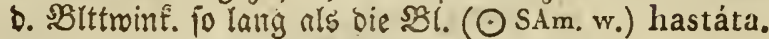

2. runol. = herz, ftumpf, faft 5 edt.: b. obern

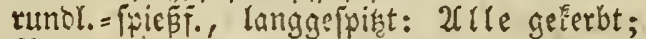

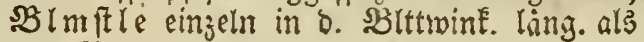
Das SBl. ( $\odot$ SAm.)

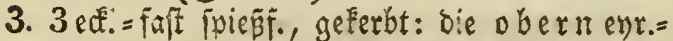

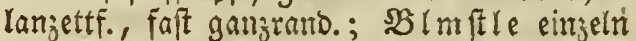

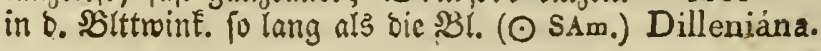

14. SIDERITIS. 469 .

\section{Ohne Nebenblätter.}

Bldecke :

1. bornig,

a. grós. als die SBlE.; Slippe ber BlE. 4227. 3 ipalt. ( $\odot$ gb., mit braun. Rande)

b. Stgl niederlieg.; Slippe o. SB!t. enr. 4228. ( $\sigma^{\top} \odot$, w.) romána.

2. mit faft gleich., feinburn. Rappen; Stgl 4229.

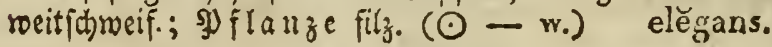

\section{II. mit Nebenblättern.}

Nebenbl.:

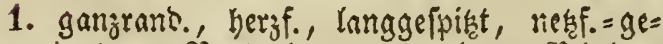
rippt, am Sande baar.; Die obern $\$ 81$. lan= zettf., umfafi., feingezahnt; \$ु flanje haar.; Gacir. (4 As. w., mit braun. Strichen) 


\section{Sideritis, - Silene.}

2. gejahnt=bornig; bic langettf. Bl.:

a. auf kenb. Ecit. banr., ftumpf, gezabnt; 4231. (Stgl rauds, nieberlieg. (4 SE: gb, u. w.) hirstita.

b. oben unbehsart, etwas gezahnt; Reben= 4232. blátt. eyr.; Sblb. gleichf. (4 SE. gb.) Scordioídes.

c. auf beyo. Seiten unbehanrt, ganzrano.; 4233. Reberbl. her fr. ; $\$$ ID gleichf. (4SE, gb.) hyssopifolra.

\section{SIEGESBECKİA, 068.}

Die eyr. Blätter:

1. geftielt, ungleid)geashnt, am Brrunte faft

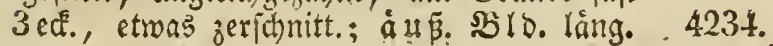
als ọ. innere. $(\odot$ As. gb.) orientális.

2. flicllos, gezahnt; $\mathfrak{B l}$ lutben b. Scheibe 4235.

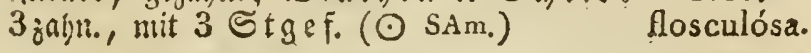

10. SILÉNE. 357. (84.2. $305^{2}$. $321^{x} \cdot 362^{2} .805^{3}$.)

\section{Blüthen zur Seite stehend,} A. einzeln.

Blumenblätter:

I. gamjranbig,

1. vund.; Fruำt te wechj. alfr. $(\odot 26,32.4236$. dr. mit w. Rande) quinquevulněra.

2. Frith te wechi untgefblagen, geftielt; 4237.

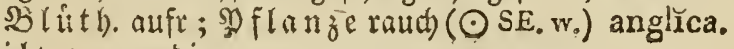
II. nicht gangranbig,

A. ungetheit,

1. gejahnt; B Slut then aufr.; Frudch te

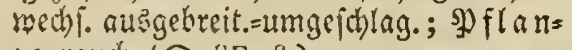

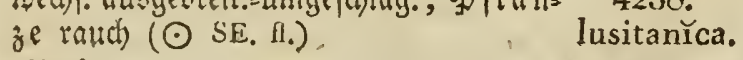

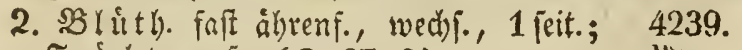
Frud te aufr. $(\odot$ SE, A.)

B. ausgeranto; Frichte aufr.; $B$ lob. faft fticllos, ctwas baar.; \$ fla llze raud) 4240 . $(\odot$ SE.) cerastivides.

C. 2 fpaltig; Stengel: 
1. faubig, am Grunoe áft., niebcrlieg.; $\mathfrak{B} \mathrm{I}$. gleichbr., icharfgeipigt, frachelhaar.; BB luth.

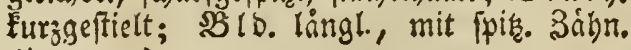
(ち As. w.)

2. Erautartig; Blätter:

a. gleichformig,

Q. lanjettf.; SBlủthen åfrenf., mechí, 4242. 1 feit., fitillos ( $\odot$ SE. A., unten grau) noctúrna.

b. langl. = fpatelfo, feitenart. =grau; $\mathfrak{S} l \mathfrak{L}=$ then entgegengei., gefticlt, aufr. (๑) 4243 . SE. I.)

c. gleichbr. = fpatelf.; $\$ 2$ ro. walzenf. = Eet! = letr., 10 1treif.; mit Enobluth. in b.

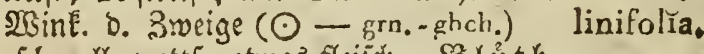

o. fchmallanjettf., etras fteifa).; $\mathfrak{B} \mathfrak{l} \mathfrak{u} t \mathfrak{h}$. traubenf., 1 feit.; $\$ 210$. bauci), 10 ede., gefâtbt ( 4 NAs. w., ausw, ugb.)

e. ellipt. = fpatelf., runol.: bie oberfien etros feimhanr; $\mathfrak{B}$ lo. feulenf., 10freif; $\mathfrak{B}$ lù th. traubenf,, 1 feit, übergebog. $\left(\odot-p_{0}\right)$ sericěa.

4241.
supina.

\section{sericea.}

4244.

4245. jeniseénsis.

b. ungleidjơrmig: bie unt. ellipt. = ipatelf.: bie obern lanjettf.; SBlbece 10 ftreif́; Blithen:

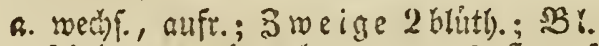
feinbant.: bie obern etras frumpf $\left(\odot-p_{0}\right.$, ausw. up.)

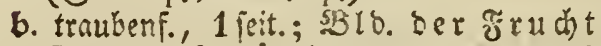
frumpi 3 cá., lángl. $(\odot-g m .-g b c h$.$) nyctántha.$

B. geh äuft;

1. Blblätter 2 spaltig;

a. strauchig.

B 1 . vert. enr. = langettf., langgepist, gegen $\delta$. (3rund Eeilf., am Pande pebi peirgeivimpert, anf bevo. Seit. unbebart; $\mathfrak{B}$ luth. in immer 3 fach) geth. Endrifpe; SBY. Keulcnf. (5 SE. blch.. lila $\Delta$ )

4249. fruticósa.

b. Erautartig.

Blüthen :

1. ủbergebogen; 273 Ibl. am Ranbe 倘arf; 4250 . $\$ 3161$. gleichbr. (4 - gbch.) 
2. in 1 frit. Rispe:

a. úberbangeno; Stgl:

ค. auffeig.; $\$ 2$ l. langettf., fharf (4 3. 4251.

. 1. w. -rch., Bld. r.)

b. hin u. Kergebog. = eingebrochen; $\mathfrak{B} l$. lángl. = lanzettf., nebft Etgl frirbaar. (4 NAm. ugrn.. inw. w.)

(nulans.)

4252.

livĩa.

b. aufr.; $\mathfrak{B} \mathfrak{l} m$ fit le entgegenger., long. als

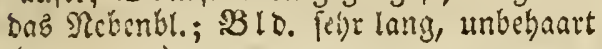
(4 SE. w.)

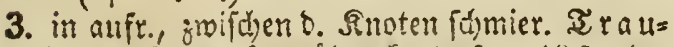
ben; BS loede rógr. = feulenf., 10 furd).; Stgef. julest aus D. sgluthe berborrag. (4, SE. wcb., IVachts entfaltet u. wohlriech.).

4253.

lungitlóra.

2. Blbl. 2 theilig.

Die rifpenf. Blithen:

1. aufr.; Rebenbl. am Rande häut., ges 4255. wimp; 23 l. Ğmallanzcttf. (4 NAs. ugb.-w.) teuùis.

2. uberhang., 1 frit.; S゙tgl น. B I. unkebaart 4256. (4 SE.) infrácta.

\section{Bliuthen in d. Winkeln d.} gabelf. Ziveige;

A. Bl. bïschelsueisstehend.

281. borftenf: Die an o. blubens. 3roei 4257 : gen entgegengefegt (4)

polyphjilla.

B. nicht;

1. Bldecke aufgeblasen.

Bldecke:

1. unbehaart, enr., aber. =nesf.; Stapleln, 4258. 3 faid. ( $\odot$ SE. r.)

Beilen.

4259.

2. bangeno, mit 10 fünrf. Eden ( $\odot$ SE. r.) pendüla.

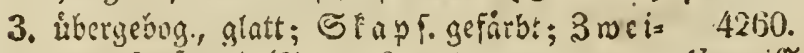
ge nusgelpert $(44.26$. w.)

(baccifẽra.)

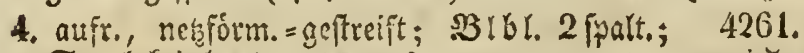
Stgl Eriect). (4 NE. w.)

mariť̆ma. 
5. faft 10 edf., fahar; $\mathfrak{B} \mathfrak{l b l a ́ t t . ~ a u s g e r a n o . ; ~}$ Stgl nicoerlieg.; $\mathbb{B}$ !. ffiellos, fathallan= zettf. (4 NAs, wch.)

4262. procúmbens.

\section{2. nicht,}

Blumenblätter;

A. ungeth.; $\mathfrak{B}$ lo. o. $F r u$ d t fugel., langges 4263. fpiat, 30 ftreif.; $\$ 3$ l. unbebaart $(\odot 30$ r.) (conoidéa.)

B. ausgeranbet; Bld.:

1. unbeboart, nekí. =aber., langgeipint, lảng. als ber \$lmitt; Stgl gabelf., ganz gerabe (O) $S E$, rch.)

2. eyr.; $\$ B$ l. lanjettform, etwas gewimp.; SB Imifte 3 ppalt. $1 \odot$ NAm.)

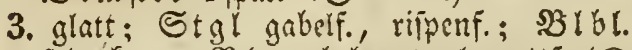

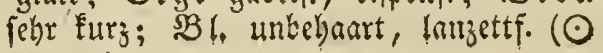
SE. wch,)

C. 2 proltig; Hldecke:

1. Eegelf., 30 fitreif.; $B$ Y. weith $(\odot$ 29. $r \mathrm{ch}$.) 4264. stricta. 4265. antirrhũa.

2. eyruno,

a. fomirr. =baar., aufr.; Irauben u. Sittelblatbe wie vorber; sbl. ge= ftielt, evr.s lanjettf, am (S)runde ge= wimp. ( $\odot \sigma^{>}$SE. w.)

b. feinhant., 10 eq.; $\$ 9 l b l$ aufr, wenig láng. als o. SBlo.; NB K. lángl. lanjettf., gerwimp. ( $\odot$ SAf.)

3. waljenf.=Eegelf., feinhanr., aufr.; II $\mathfrak{r} a$ us ben gepart: Die am (ande itehenden 1 feit: NR it telblutbe gefticlt; 23 l. lan gettf., feinhaar. ( $\odot)$

4. Éculenformig,

a. feinbaar., aufr.; Rect. Rranz zige=

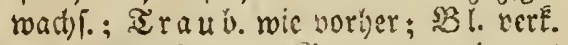
ev)r.=larigettf., am Girumbe gewimpert $\left(\odot r_{.}\right)$, vespertína.

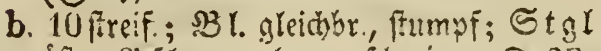
áft.; Dilanje bar. = f wch.)

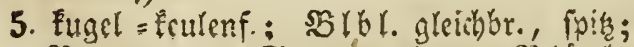

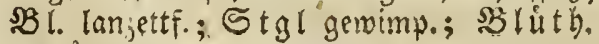

georångt $(\odot$ rch, , sehr kleir) disticha.

4268.
dichotŏma.

inapérta. 4267.

conica.

4269.

clandestína.

6. geftreift; Stgl gabelf., rifpenf.; $\mathfrak{B l}$ l. 4274. gleichbr.(๑SE.Bld, rch., mit wechs, w.Streif.) porténsis. 


\section{Silene.}

7. 10 ecfig,

4275.

a. aufr.; $\subseteq$ tgl aufr., glatt $(\odot$ SE. r.) cretǐca.

b. mit io langen Bábnen, als D. Róbre; Stgl gabelf. ( $\odot$ w, ; r., Bld, r, streif., nur Nachts geöffuet)

D. Fel)r. Elein; $\mathfrak{B}$ IE. nidbt geoffinet; $\mathfrak{B}$ lo. fałt fugel., unbebart, aber.; Sigl aufr., glatt (广 SE. rch.)

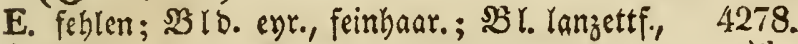
feinbaat. ( $\odot)$

4276. (noctiflóra.)

4277. rubélla. apetăla.

III. Blüth. stiellos in d. Blttwink.

B I Gl. 2 fpalt.; Stgl gabelf.; SBl. unbehaart 4279. ( $\odot \mathrm{SE}$, rch.)

Muscipŭla.

\section{V. Blïthen am Ende stehend,}

A strauchig.

Brbt. balb 2 palt.; $\Re$ ro. Feulenf., untehnart;

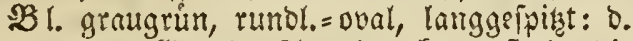
unt. an (S)runde fí)maler, Eurzgeftelt: Die obern am Grumbe beriff, umfanl. (ち As. $\nabla_{0}$, ausw, rcl. $\Delta$ )

4280.

chloraefolía.

B. krautartig.

Stengel :

1. feblt; SBlet. ausgeranb.; YJflange nies 4281. Dergedrúcet (4 9, r.)

2. meift 1 blitthig,

a. nieberlieg.; $: B$ I. lanzettf., fitz., fo lang 4282. als ๖. 33L. (4 SE. P., Bld, braun) vallesiăca.

b. nicht länger als b. SKimfte; $\mathfrak{B} 1$. unbe= 4283 . haart; mit Z. u W. S3lütí). (4 3. w.) Saxifrŭga.

3. melorbtithig; Blblätter:

2. ungeth.; $\mathfrak{B}$ luth. gebuif felt, g'eithlod): bie obern $\mathfrak{B}$ l. berzí, unbehaart $(\odot$ 4. $\left.r_{0} ; w_{0} ; f_{.}\right)$

b. ausgeranoet,

a. auf beyd. Seit. gezalynt; $\$ 2$ l. etwas 4285. filly. (NAf, flo)

b.

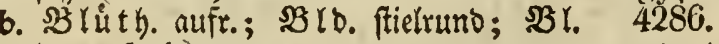
lanjettf. ( $\left.\sigma^{\top} 23 . w_{0}\right)$

rupéstris. 
c. 2 palt.; $B$ Ib. waljenf., an Ente etwas ecé; $B$ l. lanjettf.; sy flanje zott.=grau (4 SE. gbch.)

4287.

flavéscens.

d. 2 lapp., am Grunoe jeces \&app. auf beyb. Seit. mit e pfriemenf. 2rnjals; $B B$ l. glatt: bie unt. runol. = fpatelf.; $331 t$ tffle ge= roimp. ( $\odot$ As. hr.)

e. 4 jabn.; Sial:

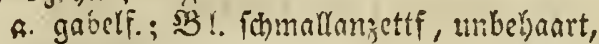

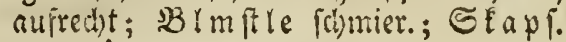
eyr. = lángl. (4 \%. w.)

b. aufr., meift 3 igluth.; $\mathfrak{B}$ l. glcidbreit, fumpf, geroimp.; Sfapf. ev)r. (4 SE.) pusilla.

5. SILER. 203.

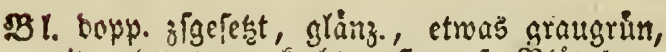
mit gelappt, geferbt., ffumpf. SBláttben; $\$ 3$ เ

4291.

aquilegifolium.

\section{SILPHĬUM. 701.}

Blätter:

1. im Satirl, 3 gåhl., eyr., gezahnt; Ri ipe immer 3 fach sertbeilt; Stenget 6ed. (4 4292. NAm. gb.)

2. rocd) jelşweisfteh̆eno,

a. Uite u.

R. ficoetform.; Stengel fachelfaar. (4 NAm. gb.)

trifoliátum.

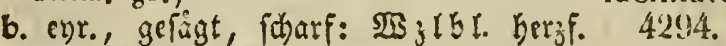
(4 NAm.)

b. nur bie obern u. untern: bie mitt= cěun.

leren 3jabl. im suirl: Ulle feinge= jabnt; S:gl ftierruno; Rifpe gabelf. 4295. ( $4 \mathrm{NAm}$ )

3. 'entgegengeiest,

a. Lile, u. sigemachi.,
a. ungl. gejaint; Etgl glatt, 4 peit.; $b$. - 4 a 4 . S3lo ibup innere $2 B 10$. ( 4 NAm.)
b. entferntgejägt; Stgl:
4296. conjúnctum:




\section{Silphium. - Sinapis.}

1) glatt, 4 jeit.; bie 4 åu pen fo lang als bie innere solio. (4 NAm. gb.)

2) backs, ftielruno (4 NAm gb.)

b. eitrige entgegengefekt: andere wethi., ftiellos, långl., Nlachelhanr.: bie unt. ge= 4299. fägl; Stgl fieltruno, baftr. (4 NAm.) Asteriscus.

\section{SINÁPIS. 536.}

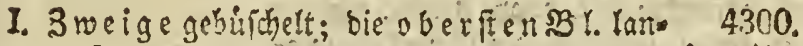

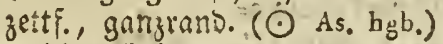
II. nidit; Schoten:

1. vielecf., frot. = aufgeidhosll., ling. als b. 2 ithneio. Echnabel $(\odot$ 29. gb. - $\Varangle$ Sal, unir.)

juncěa.

2. hadirig,"

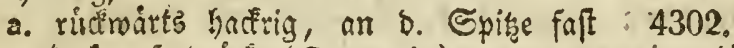

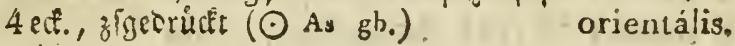

b. nicht, $u$.

a. a!fr.; SB 1. lencrform., fehr fdharf; Stgl hadr. ( $\odot$ Nat)

b. Eurs. als D. Jigeoridte, fety icharfe

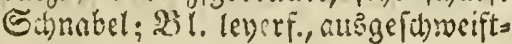
eft., unbei)aart $(\odot$ As. $)$

4301: ซq

(arvénsis.)

perfoliátum.

$4: 98$.

connátum.

c. mit frbief., febr lang., "fobrerotförm.

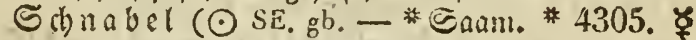
- ఫ Del, Birb., Sivich.). alba.

3. icharf, gellreift; SBI. fdrotfagef., glatt 4306 . (o' SE. gb.)

4. glatt, an bie Iraube angeoridit gb. - Enam. - ఫ Det, Sitid.) (nigra)

5. glatt; Blätter:

a. ficoerf., mit jerichnitt. Lapp.; Sd ot. 4308. cyr. =langl., etwas abftcheno $(\odot-$ gb.) Allióni. b. lenerformig,

a. Zlle u. langl, unbehart; Stgl 4309. litharf; $S_{\text {d) }}$ t. gleith $(\odot \mathrm{SE}$. w.) erucoides.

b. Die oberften lanjettf.: bie ib $\mathrm{ri}=$ gen leverf. unbebaart; $S_{\text {d }}$ oten ctwas abficbeno ( $\sigma^{x} S E$. gb.)

c. mur bie unt. leserf., fcharf: bie oberfan lanzettf.; Stgl idarf;

4310. laevigáta. 
Sd) oten an bie 2raube angebrudt ( $\sigma^{\top} S E$. hgb.)

4311.

incána.

c. leverf. = leyrotiagef., etwas rauds; $S_{\text {ch }} \mathrm{O}=$ ten etwas gegliedert, und abjteheno $(\odot$ Ás. w.)

4312.

chinénsis.

5. SISON. 187.

Stengel:

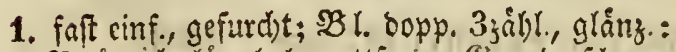

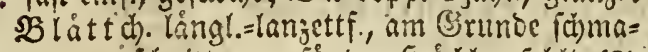
ler, jerichnitten=gelägt; Sgulle fehlt (4) NAm. $g^{b}$.)

2. gahelform.; $B \mathfrak{B l} 3$ fach $=$ onpp. zigefest, mit

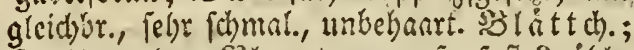
Doloen ben sol. entgegengei. faft 3 jábl., * 4314.

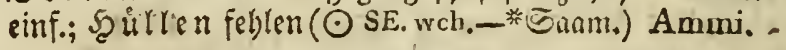

3. ganz einfach; $\mathbb{W Z}_{z}$ l bl. langgeftielt, herjf.= rundl, gelappt, zerichnitt.=gciagt: D. obern fieterf, lanzettf., feilf : D. oberffen 3ipalt., gleichbr, gangrano.: gem. Şülle fehlt 4315 . ఫ

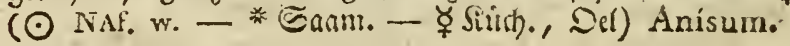

4. åtig,

a. gefurd)t; 58 l. oopp. u. oreyfach 3 zábl.: $\mathfrak{S}$ lát toben langl., gejägt, an Girumbe ungl.: bie unt. 2 zabl; Şüllen fehlen (4 26. 32. w.)

b. rutbenf.: sic unt. $\mathfrak{B l}$ l. gefico.: :R̊latt= chen faft ftiellos, fieoerform. = jerichnitt., icharfgefpist=gejabnt, mit angecrufft. uns terft. Rapp.: bie ob sol. gleichor.; Sy últe wenigblattr.; Dolbe iberhang., wenig. blitb): Strahl ungl., verling. ( $\sigma^{7} \mathrm{SE}$. w.; f.)

5. jefr åft., rifpenf.; \$ేl. gefieb.: bie unt. Blat toh. runol., gelappt $=$ jeridunitt., gez zabnt: bie obern lanjettf.=gleidbr.; So ille wenigbláttr.; Dolb enftrabl verlang. ( $\sigma^{7}$ 8. 48. w.j

\section{7. segĕtum.}




\section{SISYMBRIUUM. 537.}

\section{Schoten abwärtsgebog., kurz.}

\section{Blätter :}

1. gefiebert: Blättchen:

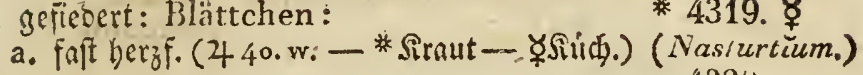

b. lanjettf., gefägt ( $4,22, g b$.)

4320.

(sylivéstre.)

2. fieocrfermig: einige:

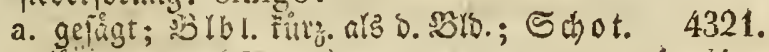
langl.=é)r. ( $\odot$ 47.) (palústre.)

b. lang! = lantactt., gefigt; sE I bl. lång. als o. salo.; Schot. mie vortser (4 47. gb. * 4.322. \% - Sirnut - ఫ lluftr)

c. oopp, ficoerf : 2ulle unbelinart, faft gang= rano.: Die oberfen ungeth.; Ed) oten aufr. (24 14.24.26. gl.)

3. die unt. leyerf. : bie obern bows. fieberf., umfâf.: 2ílle faft ext.; Giriff. fadenf. (4 SE. 9. gb.)

(amphibium.)

4323. tenuifulăum.
4324.

pyrenăcum.

\section{Schot. stiellos, in d. Blttwink.}

\section{Blätter:}

1. gezahnt=butibt.; 5 d o ot. eingeln, faft ftiellos 4325 . (○ SE.) supínum.

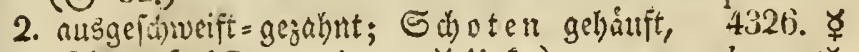

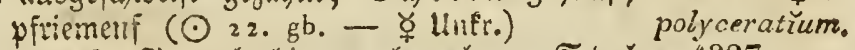

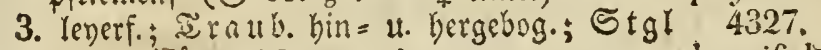
aufr., blitttr. $\left(\odot S E_{\text {w. }}\right)$ bursifulüum.

\section{I. fast ohne Stengel.}

B L. Lamzettförm., butht. =geingt, foft glatt; 4328.

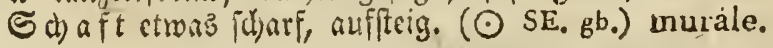

\section{Bl. gefiedert, fiederf.}

\section{Blätter:}

I. gleibfớcmig,

1. fieserformig,
a. ftumpf, gezahnt, umialt; Stgl rưd"s waits bader. ( $\odot$ SE. gb.)
4329.
obtusangŭlum. 


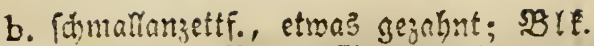
lang a!s 5. Sold.; Sotot. litarf $(\odot$ SE. gb.)

c. grob=uno frumpigezahnt, untebaart; ङ hot. noffebents, gleidjbr., mit bour.

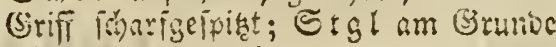
frimbaar. ( $\odot$ SE.)

2. Fotrotogeformig,

\section{0.} aspěrum.

4331. gallicum.

a. roeit,

a. am Siotice icharf, ganjrano.; $S_{\text {di }}$ de ten fatomf, eingebeg. = abftebeno $(\odot$ - gh.)

b. mit faft glcichbr., ginjrand. Eapp.; SBlmple lobloff $(\odot$ 26.)

b. jott., gezabnt, toeisirait; Stgl zott,

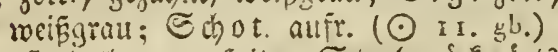

c. ftathethaar., fwis; Stgl ructrórte bactr. (广 4 8.)

d. nacát, gegabut; Stgl glott; $S_{\text {djo }}$ t. aufr. $(\odot=9$. gb.)

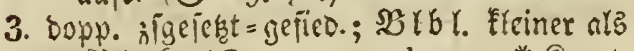

D. SBblocere $(\odot 24.25 . \mathrm{gb}$. - * Sirnut, Saam. - ૪ Del, llnfr.)

4. mel)e als bopp. șigeictet, filz.; SBlbl. grip̄. als o. $\$ 3 ! 0$. (5 Af.)

4332.

austriücum. 4333.

altissimum. 43.34.

Colúmnae.

4335.

(Loeselü.)

4336.

(Irio.)

* 4337.

(Sophía.)

4338 .

milletolŭum.

\section{Unigieichformig,}

1. গ⿰彳 Stglbl. lanjettī., "Durch e 2tribang falt

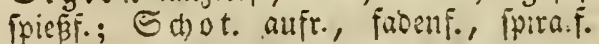
georebt $(\odot$ SE.)

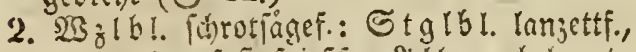
ganzranb., falt fipiest.: Ulle unbebacrt;

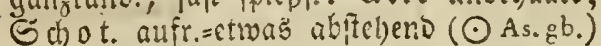

3. Lie unt. jotrotfágef, gezahnt; D. obern gefieb., mit gleichbr., ganztano. Jiebern; 4341 . Sゅot. rectitwintl. abftebeno $(\odot \mathrm{SE}$. gb.) pannonicum.

\section{Blätter einfach.}

\section{Blätter :}

1. Fateif. = evr., gejabnt, umfall.; Stgl nact 4342. (4 As.)

2. lingl.=lamjettr., gejabnt, feinbaar., geftielt; 4343 . Schot. abftetend (4 1.8.21. gb.)

3. lanjettf., gejahnt,

4339. contórtum.

4340. subhastátum. 


\section{Sisymbrium, - Sium.}

a. ftiellos, unbebaart; Schot. angebricat; 4344 . S‡gl aft., ausgebreit. ( $\sigma^{\prime} \mathrm{SE}$. gb.) hispanĭcum;

b. umfaif., feinl)aar., pfeilf.; S d)ot. aufr. $=4345$.

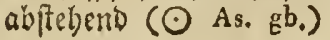

pumilum.

\section{SISYRINCHIUM. 540. (46²)}

Schaft 2 idancio., geflug., cinf., faft blattlos;

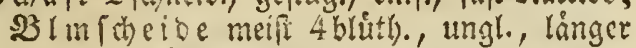

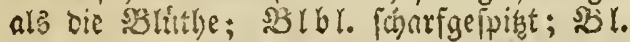
fatwerotf. (4 NAm. bl.)

4346.
anceps.

\section{SIUM. 216.}

\section{Blätter:}

I. glciö:fơrmig,

1. gefiesert: Blättchen:

a. ungl. gefägt, jerfichnitt.; Stgl ftiels rumo, aufr.; Solben geftielt, Den \$Bl.

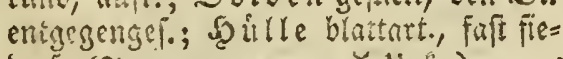

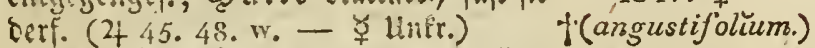

b. gleiỏgelâgt, långl.; Stgl nieverlicg., geflecitt; Dorben sefitielt, in o. SBltt= wink.; 5 irlte foglt (24 38. w.)

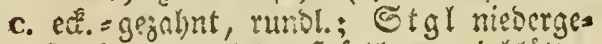

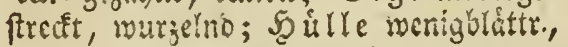
lanzet

d. vielpalt. =gleichbr. = Fîbenf., im Suirl 4350 . fiebeno; $\mathbb{S S S}_{\text {z }}$ f fnoil. (4 SE. w.) verticillátum.

2. berablauf. gefieb., grautgrunl.: $B$ la $t$ thd. (d) mallanzetif., id)arf = enoripelart. = feinge. fägt; Stgl geffreift; 3weige aujge= breit, Shullten pfriemenf. (43.30: wch. - ६ (Infr.)

3. Dopp. zfgefest: B 1 lattch. gleichbre, fte 4348. (nodiftorum.)

4349. repens. chenofpis, ganzrant, rimenf., faft 2 fralt.;

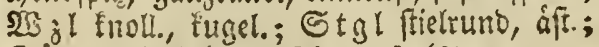
Jitile vielipalt., pirientenf. (4 28. 29 . w. - ૪ Siti(i).)

II. untgleichformig,

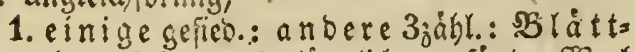

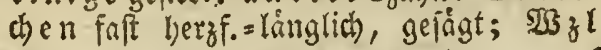

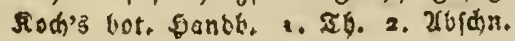

4351. ఫ

(Falcaria.)
4352. ్ㅜ

(Bulbocastănum. 
Enoll., fpinbelf., gebůibelt; mit En no ol= * 4353. ช ben (21. As. w. - *arit. - ఫ Sitch.)

2. Die utrt. b. 23 a ffer bopp. ficoerf.: bie (Sisŭrum.) unterften $B$ latto. Ercuzweisfichento, fieberf., langgefpist: bie uber 0 . SRB a $=$ fer gefiebert, mit lanjett, am Sirunee ungl., icharf $=\mathfrak{u}$. feingelingt. SBlattchen; mit (5) DDOLL. (4 4r. 47. w. - ఫ Futt.) Ulift.)

22. SMILAX, 787. (W. 77.)

\section{Stengel:}

1. edig,

1. (tachl.; Blätter:

a. 7-9 ripp., leserart., tief ber form. od. ipieff. = langettf., mit ohrf. Ŕnbang, am Sande ftadbl., unten an D. Mittelrippe

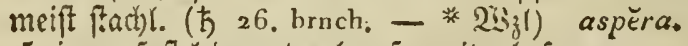

b. 5 ripp.; faft leberart., hersf., mit ohrf. 2rnbang, am Grrunde etroas aígefífect, am Ranoe gewimp. = ftachl., oben sunt, unten an 0 . I) Rittelrippe furw. (tad)lid) (ち NAm: $\Delta$ )

2. wenig ftad)l.; bie leberart., 5 ripp. Bl.:

a. långl., bergf., mit obrf. 2lnbang, am Raride gewimp. = (tachl., unten an ser Mittelripue ohne Etadbein (ち NAf. $\triangle$ )

b. eyr.= kerof., ftumpf, foharigeipitat, ge= gen ben (srund fted)enofpis = gezabnt (ち NAm: $\triangle$ )

3. Faft obne Stadheln; $\mathfrak{B}$ l. Yeberart., enr., 7 ripp. am Rante uns an D. Sittelrippe etwaz frachl. (方 SE. $\triangle$ )

II. faft 4 feit., wentig frachl.; $\$ \mathbb{3}$ \%. leberart., olne Stachaln, eyrumb = lanjettf., feinipisig, undeutl. 5 ripp., unten etw6.5 weiß̈grún ( NAm. $\triangle$ - $\mathfrak{W S}_{\mathrm{g}}$ )

III. meift fitelruno, ftachl.; $\$ 3$ l. ent. = runol., langgefpist, am Sante ichari, faft 5 ripp.: bie unt. fỗ berjf. ( $5 \mathrm{NAm}$, braun $\triangle$ )

4356.

hastáta.

4355 .

4357.

Aipíni.

4358.

rubens.

4359.
nigra.

* 4360 .

Sarsaparilla.

4361. rotundifolia.

IV. gang ficlruno, ftad)l.; $\$ B l$. eyr., unge= Erum!nt=icharfgeipist; die o. Frúbling 4362. 3ripp.: 0. Des Gom mer 3 5riph. (ちNAm, $\triangle$ ) cadúca. 
5. SMYRNYUM. 199.

20 ; Ibl. 3 fach 3 zadhl., ungeth.: Die obern Etglbr. enr., fcingezalynt, umfall.; Stgl oben 4 fliigel. $\left(\sigma^{7}\right.$ SE. gb.)

4363.

Dioscorrdis.

\section{SOLANUM. 139.}

\section{Strauchig.}

Stgl ohne Stacheln, Fletternd; BI. ganj= rant.: sie unt. hersf. =eyr.: bie obern faft fpieff.; $3 \mathfrak{B}$ lit $\mathfrak{h}$. in 2 theil. Doldentraus. in

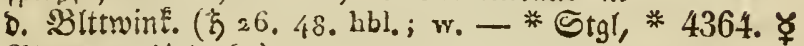
Rinde - $\checkmark$ tinfi.)

† (Dulcamára.)

II. kráutartig.

Shne Stacheln; Blätter:

A. geficiert,

1. ganzrand.; mit Endoolsentrauben:

B I mftle etwas geth. (4 SAm. w.; v.; 4365. ఫ

bl.; p. - ఫ Sitidy., Futt., Farb. get(b) tuberósum.

2. zerichnitten; Trauben:

a. 2 theil., blattlos; Frit tht te unbebart 4366. ซ̧

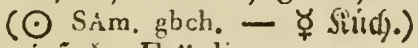

b. einfont Frülite:

tLycopersǐcum.

a. ctruas jott.; sgiolapp. faft folang ats D. SBIf. $(\odot)$

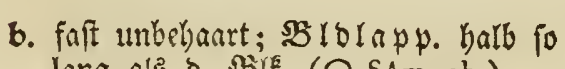

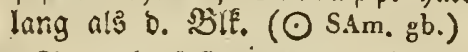

4367.
Pseudo-Lycopersicum.

4368.

Hunboldtri.

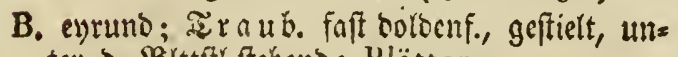
ter 8 . Bittifft féheno; Blätter:

1. ganzrant., langgefpiazt; 3 to eige unbes 4369 . haart, cd.., gezahnt ( $\odot$ NAf. w. grnch.) guineènse.

2. ausgeichreift:

a. Ulle ausgeläbleift, faft unbehaart; 3weige ftriegl.=feinbaar., ef́., gejabnt $(\odot S E)$

b. bie obern gangrand.: bie : etras altsgefdrweift; 3 roeige unbe=

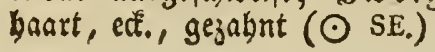

4370.

miniátum. 
3. gejabnt = ced.,
a. unbebart: Zweige:
ค. wie trorbit $(\odot$ SE.) melanocerăsum.
b. nicht $(\odot 22.29$. w., wohlriech. .- * 43i3. ఫ્ * Siraut - ఫ Unfr.)

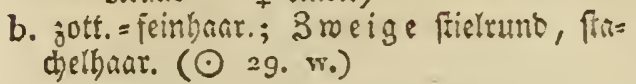
$+($ nigrum.)
villósum.

\section{SOLDANÉLLA. 113.}

\section{Blkrone :}

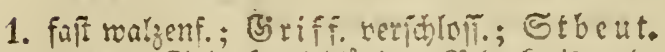

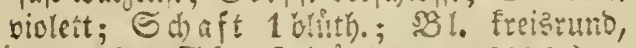

'ganjtains.; EEapf. blául (4 g. hblch.) minima.

2. malgenf. =glodenfo, Faum bis jur Şaifte jer= rifi.; SBloranp. abfebend; Sriff. langer

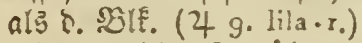

3. erien=tribterf., uiber sie Soúffte jerrifien; Biblapp. an D. sRif. angetricet; (Griff. folang als \$. SBIf. (4 9. lila-bl.)

$43 \div 6$.

montána.
4375.

4377.
alpina,

\section{SOLIDÁGO. 682}

I. Mit 1 seit. Traub. u. Rispen. A. Bl. 3 fachgerippt.

Trauben:

I. rifpenfórmig; Stengel:

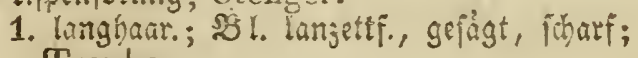
Trauben:

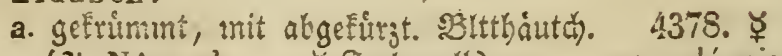
(4 NAm. gb. - o ₹rrb. gelb) canadénsis.

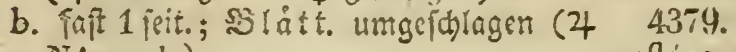
NAm。 gb.)

2. etwas haar.; $\Re$ l. Lamjettí, unbehaart,

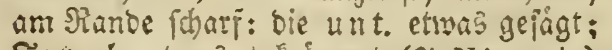

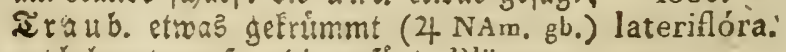

3. unbehacrt, aufr.; bie gejaght. Blätter:

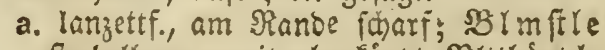
fradellhaar., mit abgeturest. SBlttháuth. (4 NAm, gb.) refléxa. 4380. 
b. ichatlanjettf:, unbehart, am SRanbe 4382. rauh (4 Nam, gb.) serotrna.

II. äbrenir., auTr., vor ๖. SBefrechtung ủber=

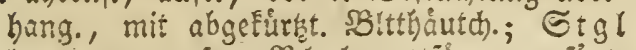
langbaar., aufr.; $\mathfrak{B}$ l. lânjettifótm., gejägt, foharf, unten zott. (4 NAm, gb.)

4383.

procĕra.

\section{B. Bl. aderig.}

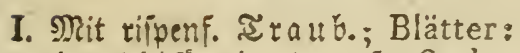

A. gleiviformig; D. alifr. Stgl:

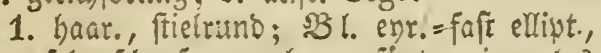

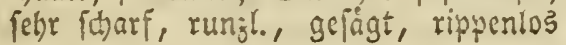
(4 NAm.g.)

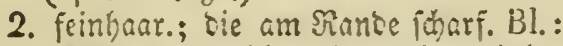

a. gangrano., fdi)mallanzettf., unbehaart 4385. (4 NAm, gb.)

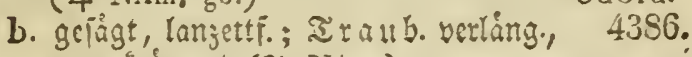
umgefrummit (4 $\mathrm{NAm}$.) recurváta.

3. unbehaart; ס. gintt. Blätter:

a. ithmallanjetrf., etroas fícijh., ganjs=

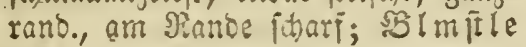
haar. (4 NAm. gb.)

b. elliptifon, gefógt; mit mápiggros. SBlttlántut). (4 NAm. bgb.)

B. ungleichformig; D. aufr. Sigl:

4384.
asperrá. odóra.

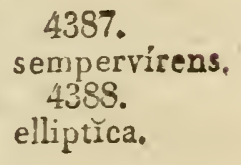

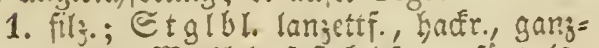

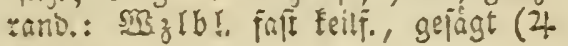
N.Am,gb.)

2. unbehaurt; Blätter:

a. unten zott., ellint., tiẹgeiagt, lang=

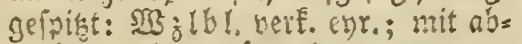

b. unte')aart; Tralbl.:
a. lánglid = fpateff.; sie irbrigen

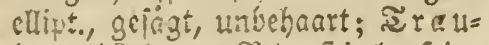 ben abifeteno; SBlmficte fein= baur. (4 NAm.)
b. verE. evr.=lángl.: Etglb! ellipt.: zclle unbegaart, iowri = 1t. ungl.

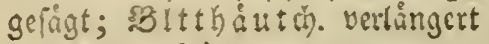 (24 NAm, gb.)

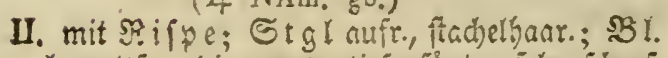
langetty.: Die unt. ticfigfägt, iefre icharf, tunjl. (4 NAm. gb.)

4389,
nemorális, 4390. 


\section{1. mit aufrechten Traub.;}

A. Bl. gleichförmig.

\section{Stengel :}

I. baarig; Blätter:

1. ellipt., baar.: bie unt. gefågt; 3meige mit flein. SBláttch); $\mathfrak{B}$ loblát $t$ d). \{tumpf (4 NAm. w.)

2. lanjettF., fpik, gefiagt, unbelaant; Stgl ganz cinf.; Enotraub. eiuf., mit ver= lång. Sittthåutch). (4 9. gb.)

II. zott.; $\mathfrak{B}$ l. elliptifoh, etwas icharf, geftielt; $\mathfrak{B}[t t \mathfrak{b} u t \mathfrak{b}$. wie vorber (4 NAm. gb.) petioláris.

III. Feingaarig; Blätter:

1. Ianzettf., auf beys. Eeit. fdjarf, an beys. Eno. Fchmaler, 3 fachgerippt, ganjrand.; 4397.

$\mathfrak{S}(t \mathfrak{t}) \mathfrak{a} u t d$. wie vorber (4 NAm.) lithospermifolla.

2. fchmallanjettF., båut, am (Sirunde id)ma= ler, unbebaart, am Ranoe fobarf: $\delta$. $u n=$ terften ctwas gejagt; - St Engel aufr.; Błttháutch. wie vorher ( 4 NAm, gb.) viminèa.

3. Eeilf.=lanjettfórm., gejågt, etwas baar.;

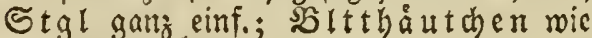
vorber (4 WE.)

IV. unbehaart,

1. nufrecht; Blätter:

a. Ianzettfórmig,

a. unbebaart; mit nicht lang. SBlttbåut. then (4 NAm. gb.)

b. auf berd. Seit. glatt, fleitch., ganz= rano.; IIraub. ripenf.; $\mathbb{S l}$ Infle

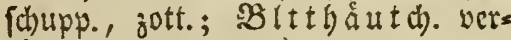
ling. ( 4 NAm, gb.)

b. ellipt. = lanjettform. : bic unt. sefägt; Iraub. zlgejog., furrer als bie SBl.; B Ittháath. wie vorber (4 9.)

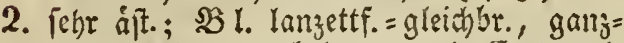
rano., 3 ripp., unbehaart; mit Enodol= bentraub. u. B Ittháth. fo lang als b. Scheibe (4 NAm. gb.)

3. rifpenf.; $\Re B$ I. lanjettf., gefingt, unbel)aart,

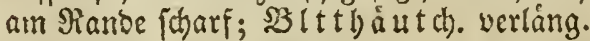
(4 NAm.)

4. fhrågftebend; 3 I. lanzettF., eitwas fleilch., 4394. bicŏlor.

439.5. minita.

4396.

\section{8.}

4399. cambrǐca.

4400. caesia.

4401. lâevigáta.

4402. alpéstris. ganzrand., auf bend. Eeit. glatt; $\mathfrak{I} r a u b$.

4403.

lanceoláta. livĩda. 
rifpenform.; BImfte fohupp., unbel)aart; 4405 .

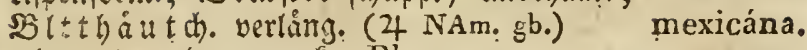

5. hin u. horgebogen, edf.; Bl.:

a. enrunb langgeinist, gciăgt, unbehanrt; 4406.

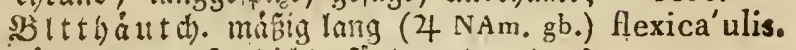

b. Loingl.=lanjettf., bichtgefágt, uitten etwas

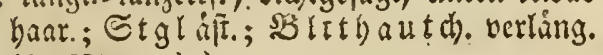
(4 NAm, gb.)

4407. ambigŭa.

\title{
B. Bl. ungleichförmig.
}

Stengel :

1. Ftachelhaax., rifpenf.; BSt. Lamzettform., auf beub. Scit. fitarf: Stglbl. gejägt: 3 we i=

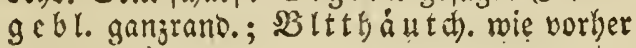
(4 NAm.)

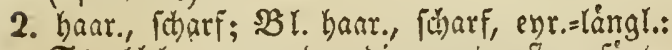
Etglol. ganzrano.: Die unterft. gefágt; bie blutbetrag. Bueige rifpenf.; Tralts ben georạngt; : șittháat d)en wie vorher (4 NAm, gb.)

3. oben foinhaar. ffielrunb, aufr., oben åf.; 4403. lirita.

Etgibl. lamjettf., an bero. Ento ichnaler, gefagt: f. unt. ellipt., etroas Gaar.; $\$ B l t t=$

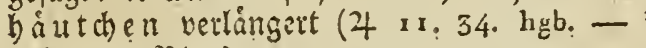
* Sirmut, B̉im.)

4. unbehaart, aufr.; StgIbl. lanzettf., ganz= rand., unbehaart, am Siande fibarf: $23 j l=$

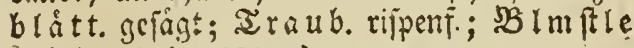
unbebarart (4 NAm.)

\section{SONCHUS. 615.}

\section{Blätter umfassend,}

A. gleichförmig.

\section{Blätter :}

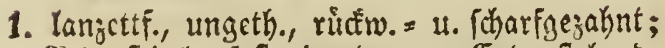
Slinftiele faft cinzern, am Enbe fteheno, nacft (4 45.47. gb.)

4412.

maritĭmus.

2. lángl. =lanzettf., feingejalhnt, etwas buat)t.; Blinftle etwas fitz., Doldenf.; $B$ lo. uns behaart $(\odot$ 29. hgb. - * Siraut - $\Varangle$ Futt., unfr.) 
3. längl.s fieterf., gejubnt,

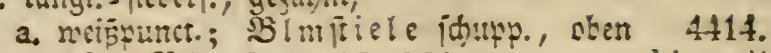

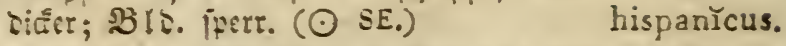

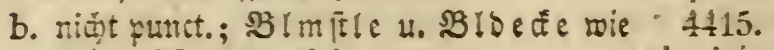
vorher $(\odot$ Nis. obs, ) - tingitánus.

B. ungleichförmig.

Etglblatt. umfoñ., lingl., fait gangrunb.:

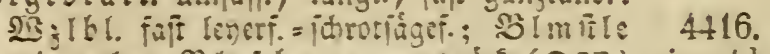
rie vother; $\$$ Is

\section{Bl. nicht umfassend,}

A. gieichförmig.

Blumenstiele:

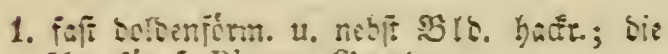

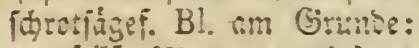

a. iffelf., (4 5. 4i. sb.)

b. Ferjf. (4t 29. gb. -

2. soism . Filj.; Bld.:

4117.
(palisstris.)
$4 \pm 18$. o
(arbensess.)

a. unbeguart; $\mathbb{B}$ l. Fitcirfo, gejahat, am 4419.

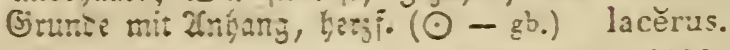

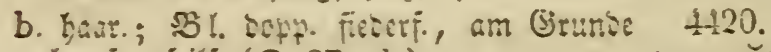

3. rifichictmig,

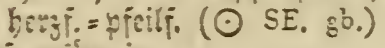

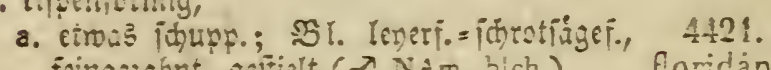
feingejahnt, geffielt ( $\sigma^{7}$ N.Am, blcb.) foridaus.

b. nidit iutupis; Bl.:

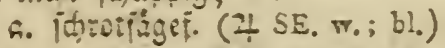

422.

Plumiéri.

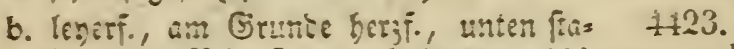

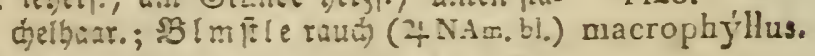

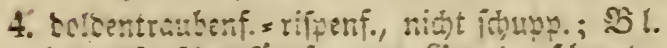

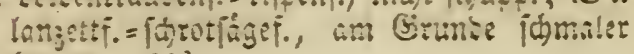
(4 N.As. bl)

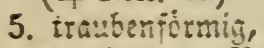

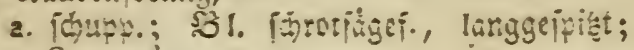
Stengel:

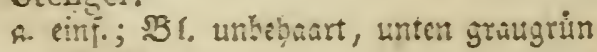
( $\sigma^{2}$ NE. bl.)

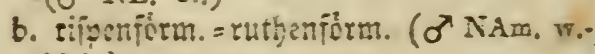
blcb.)

4424.

tatarǐcus. 
b. nidt (chusp., raud); mit Rebenbl.; BI. Throtiagefo, am Grunte pfeilfo, uns behaart, unten graugrin (4 9. bl. -

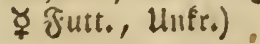

4427. $\not$ alpinus.

\section{B. ungleichförmig.}

\section{BBImftre forusp.; Blïthen:}

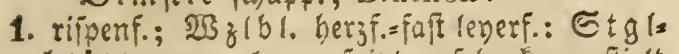
biát t. eyr., langgeipiett, fegr Eurgeffitieit

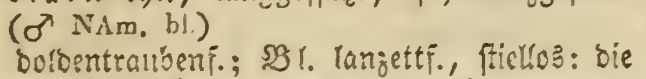
unt. jubrotiazer. = gezabnt: bie obern ganzz ramb. (4 NE. blo; w.)

4128. acurninátus.

sibiricus.

\section{SOPHÖRA. 334.}

1. SBaumartig; SB 1. fieterf., mit bielen eyr., 4430. unbehaart. SBlätth. ( 5 As. gbch.-w. $\Delta$ ) japonìca.

2. frautart.; B 1. gefies., mit vieien láng!., 4131 . gott. $\$ B$ !áttch. (4 As.) alopecuroídes.

\section{SORBUS. 392. $\left(3 \mathrm{~g}^{\circ}.\right)$}

\section{Blätter:}

1. ungepanttgef.ebert; gem. Blattstiel:

a. zott. = Fembaar.; $\mathfrak{B}$ (åt tdo. bopp. geiägt,

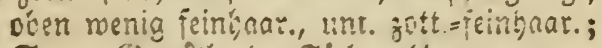

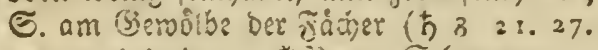
w.., starkriech. - * iepro, Ehwwammఛ̧ (Berb.)

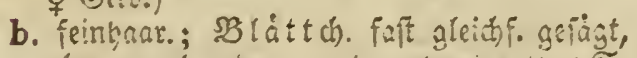
oben rumito = acer, unten etroas jott.;

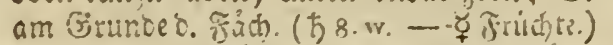

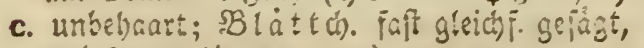
unbelyaat. (5 Nidm. $w_{0}$ )

2. zufunmenbang. = gefiebert, oben unkehart,

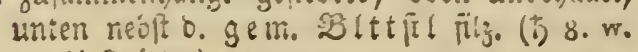

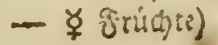

* 4.9.2. ซ్

(aucuparia.)

4.433. Ұ

(d)mesica.)

$4: 3.3$.

americána.

4.435. ซ్ hybrida. 
21. SPARGANIUUM. 733. (545, W. 20.)

Blättคr :

1. flach, nicberlieg.; gem. B $\operatorname{lm}$ ft $\mathfrak{l}$ cinfach; Narbe enr., felor furz; M. Nóp fó. meift einjeln (4 42. 44)

a. áf.; ; $B$ l. an b. Eeiten bobl (4 44: w.) (ramósum.)

4438.

b. cinf.; $B$ I. an b. Seiten flach (4 44.) (simplex.)

\section{SPARTIÁNTHUS. 561. $\left(542^{5}\right.$.)}

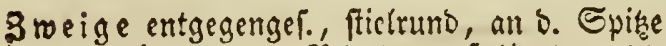

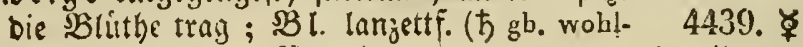
riecb. - $\Varangle$ jarb. 23eber) juncěum.

\section{SPARTIUUM. $560 .\left(542^{4}\right.$.)}

\section{Blätter:}

1. Xlte $3 j_{j}$ åhl.,

a. gleichbr.; 3 weig e entgegengef., georängt, e:t.; $\mathfrak{g}$ lttft le oben breiter, onur.; End traub. Fopfi. (5 4. 21. gb.)

b. rerk, eyr., geftielt; 3 weige ftielrumb, geftreift; Seitenbluth. gepaart, über= bang. i $5 E$. gb.)

2. 3 zábl. u. cinf.,

4440.
radcátum.

4441. patens.

a. etmas feicenart.; 3roeige gan gerabe, 4442. geftreift, uberall blutíetrag. ( $5 \mathrm{SE} . \mathrm{w}$.) multiflórum.

b. longl.; 3 weige eff.; $3 \mathfrak{B}$ t 1 then in ben Slttwinf.; Şúlfen am Rambe haar. (ち 19. gb. - * Jiraut, 3ilm., ভaam. - *443. ఈ ఫุ Siú, , Jabr. 2bsber, Farb. gelb, (jerb.) (scoparüum.)

10. SPERGǓLA. $370 .\left(305^{*}.\right)$

Blätrer:

1. im Suirl;

a. mit 10 Stgef.; o frudttrag. BI me

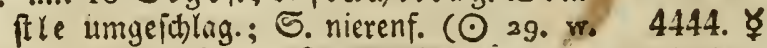
- ૪ Mibl, jutt., Der, Lutr.) arvénsis. 
b. mit 5 Stgef.; S. an b. Spitse einges brifft, gefing. $(\odot$ 11. 19. w. - ఫ Jutt., Del, Uhitr.)

2. entgegengelest,

a. glatt,

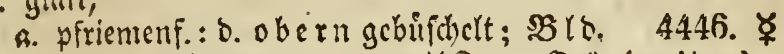

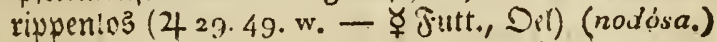

b. gleichbreit; siglmfiele eimzeln, iebr lang; mit 5 Stgef.; Stgl trich). (4 4447. SE. 9.)

b. getoimp., fait 1 feit., gleichbr.=pfriemenf., $4+48$. nactenf.; mit 10 Stgef. $(\odot, 34$.$) subuláta.$

\section{SPERMACÓCE. 69.}

231. lanjettform., ctwas geftielt, oben liharf; Etengel unbebaart; Stgef. veriblofien; B! thth. falt im Suirl; S. ftachelhaar. ( $\odot$ Am. rch.- wch.)

4445. $ఛ$

(pentándra.) 
2. ausgebreitet = rifpenf.; $\mathfrak{B l}$ l. enr. =ellipt., an beyo. (Enoen fpit, unbeharat, grobgelägt (万) NAm. rch.)

3. gebrángt=rifpenf.; $\mathfrak{B} l$. längl. = langettf., etwas isis, am (3runde teilf., gejägt, unbebart ( $h \mathrm{hr}$. )

4454.

carpinifoľa.

4455.

(salicifolia.)

\section{II. in Doloentrauben; Blätter:}

A. gleidfformig,

1. lang fógt, 3 fad gerippt, unbegnart; mit Endodoldentrauts. an b. Eitr., jưn= gern 2effthen (ち N.As, wo)

2. Fpatelf., fois, 3 fachgerippt, gomzrant., untehaart; Doldentraub. Piellos in b. SBttwine, 6-12 b'ưth., am stumbe iduup. ( 5 - w., sehr klein)

3. vett. enruno, unbehaart,

a. an b. Epife jeridnitt, = gefagt, we= nig gewimp.; De!sentraub. lang= gefticlt, ant 0 . jungern ziefteden ( NAs, w.)

b. gangranoig, fumpf, 3 fachscripht; Doloentraub. Ficílos in D. SBitt= mint. (5 $\mathrm{NAm}, \mathrm{w}$.

4. ereunt, unowhaart,

a. 3 Yapp, ungleid $=0$ ob faft bopp. zets jămitt. =gefagt; croboloentralts ben geffielt, vieibluth. (方 NAm。 wch. u. hr.)

b. faft bopy. = gefigt; E Enoboloen= traub. Langsefficect, faft traubenform. ( 5 w.)

B. ungleidyfermig,

1. ver\%. ent., 3 fachgeripnt, unkebarat,

a. Fois: an o. unfu

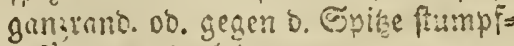
gefingt: an $\delta$. blumentrag. gantz=

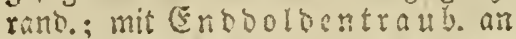
D. vielyabl., zur Seite fachento, Eurz., juing. Jeffechen (b stow.

4462.

crenáta.

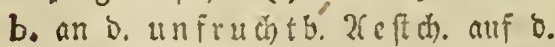

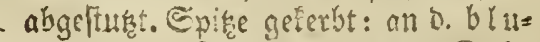
mentrag. ftumpi, ganzrani.; (E) boroentrauber mie yorber (b SE. w.)

4456.

alpína.

4457.

acutifolia."

4458.

chárnaedrifola.

$4459^{\circ}$

hypericifolia.
4460.

opulifolǐa.

4461.

ulmifolia. 


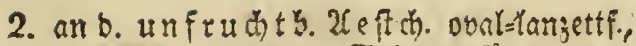
ganz̧rand. vb. an b. Spitze gejăgt: an $\delta$. blumentrag. lanjettip. und ganjrant.: acre foik, gemintp., feinhar.; D olden= t: $\cap 4 b$. wie vorther ( $\hbar$ i. w. w.)

III. in geftielt. Enoontoen; s3i. rund, faft bergf., flumpfociapst, gejagnt, 3 u. 5 ripp., unbelgart (bNAs. w.)

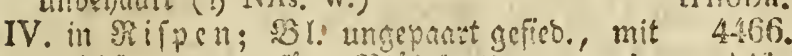

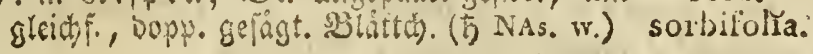

\section{Vrautartig.}

\section{Blätter:}

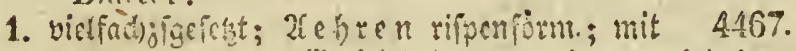
ganzgetrentito (s)efitl. (4 8. w.) (Arúncus.)

2. cinfabgefroscrt: Blärrchen:

a. 2urfe gleidif., gefigt; mit Dolden= traub. (4 5.54. w, ; rch,, wohlriech, -. * 4468. 8

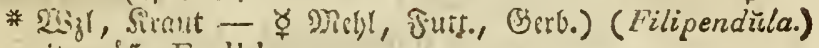

b. mit gróp. Endblatt:

a. 3!app: Seitentlatt doen ungeth:

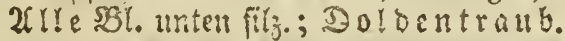

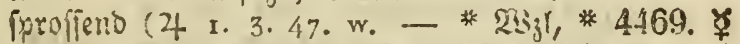
Siraut, Bim. - $¥$ Meli, Fintt., Sirtb.) (Ulinaria.)

b. 7 lapp.; Seitenblättchen:

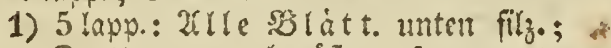
Dalbentraub. áf., zfgezog. (4 4470 . NAs. w.) digitáta.

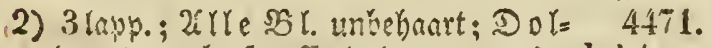
Dentraub. purofiento (4 NAm, r.) lobáta.

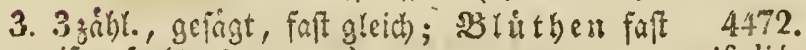
rifpenf. (4 NAm. w.)

trifoliáta:

\section{STACHYS. 467.}

Quirle:

1. 6 blitthig; Blälter:

A. gleithformig,

1. Geriform, geftielt,

a. langgeppint $(\odot$ ×. 4. r.; braun - $4473 . \not$ $\Varangle$ ঔarb. ger(b) 
b. aberig, haar.; $\subseteq$ tgl niebergc|frecti ; Slippe ber 351 f. 2 fpalt., ausges breit., umgefothlag. (4 SE. p.) hirta.

2. enr. =lanjettf., 3 ripp., glatt, geftielt; Etgl aufred)t $(\odot$ 15. w. u. r.; sb.u. braun - Siraut - ఫ llutr.)

3. ftumpf bersfo, faft nadt; $B$ IE, fo lang

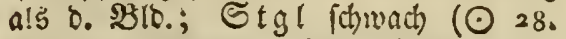
wch, u. rch.; 月. -

B. ungleichf., langl., feinhare, ftumpfges jabnt, am Brunce fcilf.: $\mathbb{N B}_{3} l \mathrm{bl}$ l. falt berzf.; Siebenbl. fitellos, lỏngl., ganj= rano. (4 SE. sbch., wohlriech.)

4475. ఫ (annǐa.)

4476. 후 (arvénsis.)

4477. maritrma:

II. meift 6 thuthig; Blätter:

1. iama!!anzettf., balbumfafi., fiellos (4 29.38. 49. p. u. w., übelriech. - ఫ Unfr.)

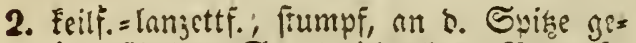
fágt, ftiellos; St gl nicberlieg.; ßJ Ibede fait frechento (4 NAm, p.)

3. langettf., ftachelhaar.; ftumpfgefiagt, faft fliellos; Stgl aufiteigeno; Suirl fajt ábrenf.; $B$ ID. fecti)eno (4 4 x. I 3.)

4478. 후 (paliustris.)

4479. scordifolia.

4480. recta.

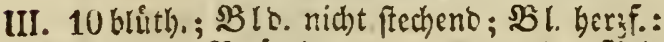

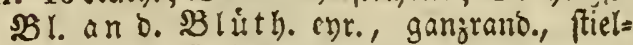
los; Stgl ftathelloar. (4 As. r.) 4481. heracléa. IV. vielbluthig; Blätter:

1. friritgarig,

a. ftumprgejabnt, rumbli, 3 lo jånne lanzettform., ftechenoipis; Slippe d. Ble langl., gemỏlbt, julekt Hinges fohlag ( 4 As.)

4482. cretřca.

b. Tpisgezahnt; SBlozả hne enr., ftecheno= fpić; Slippe s. SBlE. língl., ausge= rario. (4 9. up.)

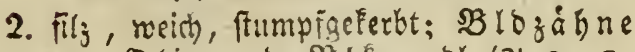
ent.: SIlipue D. SB IE. runol. (4 8. 23. $x_{0} ;$ w.)

3. jott., lingl., faft herjf., geferbt; $\mathfrak{B}$ \b.

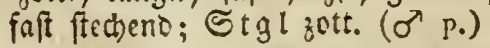

4483.

(alpina.)

4484.

(germanǐca.)

4485. : intermedĩa. 4456. lanáta. 
5. STAPHYLEA. 243.

İaub. hamg.: Blätter:

1. geflecert; mit '2 Etweg. (古 4. 8: 26. w. u. 4487. ఫ̧ sch. - $\Varangle D e l)$

2. 3 zágh; mit 3 Etreg. (5 NAm. T.)

(pinnáta.)

4483.

trifolìa.

\section{STATICE. 253.}

I. Mit Schaft:

A. ripenfórmig; Dlätter:

1. gleidbr., rimenf; 3 weige 3 lant. 4489.
(4 NAs, r.)

2. länglith,

a. unbeţart, an Rante wellenform.

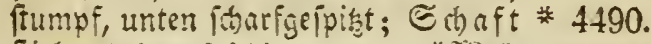

ftielruno (4 56 hbl.: r.; w. - *2:

b. Feingiar., mit bưicheif = fternf. gॄ= fteilt. Scaur.; હ (4 As. bl) ) latifolis.

3. ellipt., fait unbehaurt, unten iharfase 4492 . fpitst; Ext) a ft feinbaar. (4 NAs, dbl) Gmelini.

4. langl. = juateliơrm, foumpi, glatt, faft

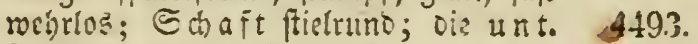
3reeige unfruchtb. (4 As. r.) oleiforia.

5. Fuateliormig, 4494.

a. an o. Epige eingerridet (4 SE. b1.) cordáta.

b. nicht: $\odot$ cha it jielrumo, geglietert, pehr aft., hin u. bergésg., ausges breit.; yjflanje punct. = itharf, bes 4495. reift (4 As, hblo, mit e, r. Streif, $)$ echioides.

B. gabe!förm.; $\mathfrak{B l}$ l. lanjettfórm., fibarfiges fpikst; Blutheal tochi., entierntiteheno (4 As. ros.)

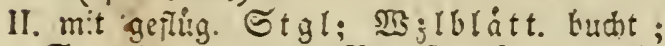
Etglbl. lanjettF.; SBlmitle Esili, 3 Fat)=

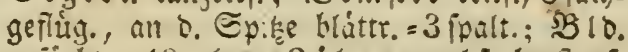
gefürbt, 10 jafin.: 3ábnc medjo. borfienf. (- hgb., Bld. bblch.)

\section{6. \\ tatarǐca.}




\section{STELLARĬA. 359.}

\section{Blätter:}

1. Kerzf., geftielt; Rifpe mit åf. SOInffielen ( 44 4. vr.)

2. enr., ftiellos; Etgl gabelf; ; Slith. ein=

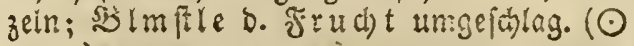
9. w.)

3. eyr. =lanjettf., unten aberles; Stgletwas

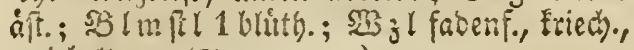
zovieseltrag. (4 I. 9. 48.)

4. lanjettformig,
a. feingefagt; 3 rbt. 2 pralt. (4 4. w.)
b. unbel)aart; 3reige aufr., ganj cinf.; Enoblinfit meift einjeln; SZlbl. groúp. als D. $2810 .(2+9$.

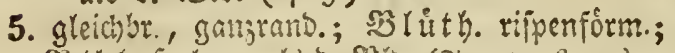
SBlbl. ó lang als b. SB:D. (4 25. 26. w.) graminĕa.

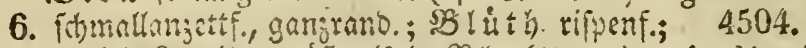

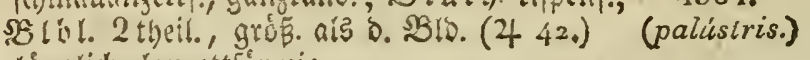

7. långlid) = ramgettférmig,

a. etwas bia, grangrim; $B$ Im mite 1 britt),

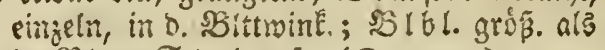

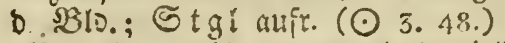

b. SBImftie meif gepart, 1 oo. vielblith.;

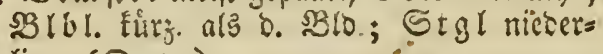
lieg. () 42.)

8. Iángl, feinthaar.; Stgl nieberlieg.; $3 \mathrm{I} \mathrm{m}=$

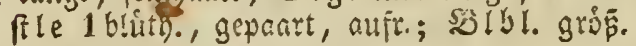
cls จ. $\mathfrak{B} l$ lo. (24 SE. 9.)

9. fpatelf., Etg! aulfr., 2 part.; 3 3 weige

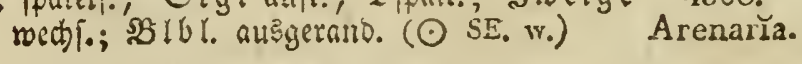

(nemorum.)

(dicholóma.)

4500.
bulhosa.
4501.

(Fiolostěa.)

4502. multica'ulis. 4503. 4505 . crassifolía. 4506. (Alsine.) 4507.
cerastoides.
4508.

\section{STELLE'RA. 318.}

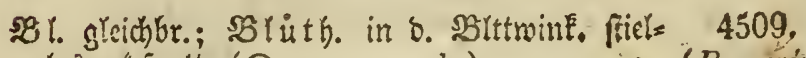
lo5, 4 ipalt. $(\odot$ 2.29. grnch. $)$

\section{STERNBERGIA. 272.}

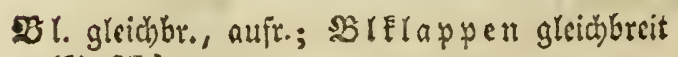
(4) SE.)

4510. colchicifóra. 


\section{STRATIÓTES. 795. (W. 86.)}

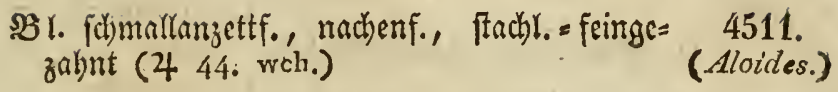

\section{SUBULARİA. 517.}

Stengel:

1. åft.; SBY. vert: ent. (4 9.)

2. fefflt; 3 B. pfriemenf; $(\odot 57: 42: 50$.
4512.

alpina.

4513 .

aquatica:

\section{SWERTIA 1 175. $\left(57^{x} \cdot 82^{\mathrm{x}}\right.$.)}

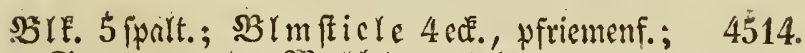
Stgl ungeti).; 933 ł bl. oval (4 9. dbl.) perénnis.

\section{SYMPHÓORICÁRPOS. 150 .}

Bï̈üthen:

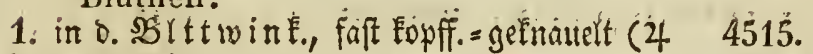
NAm. w.)

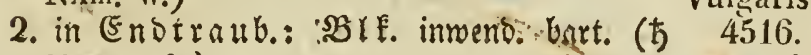
NAm, rch.)

racemósus:

\section{ร. SYMPHYัтU்M. 95.}

\section{Blätțer:}

1. enr. = lanjettf., herablauf, wect)i. (4 r: 49 :

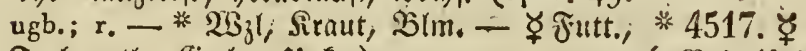
Farb. roth, (jerb., Iluft.)

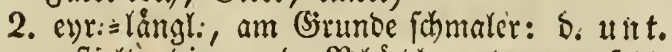
(officincile.)

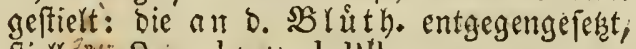
fitielläss Saumlapp. d. Blk.:

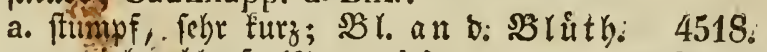
wenigberablauf. ( 4 4. ugb.)

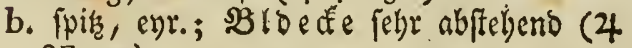
SE. $w_{0}$ )

3. berif $f_{0} \geq$ enyr., geffielt, tuberósuni.

a. ftachelhaar.: Die an ร. SBluthen entges gengef., ftietlos; Stgl:

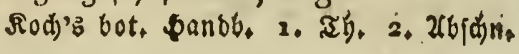


6. åt. ; Saumlappen D. SElE. frumpf 4520 . (4 As.) tauricun.

b. cinf.; $\$ B \mathrm{l}$. langgeipist, ganzlant. (4) 4521 . $S E$. ugb.)

b. ftriegl., febr raub, langgefpizt, fum. lan= jettf.: S. oberfien entgegenger, faft fiele

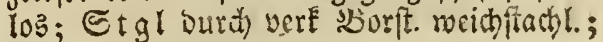
SB IE faum gloden?. (2+ As. b..) cordátum.

2. SYRÍNGA. 12.

Blätter :

1. her $f_{-}=$ey r. $\left(\hbar S E, b l_{0}-r_{0} ; r_{0}-b l . ; w_{0}\right)$

2. ey)r. =laugettf. (†) As. v. -bl.)

3. ไanjettf. (方 As, r. - bl.; w.)

4523. vulgaris. 4524.

chinénsis. 4525 . persica.

\section{TABERNAEMONTÁNA. 136.}

\$81. lanjettf. = eur.; 3weige ausgefpert (b As. w. $\Delta$ )

4526. divaricata.

\section{- 19. TAGÉTES. 675.}

281. gefied.: bie lanzettf. Blättch. :

1. geiágt: Enobląt tben faft beroblant.;

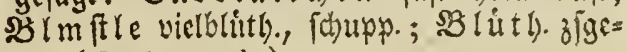

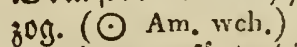

2. gcump. sciágt; bie 1 blưth. Blmstle:
a. oben etwas bifer; Bro. eben; Stgl etwas abftebind ( $\odot$ SAm, braun u. dgb., übelriech. - ఫ̧ jarb. get(6)
b. baud). =verdict; ; SB lo. eff.; Stgl aufr. ( $\odot$ SAm, gb., übelriech.)
452S. $\nsucceq$ patula: 4529 ; erécta.

4527. minúta.

\section{TAMĂRIX. 244. (358 ${ }^{3}$ )}

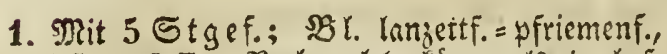
faft umant.; Rebenbl. turro. als b. bef. 


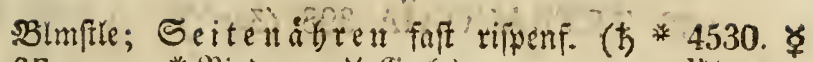

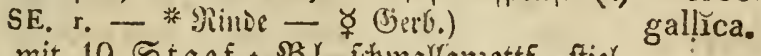

2. mit 10 Stgef.; B. . inmallanjettfo, ftiel= los; Si c benbl. longcr als o. bej., Bblmftl;

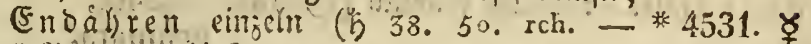
* 3inte -

22. TAMUS. 785. (W. 3o.)

BBI. hersf., ungeth. (4 SE. gbcb.)

4532.

commúnis.

\section{TANACÉTUM. 661.}

\section{Blätter:}

1. ficberf., mit lanzettf., gefïgt. 趾p.; Dola 4533 .

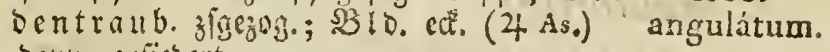

2. bopp. gefievert,

a. 2rile th.

a. fils.: bef. Ficbernber Stglbl. ge= 4534.

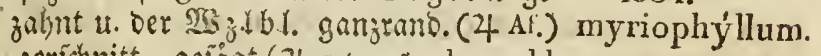

b. zerichnitt., geiógt (4 14. 15. gb., wohlriech. - * Sirant, : Din., ๔anm. - * 4535. ఫ

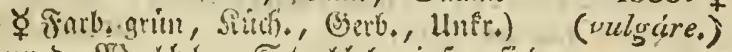

b. mur o. Sigz zlol.: Etglbl. einf. gefico.,

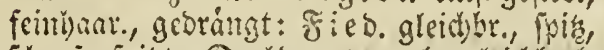

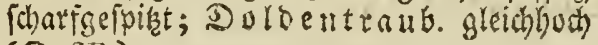
(๑ SE.)
4536. annuัum.

\section{TAXUS. 799. (WT. 46.)}

M. $B \in f r u$ ditung bुoben fugel,; bie gleidhbr., 2 zeit. Blätter:

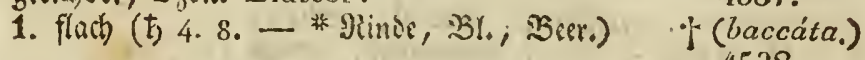

2. an Rande ungerollt ( $5 \mathrm{NAm}$.) - canadénsis.

\section{TELEPHIUM. 246.}

BS \%. vedti. (4, SE, w.)

4539.

Imperáti. 


\section{TETRAGONIA. 398 ; $\left(392^{\circ}\right.$.)}

\section{Blätter :}

1. rautenf.=eyr., geftielt; mit $3-4$ Stgcf.: Frudte blattr. = runbum ftachlid) (๑ SAf. 4540. grün)

echináta.

$$
\text { grn, } \left.u_{0} b_{0}\right) \text {, expánsa. }
$$

(4 $\left.\mathrm{NAm}_{\mathrm{i}}\right)$

\section{TETRAGONOTHĹCA. 671}

4542 .

helianthoides.

\section{TEUCRIUM. $45 \%$ \%}

\section{Krautartig;}

A. Bl. vielspaltig:

\section{Blüthen:}

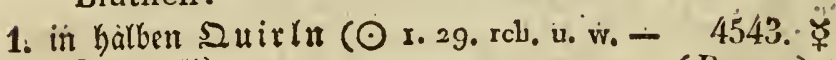

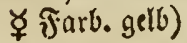
(Botrys:)

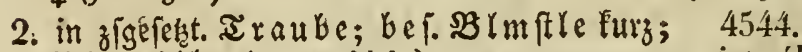
56r. gleichbr. (4 As. blch.).

orientále:

\section{B. Bl. ungetheilt.}

\section{Blätter :}

1. enrunb,
a. ungl. gefigt; mit Énbtrauben; $\mathfrak{R} e=4545$.

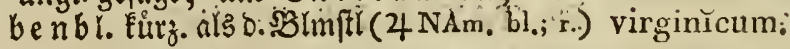
b. zerfchnitt. = gejågt, unbebaart, mit balben 4546 . इuirln; Etgl aufro, glatt (4 SE. r.) lucrdum.
2. feirf. = eyruno, zerfichnitt., geferbt, geffielt; B hith. 3 záhl.; S tgl nieberlieg., ctwas

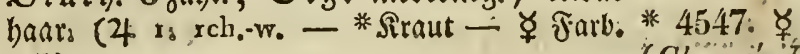 gelb) (Chama' edrys.)

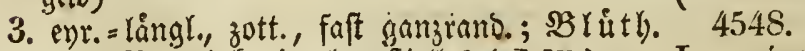 in b. SBlttwinte. cinzeln, fitiellos ( $\sigma^{\prime \prime} S E_{\text {. }}$ ) Laxmánni.
4. cyr. = lamzettf., gclágt; S tgl aufr.; E End = traub. ftietruno, mit 6 bráttr. \$uirtu (4 4549 . NAm. $g$.) 
5. Kerzfo, etwas feinhaar., gezabnt, geftielt; Ir a u b. in D. Blttwint., 1 leit.; Stgl aufr.

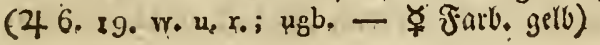

\section{II. strauchig}

\section{Blätter:}

1. langl., ftielloz, gezahnt, faft nadét; Bzlñth. in oen sBlttwink:, geftiekt, gepaart; Stgl weitichweif., feinbaar. (5 47. hro - * Siraut * 4551. ఫ

- ૪ Futt., Farb. gerb)

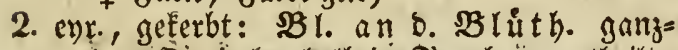
tano.; Sutrte halb in arnubert vertbeilt;

Stgl feintbanr. (5 6.2x.wch. -

3. lanzettfórmia, a:' ganzrano,, unten fil

frummt; mit Enoboloentraub.; 3 lo.

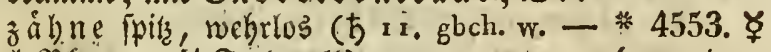

* 23 (m. - Ұ Farb. geib)

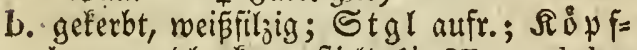

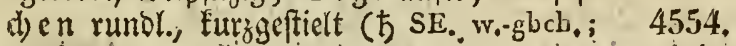
rch.)

4. gleichbr., ganzrant., am Riante umgeroult; (Scordium.)

4552. unten filfo, mit Enobolbentrauben;

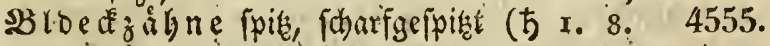
sbch.-w.)

capitátum. (montánum.). supinum.

\section{THALÍCTRUM. 437. (3824;}

$$
437^{2} \cdot 79^{5} \cdot \mathrm{W}_{;} 9^{3 .)}
$$

\section{Friichte 3 eckig, geflügelt}

Frůb̆te bang.; gerabe; Stgl fteftrunte (4 4356:
3. 8. . $\mathrm{chl}$.)
(aquilegifolium.)

\section{Frïchte gestreift;}

A. mit gọnzgetrennt. Geschl.

1. mit (E) (4 $\mathrm{NAm}_{0} \mathrm{w}_{0}$ )

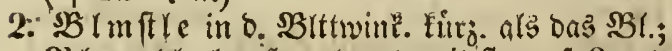
$\mathbf{B}$ l. rtmol., hycrf., gclappt, mit ftumpf. \&app. (4 NAm. gruch.).

Cornúti. 4558. dioùcun. 
B. mit Zwitterblïthen.

I. Sdift faft Dolbenf; ' Stfád. vere. lants. 4559 ;

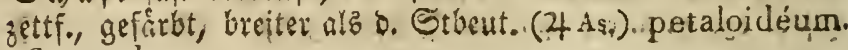
II. Stengel:

1. ganz einfach,

(Endtraub. einf. (4 NE.) alpinun,

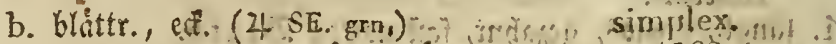

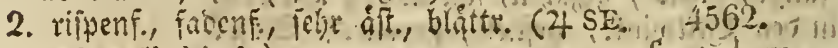
wch., übelriech.).

3. gefurdit, blattrig:

a. mit Rispe:

g. vielfub), nurr. (4 49. gbch,-grn, T 4563.8

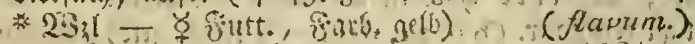

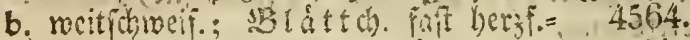
c cyr, ftump fiffalt, graugrun (2-SE.) speciosum.

c. gans gerabe, nit litertgang. Solith. ; Etglgangerabe; s及lattci). 3jab)!., gleidher., am Sianoe unigerollt (2t 4565. $S E$, woblriech.)

b. mit Dolocntrnub.; 913 zlblát do. feilf., 3 ipalt.; Stglblat breit (2) galioides, $456 \%$.

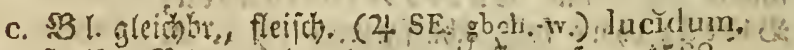

4. geftreift; $\mathfrak{B}$ l. rungl, abcr., mit thmp\%. Eopp. (4 NAm.)

III. S3luthen 5 blattr.; 938 frolt. (4 SE. w.)

IV. Blättchen:

45635.

rugósum.
4566.

nigricins

tuberósum.

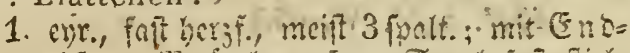

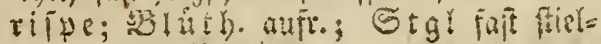
runb (2I SES)

4570.

elátum.

2. numol, 3 fwait.,

a. faft herf: Inten gramgrin; Mifpe, 4571 . blittr.; Şlith. úbergevog. (4 5.) majus.

b. jerfonith; s5itits. wie vorber $(4,3$. gb, - rch.; grnch. - ఫ Farb. grlú).

$4572, .7$

3. lengl, stalf, fuic, 3ipalt.: bie oberf.

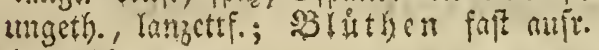
(4 SE.)

(minus.)

4. ganj feif , ivis, 3 palt.; Etgr ruthanf., 457 . edf.; $\$ B$ lut th. wie vorber (4)

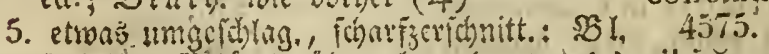

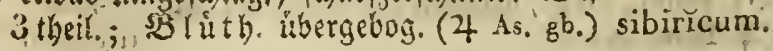




\section{Thalictrum. - Thlaspi.}

6. lanzettform. = gleichbr., ganzrano. (4 3.47. 4.576 . bob.; w.)

\section{THAPSĬA. 228.}

BI. 3 fach bopp. zigcient u. mebit $B$ I a tit fiten gott.: SBlåttch. lángl., bucht. = fieberf.: bie unterften nbrairtsgevogen, mit frumpfon,

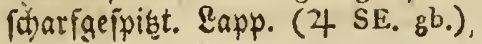

4577.

villósa.

\section{THELIGOัNUM. 755. (W, 31.)}

$45 \%$.

$\left(\odot S \bar{E} \cdot w_{\imath} \cdot g^{r n c h}.\right)$
Cynocrámbe.

5. THESİUM. $170 \cdot\left(40^{x} \cdot 8 \mathrm{a}^{\mathrm{x}}\right.$ )

\section{Bliithen :}

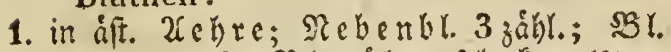

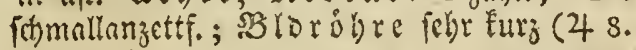
II. wch.)

2. in blattr. $\Re$ ifpe; bie 3 ripp. Bl.:

a. fchmallnmzettf.; 'Etgl ganj gerabs (4) (intermedzum.)

b. Ianjettf.; Stgl werfe (24 8.)

3. in Ghittr. Traub.; mit פebenbl.; Stgl:

a. nicbergeftreft, einf.; Enotraub. 1 peit.; SStith. ftiellos (4 8.)

b. aufrecht,

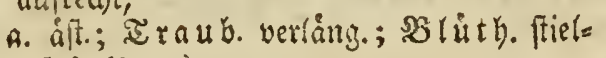 I0马 (4 4.)
4583.
ramóslem.
4584.

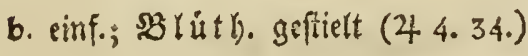
(ebráctedtum.)

(montánum.)

4579.

(Linophýllum.)

4580.

4581.

$45 \$ 2$.

(alpinum.)

15. THLASPI. 509.

Schütchen:

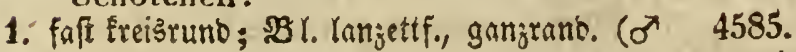
6. II. P) peregrinum. 
2. gुanz freistund; $\mathfrak{B}$ l. langl., gejabnt, unbes baart (4 29. w.; p., Bl. wie Knoblauch 4596. భ̧ riech. - * Saam. - ఫ Del, llufre)

3. Faft ey)r., bauch.; $B \mathbb{B}$ r. långl., ftumpf, ge= zaknt, unbehaart $(\odot$ 22: 23: w.)

4. runolich); Blätter:

(arvénse.)

4587.

alliacěum. 4588.

a. lanjettf.=gleichbr., ftumpf, fleiich. ( $\odot 2$ r. A.) saxatile.

b. pfeilf., gezabnt, grau (OT.2. 4.28 . w. - $ซ$ unfr.)

c. Stglbl. pfeilf., jott.; Scbitch. baar. ( $\left.\sigma^{\pi} \mathrm{w}.\right)$

5. verk. berfformig;

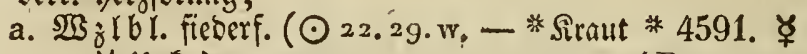

-

b. Stengelbl.:

6. långl., umfaff., faft pfeilf.: $\mathbb{2 B}_{3} \mathfrak{l}$ bl: etwas fleifch., verk. enrunt, ganjrano.: Zlle unbehant; SBlf. gróp. als bie Blo. (4 5. 23. w.)

b. berzf., unbehaart,

4589. భ (campestre.)

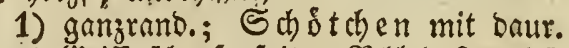
(Sriff. jharfgeipist: $B|b| 2$ mal io lang alb D. SBlb.; Stgl cinf. (4 9.)

2) etwas gejabnt; 5 ch $5 t c h$. mit $a b=$ fall. Briff.; Błlbl. fo lang ats bie SBlo.; Stgl âf. $\left(\sigma^{7} 2,29\right.$. w. ช unf $_{\text {t) }}$

6. umfall.: 2irle etwas gezahnt; Sch of $t=$ chen mit oaur. (5) riff. Fharfgefpint; SBlbl. wie vorber; $\subseteq$ tgl einf. $(\odot 8$. 29. w.)

6. 2 lappig; Stgl gefurdt; $\mathfrak{E l}$. pfeilf., lan= zetff., etraas gefágt; $\mathfrak{N}$ fla na ge ganz unbe= baart ( $\odot$ NAs, w.)

(Bursa pastóris.)

4590.

hirtum.

4592.

(montạ́num.)

4593.

alpinum.

4594. ఫ్

(perfolicuturn.)

4595.

(alpéstre.)

4596.

ceratọárpum.

\section{THRINCĬA. $60 \%$.}

Sdbaft 1 blith.; Bld.:

1. faft unbebaart, mit e. 2(nbang yon Kleinen Schupp.; $B$ l. . lanzettf., bulcht:=gezahnt, mit einf. Soaren (4 3.47. gb.)

2. etwas grau, ohne 2 nnbang; Schaft haar.; $\mathfrak{B r}$, lanjettf. fttumpf, sezahnt, mit gabelf, Şnaren $\left(\odot\right.$ SE. $\left.^{2}\right)$

4597. (hirta.) 4598. hispřda. 


\section{THUJA, 768.' (W, 23.)}

Die bef. 3 weige zigeoridut $=2$ fthneit.; bie 4 reihig: $=$ bachjiegelf., evr. $=$ rautenf., $_{\text {, }}$ ange= orúct. Blätter:

1. int b. Jlitte mit e. Şôder; 3apfen vert. cur., mit angedrúdf́t. Sthupp.: bie innern Schuppen abgeftukt, u. unter b. Spifge hơdr. (t NAm. - * Siraut)

2. in ठ. Mitte mit e. Langenfurd) ; $3 \propto p$ fen cllipt.: bie itnt. S ch upp. ftumpf, u. unter \$. Spitse ftechendipits (万 As. $\triangle$ )

* 4599 .

occidentális.

4600 orientális.

\section{THYMUS, 446 ,}

\section{Strauchig:}

Bliithenquirle:

I. fopffơ rmig; Blätter:

1. ovallångl. u. fommallamjettf., ftumpf, un= behant, am Sirunbe etwas gewimpert; Stgl auffiteig., nieverlieg. oo. friech., meift ftielrund; 2 2 efte 4 [eit., etwas feinluar.;

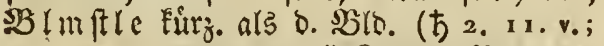

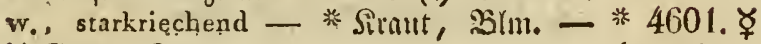

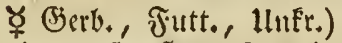

2. Ianjettf., ftumpf, unbebart, am (3runbe ctwas gewimp.; St gl niebcrlieg.; Ue fte 4 feit., Furjhanr.; $B$ lmftle meift lángev

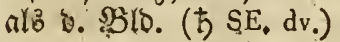

3. runolich=elliprifa), am (sormbe ichnaler, ftumpf, gewimp., oben zott., unten meift unbehaart; Stgl kriech.; 2 (efte wie vor= her ( 523. lila $-\dot{x}_{0}$ )

4. långl., ftumpf, auf beyden Geiten zott.; Stgl aufferig.; X 2 fte unoentlich 4 feit., furghar. († SE.)

5. rundr. = eyruno: oie, unt. faft herzf., am (Srumbe etwas haar.; Stgl neben d. Febr langen $\Re a n k e n$ turghan. (5 As.)

(Serpýllum.)

4602.

glabréscers.

4603.

lanueginósus.

4604.

pannonǐcus.

4605.

nummularŭus,

II. ábrenf. = traubenf. : Blätter:

1. ent., ftumpi anbelbart, an Sirunbe etwas gewimp.; Stgl nufiteig.; 2 e efite 4 peit., 4606. an o. Santen furbjanr. (†) SE. hv.)

montánus: 
2. langellint., flach, ctros frinkanrig, auf bero. Seit. funct., am Brunbe gewim.; Stgl aufr. Do. niebertirg., mit aufftrig. Jeiten; Slippe o. SBID. fait 4 eá., mit pfriemenf., faft gleich., ant (Sirunce gex roimp. Babnen (亏 NAs.)

III. traubenif.; Die unbebant. Blätter:

1. oval, etwas ppif́, oben glamjeno; Stgl -4608 . aufr., sift. ( $5 \Delta$ )

lucìdus.

2. eyr., ant Sande umgerolyt, meift 7 fad) gerippt; Stgl wie worker. (5.SE. hr., * 4609. wohlriech. - * Siraut, L̇im. - ఫ (jerb.) vulgáris.

\section{II. krautartig.}

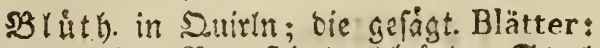

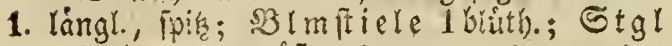
aufrect), ctwas aft. $(\odot$ r. 18. hv. u. gb.; 4010. braun, wohlriech.)

2. eyr.; $\$ 3$ IE $i$ chlu no aufgeblar., lang. als b. SBlD; Stgl ftaub. ( $\odot \sigma^{7} \mathrm{SE}$.) patavinus;

3. runol., etras fumpf, hogl; Du irle Gblith; ; 4612 ,

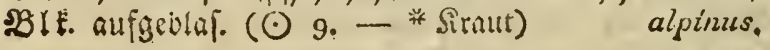

\section{THYSSELINUM. 194.}

Etgl faft ftielruno, ctwas et., ift.; SBl. 3fach bopp. ofgelest: siz lat the. entzegenger. fies

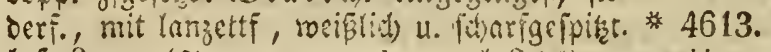
bej. Iapp. (4 42, w, u. xch. - $24 j i)$ pralistre.

10. TIARÉLLA. 352.

D1. bergf. (24 NAm, wch.-grach.)

4614. cordifolra.

\section{TILIA. 414.}

Die Kerźf. Blätter:

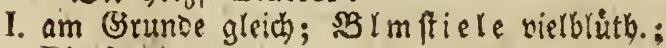
Bliithen: 
1. ohne Reftar, Birl, unbegant, unten in b. 2loctmintelt bart.; SBlattifle b.

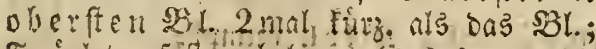

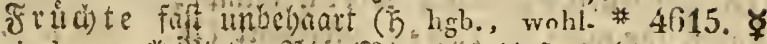

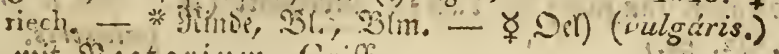

2. nit Sictarinul Griff:

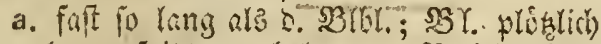
imagernist; unbelgant; $5316 \%$ abge=

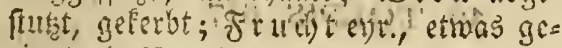

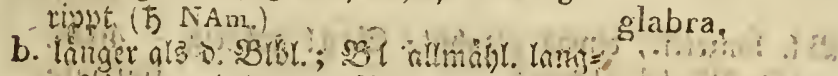

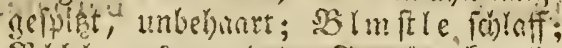
solbl. augeranost; Frutht Eugetig (

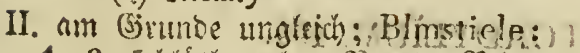

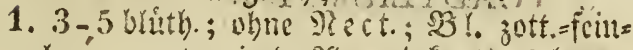

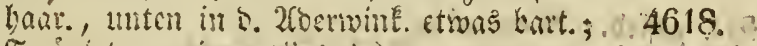
Fridote gcripst (bigb.)

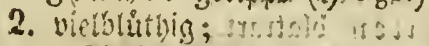

4617 .

laxiflóra.

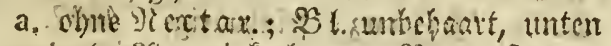

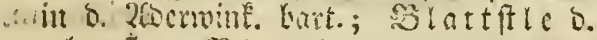
oberiten 98 l. methe als haits io ling

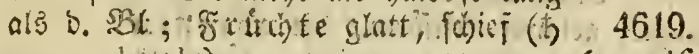
w. grnch.-gb.) (parvifolia.)

b. thit Petar.; Bl.:

(panciflora). i

a. 1enten feinhaar., am Gibunte abges

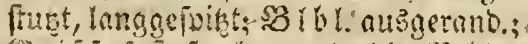

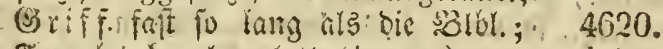
Frud th fagel., glatt (t) Am.) pubéscens.

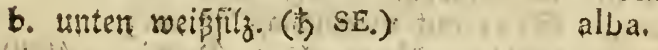
4021.

\section{TILLA'EA. 86. ( $\left.47^{\circ}\right)$}

Stengel:

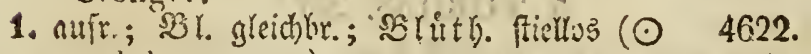
50. gbch.- w.; ros.)

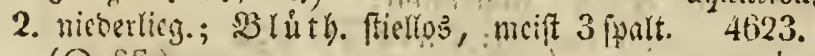
$(\odot$ SE.)

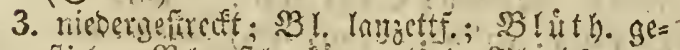

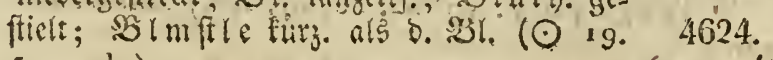
50. wch.)

(prostráta.) 


\section{TOFIELDİ. 293.}

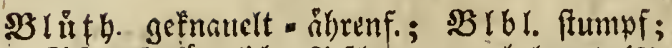
SEapf. l'nultid); SJ flanze unbebaart (4 9. 42 . gb.)

\section{5. paliestris:}

\section{TOLPIS, 617.}

RI. lanzettf., ctwas gezahnt; Stgl auffteig: (○ SE, dgb. - braun)

\section{TORDYLIUUM. 198.}

\section{sBl. gefico.; Stengel:}

1. weitjobeif., nicoertieg.; $\mathfrak{B}$ låt d d). rumbl., atsegefhweift = gejahnt; Şullen blattart., langer als o. Solde; J flanze fcinbaarig
$(\odot$ As. w.) .

2. aufred)t; Blättchen:-

a. lángl.; gelappt = Eeifform.: bie oberften

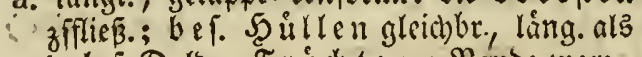

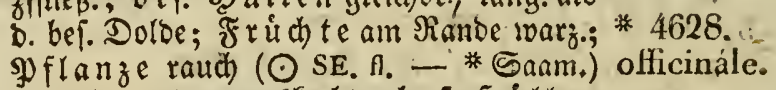
b. runbl., gelappt=geferbt; bef. Şưlten boritenf., fo lang als b. bciono. Dolden; 4629. D flanze zott. ( $\odot$ SE. wo stink.) apŭlum.

c. laniettf., ftumpfgefógt: bas ỉ üerfte verláng.; Stgl mit uningeichling: Shar.; Șílten gleichbr., furrer als D. Dolbe; ญू fla

\section{TORĬLIS. 207;}

IZ1. bowp. gefied.; Blättchen :

1. långl., zerfobnitt. = gefigt: (Enoblát tchen verlång.; Şúllen lebr Flein; Stgl aufr.,

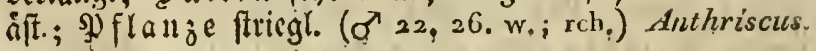

2. c)rutb,
a. zerficlyitt.=ficoerf., gejågt: bas a u $\beta$ er fite

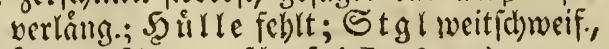

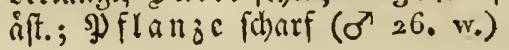
4632. helvetı̌ca. 
b. fieberf ; mit lanzettf.; fuig:; bef. Eapp.; Dorben in 8. SBlttwint. gefnnult, fait 4633.

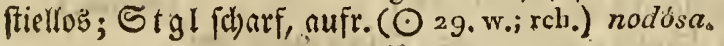

\section{TORMENTÍLLA. $402 .\left(38^{\circ}\right.$.)}

Stengel:

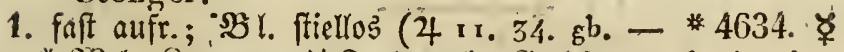

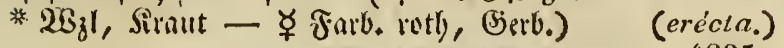

2. friet).; $\mathfrak{B l}$. geftielt (4t t. 4. gb.) (replans.)

14. TOZZIA. 487.

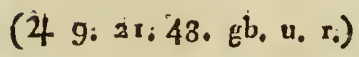

4636.

alpina.

\section{TRADESCANTIัA. 261.}

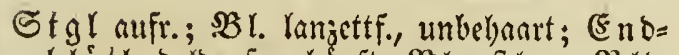

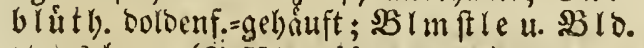
etwas bant. (24 NAmo bl.; P.; w.)

$463 \%$ virginǔca:

\section{TRAGOPÓGON. 610.}

Blumendecken:

1. fo lang ats b. SBlffifrabl; bie ganz gernben ungetheilt. Blätter:

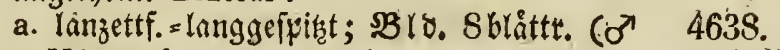

NAs; wch.; ros.; gbch.)

mutabilis. * 4639. ఫ̧

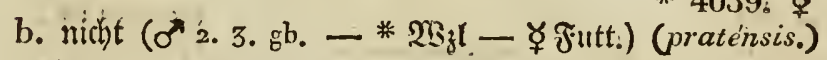

2. faft fo lang als o. Strabl, 8 blăttr. u. mebft D. SBImftlen filz:; $\mathfrak{B l}$. gleicbbreit, ganz 4640 . gernde (ot SE. hob.) :

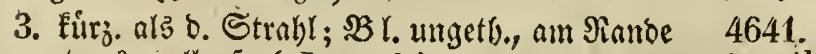
etruas wellenf. ( $\sigma^{2} \mathrm{As}$, gb.) orientấlis,

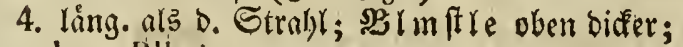
bes. Blk.: 
a. gegen bie Evike runor.; Nit. ungetheit, 4642 . ganj gerabe ( $\sigma^{7} 3.31 .5$.

b. Febr ichmal, abgefturt; Stgl ganz ge=

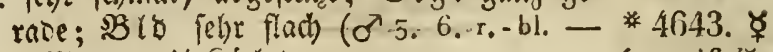
* $2{ }_{31}$ - ६ Sitid.) (porrifolius.)

5. noch balb mal fo long als.t. Strabl; Stgh 4644. u. B I. jott. (ơ SE. hgb.) villósus.

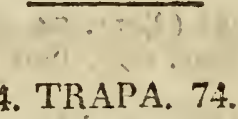

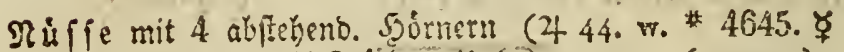

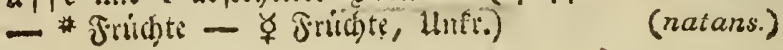

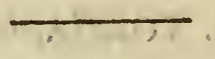

10. TRIBǓLUS. 342 .

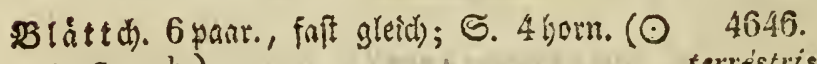
8. $30, g^{b}$.) terréstris.

\section{TRICHOSTÉMMA. 448.}

Staubgefaifse:

1. turz, verjalolien ( $\odot$ NAm. p.)

2. febr lang, bervorrag. ( $\odot$ INAm. p.)
4647. brac hiàtum. 4648. dichotŏmum.

7. TRIENTÁLIS. 304.

(4 5. m.)

4649.

(europáea.)

\section{TRIFOLIUM. 595.}

\section{Blätter 5 zählig.}

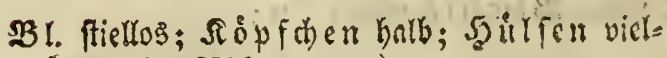
fanm. (4 NAf. p. u, w.)

4650.

Lupináster. 


\section{Trifolium.}

\section{Blätter 3zählig;}
A. Bld. nach d. Aufblühon
a ufgeblasen-bauchig.

Köpfchen :

1. evruno; Stgl aufr.; Bld. d. Frucht:

a. ent., bauth., unbehaart, mit borfienf., umgefrümmen Sábnen; gem. Sớlten

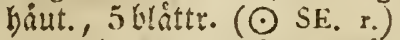

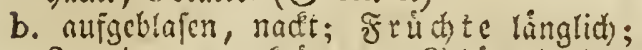

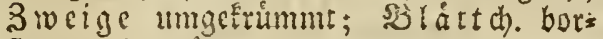
ftenf. = fringefógt $\left(\sigma^{T} S E\right.$. $)$

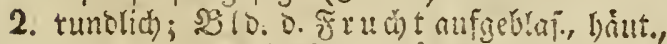
foinbaur., mit borftent. Bingen; Stgl:

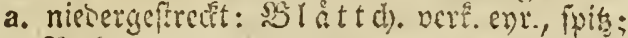
4653. $\not$

BlE. ganj umgewandt $(\odot-\gamma$ filtt.) resupinitum.

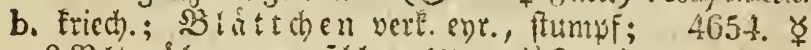

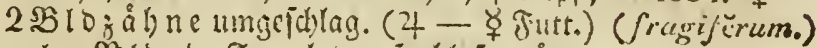

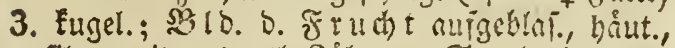
fill., mit moutl. Babnen; Stgl niescrge= ftredft $\left(\odot S E_{0}\right)$

4655 .

tomentósum.

B. nicht;

1. mit Nebenblättern;

a. Blüthen in Köpfchen.

Küpfchen :

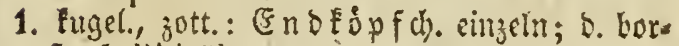
ften $\bar{r}$. Bldzähne:

a: lang. als o. SBlf.; Stgt nieserliegent;

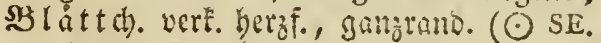
wch.)

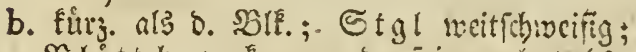

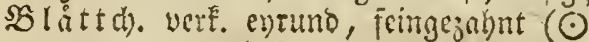
Blättch, r. gefleckı)

2. Galbfugd., in D. SBlttwint. fíeltỏ; $\mathfrak{B} 18=$ óhne gleid), pfriemenf., ctmas abitebend,

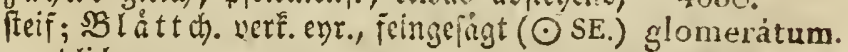

3. illnolich,

a. geftielt, v. e. frciaruno., gejahnt. Sult le umgcben: 2ffterbl. lingl, bcgrannt, ge= zabut; Stgl aufr., âf. ( $\odot)$

b. ftiellos, zul Eeite fiebrno, faft unbrhart;

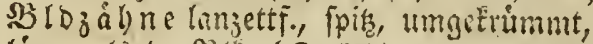

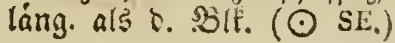

4659 . involucrátum. sulfocatum. 
4. lingl.; B̉ Is. jott., mit ungl. 3̂́fnt.; Stgl:

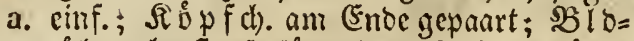

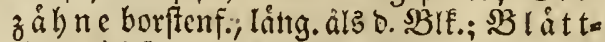
d) en keilf., antageranto, an o. Spitge fein= gejabut $(\odot$ SE.)

b. bin u. bergebog. = aufr.; $\Omega \dot{0} \not f \mathfrak{h}$. geftielt ;

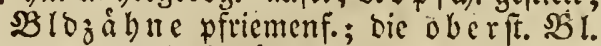
entgegengef.: :Blatt d). ellipt., feingezalynt

5. enritino, $\left(\odot \cdot N A f_{0}\right)$

a. in D. Slttwinfelti, ftiellos; SBlojă ungl, lantzettf., icharfgeipiat, fteif, unn=

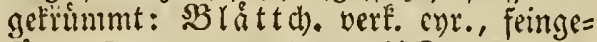
fight $(\odot$ I. 34. wch. -

b. ain (Ende $\mathfrak{u}$. in $\mathfrak{b}$. ßlttrinint., faft einzelu

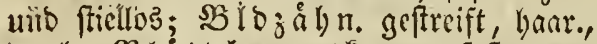

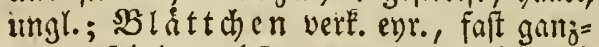

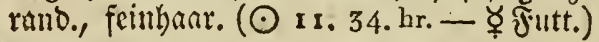

4661.

geméllum.

4662.

alexandrínum:

\section{b. Bliuthen in feliren.}

Áehren:

1. fugel., an (Ende ftelyent, raut), faft fticle

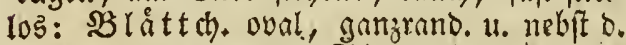
nicoerlieg., gamz dinf. Stgl bichtjottig (4 9. w. - ఫ Э.utt.)

2. faft fugelig; ber unterfte $2 B$ rogahn fo latig als 0 . Sislyre Der 1 bláttr., faft gleichf. Biff: ; Slåttch. felyr jarit gefágt, geftreift= aber.; Stgl:

a. aft., hin u. hergebog.; Uehren einjelit,

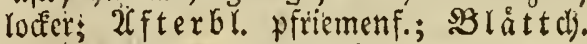
ellipt. (4 4. - ఫ Jutt.)

4666. \$ medüım:

b. ganz einf. u. gërabe; $\mathcal{X}$ elsen meift ge=

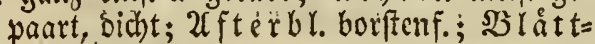
chen lanjettf. (4 4. 8, dbraun, daan $\mathrm{p}$ : - ఫ jutt.)

3. rutiol., cinzeln; $\mathfrak{A}$ fterbl. båat.: o. ob ber=

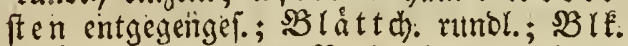
1 blittr.; $\mathfrak{B}$ I b. am $\Re a n d e$ inweno. bart., mit faft gleich. Sálfin. ( $\odot$ SE. w:)

4. runol. =eyyr., jott:; \$Blozáline ungl., bor = ftenform., braunbaar., fo lang als b. SBlE.;

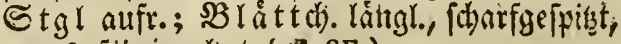
etwas féingezaljnt (o $S E_{\text {: }}$ )

4665. ชุ noricum.
4067. ఫ
(alpéstre:)

4668.

pailìdum.

4669.

diffúsum. 
5. ganz eyr, bicht; ber unterfte Brozahn

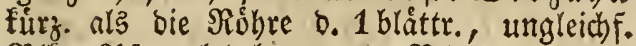
SBle.; 2Afterbl. begramt; $B$ l. oval, ganj= rani.; Stgl auffteig. (4 3. so. p.; w. ఫ̛ Futt., æarb. griut)

6. evrr. = legelf., bicht, cinzcln, fticlloz; $\$ 3 \mathfrak{r}$.

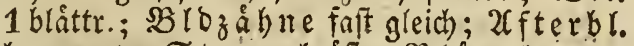
begrannt; Stengel áft.; SBláttch. eyr., ftumpf, unteutl. feingejahnt ( $\odot \sigma^{7}$ NAf. p.) bracteátum.

7. eyr. =walzenf., bicht, einzeln; ber u nter fte

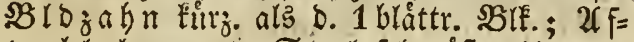
terbr. begrannt; Ștgl fehr åft, bin unb hergebog.; 3 la a tt dh. evr. ellipt., ftumpf, 4672 . ganzramb. (4 NAm.) , pensylvanicum.

2. oḷne Nebenblätter;

a. Blüth. nach d: Blühen nach allen Seit. abwärtsgebogen;

a. Blk, roth od, weils.

Bliithen :

I. in faft bachziegelf., meift 3 zahlig. 2(ehren;

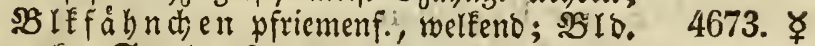

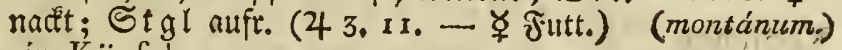
II. in Köpfchen:

1. Coldenf. ; J

a. niebertieg., ect., gefurcht, bin ut. bers gebog.; $\Re^{\circ}$ p f fh. nach b. SBefruchtung umgerdhlag. ( $\odot \mathrm{SE}_{\text {. }}$ )

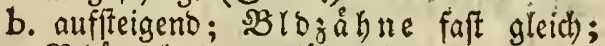

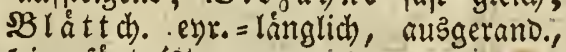
feingefígt (4 29 . wo, dann p.o zuletzt braun - $\Varangle$ Futt.)

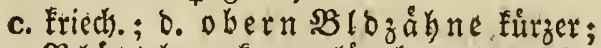

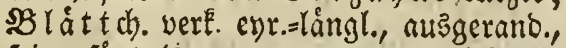

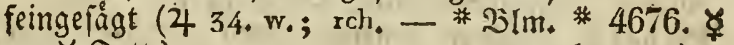

$-\forall$ Futt.)

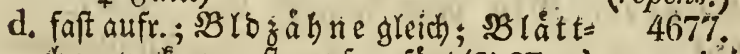
chen verf. eur., ftumpf, gefágt (4 SE. 9.) caespitósum.

2. 5 blúth., zott., in $D$. Nitte mit e. umges fhlag., fteif., bie Frucht verbed. Stjopf $(\odot \mathrm{SE}$.

3. Fugel., in b. sglttwint. geftielt; $5318=$

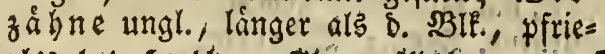

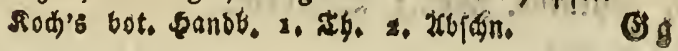


menform., etwas abjtekeno, ungchrinmit: "4679. $\mathfrak{B l a ́ t t} \omega$. verk. evr., feingeriggt $(\odot \cdot S E$.) parviflórum.

\section{b. Blk, gelb.}

Aehren :

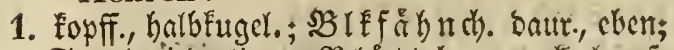
Stgl nieberlieg.; SBlatt dben verE: herzf.

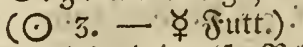

2. oval, Dachziegelf.; $\mathfrak{B l}$ lef ẩ in ch. Daur.; Stgl:

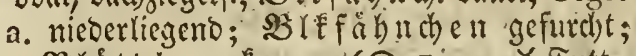

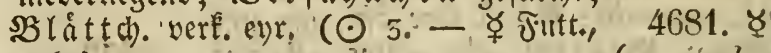
llnfr.)

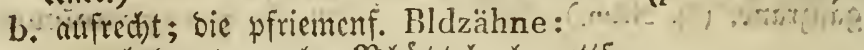

a. Imbchaart, ungl.; B Blattch. ranjettf.= feilf.: bas mittlere ftiellos $(\odot 3 .-4682$. ఫ

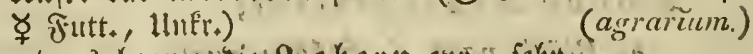

b. etwas haar: "oic 2 obern cyr., febt

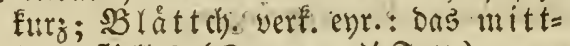

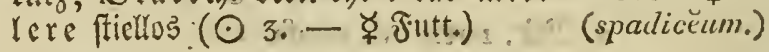

\section{b. nicht;}

a. mit Köpfchen.

\section{Köpfchen:}

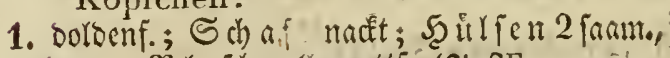

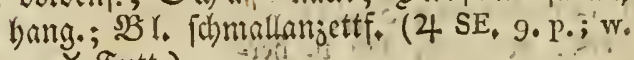

2. fugelig,

\section{- ఛ (jitt.)}

4650. 후

(filifórme.)

a. badfr, faft fuget.; SBro a åb ne borftent., fo lang als o. Blle.; Stgl weitichmeif.; BI. verE. eyt. "an D. Spitce cingerritut (广 $\left.S E_{.}\right)$

b. zottig;

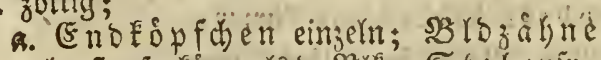

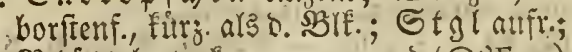

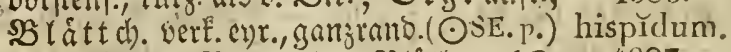

b. Die obern. Blo. obne Slithen $(\odot$. A s's')

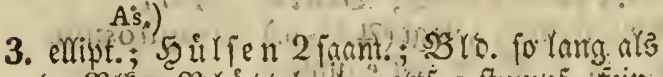
$4684 . \gamma$ alpínum. 


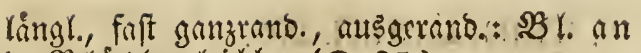
b. SB lit th. gleichir. ( $\odot$ SE.)

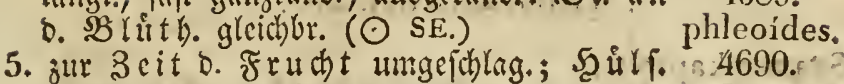
3 jaamt. (4 NAm.)

4689. refléxum.

\section{b. mit Aehren.}

Aehren:

1. lónglich),

a. zott., ftumpf, blattlob: $\mathfrak{B l}$ láttch. runot.= verk. herzfo =eyr., geferbt, ott. $(\odot$ S.

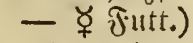

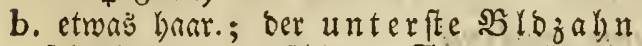
fehr lang, umgefblag.; Stengel nufr. $(\odot \mathrm{SE}$.

2. faft Fugelig,

a. geftielt; $S B$ to abfelyeno: ber unterfte Babn pfriemenf., gleictbre; $\mathcal{C}$ fterbl.

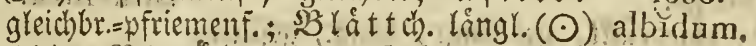

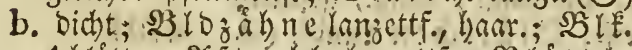

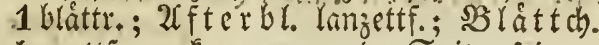

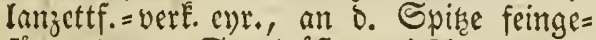

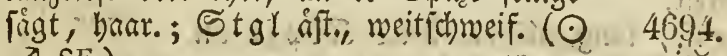
O' SE.) $\therefore$ maritumum.

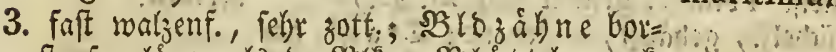
$4691 . \not ̧$
incarnátum.

4692. squarrósum.

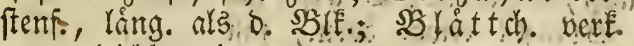

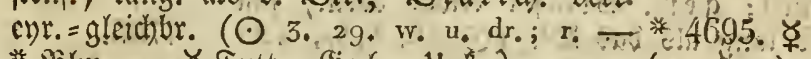

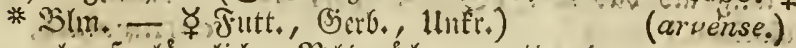

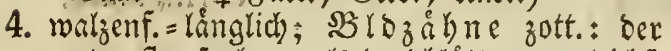
unterfte fo lang als o. 1 blattr., ungleids.

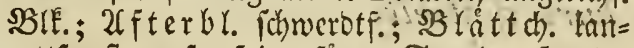
zettf., ftumpf, feingerägt; Stgl aufr. (4

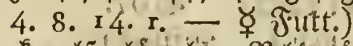

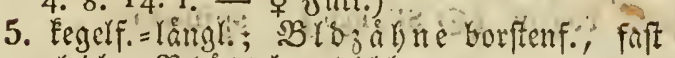
gleich; SBlattch. gleichbr. (๑ Ir. 34. hr. - $\not \gamma^{\prime \prime u t t .)}$

6. cllipt., zott.; Stgl aufr., dift, feinhaar.;

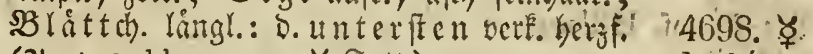
(4 4. 8. hbraun - ఫ Jutt.) ochrolé'ucum.

7. eyrund ; Blättch: :

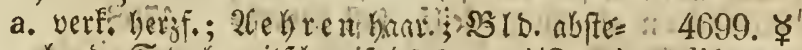

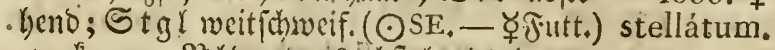

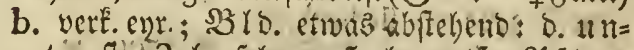
terfte Sagn fehr grof, fruzettf; ; 2 fter=

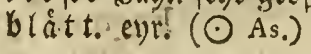
4700. olypeátum. 
Saamenkapseln:!

1. 3 fách., eben; gleidbbr., am (srumbe fama= 4701. \&̧ ler (ơ 47.50. grncl. - ఫ Fitt.) (palústre.)

2. 6 fåd., gefurdst, eyrund (436. bracb. - 4702. ชุ Futt.)

(mariťmum.)

\section{TRIGONÉLLA. 570:}

\section{Blüthen gestielt.}

\section{Hülsen :}

1. långl = gleidbbr., gerabe, gebrångt, geffielt;

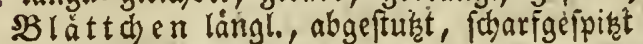
(4 SE, gb.)

2. oval, zfgeorudt, georångt, bang., geftielt; Stgl weitidweif.; RI NAs. gbch, $\left.-w_{\text {, }}\right)$

3. gleidbreit,

a. bogenf., lång, als bas $3 B l$; J Jo p fden meift 4 bluth, geftielt ( $\odot$ Af.gb.)

b. fichelf., gebauft, abftebend; İ a ub. ges ftielt; $g$ em. sB I m ft l etwas born., fâft fo. lang als bas $\mathfrak{B l}$ : ( $\odot$ SE. bgb., sehr woblriech.)

4703.

ruthenica.

4704.

platycárpos.

4705. striáta.

\section{II. sehr kurz gestielt.}

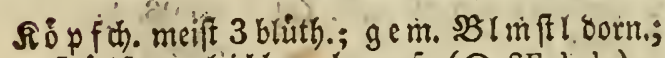
- Şullen gleidbr., bogenf. (O SE. hgb.)
4706. corniculáta.

\section{iII. stiellos.}

\section{Die friellof. Hülsen:}

1. gleidbreit,

a. etwảs aufr. u. faft fithelf., meift 3 zăbr.; soláttchen abgeftutht= feilf, faft fiederfo= gezahnt ( $\odot$ SE.)

b. ganz gerade, meift gepant, frechendipiz;

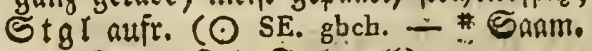
- Ұ Futt., Dir, Jarb. ge(b)

4707 : spinósa.
4708. pinnatifüla.

\footnotetext{
* 4709. 후

Foenum graecum.
} 
c. gerabe, $\Omega \& p f$ h. fiellos, meift 3 blüth.; 4710 .

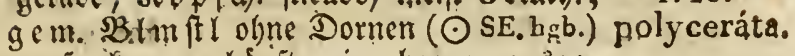

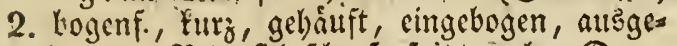

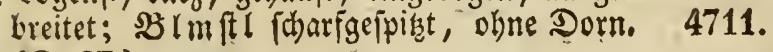

$(\odot S E$.

monspeliăca.

\section{TRILLIUM. 294.}

Blüthe:

1. geftielt,

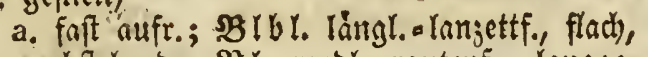

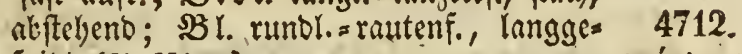
pirat (4 NAm.) eréctum.

b. ubergebog.; B B bl. eyr., flach, abfebend; 4713. sBl. wie vorher (4 NAm, w. -grn。 u. p.) pendŭlum,

2. ftieflob, oufr. (4 NAm. dp. u. grn.) sessŭle, 4714.
sessile.

\section{TRIOSTĔUM. 149.}

\section{Blätter:}

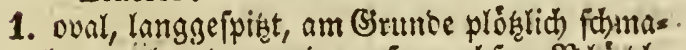
ler, mebr 0o. weniger zigewach); 3 lüth. in ben Bitttwink. ftiello3, 1 oo. vielblítbig; B IE. Dunfelroth) (4 NAm.)

2. oval=lamgettf., etwas zigewactif.; $B$ Imft le

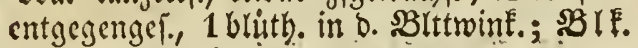
gelb (4 NAm.)

4715. perfoliátum.

4716. angustifolium.

\section{TROLLIUS. 443 ;}

\section{Blumenkronen:}

1. 3[geneigt; Rect. io lang als b. Stgef. (4 9. gb., Nect. gb. - ఫ ₹utt.)

2. ctrvas abfteheno; Rect. láng. als b. Stgef. (4 NAs. dgb., Nẹct. dr.)

4717. ఫ్ † (europá eus:) 4718. asiatı̌cus:

\section{TROPAEరLLUM. 314.}

sBl. fchiltof.; Blblätter:

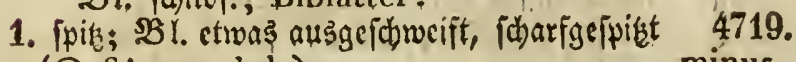
$(\odot$ SAm. r.; hgb.) minus. 


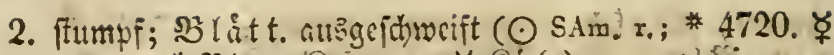

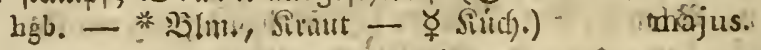

\section{Stengel:}

I. 1 Gruttrig,

1. umbehaart; Bliithe:

a. aufrect); bie unbehaart. Blbl.:

G. ftumpf; 30 1. eyt: =lanjettf. (4 As. 4721 . sehr verschiedenfarb.) gesneriána.

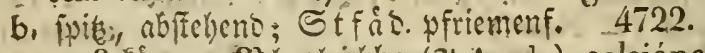

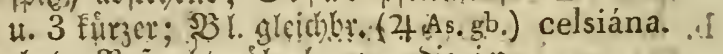

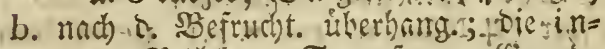

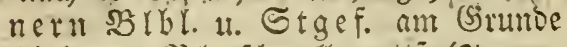

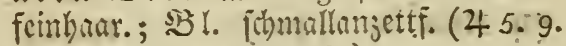
gb. u. grnch., wohlriech.)

2. Feinhar.; 3 lúth. aufr.; $B$ lol. frumpf,

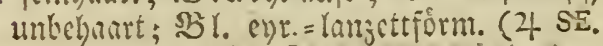
wrohlriech.) : suaveŏlens.

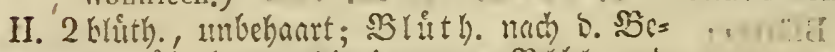

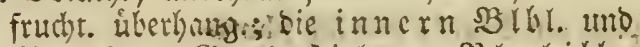
ङtgef. am (j) rumbe feinbanr.; .33 l. gleid)br. (4 As. ginch. u. ivo u. gb.) :

4725. bifóra.

\section{TURRITIS. 535.}

1. $\mathfrak{T Z}_{3}$ lbl. gegahnt, hader.: Stglbl. ganj= rano., umfall, unbehaurt ( $\sigma^{7}$ 2. 3. Ir. grn.wch., Bld. w. - ૪

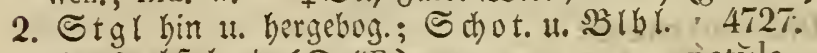

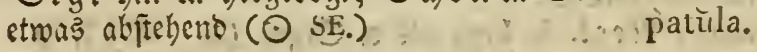

\section{TUSSILÁGO. 684.}

\section{Blïthen :}

I. am 1 blith. Sobaft u.

A. geftrah!t; Blätter:

1. lángl., am (3runde ctwas buctst. unto

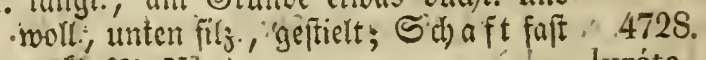
nadét (4 NAs.) 


\section{Tussilago. - Typha.}

2. kersf., efa., gezalynt, unten feinthaar.;

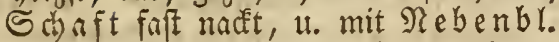

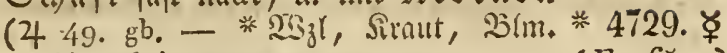
- ૪ lnfr.) (Farfüra.)

B. Fheibenformig, faft nadt; bie nierenf. Bl.: 1. utrbehaart,

a. gejabnt ( 4 9. w., Bld, r.)

b. Fchach 7 lapp.: bie mittl. $\mathfrak{R} a p p$. 3 zabn.; Schaft meift 1 blutb). (4 5. 9.)

2. unten filżig, gezabnt (4 9.) 4730. alpina.

4731. syluéstris. 4732. discōlor.

II. in e. Strauk umb

1. geftrablt; Stralle gleidhody; D. rundl.= herzf. Blätter:

4733.

a. ungl. gejalgut, unten filł. (4 NE. 9.) friğda.

b. gleid $=$ u. feingejabnt, unten feinbaat. 4734 . (4 SE.)

2. Fiteibenformig; Straufs:

fragrans,

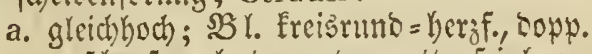

u. Fdyarfigezabnt, unten jott. = Feinhaar.

- (4 8. w. - gbch.)

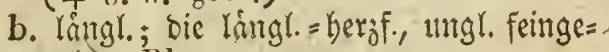

4735.

(alba.)

jahnt. Bl. unten

a. feinthaar., mit angenályert. app: (4

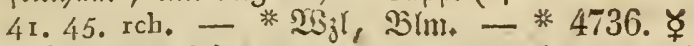

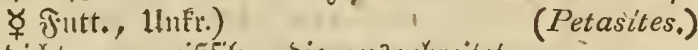

b. Did)t $=$ u. weisfil Lappen:

1) 2 ไap⿰. (4 38.)

2) nicht (4. 8.)

4737.

(spuria.)

4738 .

nivěa.

21. TYPHA. 732. (W. 25.)

\section{Blätter:}

1. fladd), gleicbbr.; b. M. u. W. Aehre walganf. ut.

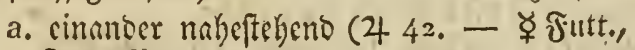

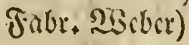
4739. ్ㅜ (latifolia.)

b. vort cirtanber entfernt; 5 a a m 2 mal fo 4740 . lang als D. 2 Bl. (4 SE. 42.) minor.

2. rinnenf.; M. u. W. Uchre entfernt: 4741.

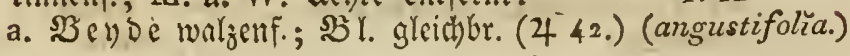


b. W. Ue Lre ellipt.; $\mathfrak{B l}$ l. borftenf $=$ gleid $=4742$, breit, fâft fo lang als D. Şalm $(4 \quad 9.47$.$) minima.$

\section{- 27. ULEX. 565. (543.)}

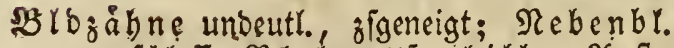
eyr, , fhlaff; $\mathfrak{B l}$. lanjettr. =gleid)br.; $\mathcal{X}$ efte mit bichtjebenden, zigejegt. Dorn.: bie a $l=4743$.

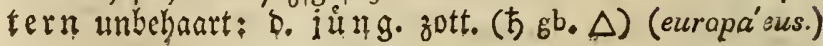

5. ULMUS. 235. $\left(82^{4} .319^{3}\right.$.)

\section{Blätt, am Grunde ungleich.}

\section{Blätter :}

I. bopp. gefägt u.

1. langgefpiezt, mit jobarf. Sảgejähn., am (Sinunde febr ungl., unten jebr blas, u. an D. 2Coern u. D. sBittift mit Fleinen Eraú. Şaaren (ち SE.)

2. Bliithen:

a. ganz ftiellos; $\mathfrak{B} \mathfrak{l}$. oval = långl. (felten her $f_{0}=$ oval) jehr langgeipist, auf bend. Seiten feinbaar. u. Felgr idjarf u. an

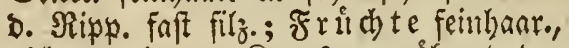

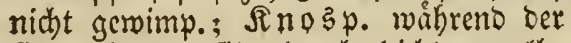
Entrwifelung fily. Durd) Sidte, gelbe Siolle (ち NAm.)

4744. alba.

b. faft ftiellos, gefnauelt, meift mit 5 Stgef.; Fruichte:

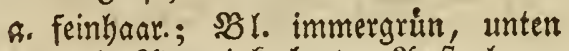
an D. 2roerwink, bart.; 2A efte hang. (ち NAm. $\triangle$ )

b. unbehaart; $\mathfrak{X}$ efte glatt: bie $1 \mathrm{jåhr}$. u. jüngern 2 eft

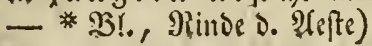

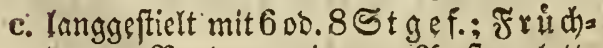
te am Rande gemimp.; Xefte glatt:

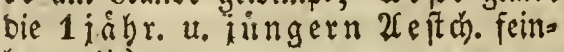
haar. (5)

4746. pendǔla,

\section{* 4747 . \\ (campéstris.)}

4745.

fulva. 


\section{Ulmus,}

(26)arten:

I. mit 8 Stgef.; B lblapp. rotslid, Stgef. Eaum láng. als D. \$Slo.; Fl Fis gelf $\mathrm{r}$ if do te am Erunde fomaler, mit b. Spitzer zieml. gerabe

2. mit 6 @ $\mathrm{tgef}$;

2. Stgcf. fait $3 \mathrm{mal}$ fo lang, toie bie

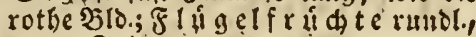
an D. Spizen gerabe

rotundáta,

b. Stgef. Dopp. fo lang als b. rothl.

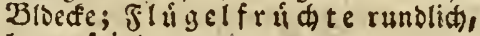
lauggépitizt,

a. mit geraben Spitzen

b. mit cingebog.

oblongáta.

acumináta

inflexáta.)

II. faft bopp. gejägt, unten feinhaar, unb b. 2fftrink. D. 210ern ourch e. Şaut verbunden; ISl it then langgeftielt, mit 5 oo. S Stger.; Fra

(H)arten:

1. 2 efte roth; ; '5 l. eyr., rumgl., idarf rubra.

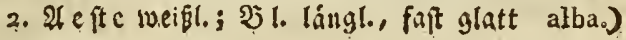

\section{II. fast gleich.}

\section{Blätter :}

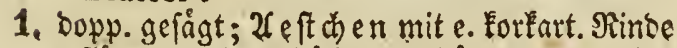
gefligelt: bie 1 íáhr. u. júngern nidht; \$B.rithen faft fticllos, zigefnauelt, mit 4750. 4 Stgef.; $\mathfrak{F}$ rủ the unbehant (b) suberósa. (abarten:

x. mit runzlo, fdyluargl.s graner Rinbe parvifolia.

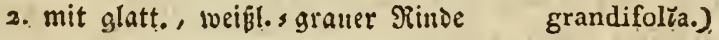

2. bopp. geferbt, långl.=eyr., nach u. nach fpiá, nicht langgefpint; 2 effe bie u. Da von beno. Ceit. in e. torfart. Flugel aušgebehnt; $\mathfrak{F} r$ il $d$ ) $=$ te feinhar., bichtgewimp. (5 NAm.)

3. $\sin F a c h=g r o b=$ u. $i d$ arfgekerbt, faft ftiellos,

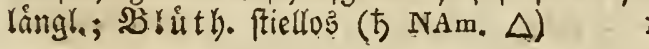

4751. aláta. 4752. nemoráliṣ.

\section{III. ganz gleich.}

Blätter:

1. ungl. bopp. gelågt, långl. = lanzettf., fehe langgefpiat, wellenf., oben itharf, unten fcinbaat. = socich) (t) NAm.)

4753.

crispa. 
2. gleidhgefågt, eyr. = Yanzettf., auf bend. Seit. unbchanrt, glánz.; B li th. Entrzeftielt, mit 4 Stgef.; Fra dite unbelsant (ந)

21. URTÍCA. 738. $\left(730^{x}\right.$. $77^{\text {. W. } 36 .)}$

\section{Blätter entgegengesetzt.}

\section{Blätter:}

1. eyruno,
a. gejôgt,
a. langgeipieft, 3 ripp.; bie unt. $2 B(t t=$

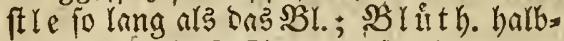 getrennt, mit 3 Stgef., fopff:=bolocn= 4755 . traubenf., tưrz. als o. \$3tttfft $(\odot$ NAm.) pumìla.

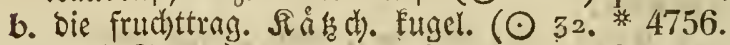

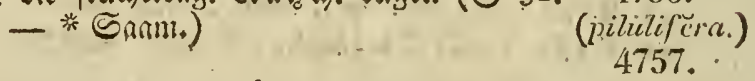

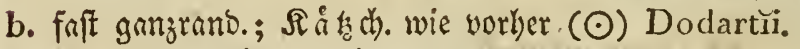

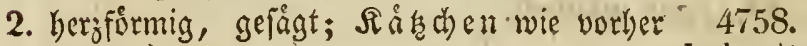
$(\odot$ As.)

balearǐca.

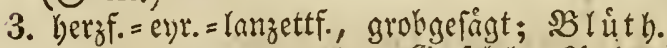
mit ganzetrentent (Scf l) rifpenf., getnauelt, gepant, lainger als ber Blttffl' (4 22. - * $23 i j l$, Siraut, 巨aam. * 4759. ఫ̧

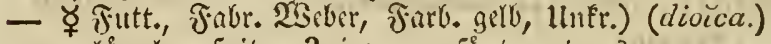

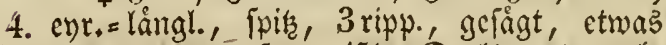
bide, unter netge, weipl.; Dordentraub.

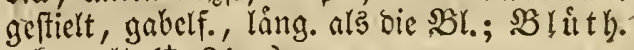
gefnauelt († SAm.)

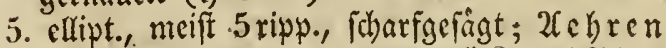

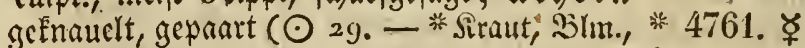
Eaant. - ఛ lnfr.)

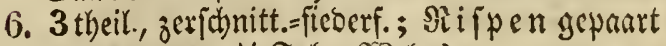

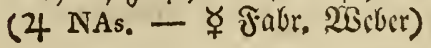

(urens.)

4762.

cannabǐna.

\section{II. wechselsweisstehend.}

Die Yanggefpitat. Blätter:

1. eyr., gefágt; $\mathfrak{x} \mathfrak{r} \mathfrak{a} \mathfrak{u} \mathfrak{b}$. rifpenf., ausgebreit.: bie unt. M. ftiellos: bie obern W. geftielt (4 NAm.).

4763. canadénsis. 
2. rundr.=evtr., langgeipiat, gezabnt, am Sirunde formaler il. ganjtand., Eeilf. 3 riph., aber., unten weipfill. (ち As. $\Delta-\not$ Del, Jakr. DSeber)

\author{
4764. ఫ్ధ \\ nivěa.
}

\title{
2. UTRICULARİA, 21.
}

W3 z lbl. zigejest; S (d) a ft blattlos; Nect: 1. Eegelf.; bie ungeth. Olippe:

a. eben fo lang als b. Saumen; Br. gefied,= vieltheil. (4.42. 45 . gb.)

b. 2 mal fo lang; $\mathfrak{B}$ l. 3 theil. $=$ gaberf. (4 42.45. (g. b.)

2. mad)enf.; Slippe ausgermo.; fo lang als

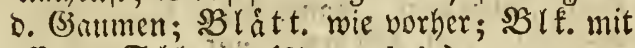
offnem Schlunoe ( 4 45. hgb.)

4765:

(vulgáris.)

4766. (intermedüa.)

4767:

(minior.)

\section{Blätter:}

1. impaffend a. nebft $5 \operatorname{tgl}$ unbel)ant (48. grn. -w.; gb.)

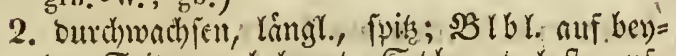
ben Seiten unbebart; $\mathbb{S}$ tbeutel ftumpf; Rect. rumbl. (4 NAm.: gb.)

\section{UVULARIA 284.}


b. ganzrans., unten fitz.; Blmftle einf.; St beut. unbegrannt, fing. als b. glodenf. IBIE. (

4772.

2. verE. erruno,

a. ganzrand., abgeruno.; Stgl fielrund; sBl mft le 1 blutth. (ち 9.47. rch,, Fr, schw.: b., mit w. Fleische - ఫ Fridhte, (jêtb.) (uliginósum.)

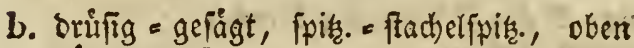

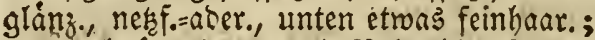
Ir a ub. ubberbang.; mit Rebenbl.; $\mathfrak{S} \mathfrak{I m}$ fitele in $b$. Slttwink., eingeln, fabenf.; Stbeut. begrannt, Fur. als D. Sg(E. mit

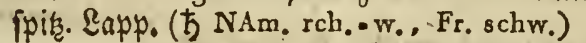

4774. arborěum,

3. verk. eytuno = lóngl., abgerund., ganzrano,;

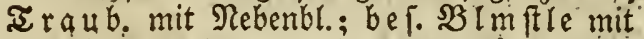
2ffterbl.; Stbeut. unbegrannt, für. als $b$. glodenf. SBIE. (ち NAm. w., Fr. schw. - bl.)

4775.

frondósum,

4. oval, etwas feingefågt, unten an $\delta$. 2rocrn feimbaar.; I $r a \mathfrak{b}$ b. mit Nebenbl.; ' $B$ IE. faft

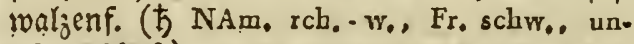
schmackbaft)

.4776 .

amo'enum.

5. oval = lanjettf.,

a. unbehaart, urten an $\delta .21$ bern u. am Ranbe feinbaar.,' ganjrand. oo. unteutl. gejägt; SI I f f le eingeln, 1 blieth., febe furz (b NAm. Fr. bl.- - schw.)

4777.

myrtilloídes.

b. mit hargigen Sुunct. bidht bejekt, etwas fpis, ganzrano.; Iraub. mit Rebent.; Etbeut. Kurzer als bie Engf. sBlf.' (t) NAm, w., Fr, schw, mit wch, Fleische)

4778.

resipósum.

6. längl. = oval, gefágt, fwik, fehr furz, frachels fpits.; İaub. uberbang., mit Nebenbl.; Stbeut. unbegrannt, turz. als D. fugel.s glodfenf. SBlE. mit 5 palt. Saum. († NAm, Fr. blch.-schw.)

4779.

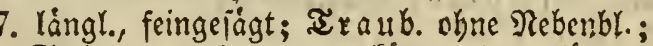
Stbzut. unbegrannt, fưrz. als $\delta$. lángl. eyr. \&BlE. (ち NAm, w., Fr. schw.) tenéllum.

8. langl.seyr., fpits, ganzrano., unten meis= grum; $B I_{m}$ ftiele 1 bluth.; Stbeut. bes graint, láng. als b. glodécnf. RIF. (ち NAm. - š.,-Fr.-grün, vuletzt w.)

4780 . ligustrŭnum. 4781. staminĕum. 


\section{Vaccinium, - Valeriana,}

\section{Bl. immergrün.}

\section{Stengel:}

1. aufrecht: bie âft: Aeste:

a. wed) jelsweizftehend; $\mathfrak{B l}$ l. verf, enr., ganj= rano., unngerollt, unten purictirt; $E$ it $b=$ trauben uberhang. (ち 5. w., Fr. r. * Sieer., Siraut - ఫ̧ Jrrichte", (Serb.)

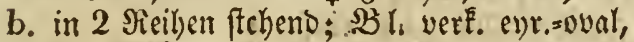
fpis, gefingt, unbelgart; ; notraben Doloentraubenf., úberhang., mit গlebenbl.;

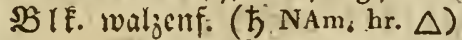

* 4782. ซ్ధ

(Vitis ida'ea.)

2. Friç), i fabenf.; Blätter:

a. faft lerafi.eenr., am Siante umgerollt;

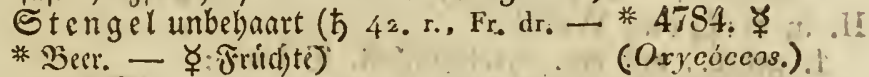

b. ovalelaingl., ganjrano.; flach) (ち NAm. 4785 . $\mathrm{s}_{\mathrm{s}}, \mathrm{Fr}_{\mathrm{o}} \mathrm{dr}$.) macrocárpos.

\section{VAILLANTIA. 66.}

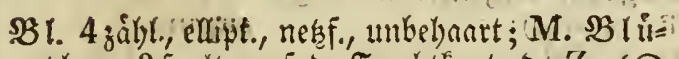
then 3 falt. auf $D$. Fruchtenot. D: Z: $(\odot$ SE. $\left.g r_{i}=g b c h.\right)$

4783 .
nițudumm. 
2. långlids, etwas gejabnt: bie interften ftumpf; bie obertien ipiz; Stgl gernoe;

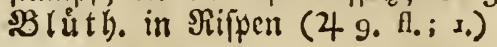

\section{B. Bl. üngleichförmig.}

\section{Blittlen:}

I. in Dolbentrhuben; Blätter:

1. berzf., ungl. gejafunt: bie unt. cinf.: o. obern 3 zábl. u. gefied.; Stgl geftreift 4790. (4 SE. 9. hr.,.,dann.w.) pyrenăca.

2. Stglbl. gefies.: $\mathfrak{N B}_{3}$ lblitt. ungeth.; Stgl glatt, ftielrund (4 3. 8. wo, wohl- * 4791. riech. = * $\mathfrak{B S}_{3} \mathrm{l}$, Siratut)

Phine.

II. in $\Re$ ifpen; Wzlbl..:

1. gefieo, mit ento, grobgejabnt. SBlattch: Stgibl:-berablauf. geftes., mit lanjettf., gezalint. Sapp. (4 9.)

2. herzf., cinf.: Stglbl. 3zoilhl, curums= längl: : Seitenblattch. lanzettf.; $2 \mathfrak{r l e} 4793$. gejal)nt; $\widetilde{t g l}$ gerabe (24 9: wi, zuerst rcll.) triptěris.

3. verf. enr.; Stglbl. langette: 2Alle ge= wimp. (24 SE: 9. rchli)

III. in arauben; Wzlbl.:

1. ent.; Stglbl. Kerzfo, ftiellos, zerfdnitt.= faft fpiesf.; Stgl e. Spanme. long (4 9. ugb.)

2. ellipt., 3 u. 5 ripp., gamzrano, u. etwas gezabut; Stglbl. gleichbr.: 2 rre un= 4796. geth.; Stgl gerabe (4 . . w.) saxatilis.

3. Feilf. = lơngl.: Stglbl. gleid)br.: 2crke ungeth., ganzrano., ftumipf; Stgl auf=. fteig., unbehaart (4 SE. 9. hgb., ausw。 * 4797. rch. - \# $2 \vdots$ l

\section{VELLA. 518.}

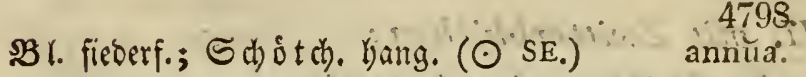

\section{VERÁTRUM. S04. (301.)}

Trauben:

1. rifpenf.; Nebenbl, an d, Zweigen: 
a. langl: bic bef. faft fo lang ats s. hatr.

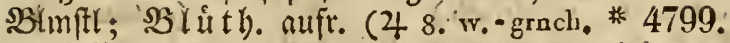

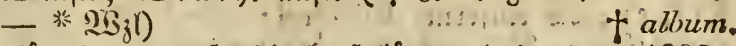

b. lángl. = lanzettf.: bie be f. long. als o. et= 4800 .

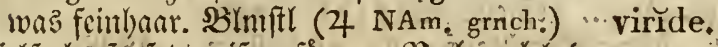

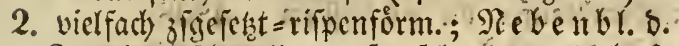
3weige fatmallanzettf., febre lang: o. bef. 4801.

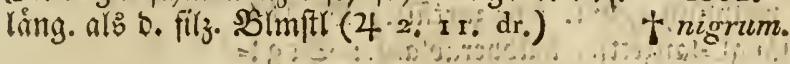

$\therefore$ at

\section{VERBÁSCUM. 125.}

I. Bl. hérablaufend, filzig,

A. gleichförming

Blätter:

1. gan beriblauferts, feingeferbt; Stbeut.:

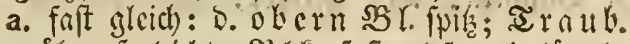

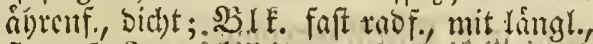
ftumpf. Lapp: (O' 1 3. 1 8. gb.., u. wohlriech.; wo geruchlos - * Sitait, Sllin. - * 4802. Ұ

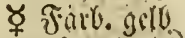

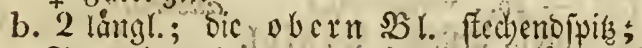
İaub. mit etwas entforiten sbiufdeln ( $\sigma^{7}$ 4. 8. hgrn.)

2. balbhcrabrauf, geferbt, weisfilf.: Iraub.

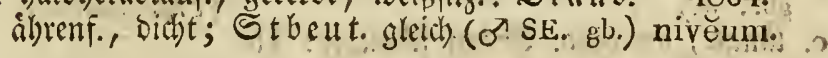

B. ung leichförmig.

Staubbeutel:

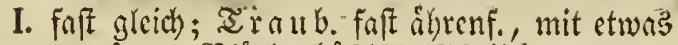
georaingt. SBluthenbuifchet; Walbl.:

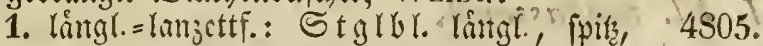
herablauf.: 2Crle fcingeferot (o SE. gh.) Thapsoídes.

2. Yaingl. = cllipt., geterbt, gefticlt: Stglbr. laingl., etwas fpis, untocutt. = ut. feinge= ferbt: bic d bern fipis, fdwadh hernblumf. ( $\sigma^{7} 9 \cdot \mathrm{gb}$. , wohlriech.)

(Thapsiss.)

4803.

cuspidaitum.

4804. . 


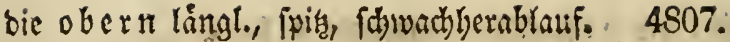

(4 6.33. gb., wohlriech.)

2. in sifpen: Die entfernt. Büschel:

a. wenigbluth.; $\$ B l$. geterbt: bie unt. cllipt.=längl., geftielt: Et glbl. längl., ipis, fow watherablauf.: bie oberften langgeipitst ( 4 1 9. rch.)

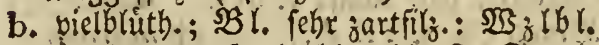
langl.:lanfettf., halbberablauf.: Stgl: blàtt. lángl. ipis, bopp. geferbt, balb= berablauf: : bie oberften langgefpikt (o' - gb.)

(collinum.)

III. unglech, 2 lângl.; Traubén:

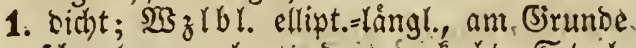
fohmaler, ungl. = u. Dopp. geferbt: Stgl= blátter långl, , ipí, cinf: geferbt: bie o bern runbl. =evr., ftechendipis, (d)wad)= herablauf. ( $\sigma^{\top} 22 . \mathrm{gb}$.)

2. mit entfernten Büfbeln; $\mathfrak{B}$ l. gelerbt: Wzlblätter:

a. lángl. =langettf., in b: \$lttefft serobunt: Stglbl. langl., fpik, ganzherablauf: bie obern breiterr., ftechendpis, $\mathfrak{h a l b}=$ berablauf.' ( $\sigma^{T}-$ gb., wohlriech.)

b. ellipt. oD. enr.=ellipt., geftielt: Stglbl. lángl., fpis: Die obern breitent., ite=

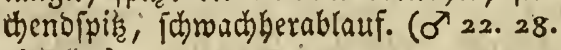
gb. ; "w.)

c. Yångl., an beyb. Enden fomaler: bie 4808. persifórum. unt. Stglbl. lanjettf, am Sirunde (d)maler: bie mittl. lăngl:=lanzettf.: bie obern langl., fchrwadberablaufeno $\left(\sigma^{7} 5 . \mathrm{gb}.\right)$

4810. conḋensátum.
4811: austrále.

(Phlomoides.)

4813.

nernorósum.

\section{Bl. umfassend.}

\$BY. werl. èr. långl, unbebaart, bopp. gefägt; BI mftle 1 blith)., einjelt $\left(\odot \sigma^{7} 14.49\right.$. gb., mit r. Strichen)

4814.

(Blattaria.)

\section{Bl. gestielt.}

1. Xrle'szl. geftielt, långl. = bersf., wellenf.= geferbt, etwas feinbaar. (4 14.26. gb. - 4815 . * $\left.2 b_{j} l\right)$ (nigrum.) 
2. mur $\delta$. Stglbl. geftelt u. eyr. = Yanzettfo; unten etwas fil $z_{\text {. }}$ feingeferbt: Die o berften fitel'os, oben unbehaart: 293 z lbl. eyr., bopp. geferbt, faft nadit; Stgl eck., rippenform.; Etgef. mit roth). Fillz (o 6. gb.)

4816 .

austriăcum.

\section{Bl. stiellos,}
A. gleichförmig。

\section{Blătter:}

1. faft Keiff.=långl., oben nacăt; Stgl eâ.; 4817. rifpenf. ( $0^{x} 13.23$. w.)

2. cyr. = lingl., etwas gefígt, auf beyd. Seit. wie mit Megl beftrent; Stengel ftielrund,

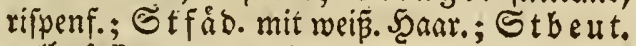
gelb ( $\left.\sigma^{7} 22,26 . \mathrm{gb}.\right)$

3. enr., unten bidht = woll. = fillz; Stgl åfig; Stfád. fámmtl. bart. (o'sE. gb.)

B: ungleichförmig.

Blithen:

1. geF̂nauelt, ftiellog; $\mathfrak{Z}$ 1. fil bopp. fieberf.: Stglbl. einf. fieberf. (ơ SE.) pinnatifidum.

2. zerftreut, faft cinzeln in rifpenf. Irauben;

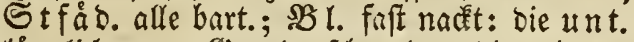
långlid, am Grumbe fdhmaler: bie obern bergfo, langgefpicist, faft ftiellos $\left(\sigma^{\top} \mathrm{As} ; \mathrm{gb}\right.$.) pyramidátum.

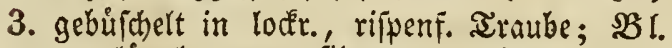
enr. =lángl., unten filf. = grau: Die $\mathfrak{u}$ t. am Brumbe fommaler: bie obern faft berff. (4 As. $g$ b.)

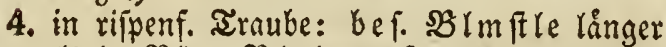
als D. SBlo.; $B$ K.: Kanjettf., ganzeand., filz: :

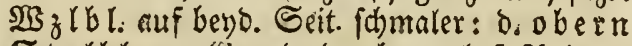
Etglbl. am (sirunde burch e. obrf. 2tnbang berzf. $\left(-g_{b}\right.$. $)$

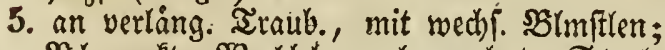

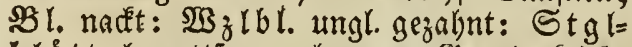
blåt t. lanzettf., gezahnt, am Srunbe feilf.; Stgl faft nactit (o' SE. dp.)

6. in åhrenf., menigblutth. Irraube; $B$ l. etwas zott., rungl.: $\mathbb{S}_{\mathrm{t} g} \mathfrak{l b l}$. faft ftiellos, gleich)=

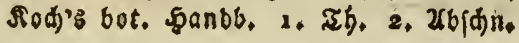

4822. orientále.
4819.

floccósum,

\section{1.} orientale.

4823. speciósum: 4824. phoenicěum. $\sqrt{2} 5$ 
geferbt; 23 glbl. langl., hergf., bopp. ge $=4825$. ferbt (4 SE. brauo)

ferruginĕum.

\section{VERBÉNA. 471. $\left(62^{2}.\right)$}

\section{Blätter :}

1. 3 jpalt., mit långl., jeridnnitt. = gejabnten Eapp.; Stgl aufr.; 2 ebr. fabenf., rippenf. * 4826.
(4 22, bl, ; rch. - * Siraut)
(oficincilis.).

2. 3 theilig,
a. zerihnitt.; ftumpf; Stgl åft., auffteig.;' 4827. Uebren fadenf., cinzeln (4 NAm. bl.) spuria.

b. zorrifl. mit fomallanjettf. Lapp. u. nebit

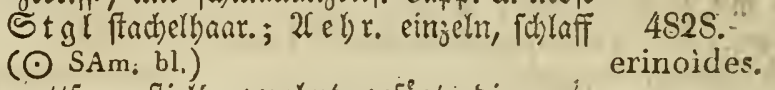

3. lanzettf.; geftielt, gezalunt =gefågt: bie unt. am Srunbe zerichntitt.=faft ipieśf.; 2 Uebren

gebufichelt (4 NAm, vo; r.) hastáta:

4. långlich, ungl. gefăgt, etwas ftumtp àm (Sirunde feilform., foit ftiellos, unten raucb);

2lehren fabenf., rifpenf. (4 NAm. w.) caroliniánà.

5. enruno,

a. Dopp. gezahnt, ftiellos u. nebft o. ganz geraben $\subseteq$ tgl jebr rauds; 2 ebren ge= buifhelt ( 4 NAm. bl.)

b. 3 fpalt., jeridnitt. =gezalnet, faft ün be $=$ baart; Stgl idarf; Uehren eingeln; fblaff ( $\odot$ NAm.p.)

$4 S 31$.

stricta.

4829.

$4 \$ 30$.

\section{VERNONIA. 643.}

\section{Blätter:}

1. lamjettf., juarf, feingeiagt; Dolbentraul= ben gleichbod); $B$ Iojd upp. an D. Spite fabenf. ( 4 NAm. p.)

2. eyr.=lanzettfórm., gejägt, unter feinbar.; Dolsentraub. mie vorber; BS rofbupp. evr., langgeipitit (4 NAm. p.)

3. oval, ftumpf, gefagt=geferbt: bie untern mit geflugelt., umfall. Blttft; $B$ lut ben faft doldentraubenf. ( 4 - p.)

4833:

noveboracénsis.

Aubletra: 


\section{VERONÍCA. 15.}

\section{Bldecke 2 blätırig.}

BImfte fo lang als die SBl.; Blåtthen 4836 .

2 lapp., gefágt $\left(\odot \sigma^{>}\right.$As. bl., klein) Crista galli.

\section{Bld. 1 blättr, 4-5theilig;,}

A. Blk. trichterförmig.

\section{Blätter:}

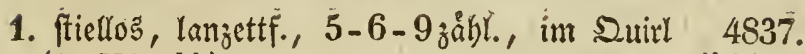
(4 NAs. bl.) sibiř̌ca.

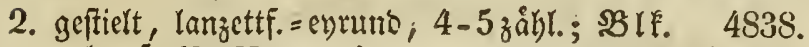
walzenf. (4 NAm. w.) virginı̆ca:

B. Blk. radförmig;

1. mit Endähren od. Endtrauben; Bl. im Quirl o d. entgegenges.;

a. Bl. gleichförmig.

\section{Blätter:}

I. gejågt,

A. einfachgefingt,

1. Ulle uno

a. ไanzettformig,

a. 3 zåhl. ; Stgl aufíteig. (4 As.bl.) paniculáta。

b. einige 3 , andere 4 zabl., faft $4 S 40$. ftiellos, glèiágejågt (4 r. 3. bl.; ; fl.) (spurǔa.)

c. entgegengef. do. 3 zaghl., fpich,

1) entferntgejågt, etroas fleifo,., am Grumbe keilf.; Stgl fein= 4841 . haar. ( - bl.)

2) bidit $=$ u. ungl. gefigt, ut. nebft Stgl feinhaar. $=30 t t . \quad(43.26$. 59. bl.; w.)

b. långl. = lavizettf., 3 żăhl. ut. entgegen= amethystǔna.

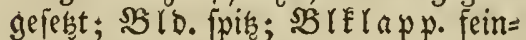
geferbt (4 - bl. u. w.)

c. faft berzf. =lanzettf., entgegenger., ut.

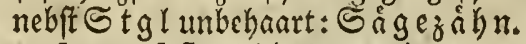
entfernt, faft gleid) (4 $\mathrm{SE}_{\text {. }}$ )

4842. media.

\section{3.} crenuláta。 4844.
glabra. 旅 2 
d. hergf.=eyr., ftiellos, febr ftumipf u; nebit $\delta$. ganz einf. Stgl haar.; I $r$ a $u=$ ben wenigbluth.; 3 I 0 . unbebaart (4 $\mathrm{SE}, \mathrm{bl}$.)

$4 S 45$.

e. Die obern langlich, etmos geiaght; Stgl aufr., ftaud.; $\mathfrak{x} \mathfrak{a}$ u b. vielblüth.;

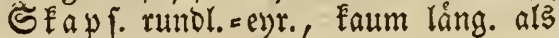
D. SRlb. ( 5 9. rch.)

4846.

fruticulos $\alpha$.

f. bie obern longl. = verk. eyr., ctwas ge= fågt; Skap Stgl ftrauch., weitichmeif.; Endo ols bentraub. wenigbruth. (ち 9. bl.) saxatelis:

2. gefågt u. ganzranbig,

a. an b. Spitze ganzranb.,

ค. lanjettf., ipic, mit entfernt., gleich., einf. Sågezăhn. (-)

b. eyr. = lanzettf. u. nebft Stgl fein= haar., mit bicht., ungleid). Sagges zåhn. (4 SE.)

b. am Grunto ganzrano. u. Fcilfo, fpic; Stglaufr. \$ D) fla nze weisfil. ( 4 SE.) neglécta.

c. einige gefágt, andere ganjrandig, unbehaart, ellipt. =eyr.; End Dolden= tra $u$ b. faft ábrenf.; $\mathfrak{B}$ ld. gerimp.; SEap f. vert. eyr.; Stgl einf., didyt= murzelnd, frautart. (4 9. r.)

3. boppeltgefágt,

1. eyr. ob. eyr. =lanzettf., $3-4$ záhl., faft

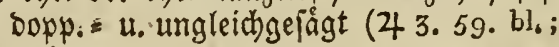
lila)

2. Fhmallangettfórm., am Girunde eyt., $3-4$ zól.t., langgefpist, tiefgefágt uno nebit $D$. Stgl etwas feinthar.; $\$ B I D$. faft gleichf., kinr. als ๖. Skapf. (42. 36. bl.; f.; w.)

3. Yanzettif, am Sirunoe fajt berzf., 3 z̧áhl., langgeipitst, mit am Sirunoe tiefern Sảgezáhn.; Sু) flanze (chmad) feintaar. (4 SE. bl.)

4. herzf.=lanjettr., entgegengef., 3-4 jåbl., ranggeppist, neblt b. Stgl feinhaar.s filz. (4 3. 59. bl.; f.; w.)

II. gezabnt, entgegenger., zigefalt., mit bice. Sạh.; Seitentrauben Eurz., faft uber= hang.; BlElapp. gangrano: (4 - bl.) complicáta:

4853.

(mariťma.)

4854. elaťor. 4855 . (longifolia.)

4856. 
III. geferbt,

1. 2 rre geferbt u.

a. vert. evr. u. neblt o. ganz cinf, auf= fteig. Stgl haar.: Stglbl. entfern= ter; 王raub. borbentraubenf., raudh, meif 5 blutth. u. nebft SEapf. verf́. evr. (4 9. bl. u. w.)

b. rundr. = eyrr., georangt; $\mathfrak{x} r a \mathfrak{c}$ b. Fittr; geerángt; $\mathfrak{B}$ lo $\mathfrak{l}$ a p p. ftumpf, gewimp.; Stgl ftrauch., niebergeftrecêt (4 $\mathrm{SE}$. 9. bi. .)

c. längl., entgergengef. ut. nebit $\mathfrak{B}$ [d. un= bebart; $\mathfrak{x} \mathfrak{i} \mathfrak{a}$ b. verlang.; 3 lit then v. cinanber entferntfteheno; Stgl autfs fteig. (4 14. 24 . blch.; f.- - wch.)

2. einige geferbi, andere fáf ganjrando: 2Crle lanjettf., ftumpf; Stgl aufrectit; Jy flanze weífilz. (4 SE.)

IV. fieberformig, etwas gebuifchelt,

4857:
bellidioides.

4858.

nummularĭa:

4859.

(serpyllifolia.)

4860. incána.

1. lanzettfórm., geffielt, mit fáft ganzrand.

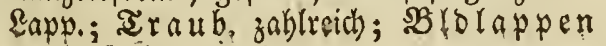
Lanzettf. (4 NAs.)

2. gleichbr., furzgefticlt, mit ganzrano. Iapp.;

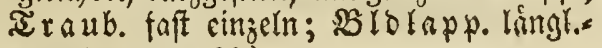
e.jr. (4 NAs. bl.)

4861. incísa.

4862.

laciniátạ.

\section{b. Bl. ungleichförmig.}

\section{Blätter :}

1. gefågt: $\mathfrak{N}_{3}$ glbl. eyr.: Stglbl. länglicy; fipit, gefficlt, am Srunde u. an b. Spise ganzranto; sB líth. traubenf.; Rebenbl. gleid) br.; sp franze zott. (-)

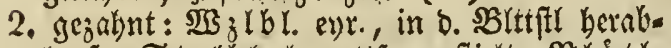
lauf.: Stglbr. lanzettf., geftielt; $B \mathfrak{l}$ it th. áhrenfơrm.; Rebenbl. u. SB lo. gewimp. \$) flanze filz., mit geftielt. Drifien (4).

3. ungl. gefógt=gejahnt u. nebft Sigl feint= baar.: $\mathfrak{S B}_{\text {z }}$ lbl. geftielt, eyr.: St glbl. faft ftiellos, ellipt. = längl.; $B$ lut th. faft abrenf.; Rebenbr. gleichbr, láng. ats D. $\$$ Blb. (4 8. hbl.)

4. geferbt; Walbl. :

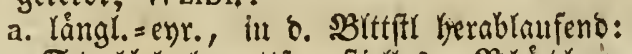
Stglbl. lanzettf., ftiellos; $\mathfrak{B l u}$ the

4863. menthaefolia.

4864. Clusiit. 
åbrenf.; Rebenblått. u. Blo. haar.;

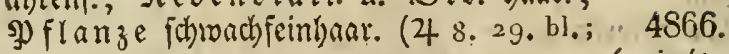
w.; ..)

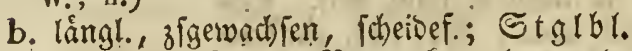
(spicáta.) eyr.= lanjettf., am Sanbe Enorpl., an D. Spike geferbt, am (Sirumbe famaler: bie oberiten fpic, gangrand.; Stgl einf., aufitcig.; (5not tạben boldentraubenf: (4 As. hbl.; w.)

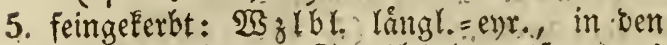
sittitl herablauf.; Stglbl. lanzettf., etwas fpis, faft ftiellos; sB lut hen biditábrenf.; Blélappen íbntallangetti., läng. als ber Stbeut.; Sु fla dbl.)

6. bie unt. gefied.: bie obern fiederf. u. einf., mit fabenf., ausgebreit. SBlôttâ). u. Eapp.: Ulle etwas gebiujhelt; Stgl aufiteig. (4 NAs. w.)

4868. orchidéa: gentianoídes.

2. mit Seiten-Trauben od. Aehren;

a, Bl. gleichförmig,

\section{Blätter :}

1. faft ganjrano., fomallanzettf.; bef. $\mathfrak{B l} l \mathrm{~m}=$ friele hang. od. Febr abfebeno; SBldece 4 theil.; Stgl faft aufred)t (4 50. hfl. - * 4870. * Sirnut)

2. gejågt, (scutellata.)

a. lamettf., unfall.; SB!o. 4 theil.; Stg! 4871. aufr. ( 4 41. 45. rch. - hbl.)

b. långl, = rumol., fteif, glänz. u. nebjt ben friect., nieberlieg. Stengel unbehaart; Bł luth. ibrenf., fehr georångt (4 $8 \mathrm{E}$. 9. bl.)

(Anagállis.)

pinnáta.

4871. Allióni. 4873.

Tournefortịi. aufr. (4 SE, bl:)

d. hergf. =eyrums,

ค. ftiellos; Lld.:

1) 4 theil.; $B$ l. fpit; Stgl ganz ges rabe ( 4 bl. - p.; bbl.)

4874.

urticaefolia.

2) 5 bláttr.; $\mathfrak{B l}$ r. rungl., ftumpfigelågt; Stgl ivic worker (4 8. 9. bl.) 
b. geftielt, ftumpf, grobgefägt; $\subseteq t g l u$.

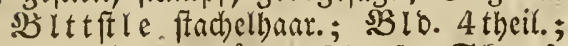
Irauben verlang., fabenf.; SEapf. frcišruno $=2$ Enopf. (4 I. wch. od. hbl., mit p. Adern)

3. feinge lägt, ellipt., ftumpf, Eurzgeftielt; SBlo. 4 theil,; Stgl nieberlieg., unterbalb wux= jelno (4 40. bl. - * Siraut - ఫ Futt., Linfr.).

4. zerfd)nitt. = gefagt; eyr.; Stgl fachelhaar., in 2 sieiken mebrharig; bef. SBlmftiele o. Frud t harar., 2 mal fo lang als bas

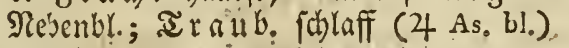

5. gejalint,
a. tiefgezalut, eyr., ftumpf, gefalt.; Stgl nizbergeftrectet' in' 2 Sieiben haar. (4) pilósa.
b. feingezafnt, fomallanjettf., gewimpert; Stgl fait aufr., rauch (4) Parmularia.

6. bopp. fieberf., mit lamjettf. u. gleichbreit. Eapp.; Brolapp. ungl., priemenf.; Stgl

niederlieg., am (S)moe ctwas $\mathfrak{y o l}_{3}$. (4 NAs.) multifida.

4876.

(montána.)

* 4877. 후

(Beccabinga.)

4878 . pedunculáris.

\section{1.}

\section{b. Bl, ungleichförmig:}

\section{Blätter:}

1. feblen am Schaft: einige $\mathscr{Z} z \mathfrak{l} \mathfrak{l}$ l. langl.; andere rumbl.; Stgl nadt, febr furz; BImftiel fobaftf., meift 3 bluth. (49. bl.) aphilla.

2. gefagt,

a. einige vert. eyr., anbere verf. evr.= runol.; $\$ 318.4$ theil.; Stgl unterbalb wurjelno (4 5. 13. hbl, mit dbl. Adern - * Siraut, Bim.)

b. långl., ftiellos, ftumpf: die obern lan=

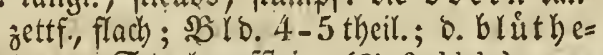

3. bie untern grobgefógt, lángl., jott.: bie obcrn gezabut, erie, rumjl.; Stgl jot̂. (4 3. s. 26. bl. - - 浩 Siraut)

* 4883.

(officinális.)

4854.

(prostráta.)
(Teucrium.)

4. entferntgẹagt, ichmallamzettf.: bic andern ganjrant., gleichbr., am Ranbe umgeröllt;

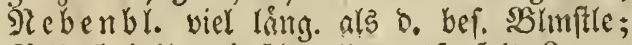
Blo. 5 their, mit fibmallam jettf., ipis. Eapp.; Stgl auffteig. $(4-b l$.

4856.

Schmidtü. 
5. zeridunitt. = gefágt,

a. Ufre: D. oberfen hersf. =cyr., ftiellog:

s. unterftengefrielt, ent.; $\mathfrak{B}$ Io. 4theil.; Stgl in 2 Neiben baar.; $\mathfrak{x} a \mathfrak{u b}$. in $b$. sglttwinte. entgegengei., läng. als ๖. Stgl * 4887. (4 3. 26. bl.; H. ; w, - * Sirant)

b. einige fieberf.: 2crle ftiellos, lanjsttf.; Nebenbl. ungetheilt, Eurr. als Der ber.

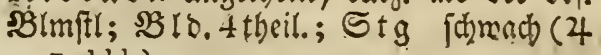
I. 3. hbl.)

(Chama'edrys.)

6. fieberfêrmig, $u$.

a. bopp. fieberf., ftiellos; bie unt. Rebens blát t. 3 ipalt, lång, als ber bej. SBlmft; SB Ib. 5 theil.; Stgl faft aufr. (4)

b. nur bie unt. Eammf. e fieberf.: s. obern

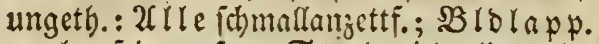
ungl. pfriement.; Ștgl nieberlieg. (4) As, bbl.)

4888.

austriüca,

4889.

lacquini, A.

3. mit 1tlüth. Blmstlen in d. Blttwink.

a. Bl. gleichförmig.

\section{Blätter:}

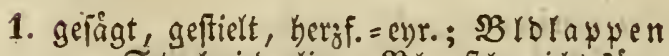
evr.; Et g I nieberlieg.; B Bl mftle nidyt fúr.

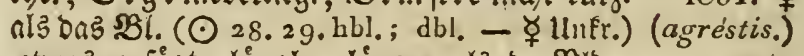

2. etwas gefágt, långl., lánger als $\mathrm{b}$. SBlb.; Stgl aufr.; SBluth. ftiellos $(\odot 28,32$, blch.; w.)

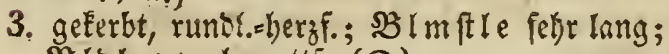

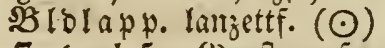

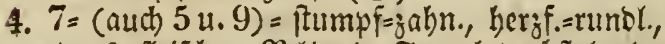
etwas fleifot.; SB!s. o. F) ভ́

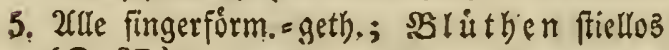
(๑. SE.)

4891. ఫ

4892.

peregrina. 4893.

filifórmis,
Cymbalaria, 4895. digitáta,

b. Bl, ungleichförmig:

Bläțter:

1. bie unt. gefågt, geftielt, bersf. = ệr.: Bl. an d. Bliith.: 


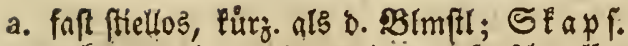
verk. eyrunt, ausgerano.; aufgejdroull.; ङtgl faft aufr. $(\odot$ 28. bl.)

b. ftiellos, lanzettfo, lång. als b. BImft; Stglblatt. geferbt; SBIDlapp. ungl.; B I

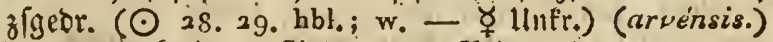

2. Die unt. gefétbt, geffielt, enr: : $\mathfrak{B l}$ l. and.

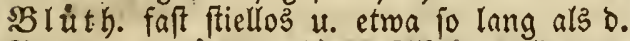

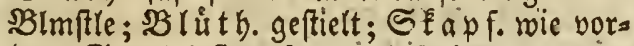
her; Stgl faft aufr. u. einf. (๑ 29. hbl,, mit dbi: Adern)

3. 5 lapp:, berzf $=$ runbl., fo lang als $b$. $\mathfrak{B l m}=$ 4896. praecox:

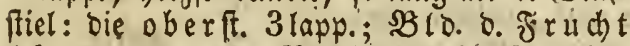
4 Eant., zigeneigt; SBlolapp. bersf., ipic; Stgl nieberlieg. ( $\odot 22,29,32$, hbl., dann $w_{0},-$ ఫ̧ UnRr.)

4. bie unterften ungeth.: bie mittrern fine gerf. = getf.; bie obern 3 ipalt.; B B Im ft le läng. als. D. $\$ B I_{b}$; Stgl aufr., etwas abs ftehend $(\odot$ 29. bl. u. wch. - ४ Hunfr.)

5. fingerform. = geth.: bie ob erften ungeth.; SS li t $t \mathfrak{b}$. faft frielloz; sB Im ft le furr. als b.

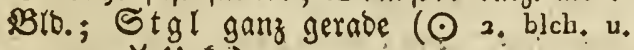
$\mathrm{grn}^{\mathrm{rn}},-\not{t} \mathrm{Unf}$.)

4899. 후 (lederifolĭa.)

4900. ఫ్ధ (triphylllos.) (verna.)

\section{VIBÚRNUM. 239.}

\section{Blătter:}

1. fdimallanzettf., unbeutl. gefägt ob. ungeth., oben glänzeno, unbehaart; Xfe fte 4 jeit. (5) NAm.)

2. Yånglitì,

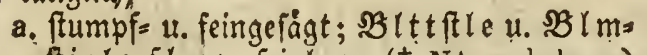
ftiele folupp.=feinlaar. (ち NAm. gbch.-w.) squamátum.

b. am Srunbe fotmaler, etras frumpf, ganz? ranb., am Rande umgerollt, unbehaart, oben glånz., mekgf. × aber., unten blafler, 4904. zartpunct.; 2 e eft th. 4 jeit. ( $\hbar \mathrm{NAm}$. gbch. w.) nudum.

3. langl. $=$ lanzettf., ungl. $=\mathfrak{u}$. faharfige fägt, am Brunce Eeilfớrm., ganzrano.; Ul ête 4 feit. * 4905,

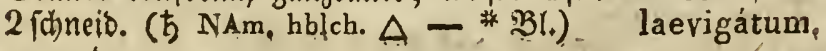
4. eyrunt,

a, gamz eyrund, 
ค. gezabnt = gefägt, gefalt.; furģgeipingt, oben unbebaart, unten an o. Slippen feinbaar. ( 5 NAm. gbch.- w.)

4906. dentảtum.

b. etras gefågt u. ipis, unbebaart; $\mathbb{S}$ It $t=$ ftiele glatt; $\mathfrak{F} \mathfrak{x} \mathfrak{u} d t$ te cyr. =läglich; 4907. 2ifteroold. etwas geftielt (ち NAm.) pyrifolium:

b. breitenr., langgeipict, id)arfgelägt, unbe= baart; $B$ lattfiele frauggerandet ( NAm. w.)

c. evr. lơngl., ganzrand; unten an s. 20oers after neblt D. bef. 3weigen oruf. haar.

(b $\mathrm{SE}$. w., vor d. Aufblühen ausw, rch. gestreift)

d. ent. =långl. = herzf, gejägt, oben jammets art. $=$ weich), unten rumgl. =aber. $=$ fil $l_{3}$. (h) SE. w.; rch.)

4908.
Lentágo:

4909.

Tinus.

e. enr. =lanjettfo, an beyd. Eno. "ipiti, am Rante etwás umgerout: bie allt. gefägt= gejabnt: Die ober u ganjrand.: D. ober= fien lanjettf.; $B$ l tt it le nachenf., brir.;

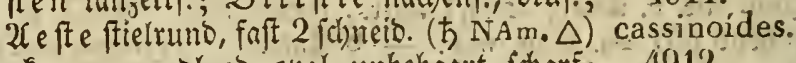

5. verf. evr. = rundl. ob. oval, unbehaart, faarf 4912 . gefagt; $\mathfrak{B}$ lttfle gerånbelt' (

6. 3 lappig,

a. grobgeragt, zuw. herzf. =evr.; sblttitle obne Druien, etwas haar., am Sirunde mit e. Spur v. Ufterbl. u. etwas filz.;

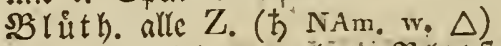

b. alle langgeipitst = gejahint; $B$ ltt title unz behaart, mit in D. Nitte vertieft., ftielluj. Drữ.; 2 fterdold geftrablt, im Strable geidlechtlos (5 3. 49. w.)

c. Die unt. Eurggezahnt: Die oberften fajt ganzrano.: Olle langgeipitst; BIttitle unbehaart, mit feulenf. Srujen: bie unt: Drúfen meift geftielt (

4913. acerifoluum.

4914. (Opülus.) 4915. Oxycóccos.

\section{VICIA: 576.}

\section{Blmstiele mehr als 2 blüthig.}

Blumenstiele :

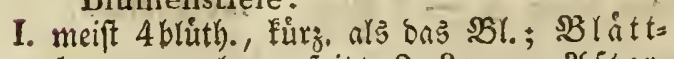
den enr., langgerpiet, $2-3$ paar,; $\mathfrak{X} f \in t=$ 
blätter harbufeilformig, am Branbe ge= ...4916. zabnt $(49$ !)

II. mebrbblittbig; Blättchen:

1. evrutio,

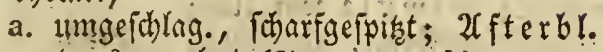
etrags gejabnt (4 4. 8. p.-bl.; w. - 4917. ఫุ $\Varangle$ Futt.)

b. Die unterfien fiellos; SBattifiele

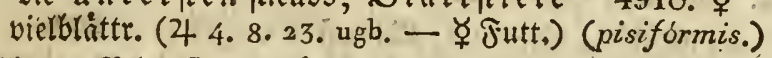

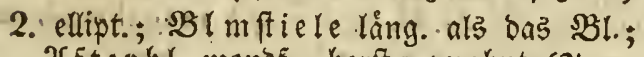
afterbl. monof., borft. =gezahnt (4 5. w. u, bl.,..übelriech, -

3. långlich; Afterble:

a. balkpfeilf., ganzrant., lanzetfform.;

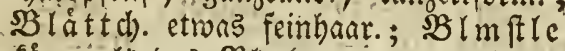

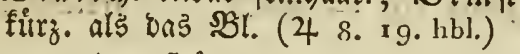

b. ungeth.; Şill len zott., ent. =långl. $(\odot$ As. dr. $)$

4. långl. = eyr., 4919. ళ̧ (sylpaticu.)

(dumetórum.)

4918. ఫ๐

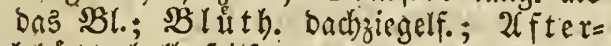
blått. barbpfeilf., cyruno; am (srunbe ftumpfgezabnt $\left(\odot-p_{0} \cdot\right.$ v. $)$

4920.

(cassubica.)

4921:

nissoliảna:

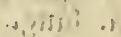

4922.

pillósia.

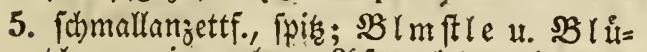

then wie vorber; 2 fterbl. halbpfeilf.; lanzettf., ganzrano. (SE.).

6. lanjettformig; sie Galbpfeilf. Afterbl.:
4923.
polyphýlla.

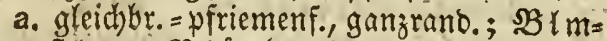
ftle u; sBluth. wie vorber; $B$ Iatt d)en ftumpf (4 3: 29. p.-bl.; w: ఫ̧ juttt., lutfr.)

b. geftielt; $B$ Iat t fticle gefurdht, meift 12 blåttr.; B B lát tho. unbehaart ( $\sigma^{\top} 38$. bl. u. w. - $\Varangle$ jutt.)

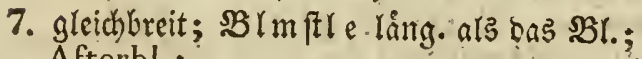
Afterbl, :

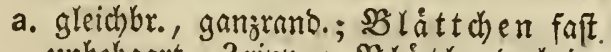
unbehaart, 3 ripp.;' $B \mathfrak{l}$ it th. Dachjic= gelf. (4 6. $19 . v$.)

b. halbpfeilf., fdomallangettf., am Grunde

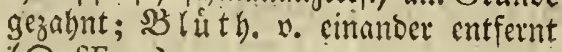
(○ SE, p.)

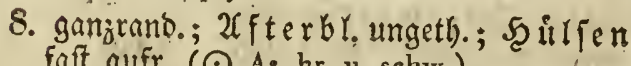
4924. 모. (Cracca.) 4925. ఫ্ (biénnis.) 4926.
(ienuifolia.) 4927. onobrychioídes faft alfr. ( $\odot$ As, hr, u. schw.) benghalénsis. 


\section{Blmstlc 1 blïth. od. fehlen.}

I. $\mathfrak{B I}$ Imftle 1 bhìthig; Afterbl.:

1. ungeth., lanzettf.; $\mathfrak{B l m}$ ftle felly rurz;

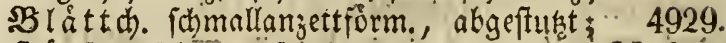

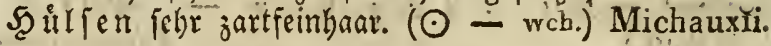

2. 2 \{paltig; Blättch.:

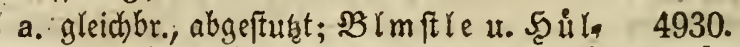

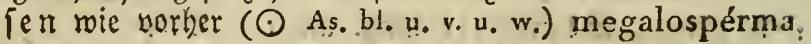

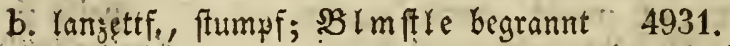

$(\odot$ r.v.)

(monúntha.)

3. vielfwalt.; Brmfte Das sBl., begramnt; B B åt tch. greid)br., ftumpf, idharfgeipigt $(\odot-$ wch. $)$

4932.

articuláta,

II. Hülsen :

A. ganz ftielloz,

1. meift 2 záall.; Blättchen:

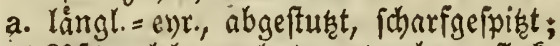

"2lfterbl. gejabnt, unten braunflect.

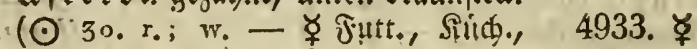
Unfti.)

(sațiva.).

b. bie unterften eyr., an b. Spitze eingebrúteft, ausgerand.: bie obern gleichbr., an ber Spitze ganzrano.; Jeulfen abjtebeno; afterbl. ge*

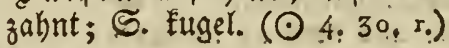

4934.

(angustifolǐa.)

2. einzeln,

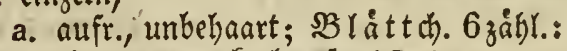
bie unt. verf. berź $(\odot$ 2. 8. 19. p. - bl.)

b. umgẹalalagen, haar.; Fähnchen:

4935. (latihyroides.)
a. unbehnart; Rffterbl. gefårbt; Etgl weitj̄dneif. $(\odot 29$. hgb.) lutěa.

b. zott.; Şúlfen 5 jaam. $(\odot-)$
gb.) hylruda.

B. faft fitiellos,

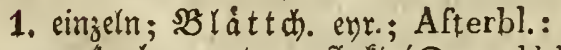
4938.

a. 4 zahn., unten gefledt $(\odot-$ blch.) globósa.

b. gemimpert = gejafint; Ș úl len jige= brưutt, etwas aufgeblaf.; 8 lát tø.

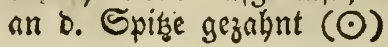

4939. platycárpos. 
2. gepaart, umgeichlag., unbchaart; $B \mathfrak{g} \mathfrak{a} t \mathrm{t}=$

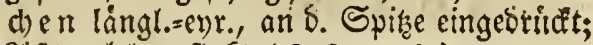
Afterbl. gefledít ( $\odot$ SE. ugb.)

4940. sordǐda.

3. 3 şáblig; Blättchen:

a. ganzrand., eyr.; SBltfite oline Siari= fen; Ş úlfen Enot.; $\{$ fter b l. pfeilf., am STunbe gejahnt $(\odot$ As. w. u. schw. 4941 . ఫ

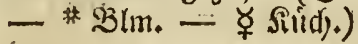

Faba. 4942.

b. nébft. b. Xfterbl. gefagt ( $\odot$ SE.) serratifolia.

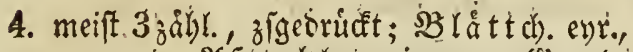
ganzrand.; $\mathcal{A} f$ ter bl. gemimp., am (Srunbe

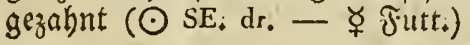

5. 4 faam, bang., unbebart; B̉ láttchen 4944. gleichbr., ausgerano. ( $\odot$ SE. $\left.p_{*}-v_{b}\right)$ peregrina.

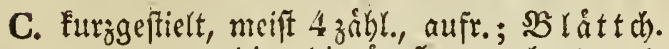
evr., ganzrandig: bie åu Bern abnebmeno (4 26 . dbl, ; w. -

4943: భৃ narbonénsis.

D. geftielt,

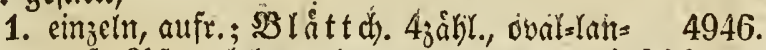
jettf.; $\mathfrak{A} f$ ter $b$ l. gezabnt ( $\odot$ SE.v.u. wch.) bithynica:

2. meifi 3 zábl. u. nebft o. Fibnch. Gaar.; 4947. 2ffterbl. lanzettf., gefledt (๑ 3.w.; v.) pannonca.

\section{VINCA, 137.}

SBI $\mathfrak{H}$ th. geftielt; Stengel :

1. niebertieng, rank.: $\mathcal{X}$ efte nufr., 4 feit.; $B Y_{\text {. }}$ ellipt.=lanjettf., am $\Re a n b e$ unbebaart; $\mathfrak{B} \backslash \mathbb{D}=$ zåbne lanjettf., unbehaart (ち r. 8. hbl.; * 4948. ఫ uv.; w. ; bunt - * Sirant - $\Varangle($ (Serb.) (minor.)

2. niebergeftredit, frautart.; $\$ 2$ I. wie vorher; 4949. B I O. gewimp. (4 SE.v.; w.) herbacĕa:

3. faft aufr. $\mathfrak{X}$ efte gerade, 4 feit.; $\mathfrak{B l}$ l. eyr.:

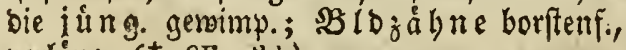
verláng. (ち SE. dbl.)

4950.

major. 


\section{VIOัLA. 158.}

\section{Afterblätt. häutig;}

A. ohne Stengel.

Blätter :

1. hanof., 5 lapp., gezahnt: einige ungeth.; 4951.

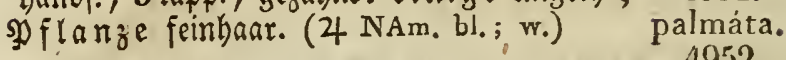

2. fußf., 7 theil. (4 NAm. bl, ; hbl. u. dr.) pedáta.

3. långl., fpich, herźf. =pfeilf.; etwas gejågt, am Sirunde zerichintt.; SB lut then umgewand; BI I m ft le läng. als bie Bl.; B Blorappen gleidbbr., unbelaart; bie 3 innern $\mathfrak{B l b l}$ am (Srunbé bart. (4 NAm. bl. u. w.) sagittáta.

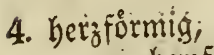

a. ganjher formigi,

a. unbehaart,

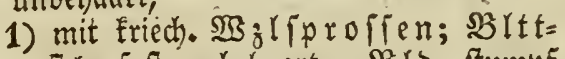
it te faft unbehaart; SBlo. ftumpf (4 4. v.; w., wohlriech. - * $\mathfrak{W B}_{3} l_{\text {, }}$

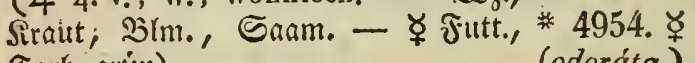
Farb. grim)

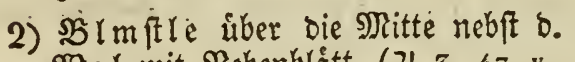
$\mathfrak{M}_{3}$ zl mit Nebenblatt. (4 3. 47. v.* 4955. rch: $u, w_{\text {. }}$ )

3) nidit; ; Bl.:

a) geiånt, am Brunde Eappenform. ; SBlmitle fo lang als D. SBlátt.; sBlbl. íhiefgebog., an d. Seitert bartig; Splanze unbebaart (4 NAm. bl. u. wo u. vi)

b) geferbt = gefägt, fpic, faft flach;

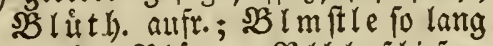
áls bie SBlátt.; $B$ lbl. pothiefge= bog.; an $D_{0}$. Seitent fénmarer u. län= ger, unterbalb ber Mitte bartig; sू fla $\mathfrak{a} \mathfrak{n} z \dot{e}$ unbebaart (4 NAm。

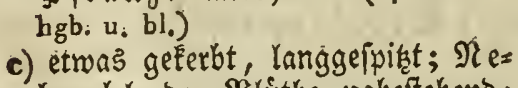
benbl. Der sglutthe nabeftehento;

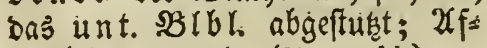
terbl. ganjanto. (4 - bl.) Schmiditána. 


\section{Viola.}

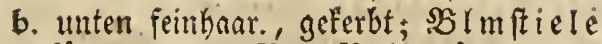

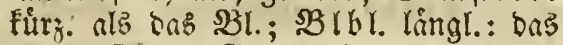
unterfte am Sirunde bart. (4 NAm. v. u. w.)

b. långl. = her fípormig,

ค. nadet, etwas ftumpf, geferbt, am Sirumbe mit ungleich., einwaertsgebog., fappenf. Rapp. (4 SE. hv., wohlriech.)

4960. ambigŭa.

b. nebft o. SBlttiflen haar.=bactr.; Bro. 4961. ftumpf ( 4 4. bl., gerucblos)

(hirta.)

c. nicrenf. $=$ kerzf., nebft s. SBlttften haar. $=4962$.

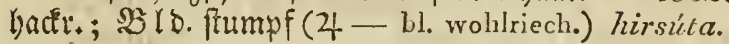

5. ganz nierènf., unbebnart; 925 z friechend; 4963 : B ID. ftumpf ( 4 42. hbl.; fl.)

6. rumbl. =ellipt.; geferbt, geftielt; $2 \mathfrak{f t e r b r}$. gleichbr., gefagt, fo breit als ber B!attfiel; SB Ibl. 3 mal fo lang als o. SBlo.: bas cin= zelne breit, abgeftukt, an b. Spise einge= orickt: Sporn faft fo lang als b. SBlb. (4 9. dv. u. wch.)

4964.

alpina.

\section{B. mit Stengei.}

Stengel :

1. fchwach, meift 2 blưtl.; $: B$ l. nierenf., gelägt; 4965 . BID. fpic; 2Cfterbl. ganzrant. (4 9. gb.) biflora.

2. einfach; Blätter:

a. herzf.=runol., faft unbebaart, feingeferbt; Stgl etwas fcinbar.; Ufterbl. lan= jettf.; gezahnt; SBlo. Fpiç (4 - bl.) Allióni.

b. faft herzfo, longgefpist, gefägt; sBImftle fo lang ols die $\mathfrak{B l}$.; 2l fterbl. Furz, une geth.; Sl flanze faft unbebaart (4 NAm; wch. Jgbch.)

4967. canadénsis.

3. åft., mit abfelend. Smeigen; 2 fterbl. ge=

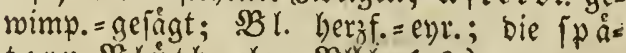
tern $\mathfrak{B l} \mathfrak{l}$ th. olne $B \mathfrak{b l}$. (36.)

4968.

littorális.

4. aufrecht; Blätter!

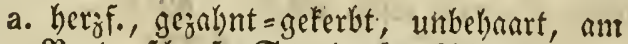
Ranbe fitarf; Stgled.; 2f terbl. an 
e. Seite gezahnt; Rebenblåt t. ủber ber Mitte bes Blmitiz; SB lùth. fámmtl. ohne Blo. (4 - hbl.)

4969.

neglécta:

b. herzf.:långl.; Ifterblàtt. wie vorher; 4970.

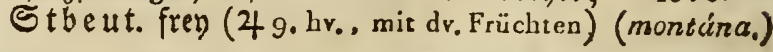

c. nierenf. = herzf., unkehaart, gefterbt, fpits,

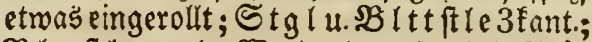

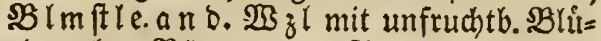
then ohne SBlb.: an D. Stgl mit frucht= bar. ofhe SBlbl.; Xf fter bl. langettf., wels feno (4 4. 8. wch, u. v.; lila, wohlriech.) 4971:

(mirabilis.)

d. eyr. 三ellipt., gefetbt, unbehaart; 2f fter 4972. blát, gelágt (4 SE, hb.)

5. abfteheno, êtwas zifgeoruoft; die unt. $\mathfrak{B} l$. faft bers $f_{0}=$ enr.: bie obern evr. = lanzettf.; feingeferbt; 2 fterbl: lanzettf., von beyd. Seit. gemimp.=gejahnt (4 SE. bl.-v., geruchlos) glauca,

6. aufiteigento; Blätter:
a. långl. = berzf., ftumpf, punct:; Xfterbl.

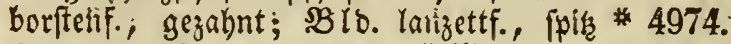 (4 2. 26. bl, geruchlos — * $2(3 j)$
b. enr.=lanzettf.; St gl ftielruno; $\mathfrak{A}$ fter bl. zeridunitt. =gejågt (4 3.7. 59, bl. - rch.; hbl. u. p.)
4975:
(lactěa.)

7. nieberlieg.; Blätter:

a. herff., 位umpf, geterbt, feinhaar.; $2 \mathfrak{f}=$ terbl. fdimallanjettf., ganjrano.; $\mathfrak{i e =}$ benbl. über D. Mitte D. SBlmfile (4 3. 59. $r_{0}$-lila, geruchlos)

4976 ;

Krockéri. 4977.

b. långl.; ganjrano., gemimp. hadfr; 2 fters blátt. ungeth.; $B$ ID. \{pic $(\odot S E$. p. u.gb.) Valderĭa.

\section{Afterbl.flederf.; Narbe krugf.}

\section{Stengel:}

1. feblt'faft; $\mathfrak{B l}$ l. rundl. = ellipt., gefferbt, ges ftielt; 2 f te $\mathfrak{b l}$. gleichbt, ganzrano., fo breit

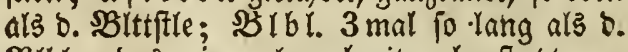
SBlbl.: bas eingelne breit, abgeftust, an o. Spine eingeorudt; Sporn lâng als D. SBIo. (4 9. $\left.g b_{i}\right)$ 
2. fur ; : ęct. pfriemenf., läng. ałs b. $\$ B$ tbt.; 2B r. faft eyr.; Xfferbl. gezabnt (4 SE. 9. p.; bl.; gb.)

3. einfach, 3 entig; Afterbl.:

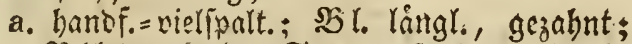

4979.

calcaráta 28 lol. geferbt: Sporn fo lang als bie Bglo. (4 9.)

b. hanof. = zeridnitt.; şl. enr.=längl., ge= 4981. ferbt, gerwimp. (4 SE. sb, u. schw.; blo; v.) lutěa.

c. fieberfơrm.; $\mathfrak{B l}$ l. faft lảnglide) (24 9. gb., 4982. grofs)

4. ecểig; Blätter :

a. länglict), gezaknt=geferbt; $S t g l$ weits

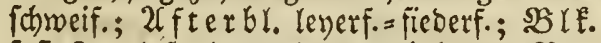
faft 2 mal po lang als b. unbetyaarte BBro. $(\odot$ 29. gb. u. v.; w. u. gb., od. hbl. - * 4983. ఫ్

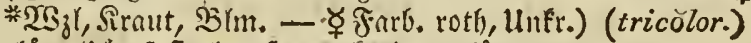

b. länglich = faft her子f., geferbt $=$ gefågt, u. nebft D. weitichneif. Stgl ftachelhaar.; 2ffterbl. leverf. = fieberf.: Der áüerfte

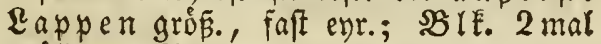

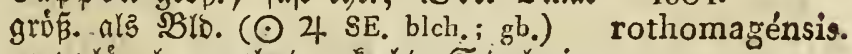

c. ent. = längl., gezallynt = geferbt; Stgl nies

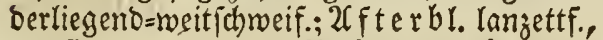
ain Srunbe fieberf.; $\mathfrak{B}$ lḱ. Eaum lång. nls D. Kaar. SBlo. (○ 28.gb., dann lila-bunt; * 4985. そ bl.-v. u. gb. - * Siraut - $\mathfrak{u}_{\mathrm{unft}}$.) (arvénsis.)

5. aufferig., 3 jeit.; $\mathfrak{B}$ l. herzf., geferbt; $\mathfrak{R e c t .}$ pfriemenf., läng. als b. SBlo.; bas ober fte B lbl. etwas langgeipiatat (4 SE. 9. bl.) grandiflóras

4980. sudetica:

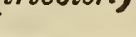

4986.

cornúta:

6. aufr., 4 feit.: bie unt. $\$$ l. evr., ffumpf, grob $=$ u. rundl. =geferbt: Die obern langettf., geferbt, gejägt; 2 f f terbl. fieberf. : ber $\mathfrak{M}$ it:

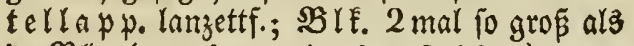
จ. SBlb. (23. gb. u. mit schw, Strichen)

\section{VISCUM. 777. (W. 38.)}

Stgl gaberf.; $\$ S$ r. verl. ey)r.=Lanjettf., ftumpf, 3 ripp.;' SB hit th. meift 3 záhl., ftiellos, ges * 4988.

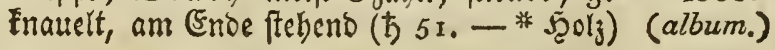




\section{VITIS. 155.}

\section{Blätter :}

1. bud)t. $=5$ lapp., ungl. $*$ u. grokge lägt, unbe=

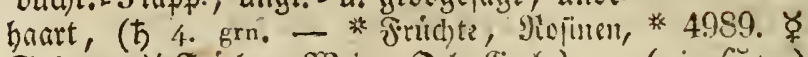

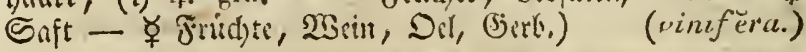

2. faft bersf. u. beltaf, befägt, unten on b. 4990. 2(bern ftachelbaar. (\$ NAm.)

3. Gerfförmig,
a. fulwach 3 it. 5 laps., ingl. gefägt, unten meipgeibfilts. (方 NAm. grn.)
4991.
Labrúsca.
b. 3 u. faft 5 lapp., ungl. = u. fehre grobgefágt, 4992.

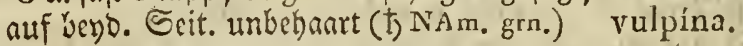

4. eyr. = berzf., faif 3 part. grobgejågt, an 8 . xeern u. an sante nebir ben $B$ lttfifen feingaar. (ち NAm, wohlriech.)

4993. ripařa.

5. faft 5 záhl., mit vielipalt., unbelnart. 2 blått $=$ d)en (h grn.)

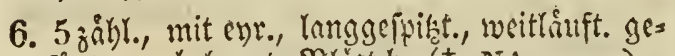
fágt., unbehaart. Blăttch. (ち NAm. grn.)

7. Dopp. zigefefgt = gefico., mit grobgefingt., un= behaart., nue unten ant b. 2(bern ftac)erbaar. Fieberch., woyond. unterft. bersfo, b. obern faft rautenf. = enr. jint (ち NAm。 grnch.-w. $\Delta$ ) arborěa.

\section{WALDSTEINIA. 391.}

(4 $\mathrm{SE}$. )

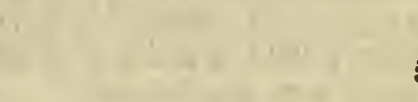

4997.

geoídes.

19. WEDELİA. 700.

Stgl frautart.; $B$ r. rautenf., am Brunte idimaler u. zigewadjf. ( $\odot$ SAm.) 
Wulfeñia, - Xerophyllum.

2. WULFENIA, 17.

(4 9. 12, bl.)

\author{
4999. \\ carinthiăca,
}

21. XANTHIUUM.1744. (W. 64.)

Stengel :

5000.

1. mit 3 záhl. Dornen; $\$ B l .3$ lapp. ( $\odot$ SE.) spinósum.

2. ohne Dornen; Blätter:

a. berzf., 3 fachgerippt $(\odot$ 14.22. gb. - 5001.

* Siraut, 巨aam. - ఛ̧ Farb. getb)

b. Feilf.=eyr., faft 3 lapp. $(\odot$ As. $), \quad$ orientále.

5. XANTHORHÍZA. 257.

( 5 NAm. dv.)

5003.

apiifolYa.

\title{
19. XERANTHӖMUM. 653.
}

Bldschuppen :

1. ftumpf, trode: die innern bes Strahls lanjettf., ftumpf, abjtebeno $(\odot-$ w.; p. 5004. u. w.)

2. Tpis, am Rande bout.: die innern bes 5005. Strabls lanjettf., fpic, zigeneigt $(\odot)$ inapértum.

3. rund., ganz trod.: D. innernd. Strahls cyr., langgeipiat, aufr.; Sł reublátt. b. Strotte eyr., begrannt, lang. alb o. Blo. $(\odot$ 'As.)

5006. orientále.

6. XEROPHÝLLUM. 299.

(4 NAm.)

5007.

setifolium. 
19. XIMENESİA. 672 .

(4) SAm, Gb.)

5005.

enceloides.

\section{YUCCA. 292.}

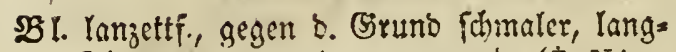
gefpigt, ganj gerabe, ganjrano. (5 NAm. w.'u. p.)

5009. gloriósa.
19. ZACÍNTHA. 618.

(○ SE. gb.)
5010. verrucósa.

\section{ZANNICHELLİA. 729. (W. 8o.)}

Stbeut. 4 fắdy.; $\mathfrak{R}$ arben ganzramo.; S. am 5011. ఫ sRuden feingejabnt $(\odot 37.45 . \div \Varangle$ Hnfr.) (palístris.)

\section{ZANTHOXY̌LUM, 783. \\ ( $77^{5}$. W. 85.)}

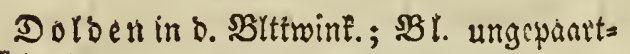
gefiebert;

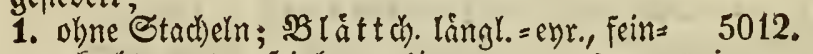
gefeerbt, unten feimhanr. (ち) NAm. grn.) mite.

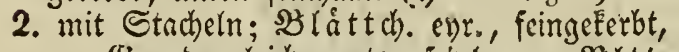
am (Srumbe gleid), unten feinbanr.; $B \mathfrak{l t}$ t 5013. fitiele ohne Stacheln ( 5 NAm. grn。 $)$ fraxinĕum:

\section{ZINNǏA. 665 .}

Blïthen :

1. ftiellos; sgit. entgegenger., hergf. = langettf., 5014. umfalf., ftiellos ( $\odot$ SAm. gb.)

2. geftielt; Blätter: pauciflóra。 
a. enţegengeiekt,

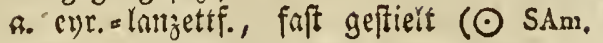 sch.; r, u. gb.)
5015. multiflóra.

b. ent. = herif., fticlloz, umfaff.; Stgl

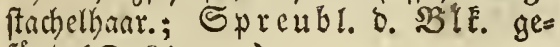
fingt $(\odot$ SAm. v. $)$.

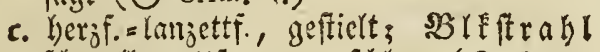
fd)mallanzcttf., tumgeidlag. ( $\odot$ SAm. sch.)

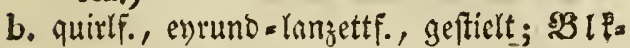
firahl Dokp. ( $\odot$ SAm. scb.)

5016. elěgans.

5017.

tenuiflóra.

5018.

verticilláta.

\section{ZIZIPHŎRA. 26 :}

\section{Bliithen :}

1. in ben Slattwinfeln,

a. 4 ob. 6 jál. ; 3 ID. borft. b hadr.; $2 B$ l. 5019. lanzettf., getvimp. ( $\odot$ As. p.)

b. meift gevanrt; $\mathfrak{B} 10$. ftachelhant; $\mathfrak{B} I t$. am Schlunbe aufgcblai., 2 mal io lang als ๖. SBlo.; $2 B$ l. lanzettf., ant Sirunde gewimp. (广 As.)

2. am Enbe ftehend u. gebirfhelt; $\mathfrak{B} 1 \mathrm{~b}$. wie vorber; $\$ B l$ l. Ianjettf., ftachelbaar.; Stgl ftaud., mit fcimbrar. Sweigen (

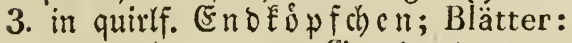

a, rund = eyr., am (Sirunbe etwas gewimp.; Stgl u. Sweige wie vorker (万 NAs. $\triangle$ ) dasyàntha.

b. oval, langgefpiget, unbehaart, punctirt; 5023.

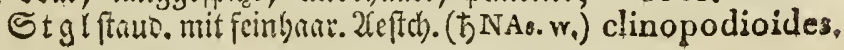

\section{ZIZY̌PHUS, 157.}

\section{Stamm:}

1. unberaffret, fo wie bie oberthalb gemund:

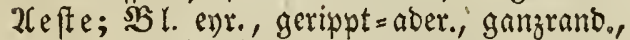
etwas wellenf.; Dolben geftielt in o. .Bltte winte; Frachte längl, = runblich (5) NAm. wch.-grn. $\triangle$ )

2. baumart: 2 efte mit gepaart., afterblattart. Stadeln: ber eine unt. Stadiel umge: frúmmt; $\mathfrak{B} l$. chr. U. Kingl., ausgerano.,

5024. volubìlis. 
mit fur . Statblipifte, u. einmwåtşgefriommt., an D. Spiase oriff. Sågezåbn., 3 ripp., un=

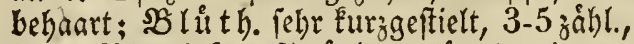
in D. SittwinE.; Frud te långl. (b SE, 5025 . gbch. $\Delta$ - Fruidte)

vulgáris.

19. ZOEGEA. 696.

$(\odot$ As, dgb.)

5026.

Leptaurĕa.

21. ZOSTÉRA. 724. (4.)

BI. gangrand., faft 3 ripp.; Stgl faft ftiel 5027. runo (4 36.)

marina.

10. ZYGOPHÝLLUM. 346.

SBl. gepaart, geftielt: $\mathfrak{B l a ̀ t t h . ~ v e r l . ~ e y r . ; ~}$

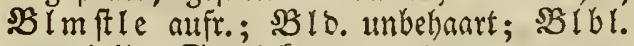
ungetbeilt; Stgl frautart. (4 As. rch. ૪ุ Silid.)

5028. ఫ๐ Fabágo. 


\title{
Regifter Der Prten
}

\author{
mit Scinweijung auf ibre Rummert.
}

A

bies 3307. Abituloides 42rg. abortivum 2590. abortivus 3652 . abrotanifolius 4I79. abrotanoides 981. 4064. Abrotanun 46r. Absinthium 494. Abutilon 4220. abyssinica 3952. acanthifolia 974, Acanthium 3064. acantlioides 960. acaulis 1256. 1946. $3195,3473$. 4095. 4281, acaulon 3068. acerifolia 3349. acerifolium 4913. Acetosa 3842. Acetosella 3148. Aclilleae II86. acidultum 3380, acinifolia 4898. Acinos 4610, aconiti 1527. aconitifolium 1986. aconitifolius 3620 , acre 1692 . 4141. acris 543. 3642. aculeata 2773 . aculeatus 3847. acuminata 274. 1846. 2730. 3906 . acuminatus 4428 . acutifolia $387^{2}$. 4457. acutifolius 3420 . acutum 147 . acutus 3826 . Adami 1051. adgregata 976 . adiantifolia 3859. adscendens 3444. 4014. adsurgens 625 . adulterinus 565 , advena 3018 . aethnensis 4191. aegyptia 2740. aegyptiaca 58.-2068. 3732. 3926. 4285. aestiva 1950. aestivalis 79 . aestivum 2565. aestivus $53 \mathrm{I}$. Aethiopis 3950 . afer 1260. africana 1487.2181 . ageratoides 1755. Ageratum 30. Ageria 3151. aggregata 877. agrarium 4682. agrestis 1417. 2279. 4054. 489 1. Agrimonoides 97. Ajacis 1526. Aipini 4357. ajugifolia 4019 . aizoides 1607.4009 . Aizoon 4003.4123 . alata 1028. 4497. 4751 . Alaternus 3684 . alatum 2575 . alatus 3215 , alba $7 \mathbf{3} .781$. 1074. 1363. 1849. 2924. 3014. 3126. 3145. 3305. 3393. 3438. 3463. 3604. 3665 . 3785. 3877.4305 .4452 .4621 .4735 .4744 . albescens 4030 albida 383. I413. 3101. 4115. albidum 4693. albiflora 3155. albifroms 827. album 67. II50. 2004. 2478.4143 .4772 .4799 .4988 . albus 200 . 1572. 2278 . 2695.313 . Alcea 2\%42. alexandrinum 4662. Alkekengi 3250, alliacea 452. 3 I98. alliaceum 4587. Alliaria 1730. Allioni 390. 902. 4308.4872 . 4966 . alnifolia 1245. 1823. alnifolius 3679 . Aloides 4511. alopecuroides 612. 1505. 3057. 4431. alopecuros 7 I0. alpestre 187. 1666. 1898. 2194. 3206. 4595. 4667. alpestris 746.965. 2940. 3623. alpigena 2656 . alpina 35. 106. 116. 291. 310. 355. 373. 382. 45 T. 658. 693. 757. 773. 826.903 .938 , 1077. 1213. 1508. 1608. 2597 . 2698. 3072 . 3208 . 3235, 3282. 3326. 3566. 376r. 4026. $4117 \cdot 4377$. 4456.4483 .4512 .4636 .4730 .4851 .4964 . alpinuin 1090. 1673. 1677 . 1701. 1712. 1724. 2050. $2102.2166 .2184 .2195 \cdot 2552.2625 \cdot 3165 \cdot 3383$. 3542. 3722 . 4560.4582 . 4593 . 4684. alpinus 528. 647. 1137. 1489. 1542. 1706. 3683. 384 T. 442\%. 46I2. Alsine 4506. Altagana 3740. altaica 1510.4103 . altaicus 542 . altaiense $\mathrm{I}_{32}$. alternitolia 1343 . 1358. alternifolium 1193. althaeoides 1316. altissima 313. 2072. 2916. 3338. 3464. 4393. altissimum 1147. 1747. 4333. altissinus 
2138. alsaticum 1277. Alsinastrum 1657. Alypum 2026. Alyssum 2757. Amaniana 3881. amara 240. 946. 2336. 3363. amaranthoides 678. Amarella 1944. amligua 1579. 1768. 2858. 4407. 4960. ambiguum 1528. ambiguus 606. ambrasioides 1163 . Amelanchier 3578 . Amellus 548. americana 898. 983. I003. I386. I80 r. 1842. 4434, 4749. amethystina 484I. am thystinum 1728. amethystinus 2259. Amni 4314. ammoides 4209. amoena 506. 2395. 4072. amoenum 4776. Amomum 43r8. Ampeloprasum 127. amphibium 3370. 4322. amplexicaule 2233. 2480. amplexicaulis 2144. 3341. 3615. 3939. amplexifolia 3815, 4768. amygdaloides 1787. Anacampseros 4125 , Anagallis $487 \mathrm{r}$, anceps 4346. androsacea 1615.3986 . androsaemifolium 364. Androsaeınum 2308. andryaloides 3787. Anethum 3181 . anglica 1279. 1932, 4237. anglicum 1908. angulata 1770. 3254. 3405 . 4214. angulatum I981. 4533. 4674. angulatus 2502 . angulosum 153. 814. angustifulia 263. 664. 1237. 1430. 1653. 3523. 1822. 2446.3229. 3575. $381 \mathrm{r} .4090 .4741$. 4934. angustifolium 1642. 1674. 1739. $200 \mathrm{I}$. 2169.2629 .4347 .4576 .4697 .4716 . angustifolius 204. 1084. 2140. 2693. 2975. 3132. angustissimum 1675, 2673. Anisum 4315. annua 656. 694. 786. 788 . 2442. 2685. 2859. 4475. 4798. anutularis 629. annuurn 4142. 4206. 4536. 5004. annuus 59r. $1132.2126 .25 \mathrm{cg} .4073$. anomala 3157. anomalum 1096 . Auserina 3423. anthemifolia $207 \mathrm{r}$. anthemoides 57. 414. 1392. Anthora 66. Anthriscus 4631. anthropophora 3073 . antiquorum 3050. antirrhina 4265 . apargioides $142 \mathrm{I}$. Aparine 521. 1914. apennina 83. 294. apenninum 1477.2117. apetala 3852.4278 . apetalum 2863. Aphaca 2496, aphylla 1871. 4882. apiculata 2774. apifera 3075. apuifolia 290. 5003. Apios 2034. apula 739. apulum 2642. 4629, aquatica 2592. 2845 . 3312. 3911. 4104.4513 .4622 . aquaticum 805 . $110 \mathrm{r}$. $172 \mathrm{I}$. aquaticus 3885.4178 . aquatilis 3613. Aquifolium 2341. aquilegifolium 4291. 4556 . aquilegoides 2424. arabica 333. 124\%. arabicum 306\% arabicus 957.2675 . arachnoideum 4157. arachnites 3076 . arborea 269.4996 . arborescens 820. 1290. 2265. arboreum 4774. Archangelica 302. 2490. arbutifolia 3569 . arcticum 1177 . arcticus 3790 . arctioides 961 . al:cuatus 3660 . arenaria 2077 . 2 r 82.2286 . 2401. 2451 . 3345. 4508. arenarium 124. 1287, 2044. 3205. arenarius 644. I105. 1543, arenosa 399. Argemone 3164. argentatus 953. argentea 3434. 3909. 4043. argenteum 186. I183. 1978. argenteus 1501. 1654. arguta 4392.4848. Aria 3568. aristatum II68. aristatus 599. armena 2420. Armeniaca 35 II. Armeria 1548. 4284. Armoracia 1284. aromatica 2956. 3717 . aromaticum 797. 1754. artemisiaefolia $3 \mathrm{Ir}$, artemisifolia 229, articulata 4932. articulatus 2507. Aruncus 446\%, arvense rogi. $2067^{\circ}$ 2641. 2803. 3309. 3361. 4586. 4695. arvensis I17. 326. 522. 772.837 . 1311. 2614. 2707. 2852. 2941, 3010, 3655. 3750. 4057. 4197. 4211 . 4301 . 4418. 4444. 4476. 4897. 4935. Asarina 352. ascalonicum 145. asclepiadea 1962. Ascyron 2294, asiatica 3335. asiaticus 3643. 47 r8. asper 6I5. I553. aspera 353. 385. 762. 791. I055. III3. 4005. 4355. 4384. asperrima 4093. asperrimum 4522. asperum 4330. asplenifolia 37. 1298. 3185, Asteriscus 4299. asteroides 754. astracanica 3437. Atamasco 224. athamanticum 2889. athamantoides 2572 . athinticum 2001. atrata 52. atratum 1179. 4144. atriplicifolia 830. atropurpurea 303. 37 r. 1025, 1800. 4040. atropurpureum 157. atrorubens 1555. 2139 . Aubletia 4832. augustana 2460. aurantia 1451. aurautiaca 1204, aurantiacum 2205. Aurantium 1221. aurea 917. 1382. I39I. 1956. 2951,3445 , aureus $245.4177 \cdot 4313$, aureum 2 19I. 3723. Auriçula 2204. 3483. auriculata 743. 1344. 2731 . 4108 . aurieomus $365 \%$. aurita 3912 . australe 4811 . australis $1007 \cdot 3160.3210$ 
3354. 4034. 4849. austriaea 322. 428. 485. 770. 858. xo13. 2853. 3605. 3937. 4084. 4888." austriacum 21. I60I. 1622.1891. 2171.2626 .3351 . 4151. 4332. 4816. austriacus 643. 1495. autumnale 1286. 2112.2566. autumnalis 90.354 .876 .4008 .407 r. aucuparia 4432. Avellana 1384. aviculare 3388 avium 3503 . axillaris $27 \%$. 1031. azaleoides 3694 . Azarolus 2887. azurea 3524 .

Babylonica 1069. 3870. baccata 3582. 4537. Baccifera 4260. Baetica $320 \%$. breticus 638. baicalense 161. 4149. balearica 796. 822 . 4758. balearicum 2291. balsamea 2838. 3303. balsamifera 3401 . Balsamina 2347 . banatica 4052 . barbadensis 3253 . barbara 1524. Barbarea 1740. barbareae 434.2. barbarum 2705. barbata 904. 1146. 4626. barbatum 10. 2314. 3375. barbatus 1547. Bardana 407. Basilicum 3023. bavarica 430. 1953. Beccabunga 4877. Belien r436. 4258. Belladonna 676. Bellardi 3324. Bellidiastrum 453. bellidiflorus 570. bellidifolia 378. 937. 4270 . bellidifolium 253. bellidioides 4857. benedicta 1061. benghalensis 4928. Benzoin 2525. betonicaefolia 907. 3940. 4110 . betonicifolium 3266. Betulus 982 . bicolor 2962. 1481. 3954. 4394. bicorne 1736. bicuspidatus 1131. 1313. biennis 495. 1412. 1918. 3035. 4925. biflora 2386. 3021. 4725.4965. biflorus 530. 1424. 1499. 2974. bifolia 1310. 3077. 4070. bifrons 1335. 2354. bipinnata 733. 1389. hipinnatum 3554. bipartita 2600. Bistorta 3377. bithynica 4946. blanda 3756 . Blattaria 4814 . Blitum 205. Bocconi 1890. bohenica 2383. bolsemicum 1987. 3106. bonariense 1703. Bonarota 3152. bononiensis $86 \pi$. bonus Henricus 1152. boreale 1911, borealis 2620. Botryapium 3576. botryoîdes 2932. 2980. Botrys 1161. 4543. Bourgati 1727. brachiatum 4647. bracteatum 467 I. brigantica 3514 . britannica 2360.3820 . bryoides 3999. Bubonium 2364. buchtormensis 783. bucephalophorus 3934 . bulbifera I540. bulbiferum 2584. Bulbocastanum 4352. Bulbocodium 2960. bulbosa 1376. 2953. 4500 . bulbosus 364 . bullata 731 . 2162 . buphthalmoides 33 r. Bursa pastoris 459 r. bursifolia 2473 . bursifolium 4327 .

C

aduca 4362. caesia 3995: 4400. caesius 1541. 3796. caespitosa 4024. caespitosum 4677, calabrica 520. Calamintha 2824. calcarata 4979. Calceolus I484. Calcitrapa 1060. 1085. calendulacea 408. 681. calocephala 1026. calycinum 194. 2293. calyculata 277 . cambrica $43990^{\circ}$ cambricum 3170. Cammarum 7 I, campanulata 1959. 4068. campanulatum 1903. 2630. campestre 20. 196. 1726. 1906. 4589. campestris 475. 604. 769. 1206. 1565. 1941. 2900. 3947.4747 . camtschatcense 2579. cantschatica 3747 . camtschaticum 3700 . canadense 159. 503. 1698. 209.5. 2271. 2310. 2578. 2827. 4549. canadensis 374.628 .698 . 1111 . 1216.1289 .1355 .1574 .2070 .2250 .2266$. 2344. 3304. 3436. 3609. 37\%6. 3960. 3965. 3968. 4378. 4538.4763 .4967 . canariense 110 . canariensis 1919 . cancellata 6 . candicans 963.1920. 3019. 3402 . candida 3896 . candidissimum 2760 . candidum 2583. canescens 1351. 1627. 2097. 2686 3133. 3270. 3392, 3663. 4053. canina $377^{2} \cdot 4412.4974$. cannabina 175 . $1515 \cdot 1878.4762$. cannabinums $365.175 \mathrm{r}$. cąntabrica 1330 . canus 541 . 1252. 4640 . capensis 3220 . capillata 4055. oapitatum 750. 4554. Capnoides 1381. caprea 3910. capreolata 737. 1868. Caput galli 2106. Caragana 3739. Cardiaca 2545. Cardinalis 2649. Carduncellus 988 . Cardunculus $1474 . \mathrm{Ca}$ rica 1818. carinatum 126 . 1184 . carinthiaca 1937.4999. carlinae- 
folius 951. carlinoides 956. carnea 86. 285. carneum 440 . camiolioa 651. 1769. 3484. carniolicus 1271. 4175. carnosa 2335 . carolina 3241. 3755. carolinianj 3. $1837 \cdot 2750.3500 .4830$. carolinianum 1694. 1990. carolinianus 618. Caruta 1522. carpinifolia 722.4454. carpathica-317. 893. carpaticum 2111 . carstiensis 2785 . carthusianorum 1554. Carvi 989. Carvifolia 306. caryophyllacea 3124. Caryophyllus 1556, Casabonae 1258. Cassine 2343. cassinoides $491 \mathrm{r}$. cassubica 4920. cassubicus 3650. Castanea 3591. Cataria 2981. catariaefolium $276 \mathrm{t}$. catawbiense 3698. Cateshaei 276.2585. catharticum 2636. catharticus 3674. catholicus 1439. calicasica 3771.4050. caucasioum 3550.3704. caucasicus 1567. caudatum II65. "caudatus 219. caulescen6 3439. ceanothifolium 1750. Cedrus 3298. celsiana 4722. celtica 4797. Cembra 3296. cenisia 879. Centaurium 1078. x742. centauroides ro3y. 4031. 4r98. centilolia 3776. Cера 148. Cepaea 4124. cepeaefolia 2334. cephaloideum 2060. cesfritosa 900. cerastoides 4240. 4507 . Cerasu8 3502. cerasifera 3517. ceritocarfum 4596. ceratocaula I52I. ceratophylloides 354I. cerifera 2948. cerinthoide 972.2239 .3003 . cernua 732 . cernum 163.980 . cernuus 1272. 2258. 4162. Cerris 3602 . Cervaria 2570. Cerviana 3211. Cervicaria 873. cervina 2848. Chabraei 2350. chaerophyllus 3639. Chaixi $247^{\circ}$. chalcedonica 2696 . chalcedonicuu $258 \%$ chalepensis 2602. Chamaebuxus 336\%. Chamaecerasus 3510. Chamaecistus 3702. Chamaesyparissus 3971. chamaedrifolia 4458. Chamaedrys 4547. 488\%. Clianaemespilus 2883. Chamaemorus 3792. Chamaepitys 108. Chamliggu 3737. Chamomilla 316.2764. cheiranthoides 1731. Cheiranthus 778. Cheiri 1121. chelidonia 943. Cherleri 465t. chiloensis 1829. chinensis 534, 736. 774. 1560. 2921 . 4312. 4524. chium 1709. Chius 1139. cliloraefolia 4280. chlorantha 3557. 4250. clilorostachys 216. chondrilloides 2242. chrysanthe. moides 753. Clirysauthum 3705. Cicer 639. Cicera 2497. Cicla 703. ciconium 1713. cicutarinm. 1714. ciliaris 394. 1611. 2439. 2777. ciliata 1964. 2917. 3478. ciliatus 540. 1261. 1320. Cineraria 1024. cinerea 1034. 1688. 2428. cinnanomea 3757. Circaeoides 2146. circinnata 1359. circimalus 2273. cirrlosa 2594. citrata 2846 . cladostemma 3915. clandestina 4269. clavata 323. claviculata 1383. 1869. Clematitis 445. Clavemnae 32. Clilfortiana $265 \mathrm{I}$. clinopodia 2912. clinopodioides 5023. clusiana 3440. Clusii 3685. 4864. Clymenum 2511. clypeatum 4700. Cneorum 1327. 1509. coccinea 2867. 3594. coccineum 1182 . cochlearifolià 916 . cochlearioides 794. coelestina 2443. coelestinum 1756. Coeli rosa. Ior, coerulea 230. 249. 994. 2657. 2813.2989 .3127$. coerulescens 47 . coeruleum 3142. 3357. 4140. coimbricensis 2670 . colchiciflora 4510 . collina 869 . 1043. 1828 collinum 4807. collinus 1552. colorata 2957.' 2992. coloratum 1668. columbaria 4047. colmmbinum 1993. 3197. Columnae 3055. 4334. Cotuna 1388. comosa 3194. 2032. 2248. co. mosum 2930. 3rI7. 3269. communis $=38$. 1296. 2011.2436 .2483$. 2958. 35t2. 3731. 4532., compacta 43. compactum 3686. complicata 4856. compressa 2431 . compressus 3119 . 3418 . concinnum 4574. concinnus 569. concolor 552. condensatum 4810 . confertus 3832 . confusa 1618. conica 4267. conifera 1075. 3892. conjugatus 2671. conjunctum 4296. connatum 4298. conoidea 4263. conopsea 3099. Consolida 1525. contorta 1619 , contortum 4339. contortuplicatus 642. 1125. controversum 125. Convolvulus 3389. conyzoides 93. 585. Copallinum 3708. copticum 798. 2864. corallina 3r54. cordata 170. $1686,2087.2734 .2872 .4494$. cordatum 4521. cordifolia 1214. 2029. 4223. 4614. cordifolium 808. coriacee 1036. coriacens 
4184. coridifolius 551. Coriaria 37\%9. Corindum 949. coriolshora 3084. cormicina 347. corniculata 3150. 4706 . cormiculatus 2683. Cornucopiae 1806. cornuta 4986. Cornuti 3315. 4557. coromandeliana 1006. Coronaria 99. 300. 357 I. coronarimm 1190. 2180 , coronarius 3226. coronata 1341. 1366. 1812. 2791. 4193. coronopifolia 36. 309. 744. 1040. 1393. 1419. Coronopus 1280. 3328. cortu. soides 3468. corylifolitis 3809. corymbosa 329. 779.979 . corym. bosum 2313. 3544. corymbosus 596. Cota 315. Cotoneaster 2882. Cotinus 37 18. Cotula 327. Cotyledon 4001. Cracca 4924. crantziana 396. crassifolia 3932. 3994. 4505. 4760. crassifolium 2563. crassifolius 968. crenata 3470,4462 . crenulata 4843. cretensis 655 . cretica 700. 1370. 2094. 2530.2817.3175. 3927.4275.4482. creticum 8. 1649. 2759. 3102 creticus 987. crispa 360. 1335. 2458. 2484. $2747 \cdot 2844 \cdot 2982.4753$. crispata 386.2832 . crispum 3024. crispus 958. 2544. 3414. 3822. Crista galli 2105.3693 .4836 . cristata 29. 1659. 2394.4225 . cristatum 2802. crithmifolia 48. 479. 2148.2368 . Critonia 2456. croaticum I300, 2245. crocata 3031. Crocodylium 1065. Cruciata 1880. 1963. cruenta 1292. cruentus 218. Crupina I081. Crus galli 2875. Crux Andreae 512. crystallinum 2862. Ciscullaria 1374 . cucullata 4956 . cuminoides 2472 . cuneata 3348 . cuneifolia 3,96. cuspidatum 4803. cuspidalus x 130 . cyanoides 954. Cyanus 1032. cydoniaefolium 2227. cylindrica 1231. Cymbalaria 2593. 4894. Cyminum 1456. cymosimi 2207. 2346. cynanchica 523. Cynocrambe 4578. Cynapium 92. Cynops 3340. Cyparissias 1784. cytisoides $349 \cdot 3682$.

D

alechampii 459 . dalmatica 2615 . damascena 3008 . 3775. danica 1278. dasyautla 5022. dasycarpa 3513. dasycarpum 22. dasy. phyllum 4139. daucoides 999. dauricum 3701. davurica 719 . de. albata 1035 . dealbatus $61 \%$. debilis 2896 . decandla 3271 . decapetalus 2 130. decolorans 27. decumana 1222. decumbens 1 134. I93 I. deflexum 130. deflexus 210 . defloratus 966. deltoides 1558. demersuin 1108. dendroides 2353. Dens canis 1744. densus 3410. dentata 413. 857. 1811. 2088. 2815. 2947. dentatum 1603. 4906. denticulata 2792 . 3020. denticulatmm 1624. depressa 742. 3318. depressus 646. descendens 141. Diacantha 3727. dichotoma 1404 . 2899. 4035. 4268. 4499. diclotomum 1093. 1301, 4648. didyma 741 . 2915. dirlymum 2550. diflormis 1641 . diffusa 1765.3430. diffusum 1738. 4669. diffusus 576. 2674. digitata 3818. 4470.4895. digynus 3839. dilatata 3399. Dilleniana 4226. dioica 782. I 512.2702 .3280 . 3583. 4217.4759 . 4787. dioicun 933. 2047. 4558. Dioscoridis 1418 . 1478. 4363. diphylla 2903. diptera 2084. discoidea 334. I813. di. color 1254. 3596.14732. dispermum 2645. dissectum I992. disstacliya 1662. distans 45. disticha 1461. 4273. diutinus 1549. divaricata 3244. 4526. divaricatum II99 I892. 1988. 338 I. divaricatus 1491. 2135. 3828. divergens 908. Dodartii 4757. domestica 3516. 4433. Doronicum 456. 4182 . Doria 4188. Dortmanna 2647 . Draba 195. 1281. Dracontium 497. dracunculöides 593. Dracunculns 469. 496. dubia 356. dubium 2203. 2312.3168. 4202. dubius 2971. Dulcanuara 4364 . dulcis 1767 . dumetorum 2158.3390 .3773 . 4917. dumosus 555. durus 3120 . dysenterica 2357.

Ebracteatum 4584. Ehulus 3959. echinata 1807. 2036. 2351. 4540. echioides $2161.2215 .3069 .44950^{\circ}$ edentulum 188. edulis 2669.3567. 3673. effusa 4748 . elata 235. Elaterium 290\%. elatior 227 . 1825. 
3125. 3475. 4854. elatum 1534. 2304. 4204. 4570. elegans 590. 2302. 2767.37 ×3. 4229. 5016. elliptioa 1840. 2873. 4388. ellipticum 3263 . elongata $279.240 \%$. 4795. elongatus 1502 . elodes 2300. emarginata 699. emarginatuin 3385. emarginatus 1314. Emerus $\times 365$. eminens 580. enceloides 5008. Lndivia 1200. enneaphylla 1537. 1864. 2352. ensifolia 1681. 2369. Ephemerum 2717. Epipactis 1598. epiglottis 649. Epigogium 2591. epithymoides 1 766 . Epithymum 1463. erecta 666. 692. 1238. 1297. 3853. 4529. 4634. erectum 2290, 4712. erectu 2895. ericoides 554. Erinoides 2654. 4828. Erinus 9I4. 2653. eriophora 1062, eriophorus 1253. eriosperma 4088. Erisithales 1262. Eruca 777. Elucago 792. Erucastrum 776. erucifolius 4173. erucoirles 4309. erysimoides ir28. Esculus 3601. Esula 1780. etruseum 928. Eupatoria 94. Eupatorium 40. europaea I462. 1799. 3352. 3970. 4213. 4649. europaeum 502. 1465. 2150. 2706. europaeus 2665. 2709 . 4717 . 4743. exaltata 3314. exaltatum 532. exaltatus 2711 . excelsa 716 . excelsior 1853 excelsus 2136 . excisa 885 . exigua 1763. expansa 1836. 4541 . exscajus 610 .

$\mathbf{F}$

Taba 4941. fabacea 1377. Fabago 5028. Fagopyrum 3386. Falcaria 4351. falcata 56. 1762. 2798. falcatum 815. falcatus 1106. 1500. fallax 321. 2206. Farfara 4729. farinosa 347 r. farinosus 1318. fasciculata 436. fasciculatum 2306. fastigiata 1338. 2082. fastuosa 411 . ferox 1516. 1594. 3748. ferruginea 271. 1582. 1805 . ferrugineum 3696. 4825 . ferrugineus 1546. Ferulago 1815. Ficaria 3629. ficifolia 179. ficifolium II49. filicaulis 3147 . 1321. filiforme 1803. 4680. filiformis 4893. Filipendula 4468. fimbriatum 2296 , finmarchica 3879. Fischeri 1037. fissa 115. fistulosa 2908. 3029. fistulosum 149. flagellaris 3793. flammea 81. 3190. Flammula 1232. 3626. flava 88, 1949 2163. 2868. 3981 . flavescens 4287. flavissima 2382. flavum 142. 934. 2635. 4552. 4563. flavus 215. flexicaulis 4406. flexilis 1120. flexuosa 2922. flexuosum 2109. 2244. floccosum 4819. Floerkeana 3479. florentina 2384. florentinum 2208. floribundus 568. florida 1233. 1356. 1928. floridanus 472I. floridum 3725. floridus 849. Flos Cuculi 2700. flosculosil 4235. Flos Jovis 100. fluitans 3413. fluviatilis 36r9. Foeniculum 2891: Foenum graecum 4709 . foetida 252. $700.1203 .2037 .2481 .2755^{\circ}$. foetidissima 2403. 3256 . foetidum 1164. 2048. 4562 . foetidus 2159. foliosa 876.3189 .4304 . 4852 , foliolosus 557 . foliosum 2232 . for mosa 3878 . formosissima 225. fragarioides 1295. fragiferum 4654. fragilis 239. 594. 1001. 3873. fragrans 156. 4734. Frangula 3681 . Fraxinella 1573 . fraxineum 5013. fraxinifolia 2427.3760 . frigida $1960,3209.4733$. frigidus 3617 . frondosa 729 . frondosum 4775 . frutescens $799 \cdot 2035 \cdot 2098.2441 .3368 .3742$. fruticans 2327 . fruticosa 234. 723. 3044. 3048. 3422. 3856. 4249. fruticosum 818. 2190. fruticosus 38 ro. fruticulosa 3666.4846 . fruticulosus I I22. fulgens 2648. fulgida 3814. fullonum 1590. fulva 2164.4745 . fulrum 2017. Fumana 2122. fumarioides 2422. fungosa 1373 , fusca 3086. 3907. fuscata 325. fuscescens 1583. fuscum 2057 .

Gale 2947. galactites 1064. galegiformis 623. galericulata 4120 . galioides 4565 . gallica 492. 3777.4239 .4530 . gallicum 2062. 2631 . 4331. gangeticus 203. garganicum 2476 . gemella 3759 . gemellum 4661. geminiflora 4247. gemonense 199. genevensis 10\%. genistifolia 2601 , gentianoides 4867 . gentilis 2850 . geoides 4997 . gera. 
noides 4018. Gerspdiana 1783. Gerardi 440, 2780. Gerber1 39. germanica 1933. 2362. 2388. 2884. 4484. 453\% germanicum 2061 . gesneriana 4721 . gibba 2536. gigantea 4381. giganteus $2137 \%$. Gingidium 1523. Githago 98. glabellum I670. glaber tog9. 3488. gla: berrina 3243. glabra 89. 1145. 2040. 2175 . 2318. 2433. 3712. 4616. 4726.4844 . glabrata 4037 . glabratum 2196 . glabrescens 4602 . glacialis 457.463 .1940 .3622 . glandulifera 2038. glandulosa 102. 360 . 424. 1536. 2363.2874 .4105 . glandulosus 3795. glastifolia 755. 1068 . 1282. 4083. glauca 484. 686. 1210. 1229.2445 .2728 .3664 .4973 . glaucum-I153. 1882. 2217.4208 . glaucus 784. 850, 1559. Glaux 63r. globosa 3083. 4938. globosum 4687. globilerum 4155. globulariaefolium 3259. glonerata 875.1405 .2799 . glomeratum 4658 . gloriosa 5009. glutinosa 165. 2800. 3002. 3482.3925. glutinosim 1697. glycyphyllos 640. Gmelini 4492. Gouani 3648 . gracile 4200 . gra. cilis 2677 . graeca $945 \cdot 2790.3203 .3397 \cdot$ graecizans 201. graecum 3063. graminea 2408.4503 . gramineus $3417 \cdot 3628$. graminifolia 431. 871. 4041. 4091. 4489. graminifolium 816. 1181. 2553. granadensis $27799^{\circ}$ Granatum 3535 . grandidentata 3394 . grandiflora 25.

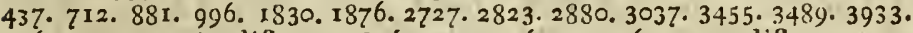
4769. 4982. grandiflorum 806. 1529. I 525.2236 . grandiflorus 532. 608. 3227. 3645. 4075. granulata 4011 . gratum 929. graveolens 362. 758. I I 51. 1696. 2995.3849. grisea 3889. Gronovii 2200. Grosd sularia 3728 . grinum 1711. guineense 4369. gummifera 909. guttatum 2125. guttatus 1564, gymnorhiza 3030 .

Habliziana 39rg. halepensis 3285. Halicacabum 948. halimifolium 183. Halimus 66r. halimifolia 688. Halleri 393. 1378. 2238. 3268. 3532.3539 . Halodendron 3736. halophila 2402: hamosus 641 . hamulosus 952. Harmala 3196. hastata 672. 824. 3883. 4224. 4356. 4829. hastifolia 4122. hastilis 359. hederacea 915. 2373 . hederaceum 2020. hederaceus 3612 , hederaefolium 1467 . hederifolia 2374 . 4899. helianthoides 800,4542 . Helenium 2356. helioscopia 1774. Helix 2089. 2769.3868 . helvetica 287.23 I5. 4632 . helveticus II29. helvolus $32 \mathrm{r} 4$. hemisphaericum 3260. heraclea $448 \mathrm{I}$. herbacea 493 . 1691.3857 .4949 . herbaceum 1606 . Herba rota 24. Herba venti 3232 . heterophylla 2876.3404 . heterophyllum 7.1173 .2892 . heterophyllus 589. 1270. 2520.312 r. 3408. 3808. hiberna 1786. hieracifolia 3467. 3990. hieracifolium 1733. hieracifolius 416r. hieracioides 3276. hierochuntica 254. Hippocastanum 85. Hippoinarathrnm 4201. hircina 3053. 3080. hircinum 2307. Hirculus 4006. hirsuta I76. 387. 7 II. 936. 2039. 2176. 2955. 2983. 3921.3993. 4089. 423 r。 4962. hirsutum 1720. 1664. 2019. 2298. 2493. 2621. 3695. hirsutis 1503. 1998. 2513.2679 .2691 .3130 . hirta 921. 1514. 1614. 2366.2616. 2837. 3449. 3812.4408. 4474. 4597. 4961.' hirtum 4158.4590. hirtus 1325. 2501,3803 . hirtella 3100 . hispanica 3922. 4082. hispanicum 4145. 4344. hispanicus 4077. 4414. hispida 361. 76I. IIIT. 2282. 3427. 3461. 3735, 4303. 4598. hispidum I913. hispanica 1400. 1935. 2655. 3011. 3608. hispanicum II . hispidun 4686. hispidus 3794 . holosericea 389I. Holostea 450r. Holosteum 3334. Hoppeana 3862. Horminum 3944. horrida 2024. hortensis 301.667. 1568.2262. 3984 . hudsonica 3400. Humboldtii 4368 . humifusum 2309. litumile 221 r. 4371. humilis $562.657 \cdot 3156.3898 .4085$. hungarica 2398. hybrida 679. 842. 922. 1046. 3572. 4435. 4865. 4937. hybridum 1158. 1531 . 3163. 3459. 3688. 4128. 4675. hybridus 217. 3682. Hydrolapathum 3821. Hydropiper 1656. 3372. hyemalis 2152. 3507. hypericifolia 
4459. hypericifolium 366. hyproides 4020. hypochondriaca 409. hypochondriacus 213. hypogaea 40I. Hypoglossum 3846. hypoglottis 635. Hypophyllum 3848. Hypopithys 2918. hyssopifolia 2453. $2723.3492,4233$. hyssopifolium 1350. hyssopifolius 537 .

J Jacea ro73. Jacobaea 4176. Jacquini 4889. japonica 677. 2619.4430. iberica 2658. ibericum 1977. Iberis 2558. Idaeus 3805 . jeniseensis 4245. ilicifolia 3598. illyricum 154.3162. 3066 . illyricus 3635 . imberbe 2638 . imbricaria 3586 . inbricata $105 \mathrm{I}$. inpatiens 34. 942. Imperati 1324. 4539. imperialis 1857. implexum 930. inamoenus 3216 . inaperta 4266. inapertum 5005. incana 167.243. 357.389. 707. 284I. 2990. 431 I. 4860. incanum 189. x248.3374. incanus II23. 4174. incarnata 510.3096 .3186 . incarnatum 2209. 4691 . incisa 4189. 4861. inclinata 3446. incomparabilis 2965. inconspicuus 2499. incubacea 3890 . incurvus 2522 . indica 473.2807 . indicus 2127. indivisa 4990. inermis 3798 . infectoria 3587 . infectorius $3677^{\circ}$ infestum 1884. inflata 2652 . infracta 4256 . inodora 470.2179 . inodortum $355 \%$ inodorus 3228 . inops $329 \mathrm{r}$. insiticia 3515 . intacta 4004. integrifolia $1207.1240 .2145 \cdot 3485.4060$. integrifolium 2199. 3178. intermedia $1219.3443 .3565: 4485.4766$. intermedium 1352 . 1530. 2009. 4580. intertexta $277^{6}$. intybacea 1415 . intybaceum 2216 . Intybus 1 197. inundatum 2893. involucratum 4659. Jonquilla $297^{6}$. Jonthlaspi 1249. Irio 4336. Isnardi 1056. italica 2816. 2985.4067. italicum 500. 932. I 189. 1644. 2043. italicus 1437. jubata 3741. judaica 3173 . juglandifolia 1843 . Julibrissin 1 . juncea II 72.4300. juncetm 812. 1737. 2099. 4439. junceus 588. Jva rog.

$\mathrm{K}_{\text {aki 1589. Fiali 3916. kalmianum 2292. Kralmii 2218. Kochiana }}$ 2819. Krockeri 4976.

Lablab 1597. Labrusca 499r. Laburnum r488. lacera 2177. 1a. ceru's 4419. laciniata 46r. 668. 2782. $3158.3230 .3817 \cdot 4096.4109$. 4862. laciniatum 1143.4293 . laciniatus 1592.3806. laciniosa 4494. lactea 284. 4975. lactiflora 282. 862. lacunosa 2376. lacustris 2646. Ladanum 1875. laeta 2697. laetus 212 . laevigata 519. 745. 1581 . 2833. $4310.440 \mathrm{r}$. 445 I. laevigatum 2220. 4905. laevigatus $85 \mathrm{I} .2543$. 320r. laevis 563. 1520. 2g06. lagenaria 1450. Lagopus 1506. lamiifolia 910. lanata 49. 289. 977. 1580. 4486. laiatum 1089. lanatiis 986. lancea 1850. lanceolata 1342. 253 I. 3337. 387 r. 3920. 4403. lanceolatum 765. I 67 . lanceolatus 587. 1251. Langsdorfii 3007 . lantana 690.4910. lanuginosum 1991.2299. lanuginosus 3634. 4603 . Lappa 406. lappaceum 4685. lapponicus 4425. Lappala 3745. lajsanoides 2214. laricifolia 432. Laricio 3287. Larix 3301. Lasianthus 2068. lateriflora 4119.4380 . latifolia 236. 250. 859. 997. 1307. 1431. 1678. 179 I. 1 802. I862. 2444. 2528. 2609. 3095. 3855. 449 I. 4739 . 4875. latifolium I IO2. 1605. I676. $2486.2534 .2557 \cdot 3536.4153 \cdot 4354$. latifolius 1550, 2518. 3680. Lathyris 1764. lathyroides 3129.3224. 4935. Laureola 1513. laurina 3900. Lauro-Cerasus 3498. lavandulacea 2836 . laxiflora $46 \mathrm{z}$. Laxmanni 637. 4548. laxus 592. ledifolium 2120 . lenta 721 . Lentago 4908. lentiscifolia 1838. . leontinus 636. Leontopodium 2055. leontopodoides 1407. Leptaurea 5026 . leptophylla 1000. leucantha $735: 4028$. leucanthemoides 415. Leucanthemum 1180 . leucanthemus II26. lencanthus 1496. leucographus 950, leucophaeus 645. 4426. leucophylla 3905. leuco. spermum 4207. Levisticum 2573. Libanotis 653. 2487. ligustica 46. 
ligusticifolium 2874. ligustrinum 4780. Lilingo 338. Liliastrum 339. liliifolia 890. Lilio-Hyacinthus 4066. limensis 2745. Limonium 4490. linariifolius 546. lineare 129. 4152 . lineatus 1328. Lingua 3627. 4190. lingulata 906. linifolia 882.3274. 4244. linifolium 764 . 1704. I896. linifolius 544. 2692 . linoides 36 ro Linophyllum 4579. Linosyris r I9i. Linum stellatum 2714. Lippii 1082. literata 1779. lithospermifolia 2440.4397 . littorale 2287 . littotalis 669. 1371. 2607. 4968. littoreus 1133 . livida 4252. 4404. lividun I984. lividus 206. 2160.2542 .4165 . lobata $1211.447 \%$. lobelioides 892 . Loeffingii 3327. Loeselii 2736. 4335. longa 443. longillora 2991. 3046. 348 r. 4253. longillorus 2968. longifolia 1208. I 634. 4955. longifolium 813. 1702. 2801. Lophanthus 2324. Lorei 870. Lotus 1588. 3016. lotoides 2025. lucens $34 \mathrm{Ir}$, lucida 377.2284 .3180 .3758 . 4048. lucidum 1895. 1989. 4546. 456\%. lucidus 4608. Ludwigii 178. lunatus 3217. Lupinaster 4650. lupinoides 3355. lupulina 2794. 4 I 18. Lupulus 2256. lurida 2390 . lusitanica 1244. $2415 \cdot 3339.3499 .4238$. lusitanicum 1647. lutea 223. 995. 1380. 1377. 1578. 1797. 1936. 3017. 367 r 3779. 3977. 4936.498I. luteo-album 20 45. luteola 3026.366r. lutescens 2400. 3766. Iutetiana $121 \%$. luteum $1874 \cdot$ ' $^{2} 016.3108$. lu. teus 526. 1429.2689 .2898 .3138 . Lychnitis 4817. Lycoctonum 63. Lycopersicum 4366. lycopifolius 970. lyrata 2485. 3958. 4728. lyratum 2556.

$\mathrm{M}$ acqui 446. macracantha 2023. macrocarpa 3603. I285. macro. carpos 4785. macrocephala 1022. macrophylla 55.2733.2999. ma. crophyllum 1760. 2005. 3540. macrophyllus 597. 2 132.4423. ma: crorhiza 2810 . macrorhizum 1972 . macrostacliya. yI. maculata 1202. 2316.2778 . 3094. 3239. 356r. maculatum 499. I299. I 97 I. 2477 . maculatús 4076. maderense 477I. magellanicim 2269. magicum 136. magna 5r. 3278. Alahaleb 350r. majalis r 302 . major 650. II12. $3123.3313 \cdot 3365 \cdot 4642.4950$. Majorana 3104. majus 231. 350. I142. 457 r: 4720 . malacoides 17 10. 2739. Malus 3579. manticum rog8. margaritaceum 2052. màginata 3764. mariana 266 . maria. nus 973 . marina 418. 2775 . 5027. nuaritima 228. 312. 449. 483. 704. 831. 834. III5. 1236. 1398. I 433. $2018.3186 .3333 .3518 .352 \% 3944$. 4261. 4477. 4853. maritimum 182. 807. 1 148. 1708. 1725. 1915. 2633. 316r. 33 II. 3387. 3552. 4694. 4702. maritimus II $40.2666 .3825 \cdot 4412$. Marrubiastrum 2546. marrubioides 2984. Narschallianus $460 \%$. Mlartagon 2577. - martinicensis 3234. marylandica 990. 3969. 4100. mascula $1357 \cdot 3089$. Niatthioli 654. 1372. matronalis 2.178, mauritiana 2746. Mauritanica 2090. 2812 .- Nax 3223. maxima 278.3317. Maxjmum 3703. 4630. Meadia 1596. media 171. 417. I291. 2914. 3316. 3967. 4842. medica 1223.. mediterranea 427. 1690. 3669. Medium 913. 4573. 4666. megalosperma 4930. melancholicus 202, melanocerosum 4372 . melanocarpa 2879.3570 . Meleagris 1860. melilotoides 621.' Melissophyllum 2826. meliterisis 1050. mellosa 2725. Melo r 448. Melopepo 1455, menthaefolia 4863. ntessanensis 2809 . Metel I 5 19. mexicana 441. 4405. Meyeriana 3865. Mezereum $150 \%$. Michauxii 4929. micrantha 4I. 4045. microcarpa 3300. microphylla 1680. microphyllus 634. microsperma 67 r. millefoliatum 3553 . Millefolium 59. 4338. Milleri 259. militaris 3081. miniatum 4370. minima 1367. $2317 \cdot 2789.3477 \cdot 4375.4742$. minimum rgr. 3025. 3110. minimus 1086. 2946 . minor 652. II 14. 1576. 2537. 2606. 2919. 412 r. 2963. 3558. 4740.4767 .4948 . minus 337 r. 4572.47 r 9 . minuta 1395. 4395. 4527. minutissima 3054. minutum 696. 1900. mirabi- 
lis 497 . mite 5012. mitissina 3058. mixta 308. Moldavica 1626. molle i 995.2237 . mollis 5. 969. 2133.2909 .3526 .3743 . mollíssima 3039. 3894 . molluginoides 1435. Mollugo 1902. Moly 164. monantha 493 I. monanthos 2506 . Ionelli 248. monilifera 3403. moniliferum $314 \mathrm{I}$. nonogyna 491. 1464. 2878. monophylla 1827. monophyllos 2735. Monorchis $307 \mathrm{I}$. monosperma 2021. 2959. monostachya 166r. mon. regalense 4I35. monspeliaca 1432. 3366. 47 II. monspeliacus 1570 . monspeliense i604. monspeliensis! 3452. 1349. 2092. monspessulanum 12. monspessulanus 6ri. montana 113. 319. 344. 423. 454. 525. 1021. 2325. 2372. 290 r. 3590. 3850. 3985. 4058. 4227.4376 . 4789.4876 .4970 . montanum 17. 192. I174. 1671. 1886. 2000. 2063. $222 \mathrm{I} .2297 .4159 .4205 .4553$. $458 \mathrm{I}$. 4592. 4673 . 4806. montanus 602. 3647. 4171.4606. Mlorio 3087. Morinsonii 832. Morsus Ranae 2267. moschata 53. 1080. 2743 . 3751.4023. Móschatellina 84. moschatum 143. I716. 2929 . moschatus 2964. Mtughus 3292. multicaulis 421 . 26 Ir. 4502. multifida 2979. 3431. 4881. multiflora 1306. 1689. 5015. multiflorum 4442. multiflorus 560. 1440. 2142.3213 . multisiliquosa 2247. Mungo 3221. murale 184. I157. 4328. muralis I617. 2079. 3466. 4786. muricata 1067. 2786. 3038. 40\%9. múricatus 3632 . " murorum 2213 . Miscipula 4279. muscoides 4025 . muscosa 2904.4623. mutabile 2II4. 'mutabilis 583. 4638. 'mutata 4002 . mutellina 464. 2890. muticus 2275. Myconis I188. myodes 3074 . myriacantha 3770. myrinphylla 60. myriophyllum 4534. myrsinites 178r. 3882. myrtifolia 1348. myrtilloides 4777 . Myrtillus 4770.

N ana 54. 244. 280. 725. 1854. 1939. 243\%. nanus 1493. 3222. Na. paea 42 I6. Napellus 68. napifolia 3953. 1059. Napus 766. narbonense 2622. 3115. narbonensis 174. 4943. narcissiflora 298. Nasturtium 4319. Natrix 306I. natans I I9. 3407. 4436. 4645. neapolitanum 1466. neglecta 478.896 .2392 .4850 .4969 . neglectus 2012. Negundo 23. nemausensis 1406. Nèmolapathum 3824. nemoralis 545. 1616. 4389. 4752 . nemorensis 4186. nemorosa 293.2834. 3948. nemorosum 4813. nemorosus 3802. 3829. nemorum 2713. 4498. neomontanum 69. Nepeta 2825. Nepetella 2994. nepetifolia 3231. nepetoides 2322. nervosa 1014. 1861. nicaeensis 759. 1782. $275 \mathrm{I}$. 4272. Nidus avis 1684. Nigellastrum 1916. niger 2154. 2281.3135. nigra 689. 720. 1029. 2429. 2660. 2781 . 2888. 2926. 3092. 3306.3398. 3506. 386r. 3962.4307 .4359 . nigrescens 1030. 2225. nigricans 1490. 3899. 4566. nigrum 158. 1472. 1660. 3265. 3724. 4373. 4801. 4815. niliaca 2839. nilotica 3956. Nissolia 2495. nissoliana 2921. nitens 670. 107 I. nitida 273. 687. 3272. 3447. 3754. nitidum 4783، 4902 . nivalis 1612. 1870. 1966. 3563. 3646. nivea 486. 3454. 4738 . 4764 . niveum 4804. nireus $297^{\circ}$. nobilis 50. 328. 1375. 2524 . noctiflora 4276. nocturna 3040. 4242: nodiflora 727 : 1817 . nodiflorum 257 . 2865. 4348. nodiflorus 3624. nodosa 343. 4106.4446 .4633 . nodosum 1982. Noli tangere 2348. non scriptus 2257. noricum 4665. norvegica 3453. novae angliae 535. noveboracensis 4833 . novi belgii 567 . nubia 3945. nuda 2997. nudicaule 3166 . nudicaulis 404 . 843. 2027. 2337. nudiflora 682. nudum 4904. Nummularia 2715. 4858. nummularius 4605. nutans 151. 379. 962. 1629. 3111.3938. 4251. nyctantha 4248. Nyctelea 1658. nymphoides 2856 .

Obliqua 1r44. 4957. obliquum 134. oblongata 166. 2910. oblongifolia 4464 . obovata 4463 . obovatüs 254 r. obscura 1585. $2795^{\circ}$ : 9451. obscurum 2107. obscurus 3412 , obtusangulum $4329^{\circ}$ obtu- 
satum 19. obtusifolia 286. 1942. 4246. obtusifolium 2051. obtusifolius 3801.3827 . obvallatus 1265. occidentalis 1008. 1087. 3350 . 3797 . 4599. ochroleuca 47.256. 1018. 1239. 2409. 3767. 4049. ochroleucum 64, 4698. ochroleucus 1266. 3140 . Ochrus 3308. octangularis 4099. octopetala 1635. Oculus Christi 2358. Ocymoides 3978. odontalgica 833 . Odontites 1794 . odora 2355.4385 . odorata 62. 95 . 515. $734.2333 .2954 \cdot 3015 \cdot 3670.4954$. odoratissima 3098. odoratum 1735. odoratus 2508.3800 . odorum 160., odorus 2156.2966 . oelandicum 2124. officinale 1479. 1729. 2328. 2640.3204.4517.4628. officinalis 173. 257. 513. 706. 756. 836. 1283. 1792. 1866. 1872. 2069.2320. 2754. $2814.2822 .3153 .3172 .3525 \cdot 3786.3928$. 3966. 3975. 4788.4826 . 4883. Oleander 2998. oleifolia 4493. oleracea 768.3406.4450. oleraceum 138. oleraceus 208. 1269. 4413. olitoria 1810. olivaeformis 2430 . onobryehioides 345.4927 . Onobrychis 633.2103. opaca 2342. 3448. Opalus i8. oppositifolia 4007. oppositifolium 1 194. opulifolia 4460 . Opulus 4914 . orbiculare 3262 . orbicularis 2766 . orchidea 4288. 4868. Oreoselinum 4150. Orientale 1602. 1652. 2935. 3171. 337.6. 4519.4544 .4822 . 5002. 5006. orientalis 104. 242. 709. 771. 793.984. 1005. 1010. 1220. 1226. 1399. 1595. 1655. 1873. $226 \mathrm{r}$ : 2321.2396 .2419 .2449 .2659 .2866 .2969 .3012 .3347 .3979 .4096 .4111$. $4 \mathrm{Ir}$ 4. 423.4. 4302. 4600. 4641. 4890. origanifolia 2605 . ornithis $309 \mathrm{I}$. ornithopodioides, 1242. 2676 . 2821 . 3062. Oruus 1832. orolooides 4916. Urontiun 351. orthoceras 1107. Orvala $2475 . \quad$ ossifragum 341. Ostruthium 2349. Otites 1445. ovalis 3577 . Ovata 718 . 1685. 184I. 1921. 3242. ovatum. 925. 1103. ovatus 4187 . ovifera 1452. ovina 1027. ovirensis 400. Uxyacantha 2877. oxycarpa 1852. Oxycedrus 2438. Oxycoccos 4784.4915 , oxyihyllus 600 .

$P_{a}$ Padifolins 3487. Padus 3493. pallens 1683. 3090. pallescens 2231 * pallida 90. 180. 1790. 1855. 2387. pallidum 4668. pallidıs 2280. palınata 2463. 4951. palmatun 3691. paludosa 2737. paludósum 1178. 2212 . paludosus 4183. palustre 1294. 1672. 1888. 1973. 2533. 4321.4613 .4701 . palustris 844. 848. 1212. 1250. 1679. 1789. 2186. 2254. 242 I. 2521 . 2938. 3088. 3177. $3182.3294 \cdot 3597 \cdot 4063 \cdot 4417 \cdot 4478$. 4504. 4625. 4763. 50II. Panaces 2172. pandurata 4835. janduratus, 1315. paniculata 255. 268. 1033. 1364. 1833. 2075. 2454. 3005. 3237. 4839. paniculatum 144. 190.2096. paniculatus 221.598 . pannonica 1957. 2993. 4947. pannoniicum 434 I. pamnonicus 550. 967. 4604. papilionacea 3078 . papposa 4033 . papyracea 717 . papyrifera 780 . paradoxa 4254. Paralias 1772 . Pardalianches 1 599. parisiense r885. Parmularia 4880. parnassifolia 120. parnassifolius 3616, partheni. folium 3546. Parthenium 3545. parviflora 728. 944. 1041. 1205. J390. 1577. 1867. 1879. 2749. 3036. 3753. 4086. parviflorum 1485. 1645. 4679. parviflorus 626. II 35. I 446. 268I, 3633. parvifolia I835. $266 \mathrm{I}$. 2869. 3778. 4619.4754. Passerina 4509. patavinus 4611 . patens 534. 3530. 4441. Patientia 3830. patula 674. 895. 1434. 4391. 4528. 4727. pauciflora $477^{\circ} 3277^{\circ} 4618.5014$ pauciflorum $3257^{\circ}$ Pavia 87 . Pe* cten 4061 . pectinata 38.465 . 10r7. pectinatus 3421 . pedata 4952 . pedatus 3653. peduncularis 4878. pedunculata 675.3600. Pelecinus 748. pelisseriana $261 \%$. peloponnense 2574 . peltatum 1630.3356. pendula 375.425 .2091 .2147 .3299 .3941 .4259 .4746 . pendulum $228 \mathrm{c}$. 4713. pendulus 577. pensylvanica $3174 \cdot 3251.3426 .3491,3504.3989$. pensylvanicun 11. 2065.467\%. pensylvanicus 3636 . pentadactyla 4022. pentaglottis 648. peptagonia 920. pentagyna 2870. pentupetaloides r 329. pentandra 3860. 4445. pentapliyllos 1538. Peplus

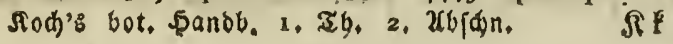


176r. peploides 4r9. Pepo 1453. perennis 4074. peregrina 864. 3789. 4107.4116 .4892 .4944 . peregrinum 1620.2758. 4585 . peregrinus 955.2672 . perenne 924. 2628.2933 . perennis 695. 787.2326. 246r. 2694. 2857. 4514. perfoliata 923. I1 7 r. I225. 4230.4998 . perfoliatum 1088. $1752.2560 .2936 .42970^{\circ} 4594.4715$. perfoliatus 3409. perforatum 2308. Periclymenum 931. perpusillus 3118. Persica $237^{\circ}$ 1858. 2412. 2923. 4525. Persicaria 3373. persicarioilles 3823. persicifolia 901. Personata 964. peruviana 2744. peruviainum 2r49. petaloideum 4559. Petasites 4736. petiolare 752. petiolaris 3888. 4396. Petitpierreana 2811. petraea 392. 4021 . petraeum 2104. 2554. 3720. petraeus 1544. Petroselinum 363. peucedanoides 2489. peucedanifolia 3033. phåum 1983. Phellandrium 3034. Phellos 3585. philadelphicum 1705. 2580 . phillyraeoides 1820. Philonotis 3637 . phleoides 4689. phlogifolius 533. Phlomoides 4812. phoenicea 247 . phoeniceum 2014. 4824. phrygia 1012. Phu 4791. pliylícaefolia 3880. pliysodes 605. physaloides 2276. Phyteuma 3668. Picea 3302. picroides 458.4416 . pictum 1480. $4657^{\circ}$ pilosa 730 . 1776 . 1922. 3245. 3433. 4879. Pilosella 2197. pilosum 2491. pilosus 553. 613. I 439. I 593. 2690 . pilulifera 4756. pimpinellifolium 1715 . pimpinellifolium 17 15. pimpinelloides 3032. Pinastor 3290. Pinea 3288. pinnata 749. 835 . $1539.2338 .3816 .4487 \cdot 4869$. pinnatifida 1409.4062. 4708. pinnatifidum 2198. 286 .3543 .4820 . pinnatum 2183 . piperita 2843. pisiformis 2523. 4918 . planiflora 889. plantaginea 450 . plantagineum 1650. plantaginifolium 2046. Plantago I I8. planum 1722. platanifolius 3621 . platanoides 15. platycarpa 1839. platycarpos 998. 4704 . 4939. platyphyllos 1778. plicata 2393. plumarius 1569. Plumieri 4422. plumosa 2449. pluvialis 841. Pneumonanthe 1961. Pocockii 1293. Podagraria 4316. poëticus 2973. polifolia 272. Pollveria 3564. polonica 2818. polyanthemos 3638. polycerata 4710. polyceratium 4326. polygamum 3458. Polygonatum 1304. polygonoides 426 . polymorpha 775 . polyphylla 4257.4923 . polyphyllus 595. polyrhiza 2538. polyspermum I 166 . pomifera 3918. pomponium 2586. Ponae 4845. pontica 489.683. I5 II. ponticum 3699 . populifolia 7 I5. populifolium 4127 . porrifolium 2234. porrifolius 4643. porrigens 3976 . Porrum 128. portensis 4274. Portula 3202 . portulacoides 660.4132 . potentilloides 2002 . praealta 4834. praecox 1741. 3874. 4069. 4896. praemorsum 2210 . pratense 1974. $2805.4670^{\circ}$ pratensis $305.947 \cdot 2517 \cdot 3533 \cdot 3934.4639$. prenanthoides 222. Prinus 3589. prismatocarpus 918. procera 462. 4383. procumbens 514. 1458. 1917. 1930. 2288. 2562. 3851. 3858. 3973. 4212.4262 .4681 . procurrens 398 . prolifer 1551. prolifera 4042. prolificum 2305. prophetarum 1447. prostrata 680. I954. 2073. 2450. 2797.3521 .4624 .4884 . prostratum 3721. prostratus 209. 2141 . provincialis 1773 . pruinosa 3255 . prunellaefolium 2229. prunifolia 3581. prunifolium 4912. pruthenicum 2492. purpurea 3982. Pseudacacia 3733. Pseud-Acorus 2406. Pseudo-Lycopersicum 4367. Pseudo-Narcissus 2961. Pseudo-Platanus 13. pseudosiculus 1323. Psyllium 3346. Ptarmica 26. pubers 3961. pubescens I12. 233. 324. 714. 883. 1246. 1305. I486. I665. 1746. 1847. 2129.2167 .2432 .2842 . 3200. 3252 . 3607 . $3469.4059 \cdot 4620$. pulchella 1743. pulchellus 529. pulcher 3833. pulchra 509. pulchram 2301. Pulegium 2854. Pulicaria 2359. pulla 880. pullata 1057. pulverulenta 1831. 378 $\mathrm{r}$. pulverulentum 3547. 4818. pumila 241. 724. 992. 1948. 2399. 3043. 3344. 3508. 4755. 4972. Pumilio 3289. pumilum I I85. 2192.4345. pumilus 3678 . punctata 912. 1958. 27I2. 2880. 2911. punctatum 1753. $2119.3697^{\circ}$ punctatus 538. puniceuin 1533. puniceus 564. 
purpurascens 507. 2r55. purpurea r575. r955.2255.2380.2596.3042. 3462. 3813. 3867. 4092. purptureo-coeruleum 2643. pupureum 1700. 1757. 1883. 2053.2479. purpureus 1 497. pusilla 899. 2482.2763. 3000. 4290. pusillum 1899. 1994. 2056. pusillus 2277.3419. pycnocephalus 959. pygmaea 1475. 1819. 3505. 3744. 4199. Pyracantha 287x. pyramidale 3116. pyramidalis 105.887 . 3079. pyramidata 2732. pyramidatum 2064. 2295. 4821. pyrenaeus 36r4. pyrenaica 307. 517. 1610. 1863. 1947. 2664. 3611. 4044.4306.4790. pyrenaicum 65. 1643. 1979. 2010. 2168.2243 .2253 .2588 . 3113.4324 . Pyrethrum 330. pyrifolia 2885. pyrifolium 490\%. Pyxidaria 2618.

Quadrangulare 2311. quadrangulata 185 r. quadrangulus 1138 . quadridentata 2699 . quadrifolia 3176 . quadrivalvis 3006. querci. folia 2264. quercina 2468. quinquefolia 4192. 4995. quinquevulnera 4236 . $\mathbf{R}_{\text {acemosa 78. 270. 403. r308. 3963. racemosum 293r. 2223. race- }}$ mosus 3845.4516 . radiata 2263.2283 . radiatum 4440 . radiatus $97 \mathrm{I}$. 2272 . radicans 738 . radicata 2319 . ramigerum 4809 . ramosa 3128 . ramosum $337 \cdot 2230.44 j 7 \cdot 4583$. ramosus 527 . ranunculoides $12 \mathrm{I}$. 296. Rapa $76 \%$ raphanifolia $747 \cdot 2474$. Raphanistrum 3657. rapunculoides 860. Rapunculus 897. reclinata 3059. recta 3450.4480. rectum 2058. rectus 2680 . recurva 438 . recurvata 3331.4386 . recurvum 3362.4652 . recutita 3188 . rediviva 2684 . reflexa 1042.3886 . $437 \%$. reflexum $1985.4147 \cdot 4690$. reflexus 1442 . retroflexus 222 . regia 2426 . repanda $23799^{\circ}$ repandum 1734. repens 96. 481 . 1097. 1663 , 2074. 2101.2604 . 2902. 2978.3051 .3641 .3908 .4349 .4676 . reptans $103.3247 .3358 .344 \mathrm{I}$. 3630.4635 . resedifolia 940.4087 . resinosa 3284. resinosuin 4778 . resupinatum 4653 . reticuiata 2986. 3903. reticulatus 1426 . retusa 3887 . reversa 3763 . revoluta 412 . rhagadioloides 1408. rhagadiolus 2093. Rhamnoides 2249. Rhapoutica 1070. Rhaponticum 3689. Rhodiola 4131. Rhoeas 3167 . rhombifolium 1155, rhomboidea 89r. Ribes 3692. Richardi 1844. rigeseens 314. rigens 1255 . rigida 559. 1045. 1411.3293 .4409 . rigidula 2793. rigidum 817 . ringens 2897 . riparia 3876.4993 . Ritro 1640. rivale 2008. rivularis 1274. 2920. 4017 . robertianum r997. Robur 3599. romana 1058.4228 . romanum 17 I7. romanus 2260. rosea 177.662 . 3191.4097 . roseum 1667.3548 . roseus 3837. rosmarinifolia 3895 . rostrata 439.1387 .3192 . rothomagensis 4984. rotunda 444 . rotundifolia 894 . 1633 . 1834.2339 .2753 .2835 .2840$. 3049. 3556. 4010.4361 . rotundifolium 809 . I175. 1912. 1996. rotundifolius 2516 . rotundum 131. rubella 3762 . 4277 . rubens 155 . 1403. 4358. 4696. ruber 1083. rubiginosa 3780. rubioides 1887. rubra 77.416 .763 .1682 .2849 .2928 .3495 .3593 .3893 . rubrifolia 3774. rubrum 16. II56. 1651. 1881. 37 I9. ruderale 2559 . rugosa 2913. rugosum 2934. 4568 . rugulosa 2820 . rupestre 2193.4146. rupestris 1044. 1334. 1809. 3428. 3983. 4012. 4170. 4286. Russeliana 3864. rustica 3004 . rutaefolius 3644 . ruthenica 868.1079 .3429$. 4703. ruthenicum 2704. Ruyschiana 1621.

Sabaudum 2226. Sabina 2434. saccharatum 1893. saccharinum 14. saginoides $4447^{\circ}$ sagittalis 1923. Salicaria $27^{20}$. sagittata 829.4953 . -sagittifolia 3854. salicifolia 1011. 1785. 3584. 4455. salicifolium 804 . 212r. salicifolius 558. salicina 2365. saligna $246 \%$ salignum 3379 . 
salignus 5St. salina 48:. salinus I136. salisburgensis 1273. 1793. salmantica 1066 . salsa 3325. salsuginosus 3625. sambucifolia 1848 . 4792. sambucina 2389.3097 . sancta 840 . sanguinea 1361 . sanguineum 1970. sanguineus 220. 3831. Sanguisurba 345\%. Saponaria 1967. saracenica $8=3$. saracenicus 4r85. sarmentosa 2030.3442. 4000. Sarsaparilla 4360 . Sassafras 2526 . sativa $856.9=6.17$ r8. 2462 . 2796. 2851.3009 .3047 .3179 .4933 . sativum 122. 1116.1346 .2555 . 3310. sativus 1 $\div 22$. 1449. 2503.3656 . saxatile 185. 1909. 2219.4137. 4589. saxatilis 318. 384. 435. 480. 740. 1331. 1333. 1771.2331.3321. 3676. $3791.4796 .4847 \cdot 4987$. Saxifraga 2081. 3279. 4283. Scabiosa I023. Scabra 2285.2927 .2375 . scabrum 1894. 4663 . Scammonia 1312. scandens 1004. Scariola 2459. sceleratus 3654. Sceptrum Carolinum 3184. Scheuchzeri 3258. Schmidtiana 4958. Schmirtii 4886. Schoberi 3013. Sch enoprasum 150. Sohraderianum 1162. Sclarea 3943. Scolymus 1,76 . scoparia 776,2452 . scoparium 4443 . scordifolia 4479. Scordioides 4232. Scordium 4551. Scorodonia 4102. 4550. Scorodoprasum 123. scorpioides 455. 624. 16c0. 2937. 3122.3275 . scorzonerifolia 448. scorzonerifolium 3264. scoticum 2569. scrophularoides 2323 . scutatus 3836. scutellata $2758.48 \% 0$. secunda 3555. Securidaca 1368 . sediforme 4160. sedoides 1169 . 3199. 398\%. segetalis 172 . I775. segetum II8?.2013.4317. Seguieri 3618. segusiana $246 \div$. semicompositum 302\%. semidecandrum 1095 . semierectus 3218. semperflorens 2329. 3496. sempervirens 261.935. 1379. 1459. 2330.3752 .4387 . senescens I52. sepium 852. 1159.4945. septentrionalis 281 . sericea $474.1362 .1929 .3319 \cdot 3432.3904 .4243$. sericeurn 801 . sericens 2514.3649 . seridis 1054 . serotina 2080 . 2457. 3497. 3923. 4382. serotinum 336. 1160.3537. serotinus 572 : 1545. 2540. serpyllacea 5021. serpyllifolia 397.422. 4859. Serpyllum 4601. Serraria 3322. serrata 31. serratifolia 4942, serratuloides 1257. serratus 3415. serrulata 169.3894 . sesameus 632. sesamoides 3662. sessile 47 I . sessilifolium 1745. sessilifolius 1492. setacea 6r. 3246. setaceum I47. setaceus 3416. setifera 4051 . setifolium 5007. setifolius 2500 . setosa 4195 . sexangulare 4148 . sexfidum 4138. slierartiana 2752 . sibirica 659. 663. 70r. 905. 1038., 1209. 1927. $2410.3249 .3512 .3529 .4837^{\circ}$ sibiricum 146. 1631 . $19699^{\circ}$ 2I73. 224I. 4575. sibiricus 566. I 44I. 2548. 3658. 4429. sicula 838 . 1052. 1336. 4038 . siculus 785. 1322. Silaus 1276. Siler 2488. Siliquastrum I110. siliquosus 2667 . simplex 579. 26I3. 4438. 4561. simplicifolia I856. simplicissimum 3070. sinaica 3574. sinensis 1469. sinuata 3045. sinuatum I97. sinuatus II27. Sipho 442: Sisarum 4353. Sisyrinchium 2416. siversiana 487. Soldanella $855^{\circ}$ solidaginoides $5390^{\circ}$ solstitialis 1049 . somniferum 3169 . sonchifolia 828. 1053.2465 . Sonneratiana 4222. Sophia $4337^{\circ}$ sorbifolia 4466. sordida 4940. sororia 4959. spadiceum 4683. sparsiflora 2912. 3320. sparsiflorus 556 . spartea 2599 . spathaceum 3105. spathulata 884. 3914. spathulatum 413\%. speciosa 29.267 .685 . 874.1470 . speciosissimum 802 . speciosum 4564.4823 . spectabile 2582 . spectabilis 578. 3580. Speculum 9ig. sphaerica 3520. Sphaericus 2498. Sphaerocephalon 39 . sphaerocephalus 1638 . sphaerosjermus 3225 . Spica 2527. spicata 76.468 .911 .1865 .2549 .2568 .4866 . spicatum $=949.3267$. Spielmani 3930. spinosa 402. $790.927 \cdot 1636.1637$. $3052.3519 \cdot 3738.3949 \cdot 470 \%$. spinosissima 3768. spinosissimum 2 I IO. spinosissimus 1264. spinasum 181. 803. 1198. 2561. 4133: 5000. spinosus 4. 214. spinulosa 410. spiralis 2977. spithameus 854. splendens 1072. Spondylium 2170 . sprengeriana 1410. spumosum 4651. spmria $=411.4737 \cdot 4827 \cdot 4840$. spurium $1907 \cdot 2595 \cdot 4129$. 
spurius 536. 2505 : squalens 239r. squalidus 4172. Squamaria 2494. squimata 460. squamatum 4903. squarrosa 33. 1337. 236r. 2567. 3342. 3746. $397^{2}$. squarrosum I 353. 4692. stachyoides 31 I4. stainineum 478r. Staphisagria 1535. staticifolium 2202. Stella 630. stellaris 3988. stellata 839. 1309. 1613. 3606. 4039. stellatum 8I1. 4130.4156 .4699 . stellatus 1259.3672 . stenopetala 388 . Stephanianum 1707. Sterubergii 4015. Stoochas 2042. straminea.10+8. Stramonium 1517. striata 429.4664. 4705. striatum 1975 . striatus 1425 . stricta 395. 708. 1360. 247 I. 27I8. 2943. 314.9. 3343. 3474. 4264. 4411. 4831. strictissimum 4343. strictum 1099. 2006. 2632. 4.688. strictus 211. strigosus 1639. 3807. Strobus 3297. Strumarium 5001. strunosus 2131. Struthim 2078. Styraciflua 2637. subacaulis 3456 . suberosa 4750. subhastatum 4340. submersum I 109 . subovatum 810. subterraneum 4678. subulata 348. 3332. 4448. subvillosa 408 r. Succisa 4036. sudetica 4980. sulfocatum 4660. suflruticosa 82 r. sufiruticosıun I 100. sulcata 4080. sulcatus 6 rg. sulphurea 3765. ro47. sulpliureum 340. 2115. sulphureus 1428. supina 5 r8. 3424. 4241. 4794. supinum 2059. $2151.2762 .4325 \cdot 4555$. supinus 1498. $299 . \%$ superbum 2581. superbus $157 \mathrm{r}$. susiana $238 \mathrm{r}$. susianus 1427. Susquehanae 3509. suaveolens 132.825.1076. 2371.2765 . 2939. 3240.3716 .4724 . suavis 3769 . svecica 1354. Sivertii 2385 . sylvatica 1788 . 1804. 2945. 3183. 4056. 4473. 4919. sylvaticum 1483 . I905. I980. 2054. 2224. 2806. 3109 . sylvaticus 1566. 3139.4168. sylvestre III5. 1176. 4320. sylvestris 295. 304. 853. 1319. 1557. 1591 . 1934. 2519. 2703. 2748. 2830. 3283. 3942. 4723 . 4731 . syphilitica 2650. syriaca 505.795 . 4027. syriacum $1749 \cdot 4627$. syriacus 1267 . 2185. syringaefolia 993 . systyla 3749.

T Tabacum 3001. Taeda 3295. Tagetes 3146. tamnifolia 2378. tanacetifolia 44. 466. 1394. 'Iaraxaci 358. taraxacilolia 4094. 'Taraxacum 2539. tardiflorus 574. tatarica 665. rorg. 1401. 2663. 2925, 4029. 4496. tataricm 9. I35. 3384. 3687. tataricus 1268. 1443. $-254 \%$. 4424. 'Tatula' I518. taurica 490. 5020. tauricum 70. 3065. 4520. tauricus 627 . taurina 516 . taurinensis 1414 . 'Tazetta 2972 . tectorum I416. 4154. telephioides 265. Telephium 4126. temula 2952. tenacissima $182 \mathrm{I}$, tenella $25 \mathrm{I}, 262.3998$. tenellum 4779 . tenellus 3659. tenerrimus4420. tentaculaca 2783 . tenuiflora 3329.5017 . tenuifiorum 2644. tenuifolia 434.872 . $1340.3159 \cdot 3460.4926$. tenuifolium 2623. 3549. 4323. tentilolius 549. 2512 . 4180 . tenuior 4449.5019. tenuis $2678.4255^{\circ}$ tenuissima 3028 . Terebellum 2787 . ternatum 4295 . terrestris 4646 . testiculatum 1347 . testiculatus 603 . tetragonolobus 2668. tetragnum 1669. Tetralit 1877. 'Tetralix 1687. tetraphylla 348. tetraphyllum 3360. tetraptera 2083. 30 4. tetraspermum $17 \mathbf{1 9}$. Tencrium 4885. thaliana 391. thalictroides 299. 941. I002. 2423. 'Ihapsi 1584. 'Thapsoides 3332 . 4805. "Whapsus-4802. therebinthinaceun 429.' Thora 3631. thuringiaca 252g. thymiflorum 1628 . Thymifolia 2724 . thyoiles 1460 . thyrsiftora 2719 . thyrsoidea 863 . tigrinum 2589. tiliaceus, 1317. tiliaefolia 3924. 4221. tinctoria 260 . 332. 524. 1925, 2418 . 3353. 3595. 4194. tilctorius 985.3675 . till. ctorum 3788. tingitana 1816. tingitanus 2510. 4415. Tinus 4909 tomentosa 42. 320. 1118. 2033. 2881. 3784. 4453. tomentosum 1104 4655. tomentosns 3799. torninalis 3573 . tormata 2772. tortuosum 72. 193. 4203. tommefortiana 2741; Touncfortii 1009. 4873. Toxi oodendrum 3714. traolelifolius 2134. tracheloides 867. "Trachelimin 866. 'Tradescanti 573. 'Tragus 3917. transsylvanica 4032. tre 
mula 3396. trepida 3395. triacanthos 2022. triandra 3863. trian. drus 2967. triangularis 1924. 4218. tribuloides 2784. trichocephala 1015. tricolor 1326. 2860. 4983. tricorne 1897. tricuspidata 1796. tricuspidatum I723. tricuspidatus II4I. 'Tridactylites 40I3. trifida 226. 2739 . trifidum 1889. triflorum 3726. trillorus 4166 . trifolia 292. 939. 4488. trifoliata 2855.3522 .4113 .4472 . trifoliatum 1748 . 4292. trifolius 2153 . trigyna 705. triloba 2165. 3592. $3819.3957^{\circ}$ 4465. trilobus 3219. trimestris 2532. trinervia 420. 'Trionum 2189. triornitliopophora 2610 . tripartita 726 . tripetala 2729 . triphylla 2608. triphyllos 4900. triphyllum 498. Tripolium 581. tripteris I345. 4793. tristis I I 24. 2180.2612 . trisulca 2535. 'Triumfetti 1020 . trivialis 3804 . truncata 3476 . tubaeformis 2128 . tuberculata 2771 . tuberosa $511.2370,2417.2987 .3193 .3236 .3359$. tuberosum 1695 . 4365. 4518. 4569. tuberosus 1263. 2143. 2515 . 3137 . tubulosa I385. Tulipifera 2639. tumidus 2504. turbinata 2770. 3782. Turneri 3588. Turrita 376. typhina 37 I I. tyrolense 1904.

$\mathrm{U}$ cranica 2996. 4046. uliginosa 1945. 3913. 4955. uliginosum 1910. 2066. 3538. 4773. uliginosus 617. Ulmaria 4469. ulmifolia 4390. 446I. umbellata 2031. 2340. 3559. umbellatum 2235. 2252. 3112. umbellatus $547.8 \mathrm{rg}$. umbrosa 3992 . umbrosum 1976 . umbrosus 4181. uncinata 1814.2788 . uncinatum 75. undata 3667 . undulata 168. 258. 38I. 283 r. 3238. 3866. undulatum 2049, 2228. 3382. 3690 . undulatus 582. uniflora 878. 1016. I938. 3580. uniflorum 1693. unisiliquosa 2246. uralensis $60 \%$ urbanum 2007. urbicum II54. urens 476r. ursinum 162. urticaefulia 865. 3929. 4874. urticaefolium 1759. usitatissimum 2627. ustulata 3085. utriculatum 198. utriculosa 1965. Uva crispa 3729. Ura ursi 405. Uvedalia 3391.

$\mathrm{V}$ accaria 3980. Vaillantii 2367. Valderia 497\%. valentiana 335. valentinus 246. Valerandi 3964 . vallesiaca 488.4282 . varia 1369. variabilis 1968. variegata 508. 2397. 2708. 3082. 3935. variegatum 74. 1288 . varius 2688.3134 . veneta 673 . venetum 367 . Verbenaca 3955 . verbenaefolius 4163 . veris 3472 . vermiculata 2332.4078 . verna 380. 433. 845.1609 . 1952. 2407.3273 . 3435. 4065. 4901 . vernalis 82. 3531. 3991. 4101. 4169. Vernix 3707. vernum 789. 2564. vernus 561. 1423. 3136 . verrucosa 264. 1777. 1798. 1454. 5010. versicolor 57 . I 562. 2405. 2598. 2944. versiflorum 4808. verticillaris 601. 1402. 342 j. verticillata 888. 1303. 1339. 1859.2905. 3187.3931 . 5018. verticillatum 1758.2345 .2722 .2950 .4210 .4350 . verticillatus 3486. verum з gor. Verutum 1063. vesca 99 ז. 1824. vesicaria 1808. vesicarius 2188 . 3838. vespertina 4271 . Victorialis 133. 3323. Villarsii I699. villosa 283. I586. 3022, 3480, 3783. 4016. 4577. 4922 . villosissima 3056 . villosum 2240.3107 .4136 .4374 . villosus 2687 . 4644. viminạlis 3897 . viminea 3465 . 4398 . vimineus 575 . Vincetoxicum 1473. vineale 137.2123 . vinifera 4989 . violacea 1243.2377 . 2988. 3902. violaceum 1648. 2015. 2100. Viorna 1230. virens 1420. virescens 620. virgata 1926. 3946. virgatum, 751. 1732. 2721 . 3261. virgatus 622 . Virgaurea 4710 . virgineus 1561 . virginiana 297. 1587 . 1826. 2435. 3494. virginianmm 1623.2003 .2634 .3369 . virginica 2 . I 170. 1224. 2085 . 2404. 2425. 2455. 3144. 3336. 3528. 4637. 4838 . virginicum 50I. 504. 1482. 2270. 255 I. 2828. 4545. virginicus 2187. 2710 . viride 4800 . viridillora 372.3710 . viridis 207.1845 .2157 . 2829. 3093. 3730. 3951. 3973. virosa 1201. 2466. Viscaria 2701. 
viscosa 370, 684. $-1396.1795,2076,2726.3060 .3734 \cdot 3936$. viscosissima 1457. viscosum 1094. 2624. viscosus 609. 1444. 416?. Visnaga 232. Vitalba 1228. Vitaliana 288 , vitellina 3869. Viticella 1234. vitifolia $2447 \cdot 4215$. Vitis idaea 4782 , viviparum 3378 . voInbile 73. volubilis 3715,5024 . vulgare $1247,1646.2113 .2576 .2756$. 3103. 4535. vulgaris II4. 342. 368. 447. 472, 691.697. 702. $847 \cdot 978$. I119. 1468. 2028. 2251.2268.2603.2716. 2808 . 3143. 3212.3281 .3364 . 3490. 3534. 4164. $4515 \cdot 4525 \cdot 4609$. $4615 \cdot 4765 \cdot 5025$. vulgatum 1092. Vulneraria 346 . vulpina 4992 . vulpinus 616 .

Weigeliana 390r. Wilidenowii 1563. wolgaricus 1504. Wulfeni 3330. Wulfeniana 3885.

$X_{\text {ipllioides 2413. Xiphium 2414. Xylosteum } 2662 .}$

Zeylanica 3233. Zoysii 886. 4978. 


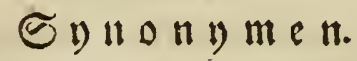

anmerf. Die officiellen Samen finb mit farn̈ger Schrift gebutuct.

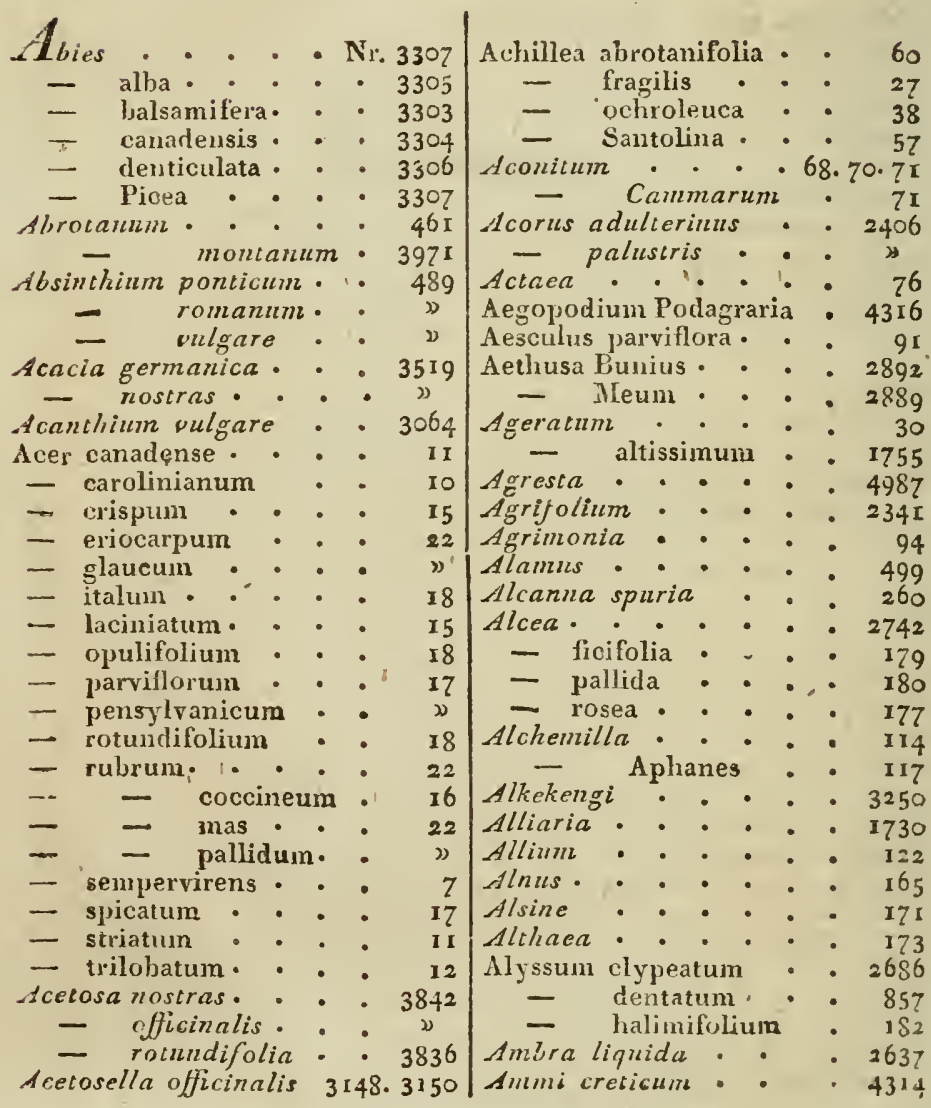


Sinmi verwm . . .4314 - vilgare. . . 231 Alaorpha fruticosa. . $\quad 235$ - herbacea - $\quad 233$ - xionperforata - 234 - perforata. '. 235 - jumila.. . 233 Ampelopsis bipiunata . 4996 cordata $\quad 4990$ quinquefolia - 4995

Anydalae . . . . 238 - Imygdalus argentea . . 242 communis amara 238 Arsagalis • . • . 247 -1nchuse: Andrometa axillaris

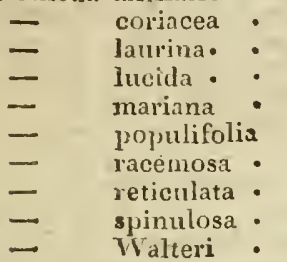

Anemone Halleri . . .

- 1lepatica - patens. -

— pratensis.

- Pulsatilla. - . Anethunz . . . . . 3531 - Focniculum . . 2891 Anvelica graveolens - 2889 - sylsestris. . Anisum vilgare. Anonymos aquatica. Anserina . . . . . Antlerieam annumm . calyculatum . Anthora • . . . . Anthos . . • . . . Antirrhinum alpintum - .

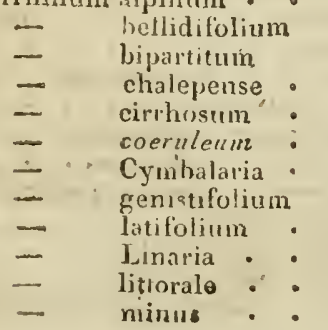
302 304 4315 3312 3423 788 4625 66 3786 2533 2597 253 2600 2602 2504 1961 2593 2609 2 too 2603 2607 2606 Astragalus arenarius
Antirrhinum origanifolium 2605 2596 2604 2613 2599 2595 2608 2598 2603

Apios frutescens . . 2035

- tuberosa • . . 2034 Apium . . . . 362 - montanim . - 4T50 Aquatica . . - . 4645 Aquifolium -. • • $234 \mathrm{I}$ Aquilegia . . . . 368 Arbor vitae . . . • 4599 Arctium carduelis . . 96 I - Personata. . 960 Arenaria caesjitusa : • 433 431 418 287 288 3423 द 376 445 $137^{6}$ 442 443 442 444 445

Ariscologia vulgaris . • • • ग

Armoracia . . • . 1284 Arnica - - . . 454 - svedensis - . 2357 Amoseris pusilla • . 2482 Aroll . . . . . 499 Artemisia . . . . 472 475 495 462 476 Arthanica . . . 1465 Arum . . . . . 499 Asa dulcis . . . . . 2525 Asarmin - . • . 502 Asclevias nigra • . 1472 - Vincetoxicum - 1473 Aisparaglls . . . . 513 Asphodelus . . . 526.527 Aater pumilus • • • 542 — salicifolius . . 586 586
635 
Astragalus cristatus - .

danicus $:$
dasyglottis :
echinatus
falcatus :
二 $\begin{aligned} & \text { hians }: \\ & \text { isetensis }:\end{aligned}$
retroflexus:
varius

Astrantia Epipactis . : .

Athamantha Cervaria $\cdot$

Atriplex alba . .

= $\begin{gathered}\text { foetida } \\ \text { marina } \\ \text { = } \\ \text { mexicana: } \\ \text { - } \\ \text { rubra } \\ \text { syluestris: }\end{gathered}$

Atropa Mandragora • .

Aurantiun $\cdot$ : $^{\circ} \cdot$

Auricula muris. . .

Avellana. $\cdot \cdot \cdot \cdot$

Azalea nudiflora coccinea.

- viscosa

Baccae Norlandicae.

Balsamica maris

Balsamum carpathicum

Libani. .
A pyrenaica.

648 Betonica aqualica. • . 4104

635 Betula •. •. • • 713

648 - Alnus. . • . 165

2) - - crispa. • 168

620 - - serrulata. 169

626 - crispa. . . 168

620 - excelsa canadensis. 719

626 - humilis . . . $7^{23}$

.622 - incana .... 167

1598 - lanceolata $\bullet \cdot 7_{17}$

2570 - lenta • *. 715

$4150-$ macrocarpa $\cdot \cdot{ }^{113}$

653 - nigra... 716.721

6 - odorata - • $\quad 714$

I164 - papyrifera $\bullet \cdot 7^{17}$

$669=$ pendula

- pumila brockenbergensis 714

1163 - quebeccensis. - 723

1164 - rugosa $\cdot . \cdot 169$

667 - serrulata...

1156 - vulgaris • • • 713

2754 Bidens • • • • • 732

$122 \mathrm{I}$ - macrosperma * 728

2197 Bifolinm • • • • 1685

1384 Bignonia Catalpa • • 993

685 Bismalva. . • • 173

686 Bistorta • • • • • 3377

Blitnm - . . . . 205

Bonus Henricus. . • 1152

Borrago - • • • • 756

Botrys vulgaris. . . 1 $16 \mathbf{1}$

$\begin{array}{cr}3296 \text { - mexicana. : } & 1163 \\ \text { Brachystemum virginicum } & 764\end{array}$

4469 Branca germanica. • 2170

Barba caprina : : 4.4469

Barbarea.: : 4639. 1740

Bardana • . - 406.407

- minor. . . 4999

Basilicum • - 3023. 3025

Baccabunga. - : :

Bedeguar. • • • $377^{2}$

Belien album • • • 1436

- rubrum . . . 4490

Belladonna • • • . 676

Bellis hortensis. • . 695

- minor. . . . 1)

- major : : : 1180

- pracensis. . . "

Bencdicta syluestris : 2008

Benzoes . . . . . 2525

Berberis • • • • 697

- vulgaris canadensis

703

703
702

7.02
3

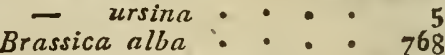

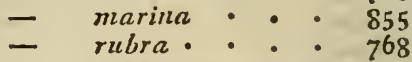

- subhastata • 4340

Britannica herba • - 3835

Brunella • • . . 3490

Bryonia alba • • • $78 \mathrm{r}$

Buechnera foetida * * 2755

Buglossum • • • • 257

Bugula : : : : 103.105

Bunias . . . * 766

Bunium Bulbocastanum- - 4352

Bupthalmum • • • • • 332

Bupleurum minimum • 3028

Odontites - . 3026

semicompositum 3027

Bursa pastoris ! . • 4591

Buxus angustifolia. * : 820

- sempervirens. . 821

suffruticosa 
$C$

lanintha montana 2824.2825 Calcatrippa $\cdots \cdot{ }^{2525}$ Calcitrapa..$*{ }^{0}{ }^{060}$ Calendula $: .:{ }^{836}$ Calla . . .. . ${ }_{844}^{84}$ Callitriche caespitosa .845 Calycanthus ferax ${ }^{\text {inta }}:{ }^{85}$ Camphorata .. .924 Cannabis. . . . ${ }_{926}$ Capparis. . . . $\quad{ }_{927}$ Caprifolinm. . . ${ }_{933}^{92}$

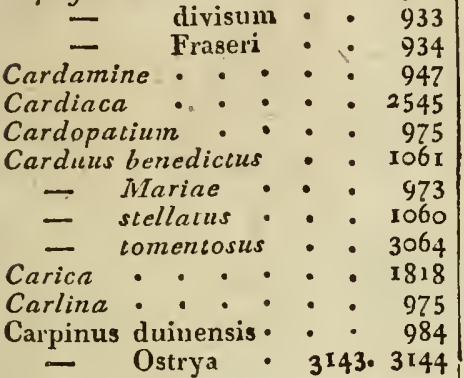
Carchamus virginica - . : : : 9885 Caryophyllata. . . ${ }_{2007}^{989}$ aquatica - 2009 Cassine Peragua . * . 4904 Castanlea. . . . 991 c- equina $: 885$ Cataputia nzajor . . $373 \mathrm{I}$ - minor :- . 1764 Cataria . . . 2981 Caucalis Anthriscus . 4631 二

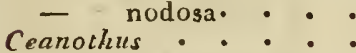
Cedronella . . . 1626 Centauréa Amberboi : . I076

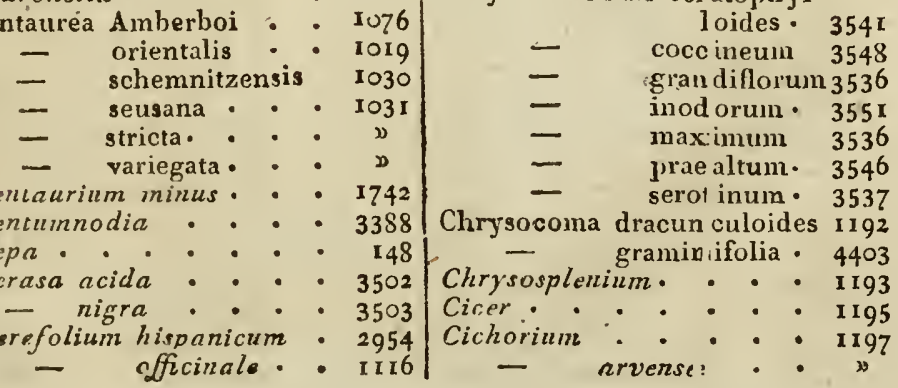
Chaerophyllum .

aromaticum 2956 aureum - 295 I bulbosum. 2953 coloratum. 2957 Jairsutum - 2955 snodosum - 343 ttemulum - 2952

Chamaedrys. - 3792. 4547 Chaneleon albiz • • 975 Chamaemelum nostras. : 2764 Chamaemorus ivulgare: : Chamaepitys . . : Chamomilla foetida : 327 - nobilis . $\quad 328$ - nostras: 2764 - romana. 328 - vulgaris $\cdot 2764$ Cheiranthus Cheiri fruticulosus. • • • I II22 Cheiri. . . . . - I12I Chelidonium ersmiculatum 2014 -- Grlancium 2016.2017 - $\quad \begin{aligned} & \text { hybridum } \\ & \text { miajus }: 2015\end{aligned}$ - nzinus • 3629 Chelone formosa. - 1146 Chenopodium : : : I Chenopodium concatenatum 1167 - mexicanum 1163 - $\quad$ Viride $:$ Vulvaria: 1550 Chimaphila corymbosa • 3559 Chionanthus maritirta. - I170 Chironia Centauriumı : : 1742 - pulchella • 1743 Chondrilla • • • 3466 Christophoriana . • ${ }^{26}$ Chrysanthiemum ceratophyl-

Chaerefolium officinale - I116

(1)


Cichorium syluestre • - II97

Cicuta aqualica - . - 120I

- major • • . 1299

- cerrestris - . - ग

Cicularia.

Cissus Ampelopisis • • 4990

- hederaceus • • 4995

Cistus aegyptiacus • • 2118

- alpestris • $\cdot$ - 2124

- apenninus. . 2117

- Fumana. •. 2122

- Helianthemum . . 2113

- ledifolius. • . 2120

- mutabilis. . . 2114

- oelandicus . . 2124

- junctafus • . 2119

- salicifolius • • 2121

Citrus. viliealis : * : : 2123

Claytonia cubensis. . . I225

Clematis aljuina • • • 658

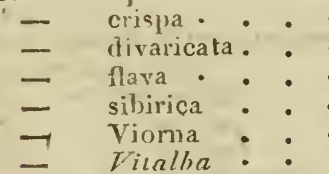

Clcnopodium montanum. Clethra tomentosa • •

Clinopodium variegatum .

Clypeola maritima - • 182

Cnidium • . • • 1507

Coccognidium . • • • य

Coccumeniclium - • 》)

Cochlearia Jortensis - 1283

- ' hmmifusa. * 4189

vnlgaris - 1283

Colchicum . • • 1286

Colubrina • • • • 3377

Colutea halejica • • • 1293

- humilis. • 1292

- istrica . . . 1293

- orientalis . 1242

- sanguinea... »

Comarum pallustre. . 1294

Conium • • • • 1299

Consolida major • . 4517

- media..103.105

- minor : $\quad$ reulis. 3490

Convolvulus folongatile. - -4410

- nuajor albus. 852

- spium - D D
Convolvulus spithameus - 854

- $\quad 853$

undulatus -1320

$5 \$ 5$

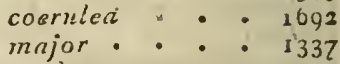

media : - :

minor • • • 1692

Copal. • • • - 3708

Coreopsis parviflora . - 1390

Coriandrum : • . . 1346

Cornus : $:$ : • . 1357

- allida . . 1364

- Anomum * : 1362

- foemina $\cdot . \quad 1364$

-. rubiginosa... . 1362

Coronopus didyma: •. 2550

Corylus : • : • . 1384

- cotnuta • . 1387

- níaxima • . 1385

Costa mlgaris . . . 2316

Costum nostras. . . .

Cosins hortoriun : . $69 \mathrm{r}$

Cotula foetida - • - 327

Cotyledon spinosa. . . 4133

Crambe Corvini • . 794

Crassula major. • . 41276

- spinosa.. 4133

Crataegus alpina . . 3566

- Aria . . 3568

- $\quad$ - svecica. 3565

- Azarolus. - 2887

- cerasifolia - 3581

— coccinea. - . 2867

- Crus galli 2875.2886

- edulis . 2885.3567

- Hlava. . . 2868

- glandulosa - 2874

- heterophylla . 2876

- leucophaeos. . 2885

- lncida • . 2875

- Mlichauxii . 2868

- monugyna. 2878

- nigra. . . 2888

- orientalis . . 2866

- Oxyacantha. . 2877

- jarvifulia . 2885

- jentazyna. . 2870

- populifolia. . 2872

- promifíslia . 28-3

- puncrita - . 2886

- prifulia. . 2885

- racentosa. $355^{5}$

- sanguinea . . 2874

spicata * . 3577 
Crataegus tomentosa - 2885 Digitalis epiglottis ..' • $158 \mathrm{r}$

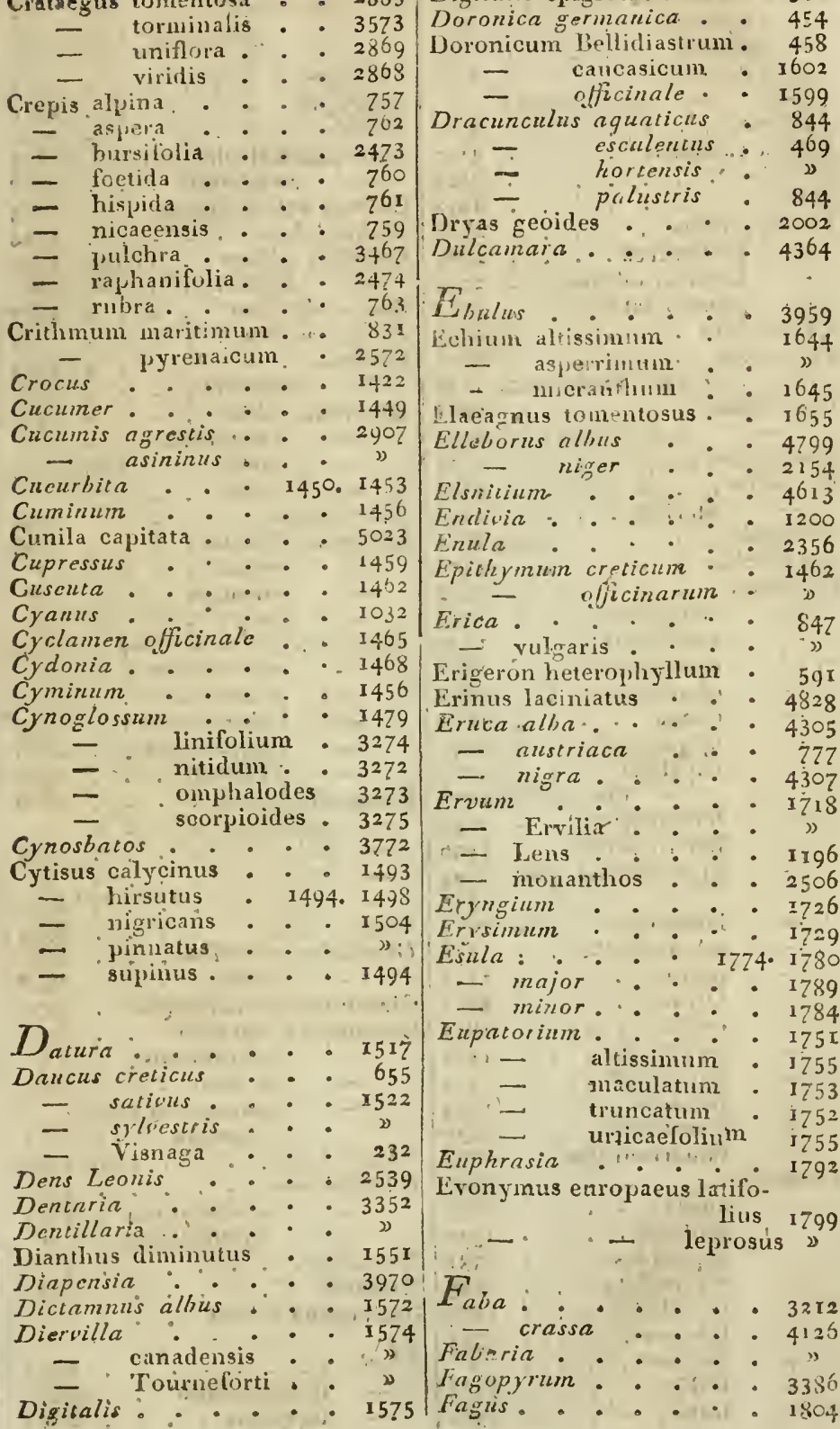


Fagus americana Jatifolia . 1805

- Castanea . : . : 991

- sylvatica . . . 1805

Farfara - atroptmicea " ". . 4729

Ferula rablensis : . . 4152

Ficaria . . . . - 3629

Ficus . . . . . 1818

Filago gallica .. . . . 2062

- germanica. . . 2061

- Leontopjodium - 2055

Filipendula. : : : : 2064

Flammula . . . . 3626

- Joviis . 1228. 1232. 1238

Foeniculum .. . . . . 2891

- aquaticum. . 3034

Foenugracc um . . . . 4709

Fragaria . . . . . 1824

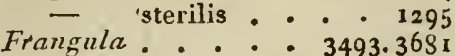

Fraxineli'a . . . . 1572

Fraxinuis . . . . . I853

- acuminata . . I842

- americana.: 1846

- carolinensis : . 1837

- caroliniana . 1836

- crispa . . . 1853

-. epiptera. • • 1847

-- excelsior - : 1837

-_ - diversifolia 1856

- halepensis . . $\mathbf{1 8 3 8}$

- heterophylla . . 1856

- juglandifolia 1841. 1844

- lanceolata . . 1845

- movophylla.: 1837

- nigra ... . . 1847

- Ornus . . . 1852

- ovalis . . 184t

- oxyphylla.. . 1852

- parvifolia... 1838

- pendula . . 1853

- serratifolia. . 1837

Fumaria tamariscifolia : . - 1838

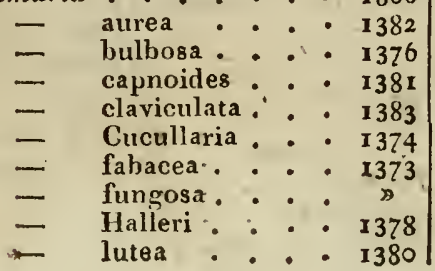

Pumaria nobilis . . 1325

sempervirells • 1379

Galega . . . . . 1872

Gruleopsis . .. . 2478

Gallae . : 3602

Galeopsis Galeobdolon : 1874

- versicolor . . 1878

Galitum scaberrimum . . 1913

Gallitrichuin • . • 3943

Gallium album . 190r.1902

Garyophyllata : : . 2007

Genipi verun . . . . 52

Genista . ... . : 4443

- tinctoria... 1925

Gentiana alba . . . 2486

- Centaurium . ${ }^{1742}$

- lutea:

Gentianella : . . . I I944

Georgina lilacina . • . 1968

- pallida....

- purpurea $: \therefore$

Geranium batrachioides : 1974

- moschatun . : 1716

- jrostratum . 1970

- robertianum. . 1997

- sanguinenm . . 1970

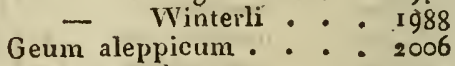

- canadense . . .

- palustre. . . . 2008

- rivale . . . . "

- urbanum ... 2007

Ginkgo biloba . . . 3859

Gladiolus coeruleus . : 2388

- luteus. . . 2406

Glandularia carolinensis - 4932

Gleditschia aquatica . . 202 I

- carolinensis. .

- sinensis . . . 2024

- sperma. . 2021

Glycirrhiza . . 2036.2040

Gnaphalium. . . . 2047

- americanum . 2065

Granatus . . . 3535

Gratiola ... . . . 2069

Guilandina dioica .. 2070

$H_{\text {edera . . . . . } 2089}$

- quinquefolia: : 4995 
Helenium . . . . 2356| Inula germanica... . 2362

Helianthemum . . . . 2113

Helianthus eanescens . 2129

- laevis.: $\quad$ mollis.: 800

- tomentosus . . 2133

Helleboraster. . . . 2159

Halleborus allus. . . 4799

- foetidus:. 2159 niger. . . 2154 viridis . . . 2157

Helonias asphodeloides . 5007 borealis. . . 4625

Helxins latifolia. . . 2162

$\cdot \cdot \cdot \cdot 3172$

Hopalica alba. . • 3177

- nobilis... 2165

Heracancha stlata - • 515

Herba Britannica • 978

- Cosia . . . . 2316

Hermod - wulgaris . . 2319

Hermodactylus. . . 2417

Herniaria . . . . . 2175

Hesperis verna.. •. . 380

Hieracium coronopifolium 2235

Hippocastanum • . . 85

Hirundinaria . • . . 1473

Horminum : • • 3943. 3944

Hortensia opiuloides . . 2262

Hyacinthus botryoides . . 2932

- comosus... 2930

— Monstrosus . •

Hydrangea nivea.:.$: 2931$

Hydropiper . . . . . 3372

Hyoseyanus allus . . . 2278

Hyoserig niger • • 2281

Hoseris foetida • • - $248 \mathrm{I}$

- virginica • . 2455

Hyperanthera dioica . 2070

Hypericum .. . . 2308.2311

Hyssopus . . • • 2320 ocymifolius : $\quad 1659$

$J_{\text {acea . . . . . } 4983}$

Jacolaea . . . . 4176

Jasminuni album . . : 3226

Ilseris . : . . . . 2558

Ilex . . . . . . . 2341

Illecebrum vermiculare - 414I

Imperatoria. • • . . 2349

Inula . . . . . 2356

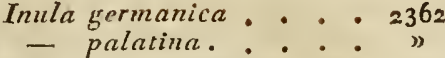

Intybus augustus . . 2459. 2466

Iris florencina . . . 2384

- nostras. . . • . 2388

- odoratissima . . 2387

- palustris . . . . 2406

Isatis lusitanica . . . 2419

Inglaus . . . . . 2426

- alba . . . 2431

- angustifolia . . 2430

- Pecan . . . D

rubra.... . ग

Junjuerus • • • • 5025

* * $\cdot 2436$

inntana 2437

- sibirica saxatilis "

Justicia ladanoides • : 2440

Iva arthritica: . . . 108

Ixia chinensis . . . . 2921

Tsacuca ...... 2462

- longifolia. . 2469

- syliestris . . 2459

- virnsa. . . 2466

Lagophthalutus . . . 2007

Lagopus . . . . . 4695

Lamiuni album . • . 2478

- Galeobdolon .' 1874

Lapathum acutum . . 3829

- aquaticnm • 3835

- sanguineum . 3831

Lappa major : . . 406.407

- uninor . . . 500 I

Lapsana lilıagadiolus - 3673

- stellata . . . 3672

- Zacintha. . . 50го

Larix americana . . . 3300

Latlıyrus axillaris . . 2498

- coccineus . .

- siculus •. • 2508

Laurentia zeylanicus. • ग

Laurentia atroptrpurea - 3974

Lautreola . . • •. 1507

Laurocerasus . . • . 3498

Lauris . • • • . 2524

- aestivalis . . . 2525

- alexandrina : - 3848

Lavandula angustifolia. 2527

- Spica latifolia . 2528

Lavendula . . . . . 2527

Ledum groenlandicum . 2534

Lens . • • . • . 1196 2537 
Leontice thalictroides : : $\mathbf{1 0 0 2}$

Leonurus Galeobdolon . . 1874

Lepidium fragrans . . 182

- Pollichii . . 2558

Levisticum . • • . . 2573

Lignum Sassafras . . . 2526

Ligusticum austriacum . : 3551

Ligustrum . . . . . 2576

- germanicum :

Lilium album : . . : 2583

- convallium: . 1302

Linaria,. . . . . 2603

lingua avis.. . . . . 1853

Linnaca . . . . 2620

Linum . . . . . 2627

- cacharlicum. . 2639

- monopetalum . . 2635

- Radiola. . . 3610

- sativum ... 2627

Liquidambar . ... 2637 - asplenifolium 1298

Liquiritia. peregrinum " 2036.2040

Lithospermuin : . 26402643 - nigrum . 2641

Lobelia . . . . 2650

Lolium offcinarum . . 98

Lonicera Caprifolium . 932

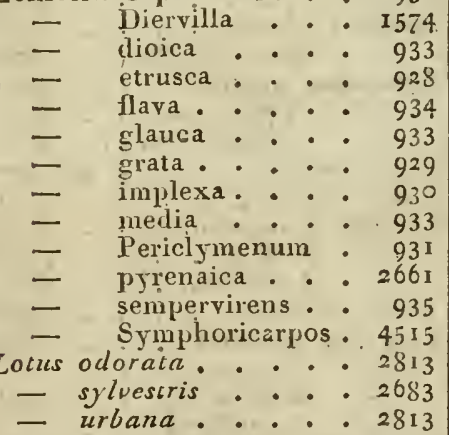

Lujula. . . . 3148.3150

Lupinus : . . . . 2695

Lupulus : . . 2256

Luteola : * 366r

Lysimachía bulbifera : 2718 lutea. : : 2716

- purpurea $:$ racemosa 2720

$\mathrm{M}$

agnolia a uriculata . 2732

- frondosa. . 2729
Majorana . . . 3104

Malicornium . . . . 3535

Malus non florens . . 3583

- syluestris . . . 3579

Malva arborea . . . 177

- horcensis. . .

- minor.... 275

- rosea . . . . 177

- syluestris : . . I73

- rotundifolia. . 2753

- vulgaris . . . "

Mandraggra . . . 2754

Marrubium album . . 2756

:- nigrım... 689

Matricaria . . . . 3545

- maritima . 3552

Matrisylva Parthenium - 3545

Médicago circinnata . . 2273

- cordata . . 2778

- polymorpha arabica $\pi$

- jinnatifida 2779

radiata. . 2272

rugosa . .. 2767

Melampodium . . . 2157

Melimpyrun nemoiosum., 2804

Melanthinm. : . . 3009

Melilotus atha $\because \because 2814$

- citrina. coerulea $\because 28 \mathrm{I} 3$

- sulcata... 2812

Melíssa citrata . . . 2822

- citrina, . . .

- ioriensis.:

Melissophyllım : • : 2826

Melo . . . . . . 1448

Mentha adsjersa . . 3846

- aquatica. . . 2845

- balsamina. . 2850

- crispa. . 2844.285 I

- equina . . 2852

- hirsuta. . . 2845

- longifolia . $\because 2830$

- moñtana. 2824.2825

- odorata . . 2846

- ovata . . . 1659

- Patrini : . . 》

- piperitls : . . . 2843

- romana : . 6gI

saracenica : .

syluestris: : 2830.2852

Menthastrum . . . . 2829

Mercurialis . . . . 859 
Mesembryanthémum crysiallinulun. . . . 2862 Mespilus . . • . 2884 acerifolia * . 2872 Amelanchier $3577 \cdot 3578$ arbutifolia . : 3569 axillaris . . 2869 Calpodendron. . 2885 canadensis . . 3576 caroliniana . . 2868 constantinopolitana 2876 cuneifolia . . 2886 cuneifornis : . ” eriocarpa . . 2881 erythrocarpa . 3569 flexispina . . 2868 laciniata. : 2869 lucida . . . 2875 Phaenopyrum, . 2872 pygnaea • . 2881 rotundifolia * 2874 tanacetifolia . 2866 Xanthocarpos. 2869

Merum . . . . 2889 Mezererun athanticum : : ${ }^{\prime \prime}$ Milium solis : 2640.2643 Millefoliuin nobile: . 50

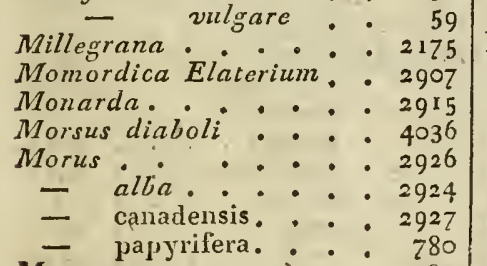
Mustum : : : . . 4989 Nifyagrum austriacum: 858

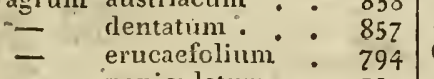
- paniculatum. . 190 - sativum . . 856 - saxatile • . 858 - squarrosa: : 3745 Myrtillus...... 4770 Myrtus :. . . 2958 - brabantica. . : 2947

$\mathrm{N}$ Napaea dioica : * 2959 - hermaphrodita. 4216 - laevis.... - scabra.... 42I7 Oreoselinum

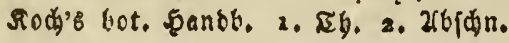

Napellus . . . . 68. $7^{0.71}$

Naplua...... 1221

Napits . . . . 766

Narcissus angustifolius. 2973

Nordus tenuior. • 2975

Nardus celtica. . . . 4797

Nasturtiunn aquaticum. . 4319

$$
\text { - hispanicum. : } 4720
$$

- hortense. . . 2555

- indicum : 4720

- pecraeum. . $1 \mathbf{I} 93$

- $\begin{aligned} & \text { pratense } \\ & \text { syluestre : } 942 \\ & 1280\end{aligned}$

Nenuphar ..... 3014

Nepeta . . . . $298 \mathrm{I}$

- annua.... 2980

- hipinnata... ग

- lavandulacea . 2979

- melissaefolia . 2992

- multifida.. . . 2980

- salviaefolia . . 2991

- teucrioides . . 2990

Nicotiana . . . . 300

Nigella . : : : 3009

Ninsi, Ninsing, Ninzin : 4353

Numinularia . . . 2715

Nux aquatica. . . 4645

Nymphaea alba : : 3014

Nyssa angulisans: • 3017

aquática $: \cdot 3020$

- integrifolia.. 3021

- multiflora.: : 3022

- uniflora. . : : 3020

$O_{\text {cymum citratum . 3023. } 3025}$

Oelsuitium . : : 4613

Oenanthe. Sylvestre. : :

Oenoplia volubilis : 5024

Olea, Olivae . . . 3047

Olsnitium. . . . . 4613

Ononis. . . . 3052. 3053

- altissima ... 3053

- arvensis . . .

- barbata ... 3054

- foetens. . . . 3053

- saxatilis ... 3054

- spinosa mitis : . 3053

Opium . . . . 3169

Ophris Loeselii . : : 2736

- ovata - . . 1685

Orchis $3077 \cdot 3079 \cdot 3081 \cdot 3087 \cdot 3089$

3095

4150 
Origanum creticum . . 3102

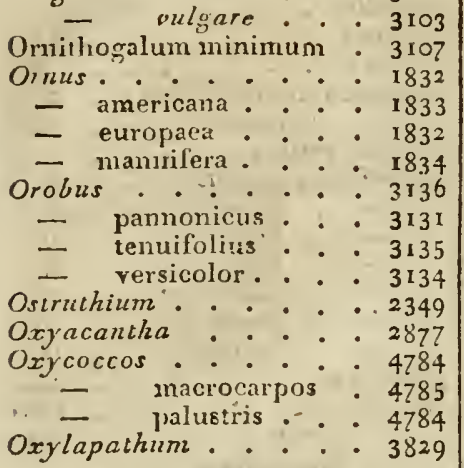

Padus . . . . . 3493

Paconia .. : . . . 3153

Palmata. . . 3094.3095

Papaver ... . . 3171

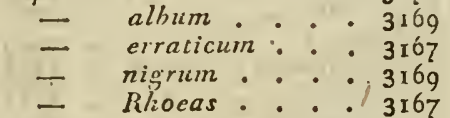

Paralysis . . . $347^{2} 3475$

Parietaria . . . . 3172

Paris . . . . . 3176

Parthenium . . . . 3545

Passulae majores . . 4989

Pastinaca . . : : 3179

Patientia. . . . . 3830

Paulinia aurea . . . . 2454

Pedicularis aquatica : 3182

Penthaphyllum : . . 344I

- aquaticum

Peragua . . . . . 4904

Perfoliata . . . . 809

Persica . . . . . 237

Persicaria . . . . 3373

Pes anserinus secisndus: 3372

- Cali.. . . . . 2047

- columbi ... . . 1996

Petasites . : . . . 4736

Petroselinum $\because \quad . \quad 363$

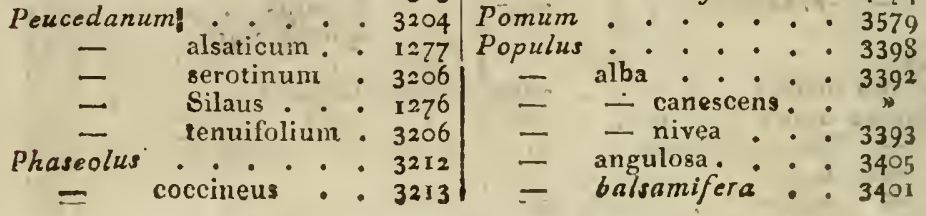

Phellandrium aquaticum. 3034

Philaidelphus . . . 3226

Phlox oborata. . . . 3247

Phu. 'stolonifera . . : . . 479

- ponticum . . . .

Phytolacica. . . . 3271

Pilosella . . . . . 2397

Pimpinella alba . . . 3279

- Anisum : $: 4315$

- italica . . 396ó

- $\quad$ minor.: $\cdot$ nigra $^{-} \cdot 3278$

- nostras.. 3279

Pinea, Pineoli . . . 3288

Pinus . . . . . . 3283

- Abies . . . 3302

- americana . . 3304

- canadensis... 3305

- laricina. . . 3300

- Larix nigra . . .

- laxa.... 3305

- mariana. . . 3306

- pendula. . . 3300

- Picea. . . . 3307

- tenuifolia . . 3286

- virginiana . . 3291

Planera Gmelini . . 3312

Plantago acuta . . • $333 \pi$

- aegyitiaca. . 3342

- aquatica... I1s

- exigua. . . 3344

- latifolia . . 3313

- major . . :

- vulgaris - : 3313

Platanus orientalis acerifolia 3349

- - elongata 3347

Polium monianum 3348

Pollichia Galeobdolon : 4553

Polygala amara. . . 3363

Polyoonaumlgaris - $\cdot 3364$

Polygonum . . . . 3388

- amphibium. $337^{\circ}$

- cocciferum. 4074

Pomim . . . . . 3579

1277 Populus alba : *: $: 3398$

${ }_{32} 27^{6}-\quad \doteqdot$ canescens -

$\begin{aligned} & 3212 \\ & 3213\end{aligned}-$ angulosa $:$ balsamifera $: 3405$ 
Populus betulifolit . . 3400/Quercus nigra . . . 3595

- canadensis. - 3403 - obtusiloba . . 3606

- cordifolia . . 3404

- carolinensis . 3399.3403

- heterophylla : 3405

- italica. . . . 3399

- latifolia... 3402

- major.... . 3393

- nigra . . . 3400

- tremuloides: - $\mathbf{3 3 9 5}$

Portulaca . . . . 3406

Potentilla . . . . 3441

Prasium . . . . . 2756

Prinulala veris . . . . 3472

Prosopis . . . . 406. 407

Pruna Damascena * . 3516

Prunella . . . . . 3490

Prunus . . . . . 3516

- domestica myrobalan. 3517

$R_{\text {anunculus acris . } 3^{642}}$

- Phellos sylvatica 3585

- Prinus acuminata 3591

- - monticola 3590

- $\quad$ racemosa palustris 3589

- Lobur lanuginosa 3607

- rubra . . . 3595

- sessiliflora.. . 3607

- sessilis . . . 3599

- tinctoria angulosa 3595

- - sinuosa. 3596

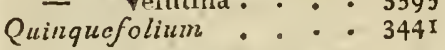

- albus . . 293

- bulbosus : 3640

Pscudacorus. . . . . 2496

Psgdium . . . . . . 3535

Psyllium . . . . . . 3346

Piarmica. . . . . . 26

Pulegium . . . . . 2854

Pulinonaria gallica • - 2213

\begin{tabular}{l} 
Pulsacilla nigricans : $\quad 3525$ \\
\hline
\end{tabular} vulgaris . . 3534

Pyrethrum fruticulosum - 3547

— ptarmicaefolium $\begin{array}{r}25 \\ \text { - verum. . } 330\end{array}$

Pjrola. . . : : 3556

- media . . 3557

- rotundifolia . . .

Pyrus apetala . . . 3583

- arbutifolia nigra. - $357^{\circ}$

- Chamaemespilus. . 2883

- coronaria. . . 3575

- Cydonia . : I468

- japonica sinensis: .1469

- irregularis : : 3564

- montis Sinai . . 3574

- órientalis . . :

- pinnatifida.: : 4435

- rotundifolia . . 3557

- sinensis. . . . 1469

- sylvestris . . : 3562

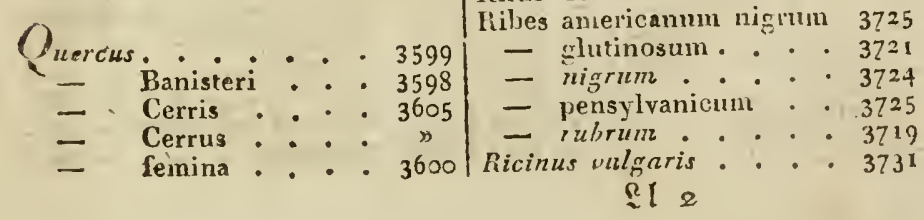

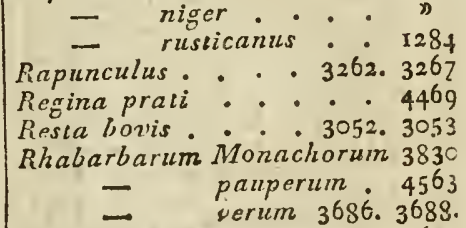

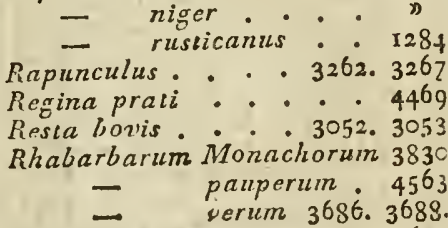

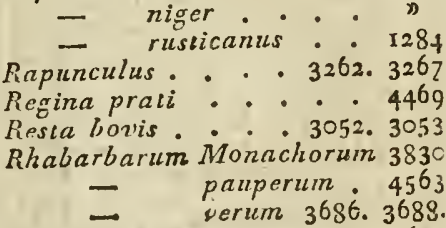

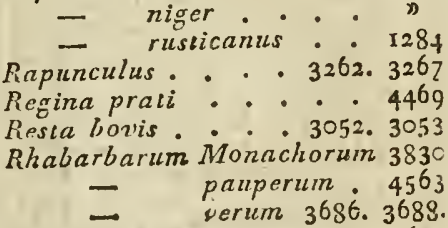

Rapa . ..... 767

Raphanus hortensis . : 3656

Phamnoides florifera 369 I. 3699

Rhamnus catharticus: : 3674

— Paliurus . . 3160

- volubilis . . 5022

- Zizyphus.. 5023

Rhaponticum ierum. • 3689

Rhenm . 3686.3688.3690. 369 r

Rloodia . . . . . 4 4 $3 \mathrm{I}$

Thlodiola rosea. . . . v

Rhodinm lignumz . . . 1919

Rhododendrnm • . - 3705

Rlooeas fuscum $: 3696$

Rhoeas Cacodendron : * 3102

Hibes antericanmm nigrum 3725

- glutinosum . • . 3721

- nigrum •. • • 3724

- rubrume . . . 3719

RI 2 
Robinia ferox . . . 3738

- inemis . • 3733

Roriella, Ros Solis : : 3738

Rosa . . : . 3776

- aciphylla: : : $377^{2}$

- alpina: : 3760.3762

- altaica... . 3768

- austriaca . . . 3777

- bicolor .... 3779

- blanda . . . 3754

- brevistyla : ${ }_{\text {- }}$ - 3749

- campanulata.: 3776.3778

- carolina . : 3753. 3758

- chlorophylla. .3779

- collina. . 3749.3768

- corymbosa . . 3755

- damascena..: 3776

- dibracteata . . 3749

- divionensis . . 3776

- dumalis .... 3772

- eglanteria . . $\quad 3765$

- foecundissima . . 3757

- francofurtana : : 3782

- glaucophylla.. . 3765

- gracilis . . . . 3783

- hispida : : 3766

- horrida . . . 3748

- humilis . : : 3753

- hybrida $: \therefore 376 \mathrm{I}$

- kamchatica . : $: 3748$

- lagenaria : . . 3761

- lutea multiplex. . 3765

- mollis : . . . 3784

- Obllissima $::{ }^{3}$

- Pallásii : : : $375 \mathrm{I}$

- pallida . : $: 3776$

- pendula..: : 3762

- pendulina . : $376 \mathrm{r}$

- pensylvanica : : 3755

- pimpinellifolia . 376r. 3768

- polyphylla .. 3762

- pomifera..: 3783

- pygmaea . . . 376I

- pyrenaica . . . .

- Redoutea rubescens . 3754

- repens . . . . . 3750

- rubra..... 3777

- mpestris . . . 376I

- scandens .... . 3752

- scotica .... 3768

- stylosa :. . . 3749

- sylvestris.... 3772
Rosa tuguriorum . . . 3750

- virginiana . 3755. 3760

Rosmarinus . . • 3786

hortensis :

- sylvestris : 2533

Rubia cinctorum . . . 3788

Rubus caesins : : : 3796

- frulicosus. . . 3810

- idaeus . . 3805

- niger. . . . 3810

- procumbens . . 3804

- triphyllus . . 3799

Rudbeckia odorata : 3816

Rumex oppositifolia : 800

Rumex aquaticus . • $382 \mathrm{I}$

Rupertus . . . . . I 997

Ruscus. . . . . 3847

Ruca capraria.: : $187^{2}$

- hortensis ... 3849

- major : : : :

Sabina

Salab sa. • • . 2434

Salab, Salap, Salep 3087.3089

Salisburya Ginkgo . - 3859

Salicaria. - . . 2720

Salix alba 3860. 3869. 3873. 3877

- - vitellina .. 3869

- amygdalina. . 3863

- angustifolia * . 3890

- arbuscula - 3879.3890

- bigemmis . . 3874

- caroliniana . . $386 \mathrm{r}$

- caspica . . . 3872

- daphnoides. . . 3875

- depressa. . 3890. 3908

- fissa ..... 3893

- flavovirens . . . 386 r

- fragilis .. 3864.3874

- glauca..... 3904

- hastata . . . 3881

- incana $\cdot 3876$

- lavandulaefolia . "

- longirostris . . . 3892

- monandra . 3867.3868

- myrsinites . . 3881

- pendula . * 3864. 3873

- pentandra $\therefore 3861$

- phylicifolia . . 3899

- polymorpha . . 3908

- propendens . • 3870

- rosmarinifolia . . 3876

- rugosa . . . 3912

- stylaris ... 3881

- triandra androgyna. 3862

Salix viminalis . . $387 t$ 
Salix virescens . . . 3893 / Sedum ternatum : . , 4132

Salsola altissima . . . $1147 \rightarrow$ vermiculare. . 4141

- arenaria . . $245 \mathrm{I}$ Selinum Chabraei. . 2350

- hyssopifolia.. 2453 - Monnieri : 1275

- prostrata :. . 2450 - Ostruthium . 2349

- Scoparia . . 2452 \& palustre . . 2350

Salvia . . . . . 3928 Sempervivum . . . : 4154

- minor... - 3921 Senecio . . . . . 4164

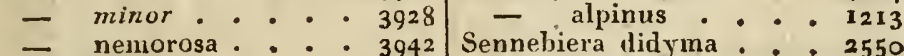

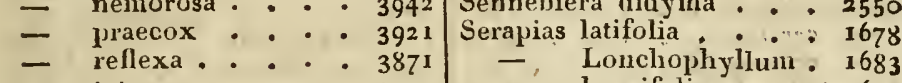

- tetragona... 3921 - longifulia . . 1679

Sambucus sulgaris * • : 3928 Serpyllum alpina • • . 2184

Sampsuchum : . - 3104 Serracula : : : : 4601

Sandaraca . . . - 2436 - alpina ... . 2184

Sangninalis - - - 3388 - heterophylla. 970

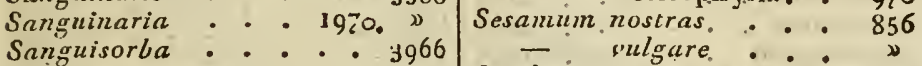

Srinicula. . . . . 3970 Seseli crecicum $\therefore .: 4628$

Santolina ..... 3971 - massiliense.1. . 4203

Saponaria . . - . - 3972 3975 Sida moncanum. ... 4628

- alla : : : 2702 - 275 suberosa : : : 4218

Sapindus chinera. • • 3975 Sideritis . •.ं • . . 4475

Sassafras chinensis • - 2454 Sigillum Sigricans .. . 4229

Sassafras • • • - 2526 Sigillmn Salomonis ... 1304

Sassaparilla . . . . 4360 Silene liispida . . . . 4273

Satureja. . . . 3984 - juncea .... . ע

Satyrium . . . ' 3077 - pilosa . . . \

- repens - . - 2978 Siler montanuin •.: 2488

Saxifraga alla. . - 40 I Silphimm solillaginoides: 800

- Aizoides.. 4068 Sinapis . . 4305.4307

- palmata . - 4016 Sisymbrium amphibium . 4322

- rnbra.: - 4468 Sisyrinchium angustifoliun 4322

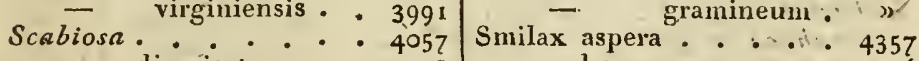

- divaricata:. 4038 - bona nox. - . 4356

Scammonium parviflora - . 4035 - glauca . . . 4360

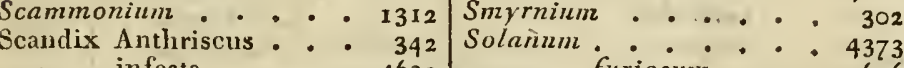

- infesta ... 4632

- nodosa $\cdot$ - 343

Scariola.: $: 245924$

Schollera Oxycoccos .4784

Sclareh •. . . . 3943

Scordium. - . . . $455 \mathrm{I}$

Scorzonera: . 4082.4085

Scrophularia aquatica. . 4104

- foelida. vulgaris: 4106

Sedum deficiens?. . 4132

- majus. . . . 4154

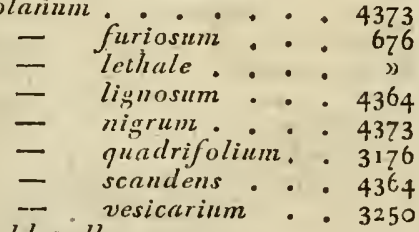

Soldanella . . . . 855

- Clusii .. . 4377

Sonchus asper. . . . 4413

- laevis . . . "

- chirurgorim • : 4337

Sorbus. . . . : 4432 
Sorbus Amelanohier . . 3578

- Aria. . . . 3565

- turminalis . . 3573

Spartium album . . 4442

- dispeimum $_{\text {scoparium }}:{ }^{4}$

Spathula foetida. . 2403

Spica . . . . . : 2527

- celtica .... 4797

Spina alba... . 2877

- cervina... 3674

Spinachia, Spinacia : : 4450

Spiraea altaica : . . 445 t

- chamaedrifolia. . 4461

- salicifolia latifolia 4454

Spondylium. paniculata 4452

Squamaria . : : 2494

Staphisagria . . I 1535

Statice Armeria * 447. 449

Stoechas citrina : . . 2044

Stramonium. . . . 1517

Succisa ......4 4036

Sumach . . . . 3709

Symphoria conglomerata - 4515

Symphoricarpos conglome-

rata ..... 4515

Symphytum .. .. . 4517

Syringa alba $\because \cdots \quad 3226$

- babylonica: : 4525

- laciniata. . . $\gg$

Tabacum . . 3001

Tacamahaca vulgaris . 3401

Tamariscus, Tamarix 4530.453 I

Tanacetum . . . 4535

- hortense. $69 \mathrm{~g}$

Taraxacum vulgare: : 4535

Taxus baccata : . 4537

- - minor. 4538

Telephium .... 4126

Tenoria fruticosa .. 818

Terebinthina argentinensis 3302

- canadensis $: 3303$
- $\quad$ communis $: 3283$
- larigna $: 3301$

Thlaspi . . .

Thymm prateox ... 4593

- horiensis: : 4609

- Serpyllum : 4603

- virginicus . 764

Thysselingis . : . 4 4rot

: : . 4613

Tilia . . : . . 46I5

- americana 4616.4620.4621

- canadensis . . 4656

- caroliniana . . . 4620

- europaea.:4615.4618

- grandifolia . .

- microphylla . 4619. 2

- platypihytlos :... 4615

- tomentosa : . $462 \mathrm{r}$

- triflora . : . . 4618

Tithymalum. . . . 5774

Tophea lucida. . . 446

Tordylium Anthriscus . . 4631

- latifolium $:{ }_{4633}$

Tormentilla. . . . 4634

Toxicodendrum volubile . 37 I5

Tragopogon ... 4639

Tribulus a quaticus . . 4645

Trifoliun album. . . 4676

- aquaticum . . 2855

- coeruleum . . 2813

- creticum . . 2817

- dentatum. . 2815

一 fibrinum . . 2855

- indicum .. 2807

- italicum . . 2816

- macrorhizum . 2810

- mauritanicum . 2812

- messanense . 2809

- officinale . 2808.2814

- ornithopodioides 2821

- paludosum . 2855

Trilliun erythrocarpum : $47^{13}$

Trinicas . . . . 4983

'Tulipifera arbor virginiana 2639

Tunica . . . . 1556

- sylvestris. . 1554

I'urritis minor . . . 389

- Tiaji . : : 395

Tussilago : : : : 4729

- hybrila : 4736

- venela. :

Tertianaria. . . - 4120

Tetragonia halimifolia • . 454I

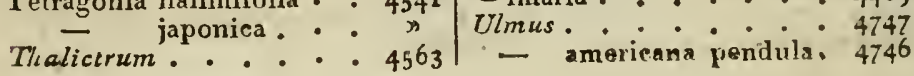




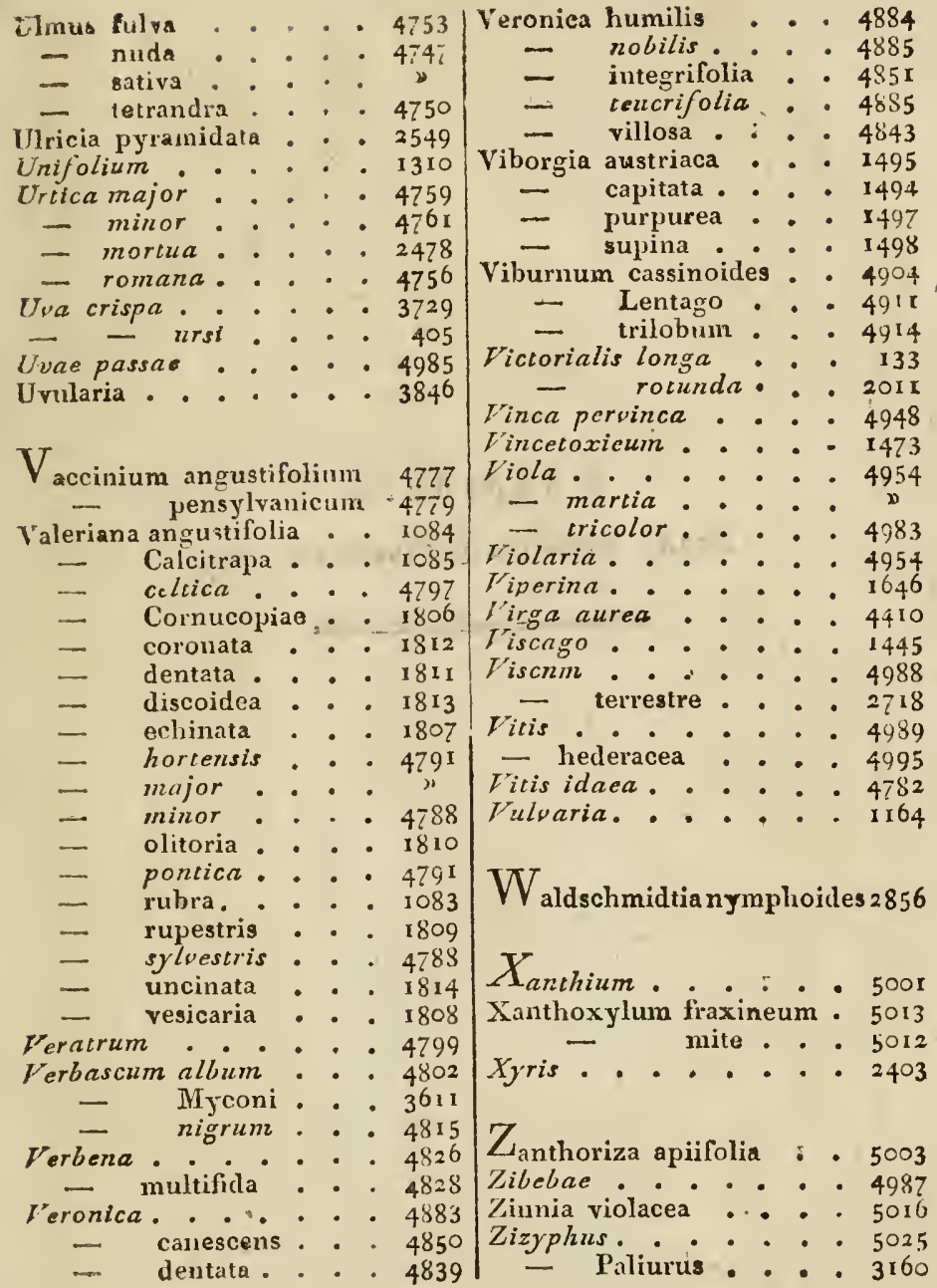




$$
\text { In a go e burg, }
$$

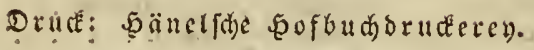


- $\quad+$

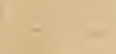

$\rightarrow$

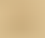

$-$

\section{,}
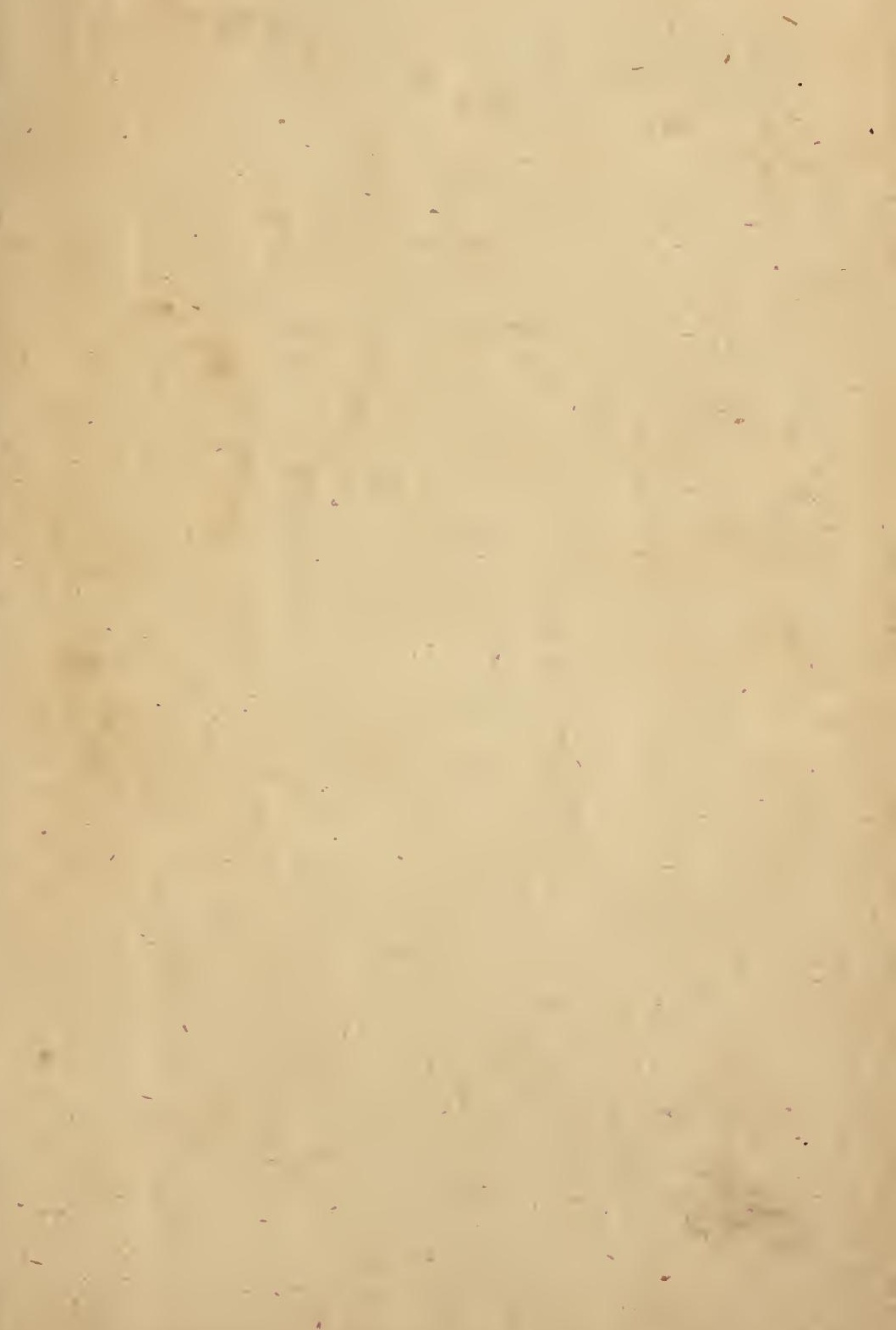

New York Botanical Garden Library

QK45 .K6 1824 Th 1

Koch Johann Friedr/Botanisches Handbuch

.

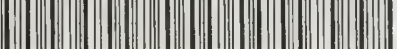

3 5185001018587 


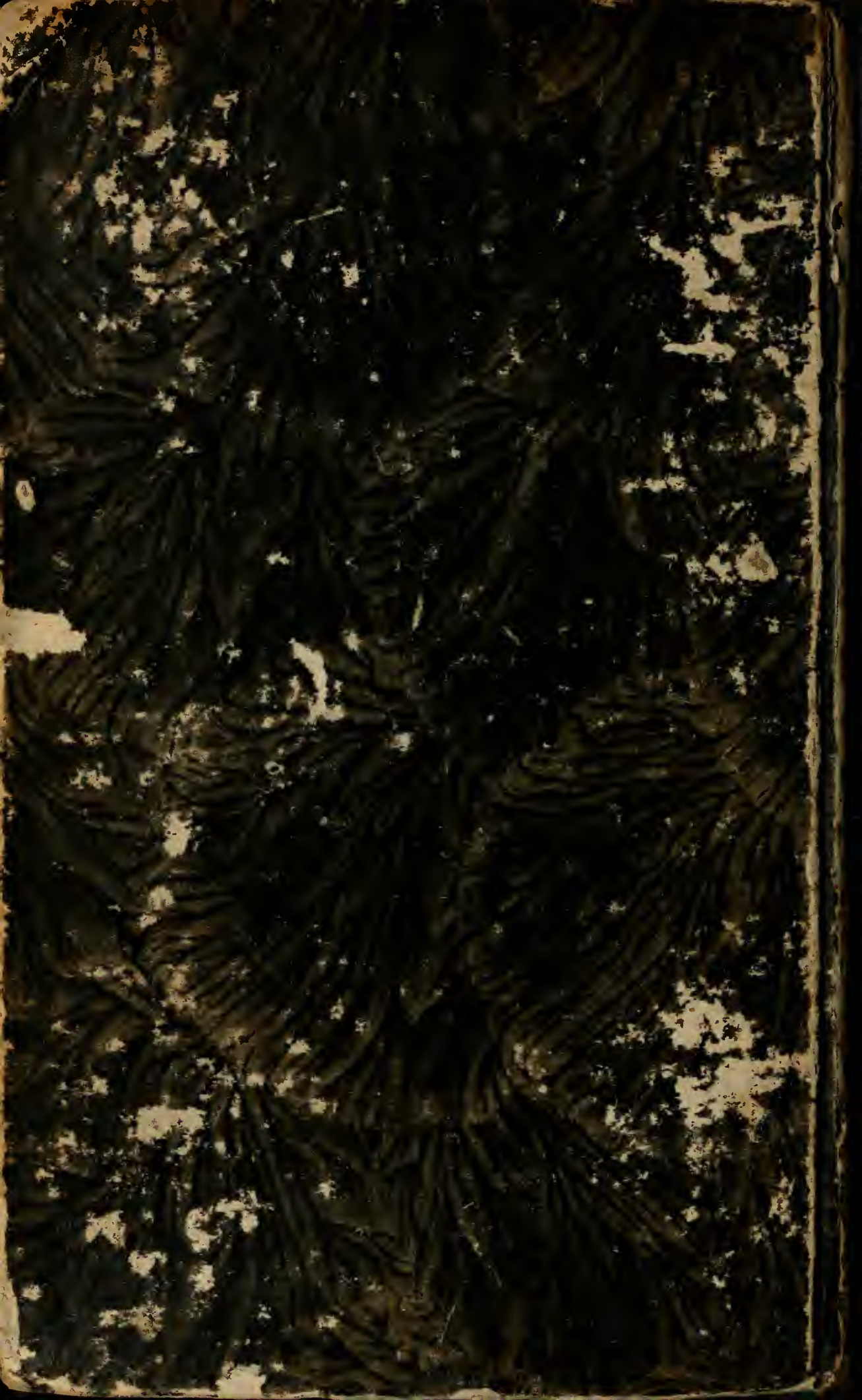

